\title{
Supplemental Information Related to Risk Assessment for the Off-Site Transportation of Low-Level Waste for the U.S. Department of Energy Waste Management Programmatic Environmental Impact Statement
}

by F.A. Monette, B.M. Biwer, D.J. LePoire, and S.Y. Chen

Environmental Assessment Division, Argonne National Laboratory, 9700 South Cass Avenue, Argonne, Illinois 60439

\section{DISCLAIMER}

This report was prepared as an account of work sponsored by an agency of the United States Government. Neither the United States Government nor any agency thereof, nor any of their employees, makes any warranty, express or implied, or assumes any legal liability or responsibility for the accuracy, completeness, or usefulness of any information, apparatus, product, or process disclosed, or represents that its use would not infringe privately owned rights. Reference herein to any specific commercial product, process, or service by trade name, trademark, manufacturer, or otherwise does not necessarily constitute or imply its endorsement, recommendation, or favoring by the United States Government or any agency thereof. The views and opinions of authors expressed herein do not necessarily state or reflect those of the United States Government or any agency thereof.

December 1996

Work sponsored by United States Department of Energy, Assistant Secretary for Environmental Management 
The WM PEIS assessment of LLW considers LLW generated from WM activities. A description of the LLW inventory, characteristics, and treatment options is presented in Goyette and Dolak (1996). This analysis does not include the updated LLW volumes that are presented in Appendix I of the WM PEIS. This report includes definitions of the LLW alternatives considered in the WM PEIS and presents data related to the inventory and to the physical and radiological characteristics of WM LLW that are used in the transportation assessment (Section 2). An overview of the risk assessment method is provided in Section 3. In addition, supplemental assumptions and parameters for the risk assessment calculations are provided (Section 4), and detailed results of the assessment are presented for each WM LLW alternative considered (Section 5). 


\section{A.3 WM LLW DECENTRALIZED ALTERNATIVE (CASE 2): HETEROGENEOUS SOLIDS}

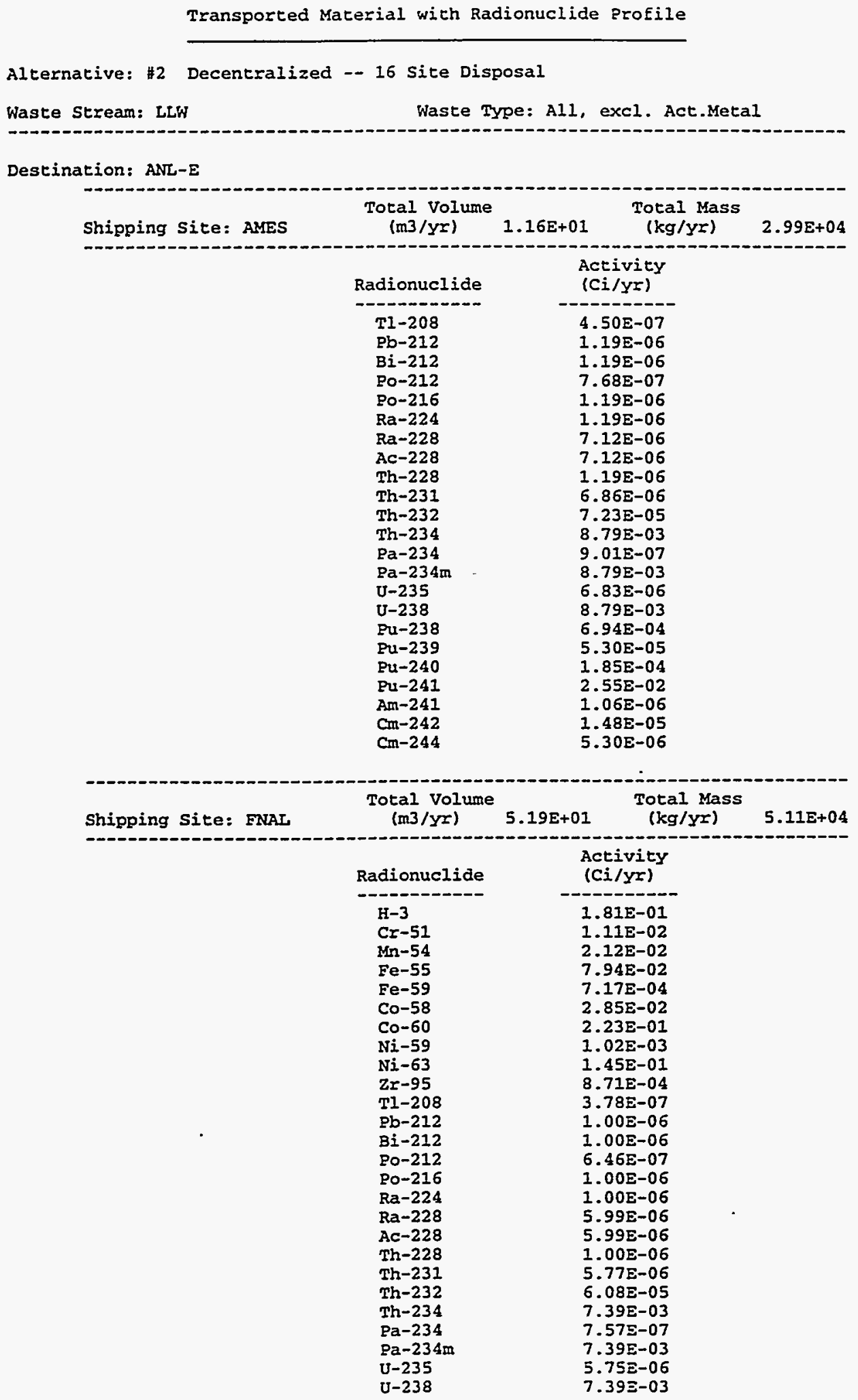


A-2I

Destination: INEL

\begin{tabular}{|c|c|c|c|}
\hline Shipping site: ANL-W & $\begin{array}{l}\text { Total Volume } \\
(\mathrm{m} 3 / y x)\end{array}$ & $\begin{array}{l}\text { Total Mass } \\
(\mathrm{kg} / \mathrm{yr})\end{array}$ & $2.12 E+05$ \\
\hline & $\begin{array}{l}\text { Radionuclide } \\
\mathrm{H}-3 \\
\mathrm{Cr}-51 \\
\mathrm{Mn}-54 \\
\mathrm{Fe}-55 \\
\mathrm{Fe}-59 \\
\mathrm{Co}-58 \\
\mathrm{Co}-60 \\
\mathrm{Ni}-59 \\
\mathrm{Ni}-63 \\
\mathrm{Sr}-90 \\
\mathrm{Y}-90 \\
\mathrm{Zr}-95 \\
\mathrm{Tc}-99 \\
\mathrm{Ru}-106 \\
\mathrm{Rh}-106 \\
\mathrm{Sb}-125 \\
\mathrm{Te}-125 \mathrm{~m} \\
\mathrm{Cs}-134 \\
\mathrm{Cs}-137 \\
\mathrm{Ba}-137 \mathrm{~m} \\
\mathrm{Ce}-144 \\
\mathrm{Pr}-144 \\
\mathrm{Pr}-144 \mathrm{~m} \\
\mathrm{Pm}-147 \\
\mathrm{Sm}-151 \\
\mathrm{Eu}-152 \\
\text { Eu-154 } \\
\mathrm{Eu}-155 \\
\mathrm{Pu}-238 \\
\mathrm{Pu}-239 \\
\mathrm{Pu}-240 \\
\mathrm{Pu}-241 \\
\mathrm{Am}-241 \\
\mathrm{Cm}-242 \\
\mathrm{Cm}-244\end{array}$ & 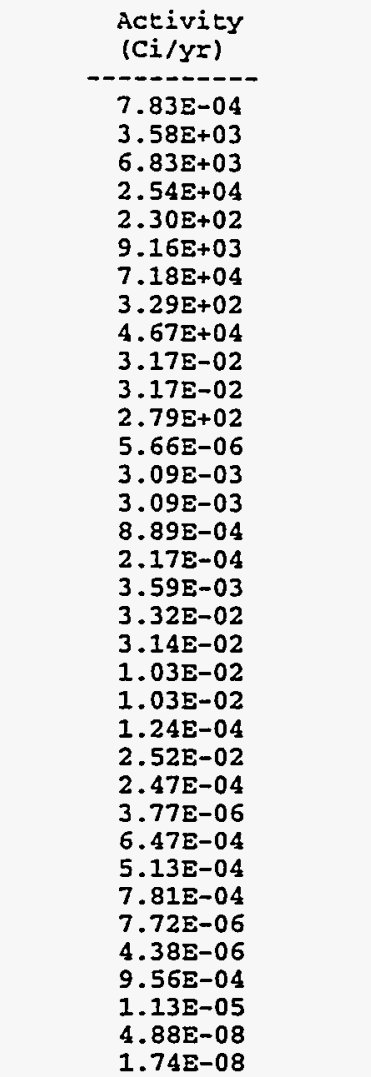 & \\
\hline
\end{tabular}




\begin{tabular}{|c|c|c|c|}
\hline Shipping site: LBL & $\begin{array}{l}\text { Toral Volume } \\
(\mathrm{m} 3 / \mathrm{yr})\end{array}$ & $\begin{array}{c}\text { Total Mass } \\
(\mathrm{kg} / \mathrm{yx})\end{array}$ & $4.51 E+04$ \\
\hline & $\begin{array}{l}\text { Radionuclide } \\
\mathrm{H}-3 \\
\mathrm{C}-14 \\
\mathrm{Mn}-54 \\
\mathrm{Co}-58 \\
\mathrm{Co}-60 \\
\mathrm{~S}-90 \\
\mathrm{Y}-90 \\
\mathrm{TC}-99 \\
\mathrm{Cs}-134 \\
\mathrm{Cs}-137 \\
\mathrm{Ba}-137 \mathrm{~m} \\
\mathrm{U}-238 \\
\mathrm{Cm}-244\end{array}$ & 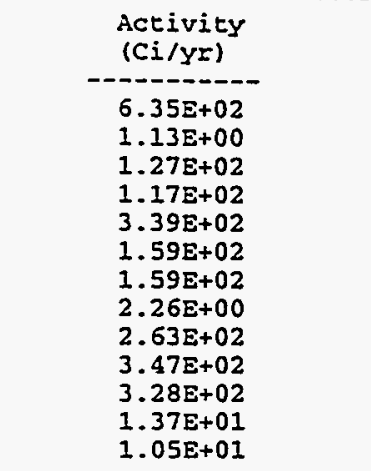 & \\
\hline Shipping Site: SLAC & $\begin{array}{l}\text { Total volume } \\
\text { (m3/yr) }\end{array}$ & $\begin{array}{cc}2.73 \mathrm{E}+02 & \begin{array}{c}\text { Total Mass } \\
(\mathrm{kg} / \mathrm{yr})\end{array}\end{array}$ & $7.45 \mathrm{E}+05$ \\
\hline & $\begin{array}{l}\text { Radionuclide } \\
\mathrm{Cr}-51 \\
\mathrm{Mn}-54 \\
\mathrm{Fe}-55 \\
\mathrm{Fe}-59 \\
\mathrm{Co}-58 \\
\mathrm{Co}-60 \\
\mathrm{Ni}-59 \\
\mathrm{Ni}-63 \\
\mathrm{Zr}-95\end{array}$ & $\begin{array}{c}\begin{array}{c}\text { Activity } \\
\text { (Ci/yr) }\end{array} \\
3.86 E-03 \\
7.36 \mathrm{E}-03 \\
2.74 \mathrm{E}-02 \\
2.48 \mathrm{E}-04 \\
9.88 \mathrm{E}-03 \\
7.74 \mathrm{E}-02 \\
3.54 \mathrm{E}-04 \\
5.04 \mathrm{E}-02 \\
3.01 \mathrm{E}-04\end{array}$ & \\
\hline Shipping Site: SNLL & $\begin{array}{l}\text { Total Volume } \\
(\mathrm{m} 3 / \mathrm{yr})\end{array}$ & $\begin{array}{cc}3.68 \mathrm{E}+01 & \text { Total Mass } \\
(\mathrm{kg} / \mathrm{yr})\end{array}$ & $9.73 E+04$ \\
\hline & \begin{tabular}{l} 
Radionuclide \\
\hdashline $\mathrm{H}-3$ \\
$\mathrm{Fe}-55$ \\
$\mathrm{Co}-60$ \\
$\mathrm{~T} 1-208$ \\
$\mathrm{~Pb}-212$ \\
$\mathrm{Bi}-212$ \\
$\mathrm{PO}-212$ \\
$\mathrm{Po}-216$ \\
$\mathrm{Ra}-224$ \\
$\mathrm{Ra}-228$ \\
$\mathrm{AC}-228$ \\
$\mathrm{Th}-228$ \\
$\mathrm{Th}-231$ \\
$\mathrm{Th}-232$ \\
$\mathrm{Th}-234$ \\
$\mathrm{~Pa}-234$ \\
$\mathrm{~Pa}-234 \mathrm{~m}$ \\
$\mathrm{U}-235$ \\
$\mathrm{U}-238$
\end{tabular} & \begin{tabular}{c}
$\begin{array}{c}\text { Activity } \\
(C i / y r)\end{array}$ \\
\hdashline $2.78 E+04$ \\
$3.15 E-01$ \\
$3.15 E-01$ \\
$6.40 E-05$ \\
$1.69 E-04$ \\
$1.69 E-04$ \\
$1.09 E-04$ \\
$1.69 E-04$ \\
$1.69 E-04$ \\
$1.01 E-03$ \\
$1.01 E-03$ \\
$1.69 E-04$ \\
$9.75 E-04$ \\
$1.02 E-02$ \\
$1.25 E+00$ \\
$1.28 E-04$ \\
$1.25 E+00$ \\
$9.71 E-04$ \\
$1.25 E+00$
\end{tabular} & v \\
\hline
\end{tabular}


Destination: ORNL

\begin{tabular}{|c|c|c|c|}
\hline Shipping Site: $\mathrm{k}-25$ & $\begin{array}{l}\text { Total Volume } \\
(\mathrm{m} 3 / \mathrm{Yr})\end{array}$ & $\begin{array}{c}\text { Total Mass } \\
(\mathrm{kg} / \mathrm{yr})\end{array}$ & $4.67 E+07$ \\
\hline & $\begin{array}{l}\text { Radionuclide } \\
\mathrm{T} 1-208 \\
\mathrm{~Pb}-212 \\
\mathrm{Bi}-212 \\
\mathrm{Po-212} \\
\mathrm{Po-216} \\
\mathrm{Ra}-224 \\
\mathrm{Ra}-228 \\
\mathrm{Ac}-228 \\
\mathrm{Th}-228 \\
\mathrm{Th}-231 \\
\mathrm{Th}-232 \\
\mathrm{Th}-234 \\
\mathrm{~Pa}-234 \\
\mathrm{~Pa}-234 \mathrm{~m} \\
\mathrm{U}-235 \\
\mathrm{U}-238\end{array}$ & \multicolumn{2}{|l|}{$\begin{array}{l}1.06 \mathrm{E}-04 \\
2.82 \mathrm{E}-04 \\
2.82 \mathrm{E}-04 \\
1.82 \mathrm{E}-04 \\
2.82 \mathrm{E}-04 \\
2.82 \mathrm{E}-04 \\
1.68 \mathrm{E}-03 \\
1.68 \mathrm{E}-03 \\
2.82 \mathrm{E}-04 \\
1.62 \mathrm{E}-03 \\
1.71 \mathrm{E}-02 \\
2.08 \mathrm{E}+00 \\
2.13 \mathrm{E}-04 \\
2.08 \mathrm{E}+00 \\
1.62 \mathrm{E}-03 \\
2.08 \mathrm{E}+00\end{array}$} \\
\hline \multirow[t]{2}{*}{ Shipping Site: ORISE } & $\begin{array}{l}\text { Total volume } \\
(\mathrm{m} 3 / \mathrm{yr})\end{array}$ & $6.07 \mathrm{E}+01 \quad \begin{array}{c}\text { Total Mass } \\
(\mathrm{kg} / \mathrm{yr})\end{array}$ & $7.36 \mathrm{E}+04$ \\
\hline & \begin{tabular}{l} 
Radionuclide \\
\hdashline $\mathrm{H}-3$ \\
$\mathrm{C}-14$ \\
$\mathrm{Mn}-54$ \\
$\mathrm{Co}-58$ \\
$\mathrm{Co}-60$ \\
$\mathrm{Sr}-90$ \\
$\mathrm{Y}-90$ \\
$\mathrm{Tc}-99$ \\
$\mathrm{Cs}-134$ \\
$\mathrm{Cs}-137$ \\
$\mathrm{Ba}-137 \mathrm{~m}$ \\
$\mathrm{~T} 1-208$ \\
$\mathrm{~Pb}-212$ \\
$\mathrm{Bi}-212$ \\
$\mathrm{Po}-212$ \\
$\mathrm{Po}-216$ \\
$\mathrm{Ra}-224$ \\
$\mathrm{Ra}-228$ \\
$\mathrm{Ac}-228$ \\
$\mathrm{Th}-228$ \\
$\mathrm{Th}-231$ \\
$\mathrm{Th}-232$ \\
$\mathrm{Th}-234$ \\
$\mathrm{~Pa}-234$ \\
$\mathrm{~Pa}-234 \mathrm{~m}$ \\
$\mathrm{U}-235$ \\
$\mathrm{U}-238$
\end{tabular} & \multicolumn{2}{|l|}{$\begin{array}{l}2.00 \mathrm{E}-02 \\
1.19 \mathrm{E}-05 \\
1.34 \mathrm{E}-03 \\
1.24 \mathrm{E}-03 \\
3.59 \mathrm{E}-03 \\
1.69 \mathrm{E}-03 \\
1.69 \mathrm{E}-03 \\
2.39 \mathrm{E}-05 \\
2.79 \mathrm{E}-03 \\
3.68 \mathrm{E}-03 \\
3.48 \mathrm{E}-03 \\
3.39 \mathrm{E}-07 \\
8.98 \mathrm{E}-07 \\
8.98 \mathrm{E}-07 \\
5.78 \mathrm{E}-07 \\
8.98 \mathrm{E}-07 \\
8.98 \mathrm{E}-07 \\
5.36 \mathrm{E}-06 \\
5.36 \mathrm{E}-06 \\
8.98 \mathrm{E}-07 \\
5.16 \mathrm{E}-06 \\
5.44 \mathrm{E}-05 \\
6.62 \mathrm{E}-03 \\
6.78 \mathrm{E}-07 \\
6.62 \mathrm{E}-03 \\
5.14 \mathrm{E}-06 \\
6.77 \mathrm{E}-03\end{array}$} \\
\hline \multirow[t]{2}{*}{ Shipping Site: $Y-12$} & $\begin{array}{l}\text { Total Volume } \\
(\mathrm{m} 3 / \mathrm{y} x)\end{array}$ & $\begin{array}{l}\text { Total Mass } \\
(\mathrm{kg} / \mathrm{yr})\end{array}$ & $\begin{array}{r}8.27 E+07 \\
-\end{array}$ \\
\hline & $\begin{array}{l}\text { Radionuclide } \\
\mathrm{Tl}-208 \\
\mathrm{~Pb}-212 \\
\mathrm{Bi}-212 \\
\mathrm{Po}-212 \\
\mathrm{Po}-216 \\
\mathrm{Ra}-224 \\
\mathrm{Ra}-228 \\
\mathrm{Ac}-228 \\
\mathrm{Th}-228 \\
\mathrm{Th}-231 \\
\mathrm{Th}-232 \\
\mathrm{Th}-234 \\
\mathrm{~Pa}-234 \\
\mathrm{~Pa}-234 \mathrm{~m} \\
\mathrm{U}-235 \\
\mathrm{U}-238\end{array}$ & $\begin{array}{c}\begin{array}{c}\text { Activity } \\
(\mathrm{Ci} / \mathrm{y})\end{array} \\
6.80 \mathrm{E}-05 \\
1.80 \mathrm{E}-04 \\
1.80 \mathrm{E}-04 \\
1.16 \mathrm{E}-04 \\
1.80 \mathrm{E}-04 \\
1.80 \mathrm{E}-04 \\
1.07 \mathrm{E}-03 \\
1.07 \mathrm{E}-03 \\
1.80 \mathrm{E}-04 \\
1.03 \mathrm{E}-03 \\
1.09 \mathrm{E}-02 \\
1.32 \mathrm{E}+00 \\
1.36 \mathrm{E}-04 \\
1.32 \mathrm{E}+00 \\
1.03 \mathrm{E}-03 \\
1.32 \mathrm{E}+00\end{array}$ & \\
\hline
\end{tabular}




\begin{tabular}{|c|c|c|c|}
\hline Shipping Sice: BAPL & $\begin{array}{l}\text { Total Volume } \\
(\mathrm{m} 3 / \mathrm{y} z)\end{array}$ & $\begin{array}{l}\text { Total Mass } \\
\text { (kg/yr) }\end{array}$ & $1.08 \mathrm{E}+06$ \\
\hline & 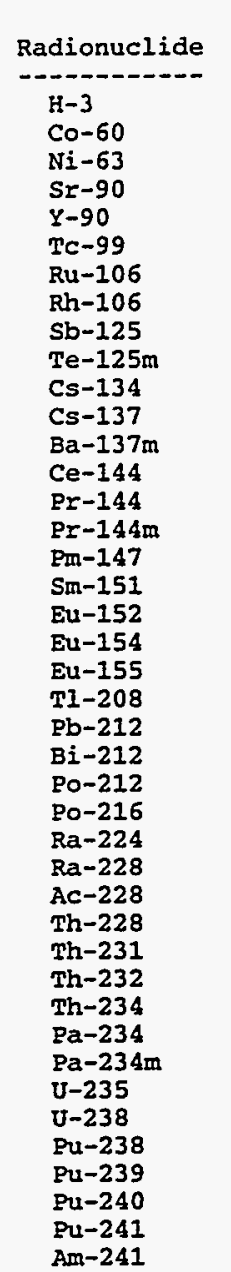 & $\begin{array}{c}\text { Activity } \\
(C i / Y \mathrm{t}) \\
-51 \mathrm{E}-03 \\
2.51 \mathrm{E}-03 \\
1.19 \mathrm{E}-02 \\
2.38 \mathrm{E}-02 \\
2.11 \mathrm{E}+00 \\
2.11 \mathrm{E}+00 \\
3.77 \mathrm{E}-04 \\
2.06 \mathrm{E}-01 \\
2.06 \mathrm{E}-01 \\
5.93 \mathrm{E}-02 \\
1.44 \mathrm{E}-02 \\
2.40 \mathrm{E}-01 \\
2.21 \mathrm{E}+00 \\
2.09 \mathrm{E}+00 \\
6.91 \mathrm{E}-01 \\
6.91 \mathrm{E}-01 \\
8.31 \mathrm{E}-03 \\
1.68 \mathrm{E}+00 \\
1.65 \mathrm{E}-02 \\
2.51 \mathrm{E}-04 \\
4.32 \mathrm{E}-02 \\
3.42 \mathrm{E}-02 \\
3.80 \mathrm{E}-06 \\
1.00 \mathrm{E}-05 \\
1.00 \mathrm{E}-05 \\
6.49 \mathrm{E}-06 \\
1.00 \mathrm{E}-05 \\
1.00 \mathrm{E}-05 \\
6.02 \mathrm{E}-05 \\
6.02 \mathrm{E}-05 \\
1.00 \mathrm{E}-05 \\
5.80 \mathrm{E}-05 \\
6.11 \mathrm{E}-04 \\
7.43 \mathrm{E}-02 \\
7.61 \mathrm{E}-06 \\
7.43 \mathrm{E}-02 \\
5.77 \mathrm{E}-05 \\
7.43 \mathrm{E}-02 \\
5.20 \mathrm{E}-02 \\
5.03 \mathrm{E}-04 \\
2.51 \mathrm{E}-04 \\
5.81 \mathrm{E}-02 \\
7.55 \mathrm{E}-04\end{array}$ & \\
\hline
\end{tabular}


A-25

\begin{tabular}{|c|c|c|c|}
\hline Shipping Sice: KAPL & $\begin{array}{l}\text { Total volume } \\
\left(\mathrm{m} 3 / Y^{\mathrm{r}}\right)\end{array}$ & $\begin{array}{l}\text { Total Mass } \\
\text { (kg/Yr) }\end{array}$ & $5.36 E+05$ \\
\hline & \begin{tabular}{l} 
Radionuclide \\
\hdashline------- \\
C-14 \\
Cr-51 \\
Mn-54 \\
Fe-55 \\
Fe-59 \\
Co-58 \\
Co-50 \\
Ni-59 \\
Ni-63 \\
Sr-90 \\
Y-90 \\
Zr-95 \\
Tc-99 \\
Ru-106 \\
Rh-106 \\
Sb-125 \\
Te-125m \\
Cs-134 \\
Cs-137 \\
Ba-137m \\
Ce-144 \\
Pr-144 \\
Pr-144m \\
Pm-147 \\
Sm-151 \\
Eu-152 \\
Eu-154 \\
Eu-155 \\
I $1-208$ \\
Pb-212 \\
Bi-212 \\
Po-212 \\
Po-216 \\
Ra-224 \\
Ra-228 \\
Ac-228 \\
Th-228 \\
Th-231 \\
Th-232 \\
Th-234 \\
Pa-234 \\
Pa-234m \\
U-235 \\
U-238 \\
Pu-238 \\
Pu-239 \\
Pu-240 \\
Pu-241 \\
Am-241
\end{tabular} & 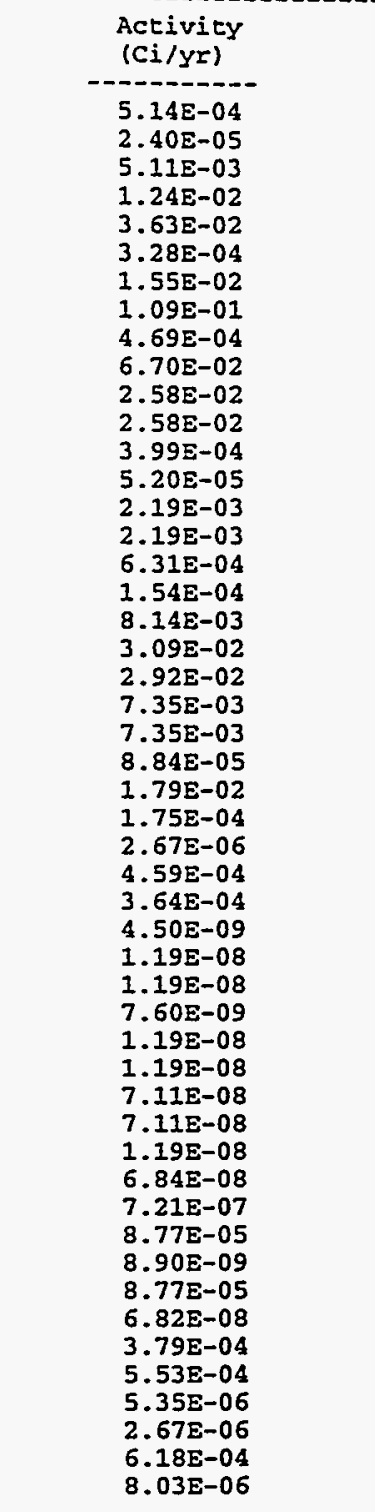 & \\
\hline Shipping Sice: MOUND & $\begin{array}{l}\text { Total Volume } \\
\text { (m3/yx) }\end{array}$ & $\begin{array}{l}\text { Total Mass } \\
\text { (kg/Yr) }\end{array}$ & $9.67 \mathrm{E}+06$ \\
\hline & $\begin{array}{l}\text { Radionuclide } \\
\mathrm{H}-3 \\
\mathrm{Pu}-238 \\
\mathrm{Pu}-239 \\
\mathrm{Pu}-240 \\
\mathrm{Pu}-241 \\
\mathrm{Am}-241 \\
\mathrm{Cm}-242 \\
\mathrm{Cm}-244\end{array}$ & $\begin{array}{c}\begin{array}{c}\text { Activity } \\
\text { (Ci/YI) }\end{array} \\
-1.34 \mathrm{E}+04 \\
1.60 \mathrm{E}-01 \\
1.22 \mathrm{E}-02 \\
4.28 \mathrm{E}-02 \\
5.90 \mathrm{E}+00 \\
2.44 \mathrm{E}-04 \\
3.42 \mathrm{E}-03 \\
1.22 \mathrm{E}-03\end{array}$ & \\
\hline
\end{tabular}




\begin{tabular}{|c|c|c|c|}
\hline Shipping Site: PPPL & $\begin{array}{l}\text { Total Volume } \\
(\mathrm{m} 3 / \mathrm{yr})\end{array}$ & $\begin{array}{l}\text { Total Mass } \\
(\mathrm{kg} / \mathrm{Y} r)\end{array}$ & $6.10 \mathrm{E}+03$ \\
\hline & $\begin{array}{l}\text { Radionuclide } \\
\mathrm{H}-3 \\
\mathrm{Cr}-51 \\
\mathrm{Mn}-54 \\
\mathrm{Fe}-55 \\
\mathrm{Fe}-59 \\
\mathrm{Co}-58 \\
\mathrm{Co}-60 \\
\mathrm{Ni}-59 \\
\mathrm{Ni}-63 \\
\mathrm{Zr}-95\end{array}$ & $\begin{array}{c}\begin{array}{l}\text { Activity } \\
\text { (Ci/yr) }\end{array} \\
-1.01 \mathrm{E}-01 \\
2.01 \\
8.50 \mathrm{E}-04 \\
1.61 \mathrm{E}-03 \\
6.04 \mathrm{E}-03 \\
5.46 \mathrm{E}-05 \\
2.17 \mathrm{E}-03 \\
1.70 \mathrm{E}-02 \\
7.80 \mathrm{E}-05 \\
1.10 \mathrm{E}-02 \\
6.63 \mathrm{E}-05\end{array}$ & \\
\hline hipping Site: RMI & $\begin{array}{l}\text { Total volume } \\
\text { (m3/yr) }\end{array}$ & $\begin{array}{l}\text { Total Mass } \\
(\mathrm{kg} / \mathrm{Y}=)\end{array}$ & $1.47 \mathrm{E}+07$ \\
\hline
\end{tabular}

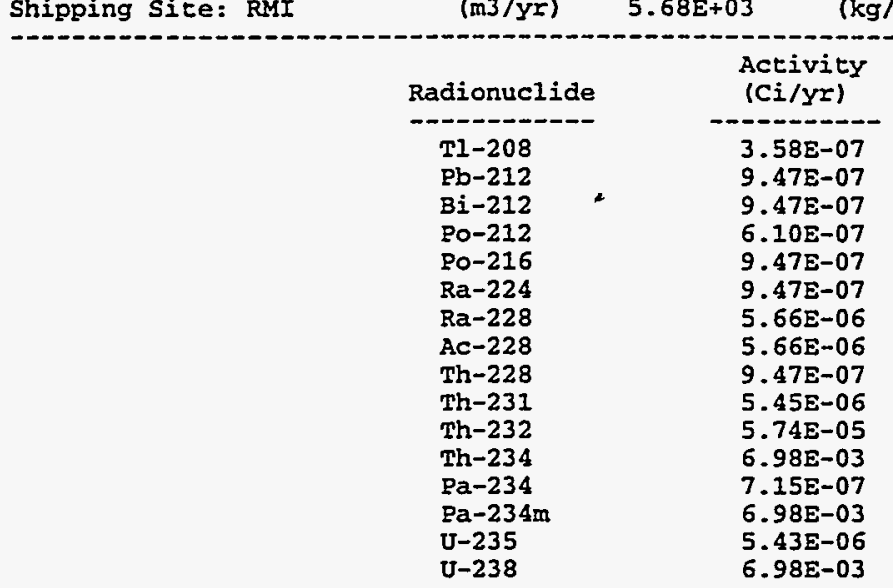

Destination: Paducah

\begin{tabular}{|c|c|c|c|}
\hline Shipping Site: KCP & $\begin{array}{c}\text { Total Volume } \\
(\mathrm{m} 3 / \mathrm{yr})\end{array}$ & $\begin{array}{c}\text { Total Mass } \\
(\mathrm{kg} / \mathrm{Yr})\end{array}$ & $7.23 E+03$ \\
\hline & Radionuclide & $\begin{array}{l}\text { Activity } \\
(\text { Ci/yx) }\end{array}$ & \\
\hline & $\begin{array}{l}\mathrm{H}-3 \\
\mathrm{C}-14 \\
\mathrm{Mn}-54 \\
\mathrm{Co}-58 \\
\mathrm{Co}-60 \\
\mathrm{Sr}-90 \\
\mathrm{Y}-90 \\
\mathrm{TC}-99 \\
\mathrm{Cs}-134 \\
\mathrm{Cs}-137 \\
\mathrm{Ba}-137 \mathrm{~m} \\
\mathrm{U}-238\end{array}$ & $\begin{array}{l}2.70 \mathrm{E}-02 \\
1.44 \mathrm{E}-04 \\
1.62 \mathrm{E}-02 \\
1.50 \mathrm{E}-02 \\
4.34 \mathrm{E}-02 \\
2.04 \mathrm{E}-02 \\
2.04 \mathrm{E}-02 \\
2.89 \mathrm{E}-04 \\
3.37 \mathrm{E}-02 \\
4.44 \mathrm{E}-02 \\
4.20 \mathrm{E}-02 \\
1.76 \mathrm{E}-03\end{array}$ & \\
\hline
\end{tabular}

Destination: SNLA

\begin{tabular}{|c|c|c|c|}
\hline Shipping Site: ITRI & $\begin{array}{l}\text { Total Volume } \\
(\mathrm{m} 3 / \mathrm{y} x)\end{array}$ & $\begin{array}{c}\text { Total Mass } \\
(\mathrm{kg} / \mathrm{yr})\end{array}$ & $1.64 E+05$ \\
\hline & \multicolumn{3}{|c|}{$\begin{array}{l}\text { Activity } \\
(\mathrm{C} i / \mathrm{Y})\end{array}$} \\
\hline & $\begin{array}{l}\mathrm{H}-3 \\
C-14 \\
M n-54 \\
C o-58 \\
\mathrm{C}-60 \\
S z-90 \\
Y-90 \\
\mathrm{~T}-99 \\
\mathrm{C}-134 \\
\mathrm{C}-137 \\
\mathrm{Ba}-137 \mathrm{~m} \\
\mathrm{U}-238\end{array}$ & $\begin{array}{l}6.17 \mathrm{E}+00 \\
3.03 \mathrm{E}-01 \\
3.41 \mathrm{E}+01 \\
3.15 \mathrm{E}+01 \\
9.12 \mathrm{E}+01 \\
4.28 \mathrm{E}+01 \\
4.28 \mathrm{E}+01 \\
6.07 \mathrm{E}-01 \\
7.07 \mathrm{E}+01 \\
9.33 \mathrm{E}+01 \\
8.82 \mathrm{E}+01 \\
3.69 \mathrm{E}+00\end{array}$ & \\
\hline
\end{tabular}


Destination: SRS

\begin{tabular}{|c|c|c|c|}
\hline Shipping Site: Pinellas & $\begin{array}{l}\text { Total Volume } \\
(\mathrm{m} 3 / \mathrm{yr})\end{array}$ & $\begin{array}{c}\text { Tocal Mass } \\
(\mathrm{kg} / \mathrm{yr})\end{array}$ & $1.91 E+04$ \\
\hline & Radionuclide & $\begin{array}{c}\begin{array}{c}\text { Activity } \\
\text { (Ci/yr) }\end{array} \\
1.99 \mathrm{E}+04\end{array}$ & \\
\hline
\end{tabular}




\section{A.4 WM LLW DECENTRALIZED ALTERNATIVE (CASE 2): ACTIVATED METALS}

Transported Material with Radionuclide Profile

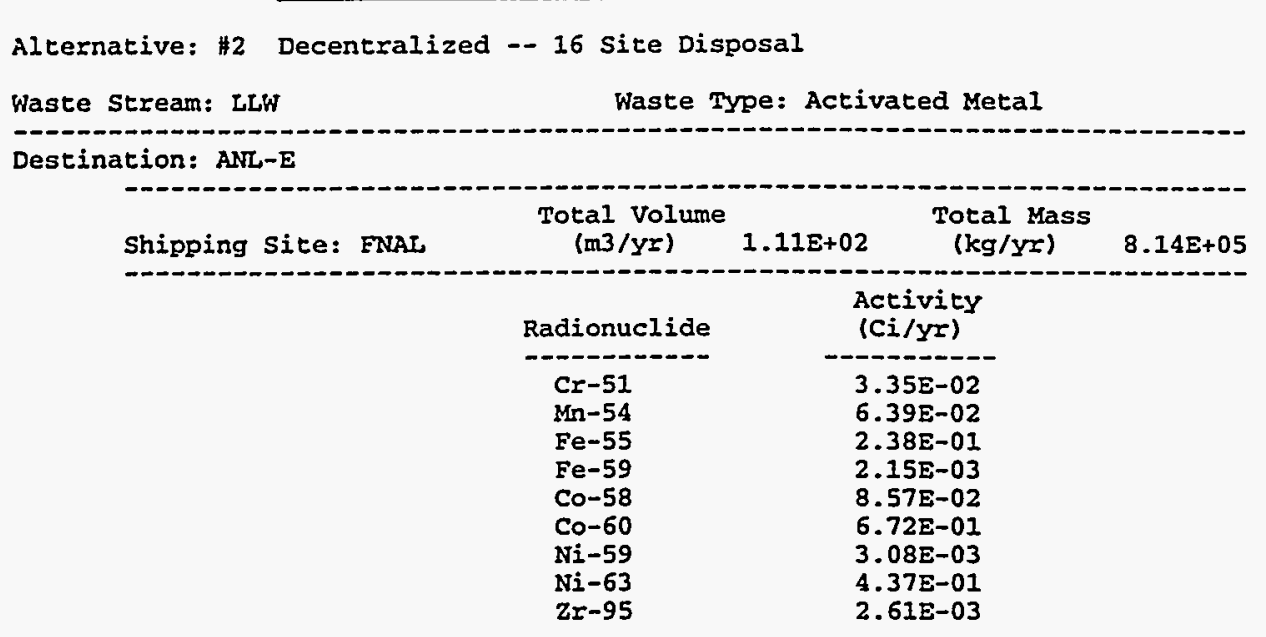


Destination: INEL

\begin{tabular}{|c|c|c|c|}
\hline Shipping Site: ANL-W & $\begin{array}{l}\text { Toral Volume } \\
(\mathrm{m} 3 / \mathrm{y})\end{array}$ & $\begin{array}{c}\text { Total Mass } \\
(\mathrm{kg} / \mathrm{y} x)\end{array}$ & $1.64 E+05$ \\
\hline & $\begin{array}{l}\text { Radionuclide } \\
\mathrm{H}-3 \\
\mathrm{Cr}-51 \\
\mathrm{Mn}-54 \\
\mathrm{Fe}-55 \\
\mathrm{Fe}-59 \\
\mathrm{Co}-58 \\
\mathrm{Co}-60 \\
\mathrm{Ni}-59 \\
\mathrm{Ni}-63 \\
\mathrm{Sr}-90 \\
\mathrm{Y}-90 \\
\mathrm{Zr}-95 \\
\mathrm{Tc}-99 \\
\mathrm{Ru}-106 \\
\mathrm{Rh}-106 \\
\mathrm{Sb}-125 \\
\mathrm{Te}-125 \mathrm{~m} \\
\mathrm{Cs}-134 \\
\mathrm{Cs}-137 \\
\mathrm{Ba}-137 \mathrm{~m} \\
\mathrm{Ce}-144 \\
\mathrm{Pr}-144 \\
\mathrm{Pr}-144 \mathrm{~m} \\
\mathrm{Pm}-147 \\
\mathrm{Sm}-151 \\
\mathrm{Eu}-152 \\
\mathrm{Eu}-154 \\
\mathrm{Eu}-155 \\
\mathrm{Pu}-238 \\
\mathrm{Pu}-239 \\
\mathrm{Pu}-240 \\
\mathrm{Pu}-241 \\
\mathrm{Am}-241\end{array}$ & $\begin{array}{c}\begin{array}{c}\text { Activity } \\
(C i / y z)\end{array} \\
1.71 \mathrm{E}-06 \\
1.24 \mathrm{E}-04 \\
2.37 \mathrm{E}-04 \\
8.86 \mathrm{E}-04 \\
8.00 \mathrm{E}-06 \\
3.18 \mathrm{E}-04 \\
2.50 \mathrm{E}-03 \\
1.14 \mathrm{E}-05 \\
1.64 \mathrm{E}-03 \\
1.43 \mathrm{E}-03 \\
1.43 \mathrm{E}-03 \\
9.72 \mathrm{E}-06 \\
2.56 \mathrm{E}-07 \\
1.40 \mathrm{E}-04 \\
1.40 \mathrm{E}-04 \\
4.03 \mathrm{E}-05 \\
9.84 \mathrm{E}-06 \\
1.63 \mathrm{E}-04 \\
2.00 \mathrm{E}-01 \\
2.00 \mathrm{E}-01 \\
4.69 \mathrm{E}-04 \\
4.69 \mathrm{E}-04 \\
5.64 \mathrm{E}-06 \\
1.14 \mathrm{E}-03 \\
1.12 \mathrm{E}-05 \\
1.71 \mathrm{E}-07 \\
2.93 \mathrm{E}-05 \\
2.32 \mathrm{E}-05 \\
3.53 \mathrm{E}-05 \\
3.42 \mathrm{E}-07 \\
1.71 \mathrm{E}-07 \\
3.95 \mathrm{E}-05 \\
5.13 \mathrm{E}-07\end{array}$ & \\
\hline \multirow[t]{2}{*}{ Shipping Site: NRF } & $\begin{array}{l}\text { Total Volume } \\
\text { (m3/yr) }\end{array}$ & $\begin{array}{l}\text { Total Mass } \\
(\mathrm{kg} / \mathrm{yr})\end{array}$ & $9.40 E+06$ \\
\hline & $\begin{array}{l}\text { Radionuclide } \\
\mathrm{Cr}-51 \\
\mathrm{Mn}-54 \\
\mathrm{Fe}-55 \\
\mathrm{Fe}-59 \\
\mathrm{Co}-58 \\
\mathrm{Co}-60 \\
\mathrm{Ni}-59 \\
\mathrm{Ni}-63 \\
\mathrm{Z}-95\end{array}$ & \multicolumn{2}{|l|}{$\begin{array}{l}2.31 \mathrm{E}+03 \\
4.40 \mathrm{E}+03 \\
1.64 \mathrm{E}+04 \\
1.48 \mathrm{E}+02 \\
5.91 \mathrm{E}+03 \\
4.63 \mathrm{E}+04 \\
2.12 \mathrm{E}+02 \\
3.01 \mathrm{E}+04 \\
1.80 \mathrm{E}+02\end{array}$} \\
\hline
\end{tabular}




\section{LOW-LEVEL WASTE CHARACTERISTICS, ALTERNATIVES, AND ASSESSMENT SCOPE}

The risks of transporting LLW have been assessed for $14 \mathrm{WM}$ alternatives in the WM PEIS. The transportation risks for each case depend on the amount of waste transported, the physical form of the waste, and the quantity of radioactivity (curies) in each shipment. This section of the report describes the alternatives considered for WM LLW in the WM PEIS, presents important LLW inventory and characterization data used in the transportation assessment, and defines the scope of the human health risk assessment.

\subsection{DEFINITION OF LOW-LEVEL WASTE}

Low-level waste is radioactive waste that is not high-level waste, spent nuclear fuel, transuranic waste (greater than $100 \mathrm{nCi} / \mathrm{g}$ ), or by-product material as defined in Section $11 \mathrm{e}(2)$ of the Atomic Energy Act of 1954. Low-level waste is produced in a variety of forms by defense and research activities. The DOE LLW results from daily handling and processing of radioactive materials by DOE defense activities, uranium enrichment operations, the naval nuclear propulsion program, and various research-and-development activities. Low-level waste can contain many different radionuclides in activities ranging from trace quantities to several thousand curies. Low-level waste is present in several physical forms as well, including liquids, wet solids or sludge, and dry solids. Dry solids range from contaminated compactible and combustible materials to contaminated equipment and irradiated hardware. Solid LLW can also consist of soil and sediment and concrete, brick, and rock. Liquid $L L W$ is treated to produce a sludge or solid that may also require packaging before disposal.

\subsection{LOW-LEVEL WASTE ALTERNATTVES FOR THE WM PEIS}

For the purposes of the WM PEIS, the DOE is considering a range of alternatives for the configuration of new or expanded WM facilities and the potential consolidation of existing facilities. In general, the WM PEIS alternatives reflect decentralized, regionalized, and centralized approaches. For each type of waste, several options have been defined for each broad alternative. The individual alternatives differ in the number, location, and types of facilities for treatment, storage, and disposal (TSD) that are being considered.

Transportation risks have been calculated for 14 WM LLW alternatives. The alternatives range from decentralized to centralized approaches to TSD. The number of disposal sites varies from 16 for decentralized disposal to one for centralized disposal. Options for treatment also vary from decentralized to centralized approaches. In general, sites not having the capability for treatment or 
Destination: LLNL

\begin{tabular}{|c|c|c|c|}
\hline Shipping Site: LBL & $\begin{array}{l}\text { Total Volume } \\
(\mathrm{m} 3 / \mathrm{Y} Y)\end{array}$ & $\begin{array}{c}\text { Total Mass } \\
(\mathrm{kg} / \mathrm{y} z)\end{array}$ & $2.87 \varepsilon+05$ \\
\hline & $\begin{array}{l}\text { Radionuclide } \\
\mathrm{Cr}-51 \\
\mathrm{Mn}-54 \\
\mathrm{Fe}-55 \\
\mathrm{Fe}-59 \\
\mathrm{Co}-58 \\
\mathrm{Co}-60 \\
\mathrm{Ni}-59 \\
\mathrm{Ni}-63 \\
\mathrm{Zr}-95\end{array}$ & $\begin{array}{c}\begin{array}{c}\text { Activity } \\
\text { (Ci/yr) }\end{array} \\
6.32 \mathrm{E}-02 \\
1.20 \mathrm{E}-01 \\
4.49 \mathrm{E}-01 \\
4.06 \mathrm{E}-03 \\
1.61 \mathrm{E}-01 \\
1.26 \mathrm{E}+00 \\
5.80 \mathrm{E}-03 \\
8.24 \mathrm{E}-01 \\
4.93 \mathrm{E}-03\end{array}$ & \\
\hline
\end{tabular}

Destination: PORTS

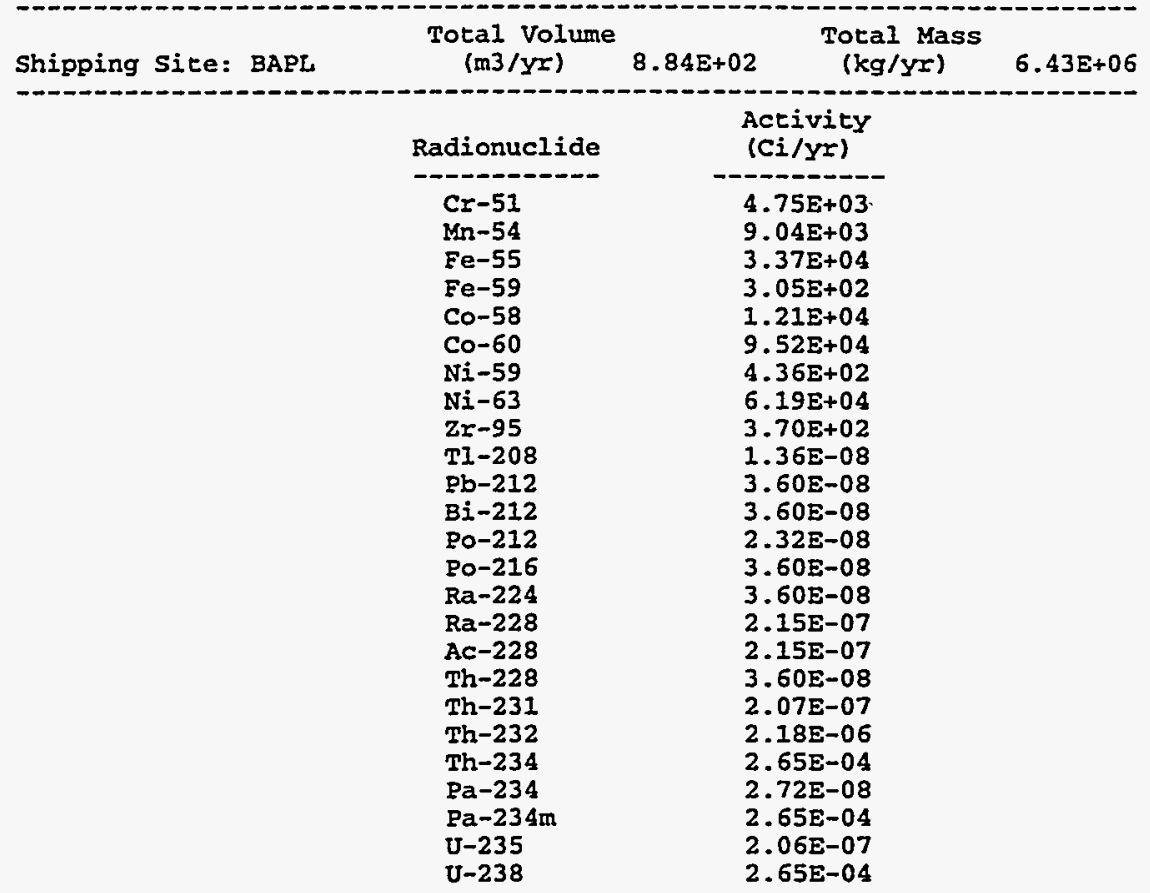




\section{A-3I}

\begin{tabular}{|c|c|c|c|}
\hline Shipping Site: KAPL & $\begin{array}{l}\text { Total Volume } \\
(\mathrm{m} 3 / \mathrm{yr})\end{array}$ & $\begin{array}{c}\text { Total Mass } \\
(\mathrm{kg} / \mathrm{yr})\end{array}$ & $1.33 E+07$ \\
\hline & $\begin{array}{l}\text { Radionuclide } \\
-\mathrm{H}-3 \\
\mathrm{C}-14 \\
\mathrm{Cr}-51 \\
\mathrm{Mn}-54 \\
\mathrm{Ee}-55 \\
\mathrm{Fe}-59 \\
\mathrm{Co}-58 \\
\mathrm{Co}-60 \\
\mathrm{Ni}-59 \\
\mathrm{Ni}-63 \\
\mathrm{Sr}-90 \\
\mathrm{Y}-90 \\
\mathrm{Z} \mathrm{-}-95 \\
\mathrm{TC}-99 \\
\mathrm{Ru}-106 \\
\mathrm{Rh}-106 \\
\mathrm{Sb}-125 \\
\mathrm{Te}-125 \mathrm{~m} \\
\mathrm{Cs}-134 \\
\mathrm{Cs}-137 \\
\mathrm{Ba}-137 \mathrm{~m} \\
\mathrm{Ce}-144 \\
\mathrm{Pr}-144 \\
\mathrm{Pr}-144 \mathrm{~m} \\
\mathrm{Pm}-147 \\
\mathrm{Sm}-151 \\
\mathrm{Eu}-152 \\
\mathrm{Eu}-154 \\
\mathrm{Eu}-155 \\
\mathrm{Tl}-208 \\
\mathrm{~Pb}-212 \\
\mathrm{Bi}-212 \\
\mathrm{PO}-212 \\
\mathrm{PO}-216 \\
\mathrm{Ra}-224 \\
\mathrm{Ra}-228 \\
\mathrm{Ac}-228 \\
\mathrm{Th}-228 \\
\mathrm{Th}-231 \\
\mathrm{Th}-232 \\
\mathrm{Th}-234 \\
\mathrm{~Pa}-234 \\
\mathrm{~Pa}-234 \mathrm{~m} \\
\mathrm{U}-235 \\
\mathrm{U}-238 \\
\mathrm{Pu}-238 \\
\mathrm{Pu}-239 \\
\mathrm{Pu}-240 \\
\mathrm{Pu}-241 \\
\mathrm{Am}-241 \\
\end{array}$ & $\begin{array}{c}\text { Activity } \\
\text { (Ci/yr) } \\
-3.15 \mathrm{E}-04 \\
2.40 \mathrm{E}-05 \\
1.13 \mathrm{E}+04 \\
2.15 \mathrm{E}+04 \\
8.05 \mathrm{E}+04 \\
7.28 \mathrm{E}+02 \\
2.89 \mathrm{E}+04 \\
2.27 \mathrm{E}+05 \\
1.04 \mathrm{E}+03 \\
1.47 \mathrm{E}+05 \\
2.62 \mathrm{E}-02 \\
2.62 \mathrm{E}-02 \\
8.84 \mathrm{E}+02 \\
5.20 \mathrm{E}-05 \\
2.22 \mathrm{E}-03 \\
2.22 \mathrm{E}-03 \\
6.40 \mathrm{E}-04 \\
1.56 \mathrm{E}-04 \\
8.18 \mathrm{E}-03 \\
3.13 \mathrm{E}-02 \\
2.96 \mathrm{E}-02 \\
7.46 \mathrm{E}-03 \\
7.46 \mathrm{E}-03 \\
8.97 \mathrm{E}-05 \\
1.82 \mathrm{E}-02 \\
1.78 \mathrm{E}-04 \\
2.71 \mathrm{E}-06 \\
4.66 \mathrm{E}-04 \\
3.69 \mathrm{E}-04 \\
2.96 \mathrm{E}-08 \\
7.83 \mathrm{E}-08 \\
7.83 \mathrm{E}-08 \\
5.05 \mathrm{E}-08 \\
7.83 \mathrm{E}-08 \\
7.83 \mathrm{E}-08 \\
4.68 \mathrm{E}-07 \\
4.68 \mathrm{E}-07 \\
7.83 \mathrm{E}-08 \\
4.50 \mathrm{E}-07 \\
4.75 \mathrm{E}-06 \\
5.77 \mathrm{E}-04 \\
5.92 \mathrm{E}-08 \\
5.77 \mathrm{E}-04 \\
4.48 \mathrm{E}-07 \\
8.69 \mathrm{E}-04 \\
5.61 \mathrm{E}-04 \\
5.43 \mathrm{E}-06 \\
2.71 \mathrm{E}-06 \\
6.28 \mathrm{E}-04 \\
8.15 \mathrm{E}-06\end{array}$ & \\
\hline
\end{tabular}




\section{A.5 WM LLW REGIONALIZED 1 ALTERNATIVE (CASE 3): HETEROGENEOUS SOLIDS}

Transported Material with Radionuclide Profile

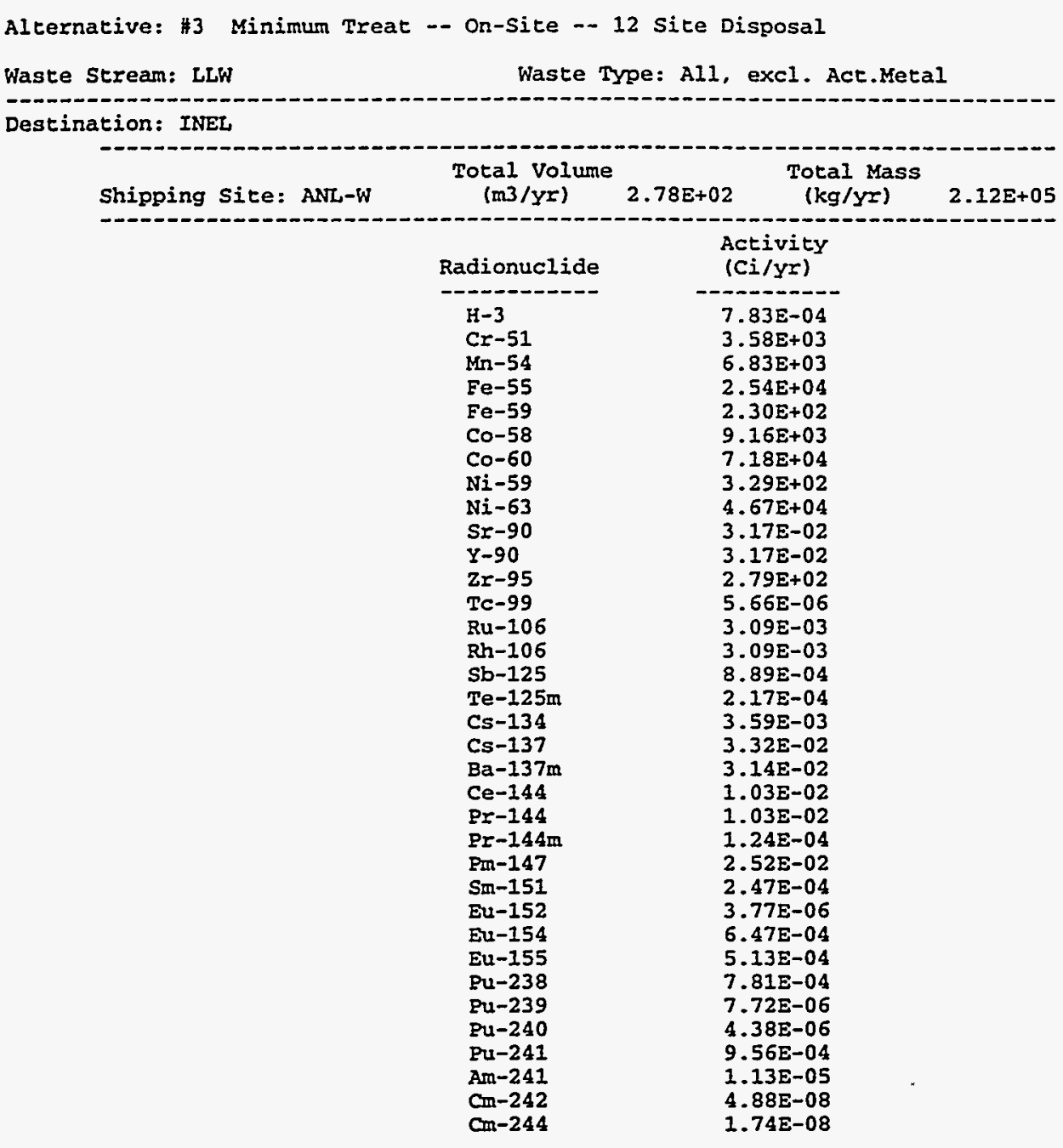




\section{A-33}

Destination: LARE

\begin{tabular}{|c|c|c|c|}
\hline Shipping Site: ITRI & $\begin{array}{l}\text { Total Volume } \\
(\mathrm{m} 3 / \mathrm{yr})\end{array}$ & $\begin{array}{l}\text { Total Mass } \\
(\mathrm{kg} / \mathrm{yr})\end{array}$ & $1.64 \mathrm{E}+05$ \\
\hline & $\begin{array}{l}\text { Radionuclide } \\
\mathrm{H}-3 \\
\mathrm{C}-14 \\
\mathrm{Mn}-54 \\
\mathrm{Co}-58 \\
\mathrm{Co}-60 \\
\mathrm{~S}-90 \\
\mathrm{Y}-90 \\
\mathrm{Tc}-99 \\
\mathrm{Cs}-134 \\
\mathrm{Cs}-137 \\
\mathrm{Ba}-137 \mathrm{~m} \\
\mathrm{U}-238\end{array}$ & $\begin{array}{c}\text { Activity } \\
(C i / y Y) \\
6.17 E+00 \\
3.03 E-01 \\
3.41 E+01 \\
3.15 E+01 \\
9.12 E+01 \\
4.28 E+01 \\
4.28 E+01 \\
6.07 E-01 \\
7.07 E+01 \\
9.33 E+01 \\
8.82 E+01 \\
3.69 E+00\end{array}$ & \\
\hline Shipping Site: SNLA & $\begin{array}{l}\text { Total Volume } \\
(\mathrm{m} 3 / \mathrm{y} x)\end{array}$ & $1.19 \mathrm{E}+02 \quad \begin{array}{c}\text { Total Mass } \\
(\mathrm{kg} / \mathrm{yr})\end{array}$ & $3.28 \mathrm{E}+05$ \\
\hline & $\begin{array}{l}\text { Radionuclide } \\
\mathrm{H}-3 \\
\mathrm{Co}-60 \\
\mathrm{Ni}-63 \\
\mathrm{Sr}-90 \\
\mathrm{y}-90 \\
\mathrm{Tc}-99 \\
\mathrm{Ru}-106 \\
\mathrm{Rh}-106 \\
\mathrm{Sb}-125 \\
\mathrm{Te}-125 \mathrm{~m} \\
\mathrm{Cs}-134 \\
\mathrm{Cs}-137 \\
\mathrm{Ba}-137 \mathrm{~m} \\
\mathrm{Ce}-144 \\
\mathrm{Pr}-144 \\
\mathrm{Pr}-144 \mathrm{~m} \\
\mathrm{Pm}-147 \\
\mathrm{Sm}-151 \\
\mathrm{Eu}-152 \\
\mathrm{Eu}-154 \\
\mathrm{Eu}-155 \\
\mathrm{TI}-208 \\
\mathrm{~Pb}-212 \\
\mathrm{Bi}-212 \\
\mathrm{PO}-212 \\
\mathrm{Po}-216 \\
\mathrm{Ra}-224 \\
\mathrm{Ra}-228 \\
\mathrm{Ac}-228 \\
\mathrm{Th}-228 \\
\mathrm{Th}-231 \\
\mathrm{Th}-232 \\
\mathrm{Th}-234 \\
\mathrm{~Pa}-234 \\
\mathrm{~Pa}-234 \mathrm{~m} \\
\mathrm{U}-235 \\
\mathrm{U}-238 \\
\mathrm{Pu}-238 \\
\mathrm{Pu}-239 \\
\mathrm{Pu}-240 \\
\mathrm{Pu}-241 \\
\mathrm{Am}-241 \\
\mathrm{Cm}-242 \\
\mathrm{Cm}-244 \\
\end{array}$ & 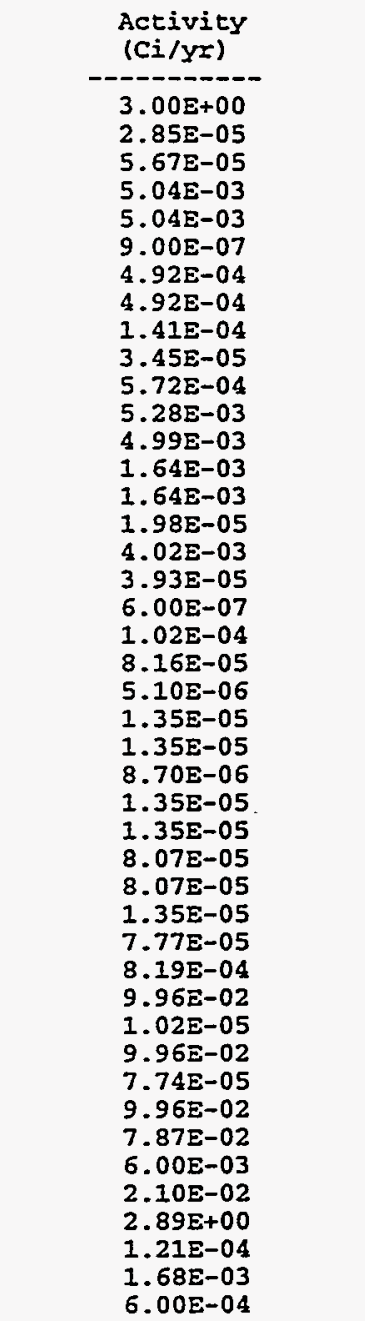 & . \\
\hline
\end{tabular}


Destination: LLNL

\begin{tabular}{|c|c|c|c|}
\hline Shipping Sice: LBL & $\begin{array}{l}\text { Tocal volume } \\
(\mathrm{m} 3 / \mathrm{yr})\end{array}$ & $\begin{array}{c}\text { Total Mass } \\
(\mathrm{kg} / \mathrm{yr})\end{array}$ & $4.51 E+04$ \\
\hline • & $\begin{array}{l}\text { Radionuclide } \\
\mathrm{H}-3 \\
\mathrm{C}-14 \\
\mathrm{Mn}-54 \\
\mathrm{Co}-58 \\
\mathrm{Co}-60 \\
\mathrm{Sr}-90 \\
\mathrm{Y}-90 \\
\mathrm{Tc}-99 \\
\mathrm{Cs}-134 \\
\mathrm{Cs}-137 \\
\mathrm{Ba}-137 \mathrm{~m} \\
\mathrm{U}-238 \\
\mathrm{Cm}-244\end{array}$ & \multicolumn{2}{|l|}{$\begin{array}{l}6.35 \mathrm{E}+02 \\
1.13 \mathrm{E}+00 \\
1.27 \mathrm{E}+02 \\
1.17 \mathrm{E}+02 \\
3.39 \mathrm{E}+02 \\
1.59 \mathrm{E}+02 \\
1.59 \mathrm{0}+02 \\
2.26 \mathrm{E}+00 \\
2.63 \mathrm{0}+02 \\
3.47 \mathrm{E}+02 \\
3.28 \mathrm{E}+02 \\
1.37 \mathrm{E}+01 \\
1.05 \mathrm{E}+01\end{array}$} \\
\hline \multirow[t]{2}{*}{ Shipping Site: SLAC } & $\begin{array}{l}\text { Total Volume } \\
\text { (m3/yr) }\end{array}$ & $\begin{array}{c}\text { Total Mass } \\
(\mathrm{kg} / \mathrm{yr})\end{array}$ & $7.45 \mathrm{E}+05$ \\
\hline & $\begin{array}{l}\text { Radionuclide } \\
\mathrm{Cr}-51 \\
\mathrm{Mn}-54 \\
\mathrm{Fe}-55 \\
\mathrm{Fe}-59 \\
\mathrm{Co}-58 \\
\mathrm{Co}-60 \\
\mathrm{Ni}-59 \\
\mathrm{Ni}-63 \\
\mathrm{Zr}-95\end{array}$ & \multicolumn{2}{|l|}{$\begin{array}{l}3.86 \mathrm{E}-03 \\
7.36 \mathrm{E}-03 \\
2.74 \mathrm{E}-02 \\
2.48 \mathrm{E}-04 \\
9.88 \mathrm{E}-03 \\
7.74 \mathrm{E}-02 \\
3.54 \mathrm{E}-04 \\
5.04 \mathrm{E}-02 \\
3.01 \mathrm{E}-04\end{array}$} \\
\hline \multirow[t]{2}{*}{ Shipping Site: SNLL } & $\begin{array}{l}\text { Total Volume } \\
(\mathrm{m} 3 / \mathrm{yr})\end{array}$ & $\begin{array}{c}\text { Total Mass } \\
(\mathrm{kg} / \mathrm{y})\end{array}$ & $9.73 E+04$ \\
\hline & $\begin{array}{l}\text { Radionuclide } \\
\mathrm{H}-3 \\
\mathrm{Fe}-55 \\
\mathrm{Co}-60 \\
\mathrm{Tl}-208 \\
\mathrm{~Pb}-212 \\
\mathrm{Bi}-212 \\
\mathrm{Po-212} \\
\mathrm{Po-216} \\
\mathrm{Ra-224} \\
\mathrm{Ra-228} \\
\mathrm{Ac-228} \\
\mathrm{Th}-228 \\
\mathrm{Th}-231 \\
\mathrm{Th}-232 \\
\mathrm{Th}-234 \\
\mathrm{Pa-234} \\
\mathrm{Pa}-234 \mathrm{~m} \\
\mathrm{U}-235 \\
\mathrm{U}-238\end{array}$ & \begin{tabular}{c}
$\begin{array}{c}\text { Activity } \\
(C i / y I)\end{array}$ \\
\hdashline $2.78 E+04$ \\
$3.15 E-01$ \\
$3.15 E-01$ \\
$6.40 E-05$ \\
$1.69 E-04$ \\
$1.69 E-04$ \\
$1.09 E-04$ \\
$1.69 E-04$ \\
$1.69 E-04$ \\
$1.01 E-03$ \\
$1.01 E-03$ \\
$1.69 E-04$ \\
$9.75 E-04$ \\
$1.02 E-02$ \\
$1.25 E+00$ \\
$1.28 E-04$ \\
$1.25 E+00$ \\
$9.71 E-04$ \\
$1.25 E+00$
\end{tabular} & \\
\hline
\end{tabular}




\section{A-35}

Destination: ORNL

\begin{tabular}{|c|c|c|c|}
\hline Shipping Site: $\mathrm{K}-25$ & $\begin{array}{l}\text { Total Volume } \\
(\mathrm{m} 3 / \mathrm{yr})\end{array}$ & $\begin{array}{l}\text { Total Mass } \\
(\mathrm{kg} / \mathrm{Y} \tau)\end{array}$ & $4.67 E+07$ \\
\hline & $\begin{array}{l}\text { Radionuclide } \\
\text { Tl-208 } \\
\mathrm{Pb}-212 \\
\mathrm{Bi}-212 \\
\mathrm{Po}-212 \\
\mathrm{Po}-216 \\
\mathrm{Ra}-224 \\
\mathrm{Ra}-228 \\
\mathrm{Ac}-228 \\
\mathrm{Th}-228 \\
\mathrm{Th}-231 \\
\mathrm{Th}-232 \\
\mathrm{Th}-234 \\
\mathrm{~Pa}-234 \\
\mathrm{~Pa}-234 \mathrm{~m} \\
\mathrm{U}-235 \\
\mathrm{U}-238\end{array}$ & \multicolumn{2}{|l|}{$\begin{array}{l}1.06 \mathrm{E}-04 \\
2.82 \mathrm{E}-04 \\
2.82 \mathrm{E}-04 \\
1.82 \mathrm{E}-04 \\
2.82 \mathrm{E}-04 \\
2.82 \mathrm{E}-04 \\
1.68 \mathrm{E}-03 \\
1.68 \mathrm{E}-03 \\
2.82 \mathrm{E}-04 \\
1.62 \mathrm{E}-03 \\
1.71 \mathrm{E}-02 \\
2.08 \mathrm{E}+00 \\
2.13 \mathrm{E}-04 \\
2.08 \mathrm{E}+00 \\
1.62 \mathrm{E}-03 \\
2.08 \mathrm{E}+00\end{array}$} \\
\hline \multirow[t]{2}{*}{ Shipping site: ORISE } & $\begin{array}{l}\text { Total Volume } \\
\left(\mathrm{m}^{3} / \mathrm{yr}\right)\end{array}$ & $\begin{array}{cc}6.07 \mathrm{E}+01 & \begin{array}{c}\text { Total Mass } \\
(\mathrm{kg} / \mathrm{yz})\end{array}\end{array}$ & $7.36 \Xi+04$ \\
\hline & \begin{tabular}{l} 
Radionuclide \\
\hdashline $\mathrm{H}-3$ \\
$\mathrm{C}-14$ \\
$\mathrm{Mn}-54$ \\
$\mathrm{Co}-58$ \\
$\mathrm{Co}-60$ \\
$\mathrm{~S} r-90$ \\
$\mathrm{Y}-90$ \\
$\mathrm{Tc}-99$ \\
$\mathrm{Cs}-134$ \\
$\mathrm{Cs}-137$ \\
$\mathrm{Ba}-137 \mathrm{~m}$ \\
$\mathrm{~T}-208$ \\
$\mathrm{~Pb}-212$ \\
$\mathrm{Bi}-212$ \\
$\mathrm{PO}-212$ \\
$\mathrm{Po}-216$ \\
$\mathrm{Ra}-224$ \\
$\mathrm{Ra}-228$ \\
$\mathrm{Ac}-228$ \\
$\mathrm{Th}-228$ \\
$\mathrm{Th}-231$ \\
$\mathrm{Th}-232$ \\
$\mathrm{Th}-234$ \\
$\mathrm{~Pa}-234$ \\
$\mathrm{~Pa}-234 \mathrm{~m}$ \\
$\mathrm{U}-235$ \\
$\mathrm{U}-238$
\end{tabular} & \multicolumn{2}{|l|}{$\begin{array}{l}2.00 \mathrm{E}-02 \\
1.19 \mathrm{E}-05 \\
1.34 \mathrm{E}-03 \\
1.24 \mathrm{E}-03 \\
3.59 \mathrm{E}-03 \\
1.69 \mathrm{E}-03 \\
1.69 \mathrm{E}-03 \\
2.39 \mathrm{E}-05 \\
2.79 \mathrm{E}-03 \\
3.68 \mathrm{E}-03 \\
3.48 \mathrm{E}-03 \\
3.39 \mathrm{E}-07 \\
8.98 \mathrm{E}-07 \\
8.98 \mathrm{E}-07 \\
5.78 \mathrm{E}-07 \\
8.98 \mathrm{E}-07 \\
8.98 \mathrm{E}-07 \\
5.36 \mathrm{E}-06 \\
5.36 \mathrm{E}-06 \\
8.98 \mathrm{E}-07 \\
5.16 \mathrm{E}-06 \\
5.44 \mathrm{E}-05 \\
6.62 \mathrm{E}-03 \\
6.78 \mathrm{E}-07 \\
6.62 \mathrm{E}-03 \\
5.14 \mathrm{E}-06 \\
6.77 \mathrm{E}-03\end{array}$} \\
\hline \multirow[t]{2}{*}{ Shipping Site: $Y-12$} & $\begin{array}{l}\text { Total volume } \\
\text { (m3/Yx) }\end{array}$ & $\begin{array}{l}\text { Total Mass } \\
(\mathrm{kg} / \mathrm{yr})\end{array}$ & $8.27 \mathrm{E}+07$ \\
\hline & $\begin{array}{l}\text { Radionuclide } \\
\mathrm{TI}-208 \\
\mathrm{~Pb}-212 \\
\mathrm{Bi}-212 \\
\mathrm{Po-212} \\
\mathrm{PO-216} \\
\mathrm{Ra-224} \\
\mathrm{Ra}-228 \\
\mathrm{Ac-228} \\
\mathrm{Th}-228 \\
\mathrm{Th}-231 \\
\mathrm{Th}-232 \\
\mathrm{Th}-234 \\
\mathrm{~Pa}-234 \\
\mathrm{~Pa}-234 \mathrm{~m} \\
\mathrm{U}-235 \\
\mathrm{U}-238\end{array}$ & $\begin{array}{c}\text { Activity } \\
\text { (Ci/yr) } \\
6.80 \mathrm{E}-05 \\
1.80 \mathrm{E}-04 \\
1.80 \mathrm{E}-04 \\
1.16 \mathrm{E}-04 \\
1.80 \mathrm{E}-04 \\
1.80 \mathrm{E}-04 \\
1.07 \mathrm{E}-03 \\
1.07 \mathrm{E}-03 \\
1.80 \mathrm{E}-04 \\
1.03 \mathrm{E}-03 \\
1.09 \mathrm{E}-02 \\
1.32 \mathrm{E}+00 \\
1.36 \mathrm{E}-04 \\
1.32 \mathrm{3}+00 \\
1.03 \mathrm{E}-03 \\
1.32 \mathrm{E}+00\end{array}$ & \\
\hline
\end{tabular}


Destination: PORTS

\begin{tabular}{|c|c|c|c|}
\hline Shipping Site: AMES & $\begin{array}{l}\text { Total Volume } \\
(\mathrm{m} 3 / \mathrm{y} x)\end{array}$ & $\begin{array}{l}\text { Total Mass } \\
(\mathrm{kg} / \mathrm{yr})\end{array}$ & $2.99 \mathrm{E}+04$ \\
\hline & $\begin{array}{l}\text { Radionuclide } \\
\mathrm{T} 1-208 \\
\mathrm{~Pb}-212 \\
\mathrm{Bi}-212 \\
\mathrm{Po-212} \\
\mathrm{Po-216} \\
\mathrm{Ra}-224 \\
\mathrm{Ra}-228 \\
\mathrm{Ac}-228 \\
\mathrm{Th}-228 \\
\mathrm{Th}-231 \\
\mathrm{Th}-232 \\
\mathrm{Th}-234 \\
\mathrm{~Pa}-234 \\
\mathrm{~Pa}-234 \mathrm{~m} \\
\mathrm{U}-235 \\
\mathrm{U}-238 \\
\mathrm{Pu}-238 \\
\mathrm{Pu}-239 \\
\mathrm{Pu}-240 \\
\mathrm{Pu}-241 \\
\mathrm{Am}-241 \\
\mathrm{Cm}-242 \\
\mathrm{Cm}-244\end{array}$ & 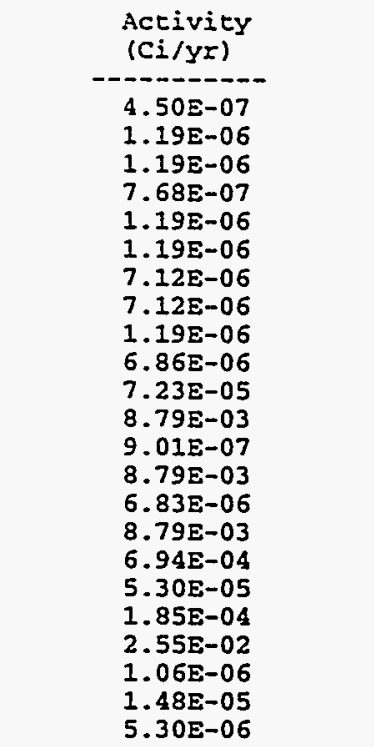 & \\
\hline Shipping Site: ANL-E & $\begin{array}{l}\text { Total Volume } \\
(\mathrm{m} 3 / \mathrm{yr})\end{array}$ & $\begin{array}{cc}\text { Total Mass } \\
(\mathrm{kg} / \mathrm{yr})\end{array}$ & $2.00 \mathrm{E}+06$ \\
\hline & 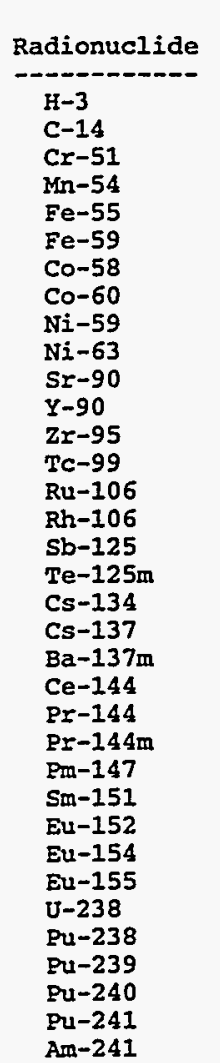 & \begin{tabular}{c} 
Activity \\
$(C i / y r)$ \\
\hdashline $1.33 E+02$ \\
$7.91 E-02$ \\
$2.87 E+00$ \\
$1.43 E+01$ \\
$2.04 E+01$ \\
$1.84 E-01$ \\
$1.55 E+01$ \\
$8.15 E+01$ \\
$2.63 E-01$ \\
$3.77 E+01$ \\
$3.33 E+01$ \\
$3.33 E+01$ \\
$2.24 E-01$ \\
$1.62 E-01$ \\
$2.16 E+00$ \\
$2.16 E+00$ \\
$6.21 E-01$ \\
$1.51 E-01$ \\
$2.09 E+01$ \\
$4.75 E+01$ \\
$4.50 E+01$ \\
$7.24 E+00$ \\
$7.24 E+00$ \\
$8.70 E-02$ \\
$1.76 E+01$ \\
$1.72 E-01$ \\
$2.63 E-03$ \\
$4.52 E-01$ \\
$3.58 E-01$ \\
$9.63 E-01$ \\
$5.44 E-01$ \\
$5.27 E-03$ \\
$2.63 E-03$ \\
$6.09 E-01$ \\
$7.91 E-03$
\end{tabular} & \\
\hline
\end{tabular}




\begin{tabular}{|c|c|c|c|}
\hline Shipping Sice: BAPL & $\begin{array}{l}\text { Total Volume } \\
(\mathrm{m} 3 / \mathrm{yr})\end{array}$ & $\begin{array}{c}\text { Total Mass } \\
\text { (kg/yr) }\end{array}$ & $1.08 \mathrm{E}+06$ \\
\hline & $\begin{array}{l}\text { Radionuclide } \\
\mathrm{H}-3 \\
\mathrm{Co}-60 \\
\mathrm{Ni}-63 \\
\mathrm{Sr}-90 \\
\mathrm{Y}-90 \\
\mathrm{Tc}-99 \\
\mathrm{Ru}-106 \\
\mathrm{Rh}-106 \\
\mathrm{Sb}-125 \\
\mathrm{Te}-125 \mathrm{~m} \\
\mathrm{Cs}-134 \\
\mathrm{Cs}-137 \\
\mathrm{Ba}-137 \mathrm{~m} \\
\mathrm{Ce}-144 \\
\mathrm{Pr}-144 \\
\mathrm{Pr}-144 \mathrm{~m} \\
\mathrm{Pm}-147 \\
\mathrm{Sm-151} \\
\mathrm{Eu}-152 \\
\mathrm{Eu}-154 \\
\mathrm{Eu}-155 \\
\mathrm{~T} 1-208 \\
\mathrm{~Pb}-212 \\
\mathrm{Bi}-212 \\
\mathrm{Po}-212 \\
\mathrm{Po-216} \\
\mathrm{Ra}-224 \\
\mathrm{Ra}-228 \\
\mathrm{Ac}-228 \\
\mathrm{Th}-228 \\
\mathrm{Th}-231 \\
\mathrm{Th}-232 \\
\mathrm{Th}-234 \\
\mathrm{~Pa}-234 \\
\mathrm{~Pa}-234 \mathrm{~m} \\
\mathrm{U}-235 \\
\mathrm{U}-238 \\
\mathrm{Pu}-238 \\
\mathrm{Pu}-239 \\
\mathrm{Pu}-240 \\
\mathrm{Pu}-241 \\
\mathrm{Am}-241 \\
\end{array}$ & 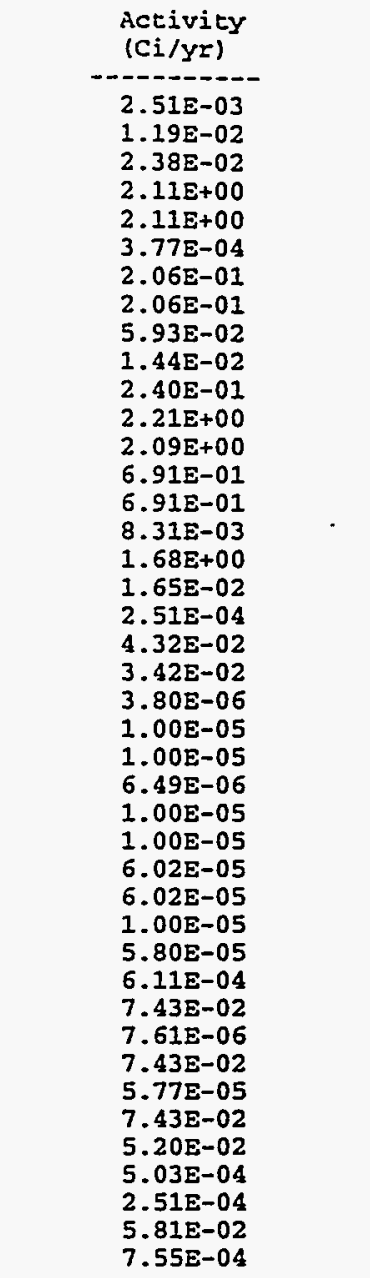 & \\
\hline & $\begin{array}{l}\text { Total Volume } \\
(\mathrm{m} 3 / \mathrm{YY})\end{array}$ & $\begin{array}{cc}\text { Total Mass } \\
(\mathrm{kg} / \mathrm{y})\end{array}$ & $5.11 E+04$ \\
\hline & $\begin{array}{l}\text { Radionuclide } \\
---3 \\
\mathrm{E}-3 \\
\mathrm{Cr}-51 \\
\mathrm{Mn}-54 \\
\mathrm{Fe}-55 \\
\mathrm{Fe}-59 \\
\mathrm{Co}-58 \\
\mathrm{Co}-60 \\
\mathrm{Ni}-59 \\
\mathrm{Ni}-63 \\
\mathrm{Z}-95 \\
\mathrm{~T}-208 \\
\mathrm{~Pb}-212 \\
\mathrm{Bi}-212 \\
\mathrm{Po-212} \\
\mathrm{Po-216} \\
\mathrm{Ra}-224 \\
\mathrm{Ra}-228 \\
\mathrm{Ac}-228 \\
\mathrm{Th}-228 \\
\mathrm{Th}-231 \\
\mathrm{Th}-232 \\
\mathrm{Th}-234 \\
\mathrm{~Pa}-234 \\
\mathrm{~Pa}-234 \mathrm{~m} \\
\mathrm{U}-235 \\
\mathrm{U}-238\end{array}$ & 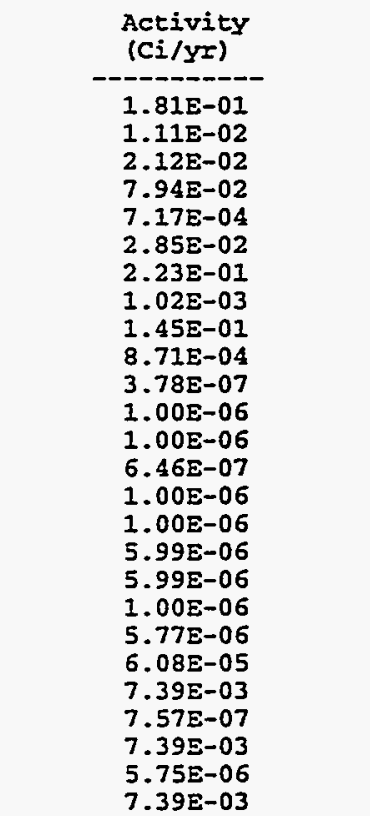 & \\
\hline
\end{tabular}




\begin{tabular}{|c|c|c|c|}
\hline Shipping Sice: KAPL & $\begin{array}{l}\text { Total Volume } \\
(\mathrm{m} 3 / \mathrm{yc})\end{array}$ & $\begin{array}{l}\text { Total Mass } \\
\text { (kg/y }\end{array}$ & $5.36 \mathrm{E}+05$ \\
\hline & 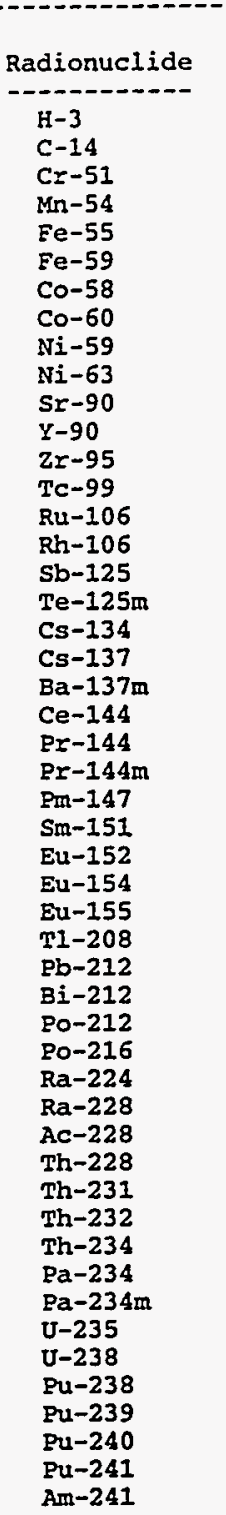 & 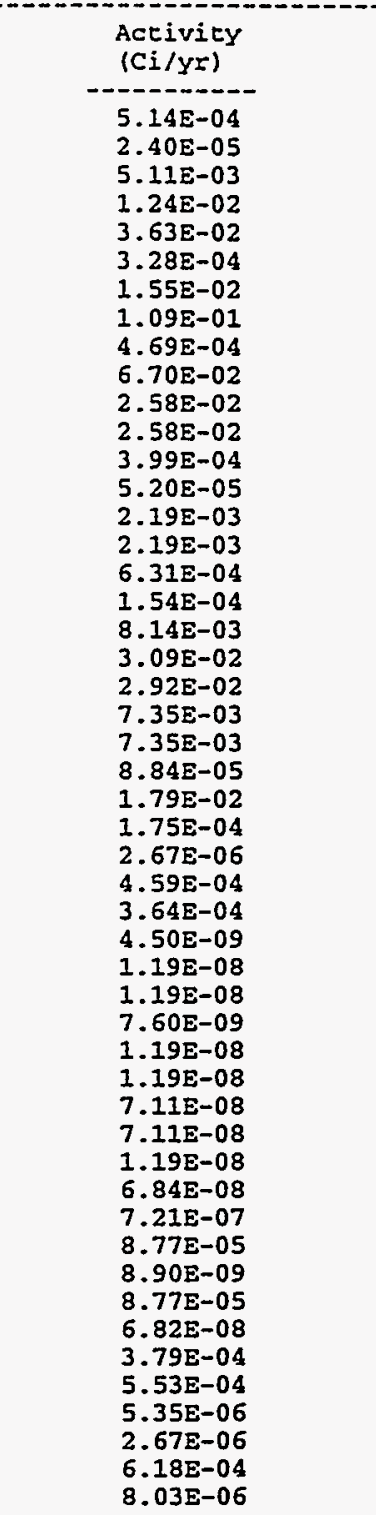 & \\
\hline Shipping Site: MOUND & $\begin{array}{l}\text { Total Volume } \\
\text { (m3/yr) }\end{array}$ & $\begin{array}{l}\text { Total Mass } \\
\text { (kg/yr) }\end{array}$ & $9.67 \mathrm{E}+06$ \\
\hline & $\begin{array}{l}\text { Radionuclide } \\
\mathrm{H}-3 \\
\text { Pu-238 } \\
\text { Pu-239 } \\
\text { Pu-240 } \\
\text { Pu-241 } \\
\text { Am-241 } \\
\text { Cm-242 } \\
\text { Cm-244 }\end{array}$ & $\begin{array}{l}\text { Activity } \\
\text { (Ci/YI) } \\
1.34 E+04 \\
1.60 E-01 \\
1.22 E-02 \\
4.28 E-02 \\
5.90 E+00 \\
2.44 E-04 \\
3.42 E-03 \\
1.22 E-03\end{array}$ & \\
\hline
\end{tabular}




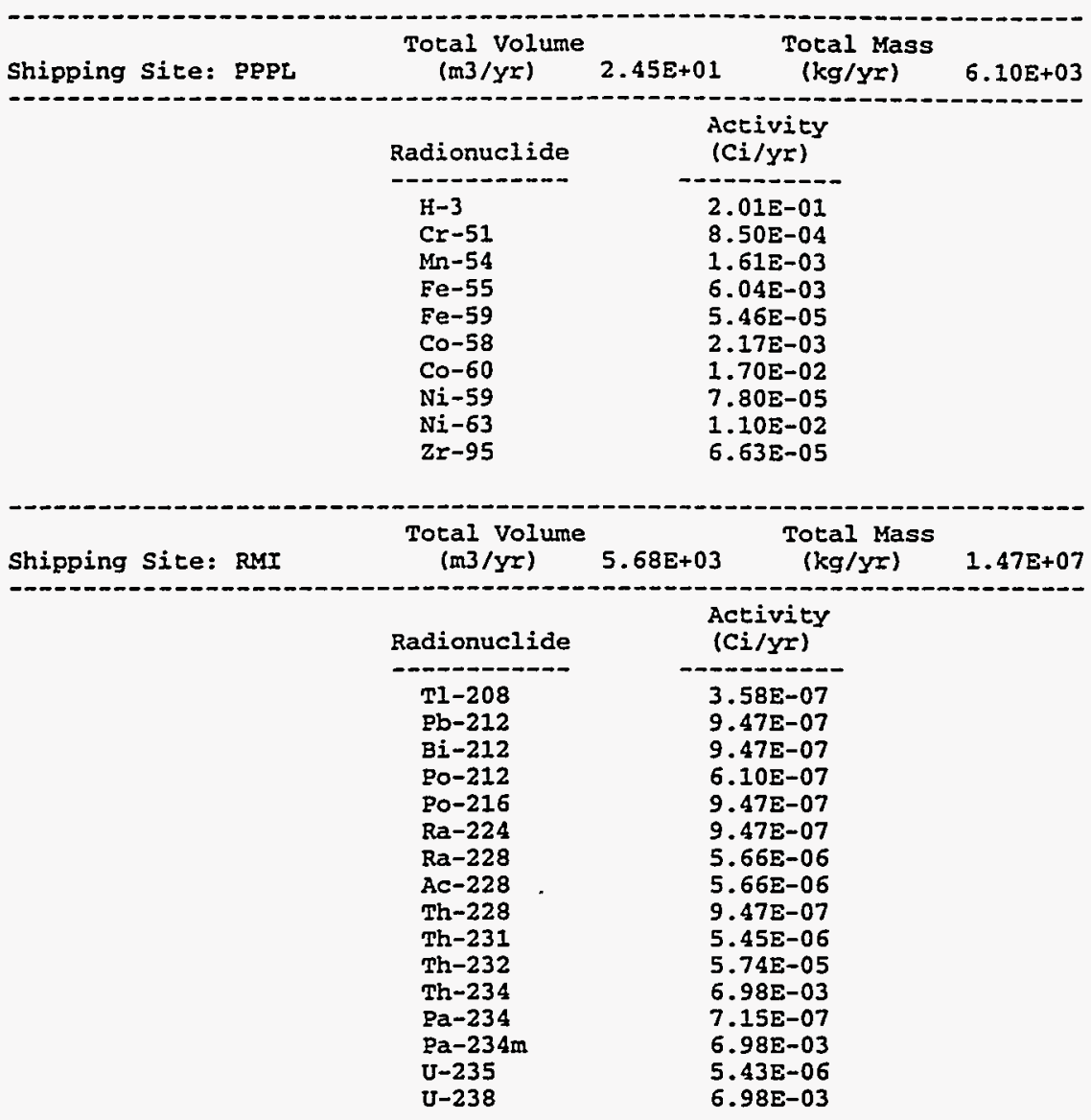

Destination: Paducah

\begin{tabular}{|c|c|c|c|}
\hline Shipping Site: KCP & $\begin{array}{c}\text { Total Volume } \\
(\mathrm{m} 3 / \mathrm{Yr})\end{array}$ & $\begin{array}{l}\text { Total Mass } \\
(\mathrm{kg} / \mathrm{Yr})\end{array}$ & $7.23 E+03$ \\
\hline - & $\begin{array}{l}\text { Radionuclide } \\
\mathrm{H}-3 \\
\mathrm{C}-14 \\
\mathrm{Mn}-54 \\
\mathrm{Co}-58 \\
\mathrm{Co}-60 \\
\mathrm{~S}-90 \\
\mathrm{Y}-90 \\
\mathrm{TC}-99 \\
\mathrm{Cs}-134 \\
\mathrm{Cs}-137 \\
\mathrm{Ba}-137 \mathrm{~m} \\
\mathrm{U}-238\end{array}$ & $\begin{array}{l}2.70 \mathrm{E}-02 \\
1.44 \mathrm{E}-04 \\
1.62 \mathrm{E}-02 \\
1.50 \mathrm{E}-02 \\
4.34 \mathrm{E}-02 \\
2.04 \mathrm{E}-02 \\
2.04 \mathrm{E}-02 \\
2.89 \mathrm{E}-04 \\
3.37 \mathrm{E}-02 \\
4.44 \mathrm{E}-02 \\
4.20 \mathrm{E}-02 \\
1.76 \mathrm{E}-03\end{array}$ & \\
\hline Shipping Site: Pinellas & $\begin{array}{l}\text { Total Volume } \\
(\text { (m3/yr) }\end{array}$ & $\begin{array}{c}\text { Total Mass } \\
(\mathrm{kg} / \mathrm{Yr})\end{array}$ & $1.91 E+04$ \\
\hline & $\begin{array}{l}\text { Radionuclide } \\
\mathrm{H}-3\end{array}$ & $\begin{array}{c}\begin{array}{c}\text { Activity } \\
\text { (Ci/Yr) }\end{array} \\
1.99 \mathrm{E}+04\end{array}$ & \\
\hline
\end{tabular}


disposal ship to the nearest site that has such capability. The alternatives are summarized in Table 2.1 and are described as follows:

- No Action (Case 1). All sites would treat LLW using existing, planned, and approved treatment facilities and dispose of LLW at the six current disposal sites in accordance with current arrangements.

- Decentralized (Case 2). All sites would minimally treat LLW, stabilizing fines and liquids, and dispose of LLW at 16 sites (Argonne National LaboratoryEast [ANL-E], Brookhaven National Laboratory [BNL], Fernald Environmental Management Project [FEMP], Hanford Site [Hanford], Idaho National Engineering Laboratory [INEL], Lawrence Livermore National Laboratory [LLNL], Los Alamos National Laboratory [LANL], the Nevada Test Site [NTS], Oak Ridge Reservation [ORR], Paducah Gaseous Diffusion Plant [PGDP], Pantex Plant [Pantex], Portsmouth Gaseous Diffusion Plant [PORTS], Rocky Flats Environmental Technology Site [RFETS], Sandia National Laboratory-New Mexico [SNL-NM], Savannah River Site [SRS], and West Valley Demonstration Project [WVDP].

- Regionalized I (Case 3). All sites would minimally treat LLW, stabilizing fines and liquids, and dispose of LLW at 12 sites (Hanford, INEL, NTS, LANL, ORR, SRS, PORTS, PGDP, FEMP, LLNL, Pantex, and RFETS).

- Regionalized 2 (Case 9). Eleven sites (Hanford, INEL, LANL, ORR, SRS, PORTS, PGDP, FEMP, LLNL, Pantex, and RFETS) would thermally treat, supercompact, reduce the size of, and grout volume-reducible waste; all sites would minimally treat other waste; disposal would occur at 12 sites (Hanford, INEL, NTS, LANL, ORR, SRS, PORTS, PGDP, FEMP, LLNL, Pantex, and RFETS).

- Regionalized 3 (Case 4). All sites would minimally treat LLW, stabilizing fines and liquids, and dispose of LLW at the nearest of six sites (Hanford, INEL, NTS, LANL, ORR, and SRS).

- Regionalized 4 (Case 12). Seven sites (Hanford, INEL, LANL, ORR, PORTS, RFETS, and SRS) would thermally treat, supercompact, reduce the size of, and grout volume-reducible waste; all sites would minimally treat other waste; disposal would occur at six sites (Hanford, INEL, NTS, LANL, ORR, and SRS). 


\section{A.6 WM LLW REGIONALIZED 1 ALTERNATIVE (CASE 3): ACTIVATED METALS}

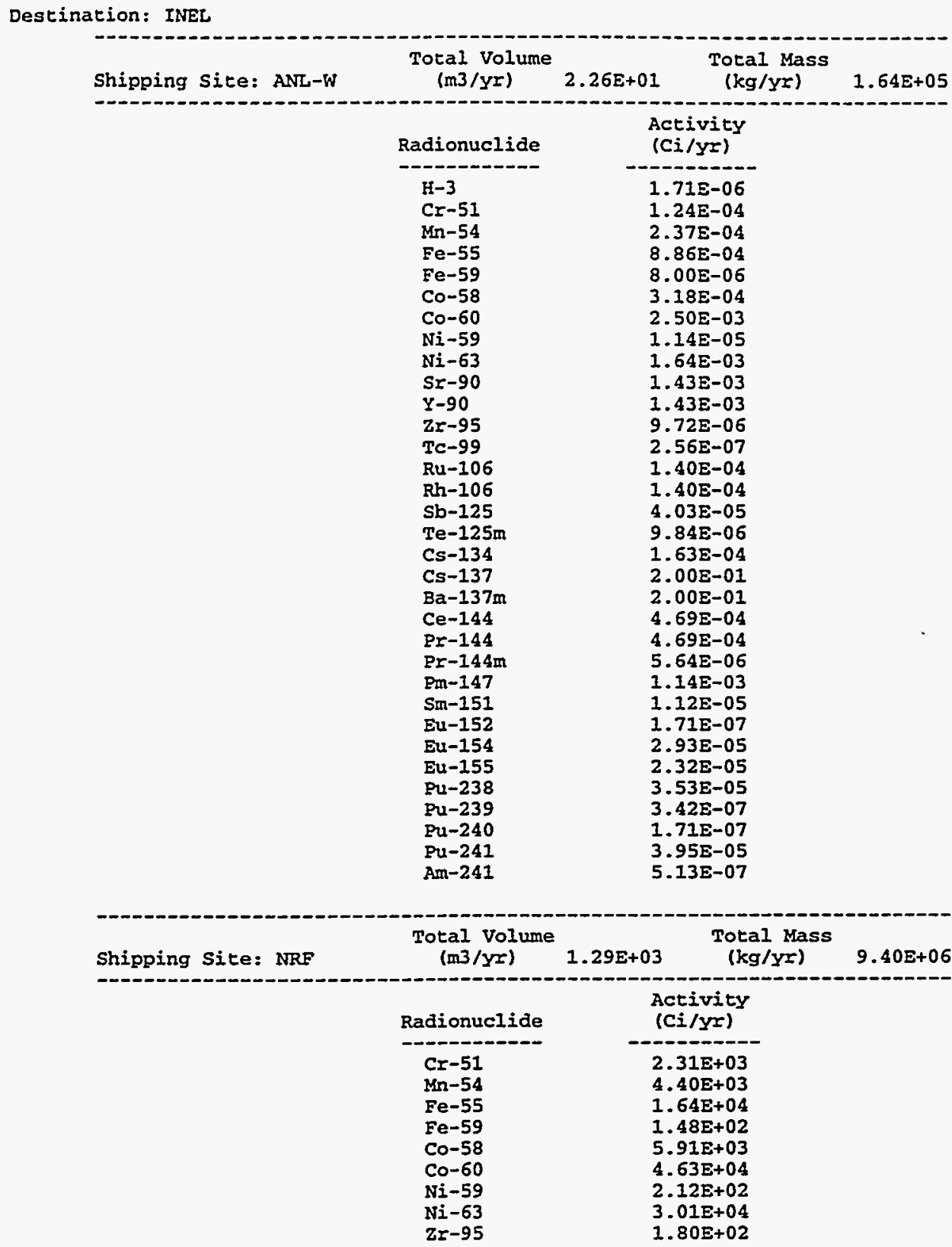


Destination: LANL

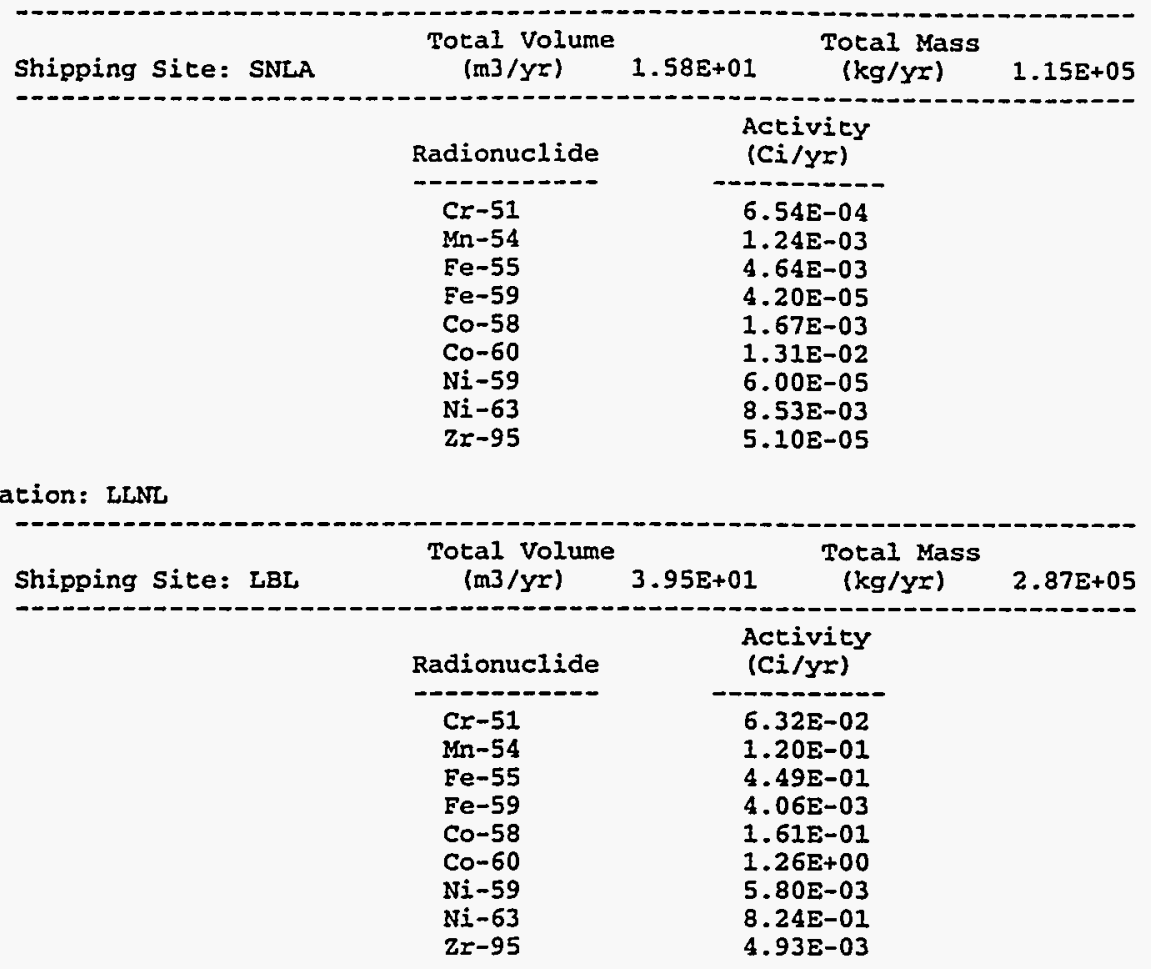




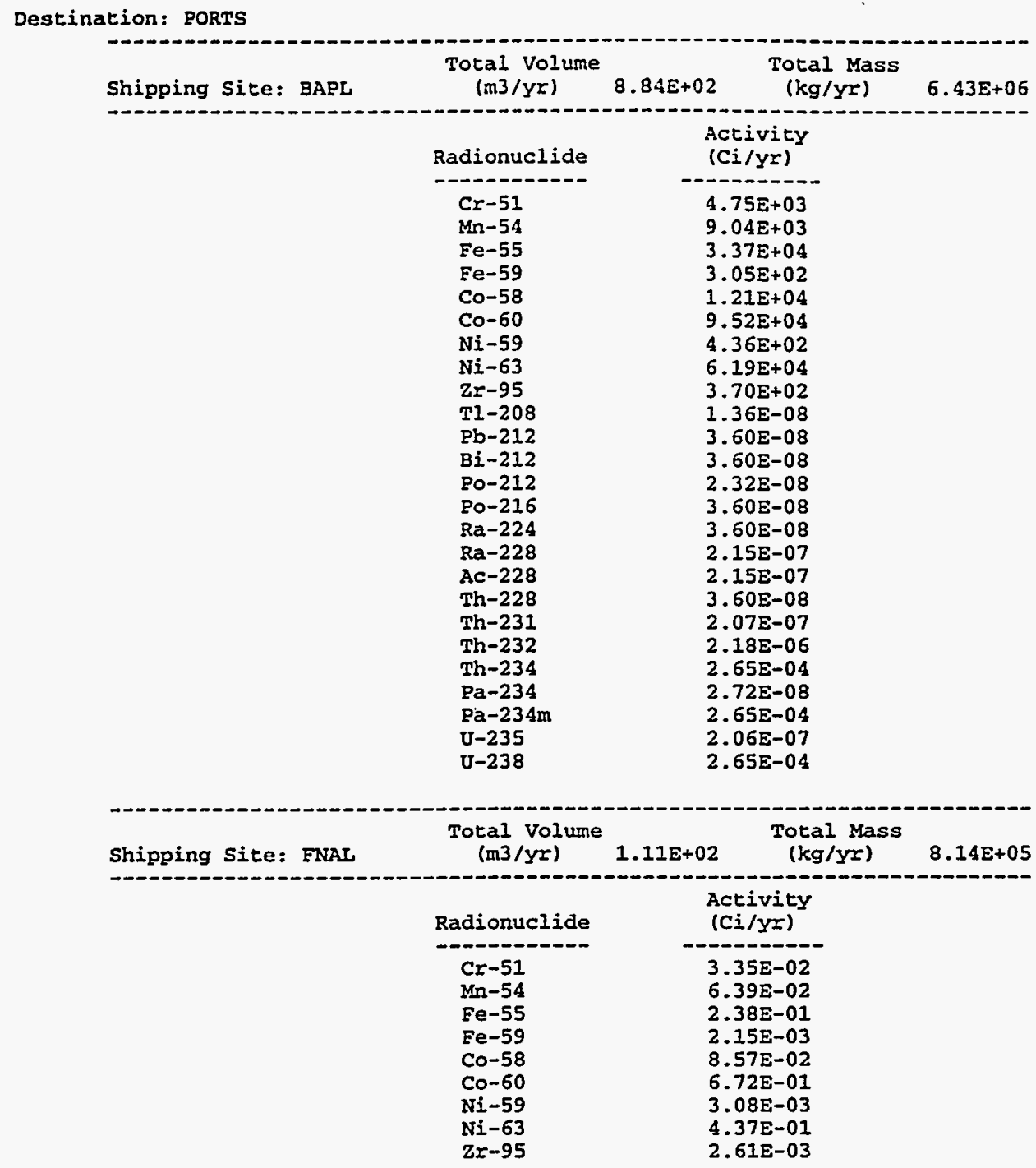


$A-43$

\begin{tabular}{|c|c|c|c|}
\hline Shipping Site: KAPL & $\begin{array}{l}\text { Total volume } \\
(\mathrm{m} 3 / \mathrm{yr})\end{array}$ & $\begin{array}{l}\text { Total Mass } \\
(\mathrm{kg} / \mathrm{Yr})\end{array}$ & $1.33 E+07$ \\
\hline & 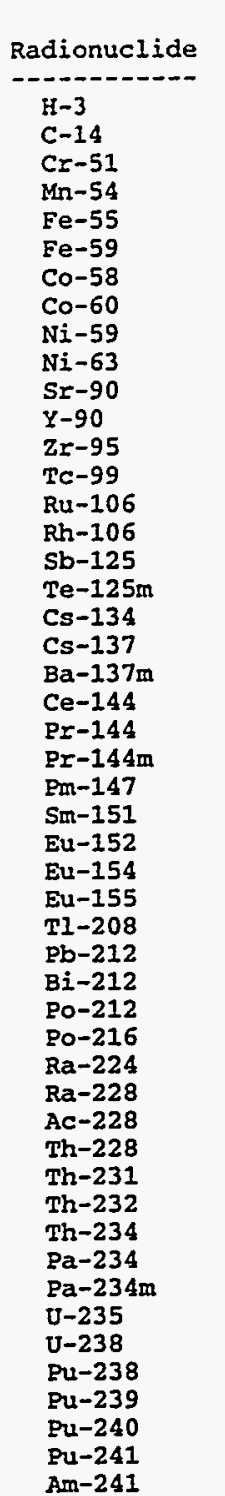 & 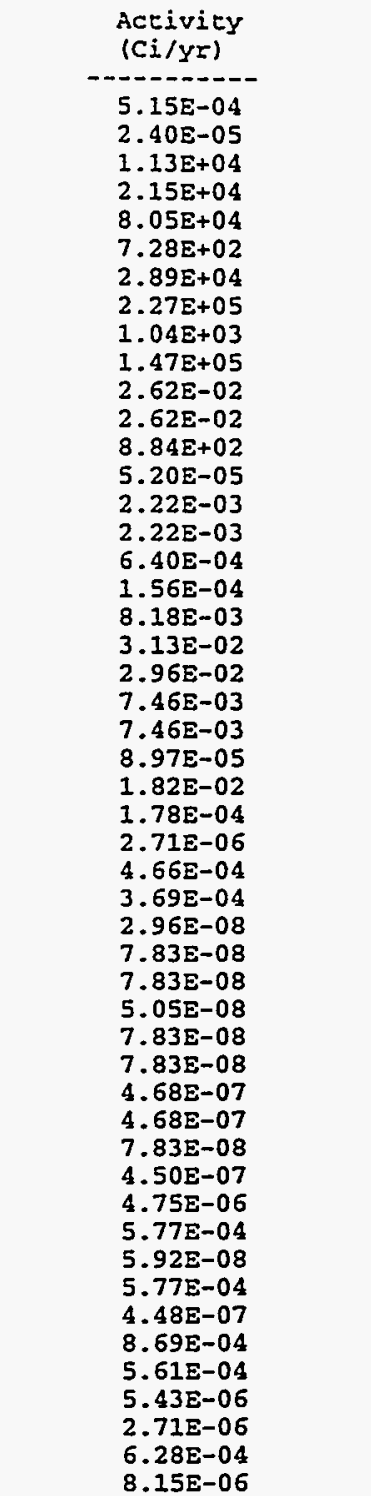 & \\
\hline
\end{tabular}




\section{A.7 WM LLW REGIONALIZED 2 ALTERNATIVE (CASE 9): HETEROGENEOUS SOLIDS}

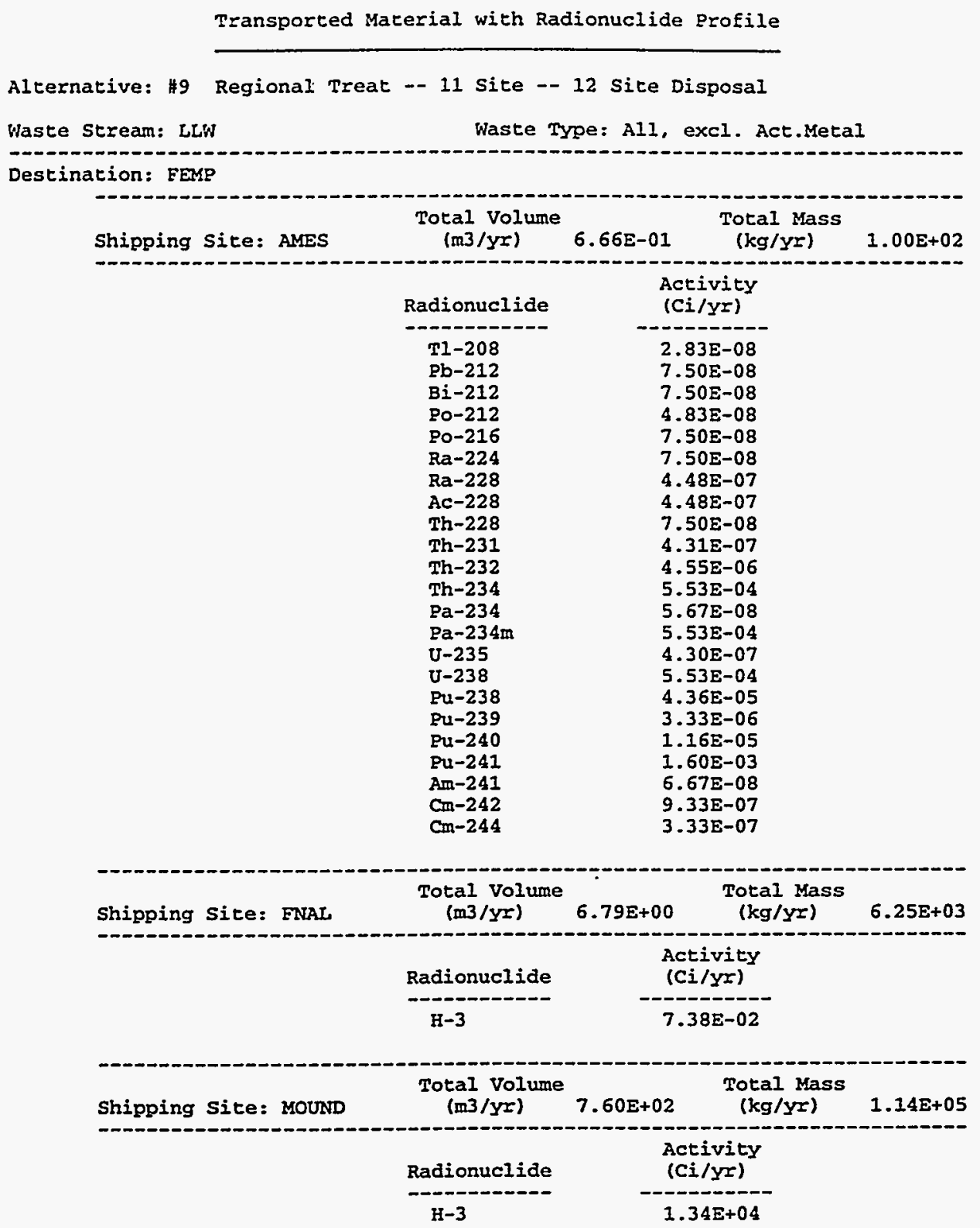


A- -45

Destination: INEL

\begin{tabular}{|c|c|c|c|}
\hline Shipping Site: ANL-W & $\begin{array}{l}\text { Total volume } \\
(\mathrm{m} 3 / \mathrm{yr})\end{array}$ & $\begin{array}{l}\text { Total Mass } \\
(\mathrm{kg} / \mathrm{yr})\end{array}$ & $2.12 \mathrm{E}+05$ \\
\hline & $\begin{array}{l}\text { Radionuclide } \\
\mathrm{H}-3 \\
\mathrm{Cr}-51 \\
\mathrm{Mn}-54 \\
\text { Fe-55 } \\
\text { Fe-59 } \\
\mathrm{Co}-58 \\
\mathrm{Co}-60 \\
\mathrm{Ni}-59 \\
\mathrm{Ni}-63 \\
\mathrm{Sr}-90 \\
\mathrm{Y}-90 \\
\mathrm{Zr}-95 \\
\mathrm{Tc}-99 \\
\mathrm{Ru}-106 \\
\mathrm{Rh}-106 \\
\mathrm{Sb}-125 \\
\mathrm{Te}-125 \mathrm{~m} \\
\mathrm{Cs}-134 \\
\mathrm{Cs}-137 \\
\mathrm{Ba}-137 \mathrm{~m} \\
\mathrm{Ce}-144 \\
\mathrm{Pr}-144 \\
\mathrm{Pr}-144 \mathrm{~m} \\
\mathrm{Pm}-147 \\
\mathrm{Sm}-151 \\
\mathrm{Eu}-152 \\
\mathrm{Eu}-154 \\
\mathrm{Eu}-155 \\
\mathrm{Pu}-238 \\
\mathrm{Pu}-239 \\
\mathrm{Pu}-240 \\
\mathrm{Pu}-241 \\
\mathrm{Am}-241 \\
\mathrm{Cm}-242 \\
\mathrm{Cm}-244\end{array}$ & $\begin{array}{c}\text { Activity } \\
(C i / y r) \\
7.83 \mathrm{E}-04 \\
3.58 \mathrm{E}+03 \\
6.83 \mathrm{E}+03 \\
2.54 \mathrm{E}+04 \\
2.30 \mathrm{E}+02 \\
9.16 \mathrm{E}+03 \\
7.18 \mathrm{E}+04 \\
3.29 \mathrm{E}+02 \\
4.67 \mathrm{E}+04 \\
3.17 \mathrm{E}-02 \\
3.17 \mathrm{E}-02 \\
2.79 \mathrm{E}+02 \\
5.66 \mathrm{E}-06 \\
3.09 \mathrm{E}-03 \\
3.09 \mathrm{E}-03 \\
8.89 \mathrm{E}-04 \\
2.17 \mathrm{E}-04 \\
3.59 \mathrm{E}-03 \\
3.32 \mathrm{E}-02 \\
3.14 \mathrm{E}-02 \\
1.03 \mathrm{E}-02 \\
1.03 \mathrm{E}-02 \\
1.24 \mathrm{E}-04 \\
2.52 \mathrm{E}-02 \\
2.47 \mathrm{E}-04 \\
3.77 \mathrm{E}-06 \\
6.47 \mathrm{E}-04 \\
5.13 \mathrm{E}-04 \\
7.81 \mathrm{E}-04 \\
7.72 \mathrm{E}-06 \\
4.38 \mathrm{a}-06 \\
9.56 \mathrm{E}-04 \\
1.13 \mathrm{E}-05 \\
4.88 \mathrm{E}-08 \\
1.74 \mathrm{E}-08\end{array}$ & \\
\hline
\end{tabular}


Destination: LANL

\begin{tabular}{|c|c|c|c|}
\hline Shipping Site: ITRI & $\begin{array}{l}\text { Total Volume } \\
(\mathrm{m} 3 / \mathrm{yr})\end{array}$ & $\begin{array}{c}\text { Total Mass } \\
(\mathrm{kg} / \mathrm{yr})\end{array}$ & $1.64 E+05$ \\
\hline & $\begin{array}{l}\text { Radionuclide } \\
H-3 \\
\mathrm{C}-14 \\
\mathrm{Mn}-54 \\
\mathrm{Co}-58 \\
\mathrm{Co}-60 \\
\mathrm{Sr}-90 \\
\mathrm{Y}-90 \\
\mathrm{TC}-99 \\
\mathrm{Cs}-134 \\
\mathrm{Cs}-137 \\
\mathrm{Ba}-137 \mathrm{~m} \\
\mathrm{U}-238\end{array}$ & $\begin{array}{c}\begin{array}{c}\text { Acrivity } \\
\text { (Ci/yr) }\end{array} \\
6.17 \mathrm{E}+00 \\
3.03 \mathrm{E}-01 \\
3.41 \mathrm{E}+01 \\
3.15 \mathrm{E}+01 \\
9.12 \mathrm{E}+01 \\
4.28 \mathrm{0}+01 \\
4.28 \mathrm{E}+01 \\
6.07 \mathrm{E}-01 \\
7.07 \mathrm{E}+01 \\
9.33 \mathrm{E}+01 \\
8.82 \mathrm{E}+01 \\
3.69 \mathrm{E}+00\end{array}$ & \\
\hline Shipping Site: SNLA & $\begin{array}{l}\text { Total Volume } \\
(\mathrm{m} 3 / \mathrm{Yr})\end{array}$ & $\begin{array}{cc}1.19 \mathrm{E}+02 & \begin{array}{c}\text { Total Mass } \\
(\mathrm{kg} / \mathrm{yr})\end{array}\end{array}$ & $3.28 \mathrm{E}+05$ \\
\hline & 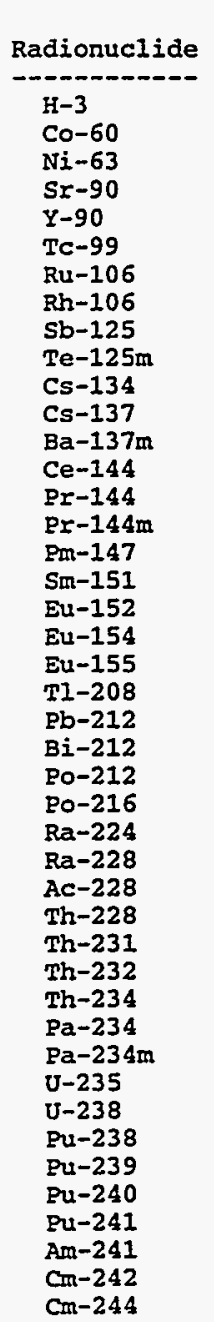 & $\begin{array}{c}\text { Activity } \\
\text { (Ci/ys) } \\
3.00 \mathrm{E}+00 \\
3.85 \mathrm{E}-05 \\
5.67 \mathrm{E}-05 \\
5.04 \mathrm{E}-03 \\
5.04 \mathrm{E}-03 \\
9.00 \mathrm{E}-07 \\
4.92 \mathrm{E}-04 \\
4.92 \mathrm{E}-04 \\
1.41 \mathrm{E}-04 \\
3.45 \mathrm{E}-05 \\
5.72 \mathrm{E}-04 \\
5.28 \mathrm{E}-03 \\
4.99 \mathrm{E}-03 \\
1.64 \mathrm{E}-03 \\
1.64 \mathrm{E}-03 \\
1.98 \mathrm{E}-05 \\
4.02 \mathrm{E}-03 \\
3.93 \mathrm{E}-05 \\
6.00 \mathrm{E}-07 \\
1.02 \mathrm{E}-04 \\
8.16 \mathrm{E}-05 \\
5.10 \mathrm{E}-06 \\
1.35 \mathrm{E}-05 \\
1.35 \mathrm{E}-05 \\
8.70 \mathrm{E}-06 \\
1.35 \mathrm{E}-05 \\
1.35 \mathrm{E}-05 \\
8.07 \mathrm{E}-05 \\
8.07 \mathrm{E}-05 \\
1.35 \mathrm{E}-05 \\
7.77 \mathrm{E}-05 \\
8.19 \mathrm{E}-04 \\
9.96 \mathrm{E}-02 \\
1.02 \mathrm{E}-05 \\
9.96 \mathrm{E}-02 \\
7.74 \mathrm{E}-05 \\
9.96 \mathrm{E}-02 \\
7.87 \mathrm{E}-02 \\
6.00 \mathrm{E}-03 \\
2.10 \mathrm{E}-02 \\
2.89 \mathrm{E}+00 \\
1.21 \mathrm{E}-04 \\
1.68 \mathrm{E}-03 \\
6.00 \mathrm{a}-04\end{array}$ & \\
\hline
\end{tabular}


Destination: LLNL

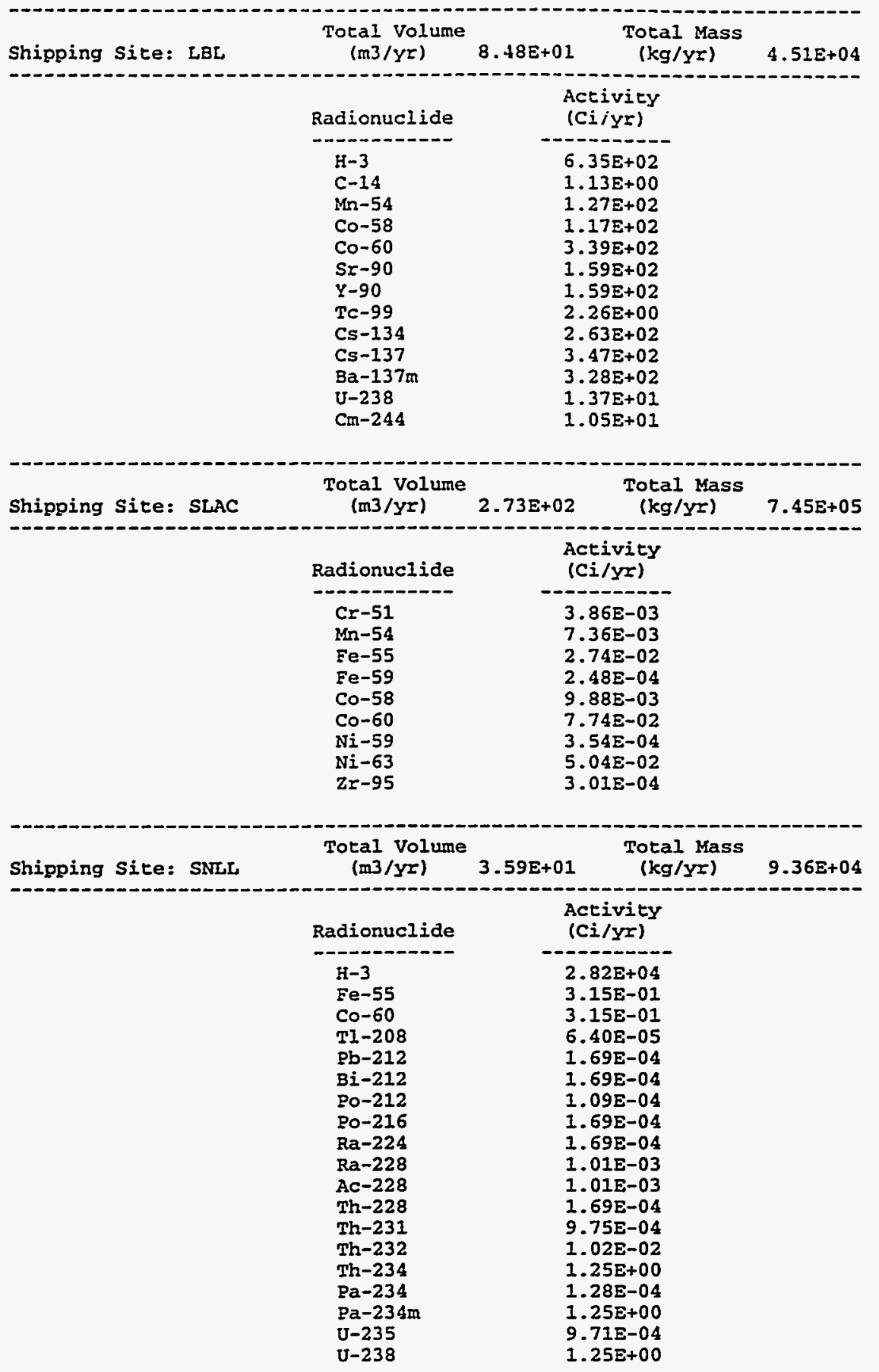


Destination: ORNL

\begin{tabular}{|c|c|c|c|}
\hline Shipping Site: K-25 & $\begin{array}{l}\text { Total Volume } \\
(\mathrm{m} 3 / \mathrm{yr})\end{array}$ & $\begin{array}{c}\text { Total Mass } \\
(\mathrm{kg} / \mathrm{y} \tau)\end{array}$ & $4.67 \varepsilon+07$ \\
\hline & $\begin{array}{l}\text { Radionuclide } \\
-\mathrm{Tl}-208 \\
\mathrm{~Pb}-212 \\
\mathrm{Bi}-212 \\
\mathrm{Po}-212 \\
\mathrm{Po}-216 \\
\mathrm{Ra}-224 \\
\mathrm{Ra}-228 \\
\mathrm{Ac}-228 \\
\mathrm{Th}-228 \\
\mathrm{Th}-231 \\
\mathrm{Th}-232 \\
\mathrm{Th}-234 \\
\mathrm{~Pa}-234 \\
\mathrm{~Pa}-234 \mathrm{~m} \\
\mathrm{U}-235 \\
\mathrm{U}-238\end{array}$ & \multicolumn{2}{|l|}{$\begin{array}{l}1.06 \mathrm{E}-04 \\
2.82 \mathrm{E}-04 \\
2.82 \mathrm{E}-04 \\
1.82 \mathrm{E}-04 \\
2.82 \mathrm{E}-04 \\
2.82 \mathrm{E}-04 \\
1.68 \mathrm{E}-03 \\
1.68 \mathrm{E}-03 \\
2.82 \mathrm{E}-04 \\
1.62 \mathrm{E}-03 \\
1.71 \mathrm{E}-02 \\
2.08 \mathrm{E}+00 \\
2.13 \mathrm{E}-04 \\
2.08 \mathrm{E}+00 \\
1.62 \mathrm{E}-03 \\
2.08 \mathrm{E}+00\end{array}$} \\
\hline \multirow[t]{2}{*}{ Shipping Site: ORISE } & $\begin{array}{l}\text { Total Volume } \\
(\mathrm{m} 3 / \mathrm{yr})\end{array}$ & $\begin{array}{c}\text { Total Mass } \\
(\mathrm{kg} / \mathrm{yr})\end{array}$ & $7.36 \mathrm{E}+04$ \\
\hline & $\begin{array}{l}\text { Radionuclide } \\
\mathrm{H}-3 \\
\mathrm{C}-14 \\
\mathrm{Mn}-54 \\
\mathrm{Co}-58 \\
\mathrm{Co}-60 \\
\mathrm{Sr}-90 \\
\mathrm{Y}-90 \\
\mathrm{TC}-99 \\
\mathrm{Cs}-134 \\
\mathrm{Cs}-137 \\
\mathrm{Ba}-137 \mathrm{~m} \\
\mathrm{Tl}-208 \\
\mathrm{~Pb}-212 \\
\mathrm{Bi}-212 \\
\mathrm{PO}-212 \\
\mathrm{PO}-216 \\
\mathrm{Ra}-224 \\
\mathrm{Ra}-228 \\
\mathrm{Ac}-228 \\
\mathrm{Th}-228 \\
\mathrm{Th}-231 \\
\mathrm{Th}-232 \\
\mathrm{Th}-234 \\
\mathrm{~Pa}-234 \\
\mathrm{~Pa}-234 \mathrm{~m} \\
\mathrm{U}-235 \\
\mathrm{U}-238\end{array}$ & \multicolumn{2}{|l|}{ 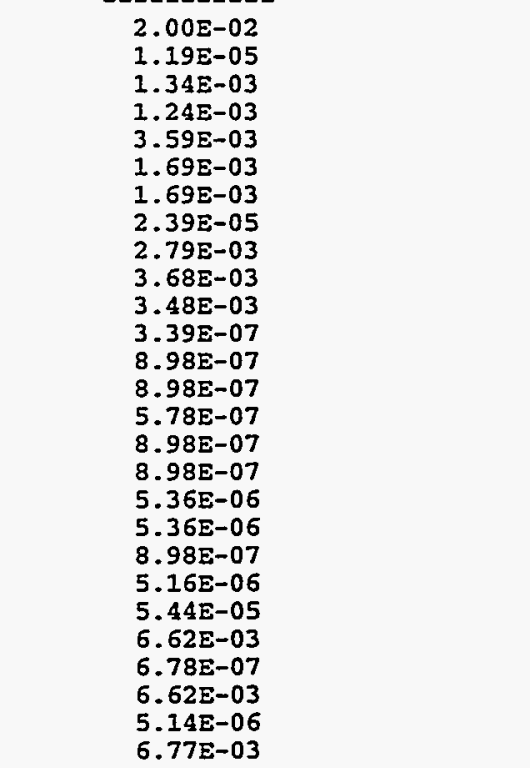 } \\
\hline \multirow[t]{2}{*}{ Shipping Site: $Y-12$} & $\begin{array}{l}\text { Total volume } \\
\text { (m3/yr) }\end{array}$ & 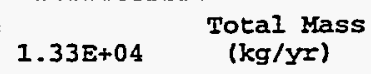 & $8.27 \mathrm{E}+07$ \\
\hline & $\begin{array}{l}\text { Radionuclide } \\
\mathrm{TI}-208 \\
\mathrm{~Pb}-212 \\
\mathrm{Bi}-212 \\
\mathrm{Po-212} \\
\mathrm{Po-216} \\
\mathrm{Ra-224} \\
\mathrm{Ra}-228 \\
\mathrm{Ac}-228 \\
\mathrm{Th}-228 \\
\mathrm{Th}-231 \\
\mathrm{Th}-232 \\
\mathrm{Th}-234 \\
\mathrm{~Pa}-234 \\
\mathrm{~Pa}-234 \mathrm{~m} \\
\mathrm{U}-235 \\
\mathrm{U}-238\end{array}$ & $\begin{array}{c}\begin{array}{c}\text { Activity } \\
(C i / y z)\end{array} \\
6.80 \mathrm{E}-05 \\
1.80 \mathrm{E}-04 \\
1.80 \mathrm{E}-04 \\
1.16 \mathrm{E}-04 \\
1.80 \mathrm{E}-04 \\
1.80 \mathrm{E}-04 \\
1.07 \mathrm{E}-03 \\
1.07 \mathrm{E}-03 \\
1.80 \mathrm{E}-04 \\
1.03 \mathrm{E}-03 \\
1.09 \mathrm{E}-02 \\
1.32 \mathrm{E}+00 \\
1.36 \mathrm{E}-04 \\
1.32 \mathrm{E}+00 \\
1.03 \mathrm{E}-03 \\
1.32 \mathrm{E}+00\end{array}$ & . \\
\hline
\end{tabular}


Destination: PORTS

\begin{tabular}{|c|c|c|c|}
\hline Shipping Site: AMES & $\begin{array}{l}\text { Total Volume } \\
\text { (m3/yx) }\end{array}$ & $\begin{array}{l}\text { Total Mass } \\
\text { (kg/yr) }\end{array}$ & $2.98 \mathrm{E}+04$ \\
\hline , & $\begin{array}{l}\text { Radionuclide } \\
\mathrm{Tl}-208 \\
\mathrm{~Pb}-212 \\
\mathrm{Bi}-212 \\
\mathrm{Po}-212 \\
\mathrm{Po}-216 \\
\mathrm{Ra}-224 \\
\mathrm{Ra}-228 \\
\mathrm{Ac}-228 \\
\mathrm{Th}-228 \\
\mathrm{Th}-231 \\
\mathrm{Th}-232 \\
\mathrm{Th}-234 \\
\mathrm{~Pa}-234 \\
\mathrm{~Pa}-234 \mathrm{~m} \\
\mathrm{U}-235 \\
\mathrm{U}-238 \\
\mathrm{Pu}-238 \\
\mathrm{Pu}-239 \\
\mathrm{Pu}-240 \\
\mathrm{Pu}-241 \\
\mathrm{Am}-241 \\
\mathrm{Cm}-242 \\
\mathrm{Cm}-244\end{array}$ & $\begin{array}{l}\text { Activity } \\
(\mathrm{Ci} / Y \mathrm{Y}) \\
4.22 \mathrm{E}-07 \\
1.11 \mathrm{E}-06 \\
1.11 \mathrm{E}-06 \\
7.20 \mathrm{E}-07 \\
1.11 \mathrm{E}-06 \\
1.11 \mathrm{E}-06 \\
6.68 \mathrm{E}-06 \\
6.68 \mathrm{E}-06 \\
1.11 \mathrm{E}-06 \\
6.43 \mathrm{E}-06 \\
6.77 \mathrm{E}-05 \\
8.24 \mathrm{E}-03 \\
8.44 \mathrm{E}-07 \\
8.24 \mathrm{E}-03 \\
6.40 \mathrm{E}-06 \\
8.24 \mathrm{E}-03 \\
6.50 \mathrm{E}-04 \\
4.96 \mathrm{E}-05 \\
1.73 \mathrm{E}-04 \\
2.39 \mathrm{E}-02 \\
9.93 \mathrm{E}-07 \\
1.39 \mathrm{E}-05 \\
4.96 \mathrm{E}-06\end{array}$ & \\
\hline Shipping Sice: ANL-E & $\begin{array}{l}\text { Total Volume } \\
\text { (m3/yx) }\end{array}$ & $\begin{array}{l}7.35 E+02 \quad \text { Total Mass } \\
(\mathrm{kg} / \mathrm{Y})\end{array}$ & $2.005+06$ \\
\hline & $\begin{array}{l}\text { Radionuclide } \\
\mathrm{H}-3 \\
\mathrm{C}-14 \\
\mathrm{Cr}-51 \\
\mathrm{Mn}-54 \\
\mathrm{Fe}-55 \\
\mathrm{Fe}-59 \\
\mathrm{Co}-58 \\
\mathrm{Co}-60 \\
\mathrm{Ni}-59 \\
\mathrm{Ni}-63 \\
\mathrm{Sr}-90 \\
\mathrm{Y}-90 \\
\mathrm{Zr}-95 \\
\mathrm{Tc}-99 \\
\mathrm{Ru}-106 \\
\mathrm{Rh}-106 \\
\mathrm{Sb}-125 \\
\mathrm{Te}-125 \mathrm{~m} \\
\mathrm{Cs}-134 \\
\mathrm{Cs}-137 \\
\mathrm{Ba}-137 \mathrm{~m} \\
\mathrm{Ce}-144 \\
\mathrm{Pr}-144 \\
\mathrm{Pr}-144 \mathrm{~m} \\
\mathrm{Pm}-147 \\
\mathrm{Sm}-151 \\
\mathrm{Eu}-152 \\
\mathrm{Eu}-154 \\
\mathrm{Eu}-155 \\
\mathrm{U}-238 \\
\mathrm{Pu}-238 \\
\mathrm{Pu}-239 \\
\mathrm{Pu}-240 \\
\mathrm{Pu}-241 \\
\mathrm{Am}-241 \\
\end{array}$ & $\begin{array}{c}\text { Activity } \\
\text { (Ci/y工) } \\
-1.33 E+02 \\
1.91 \mathrm{E}-02 \\
2.87 \mathrm{E}+00 \\
1.43 \mathrm{E}+01 \\
2.04 \mathrm{E}+01 \\
1.84 \mathrm{E}-01 \\
1.55 \mathrm{E}+01 \\
8.15 \mathrm{E}+01 \\
2.63 \mathrm{E}-01 \\
3.77 \mathrm{E}+01 \\
3.33 \mathrm{E}+01 \\
3.33 \mathrm{E}+01 \\
2.24 \mathrm{E}-01 \\
1.62 \mathrm{E}-01 \\
2.16 \mathrm{E}+00 \\
2.16 \mathrm{E}+00 \\
6.21 \mathrm{E}-01 \\
1.51 \mathrm{E}-01 \\
2.09 \mathrm{E}+01 \\
4.75 \mathrm{E}+01 \\
4.50 \mathrm{E}+01 \\
7.24 \mathrm{E}+00 \\
7.24 \mathrm{E}+00 \\
8.70 \mathrm{E}-02 \\
1.76 \mathrm{E}+01 \\
1.72 \mathrm{E}-01 \\
2.63 \mathrm{E}-03 \\
4.52 \mathrm{E}-01 \\
3.58 \mathrm{E}-01 \\
9.63 \mathrm{E}-01 \\
5.44 \mathrm{E}-01 \\
5.27 \mathrm{E}-03 \\
2.63 \mathrm{E}-03 \\
6.09 \mathrm{E}-01 \\
7.91 \mathrm{E}-03\end{array}$ & \\
\hline
\end{tabular}


TABLE 2.1 Summary of WM LLW Cases

\begin{tabular}{|c|c|}
\hline Alternative & Description \\
\hline No Action (Case 1) & $\begin{array}{l}\text { All sites use existing and approved } \\
\text { treatment facilities and dispose of } \\
\text { waste at } 6 \text { sites per current } \\
\text { arrangements. }\end{array}$ \\
\hline Decentralized (Case 2) & $\begin{array}{l}\text { All sites minimally treat and } \\
\text { stabilize liquids and fines and } \\
\text { dispose of waste at } 16 \text { sites. }\end{array}$ \\
\hline Regionalized 1 (Case 3) & $\begin{array}{l}\text { Same as Case } 2 \text {, except dispose of } \\
\text { waste at } 12 \text { sites. }\end{array}$ \\
\hline Regionalized 2 (Case 9) & $\begin{array}{l}\text { Regional treatment of volume- } \\
\text { reducible wastes at } 11 \text { sites (thermal } \\
\text { treatment, supercompaction, and } \\
\text { size reduction); all sites minimally } \\
\text { treat other wastes and dispose of } \\
\text { waste at } 12 \text { sites. }\end{array}$ \\
\hline Regionalized 3 (Case 4) & $\begin{array}{l}\text { Same as Case } 2 \text {, except dispose of } \\
\text { waste at } 6 \text { sites. }\end{array}$ \\
\hline Regionalized 4 (Case 12) & $\begin{array}{l}\text { Regional treatment of volume- } \\
\text { reducible wastes at } 7 \text { sites; all sites } \\
\text { minimally treat other wastes and } \\
\text { dispose of waste at } 6 \text { sites. }\end{array}$ \\
\hline Regionalized 5 (Case 19) & $\begin{array}{l}\text { Regional treatment of volume- } \\
\text { reducible waste at } 4 \text { sites; all sites } \\
\text { minimally treat other wastes and } \\
\text { dispose of waste at } 6 \text { sites. }\end{array}$ \\
\hline Regionalized 6 (Case 5) & $\begin{array}{l}\text { Same as Case } 2 \text {, except dispose of } \\
\text { waste at } 2 \text { sites. }\end{array}$ \\
\hline Regionalized 7 (Case 6) & $\begin{array}{l}\text { Same as Case } 2 \text {, except dispose of } \\
\text { waste at } 2 \text { sites. }\end{array}$ \\
\hline Centralized 1 (Case 7) & $\begin{array}{l}\text { Same as Case 2, except dispose of } \\
\text { waste at } 1 \text { site. }\end{array}$ \\
\hline Centralized 2 (Case 8) & $\begin{array}{l}\text { Same as Case } 2 \text {, except dispose of } \\
\text { waste at } 1 \text { site. }\end{array}$ \\
\hline Centralized 3 (Case 14) & $\begin{array}{l}\text { Same as Case } 12 \text {, except dispose of } \\
\text { waste at } 1 \text { site. }\end{array}$ \\
\hline Centralized 4 (Case 14a) & $\begin{array}{l}\text { Same as Case } 12 \text {, except dispose of } \\
\text { waste at } 1 \text { site. }\end{array}$ \\
\hline Centralized 5 (Case 21) & $\begin{array}{l}\text { One site thermally treats, } \\
\text { supercompacts, and reduces size of } \\
\text { volume-reducible waste; all sites } \\
\text { minimally treat other wastes; } 1 \text { site } \\
\text { disposes of waste. }\end{array}$ \\
\hline
\end{tabular}


A-50

\begin{tabular}{|c|c|c|c|}
\hline Shipping Site: BAPL & $\begin{array}{l}\text { Total Volume } \\
\text { (m3/yr) }\end{array}$ & $\begin{array}{c}\text { Total Mass } \\
(\mathrm{kg} / \mathrm{Y} \mathrm{r})\end{array}$ & $1.08 \mathrm{E}+06$ \\
\hline & $\begin{array}{l}\text { Radionuclide } \\
\mathrm{H}-3 \\
\mathrm{Co}-60 \\
\mathrm{Ni}-63 \\
\mathrm{Sr}-90 \\
\mathrm{Y}-90 \\
\mathrm{Tc}-99 \\
\mathrm{Ru}-106 \\
\mathrm{Rh}-106 \\
\mathrm{Sb}-125 \\
\mathrm{Te}-125 \mathrm{~m} \\
\mathrm{Cs}-134 \\
\mathrm{Cs}-137 \\
\mathrm{Ba}-137 \mathrm{~m} \\
\mathrm{Ce}-144 \\
\mathrm{P}-144 \\
\mathrm{Pr}-144 \mathrm{~m} \\
\mathrm{Pm}-147 \\
\mathrm{Sm}-151 \\
\mathrm{Eu}-152 \\
\mathrm{Eu}-154 \\
\mathrm{Eu}-155 \\
\mathrm{~T} 1-208 \\
\mathrm{~Pb}-212 \\
\mathrm{Bi}-212 \\
\mathrm{Po}-212 \\
\mathrm{Po}-216 \\
\mathrm{Ra}-224 \\
\mathrm{Ra}-228 \\
\mathrm{Ac}-228 \\
\mathrm{Th}-228 \\
\mathrm{Th}-231 \\
\mathrm{Th}-232 \\
\mathrm{Th}-234 \\
\mathrm{~Pa}-234 \\
\mathrm{~Pa}-234 \mathrm{~m} \\
\mathrm{U}-235 \\
\mathrm{U}-238 \\
\mathrm{Pu}-238 \\
\mathrm{Pu}-239 \\
\mathrm{Pu}-240 \\
\mathrm{Pu}-241 \\
\mathrm{Am}-241 \\
\end{array}$ & $\begin{array}{l}\text { Activicy } \\
\text { (Ci/yr) } \\
-2.51 \mathrm{E}-03 \\
1.19 \mathrm{E}-02 \\
2.38 \mathrm{E}-02 \\
2.11 \mathrm{E}+00 \\
2.11 \mathrm{E}+00 \\
3.77 \mathrm{E}-04 \\
2.06 \mathrm{E}-01 \\
2.06 \mathrm{E}-01 \\
5.93 \mathrm{E}-02 \\
1.44 \mathrm{E}-02 \\
2.40 \mathrm{E}-01 \\
2.21 \mathrm{E}+00 \\
2.09 \mathrm{E}+00 \\
6.91 \mathrm{E}-01 \\
6.91 \mathrm{E}-01 \\
8.31 \mathrm{E}-03 \\
1.68 \mathrm{E}+00 \\
1.65 \mathrm{E}-02 \\
2.51 \mathrm{E}-04 \\
4.32 \mathrm{E}-02 \\
3.42 \mathrm{E}-02 \\
3.80 \mathrm{E}-06 \\
1.00 \mathrm{E}-05 \\
1.00 \mathrm{E}-05 \\
6.49 \mathrm{E}-06 \\
1.00 \mathrm{E}-05 \\
1.00 \mathrm{E}-05 \\
6.02 \mathrm{E}-05 \\
6.02 \mathrm{E}-05 \\
1.00 \mathrm{E}-05 \\
5.80 \mathrm{E}-05 \\
6.11 \mathrm{E}-04 \\
7.43 \mathrm{E}-02 \\
7.61 \mathrm{E}-06 \\
7.43 \mathrm{E}-02 \\
5.77 \mathrm{E}-05 \\
7.43 \mathrm{E}-02 \\
5.20 \mathrm{E}-02 \\
5.03 \mathrm{E}-04 \\
2.51 \mathrm{E}-04 \\
5.81 \mathrm{E}-02 \\
7.55 \mathrm{E}-04\end{array}$ & \\
\hline Shipping Site: FEMP & $\begin{array}{l}\text { Total Volume } \\
(\mathrm{m} 3 / \mathrm{Y})\end{array}$ & $\begin{array}{c}\text { Total Mass } \\
4.99 \mathrm{E}+01\end{array}$ & $1.30 \mathrm{E}+05$ \\
\hline ' & $\begin{array}{l}\text { Radionuclide } \\
\mathrm{H}-3 \\
\mathrm{Tl}-208 \\
\mathrm{~Pb}-212 \\
\mathrm{Bi}-212 \\
\mathrm{Po}-212 \\
\mathrm{Po}-216 \\
\mathrm{Ra}-224 \\
\mathrm{Ra}-228 \\
\mathrm{Ac}-228 \\
\mathrm{Th}-228 \\
\mathrm{Th}-231 \\
\mathrm{Th}-232 \\
\mathrm{Th}-234 \\
\mathrm{~Pa}-234 \\
\mathrm{~Pa}-234 \mathrm{~m} \\
\mathrm{U}-235 \\
\mathrm{U}-238 \\
\mathrm{Pu}-238 \\
\mathrm{Pu}-239 \\
\mathrm{Pu}-240 \\
\mathrm{Pu}-241 \\
\mathrm{Am}-241 \\
\mathrm{Cm}-242 \\
\mathrm{Cm}-244\end{array}$ & $\begin{array}{l}\text { Activity } \\
\text { (Ci/yr) } \\
-2.24 \mathrm{E}+02 \\
2.83 \mathrm{E}-08 \\
7.50 \mathrm{E}-08 \\
7.50 \mathrm{E}-08 \\
4.83 \mathrm{E}-08 \\
7.50 \mathrm{E}-08 \\
7.50 \mathrm{E}-08 \\
4.48 \mathrm{E}-07 \\
4.48 \mathrm{E}-07 \\
7.50 \mathrm{E}-08 \\
4.31 \mathrm{E}-07 \\
4.54 \mathrm{E}-06 \\
5.53 \mathrm{E}-04 \\
5.67 \mathrm{E}-08 \\
5.53 \mathrm{E}-04 \\
4.30 \mathrm{E}-07 \\
5.53 \mathrm{E}-04 \\
4.36 \mathrm{E}-05 \\
3.33 \mathrm{E}-06 \\
1.16 \mathrm{E}-05 \\
1.60 \mathrm{E}-03 \\
6.67 \mathrm{E}-08 \\
9.33 \mathrm{E}-07 \\
3.33 \mathrm{E}-07\end{array}$ & 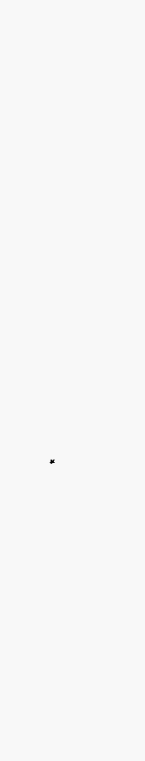 \\
\hline
\end{tabular}


A-5l

\begin{tabular}{|c|c|c|}
\hline Shipping Site: FNAL & $\begin{array}{l}\text { Total volume } \\
\text { (m3/yr) }\end{array}$ & $\begin{array}{l}\text { Total Mass } \\
(\mathrm{kg} / \mathrm{Yr})\end{array} \quad 3.26 \mathrm{E}+04$ \\
\hline & \begin{tabular}{l} 
Radionuclide \\
\hdashline$H-3$ \\
$\mathrm{Cr}-51$ \\
$\mathrm{Mn}-54$ \\
$\mathrm{Fe}-55$ \\
$\mathrm{Fe}-59$ \\
$\mathrm{Co}-58$ \\
$\mathrm{Co}-60$ \\
$\mathrm{Ni}-59$ \\
$\mathrm{Ni}-63$ \\
$\mathrm{Zr}-95$ \\
$\mathrm{~T} 1-208$ \\
$\mathrm{~Pb}-212$ \\
$\mathrm{Bi}-212$ \\
$\mathrm{Po}-212$ \\
$\mathrm{Po}-216$ \\
$\mathrm{Ra}-224$ \\
$\mathrm{Ra}-228$ \\
$\mathrm{Ac}-228$ \\
$\mathrm{Th}-228$ \\
$\mathrm{Th}-231$ \\
$\mathrm{Th}-232$ \\
$\mathrm{Th}-234$ \\
$\mathrm{~Pa}-234$ \\
$\mathrm{~Pa}-234 \mathrm{~m}$ \\
$\mathrm{U}-235$ \\
$\mathrm{U}-238$
\end{tabular} & $\begin{array}{l}\text { Activity } \\
\text { (Ci/yr) } \\
1.08 \mathrm{E}-01 \\
1.11 \mathrm{E}-02 \\
2.12 \mathrm{E}-02 \\
7.94 \mathrm{E}-02 \\
7.17 \mathrm{E}-04 \\
2.85 \mathrm{E}-02 \\
2.23 \mathrm{E}-01 \\
1.02 \mathrm{E}-03 \\
1.45 \mathrm{E}-01 \\
8.71 \mathrm{E}-04 \\
3.78 \mathrm{E}-07 \\
1.00 \mathrm{E}-06 \\
1.00 \mathrm{E}-06 \\
6.46 \mathrm{E}-07 \\
1.00 \mathrm{E}-06 \\
1.00 \mathrm{E}-06 \\
5.99 \mathrm{E}-06 \\
5.99 \mathrm{E}-06 \\
1.00 \mathrm{E}-06 \\
5.77 \mathrm{E}-06 \\
6.08 \mathrm{E}-05 \\
7.39 \mathrm{E}-03 \\
7.57 \mathrm{E}-07 \\
7.39 \mathrm{E}-03 \\
5.75 \mathrm{E}-06 \\
7.39 \mathrm{E}-03\end{array}$ \\
\hline
\end{tabular}


A-52

\begin{tabular}{|c|c|c|c|}
\hline Shipping Sice: KAPL & $\begin{array}{l}\text { Total volume } \\
\text { (m3/yr) }\end{array}$ & $2.11 E+02 \quad \begin{array}{c}\text { Total Mass } \\
(\mathrm{kg} / \mathrm{Yr})\end{array}$ & $5.36 E+05$ \\
\hline & 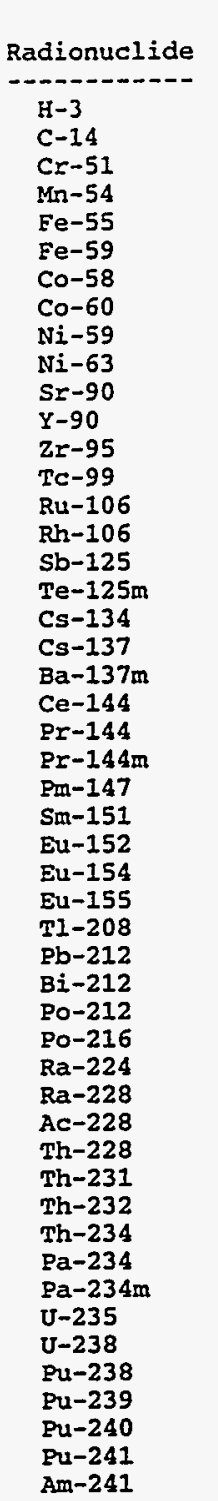 & $\begin{array}{l}\text { Accivicy } \\
\text { (Ci/Yr) } \\
-3.14 \mathrm{E}-04 \\
2.40 \mathrm{E}-05 \\
5.11 \mathrm{E}-03 \\
1.24 \mathrm{E}-02 \\
3.63 \mathrm{E}-02 \\
3.28 \mathrm{E}-04 \\
1.55 \mathrm{E}-02 \\
1.09 \mathrm{E}-01 \\
4.69 \mathrm{E}-04 \\
6.70 \mathrm{E}-02 \\
2.58 \mathrm{E}-02 \\
2.58 \mathrm{E}-02 \\
3.99 \mathrm{E}-04 \\
5.20 \mathrm{E}-05 \\
2.19 \mathrm{E}-03 \\
2.19 \mathrm{E}-03 \\
6.31 \mathrm{E}-04 \\
1.54 \mathrm{E}-04 \\
8.14 \mathrm{E}-03 \\
3.09 \mathrm{E}-02 \\
2.92 \mathrm{E}-02 \\
7.35 \mathrm{E}-03 \\
7.35 \mathrm{E}-03 \\
8.84 \mathrm{E}-05 \\
1.79 \mathrm{E}-02 \\
1.75 \mathrm{E}-04 \\
2.67 \mathrm{E}-06 \\
4.59 \mathrm{E}-04 \\
3.64 \mathrm{E}-04 \\
4.50 \mathrm{E}-09 \\
1.19 \mathrm{E}-08 \\
1.19 \mathrm{E}-08 \\
7.60 \mathrm{E}-09 \\
1.19 \mathrm{E}-08 \\
1.19 \mathrm{E}-08 \\
7.11 \mathrm{E}-08 \\
7.11 \mathrm{E}-08 \\
1.19 \mathrm{E}-08 \\
6.84 \mathrm{E}-08 \\
7.21 \mathrm{E}-07 \\
8.77 \mathrm{E}-05 \\
8.90 \mathrm{E}-09 \\
8.77 \mathrm{E}-05 \\
6.82 \mathrm{E}-08 \\
3.79 \mathrm{E}-04 \\
5.53 \mathrm{E}-04 \\
5.35 \mathrm{E}-06 \\
2.67 \mathrm{E}-06 \\
6.18 \mathrm{E}-04 \\
8.03 \mathrm{E}-06\end{array}$ & \\
\hline Shipping Sice: MOUND & $\begin{array}{l}\text { Total Volume } \\
(\mathrm{m} 3 / \mathrm{yx})\end{array}$ & $\begin{array}{c}\text { Total Mass } \\
\text { (kg/Yr) }\end{array}$ & \\
\hline & $\begin{array}{l}\text { Radionuclide } \\
\mathrm{H}-3 \\
\mathrm{Pu}-238 \\
\mathrm{Pu}-239 \\
\mathrm{Pu}-240 \\
\mathrm{Pu}-241 \\
\mathrm{Am}-241 \\
\mathrm{Cm}-242 \\
\mathrm{Cn}-244\end{array}$ & $\begin{array}{l}\text { Activity } \\
\text { (Ci/yr) } \\
1.59 \mathrm{E}+00 \\
1.60 \mathrm{E}-01 \\
1.22 \mathrm{E}-02 \\
4.28 \mathrm{E}-02 \\
5.90 \mathrm{E}+00 \\
2.44 \mathrm{E}-04 \\
3.42 \mathrm{E}-03 \\
1.22 \mathrm{E}-03\end{array}$ & \\
\hline
\end{tabular}


$A-53$

\begin{tabular}{|c|c|c|c|}
\hline Shipping Sice: PPPL & $\begin{array}{l}\text { Total volume } \\
\qquad\left(\mathrm{m} 3 / y^{\prime}\right)\end{array}$ & $\begin{array}{cc}\text { Tolal Mass } \\
(\mathrm{kg} / \mathrm{Yr})\end{array}$ & $4.97 \mathrm{E}+03$ \\
\hline & $\begin{array}{l}\text { Radionuclide } \\
\mathrm{H}-3 \\
\mathrm{Cr}-51 \\
\mathrm{Mn}-54 \\
\mathrm{Fe}-55 \\
\mathrm{Fe}-59 \\
\mathrm{Co}-58 \\
\mathrm{Co}-60 \\
\mathrm{Ni}-59 \\
\mathrm{Ni}-63 \\
\mathrm{Z}-95\end{array}$ & $\begin{array}{l}\text { Activicy } \\
\text { (Ci/ys) } \\
2.01 \mathrm{E}-01 \\
8.50 \mathrm{E}-04 \\
1.61 \mathrm{E}-03 \\
6.04 \mathrm{E}-03 \\
5.46 \mathrm{E}-05 \\
2.17 \mathrm{E}-03 \\
1.70 \mathrm{E}-02 \\
7.80 \mathrm{E}-05 \\
1.10 \mathrm{E}-02 \\
6.63 \mathrm{E}-05\end{array}$ & \\
\hline Shipping Site: RMI & $\begin{array}{l}\text { Radionuclide } \\
\mathrm{Tl}-208 \\
\mathrm{~Pb}-212 \\
\mathrm{Bi}-212 \\
\mathrm{Po}-212 \\
\mathrm{Po}-216 \\
\mathrm{Ra}-224 \\
\mathrm{Ra}-228 \\
\mathrm{Ac}-228 \\
\mathrm{Th}-228 \\
\mathrm{Th}-231 \\
\mathrm{Th}-232 \\
\mathrm{Th}-234 \\
\mathrm{~Pa}-234 \\
\mathrm{~Pa}-234 \mathrm{~m} \\
\mathrm{U}-235 \\
\mathrm{U}-238\end{array}$ & $\begin{array}{c}5.63 \mathrm{E}+03 \quad \begin{array}{c}\text { Total Mass } \\
(\mathrm{kg} / \mathrm{yr})\end{array} \\
\begin{array}{c}\text { Activity } \\
\text { (Ci/yr) }\end{array} \\
3.58 \mathrm{E}-07 \\
9.47 \mathrm{E}-07 \\
9.47 \mathrm{E}-07 \\
6.10 \mathrm{E}-07 \\
9.47 \mathrm{E}-07 \\
9.47 \mathrm{E}-07 \\
5.66 \mathrm{E}-06 \\
5.66 \mathrm{E}-06 \\
9.47 \mathrm{E}-07 \\
5.45 \mathrm{E}-06 \\
5.74 \mathrm{E}-05 \\
6.98 \mathrm{E}-03 \\
7.15 \mathrm{E}-07 \\
6.98 \mathrm{E}-03 \\
5.43 \mathrm{E}-06 \\
6.98 \mathrm{E}-03\end{array}$ & $1.46 \mathrm{E}+07$ \\
\hline
\end{tabular}

Destination: Paducah

\begin{tabular}{|c|c|c|c|}
\hline Shipping Site: KCP & $\begin{array}{c}\text { Total Volume } \\
(\mathrm{m} 3 / \mathrm{yr})\end{array}$ & $\begin{array}{c}\text { Total Mass } \\
(\mathrm{kg} / \mathrm{Y})\end{array}$ & $7.23 E+03$ \\
\hline & $\begin{array}{l}\text { Radionuclide } \\
H-3 \\
\text { C-14 } \\
\text { Mn-54 } \\
\text { Co-58 } \\
\text { Co-60 } \\
\text { Sr-90 } \\
\text { Y-90 } \\
\text { TC-99 } \\
\text { Cs-134 } \\
\text { Cs-137 } \\
\text { Ba-137m } \\
\text { U-238 }\end{array}$ & $\begin{array}{c}\text { Activity } \\
\text { (Ci/yx) } \\
2.70 \mathrm{E}-02 \\
1.44 \mathrm{E}-04 \\
1.62 \mathrm{E}-02 \\
1.50 \mathrm{E}-02 \\
4.34 \mathrm{E}-02 \\
2.04 \mathrm{E}-02 \\
2.04 \mathrm{E}-02 \\
2.89 \mathrm{E}-04 \\
3.37 \mathrm{E}-02 \\
4.44 \mathrm{E}-02 \\
4.20 \mathrm{E}-02 \\
1.76 \mathrm{E}-03\end{array}$ & \\
\hline
\end{tabular}

Destination: SRS

\begin{tabular}{|c|c|c|c|}
\hline Shipping Site: Pinellas & $\begin{array}{l}\text { Total Volume } \\
(\mathrm{m} 3 / \mathrm{yr})\end{array}$ & $\begin{array}{c}\text { Total Mass } \\
(\mathrm{kg} / \mathrm{yr})\end{array}$ & $1.91 E+04$ \\
\hline & $\begin{array}{l}\text { Radionuclide } \\
\mathrm{H}-3\end{array}$ & $\begin{array}{c}\begin{array}{c}\text { Activity } \\
(C i / y r)\end{array} \\
1.99 \Xi+04\end{array}$ & \\
\hline
\end{tabular}




\section{A.8 WM LLW REGIONALIZED 2 ALTERNATIVE (CASE 9): ACTIVATED METALS}

Transported Material with Radionuclide Profile

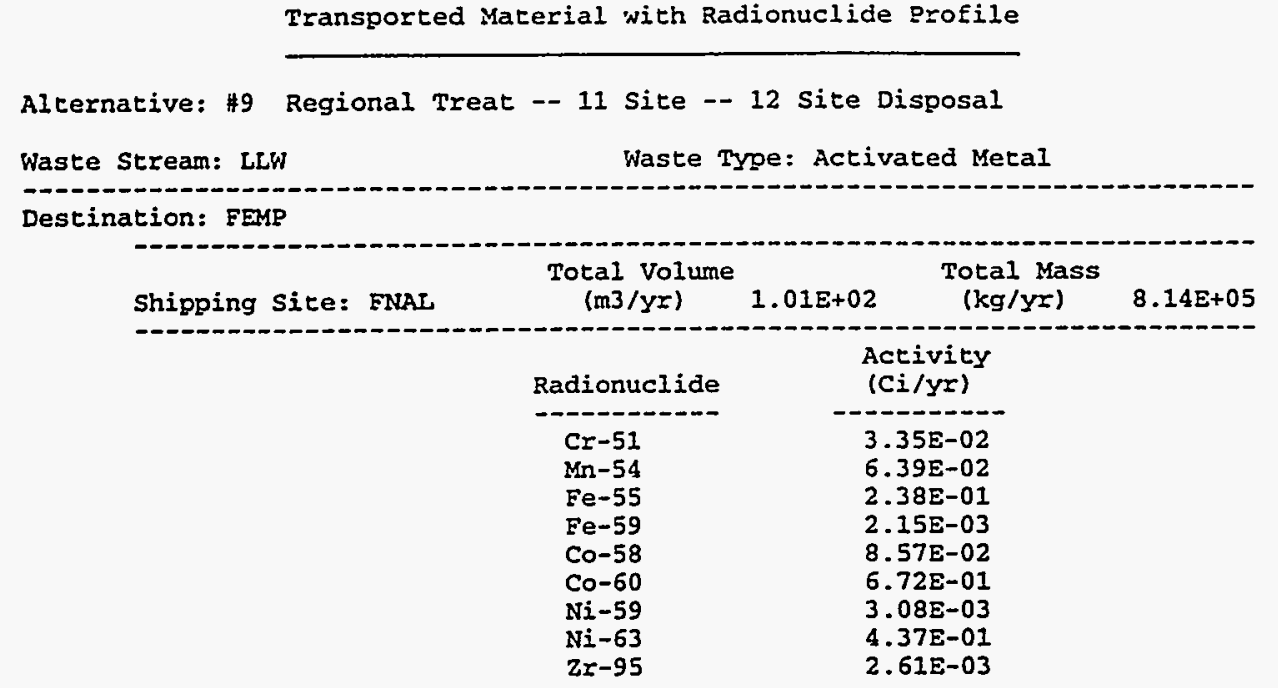

Destination: INEL

\begin{tabular}{|c|c|c|c|}
\hline Shipping Site: ANL-W & $\begin{array}{l}\text { Total Volume } \\
\qquad(\mathrm{m} 3 / \mathrm{yr})\end{array}$ & $\begin{array}{c}\text { Total Mass } \\
\text { (kg/yx) }\end{array}$ & $1.64 \mathrm{E}+05$ \\
\hline & $\begin{array}{l}\text { Radionuclide } \\
\mathrm{H}-3 \\
\mathrm{Cr}-51 \\
\mathrm{Mn}-54 \\
\mathrm{Fe}-55 \\
\mathrm{Fe}-59 \\
\mathrm{Co}-58 \\
\mathrm{Co}-60 \\
\mathrm{Ni}-59 \\
\mathrm{Ni}-63 \\
\mathrm{Sr}-90 \\
\mathrm{Y}-90 \\
\mathrm{Zr}-95 \\
\mathrm{Tc}-99 \\
\mathrm{Ru}-106 \\
\mathrm{Rh}-106 \\
\mathrm{Sb}-125 \\
\mathrm{Te}-125 \mathrm{~m} \\
\mathrm{Cs}-134 \\
\mathrm{Cs}-137 \\
\mathrm{Ba}-137 \mathrm{~m} \\
\mathrm{Ce}-144 \\
\mathrm{Pr}-144 \\
\mathrm{Pr}-144 \mathrm{~m} \\
\mathrm{Pm}-147 \\
\mathrm{Sm}-151 \\
\mathrm{Eu}-152 \\
\mathrm{Eu}-154 \\
\mathrm{Eu}-155 \\
\mathrm{Pu}-238 \\
\mathrm{Pu}-239 \\
\mathrm{Pu}-240 \\
\mathrm{Pu}-241 \\
\mathrm{Am}-241 .\end{array}$ & $\begin{array}{l}\text { Activity } \\
\text { (Ci/Yr) } \\
-1.71 E-06 \\
1.24 \mathrm{E}-04 \\
2.37 \mathrm{E}-04 \\
8.86 \mathrm{E}-04 \\
8.00 \mathrm{E}-06 \\
3.18 \mathrm{E}-04 \\
2.50 \mathrm{E}-03 \\
1.14 \mathrm{E}-05 \\
1.64 \mathrm{E}-03 \\
1.43 \mathrm{E}-03 \\
1.43 \mathrm{E}-03 \\
9.72 \mathrm{E}-06 \\
2.56 \mathrm{E}-07 \\
1.40 \mathrm{E}-04 \\
1.40 \mathrm{E}-04 \\
4.03 \mathrm{E}-05 \\
9.84 \mathrm{E}-06 \\
1.63 \mathrm{E}-04 \\
2.00 \mathrm{E}-01 \\
2.00 \mathrm{E}-01 \\
4.69 \mathrm{E}-04 \\
4.69 \mathrm{E}-04 \\
5.64 \mathrm{E}-06 \\
1.14 \mathrm{E}-03 \\
1.12 \mathrm{E}-05 \\
1.71 \mathrm{E}-07 \\
2.93 \mathrm{E}-05 \\
2.32 \mathrm{E}-05 \\
3.53 \mathrm{E}-05 \\
3.42 \mathrm{E}-07 \\
1.71 \mathrm{E}-07 \\
3.95 \mathrm{E}-05 \\
5.13 \mathrm{E}-07\end{array}$ & \\
\hline
\end{tabular}


A-55

\begin{tabular}{|c|c|c|c|}
\hline Shipping Site: NRE & $\begin{array}{l}\text { Total Volume } \\
\text { (m3/yr) }\end{array}$ & $\begin{array}{l}\text { Total Mass } \\
(\mathrm{kg} / \mathrm{Yr})\end{array}$ & $9.40 E+06$ \\
\hline & $\begin{array}{l}\text { Radionuclide } \\
\mathrm{Cr}-51 \\
\mathrm{Mn}-54 \\
\mathrm{Fe}-55 \\
\mathrm{Fe}-59 \\
\mathrm{Co}-58 \\
\mathrm{Co}-60 \\
\mathrm{Ni}-59 \\
\mathrm{Ni}-63 \\
\mathrm{Zr}-95\end{array}$ & $\begin{array}{c}\begin{array}{l}\text { Activity } \\
(C i / Y I)\end{array} \\
-2.31 E+03 \\
4.40 E+03 \\
1.64 E+04 \\
1.48 E+02 \\
5.91 E+03 \\
4.63 E+04 \\
2.12 E+02 \\
3.01 E+04 \\
1.80 E+02\end{array}$ & \\
\hline
\end{tabular}

Destination: LANL

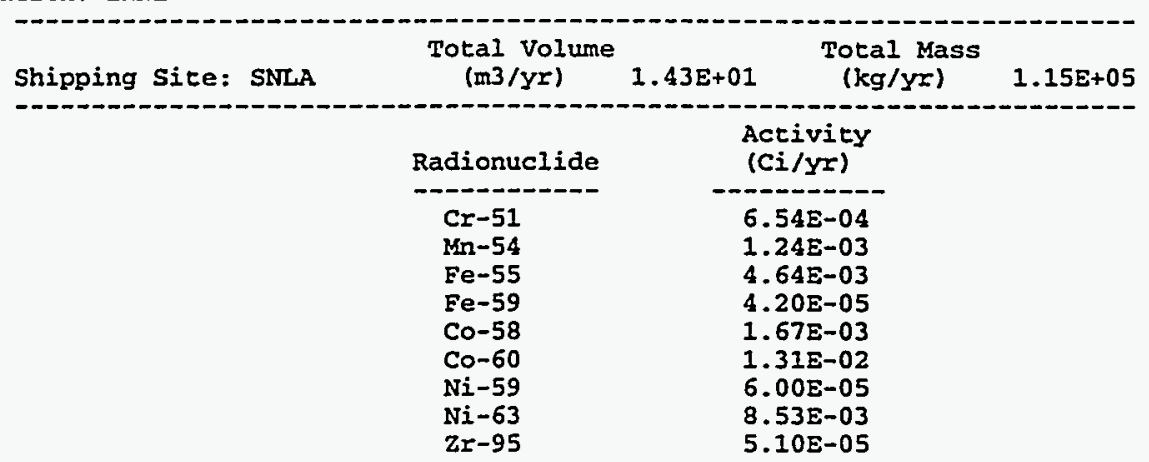

Destination: LLNL

\begin{tabular}{|c|c|c|c|}
\hline Shipping Site: IBL & $\begin{array}{l}\text { Total Volume } \\
\text { (m3/yx) }\end{array}$ & $\begin{array}{l}\text { Total Mass } \\
(\mathrm{kg} / \mathrm{Yr})\end{array}$ & $2.87 E+05$ \\
\hline & Radionuclide & $\begin{array}{l}\text { Activity } \\
\text { (Ci/yr) }\end{array}$ & \\
\hline & $\begin{array}{l}\text { Cr-51 } \\
\text { Mn-54 } \\
\text { Fe-55 } \\
\text { Fe-59 } \\
\text { Co-58 } \\
\text { Co-60 } \\
\text { Ni-59 } \\
\text { Ni-63 } \\
\text { Zr-95 }\end{array}$ & $\begin{array}{l}6.32 \mathrm{E}-02 \\
1.20 \mathrm{E}-01 \\
4.49 \mathrm{E}-01 \\
4.06 \mathrm{E}-03 \\
1.61 \mathrm{E}-01 \\
1.26 \mathrm{E}+00 \\
5.80 \mathrm{E}-03 \\
8.24 \mathrm{E}-01 \\
4.93 \mathrm{E}-03\end{array}$ & \\
\hline
\end{tabular}




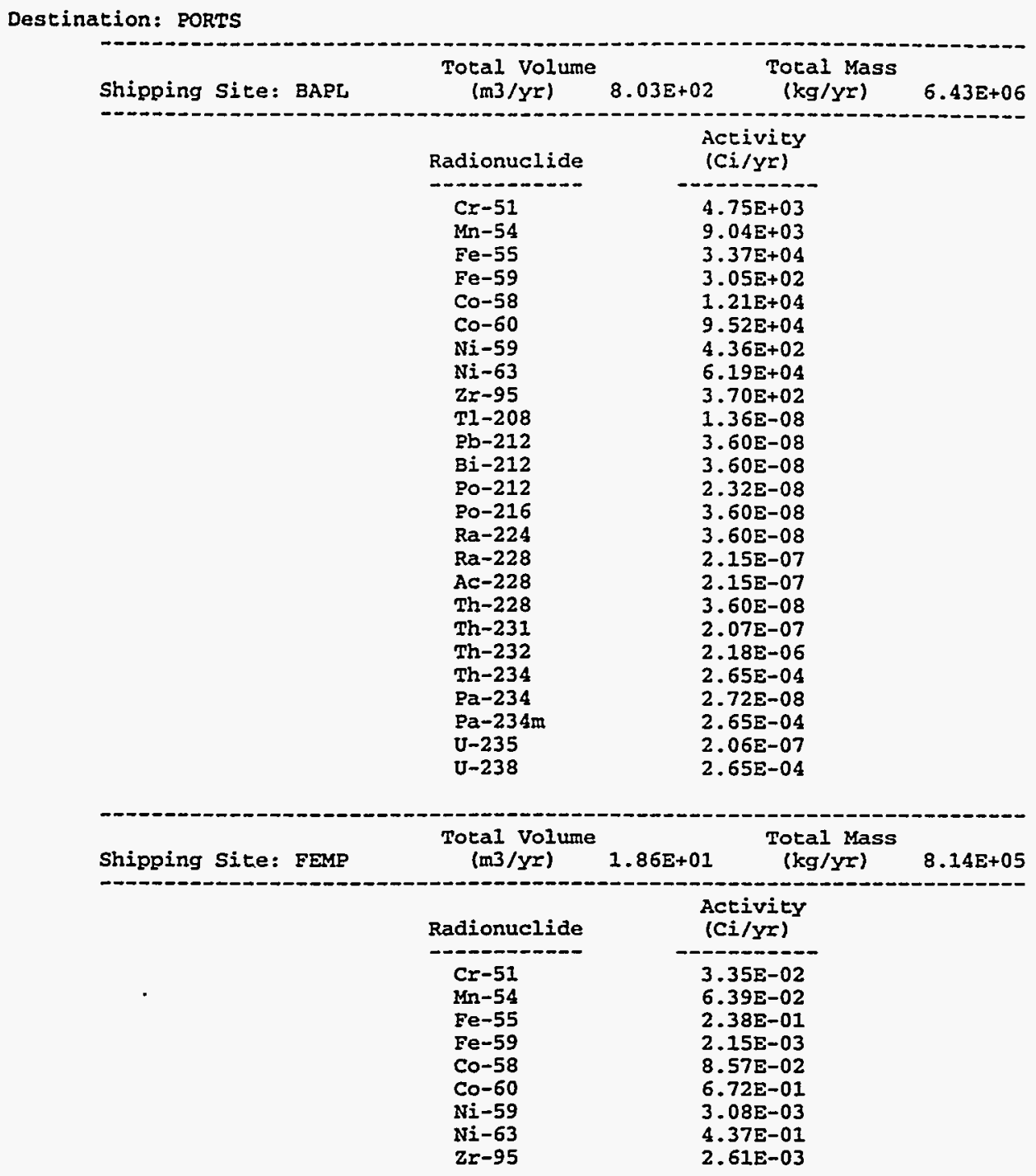


A-57

\begin{tabular}{|c|c|c|c|}
\hline Shipping Sice: KAPL & $\begin{array}{l}\text { Total volume } \\
\text { (m3/yr) }\end{array}$ & $\begin{array}{c}\text { Total Mass } \\
(\mathrm{kg} / \mathrm{yr})\end{array}$ & $1.33 E+07$ \\
\hline & 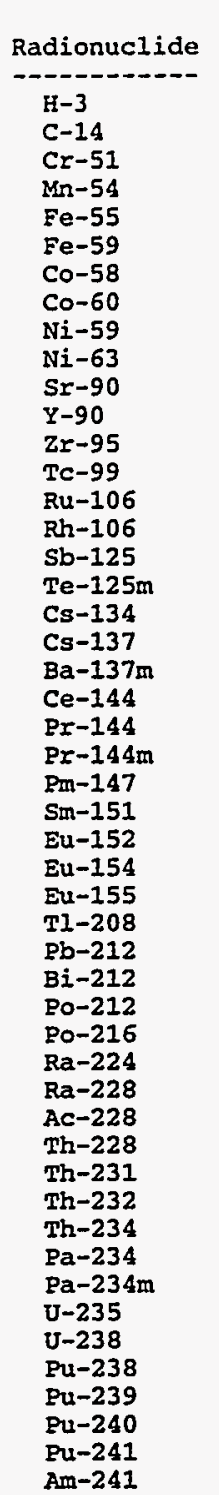 & $\begin{array}{c}\text { Activity } \\
\text { (Ci/yr) } \\
-3.15 \mathrm{-}-04 \\
5.15 \mathrm{E}-04 \\
2.40 \mathrm{E}-05 \\
1.13 \mathrm{E}+04 \\
2.15 \mathrm{E}+04 \\
8.05 \mathrm{E}+04 \\
7.28 \mathrm{E}+02 \\
2.89 \mathrm{E}+04 \\
2.27 \mathrm{E}+05 \\
1.04 \mathrm{E}+03 \\
1.47 \mathrm{E}+05 \\
2.62 \mathrm{E}-02 \\
2.62 \mathrm{E}-02 \\
8.84 \mathrm{E}+02 \\
5.20 \mathrm{E}-05 \\
2.22 \mathrm{E}-03 \\
2.22 \mathrm{E}-03 \\
6.40 \mathrm{E}-04 \\
1.56 \mathrm{E}-04 \\
8.18 \mathrm{E}-03 \\
3.13 \mathrm{E}-02 \\
2.96 \mathrm{E}-02 \\
7.46 \mathrm{E}-03 \\
7.46 \mathrm{E}-03 \\
8.97 \mathrm{E}-05 \\
1.82 \mathrm{E}-02 \\
1.78 \mathrm{E}-04 \\
2.71 \mathrm{E}-06 \\
4.66 \mathrm{E}-04 \\
3.69 \mathrm{E}-04 \\
2.96 \mathrm{E}-08 \\
7.83 \mathrm{E}-08 \\
7.83 \mathrm{E}-08 \\
5.05 \mathrm{E}-08 \\
7.83 \mathrm{E}-08 \\
7.83 \mathrm{E}-08 \\
4.68 \mathrm{E}-07 \\
4.68 \mathrm{E}-07 \\
7.83 \mathrm{E}-08 \\
4.50 \mathrm{E}-07 \\
4.75 \mathrm{E}-06 \\
5.77 \mathrm{E}-04 \\
5.92 \mathrm{E}-08 \\
5.77 \mathrm{E}-04 \\
4.48 \mathrm{E}-07 \\
8.69 \mathrm{E}-04 \\
5.61 \mathrm{E}-04 \\
5.43 \mathrm{E}-06 \\
2.71 \mathrm{E}-06 \\
6.28 \mathrm{E}-04 \\
8.15 \mathrm{E}-06\end{array}$ & \\
\hline
\end{tabular}




\section{A.9 WM LLW REGIONALIZED 3 ALTERNATIVE (CASE 4); HETEROGENEOUS SOLIDS}

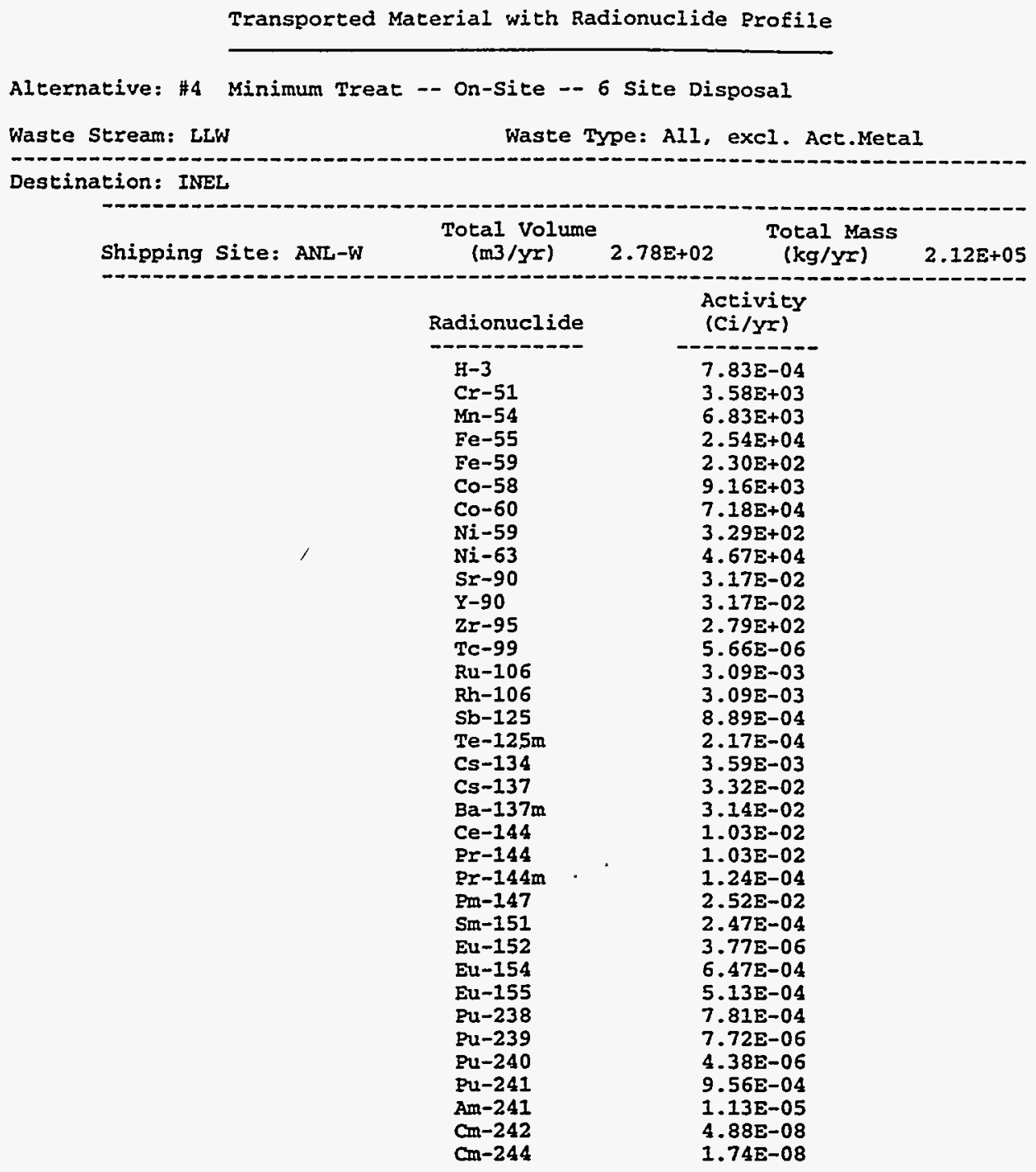




\begin{tabular}{|c|c|c|c|}
\hline Shipping Sice: ITRI & $\begin{array}{l}\text { Total Volume } \\
(\mathrm{m} 3 / \mathrm{yr})\end{array}$ & $\begin{array}{l}\text { Total Mass } \\
(\mathrm{kg} / \mathrm{yr})\end{array}$ & $1.64 \mathrm{E}+05$ \\
\hline & $\begin{array}{l}\text { Radionuclide } \\
-\mathrm{H}-3 \\
\mathrm{C}-14 \\
\mathrm{Mn}-54 \\
\mathrm{Co}-58 \\
\mathrm{Co}-60 \\
\mathrm{Sr}-90 \\
\mathrm{Y}-90 \\
\mathrm{~T}-99 \\
\mathrm{Cs}-134 \\
\mathrm{Cs}-137 \\
\mathrm{Ba}-137 \mathrm{~m} \\
\mathrm{U}-238\end{array}$ & $\begin{array}{l}6.17 \mathrm{E}+00 \\
3.03 \mathrm{E}-01 \\
3.41 \mathrm{E}+01 \\
3.15 \mathrm{E}+01 \\
9.12 \mathrm{E}+01 \\
4.28 \mathrm{E}+01 \\
4.28 \mathrm{E}+01 \\
6.07 \mathrm{E}-01 \\
7.07 \mathrm{E}+01 \\
9.33 \mathrm{E}+01 \\
8.82 \mathrm{E}+01 \\
3.69 \mathrm{E}+00\end{array}$ & \\
\hline \multirow[t]{2}{*}{ Shipping Site: PANT } & $\begin{array}{l}\text { Total Volume } \\
(\mathrm{m} 3 / \mathrm{yr})\end{array}$ & $\begin{array}{l}\text { Total Mass } \\
(\mathrm{kg} / \mathrm{yx})\end{array}$ & $2.88 E+07$ \\
\hline & \begin{tabular}{l} 
Radionuclide \\
\hdashline $\mathrm{H}-3$ \\
$\mathrm{Tl}-208$ \\
$\mathrm{~Pb}-212$ \\
$\mathrm{Bi}-212$ \\
$\mathrm{Po-212}$ \\
$\mathrm{Po}-216$ \\
$\mathrm{Ra}-224$ \\
$\mathrm{Ra}-228$ \\
$\mathrm{Ac}-228$ \\
$\mathrm{Th}-228$ \\
$\mathrm{Th}-231$ \\
$\mathrm{Th}-232$ \\
$\mathrm{Th}-234$ \\
$\mathrm{~Pa}-234$ \\
$\mathrm{~Pa}-234 \mathrm{~m}$ \\
$\mathrm{U}-235$ \\
$\mathrm{U}-238$
\end{tabular} & $\begin{array}{l}-3.34 \mathrm{E}+02 \\
2.21 \mathrm{E}-04 \\
5.86 \mathrm{E}-04 \\
5.86 \mathrm{E}-04 \\
3.78 \mathrm{E}-04 \\
5.86 \mathrm{E}-04 \\
5.86 \mathrm{E}-04 \\
3.50 \mathrm{E}-03 \\
3.50 \mathrm{E}-03 \\
5.86 \mathrm{E}-04 \\
3.37 \mathrm{E}-03 \\
3.56 \mathrm{E}-02 \\
4.32 \mathrm{E}+00 \\
4.43 \mathrm{E}-04 \\
4.32 \mathrm{E}+00 \\
3.36 \mathrm{E}-03 \\
4.32 \mathrm{E}+00\end{array}$ & \\
\hline \multirow[t]{2}{*}{ Shipping Site: RFP } & $\begin{array}{l}\text { Total Volume } \\
\text { (m3/Yr) }\end{array}$ & $\begin{array}{cc}\text { 4.56E+03 } & \begin{array}{c}\text { Total Mass } \\
(\mathrm{kg} / \mathrm{yr})\end{array}\end{array}$ & $6.49 E+06$ \\
\hline & $\begin{array}{l}\text { Radionuclide } \\
\mathrm{T} 1-208 \\
\mathrm{~Pb}-212 \\
\mathrm{Bi}-212 \\
\mathrm{PO}-212 \\
\mathrm{Po}-216 \\
\mathrm{Ra}-224 \\
\mathrm{Ra}-228 \\
\mathrm{Ac}-228 \\
\mathrm{Th}-228 \\
\mathrm{Th}-231 \\
\mathrm{Th}-232 \\
\mathrm{Th}-234 \\
\mathrm{~Pa}-234 \\
\mathrm{~Pa}-234 \mathrm{~m} \\
\mathrm{U}-235 \\
\mathrm{U}-238 \\
\mathrm{Pu}-238 \\
\mathrm{Pu}-239 \\
\mathrm{Pu}-240 \\
\mathrm{Pu}-241 \\
\mathrm{Am}-241 \\
\mathrm{Cm}-242 \\
\mathrm{Cm}-244\end{array}$ & $\begin{array}{c}\text { Activity } \\
(C i / y r) \\
-3.60 E-05 \\
3.65 E-05 \\
9.55 E-05 \\
9.55 E-05 \\
6.15 E-05 \\
9.55 E-05 \\
9.55 E-05 \\
5.70 E-04 \\
5.70 E-04 \\
9.55 E-05 \\
5.49 E-04 \\
5.79 E-03 \\
7.04 E-01 \\
7.21 E-05 \\
7.04 E-01 \\
5.47 E-04 \\
7.04 E-01 \\
5.56 E-01 \\
4.24 E-02 \\
1.48 E-01 \\
2.04 E+01 \\
8.48 E-04 \\
1.18 E-02 \\
4.24 E-03\end{array}$ & \\
\hline
\end{tabular}


- Regionalized 5 (Case 19). Four sites (Hanford, INEL, ORR, and SRS) would thermally treat, supercompact, reduce the size of, and grout volume-reducible waste: all sites would minimally treat other waste; disposal would occur at six sites (Hanford, INEL, NTS, LANL, ORR, and SRS).

- Regionalized 6 (Case 5). All sites would minimally treat LLW, stabilizing fines and liquids, and dispose of LLW at the nearer of two sites (Hanford and SRS).

- Regionalized 7 (Case 6). All sites would minimally treat LLW, stabilizing fines and liquids, and dispose of LLW at the nearer of two sites (NTS and SRS).

- Centralized 1 (Case 7). All sites would minimally treat LLW, stabilizing fines and liquids, and dispose of LLW at one site (Hanford).

- Centralized 2(Case 8). All sites would minimally treat LLW, stabilizing fines and liquids, and dispose of LLW at one site (NTS).

- Centralized 3 (Case 14). Seven sites (Hanford, INEL, LANL, ORR, SRS, PORTS, and RFETS) would thermally treat, supercompact, reduce the size of, and grout volume-reducible waste; all sites would minimally treat other waste; disposal would occur at one site (Hanford).

- Centralized 4 (Case 14a). Seven sites (Hanford, INEL, LANL, ORR, SRS, PORTS, and RFETS) would thermally treat, supercompact, reduce the size of, and grout volume-reducible waste; all sites would minimally treat other waste; disposal would occur at one site (NTS).

- Centralized 5 (Case 21). One site (Hanford) would thermally treat, supercompact, reduce the size of, and grout volume-reducible waste; all sites would minimally treat other waste; disposal would occur at one site (Hanford).

\subsection{CHARACTERISTICS OF LOW-LEVEL WASTE}

To support the WM PEIS analysis of LLW alternatives, a report has been prepared describing the LLW inventory and characteristics at each DOE site (Goyette and Dolak 1996). In addition, Goyette and Dolak (1996) describe the current LLW TSD capabilities of the DOE complex and presents the important assumptions associated with the analysis of LLW alternatives. The 
A-60

\begin{tabular}{|c|c|c|c|}
\hline Shipping Site: SNLA & $\begin{array}{l}\text { Total Volume } \\
(\mathrm{m} 3 / \mathrm{yr})\end{array}$ & $\begin{array}{c}\text { Total Mass } \\
(\mathrm{kg} / \mathrm{yr})\end{array}$ & $3.28 \mathrm{E}+05$ \\
\hline & 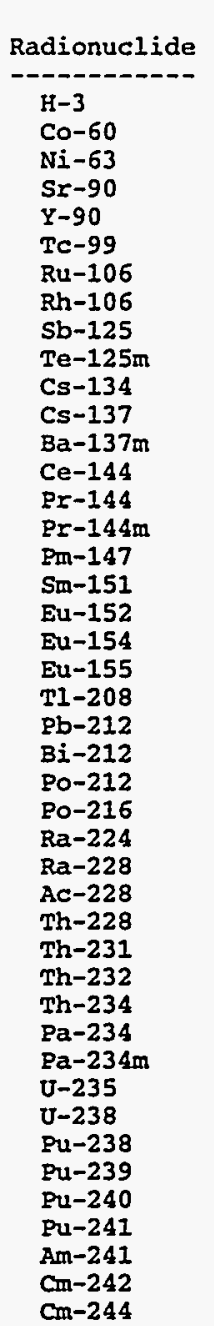 & 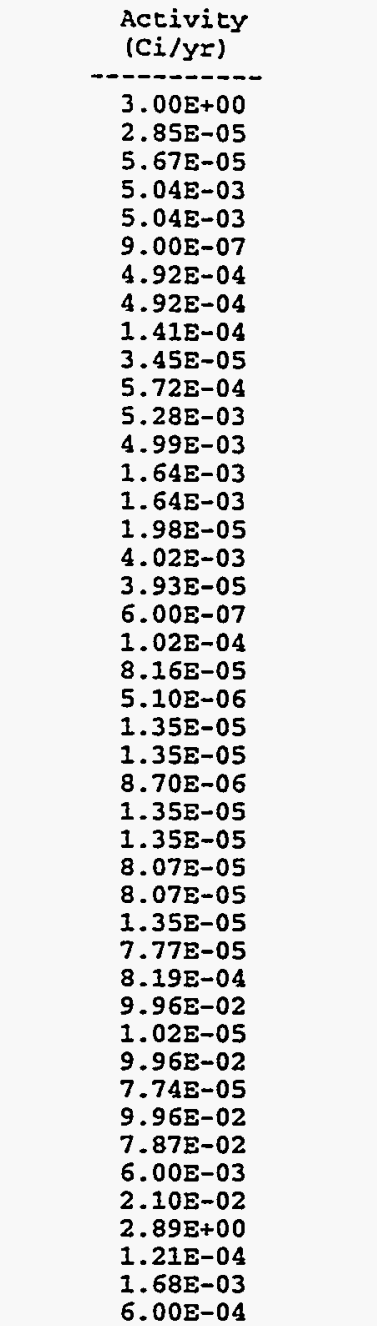 & . \\
\hline
\end{tabular}


Destination: NTS

\begin{tabular}{|c|c|c|c|}
\hline Shipping Site: LBL & $\begin{array}{l}\text { Total Volume } \\
\left(\mathrm{m} 3 / Y^{5}\right)\end{array}$ & $\begin{array}{l}\text { Total Mass } \\
(\mathrm{kg} / \mathrm{yr})\end{array}$ & $4.51 E+04$ \\
\hline & $\begin{array}{l}\text { Radionuclide } \\
\mathrm{H}-3 \\
\mathrm{C}-14 \\
\mathrm{Mn}-54 \\
\mathrm{Co}-58 \\
\mathrm{Co}-60 \\
\mathrm{~S}-90 \\
\mathrm{Y}-90 \\
\mathrm{Tc}-99 \\
\mathrm{Cs}-134 \\
\mathrm{Cs}-137 \\
\mathrm{Ba}-137 \mathrm{~m} \\
\mathrm{U}-238 \\
\mathrm{Cm}-244\end{array}$ & $\begin{array}{l}6.35 \mathrm{E}+02 \\
1.13 \mathrm{E}+00 \\
1.27 \mathrm{E}+02 \\
1.17 \mathrm{E}+02 \\
3.39 \mathrm{E}+02 \\
1.59 \mathrm{E}+02 \\
1.59 \mathrm{E}+02 \\
2.26 \mathrm{E}+00 \\
2.63 \mathrm{E}+02 \\
3.47 \mathrm{E}+02 \\
3.28 \mathrm{E}+02 \\
1.37 \mathrm{E}+01 \\
1.05 \mathrm{E}+01\end{array}$ & \\
\hline \multirow{2}{*}{ Shipping Site: LLNL } & $\begin{array}{l}\text { Total volume } \\
(\mathrm{m} 3 / \mathrm{yr})\end{array}$ & $\begin{array}{cc}4.20 \mathrm{E}+02 & \begin{array}{c}\text { Total Mass } \\
(\mathrm{kg} / \mathrm{yr})\end{array}\end{array}$ & $1.06 \mathrm{E}+06$ \\
\hline & $\begin{array}{l}\text { Radionuclide } \\
\mathrm{H}-3 \\
\mathrm{C}-14 \\
\mathrm{Mn}-54 \\
\mathrm{Co}-58 \\
\mathrm{Co}-60 \\
\mathrm{Ni}-63 \\
\mathrm{~S}-90 \\
\mathrm{Y}-90 \\
\mathrm{Tc}-99 \\
\mathrm{Ru}-106 \\
\mathrm{Rh}-106 \\
\mathrm{Sb}-125 \\
\mathrm{Te}-125 \mathrm{~m} \\
\mathrm{Cs}-134 \\
\mathrm{Cs}-137 \\
\mathrm{Ba}-137 \mathrm{~m} \\
\mathrm{Ce}-144 \\
\mathrm{Pr}-144 \\
\mathrm{Pr}-144 \mathrm{~m} \\
\mathrm{Pm}-147 \\
\mathrm{Sm}-151 \\
\text { Eu-152 } \\
\text { Eu-154 } \\
\text { Eu-155 } \\
\mathrm{U}-238 \\
\mathrm{Pu}-238 \\
\mathrm{Pu}-239 \\
\mathrm{Pu}-240 \\
\mathrm{Pu}-241 \\
\mathrm{Am}-241 \\
\mathrm{Cm}-242 \\
\mathrm{Cm}-244\end{array}$ & $\begin{array}{l}3.33 \mathrm{E}+04 \\
1.55 \mathrm{E}-03 \\
1.74 \mathrm{E}-01 \\
1.61 \mathrm{E}-01 \\
4.69 \mathrm{E}-01 \\
4.89 \mathrm{E}-03 \\
6.54 \mathrm{E}-01 \\
6.54 \mathrm{E}-01 \\
3.18 \mathrm{E}-03 \\
4.24 \mathrm{E}-02 \\
4.24 \mathrm{E}-02 \\
1.21 \mathrm{E}-02 \\
2.97 \mathrm{E}-03 \\
4.11 \mathrm{E}-01 \\
9.33 \mathrm{E}-01 \\
8.82 \mathrm{E}-01 \\
1.42 \mathrm{E}-01 \\
1.42 \mathrm{E}-01 \\
1.70 \mathrm{E}-03 \\
3.46 \mathrm{E}-01 \\
3.39 \mathrm{E}-03 \\
5.17 \mathrm{E}-05 \\
8.87 \mathrm{E}-03 \\
7.03 \mathrm{E}-03 \\
1.88 \mathrm{E}-02 \\
7.85 \mathrm{E}-02 \\
5.28 \mathrm{E}-03 \\
1.81 \mathrm{E}-02 \\
2.50 \mathrm{E}+00 \\
2.58 \mathrm{E}-04 \\
1.44 \mathrm{E}-03 \\
5.17 \mathrm{E}-04\end{array}$ & - \\
\hline \multirow[t]{2}{*}{ Shipping Site: SLAC } & $\begin{array}{l}\text { Total Volume } \\
\text { (m3/yr) }\end{array}$ & $\begin{array}{l}\text { Total Mass } \\
(\mathrm{kg} / \mathrm{yr})\end{array}$ & $7.45 E+05$ \\
\hline & $\begin{array}{l}\text { Radionuclide } \\
\text { Cr-5I } \\
\text { Mn-54 } \\
\text { Fe-55 } \\
\text { Fe-59 } \\
\text { Co-58 } \\
\text { Co-60 } \\
\mathrm{N} i-59 \\
\mathrm{Ni}-63 \\
\mathrm{Zr}-95\end{array}$ & $\begin{array}{c}\text { Activity } \\
\text { (Ci/YI) } \\
3.86 \mathrm{E}-03 \\
7.36 \mathrm{E}-03 \\
2.74 \mathrm{E}-02 \\
2.48 \mathrm{E}-04 \\
9.88 \mathrm{E}-03 \\
7.74 \mathrm{E}-02 \\
3.54 \mathrm{E}-04 \\
5.04 \mathrm{E}-02 \\
3.01 \mathrm{E}-04\end{array}$ & \\
\hline
\end{tabular}




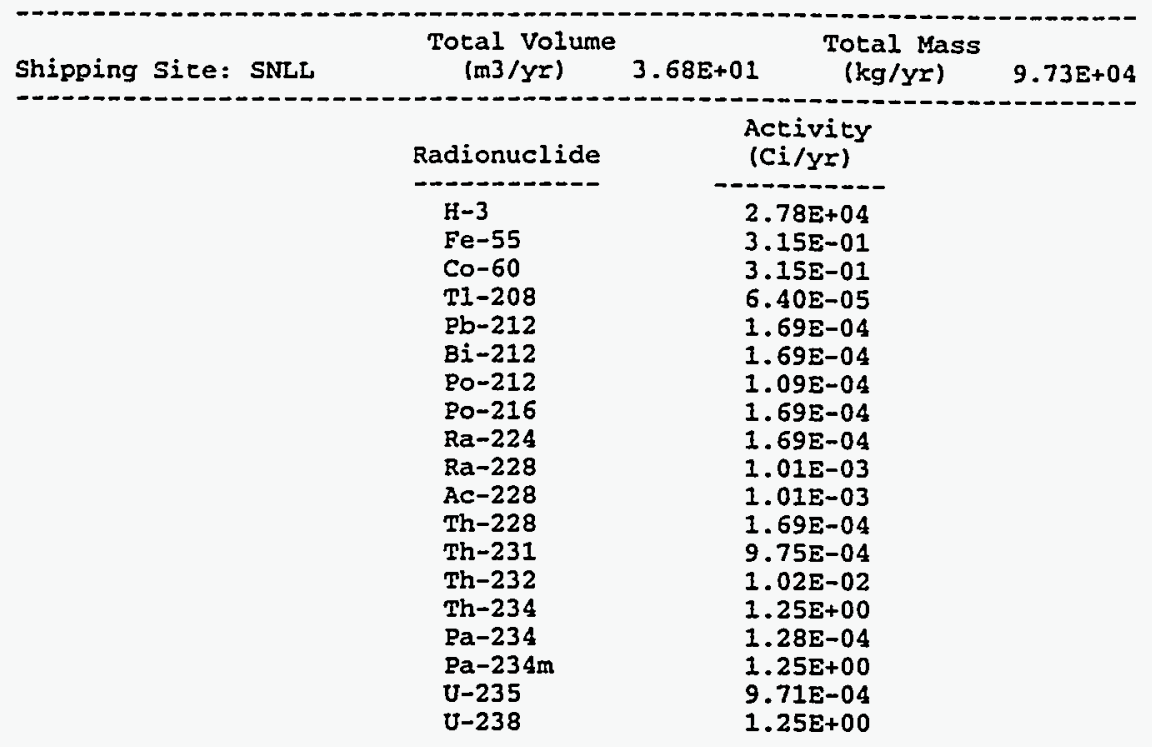


Destination: ORNL

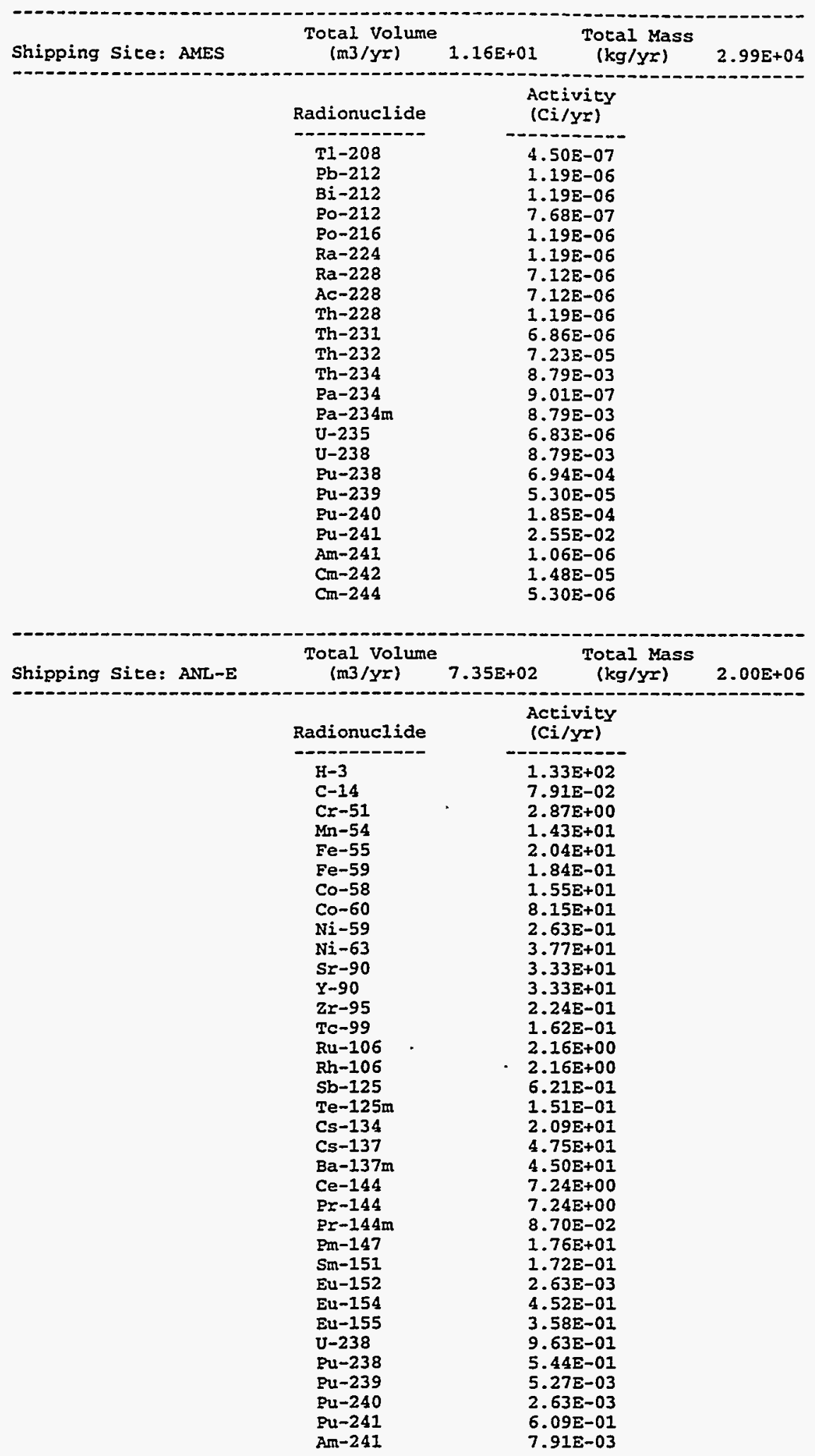


A-64

\begin{tabular}{|c|c|c|c|}
\hline Shipping Site: BAPL & $\begin{array}{l}\text { Toral volume } \\
\{\mathrm{m} 3 / \mathrm{y} z)\end{array}$ & $\begin{array}{c}\text { Total Mass } \\
(\mathrm{kg} / \mathrm{y})\end{array}$ & $1.08 E+06$ \\
\hline & 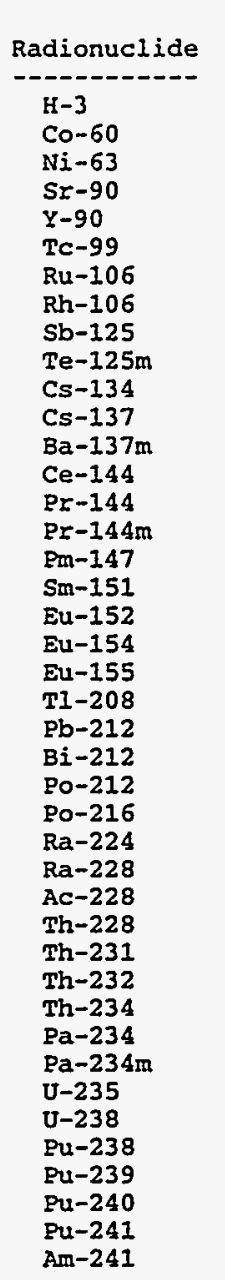 & $\begin{array}{l}\text { Activity } \\
\text { (Ci/Yr) } \\
-9-0-- \\
2.51 \mathrm{E}-03 \\
1.19 \mathrm{E}-02 \\
2.38 \mathrm{E}-02 \\
2.11 \mathrm{E}+00 \\
2.11 \mathrm{E}+00 \\
3.77 \mathrm{E}-04 \\
2.06 \mathrm{E}-01 \\
2.06 \mathrm{E}-01 \\
5.93 \mathrm{E}-02 \\
1.44 \mathrm{E}-02 \\
2.40 \mathrm{E}-01 \\
2.21 \mathrm{E}+00 \\
2.09 \mathrm{E}+00 \\
6.91 \mathrm{E}-01 \\
6.91 \mathrm{E}-01 \\
8.31 \mathrm{E}-03 \\
1.58 \mathrm{E}+00 \\
1.65 \mathrm{E}-02 \\
2.51 \mathrm{E}-04 \\
4.32 \mathrm{E}-02 \\
3.42 \mathrm{E}-02 \\
3.80 \mathrm{E}-06 \\
1.00 \mathrm{E}-05 \\
1.00 \mathrm{E}-05 \\
6.49 \mathrm{E}-06 \\
1.00 \mathrm{E}-05 \\
1.00 \mathrm{E}-05 \\
6.02 \mathrm{E}-05 \\
6.02 \mathrm{E}-05 \\
1.00 \mathrm{E}-05 \\
5.80 \mathrm{E}-05 \\
6.11 \mathrm{E}-04 \\
7.43 \mathrm{E}-02 \\
7.61 \mathrm{E}-06 \\
7.43 \mathrm{E}-02 \\
5.77 \mathrm{E}-05 \\
7.43 \mathrm{E}-02 \\
5.20 \mathrm{E}-02 \\
5.03 \mathrm{E}-04 \\
2.51 \mathrm{E}-04 \\
5.81 \mathrm{E}-02 \\
7.55 \mathrm{E}-04\end{array}$ & \\
\hline \multirow[t]{2}{*}{ Shipping Site: FNAL } & $\begin{array}{l}\text { Total Volume } \\
(\mathrm{m} 3 / \mathrm{yx})\end{array}$ & $\begin{array}{c}\text { Total Mass } \\
(\mathrm{kg} / \mathrm{yr})\end{array}$ & $5.11 E+04$ \\
\hline & \begin{tabular}{l} 
Radionuclide \\
\hdashline $\mathrm{H}-3$ \\
$\mathrm{Cr}-51$ \\
$\mathrm{Mn}-54$ \\
$\mathrm{Fe}-55$ \\
$\mathrm{Fe}-59$ \\
$\mathrm{Co}-58$ \\
$\mathrm{Co}-60$ \\
$\mathrm{~N} \mathrm{-}-59$ \\
$\mathrm{Ni}-63$ \\
$\mathrm{Zr}-95$ \\
$\mathrm{TI}-208$ \\
$\mathrm{~Pb}-212$ \\
$\mathrm{Bi}-212$ \\
$\mathrm{PO}-212$ \\
$\mathrm{PO}-216$ \\
$\mathrm{Ra}-224$ \\
$\mathrm{Ra}-228$ \\
$\mathrm{Ac}-228$ \\
$\mathrm{Th}-228$ \\
$\mathrm{Th}-231$ \\
$\mathrm{Th}-232$ \\
$\mathrm{Th}-234$ \\
$\mathrm{~Pa}-234$ \\
$\mathrm{~Pa}-234 \mathrm{~m}$ \\
$\mathrm{U}-235$ \\
$\mathrm{U}-238$
\end{tabular} & $\begin{array}{c}\begin{array}{c}\text { Activity } \\
\text { (Ci/yr) }\end{array} \\
-1.81 \mathrm{E}-01 \\
1.11 \mathrm{E}-02 \\
2.12 \mathrm{E}-02 \\
7.94 \mathrm{E}-02 \\
7.17 \mathrm{E}-04 \\
2.85 \mathrm{E}-02 \\
2.23 \mathrm{E}-01 \\
1.02 \mathrm{E}-03 \\
1.45 \mathrm{E}-01 \\
8.71 \mathrm{E}-04 \\
3.78 \mathrm{E}-07 \\
1.00 \mathrm{E}-06 \\
1.00 \mathrm{E}-06 \\
6.46 \mathrm{E}-07 \\
1.00 \mathrm{E}-06 \\
1.00 \mathrm{E}-06 \\
5.99 \mathrm{E}-06 \\
5.99 \mathrm{E}-06 \\
1.00 \mathrm{E}-06 \\
5.77 \mathrm{E}-06 \\
6.08 \mathrm{E}-05 \\
7.39 \mathrm{E}-03 \\
7.57 \mathrm{E}-07 \\
7.39 \mathrm{E}-03 \\
5.75 \mathrm{E}-06 \\
7.39 \mathrm{E}-03\end{array}$ & \\
\hline
\end{tabular}




\begin{tabular}{|c|c|c|c|}
\hline Shipping Site: $k-25$ & $\begin{array}{l}\text { Total Volume } \\
(\mathrm{m} 3 / \mathrm{yr})\end{array}$ & $\begin{array}{c}\text { Total Mass } \\
(\mathrm{kg} / \mathrm{yr})\end{array}$ & $4.67 \mathrm{E}+07$ \\
\hline & $\begin{array}{l}\text { Radionuclide } \\
\mathrm{Tl}-208 \\
\mathrm{~Pb}-212 \\
\mathrm{Bi}-212 \\
\mathrm{Po}-212 \\
\mathrm{Po}-216 \\
\mathrm{Ra}-224 \\
\mathrm{Ra}-228 \\
\mathrm{Ac}-228 \\
\mathrm{Th}-228 \\
\mathrm{Th}-231 \\
\mathrm{Th}-232 \\
\mathrm{Th}-234 \\
\mathrm{~Pa}-234 \\
\mathrm{~Pa}-234 \mathrm{~m} \\
\mathrm{U}-235 \\
\mathrm{U}-238\end{array}$ & 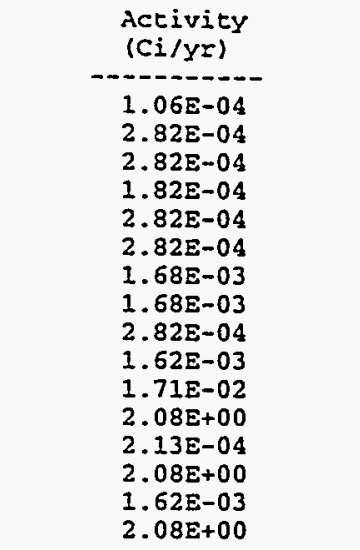 & . \\
\hline Shipping Site: KAPL & $\begin{array}{l}\text { Total Volume } \\
(\mathrm{m} 3 / \mathrm{yr})\end{array}$ & $\begin{array}{c}\text { Total Mass } \\
(\mathrm{kg} / \mathrm{yr})\end{array}$ & $5.36 E+05$ \\
\hline - & 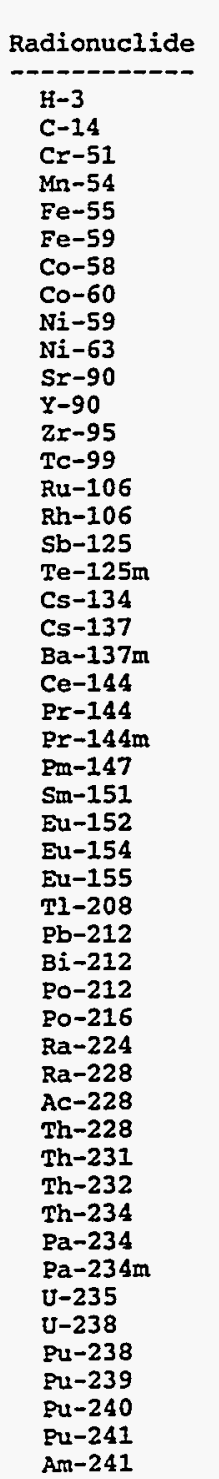 & 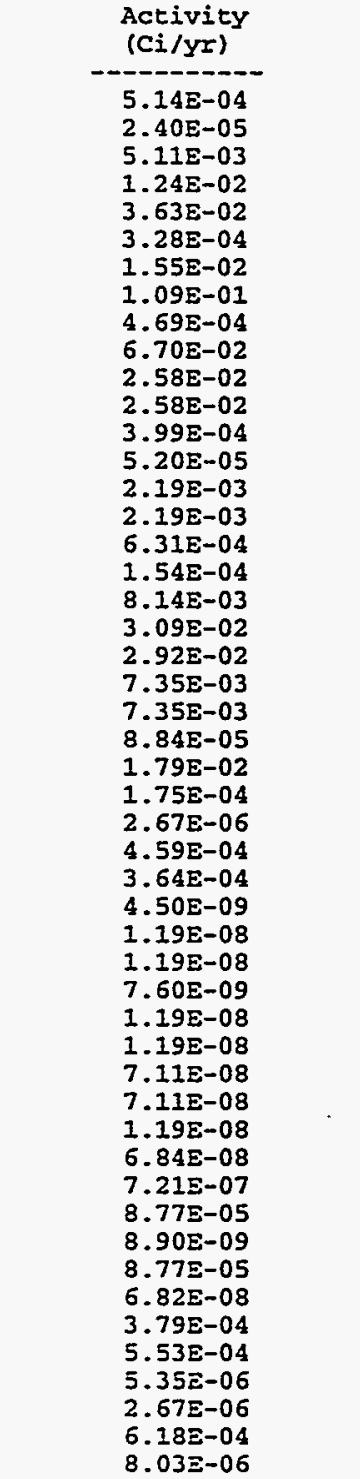 & $\omega$ \\
\hline
\end{tabular}




\begin{tabular}{|c|c|c|c|}
\hline Shipping sice: KCP & $\begin{array}{l}\text { Total Volume } \\
(\mathrm{m} 3 / \mathrm{yr})\end{array}$ & $\begin{array}{cc}2.65 \mathrm{E}+00 & \text { Total Mass } \\
(\mathrm{kg} / \mathrm{yr})\end{array}$ & $7.23 \mathrm{E}+03$ \\
\hline & $\begin{array}{l}\text { Radionuclide } \\
\mathrm{H}-3 \\
\mathrm{C}-14 \\
\mathrm{Mn}-54 \\
\mathrm{Co}-58 \\
\mathrm{Co}-60 \\
\mathrm{Sr}-90 \\
\mathrm{Y}-90 \\
\mathrm{TC}-99 \\
\mathrm{Cs}-134 \\
\mathrm{Cs}-137 \\
\mathrm{Ba}-137 \mathrm{~m} \\
\mathrm{U}-238\end{array}$ & 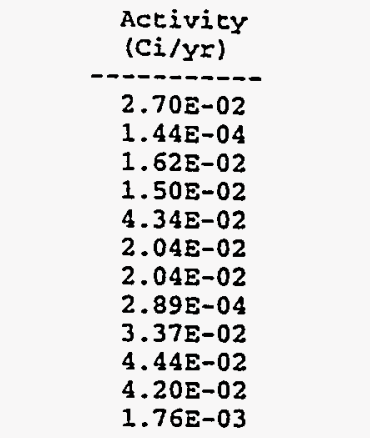 & \\
\hline Shipping Site: MOUND & $\begin{array}{l}\text { Total volume } \\
(\mathrm{m} 3 / \mathrm{yr})\end{array}$ & $\begin{array}{c}\text { Total Mass } \\
\text { 4.40E }+03 \quad \mathrm{~kg} / \mathrm{yr})\end{array}$ & $9.67 E+06$ \\
\hline 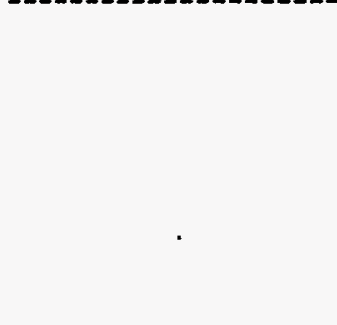 & $\begin{array}{l}\text { Radionuclide } \\
\mathrm{H}-3 \\
\mathrm{Pu}-238 \\
\mathrm{Pu}-239 \\
\mathrm{Pu}-240 \\
\mathrm{Pu}-241 \\
\mathrm{Am}-241 \\
\mathrm{Cm}-242 \\
\mathrm{Cm}-244\end{array}$ & $\begin{array}{c}\begin{array}{c}\text { Activity } \\
\text { (Ci/yr) }\end{array} \\
1.34 \mathrm{E}+04 \\
1.60 \mathrm{E}-01 \\
1.22 \mathrm{E}-02 \\
4.28 \mathrm{E}-02 \\
5.90 \mathrm{E}+00 \\
2.44 \mathrm{E}-04 \\
3.42 \mathrm{E}-03 \\
1.22 \mathrm{E}-03\end{array}$ & \\
\hline Shipping Site: ORISE & $\begin{array}{l}\text { Total volume } \\
(\mathrm{m} 3 / \mathrm{yr})\end{array}$ & $\begin{array}{l}\text { Total Mass } \\
(\mathrm{kg} / \mathrm{Yx})\end{array}$ & $7.36 \mathrm{E}+04$ \\
\hline " & \begin{tabular}{l} 
Radionuclide \\
\hdashline $\mathrm{H}-3$ \\
$\mathrm{C}-14$ \\
$\mathrm{Mn}-54$ \\
$\mathrm{Co}-58$ \\
$\mathrm{Co}-60$ \\
$\mathrm{Sr}-90$ \\
$\mathrm{Y}-90$ \\
$\mathrm{~T} \mathrm{C}-99$ \\
$\mathrm{Cs}-134$ \\
$\mathrm{Cs}-137$ \\
$\mathrm{Ba}-137 \mathrm{~m}$ \\
$\mathrm{~T} 1-208$ \\
$\mathrm{~Pb}-212$ \\
$\mathrm{Bi}-212$ \\
$\mathrm{Po}-212$ \\
$\mathrm{Po}-216$ \\
$\mathrm{Ra}-224$ \\
$\mathrm{Ra}-228$ \\
$\mathrm{Ac}-228$ \\
$\mathrm{Th}-228$ \\
$\mathrm{Th}-231$ \\
$\mathrm{Th}-232$ \\
$\mathrm{Th}-234$ \\
$\mathrm{~Pa}-234$ \\
$\mathrm{~Pa}-234 \mathrm{~m}$ \\
$\mathrm{U}-235$ \\
$\mathrm{U}-238$
\end{tabular} & $\begin{array}{c}\text { Activity } \\
\text { (Ci/yr) } \\
2.00 \mathrm{E}-02 \\
1.19 \mathrm{E}-05 \\
1.34 \mathrm{E}-03 \\
1.24 \mathrm{E}-03 \\
3.59 \mathrm{E}-03 \\
1.69 \mathrm{E}-03 \\
1.69 \mathrm{E}-03 \\
2.39 \mathrm{E}-05 \\
2.79 \mathrm{E}-03 \\
3.68 \mathrm{E}-03 \\
3.48 \mathrm{E}-03 \\
3.39 \mathrm{E}-07 \\
8.98 \mathrm{E}-07 \\
8.98 \mathrm{E}-07 \\
5.78 \mathrm{E}-07 \\
8.98 \mathrm{E}-07 \\
8.98 \mathrm{E}-07 \\
5.36 \mathrm{E}-06 \\
5.36 \mathrm{E}-06 \\
8.98 \mathrm{E}-07 \\
5.16 \mathrm{E}-06 \\
5.44 \mathrm{E}-05 \\
6.62 \mathrm{E}-03 \\
6.78 \mathrm{E}-07 \\
6.62 \mathrm{E}-03 \\
5.14 \mathrm{E}-06 \\
6.77 \mathrm{E}-03\end{array}$ & \\
\hline
\end{tabular}


A-67

\begin{tabular}{|c|c|c|c|}
\hline Shipping Sice: PORTS & $\begin{array}{l}\text { Total Volume } \\
(\mathrm{m} 3 / \mathrm{yr})\end{array}$ & $\begin{array}{c}\text { Total Mass } \\
(\mathrm{kg} / \mathrm{yr})\end{array}$ & $6.73 E+07$ \\
\hline & $\begin{array}{l}\text { Radionuclide } \\
\text { Tl-208 } \\
\text { Pb-212 } \\
\text { Bi-212 } \\
\text { Po-212 } \\
\text { Po-216 } \\
\text { Ra-224 } \\
\text { Ra-228 } \\
\text { Ac-228 } \\
\text { Th-228 } \\
\text { Th-231 } \\
\text { Th-232 } \\
\text { Th-234 } \\
\text { Pa-234 } \\
\text { Pa-234m } \\
\text { U-235 } \\
\mathrm{U}-238\end{array}$ & \multicolumn{2}{|l|}{$\begin{array}{l}3.45 \mathrm{E}-06 \\
9.14 \mathrm{E}-06 \\
9.14 \mathrm{E}-06 \\
5.89 \mathrm{E}-06 \\
9.14 \mathrm{E}-06 \\
9.14 \mathrm{E}-06 \\
5.46 \mathrm{E}-05 \\
5.46 \mathrm{E}-05 \\
9.14 \mathrm{E}-06 \\
5.26 \mathrm{E}-05 \\
5.55 \mathrm{E}-04 \\
6.74 \mathrm{E}-02 \\
6.91 \mathrm{E}-06 \\
6.74 \mathrm{E}-02 \\
5.24 \mathrm{E}-05 \\
6.74 \mathrm{E}-02\end{array}$} \\
\hline \multirow[t]{2}{*}{ Shipping Site: PPPL } & $\begin{array}{l}\text { Potal Volume } \\
\text { (m3/yr) }\end{array}$ & $\begin{array}{l}\text { Total Mass } \\
(\mathrm{kg} / \mathrm{yr})\end{array}$ & $6.10 \mathrm{E}+03$ \\
\hline & $\begin{array}{l}\text { Radionuclide } \\
\mathrm{H}-3 \\
\mathrm{Cr}-51 \\
\mathrm{Mn}-54 \\
\mathrm{Fe}-55 \\
\mathrm{Fe}-59 \\
\mathrm{Co}-58 \\
\mathrm{Co}-60 \\
\mathrm{Ni}-63 \\
\mathrm{Zr}-95\end{array}$ & \multicolumn{2}{|l|}{$\begin{array}{l}2.01 \mathrm{E}-01 \\
8.50 \mathrm{E}-04 \\
1.61 \mathrm{E}-03 \\
6.04 \mathrm{E}-03 \\
5.46 \mathrm{E}-05 \\
2.17 \mathrm{E}-03 \\
1.70 \mathrm{E}-02 \\
1.10 \mathrm{E}-02 \\
6.63 \mathrm{E}-05\end{array}$} \\
\hline \multirow[t]{2}{*}{ Shipping Site: Paducah } & $\begin{array}{l}\text { Total Volume } \\
\left(\operatorname{m} 3 / \mathrm{Y}^{2}\right)\end{array}$ & $5.39 E+03 \quad \begin{array}{c}\text { Total Mass } \\
(\mathrm{kg} / \mathrm{Yx})\end{array}$ & $1.20 E+07$ \\
\hline & $\begin{array}{l}\text { Radionuclide } \\
\text { Tc-99 } \\
\text { Tl-208 } \\
\mathrm{Pb}-212 \\
\mathrm{Bi}-212 \\
\mathrm{Po-212} \\
\mathrm{Po-216} \\
\mathrm{Ra}-224 \\
\mathrm{Ra}-228 \\
\mathrm{Ac-228} \\
\mathrm{Th}-228 \\
\mathrm{Th}-231 \\
\mathrm{Th}-232 \\
\mathrm{Th}-234 \\
\mathrm{~Pa}-234 \\
\mathrm{~Pa}-234 \mathrm{~m} \\
\mathrm{U}-235 \\
\mathrm{U}-238 \\
\mathrm{~Np}-237 \\
\mathrm{Pu}-239\end{array}$ & 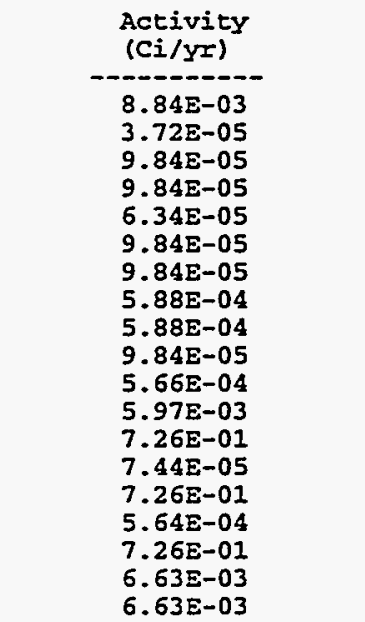 & \\
\hline
\end{tabular}




\begin{tabular}{|c|c|c|c|}
\hline Shipping Sice: RMI & $\begin{array}{c}\text { Total Volume } \\
(\mathrm{m} 3 / \mathrm{yr})\end{array}$ & $\begin{array}{c}\text { Total Mass } \\
(\mathrm{kg} / \mathrm{yr})\end{array}$ & $1.475+07$ \\
\hline & $\begin{array}{l}\text { Radionuclide } \\
\text { Tl-208 } \\
\mathrm{Pb}-212 \\
\mathrm{Bi}-212 \\
\mathrm{Po-212} \\
\mathrm{Po-216} \\
\mathrm{Ra}-224 \\
\mathrm{Ra}-228 \\
\mathrm{Ac}-228 \\
\mathrm{Th}-228 \\
\mathrm{Th}-23 \mathrm{I} \\
\mathrm{Th}-232 \\
\mathrm{Th}-234 \\
\mathrm{~Pa}-234 \\
\mathrm{~Pa}-234 \mathrm{~m} \\
\mathrm{U}-235 \\
\mathrm{U}-238\end{array}$ & $\begin{array}{c}\begin{array}{c}\text { Activity } \\
(C i / y r)\end{array} \\
-3.58 \mathrm{E}-07 \\
9.47 \mathrm{E}-07 \\
9.47 \mathrm{E}-07 \\
6.10 \mathrm{E}-07 \\
9.47 \mathrm{E}-07 \\
9.47 \mathrm{E}-07 \\
5.66 \mathrm{E}-06 \\
5.66 \mathrm{E}-06 \\
9.47 \mathrm{E}-07 \\
5.45 \mathrm{E}-06 \\
5.74 \mathrm{E}-05 \\
6.98 \mathrm{E}-03 \\
7.15 \mathrm{E}-07 \\
6.98 \mathrm{E}-03 \\
5.43 \mathrm{E}-06 \\
6.98 \mathrm{E}-03\end{array}$ & \\
\hline \multirow[t]{2}{*}{ Shipping Site: $Y-12$} & $\begin{array}{l}\text { Total Volume } \\
\text { (m3/yr) }\end{array}$ & $\begin{array}{c}\text { Total Mass } \\
(\mathrm{kg} / \mathrm{yr})\end{array}$ & $8.27 E+07$ \\
\hline & $\begin{array}{l}\text { Radionuclide } \\
\mathrm{Tl}-208 \\
\mathrm{~Pb}-212 \\
\mathrm{Bi}-212 \\
\mathrm{Po-212} \\
\mathrm{Po-216} \\
\mathrm{Ra-224} \\
\mathrm{Ra-228} \\
\mathrm{Ac-228} \\
\mathrm{Th}-228 \\
\mathrm{Th}-231 \\
\mathrm{Th}-232 \\
\mathrm{Th}-234 \\
\mathrm{Pa-234} \\
\mathrm{Pa-234m} \\
\mathrm{U}-235 \\
\mathrm{U}-238\end{array}$ & $\begin{array}{c}\begin{array}{c}\text { Activity } \\
\text { (Ci/yr) }\end{array} \\
6.80 \mathrm{E}-05 \\
1.80 \mathrm{E}-04 \\
1.80 \mathrm{E}-04 \\
1.16 \mathrm{E}-04 \\
1.80 \mathrm{E}-04 \\
1.80 \mathrm{E}-04 \\
1.07 \mathrm{E}-03 \\
1.07 \mathrm{E}-03 \\
1.80 \mathrm{E}-04 \\
1.03 \mathrm{E}-03 \\
1.09 \mathrm{E}-02 \\
1.32 \mathrm{E}+00 \\
1.36 \mathrm{E}-04 \\
1.32 \mathrm{E}+00 \\
1.03 \mathrm{E}-03 \\
1.32 \mathrm{E}+00\end{array}$ & \\
\hline \multicolumn{4}{|l|}{ ation: SRS } \\
\hline \multirow[t]{2}{*}{ Shipping Site: Pinellas } & $\begin{array}{c}\text { Total Volume } \\
(\mathrm{m} 3 / \mathrm{yr})\end{array}$ & $\begin{array}{c}\text { Total Mass } \\
(\mathrm{kg} / \mathrm{Y} x)\end{array}$ & $1.91 E+04$ \\
\hline & $\begin{array}{l}\text { Radionuclide } \\
\mathrm{H}-3\end{array}$ & $\frac{\begin{array}{c}\text { Activity } \\
\text { (Ci/Yr) }\end{array}}{1.99 E+04}$ & \\
\hline
\end{tabular}




\section{A.10 WM LLW REGIONALIZED 3 ALTERNATIVE (CASE 4): ACTIVATED METALS}

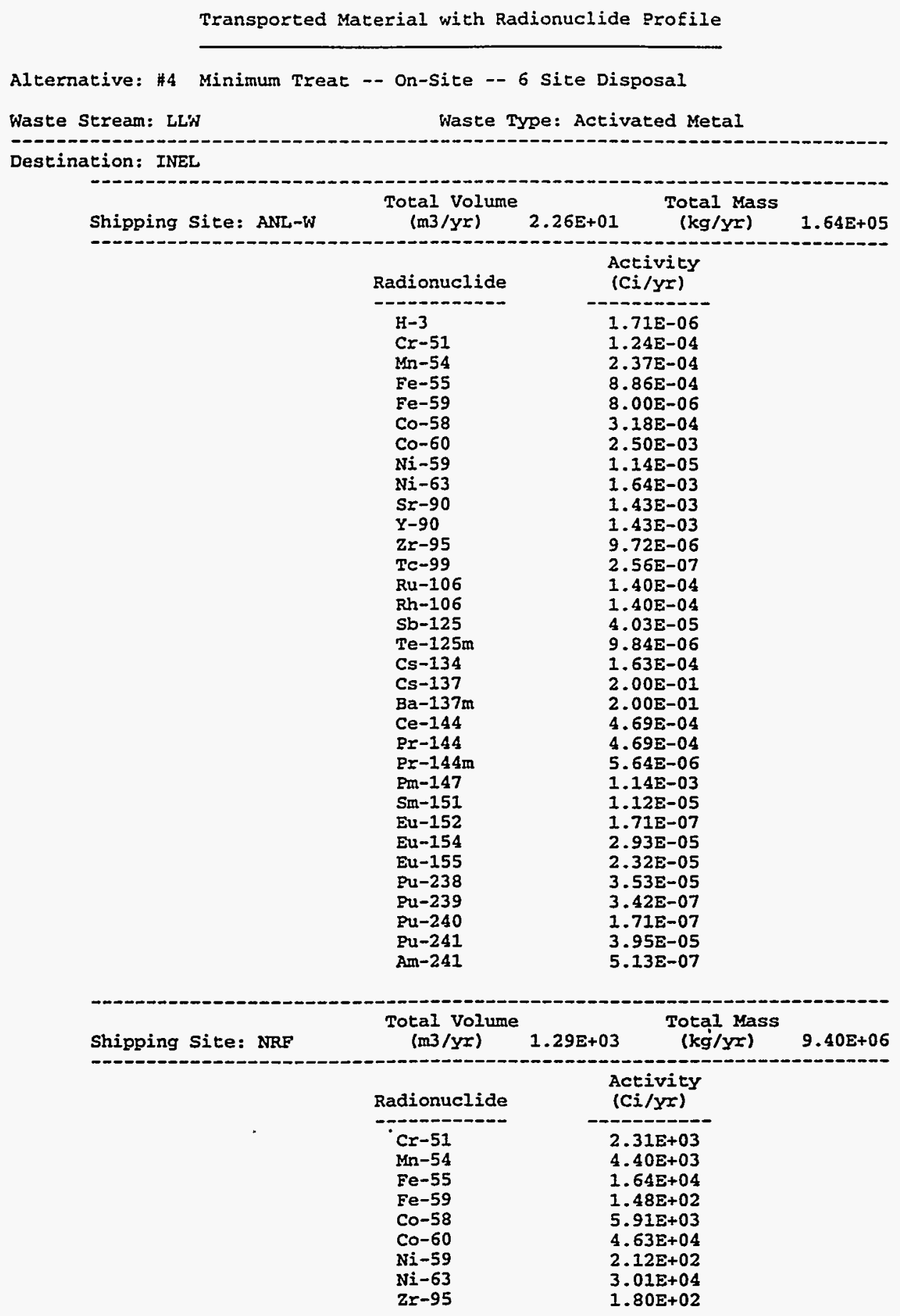


information in the report by Goyette and Dolak (1996) was used as the primary source of information for the transportation assessment.

In the report by Goyette and Dolak (1996), the current inventories and annual generation rates of LLW are reported for about 30 sites in the DOE complex. For the purposes of assessment, the WM PEIS considers current inventories plus 20 years of generation for LLW generated from WM activities. For each site, information on inventory is presented in terms of projected waste volumes and masses. In addition, the physical and radiological characteristics of the waste at each site are presented.

\subsubsection{Physical Waste Forms}

The LLW at each site is characterized as a function of the potential options for treatment of the various waste forms in the report by Goyette and Dolak (1996). For WM LLW, 10 different LLW treatability categories were developed for WM PEIS assessment on the basis of the physical characteristics of the waste. Treatability categories for solid waste (both wet and dry) are (1) combustible, (2) noncombustible and noncompactible, (3) noncombustible and compactible,

(4) surface-contaminated bulk metal or equipment, (5) activated bulk metal or equipment, (6) sludge or resin, and (7) other (wastes that do not fit into the previous categories). Treatability categories for liquid wastes are (8) dilute or aqueous and (9) organic. Low-level waste can also be remote-handled, although no liquid waste at the installations falls into this category. The 10th LLW treatability category is identified simply as remote-handled, independent of the physical characteristics of the waste. For the WM PEIS, all aqueous LLW is assumed to be treated at the site of generation before transportation (Goyette and Dolak 1996). Residuals are then managed as solid LLW.

For purposes of the transportation assessment, two physical waste forms were defined on the basis of the 10 treatability categories listed previously: (1) heterogeneous solids, and (2) activated metals. The heterogeneous solids include all solid-waste categories with the exception of Category 5, plus the solid residuals of liquid-waste treatment. The activated metals consist of waste in treatability Category 5. Activated metals were treated separately in the transportation assessment because the radioactive contamination is not readily released to the environment in a dispersible form during transportation-related accidents.

\subsubsection{Radiological Profiles}

The radiological characteristics of LLW at each site are divided into six categories by Goyette and Dolak (1996): (1) uranium and thorium - waste materials for which the principal hazard results from naturally occurring uranium and thorium isotopes; (2) fission products - waste materials contaminated with beta- or gamma-ray-emitting radionuclides that originate as a result of 
Destination: LANL

\begin{tabular}{|c|c|c|c|}
\hline Shipping Site: SNLA & $\begin{array}{l}\text { Total Volume } \\
(\mathrm{m} 3 / \mathrm{Yr})\end{array}$ & $\begin{array}{c}\text { Tocal Mass } \\
(\mathrm{kg} / \mathrm{Yr})\end{array}$ & $1.15 \mathrm{E}+05$ \\
\hline & $\begin{array}{l}\text { Radionuclide } \\
\mathrm{Cr}-51 \\
\mathrm{Mn}-54 \\
\mathrm{Fe}-55 \\
\mathrm{Fe}-59 \\
\mathrm{Co}-58 \\
\mathrm{Co}-60 \\
\mathrm{Ni}-59 \\
\mathrm{Ni}-63 \\
\mathrm{Z}-95\end{array}$ & $\begin{array}{c}\begin{array}{c}\text { Activity } \\
\text { (Ci/yr) }\end{array} \\
6.54 \mathrm{E}-04 \\
1.24 \mathrm{E}-03 \\
4.64 \mathrm{E}-03 \\
4.20 \mathrm{E}-05 \\
1.67 \mathrm{E}-03 \\
1.31 \mathrm{E}-02 \\
6.00 \mathrm{E}-05 \\
8.53 \mathrm{E}-03 \\
5.10 \mathrm{E}-05\end{array}$ & \\
\hline
\end{tabular}

Destination: NTS

\begin{tabular}{|c|c|c|c|}
\hline Shipping site: LBL & $\begin{array}{l}\text { Total Volume } \\
\text { (m3/yr) }\end{array}$ & $\begin{array}{l}\text { Total Mass } \\
(\mathrm{kg} / \mathrm{yr})\end{array}$ & $2.87 \mathrm{E}+05$ \\
\hline & $\begin{array}{l}\text { Radionuclide } \\
\mathrm{Cr}-51 \\
\mathrm{Mn}-54 \\
\mathrm{Fe}-55 \\
\mathrm{Fe}-59 \\
\mathrm{Co}-58 \\
\mathrm{Co}-60 \\
\mathrm{Ni}-59 \\
\mathrm{Ni}-63 \\
\mathrm{Zr}-95\end{array}$ & 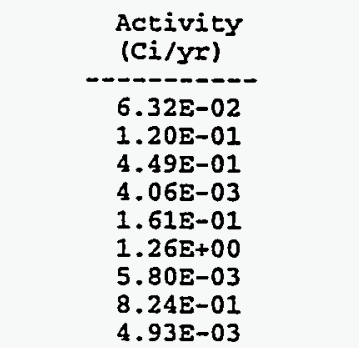 & \\
\hline
\end{tabular}

Destination: ORNL

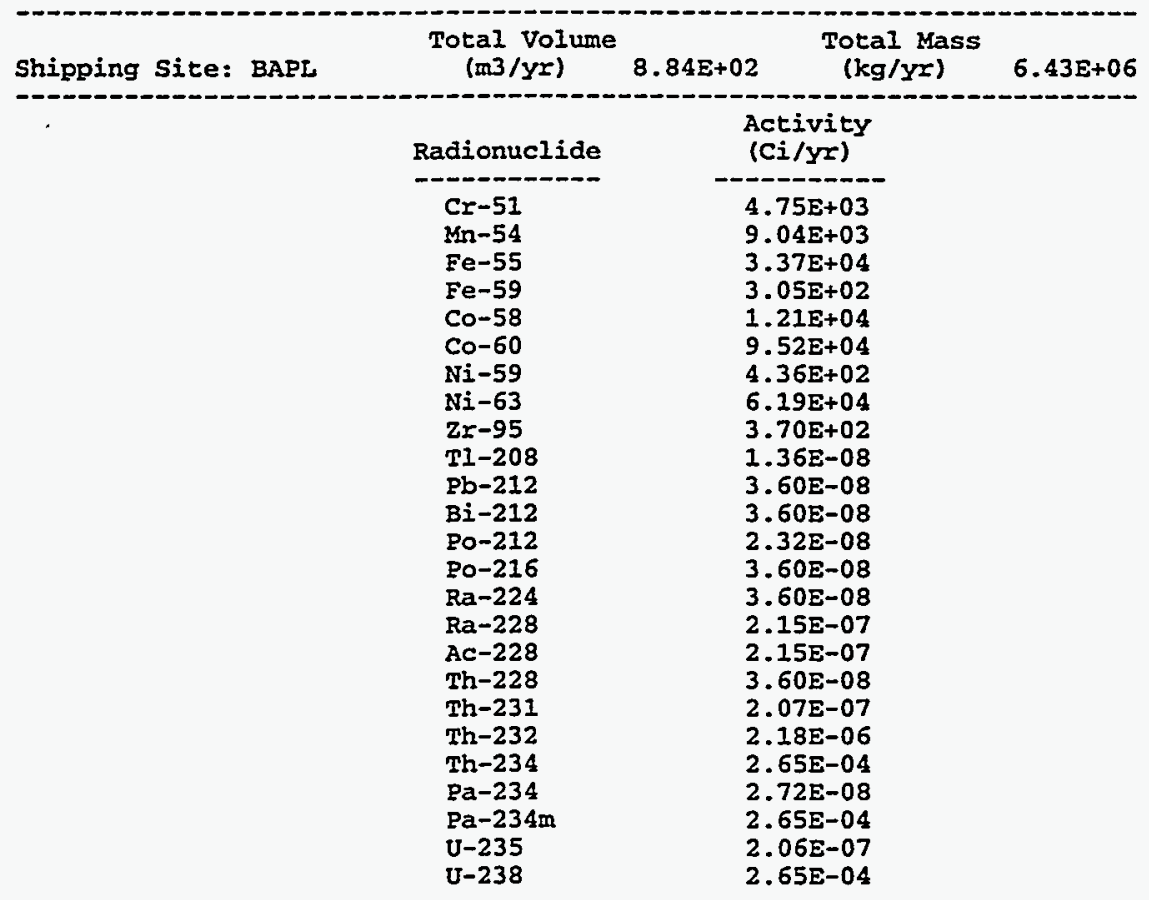




\begin{tabular}{|c|c|c|c|}
\hline Shipping Site: FNAL & $\begin{array}{l}\text { Total volume } \\
(\mathrm{m} 3 / y r)\end{array}$ & $\begin{array}{l}\text { Total Mass } \\
\text { (kg/yr) }\end{array}$ & $8.14 \mathrm{E}+05$ \\
\hline & $\begin{array}{l}\text { Radionuclide } \\
\mathrm{Cr}-51 \\
\mathrm{Mn}-54 \\
\mathrm{Fe}-55 \\
\mathrm{Fe}-59 \\
\mathrm{Co}-58 \\
\mathrm{Co}-60 \\
\mathrm{Ni}-59 \\
\mathrm{Ni}-63 \\
\mathrm{Z}=-95\end{array}$ & $\begin{array}{l}\begin{array}{c}\text { Activity } \\
\text { (Ci/yr) }\end{array} \\
-3.35 \mathrm{E}-02 \\
6.39 \mathrm{E}-02 \\
2.38 \mathrm{E}-01 \\
2.15 \mathrm{E}-03 \\
8.57 \mathrm{E}-02 \\
6.72 \mathrm{E}-01 \\
3.08 \mathrm{E}-03 \\
4.37 \mathrm{E}-01 \\
2.61 \mathrm{E}-03\end{array}$ & \\
\hline Shipping Site: KAPL & $\begin{array}{l}\text { Total Volume } \\
\text { (m3/yr) }\end{array}$ & $\begin{array}{l}\text { Total Mass } \\
\text { (kg/Yr) }\end{array}$ & $1.33 E+07$ \\
\hline & 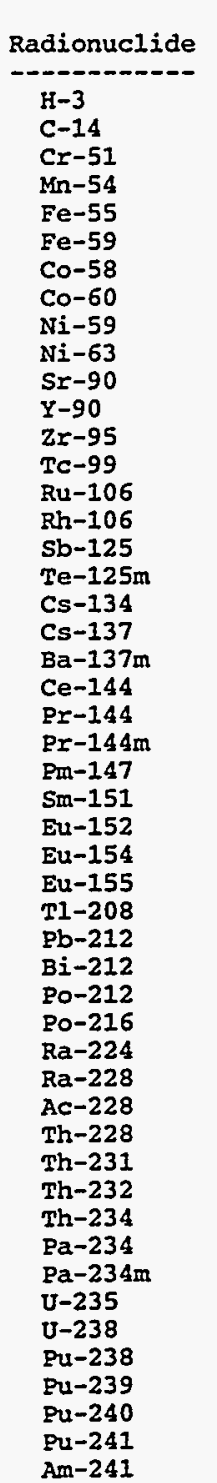 & 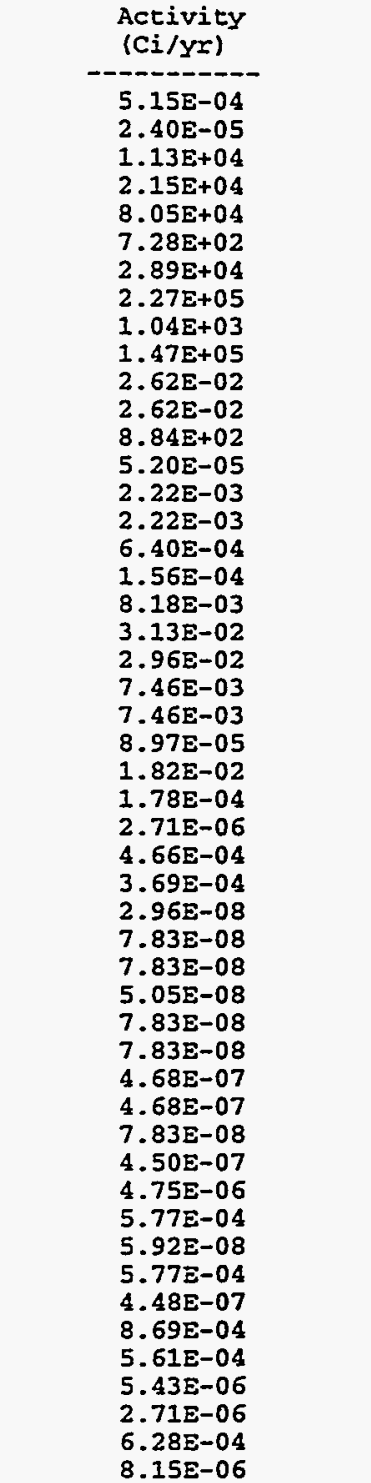 & \\
\hline
\end{tabular}




\section{A.11 WM LLW REGIONALIZED 4 ALTERNATIVE (CASE 12): HETEROGENEOUS SOLIDS}

Transported Material with Radionuclide Profile

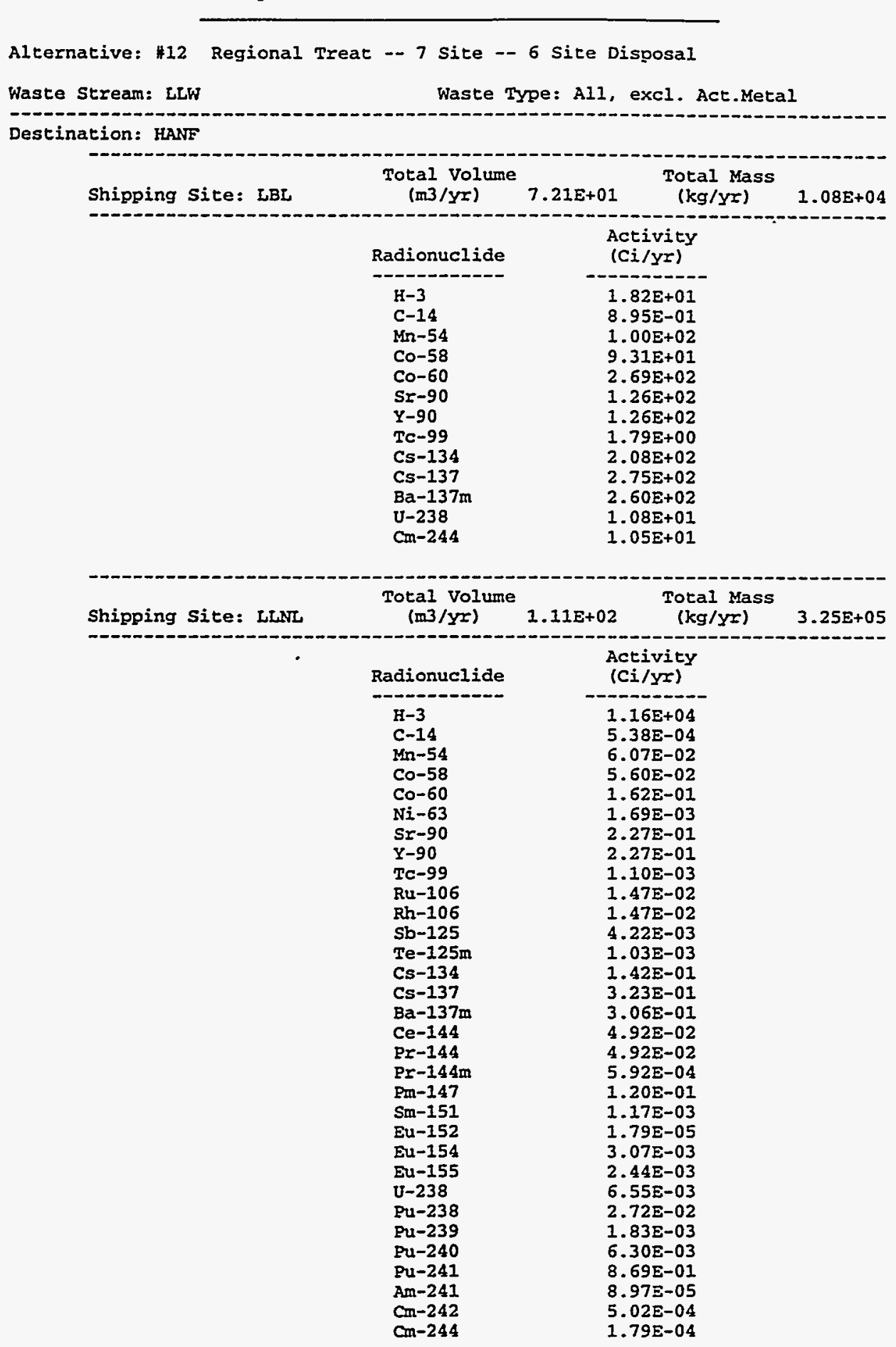


A-73

\begin{tabular}{|c|c|c|c|}
\hline Shipping Site: SLAC & $\begin{array}{l}\text { Total Volume } \\
(\mathrm{m} 3 / \mathrm{Yr})\end{array}$ & $\begin{array}{c}\text { Total Mass } \\
(\mathrm{kg} / \mathrm{yr})\end{array}$ & $1.24 \mathrm{E}+01$ \\
\hline & $\begin{array}{l}\text { Radionuclide } \\
\mathrm{Cr}-51 \\
\mathrm{Mn}-54 \\
\mathrm{Fe}-55 \\
\mathrm{Fe}-59 \\
\mathrm{CO}-58 \\
\mathrm{Co}-60 \\
\mathrm{Ni}-59 \\
\mathrm{Ni}-63 \\
\mathrm{Zr}-95\end{array}$ & \begin{tabular}{c}
$\begin{array}{c}\text { Activity } \\
\text { (Ci/YY) }\end{array}$ \\
\hdashline $2.10 \mathrm{E}-07$ \\
$4.00 \mathrm{E}-07$ \\
$1.49 \mathrm{E}-06$ \\
$1.35 \mathrm{E}-08$ \\
$5.38 \mathrm{E}-07$ \\
$4.21 \mathrm{E}-06$ \\
$1.93 \mathrm{E}-08$ \\
$2.74 \mathrm{E}-06$ \\
$1.64 \mathrm{E}-08$
\end{tabular} & \\
\hline Shipping Site: SNLL & $\begin{array}{l}\text { Total volume } \\
\quad(\mathrm{m} 3 / \mathrm{yr})\end{array}$ & $2.28 \mathrm{E}+00 \quad \begin{array}{c}\text { Total Mass } \\
(\mathrm{kg} / \mathrm{yr})\end{array}$ & $1.92 \mathrm{E}+03$ \\
\hline - & $\begin{array}{l}\text { Radionuclide } \\
\mathrm{H}-3 \\
\mathrm{Fe}-55 \\
\mathrm{Co}-60 \\
\mathrm{Tl}-208 \\
\mathrm{~Pb}-212 \\
\mathrm{Bi}-212 \\
\mathrm{Po-212} \\
\mathrm{Po-216} \\
\mathrm{Ra}-224 \\
\mathrm{Ra}-228 \\
\mathrm{Ac}-228 \\
\mathrm{Th}-228 \\
\mathrm{Th}-231 \\
\mathrm{Th}-232 \\
\mathrm{Th}-234 \\
\mathrm{~Pa}-234 \\
\mathrm{~Pa}-234 \mathrm{~m} \\
\mathrm{U}-235 \\
\mathrm{U}-238\end{array}$ & \begin{tabular}{c}
$\begin{array}{c}\text { Activity } \\
\text { (Ci/yr) }\end{array}$ \\
\hdashline $2.82 \mathrm{E}+04$ \\
$3.15 \mathrm{E}-01$ \\
$3.15 \mathrm{E}-01$ \\
$5.35 \mathrm{E}-06$ \\
$1.41 \mathrm{E}-05$ \\
$1.41 \mathrm{E}-05$ \\
$9.13 \mathrm{E}-06$ \\
$1.41 \mathrm{E}-05$ \\
$1.41 \mathrm{E}-05$ \\
$8.47 \mathrm{E}-05$ \\
$8.47 \mathrm{E}-05$ \\
$1.41 \mathrm{E}-05$ \\
$8.15 \mathrm{E}-05$ \\
$8.59 \mathrm{E}-04$ \\
$1.04 \mathrm{E}-01$ \\
$1.07 \mathrm{E}-05$ \\
$1.04 \mathrm{E}-01$ \\
$8.12 \mathrm{E}-05$ \\
$1.04 \mathrm{E}-01$
\end{tabular} & \\
\hline
\end{tabular}


Destination: INEL

\begin{tabular}{|c|c|c|c|}
\hline Shipping Site: ANL-W & $\begin{array}{l}\text { Total volume } \\
(\mathrm{m} 3 / \mathrm{yr})\end{array}$ & $\begin{array}{c}\text { Tocal Mass } \\
(\mathrm{kg} / \mathrm{yr})\end{array}$ & $2.12 E+05$ \\
\hline . & $\begin{array}{l}\text { Radionuclide } \\
\mathrm{H}-3 \\
\mathrm{Cr}-51 \\
\mathrm{Mn}-54 \\
\mathrm{Fe}-55 \\
\text { Fe-59 } \\
\mathrm{Co}-58 \\
\mathrm{Co}-60 \\
\mathrm{Ni}-59 \\
\mathrm{Ni}-63 \\
\mathrm{Sr}-90 \\
\mathrm{Y}-90 \\
\mathrm{Zr}-95 \\
\mathrm{Tc}-99 \\
\mathrm{Ru}-106 \\
\mathrm{Rh}-106 \\
\mathrm{Sb}-125 \\
\mathrm{Te}-125 \mathrm{~m} \\
\mathrm{Cs}-134 \\
\mathrm{Cs}-137 \\
\mathrm{Ba}-137 \mathrm{~m} \\
\mathrm{Ce}-144 \\
\mathrm{Pr}-144 \\
\mathrm{Pr}-144 \mathrm{~m} \\
\mathrm{Pm}-147 \\
\mathrm{Sm}-151 \\
\mathrm{Eu}-152 \\
\text { Eu-154 } \\
\text { Eu-155 } \\
\mathrm{Pu}-238 \\
\mathrm{Pu}-239 \\
\mathrm{Pu}-240 \\
\mathrm{Pu}-241 \\
\mathrm{Am}-241 \\
\mathrm{Cm}-242 \\
\mathrm{Cm}-244\end{array}$ & $\begin{array}{c}\text { Acrivicy } \\
\text { (Ci/yr) } \\
7.83 E-04 \\
3.58 E+03 \\
6.83 E+03 \\
2.54 E+04 \\
2.30 E+02 \\
9.16 E+03 \\
7.18 E+04 \\
3.29 E+02 \\
.4 .67 E+04 \\
3.17 E-02 \\
3.17 E-02 \\
2.79 E+02 \\
5.66 E-06 \\
3.09 E-03 \\
3.09 E-03 \\
8.89 E-04 \\
2.17 E-04 \\
3.59 E-03 \\
3.32 E-02 \\
3.14 E-02 \\
1.03 E-02 \\
1.03 E-02 \\
1.24 E-04 \\
2.52 E-02 \\
2.47 E-04 \\
3.77 E-06 \\
6.47 E-04 \\
5.13 E-04 \\
7.81 E-04 \\
7.72 E-06 \\
4.38 E-06 \\
9.56 E-04 \\
1.13 E-05 \\
4.88 E-08 \\
1.74 E-08\end{array}$ & \\
\hline \multicolumn{4}{|l|}{ lation: LANL } \\
\hline \multirow[t]{2}{*}{ Shipping site: ITRI } & $\begin{array}{l}\text { Total Volume } \\
(\mathrm{m} 3 / y r)\end{array}$ & $\begin{array}{c}\text { Total Mass } \\
(\mathrm{kg} / \mathrm{y} x)\end{array}$ & $1.64 E+05$ \\
\hline & $\begin{array}{l}\text { Radionuclide } \\
\mathrm{H}-3 \\
\mathrm{C}-14 \\
\mathrm{Mn}-54 \\
\mathrm{Co}-58 \\
\mathrm{Co}-60 \\
\mathrm{Sr}-90 \\
\mathrm{X}-90 \\
\mathrm{Tc}-99 \\
\mathrm{Cs}-134 \\
\mathrm{Cs}-137 \\
\mathrm{Ba}-137 \mathrm{~m} \\
\mathrm{U}-238\end{array}$ & 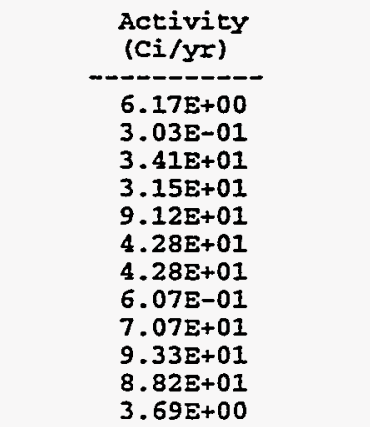 & \\
\hline
\end{tabular}




\section{A-75}

\begin{tabular}{|c|c|c|c|}
\hline Shipping site: PANT & $\begin{array}{l}\text { Total Volume } \\
\text { (m3/yr) }\end{array}$ & $\begin{array}{l}\text { Total Mass } \\
\text { (kg/Yr) }\end{array}$ & $2.88 \mathrm{E}+07$ \\
\hline & $\begin{array}{l}\text { Radionuclide } \\
\mathrm{H}-3 \\
\mathrm{Tl}-208 \\
\mathrm{~Pb}-212 \\
\mathrm{Bi}-212 \\
\mathrm{Po}-212 \\
\mathrm{Po}-216 \\
\mathrm{Ra}-224 \\
\mathrm{Ra}-228 \\
\mathrm{Ac}-228 \\
\mathrm{Th}-228 \\
\mathrm{Th}-231 \\
\mathrm{Th}-232 \\
\mathrm{Th}-234 \\
\mathrm{~Pa}-234 \\
\mathrm{~Pa}-234 \mathrm{~m} \\
\mathrm{U}-235 \\
\mathrm{U}-238\end{array}$ & $\begin{array}{l}\text { Activity } \\
\text { (Ci/yr) } \\
2.34 E+02 \\
2.21 E-04 \\
5.86 E-04 \\
5.86 E-04 \\
3.78 E-04 \\
5.86 E-04 \\
5.86 E-04 \\
3.50 E-03 \\
3.50 E-03 \\
5.86 E-04 \\
3.37 E-03 \\
3.56 E-02 \\
4.32 E+00 \\
4.43 E-04 \\
4.32 E+00 \\
3.36 E-03 \\
4.32 E+00\end{array}$ & \\
\hline Shipping Site: RFP & $\begin{array}{l}\text { Total Volume } \\
\text { (m3/yr) }\end{array}$ & $\begin{array}{cc}\text { Total Mass } \\
(\mathrm{kg} / \mathrm{yr})\end{array}$ & $6.52 E+06$ \\
\hline & $\begin{array}{l}\text { Radionuclide } \\
\text { Tl-208 } \\
\text { Pb-212 } \\
\text { Bi-212 } \\
\text { Po-212 } \\
\text { Po-216 } \\
\text { Ra-224 } \\
\text { Ra-228 } \\
\mathrm{Ac}-228 \\
\mathrm{Th}-228 \\
\mathrm{Th}-231 \\
\mathrm{Th}-232 \\
\mathrm{Th}-234 \\
\mathrm{~Pa}-234 \\
\mathrm{~Pa}-234 \mathrm{~m} \\
\mathrm{U}-235 \\
\mathrm{U}-238 \\
\mathrm{Tu}-238 \\
\mathrm{Pu}-239 \\
\mathrm{Pu}-240 \\
\mathrm{Pu}-241 \\
\mathrm{Am}-241 \\
\mathrm{Cm}-242 \\
\mathrm{Cm}-244\end{array}$ & $\begin{array}{c}\begin{array}{l}\text { Activity } \\
\text { (Ci/yr) }\end{array} \\
-0.60 \mathrm{E}-05 \\
3.55 \mathrm{E}-05 \\
9.5 \mathrm{E}-05 \\
9.55 \mathrm{E}-05 \\
6.15 \mathrm{E}-05 \\
9.55 \mathrm{E}-05 \\
9.55 \mathrm{E}-05 \\
5.70 \mathrm{E}-04 \\
5.70 \mathrm{E}-04 \\
9.55 \mathrm{E}-05 \\
5.49 \mathrm{E}-04 \\
5.79 \mathrm{E}-03 \\
7.04 \mathrm{E}-01 \\
7.21 \mathrm{E}-05 \\
7.04 \mathrm{E}-01 \\
5.47 \mathrm{E}-04 \\
7.04 \mathrm{E}-01 \\
5.56 \mathrm{E}-01 \\
4.24 \mathrm{E}-02 \\
1.48 \mathrm{E}-01 \\
2.04 \mathrm{E}+01 \\
8.48 \mathrm{E}-04 \\
1.18 \mathrm{E}-02 \\
4.24 \mathrm{E}-03\end{array}$ & \\
\hline
\end{tabular}




\section{A-76}

\begin{tabular}{|c|c|c|c|}
\hline Shipping Site: SNLA & $\begin{array}{l}\text { Total Volume } \\
(\mathrm{m} 3 / \mathrm{yr})\end{array}$ & $\begin{array}{c}\text { Total Mass } \\
(\mathrm{kg} / \mathrm{Yr})\end{array}$ & $3.28 \mathrm{E}+05$ \\
\hline & 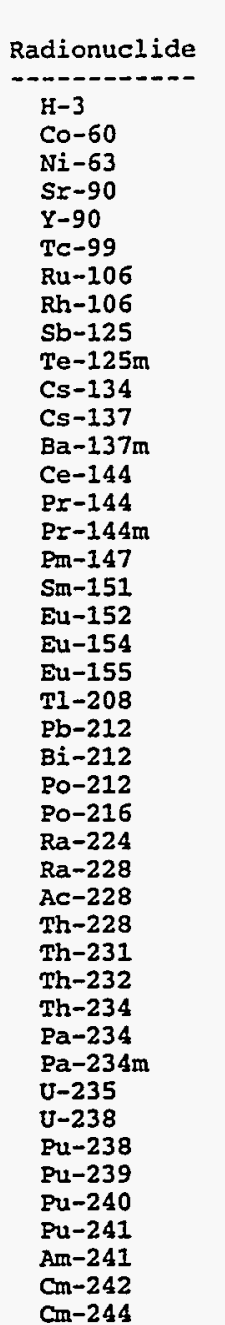 & $\begin{array}{c}\text { Activity } \\
\text { (Ci/Yr) } \\
3.00 \mathrm{E}+00 \\
3.85 \mathrm{E}-05 \\
5.67 \mathrm{E}-05 \\
5.04 \mathrm{E}-03 \\
5.04 \mathrm{E}-03 \\
9.00 \mathrm{E}-07 \\
4.92 \mathrm{E}-04 \\
4.92 \mathrm{E}-04 \\
1.41 \mathrm{E}-04 \\
3.45 \mathrm{E}-05 \\
5.72 \mathrm{E}-04 \\
5.28 \mathrm{E}-03 \\
4.99 \mathrm{E}-03 \\
1.64 \mathrm{E}-03 \\
1.64 \mathrm{E}-03 \\
1.98 \mathrm{E}-05 \\
4.02 \mathrm{E}-03 \\
3.93 \mathrm{E}-05 \\
6.00 \mathrm{E}-07 \\
1.02 \mathrm{E}-04 \\
8.16 \mathrm{E}-05 \\
5.10 \mathrm{E}-06 \\
1.35 \mathrm{E}-05 \\
1.35 \mathrm{E}-05 \\
8.70 \mathrm{E}-06 \\
1.35 \mathrm{E}-05 \\
1.35 \mathrm{E}-05 \\
8.07 \mathrm{E}-05 \\
8.07 \mathrm{E}-05 \\
1.35 \mathrm{E}-05 \\
7.77 \mathrm{E}-05 \\
8.19 \mathrm{E}-04 \\
9.96 \mathrm{E}-02 \\
1.02 \mathrm{E}-05 \\
9.96 \mathrm{E}-02 \\
7.74 \mathrm{E}-05 \\
9.96 \mathrm{E}-02 \\
7.87 \mathrm{E}-02 \\
6.00 \mathrm{E}-03 \\
2.10 \mathrm{E}-02 \\
2.89 \mathrm{E}+00 \\
1.21 \mathrm{E}-04 \\
1.68 \mathrm{E}-03 \\
6.00 \mathrm{E}-04\end{array}$ & \\
\hline
\end{tabular}


Destination: NTS

\begin{tabular}{|c|c|c|c|}
\hline Shipping Site: LBL & $\begin{array}{l}\text { Total Volume } \\
(\mathrm{m} 3 / \mathrm{yr})\end{array}$ & $\begin{array}{l}\text { Total Mass } \\
\text { (kg/Yr) }\end{array}$ & $3.43 \mathrm{E}+04$ \\
\hline & $\begin{array}{l}\text { Radionuclide } \\
\mathrm{H}-3 \\
\mathrm{C}-14 \\
\mathrm{Mn}-54 \\
\mathrm{C}-58 \\
\mathrm{C}-60 \\
\mathrm{~S}-90 \\
\mathrm{Y}-90 \\
\mathrm{Tc}-99 \\
\mathrm{Cs}-134 \\
\mathrm{Cs}-137 \\
\mathrm{Ba}-137 \mathrm{~m} \\
\mathrm{U}-238\end{array}$ & $\begin{array}{c}\begin{array}{c}\text { Activity } \\
\text { (Ci/Yr) }\end{array} \\
6.17 \mathrm{E}+02 \\
2.35 \mathrm{E}-01 \\
2.65 \mathrm{E}+01 \\
2.45 \mathrm{E}+01 \\
7.08 \mathrm{E}+01 \\
3.33 \mathrm{E}+01 \\
3.33 \mathrm{E}+01 \\
4.71 \mathrm{E}-01 \\
5.49 \mathrm{E}+01 \\
7.25 \mathrm{E}+01 \\
6.85 \mathrm{E}+01 \\
2.86 \mathrm{E}+00\end{array}$ & \\
\hline \multirow{2}{*}{ Shipping Site: LLNL } & $\begin{array}{l}\text { Total Volume } \\
\text { (m3/yr) }\end{array}$ & $\begin{array}{cc}\text { Total Mass } \\
(\mathrm{kg} / \mathrm{Yr})\end{array}$ & $7.42 \mathrm{E}+05$ \\
\hline & $\begin{array}{l}\text { Radionuclide } \\
\mathrm{H}-3 \\
\mathrm{C}-14 \\
\mathrm{Mn}-54 \\
\mathrm{Co}-58 \\
\mathrm{Co}-60 \\
\mathrm{Ni}-63 \\
\mathrm{Sr}-90 \\
\mathrm{Y}-90 \\
\mathrm{Tc}-99 \\
\mathrm{Ru}-106 \\
\mathrm{Rh}-106 \\
\mathrm{Sb}-125 \\
\mathrm{Te}-125 \mathrm{~m} \\
\mathrm{Cs}-134 \\
\mathrm{Cs}-137 \\
\mathrm{Ba}-137 \mathrm{~m} \\
\mathrm{Ce}-144 \\
\mathrm{Pr}-144 \\
\mathrm{Pr}-144 \mathrm{~m} \\
\mathrm{gm}-147 \\
\mathrm{Sm}-151 \\
\mathrm{Eu}-152 \\
\mathrm{Eu}-154 \\
\mathrm{Eu}-155 \\
\mathrm{U}-238 \\
\mathrm{Pu}-238 \\
\mathrm{Pu}-239 \\
\mathrm{Pu}-240 \\
\mathrm{Pu}-241 \\
\mathrm{Am}-241 \\
\mathrm{Cm}-242 \\
\mathrm{Cm}-244\end{array}$ & $\begin{array}{c}\text { Activity } \\
\text { (Ci/yI) } \\
2.16 E+04 \\
1.01 E-03 \\
1.14 E-01 \\
1.05 E-01 \\
3.06 E-01 \\
3.19 E-03 \\
4.27 E-01 \\
4.27 E-01 \\
2.07 E-03 \\
2.77 E-02 \\
2.77 E-02 \\
7.96 E-03 \\
1.94 E-03 \\
2.68 E-01 \\
6.09 E-01 \\
5.76 E-01 \\
9.27 E-02 \\
9.27 E-02 \\
1.11 E-03 \\
2.26 E-01 \\
2.21 E-03 \\
3.38 E-05 \\
5.79 E-03 \\
4.59 E-03 \\
1.23 E-02 \\
5.12 E-02 \\
3.44 E-03 \\
1.18 E-02 \\
1.63 E+00 \\
1.69 E-04 \\
9.46 E-04 \\
3.38 E-04\end{array}$ & \\
\hline \multirow[t]{2}{*}{ Shipping Site: SLAC } & $\begin{array}{l}\text { Total Volume } \\
(\mathrm{m} 3 / \mathrm{yr})\end{array}$ & $\begin{array}{l}\text { Total Mass } \\
(\mathrm{kg} / \mathrm{yx})\end{array}$ & $7.45 E+05$ \\
\hline & $\begin{array}{l}\text { Radionuclide } \\
\text { Cr-51 } \\
\text { Mn-54 } \\
\text { Fe-55 } \\
\text { Fe-59 } \\
\mathrm{Co}-58 \\
\mathrm{Co}-60 \\
\mathrm{Ni}-59 \\
\mathrm{Ni}-63 \\
\mathrm{Z}-95\end{array}$ & $\begin{array}{c}\begin{array}{c}\text { Activity } \\
\text { (Ci/yz) }\end{array} \\
3.86 \mathrm{E}-03 \\
7.36 \mathrm{E}-03 \\
2.74 \mathrm{E}-02 \\
2.48 \mathrm{E}-04 \\
9.88 \mathrm{E}-03 \\
7.74 \mathrm{E}-02 \\
3.54 \mathrm{E}-04 \\
5.04 \mathrm{E}-02 \\
3.01 \mathrm{E}-04\end{array}$ & \\
\hline
\end{tabular}


A-78

\begin{tabular}{|c|c|c|c|}
\hline Shipping Site: SNLL & $\begin{array}{c}\text { Toral Volume } \\
(\mathrm{m} 3 / \mathrm{y} x)\end{array}$ & $\begin{array}{l}\text { Total Mass } \\
(\mathrm{kg} / \mathrm{y}=)\end{array}$ & $9.17 E+04$ \\
\hline & $\begin{array}{l}\text { Radionuclide } \\
\mathrm{H}-3 \\
\mathrm{Tl}-208 \\
\mathrm{~Pb}-212 \\
\mathrm{Bi}-212 \\
\mathrm{PO-212} \\
\mathrm{PO}-216 \\
\mathrm{Ra}-224 \\
\mathrm{Ra}-228 \\
\mathrm{Ac}-228 \\
\mathrm{Th}-228 \\
\mathrm{Th}-231 \\
\mathrm{Th}-232 \\
\mathrm{Th}-234 \\
\mathrm{~Pa}-234 \\
\mathrm{~Pa}-234 \mathrm{~m} \\
\mathrm{U}-235 \\
\mathrm{U}-238\end{array}$ & $\begin{array}{c}\begin{array}{c}\text { Activity } \\
(C i / y r)\end{array} \\
1.38 E+01 \\
5.86 E-05 \\
1.55 E-04 \\
1.55 E-04 \\
1.00 E-04 \\
1.55 E-04 \\
1.55 E-04 \\
9.28 E-04 \\
9.28 E-04 \\
1.55 E-04 \\
8.93 E-04 \\
9.42 E-03 \\
1.14 E+00 \\
1.17 E-04 \\
1.14 E+00 \\
8.90 E-04 \\
1.14 E+00\end{array}$ & \\
\hline
\end{tabular}


Destination: ORNL

\begin{tabular}{|c|c|c|c|}
\hline Shipping Site: AMES & $\begin{array}{l}\text { Total volume } \\
(\mathrm{m} 3 / \mathrm{yr})\end{array}$ & $\begin{array}{c}\text { Total Mass } \\
(\mathrm{kg} / \mathrm{yr})\end{array}$ & $2.98 E+04$ \\
\hline & $\begin{array}{l}\text { Radionuclide } \\
\mathrm{T} 1-208 \\
\mathrm{~Pb}-212 \\
\mathrm{Bi}-212 \\
\mathrm{Po}-212 \\
\mathrm{Po}-216 \\
\mathrm{Ra}-224 \\
\mathrm{Ra}-228 \\
\mathrm{Ac}-228 \\
\mathrm{Th}-228 \\
\mathrm{Th}-231 \\
\mathrm{Th}-232 \\
\mathrm{Th}-234 \\
\mathrm{~Pa}-234 \\
\mathrm{~Pa}-234 \mathrm{~m} \\
\mathrm{U}-235 \\
\mathrm{U}-238 \\
\mathrm{Pu}-238 \\
\mathrm{Pu}-239 \\
\mathrm{Pu}-240 \\
\mathrm{Pu}-241 \\
\mathrm{Am}-241 \\
\mathrm{Cm}-242 \\
\mathrm{Cm}-244\end{array}$ & 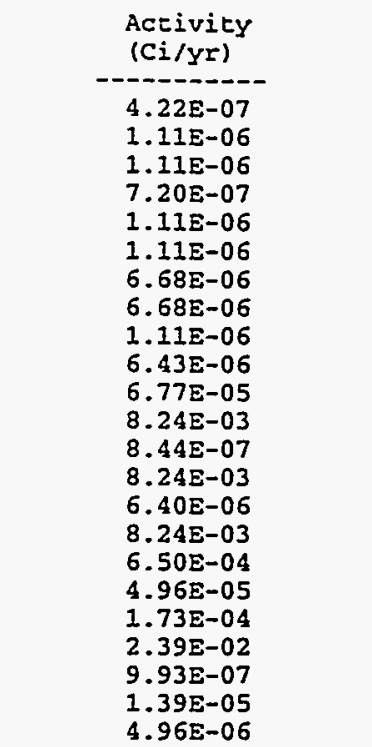 & \\
\hline Shipping Site: ANL-E & $\begin{array}{l}\text { Total volume } \\
(\mathrm{m} 3 / \mathrm{yr})\end{array}$ & $7.35 \mathrm{E}+02 \quad \begin{array}{c}\text { Total Mass } \\
(\mathrm{kg} / \mathrm{yr})\end{array}$ & $2.00 \mathrm{E}+06$ \\
\hline & $\begin{array}{l}\text { Radionuclide } \\
\mathrm{H}-3 \\
\mathrm{C}-14 \\
\mathrm{Cr}-51 \\
\mathrm{Mn}-54 \\
\mathrm{Fe}-55 \\
\mathrm{Fe}-59 \\
\mathrm{Co}-58 \\
\mathrm{Co}-60 \\
\mathrm{Ni}-59 \\
\mathrm{Ni}-63 \\
\mathrm{Sr}-90 \\
\mathrm{Y}-90 \\
\mathrm{Zr}-95 \\
\mathrm{Tc}-99 \\
\mathrm{Ru}-106 \\
\mathrm{Rh}-106 \\
\mathrm{Sb}-125 \\
\mathrm{Te}-125 \mathrm{~m} \\
\mathrm{Cs}-134 \\
\mathrm{Cs}-137 \\
\mathrm{Ba}-137 \mathrm{~m} \\
\mathrm{Ce}-144 \\
\mathrm{Pr}-144 \\
\mathrm{Pr}-144 \mathrm{~m} \\
\mathrm{Pm}-147 \\
\mathrm{Sm}-151 \\
\mathrm{Eu}-152 \\
\mathrm{Eu}-154 \\
\mathrm{Eu}-155 \\
\mathrm{U}-238 \\
\mathrm{Pu}-238 \\
\mathrm{Pu}-239 \\
\mathrm{Pu}-240 \\
\mathrm{Pu}-241 \\
\mathrm{Am}-241\end{array}$ & 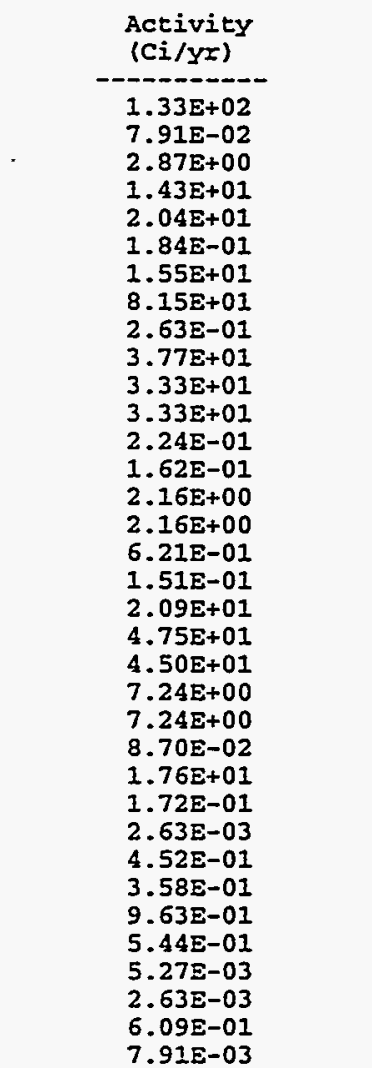 & - \\
\hline
\end{tabular}


fission processes (primary examples are cesium-137 and strontium-90); (3) induced activity - waste materials contaminated with beta- or gamma-ray-emitting isotopes that are generated through neutron activation (of major concern is cobalt-60); (4) tritium - waste materials for which the principal hazard results from tritium; (5) alpha - waste material contaminated with low levels (between 10 and $100 \mathrm{nCi} / \mathrm{g}$ ) of transuranic isotopes, excluding alpha-emitting radionuclides listed under uranium and thorium; and (6) other - mixture or not defined. For each of the six categories, a representative radionuclide profile is defined that specifies the relative mix of radionuclides in the category (Goyette and Dolak 1996). These data are combined with activity levels reported by generating sites to determine the activities of individual radionuclides in the LLW at each site.

\subsection{QUANTITIES AND CHARACTERISTICS OF TRANSPORTED LOW-LEVEL WASTE BY CASE}

The LLW inventory and characterization data described previously are used as the primary input to a computational model called WASTE_MGMT (Kotek et al. 1996). The WASTE_MGMT model has been developed at ANL-E to support analyses of risks and costs for all alternatives for each waste type considered in the WM PEIS (Kotek et al. 1996). Input to the model includes not only data on the waste inventory and characterization, but also data on operations for the TSD facilities used for the wastes, and the definitions of the various alternatives. Specific modeling parameters for the LLW assessment are discussed by Goyette and Dolak (1996).

One output of the WASTE_MGMT model consists of the annual quantity, physical form, and radiological characteristics of the waste shipped between sites for each case. The output of the model consists of a set of origin-and-destination pairs that represent shipping linkages among generator, treatment, and storage sites. For each origin-and-destination pair, the model computes the annual volume (cubic meters), weight (kilograms), and radioactivity (curies) of the LLW transported. In addition, the radioactivity is computed for each individual radionuclide present in the LLW. The effects of potential waste treatment, such as volume reduction or incineration, are considered in the model and are reflected in changes in waste density and activity concentrations. The WASTE_MGMT output files are used directly as input to the transportation risk assessment.

The WASTE_MGMT transportation summary files generated for the LLW transportation assessment are presented in Appendix A of this report. For each of the $14 \mathrm{WM}$ alternatives, shipments are summarized separately for heterogeneous solids and for activated metals. The total quantity of shipments for a case can be found by summing the quantities for heterogeneous solids and activated metals. In general, the quantity of activated metals transported is insignificant compared with the quantity of heterogeneous solids for each case. 


\begin{tabular}{|c|c|c|c|}
\hline Shipping Site: BAPL & $\begin{array}{l}\text { Total Volume } \\
(\mathrm{m} 3 / \mathrm{yr})\end{array}$ & $\begin{array}{c}\text { Total Mass } \\
(\mathrm{kg} / \mathrm{yr})\end{array}$ & $1.08 E+06$ \\
\hline & $\begin{array}{l}\text { Radionuclide } \\
\mathrm{H}-3 \\
\mathrm{Co}-60 \\
\mathrm{Ni}-63 \\
\mathrm{Sr}-90 \\
\mathrm{Y}-90 \\
\mathrm{Tc}-99 \\
\mathrm{Ru}-106 \\
\mathrm{Rh}-106 \\
\mathrm{Sb}-125 \\
\mathrm{Te}-125 \mathrm{~m} \\
\mathrm{Cs}-134 \\
\mathrm{Cs}-137 \\
\mathrm{Ba}-137 \mathrm{~m} \\
\mathrm{Ce}-144 \\
\mathrm{P}-144 \\
\mathrm{Pr}-144 \mathrm{~m} \\
\mathrm{Pm}-147 \\
\mathrm{Sm}-151 \\
\mathrm{Eu}-152 \\
\mathrm{Eu}-154 \\
\mathrm{Eu}-155 \\
\mathrm{Tl}-208 \\
\mathrm{~Pb}-212 \\
\mathrm{Bi}-212 \\
\mathrm{Po}-212 \\
\mathrm{Po}-216 \\
\mathrm{Ra}-224 \\
\mathrm{Ra}-228 \\
\mathrm{Ac}-228 \\
\mathrm{Th}-228 \\
\mathrm{Th}-231 \\
\mathrm{Th}-232 \\
\mathrm{Th}-234 \\
\mathrm{~Pa}-234 \\
\mathrm{~Pa}-234 \mathrm{~m} \\
\mathrm{U}-235 \\
\mathrm{U}-238 \\
\mathrm{Pu}-238 \\
\mathrm{Pu}-239 \\
\mathrm{Pu}-240 \\
\mathrm{Pu}-241 \\
\mathrm{Am}-241 \\
\mathrm{~A}\end{array}$ & $\begin{array}{c}\text { Activicy } \\
(C i / y r) \\
2.51 \mathrm{E}-03 \\
1.19 \mathrm{E}-02 \\
2.38 \mathrm{E}-02 \\
2.11 \mathrm{E}+00 \\
2.11 \mathrm{E}+00 \\
3.77 \mathrm{E}-04 \\
2.06 \mathrm{E}-01 \\
2.06 \mathrm{E}-01 \\
5.93 \mathrm{E}-02 \\
1.44 \mathrm{E}-02 \\
2.40 \mathrm{E}-01 \\
2.21 \mathrm{E}+00 \\
2.09 \mathrm{E}+00 \\
6.91 \mathrm{E}-01 \\
6.91 \mathrm{E}-01 \\
8.31 \mathrm{E}-03 \\
1.68 \mathrm{E}+00 \\
1.65 \mathrm{E}-02 \\
2.51 \mathrm{E}-04 \\
4.32 \mathrm{E}-02 \\
3.42 \mathrm{E}-02 \\
3.80 \mathrm{E}-06 \\
1.00 \mathrm{E}-05 \\
1.00 \mathrm{E}-05 \\
6.49 \mathrm{E}-06 \\
1.00 \mathrm{E}-05 \\
1.00 \mathrm{E}-05 \\
6.02 \mathrm{E}-05 \\
6.02 \mathrm{E}-05 \\
1.00 \mathrm{E}-05 \\
5.80 \mathrm{E}-05 \\
6.11 \mathrm{E}-04 \\
7.43 \mathrm{E}-02 \\
7.61 \mathrm{E}-06 \\
7.43 \mathrm{E}-02 \\
5.77 \mathrm{E}-05 \\
7.43 \mathrm{E}-02 \\
5.20 \mathrm{E}-02 \\
5.03 \mathrm{E}-04 \\
2.51 \mathrm{E}-04 \\
5.81 \mathrm{E}-02 \\
7.55 \mathrm{E}-04\end{array}$ & \\
\hline Shipping Site: FNAI & $\begin{array}{l}\text { Total volume } \\
(\mathrm{m} 3 / \mathrm{yr})\end{array}$ & $\begin{array}{l}\text { Total Mass } \\
(\mathrm{kg} / \mathrm{Yr})\end{array}$ & \\
\hline & \begin{tabular}{l} 
Radionuclide \\
\hdashline $\mathrm{H}-3$ \\
$\mathrm{Cr}-51$ \\
$\mathrm{Mn}-54$ \\
$\mathrm{Fe}-55$ \\
$\mathrm{Fe}-59$ \\
$\mathrm{Co}-58$ \\
$\mathrm{Co}-60$ \\
$\mathrm{Ni}-59$ \\
$\mathrm{Ni}-63$ \\
$\mathrm{Zr}-95$ \\
$\mathrm{Tl}-208$ \\
$\mathrm{~Pb}-212$ \\
$\mathrm{Bi}-212$ \\
$\mathrm{Po}-212$ \\
$\mathrm{PO}-216$ \\
$\mathrm{Ra}-224$ \\
$\mathrm{Ra}-228$ \\
$\mathrm{Ac}-228$ \\
$\mathrm{Th}-228$ \\
$\mathrm{Th}-231$ \\
$\mathrm{Th}-232$ \\
$\mathrm{Th}-234$ \\
$\mathrm{~Pa}-234$ \\
$\mathrm{~Pa}-234 \mathrm{~m}$ \\
$\mathrm{U}-235$ \\
$\mathrm{U}-238$
\end{tabular} & 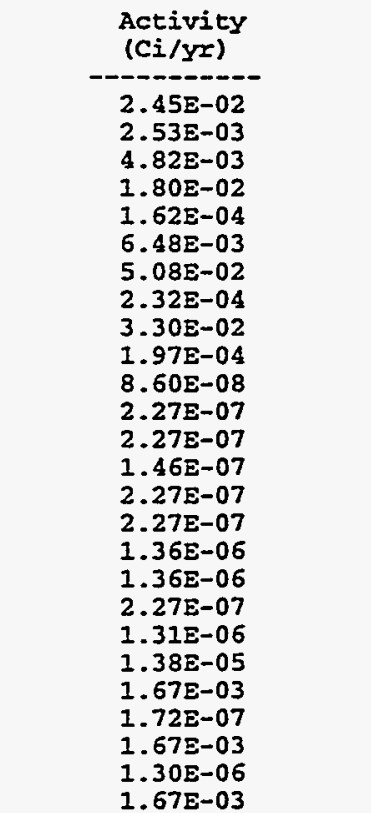 & \\
\hline
\end{tabular}


A-8I

\begin{tabular}{|c|c|c|c|}
\hline Shipping Site: $\mathrm{K}-25$ & $\begin{array}{c}\text { Total Volume } \\
(\mathrm{m} 3 / \mathrm{yr})\end{array}$ & $\begin{array}{l}\text { Total Mass } \\
(\mathrm{kg} / \mathrm{y}=)\end{array}$ & $4.67 E+07$ \\
\hline & $\begin{array}{l}\text { Radionuclide } \\
\mathrm{T} 1-208 \\
\mathrm{~Pb}-212 \\
\mathrm{Bi}-212 \\
\mathrm{Po}-212 \\
\mathrm{Po}-216 \\
\mathrm{Ra}-224 \\
\mathrm{Ra}-228 \\
\mathrm{Ac}-228 \\
\mathrm{Th}-228 \\
\mathrm{Th}-231 \\
\mathrm{Th}-232 \\
\mathrm{Th}-234 \\
\mathrm{~Pa}-234 \\
\mathrm{~Pa}-234 \mathrm{~m} \\
\mathrm{U}-235 \\
\mathrm{U}-238\end{array}$ & \begin{tabular}{c}
$\begin{array}{l}\text { Activity } \\
\text { (Ci/yr) }\end{array}$ \\
\hdashline $1.06 \mathrm{E}-04$ \\
$2.82 \mathrm{E}-04$ \\
$2.82 \mathrm{E}-04$ \\
$1.82 \mathrm{E}-04$ \\
$2.82 \mathrm{E}-04$ \\
$2.82 \mathrm{E}-04$ \\
$1.68 \mathrm{E}-03$ \\
$1.68 \mathrm{E}-03$ \\
$2.82 \mathrm{E}-04$ \\
$1.62 \mathrm{E}-03$ \\
$1.71 \mathrm{E}-02$ \\
$2.08 \mathrm{E}+00$ \\
$2.13 \mathrm{E}-04$ \\
$2.08 \mathrm{E}+00$ \\
$1.62 \mathrm{E}-03$ \\
$2.08 \mathrm{E}+00$
\end{tabular} & \\
\hline Shipping Site: KAPL & $\begin{array}{l}\text { Total volume } \\
(\mathrm{m} 3 / \mathrm{y} x)\end{array}$ & $2.11 E+02 \quad \begin{array}{c}\text { Total Mass } \\
(\mathrm{kg} / \mathrm{yr})\end{array}$ & $5.36 \pm+05$ \\
\hline . & $\begin{array}{l}\text { Radionuclide } \\
\mathrm{H}-3 \\
\mathrm{C}-14 \\
\mathrm{Cr}-51 \\
\mathrm{Mm}-54 \\
\mathrm{Fe}-55 \\
\mathrm{Fe}-59 \\
\mathrm{Co}-58 \\
\mathrm{Co}-60 \\
\mathrm{Ni}-59 \\
\mathrm{Ni}-63 \\
\mathrm{Sr}-90 \\
\mathrm{Y}-90 \\
\mathrm{Zr}-95 \\
\mathrm{Tc}-99 \\
\mathrm{Ru}-106 \\
\mathrm{Rh}-106 \\
\mathrm{Sb}-125 \\
\mathrm{Te}-125 \mathrm{~m} \\
\mathrm{Cs}-134 \\
\mathrm{Cs}-137 \\
\mathrm{Ba}-137 \mathrm{~m} \\
\mathrm{Ce}-144 \\
\mathrm{Pr}-144 \\
\mathrm{Pr}-144 \mathrm{~m} \\
\mathrm{Pm}-147 \\
\mathrm{Sm}-151 \\
\mathrm{Eu}-152 \\
\mathrm{Eu}-154 \\
\mathrm{Eu}-155 \\
\mathrm{Tl}-208 \\
\mathrm{~Pb}-212 \\
\mathrm{Bi}-212 \\
\mathrm{PO}-212 \\
\mathrm{PO}-216 \\
\mathrm{Ra}-224 \\
\mathrm{Ra}-228 \\
\mathrm{Ac}-228 \\
\mathrm{Th}-228 \\
\mathrm{Th}-231 \\
\mathrm{Th}-232 \\
\mathrm{Th}-234 \\
\mathrm{~Pa}-234 \\
\mathrm{~Pa}-234 \mathrm{~m} \\
\mathrm{U}-235 \\
\mathrm{U}-238 \\
\mathrm{Pu}-238 \\
\mathrm{Pu}-239 \\
\mathrm{Pu}-240 \\
\mathrm{Pu}-241 \\
\mathrm{Am}-241 \\
\end{array}$ & 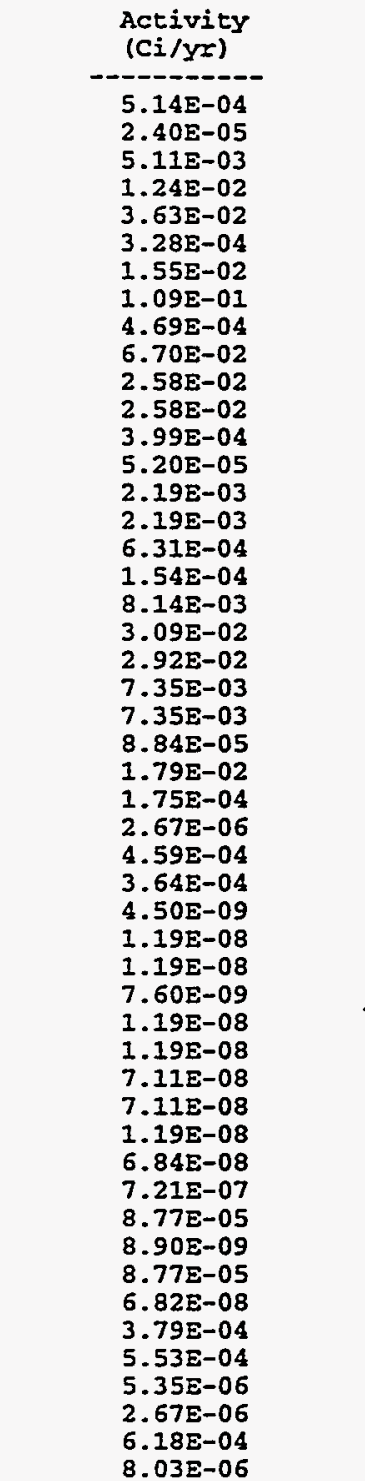 & . \\
\hline
\end{tabular}




\begin{tabular}{|c|c|c|c|}
\hline Shipping Site: KCP & $\begin{array}{l}\text { Total Volume } \\
(\mathrm{m} 3 / \mathrm{y}=)\end{array}$ & $\begin{array}{l}\text { Total Mass } \\
(\mathrm{kg} / \mathrm{Yr})\end{array}$ & $7.23 E+03$ \\
\hline & $\begin{array}{l}\text { Radionuclide } \\
\mathrm{H}-3 \\
\mathrm{C}-14 \\
\mathrm{Mn}-54 \\
\mathrm{Co}-58 \\
\mathrm{Co}-60 \\
\mathrm{Sr}-90 \\
\mathrm{Y}-90 \\
\mathrm{Tc}-99 \\
\mathrm{Cs}-134 \\
\mathrm{Cs}-137 \\
\mathrm{Ba}-137 \mathrm{~m} \\
\mathrm{U}-238\end{array}$ & \multicolumn{2}{|l|}{$\begin{array}{l}2.70 \mathrm{E}-02 \\
1.44 \mathrm{E}-04 \\
1.62 \mathrm{E}-02 \\
1.50 \mathrm{E}-02 \\
4.34 \mathrm{E}-02 \\
2.04 \mathrm{E}-02 \\
2.04 \mathrm{E}-02 \\
2.89 \mathrm{E}-04 \\
3.37 \mathrm{E}-02 \\
4.44 \mathrm{E}-02 \\
4.20 \mathrm{E}-02 \\
1.76 \mathrm{E}-03\end{array}$} \\
\hline \multirow[t]{2}{*}{ Shipping Site: MOUND } & $\begin{array}{l}\text { Total volume } \\
(\mathrm{m} 3 / \mathrm{yx})\end{array}$ & $\begin{array}{c}\text { Total Mass } \\
(\mathrm{kg} / \mathrm{Yr})\end{array}$ & $9.56 \mathrm{E}+06$ \\
\hline & $\begin{array}{l}\text { Radionuclide } \\
\mathrm{H}-3 \\
\mathrm{Pu}-238 \\
\mathrm{Pu}-239 \\
\mathrm{Pu}-240 \\
\mathrm{Pu}-241 \\
\mathrm{Am}-241 \\
\mathrm{Cm}-242 \\
\mathrm{Cm}-244\end{array}$ & \multicolumn{2}{|l|}{$\begin{array}{l}1.59 \mathrm{E}+00 \\
1.60 \mathrm{E}-01 \\
1.22 \mathrm{E}-02 \\
4.28 \mathrm{E}-02 \\
5.90 \mathrm{E}+00 \\
2.44 \mathrm{E}-04 \\
3.42 \mathrm{E}-03 \\
1.22 \mathrm{E}-03\end{array}$} \\
\hline \multirow[t]{2}{*}{ Shipping Site: ORISE } & $\begin{array}{l}\text { Total volume } \\
(\mathrm{m} 3 / \mathrm{yr})\end{array}$ & $\begin{array}{l}\text { Total Mass } \\
(\mathrm{kg} / \mathrm{YI})\end{array}$ & $7.36 \varepsilon+04$ \\
\hline & $\begin{array}{l}\text { Radionuclide } \\
H-3 \\
\mathrm{C}-14 \\
\mathrm{Mn}-54 \\
\mathrm{Co}-58 \\
\mathrm{Co}-60 \\
\mathrm{~S} r-90 \\
\mathrm{Y}-90 \\
\mathrm{Tc}-99 \\
\mathrm{Cs}-134 \\
\mathrm{Cs}-137 \\
\mathrm{Ba}-137 \mathrm{~m} \\
\mathrm{TI}-208 \\
\mathrm{~Pb}-212 \\
\mathrm{Bi}-212 \\
\mathrm{Po}-212 \\
\mathrm{PO}-216 \\
\mathrm{Ra}-224 \\
\mathrm{Ra}-228 \\
\mathrm{Ac}-228 \\
\text { Th-228 } \\
\text { Th-231 } \\
\text { Th-232 } \\
\text { Th-234 } \\
\text { Pa-234 } \\
\text { Pa-234m } \\
\mathrm{U}-235 \\
\mathrm{U}-238\end{array}$ & $\begin{array}{c}\begin{array}{c}\text { Activity } \\
\text { (Ci/yI) }\end{array} \\
2.00 \mathrm{E}-02 \\
1.19 \mathrm{E}-05 \\
1.34 \mathrm{E}-03 \\
1.24 \mathrm{E}-03 \\
3.59 \mathrm{E}-03 \\
1.69 \mathrm{E}-03 \\
1.69 \mathrm{E}-03 \\
2.39 \mathrm{E}-05 \\
2.79 \mathrm{E}-03 \\
3.68 \mathrm{E}-03 \\
3.48 \mathrm{E}-03 \\
3.39 \mathrm{E}-07 \\
8.98 \mathrm{E}-07 \\
8.98 \mathrm{E}-07 \\
5.78 \mathrm{E}-07 \\
8.98 \mathrm{E}-07 \\
8.98 \mathrm{E}-07 \\
5.36 \mathrm{E}-06 \\
5.36 \mathrm{E}-06 \\
8.98 \mathrm{E}-07 \\
5.16 \mathrm{E}-06 \\
5.44 \mathrm{E}-05 \\
6.62 \mathrm{E}-03 \\
6.78 \mathrm{x}-07 \\
6.62 \mathrm{E}-03 \\
5.14 \mathrm{E}-06 \\
6.77 \mathrm{E}-03\end{array}$ & \\
\hline
\end{tabular}


A-83

\begin{tabular}{|c|c|c|c|}
\hline Shipping site: PORTS & $\begin{array}{l}\text { Total Volume } \\
\text { (m3/yx) }\end{array}$ & $\begin{array}{l}\text { Total Mass } \\
\text { (kg/yr) }\end{array}$ & $6.77 \mathrm{E}+07$ \\
\hline & $\begin{array}{l}\text { Radionuclide } \\
\mathrm{H}-3 \\
\mathrm{Cr}-51 \\
\mathrm{Mn}-54 \\
\mathrm{Fe}-55 \\
\mathrm{Fe}-59 \\
\mathrm{Co}-58 \\
\mathrm{Co}-60 \\
\mathrm{Ni}-59 \\
\mathrm{Ni}-63 \\
\mathrm{Zr}-95 \\
\mathrm{~T} 1-208 \\
\mathrm{~Pb}-212 \\
\mathrm{Bi}-212 \\
\mathrm{PO}-212 \\
\mathrm{Po}-216 \\
\mathrm{Ra}-224 \\
\mathrm{Ra}-228 \\
\mathrm{Ac}-228 \\
\mathrm{Th}-228 \\
\mathrm{Th}-231 \\
\mathrm{Th}-232 \\
\mathrm{Th}-234 \\
\mathrm{~Pa}-234 \\
\mathrm{~Pa}-234 \mathrm{~m} \\
\mathrm{U}-235 \\
\mathrm{U}-238 \\
\mathrm{Pu}-238 \\
\mathrm{Pu}-239 \\
\mathrm{Pu}-240 \\
\mathrm{Pu}-241 \\
\mathrm{Am}-241 \\
\mathrm{Cm}-242 \\
\mathrm{Cm}-244\end{array}$ & $\begin{array}{l}\text { Activity } \\
\text { (Ci/yr) } \\
2.24 E+02 \\
8.63 \mathrm{E}-03 \\
1.64 \mathrm{E}-02 \\
6.13 \mathrm{E}-02 \\
5.54 \mathrm{E}-04 \\
2.20 \mathrm{E}-02 \\
1.73 \mathrm{E}-01 \\
7.92 \mathrm{E}-04 \\
1.12 \mathrm{E}-01 \\
6.73 \mathrm{E}-04 \\
3.79 \mathrm{E}-06 \\
1.00 \mathrm{E}-05 \\
1.00 \mathrm{E}-05 \\
6.47 \mathrm{E}-06 \\
1.00 \mathrm{E}-05 \\
1.00 \mathrm{E}-05 \\
6.00 \mathrm{E}-05 \\
6.00 \mathrm{E}-05 \\
1.00 \mathrm{E}-05 \\
5.78 \mathrm{E}-05 \\
6.09 \mathrm{E}-04 \\
7.41 \mathrm{E}-02 \\
7.59 \mathrm{E}-06 \\
7.41 \mathrm{E}-02 \\
5.76 \mathrm{E}-05 \\
7.41 \mathrm{E}-02 \\
4.36 \mathrm{E}-05 \\
3.33 \mathrm{E}-06 \\
1.16 \mathrm{E}-05 \\
1.60 \mathrm{E}-03 \\
6.67 \mathrm{E}-08 \\
9.33 \mathrm{E}-07 \\
3.33 \mathrm{E}-07 \\
\end{array}$ & . \\
\hline Shipping Site: PPPL & $\begin{array}{l}\text { Total Volume } \\
\text { (m3/yr) }\end{array}$ & $\begin{array}{l}\text { Total Mass } \\
\text { 4.51E-01 }\end{array}$ & $1.23 \mathrm{E}+03$ \\
\hline & $\begin{array}{l}\text { Radionuclide } \\
\mathrm{H}-3 \\
\mathrm{Cr}-51 \\
\mathrm{Mn}-54 \\
\mathrm{Fe}-55 \\
\mathrm{Fe}-59 \\
\mathrm{Co}-58 \\
\mathrm{Co}-60 \\
\mathrm{Ni}-59 \\
\mathrm{Ni}-63 \\
\mathrm{ZI}-95\end{array}$ & $\begin{array}{c}\begin{array}{c}\text { Activity } \\
(\mathrm{Ci} / \mathrm{yr})\end{array} \\
-3.74 \mathrm{E}-03 \\
8.50 \mathrm{E}-04 \\
1.61 \mathrm{E}-03 \\
6.04 \mathrm{E}-03 \\
5.46 \mathrm{E}-05 \\
2.17 \mathrm{E}-03 \\
1.70 \mathrm{E}-02 \\
7.80 \mathrm{E}-05 \\
1.10 \mathrm{E}-02 \\
6.63 \mathrm{E}-05\end{array}$ & \\
\hline
\end{tabular}


A-84

\begin{tabular}{|c|c|c|c|}
\hline Shipping Site: Paducah & $\begin{array}{l}\text { Total Volume } \\
(\mathrm{m} 3 / \mathrm{yr})\end{array}$ & $\begin{array}{c}\text { Total Mass } \\
\text { (kg/Yr) }\end{array}$ & $1.19 \mathrm{E}+07$ \\
\hline & $\begin{array}{l}\text { Radionuclide } \\
------.-- \\
\text { Tc-99 } \\
\text { Tl-208 } \\
\mathrm{Pb}-212 \\
\mathrm{Bi}-212 \\
\mathrm{PO}-212 \\
\mathrm{Po-216} \\
\mathrm{Ra}-224 \\
\mathrm{Ra}-228 \\
\mathrm{Ac-228} \\
\mathrm{Th}-228 \\
\mathrm{Th}-231 \\
\mathrm{Th}-232 \\
\mathrm{Th}-234 \\
\mathrm{~Pa}-234 \\
\mathrm{~Pa}-234 \mathrm{~m} \\
\mathrm{U}-235 \\
\mathrm{U}-238 \\
\mathrm{~Np}-237 \\
\mathrm{Pu}-239\end{array}$ & 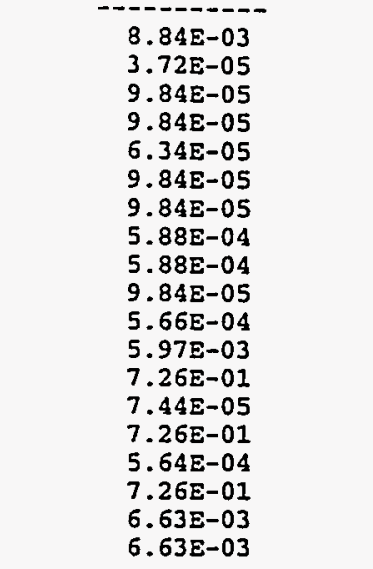 & \\
\hline \multirow[t]{2}{*}{ Shipping Site: RMI } & $\begin{array}{l}\text { Total Volume } \\
(\mathrm{m} 3 / \mathrm{yx})\end{array}$ & $\begin{array}{c}\text { Total Mass } \\
(\mathrm{kg} / \mathrm{Yr})\end{array}$ & $1.45 E+07$ \\
\hline & $\begin{array}{l}\text { Radionuclide } \\
\mathrm{T} 1-208 \\
\mathrm{~Pb}-212 \\
\mathrm{Bi}-212 \\
\mathrm{Po-212} \\
\mathrm{Po-216} \\
\mathrm{Ra}-224 \\
\mathrm{Ra}-228 \\
\mathrm{Ac}-228 \\
\mathrm{Th}-228 \\
\mathrm{Th}-231 \\
\mathrm{Th}-232 \\
\mathrm{Th}-234 \\
\mathrm{Pa-234} \\
\mathrm{Pa-234m} \\
\mathrm{U}-235 \\
\mathrm{U}-238\end{array}$ & $\begin{array}{l}3.38 \mathrm{E}-07 \\
8.96 \mathrm{E}-07 \\
8.96 \mathrm{E}-07 \\
5.77 \mathrm{E}-07 \\
8.96 \mathrm{E}-07 \\
8.96 \mathrm{E}-07 \\
5.35 \mathrm{E}-06 \\
5.35 \mathrm{E}-06 \\
8.96 \mathrm{E}-07 \\
5.15 \mathrm{E}-06 \\
5.43 \mathrm{E}-05 \\
6.61 \mathrm{E}-03 \\
6.77 \mathrm{E}-07 \\
6.61 \mathrm{E}-03 \\
5.13 \mathrm{E}-06 \\
6.61 \mathrm{E}-03\end{array}$ & \\
\hline \multirow[t]{2}{*}{ Shipping Site: $Y-12$} & $\begin{array}{l}\text { Total Volume } \\
(\mathrm{m} 3 / \mathrm{y} x)\end{array}$ & $\begin{array}{c}\text { Total Mass } \\
(\mathrm{kg} / \mathrm{y})\end{array}$ & \\
\hline & $\begin{array}{l}\text { Radionuclide } \\
\mathrm{T} 1-208 \\
\mathrm{~Pb}-212 \\
\mathrm{Bi}-212 \\
\mathrm{Po}-212 \\
\mathrm{Po}-216 \\
\mathrm{Ra}-224 \\
\mathrm{Ra}-228 \\
\mathrm{Ac}-228 \\
\mathrm{Th}-228 \\
\mathrm{Th}-231 \\
\mathrm{Th}-232 \\
\mathrm{Th}-234 \\
\mathrm{~Pa}-234 \\
\mathrm{~Pa}-234 \mathrm{~m} \\
\mathrm{U}-235 \\
\mathrm{U}-238\end{array}$ & \begin{tabular}{c}
$\begin{array}{c}\text { Activity } \\
(C i / Y I)\end{array}$ \\
\hdashline $6.80 E-05$ \\
$1.80 E-04$ \\
$1.80 E-04$ \\
$1.16 E-04$ \\
$1.80 E-04$ \\
$1.80 E-04$ \\
$1.07 E-03$ \\
$1.07 E-03$ \\
$1.80 E-04$ \\
$1.03 E-03$ \\
$1.09 E-02$ \\
$1.32 E+00$ \\
$1.36 E-04$ \\
$1.32 E+00$ \\
$1.03 E-03$ \\
$1.32 E+00$
\end{tabular} & \\
\hline
\end{tabular}


Destination: PORTS

\begin{tabular}{|c|c|c|c|}
\hline Shipping Site: AMES & $\begin{array}{l}\text { Total volume } \\
(\mathrm{m} 3 / \mathrm{yr})\end{array}$ & $\begin{array}{l}\text { Total Mass } \\
(\mathrm{kg} / \mathrm{yr})\end{array}$ & $1.00 \mathrm{E}+02$ \\
\hline & $\begin{array}{l}\text { Radionuclide } \\
\mathrm{Tl}-208 \\
\mathrm{~Pb}-212 \\
\mathrm{Bi}-212 \\
\mathrm{PO}-212 \\
\mathrm{PO}-216 \\
\mathrm{Ra}-224 \\
\mathrm{Ra}-228 \\
\mathrm{Ac}-228 \\
\mathrm{Th}-228 \\
\mathrm{Th}-231 \\
\mathrm{Th}-232 \\
\mathrm{Th}-234 \\
\mathrm{~Pa}-234 \\
\mathrm{~Pa}-234 \mathrm{~m} \\
\mathrm{U}-235 \\
\mathrm{U}-238 \\
\mathrm{Pu}-238 \\
\mathrm{Pu}-239 \\
\mathrm{Pu}-240 \\
\mathrm{Pu}-241 \\
\mathrm{Am}-241 \\
\mathrm{Cm}-242 \\
\mathrm{Cm}-244\end{array}$ & \multicolumn{2}{|l|}{$\begin{array}{l}2.83 \mathrm{E}-08 \\
7.50 \mathrm{E}-08 \\
7.50 \mathrm{E}-08 \\
4.83 \mathrm{E}-08 \\
7.50 \mathrm{E}-08 \\
7.50 \mathrm{E}-08 \\
4.48 \mathrm{E}-07 \\
4.48 \mathrm{E}-07 \\
7.50 \mathrm{E}-08 \\
4.31 \mathrm{E}-07 \\
4.55 \mathrm{E}-06 \\
5.53 \mathrm{E}-04 \\
5.67 \mathrm{E}-08 \\
5.53 \mathrm{E}-04 \\
4.30 \mathrm{E}-07 \\
5.53 \mathrm{E}-04 \\
4.36 \mathrm{E}-05 \\
3.33 \mathrm{E}-06 \\
1.16 \mathrm{E}-05 \\
1.60 \mathrm{E}-03 \\
6.67 \mathrm{E}-08 \\
9.33 \mathrm{E}-07 \\
3.33 \mathrm{E}-07\end{array}$} \\
\hline \multirow[t]{2}{*}{ Shipping Site: FNAL } & $\begin{array}{l}\text { Total Volume } \\
(\mathrm{m} 3 / \mathrm{yr})\end{array}$ & $\begin{array}{c}\text { Total Mass } \\
(\mathrm{kg} / \mathrm{Yr})\end{array}$ & $1.04 E+04$ \\
\hline & \begin{tabular}{l} 
Radionuclide \\
\hdashline $\mathrm{H}-3$ \\
$\mathrm{Cr}-51$ \\
$\mathrm{Mn}-54$ \\
$\mathrm{Fe}-55$ \\
$\mathrm{Fe}-59$ \\
$\mathrm{Co}-58$ \\
$\mathrm{Co}-60$ \\
$\mathrm{Ni}-59$ \\
$\mathrm{Ni}-63$ \\
$\mathrm{Zr}-95$ \\
$\mathrm{Tl}-208$ \\
$\mathrm{~Pb}-212$ \\
$\mathrm{Bi}-212$ \\
$\mathrm{PO}-212$ \\
$\mathrm{PO}-216$ \\
$\mathrm{Ra}-224$ \\
$\mathrm{Ra}-228$ \\
$\mathrm{Ac}-228$ \\
$\mathrm{Th}-228$ \\
$\mathrm{Th}-231$ \\
$\mathrm{Th}-232$ \\
$\mathrm{Th}-234$ \\
$\mathrm{~Pa}-234$ \\
$\mathrm{~Pa}-234 \mathrm{~m}$ \\
$\mathrm{U}-235$ \\
$\mathrm{U}-238$
\end{tabular} & \multicolumn{2}{|l|}{ 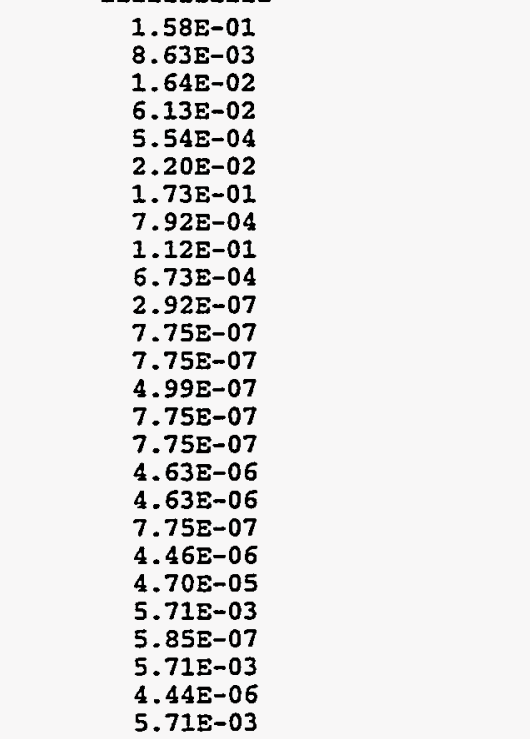 } \\
\hline \multirow[t]{2}{*}{ Shipping Site: MOUND } & $\begin{array}{l}\text { Total volume } \\
(\mathrm{m} 3 / \mathrm{Yx})\end{array}$ & $\begin{array}{cc}\text { Total Mass } \\
(\mathrm{kg} / \mathrm{yr})\end{array}$ & \\
\hline & $\begin{array}{l}\text { Radionuclide } \\
\text { H-3 }\end{array}$ & $\begin{array}{c}\text { Activity } \\
\text { (Ci/yr) }\end{array}$ & \\
\hline
\end{tabular}




\begin{tabular}{|c|c|c|c|}
\hline Shipping Sice: PPPL & $\begin{array}{l}\text { Total Volume } \\
(\mathrm{m} 3 / \mathrm{yr})\end{array}$ & $\begin{array}{c}\text { Total Mass } \\
(\mathrm{kg} / \mathrm{yr})\end{array}$ & $3.73 \mathrm{E}+03$ \\
\hline & $\begin{array}{l}\text { Radionuclide } \\
\mathrm{H}-3\end{array}$ & $\begin{array}{c}\text { Activity } \\
\text { (Ci/yr) }\end{array}$ & \\
\hline Shipping Site: RMI & $\begin{array}{l}\text { Total Volume } \\
(\mathrm{m} 3 / \mathrm{yr})\end{array}$ & $2.74 E+02 \quad \begin{array}{c}\text { Total Mass } \\
(\mathrm{kg} / \mathrm{Yr})\end{array}$ & $9.49 \mathrm{E}+04$ \\
\hline & $\begin{array}{l}\text { Radionuclide } \\
\mathrm{TI}-208 \\
\mathrm{~Pb}-212 \\
\mathrm{Bi}-212 \\
\mathrm{PO}-212 \\
\mathrm{Po}-216 \\
\mathrm{Ra}-224 \\
\mathrm{Ra}-228 \\
\mathrm{Ac}-228 \\
\mathrm{Th}-228 \\
\mathrm{Th}-231 \\
\mathrm{Th}-232 \\
\mathrm{Th}-234 \\
\mathrm{~Pa}-234 \\
\mathrm{~Pa}-234 \mathrm{~m} \\
\mathrm{U}-235 \\
\mathrm{U}-238\end{array}$ & \begin{tabular}{c}
$\begin{array}{c}\text { Activity } \\
\text { (Ci/yr) }\end{array}$ \\
\hdashline $1.94 E-08$ \\
$5.13 E-08$ \\
$5.13 E-08$ \\
$3.30 E-08$ \\
$5.13 E-08$ \\
$5.13 E-08$ \\
$3.06 E-07$ \\
$3.06 E-07$ \\
$5.13 E-08$ \\
$2.95 E-07$ \\
$3.11 E-06$ \\
$3.78 E-04$ \\
$3.87 E-08$ \\
$3.78 E-04$ \\
$2.93 E-07$ \\
$3.78 E-04$
\end{tabular} & \\
\hline
\end{tabular}

Destination: SRS

\begin{tabular}{|c|c|c|c|}
\hline Shipping Site: Pinellas & $\begin{array}{c}\text { Total Volume } \\
(\mathrm{m} 3 / \mathrm{y})\end{array}$ & $\begin{array}{c}\text { Total Mass } \\
(\mathrm{kg} / \mathrm{yr})\end{array}$ & $1.91 \mathrm{E}+04$ \\
\hline & Radionuclide & $\begin{array}{l}\text { Activity } \\
\text { (Ci/yx) }\end{array}$ & \\
\hline & $\mathrm{H}-3$ & $1.99 E+04$ & \\
\hline
\end{tabular}




\section{A.12 WM LLW REGIONALIZED 4 ALTERNATIVE (CASE 12): ACTIVATED METALS}

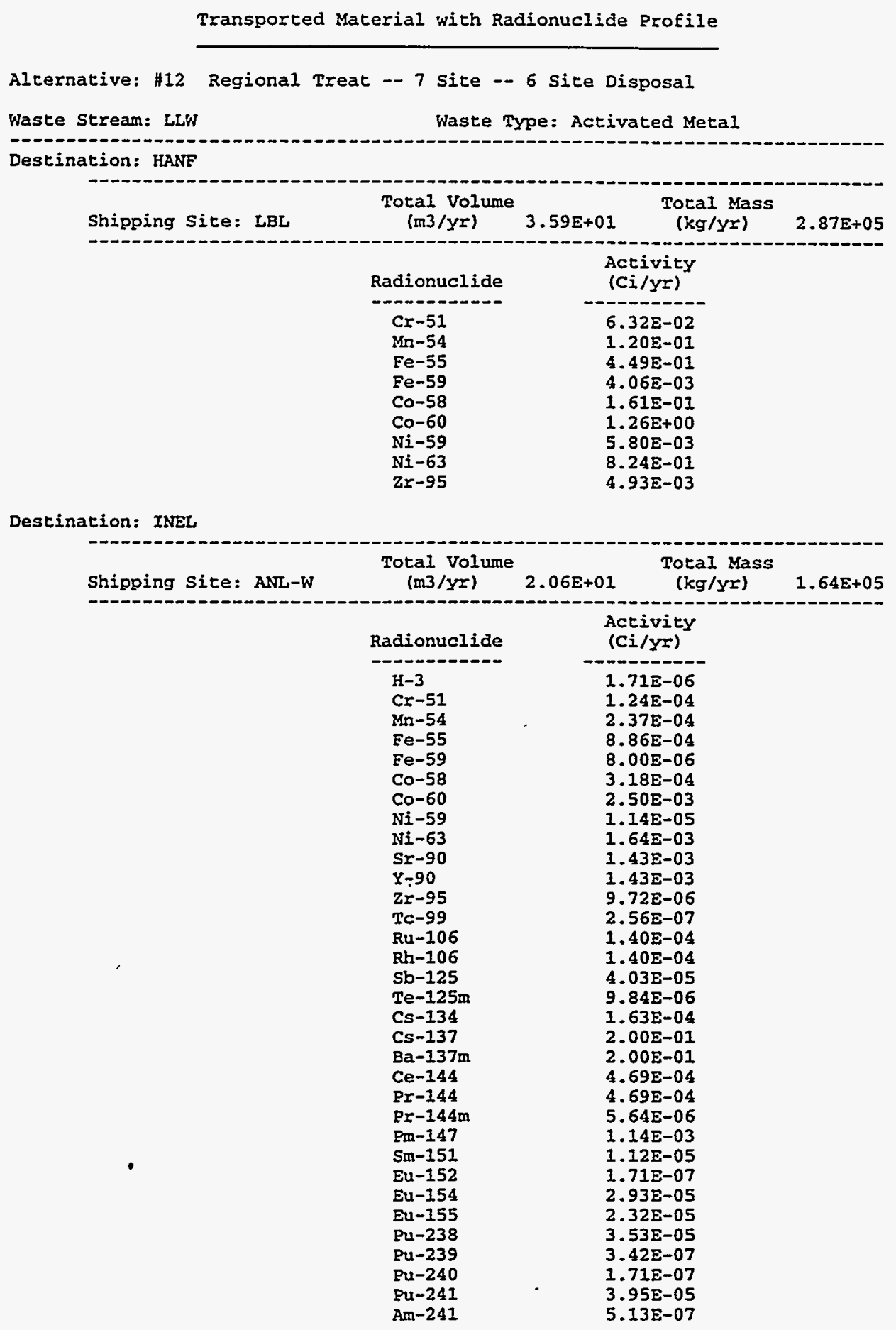


A- 88

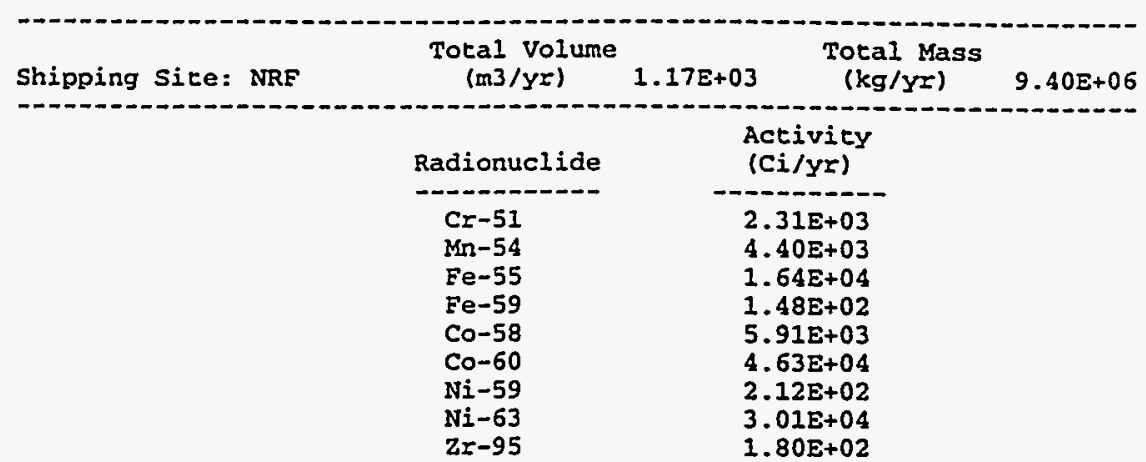

Destination: LANL

\begin{tabular}{|c|c|c|c|}
\hline Shipping Site: SNLA & $\begin{array}{c}\text { Total Volume } \\
(\mathrm{m} 3 / \mathrm{y} x)\end{array}$ & $\begin{array}{l}\text { Total Mass } \\
(\mathrm{kg} / \mathrm{yr})\end{array}$ & $1.15 E+05$ \\
\hline * & $\begin{array}{l}\text { Radionuclide } \\
\mathrm{Cr}-51 \\
\mathrm{Mn}-54 \\
\mathrm{Fe}-55 \\
\mathrm{Fe}-59 \\
\mathrm{Co}-58 \\
\mathrm{Co}-60 \\
\mathrm{Ni}-59 \\
\mathrm{Ni}-63 \\
\mathrm{Zr}-95\end{array}$ & $\begin{array}{c}\begin{array}{l}\text { Activity } \\
\text { (Ci/YI) }\end{array} \\
6.54 \mathrm{E}-04 \\
1.24 \mathrm{E}-03 \\
4.64 \mathrm{E}-03 \\
4.20 \mathrm{E}-05 \\
1.67 \mathrm{E}-03 \\
1.31 \mathrm{E}-02 \\
6.00 \mathrm{E}-05 \\
8.53 \mathrm{E}-03 \\
5.10 \mathrm{E}-05\end{array}$ & \\
\hline
\end{tabular}


Destination: ORNL

\begin{tabular}{|c|c|c|c|}
\hline Shipping site: PORTS & $\begin{array}{c}\text { Total Volume } \\
(\mathrm{m} 3 / \mathrm{y} x)\end{array}$ & $\begin{array}{l}\text { Toral Mass } \\
\text { (kg/Y } / \mathrm{k})\end{array}$ & $2.05 E+07$ \\
\hline & 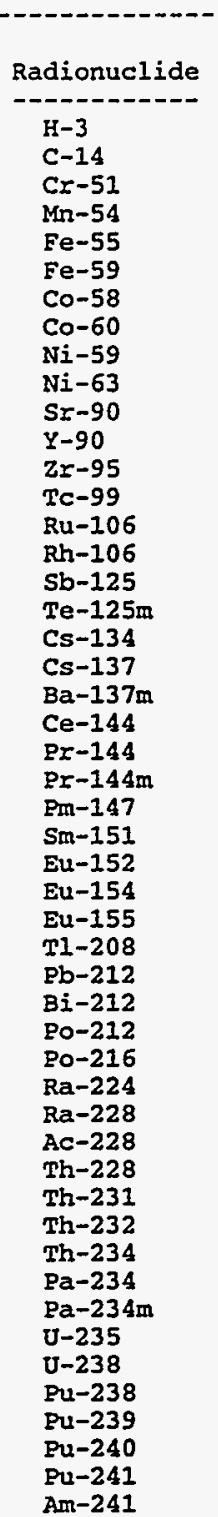 & 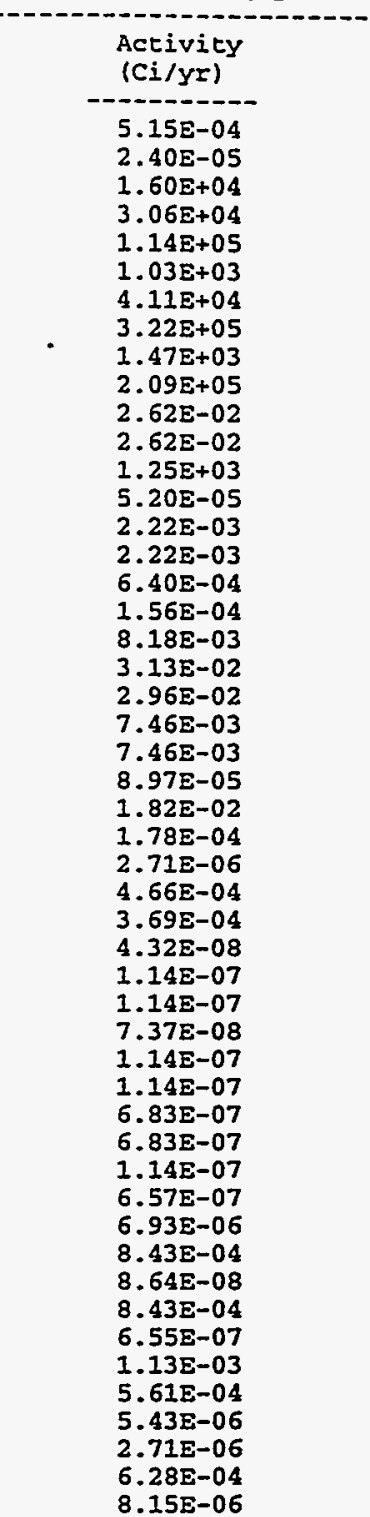 & $----\infty-\infty$ \\
\hline
\end{tabular}


Note that the WASTE_MGMT files report the quantity of waste shipped on an annual basis (cubic meters per year or kilograms per year). As stated previously, the WM PEIS considers a 20-year time frame for WM LLW. For most of the WM alternatives considered in the WM PEIS, the assumption was that shipments would occur uniformly over a 10-year period, which would allow an initial 10-year period to build TSD facilities. Sites would essentially store their own waste for the first 10 years and ship all waste over the subsequent 10 years; however, the assumption for the No Action Alternative was that shipments would be distributed uniformly over a 20-year period because no new facilities need to be built. Therefore, although the total inventory considered is the same for all WM alternatives (current inventories plus 20 years of generation), the annual amounts of waste shipped, as reported in the WASTE_MGMT files in Appendix A, are a function of the assumption concerning the duration of the shipping campaigns for each case.

Because of the differences in the time frames noted previously and uncertainties in the actual timing of future waste shipments, the impacts of transportation for the various alternatives are best compared by examining the total impacts of transporting a specific inventory of waste, independent of the annual shipping rates. The total amount of LLW transported for each alternative can be calculated by multiplying the annual shipment quantities (reported in the WASTE_MGMT files in Appendix A) by 20 years for the No Action Alternative and by 10 years for the other WM cases.

\subsection{SCOPE OF THE RISK ASSESSMENT}

The transportation risk assessment is limited to estimating the human health risks incurred during the actual transportation of LLW by truck or rail. The risks to workers or to the public during the loading, unloading, and handling of waste before or after shipment are considered as part of normal operations of the facility and are not included in the transportation assessment. Similarly, the transportation risk assessment does not address how increased transportation levels may possibly impact local traffic flow, noise levels, logistics, or infrastructure. The impacts that are addressed are discussed subsequently.

\subsubsection{Cargo-Related Impacts: Radiological}

For radioactive materials, the cargo-related impacts on human health during transportation are caused by exposure to ionizing radiation. For all cases, radiological risks (i.e., risks that result from the radioactive nature of the LLW) are assessed both for routine (normal) transportation and for accidents. The radiological risk associated with routine transportation results from the potential exposure of people to low levels of external radiation in the vicinity of a loaded shipment. The radiological risk from transportation-related accidents lies in the potential release and dispersal of radioactive material into the environment during an accident and the subsequent exposure of people 


\begin{tabular}{|c|c|c|c|}
\hline Shipping Sice: BAPL & $\begin{array}{l}\text { Total Volume } \\
(\mathrm{m} 3 / \mathrm{yr})\end{array}$ & $\begin{array}{c}\text { Total Mass } \\
\text { (kg/yr) }\end{array}$ & $6.43 \mathrm{E}+06$ \\
\hline . & $\begin{array}{l}\text { Radionuclide } \\
\mathrm{Cr}-51 \\
\mathrm{Mn}-54 \\
\mathrm{Fe}-55 \\
\mathrm{Fe}-59 \\
\mathrm{Co}-58 \\
\mathrm{Co}-60 \\
\mathrm{Ni}-59 \\
\mathrm{Ni}-63 \\
\mathrm{Zr}-95 \\
\mathrm{Tl}-208 \\
\mathrm{~Pb}-212 \\
\mathrm{Bi}-212 \\
\mathrm{Po-212} \\
\mathrm{Po-216} \\
\mathrm{Ra}-224 \\
\mathrm{Ra}-228 \\
\mathrm{Ac-228} \\
\mathrm{Th}-228 \\
\mathrm{Th}-231 \\
\mathrm{Th}-232 \\
\mathrm{Th}-234 \\
\mathrm{~Pa}-234 \\
\mathrm{~Pa}-234 \mathrm{~m} \\
\mathrm{U}-235 \\
\mathrm{U}-238\end{array}$ & $\begin{array}{c}\begin{array}{c}\text { Activity } \\
(C i / y r)\end{array} \\
-9.75 E+03 \\
4.04 E+03 \\
3.37 E+04 \\
3.05 E+02 \\
1.21 E+04 \\
9.52 E+04 \\
4.36 E+02 \\
6.19 E+04 \\
3.70 E+02 \\
1.36 E-08 \\
3.60 E-08 \\
3.60 E-08 \\
2.32 E-08 \\
3.60 E-08 \\
3.60 E-08 \\
2.15 E-07 \\
2.15 E-07 \\
3.60 E-08 \\
2.07 E-07 \\
2.18 E-06 \\
2.65 E-04 \\
2.72 E-08 \\
2.65 E-04 \\
2.06 E-07 \\
2.65 E-04\end{array}$ & \\
\hline \multirow{2}{*}{ Shipping Site: FNAL } & $\begin{array}{l}\text { Total Volume } \\
(\mathrm{m} 3 / \mathrm{yr})\end{array}$ & $\begin{array}{cc}\text { Total Mass } \\
(\mathrm{kg} / \mathrm{yr})\end{array}$ & $8.14 E+05$ \\
\hline & $\begin{array}{l}\text { Radionuclide } \\
\mathrm{Cr}-51 \\
\mathrm{Mn}-54 \\
\mathrm{Fe}-55 \\
\mathrm{Fe}-59 \\
\mathrm{Co}-58 \\
\mathrm{Co}-60 \\
\mathrm{Ni}-59 \\
\mathrm{Ni}-63 \\
\mathrm{Zr}-95\end{array}$ & $\begin{array}{l}\text { Activity } \\
\text { (Ci/yr) } \\
3.35 \mathrm{E}-02 \\
6.39 \mathrm{E}-02 \\
2.38 \mathrm{E}-01 \\
2.15 \mathrm{E}-03 \\
8.57 \mathrm{E}-02 \\
6.72 \mathrm{E}-01 \\
3.08 \mathrm{E}-03 \\
4.37 \mathrm{E}-01 \\
2.61 \mathrm{E}-03\end{array}$ & \\
\hline
\end{tabular}




\begin{tabular}{|c|c|c|c|}
\hline Shipping Site: KAPL & $\begin{array}{l}\text { Total Volume } \\
(\mathrm{m} 3 / \mathrm{yr})\end{array}$ & $\begin{array}{l}\text { Total Mass } \\
(\mathrm{kg} / \mathrm{Yr})\end{array}$ & $1.33 E+07$ \\
\hline & $\begin{array}{l}\text { Radionuclide } \\
\mathrm{H}-3 \\
\mathrm{C}-14 \\
\mathrm{Cr}-51 \\
\mathrm{Mn}-54 \\
\mathrm{Fe}-55 \\
\mathrm{Fe}-59 \\
\mathrm{Co}-58 \\
\mathrm{Co}-60 \\
\mathrm{Ni}-59 \\
\mathrm{Ni}-63 \\
\mathrm{Sr}-90 \\
\mathrm{Y}-90 \\
\mathrm{Zr}-95 \\
\mathrm{TC}-99 \\
\mathrm{Ru}-106 \\
\mathrm{Rh}-106 \\
\mathrm{Sb}-125 \\
\mathrm{Te}-125 \mathrm{~m} \\
\mathrm{Cs}-134 \\
\mathrm{Cs}-137 \\
\mathrm{Ba}-137 \mathrm{~m} \\
\mathrm{Ce}-144 \\
\mathrm{Pr}-144 \\
\mathrm{Pr}-144 \mathrm{~m} \\
\mathrm{Pm}-147 \\
\mathrm{Sm}-151 \\
\mathrm{Eu}-152 \\
\mathrm{Eu}-154 \\
\mathrm{Eu}-155 \\
\mathrm{Tl}-208 \\
\mathrm{~Pb}-212 \\
\mathrm{Bi}-212 \\
\mathrm{Po}-212 \\
\mathrm{PO}-216 \\
\mathrm{Ra}-224 \\
\mathrm{Ra}-228 \\
\mathrm{Ac}-228 \\
\mathrm{Th}-228 \\
\mathrm{Th}-231 \\
\mathrm{Th}-232 \\
\mathrm{Th}-234 \\
\mathrm{~Pa}-234 \\
\mathrm{Pa-234m} \\
\mathrm{U}-235 \\
\mathrm{U}-238 \\
\mathrm{Pu}-238 \\
\mathrm{Pu}-239 \\
\mathrm{Pu}-240 \\
\mathrm{Pu}-241 \\
\mathrm{Am}-241 \\
\end{array}$ & $\begin{array}{c}\text { Activicy } \\
(C i / y=) \\
-9.15 \mathrm{Y}-04 \\
2.40 \mathrm{E}-05 \\
1.13 \mathrm{E}+04 \\
2.15 \mathrm{E}+04 \\
8.05 \mathrm{E}+04 \\
7.28 \mathrm{E}+02 \\
2.89 \mathrm{E}+04 \\
2.27 \mathrm{E}+05 \\
1.04 \mathrm{E}+03 \\
1.47 \mathrm{E}+05 \\
2.62 \mathrm{E}-02 \\
2.62 \mathrm{E}-02 \\
8.84 \mathrm{E}+02 \\
5.20 \mathrm{E}-05 \\
2.22 \mathrm{E}-03 \\
2.22 \mathrm{E}-03 \\
6.40 \mathrm{E}-04 \\
1.56 \mathrm{E}-04 \\
8.18 \mathrm{E}-03 \\
3.13 \mathrm{E}-02 \\
2.96 \mathrm{E}-02 \\
7.46 \mathrm{E}-03 \\
7.46 \mathrm{E}-03 \\
8.97 \mathrm{E}-05 \\
1.82 \mathrm{E}-02 \\
1.78 \mathrm{E}-04 \\
2.71 \mathrm{E}-06 \\
4.66 \mathrm{E}-04 \\
3.69 \mathrm{E}-04 \\
2.96 \mathrm{E}-08 \\
7.83 \mathrm{E}-08 \\
7.83 \mathrm{E}-08 \\
5.05 \mathrm{E}-08 \\
7.83 \mathrm{E}-08 \\
7.83 \mathrm{E}-08 \\
4.68 \mathrm{E}-07 \\
4.68 \mathrm{E}-07 \\
7.83 \mathrm{E}-08 \\
4.50 \mathrm{E}-07 \\
4.75 \mathrm{E}-06 \\
5.77 \mathrm{E}-04 \\
5.92 \mathrm{E}-08 \\
5.77 \mathrm{E}-04 \\
4.48 \mathrm{E}-07 \\
8.69 \mathrm{E}-04 \\
5.61 \mathrm{E}-04 \\
5.43 \mathrm{E}-06 \\
2.71 \mathrm{E}-06 \\
6.28 \mathrm{E}-04 \\
8.15 \mathrm{E}-06\end{array}$ & 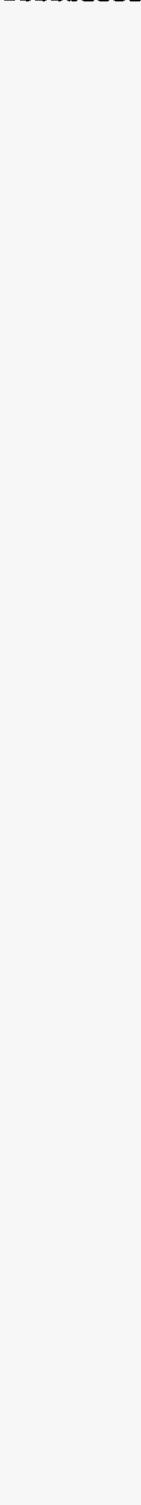 \\
\hline
\end{tabular}




\section{A.13 WM LLW REGIONALIZED 5 ALTERNATIVE (CASE 19): HETEROGENEOUS SOLIDS}

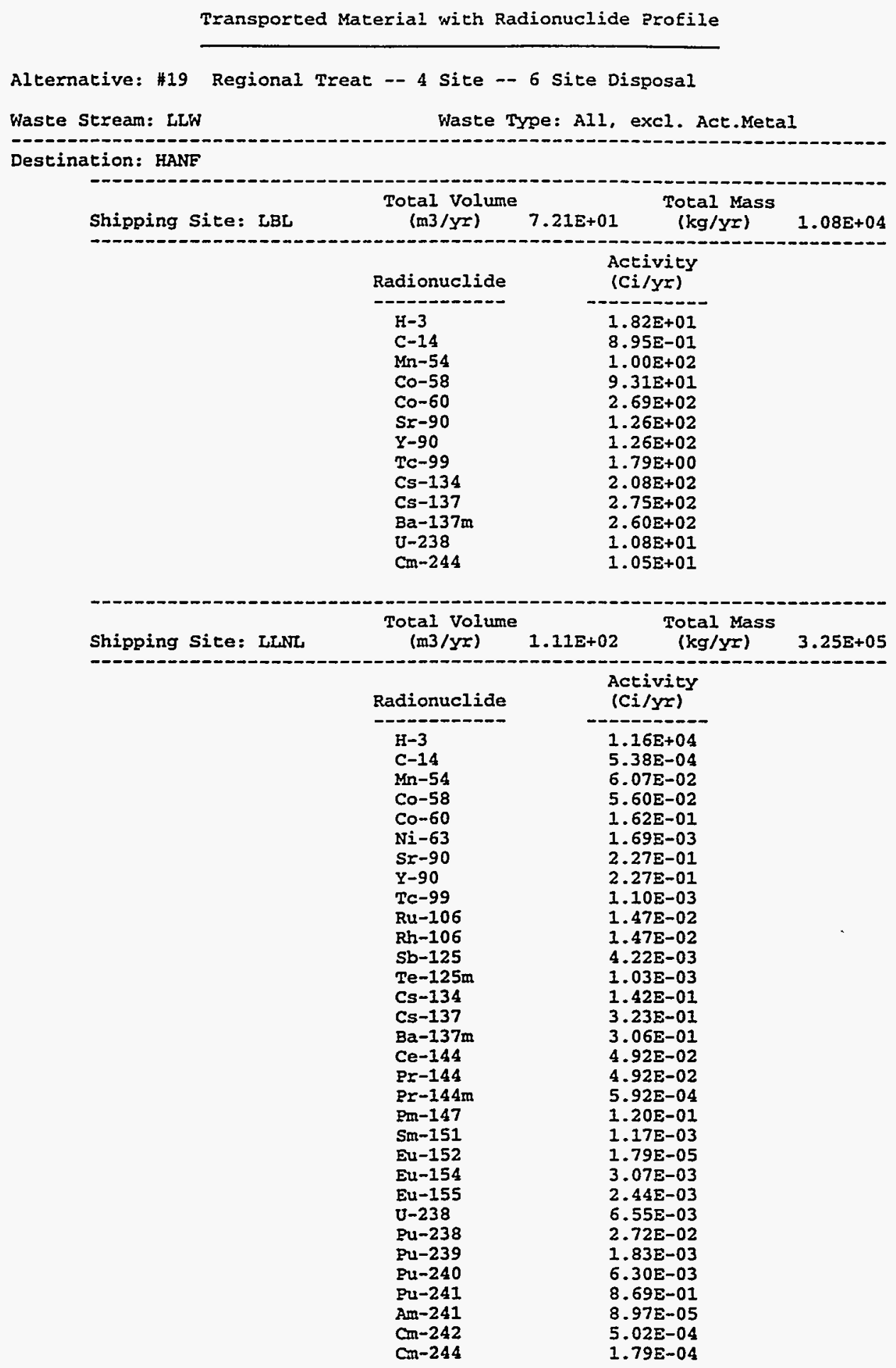




\section{A-93}

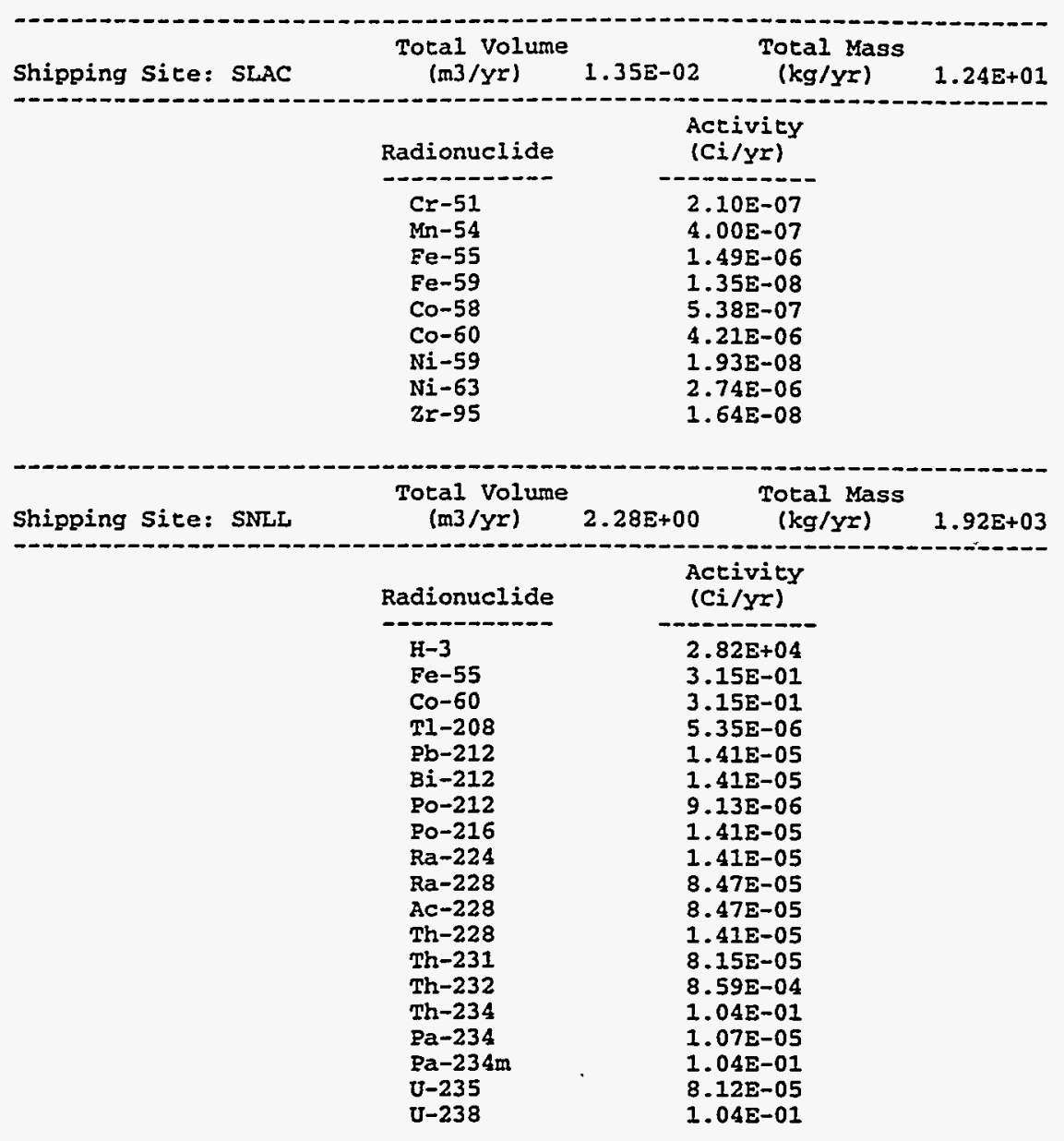


Destination: INEL,

\begin{tabular}{|c|c|c|c|}
\hline Shipping Site: ANL, $W$ & $\begin{array}{l}\text { Total Volume } \\
(\mathrm{m} 3 / \mathrm{yr})\end{array}$ & $\begin{array}{l}\text { Total Mass } \\
(\mathrm{kg} / \mathrm{yr})\end{array}$ & $2.12 E+05$ \\
\hline & $\begin{array}{l}\text { Radionuclide } \\
\mathrm{H}-3 \\
\mathrm{Cr}-51 \\
\mathrm{Mn}-54 \\
\mathrm{Fe}-55 \\
\mathrm{Fe}-59 \\
\mathrm{Co}-58 \\
\mathrm{Co}-60 \\
\mathrm{Ni}-59 \\
\mathrm{Ni}-63 \\
\mathrm{Sr}-90 \\
\mathrm{Y}-90 \\
\mathrm{Zr}-95 \\
\mathrm{Tc}-99 \\
\mathrm{Ru}-106 \\
\mathrm{Rh}-106 \\
\mathrm{Sb}-125 \\
\mathrm{Te}-125 \mathrm{~m} \\
\mathrm{Cs}-134 \\
\mathrm{Cs}-137 \\
\mathrm{Ba}-137 \mathrm{~m} \\
\mathrm{Ce}-144 \\
\mathrm{Pr}-144 \\
\mathrm{Pr}-144 \mathrm{~m} \\
\mathrm{Pm}-147 \\
\mathrm{Sm}-151 \\
\mathrm{Eu}-152 \\
\mathrm{Eu}-154 \\
\mathrm{Eu}-155 \\
\mathrm{Pu}-238 \\
\mathrm{Pu}-239 \\
\mathrm{Pu}-240 \\
\mathrm{Pu}-241 \\
\mathrm{Am}-241 \\
\mathrm{Cm}-242 \\
\mathrm{Cm}-244\end{array}$ & 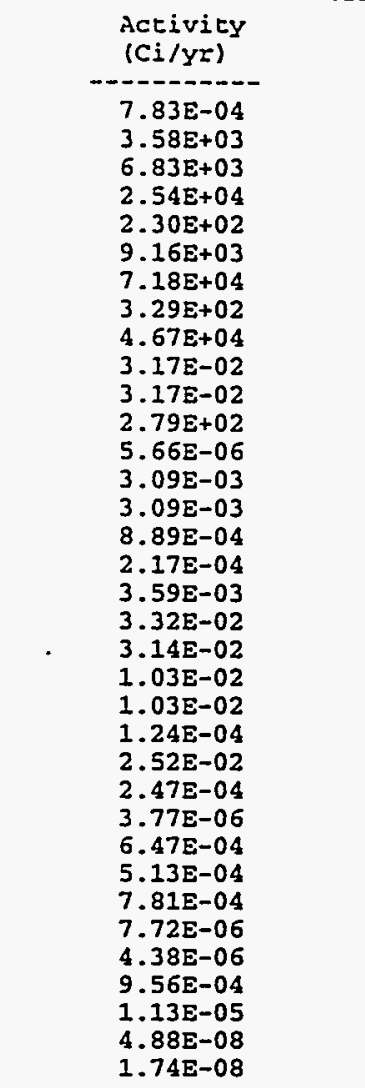 & \\
\hline \multirow[t]{2}{*}{ Shipping Site: ITRI } & $\begin{array}{l}\text { Total volume } \\
(\mathrm{m} 3 / \mathrm{yr})\end{array}$ & $\begin{array}{cc}\text { Total Mass } \\
(\mathrm{kg} / \mathrm{Yr})\end{array}$ & $1.17 \mathrm{E}+04$ \\
\hline & $\begin{array}{l}\text { Radionuclide } \\
\mathrm{H}-3 \\
\mathrm{C}-14 \\
\mathrm{Mn}-54 \\
\mathrm{Co}-58 \\
\mathrm{Co}-60 \\
\mathrm{Sr}-90 \\
\mathrm{Y}-90 \\
\mathrm{Tc}-99 \\
\mathrm{Cs}-134 \\
\mathrm{Cs}-137 \\
\mathrm{Ba}-137 \mathrm{~m} \\
\mathrm{U}-238\end{array}$ & 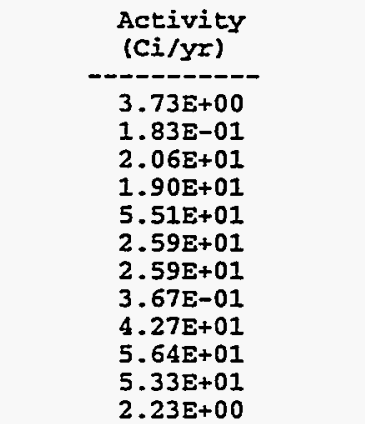 & \\
\hline
\end{tabular}




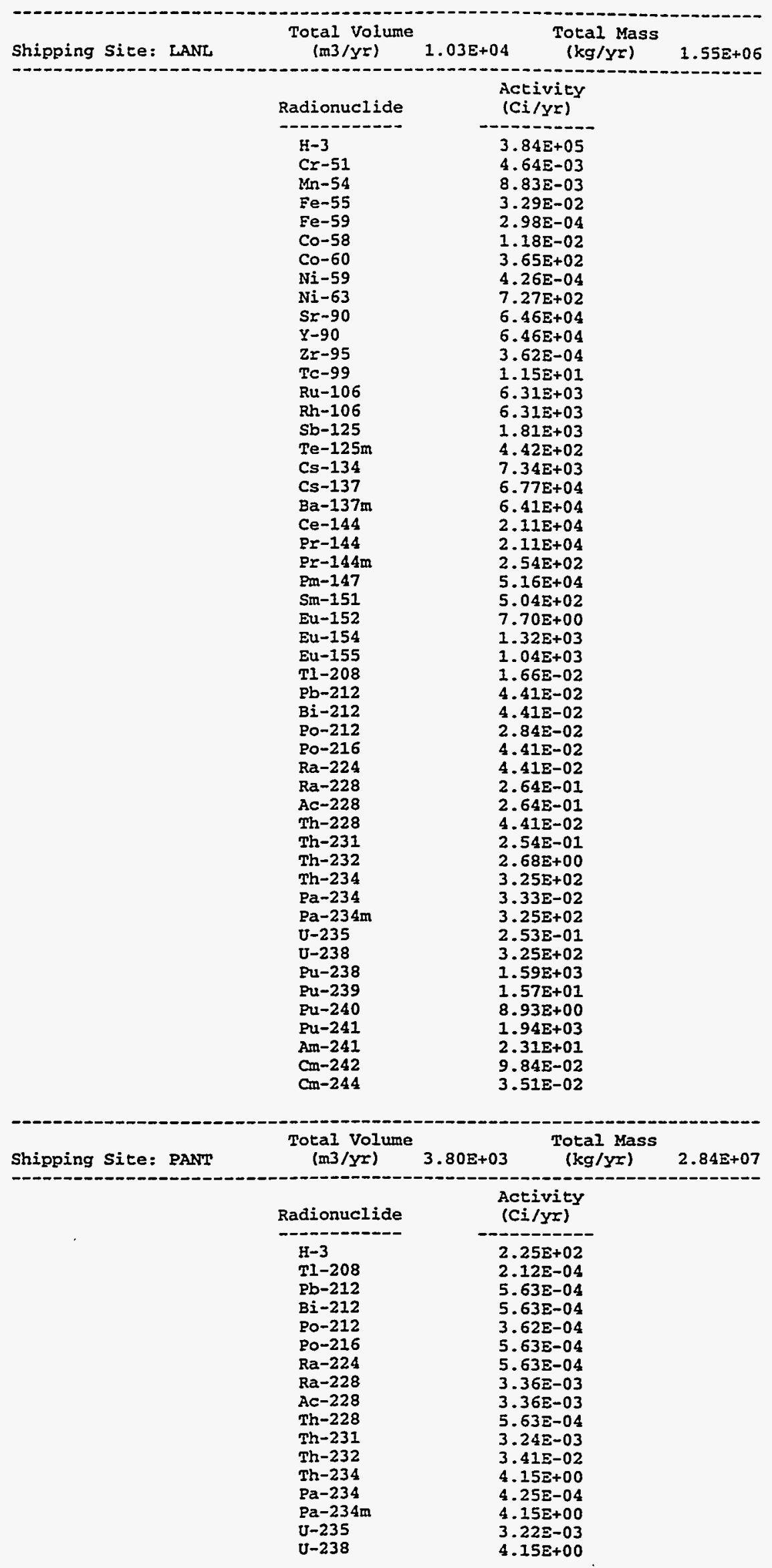




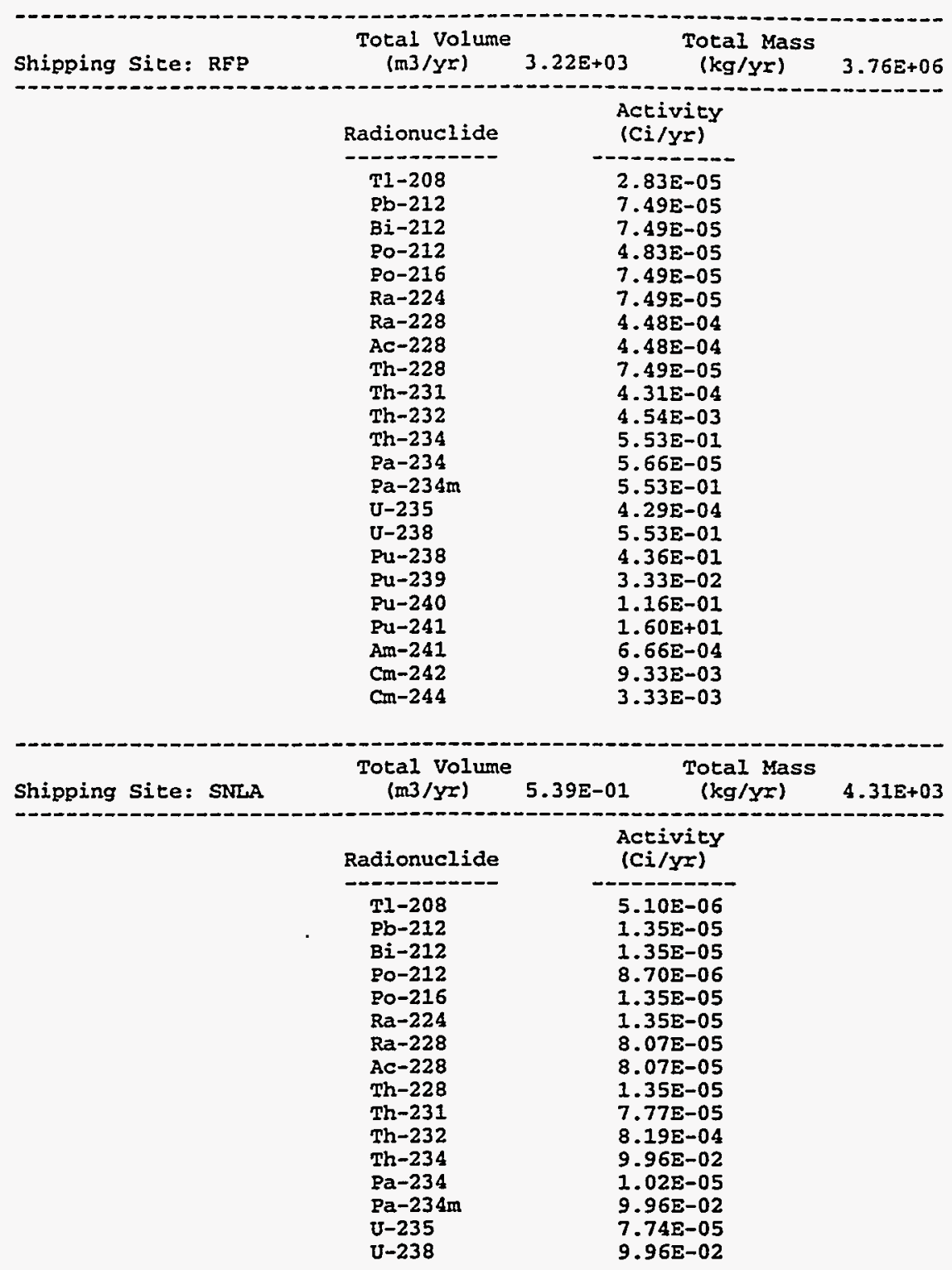


Destination: LANL

\begin{tabular}{|c|c|c|c|}
\hline Shipping Site: INEL & $\begin{array}{l}\text { Total Volume } \\
(\mathrm{m} 3 / \mathrm{yr})\end{array}$ & $\begin{array}{l}\text { Total Mass } \\
\left(\mathrm{kg} / \mathrm{yr}^{\mathrm{r}}\right)\end{array}$ & $1.66 \mathrm{z}+06$ \\
\hline & \begin{tabular}{l} 
Radionuclide \\
\hdashline $\mathrm{H}-3$ \\
$\mathrm{Cr}-51$ \\
$\mathrm{Mn}-54$ \\
$\mathrm{Fe}-55$ \\
$\mathrm{Fe}-59$ \\
$\mathrm{Co}-58$ \\
$\mathrm{Co}-60$ \\
$\mathrm{Ni}-59$ \\
$\mathrm{Ni}-63$ \\
$\mathrm{Sr}-90$ \\
$\mathrm{Y}-90$ \\
$\mathrm{Zr}-95$ \\
$\mathrm{TC}-99$ \\
$\mathrm{Ru}-106$ \\
$\mathrm{Rh}-106$ \\
$\mathrm{Sb}-125$ \\
$\mathrm{Te}-125 \mathrm{~m}$ \\
$\mathrm{Cs}-134$ \\
$\mathrm{Cs}-137$ \\
$\mathrm{Ba}-137 \mathrm{~m}$ \\
$\mathrm{Ce}-144$ \\
$\mathrm{Pr}-144$ \\
$\mathrm{Pr}-144 \mathrm{~m}$ \\
$\mathrm{Pm}-147$ \\
$\mathrm{Sm}-151$ \\
$\mathrm{Eu}-152$ \\
$\mathrm{Eu}-154$ \\
$\mathrm{Eu}-155$ \\
$\mathrm{~T}-208$ \\
$\mathrm{~Pb}-212$ \\
$\mathrm{Bi}-212$ \\
$\mathrm{PO}-212$ \\
$\mathrm{Po}-216$ \\
$\mathrm{Ra}-224$ \\
$\mathrm{Ra}-228$ \\
$\mathrm{Ac}-228$ \\
$\mathrm{Th}-228$ \\
$\mathrm{Th}-231$ \\
$\mathrm{Th}-232$ \\
$\mathrm{Th}-234$ \\
$\mathrm{~Pa}-234$ \\
$\mathrm{~Pa}-234 \mathrm{~m}$ \\
$\mathrm{U}-235$ \\
$\mathrm{U}-238$ \\
$\mathrm{Pu}-238$ \\
$\mathrm{Pu}-239$ \\
$\mathrm{Pu}-240$ \\
$\mathrm{Pu}-241$ \\
$\mathrm{Am}-241$ \\
$\mathrm{Cm}-242$ \\
$\mathrm{Cm}-244$ \\
\end{tabular} & 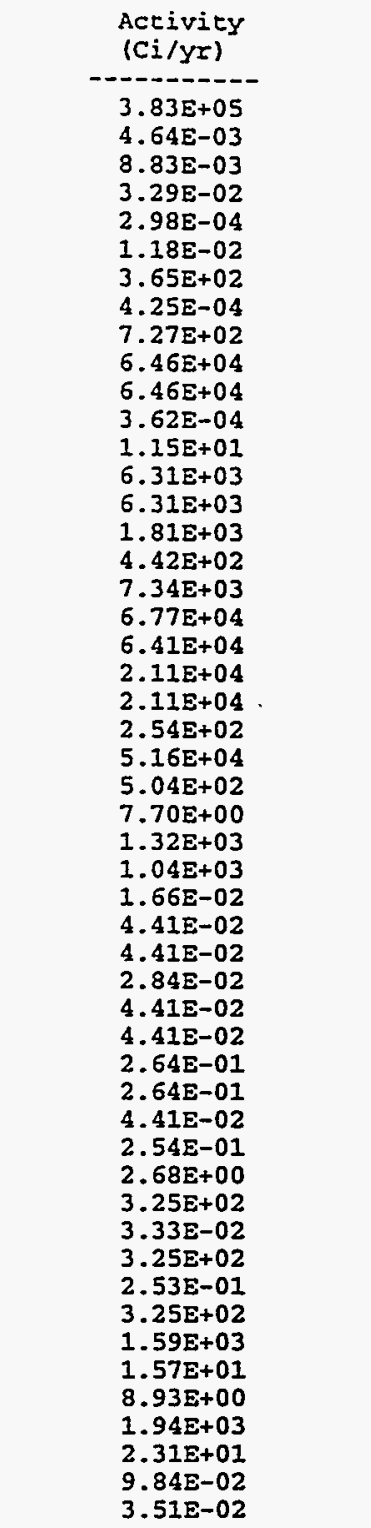 & \\
\hline Shipping Sire: ITRI & $\begin{array}{l}\text { Total Volume } \\
\text { (m3/yr) }\end{array}$ & $\begin{array}{cc}5.61 E+01 & \text { Total Mass } \\
(\mathrm{kg} / \mathrm{yr})\end{array}$ & $1.53 \mathrm{E}+05$ \\
\hline & $\begin{array}{l}\text { Radionuclide } \\
\mathrm{H}-3 \\
\mathrm{C}-14 \\
\mathrm{Mn}-54 \\
\mathrm{Co}-58 \\
\mathrm{Co}-60 \\
\mathrm{~S}-90 \\
Y-90 \\
\mathrm{~T}-99 \\
\mathrm{Cs}-134 \\
\mathrm{Cs}-137 \\
\text { Ba-137m } \\
\mathrm{U}-238\end{array}$ & 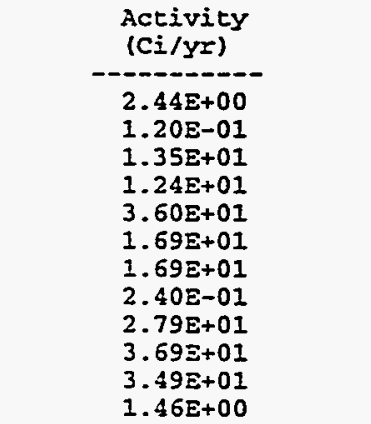 & \\
\hline
\end{tabular}




\begin{tabular}{|c|c|c|c|}
\hline Shipping Sice: PANT & $\begin{array}{l}\text { Total Volume } \\
(\mathrm{m} 3 / \mathrm{yr})\end{array}$ & $\begin{array}{cc}1.70 \mathrm{E}+02 & \begin{array}{c}\text { Total Mass } \\
(\mathrm{kg} / \mathrm{y})\end{array}\end{array}$ & $4.66 E+05$ \\
\hline • & $\begin{array}{l}\text { Radionuclide } \\
\mathrm{H}-3 \\
\mathrm{Tl}-208 \\
\mathrm{~Pb}-212 \\
\mathrm{Bi}-212 \\
\mathrm{PO}-212 \\
\mathrm{Po}-216 \\
\mathrm{Ra}-224 \\
\mathrm{Ra}-228 \\
\mathrm{Ac}-228 \\
\mathrm{Th}-228 \\
\mathrm{Th}-231 \\
\mathrm{Th}-232 \\
\mathrm{Th}-234 \\
\mathrm{~Pa}-234 \\
\mathrm{~Pa}-234 \mathrm{~m} \\
\mathrm{U}-235 \\
\mathrm{U}-238\end{array}$ & \multicolumn{2}{|l|}{$\begin{array}{l}9.18 \mathrm{E}+00 \\
8.99 \mathrm{E}-06 \\
2.38 \mathrm{E}-05 \\
2.38 \mathrm{E}-05 \\
1.53 \mathrm{E}-05 \\
2.38 \mathrm{E}-05 \\
2.38 \mathrm{E}-05 \\
1.42 \mathrm{E}-04 \\
1.42 \mathrm{E}-04 \\
2.38 \mathrm{E}-05 \\
1.37 \mathrm{E}-04 \\
1.44 \mathrm{E}-03 \\
1.75 \mathrm{E}-01 \\
1.79 \mathrm{E}-05 \\
1.75 \mathrm{E}-01 \\
1.36 \mathrm{E}-04 \\
1.75 \mathrm{E}-01\end{array}$} \\
\hline \multirow[t]{2}{*}{ Shipping Site: RFP } & $\begin{array}{l}\text { Total Volume } \\
(m 3 / y r)\end{array}$ & $\begin{array}{cc}\text { Total Mass } \\
(\mathrm{kg} / \mathrm{y} x)\end{array}$ & $2.72 \mathrm{E}+06$ \\
\hline & \begin{tabular}{l} 
Radionuclide \\
\hdashline------- \\
$\mathrm{Tl}-208$ \\
$\mathrm{~Pb}-212$ \\
$\mathrm{Bi}-212$ \\
$\mathrm{Po}-212$ \\
$\mathrm{Po-216}$ \\
$\mathrm{Ra}-224$ \\
$\mathrm{Ra}-228$ \\
$\mathrm{Ac-228}$ \\
$\mathrm{Th}-228$ \\
$\mathrm{Th}-231$ \\
$\mathrm{Th}-232$ \\
$\mathrm{Th}-234$ \\
$\mathrm{~Pa}-234$ \\
$\mathrm{~Pa}-234 \mathrm{~m}$ \\
$\mathrm{U}-235$ \\
$\mathrm{U}-238$ \\
$\mathrm{Pu}-238$ \\
$\mathrm{Pu}-239$ \\
$\mathrm{Pu}-240$ \\
$\mathrm{Pu}-241$ \\
$\mathrm{Am}-241$ \\
$\mathrm{Cm}-242$ \\
$\mathrm{Cm}-244$
\end{tabular} & \begin{tabular}{c} 
Activity \\
(Ci/YI) \\
\hdashline $7.75 E-06$ \\
$2.05 E-05$ \\
$2.05 E-05$ \\
$1.32 E-05$ \\
$2.05 E-05$ \\
$2.05 E-05$ \\
$1.22 E-04$ \\
$1.22 E-04$ \\
$2.05 E-05$ \\
$1.18 E-04$ \\
$1.24 E-03$ \\
$1.51 E-01$ \\
$1.55 E-05$ \\
$1.51 E-01$ \\
$1.17 E-04$ \\
$1.51 E-01$ \\
$1.19 E-01$ \\
$9.11 E-03$ \\
$3.19 E-02$ \\
$4.39 E+00$ \\
$1.82 E-04$ \\
$2.55 E-03$ \\
$9.11 E-04$
\end{tabular} & \\
\hline
\end{tabular}


A.99

\begin{tabular}{|c|c|c|c|}
\hline Shipping Site: SNLA & $\begin{array}{l}\text { Total Volume } \\
(\mathrm{m} 3 / \mathrm{yr})\end{array}$ & $\begin{array}{c}\text { Total Mass } \\
(\mathrm{kg} / \mathrm{yr})\end{array}$ & $3.24 E+05$ \\
\hline & \begin{tabular}{l} 
Radionuclide \\
\hdashline $\mathrm{H}-3$ \\
$\mathrm{Co}-60$ \\
$\mathrm{Ni}-63$ \\
$\mathrm{Sr}-90$ \\
$\mathrm{Y}-90$ \\
$\mathrm{Tc}-99$ \\
$\mathrm{Ru}-106$ \\
$\mathrm{Rh}-106$ \\
$\mathrm{Sb}-125$ \\
$\mathrm{Te}-125 \mathrm{~m}$ \\
$\mathrm{Cs}-134$ \\
$\mathrm{Cs}-137$ \\
$\mathrm{Ba}-137 \mathrm{~m}$ \\
$\mathrm{Ce}-144$ \\
$\mathrm{Pr}-144$ \\
$\mathrm{Pr}-144 \mathrm{~m}$ \\
$\mathrm{Pm}-147$ \\
$\mathrm{Sm}-151$ \\
$\mathrm{Eu}-152$ \\
$\mathrm{Eu}-154$ \\
$\mathrm{Eu}-155$ \\
$\mathrm{Pu}-238$ \\
$\mathrm{Pu}-239$ \\
$\mathrm{Pu}-240$ \\
$\mathrm{Pu}-241$ \\
$\mathrm{Am}-241$ \\
$\mathrm{Cm}-242$ \\
$\mathrm{Cm}-244$
\end{tabular} & $\begin{array}{c}\begin{array}{c}\text { Activity } \\
(C i / y r)\end{array} \\
-3.00 \mathrm{E}+00 \\
2.85 \mathrm{E}-05 \\
5.67 \mathrm{E}-05 \\
5.04 \mathrm{E}-03 \\
5.04 \mathrm{E}-03 \\
9.00 \mathrm{E}-07 \\
4.92 \mathrm{E}-04 \\
4.92 \mathrm{E}-04 \\
1.41 \mathrm{E}-04 \\
3.45 \mathrm{E}-05 \\
5.72 \mathrm{E}-04 \\
5.28 \mathrm{E}-03 \\
4.99 \mathrm{E}-03 \\
1.64 \mathrm{E}-03 \\
1.64 \mathrm{E}-03 \\
1.98 \mathrm{E}-05 \\
4.02 \mathrm{E}-03 \\
3.93 \mathrm{E}-05 \\
6.00 \mathrm{E}-07 \\
1.02 \mathrm{E}-04 \\
8.16 \mathrm{E}-05 \\
7.87 \mathrm{E}-02 \\
6.00 \mathrm{E}-03 \\
2.10 \mathrm{E}-02 \\
2.89 \mathrm{E}+00 \\
1.21 \mathrm{E}-04 \\
1.68 \mathrm{E}-03 \\
6.00 \mathrm{E}-04\end{array}$ & \\
\hline
\end{tabular}


through multiple exposure pathways (i.e., exposure to contaminated ground or air or the ingestion of contaminated food).

All radiologically related impacts are calculated in terms of dose and associated health effects in the exposed populations. The dose of radiation calculated is the total effective dose equivalent as specified in Title 10 of the Code of Federal Regulations (10 CFR Part 20), which is the sum of the effective dose equivalent from exposure to external radiation and the 50-year committed effective dose equivalent (International Commission on Radiological Protection [ICRP] 1977) from exposure to internal radiation. Doses of radiation are calculated in units of rem (roentgen-equivalent man) for individuals and in units of person-rem for collective populations.

The potential exposures to the general population from transportation of radioactive materials, whether during routine operations or from postulated accidents, are usually at a low dose, such that the primary adverse health effect is the potential induction of latent cancers (i.e., cancers that occur after a latency period of several years from the time of exposure). The correlation of radiation dose and human health effects for low doses has been traditionally based on what is termed the "linear/no-threshold hypothesis," which has been described by various international authorities on protection against radiation. This hypothesis implies, in part, that even small doses of radiation cause some risk of inducing cancer and that doubling the radiation dose would mean doubling the expected numbers of cancers. The types of cancer induced by radiation are similar to "naturally occurring" cancers and can be expressed later in the lifetimes of the exposed individuals.

On the basis of the analyses conducted for this report, LLW transportation-related operations are not expected to cause acute (short-term) radiation-induced fatalities or to produce immediately observable effects in exposed individuals. Acute radiation-induced fatalities occur at doses well in excess of $100 \mathrm{rem}$ (ICRP 1991), which generally would not occur for a wide range of transportation activities, including routine operations and accidents. ${ }^{1}$ For all severe accident scenarios analyzed, other short-term effects such as temporary sterility and changes in blood chemistry are not expected.

In this report, the radiological impacts are expressed as health risks in terms of the number of estimated latent-cancer fatalities, the incidence of cancer, and the genetic effects in exposed populations for each alternative. The health risk conversion factors (expected latent health effects per dose absorbed) were derived from ICRP Publication 60 (ICRP 1991).

1 In general, individual acute whole-body doses in the range of 300-500 rem are expected to cause fatality in $50 \%$ of the exposed individuals within 30-60 days (ICRP 1991). 
Destination: NTS

\begin{tabular}{|c|c|c|c|}
\hline Shipping Site: LBL & $\begin{array}{l}\text { Total volume } \\
(\mathrm{m} 3 / \mathrm{yr})\end{array}$ & $\begin{array}{c}\text { Total Mass } \\
(\mathrm{kg} / \mathrm{yr})\end{array}$ & $3.43 E+04$ \\
\hline & \begin{tabular}{l} 
Radionuclide \\
\hdashline $\mathrm{H}-3$ \\
$\mathrm{C}-14$ \\
$\mathrm{Mn}-54$ \\
$\mathrm{Co}-58$ \\
$\mathrm{Co}-60$ \\
$\mathrm{~S} r-90$ \\
$\mathrm{Y}-90$ \\
$\mathrm{Tc}-99$ \\
$\mathrm{Cs}-134$ \\
$\mathrm{Cs}-137$ \\
$\mathrm{Ba}-137 \mathrm{~m}$ \\
$\mathrm{U}-238$
\end{tabular} & $\begin{array}{l}6.17 \mathrm{E}+02 \\
2.35 \mathrm{E}-01 \\
2.65 \mathrm{E}+01 \\
2.45 \mathrm{E}+01 \\
7.08 \mathrm{E}+01 \\
3.33 \mathrm{E}+01 \\
3.33 \mathrm{E}+01 \\
4.71 \mathrm{E}-01 \\
5.49 \mathrm{E}+01 \\
7.25 \mathrm{E}+01 \\
6.85 \mathrm{E}+01 \\
2.86 \mathrm{E}+00\end{array}$ & \\
\hline \multirow[t]{2}{*}{ Shipping Site: LLNL } & $\begin{array}{l}\text { Total Volume } \\
(\mathrm{m} 3 / \mathrm{yr})\end{array}$ & $\begin{array}{c}\text { Total Mass } \\
(\mathrm{kg} / \mathrm{Yr})\end{array}$ & $7.42 \mathrm{E}+05$ \\
\hline & $\begin{array}{l}\text { Radionuclide } \\
\mathrm{H}-3 \\
\mathrm{C}-14 \\
\mathrm{Mn}-54 \\
\mathrm{Co}-58 \\
\mathrm{Co}-60 \\
\mathrm{Ni}-63 \\
\mathrm{~S}-90 \\
\mathrm{Y}-90 \\
\mathrm{Tc}-99 \\
\mathrm{Ru}-106 \\
\mathrm{Rh}-106 \\
\mathrm{Sb}-125 \\
\mathrm{Te}-125 \mathrm{~m} \\
\mathrm{Cs}-134 \\
\mathrm{Cs}-137 \\
\mathrm{Ba}-137 \mathrm{~m} \\
\mathrm{Ce}-144 \\
\mathrm{Pr}-144 \\
\mathrm{Pr}-144 \mathrm{~m} \\
\mathrm{Pm}-147 \\
\mathrm{Sm}-151 \\
\mathrm{Eu}-152 \\
\mathrm{Eu}-154 \\
\mathrm{Eu}-155 \\
\mathrm{U}-238 \\
\mathrm{Pu}-238 \\
\mathrm{Pu}-239 \\
\mathrm{Pu}-240 \\
\mathrm{Pu}-241 \\
\mathrm{Am}-241 \\
\mathrm{Cm}-242 \\
\mathrm{Cm}-244\end{array}$ & \begin{tabular}{c}
$\begin{array}{c}\text { Activity } \\
\text { (Ci/yr) }\end{array}$ \\
\hdashline $2.16 \mathrm{E}+04$ \\
$1.01 \mathrm{E}-03$ \\
$1.14 \mathrm{E}-01$ \\
$1.05 \mathrm{E}-01$ \\
$3.06 \mathrm{E}-01$ \\
$3.19 \mathrm{E}-03$ \\
$4.27 \mathrm{E}-01$ \\
$4.27 \mathrm{E}-01$ \\
$2.07 \mathrm{E}-03$ \\
$2.77 \mathrm{E}-02$ \\
$2.77 \mathrm{E}-02$ \\
$7.96 \mathrm{E}-03$ \\
$1.94 \mathrm{E}-03$ \\
$2.68 \mathrm{E}-01$ \\
$6.09 \mathrm{E}-01$ \\
$5.76 \mathrm{E}-01$ \\
$9.27 \mathrm{E}-02$ \\
$9.27 \mathrm{E}-02$ \\
$1.11 \mathrm{E}-03$ \\
$2.26 \mathrm{E}-01$ \\
$2.21 \mathrm{E}-03$ \\
$3.38 \mathrm{E}-05$ \\
$5.79 \mathrm{E}-03$ \\
$4.59 \mathrm{E}-03$ \\
$1.23 \mathrm{E}-02$ \\
$5.12 \mathrm{E}-02$ \\
$3.44 \mathrm{E}-03$ \\
$1.18 \mathrm{E}-02$ \\
$1.63 \mathrm{E}+00$ \\
$1.69 \mathrm{E}-04$ \\
$9.46 \mathrm{E}-04$ \\
$3.38 \mathrm{E}-04$
\end{tabular} & \\
\hline \multirow[t]{2}{*}{ Shipping Site: SLAC } & $\begin{array}{l}\text { Total Volume } \\
\text { (m3/yx) }\end{array}$ & $\begin{array}{c}\text { Total Mass } \\
(\mathrm{kg} / \mathrm{Y} Y)\end{array}$ & $7.45 E+05$ \\
\hline & $\begin{array}{l}\text { Radionuclide } \\
\mathrm{Cr}-51 \\
\mathrm{Mn}-54 \\
\mathrm{Fe}-55 \\
\mathrm{Fe}-59 \\
\mathrm{Co}-58 \\
\mathrm{Co}-60 \\
\mathrm{Ni}-59 \\
\mathrm{Ni}-63 \\
\mathrm{Zr}-95\end{array}$ & \begin{tabular}{c}
$\begin{array}{c}\text { Activity } \\
(C i / y z)\end{array}$ \\
\hdashline $3.86 \mathrm{E}-03$ \\
$7.36 \mathrm{E}-03$ \\
$2.74 \mathrm{E}-02$ \\
$2.48 \mathrm{E}-04$ \\
$9.88 \mathrm{E}-03$ \\
$7.74 \mathrm{E}-02$ \\
$3.54 \mathrm{E}-04$ \\
$5.04 \mathrm{E}-02$ \\
$3.01 \mathrm{E}-04$
\end{tabular} & \\
\hline
\end{tabular}




\section{A-101}

\begin{tabular}{|c|c|c|c|}
\hline Shipping Site: SNLL & $\begin{array}{l}\text { Total Volume } \\
(\mathrm{m} 3 / \mathrm{yr})\end{array}$ & $\begin{array}{cc}3.36 \mathrm{E}+01 & \begin{array}{c}\text { Total Mass } \\
(\mathrm{kg} / \mathrm{Yr})\end{array}\end{array}$ & $9.17 E+04$ \\
\hline & $\begin{array}{l}\text { Radionuclide } \\
\mathrm{H}-3 \\
\mathrm{~T} 1-208 \\
\mathrm{~Pb}-212 \\
\mathrm{Bi}-212 \\
\mathrm{Po-212} \\
\mathrm{Po-216} \\
\mathrm{Ra}-224 \\
\mathrm{Ra}-228 \\
\mathrm{Ac}-228 \\
\mathrm{Th}-228 \\
\mathrm{Th}-231 \\
\mathrm{Th}-232 \\
\mathrm{Th}-234 \\
\mathrm{~Pa}-234 \\
\mathrm{~Pa}-234 \mathrm{~m} \\
\mathrm{U}-235 \\
\mathrm{U}-238\end{array}$ & \begin{tabular}{c}
$\begin{array}{c}\text { Activity } \\
\text { (Ci/yr) }\end{array}$ \\
\hdashline $1.38 \mathrm{-}+01$ \\
$1.86 \mathrm{0}$ \\
$5.86 \mathrm{E}-05$ \\
$1.55 \mathrm{E}-04$ \\
$1.55 \mathrm{E}-04$ \\
$1.00 \mathrm{E}-04$ \\
$1.55 \mathrm{E}-04$ \\
$1.55 \mathrm{E}-04$ \\
$9.28 \mathrm{E}-04$ \\
$9.28 \mathrm{E}-04$ \\
$1.55 \mathrm{E}-04$ \\
$8.93 \mathrm{E}-04$ \\
$9.42 \mathrm{E}-03$ \\
$1.14 \mathrm{E}+00$ \\
$1.17 \mathrm{E}-04$ \\
$1.14 \mathrm{E}+00$ \\
$8.90 \mathrm{E}-04$ \\
$1.14 \mathrm{E}+00$
\end{tabular} & \\
\hline
\end{tabular}


Destination: ORNL

\begin{tabular}{|c|c|c|c|}
\hline Shipping Site: AMES & $\begin{array}{l}\text { Total Volume } \\
(\mathrm{m} 3 / \mathrm{yr})\end{array}$ & $\begin{array}{c}\text { Toral Mass } \\
(\mathrm{kg} / \mathrm{Y}=)\end{array}$ & $2.99 E+04$ \\
\hline & $\begin{array}{l}\text { Radionuclide } \\
-\mathrm{Tl-208} \\
\mathrm{Pb}-212 \\
\mathrm{Bi}-212 \\
\mathrm{Po-212} \\
\mathrm{Po-216} \\
\mathrm{Ra}-224 \\
\mathrm{Ra}-228 \\
\mathrm{Ac}-228 \\
\mathrm{Th}-228 \\
\mathrm{Th}-231 \\
\mathrm{Th}-232 \\
\mathrm{Th}-234 \\
\mathrm{~Pa}-234 \\
\mathrm{~Pa}-234 \mathrm{~m} \\
\mathrm{U}-235 \\
\mathrm{U}-238 \\
\mathrm{Pu}-238 \\
\mathrm{Pu}-239 \\
\mathrm{Pu}-240 \\
\mathrm{Pu}-241 \\
\mathrm{Am}-241 \\
\mathrm{Cm}-242 \\
\mathrm{Cm}-244\end{array}$ & \begin{tabular}{c}
$\begin{array}{c}\text { Activity } \\
(C i / y I)\end{array}$ \\
\hdashline$-1.50 E-07$ \\
$1.19 \mathrm{E}-06$ \\
$1.19 \mathrm{E}-06$ \\
$7.68 \mathrm{E}-07$ \\
$1.19 \mathrm{E}-06$ \\
$1.19 \mathrm{E}-06$ \\
$7.12 \mathrm{E}-06$ \\
$7.12 \mathrm{E}-06$ \\
$1.19 \mathrm{E}-06$ \\
$6.86 \mathrm{E}-06$ \\
$7.23 \mathrm{E}-05$ \\
$8.79 \mathrm{E}-03$ \\
$9.01 \mathrm{E}-07$ \\
$8.79 \mathrm{E}-03$ \\
$6.83 \mathrm{E}-06$ \\
$8.79 \mathrm{E}-03$ \\
$6.94 \mathrm{E}-04$ \\
$5.30 \mathrm{E}-05$ \\
$1.85 \mathrm{E}-04$ \\
$2.55 \mathrm{E}-02$ \\
$1.06 \mathrm{E}-06$ \\
$1.48 \mathrm{E}-05$ \\
$5.30 \mathrm{E}-06$
\end{tabular} & \\
\hline Shipping Site: ANL-E & $\begin{array}{l}\text { Total volume } \\
(\mathrm{m} 3 / \mathrm{yr})\end{array}$ & $7.35 \mathrm{E}+02 \quad \begin{array}{c}\text { Total Mass } \\
(\mathrm{kg} / \mathrm{yr})\end{array}$ & $2.00 E+06$ \\
\hline & $\begin{array}{l}\text { Radionuclide } \\
--3-3 \\
\mathrm{C}-14 \\
\mathrm{Cr}-51 \\
\mathrm{Mn}-54 \\
\mathrm{Fe}-55 \\
\mathrm{Fe}-59 \\
\mathrm{Co}-58 \\
\mathrm{Co}-60 \\
\mathrm{Ni}-59 \\
\mathrm{Ni}-63 \\
\mathrm{Sr}-90 \\
\mathrm{Y}-90 \\
\mathrm{Zr}-95 \\
\mathrm{Tc}-99 \\
\mathrm{Ru}-106 \\
\mathrm{Rh}-106 \\
\mathrm{Sb}-125 \\
\mathrm{Te}-125 \mathrm{~m} \\
\mathrm{Cs}-134 \\
\mathrm{Cs}-137 \\
\mathrm{Ba}-137 \mathrm{~m} \\
\mathrm{Ce}-144 \\
\mathrm{Pr}-144 \\
\mathrm{Pr}-144 \mathrm{~m} \\
\mathrm{Pm}-147 \\
\mathrm{Sm}-151 \\
\mathrm{Eu}-152 \\
\mathrm{Eu}-154 \\
\mathrm{Eu}-155 \\
\mathrm{U}-238 \\
\mathrm{Pu}-238 \\
\mathrm{Pu}-239 \\
\mathrm{Pu}-240 \\
\mathrm{Pu}-241 \\
\mathrm{Am}-241\end{array}$ & $\begin{array}{c}\text { Activity } \\
(C i / y r) \\
1.33 \mathrm{E}+02 \\
7.91 \mathrm{E}-02 \\
2.87 \mathrm{E}+00 \\
1.43 \mathrm{E}+01 \\
2.04 \mathrm{E}+01 \\
1.84 \mathrm{E}-01 \\
1.55 \mathrm{E}+01 \\
8.15 \mathrm{E}+01 \\
2.63 \mathrm{E}-01 \\
3.77 \mathrm{E}+01 \\
3.33 \mathrm{E}+01 \\
3.33 \mathrm{E}+01 \\
2.24 \mathrm{E}-01 \\
1.62 \mathrm{E}-01 \\
2.16 \mathrm{E}+00 \\
2.16 \mathrm{E}+00 \\
6.21 \mathrm{E}-01 \\
1.51 \mathrm{E}-01 \\
2.09 \mathrm{E}+01 \\
4.75 \mathrm{E}+01 \\
4.50 \mathrm{E}+01 \\
7.24 \mathrm{E}+00 \\
7.24 \mathrm{E}+00 \\
8.70 \mathrm{E}-02 \\
1.76 \mathrm{E}+01 \\
1.72 \mathrm{E}-01 \\
2.63 \mathrm{E}-03 \\
4.52 \mathrm{E}-01 \\
3.58 \mathrm{E}-01 \\
9.63 \mathrm{E}-01 \\
5.44 \mathrm{E}-01 \\
5.27 \mathrm{E}-03 \\
2.63 \mathrm{E}-03 \\
6.09 \mathrm{E}-01 \\
7.91 \mathrm{E}-03\end{array}$ & \\
\hline
\end{tabular}


A- 103

\begin{tabular}{|c|c|c|c|c|}
\hline Shipping sice: BAPL & $\begin{array}{l}\text { Total Volume } \\
(\mathrm{m} 3 / \mathrm{yr})\end{array}$ & $3.98 \mathrm{E}+02$ & $\begin{array}{c}\text { Total Mass } \\
(\mathrm{kg} / \mathrm{Yr})\end{array}$ & $1.08 \mathrm{E}+06$ \\
\hline r & $\begin{array}{l}\text { Radionuclide } \\
\mathrm{H}-3 \\
\mathrm{Co}-60 \\
\mathrm{Ni}-63 \\
\mathrm{Sr}-90 \\
\mathrm{Y}-90 \\
\mathrm{Tc}-99 \\
\mathrm{Ru}-106 \\
\mathrm{Rh}-106 \\
\mathrm{Sb}-125 \\
\mathrm{Te}-125 \mathrm{~m} \\
\mathrm{Cs}-134 \\
\mathrm{Cs}-137 \\
\mathrm{Ba}-137 \mathrm{~m} \\
\mathrm{Ce}-144 \\
\mathrm{Pr}-144 \\
\mathrm{Pr}-144 \mathrm{~m} \\
\mathrm{Pm}-147 \\
\mathrm{Sm}-151 \\
\mathrm{Eu}-152 \\
\mathrm{Eu}-154 \\
\mathrm{Eu}-155 \\
\mathrm{~T} 1-208 \\
\mathrm{~Pb}-212 \\
\mathrm{Bi}-212 \\
\mathrm{PO}-212 \\
\mathrm{Po}-216 \\
\mathrm{Ra}-224 \\
\mathrm{Ra}-228 \\
\mathrm{Ac}-228 \\
\mathrm{Th}-228 \\
\mathrm{Th}-231 \\
\mathrm{Th}-232 \\
\mathrm{Th}-234 \\
\mathrm{~Pa}-234 \\
\mathrm{~Pa}-234 \mathrm{~m} \\
\mathrm{U}-235 \\
\mathrm{U}-238 \\
\mathrm{Pu}-238 \\
\mathrm{Pu}-239 \\
\mathrm{Pu}-240 \\
\mathrm{Pu}-241 \\
\mathrm{Am}-241 \\
\end{array}$ & $\begin{array}{l}\text { A } \\
- \\
2 \\
1 \\
2 \\
2 \\
2 \\
3 \\
2 \\
2 \\
5 \\
1 \\
2 \\
2 \\
2 \\
6 \\
6 \\
8 \\
1 \\
1 \\
2 \\
4 \\
3 \\
3 \\
1 \\
1 \\
6 \\
1 \\
1 \\
6 \\
6 \\
1 \\
5 \\
6 \\
7 \\
7 \\
7 \\
5 \\
7 \\
5 \\
5 \\
2 \\
5 \\
7\end{array}$ & 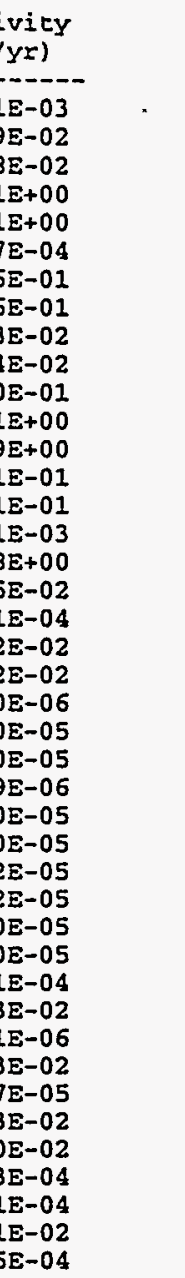 & 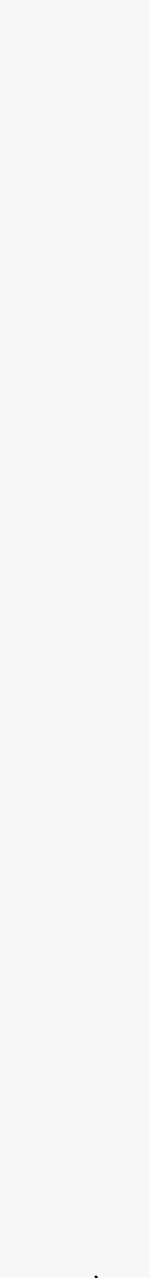 \\
\hline Shipping site: FNAL & $\begin{array}{l}\text { Total Volume } \\
\left(\mathrm{m} 3 / \mathrm{y}^{\prime}\right)\end{array}$ & \multicolumn{2}{|r|}{$\begin{array}{l}\text { Total Mass } \\
(\mathrm{kg} / \mathrm{y} x)\end{array}$} & $3.89 E+04$ \\
\hline & $\begin{array}{l}\text { Radionuclide } \\
\mathrm{H}-3 \\
\mathrm{Cr}-51 \\
\mathrm{Mn}-54 \\
\mathrm{Fe}-55 \\
\mathrm{Fe}-59 \\
\mathrm{Co}-58 \\
\mathrm{Co}-60 \\
\mathrm{Ni}-59 \\
\mathrm{Ni}-63 \\
\mathrm{Zr}-95 \\
\mathrm{~T} \mathrm{H}-208 \\
\mathrm{~Pb}-212 \\
\mathrm{Bi}-212 \\
\mathrm{Po-212} \\
\mathrm{Po-216} \\
\mathrm{Ra}-224 \\
\mathrm{Ra}-228 \\
\mathrm{Ac}-228 \\
\mathrm{Th}-228 \\
\mathrm{Th}-231 \\
\mathrm{Th}-232 \\
\mathrm{Th}-234 \\
\mathrm{~Pa}-234 \\
\mathrm{~Pa}-234 \mathrm{~m} \\
\mathrm{U}-235 \\
\mathrm{U}-238\end{array}$ & $\begin{array}{r}\text { A } \\
-1 \\
1 \\
1 \\
2 \\
7 \\
7 \\
2 \\
2 \\
1 \\
1 \\
8 \\
3 \\
1 \\
1 \\
6 \\
1 \\
1 \\
5 \\
5 \\
1 \\
5 \\
6 \\
7 \\
7 \\
7 \\
5 \\
7\end{array}$ & $\begin{array}{l}\text { ivity } \\
\text { (yI) } \\
2 E-01 \\
2 E-01 \\
2 E-02 \\
2 E-02 \\
4 E-02 \\
7 E-04 \\
5 E-02 \\
3 E-01 \\
2 E-03 \\
5 E-01 \\
1 E-04 \\
3 E-07 \\
0 E-06 \\
j E-06 \\
5 E-07 \\
j E-06 \\
0 E-06 \\
9 E-06 \\
9 E-06 \\
0 E-06 \\
7 E-06 \\
3 E-05 \\
9 E-03 \\
7 E-07 \\
9 E-03 \\
5 E-06 \\
9 E-03\end{array}$ & \\
\hline
\end{tabular}




\section{A- 104}

\begin{tabular}{|c|c|c|c|}
\hline Shipping Site: $\mathrm{k}-25$ & $\begin{array}{l}\text { Total Volume } \\
(\mathrm{m} 3 / \mathrm{yr})\end{array}$ & $\begin{array}{c}\text { Total Mass } \\
(\mathrm{kg} / \mathrm{yr})\end{array}$ & $4.67 E+07$ \\
\hline & $\begin{array}{l}\text { Radionuclide } \\
\mathrm{Tl}-208 \\
\mathrm{~Pb}-212 \\
\mathrm{Bi}-212 \\
\mathrm{PO}-212 \\
\mathrm{Po}-216 \\
\mathrm{Ra}-224 \\
\mathrm{Ra}-228 \\
\mathrm{Ac}-228 \\
\mathrm{Th}-228 \\
\mathrm{Th}-231 \\
\mathrm{Th}-232 \\
\mathrm{Th}-234 \\
\mathrm{~Pa}-234 \\
\mathrm{~Pa}-234 \mathrm{~m} \\
\mathrm{U}-235 \\
\mathrm{U}-238\end{array}$ & $\begin{array}{c}\begin{array}{c}\text { Activity } \\
(C i / y z)\end{array} \\
1.06 \mathrm{y}-04 \\
2.82 \mathrm{E}-04 \\
2.82 \mathrm{E}-04 \\
1.82 \mathrm{E}-04 \\
2.82 \mathrm{E}-04 \\
2.82 \mathrm{E}-04 \\
1.68 \mathrm{E}-03 \\
1.68 \mathrm{E}-03 \\
2.82 \mathrm{E}-04 \\
1.62 \mathrm{E}-03 \\
1.71 \mathrm{E}-02 \\
2.08 \mathrm{E}+00 \\
2.13 \mathrm{E}-04 \\
2.08 \mathrm{E}+00 \\
1.62 \mathrm{E}-03 \\
2.08 \mathrm{0}+00\end{array}$ & \\
\hline Shipping Sice: KAPL & $\begin{array}{l}\text { Total Volume } \\
(\mathrm{m} 3 / \mathrm{yr})\end{array}$ & $\begin{array}{l}\text { Total Mass } \\
(\mathrm{kg} / \mathrm{y})\end{array}$ & $5.36 \mathrm{E}+05$ \\
\hline * & $\begin{array}{l}\text { Radionuclide } \\
\mathrm{HI}-3 \\
\mathrm{C}-14 \\
\mathrm{Cr}-51 \\
\mathrm{Mn}-54 \\
\mathrm{Fe}-55 \\
\mathrm{Fe}-59 \\
\mathrm{Co}-58 \\
\mathrm{Co}-60 \\
\mathrm{Ni}-59 \\
\mathrm{Ni}-63 \\
\mathrm{Sr}-90 \\
\mathrm{Y}-90 \\
\mathrm{Zr}-95 \\
\mathrm{Tc}-99 \\
\mathrm{Ru}-106 \\
\mathrm{Rh}-106 \\
\mathrm{Sb}-125 \\
\mathrm{Te}-125 \mathrm{~m} \\
\mathrm{Cs}-134 \\
\mathrm{Cs}-137 \\
\mathrm{Ba}-137 \mathrm{~m} \\
\mathrm{Ce}-144 \\
\mathrm{Pr}-144 \\
\mathrm{Pr}-144 \mathrm{~m} \\
\mathrm{Pm}-147 \\
\mathrm{Sm}-151 \\
\mathrm{Eu}-152 \\
\mathrm{Eu}-154 \\
\mathrm{Eu}-155 \\
\mathrm{Tl}-208 \\
\mathrm{~Pb}-212 \\
\mathrm{Bi}-212 \\
\mathrm{Po}-212 \\
\mathrm{PO}-216 \\
\mathrm{Ra}-224 \\
\mathrm{Ra}-228 \\
\mathrm{Ac}-228 \\
\mathrm{Th}-228 \\
\mathrm{Th}-231 \\
\mathrm{Th}-232 \\
\mathrm{Th}-234 \\
\mathrm{~Pa}-234 \\
\mathrm{~Pa}-234 \mathrm{~m} \\
\mathrm{U}-235 \\
\mathrm{U}-238 \\
\mathrm{Pu}-238 \\
\mathrm{Pu}-239 \\
\mathrm{Pu}-240 \\
\mathrm{Pu}-241 \\
\mathrm{Am}-241 \\
.\end{array}$ & $\begin{array}{c}\text { Activity } \\
\text { (Ci/YI) } \\
5.14 E-04 \\
2.40 E-05 \\
5.11 E-03 \\
1.24 E-02 \\
3.63 E-02 \\
3.28 E-04 \\
1.55 E-02 \\
1.09 E-01 \\
4.69 E-04 \\
6.70 E-02 \\
2.58 E-02 \\
2.58 E-02 \\
3.99 E-04 \\
5.20 E-05 \\
2.19 E-03 \\
2.19 E-03 \\
6.31 E-04 \\
1.54 E-04 \\
8.14 E-03 \\
3.09 E-02 \\
2.92 E-02 \\
7.35 E-03 \\
7.35 E-03 \\
8.84 E-05 \\
1.79 E-02 \\
1.75 E-04 \\
2.67 E-06 \\
4.59 E-04 \\
3.64 E-04 \\
4.50 E-09 \\
1.19 E-08 \\
1.19 E-08 \\
7.60 E-09 \\
1.19 E-08 \\
1.19 E-08 \\
7.11 E-08 \\
7.11 E-08 \\
1.19 E-08 \\
6.84 E-08 \\
7.21 E-07 \\
8.77 E-05 \\
8.900-09 \\
8.77 E-05 \\
6.82 E-08 \\
3.79 E-04 \\
5.53 E-04 \\
5.35 E-06 \\
2.67 E-06 \\
6.18 E-04 \\
8.03 E-06\end{array}$ & \\
\hline
\end{tabular}




\section{A- 105}

\begin{tabular}{|c|c|c|c|}
\hline Shipping Site: KCP & $\begin{array}{l}\text { Total Volume } \\
\text { (m3/Yr) }\end{array}$ & $\begin{array}{c}\text { Total Mass } \\
(\mathrm{kg} / \mathrm{Yr})\end{array}$ & $7.23 \mathrm{E}+03$ \\
\hline & \begin{tabular}{l} 
Radionuclide \\
\hdashline $\mathrm{H}-3$ \\
$\mathrm{C}-14$ \\
$\mathrm{Mn}-54$ \\
$\mathrm{Co}-58$ \\
$\mathrm{Co}-60$ \\
$\mathrm{~S}-90$ \\
$\mathrm{Y}-90$ \\
$\mathrm{TC}-99$ \\
$\mathrm{Cs}-134$ \\
$\mathrm{Cs}-137$ \\
Ba-137m \\
$\mathrm{U}-238$
\end{tabular} & \multicolumn{2}{|l|}{$\begin{array}{l}2.70 \mathrm{E}-02 \\
1.44 \mathrm{E}-04 \\
1.62 \mathrm{E}-02 \\
1.50 \mathrm{E}-02 \\
4.34 \mathrm{E}-02 \\
2.04 \mathrm{E}-02 \\
2.04 \mathrm{E}-02 \\
2.89 \mathrm{E}-04 \\
3.37 \mathrm{E}-02 \\
4.44 \mathrm{E}-02 \\
4.20 \mathrm{E}-02 \\
1.76 \mathrm{E}-03\end{array}$} \\
\hline \multirow[t]{2}{*}{ Shipping Site: MOUND } & $\begin{array}{l}\text { Total Volume } \\
(\mathrm{m} 3 / Y Y)\end{array}$ & $4.33 E+03 \quad \begin{array}{c}\text { Total Mass } \\
(\mathrm{kg} / \mathrm{Yr})\end{array}$ & $9.67 \mathrm{E}+06$ \\
\hline & $\begin{array}{l}\text { Radionuclide } \\
\mathrm{H}-3 \\
\mathrm{Pu}-238 \\
\mathrm{Pu}-239 \\
\mathrm{Pu}-240 \\
\mathrm{Pu}-24 \mathrm{I} \\
\mathrm{Am}-241 \\
\mathrm{Cm}-242 \\
\mathrm{Cm}-244\end{array}$ & \multicolumn{2}{|l|}{$\begin{array}{l}1.34 \mathrm{E}+04 \\
1.60 \mathrm{E}-01 \\
1.22 \mathrm{E}-02 \\
4.28 \mathrm{E}-02 \\
5.90 \mathrm{E}+00 \\
2.44 \mathrm{E}-04 \\
3.42 \mathrm{E}-03 \\
1.22 \mathrm{E}-03\end{array}$} \\
\hline \multirow[t]{2}{*}{ Shipping Site: ORISE } & $\begin{array}{l}\text { Total Volume } \\
(\mathrm{m} 3 / \mathrm{yr})\end{array}$ & $\begin{array}{c}\text { Total Mass } \\
(\mathrm{kg} / \mathrm{Yr})\end{array}$ & $7.36 \mathrm{E}+04$ \\
\hline & $\begin{array}{l}\text { Radionuclide } \\
\mathrm{H}-3 \\
\mathrm{C}-14 \\
\mathrm{Mn}-54 \\
\mathrm{Co}-58 \\
\mathrm{Co}-60 \\
\mathrm{Sr}-90 \\
\mathrm{Y}-90 \\
\mathrm{TC}-99 \\
\mathrm{Cs}-134 \\
\mathrm{Cs}-137 \\
\mathrm{Ba}-137 \mathrm{~m} \\
\mathrm{~T} 1-208 \\
\mathrm{~Pb}-212 \\
\mathrm{Bi}-212 \\
\mathrm{Po}-212 \\
\mathrm{Po}-216 \\
\mathrm{Ra}-224 \\
\mathrm{Ra}-228 \\
\mathrm{Ac}-228 \\
\mathrm{Th}-228 \\
\text { Th-23I } \\
\text { Th-232 } \\
\text { Th-234 } \\
\mathrm{Pa}-234 \\
\mathrm{~Pa}-234 \mathrm{~m} \\
\mathrm{U}-235 \\
\mathrm{U}-238\end{array}$ & $\begin{array}{c}\begin{array}{c}\text { Activity } \\
(C i / y x)\end{array} \\
2.00 E-02 \\
1.19 E-05 \\
1.34 E-03 \\
1.24 E-03 \\
3.59 E-03 \\
1.69 E-03 \\
1.69 E-03 \\
2.39 E-05 \\
2.79 E-03 \\
3.68 E-03 \\
3.48 E-03 \\
3.39 E-07 \\
8.98 E-07 \\
8.98 E-07 \\
5.78 E-07 \\
8.98 E-07 \\
8.98 E-07 \\
5.36 E-06 \\
5.36 E-06 \\
8.98 E-07 \\
5.16 E-06 \\
5.44 E-05 \\
6.62 E-03 \\
6.78 E-07 \\
6.62 E-03 \\
5.14 E-06 \\
6.77 E-03\end{array}$ & \\
\hline
\end{tabular}




\begin{tabular}{|c|c|c|c|}
\hline Shipping Site: PORTS & $\begin{array}{l}\text { Total volume } \\
(\mathrm{m} 3 / \mathrm{yr})\end{array}$ & $\begin{array}{l}\text { Total Mass } \\
(\mathrm{kg} / \mathrm{Yr})\end{array}$ & $6.74 E+07$ \\
\hline & $\begin{array}{l}\text { Radionuclide } \\
\mathrm{Tl}-208 \\
\mathrm{~Pb}-212 \\
\mathrm{Bi}-212 \\
\mathrm{Po}-212 \\
\mathrm{Po}-216 \\
\mathrm{Ra}-224 \\
\mathrm{Ra}-228 \\
\mathrm{Ac}-228 \\
\mathrm{Th}-228 \\
\mathrm{Th}-231 \\
\mathrm{Th}-232 \\
\mathrm{Th}-234 \\
\mathrm{~Pa}-234 \\
\mathrm{~Pa}-234 \mathrm{~m} \\
\mathrm{U}-235 \\
\mathrm{U}-238\end{array}$ & $\begin{array}{c}\begin{array}{c}\text { Activity } \\
(\mathrm{Ci} / \mathrm{yr})\end{array} \\
3.45 \mathrm{E}-06 \\
9.14 \mathrm{E}-06 \\
9.14 \mathrm{E}-06 \\
5.89 \mathrm{E}-06 \\
9.14 \mathrm{E}-06 \\
9.14 \mathrm{E}-06 \\
5.46 \mathrm{E}-05 \\
5.46 \mathrm{E}-05 \\
9.14 \mathrm{E}-06 \\
5.26 \mathrm{E}-05 \\
5.55 \mathrm{E}-04 \\
6.74 \mathrm{E}-02 \\
6.91 \mathrm{E}-06 \\
6.74 \mathrm{E}-02 \\
5.24 \mathrm{E}-05 \\
6.74 \mathrm{E}-02\end{array}$ & \\
\hline Shipping Site: PPPL & $\begin{array}{l}\text { Total Volume } \\
(\mathrm{m} 3 / \mathrm{yr})\end{array}$ & $2.21 \mathrm{E}+01 \quad \begin{array}{c}\text { Total Mass } \\
(\mathrm{kg} / \mathrm{yr})\end{array}$ & $4.97 \mathrm{E}+03$ \\
\hline & \begin{tabular}{l} 
Radionuclide \\
\hdashline$-3-3$ \\
$\mathrm{Cr}-51$ \\
$\mathrm{Mn}-54$ \\
$\mathrm{Fe}-55$ \\
$\mathrm{Fe}-59$ \\
$\mathrm{Co}-58$ \\
$\mathrm{Co}-60$ \\
$\mathrm{Ni}-59$ \\
$\mathrm{Ni}-63$ \\
$\mathrm{Zr}-95$
\end{tabular} & $\begin{array}{c}\begin{array}{c}\text { Activity } \\
\text { (Ci/yr) }\end{array} \\
2.01 \mathrm{E}-01 \\
8.50 \mathrm{E}-04 \\
1.61 \mathrm{E}-03 \\
6.04 \mathrm{E}-03 \\
5.46 \mathrm{E}-05 \\
2.17 \mathrm{E}-03 \\
1.70 \mathrm{E}-02 \\
7.80 \mathrm{E}-05 \\
1.10 \mathrm{E}-02 \\
6.63 \mathrm{E}-05\end{array}$ & \\
\hline Shipping Site: Paducah & $\begin{array}{l}\text { Total Volume } \\
(\mathrm{m} 3 / \mathrm{yx})\end{array}$ & $\begin{array}{l}\text { Total Mass } \\
(\mathrm{kg} / \mathrm{yr})\end{array}$ & $1.19 E+07$ \\
\hline & $\begin{array}{l}\text { Radionuclide } \\
----99 \\
T c-99 \\
T l-208 \\
\mathrm{~Pb}-212 \\
\mathrm{Bi}-212 \\
\mathrm{PO}-212 \\
\mathrm{Po}-216 \\
\mathrm{Ra}-224 \\
\mathrm{Ra}-228 \\
\mathrm{Ac}-228 \\
\mathrm{Th}-228 \\
\mathrm{Th}-231 \\
\mathrm{Th}-232 \\
\mathrm{Th}-234 \\
\mathrm{~Pa}-234 \\
\mathrm{~Pa}-234 \mathrm{~m} \\
\mathrm{U}-235 \\
\mathrm{U}-238 \\
\mathrm{~Np}-237 \\
\mathrm{Pu}-239\end{array}$ & \begin{tabular}{c} 
Activity \\
(Ci/yI) \\
\hdashline $8.84 E-03$ \\
$3.72 E-05$ \\
$9.84 E-05$ \\
$9.84 E-05$ \\
$6.34 E-05$ \\
$9.84 E-05$ \\
$9.84 E-05$ \\
$5.88 E-04$ \\
$5.88 E-04$ \\
$9.84 E-05$ \\
$5.66 E-04$ \\
$5.97 E-03$ \\
$7.26 E-01$ \\
$7.44 E-05$ \\
$7.26 E-01$ \\
$5.64 E-04$ \\
$7.26 E-01$ \\
$6.63 E-03$ \\
$6.63 E-03$
\end{tabular} & \\
\hline
\end{tabular}




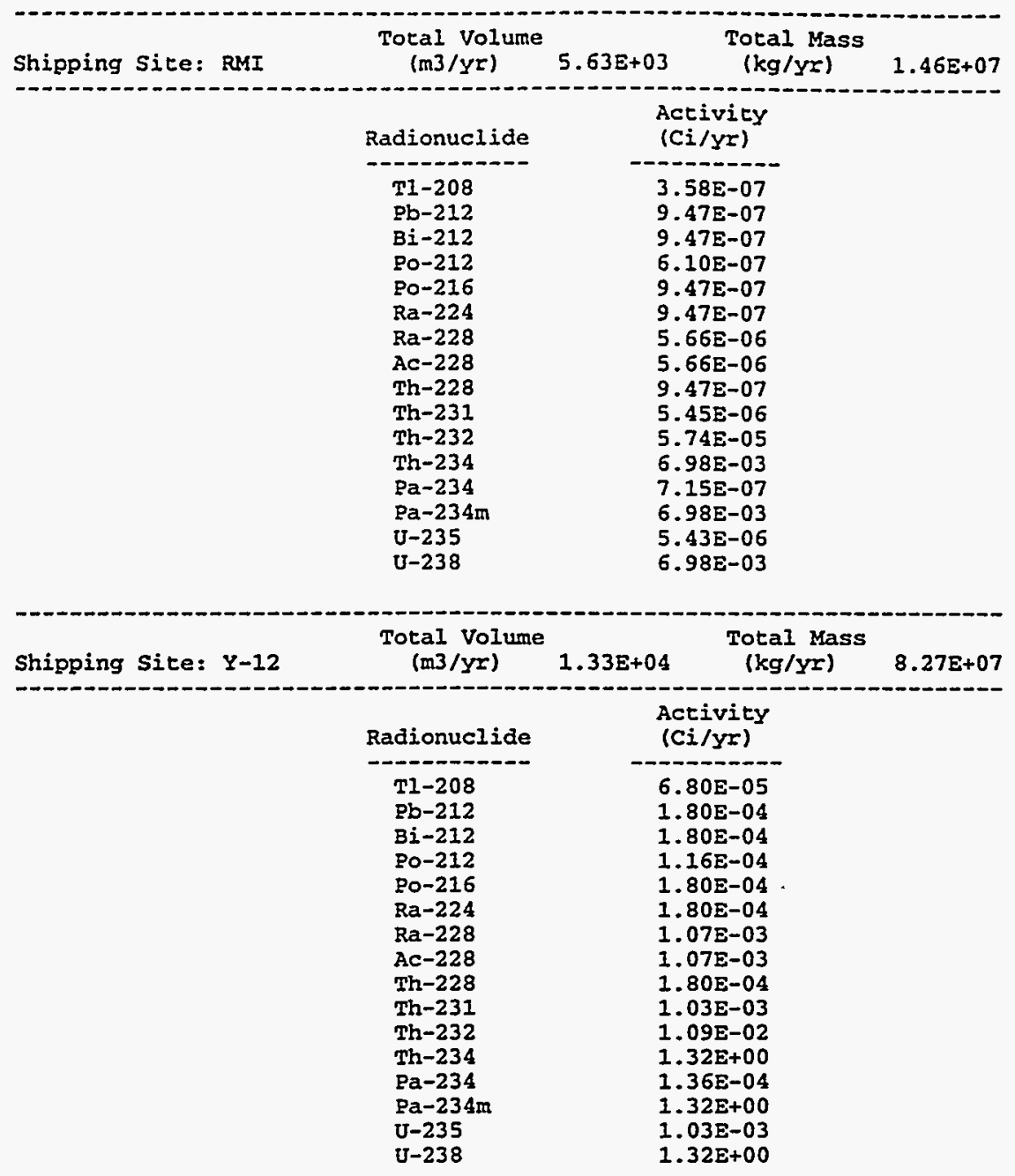

Destination: SRS

\begin{tabular}{|c|c|c|c|c|}
\hline Shipping Site: Pinellas & $\begin{array}{l}\text { Total Volume } \\
\text { (m3/yx) }\end{array}$ & $1.27 \mathrm{E}+02$ & $\begin{array}{c}\text { Total Mass } \\
(\mathrm{kg} / \mathrm{Yr})\end{array}$ & $1.91 E+04$ \\
\hline & \multicolumn{4}{|c|}{$\begin{array}{l}\text { Activity } \\
\text { (Ci/yr) }\end{array}$} \\
\hline & $H-3$ & & & \\
\hline
\end{tabular}




\section{A.14 WM LLW REGIONALIZED 5 ALTERNATIVE (CASE 19): ACTIVATED METALS}

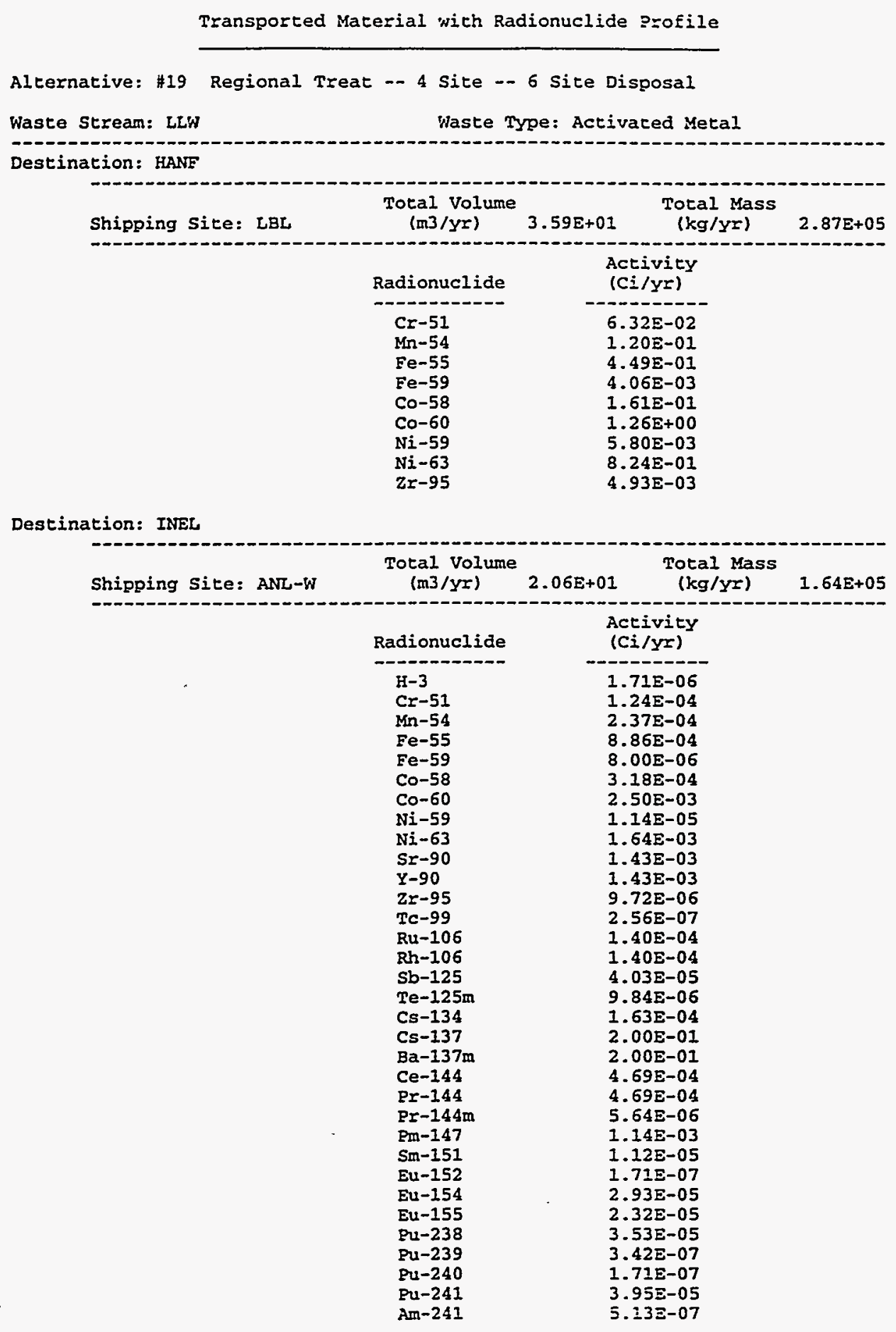




\begin{tabular}{|c|c|c|c|}
\hline \multirow{2}{*}{ Shipping Site: LANL } & $\begin{array}{l}\text { Total Volume } \\
\text { (m3/yr) }\end{array}$ & $\begin{array}{l}\text { Total Mass } \\
\left(\mathrm{kg} / \mathrm{Y}^{\mathrm{r}}\right)\end{array}$ & $8.57 E+06$ \\
\hline & $\begin{array}{l}\text { Radionuclide } \\
\text { Cr-51 } \\
\mathrm{Mn}-54 \\
\text { Fe-55 } \\
\text { Fe-59 } \\
\mathrm{Co}-58 \\
\mathrm{Co}-60 \\
\mathrm{Ni}-59 \\
\mathrm{Ni}-63 \\
\mathrm{Zr}-95\end{array}$ & $\begin{array}{l}\text { Activity } \\
\text { (Ci/yr) } \\
2.42 E+01 \\
4.62 \mathrm{E}+01 \\
1.72 \mathrm{E}+02 \\
1.55 \mathrm{E}+00 \\
6.20 \mathrm{E}+01 \\
4.86 \mathrm{E}+02 \\
2.22 \mathrm{E}+00 \\
3.16 \mathrm{E}+02 \\
1.89 \mathrm{E}+00\end{array}$ & 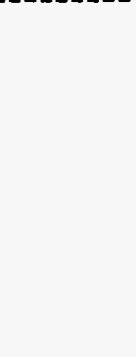 \\
\hline Shipping Site: NRF & $\begin{array}{l}\text { Total Volume } \\
(\mathrm{m} 3 / \mathrm{yr})\end{array}$ & $\begin{array}{c}\text { Total Mass } \\
(\mathrm{kg} / \mathrm{Yr})\end{array}$ & $9.405+06$ \\
\hline & $\begin{array}{l}\text { Radionuclide } \\
\text { Cr-51 } \\
M n-54 \\
\text { Fe-55 } \\
\text { Fe-59 } \\
\text { Co-58 } \\
\text { Co-60 } \\
N i-59 \\
N i-63 \\
\text { Zr-95 }\end{array}$ & $\begin{array}{l}\text { Activity } \\
\text { (Ci/yr) }\end{array}$ & \\
\hline Shipping Site: SNLA & $\begin{array}{l}\text { Total Volume } \\
(\operatorname{m} 3 / y x)\end{array}$ & $1.43 \mathrm{E}+01 \quad \begin{array}{c}\text { Total Mass } \\
(\mathrm{kg} / \mathrm{yr})\end{array}$ & $1.15 E+05$ \\
\hline & $\begin{array}{l}\text { Radionuclide } \\
\mathrm{Cr}-51 \\
\mathrm{Mn}-54 \\
\mathrm{Fe}-55 \\
\mathrm{Fe}-59 \\
\mathrm{Co}-58 \\
\mathrm{Co}-60 \\
\mathrm{Ni}-59 \\
\mathrm{Ni}-63 \\
\mathrm{Zr}-95\end{array}$ & $\begin{array}{c}\begin{array}{c}\text { Activity } \\
\text { (Ci/y工) }\end{array} \\
6.54 \mathrm{E}-04 \\
1.24 \mathrm{E}-03 \\
4.64 \mathrm{E}-03 \\
4.20 \mathrm{E}-05 \\
1.67 \mathrm{E}-03 \\
1.31 \mathrm{E}-02 \\
6.00 \mathrm{E}-05 \\
8.53 \mathrm{E}-03 \\
5.10 \mathrm{E}-05\end{array}$ & \\
\hline
\end{tabular}

Destination: LANL

\begin{tabular}{|c|c|c|c|}
\hline Shipping Site: INEL & $\begin{array}{l}\text { Total Volume } \\
\text { (m3/yr) }\end{array}$ & $\begin{array}{c}\text { Total Mass } \\
\text { (kg/yr) }\end{array}$ & $8.57 E+06$ \\
\hline & \multicolumn{3}{|c|}{$\begin{array}{l}\text { Activity } \\
(\mathrm{C} i / \mathrm{Y} x)\end{array}$} \\
\hline & $\begin{array}{l}\mathrm{Cr}-51 \\
\mathrm{Mn}-54 \\
\mathrm{Fe}-55 \\
\mathrm{Fe}-59 \\
\mathrm{Co}-58 \\
\mathrm{Co}-60 \\
\mathrm{Ni}-59 \\
\mathrm{Ni}-63 \\
\mathrm{Zr}-95\end{array}$ & $\begin{array}{l}2.42 \mathrm{~A}+01 \\
4.62 \mathrm{E}+01 \\
1.72 \mathrm{E}+02 \\
1.55 \mathrm{0}+00 \\
6.20 \mathrm{E}+01 \\
4.86 \mathrm{E}+02 \\
2.22 \mathrm{E}+00 \\
3.16 \mathrm{D}+02 \\
1.89 \mathrm{E}+00\end{array}$ & \\
\hline
\end{tabular}




\subsubsection{Vehicle-Related Impacts: Nonradiological}

In addition to the radiological risks posed by transportation-related activities, risks are also assessed for vehicle-related causes for the same routes. These risks are independent of the radioactive nature of the cargo and would be incurred for similar shipments of any commodity. The vehiclerelated risks are assessed for both routine conditions and accidents. Vehicle-related risks during routine transportation are caused by potential exposure to increased vehicular exhaust emissions. The routine risks are primarily associated with travel in urban environments. The vehicle-related accident risk refers to the potential for transportation-related accidents that result in fatalities due to physical trauma and not related to the cargo in the shipment. State-specific rates for transportation-related fatalities are used in the assessment. Vehicle-related risks are presented in terms of estimated fatalities for each alternative.

\subsubsection{Transportation Modes}

Although LLW could be transported by a variety of modes, all shipments have been assumed to take place by either truck or rail. For each alternative, risks have been calculated separately for all truck and all rail options, although the actual shipping campaigns for a selected alternative may involve a combination of the two modes. Rail shipments are assumed to take place by regular freight-train service; the use of special or dedicated rail service was not considered. Shipments by barge, although feasible for some sites, have not been explicitly considered because this mode of transportation is somewhat limited and has not been established as a major programmatic option for the WM PEIS assessment. Similarly, shipments by aircraft and other modes was not considered.

\subsubsection{Receptors}

In this report, transportation-related risks are calculated and presented separately for workers and members of the general public. The workers considered are truck and rail crew members involved in transporting LLW. The general public includes all persons who could be exposed to a shipment while it is moving or stopped en route. Potential risks are estimated for the collective populations of exposed people, as well as for maximally exposed individuals (MEIs). The collective population risk is a measure of the radiological risk posed to society as a whole by the alternative being considered. As such, the collective population risk is used as the primary means of comparing various alternatives. 


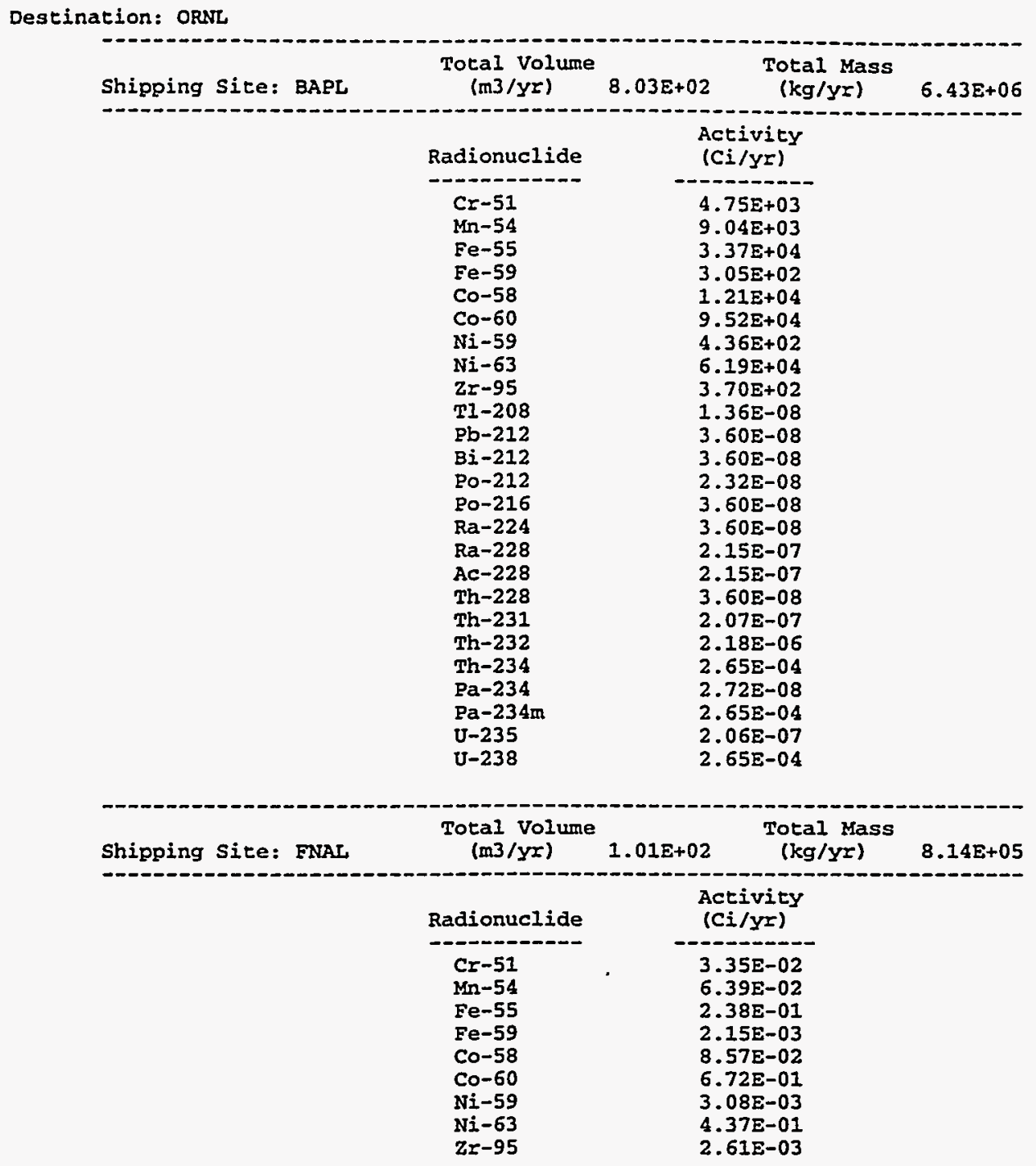


A-III

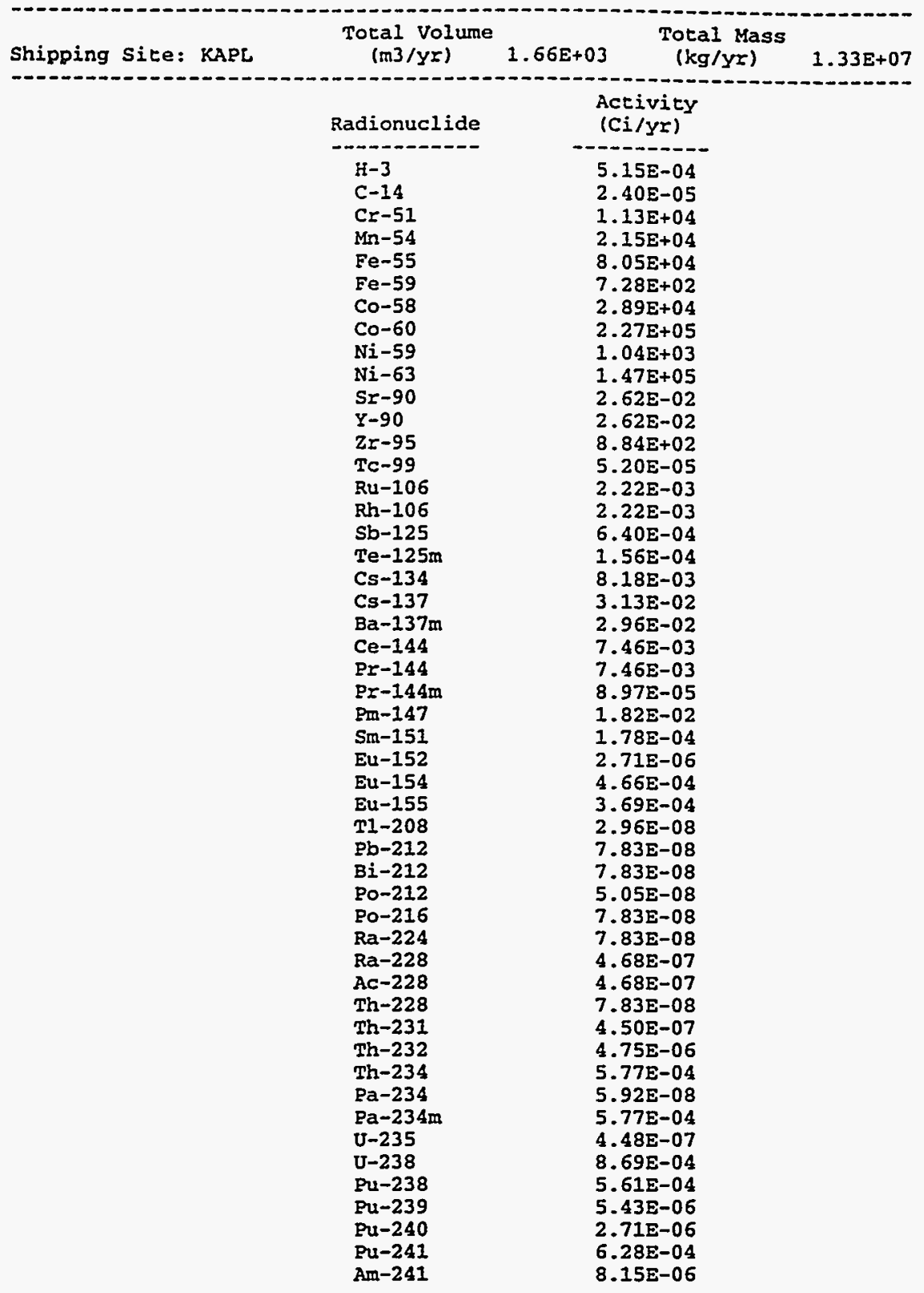




\section{A.15 WM LLW REGIONALIZED 6 ALTERNATIVE (CASE 5): HETEROGENEOUS SOLIDS}

Transported Material with Radionuclide Profile

Alternative: \#5 Minimum Treat -- On-Site -- 2 Sice (SRS, HANF) Disposal

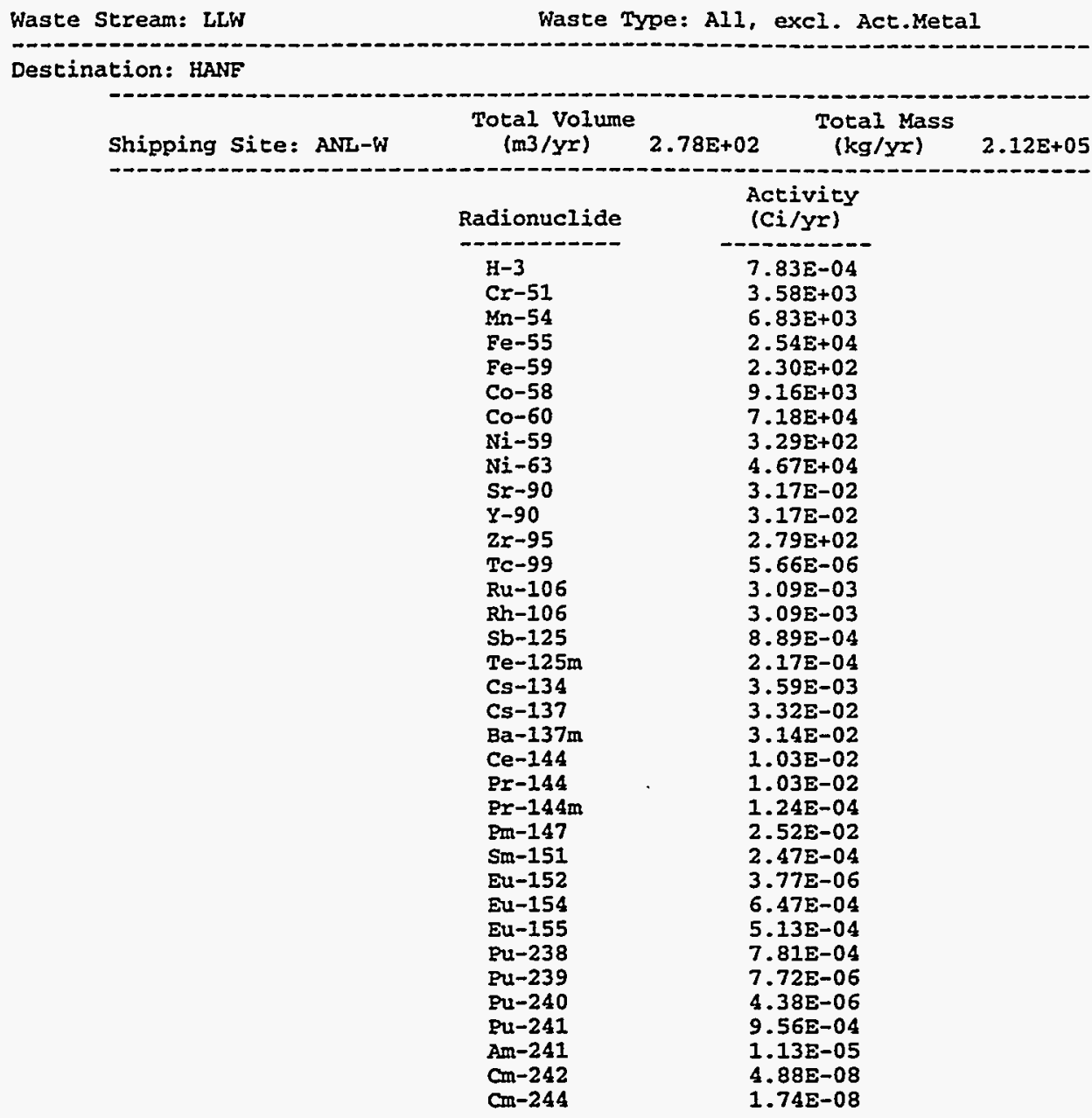


A- 113

\begin{tabular}{|c|c|c|c|}
\hline Shipping Site: INEL & $\begin{array}{l}\text { Total Volume } \\
\text { (m3/yr) }\end{array}$ & $\begin{array}{c}\text { Total Mass } \\
(\mathrm{kg} / \mathrm{yr})\end{array}$ & $1.64 E+07$ \\
\hline & $\begin{array}{l}\text { Radionuclide } \\
\mathrm{H}-3 \\
\mathrm{Cr}-51 \\
\mathrm{Mn}-54 \\
\text { Fe-55 } \\
\text { Ee-59 } \\
\mathrm{Co-58} \\
\mathrm{Co-60} \\
\mathrm{Ni}-59 \\
\mathrm{Ni}-63 \\
\mathrm{Sr}-90 \\
\mathrm{Y}-90 \\
\mathrm{Zr}-95 \\
\mathrm{Tc}-99 \\
\mathrm{Ru}-106 \\
\mathrm{Rh}-106 \\
\mathrm{Sb}-125 \\
\mathrm{Te}-125 \mathrm{~m} \\
\mathrm{Cs}-134 \\
\mathrm{Cs}-137 \\
\mathrm{Ba}-137 \mathrm{~m} \\
\mathrm{Ce}-144 \\
\mathrm{Pr}-144 \\
\mathrm{Pr}-144 \mathrm{~m} \\
\mathrm{Pm}-147 \\
\mathrm{Sm}-151 \\
\text { Eu-152 } \\
\text { Eu-154 } \\
\text { Eu-155 } \\
\mathrm{Pu}-238 \\
\mathrm{Pu}-239 \\
\mathrm{Pu}-240 \\
\mathrm{Pu}-241 \\
\mathrm{Am}-241\end{array}$ & 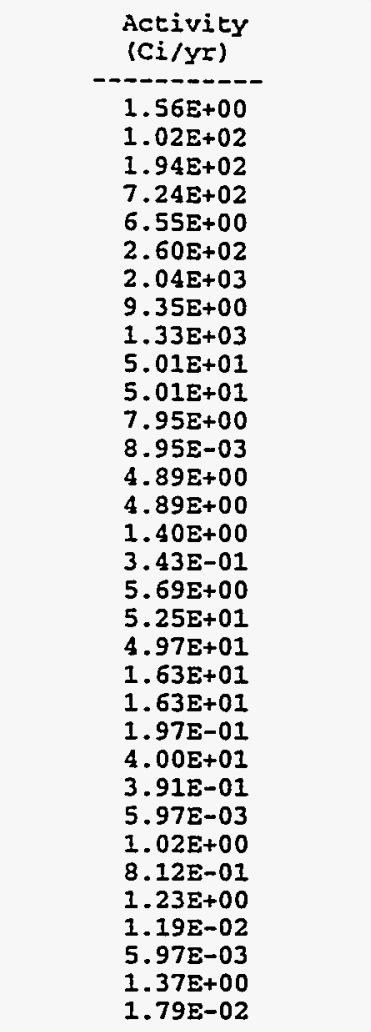 & \\
\hline \multirow[t]{2}{*}{ Shipping Site: ITRI } & $\begin{array}{l}\text { Total Volume } \\
(\mathrm{m} 3 / \mathrm{yr})\end{array}$ & $\begin{array}{c}\text { Total Mass } \\
(\mathrm{kg} / \mathrm{yr})\end{array}$ & $1.64 \mathrm{E}+05$ \\
\hline & $\begin{array}{l}\text { Radionuclide } \\
\mathrm{H}-3 \\
\mathrm{C}-14 \\
\mathrm{Mn}-54 \\
\mathrm{Co}-58 \\
\mathrm{Co}-60 \\
\mathrm{Sr}-90 \\
\mathrm{Y}-90 \\
\mathrm{TC}-99 \\
\mathrm{Cs}-134 \\
\mathrm{Cs}-137 \\
\mathrm{Ba}-137 \mathfrak{m} \\
\mathrm{U}-238\end{array}$ & $\begin{array}{c}\begin{array}{c}\text { Activity } \\
\text { (Ci/yr) }\end{array} \\
-6.17 E+00 \\
3.03 E-01 \\
3.41 E+01 \\
3.15 E+01 \\
9.12 E+01 \\
4.28 E+01 \\
4.28 E+01 \\
6.07 E-01 \\
7.07 E+01 \\
9.33 E+01 \\
8.82 E+01 \\
3.69 E+00\end{array}$ & \\
\hline
\end{tabular}




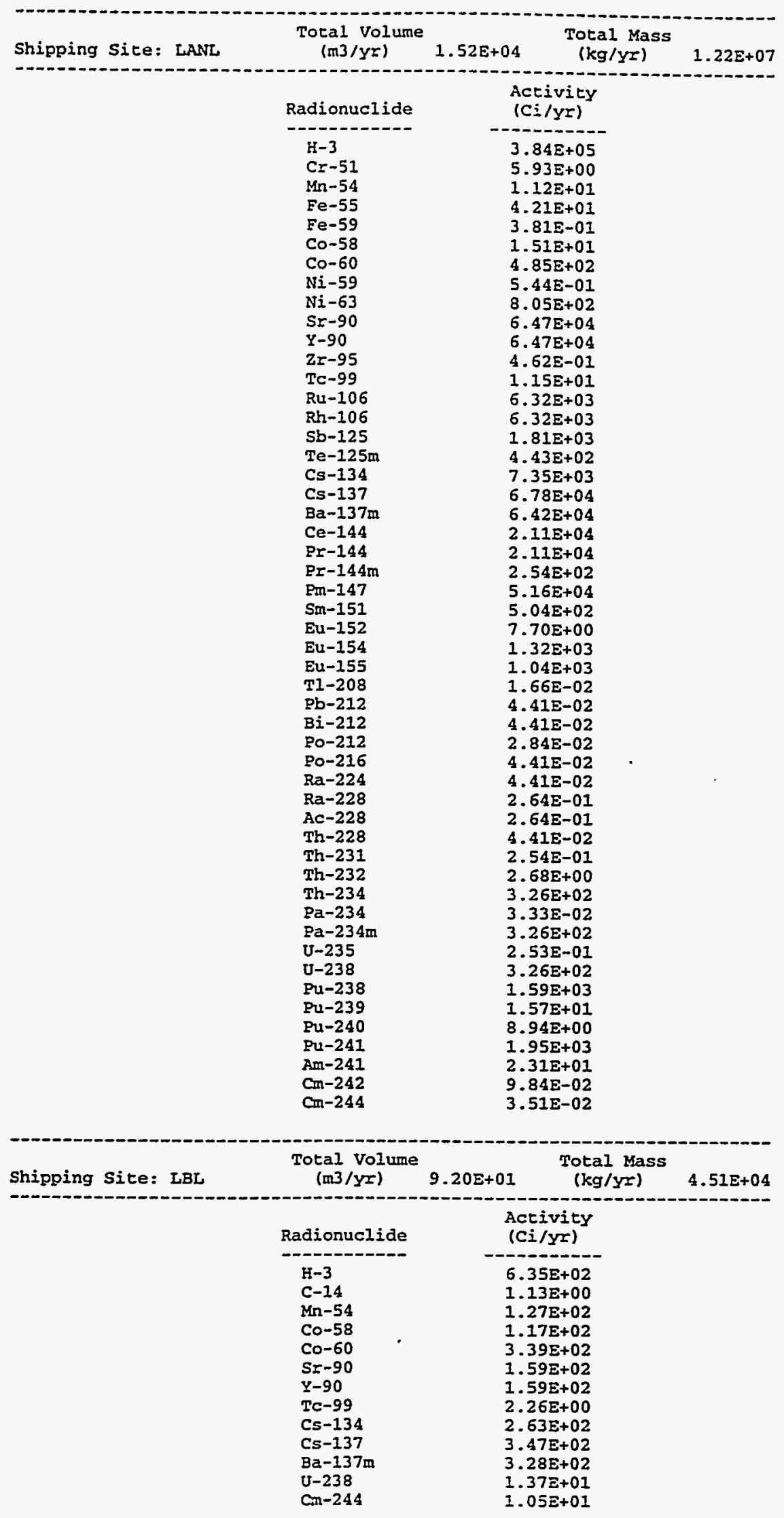




\begin{tabular}{|c|c|c|c|}
\hline Shipping Sice: LLNL & $\begin{array}{l}\text { Total Volume } \\
(\mathrm{m} 3 / \mathrm{yr})\end{array}$ & $\begin{array}{c}\text { Total Mass } \\
\left(\mathrm{kg} / \mathrm{Y}^{\prime}\right)\end{array}$ & $1.06 \Xi+06$ \\
\hline & \begin{tabular}{l} 
Radionuclide \\
\hdashline $\mathrm{H}-3$ \\
$\mathrm{C}-14$ \\
$\mathrm{Mn}-54$ \\
$\mathrm{Co}-58$ \\
$\mathrm{Co}-60$ \\
$\mathrm{Ni}-63$ \\
$\mathrm{Sr}-90$ \\
$\mathrm{Y}-90$ \\
$\mathrm{Tc}-99$ \\
$\mathrm{Ru}-106$ \\
$\mathrm{Rh}-106$ \\
$\mathrm{Sb}-125$ \\
$\mathrm{Te}-125 \mathrm{~m}$ \\
$\mathrm{Cs}-134$ \\
$\mathrm{Cs}-137$ \\
$\mathrm{Ba}-137 \mathrm{~m}$ \\
$\mathrm{Ce}-144$ \\
$\mathrm{Pr}-144$ \\
$\mathrm{Pr}-144 \mathrm{~m}$ \\
$\mathrm{Pm}-147$ \\
$\mathrm{Sm}-151$ \\
$\mathrm{Eu}-152$ \\
$\mathrm{Eu}-154$ \\
$\mathrm{Eu}-155$ \\
$\mathrm{U}-238$ \\
$\mathrm{Pu}-238$ \\
$\mathrm{Pu}-239$ \\
$\mathrm{Pu}-240$ \\
$\mathrm{Pu}-241$ \\
$\mathrm{Am}-241$ \\
$\mathrm{Cm}-242$ \\
$\mathrm{Cm}-244$
\end{tabular} & $\begin{array}{c}\begin{array}{c}\text { Activity } \\
(C i / y r)\end{array} \\
3.33 \mathrm{E}+04 \\
1.55 \mathrm{E}-03 \\
1.74 \mathrm{E}-01 \\
1.61 \mathrm{E}-01 \\
4.69 \mathrm{E}-01 \\
4.89 \mathrm{E}-03 \\
6.54 \mathrm{E}-01 \\
6.54 \mathrm{E}-01 \\
3.18 \mathrm{E}-03 \\
4.24 \mathrm{E}-02 \\
4.24 \mathrm{E}-02 \\
1.21 \mathrm{E}-02 \\
2.97 \mathrm{E}-03 \\
4.11 \mathrm{E}-01 \\
9.33 \mathrm{E}-01 \\
8.82 \mathrm{E}-01 \\
1.42 \mathrm{E}-01 \\
1.42 \mathrm{E}-01 \\
1.70 \mathrm{E}-03 \\
3.46 \mathrm{E}-01 \\
3.39 \mathrm{E}-03 \\
5.17 \mathrm{E}-05 \\
8.87 \mathrm{E}-03 \\
7.03 \mathrm{E}-03 \\
1.88 \mathrm{E}-02 \\
7.85 \mathrm{E}-02 \\
5.28 \mathrm{E}-03 \\
1.81 \mathrm{E}-02 \\
2.50 \mathrm{E}+00 \\
2.58 \mathrm{E}-04 \\
1.44 \mathrm{E}-03 \\
5.17 \mathrm{E}-04\end{array}$ & \\
\hline \multirow[t]{2}{*}{ Shipping Site: PANT } & $\begin{array}{l}\text { Total volume } \\
\text { (m3/yr) }\end{array}$ & $\begin{array}{l}\text { Total Mass } \\
(\mathrm{kg} / \mathrm{y} 工)\end{array}$ & $2.88 \mathrm{E}+07$ \\
\hline & $\begin{array}{l}\text { Radionuclide } \\
\mathrm{H}-3 \\
\mathrm{Tl}-208 \\
\mathrm{~Pb}-212 \\
\mathrm{Bi}-212 \\
\mathrm{Po}-212 \\
\mathrm{PO}-216 \\
\mathrm{Ra}-224 \\
\mathrm{Ra}-228 \\
\mathrm{Ac}-228 \\
\mathrm{Th}-228 \\
\mathrm{Th}-231 \\
\mathrm{Th}-232 \\
\mathrm{Th}-234 \\
\mathrm{~Pa}-234 \\
\mathrm{~Pa}-234 \mathrm{~m} \\
\mathrm{U}-235 \\
\mathrm{U}-238\end{array}$ & $\begin{array}{c}\begin{array}{c}\text { Activity } \\
(C i / Y r)\end{array} \\
2.34 \mathrm{E}+02 \\
2.21 \mathrm{E}-04 \\
5.86 \mathrm{E}-04 \\
5.86 \mathrm{E}-04 \\
3.78 \mathrm{E}-04 \\
5.86 \mathrm{E}-04 \\
5.86 \mathrm{E}-04 \\
3.50 \mathrm{E}-03 \\
3.50 \mathrm{E}-03 \\
5.86 \mathrm{E}-04 \\
3.37 \mathrm{E}-03 \\
3.56 \mathrm{E}-02 \\
4.32 \mathrm{E}+00 \\
4.43 \mathrm{E}-04 \\
4.32 \mathrm{E}+00 \\
3.36 \mathrm{E}-03 \\
4.32 \mathrm{E}+00\end{array}$ & \\
\hline
\end{tabular}


A- 116

\begin{tabular}{|c|c|c|c|}
\hline Shipping Site: RFP & $\begin{array}{l}\text { Total Volume } \\
(\mathrm{m} 3 / \mathrm{Yr})\end{array}$ & $\begin{array}{l}\text { Total Mass } \\
(\mathrm{kg} / \mathrm{yr})\end{array}$ & $6.49 E+06$ \\
\hline & $\begin{array}{l}\text { Radionuclide } \\
\mathrm{T} 1-208 \\
\mathrm{~Pb}-212 \\
\mathrm{Bi}-212 \\
\mathrm{Po}-212 \\
\mathrm{Po}-216 \\
\mathrm{Ra}-224 \\
\mathrm{Ra}-228 \\
\mathrm{Ac}-228 \\
\mathrm{Th}-228 \\
\mathrm{Th}-231 \\
\mathrm{Th}-232 \\
\mathrm{Th}-234 \\
\mathrm{~Pa}-234 \\
\mathrm{~Pa}-234 \mathrm{~m} \\
\mathrm{U}-235 \\
\mathrm{U}-238 \\
\mathrm{Pu}-238 \\
\mathrm{Pu}-239 \\
\mathrm{Pu}-240 \\
\mathrm{Pu}-241 \\
\mathrm{Am}-241 \\
\mathrm{Cm}-242 \\
\mathrm{Cm}-244\end{array}$ & \begin{tabular}{c} 
Activity \\
(Ci/YI) \\
\hdashline $3.60 E-05$ \\
$9.55 E-05$ \\
$9.55 E-05$ \\
$6.15 E-05$ \\
$9.55 E-05$ \\
$9.55 E-05$ \\
$5.70 E-04$ \\
$5.70 E-04$ \\
$9.55 E-05$ \\
$5.49 E-04$ \\
$5.79 E-03$ \\
$7.04 E-01$ \\
$7.21 E-05$ \\
$7.04 E-01$ \\
$5.47 E-04$ \\
$7.04 E-01$ \\
$5.56 E-01$ \\
$4.24 E-02$ \\
$1.48 E-01$ \\
$2.04 E+01$ \\
$8.48 E-04$ \\
$1.18 E-02$ \\
$4.24 E-03$
\end{tabular} & \\
\hline Shipping Site: SLAC & $\begin{array}{l}\text { Total volume } \\
(\mathrm{m} 3 / \mathrm{yr})\end{array}$ & $2.73 \mathrm{E}+02 \quad \begin{array}{c}\text { Total Mass } \\
(\mathrm{kg} / \mathrm{yr})\end{array}$ & $7.45 E+05$ \\
\hline & $\begin{array}{l}\text { Radionuclide } \\
\mathrm{Cr}-51 \\
\mathrm{Mn}-54 \\
\mathrm{Fe}-55 \\
\mathrm{Fe}-59 \\
\mathrm{Co}-58 \\
\mathrm{Co}-60 \\
\mathrm{Ni}-59 \\
\mathrm{Ni}-63 \\
\mathrm{Z}-95\end{array}$ & $\begin{array}{c}\text { Activity } \\
(C i / y x) \\
3.86 \mathrm{E}-03 \\
7.36 \mathrm{E}-03 \\
2.74 \mathrm{E}-02 \\
2.48 \mathrm{E}-04 \\
9.88 \mathrm{E}-03 \\
7.74 \mathrm{E}-02 \\
3.54 \mathrm{E}-04 \\
5.04 \mathrm{E}-02 \\
3.01 \mathrm{E}-04\end{array}$ & \\
\hline
\end{tabular}


$A-117$

\begin{tabular}{|c|c|c|c|}
\hline Shipping Site: SNLA & $\begin{array}{l}\text { Total Volume } \\
(\mathrm{m} 3 / \mathrm{yr})\end{array}$ & $\begin{array}{l}\text { Total Mass } \\
(\mathrm{kg} / \mathrm{yr})\end{array}$ & $3.28 \mathrm{E}+05$ \\
\hline • & $\begin{array}{l}\text { Radionuclide } \\
\mathrm{H}-3 \\
\mathrm{Co}-60 \\
\mathrm{Ni}-63 \\
\mathrm{Sr}-90 \\
\mathrm{Y}-90 \\
\mathrm{Tc}-99 \\
\mathrm{Ru}-106 \\
\mathrm{Rh}-106 \\
\mathrm{Sb}-125 \\
\mathrm{Te}-125 \mathrm{~m} \\
\mathrm{Cs}-134 \\
\mathrm{Cs}-137 \\
\mathrm{Ba}-137 \mathrm{~m} \\
\mathrm{Ce}-144 \\
\mathrm{Pr}-144 \\
\mathrm{Pr}-144 \mathrm{~m} \\
\mathrm{Pm}-147 \\
\mathrm{Sm}-151 \\
\mathrm{Eu}-152 \\
\mathrm{Eu}-154 \\
\mathrm{Eu}-155 \\
\mathrm{~T} 1-208 \\
\mathrm{~Pb}-212 \\
\mathrm{Bi}-212 \\
\mathrm{PO}-212 \\
\mathrm{Po}-216 \\
\mathrm{Ra}-224 \\
\mathrm{Ra}-228 \\
\mathrm{Ac}-228 \\
\mathrm{Th}-228 \\
\mathrm{Th}-231 \\
\mathrm{Th}-232 \\
\mathrm{Th}-234 \\
\mathrm{~Pa}-234 \\
\mathrm{~Pa}-234 \mathrm{~m} \\
\mathrm{U}-235 \\
\mathrm{U}-238 \\
\mathrm{Pu}-238 \\
\mathrm{Pu}-239 \\
\mathrm{Pu}-240 \\
\mathrm{Pu}-241 \\
\mathrm{Am}-241 \\
\mathrm{Cm}-242 \\
\mathrm{Cm}-244 \\
\end{array}$ & \multicolumn{2}{|l|}{$\begin{array}{l}3.00 \mathrm{E}+00 \\
2.85 \mathrm{E}-05 \\
5.67 \mathrm{E}-05 \\
5.04 \mathrm{E}-03 \\
5.04 \mathrm{E}-03 \\
9.00 \mathrm{E}-07 \\
4.92 \mathrm{E}-04 \\
4.92 \mathrm{E}-04 \\
1.41 \mathrm{E}-04 \\
3.45 \mathrm{E}-05 \\
5.72 \mathrm{E}-04 \\
5.28 \mathrm{E}-03 \\
4.99 \mathrm{E}-03 \\
1.64 \mathrm{E}-03 \\
1.64 \mathrm{E}-03 \\
1.98 \mathrm{E}-05 \\
4.02 \mathrm{E}-03 \\
3.93 \mathrm{E}-05 \\
6.00 \mathrm{E}-07 \\
1.02 \mathrm{E}-04 \\
8.16 \mathrm{E}-05 \\
5.10 \mathrm{E}-06 \\
1.35 \mathrm{E}-05 \\
1.35 \mathrm{E}-05 \\
8.70 \mathrm{E}-06 \\
1.35 \mathrm{E}-05 \\
1.35 \mathrm{E}-05 \\
8.07 \mathrm{E}-05 \\
8.07 \mathrm{E}-05 \\
1.35 \mathrm{E}-05 \\
7.77 \mathrm{E}-05 \\
8.19 \mathrm{E}-04 \\
9.96 \mathrm{E}-02 \\
1.02 \mathrm{E}-05 \\
9.96 \mathrm{E}-02 \\
7.74 \mathrm{E}-05 \\
9.96 \mathrm{E}-02 \\
7.87 \mathrm{E}-02 \\
6.00 \mathrm{E}-03 \\
2.10 \mathrm{E}-02 \\
2.89 \mathrm{E}+00 \\
1.21 \mathrm{E}-04 \\
1.68 \mathrm{E}-03 \\
6.00 \mathrm{E}-04\end{array}$} \\
\hline Shipping Site: SNLL & $\begin{array}{l}\text { Total Volume } \\
\text { (m3/yr) }\end{array}$ & $\begin{array}{cc}\text { 3.68E }+01 & \begin{array}{c}\text { Total Mass } \\
(\mathrm{kg} / \mathrm{yr})\end{array}\end{array}$ & \\
\hline$\cdot$ & $\begin{array}{l}\text { Radionuclide } \\
\mathrm{H}-3 \\
\mathrm{Fe}-55 \\
\mathrm{Co}-60 \\
\mathrm{Tl}-208 \\
\mathrm{~Pb}-212 \\
\mathrm{Bi}-212 \\
\mathrm{Po}-212 \\
\mathrm{Po-216} \\
\mathrm{Ra-224} \\
\mathrm{Ra}-228 \\
\mathrm{Ac}-228 \\
\mathrm{Th}-228 \\
\mathrm{Th}-231 \\
\mathrm{Th}-232 \\
\mathrm{Th}-234 \\
\mathrm{~Pa}-234 \\
\mathrm{~Pa}-234 \mathrm{~m} \\
\mathrm{U}-235 \\
\mathrm{U}-238\end{array}$ & \begin{tabular}{c}
$\begin{array}{c}\text { Activity } \\
\text { (Ci/yI) }\end{array}$ \\
\hdashline $2.78 E+04$ \\
$3.15 E-01$ \\
$3.15 E-01$ \\
$6.40 E-05$ \\
$1.69 E-04$ \\
$1.69 E-04$ \\
$1.09 E-04$ \\
$1.69 E-04$ \\
$1.69 E-04$ \\
$1.01 E-03$ \\
$1.01 E-03$ \\
$1.69 E-04$ \\
$9.75 E-04$ \\
$1.02 E-02$ \\
$1.25 E+00$ \\
$1.28 E-04$ \\
$1.25 E+00$ \\
$9.71 E-04$ \\
$1.25 E+00$
\end{tabular} & \\
\hline
\end{tabular}


A-118

Destination: SRS

\begin{tabular}{|c|c|c|c|}
\hline Shipping Sice: AMES & $\begin{array}{l}\text { Total Volume } \\
(\mathrm{m} 3 / \mathrm{y} r)\end{array}$ & $\begin{array}{c}\text { Total Mass } \\
(\mathrm{kg} / \mathrm{yr})\end{array}$ & $2.99 \Xi+04$ \\
\hline & $\begin{array}{l}\text { Radionuclide } \\
\mathrm{Tl}-208 \\
\mathrm{~Pb}-212 \\
\mathrm{Bi}-212 \\
\mathrm{Po}-212 \\
\mathrm{Po-216} \\
\mathrm{Ra}-224 \\
\mathrm{Ra}-228 \\
\mathrm{Ac}-228 \\
\mathrm{Th}-228 \\
\mathrm{Th}-231 \\
\mathrm{Th}-232 \\
\mathrm{Th}-234 \\
\mathrm{~Pa}-234 \\
\mathrm{~Pa}-234 \mathrm{~m} \\
\mathrm{U}-235 \\
\mathrm{U}-238 \\
\mathrm{Pu}-238 \\
\mathrm{Pu}-239 \\
\mathrm{Pu}-240 \\
\mathrm{Pu}-241 \\
\mathrm{Am}-241 \\
\mathrm{Cm}-242 \\
\mathrm{Cm}-244\end{array}$ & $\begin{array}{c}\text { Activity } \\
\text { (Ci/yI) } \\
4.50 E-07 \\
1.19 E-06 \\
1.19 E-06 \\
7.68 E-07 \\
1.19 E-06 \\
1.19 E-06 \\
7.12 \mathrm{E}-06 \\
7.12 \mathrm{E}-06 \\
1.19 \mathrm{E}-06 \\
6.86 \mathrm{E}-06 \\
7.23 \mathrm{E}-05 \\
8.79 \mathrm{E}-03 \\
9.01 \mathrm{E}-07 \\
8.79 \mathrm{E}-03 \\
6.83 \mathrm{E}-06 \\
8.79 \mathrm{E}-03 \\
6.94 \mathrm{E}-04 \\
5.30 \mathrm{E}-05 \\
1.85 \mathrm{E}-04 \\
2.55 \mathrm{E}-02 \\
1.06 \mathrm{E}-06 \\
1.48 \mathrm{E}-05 \\
5.30 \mathrm{E}-06\end{array}$ & \\
\hline Shipping Site: ANL-E & $\begin{array}{l}\text { Total Volume } \\
(\mathrm{m} 3 / \mathrm{Yr})\end{array}$ & $\begin{array}{cc}7.35 \mathrm{E}+02 & \begin{array}{c}\text { Total Mass } \\
(\mathrm{kg} / \mathrm{Y})\end{array}\end{array}$ & $2.00 \mathrm{E}+06$ \\
\hline & $\begin{array}{l}\text { Radionuclide } \\
\mathrm{H}-3 \\
\mathrm{C}-14 \\
\mathrm{Cr}-51 \\
\mathrm{Mn}-54 \\
\mathrm{Fe}-55 \\
\mathrm{Fe}-59 \\
\mathrm{C}-58 \\
\mathrm{C}-50 \\
\mathrm{~N}-60 \\
\mathrm{~N}-59 \\
\mathrm{~S}-63 \\
\mathrm{Y}-90 \\
\mathrm{Z}-90 \\
\mathrm{Tc}-95 \\
\mathrm{Ru}-106 \\
\mathrm{Rh}-106 \\
\mathrm{Sb}-125 \\
\mathrm{Te}-125 \mathrm{~m} \\
\mathrm{Cs}-134 \\
\mathrm{Cs}-137 \\
\mathrm{Ba}-137 \mathrm{~m} \\
\mathrm{Ce}-144 \\
\mathrm{Pr}-144 \\
\mathrm{Pr}-144 \mathrm{~m} \\
\mathrm{Pm}-147 \\
\mathrm{Sm}-151 \\
\mathrm{Eu}-152 \\
\mathrm{Eu}-154 \\
\mathrm{Eu}-155 \\
\mathrm{U}-238 \\
\mathrm{Pu}-238 \\
\mathrm{Pu}-239 \\
\mathrm{Pu}-240 \\
\mathrm{Qu}-241 \\
\mathrm{Am}-241\end{array}$ & \begin{tabular}{c} 
Activity \\
$(C i / y r)$ \\
\hdashline $1.33 E+02$ \\
$7.91 E-02$ \\
$2.87 \mathrm{E}+00$ \\
$1.43 \mathrm{E}+01$ \\
$2.04 \mathrm{E}+01$ \\
$1.84 \mathrm{E}-01$ \\
$1.55 \mathrm{E}+01$ \\
$8.15 \mathrm{E}+01$ \\
$2.63 \mathrm{E}-01$ \\
$3.77 \mathrm{E}+01$ \\
$3.33 \mathrm{E}+01$ \\
$3.33 \mathrm{E}+01$ \\
$2.24 \mathrm{E}-01$ \\
$1.62 \mathrm{E}-01$ \\
$2.16 \mathrm{E}+00$ \\
$2.16 \mathrm{E}+00$ \\
$6.21 \mathrm{E}-01$ \\
$1.51 \mathrm{E}-01$ \\
$2.09 \mathrm{E}+01$ \\
$4.75 \mathrm{E}+01$ \\
$4.50 \mathrm{E}+01$ \\
$7.24 \mathrm{E}+00$ \\
$7.24 \mathrm{E}+00$ \\
$8.70 \mathrm{E}-02$ \\
$1.76 \mathrm{E}+01$ \\
$1.72 \mathrm{E}-01$ \\
$2.63 \mathrm{E}-03$ \\
$4.52 \mathrm{E}-01$ \\
$3.58 \mathrm{E}-01$ \\
$9.63 \mathrm{E}-01$ \\
$5.44 \mathrm{E}-01$ \\
$5.27 \mathrm{E}-03$ \\
$2.63 \mathrm{E}-03$ \\
$6.09 \mathrm{E}-01$ \\
$7.91 \mathrm{E}-03$
\end{tabular} & \\
\hline
\end{tabular}




\begin{tabular}{|c|c|c|c|}
\hline Shipping Sice: BAPL & $\begin{array}{l}\text { Total Volume } \\
\text { (m3/yr) }\end{array}$ & $\begin{array}{cc}\text { Toral Mass } \\
(\mathrm{kg} / \mathrm{yr})\end{array}$ & $1.08 \mathrm{E}+06$ \\
\hline & $\begin{array}{l}\text { Radionuclide } \\
\mathrm{H}-3 \\
\mathrm{Co}-60 \\
\mathrm{Ni}-63 \\
\mathrm{Sr}-90 \\
\mathrm{Y}-90 \\
\mathrm{Tc}-99 \\
\mathrm{Ru}-106 \\
\mathrm{Rh}-106 \\
\mathrm{Sb}-125 \\
\mathrm{Te}-125 \mathrm{~m} \\
\mathrm{Cs}-134 \\
\mathrm{Cs}-137 \\
\mathrm{Ba}-137 \mathrm{~m} \\
\mathrm{Ce}-144 \\
\mathrm{Pr}-144 \\
\mathrm{Pr}-144 \mathrm{~m} \\
\mathrm{Pm}-147 \\
\mathrm{Sm}-151 \\
\mathrm{Eu}-152 \\
\mathrm{Eu}-154 \\
\mathrm{Eu}-155 \\
\mathrm{~T} \mathrm{l}-208 \\
\mathrm{~Pb}-212 \\
\mathrm{Bi}-212 \\
\mathrm{Po}-212 \\
\mathrm{Po}-216 \\
\mathrm{Ra}-224 \\
\mathrm{Ra}-228 \\
\mathrm{Ac}-228 \\
\mathrm{Th}-228 \\
\mathrm{Th}-231 \\
\mathrm{Th}-232 \\
\mathrm{Th}-234 \\
\mathrm{~Pa}-234 \\
\mathrm{~Pa}-234 \mathrm{~m} \\
\mathrm{U}-235 \\
\mathrm{U}-238 \\
\mathrm{Pu}-238 \\
\mathrm{Pu}-239 \\
\mathrm{Pu}-240 \\
\mathrm{Pu}-241 \\
\mathrm{Am}-241 \\
\end{array}$ & $\begin{array}{l}\text { Activity } \\
\text { (Ci/Yr) } \\
-2.51 \mathrm{E}-03 \\
1.19 \mathrm{E}-02 \\
2.38 \mathrm{E}-02 \\
2.11 \mathrm{E}+00 \\
2.11 \mathrm{E}+00 \\
3.77 \mathrm{E}-04 \\
2.06 \mathrm{E}-01 \\
2.06 \mathrm{E}-01 \\
5.93 \mathrm{E}-02 \\
1.44 \mathrm{E}-02 \\
2.40 \mathrm{E}-01 \\
2.21 \mathrm{E}+00 \\
2.09 \mathrm{E}+00 \\
6.91 \mathrm{E}-01 \\
6.91 \mathrm{E}-01 \\
8.31 \mathrm{E}-03 \\
1.68 \mathrm{E}+00 \\
1.65 \mathrm{E}-02 \\
2.51 \mathrm{E}-04 \\
4.32 \mathrm{E}-02 \\
3.42 \mathrm{E}-02 \\
3.80 \mathrm{E}-06 \\
1.00 \mathrm{E}-05 \\
1.00 \mathrm{E}-05 \\
6.49 \mathrm{E}-06 \\
1.00 \mathrm{E}-05 \\
1.00 \mathrm{E}-05 \\
6.02 \mathrm{E}-05 \\
6.02 \mathrm{E}-05 \\
1.00 \mathrm{E}-05 \\
5.80 \mathrm{E}-05 \\
6.11 \mathrm{E}-04 \\
7.43 \mathrm{E}-02 \\
7.61 \mathrm{E}-06 \\
7.43 \mathrm{E}-02 \\
5.77 \mathrm{E}-05 \\
7.43 \mathrm{E}-02 \\
5.20 \mathrm{E}-02 \\
5.03 \mathrm{E}-04 \\
2.51 \mathrm{E}-04 \\
5.81 \mathrm{E}-02 \\
7.55 \mathrm{E}-04\end{array}$ & - \\
\hline Shipping Site: ENAI & $\begin{array}{l}\text { Total Volume } \\
\text { (m3/yr) }\end{array}$ & $\begin{array}{cc}\text { Total Mass } \\
\left.\text { (kg/y } / \mathrm{I}^{2}\right)\end{array}$ & $5.11 E+04$ \\
\hline & $\begin{array}{l}\text { Radionuclide } \\
\text { H-3 } \\
\text { Cr-51 } \\
\mathrm{Mn}-54 \\
\text { Fe-55 } \\
\text { Fe-59 } \\
\mathrm{Co}-58 \\
\mathrm{CO}-60 \\
\mathrm{Ni}-59 \\
\mathrm{Ni}-63 \\
\mathrm{ZI}-95 \\
\mathrm{Tl}-208 \\
\mathrm{~Pb}-212 \\
\mathrm{Bi}-212 \\
\mathrm{PO}-212 \\
\mathrm{PO}-216 \\
\mathrm{Ra}-224 \\
\mathrm{Ra}-228 \\
\mathrm{Ac}-228 \\
\mathrm{Th}-228 \\
\mathrm{Th}-231 \\
\mathrm{Th}-232 \\
\mathrm{Th}-234 \\
\mathrm{~Pa}-234 \\
\mathrm{~Pa}-234 \mathrm{~m} \\
\mathrm{U}-235 \\
\mathrm{U}-238\end{array}$ & $\begin{array}{l}\text { Activity } \\
(C i / Y r)\end{array}$ & \\
\hline
\end{tabular}




\section{DISCLAIMER}

Portions of this document may be illegible electronic image products. Images are produced from the best available original document. 


\section{METHODS FOR CALCULATING TRANSPORTATION-RELATED RISKS}

The technical approach for conducting the transportation risk assessment was developed following a thorough and critical review of the literature and existing National Environmental Policy Act documentation for federal actions involving transportation of radioactive materials. Consideration was also given to recent DOE commitments arising from both litigation and public awareness. The approach selected uses several computer models and databases to determine the risks for each case.

The approach for the transportation risk assessment is summarized in Figure 3.1 and is discussed in detail in this section. For each case, risks are assessed for both routine transportation and accidents. For the routine assessment, risks are calculated for the collective populations of potentially exposed individuals, as well as for the MEIs. The accident assessment consists of two components: (1) an accident risk assessment, which considers the probabilities and consequences of a range of possible transportation-related accidents, including low-probability accidents that have high consequences and high-probability accidents that have low consequences; and (2) an accident consequence assessment, which considers only the radiological consequences of the severe transportation-related accidents that are postulated to result in the largest releases of radioactive material.

The RADTRAN 4 computer code (Neuhauser and Kanipe 1993) is used for the routine and accident risk assessments to estimate the impacts to collective populations. RADTRAN 4 was developed by SNL-NM to calculate population risks associated with the transportation of radioactive materials by a variety of modes, including truck, rail, air, ship, and barge. The code has been extensively reviewed, updated, and used for transportation risk assessments since it was issued in the late 1970s.

The RADTRAN 4 calculations of population risk take into account both the consequences and the probabilities of potential exposures. The collective population risk is a measure of the total radiological risk posed to society as a whole by the alternative being considered. As such, the collective population risks are used as the primary means of comparing the various cases.

As a complement to the RADTRAN calculations, the RISKIND computer code (Yuan et al. 1993) is used to estimate scenario-specific doses to MEIs for both routine operation and accidents and to estimate population impacts for the accident consequence assessment. The RISKIND computer code was developed for the DOE Office of Civilian Radioactive Waste Management specifically to analyze radiological consequences to individuals and population subgroups associated with the transportation of spent nuclear fuel. Minor modifications to the code were made for WM PEIS applications to accommodate shipments of all types of radioactive waste. 


\begin{tabular}{|c|c|c|c|}
\hline Shipping Site: $K-25$ & $\begin{array}{l}\text { Total Volume } \\
(\mathrm{m} 3 / \mathrm{yr})\end{array}$ & $\begin{array}{l}\text { Total Mass } \\
(\mathrm{kg} / \mathrm{yr})\end{array}$ & $4.67 E+07$ \\
\hline & $\begin{array}{l}\text { Radionuclide } \\
\text { Tl-208 } \\
\text { Pb-212 } \\
\mathrm{Bi}-212 \\
\mathrm{Po-212} \\
\mathrm{Po-216} \\
\mathrm{Ra}-224 \\
\mathrm{Ra}-228 \\
\mathrm{Ac}-228 \\
\mathrm{Th}-228 \\
\mathrm{Th}-231 \\
\mathrm{Th}-232 \\
\mathrm{Th}-234 \\
\mathrm{~Pa}-234 \\
\mathrm{~Pa}-234 \mathrm{~m} \\
\mathrm{U}-235 \\
\mathrm{U}-238\end{array}$ & $\begin{array}{c}\begin{array}{c}\text { Activity } \\
(C i / Y Y)\end{array} \\
1.06 E-04 \\
2.82 E-04 \\
2.82 \mathrm{E}-04 \\
1.82 \mathrm{E}-04 \\
2.82 \mathrm{E}-04 \\
2.82 \mathrm{E}-04 \\
1.68 \mathrm{E}-03 \\
1.68 \mathrm{E}-03 \\
2.82 \mathrm{E}-04 \\
1.62 \mathrm{E}-03 \\
1.71 \mathrm{E}-02 \\
2.08 \mathrm{E}+00 \\
2.13 \mathrm{E}-04 \\
2.08 \mathrm{E}+00 \\
1.62 \mathrm{E}-03 \\
2.08 \mathrm{E}+00\end{array}$ & \\
\hline Shipping Site: RAPL & $\begin{array}{l}\text { Total Volume } \\
\text { (m3/yr) }\end{array}$ & $\begin{array}{cc}\text { Total Mass } \\
(\mathrm{kg} / \mathrm{yr})\end{array}$ & $5.36 E+05$ \\
\hline & 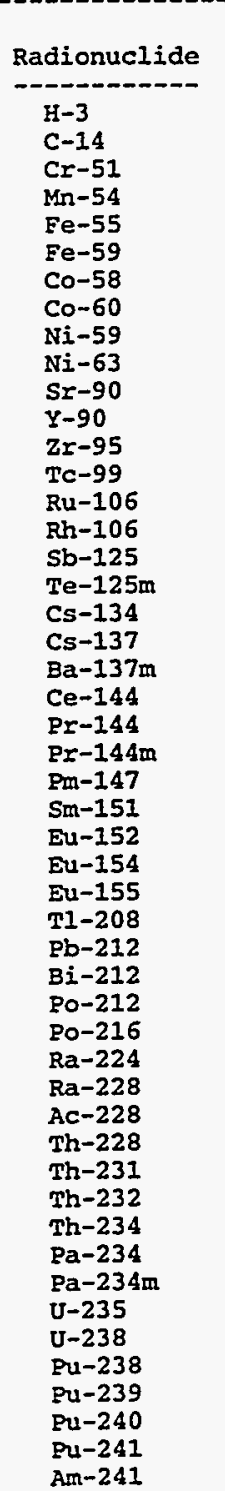 & $\begin{array}{c}\text { Activity } \\
\text { (Ci/yr) } \\
-3.14 \mathrm{E}-04 \\
2.40 \mathrm{E}-05 \\
5.11 \mathrm{E}-03 \\
1.24 \mathrm{E}-02 \\
3.63 \mathrm{E}-02 \\
3.28 \mathrm{E}-04 \\
1.55 \mathrm{E}-02 \\
1.09 \mathrm{E}-01 \\
4.69 \mathrm{E}-04 \\
6.70 \mathrm{E}-02 \\
2.58 \mathrm{E}-02 \\
2.58 \mathrm{E}-02 \\
3.99 \mathrm{E}-04 \\
5.20 \mathrm{E}-05 \\
2.19 \mathrm{E}-03 \\
2.19 \mathrm{E}-03 \\
6.31 \mathrm{E}-04 \\
1.54 \mathrm{E}-04 \\
8.14 \mathrm{E}-03 \\
3.09 \mathrm{E}-02 \\
2.92 \mathrm{E}-02 \\
7.35 \mathrm{E}-03 \\
7.35 \mathrm{E}-03 \\
8.84 \mathrm{E}-05 \\
1.79 \mathrm{E}-02 \\
1.75 \mathrm{E}-04 \\
2.67 \mathrm{E}-06 \\
4.59 \mathrm{E}-04 \\
3.64 \mathrm{E}-04 \\
4.50 \mathrm{E}-09 \\
1.19 \mathrm{E}-08 \\
1.19 \mathrm{E}-08 \\
7.60 \mathrm{E}-09 \\
1.19 \mathrm{E}-08 \\
1.19 \mathrm{E}-08 \\
7.11 \mathrm{E}-08 \\
7.11 \mathrm{E}-08 \\
1.19 \mathrm{E}-08 \\
6.84 \mathrm{E}-08 \\
7.21 \mathrm{E}-07 \\
8.77 \mathrm{E}-05 \\
8.90 \mathrm{E}-09 \\
8.77 \mathrm{E}-05 \\
6.82 \mathrm{E}-08 \\
3.79 \mathrm{E}-04 \\
5.53 \mathrm{E}-04 \\
5.35 \mathrm{E}-06 \\
2.67 \mathrm{E}-06 \\
6.18 \mathrm{E}-04 \\
8.03 \mathrm{E}-06\end{array}$ & . \\
\hline
\end{tabular}


$A-121$

\begin{tabular}{|c|c|c|c|}
\hline Shipping Sice: KCP & $\begin{array}{l}\text { Total Volume } \\
(\mathrm{m} 3 / \mathrm{yr})\end{array}$ & $\begin{array}{l}\text { Total Mass } \\
(\mathrm{kg} / \mathrm{yr})\end{array}$ & $7.23 \mathrm{E}+03$ \\
\hline & $\begin{array}{l}\text { Radionuclide } \\
\mathrm{H}-3 \\
\mathrm{C}-14 \\
\mathrm{Mn}-54 \\
\mathrm{Co}-58 \\
\mathrm{Co}-60 \\
\mathrm{Sr}-90 \\
\mathrm{Y}-90 \\
\mathrm{Tc}-99 \\
\mathrm{Cs}-134 \\
\mathrm{Cs}-137 \\
\mathrm{Ba}-137 \mathrm{~m} \\
\mathrm{U}-238\end{array}$ & \multicolumn{2}{|l|}{$\begin{array}{l}2.70 \mathrm{E}-02 \\
1.44 \mathrm{E}-04 \\
1.62 \mathrm{E}-02 \\
1.50 \mathrm{E}-02 \\
4.34 \mathrm{E}-02 \\
2.04 \mathrm{E}-02 \\
2.04 \mathrm{E}-02 \\
2.89 \mathrm{E}-04 \\
3.37 \mathrm{E}-02 \\
4.44 \mathrm{E}-02 \\
4.20 \mathrm{E}-02 \\
1.76 \mathrm{E}-03\end{array}$} \\
\hline \multirow[t]{2}{*}{ Shipping Site: MOUND } & $\begin{array}{l}\text { Total volume } \\
\quad(\mathrm{m} 3 / \mathrm{yr})\end{array}$ & $\begin{array}{l}\text { Total Mass } \\
(\mathrm{kg} / \mathrm{yr})\end{array}$ & $9.67 \mathrm{E}+06$ \\
\hline & $\begin{array}{l}\text { Radionuclide } \\
\mathrm{H}-3 \\
\mathrm{Pu}-238 \\
\mathrm{Pu}-239 \\
\mathrm{Pu}-240 \\
\mathrm{Pu}-241 \\
\mathrm{Am}-241 \\
\mathrm{Cm}-242 \\
\mathrm{Cm}-244\end{array}$ & \multicolumn{2}{|l|}{$\begin{array}{l}1.34 \mathrm{E}+04 \\
1.60 \mathrm{E}-01 \\
1.22 \mathrm{E}-02 \\
4.28 \mathrm{E}-02 \\
5.90 \mathrm{E}+00 \\
2.44 \mathrm{E}-04 \\
3.42 \mathrm{E}-03 \\
1.22 \mathrm{E}-03\end{array}$} \\
\hline Shipping Site: ORISE & $\begin{array}{l}\text { Total volume } \\
(\mathrm{m} 3 / \mathrm{yr})\end{array}$ & $\begin{array}{l}\text { Total Mass } \\
(\mathrm{kg} / \mathrm{yr})\end{array}$ & $7.36 E+04$ \\
\hline ' & $\begin{array}{l}\text { Radionuclide } \\
----3 \\
\mathrm{H}-3 \\
\mathrm{C}-14 \\
\mathrm{Mn}-54 \\
\mathrm{Co}-58 \\
\mathrm{Co}-60 \\
\mathrm{~S}-90 \\
\mathrm{Y}-90 \\
\mathrm{Tc}-99 \\
\mathrm{Cs}-134 \\
\mathrm{Cs}-137 \\
\mathrm{Ba}-137 \mathrm{~m} \\
\mathrm{~T} \mathrm{~T}-208 \\
\mathrm{~Pb}-212 \\
\mathrm{Bi}-212 \\
\mathrm{Po}-212 \\
\mathrm{Po}-216 \\
\mathrm{Ra}-224 \\
\mathrm{Ra}-228 \\
\mathrm{Ac}-228 \\
\mathrm{Th}-228 \\
\mathrm{Th}-231 \\
\mathrm{Th}-232 \\
\mathrm{Th}-234 \\
\mathrm{~Pa}-234 \\
\mathrm{~Pa}-234 \mathrm{~m} \\
\mathrm{U}-235 \\
\mathrm{U}-238\end{array}$ & $\begin{array}{c}\begin{array}{c}\text { Activity } \\
(C i / Y Y)\end{array} \\
-1.00 \mathrm{Y}-02 \\
2.19 \mathrm{E}-05 \\
1.19 \mathrm{E}-05 \\
1.34 \mathrm{E}-03 \\
1.24 \mathrm{E}-03 \\
3.59 \mathrm{E}-03 \\
1.69 \mathrm{E}-03 \\
1.69 \mathrm{E}-03 \\
2.39 \mathrm{E}-05 \\
2.79 \mathrm{E}-03 \\
3.68 \mathrm{E}-03 \\
3.48 \mathrm{E}-03 \\
3.39 \mathrm{E}-07 \\
8.98 \mathrm{E}-07 \\
8.98 \mathrm{E}-07 \\
5.78 \mathrm{E}-07 \\
8.98 \mathrm{E}-07 \\
8.98 \mathrm{E}-07 \\
5.36 \mathrm{E}-06 \\
5.36 \mathrm{E}-06 \\
8.98 \mathrm{E}-07 \\
5.16 \mathrm{E}-06 \\
5.44 \mathrm{E}-05 \\
6.62 \mathrm{E}-03 \\
6.78 \mathrm{E}-07 \\
6.62 \mathrm{E}-03 \\
5.14 \mathrm{E}-06 \\
6.77 \mathrm{E}-03\end{array}$ & \\
\hline
\end{tabular}




\begin{tabular}{|c|c|c|c|}
\hline Shipping Site: ORNL & $\begin{array}{l}\text { Total Volume } \\
(\mathrm{m} 3 / \mathrm{yr})\end{array}$ & $\begin{array}{c}\text { Total Mass } \\
(\mathrm{kg} / \mathrm{y} x)\end{array}$ & $3.30 E+06$ \\
\hline & 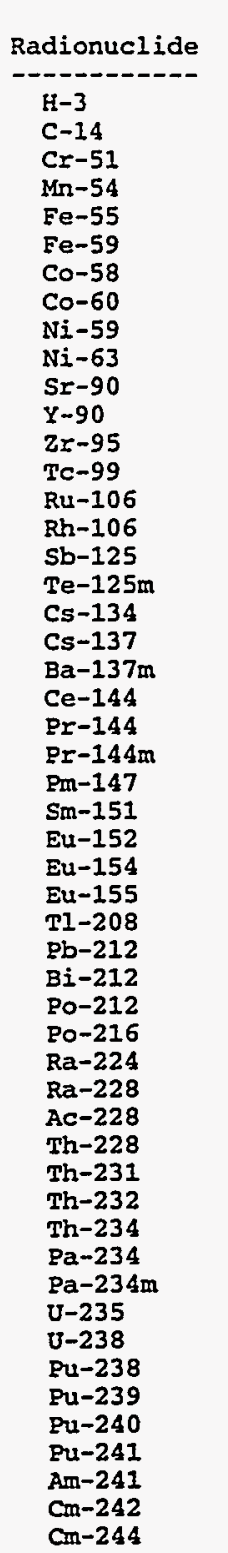 & 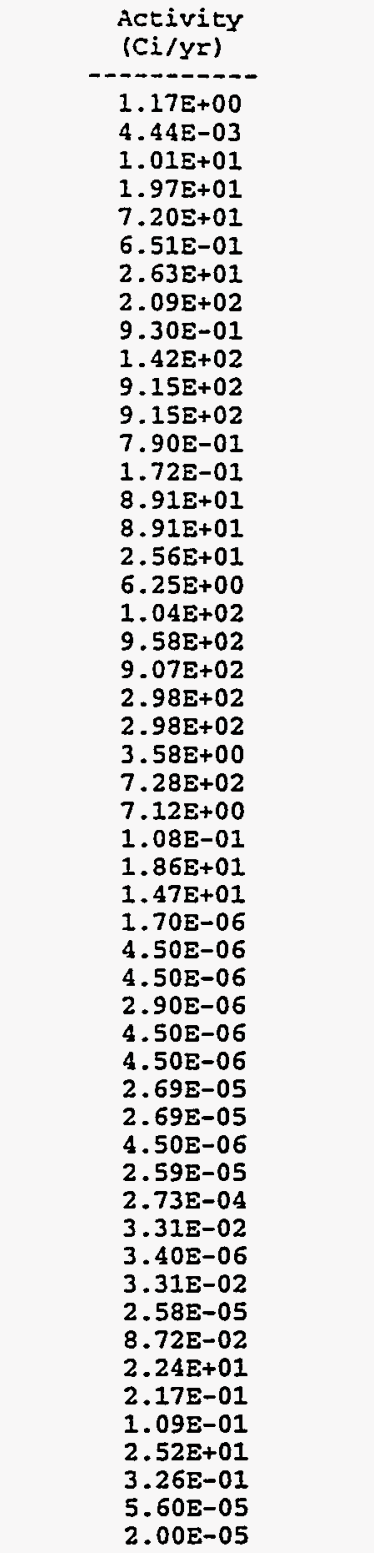 & \\
\hline Shipping Site: PORTS & $\begin{array}{l}\text { Total Volume } \\
\text { (m3/yr) }\end{array}$ & $\begin{array}{c}\text { Total Mass } \\
(\mathrm{kg} / \mathrm{Yr})\end{array}$ & $6.73 E+07$ \\
\hline & $\begin{array}{l}\text { Radionuclide } \\
\mathrm{TI}-208 \\
\mathrm{~Pb}-212 \\
\mathrm{Bi}-212 \\
\mathrm{PO}-212 \\
\mathrm{PO}-216 \\
\mathrm{Ra}-224 \\
\mathrm{Ra}-228 \\
\mathrm{Ac}-228 \\
\mathrm{Th}-228 \\
\mathrm{Th}-231 \\
\mathrm{Th}-232 \\
\mathrm{Th}-234 \\
\mathrm{~Pa}-234 \\
\mathrm{~Pa}-234 \mathrm{~m} \\
\mathrm{U}-235 \\
\mathrm{U}-238\end{array}$ & $\begin{array}{c}\begin{array}{c}\text { Activity } \\
\text { (Ci/yr) }\end{array} \\
3.45 \mathrm{E}-06 \\
9.14 \mathrm{E}-06 \\
9.14 \mathrm{E}-06 \\
5.89 \mathrm{E}-06 \\
9.14 \mathrm{E}-06 \\
9.14 \mathrm{E}-06 \\
5.46 \mathrm{E}-05 \\
5.46 \mathrm{E}-05 \\
9.14 \mathrm{E}-06 \\
5.26 \mathrm{E}-05 \\
5.55 \mathrm{E}-04 \\
6.74 \mathrm{E}-02 \\
6.91 \mathrm{E}-06 \\
6.74 \mathrm{E}-02 \\
5.24 \mathrm{E}-05 \\
6.74 \mathrm{E}-02\end{array}$ & \\
\hline
\end{tabular}




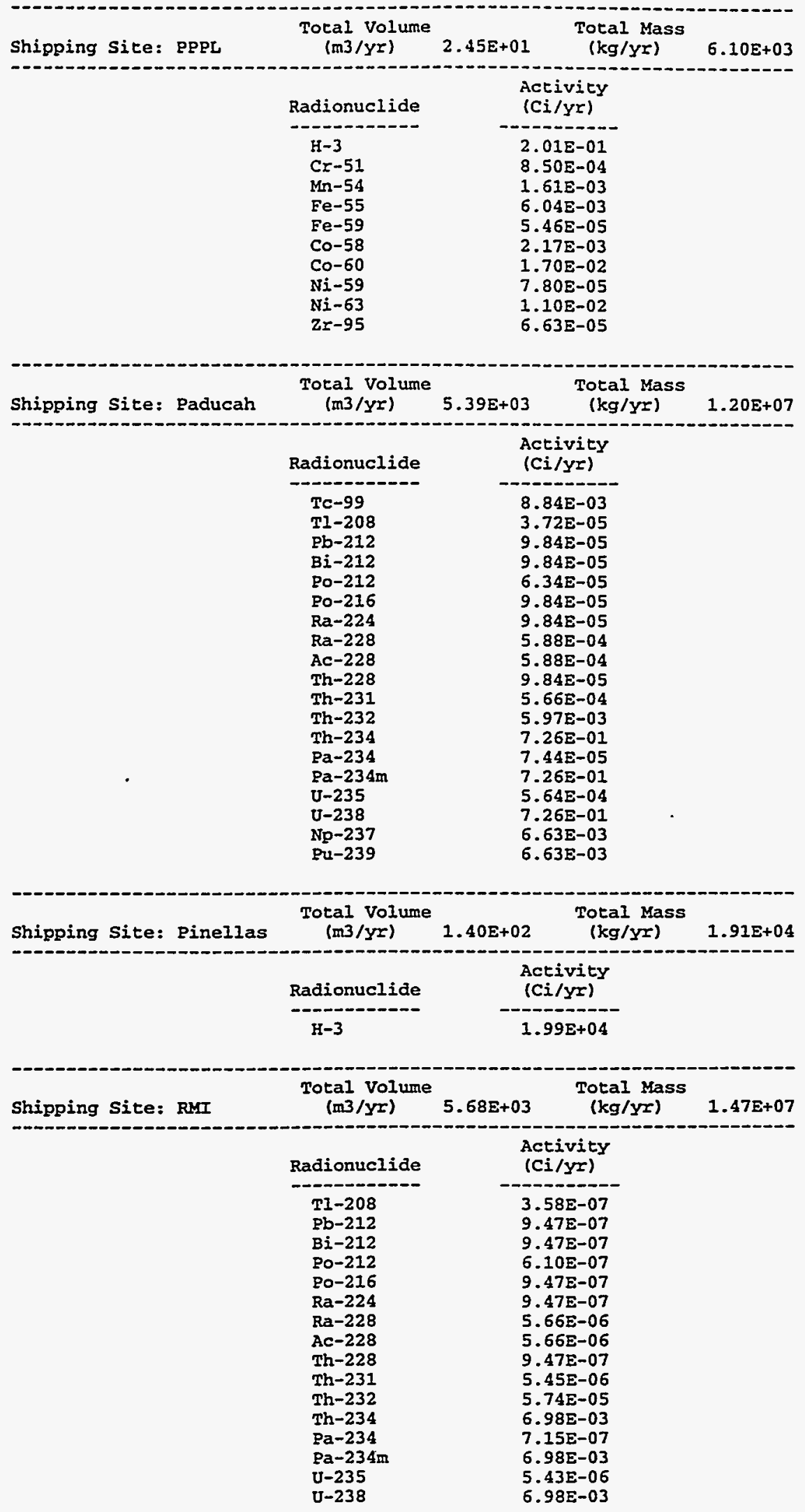


A-124

\begin{tabular}{|c|c|c|c|}
\hline Shipping Sice: $Y-12$ & $\begin{array}{l}\text { Total volume } \\
(\mathrm{m} 3 / \mathrm{yr})\end{array}$ & $\begin{array}{c}\text { Total Mass } \\
(\mathrm{kg} / \mathrm{YI})\end{array}$ & $8.275+07$ \\
\hline & $\begin{array}{l}\text { Radionuclide } \\
\text { Tl-208 } \\
\mathrm{Pb}-212 \\
\mathrm{Bi}-212 \\
\mathrm{PO}-212 \\
\mathrm{Po-216} \\
\mathrm{Ra}-224 \\
\mathrm{Ra}-228 \\
\mathrm{Ac}-228 \\
\mathrm{Th}-228 \\
\mathrm{Th}-231 \\
\mathrm{Th}-232 \\
\mathrm{Th}-234 \\
\mathrm{~Pa}-234 \\
\mathrm{~Pa}-234 \mathrm{~m} \\
\mathrm{U}-235 \\
\mathrm{U}-238\end{array}$ & \begin{tabular}{c}
$\begin{array}{c}\text { Activity } \\
\text { (Ci/yr) }\end{array}$ \\
\hdashline $6.80 \mathrm{E}-05$ \\
$1.80 \mathrm{E}-04$ \\
$1.80 \mathrm{E}-04$ \\
$1.16 \mathrm{E}-04$ \\
$1.80 \mathrm{E}-04$ \\
$1.80 \mathrm{E}-04$ \\
$1.07 \mathrm{E}-03$ \\
$1.07 \mathrm{E}-03$ \\
$1.80 \mathrm{E}-04$ \\
$1.03 \mathrm{E}-03$ \\
$1.09 \mathrm{E}-02$ \\
$1.32 \mathrm{E}+00$ \\
$1.36 \mathrm{E}-04$ \\
$1.32 \mathrm{E}+00$ \\
$1.03 \mathrm{E}-03$ \\
$1.32 \mathrm{E}+00$
\end{tabular} & \\
\hline
\end{tabular}




\section{A.16 WM LLW REGIONALIZED 6 ALTERNATIVE (CASE 5): ACTIVATED METALS}

Transported Material with Radionuclide Profile

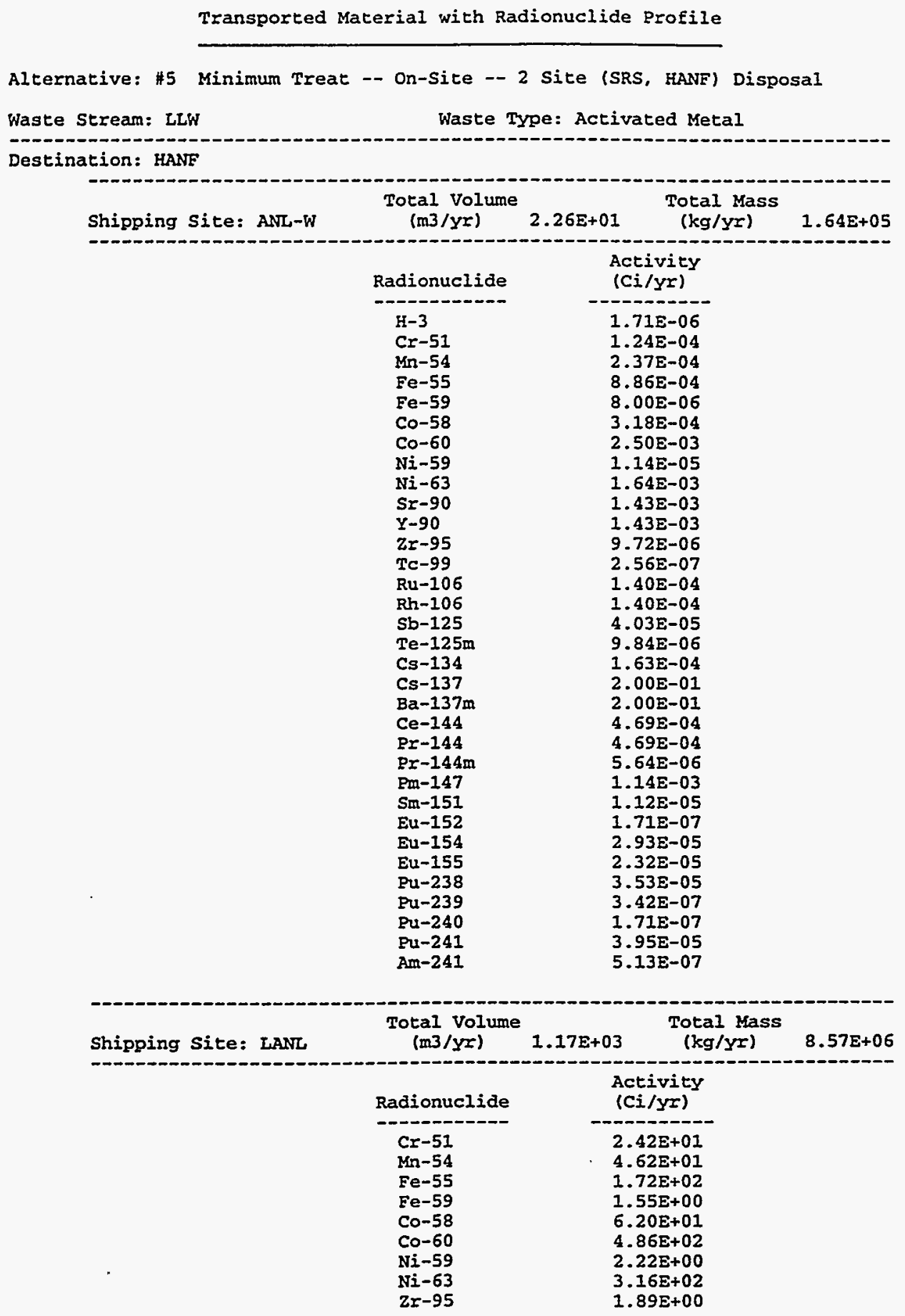




\begin{tabular}{|c|c|c|c|}
\hline Shipping Site: LBL & $\begin{array}{l}\text { Total Volume } \\
(\mathrm{m} 3 / \mathrm{Y})\end{array}$ & $\begin{array}{l}\text { Total Mass } \\
(\mathrm{kg} / \mathrm{yr})\end{array}$ & $2.87 E+05$ \\
\hline & $\begin{array}{l}\text { Radionuclide } \\
\mathrm{Cr}-51 \\
\mathrm{Mn}-54 \\
\mathrm{Fe}-55 \\
\mathrm{Fe}-59 \\
\mathrm{Co}-58 \\
\mathrm{Co}-60 \\
\mathrm{Ni}-59 \\
\mathrm{Ni}-63 \\
\mathrm{Zr}-95\end{array}$ & \multicolumn{2}{|l|}{$\begin{array}{l}6.32 \mathrm{E}-02 \\
1.20 \mathrm{E}-01 \\
4.49 \mathrm{E}-01 \\
4.06 \mathrm{E}-03 \\
1.61 \mathrm{E}-01 \\
1.26 \mathrm{E}+00 \\
5.80 \mathrm{E}-03 \\
8.24 \mathrm{E}-01 \\
4.93 \mathrm{E}-03\end{array}$} \\
\hline Shipping Site: NRF & $\begin{array}{l}\text { Total Volume } \\
(\mathrm{m} 3 / \mathrm{yr})\end{array}$ & $\begin{array}{cc}1.29 E+03 & \begin{array}{c}\text { Total Mass } \\
(\mathrm{kg} / \mathrm{Yr})\end{array}\end{array}$ & $9.40 E+06$ \\
\hline & $\begin{array}{l}\text { Radionuclide } \\
\mathrm{Cr}-51 \\
\mathrm{Mn}-54 \\
\mathrm{Fe}-55 \\
\mathrm{Fe}-59 \\
\mathrm{Co}-58 \\
\mathrm{Co}-60 \\
\mathrm{Ni}-59 \\
\mathrm{Ni}-63 \\
\mathrm{Zr}-95\end{array}$ & \multicolumn{2}{|l|}{$\begin{array}{l}2.31 \mathrm{E}+03 \\
4.40 \mathrm{E}+03 \\
1.64 \mathrm{E}+04 \\
1.48 \mathrm{E}+02 \\
5.91 \mathrm{E}+03 \\
4.63 \mathrm{E}+04 \\
2.12 \mathrm{E}+02 \\
3.01 \mathrm{E}+04 \\
1.80 \mathrm{E}+02\end{array}$} \\
\hline \multirow[t]{2}{*}{ Shipping Site: SNLA } & $\begin{array}{l}\text { Total Volume } \\
(\mathrm{m} 3 / \mathrm{yr})\end{array}$ & $\begin{array}{l}\text { Total Mass } \\
(\mathrm{kg} / \mathrm{yr})\end{array}$ & $1.15 \mathrm{E}+05$ \\
\hline & $\begin{array}{l}\text { Radionuclide } \\
\mathrm{Cr}-51 \\
\mathrm{Mn}-54 \\
\mathrm{Fe}-55 \\
\mathrm{Fe}-59 \\
\mathrm{Co}-58 \\
\mathrm{Co}-60 \\
\mathrm{Ni}-59 \\
\mathrm{Ni}-63 \\
\mathrm{Zr}-95\end{array}$ & \multicolumn{2}{|l|}{$\begin{array}{l}6.54 \mathrm{E}-04 \\
1.24 \mathrm{E}-03 \\
4.64 \mathrm{E}-03 \\
4.20 \mathrm{E}-05 \\
1.67 \mathrm{E}-03 \\
1.31 \mathrm{E}-02 \\
6.00 \mathrm{E}-05 \\
8.53 \mathrm{E}-03 \\
5.10 \mathrm{E}-05\end{array}$} \\
\hline
\end{tabular}




\begin{tabular}{|c|c|c|c|}
\hline Shipping Site: BAPL & $\begin{array}{l}\text { Total Volume } \\
(\mathrm{m} 3 / \mathrm{Y} x)\end{array}$ & $\begin{array}{l}\text { Total Mass } \\
(\mathrm{kg} / \mathrm{yr})\end{array}$ & $6.43 \mathrm{E}+06$ \\
\hline & $\begin{array}{l}\text { Radionuclide } \\
-\mathrm{Cr}-51 \\
\mathrm{Mn}-54 \\
\mathrm{Fe}-55 \\
\mathrm{Fe}-59 \\
\mathrm{Co}-58 \\
\mathrm{Co}-60 \\
\mathrm{~N} \text { i-59 } \\
\mathrm{Ni}-63 \\
\mathrm{Z} \mathrm{-}-95 \\
\mathrm{~T} 1-208 \\
\mathrm{~Pb}-212 \\
\mathrm{Bi}-212 \\
\mathrm{Po}-212 \\
\mathrm{Po}-216 \\
\mathrm{Ra}-224 \\
\mathrm{Ra}-228 \\
\mathrm{Ac}-228 \\
\mathrm{Th}-228 \\
\mathrm{Th}-231 \\
\mathrm{Th}-232 \\
\mathrm{Th}-234 \\
\mathrm{~Pa}-234 \\
\mathrm{~Pa}-234 \mathrm{~m} \\
\mathrm{U}-235 \\
\mathrm{U}-238\end{array}$ & $\begin{array}{c}\begin{array}{c}\text { Activity } \\
(C i / y z)\end{array} \\
4.75 E+03 \\
9.04 E+03 \\
3.37 E+04 \\
3.05 E+02 \\
1.21 E+04 \\
9.52 E+04 \\
4.36 E+02 \\
6.19 E+04 \\
3.70 E+02 \\
1.36 E-08 \\
3.60 E-08 \\
3.60 E-08 \\
2.32 E-08 \\
3.60 E-08 \\
3.60 E-08 \\
2.15 E-07 \\
2.15 E-07 \\
3.60 E-08 \\
2.07 E-07 \\
2.18 E-06 \\
2.65 E-04 \\
2.72 E-08 \\
2.65 E-04 \\
2.06 E-07 \\
2.65 E-04\end{array}$ & \\
\hline Shipping Site: FNAL & $\begin{array}{l}\text { TotaI Volume } \\
\text { (m3/yr) }\end{array}$ & $\begin{array}{cc}\text { Total Mass } \\
(\mathrm{kg} / \mathrm{yr})\end{array}$ & $8.14 E+05$ \\
\hline & $\begin{array}{l}\text { Radionuclide } \\
\mathrm{Cr}-51 \\
\mathrm{Mn}-54 \\
\mathrm{Fe}-55 \\
\mathrm{Fe}-59 \\
\mathrm{Co}-58 \\
\mathrm{Co}-60 \\
\mathrm{Ni}-59 \\
\mathrm{Ni}-63 \\
\mathrm{Zr}-95\end{array}$ & $\begin{array}{c}\begin{array}{c}\text { Activity } \\
\text { (Ci/yr) }\end{array} \\
3.35 \mathrm{E}-02 \\
6.39 \mathrm{E}-02 \\
2.38 \mathrm{E}-01 \\
2.15 \mathrm{E}-03 \\
8.57 \mathrm{E}-02 \\
6.72 \mathrm{E}-01 \\
3.08 \mathrm{E}-03 \\
4.37 \mathrm{E}-01 \\
2.61 \mathrm{E}-03\end{array}$ & \\
\hline
\end{tabular}


A -128

\begin{tabular}{|c|c|c|c|}
\hline Shipping Site: KAPL & $\begin{array}{l}\text { Total volume } \\
(\mathrm{m} 3 / \mathrm{yr})\end{array}$ & $\begin{array}{l}\text { Total Mass } \\
\left(\mathrm{kg} / \mathrm{y}^{\prime}\right)\end{array}$ & $1.33 E+07$ \\
\hline & $\begin{array}{l}\text { Radionuclide } \\
\mathrm{H}-3 \\
\mathrm{C}-14 \\
\mathrm{Cr}-51 \\
\mathrm{Mn}-54 \\
\mathrm{Fe}-55 \\
\mathrm{Fe}-59 \\
\mathrm{Co}-58 \\
\mathrm{Co}-60 \\
\mathrm{Ni}-59 \\
\mathrm{Ni}-63 \\
\mathrm{Sr}-90 \\
\mathrm{Y}-90 \\
\mathrm{Zr}-95 \\
\mathrm{Tc}-99 \\
\mathrm{Ru}-106 \\
\mathrm{Rh}-106 \\
\mathrm{Sb}-125 \\
\mathrm{Te}-125 \mathrm{~m} \\
\mathrm{Cs}-134 \\
\mathrm{Cs}-137 \\
\mathrm{Ba}-137 \mathrm{~m} \\
\mathrm{Ce}-144 \\
\mathrm{Pr}-144 \\
\mathrm{Pr}-144 \mathrm{~m} \\
\mathrm{Pm}-147 \\
\mathrm{Sm}-151 \\
\mathrm{Eu}-152 \\
\mathrm{Eu}-154 \\
\mathrm{Eu}-155 \\
\mathrm{TI}-208 \\
\mathrm{~Pb}-212 \\
\mathrm{Bi}-212 \\
\mathrm{Po-212} \\
\mathrm{Po-216} \\
\mathrm{Ra}-224 \\
\mathrm{Ra}-228 \\
\mathrm{Ac}-228 \\
\mathrm{Th}-228 \\
\mathrm{Th}-231 \\
\mathrm{Th}-232 \\
\mathrm{Th}-234 \\
\mathrm{~Pa}-234 \\
\mathrm{~Pa}-234 \mathrm{~m} \\
\mathrm{U}-235 \\
\mathrm{U}-238 \\
\mathrm{Pu}-238 \\
\mathrm{Pu}-239 \\
\mathrm{Pu}-240 \\
\mathrm{Pu}-241 \\
\mathrm{Am}-241 \\
\end{array}$ & $\begin{array}{c}\text { Activity } \\
\text { (Ci/yr) } \\
5.15 \mathrm{E}-04 \\
2.40 \mathrm{E}-05 \\
1.13 \mathrm{E}+04 \\
2.15 \mathrm{E}+04 \\
8.05 \mathrm{E}+04 \\
7.28 \mathrm{E}+02 \\
2.89 \mathrm{E}+04 \\
2.27 \mathrm{E}+05 \\
1.04 \mathrm{E}+03 \\
1.47 \mathrm{E}+05 \\
2.62 \mathrm{E}-02 \\
2.62 \mathrm{E}-02 \\
8.84 \mathrm{E}+02 \\
5.20 \mathrm{E}-05 \\
2.22 \mathrm{E}-03 \\
2.22 \mathrm{E}-03 \\
6.40 \mathrm{E}-04 \\
1.56 \mathrm{E}-04 \\
8.18 \mathrm{E}-03 \\
3.13 \mathrm{E}-02 \\
2.96 \mathrm{E}-02 \\
7.46 \mathrm{E}-03 \\
7.46 \mathrm{E}-03 \\
8.97 \mathrm{E}-05 \\
1.82 \mathrm{E}-02 \\
1.78 \mathrm{E}-04 \\
2.71 \mathrm{E}-06 \\
4.66 \mathrm{E}-04 \\
3.69 \mathrm{E}-04 \\
2.96 \mathrm{E}-08 \\
7.83 \mathrm{E}-08 \\
7.83 \mathrm{E}-08 \\
5.05 \mathrm{E}-08 \\
7.83 \mathrm{E}-08 \\
7.83 \mathrm{E}-08 \\
4.68 \mathrm{E}-07 \\
4.68 \mathrm{E}-07 \\
7.83 \mathrm{E}-08 \\
4.50 \mathrm{E}-07 \\
4.75 \mathrm{E}-06 \\
5.77 \mathrm{E}-04 \\
5.92 \mathrm{E}-08 \\
5.77 \mathrm{E}-04 \\
4.48 \mathrm{E}-07 \\
8.69 \mathrm{E}-04 \\
5.61 \mathrm{E}-04 \\
5.43 \mathrm{E}-06 \\
2.71 \mathrm{E}-06 \\
6.28 \mathrm{E}-04 \\
8.15 \mathrm{E}-06\end{array}$ & \\
\hline
\end{tabular}




\section{A.17 WM LLW REGIONALIZED 7 ALTERNATIVE (CASE 6): HETEROGENEOUS SOLIDS}

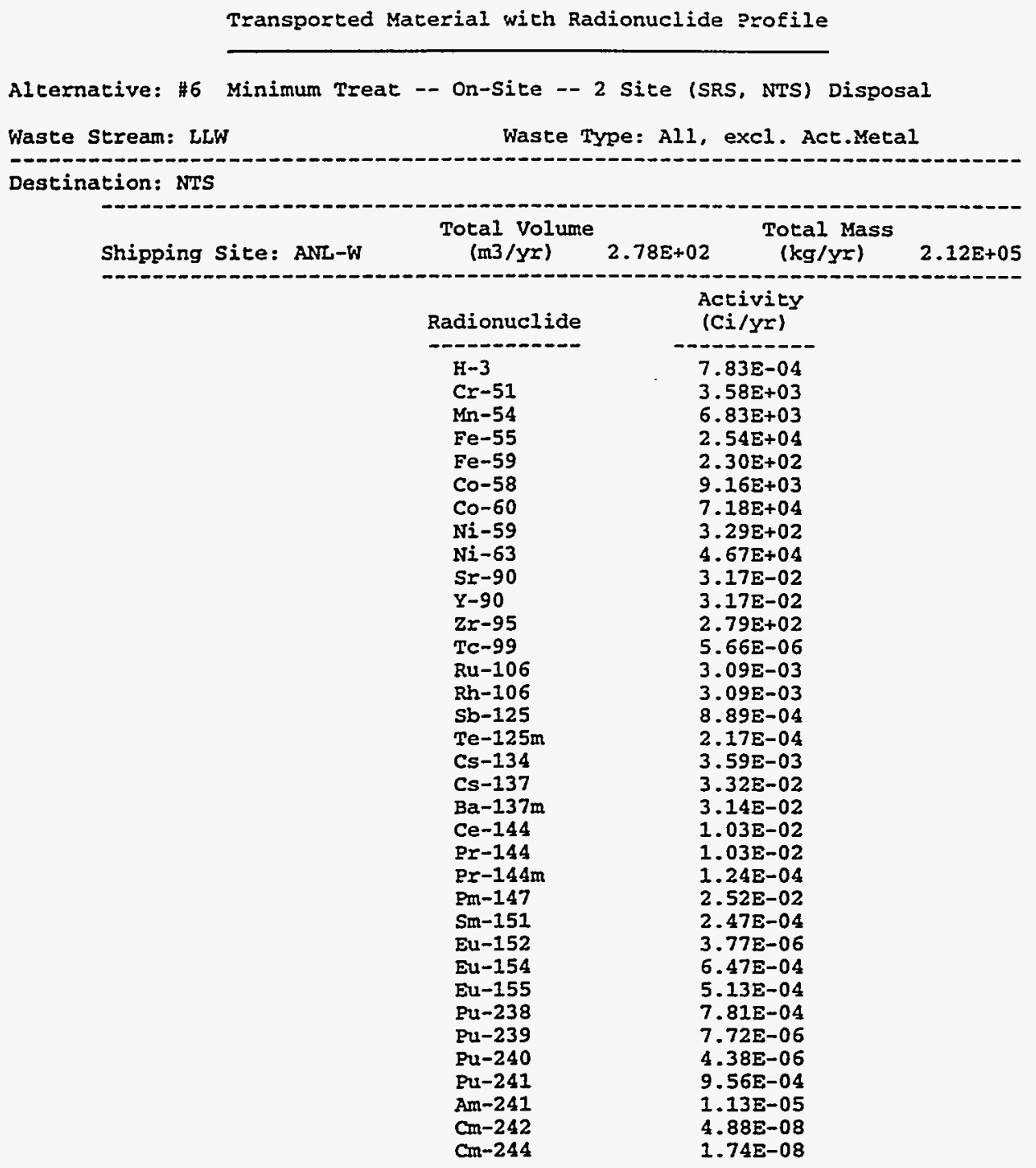



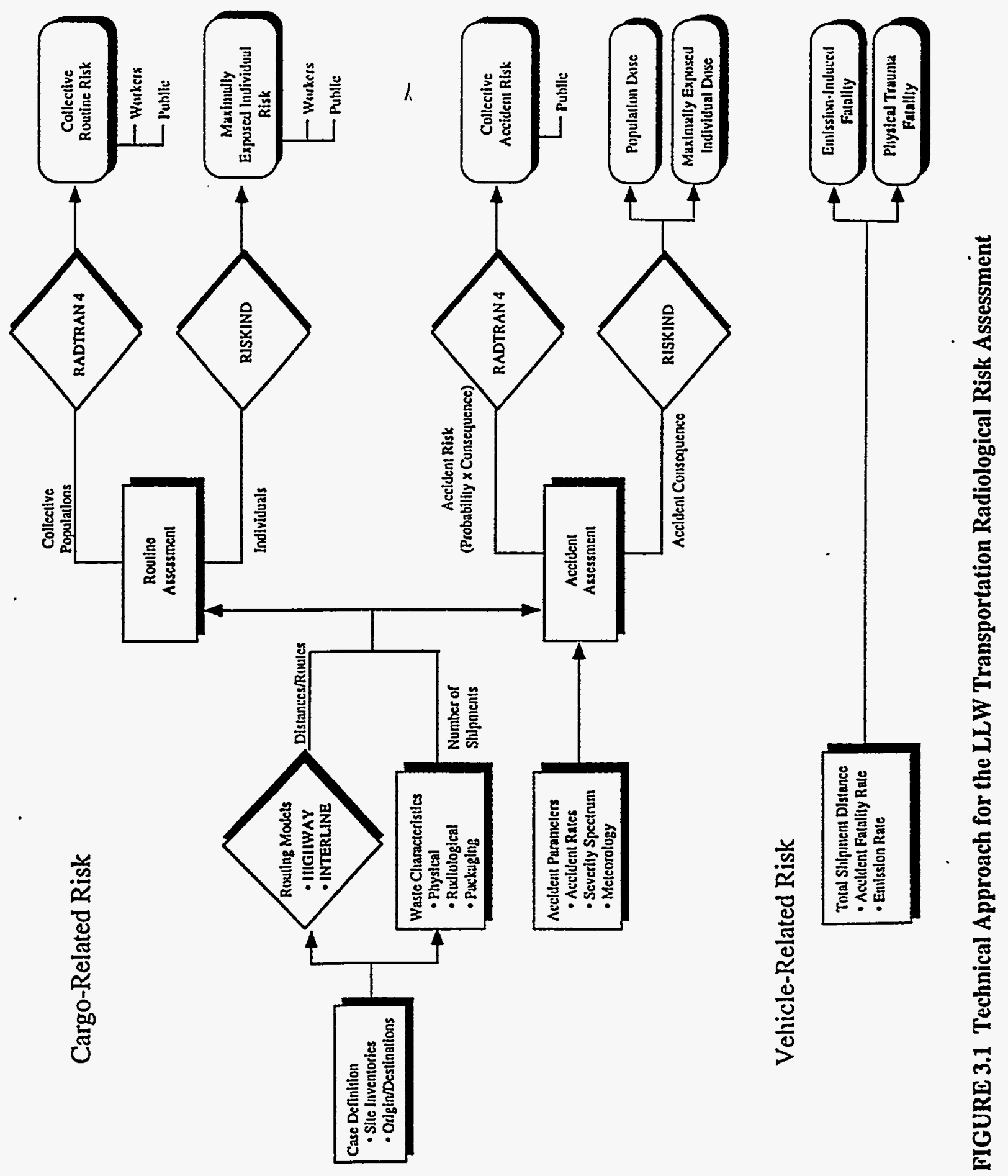


\begin{tabular}{|c|c|c|c|}
\hline Shipping Site: HANE & $\begin{array}{l}\text { Total Volume } \\
(\mathrm{m} 3 / \mathrm{yr})\end{array}$ & $\begin{array}{l}\text { Total Mass } \\
(\mathrm{kg} / \mathrm{yr})\end{array}$ & $2.75 E+07$ \\
\hline & $\begin{array}{l}\text { Radionuclide } \\
H-3 \\
\mathrm{Cr}-51 \\
\mathrm{Mn}-54 \\
\mathrm{Fe}-55 \\
\mathrm{Fe}-59 \\
\mathrm{Co}-58 \\
\mathrm{Co}-60 \\
\mathrm{Ni}-59 \\
\mathrm{Ni}-63 \\
\mathrm{Sr}-90 \\
\mathrm{Y}-90 \\
\mathrm{Zr}-95 \\
\mathrm{Tc}-99 \\
\mathrm{Ru}-106 \\
\mathrm{Rh}-106 \\
\mathrm{Sb}-125 \\
\mathrm{Te}-125 \mathrm{~m} \\
\mathrm{Cs}-134 \\
\mathrm{Cs}-137 \\
\mathrm{Ba}-137 \mathrm{~m} \\
\mathrm{Ce}-144 \\
\mathrm{Pr}-144 \\
\mathrm{Pr}-144 \mathrm{~m} \\
\mathrm{Pm}-147 \\
\mathrm{Sm}-151 \\
\mathrm{Eu}-152 \\
\mathrm{Eu}-154 \\
\mathrm{Eu}-155 \\
\mathrm{TI}-208 \\
\mathrm{~Pb}-212 \\
\mathrm{Bi}-212 \\
\mathrm{PO}-212 \\
\mathrm{Po}-216 \\
\mathrm{Ra}-224 \\
\mathrm{Ra}-228 \\
\mathrm{Ac}-228 \\
\mathrm{Th}-228 \\
\mathrm{Th}-231 \\
\mathrm{Th}-232 \\
\mathrm{Th}-234 \\
\mathrm{~Pa}-234 \\
\mathrm{~Pa}-234 \mathrm{~m} \\
\mathrm{U}-235 \\
\mathrm{U}-238 \\
\mathrm{Pu}-238 \\
\mathrm{Pu}-239 \\
\mathrm{Pu}-240 \\
\mathrm{Pu}-241 \\
\mathrm{Am}-241 \\
\mathrm{~m}-241 \\
\end{array}$ & 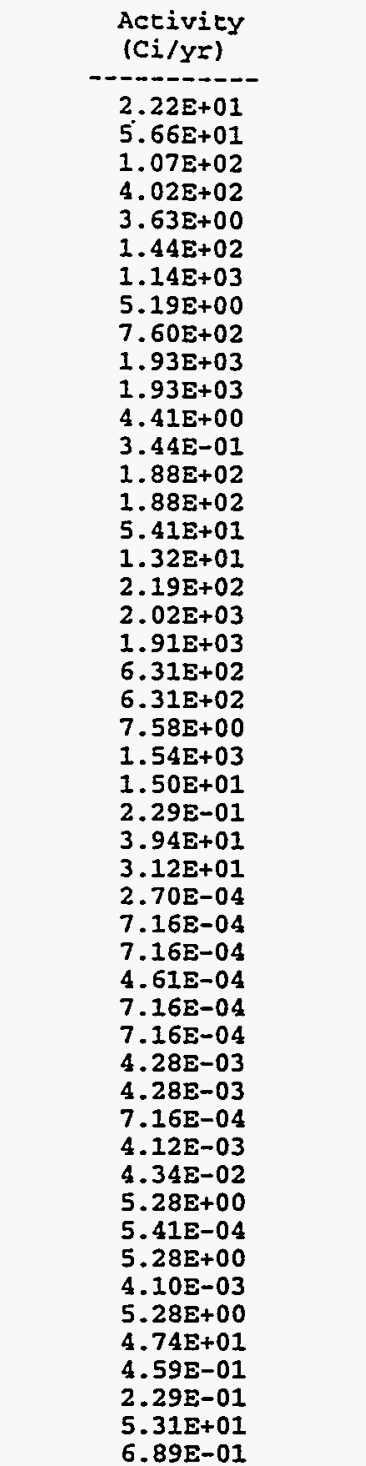 & \\
\hline
\end{tabular}




\begin{tabular}{|c|c|c|c|}
\hline Shipping Site: INEL & $\begin{array}{l}\text { Total volume } \\
\text { (m3/yr) }\end{array}$ & $\begin{array}{l}\text { Total Mass } \\
(\mathrm{kg} / \mathrm{yr})\end{array}$ & $1.64 \mathrm{E}+07$ \\
\hline . & $\begin{array}{l}\text { Radionuclide } \\
\mathrm{H}-3 \\
\mathrm{Cr}-51 \\
\mathrm{Mn}-54 \\
\mathrm{Fe}-55 \\
\mathrm{Fe}-59 \\
\mathrm{Co}-58 \\
\mathrm{Co}-60 \\
\mathrm{Ni}-59 \\
\mathrm{Ni}-63 \\
\mathrm{Sr}-90 \\
\mathrm{Y}-90 \\
\mathrm{Zr}-95 \\
\mathrm{Tc}-99 \\
\mathrm{Ru}-106 \\
\mathrm{Rh}-106 \\
\mathrm{Sb}-125 \\
\mathrm{Te}-125 \mathrm{~m} \\
\mathrm{Cs}-134 \\
\mathrm{Cs}-137 \\
\mathrm{Ba}-137 \mathrm{~m} \\
\mathrm{Ce}-144 \\
\mathrm{Pr}-144 \\
\mathrm{Pr}-144 \mathrm{~m} \\
\mathrm{Pm}-147 \\
\mathrm{Sm}-151 \\
\text { Eu-152 } \\
\mathrm{Eu}-154 \\
\text { Eu-155 } \\
\mathrm{Pu}-238 \\
\mathrm{Pu}-239 \\
\mathrm{Pu}-240 \\
\mathrm{Pu}-241 \\
\mathrm{Am}-241\end{array}$ & $\begin{array}{c}\text { Activity } \\
\text { (Ci/yr) } \\
1.56 E+00 \\
1.02 E+02 \\
1.94 E+02 \\
7.24 E+02 \\
6.55 E+00 \\
2.60 E+02 \\
2.04 E+03 \\
9.35 E+00 \\
1.33 E+03 \\
5.01 E+01 \\
5.01 E+01 \\
7.95 E+00 \\
8.95 E-03 \\
4.89 E+00 \\
4.89 E+00 \\
1.40 E+00 \\
3.43 E-01 \\
5.69 E+00 \\
5.25 E+01 \\
4.97 E+01 \\
1.63 E+01 \\
1.63 E+01 \\
1.97 E-01 \\
4.00 E+01 \\
3.91 E-01 \\
5.97 E-03 \\
1.02 E+00 \\
8.12 E-01 \\
1.23 E+00 \\
1.19 E-02 \\
5.97 E-03 \\
1.37 E+00 \\
1.79 E-02\end{array}$ & \\
\hline \multirow[t]{2}{*}{ Shipping site: ITRI } & $\begin{array}{l}\text { Total Volume } \\
\text { (m3/yx) }\end{array}$ & $\begin{array}{l}\text { Total Mass } \\
(\mathrm{kg} / \mathrm{y} /)\end{array}$ & $1.64 \mathrm{E}+05$ \\
\hline & $\begin{array}{l}\text { Radionuclide } \\
\mathrm{H}-3 \\
\mathrm{C}-14 \\
\mathrm{Mn}-54 \\
\mathrm{Co}-58 \\
\mathrm{Co}-60 \\
\mathrm{~S}-90 \\
\mathrm{Y}-90 \\
\mathrm{TC}-99 \\
\mathrm{Cs}-134 \\
\mathrm{Cs}-137 \\
\mathrm{Ba}-137 \mathrm{~m} \\
\mathrm{U}-238\end{array}$ & $\begin{array}{c}\begin{array}{c}\text { Activity } \\
(\mathrm{Ci} / \mathrm{y})\end{array} \\
6.17 \mathrm{E}+00 \\
3.03 \mathrm{E}-01 \\
3.41 \mathrm{E}+01 \\
3.15 \mathrm{E}+01 \\
9.12 \mathrm{E}+01 \\
4.28 \mathrm{E}+01 \\
4.28 \mathrm{E}+01 \\
6.07 \mathrm{E}-01 \\
7.07 \mathrm{E}+01 \\
9.33 \mathrm{E}+01 \\
8.82 \mathrm{E}+01 \\
3.69 \mathrm{E}+00\end{array}$ & \\
\hline
\end{tabular}




\section{A-132}

\begin{tabular}{|c|c|c|c|}
\hline Shipping Site: LANL & $\begin{array}{l}\text { Total Volume } \\
\text { (m3/yr) }\end{array}$ & $\begin{array}{cc}\text { Total Mass } \\
(\mathrm{kg} / \mathrm{YI})\end{array}$ & $1.22 \mathrm{E}+07$ \\
\hline “ & 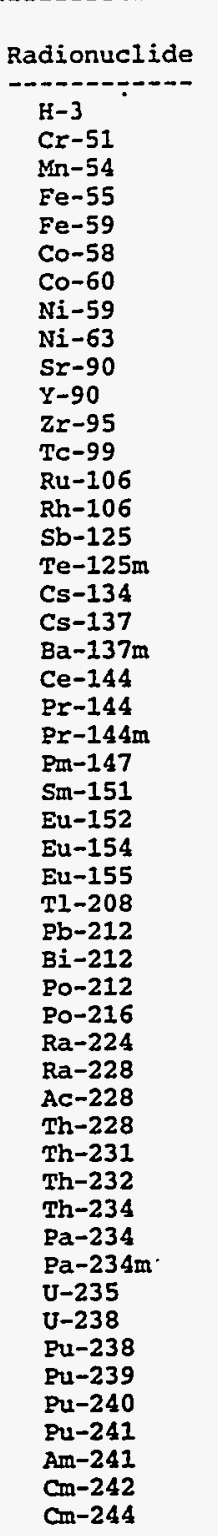 & 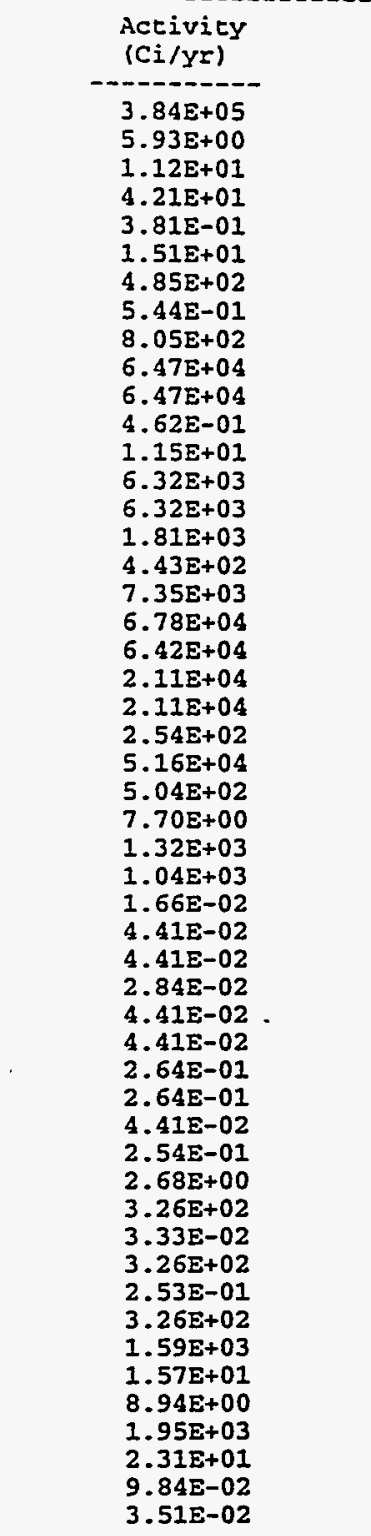 & \\
\hline Shipping site: IBL & $\begin{array}{l}\text { Total Volume } \\
\text { (m3/yx) }\end{array}$ & $\begin{array}{l}\text { Total Mass } \\
(\mathrm{kg} / \mathrm{y})\end{array}$ & \\
\hline & $\begin{array}{l}\text { Radionuclide } \\
\mathrm{H}-3 \\
\mathrm{C}-14 \\
\mathrm{Mn}-54 \\
\mathrm{Co}-58 \\
\mathrm{Co}-60 \\
\mathrm{~S} r-90 \\
\mathrm{Y}-90 \\
\mathrm{Tc}-99 \\
\mathrm{Cs}-134 \\
\mathrm{Cs}-137 \\
\mathrm{Ba}-137 \mathrm{~m} \\
\mathrm{U}-238 \\
\mathrm{Cm}-244\end{array}$ & $\begin{array}{c}\begin{array}{c}\text { Activity } \\
(C i / y \mp)\end{array} \\
-6.35 E+02 \\
1.13 E+00 \\
1.27 E+02 \\
1.17 E+02 \\
3.39 E+02 \\
1.59 E+02 \\
1.59 E+02 \\
2.26 E+00 \\
2.63 E+02 \\
3.47 E+02 \\
3.28 E+02 \\
1.37 E+01 \\
1.05 E+01\end{array}$ & \\
\hline
\end{tabular}




\begin{tabular}{|c|c|c|c|}
\hline Shipping Site: LLNL & $\begin{array}{l}\text { Total Volume } \\
\text { (m3/yr) }\end{array}$ & $\begin{array}{c}\text { Tocal Mass } \\
(\mathrm{kg} / \mathrm{Yr})\end{array}$ & $1.06 \mathrm{E}+06$ \\
\hline & $\begin{array}{l}\text { Radionuclide } \\
\mathrm{H}-3 \\
\mathrm{C}-14 \\
\mathrm{Mn}-54 \\
\mathrm{Co}-58 \\
\mathrm{Co}-60 \\
\mathrm{Ni}-63 \\
\mathrm{~S}-90 \\
\mathrm{Y}-90 \\
\mathrm{~T} \mathrm{c}-99 \\
\mathrm{Ru}-106 \\
\mathrm{Rh}-106 \\
\mathrm{Sb}-125 \\
\mathrm{Te}-125 \mathrm{~m} \\
\mathrm{Cs}-134 \\
\mathrm{Cs}-137 \\
\mathrm{Ba}-137 \mathrm{~m} \\
\mathrm{Ce}-144 \\
\mathrm{Pr}-144 \\
\mathrm{Pr}-144 \mathrm{~m} \\
\mathrm{Pm}-147 \\
\mathrm{Sm}-151 \\
\text { Eu-152 } \\
\text { Eu-154 } \\
\text { Eu-155 } \\
\mathrm{U}-238 \\
\mathrm{Pu}-238 \\
\mathrm{Pu}-239 \\
\mathrm{Pu}-240 \\
\mathrm{Pu}-241 \\
\mathrm{Am}-241 \\
\mathrm{Cm}-242 \\
\mathrm{Cm}-244\end{array}$ & $\begin{array}{c}\begin{array}{c}\text { Activity } \\
\text { (Ci/Yr) }\end{array} \\
3.33 E+04 \\
1.55 E-03 \\
1.74 E-01 \\
1.61 E-01 \\
4.69 E-01 \\
4.89 E-03 \\
6.54 E-01 \\
6.54 E-01 \\
3.18 E-03 \\
4.24 E-02 \\
4.24 E-02 \\
1.21 E-02 \\
2.97 E-03 \\
4.11 E-01 \\
9.33 E-01 \\
8.82 E-01 \\
1.42 E-01 \\
1.42 E-01 \\
1.70 E-03 \\
3.46 E-01 \\
3.39 E-03 \\
5.17 E-05 \\
8.87 E-03 \\
7.03 E-03 \\
1.88 E-02 \\
7.85 E-02 \\
5.28 E-03 \\
1.81 E-02 \\
2.50 E+00 \\
2.58 E-04 \\
1.44 E-03 \\
5.17 E-04\end{array}$ & \\
\hline \multirow[t]{2}{*}{ Shipping site: PANT } & $\begin{array}{l}\text { Total volume } \\
(\mathrm{m} 3 / \mathrm{yx})\end{array}$ & $\begin{array}{l}\text { Total Mass } \\
(\mathrm{kg} / \mathrm{yr})\end{array}$ & $2.88 \mathrm{E}+07$ \\
\hline & $\begin{array}{l}\text { Radionuclide } \\
\mathrm{H}-3 \\
\mathrm{Tl}-208 \\
\mathrm{~Pb}-212 \\
\mathrm{Bi}-212 \\
\mathrm{PO}-212 \\
\mathrm{Po-216} \\
\mathrm{Ra}-224 \\
\mathrm{Ra}-228 \\
\mathrm{Ac}-228 \\
\mathrm{Th}-228 \\
\mathrm{Th}-231 \\
\mathrm{Th}-232 \\
\mathrm{Th}-234 \\
\mathrm{~Pa}-234 \\
\mathrm{~Pa}-234 \mathrm{~m} \\
\mathrm{U}-235 \\
\mathrm{U}-238\end{array}$ & \multicolumn{2}{|l|}{$\begin{array}{l}-3.34 E+02 \\
2.21 E-04 \\
5.86 E-04 \\
5.86 E-04 \\
3.78 E-04 \\
5.86 E-04 \\
5.86 E-04 \\
3.50 E-03 \\
3.50 E-03 \\
5.86 E-04 \\
3.37 E-03 \\
3.56 E-02 \\
4.32 E+00 \\
4.43 E-04 \\
4.32 E+00 \\
3.36 E-03 \\
4.32 E+00\end{array}$} \\
\hline
\end{tabular}


A-134

\begin{tabular}{|c|c|c|c|}
\hline Shipping Sice: RFP & $\begin{array}{l}\text { Total Volume } \\
(\mathrm{m} 3 / \mathrm{yr})\end{array}$ & $\begin{array}{l}\text { Total Mass } \\
(\mathrm{kg} / \mathrm{yr})\end{array}$ & $6.49 E+06$ \\
\hline & $\begin{array}{l}\text { Radionuclide } \\
-\mathrm{Tl}-208 \\
\mathrm{~Pb}-212 \\
\mathrm{Bi}-212 \\
\mathrm{Po}-212 \\
\mathrm{Po}-216 \\
\mathrm{Ra}-224 \\
\mathrm{Ra}-228 \\
\mathrm{Ac}-228 \\
\mathrm{Th}-228 \\
\mathrm{Th}-231 \\
\mathrm{Th}-232 \\
\mathrm{Th}-234 \\
\mathrm{~Pa}-234 \\
\mathrm{~Pa}-234 \mathrm{~m} \\
\mathrm{U}-235 \\
\mathrm{U}-238 \\
\mathrm{Pu}-238 \\
\mathrm{Pu}-239 \\
\mathrm{Pu}-240 \\
\mathrm{Pu}-241 \\
\mathrm{Am}-241 \\
\mathrm{Cm}-242 \\
\mathrm{Cm}-244\end{array}$ & \multicolumn{2}{|l|}{ 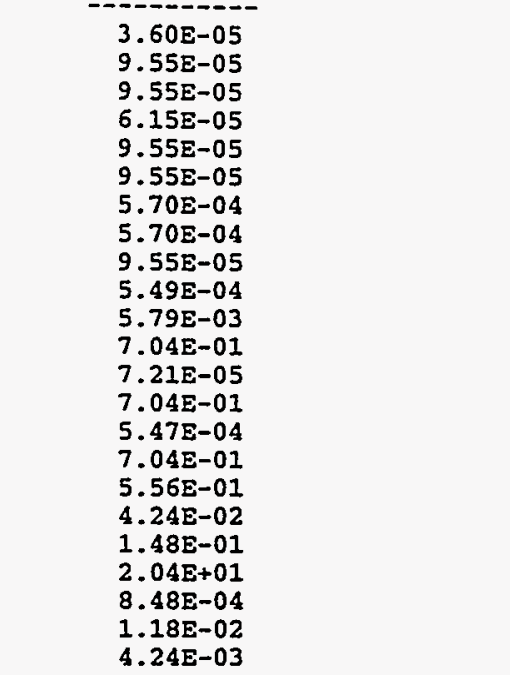 } \\
\hline \multirow[t]{2}{*}{ Shipping Site: SLAC } & $\begin{array}{l}\text { Total Volume } \\
(\mathrm{m} 3 / \mathrm{yr})\end{array}$ & $\begin{array}{cc}\text { Total Mass } \\
(\mathrm{kg} / \mathrm{yr})\end{array}$ & $7.45 E+05$ \\
\hline & $\begin{array}{l}\text { Radionuclide } \\
\text { Cr-51 } \\
\text { Mn }-54 \\
\text { Fe-55 } \\
\text { Fe-59 } \\
\text { Co-58 } \\
\text { Co-60 } \\
\text { Ni-59 } \\
\text { Ni }-63 \\
\text { Zr-95 }\end{array}$ & $\begin{array}{c}\begin{array}{c}\text { Activity } \\
\text { (Ci/yr) }\end{array} \\
3.86 \mathrm{E}-03 \\
7.36 \mathrm{E}-03 \\
2.74 \mathrm{E}-02 \\
2.48 \mathrm{E}-04 \\
9.88 \mathrm{E}-03 \\
7.74 \mathrm{E}-02 \\
3.54 \mathrm{E}-04 \\
5.04 \mathrm{E}-02 \\
3.01 \mathrm{E}-04\end{array}$ & \\
\hline
\end{tabular}


A- 135

\begin{tabular}{|c|c|c|c|}
\hline Shipping Site: SNLA & $\begin{array}{l}\text { Total Volume } \\
(\mathrm{m} 3 / \mathrm{yr})\end{array}$ & $\begin{array}{cc}\text { Total Mass } \\
1.19 \mathrm{~g}+02 & (\mathrm{~kg} / \mathrm{y})\end{array}$ & $3.28 \mathrm{E}+05$ \\
\hline - & $\begin{array}{l}\text { Radionuclide } \\
\mathrm{H}-3 \\
\mathrm{Co}-60 \\
\mathrm{Ni}-63 \\
\mathrm{Sr}-90 \\
\mathrm{Y}-90 \\
\mathrm{Tc}-99 \\
\mathrm{Ru}-106 \\
\mathrm{Rh}-106 \\
\mathrm{Sb}-125 \\
\mathrm{Te}-125 \mathrm{~m} \\
\mathrm{Cs}-134 \\
\mathrm{Cs}-137 \\
\mathrm{Ba}-137 \mathrm{~m} \\
\mathrm{Ce}-144 \\
\mathrm{Pr}-144 \\
\mathrm{Pr}-144 \mathrm{~m} \\
\mathrm{Pm}-147 \\
\mathrm{Sm}-151 \\
\mathrm{Eu}-152 \\
\mathrm{Eu}-154 \\
\mathrm{Eu}-155 \\
\mathrm{~T} \mathrm{1}-208 \\
\mathrm{~Pb}-212 \\
\mathrm{Bi}-212 \\
\mathrm{Po}-212 \\
\mathrm{Po}-216 \\
\mathrm{Ra}-224 \\
\mathrm{Ra}-228 \\
\mathrm{Ac}-228 \\
\mathrm{Th}-228 \\
\mathrm{Th}-231 \\
\mathrm{Th}-232 \\
\mathrm{Th}-234 \\
\mathrm{~Pa}-234 \\
\mathrm{~Pa}-234 \mathrm{~m} \\
\mathrm{U}-235 \\
\mathrm{U}-238 \\
\mathrm{Pu}-238 \\
\mathrm{Pu}-239 \\
\mathrm{Pu}-240 \\
\mathrm{Pu}-241 \\
\mathrm{Am}-241 \\
\mathrm{Cm}-242 \\
\mathrm{Cm}-244\end{array}$ & $\begin{array}{l}\text { Activity } \\
\text { (Ci/YE) } \\
3.00 \mathrm{E}+00 \\
2.85 \mathrm{E}-05 \\
5.67 \mathrm{E}-05 \\
5.04 \mathrm{E}-03 \\
5.04 \mathrm{E}-03 \\
9.00 \mathrm{E}-07 \\
4.92 \mathrm{E}-04 \\
4.92 \mathrm{E}-04 \\
1.41 \mathrm{E}-04 \\
3.45 \mathrm{E}-05 \\
5.72 \mathrm{E}-04 \\
5.28 \mathrm{E}-03 \\
4.99 \mathrm{E}-03 \\
1.64 \mathrm{E}-03 \\
1.64 \mathrm{E}-03 \\
1.98 \mathrm{E}-05 \\
4.02 \mathrm{E}-03 \\
3.93 \mathrm{E}-05 \\
6.00 \mathrm{E}-07 \\
1.02 \mathrm{E}-04 \\
8.16 \mathrm{E}-05 \\
5.10 \mathrm{E}-06 \\
1.35 \mathrm{E}-05 \\
1.35 \mathrm{E}-05 \\
8.70 \mathrm{E}-06 \\
1.35 \mathrm{E}-05 \\
1.35 \mathrm{E}-05 \\
8.07 \mathrm{E}-05 \\
8.07 \mathrm{E}-05 \\
1.35 \mathrm{E}-05 \\
7.77 \mathrm{E}-05 \\
8.19 \mathrm{E}-04 \\
9.96 \mathrm{E}-02 \\
1.02 \mathrm{E}-05 \\
9.96 \mathrm{E}-02 \\
7.74 \mathrm{E}-05 \\
9.96 \mathrm{E}-02 \\
7.87 \mathrm{E}-02 \\
6.00 \mathrm{E}-03 \\
2.10 \mathrm{E}-02 \\
2.89 \mathrm{E}+00 \\
1.21 \mathrm{E}-04 \\
1.68 \mathrm{E}-03 \\
6.00 \mathrm{E}-04\end{array}$ & \\
\hline Shipping Site: SNLL & $\begin{array}{l}\text { Total Volume } \\
\text { (m3/yx) }\end{array}$ & $\begin{array}{c}\text { Total Mass } \\
3.68 \mathrm{E}+01 \quad \text { (kg/yx) }\end{array}$ & $9.73 E+04$ \\
\hline & $\begin{array}{l}\text { Radionuclide } \\
\mathrm{H}-3 \\
\mathrm{Fe}-55 \\
\mathrm{Co}-60 \\
\mathrm{Tl}-208 \\
\mathrm{~Pb}-212 \\
\mathrm{Bi}-212 \\
\mathrm{Po}-212 \\
\mathrm{Po}-216 \\
\mathrm{Ra}-224 \\
\mathrm{Ra}-228 \\
\mathrm{Ac}-228 \\
\mathrm{Th}-228 \\
\mathrm{Th}-231 \\
\mathrm{Th}-232 \\
\mathrm{Th}-234 \\
\mathrm{~Pa}-234 \\
\mathrm{~Pa}-234 \mathrm{~m} \\
\mathrm{U}-235 \\
\mathrm{U}-238\end{array}$ & $\begin{array}{c}\begin{array}{l}\text { Activity } \\
(C i / Y I)\end{array} \\
2.78 \mathrm{E}+04 \\
3.15 \mathrm{E}-01 \\
3.15 \mathrm{E}-01 \\
6.40 \mathrm{E}-05 \\
1.69 \mathrm{E}-04 \\
1.69 \mathrm{E}-04 \\
1.09 \mathrm{E}-04 \\
1.69 \mathrm{E}-04 \\
1.69 \mathrm{E}-04 \\
1.01 \mathrm{E}-03 \\
1.01 \mathrm{E}-03 \\
1.69 \mathrm{E}-04 \\
9.75 \mathrm{E}-04 \\
1.02 \mathrm{E}-02 \\
1.25 \mathrm{E}+00 \\
1.28 \mathrm{E}-04 \\
1.25 \mathrm{E}+00 \\
9.71 \mathrm{E}-04 \\
1.25 \mathrm{E}+00\end{array}$ & \\
\hline
\end{tabular}


Destination: SRS

\begin{tabular}{|c|c|c|c|}
\hline Shipping Sice: AMES & $\begin{array}{c}\text { Total Volume } \\
(\mathrm{m} 3 / \mathrm{yr})\end{array}$ & $\begin{array}{l}\text { Toral Mass } \\
(\mathrm{kg} / \mathrm{y} x)\end{array}$ & $2.99 \Xi+04$ \\
\hline & $\begin{array}{l}\text { Radionuclide } \\
\mathrm{Tl}-208 \\
\mathrm{~Pb}-212 \\
\mathrm{Bi}-212 \\
\mathrm{Po}-212 \\
\mathrm{Po}-216 \\
\mathrm{Ra}-224 \\
\mathrm{Ra}-228 \\
\mathrm{Ac}-228 \\
\mathrm{Th}-228 \\
\mathrm{Th}-231 \\
\mathrm{Th}-232 \\
\mathrm{Th}-234 \\
\mathrm{~Pa}-234 \\
\mathrm{~Pa}-234 \mathrm{~m} \\
\mathrm{U}-235 \\
\mathrm{U}-238 \\
\mathrm{Pu}-238 \\
\mathrm{Pu}-239 \\
\mathrm{Pu}-240 \\
\mathrm{Pu}-241 \\
\mathrm{Am}-241 \\
\mathrm{Cm}-242 \\
\mathrm{Cm}-244\end{array}$ & $\begin{array}{l}\text { AcciviEy } \\
\text { (Ci/Yr) } \\
4.50 \mathrm{E}-07 \\
1.19 \mathrm{E}-06 \\
1.19 \mathrm{E}-06 \\
7.68 \mathrm{E}-07 \\
1.19 \mathrm{E}-06 \\
1.19 \mathrm{E}-06 \\
7.12 \mathrm{E}-06 \\
7.12 \mathrm{E}-06 \\
1.19 \mathrm{E}-06 \\
6.86 \mathrm{E}-06 \\
7.23 \mathrm{E}-05 \\
8.79 \mathrm{E}-03 \\
9.01 \mathrm{E}-07 \\
8.79 \mathrm{E}-03 \\
6.83 \mathrm{E}-06 \\
8.79 \mathrm{E}-03 \\
6.94 \mathrm{E}-04 \\
5.30 \mathrm{E}-05 \\
1.85 \mathrm{E}-04 \\
2.55 \mathrm{E}-02 \\
1.06 \mathrm{E}-06 \\
1.48 \mathrm{E}-05 \\
5.30 \mathrm{E}-06\end{array}$ & \\
\hline Shipping Sice: ANL-E & $\begin{array}{l}\text { Total volume } \\
\text { (m3/yx) }\end{array}$ & $\begin{array}{c}\text { Total Mass } \\
\text { 7.35E+02 }\end{array}$ & $2.00 E+06$ \\
\hline & 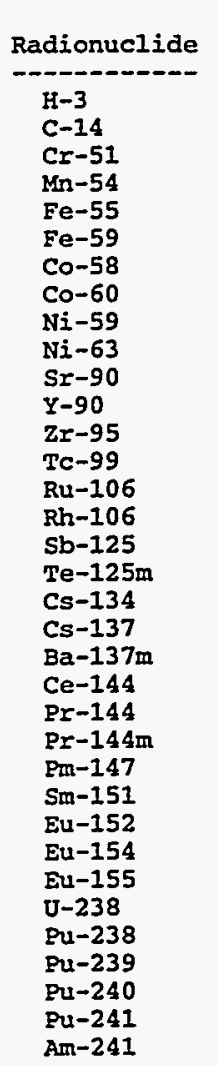 & $\begin{array}{l}\text { Activity } \\
\text { (Ci/Yr) } \\
1.33 E+02 \\
7.91 E-02 \\
2.87 E+00 \\
1.43 E+01 \\
2.04 E+01 \\
1.84 E-01 \\
1.55 E+01 \\
8.15 E+01 \\
2.63 E-01 \\
3.77 E+01 \\
3.33 E+01 \\
3.33 E+01 \\
2.24 E-01 \\
1.62 E-01 \\
2.16 E+00 \\
2.16 E+00 \\
6.21 E-01 \\
1.51 E-01 \\
2.09 E+01 \\
4.75 E+01 \\
4.50 E+01 \\
7.24 E+00 \\
7.24 E+00 \\
8.70 E-02 \\
1.76 E+01 \\
1.72 E-01 \\
2.63 E-03 \\
4.52 E-01 \\
3.58 E-01 \\
9.63 E-01 \\
5.44 E-01 \\
5.27 E-03 \\
2.63 E-03 \\
6.09 E-01 \\
7.91 E-03\end{array}$ & \\
\hline
\end{tabular}




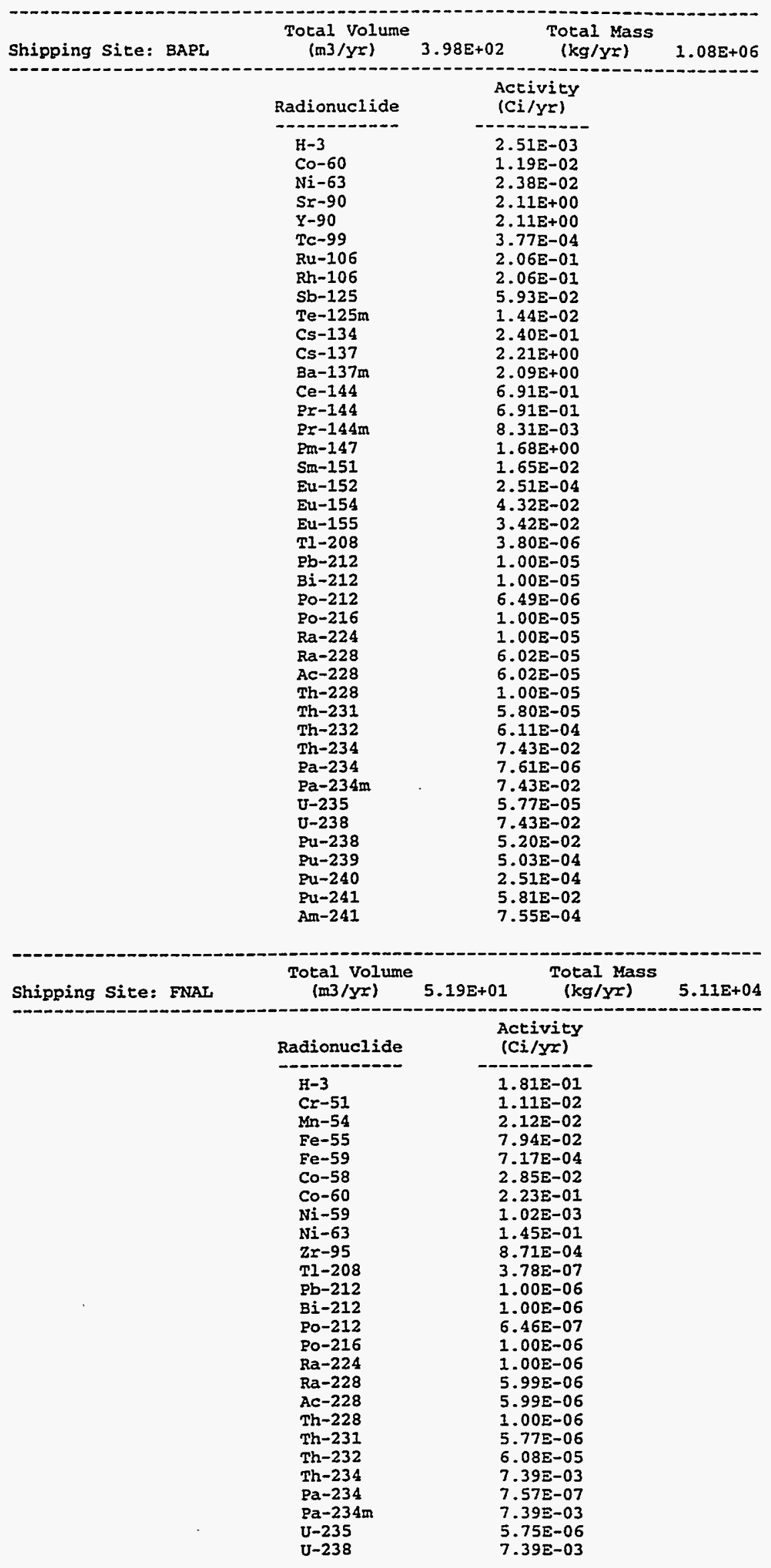




\begin{tabular}{|c|c|c|c|}
\hline Shipping Sice: $\mathrm{k}-25$ & $\begin{array}{c}\text { Total volume } \\
(\mathrm{m} 3 / \mathrm{yr})\end{array}$ & $\begin{array}{c}\text { Total Mass } \\
(\mathrm{kg} / \mathrm{yr})\end{array}$ & $4.67 E+07$ \\
\hline & $\begin{array}{l}\text { Radionuclide } \\
\mathrm{Tl}-208 \\
\mathrm{~Pb}-212 \\
\mathrm{Bi}-212 \\
\mathrm{Po}-212 \\
\mathrm{Po}-216 \\
\mathrm{Ra}-224 \\
\mathrm{Ra}-228 \\
\mathrm{Ac}-228 \\
\mathrm{Th}-228 \\
\mathrm{Th}-231 \\
\mathrm{Th}-232 \\
\mathrm{Th}-234 \\
\mathrm{~Pa}-234 \\
\mathrm{~Pa}-234 \mathrm{~m} \\
\mathrm{U}-235 \\
\mathrm{U}-238\end{array}$ & $\begin{array}{c}\begin{array}{c}\text { Activity } \\
(\mathrm{Ci} / \mathrm{yr})\end{array} \\
1.06 \mathrm{E}-04 \\
2.82 \mathrm{E}-04 \\
2.82 \mathrm{E}-04 \\
1.82 \mathrm{E}-04 \\
2.82 \mathrm{E}-04 \\
2.82 \mathrm{E}-04 \\
1.68 \mathrm{E}-03 \\
1.68 \mathrm{E}-03 \\
2.82 \mathrm{E}-04 \\
1.62 \mathrm{E}-03 \\
1.71 \mathrm{E}-02 \\
2.08 \mathrm{E}+00 \\
2.13 \mathrm{E}-04 \\
2.08 \mathrm{E}+00 \\
1.62 \mathrm{E}-03 \\
2.08 \mathrm{E}+00\end{array}$ & \\
\hline Shipping Sice: KAPL & $\begin{array}{l}\text { Total volume } \\
(\mathrm{m} 3 / \mathrm{y} x)\end{array}$ & $2.11 \mathrm{E}+02 \quad \begin{array}{c}\text { Total Mass } \\
(\mathrm{kg} / \mathrm{yr})\end{array}$ & $5.36 \mathrm{E}+05$ \\
\hline . & 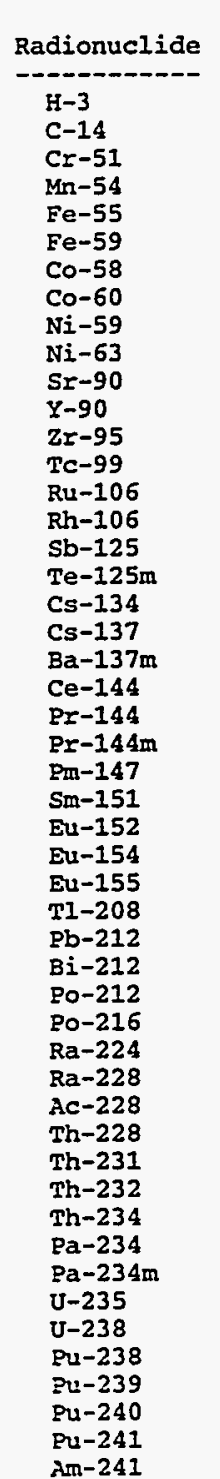 & 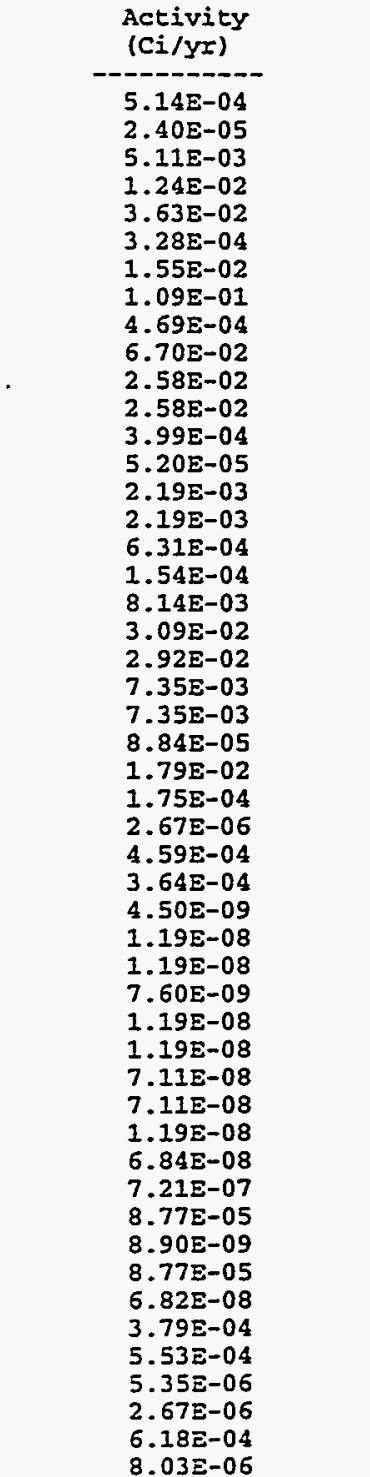 & \\
\hline
\end{tabular}


A-139

\begin{tabular}{|c|c|c|c|}
\hline Shipping Site: KCP & $\begin{array}{l}\text { Total Volume } \\
(\mathrm{m} 3 / \mathrm{y})\end{array}$ & $\begin{array}{l}\text { Total Mass } \\
(\mathrm{kg} / \mathrm{y} x)\end{array}$ & $7.23 E+03$ \\
\hline - & $\begin{array}{l}\text { Radionuclide } \\
\mathrm{H}-3 \\
\mathrm{C}-14 \\
\mathrm{Mn}-54 \\
\mathrm{Co}-58 \\
\mathrm{Co}-60 \\
\mathrm{Sr}-90 \\
\mathrm{Y}-90 \\
\mathrm{Tc}-99 \\
\mathrm{Cs}-134 \\
\mathrm{Cs}-137 \\
\mathrm{Ba}-137 \mathrm{~m} \\
\mathrm{U}-238\end{array}$ & \multicolumn{2}{|l|}{$\begin{array}{l}2.70 \mathrm{E}-02 \\
1.44 \mathrm{E}-04 \\
1.62 \mathrm{E}-02 \\
1.50 \mathrm{E}-02 \\
4.34 \mathrm{E}-02 \\
2.04 \mathrm{E}-02 \\
2.04 \mathrm{E}-02 \\
2.89 \mathrm{E}-04 \\
3.37 \mathrm{E}-02 \\
4.44 \mathrm{E}-02 \\
4.20 \mathrm{E}-02 \\
1.76 \mathrm{E}-03\end{array}$} \\
\hline \multirow[t]{2}{*}{ Shipping Site: MOUND } & $\begin{array}{l}\text { Total Volume } \\
(\mathrm{m} 3 / \mathrm{yr})\end{array}$ & $\begin{array}{c}\text { Total Mass } \\
(\mathrm{kg} / \mathrm{yr})\end{array}$ & $9.67 \mathrm{E}+06$ \\
\hline & $\begin{array}{l}\text { Radionuclide } \\
\mathrm{H}-3 \\
\mathrm{Pu}-238 \\
\mathrm{Pu}-239 \\
\mathrm{Pu}-240 \\
\mathrm{Pu}-241 \\
\mathrm{Am}-241 \\
\mathrm{Cm}-242 \\
\mathrm{Cm}-244\end{array}$ & \multicolumn{2}{|l|}{$\begin{array}{l}1.34 \mathrm{E}+04 \\
1.60 \mathrm{E}-01 \\
1.22 \mathrm{E}-02 \\
4.28 \mathrm{0}-02 \\
5.90 \mathrm{E}+00 \\
2.44 \mathrm{E}-04 \\
3.42 \mathrm{E}-03 \\
1.22 \mathrm{E}-03\end{array}$} \\
\hline \multirow[t]{2}{*}{ Shipping Site: ORISE } & $\begin{array}{l}\text { Total volume } \\
(\mathrm{m} 3 / \mathrm{yr})\end{array}$ & $\begin{array}{cc}6.07 \Xi+01 & \text { Total Mass } \\
(\mathrm{kg} / \mathrm{Yr})\end{array}$ & $7.36 \mathrm{E}+04$ \\
\hline & $\begin{array}{l}\text { Radionuclide } \\
\mathrm{H}-3 \\
\mathrm{C}-14 \\
\mathrm{Mn}-54 \\
\mathrm{Co}-58 \\
\mathrm{Co}-60 \\
\mathrm{~S}-90 \\
\mathrm{Y}-90 \\
\mathrm{TC}-99 \\
\mathrm{Cs}-134 \\
\mathrm{Cs}-137 \\
\mathrm{Ba}-137 \mathrm{~m} \\
\mathrm{Tl}-208 \\
\mathrm{~Pb}-212 \\
\mathrm{Bi}-212 \\
\mathrm{Po}-212 \\
\mathrm{PO}-216 \\
\mathrm{Ra}-224 \\
\mathrm{Ra}-228 \\
\mathrm{Ac}-228 \\
\mathrm{Th}-228 \\
\mathrm{Th}-231 \\
\mathrm{Th}-232 \\
\mathrm{Th}-234 \\
\mathrm{~Pa}-234 \\
\mathrm{~Pa}-234 \mathrm{~m} \\
\mathrm{U}-235 \\
\mathrm{U}-238\end{array}$ & \begin{tabular}{c} 
Activity \\
(Ci/yr) \\
\hdashline $2.00 \mathrm{E}-02$ \\
$1.19 \mathrm{E}-05$ \\
$1.34 \mathrm{E}-03$ \\
$1.24 \mathrm{E}-03$ \\
$3.59 \mathrm{E}-03$ \\
$1.69 \mathrm{E}-03$ \\
$1.69 \mathrm{E}-03$ \\
$2.39 \mathrm{E}-05$ \\
$2.79 \mathrm{E}-03$ \\
$3.68 \mathrm{E}-03$ \\
$3.48 \mathrm{E}-03$ \\
$3.39 \mathrm{E}-07$ \\
$8.98 \mathrm{E}-07$ \\
$8.98 \mathrm{E}-07$ \\
$5.78 \mathrm{E}-07$ \\
$8.98 \mathrm{E}-07$ \\
$8.98 \mathrm{E}-07$ \\
$5.36 \mathrm{E}-06$ \\
$5.36 \mathrm{E}-06$ \\
$8.98 \mathrm{E}-07$ \\
$5.16 \mathrm{E}-06$ \\
$5.44 \mathrm{E}-05$ \\
$6.62 \mathrm{E}-03$ \\
$6.78 \mathrm{E}-07$ \\
$6.62 \mathrm{E}-03$ \\
$5.14 \mathrm{E}-06$ \\
$6.77 \mathrm{E}-03$
\end{tabular} & \\
\hline
\end{tabular}


The RISKIND calculations are conducted for the WM PEIS to supplement the results for collective risk calculated with RADTRAN 4. Whereas the results for collective risk provide a measure of the overall risks of each case, the RISKIND calculations are meant to address areas of specific concern to individuals and subgroups of population. Essentially, the RISKIND analyses are meant to address "what if" questions, such as, "What if I live next to a site access road?" or "What if an accident happens near my town?"

\subsection{ROUTINE (INCIDENT-FREE) RISK ASSESSMENT METHOD}

\subsubsection{Collective Population Risk}

The radiological risk associated with routine transportation results from the potential exposure of people to low-level external radiation in the vicinity of loaded shipments. Because the radiological consequences (dose) occur as a direct result of normal operations, the probability of routine consequences is taken to be unity in the RADTRAN 4 code.

For routine transportation, the RADTRAN 4 computer code considers all major groups of potentially exposed persons. The RADTRAN 4 calculations of risk for routine highway and rail transportation include exposures of the following population groups:

1. Persons along the Route (Off-Link Population). Collective doses are calculated for all persons living or working within $0.8 \mathrm{~km}(0.5 \mathrm{mi})$ of each side of a transportation route. The total number of persons within the $1.6-\mathrm{km}$ $(1-\mathrm{mi})$ corridor is calculated separately for each route considered in the assessment.

2. Persons Sharing the Route (On-Link Population). Collective doses are calculated for persons in all vehicles sharing the transportation route. This group includes persons traveling in the same or opposite directions as the shipment, as well as persons in vehicles passing the shipment.

3. Persons at Stops. Collective doses are calculated for people who may be exposed while a shipment is stopped en route. For truck transportation, these stops include stops for refueling, food, and rest. For rail transportation, stops are assumed to occur for purposes of classification.

4. Crew Members. Collective doses are calculated for truck and rail transportation crew members involved in the actual shipment of waste. Workers involved in loading or unloading are not considered. 


\begin{tabular}{|c|c|c|c|}
\hline Shipping Site: ORNL & $\begin{array}{l}\text { Total Volume } \\
\text { (m3/yr) }\end{array}$ & $\begin{array}{l}\text { Total Mass } \\
(\mathrm{kg} / \mathrm{yr})\end{array}$ & $3.30 \Sigma+06$ \\
\hline - & 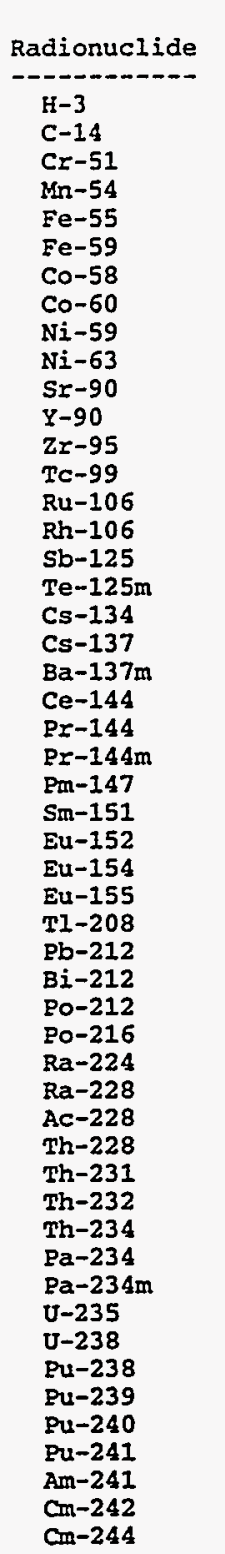 & 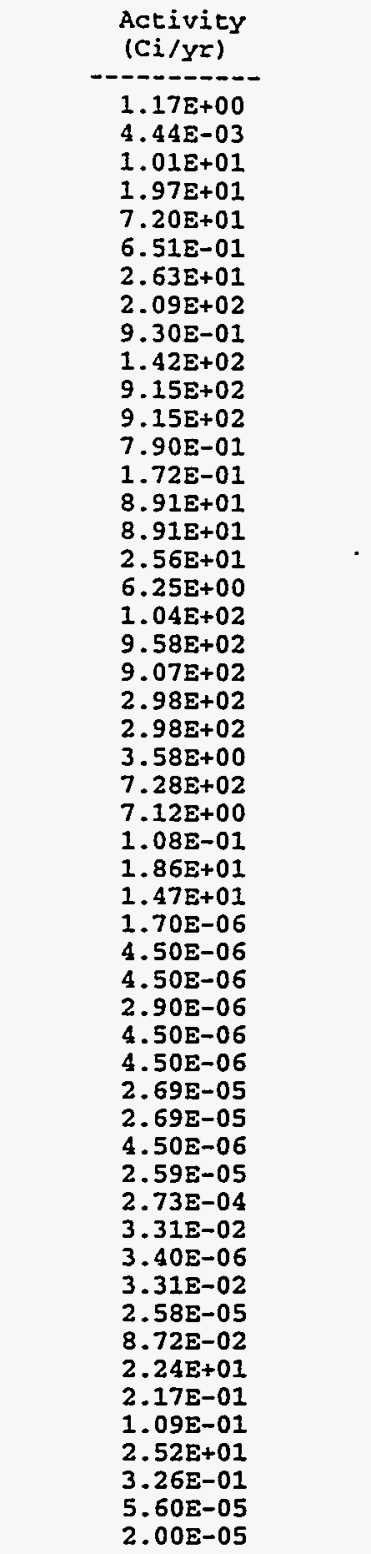 & - \\
\hline \multirow[t]{2}{*}{ Shipping site: PORTS } & $\begin{array}{l}\text { Total Volume } \\
(\mathrm{m} 3 / \mathrm{yr})\end{array}$ & $\begin{array}{c}\text { Total Mass } \\
(\mathrm{kg} / \mathrm{Yr})\end{array}$ & \\
\hline & $\begin{array}{l}\text { Radionuclide } \\
\mathrm{Tl}-208 \\
\mathrm{~Pb}-212 \\
\mathrm{Bi}-212 \\
\mathrm{PO}-212 \\
\mathrm{Po}-216 \\
\mathrm{Ra}-224 \\
\mathrm{Ra}-228 \\
\mathrm{Ac}-228 \\
\mathrm{Th}-228 \\
\mathrm{Th}-231 \\
\mathrm{Th}-232 \\
\mathrm{Th}-234 \\
\mathrm{~Pa}-234 \\
\mathrm{~Pa}-234 \mathrm{~m} \\
\mathrm{U}-235 \\
\mathrm{U}-238\end{array}$ & $\begin{array}{c}\text { Activity } \\
\text { (Ci/yI) } \\
-3.45 E-06 \\
9.14 E-06 \\
9.14 E-06 \\
5.89 \Xi-06 \\
9.14 E-06 \\
9.14 E-06 \\
5.46 E-05 \\
5.46 E-05 \\
9.14 E-06 \\
5.26 E-05 \\
5.55 \Xi-04 \\
6.74 \Xi-02 \\
6.91 \Xi-06 \\
6.74 \Xi-02 \\
5.24 E-05 \\
6.74 E-02\end{array}$ & \\
\hline
\end{tabular}




\begin{tabular}{|c|c|c|c|}
\hline Shipping Site: PPPL & $\begin{array}{l}\text { Total Volume } \\
(\mathrm{m} 3 / \mathrm{yr})\end{array}$ & $\begin{array}{l}\text { Total Mass } \\
(\mathrm{kg} / \mathrm{Yx})\end{array}$ & $6.10 \mathrm{E}+03$ \\
\hline & $\begin{array}{l}\text { Radionuclide } \\
\mathrm{H}-3 \\
\mathrm{Cr}-51 \\
\mathrm{Mn}-54 \\
\mathrm{Fe}-55 \\
\mathrm{Fe}-59 \\
\mathrm{Co}-58 \\
\mathrm{Co}-60 \\
\mathrm{Ni}-59 \\
\mathrm{Ni}-63 \\
\mathrm{Zr}-95\end{array}$ & $\begin{array}{c}\begin{array}{c}\text { Activity } \\
\text { (Ci/Yr) }\end{array} \\
2.01 \mathrm{E}-01 \\
8.50 \mathrm{E}-04 \\
1.61 \mathrm{E}-03 \\
6.04 \mathrm{E}-03 \\
5.46 \mathrm{E}-05 \\
2.17 \mathrm{E}-03 \\
1.70 \mathrm{E}-02 \\
7.80 \mathrm{E}-05 \\
1.10 \mathrm{E}-02 \\
6.63 \mathrm{E}-05\end{array}$ & \\
\hline Shipping Site: Paducah & $\begin{array}{l}\text { Total Volume } \\
(\mathrm{m} 3 / \mathrm{yr})\end{array}$ & $\begin{array}{cc}\text { Total Mass } \\
(\mathrm{kg} / \mathrm{yr})\end{array}$ & $1.20 \mathrm{E}+07$ \\
\hline$\cdot$ & $\begin{array}{l}\text { Radionuclide } \\
-\mathrm{Tc}-99 \\
\mathrm{Tl}-208 \\
\mathrm{~Pb}-212 \\
\mathrm{Bi}-212 \\
\mathrm{Po}-212 \\
\mathrm{PO}-216 \\
\mathrm{Ra}-224 \\
\mathrm{Ra}-228 \\
\mathrm{Ac}-228 \\
\mathrm{Th}-228 \\
\mathrm{Th}-231 \\
\mathrm{Th}-232 \\
\mathrm{Th}-234 \\
\mathrm{~Pa}-234 \\
\mathrm{~Pa}-234 \mathrm{~m} \\
\mathrm{U}-235 \\
\mathrm{U}-238 \\
\mathrm{~Np}-237 \\
\mathrm{Pu}-239\end{array}$ & $\begin{array}{c}\begin{array}{c}\text { Activity } \\
(C i / y r)\end{array} \\
-8.84 \mathrm{E}-03 \\
3.72 \mathrm{E}-05 \\
9.84 \mathrm{E}-05 \\
9.84 \mathrm{E}-05 \\
6.34 \mathrm{E}-05 \\
9.84 \mathrm{E}-05 \\
9.84 \mathrm{E}-05 \\
5.88 \mathrm{E}-04 \\
5.88 \mathrm{E}-04 \\
9.84 \mathrm{E}-05 \\
5.66 \mathrm{E}-04 \\
5.97 \mathrm{E}-03 \\
7.26 \mathrm{E}-01 \\
7.44 \mathrm{E}-05 \\
7.26 \mathrm{E}-01 \\
5.64 \mathrm{E}-04 \\
7.26 \mathrm{E}-01 \\
6.63 \mathrm{E}-03 \\
6.63 \mathrm{E}-03\end{array}$ & \\
\hline \multirow[t]{2}{*}{ Shipping Site: Pinellas } & $\begin{array}{l}\text { Total volume } \\
(\mathrm{m} 3 / \mathrm{yr})\end{array}$ & $\begin{array}{l}\text { Total Mass } \\
\text { (kg/Yr) }\end{array}$ & $1.91 \mathrm{E}+04$ \\
\hline & $\begin{array}{c}\text { Radionuclide } \\
\mathrm{H}-3\end{array}$ & $\begin{array}{c}\begin{array}{c}\text { Activity } \\
\text { (Ci/yI) }\end{array} \\
1.99 E+04\end{array}$ & \\
\hline \multirow[t]{2}{*}{ Shipping Site: RMI } & $\begin{array}{l}\text { Total volume } \\
\text { (m3/yx) }\end{array}$ & $\begin{array}{c}\text { Total Mass } \\
(\mathrm{kg} / \mathrm{yr})\end{array}$ & $1.47 \mathrm{E}+07$ \\
\hline & $\begin{array}{l}\text { Radionuclide } \\
\mathrm{Tl}-208 \\
\mathrm{~Pb}-212 \\
\mathrm{Bi}-212 \\
\mathrm{PO}-212 \\
\mathrm{Po}-216 \\
\mathrm{Ra}-224 \\
\mathrm{Ra}-228 \\
\mathrm{Ac}-228 \\
\mathrm{Th}-228 \\
\mathrm{Th}-231 \\
\mathrm{Th}-232 \\
\mathrm{Th}-234 \\
\mathrm{~Pa}-234 \\
\mathrm{~Pa}-234 \mathrm{~m} \\
\mathrm{U}-235 \\
\mathrm{U}-238\end{array}$ & 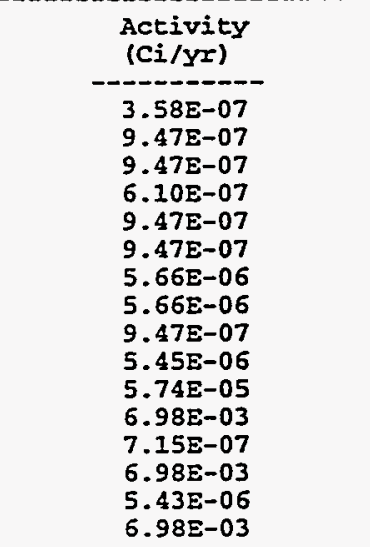 & \\
\hline
\end{tabular}




\section{A- 142}

\begin{tabular}{|c|c|c|c|}
\hline Shipping Site: $Y-12$ & $\begin{array}{l}\text { Total Volume } \\
(\mathrm{m} 3 / \mathrm{yr})\end{array}$ & $\begin{array}{c}\text { Total Mass } \\
(\mathrm{kg} / \mathrm{Yr})\end{array}$ & $8.27 \mathrm{E}+07$ \\
\hline & $\begin{array}{l}\text { Radionuclide } \\
\mathrm{Tl-208} \\
\mathrm{Pb}-212 \\
\mathrm{Bi}-212 \\
\mathrm{Po}-212 \\
\mathrm{Po-216} \\
\mathrm{Ra}-224 \\
\mathrm{Ra}-228 \\
\mathrm{Ac-228} \\
\mathrm{Th}-228 \\
\mathrm{Th}-231 \\
\mathrm{Th}-232 \\
\mathrm{Th}-234 \\
\mathrm{~Pa}-234 \\
\mathrm{~Pa}-234 \mathrm{~m} \\
\mathrm{U}-235 \\
\mathrm{U}-238\end{array}$ & $\begin{array}{c}\begin{array}{c}\text { Activity } \\
(C i / y r)\end{array} \\
-6.80 \mathrm{E}-05 \\
1.80 \mathrm{E}-04 \\
1.80 \mathrm{E}-04 \\
1.16 \mathrm{E}-04 \\
1.80 \mathrm{E}-04 \\
1.80 \mathrm{E}-04 \\
1.07 \mathrm{E}-03 \\
1.07 \mathrm{E}-03 \\
1.80 \mathrm{E}-04 \\
1.03 \mathrm{E}-03 \\
1.09 \mathrm{E}-02 \\
1.32 \mathrm{E}+00 \\
1.36 \mathrm{E}-04 \\
1.32 \mathrm{E}+00 \\
1.03 \mathrm{E}-03 \\
1.32 \mathrm{E}+00\end{array}$ & \\
\hline
\end{tabular}




\section{A.18 WM LLW REGIONALIZED 7 ALTERNATIVE (CASE 6): ACTIVATED METALS}

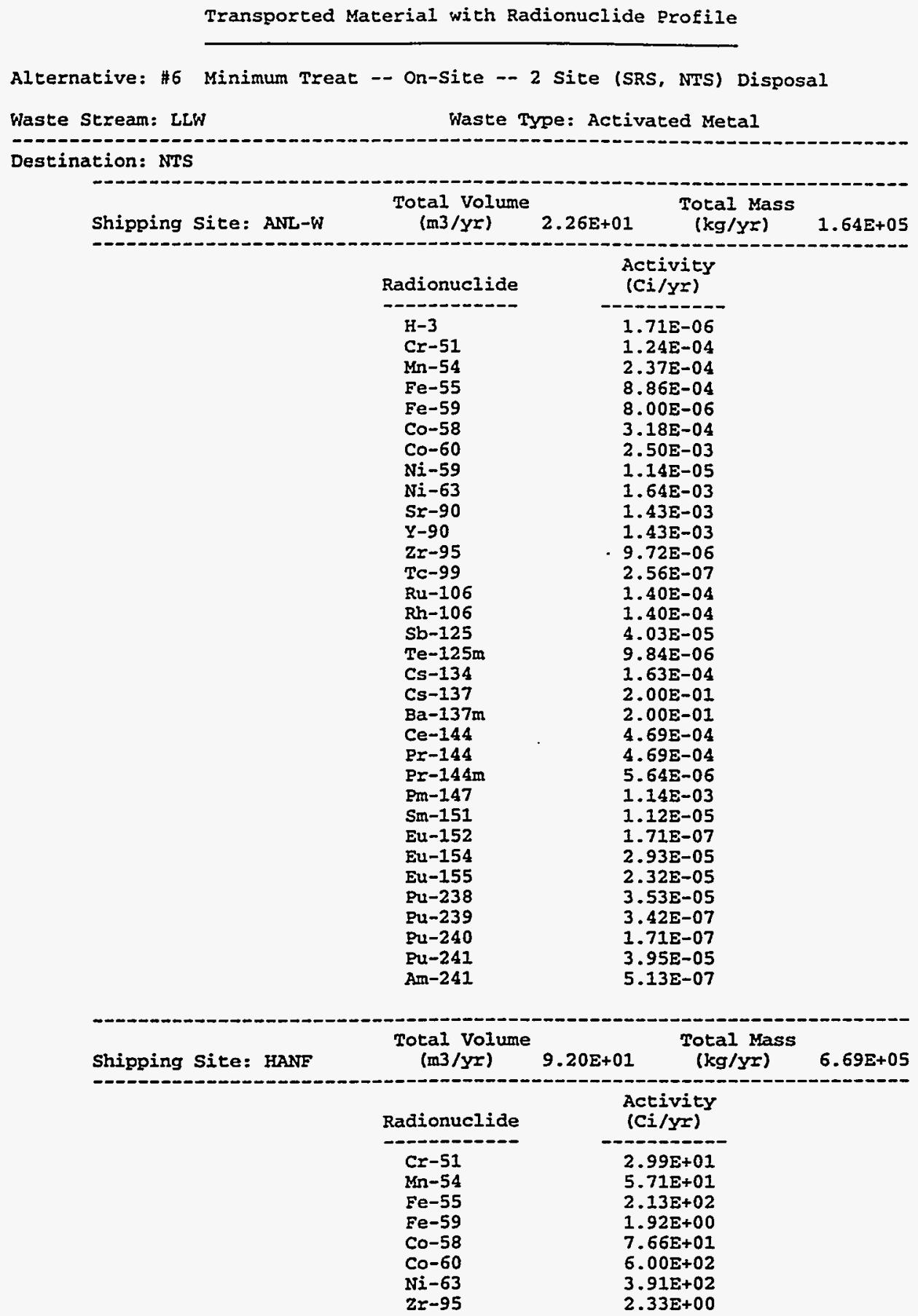




\begin{tabular}{|c|c|c|c|}
\hline Shipping Site: LANL & $\begin{array}{l}\text { Total volume } \\
(\mathrm{m} 3 / y \mathrm{r})\end{array}$ & $\begin{array}{cc}\text { Total Mass } \\
(\mathrm{kg} / \mathrm{Yr})\end{array}$ & $8.57 E+06$ \\
\hline & $\begin{array}{l}\text { Radionuclide } \\
\mathrm{Cr}-51 \\
\mathrm{Mn}-54 \\
\mathrm{Fe}-55 \\
\mathrm{Fe}-59 \\
\mathrm{Co}-58 \\
\mathrm{Co}-60 \\
\mathrm{Ni}-59 \\
\mathrm{Ni}-63 \\
\mathrm{Zr}-95\end{array}$ & $\begin{array}{l}\text { Activity } \\
\text { (Ci/yr) } \\
2.42 \mathrm{E}+01 \\
4.62 \mathrm{E}+01 \\
1.72 \mathrm{E}+02 \\
1.55 \mathrm{E}+00 \\
6.20 \mathrm{E}+01 \\
4.86 \mathrm{E}+02 \\
2.22 \mathrm{E}+00 \\
3.16 \mathrm{E}+02 \\
1.89 \mathrm{E}+00\end{array}$ & \\
\hline Shipping Site: LBL & $\begin{array}{l}\text { Total volume } \\
\text { (m3/yr) }\end{array}$ & $3.95 \mathrm{E}+01 \quad \begin{array}{c}\text { Total Mass } \\
(\mathrm{kg} / \mathrm{yr})\end{array}$ & $2.87 E+05$ \\
\hline & $\begin{array}{l}\text { Radionuclide } \\
\text { Cr-51 } \\
\text { Mn-54 } \\
\text { Fe-55 } \\
\text { Fe-59 } \\
\text { Co-58 } \\
\text { Co-60 } \\
\mathrm{Ni}-59 \\
\mathrm{Ni}-63 \\
\mathrm{Zr}-95\end{array}$ & $\begin{array}{l}\begin{array}{l}\text { Activity } \\
\text { (Ci/yr) }\end{array} \\
-6.32 \mathrm{E}-02 \\
1.20 \mathrm{E}-01 \\
4.49 \mathrm{E}-01 \\
4.06 \mathrm{E}-03 \\
1.61 \mathrm{E}-01 \\
1.26 \mathrm{E}+00 \\
5.80 \mathrm{E}-03 \\
8.24 \mathrm{E}-01 \\
4.93 \mathrm{E}-03\end{array}$ & \\
\hline Shipping Site: NRF & $\begin{array}{l}\text { Total Volume } \\
\text { (m3/yr) }\end{array}$ & $\begin{array}{cc}\text { Total Mass } \\
(\mathrm{kg} / \mathrm{yr})\end{array}$ & $9.40 E+06$ \\
\hline & $\begin{array}{l}\text { Radionuclide } \\
\mathrm{Cr}-51 \\
\mathrm{Mn}-54 \\
\mathrm{Fe}-55 \\
\mathrm{Fe}-59 \\
\mathrm{Co}-58 \\
\mathrm{Co}-60 \\
\mathrm{Ni}-59 \\
\mathrm{Ni}-63 \\
\mathrm{Zr}-95\end{array}$ & $\begin{array}{c}\begin{array}{l}\text { Activity } \\
\text { (Ci/yx) }\end{array} \\
-1.31 E+03 \\
4.40 E+03 \\
1.64 E+04 \\
1.48 E+02 \\
5.91 E+03 \\
4.63 E+04 \\
2.12 E+02 \\
3.01 E+04 \\
1.80 E+02\end{array}$ & \\
\hline Shipping Site: SNLA & $\begin{array}{l}\text { Total Volume } \\
\text { (m3/yr) }\end{array}$ & $\begin{array}{c}\text { Total Mass } \\
(\mathrm{kg} / \mathrm{Y}=)\end{array}$ & $1.15 \mathrm{E}+05$ \\
\hline . & $\begin{array}{l}\text { Radionuclide } \\
\mathrm{Cr}-51 \\
\mathrm{Mn}-54 \\
\mathrm{Fe}-55 \\
\mathrm{Fe}-59 \\
\mathrm{Co}-58 \\
\mathrm{Co}-60 \\
\mathrm{Ni}-59 \\
\mathrm{Ni}-63 \\
\mathrm{Zr}-95\end{array}$ & $\begin{array}{l}\begin{array}{c}\text { Activity } \\
\text { (Ci/yr) }\end{array} \\
-6.54 \mathrm{E}-04 \\
1.24 \mathrm{E}-03 \\
4.64 \mathrm{E}-03 \\
4.20 \mathrm{E}-05 \\
1.67 \mathrm{E}-03 \\
1.31 \mathrm{E}-02 \\
6.00 \mathrm{E}-05 \\
8.53 \mathrm{E}-03 \\
5.10 \mathrm{E}-05\end{array}$ & \\
\hline
\end{tabular}


Destination: SRS

\begin{tabular}{|c|c|c|c|}
\hline Shipping site: BAPL & $\begin{array}{l}\text { Total Volume } \\
\text { (m3/yr) }\end{array}$ & $\begin{array}{l}\text { Toral Mass } \\
(\mathrm{kg} / \mathrm{yr})\end{array}$ & $6.43 E+06$ \\
\hline & $\begin{array}{l}\text { Radionuclide } \\
\mathrm{Cr}-51 \\
\mathrm{Mn}-54 \\
\mathrm{Fe}-55 \\
\mathrm{Fe}-59 \\
\mathrm{Co}-58 \\
\mathrm{Co}-60 \\
\mathrm{Ni}-59 \\
\mathrm{Ni}-63 \\
\mathrm{Zr}-95 \\
\mathrm{TI}-208 \\
\mathrm{~Pb}-212 \\
\mathrm{Bi}-212 \\
\mathrm{Po}-212 \\
\mathrm{Po}-216 \\
\mathrm{Ra}-224 \\
\mathrm{Ra}-228 \\
\mathrm{Ac}-228 \\
\mathrm{Th}-228 \\
\mathrm{Th}-231 \\
\mathrm{Th}-232 \\
\mathrm{Th}-234 \\
\mathrm{~Pa}-234 \\
\mathrm{~Pa}-234 \mathrm{~m} \\
\mathrm{U}-235 \\
\mathrm{U}-238\end{array}$ & $\begin{array}{l}\text { Activity } \\
\text { (Ci/yr) } \\
4.75 \mathrm{E}+03 \\
9.04 \mathrm{E}+03 \\
3.37 \mathrm{E}+04 \\
3.05 \mathrm{E}+02 \\
1.21 \mathrm{E}+04 \\
9.52 \mathrm{E}+04 \\
4.36 \mathrm{E}+02 \\
6.19 \mathrm{E}+04 \\
3.70 \mathrm{E}+02 \\
1.36 \mathrm{E}-08 \\
3.60 \mathrm{E}-08 \\
3.60 \mathrm{E}-08 \\
2.32 \mathrm{E}-08 \\
3.60 \mathrm{E}-08 \\
3.60 \mathrm{E}-08 \\
2.15 \mathrm{E}-07 \\
2.15 \mathrm{E}-07 \\
3.60 \mathrm{E}-08 \\
2.07 \mathrm{E}-07 \\
2.18 \mathrm{E}-06 \\
2.65 \mathrm{E}-04 \\
2.72 \mathrm{E}-08 \\
2.65 \mathrm{E}-04 \\
2.06 \mathrm{E}-07 \\
2.65 \mathrm{E}-04\end{array}$ & \\
\hline Shipping Sice: FNAL & $\begin{array}{l}\text { Total Volume } \\
(\mathrm{m} 3 / \mathrm{y})\end{array}$ & $\begin{array}{c}\text { Total Mass } \\
\text { (kg/ } / \mathrm{Yx})\end{array}$ & $8.14 E+05$ \\
\hline & $\begin{array}{l}\text { Radionuclide } \\
\mathrm{Cr}-51 \\
\mathrm{Mn}-54 \\
\mathrm{Fe}-55 \\
\mathrm{Fe}-59 \\
\mathrm{Co}-58 \\
\mathrm{Co}-60 \\
\mathrm{Ni}-59 \\
\mathrm{Ni}-63 \\
\mathrm{Zr}-95\end{array}$ & $\begin{array}{c}\begin{array}{c}\text { Activity } \\
\text { (Ci/Yr) }\end{array} \\
-3.35 \mathrm{E}-02 \\
6.39 \mathrm{E}-02 \\
2.38 \mathrm{E}-01 \\
2.15 \mathrm{E}-03 \\
8.57 \mathrm{E}-02 \\
6.72 \mathrm{E}-01 \\
3.08 \mathrm{E}-03 \\
4.37 \mathrm{E}-01 \\
2.61 \mathrm{E}-03\end{array}$ & \\
\hline
\end{tabular}


A-146

\begin{tabular}{|c|c|c|c|}
\hline Shipping site: KAPL & $\begin{array}{l}\text { Total volume } \\
(\mathrm{m} 3 / \mathrm{yr})\end{array}$ & $\begin{array}{c}\text { Total Mass } \\
(\mathrm{kg} / \mathrm{Yr})\end{array}$ & $1.33 \mathrm{E}+07$ \\
\hline & 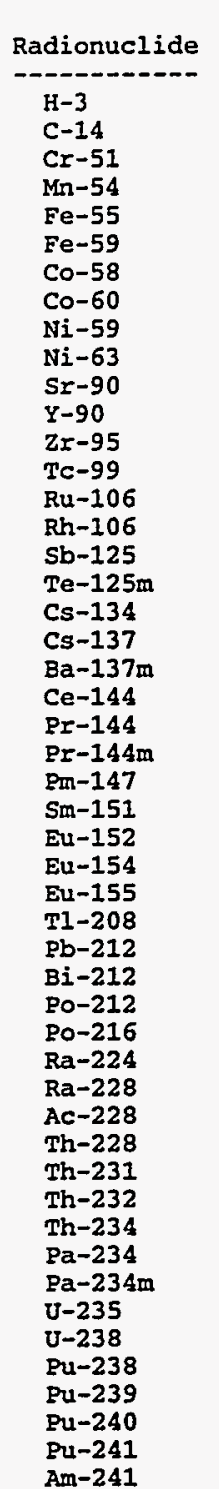 & $\begin{array}{l}\text { Activity } \\
(C i / y r) \\
--15 \mathrm{-}--- \\
5.15 \mathrm{E}-04 \\
2.40 \mathrm{E}-05 \\
1.13 \mathrm{E}+04 \\
2.15 \mathrm{E}+04 \\
8.05 \mathrm{E}+04 \\
7.28 \mathrm{E}+02 \\
2.89 \mathrm{E}+04 \\
2.27 \mathrm{E}+05 \\
1.04 \mathrm{E}+03 \\
1.47 \mathrm{E}+05 \\
2.62 \mathrm{E}-02 \\
2.62 \mathrm{E}-02 \\
8.84 \mathrm{E}+02 \\
5.20 \mathrm{E}-05 \\
2.22 \mathrm{E}-03 \\
2.22 \mathrm{E}-03 \\
6.40 \mathrm{E}-04 \\
1.56 \mathrm{E}-04 \\
8.18 \mathrm{E}-03 \\
3.13 \mathrm{E}-02 \\
2.96 \mathrm{E}-02 \\
7.46 \mathrm{E}-03 \\
7.46 \mathrm{E}-03 \\
8.97 \mathrm{E}-05 \\
1.82 \mathrm{E}-02 \\
1.78 \mathrm{E}-04 \\
2.71 \mathrm{E}-06 \\
4.66 \mathrm{E}-04 \\
3.69 \mathrm{E}-04 \\
2.96 \mathrm{E}-08 \\
7.83 \mathrm{E}-08 \\
7.83 \mathrm{E}-08 \\
5.05 \mathrm{E}-08 \\
7.83 \mathrm{E}-08 \\
7.83 \mathrm{E}-08 \\
4.68 \mathrm{E}-07 \\
4.68 \mathrm{E}-07 \\
7.83 \mathrm{E}-08 \\
4.50 \mathrm{E}-07 \\
4.75 \mathrm{E}-06 \\
5.77 \mathrm{E}-04 \\
5.92 \mathrm{E}-08 \\
5.77 \mathrm{E}-04 \\
4.48 \mathrm{E}-07 \\
8.69 \mathrm{E}-04 \\
5.61 \mathrm{E}-04 \\
5.43 \mathrm{E}-06 \\
2.71 \mathrm{E}-06 \\
6.28 \mathrm{E}-04 \\
8.15 \mathrm{E}-06\end{array}$ & \\
\hline
\end{tabular}




\section{A.19 WM LLW CENTRALIZED 1 ALTERNATIVE (CASE 7): HETEROGENEOUS SOLIDS}

Transported Material with Radionuclide Profile

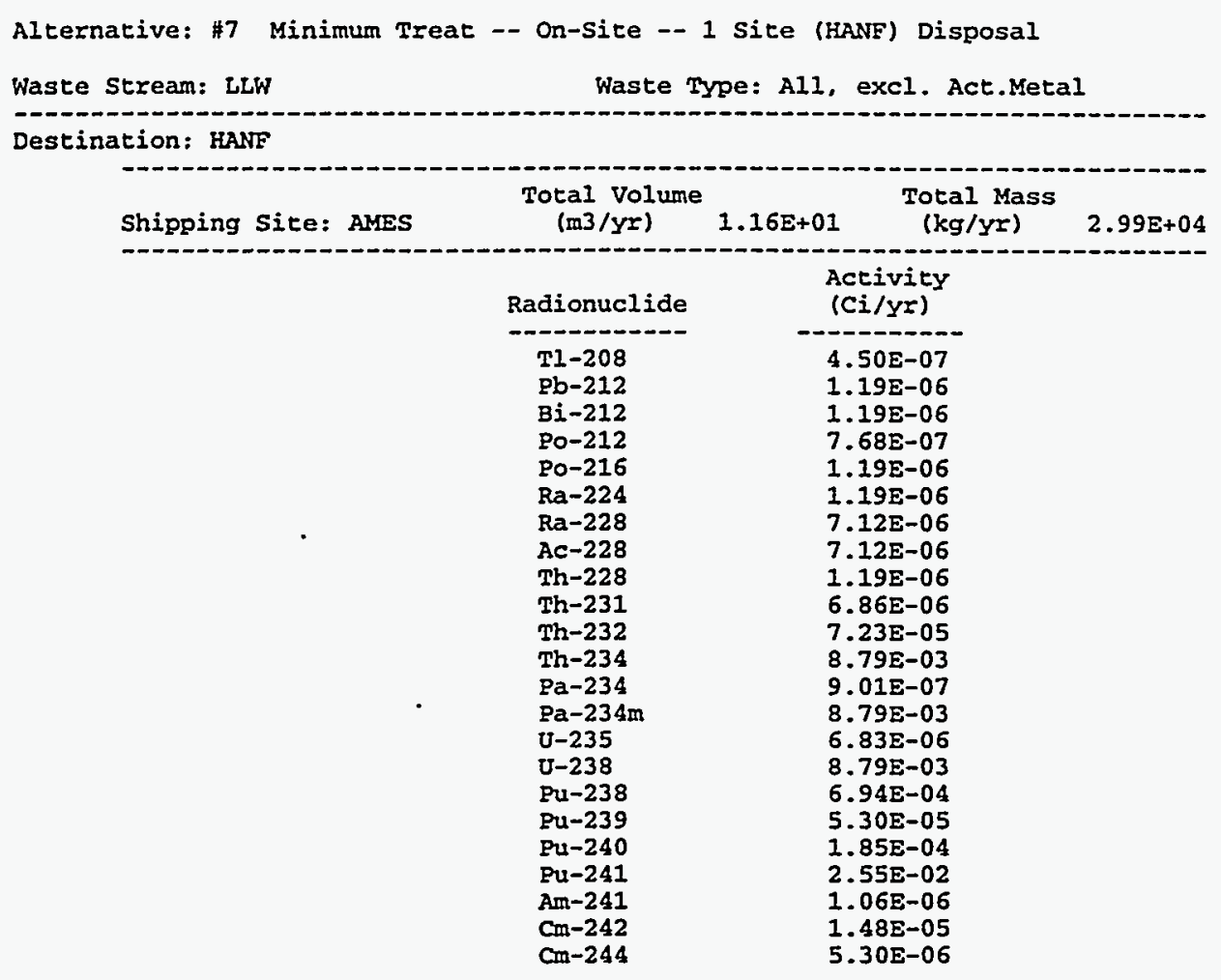


A- 148

\begin{tabular}{|c|c|c|c|}
\hline Shipping Sice: ANL-E & $\begin{array}{l}\text { Total Volume } \\
\text { (m3/yr) }\end{array}$ & $\begin{array}{l}\text { Toral Mass } \\
\text { (kg/yr) }\end{array}$ & $2.00 E+06$ \\
\hline & \begin{tabular}{l} 
Radionuclide \\
\hdashline$H-3$ \\
$\mathrm{C}-14$ \\
$\mathrm{Cr}-51$ \\
$\mathrm{Mn}-54$ \\
$\mathrm{Fe}-55$ \\
$\mathrm{Fe}-59$ \\
$\mathrm{Co}-58$ \\
$\mathrm{Co}-60$ \\
$\mathrm{Ni}-59$ \\
$\mathrm{Ni}-63$ \\
$\mathrm{Sr}-90$ \\
$\mathrm{Y}-90$ \\
$\mathrm{zr}-95$ \\
$\mathrm{~T} c-99$ \\
$\mathrm{Ru}-106$ \\
$\mathrm{Rh}-106$ \\
$\mathrm{Sb}-125$ \\
$\mathrm{Te}-125 \mathrm{~m}$ \\
$\mathrm{Cs}-134$ \\
$\mathrm{Cs}-137$ \\
$\mathrm{Ba}-137 \mathrm{~m}$ \\
$\mathrm{Ce}-144$ \\
$\mathrm{Pr}-144$ \\
$\mathrm{Pr}-144 \mathrm{~m}$ \\
$\mathrm{Pm}-147$ \\
$\mathrm{Sm}-151$ \\
Eu-152 \\
Eu-154 \\
$\mathrm{Eu}-155$ \\
$\mathrm{U}-238$ \\
$\mathrm{Pu}-238$ \\
$\mathrm{Pu}-239$ \\
$\mathrm{Pu}-240$ \\
$\mathrm{Pu}-241$ \\
$\mathrm{Am}-241$
\end{tabular} & $\begin{array}{c}\text { Activity } \\
(C i / y r) \\
1.33 \mathrm{E}+02 \\
7.91 \mathrm{E}-02 \\
2.87 \mathrm{E}+00 \\
1.43 \mathrm{E}+01 \\
2.04 \mathrm{E}+01 \\
1.84 \mathrm{E}-01 \\
1.55 \mathrm{E}+01 \\
8.15 \mathrm{E}+01 \\
2.63 \mathrm{E}-01 \\
3.77 \mathrm{E}+01 \\
3.33 \mathrm{E}+01 \\
3.33 \mathrm{E}+01 \\
2.24 \mathrm{E}-01 \\
1.62 \mathrm{E}-01 \\
2.16 \mathrm{E}+00 \\
2.16 \mathrm{E}+00 \\
6.21 \mathrm{E}-01 \\
1.51 \mathrm{E}-01 \\
2.09 \mathrm{E}+01 \\
4.75 \mathrm{E}+01 \\
4.50 \mathrm{E}+01 \\
7.24 \mathrm{E}+00 \\
7.24 \mathrm{E}+00 \\
8.70 \mathrm{E}-02 \\
1.76 \mathrm{E}+01 \\
1.72 \mathrm{E}-01 \\
2.63 \mathrm{E}-03 \\
4.52 \mathrm{E}-01 \\
3.58 \mathrm{E}-01 \\
9.63 \mathrm{E}-01 \\
5.44 \mathrm{E}-01 \\
5.27 \mathrm{E}-03 \\
2.63 \mathrm{E}-03 \\
6.09 \mathrm{E}-01 \\
7.91 \mathrm{E}-03\end{array}$ & \\
\hline
\end{tabular}


A-149

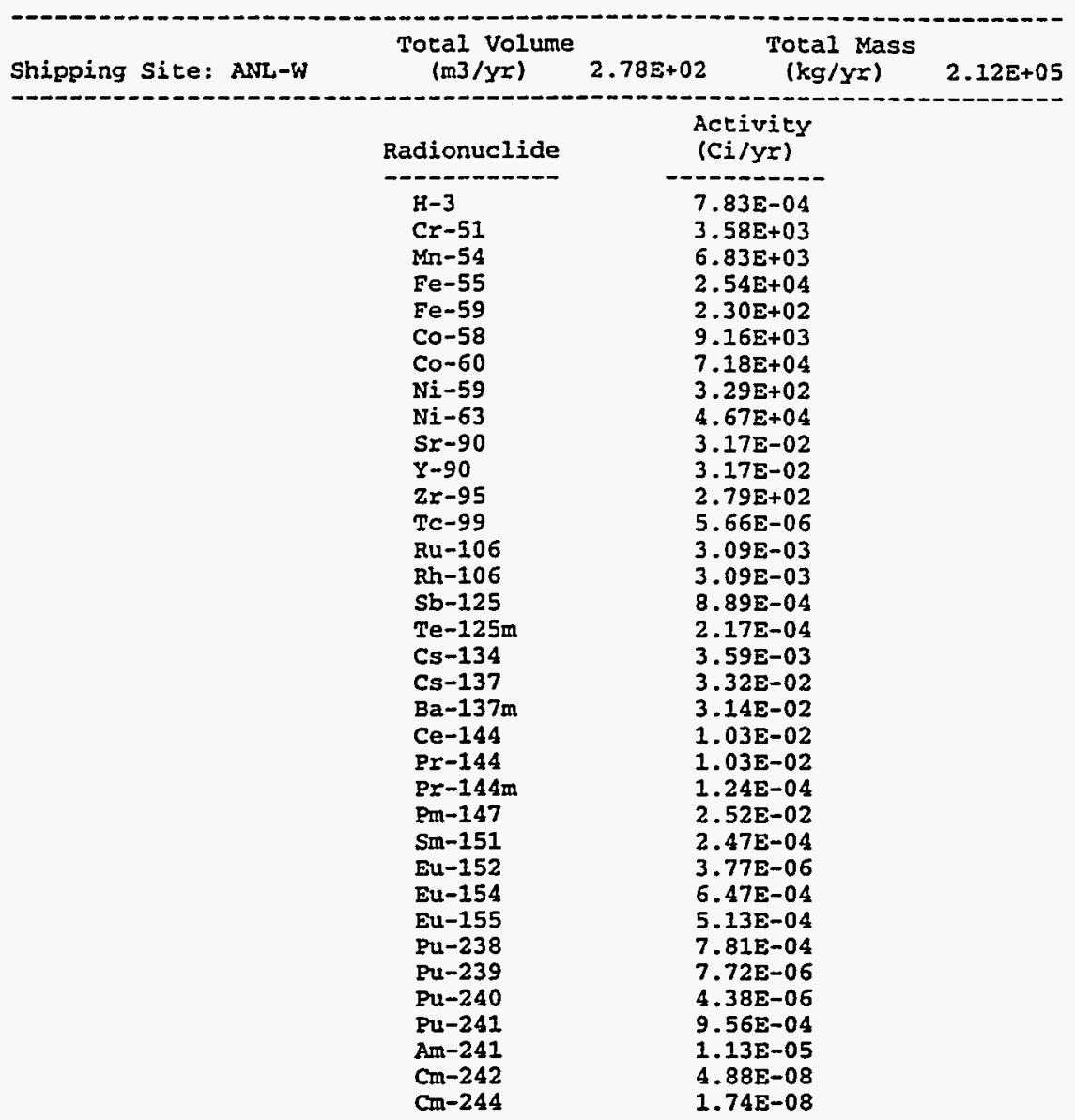


The doses calculated for the first three population groups are added together to yield the collective dose to the general public; the dose calculated for the fourth group represents the collective dose to workers. The RADTRAN 4 models for routine dose are not intended for estimating specific risks to individuals.

The RADTRAN 4 calculations for routine dose are based on generically expressing the dose rate as a function of distance from a point source (Neuhauser and Kanipe 1993). Associated with the calculation of routine doses for each exposed population group are parameters such as the radiation field strength, source-receptor distance, duration of exposure, vehicular speed, stopping time, traffic density, and route characteristics such as population density. The RADTRAN manual contains derivations of the equations and descriptions of these parameters (Neuhauser and Kanipe 1993). The values for many of the most important parameters are presented in Section 4.

The collective routine risks are calculated for each LLW alternative as follows. Each alternative is first defined as a set of origin-destination pairs, as shown in the WASTE_MGMT output files in Appendix A. Representative highway and rail routes are then determined for each unique pair. The number of shipments transported across each linkage is then calculated for both truck and rail modes by dividing the estimated site-specific LLW inventories by the assumed truck and rail shipment capacities. For shipments between each origin-destination pair, RADTRAN 4 is used to calculate the collective risks to workers and the public on the basis of representative radiological and physical properties of the LLW. The collective risks are then summed over the set of origin-destination pairs to estimate the collective routine risks associated with that alternative.

\subsubsection{Maximally Exposed Individual Risk}

In addition to the assessment of the routine collective population risk, the risk to MEIs has been estimated for a number of hypothetical exposure scenarios by using RISKIND. The receptors include transportation crew members, departure inspectors, and members of the public exposed during traffic delays, while working at a service station, or while living near a DOE site.

The dose to each MEI considered is calculated with RISKIND for an exposure scenario defined by a given distance, duration, and frequency of exposure specific to that receptor. The distances and durations of exposure are similar to those given in previous transportation risk assessments (DOE 1987b, 1990a) and are presented in Section 4. The scenarios are not meant to be exhaustive but were selected to provide a range of potential exposure situations.

The RISKIND external dose model considers direct external exposure and exposure from radiation scattered from the ground and air. RISKIND is used to calculate the dose as a function of distance from a shipment (millirem per hour for stationary exposures and millirem per event for 


\begin{tabular}{|c|c|c|c|}
\hline Shipping Sice: BAPL & $\begin{array}{l}\text { Total volume } \\
(\mathrm{m} 3 / \mathrm{yr})\end{array}$ & $\begin{array}{l}\text { Total Mass } \\
(\mathrm{kg} / \mathrm{yr})\end{array}$ & $1.08 E+06$ \\
\hline . & $\begin{array}{l}\text { Radionuclide } \\
\mathrm{H}-3 \\
\mathrm{Co}-60 \\
\mathrm{Ni}-63 \\
\mathrm{Sr}-90 \\
\mathrm{Y}-90 \\
\mathrm{Tc}-99 \\
\mathrm{Ru}-106 \\
\mathrm{Rh}-106 \\
\mathrm{Sb}-125 \\
\mathrm{Te}-125 \mathrm{~m} \\
\mathrm{Cs}-134 \\
\mathrm{Cs}-137 \\
\mathrm{Ba}-137 \mathrm{~m} \\
\mathrm{Ce}-144 \\
\mathrm{Pr}-144 \\
\mathrm{Pr}-144 \mathrm{~m} \\
\mathrm{Pm}-147 \\
\mathrm{Sm}-151 \\
\mathrm{Eu}-152 \\
\mathrm{Eu}-154 \\
\mathrm{Eu}-155 \\
\mathrm{~T} 1-208 \\
\mathrm{~Pb}-212 \\
\mathrm{Bi}-212 \\
\mathrm{Po}-212 \\
\mathrm{Po}-216 \\
\mathrm{Ra}-224 \\
\mathrm{Ra}-228 \\
\mathrm{Ac}-228 \\
\mathrm{Th}-228 \\
\mathrm{Th}-231 \\
\mathrm{Th}-232 \\
\mathrm{Th}-234 \\
\mathrm{~Pa}-234 \\
\mathrm{~Pa}-234 \mathrm{~m} \\
\mathrm{U}-235 \\
\mathrm{U}-238 \\
\mathrm{Pu}-238 \\
\mathrm{Pu}-239 \\
\mathrm{Pu}-240 \\
\mathrm{Pu}-241 \\
\mathrm{Am}-241 \\
\end{array}$ & 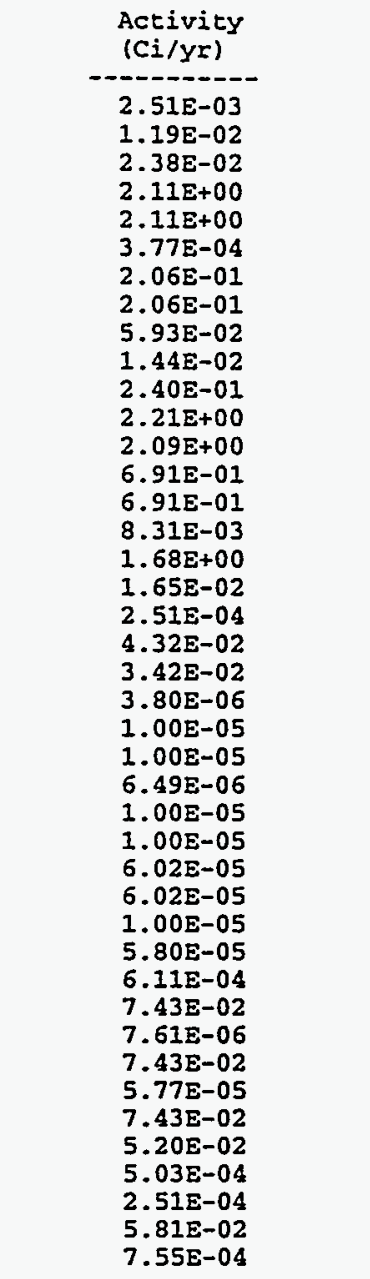 & \\
\hline \multirow[t]{2}{*}{ Shipping Site: FNAI } & $\begin{array}{l}\text { Total Volume } \\
(\mathrm{m} 3 / \mathrm{y} x)\end{array}$ & $\begin{array}{c}\text { Total Mass } \\
(\mathrm{kg} / \mathrm{yx})\end{array}$ & $5.11 E+04$ \\
\hline & $\begin{array}{l}\text { Radionuclide } \\
\mathrm{H}-3 \\
\mathrm{Cr}-51 \\
\mathrm{Mn}-54 \\
\mathrm{Fe}-55 \\
\mathrm{Fe}-59 \\
\mathrm{Co}-58 \\
\mathrm{Co}-60 \\
\mathrm{~N}-59 \\
\mathrm{Ni}-53 \\
\mathrm{Zr}-95 \\
\mathrm{~T}-208 \\
\mathrm{~Pb}-212 \\
\mathrm{Bi}-212 \\
\mathrm{To-212} \\
\mathrm{Po-216} \\
\mathrm{Ra}-224 \\
\mathrm{Ra-228} \\
\mathrm{Ac-228} \\
\mathrm{Th}-228 \\
\mathrm{Th}-231 \\
\mathrm{Th}-232 \\
\mathrm{Th}-234 \\
\mathrm{~Pa}-234 \\
\mathrm{~Pa}-234 \mathrm{~m} \\
\mathrm{U}-235 \\
\mathrm{U}-238\end{array}$ & $\begin{array}{c}\begin{array}{c}\text { Activity } \\
(C i / y I)\end{array} \\
-1.81 \mathrm{E}-01 \\
1.11 \mathrm{E}-02 \\
2.12 \mathrm{E}-02 \\
7.94 \mathrm{E}-02 \\
7.17 \mathrm{E}-04 \\
2.85 \mathrm{E}-02 \\
2.23 \mathrm{E}-01 \\
1.02 \mathrm{E}-03 \\
1.45 \mathrm{E}-01 \\
8.71 \mathrm{E}-04 \\
3.78 \mathrm{E}-07 \\
1.00 \mathrm{E}-06 \\
1.00 \mathrm{E}-06 \\
6.46 \mathrm{E}-07 \\
1.00 \mathrm{E}-06 \\
1.00 \mathrm{E}-06 \\
5.99 \mathrm{E}-06 \\
5.99 \mathrm{E}-06 \\
1.00 \mathrm{E}-06 \\
5.77 \mathrm{E}-06 \\
6.08 \mathrm{E}-05 \\
7.39 \mathrm{E}-03 \\
7.57 \mathrm{E}-07 \\
7.39 \mathrm{E}-03 \\
5.75 \mathrm{E}-06 \\
7.39 \mathrm{E}-03\end{array}$ & \\
\hline
\end{tabular}




\begin{tabular}{|c|c|c|c|}
\hline Shipping Sice: INEL & $\begin{array}{l}\text { Total volume } \\
\text { (m3/yx) }\end{array}$ & $\begin{array}{cc}\text { Total Mass } \\
\text { (kg/yx) }\end{array}$ & 1. $64 \mathrm{E}+07$ \\
\hline & $\begin{array}{l}\text { Radionuclide } \\
\text { H-3 } \\
\text { Cr-51 } \\
\text { Mn-54 } \\
\text { Fe-55 } \\
\text { Fe-59 } \\
\text { Co-58 } \\
\text { Co-60 } \\
\mathrm{Ni}-59 \\
\mathrm{Ni}-63 \\
\mathrm{Sr}-90 \\
\mathrm{Y}-90 \\
\mathrm{Zr}-95 \\
\mathrm{Tc}-99 \\
\mathrm{Ru}-106 \\
\mathrm{Rh}-106 \\
\mathrm{Sb}-125 \\
\mathrm{Te}-125 \mathrm{~m} \\
\mathrm{Cs}-134 \\
\mathrm{Cs}-137 \\
\mathrm{Ba}-137 \mathrm{~m} \\
\mathrm{Ce}-144 \\
\mathrm{Pr}-144 \\
\mathrm{Pr}-144 \mathrm{~m} \\
\mathrm{Pm}-147 \\
\mathrm{Sm}-151 \\
\mathrm{Eu}-152 \\
\mathrm{Eu}-154 \\
\mathrm{Eu}-155 \\
\mathrm{Pu}-238 \\
\mathrm{Pu}-239 \\
\mathrm{Pu}-240 \\
\mathrm{Pu}-241 \\
\mathrm{Am}-241\end{array}$ & $\begin{array}{l}\text { Activicy } \\
(C i / y r) \\
-1.56 E+00 \\
1.02 E+02 \\
1.94 E+02 \\
7.24 E+02 \\
6.55 E+00 \\
2.60 E+02 \\
2.04 E+03 \\
9.35 E+00 \\
1.33 E+03 \\
5.01 E+01 \\
5.01 E+01 \\
7.95 E+00 \\
8.95 E-03 \\
4.89 E+00 \\
4.89 E+00 \\
1.40 E+00 \\
3.43 E-01 \\
5.69 E+00 \\
5.25 E+01 \\
4.97 E+01 \\
1.63 E+01 \\
1.63 E+01 \\
1.97 E-01 \\
4.00 E+01 \\
3.91 E-01 \\
5.97 E-03 \\
1.02 E+00 \\
8.12 E-01 \\
1.23 E+00 \\
1.19 E-02 \\
5.97 E-03 \\
1.37 E+00 \\
1.79 E-02\end{array}$ & \\
\hline Shipping site: ITRI & $\begin{array}{l}\text { Total Volume } \\
\text { (m3/yx) }\end{array}$ & $\begin{array}{c}\text { Total Mass } \\
(\mathrm{kg} / \mathrm{Y})\end{array}$ & $1.64 \mathrm{E}+05$ \\
\hline . & $\begin{array}{l}\text { Radionuclide } \\
\mathrm{H}-3 \\
\mathrm{C}-14 \\
\mathrm{Mn}-54 \\
\mathrm{Co-58} \\
\mathrm{Co}-60 \\
\mathrm{Sr}-90 \\
\mathrm{x}-90 \\
\mathrm{Tc}-99 \\
\mathrm{Cs}-134 \\
\mathrm{Cs}-137 \\
\mathrm{Ba}-137 \mathrm{~m} \\
\mathrm{v}-238\end{array}$ & $\begin{array}{c}\text { Activity } \\
\text { (Ci/yr) } \\
6.17 \mathrm{E}+00 \\
3.03 \mathrm{E}-01 \\
3.41 \mathrm{E}+01 \\
3.15 \mathrm{E}+01 \\
9.12 \mathrm{E}+01 \\
4.28 \mathrm{E}+01 \\
4.28 \mathrm{E}+01 \\
6.07 \mathrm{E}-01 \\
7.07 \mathrm{E}+01 \\
9.33 \mathrm{E}+01 \\
8.82 \mathrm{E}+01 \\
3.69 \mathrm{E}+00\end{array}$ & \\
\hline Shipping Site: $\mathrm{K}-25$ & $\begin{array}{l}\text { Total volume } \\
\text { (m3/yr) }\end{array}$ & $\begin{array}{c}\text { Total Mass } \\
(\mathrm{kg} / \mathrm{yr})\end{array}$ & $4.67 E+07$ \\
\hline & $\begin{array}{l}\text { Radionuclide } \\
\mathrm{T} l-208 \\
\mathrm{~Pb}-212 \\
\mathrm{Bi}-212 \\
\mathrm{Po}-212 \\
\mathrm{Po}-216 \\
\mathrm{Ra}-224 \\
\mathrm{Ra}-228 \\
\mathrm{Ac}-228 \\
\mathrm{Th}-228 \\
\mathrm{Th}-231 \\
\mathrm{Th}-232 \\
\mathrm{Th}-234 \\
\mathrm{~Pa}-234 \\
\mathrm{~Pa}-234 \mathrm{~m} \\
\mathrm{U}-235 \\
\mathrm{U}-238\end{array}$ & $\begin{array}{c}\text { Activity } \\
(C i / y x) \\
1.06 E-04 \\
2.82 E-04 \\
2.82 E-04 \\
1.82 E-04 \\
2.82 E-04 \\
2.82 E-04 \\
1.68 E-03 \\
1.68 E-03 \\
2.82 E-04 \\
1.62 E-03 \\
1.71 E-02 \\
2.08 E+00 \\
2.13 E-04 \\
2.08 E+00 \\
1.62 E-03 \\
2.08 E+00\end{array}$ & \\
\hline
\end{tabular}




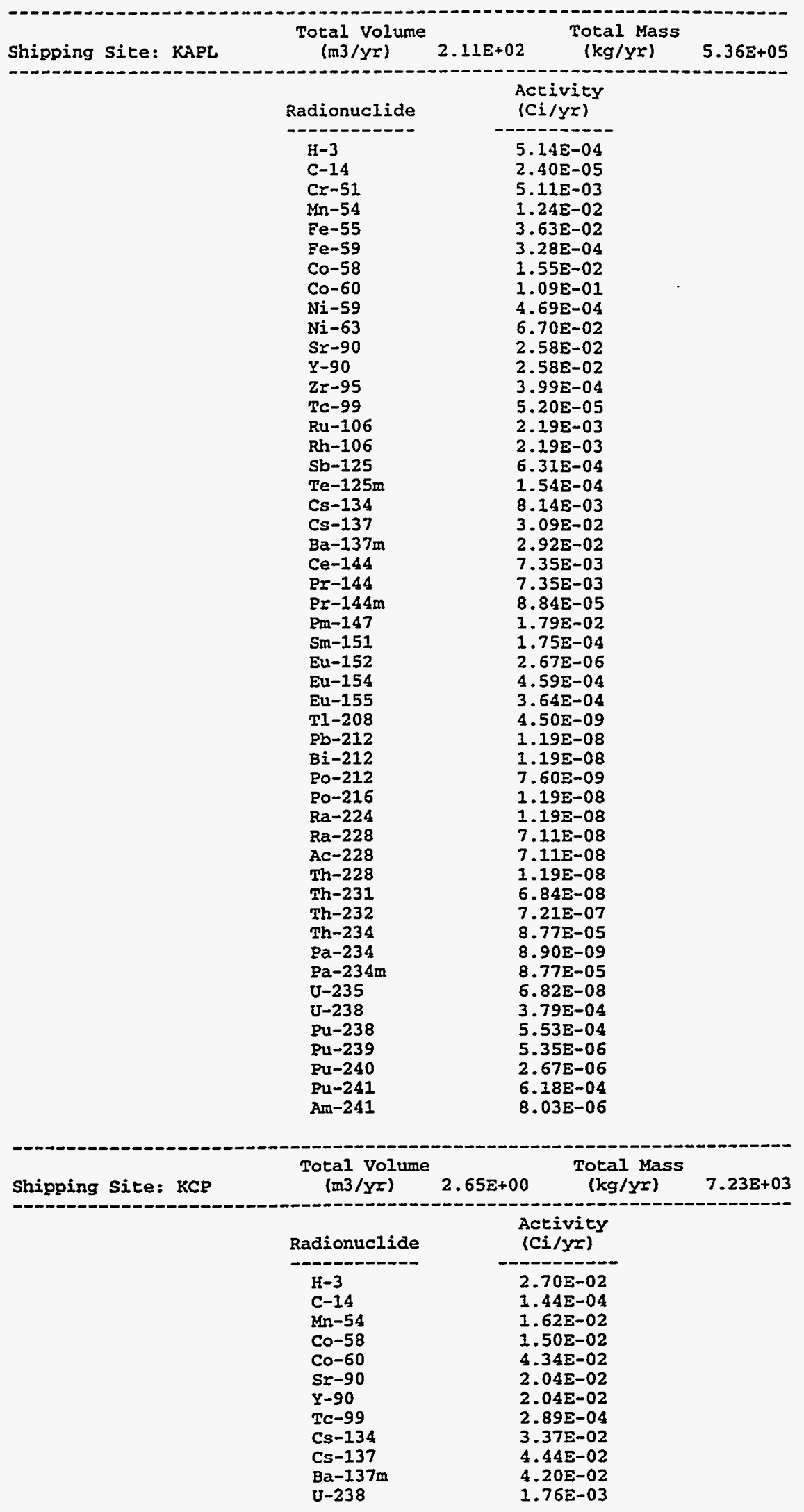




\section{A- 153}

\begin{tabular}{|c|c|c|c|}
\hline Shipping Site: LANL & $\begin{array}{l}\text { Total Volume } \\
(\mathrm{m} 3 / \mathrm{yr})\end{array}$ & $\begin{array}{c}\text { Total Mass } \\
(\mathrm{kg} / \mathrm{y} z)\end{array}$ & $1.22 \mathrm{E}+07$ \\
\hline & $\begin{array}{l}\text { Radionuclide } \\
\mathrm{H}-3 \\
\mathrm{Cr}-51 \\
\mathrm{Mn}-54 \\
\mathrm{Fe}-55 \\
\mathrm{Fe}-59 \\
\mathrm{Co}-58 \\
\mathrm{Co}-60 \\
\mathrm{Ni}-59 \\
\mathrm{Ni}-63 \\
\mathrm{Sr}-90 \\
\mathrm{Y}-90 \\
\mathrm{Zr}-95 \\
\mathrm{Tc}-99 \\
\mathrm{Ru}-106 \\
\mathrm{Rh}-106 \\
\mathrm{Sb}-125 \\
\mathrm{Te}-125 \mathrm{~m} \\
\mathrm{Cs}-134 \\
\mathrm{Cs}-137 \\
\mathrm{Ba}-137 \mathrm{~m} \\
\mathrm{Ce}-144 \\
\mathrm{Pr}-144 \\
\mathrm{Pr}-144 \mathrm{~m} \\
\mathrm{Pm}-147 \\
\mathrm{Sm}-151 \\
\mathrm{Eu}-152 \\
\mathrm{Eu}-154 \\
\mathrm{Eu}-155 \\
\mathrm{Tl}-208 \\
\mathrm{~Pb}-212 \\
\mathrm{Bi}-212 \\
\mathrm{Po}-212 \\
\mathrm{PO}-216 \\
\mathrm{Ra}-224 \\
\mathrm{Ra}-228 \\
\mathrm{Ac}-228 \\
\mathrm{Th}-228 \\
\mathrm{Th}-231 \\
\mathrm{Th}-232 \\
\mathrm{Th}-234 \\
\mathrm{~Pa}-234 \\
\mathrm{~Pa}-234 \mathrm{~m} \\
\mathrm{U}-235 \\
\mathrm{U}-238 \\
\mathrm{Pu}-238 \\
\mathrm{Pu}-239 \\
\mathrm{Pu}-240 \\
\mathrm{Pu}-241 \\
\mathrm{Am}-241 \\
\mathrm{Cm}-242 \\
\mathrm{Cm}-244 \\
\mathrm{~m} \\
\end{array}$ & 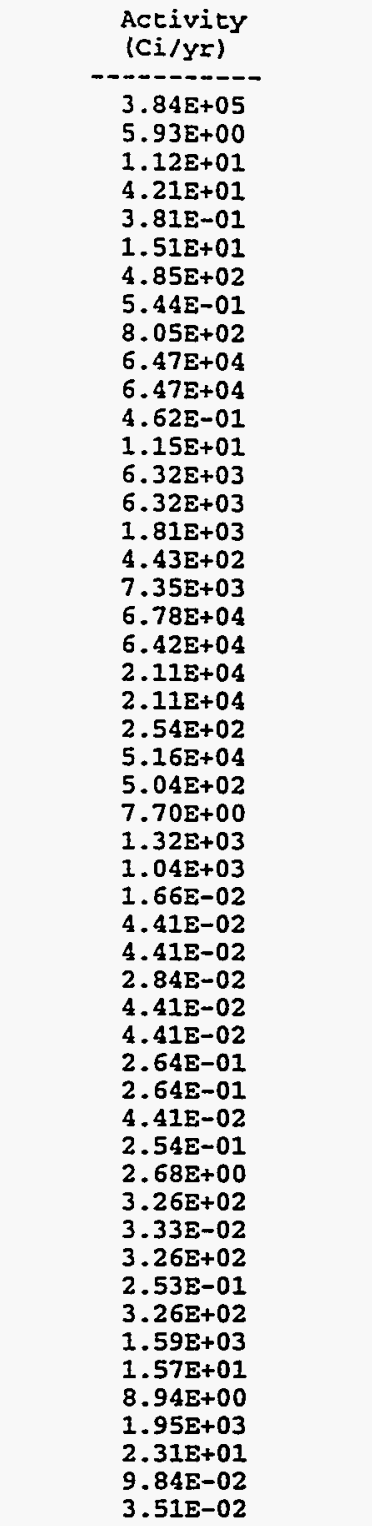 & \\
\hline Shipping Site: LBL & $\begin{array}{l}\text { Total volume } \\
\text { (m3/yr) }\end{array}$ & $\begin{array}{l}\text { Total Mass } \\
(\mathrm{kg} / \mathrm{y})\end{array}$ & $4.51 \mathrm{E}+04$ \\
\hline . & $\begin{array}{l}\text { Radionuclide } \\
\mathrm{H}-3 \\
\mathrm{C}-14 \\
\mathrm{Mn}-54 \\
\mathrm{Co}-58 \\
\mathrm{Co}-60 \\
\mathrm{Sr}-90 \\
\mathrm{Y}-90 \\
\mathrm{Tc}-99 \\
\mathrm{Cs}-134 \\
\mathrm{Cs}-137 \\
\mathrm{Ba}-137 \mathrm{~m} \\
\mathrm{U}-238 \\
\mathrm{Cm}-244\end{array}$ & $\begin{array}{c}\text { Activity } \\
(C i / y r)\end{array}$ & \\
\hline
\end{tabular}




\begin{tabular}{|c|c|c|c|}
\hline Shipping Site: LLNL & $\begin{array}{l}\text { Total volume } \\
(\mathrm{m} 3 / \mathrm{yr})\end{array}$ & $\begin{array}{l}\text { Total Mass } \\
(\mathrm{kg} / \mathrm{yr})\end{array}$ & $1.06 \mathrm{z}+06$ \\
\hline & \begin{tabular}{l} 
Radionuclide \\
\hdashline$H-3$ \\
$\mathrm{C}-14$ \\
$\mathrm{Mn}-54$ \\
$\mathrm{Co}-58$ \\
$\mathrm{Co}-60$ \\
$\mathrm{Ni}-63$ \\
$\mathrm{Sr}-90$ \\
$\mathrm{y}-90$ \\
$\mathrm{Tc}-99$ \\
$\mathrm{Ru}-106$ \\
$\mathrm{Rh}-106$ \\
$\mathrm{Sb}-125$ \\
$\mathrm{Te}-125 \mathrm{~m}$ \\
$\mathrm{Cs}-134$ \\
$\mathrm{Cs}-137$ \\
$\mathrm{Ba}-137 \mathrm{~m}$ \\
$\mathrm{Ce}-144$ \\
$\mathrm{Pr}-144$ \\
$\mathrm{Pr}-144 \mathrm{~m}$ \\
$\mathrm{Pm}-147$ \\
$\mathrm{Sm}-151$ \\
$\mathrm{Eu}-152$ \\
$\mathrm{Eu}-154$ \\
$\mathrm{Eu}-155$ \\
$\mathrm{U}-238$ \\
$\mathrm{Pu}-238$ \\
$\mathrm{Pu}-239$ \\
$\mathrm{Pu}-240$ \\
$\mathrm{Pu}-241$ \\
$\mathrm{Am}-241$ \\
$\mathrm{Cm}-242$ \\
$\mathrm{Cm}-244$
\end{tabular} & \multicolumn{2}{|l|}{ 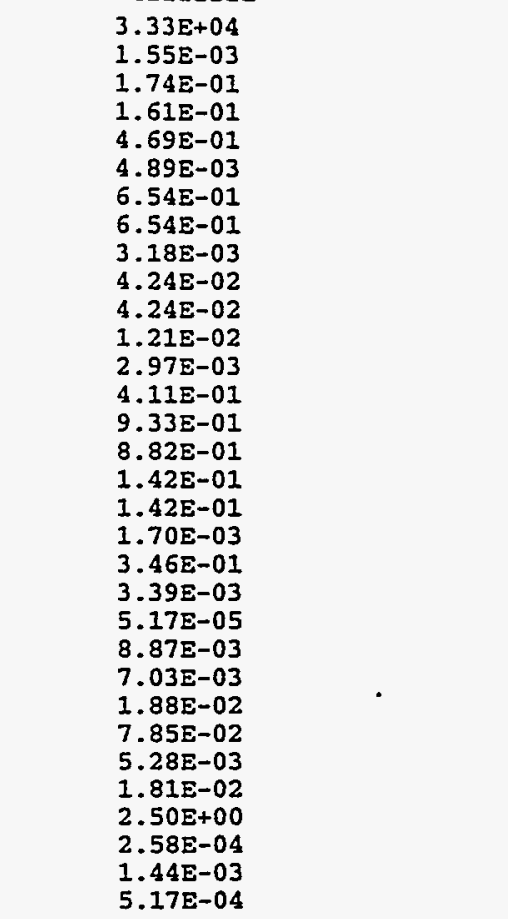 } \\
\hline \multirow[t]{2}{*}{ Shipping Site: MOUND } & $\begin{array}{l}\text { Total Volume } \\
\text { (m3/yr) }\end{array}$ & $\begin{array}{c}\text { Total Mass } \\
(\mathrm{kg} / \mathrm{Yr})\end{array}$ & $9.67 \mathrm{E}+06$ \\
\hline & $\begin{array}{l}\text { Radionuclide } \\
\mathrm{H}-3 \\
\mathrm{Pu}-238 \\
\mathrm{Pu}-239 \\
\mathrm{Pu}-240 \\
\mathrm{Pu}-241 \\
\mathrm{Am}-241 \\
\mathrm{Cm}-242 \\
\mathrm{Cm}-244\end{array}$ & $\begin{array}{c}\begin{array}{c}\text { Activity } \\
(C i / y r)\end{array} \\
1.34 \mathrm{E}+04 \\
1.60 \mathrm{E}-01 \\
1.22 \mathrm{E}-02 \\
4.28 \mathrm{E}-02 \\
5.90 \mathrm{E}+00 \\
2.44 \mathrm{E}-04 \\
3.42 \mathrm{E}-03 \\
1.22 \mathrm{E}-03\end{array}$ & \\
\hline
\end{tabular}


A- 155

\begin{tabular}{|c|c|c|c|}
\hline Shipping site: ORISE & $\begin{array}{l}\text { Total volume } \\
(\mathrm{m} 3 / \mathrm{yr})\end{array}$ & $\begin{array}{l}\text { Total Mass } \\
(\mathrm{kg} / \mathrm{yr})\end{array}$ & $7.36 \mathrm{E}+04$ \\
\hline & \begin{tabular}{l} 
Radionuclide \\
\hdashline $\mathrm{H}-3$ \\
$\mathrm{C}-14$ \\
$\mathrm{Mn}-54$ \\
$\mathrm{Co}-58$ \\
$\mathrm{Co}-60$ \\
$\mathrm{~S}-90$ \\
$\mathrm{Y}-90$ \\
$\mathrm{TC}-99$ \\
$\mathrm{Cs}-134$ \\
$\mathrm{Cs}-137$ \\
$\mathrm{Ba}-137 \mathrm{~m}$ \\
$\mathrm{~T} 1-208$ \\
$\mathrm{~Pb}-212$ \\
$\mathrm{Bi}-212$ \\
$\mathrm{Po}-212$ \\
$\mathrm{Po}-216$ \\
$\mathrm{Ra}-224$ \\
$\mathrm{Ra}-228$ \\
$\mathrm{Ac}-228$ \\
$\mathrm{Th}-228$ \\
$\mathrm{Th}-231$ \\
$\mathrm{Th}-232$ \\
$\mathrm{Th}-234$ \\
$\mathrm{~Pa}-234$ \\
$\mathrm{~Pa}-234 \mathrm{~m}$ \\
$\mathrm{U}-235$ \\
$\mathrm{U}-238$
\end{tabular} & $\begin{array}{c}\text { Activity } \\
\text { (Ci/yI) } \\
2.00 \mathrm{E}-02 \\
1.19 \mathrm{E}-05 \\
1.34 \mathrm{E}-03 \\
1.24 \mathrm{E}-03 \\
3.59 \mathrm{E}-03 \\
1.69 \mathrm{E}-03 \\
1.69 \mathrm{E}-03 \\
2.39 \mathrm{E}-05 \\
2.79 \mathrm{E}-03 \\
3.68 \mathrm{E}-03 \\
3.48 \mathrm{E}-03 \\
3.39 \mathrm{E}-07 \\
8.98 \mathrm{E}-07 \\
8.98 \mathrm{E}-07 \\
5.78 \mathrm{E}-07 \\
8.98 \mathrm{E}-07 \\
8.98 \mathrm{E}-07 \\
5.36 \mathrm{E}-06 \\
5.36 \mathrm{E}-06 \\
8.98 \mathrm{E}-07 \\
5.16 \mathrm{E}-06 \\
5.44 \mathrm{E}-05 \\
6.62 \mathrm{E}-03 \\
6.78 \mathrm{E}-07 \\
6.62 \mathrm{E}-03 \\
5.14 \mathrm{E}-06 \\
6.77 \mathrm{E}-03\end{array}$ & \\
\hline
\end{tabular}




\begin{tabular}{|c|c|c|c|}
\hline Shipping Site: ORNL & $\begin{array}{l}\text { Total Volume } \\
(\mathrm{m} 3 / \mathrm{Yr})\end{array}$ & $2.82 \mathrm{E}+03 \quad \begin{array}{cc}\text { Total Mass } \\
(\mathrm{kg} / \mathrm{y}=)\end{array}$ & $3.30 \mathrm{E}+06$ \\
\hline . & 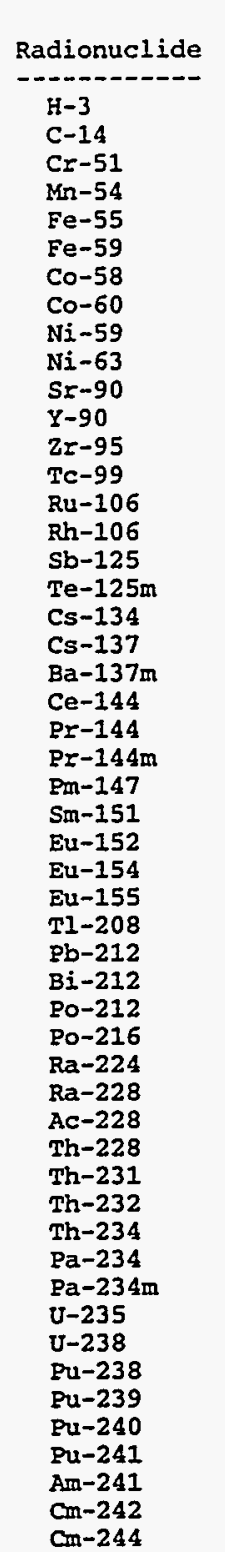 & 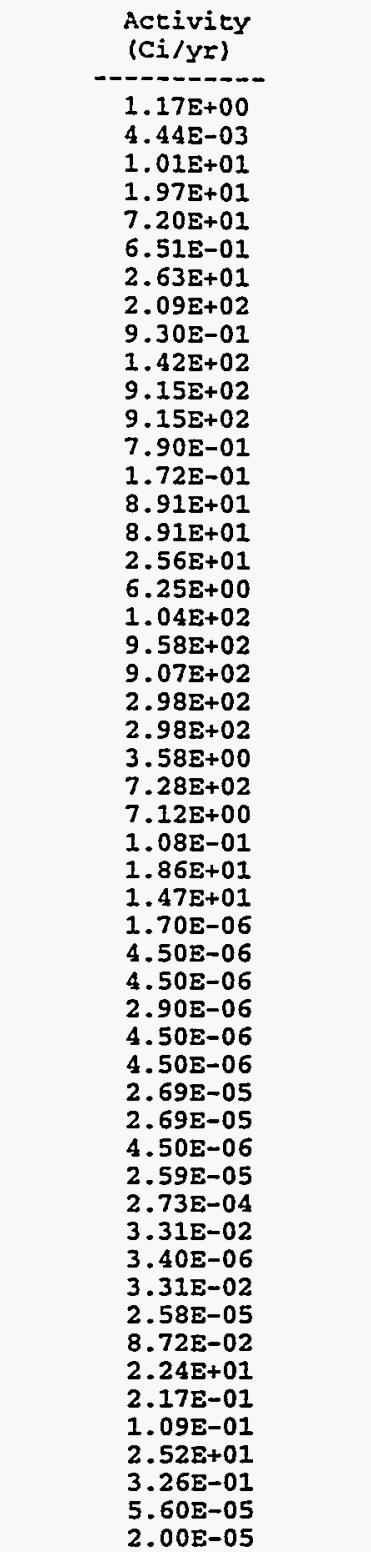 & \\
\hline
\end{tabular}




\begin{tabular}{|c|c|c|c|}
\hline Shipping Site: PANT & $\begin{array}{l}\text { Total Volume } \\
(\mathrm{m} 3 / \mathrm{yr})\end{array}$ & $\begin{array}{cc}4.35 E+03 & \text { Total Mass } \\
\left(\mathrm{kg} / \mathrm{yr}^{2}\right)\end{array}$ & 2. $88 E+07$ \\
\hline & $\begin{array}{l}\text { Radionuclide } \\
\mathrm{H}-3 \\
\mathrm{Tl}-208 \\
\mathrm{~Pb}-212 \\
\mathrm{Bi}-212 \\
\mathrm{Po}-212 \\
\mathrm{Po}-216 \\
\mathrm{Ra}-224 \\
\mathrm{Ra}-228 \\
\mathrm{Ac}-228 \\
\mathrm{Th}-228 \\
\mathrm{Th}-231 \\
\mathrm{Th}-232 \\
\mathrm{Th}-234 \\
\mathrm{~Pa}-234 \\
\mathrm{~Pa}-234 \mathrm{~m} \\
\mathrm{U}-235 \\
\mathrm{U}-238\end{array}$ & $\begin{array}{l}\text { Activiey } \\
\text { (Ci/yr) } \\
-------- \\
2.34 E+02 \\
2.21 E-04 \\
5.86 \mathrm{E}-04 \\
5.86 \mathrm{E}-04 \\
3.78 \mathrm{E}-04 \\
5.86 \mathrm{E}-04 \\
5.86 \mathrm{E}-04 \\
3.50 \mathrm{E}-03 \\
3.50 \mathrm{E}-03 \\
5.86 \mathrm{E}-04 \\
3.37 \mathrm{E}-03 \\
3.56 \mathrm{E}-02 \\
4.32 \mathrm{E}+00 \\
4.43 \mathrm{E}-04 \\
4.32 \mathrm{E}+00 \\
3.36 \mathrm{E}-03 \\
4.32 \mathrm{E}+00\end{array}$ & \\
\hline Shipping Site: PORTS & $\begin{array}{l}\text { Total Volume } \\
\text { (m3/yI) }\end{array}$ & $\begin{array}{l}\text { Total Mass } \\
\text { (kg/yr) }\end{array}$ & $6.73 E+07$ \\
\hline · & $\begin{array}{l}\text { Radionuclide } \\
\mathrm{T} 1-208 \\
\mathrm{~Pb}-212 \\
\mathrm{Bi}-212 \\
\mathrm{Po}-212 \\
\mathrm{Po}-216 \\
\mathrm{Ra}-224 \\
\mathrm{Ra}-228 \\
\mathrm{Ac}-228 \\
\mathrm{Th}-228 \\
\mathrm{Th}-231 \\
\mathrm{Th}-232 \\
\mathrm{Th}-234 \\
\mathrm{~Pa}-234 \\
\mathrm{~Pa}-234 \mathrm{~m} \\
\mathrm{U}-235 \\
\mathrm{U}-238\end{array}$ & $\begin{array}{l}\text { Activity } \\
\text { (Ci/yr) } \\
-3.45 E-06 \\
3.14 E-06 \\
9.14 E-06 \\
5.89 E-06 \\
9.14 E-06 \\
9.14 E-06 \\
5.46 E-05 \\
5.46 E-05 \\
9.14 E-06 \\
5.26 E-05 \\
5.55 E-04 \\
6.74 E-02 \\
6.91 E-06 \\
6.74 E-02 \\
5.24 E-05 \\
6.74 E-02\end{array}$ & \\
\hline Shipping Site: PPPL & $\begin{array}{l}\text { Total volume } \\
\text { (m3/yx) }\end{array}$ & $\begin{array}{l}\text { Total Mass } \\
\text { (kg/yr) }\end{array}$ & $6.10 \mathrm{E}+03$ \\
\hline$\cdot$ & $\begin{array}{l}\text { Radionuclide } \\
\mathrm{H}-3 \\
\mathrm{Cr}-51 \\
\mathrm{Mn}-54 \\
\mathrm{Fe}-55 \\
\mathrm{Fe}-59 \\
\mathrm{Co}-58 \\
\mathrm{Co}-60 \\
\mathrm{Ni}-59 \\
\mathrm{Ni}-63 \\
\mathrm{Zr}-95\end{array}$ & $\begin{array}{c}\begin{array}{l}\text { Activity } \\
\text { (Ci/yr) }\end{array} \\
-2.01 \mathrm{E}-01 \\
8.50 \mathrm{E}-04 \\
1.61 \mathrm{E}-03 \\
6.04 \mathrm{E}-03 \\
5.46 \mathrm{E}-05 \\
2.17 \mathrm{E}-03 \\
1.70 \mathrm{E}-02 \\
7.80 \mathrm{E}-05 \\
1.10 \mathrm{E}-02 \\
6.63 \mathrm{E}-05\end{array}$ & \\
\hline
\end{tabular}


A- 158

\begin{tabular}{|c|c|c|c|}
\hline Shipping Site: Paducah & $\begin{array}{l}\text { Tota1 Volume } \\
\text { (m3/yz) }\end{array}$ & $\begin{array}{l}\text { Total Mass } \\
(\mathrm{kg} / \mathrm{yr})\end{array}$ & $1.20 \mathrm{E}+07$ \\
\hline & $\begin{array}{l}\text { Radionuclide } \\
\mathrm{Tc}-99 \\
\mathrm{~T} 1-208 \\
\mathrm{~Pb}-212 \\
\mathrm{Bi}-212 \\
\mathrm{PO-212} \\
\mathrm{Po}-216 \\
\mathrm{Ra}-224 \\
\mathrm{Ra}-228 \\
\mathrm{Ac}-228 \\
\mathrm{Th}-228 \\
\mathrm{Th}-231 \\
\mathrm{Th}-232 \\
\mathrm{Th}-234 \\
\mathrm{~Pa}-234 \\
\mathrm{~Pa}-234 \mathrm{~m} \\
\mathrm{U}-235 \\
\mathrm{U}-238 \\
\mathrm{~Np}-237 \\
\mathrm{Pu}-239\end{array}$ & $\begin{array}{c}\begin{array}{c}\text { Activity } \\
(C i / y r)\end{array} \\
-8.84 E-03 \\
3.72 E-05 \\
9.84 E-05 \\
9.84 E-05 \\
6.34 E-05 \\
9.84 E-05 \\
9.84 E-05 \\
5.88 E-04 \\
5.88 E-04 \\
9.84 E-05 \\
5.66 E-04 \\
5.97 E-03 \\
7.26 E-01 \\
7.44 E-05 \\
7.26 E-01 \\
5.64 E-04 \\
7.26 E-01 \\
6.63 E-03 \\
6.63 E-03\end{array}$ & \\
\hline Shipping Site: Pinellas & $\begin{array}{l}\text { Total volume } \\
(\operatorname{m} 3 / y x)\end{array}$ & $1.40 \mathrm{E}+02 \quad \begin{array}{c}\text { Total Mass } \\
(\mathrm{kg} / \mathrm{Yr})\end{array}$ & $1.91 E+04$ \\
\hline & $\begin{array}{l}\text { Radionuclide } \\
\mathrm{H}-3\end{array}$ & $\begin{array}{c}\begin{array}{c}\text { Activity } \\
(\mathrm{Ci} / \mathrm{y})\end{array} \\
1.99 \mathrm{E}+04\end{array}$ & \\
\hline Shipping Site: RFP & $\begin{array}{l}\text { Total volume } \\
(\mathrm{m} 3 / \mathrm{yr})\end{array}$ & $\begin{array}{cc}\text { Total Mass } \\
(\mathrm{kg} / \mathrm{yr})\end{array}$ & $6.49 E+06$ \\
\hline & $\begin{array}{l}\text { Radionuclide } \\
-\mathrm{Tl}-208 \\
\mathrm{~Pb}-212 \\
\mathrm{Bi}-212 \\
\mathrm{Po}-212 \\
\mathrm{Po}-216 \\
\mathrm{Ra}-224 \\
\mathrm{Ra}-228 \\
\mathrm{Ac}-228 \\
\mathrm{Th}-228 \\
\mathrm{Th}-231 \\
\mathrm{Th}-232 \\
\mathrm{Th}-234 \\
\mathrm{~Pa}-234 \\
\mathrm{~Pa}-234 \mathrm{~m} \\
\mathrm{U}-235 \\
\mathrm{U}-238 \\
\mathrm{Pu}-238 \\
\mathrm{Pu}-239 \\
\mathrm{Pu}-240 \\
\mathrm{Pu}-241 \\
\mathrm{Am}-241 \\
\mathrm{Cm}-242 \\
\mathrm{Cm}-244\end{array}$ & $\begin{array}{c}\begin{array}{c}\text { Áctivity } \\
\text { (Ci/Yr) }\end{array} \\
-3.60 \mathrm{E}-05 \\
9.55 \mathrm{E}-05 \\
9.55 \mathrm{E}-05 \\
6.15 \mathrm{E}-05 \\
9.55 \mathrm{E}-05 \\
9.55 \mathrm{E}-05 \\
5.70 \mathrm{E}-04 \\
5.70 \mathrm{E}-04 \\
9.55 \mathrm{E}-05 \\
5.49 \mathrm{E}-04 \\
5.79 \mathrm{E}-03 \\
7.04 \mathrm{E}-01 \\
7.21 \mathrm{E}-05 \\
7.04 \mathrm{E}-01 \\
5.47 \mathrm{E}-04 \\
7.04 \mathrm{E}-01 \\
5.56 \mathrm{E}-01 \\
4.24 \mathrm{E}-02 \\
1.48 \mathrm{E}-01 \\
2.04 \mathrm{E}+01 \\
8.48 \mathrm{E}-04 \\
1.18 \mathrm{E}-02 \\
4.24 \mathrm{E}-03\end{array}$ & \\
\hline
\end{tabular}


A-159

\begin{tabular}{|c|c|c|c|}
\hline Shipping Site: RMI & $\begin{array}{l}\text { Total Volume } \\
\text { (m3/yr) }\end{array}$ & $\begin{array}{l}\text { Total Mass } \\
(\mathrm{kg} / \mathrm{yr})\end{array}$ & $1.47 \mathrm{E}+07$ \\
\hline & $\begin{array}{l}\text { Radionuclide } \\
\mathrm{T} 1-208 \\
\mathrm{~Pb}-212 \\
\mathrm{Bi}-212 \\
\mathrm{Po}-212 \\
\mathrm{Po}-216 \\
\mathrm{Ra}-224 \\
\mathrm{Ra}-228 \\
\mathrm{Ac}-228 \\
\mathrm{Th}-228 \\
\mathrm{Th}-231 \\
\mathrm{Th}-232 \\
\mathrm{Th}-234 \\
\mathrm{~Pa}-234 \\
\mathrm{~Pa}-234 \mathrm{~m} \\
\mathrm{U}-235 \\
\mathrm{U}-238\end{array}$ & \begin{tabular}{c}
$\begin{array}{c}\text { Activicy } \\
(C i / y r)\end{array}$ \\
\hdashline $3.58 \mathrm{E}-07$ \\
$9.47 \mathrm{E}-07$ \\
$9.47 \mathrm{E}-07$ \\
$6.10 \mathrm{E}-07$ \\
$9.47 \mathrm{E}-07$ \\
$9.47 \mathrm{E}-07$ \\
$5.66 \mathrm{E}-06$ \\
$5.66 \mathrm{E}-06$ \\
$9.47 \mathrm{E}-07$ \\
$5.45 \mathrm{E}-06$ \\
$5.74 \mathrm{E}-05$ \\
$6.98 \mathrm{E}-03$ \\
$7.15 \mathrm{E}-07$ \\
$6.98 \mathrm{E}-03$ \\
$5.43 \mathrm{E}-06$ \\
$6.98 \mathrm{E}-03$
\end{tabular} & \\
\hline Shipping Site: SLAC & $\begin{array}{l}\text { Total Volume } \\
\text { (m3/yr) }\end{array}$ & $\begin{array}{l}\text { Total Mass } \\
(\mathrm{kg} / \mathrm{yr})\end{array}$ & $7.45 E+05$ \\
\hline & $\begin{array}{l}\text { Radionuclide } \\
\text { Cr-51 } \\
\mathrm{Mn}-54 \\
\mathrm{Fe}-55 \\
\mathrm{Fe}-59 \\
\mathrm{Co}-58 \\
\mathrm{Co}-60 \\
\mathrm{Ni}-59 \\
\mathrm{Ni}-63 \\
\mathrm{Zr}-95\end{array}$ & $\begin{array}{c}\begin{array}{c}\text { Activity } \\
\text { (Ci/yn) }\end{array} \\
3.86 E-03 \\
7.36 \mathrm{E}-03 \\
2.74 \mathrm{E}-02 \\
2.48 \mathrm{E}-04 \\
9.88 \mathrm{E}-03 \\
7.74 \mathrm{E}-02 \\
3.54 \mathrm{E}-04 \\
5.04 \mathrm{E}-02 \\
3.01 \mathrm{E}-04\end{array}$ & \\
\hline
\end{tabular}


moving shipments) on the basis of the dimensions of the shipment. The code approximates the shipment as a cylindrical surface source, and the calculated dose includes contributions from secondary radiation scattering from buildup (scattering by waste contents), cloudshine (scattering by the air), and groundshine (scattering by the ground). The dose rates calculated by using RISKIND have been shown to be comparable with output from existing shielding codes for various waste configurations. As a conservative measure, credit for potential shielding between the waste package and the receptor is not considered.

\subsubsection{Vehicle-Related (Nonradiological) Routine Risk}

Vehicle-related health risks resulting from routine transportation may be associated with the generation of air pollutants by transport vehicles during shipment and are independent of the radioactive nature of the shipment. The health end point assessed under routine transportation conditions is the excess latent mortality due to inhalation of vehicular exhaust emissions. Risk factors for pollutant inhalation in terms of latent mortality have been generated by Rao et al. (1982). These risk factors are $1.0 \mathrm{E}-07$ mortality $/ \mathrm{km}(1.6 \mathrm{E}-07 / \mathrm{mi})$ and $1.3 \mathrm{E}-07$ mortality $/ \mathrm{km}(2.1 \mathrm{E}-07 / \mathrm{mi})$ of truck and rail travel in urban areas, respectively. The risk factors are based on regression analyses of the effects of sulfur dioxide and particulate releases from diesel exhaust on mortality rates. Excess latent mortalities are assumed to be equivalent to latent-cancer fatalities. Vehicle-related risks from routine transportation are calculated for each alternative by multiplying the total distance traveled in urban areas by the appropriate risk factor. Similar data are not available for rural and suburban areas.

Risks are summed over the entire route and over all shipments for each LLW alternative. This method has been used in several reports to calculate risks from routine transportation of radioactive wastes (DOE 1986, 1987a, 1990a). Lack of information for rural and suburban areas is an obvious data gap, although the risk factors would presumably be lower because of lower total emissions from all sources and lower population densities in rural and suburban areas.

\subsection{ACCIDENT ASSESSMENT METHOD}

\subsubsection{Radiological Accident Risk Assessment}

The risk analysis for potential accidents differs fundamentally from the risk analysis for routine transportation because occurrences of accidents are statistical in nature. The accident risk assessment is treated probabilistically in RADTRAN 4. Accident risk is defined as the product of the accident consequence (dose) and the probability of the accident occurring. In this respect, the RADTRAN 4 code estimates the collective accident risk to populations by considering a spectrum of transportation-related accidents. The spectrum of accidents is designed to encompass a range of 


\begin{tabular}{|c|c|c|c|}
\hline Shipping site: SNLA & $\begin{array}{l}\text { Toral Volume } \\
\text { (m3/yr) }\end{array}$ & $\begin{array}{c}\text { Total Mass } \\
(\mathrm{kg} / \mathrm{Yr})\end{array}$ & $3.28 \mathrm{E}+05$ \\
\hline 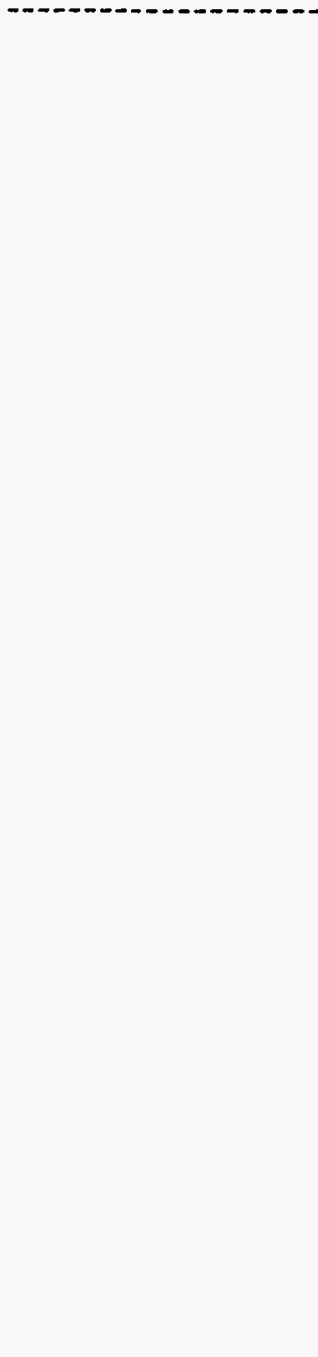 & 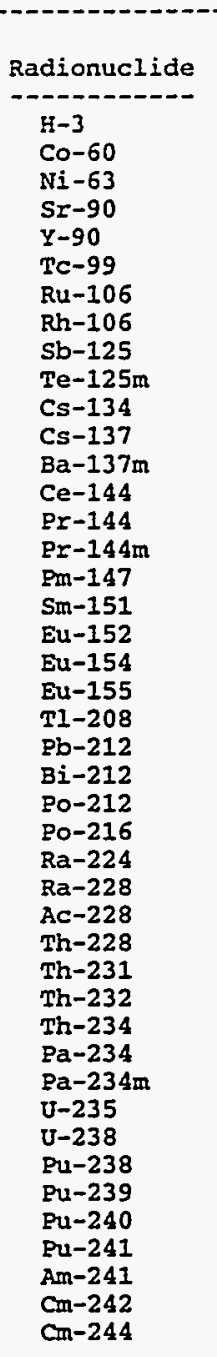 & 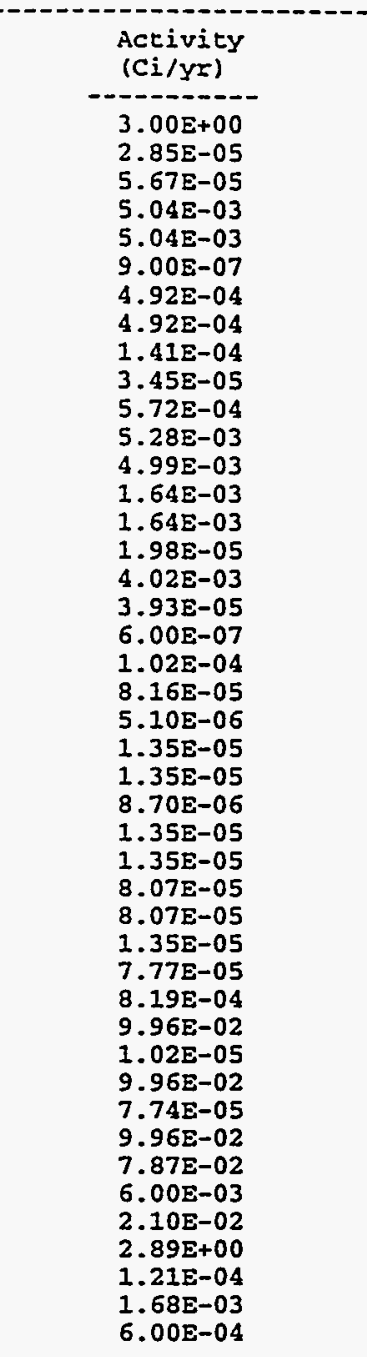 & \\
\hline Shipping site: SNLt & $\begin{array}{l}\text { Total Volume } \\
\text { (m3/yr) }\end{array}$ & $\begin{array}{cc}\text { Total Mass } \\
\text { 3.68E+01 }\end{array}$ & \\
\hline & $\begin{array}{l}\text { Radionuclide } \\
\mathrm{H}-3 \\
\mathrm{Fe}-55 \\
\mathrm{Co}-60 \\
\mathrm{~T} 1-208 \\
\mathrm{~Pb}-212 \\
\mathrm{Bi}-212 \\
\mathrm{Po}-212 \\
\mathrm{PO}-216 \\
\mathrm{Ra}-224 \\
\mathrm{Ra}-228 \\
\mathrm{Ac}-228 \\
\mathrm{Th}-228 \\
\mathrm{Th}-231 \\
\mathrm{Th}-232 \\
\mathrm{Th}-234 \\
\mathrm{~Pa}-234 \\
\mathrm{~Pa}-234 \mathrm{~m} \\
\mathrm{U}-235 \\
\mathrm{U}-238\end{array}$ & $\begin{array}{c}\begin{array}{l}\text { Activity } \\
\text { (Ci/YI) }\end{array} \\
2.78 E+04 \\
3.15 E-01 \\
3.15 \mathrm{E}-01 \\
6.40 \mathrm{E}-05 \\
1.69 \mathrm{E}-04 \\
1.69 \mathrm{E}-04 \\
1.09 \mathrm{E}-04 \\
1.69 \mathrm{E}-04 \\
1.69 \mathrm{E}-04 \\
1.01 \mathrm{E}-03 \\
1.01 \mathrm{E}-03 \\
1.69 \mathrm{E}-04 \\
9.75 \mathrm{E}-04 \\
1.02 \mathrm{E}-02 \\
1.25 \mathrm{E}+00 \\
1.28 \mathrm{E}-04 \\
1.25 \mathrm{E}+00 \\
9.71 \mathrm{E}-04 \\
1.25 \mathrm{E}+00\end{array}$ & ( \\
\hline
\end{tabular}


$A-161$

\begin{tabular}{|c|c|c|c|}
\hline Shipping Sice: SRS & $\begin{array}{l}\text { Total volume } \\
(\mathrm{m} 3 / \mathrm{y} \text { ) }\end{array}$ & $\begin{array}{c}\text { Total Mass } \\
(\mathrm{kg} / \mathrm{yr})\end{array}$ & $1.29 \mathrm{E}+08$ \\
\hline & 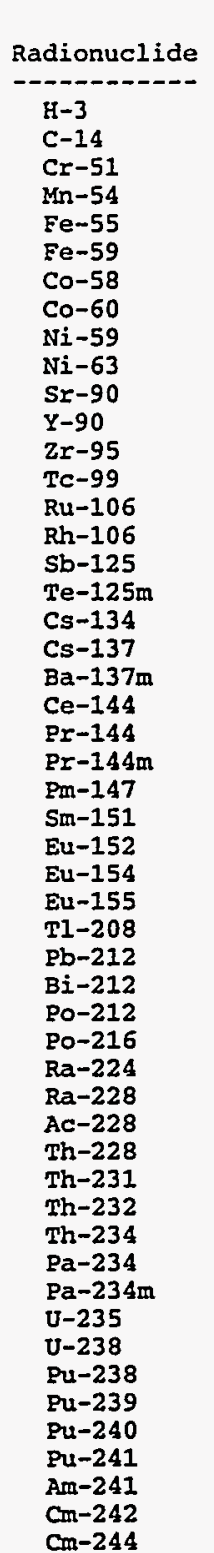 & 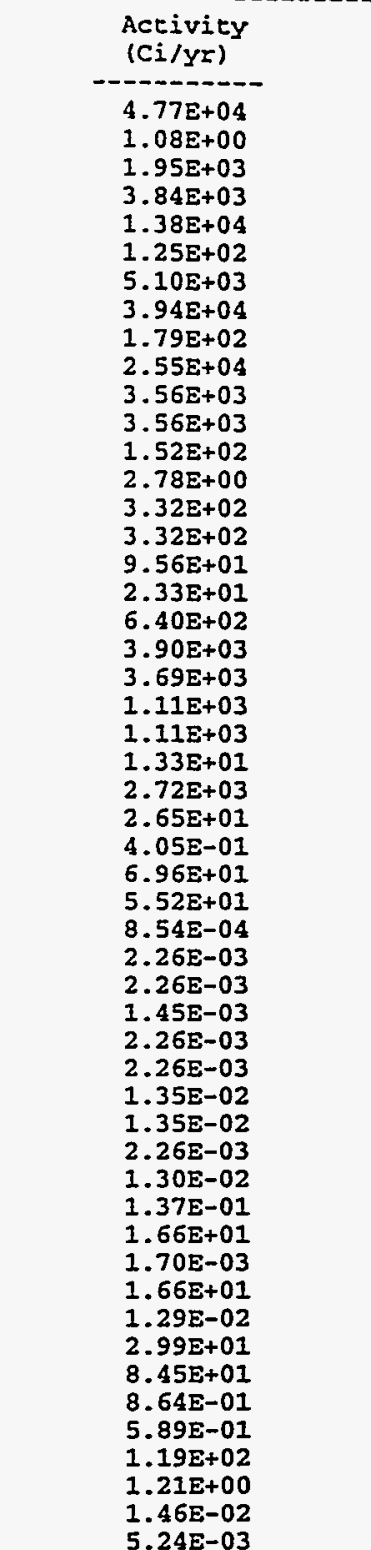 & 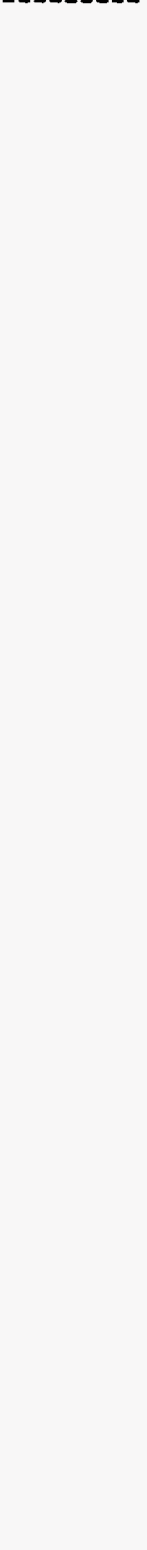 \\
\hline
\end{tabular}


A-162

\begin{tabular}{|c|c|c|c|}
\hline Shipping Site: $Y-12$ & $\begin{array}{l}\text { Total Volume } \\
(\mathrm{m} 3 / \mathrm{yx})\end{array}$ & $\begin{array}{c}\text { Total Mass } \\
(\mathrm{kg} / \mathrm{yr})\end{array}$ & $8.27 E+07$ \\
\hline & $\begin{array}{l}\text { Radionuclide } \\
\mathrm{Tl}-208 \\
\mathrm{~Pb}-212 \\
\mathrm{Bi}-212 \\
\mathrm{PO}-212 \\
\mathrm{Po}-216 \\
\mathrm{Ra}-224 \\
\mathrm{Ra}-228 \\
\mathrm{Ac}-228 \\
\mathrm{Th}-228 \\
\mathrm{Th}-231 \\
\mathrm{Th}-232 \\
\mathrm{Th}-234 \\
\mathrm{~Pa}-234 \\
\mathrm{~Pa}-234 \mathrm{~m} \\
\mathrm{U}-235 \\
\mathrm{U}-238\end{array}$ & $\begin{array}{c}\begin{array}{c}\text { Activity } \\
\text { (Ci/yr) }\end{array} \\
6.80 \mathrm{E}-05 \\
1.80 \mathrm{E}-04 \\
1.80 \mathrm{E}-04 \\
1.16 \mathrm{E}-04 \\
1.80 \mathrm{E}-04 \\
1.80 \mathrm{E}-04 \\
1.07 \mathrm{E}-03 \\
1.07 \mathrm{E}-03 \\
1.80 \mathrm{E}-04 \\
1.03 \mathrm{E}-03 \\
1.09 \mathrm{E}-02 \\
1.32 \mathrm{E}+00 \\
1.36 \mathrm{E}-04 \\
1.32 \mathrm{E}+00 \\
1.03 \mathrm{E}-03 \\
1.32 \mathrm{E}+00\end{array}$ & \\
\hline
\end{tabular}




\section{A.20 WM LLW CENTRALIZED 1 ALTERNATIVE (CASE 7): ACTIVATED METALS}

Transported Material with Radionuclide Profile

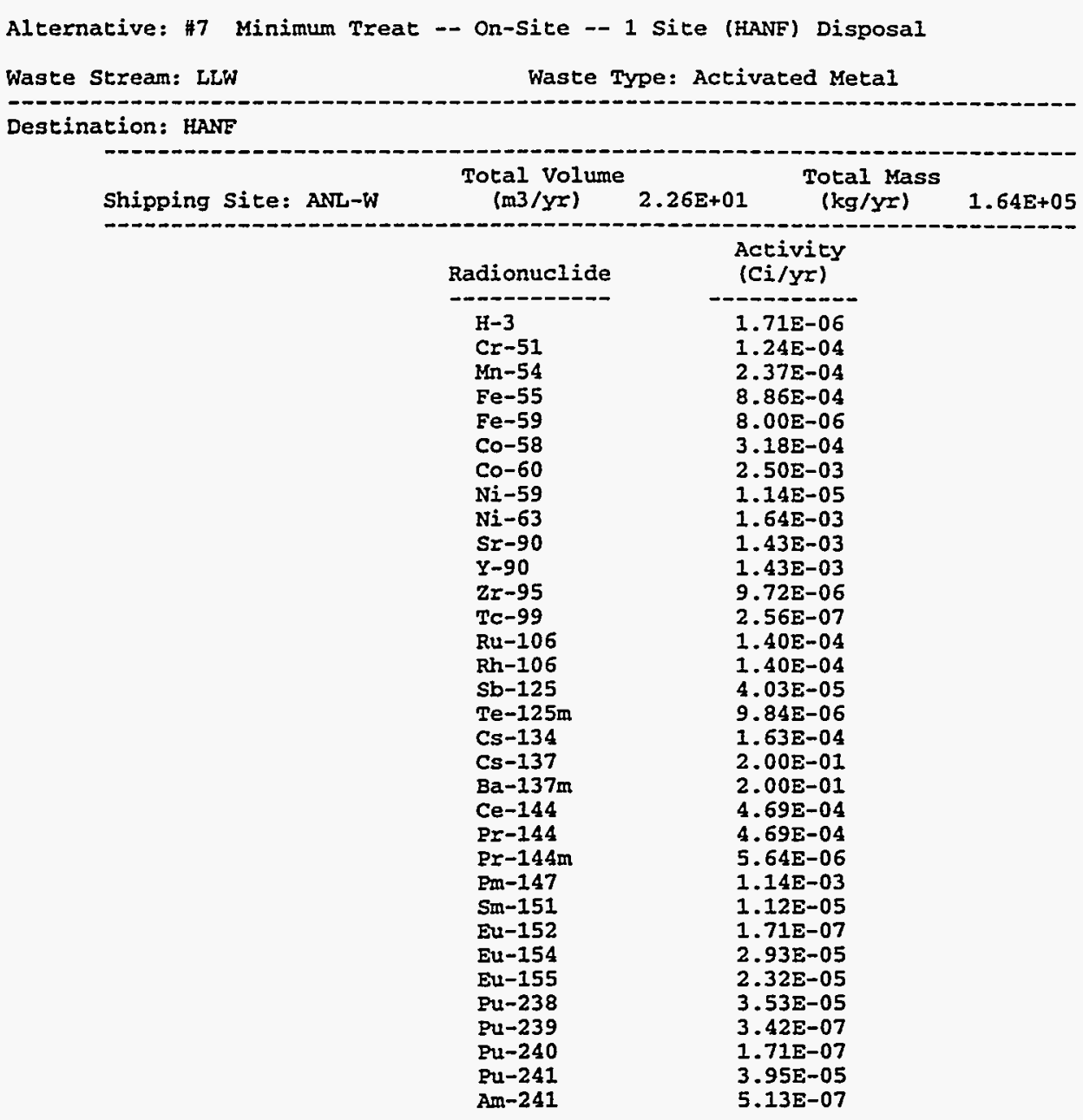


A-164

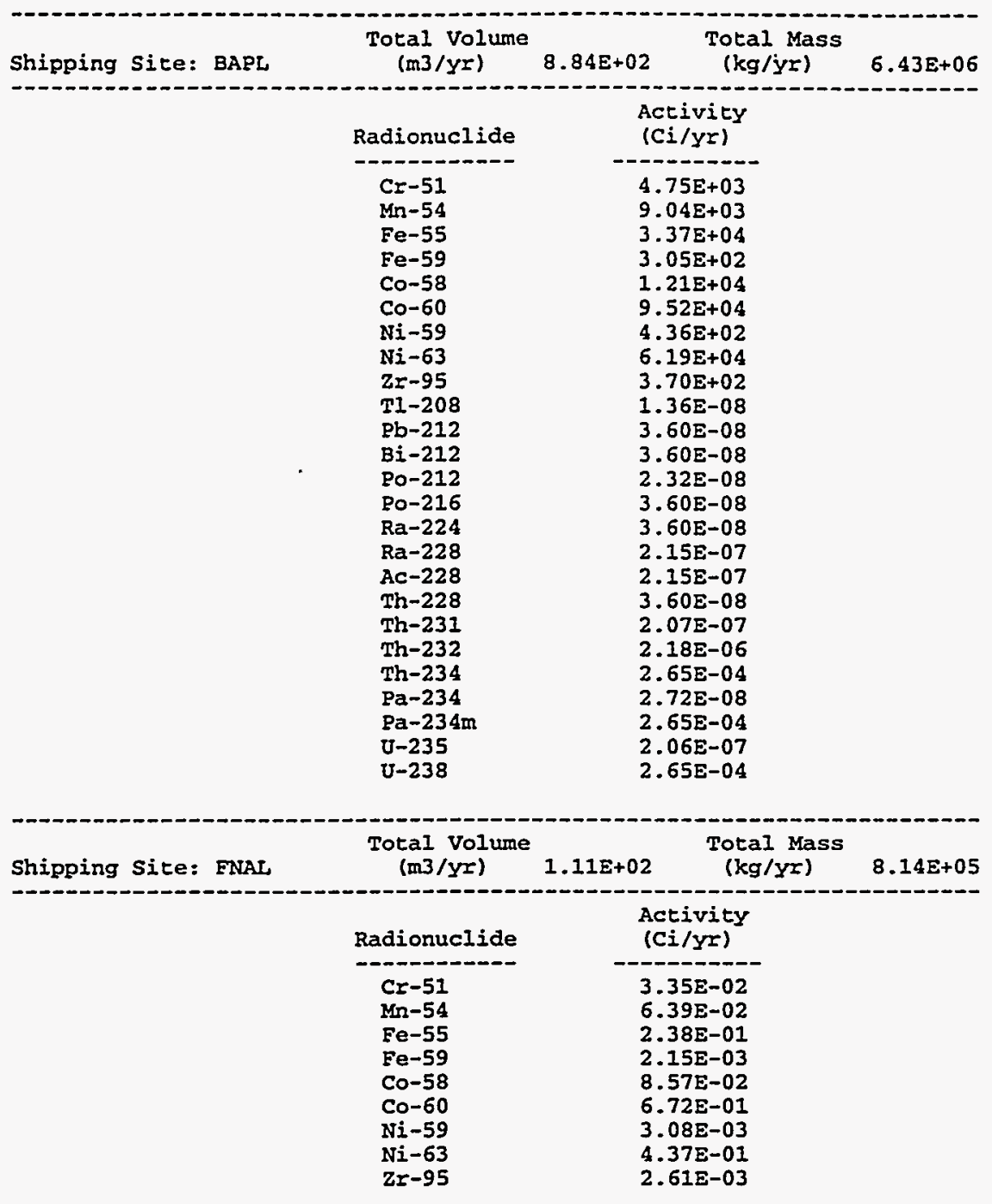




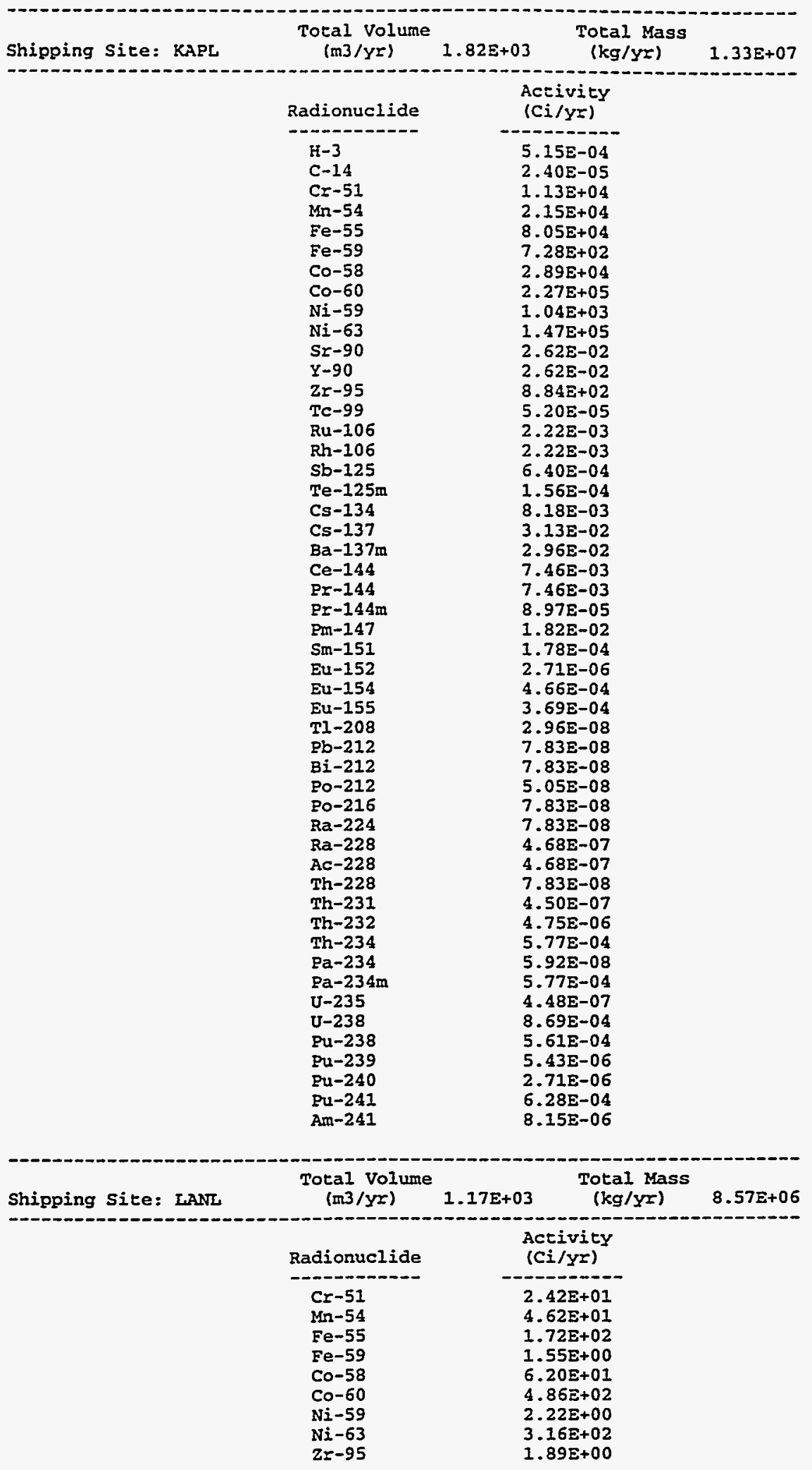


A-166

\begin{tabular}{|c|c|c|c|}
\hline Shipping site: LBL & $\begin{array}{l}\text { Total Volume } \\
(\mathrm{m} 3 / \mathrm{yr})\end{array}$ & $\begin{array}{l}\text { Total Mass } \\
(\mathrm{kg} / \mathrm{yr})\end{array}$ & $2.87 E+05$ \\
\hline & \begin{tabular}{l} 
Radionuclide \\
\hdashline $\mathrm{Cr}-51$ \\
$\mathrm{Mn}-54$ \\
$\mathrm{Fe}-55$ \\
$\mathrm{Fe}-59$ \\
$\mathrm{Co}-58$ \\
$\mathrm{Co}-60$ \\
$\mathrm{Ni}-59$ \\
$\mathrm{Ni}-63$ \\
$\mathrm{Zr}-95$
\end{tabular} & \begin{tabular}{c}
$\begin{array}{c}\text { Activicy } \\
\text { (Ci/yr) }\end{array}$ \\
\hdashline $6.32 \mathrm{E}-02$ \\
$1.20 \mathrm{E}-01$ \\
$4.49 \mathrm{E}-01$ \\
$4.06 \mathrm{E}-03$ \\
$1.61 \mathrm{E}-01$ \\
$1.26 \mathrm{E}+00$ \\
$5.80 \mathrm{E}-03$ \\
$8.24 \mathrm{E}-01$ \\
$4.93 \mathrm{E}-03$
\end{tabular} & \\
\hline Shipping Site: NRF & $\begin{array}{l}\text { Total Volume } \\
(\mathrm{m} 3 / \mathrm{yr})\end{array}$ & $\begin{array}{cc}1.29 \mathrm{E}+03 & \begin{array}{c}\text { Total Mass } \\
(\mathrm{kg} / \mathrm{y} x)\end{array}\end{array}$ & $9.40 \mathrm{E}+06$ \\
\hline & $\begin{array}{l}\text { Radionuclide } \\
-\mathrm{C} x-51 \\
\mathrm{Mn}-54 \\
\mathrm{Fe}-55 \\
\mathrm{Fe}-59 \\
\mathrm{Co}-58 \\
\mathrm{Co}-60 \\
\mathrm{Ni}-59 \\
\mathrm{Ni}-63 \\
\mathrm{Z} r-95\end{array}$ & $\begin{array}{c}\text { Activity } \\
\text { (Ci/yx) } \\
2.31 E+03 \\
4.40 E+03 \\
1.64 E+04 \\
1.48 E+02 \\
5.91 E+03 \\
4.63 E+04 \\
2.12 E+02 \\
3.01 E+04 \\
1.80 E+02\end{array}$ & \\
\hline Shipping Site: SNLA & $\begin{array}{l}\text { Total Volume } \\
(\mathrm{m} 3 / \mathrm{yr})\end{array}$ & $\begin{array}{c}\text { Total Mass } \\
(\mathrm{kg} / \mathrm{yr})\end{array}$ & $1.15 E+05$ \\
\hline & $\begin{array}{l}\text { Radionuclide } \\
\mathrm{Cr}-51 \\
\mathrm{Mn}-54 \\
\mathrm{Fe}-55 \\
\mathrm{Fe}-59 \\
\mathrm{Co}-58 \\
\mathrm{Co}-60 \\
\mathrm{Ni}-59 \\
\mathrm{Ni}-63 \\
\mathrm{Z} x-95\end{array}$ & 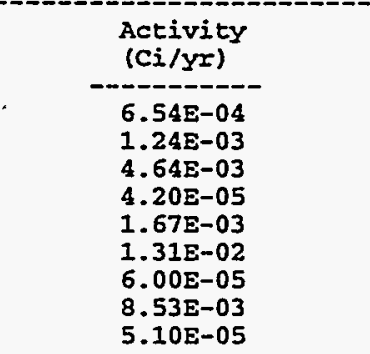 & \\
\hline
\end{tabular}




\section{A.21 WM LLW CENTRALIZED 2 ALTERNATIVE (CASE 8): HETEROGENEOUS SOLIDS}

Transported Material with Radionuclide Profile

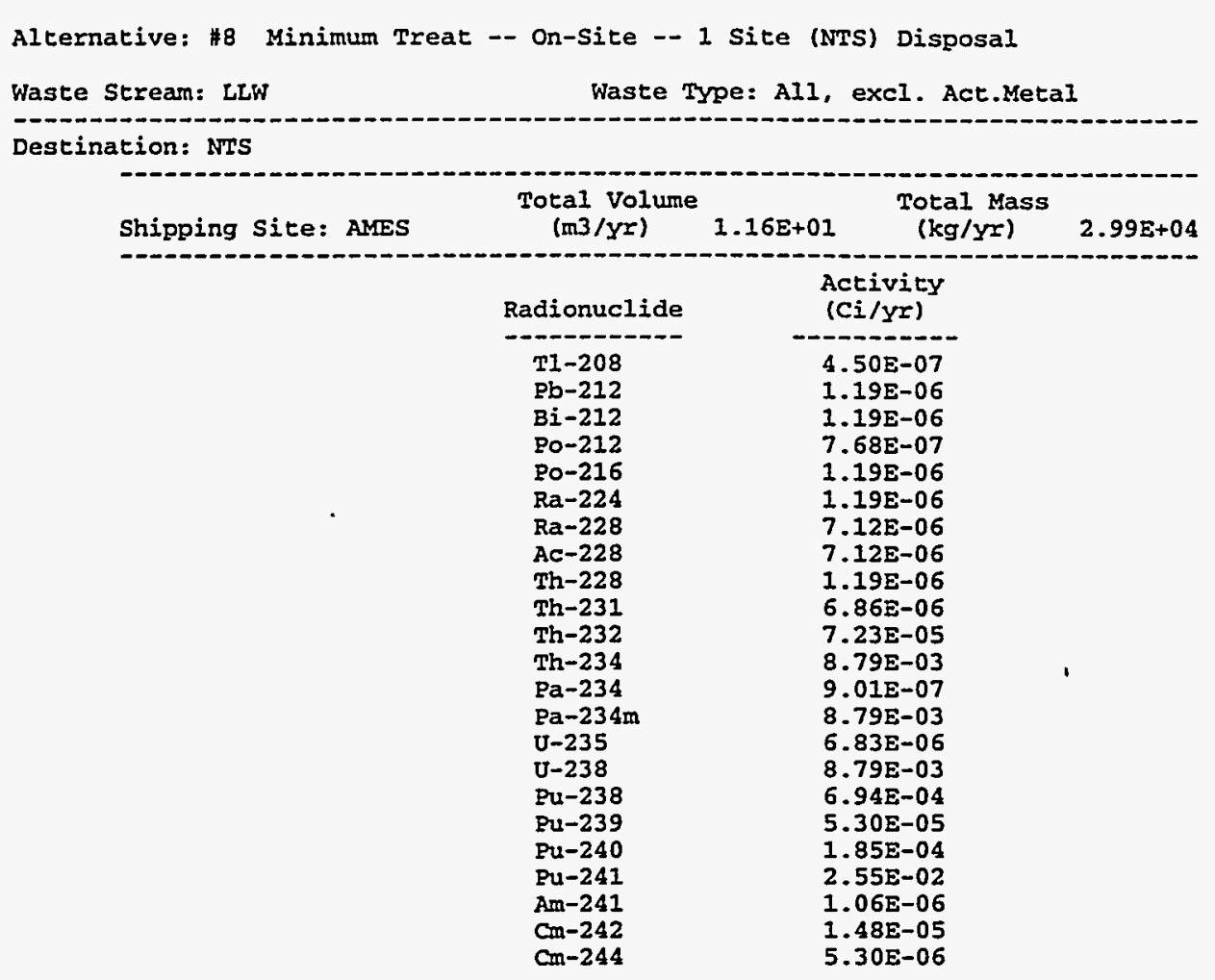


A-168

\begin{tabular}{|c|c|c|c|}
\hline Shipping Site: ANL-E & $\begin{array}{l}\text { Total Volume } \\
(\mathrm{m} 3 / \mathrm{yr})\end{array}$ & $\begin{array}{l}\text { Total Mass } \\
(\mathrm{kg} / \mathrm{yr})\end{array}$ & $2.00 \mathrm{E}+06$ \\
\hline - & $\begin{array}{l}\text { Radionuclide } \\
\mathrm{H}-3 \\
\mathrm{C}-14 \\
\mathrm{Cr}-51 \\
\mathrm{Mn}-54 \\
\mathrm{Fe}-55 \\
\mathrm{Fe}-59 \\
\mathrm{Co}-58 \\
\mathrm{Co}-60 \\
\mathrm{Ni}-59 \\
\mathrm{Ni}-63 \\
\mathrm{Sr}-90 \\
\mathrm{Y}-90 \\
\mathrm{Zr}-95 \\
\mathrm{Tc}-99 \\
\mathrm{Ru}-106 \\
\mathrm{Rh}-106 \\
\mathrm{Sb}-125 \\
\mathrm{Te}-125 \mathrm{~m} \\
\mathrm{Cs}-134 \\
\mathrm{Cs}-137 \\
\mathrm{Ba}-137 \mathrm{~m} \\
\mathrm{Ce}-144 \\
\mathrm{Pr}-144 \\
\mathrm{Pr}-144 \mathrm{~m} \\
\mathrm{Pm}-147 \\
\mathrm{Sm}-151 \\
\mathrm{Eu}-152 \\
\mathrm{Eu}-154 \\
\mathrm{Eu}-155 \\
\mathrm{U}-238 \\
\mathrm{Pu}-238 \\
\mathrm{Pu}-239 \\
\mathrm{Pu}-240 \\
\mathrm{Pu}-241 \\
\mathrm{Am}-241\end{array}$ & $\begin{array}{c}\text { Activity } \\
\text { (Ci/yz) } \\
1.33 E+02 \\
7.91 \mathrm{E}-02 \\
2.87 \mathrm{E}+00 \\
1.43 \mathrm{E}+01 \\
2.04 \mathrm{E}+01 \\
1.84 \mathrm{E}-01 \\
1.55 \mathrm{E}+01 \\
8.15 \mathrm{E}+01 \\
2.63 \mathrm{E}-01 \\
3.77 \mathrm{E}+01 \\
3.33 \mathrm{E}+01 \\
3.33 \mathrm{E}+01 \\
2.24 \mathrm{E}-01 \\
1.62 \mathrm{E}-01 \\
2.16 \mathrm{E}+00 \\
2.16 \mathrm{E}+00 \\
6.21 \mathrm{E}-01 \\
1.51 \mathrm{E}-01 \\
2.09 \mathrm{E}+01 \\
4.75 \mathrm{E}+01 \\
4.50 \mathrm{E}+01 \\
7.24 \mathrm{E}+00 \\
7.24 \mathrm{E}+00 \\
8.70 \mathrm{E}-02 \\
1.76 \mathrm{E}+01 \\
1.72 \mathrm{E}-01 \\
2.63 \mathrm{E}-03 \\
4.52 \mathrm{E}-01 \\
3.58 \mathrm{E}-01 \\
9.63 \mathrm{E}-01 \\
5.44 \mathrm{E}-01 \\
5.27 \mathrm{E}-03 \\
2.63 \mathrm{E}-03 \\
6.09 \mathrm{E}-01 \\
7.91 \mathrm{E}-03\end{array}$ & \\
\hline
\end{tabular}


A-169

\begin{tabular}{|c|c|c|c|}
\hline Shipping site: ANL-W & $\begin{array}{c}\text { Total volume } \\
(\mathrm{m} 3 / \mathrm{YY})\end{array}$ & $\begin{array}{c}\text { Total Mass } \\
(\mathrm{kg} / \mathrm{y} z)\end{array}$ & $2.12 \mathrm{E}+05$ \\
\hline & $\begin{array}{l}\text { Radionuclide } \\
\mathrm{H}-3 \\
\mathrm{Cr}-51 \\
\mathrm{Mn}-54 \\
\mathrm{Fe}-55 \\
\mathrm{Fe}-59 \\
\mathrm{Co}-58 \\
\mathrm{Co}-60 \\
\mathrm{Ni}-59 \\
\mathrm{Ni}-63 \\
\mathrm{Sr}-90 \\
\mathrm{Y}-90 \\
\mathrm{Zr}-95 \\
\mathrm{Tc}-99 \\
\mathrm{Ru}-106 \\
\mathrm{Rh}-106 \\
\mathrm{Sb}-125 \\
\mathrm{Te}-125 \mathrm{~m} \\
\mathrm{Cs}-134 \\
\mathrm{Cs}-137 \\
\mathrm{Ba}-137 \mathrm{~m} \\
\mathrm{Ce}-144 \\
\mathrm{Pr}-144 \\
\mathrm{Pr}-144 \mathrm{~m} \\
\mathrm{Pm}-147 \\
\mathrm{Sm}-151 \\
\mathrm{Eu}-152 \\
\mathrm{Eu}-154 \\
\mathrm{Eu}-155 \\
\mathrm{Pu}-238 \\
\mathrm{Pu}-239 \\
\mathrm{Pu}-240 \\
\mathrm{Pu}-241 \\
\mathrm{Am}-241 \\
\mathrm{Cm}-242 \\
\mathrm{Cm}-244\end{array}$ & 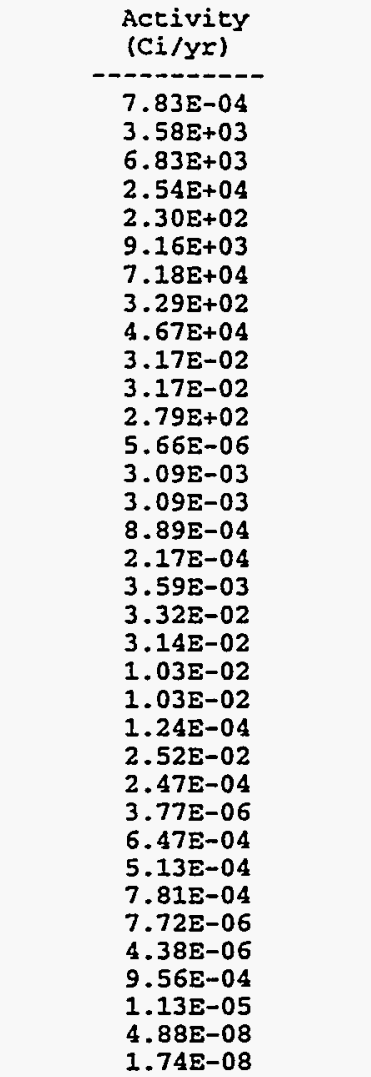 & \\
\hline
\end{tabular}


possible accidents, including low-probability accidents that have high consequences, and highprobability accidents that have low consequences (such as "fender benders"). The results for collective accident risk can be directly compared with the results for routine collective risk because the former results incorporate the probabilities of accident occurrences.

The RADTRAN 4 calculation of collective accident risk employs models that quantify the range of potential accident severities and the responses of transported packages to accidents. The spectrum of accident severity is divided into a number of categories. Each category of severity is assigned a conditional probability of occurrence-that is, the probability that an accident will be of a particular severity if an accident occurs. The more severe the accident, the more remote the chance of such an accident. Release fractions, defined as the fraction of the material in a package that could be released in an accident, are assigned to each accident severity category on the basis of the physical and chemical form of the waste material. The models take into account the mode of transportation and the type of packaging being considered. The accident rates, the definition of accident severity categories, and the release fractions used in this analysis are discussed further in Section 4.

For accidents involving the release of radioactive material, RADTRAN 4 assumes that the material is dispersed in the environment according to standard Gaussian diffusion models. For the risk assessment, default data for atmospheric dispersion were used, representing an instantaneous ground-level release and a small-diameter source cloud (Neuhauser and Kanipe 1993). The calculation of the collective population dose following the release and dispersal of radioactive material includes the following exposure pathways:

- External exposure to the passing radioactive cloud,

- External exposure to contaminated ground,

- Internal exposure from inhalation of airborne contaminants, and

- Internal exposure from the ingestion of contaminated food.

For the pathway of ingestion, state-specific food transfer factors, which relate the amount of radioactive material ingested to the amount deposited on the ground, were calculated in accordance with the methods described by the U.S. Nuclear Regulatory Commission (NRC) regulatory guide 1.109 (NRC 1977b) and were used as input to the RADTRAN code. Doses of radiation from the ingestion or inhalation of radionuclides are calculated by using standard dose conversion factors (DOE 1988a,b).

The collective accident risk for each alternative is determined in a manner similar to that described for routine collective risks. Accident risks are first calculated for each unique origindestination pair and then are summed over all pairs to estimate the total risk for the altemative. The 


\begin{tabular}{|c|c|c|c|}
\hline Shipping Site: BAPL & $\begin{array}{l}\text { Total Volume } \\
(\mathrm{m} 3 / \mathrm{y} x)\end{array}$ & $\begin{array}{l}\text { Total Mass } \\
(\mathrm{kg} / \mathrm{yr})\end{array}$ & $1.08 \mathrm{E}+06$ \\
\hline - & $\begin{array}{l}\text { Radionuclide } \\
\mathrm{H}-3 \\
\mathrm{Co}-60 \\
\mathrm{Ni}-63 \\
\mathrm{Sr}-90 \\
\mathrm{Y}-90 \\
\mathrm{Tc}-99 \\
\mathrm{Ru}-106 \\
\mathrm{Rh}-106 \\
\mathrm{Sb}-125 \\
\mathrm{Te}-125 \mathrm{~m} \\
\mathrm{Cs}-134 \\
\mathrm{Cs}-137 \\
\mathrm{Ba}-137 \mathrm{~m} \\
\mathrm{Ce}-144 \\
\mathrm{Pr}-144 \\
\mathrm{Pr}-144 \mathrm{~m} \\
\mathrm{Pm}-147 \\
\mathrm{Sm}-151 \\
\mathrm{Eu}-152 \\
\mathrm{Eu}-154 \\
\mathrm{Eu}-155 \\
\mathrm{~T} 1-208 \\
\mathrm{~Pb}-212 \\
\mathrm{Bi}-212 \\
\mathrm{Po}-212 \\
\mathrm{Po}-216 \\
\mathrm{Ra}-224 \\
\mathrm{Ra}-228 \\
\mathrm{Ac}-228 \\
\mathrm{Th}-228 \\
\mathrm{Th}-231 \\
\mathrm{Th}-232 \\
\mathrm{Th}-234 \\
\mathrm{~Pa}-234 \\
\mathrm{~Pa}-234 \mathrm{~m} \\
\mathrm{U}-235 \\
\mathrm{U}-238 \\
\mathrm{Pu}-238 \\
\mathrm{Pu}-239 \\
\mathrm{Pu}-240 \\
\mathrm{Pu}-241 \\
\mathrm{Am}-241 \\
\end{array}$ & 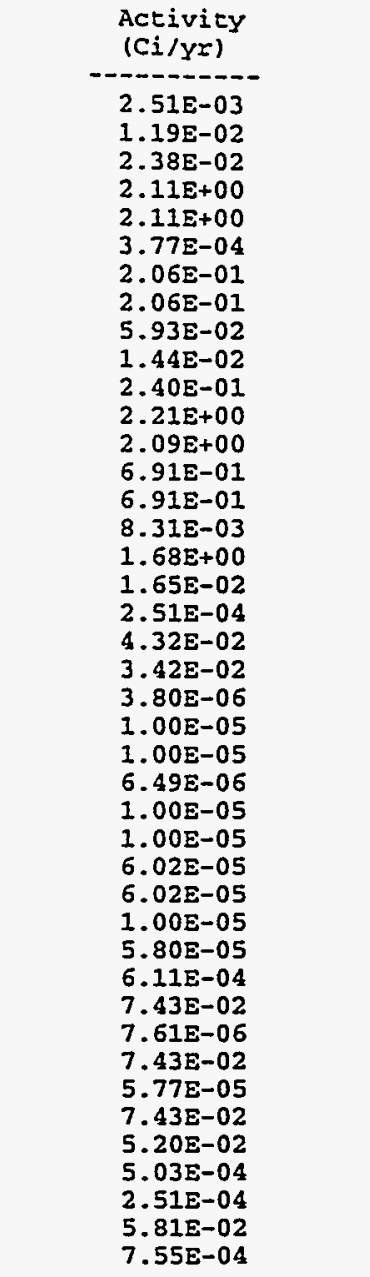 & \\
\hline \multicolumn{4}{|c|}{$\begin{array}{lclll} & \text { Total Volume } & \text { Total Mass } & \\
\text { Shipping Site: FRAL } & (\mathrm{m} 3 / \mathrm{yr}) & 5.19 \mathrm{E}+01 & (\mathrm{~kg} / \mathrm{yr}) & 5.11 \mathrm{E}+04\end{array}$} \\
\hline & \begin{tabular}{l} 
Radionuclide \\
\hdashline $\mathrm{H}-3$ \\
$\mathrm{Cr}-51$ \\
$\mathrm{Mn}-54$ \\
$\mathrm{Fe}-55$ \\
$\mathrm{Fe}-59$ \\
$\mathrm{Co}-58$ \\
$\mathrm{Co}-60$ \\
$\mathrm{Ni}-59$ \\
$\mathrm{Ni}-63$ \\
$\mathrm{Zr}-95$ \\
$\mathrm{TI}-208$ \\
$\mathrm{~Pb}-212$ \\
$\mathrm{Bi}-212$ \\
$\mathrm{PO}-212$ \\
$\mathrm{PO}-216$ \\
$\mathrm{Ra}-224$ \\
$\mathrm{Ra}-228$ \\
$\mathrm{Ac}-228$ \\
$\mathrm{Th}-228$ \\
$\mathrm{Th}-231$ \\
$\mathrm{Th}-232$ \\
$\mathrm{Th}-234$ \\
$\mathrm{~Pa}-234$ \\
$\mathrm{~Pa}-234 \mathrm{~m}$ \\
$\mathrm{U}-235$ \\
$\mathrm{U}-238$
\end{tabular} & 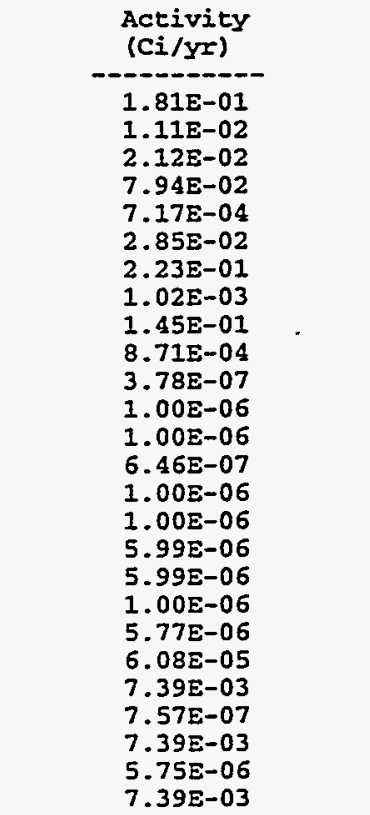 & \\
\hline
\end{tabular}




\begin{tabular}{|c|c|c|c|}
\hline Shipping site: HANE & $\begin{array}{l}\text { Total volume } \\
\left(\mathrm{m} 3 / \mathrm{y}^{\mathrm{r}}\right)\end{array}$ & $\begin{array}{c}\text { Total Mass } \\
(\mathrm{kg} / \mathrm{Yr})\end{array}$ & $2.75 E+07$ \\
\hline & 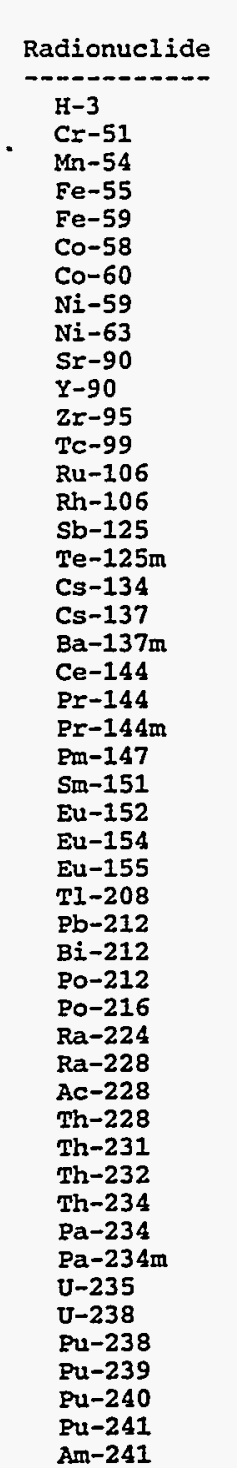 & 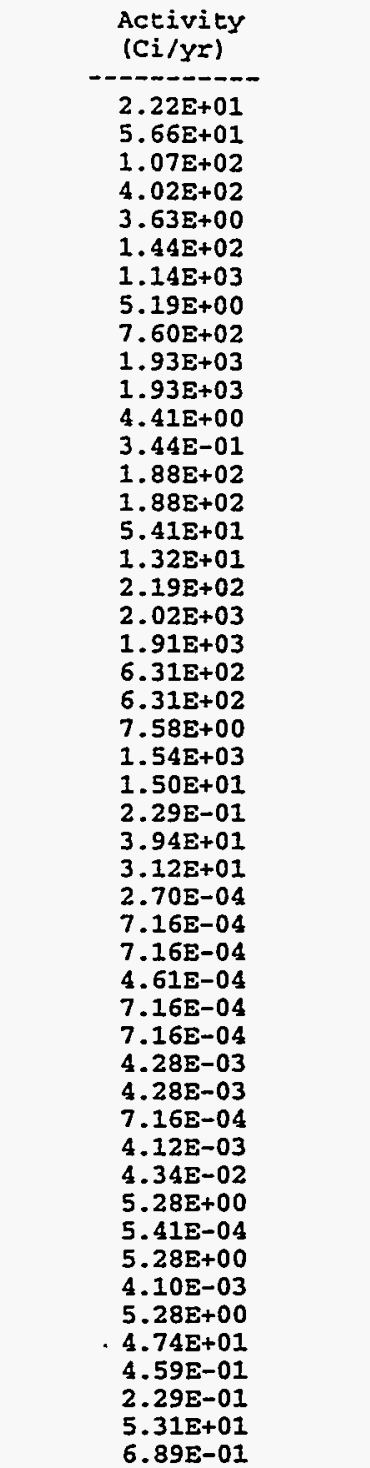 & \\
\hline
\end{tabular}




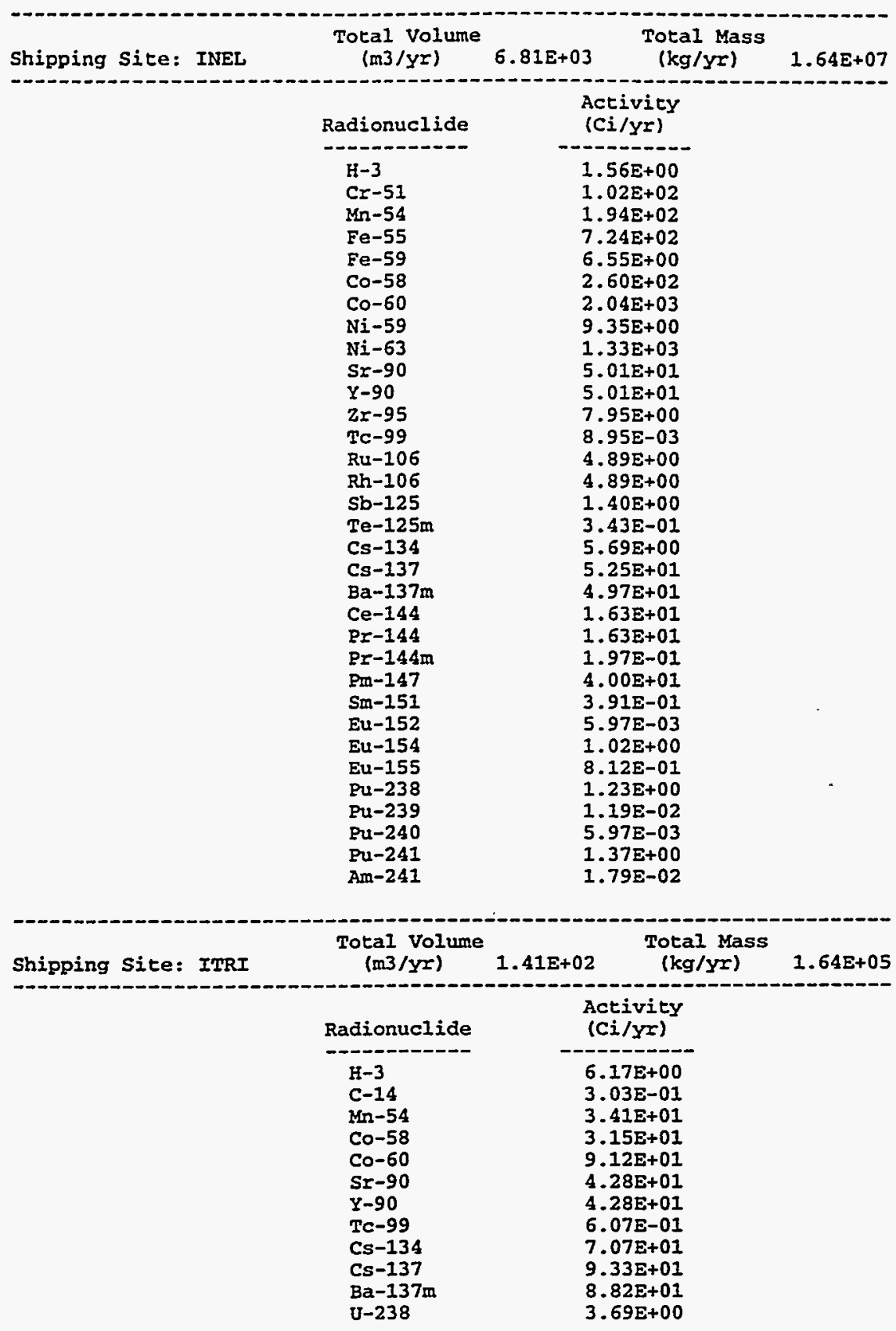




\begin{tabular}{|c|c|c|c|}
\hline Shipping Sice: $k-25$ & $\begin{array}{l}\text { Total volume } \\
(\mathrm{m} 3 / \mathrm{yr})\end{array}$ & $\begin{array}{l}\text { Total Mass } \\
(\mathrm{kg} / \mathrm{yr})\end{array}$ & $4.678+07$ \\
\hline & $\begin{array}{l}\text { Radionuclide } \\
\mathrm{T} 1-208 \\
\mathrm{~Pb}-212 \\
\mathrm{Bi}-212 \\
\mathrm{Po-212} \\
\mathrm{PO}-216 \\
\mathrm{Ra}-224 \\
\mathrm{Ra}-228 \\
\mathrm{Ac}-228 \\
\mathrm{Th}-228 \\
\mathrm{Th}-231 \\
\mathrm{Th}-232 \\
\mathrm{Th}-234 \\
\mathrm{~Pa}-234 \\
\mathrm{~Pa}-234 \mathrm{~m} \\
\mathrm{U}-235 \\
\mathrm{U}-238\end{array}$ & $\begin{array}{c}\text { Activity } \\
(C i / y z) \\
1.06 \mathrm{E}-04 \\
2.82 \mathrm{E}-04 \\
2.82 \mathrm{E}-04 \\
1.82 \mathrm{E}-04 \\
2.82 \mathrm{E}-04 \\
2.82 \mathrm{E}-04 \\
1.68 \mathrm{E}-03 \\
1.68 \mathrm{E}-03 \\
2.82 \mathrm{E}-04 \\
1.62 \mathrm{E}-03 \\
1.71 \mathrm{E}-02 \\
2.08 \mathrm{E}+00 \\
2.13 \mathrm{E}-04 \\
2.08 \mathrm{E}+00 \\
1.62 \mathrm{E}-03 \\
2.08 \mathrm{E}+00\end{array}$ & . \\
\hline Shipping Site: KAPL & $\begin{array}{l}\text { Total Volume } \\
(\mathrm{m} 3 / \mathrm{y})\end{array}$ & $\begin{array}{c}\text { Total Mass } \\
(\mathrm{kg} / \mathrm{yr})\end{array}$ & $5.36 E+05$ \\
\hline$\cdot$ & 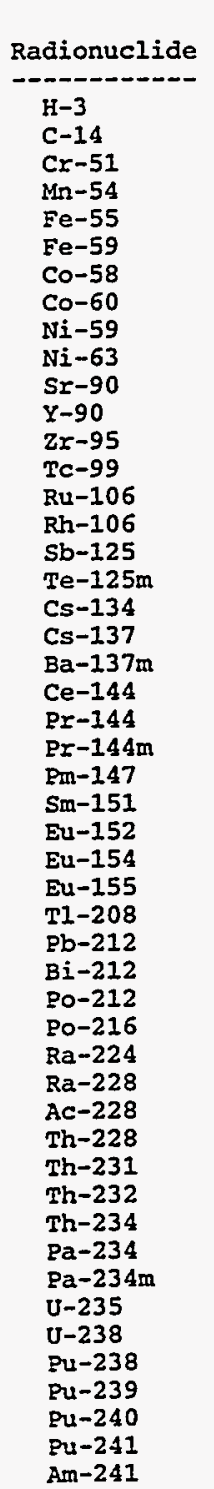 & 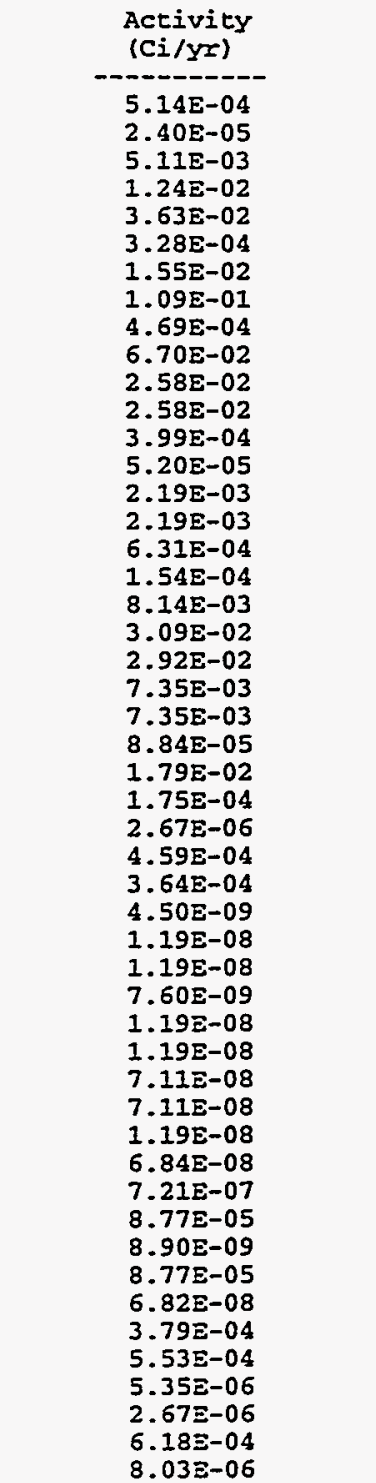 & \\
\hline
\end{tabular}




\begin{tabular}{|c|c|c|c|}
\hline Shipping Site: KCP & $\begin{array}{c}\text { Total Volume } \\
\left(\mathrm{m} 3 / \mathrm{yr}^{\prime}\right)\end{array}$ & $\begin{array}{l}\text { Total Mass } \\
(\mathrm{kg} / \mathrm{yr})\end{array}$ & $7.23 \mathrm{E}+03$ \\
\hline & $\begin{array}{l}\text { Radionuclide } \\
\mathrm{H}-3 \\
\mathrm{C}-14 \\
\mathrm{Mn}-54 \\
\mathrm{Co}-58 \\
\mathrm{Co}-60 \\
\mathrm{Sr}-90 \\
\mathrm{Y}-90 \\
\mathrm{Tc}-99 \\
\mathrm{Cs}-134 \\
\mathrm{Cs}-137 \\
\mathrm{Ba}-137 \mathrm{~m} \\
\mathrm{U}-238\end{array}$ & $\begin{array}{c}\text { Activity } \\
\text { (Ci/yr) } \\
2.70 \mathrm{E}-02 \\
1.44 \mathrm{E}-04 \\
1.62 \mathrm{E}-02 \\
1.50 \mathrm{E}-02 \\
4.34 \mathrm{E}-02 \\
2.04 \mathrm{E}-02 \\
2.04 \mathrm{E}-02 \\
2.89 \mathrm{E}-04 \\
3.37 \mathrm{E}-02 \\
4.44 \mathrm{E}-02 \\
4.20 \mathrm{E}-02 \\
1.76 \mathrm{E}-03\end{array}$ & \\
\hline Shipping Site: LANL & $\begin{array}{l}\text { Total Volume } \\
(\mathrm{m} 3 / \mathrm{yr})\end{array}$ & $1.52 \mathrm{E}+04 \quad \begin{array}{c}\text { Total Mass } \\
(\mathrm{kg} / \mathrm{yr})\end{array}$ & $1.22 E+07$ \\
\hline ' & 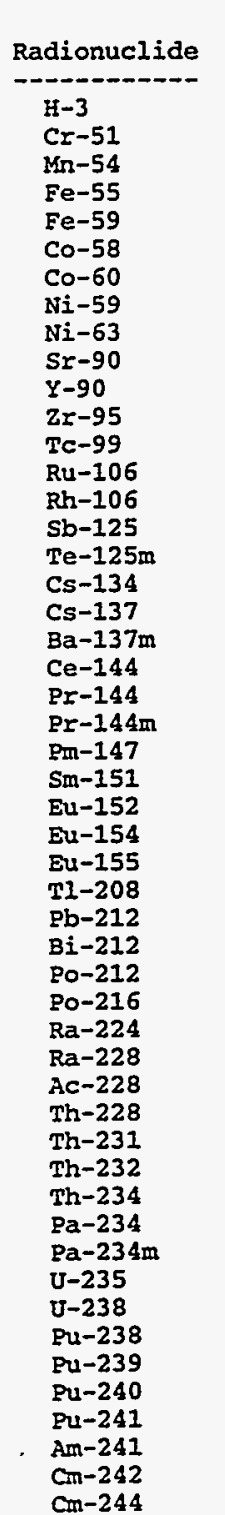 & 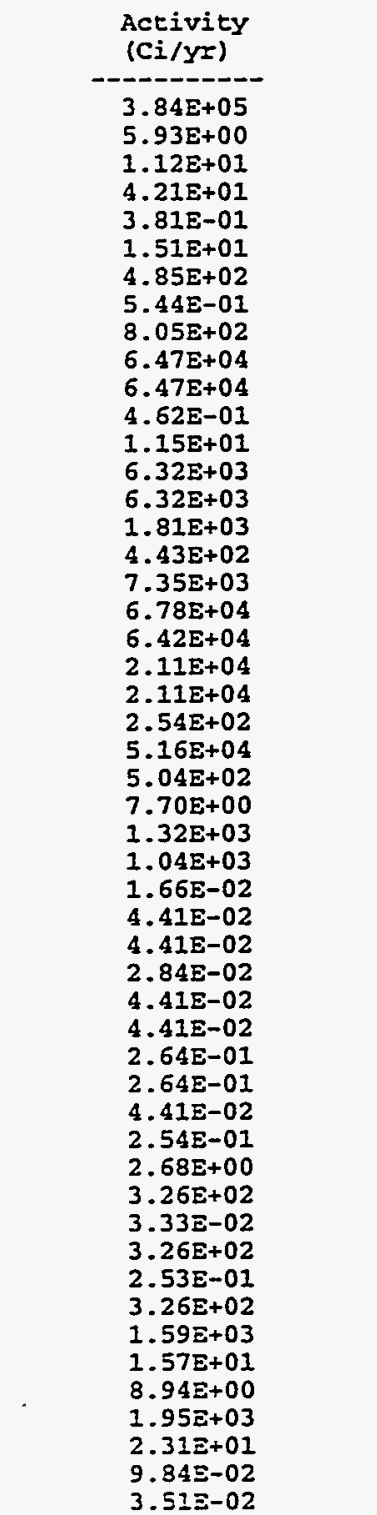 & \\
\hline
\end{tabular}




\begin{tabular}{|c|c|c|c|}
\hline Shipping Site: LBL & $\begin{array}{l}\text { Total volume } \\
(\mathrm{m} 3 / \mathrm{yx})\end{array}$ & $\begin{array}{c}\text { Toral Mass } \\
(\mathrm{kg} / \mathrm{Yr})\end{array}$ & $4.51 E+04$ \\
\hline & $\begin{array}{l}\text { Radionuclide } \\
\mathrm{H}-3 \\
\mathrm{C}-14 \\
\mathrm{Mn}-54 \\
\mathrm{Co}-58 \\
\mathrm{Co}-50 \\
\mathrm{~S} x-90 \\
\mathrm{Y}-90 \\
\mathrm{Tc}-99 \\
\mathrm{Cs}-134 \\
\mathrm{Cs}-137 \\
\mathrm{Ba}-137 \mathrm{~m} \\
\mathrm{U}-238 \\
\mathrm{Cm}-244\end{array}$ & \begin{tabular}{c} 
Activiey \\
(Ci/YI) \\
\hdashline $6.35 E+02$ \\
$1.13 E+00$ \\
$1.27 E+02$ \\
$1.17 E+02$ \\
$3.39 E+02$ \\
$1.59 E+02$ \\
$1.59 E+02$ \\
$2.26 E+00$ \\
$2.63 E+02$ \\
$3.47 E+02$ \\
$3.28 E+02$ \\
$1.37 E+01$ \\
$1.05 E+01$
\end{tabular} & \\
\hline Shipping Site: LLNL & $\begin{array}{l}\text { Total volume } \\
(\mathrm{m} 3 / \mathrm{yr})\end{array}$ & $\begin{array}{cc}\text { Total Mass } \\
(\mathrm{kg} / \mathrm{y} x)\end{array}$ & $1.06 E+06$ \\
\hline & 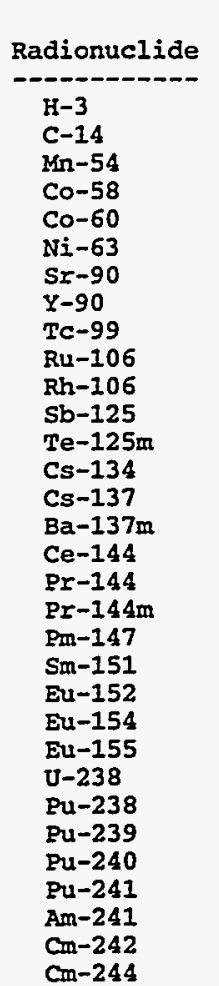 & \begin{tabular}{c} 
Activity \\
(Ci/yI) \\
\hdashline $3.33 E+04$ \\
$1.55 E-03$ \\
$1.74 E-01$ \\
$1.61 E-01$ \\
$4.69 E-01$ \\
$4.89 E-03$ \\
$6.54 E-01$ \\
$6.54 E-01$ \\
$3.18 E-03$ \\
$4.24 E-02$ \\
$4.24 E-02$ \\
$1.21 E-02$ \\
$2.97 E-03$ \\
$4.11 E-01$ \\
$9.33 E-01$ \\
$8.82 E-01$ \\
$1.42 E-01$ \\
$1.42 E-01$ \\
$1.70 E-03$ \\
$3.46 E-01$ \\
$3.39 E-03$ \\
$5.17 E-05$ \\
$8.87 E-03$ \\
$7.03 E-03$ \\
$1.88 E-02$ \\
$7.85 E-02$ \\
$5.28 E-03$ \\
$1.81 E-02$ \\
$2.50 E+00$ \\
$2.58 E-04$ \\
$1.44 E-03$ \\
$5.17 E-04$
\end{tabular} & \\
\hline
\end{tabular}




\begin{tabular}{|c|c|c|c|}
\hline Shipping Site: MOUND & $\begin{array}{l}\text { Total volume } \\
(\mathrm{m} 3 / \mathrm{yr})\end{array}$ & $\begin{array}{l}\text { Total Mass } \\
(\mathrm{kg} / \mathrm{Yr})\end{array}$ & $9.67 \mathrm{E}+06$ \\
\hline & $\begin{array}{l}\text { Radionuclide } \\
\mathrm{H}-3 \\
\mathrm{Pu}-238 \\
\mathrm{Pu}-239 \\
\mathrm{Pu}-240 \\
\mathrm{Pu}-241 \\
\mathrm{Am}-241 \\
\mathrm{Cm}-242 \\
\mathrm{Cm}-244\end{array}$ & $\begin{array}{c}\begin{array}{c}\text { Activity } \\
\text { (Ci/yr) }\end{array} \\
1.34 \mathrm{E}+04 \\
1.60 \mathrm{E}-01 \\
1.22 \mathrm{E}-02 \\
4.28 \mathrm{E}-02 \\
5.90 \mathrm{E}+00 \\
2.44 \mathrm{E}-04 \\
3.42 \mathrm{E}-03 \\
1.22 \mathrm{E}-03\end{array}$ & \\
\hline Shipping Site: ORISE & $\begin{array}{l}\text { Total volume } \\
\text { (m3/yr) }\end{array}$ & $\begin{array}{l}\text { Total Mass } \\
(\mathrm{kg} / \mathrm{yr})\end{array}$ & $7.36 \mathrm{E}+04$ \\
\hline & 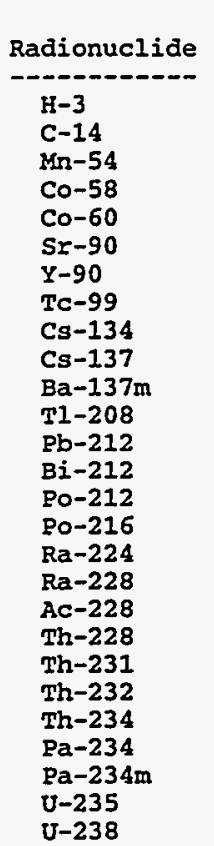 & $\begin{array}{c}\text { Activity } \\
\text { (Ci/YI) } \\
2.00 E-02 \\
1.19 E-05 \\
1.34 E-03 \\
1.24 E-03 \\
3.59 E-03 \\
1.69 E-03 \\
1.69 E-03 \\
2.39 E-05 \\
2.79 E-03 \\
3.68 E-03 \\
3.48 E-03 \\
3.39 E-07 \\
8.98 E-07 \\
8.98 E-07 \\
5.78 E-07 \\
8.98 E-07 \\
8.98 E-07 \\
5.36 E-06 \\
5.36 E-06 \\
8.98 E-07 \\
5.16 E-06 \\
5.44 E-05 \\
6.62 E-03 \\
6.78 E-07 \\
6.62 E-03 \\
5.14 E-06 \\
6.77 E-03\end{array}$ & \\
\hline
\end{tabular}


A-177

\begin{tabular}{|c|c|c|c|}
\hline Shipping Sice: ORNL & $\begin{array}{l}\text { Total Volume } \\
\text { (m3/yr) }\end{array}$ & $\begin{array}{c}\text { Total Mass } \\
(\mathrm{kg} / \mathrm{yr})\end{array}$ & $3.30 \mathrm{E}+06$ \\
\hline & $\begin{array}{l}\text { Radionuclide } \\
\mathrm{H}-3 \\
\mathrm{C}-14 \\
\mathrm{Cr}-51 \\
\mathrm{Mn}-54 \\
\mathrm{Ee}-55 \\
\mathrm{Fe}-59 \\
\mathrm{Co}-58 \\
\mathrm{Co}-60 \\
\mathrm{Ni}-59 \\
\mathrm{Ni}-63 \\
\mathrm{Sr}-90 \\
\mathrm{Y}-90 \\
\mathrm{Zr}-95 \\
\mathrm{Tc}-99 \\
\mathrm{Ru}-106 \\
\mathrm{Rh}-106 \\
\mathrm{Sb}-125 \\
\mathrm{Te}-125 \mathrm{~m} \\
\mathrm{Cs}-134 \\
\mathrm{Cs}-137 \\
\mathrm{Ba}-137 \mathrm{~m} \\
\mathrm{Ce}-144 \\
\mathrm{Pr}-144 \\
\mathrm{Pr}-144 \mathrm{~m} \\
\mathrm{Pm}-147 \\
\mathrm{Sm}-151 \\
\mathrm{Eu}-152 \\
\mathrm{Eu}-154 \\
\mathrm{Eu}-155 \\
\mathrm{Tl}-208 \\
\mathrm{~Pb}-212 \\
\mathrm{Bi}-212 \\
\mathrm{Po}-212 \\
\mathrm{PO}-216 \\
\mathrm{Ra}-224 \\
\mathrm{Ra}-228 \\
\mathrm{Ac}-228 \\
\mathrm{Th}-228 \\
\mathrm{Th}-231 \\
\mathrm{Th}-232 \\
\mathrm{Th}-234 \\
\mathrm{~Pa}-234 \\
\mathrm{~Pa}-234 \mathrm{~m} \\
\mathrm{U}-235 \\
\mathrm{U}-238 \\
\mathrm{Pu}-238 \\
\mathrm{Pu}-239 \\
\mathrm{Pu}-240 \\
\mathrm{Pu}-241 \\
\mathrm{Am}-241 \\
\mathrm{Cm}-242 \\
\mathrm{Cm}-244 \\
\end{array}$ & 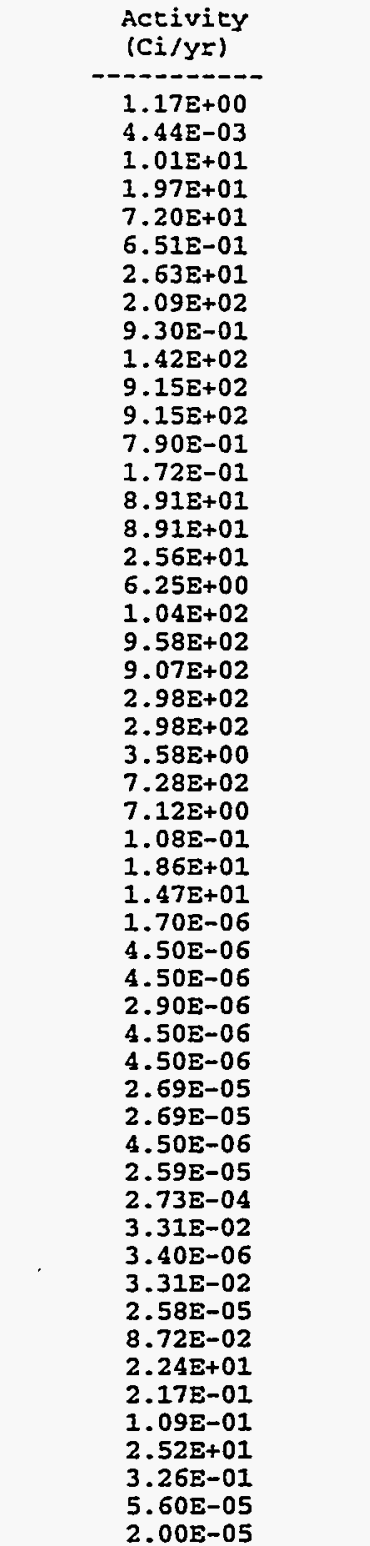 & \\
\hline
\end{tabular}


A- 178

\begin{tabular}{|c|c|c|c|}
\hline Shipping Site: PANT & $\begin{array}{l}\text { Total volume } \\
(\mathrm{m} 3 / \mathrm{yr})\end{array}$ & $\begin{array}{l}\text { Total Mass } \\
(\mathrm{kg} / \mathrm{Mr})\end{array}$ & $2.88 \mathrm{E}+07$ \\
\hline & $\begin{array}{l}\text { Radionuclide } \\
\mathrm{H}-3 \\
\mathrm{Tl}-208 \\
\mathrm{~Pb}-212 \\
\mathrm{Bi}-212 \\
\mathrm{Po}-212 \\
\mathrm{Po}-216 \\
\mathrm{Ra}-224 \\
\mathrm{Ra}-228 \\
\mathrm{Ac}-228 \\
\mathrm{Th}-228 \\
\mathrm{Th}-231 \\
\mathrm{Th}-232 \\
\mathrm{Th}-234 \\
\mathrm{~Pa}-234 \\
\mathrm{~Pa}-234 \mathrm{~m} \\
\mathrm{U}-235 \\
\mathrm{U}-238\end{array}$ & \multicolumn{2}{|l|}{$\begin{array}{l}2.34 E+02 \\
2.21 E-04 \\
5.86 E-04 \\
5.86 E-04 \\
3.78 E-04 \\
5.86 \Xi-04 \\
5.86 \Xi-04 \\
3.50 E-03 \\
3.50 E-03 \\
5.86 E-04 \\
3.37 E-03 \\
3.56 E-02 \\
4.32 E+00 \\
4.43 E-04 \\
4.32 E+00 \\
3.36 E-03 \\
4.32 E+00\end{array}$} \\
\hline \multirow[t]{2}{*}{ Shipping Site: PORTS } & $\begin{array}{l}\text { Total Volume } \\
(\mathrm{m} 3 / \mathrm{yx})\end{array}$ & $\begin{array}{cc}1.07 \mathrm{E}+04 & \begin{array}{c}\text { Total Mass } \\
(\mathrm{kg} / \mathrm{Yr})\end{array}\end{array}$ & $6.73 \mathrm{E}+07$ \\
\hline & $\begin{array}{l}\text { Radionuclide } \\
\mathrm{TI}-208 \\
\mathrm{~Pb}-212 \\
\mathrm{Bi}-212 \\
\mathrm{Po}-212 \\
\mathrm{Po}-216 \\
\mathrm{Ra}-224 \\
\mathrm{Ra}-228 \\
\mathrm{Ac}-228 \\
\mathrm{Th}-228 \\
\mathrm{Th}-231 \\
\mathrm{Th}-232 \\
\mathrm{Th}-234 \\
\mathrm{~Pa}-234 \\
\mathrm{~Pa}-234 \mathrm{~m} \\
\mathrm{U}-235 \\
\mathrm{U}-238\end{array}$ & \multicolumn{2}{|l|}{$\begin{array}{l}3.45 \mathrm{E}-06 \\
9.14 \mathrm{E}-06 \\
9.14 \mathrm{E}-06 \\
5.89 \mathrm{E}-06 \\
9.14 \mathrm{E}-06 \\
9.14 \mathrm{E}-06 \\
5.46 \mathrm{E}-05 \\
5.46 \mathrm{E}-05 \\
9.14 \mathrm{E}-06 \\
5.26 \mathrm{E}-05 \\
5.55 \mathrm{E}-04 \\
6.74 \mathrm{E}-02 \\
6.91 \mathrm{E}-06 \\
6.74 \mathrm{E}-02 \\
5.24 \mathrm{E}-05 \\
6.74 \mathrm{E}-02\end{array}$} \\
\hline \multirow[t]{2}{*}{ Shipping Site: PPPL } & $\begin{array}{l}\text { Total Volume } \\
(\mathrm{m} 3 / \mathrm{yr})\end{array}$ & $\begin{array}{l}\text { Total Mass } \\
(\mathrm{kg} / \mathrm{yr})\end{array}$ & $6.10 \mathrm{E}+03$ \\
\hline & $\begin{array}{l}\text { Radionuclide } \\
\mathrm{H}-3 \\
\mathrm{Cr}-51 \\
\mathrm{Mn}-54 \\
\mathrm{Fe}-55 \\
\mathrm{Fe}-59 \\
\mathrm{Co}-58 \\
\mathrm{Co}-60 \\
\mathrm{Ni}-59 \\
\mathrm{Ni}-63 \\
\mathrm{Zr}-95\end{array}$ & $\begin{array}{c}\begin{array}{c}\text { Activity } \\
\text { (Ci/Yr) }\end{array} \\
2.01 \mathrm{E}-01 \\
8.50 \mathrm{E}-04 \\
1.61 \mathrm{E}-03 \\
6.04 \mathrm{E}-03 \\
5.46 \mathrm{E}-05 \\
2.17 \mathrm{E}-03 \\
1.70 \mathrm{E}-02 \\
7.80 \mathrm{E}-05 \\
1.10 \mathrm{E}-02 \\
6.63 \mathrm{E}-05\end{array}$ & \\
\hline
\end{tabular}


A-179

\begin{tabular}{|c|c|c|c|}
\hline Shipping Site: Paducah & $\begin{array}{l}\text { Total Volume } \\
(\mathrm{m} 3 / \mathrm{yr})\end{array}$ & $\begin{array}{c}\text { Total Mass } \\
(\mathrm{kg} / \mathrm{yr})\end{array}$ & $1.208+07$ \\
\hline & $\begin{array}{l}\text { Radionuclide } \\
\text { Tc-99 } \\
\text { Tl-208 } \\
\text { Pb-212 } \\
\text { Bi-212 } \\
\text { Po-212 } \\
\text { Po-216 } \\
\text { Ra-2244 } \\
\text { Ra-228 } \\
\text { Ac-228 } \\
\text { Th-228 } \\
\text { Th-231 } \\
\text { Th-232 } \\
\text { Th-234 } \\
\text { Pa-234 } \\
\text { Pa-234m } \\
\mathrm{U}-235 \\
\mathrm{U}-238 \\
\mathrm{~Np}-237 \\
\mathrm{Pu}-239\end{array}$ & \begin{tabular}{c}
$\begin{array}{c}\text { Activity } \\
(\mathrm{Ci} / \mathrm{yr})\end{array}$ \\
\hdashline $8.84 \mathrm{E}-03$ \\
$3.72 \mathrm{E}-05$ \\
$9.84 \mathrm{E}-05$ \\
$9.84 \mathrm{E}-05$ \\
$6.34 \mathrm{E}-05$ \\
$9.84 \mathrm{E}-05$ \\
$9.84 \mathrm{E}-05$ \\
$5.88 \mathrm{E}-04$ \\
$5.88 \mathrm{E}-04$ \\
$9.84 \mathrm{E}-05$ \\
$5.66 \mathrm{E}-04$ \\
$5.97 \mathrm{E}-03$ \\
$7.26 \mathrm{E}-01$ \\
$7.44 \mathrm{E}-05$ \\
$7.26 \mathrm{E}-01$ \\
$5.64 \mathrm{E}-04$ \\
$7.26 \mathrm{E}-01$ \\
$6.63 \mathrm{E}-03$ \\
$6.63 \mathrm{E}-03$
\end{tabular} & \\
\hline Shipping Site: Pinellas & $\begin{array}{l}\text { Total Volume } \\
(\mathrm{m} 3 / \mathrm{yr})\end{array}$ & $\begin{array}{l}\text { Total Mass } \\
(\mathrm{kg} / \mathrm{Yr})\end{array}$ & $1.91 \mathrm{E}+04$ \\
\hline & $\begin{array}{l}\text { Radionuclide } \\
\mathrm{H}-3\end{array}$ & $\begin{array}{c}\begin{array}{c}\text { Activity } \\
\text { (Ci/Yr) }\end{array} \\
1.99 E+04\end{array}$ & \\
\hline Shipping Site: RFP & $\begin{array}{l}\text { Total Volume } \\
(\mathrm{m} 3 / \mathrm{y} r)\end{array}$ & $4.56 \mathrm{E}+03 \quad \begin{array}{c}\text { Total Mass } \\
(\mathrm{kg} / \mathrm{yz})\end{array}$ & $6.49 \mathrm{E}+06$ \\
\hline & $\begin{array}{l}\text { Radionuclide } \\
-\mathrm{TI}-208 \\
\mathrm{~Pb}-212 \\
\mathrm{Bi}-212 \\
\mathrm{PO}-212 \\
\mathrm{Po}-216 \\
\mathrm{Ra}-224 \\
\mathrm{Ra}-228 \\
\mathrm{Ac}-228 \\
\mathrm{Th}-228 \\
\mathrm{Th}-231 \\
\mathrm{Th}-232 \\
\mathrm{Th}-234 \\
\mathrm{~Pa}-234 \\
\mathrm{~Pa}-234 \mathrm{~m} \\
\mathrm{U}-235 \\
\mathrm{U}-238 \\
\mathrm{Pu}-238 \\
\mathrm{Pu}-239 \\
\mathrm{Pu}-240 \\
\mathrm{Pu}-241 \\
\mathrm{Am}-241 \\
\mathrm{Cm}-242 \\
\mathrm{Cm}-244\end{array}$ & $\begin{array}{c}\text { Activity } \\
\text { (Ci/yr) } \\
3.60 \mathrm{E}-05 \\
9.55 \mathrm{E}-05 \\
9.55 \mathrm{E}-05 \\
6.15 \mathrm{E}-05 \\
9.55 \mathrm{E}-05 \\
9.55 \mathrm{E}-05 \\
5.70 \mathrm{E}-04 \\
5.70 \mathrm{E}-04 \\
9.55 \mathrm{E}-05 \\
5.49 \mathrm{E}-04 \\
5.79 \mathrm{E}-03 \\
7.04 \mathrm{E}-01 \\
7.21 \mathrm{E}-05 \\
7.04 \mathrm{E}-01 \\
5.47 \mathrm{E}-04 \\
7.04 \mathrm{E}-01 \\
5.56 \mathrm{E}-01 \\
4.24 \mathrm{E}-02 \\
1.48 \mathrm{E}-01 \\
2.04 \mathrm{E}+01 \\
8.48 \mathrm{E}-04 \\
1.18 \mathrm{E}-02 \\
4.24 \mathrm{E}-03\end{array}$ & \\
\hline
\end{tabular}


accident risk assessment uses site-specific and waste-type-specific radiological and physical waste characteristics, as shown in the WASTE_MGMT output files in Appendix A. In addition, the assessment uses route-specific information on population density and accident rates derived for individual states.

\subsubsection{Radiological Accident Consequence Assessment}

The RISKIND code is used to provide a scenario-specific assessment of radiological consequences of severe transportation-related accidents. Whereas the RADTRAN 4 accident risk assessment considers the entire range of accident severities and their related probabilities, the RISKIND accident consequence assessment focuses on accidents that result in the largest release of radioactive material to the environment.

Accident consequences are presented for a shipment of waste that represents the highest potential radiological risk if an accident occurs. This "worst-case" waste is identified by screening the site-specific radiological waste characteristics (i.e., activity concentrations) developed for the WM PEIS, taking into account the physical forms of waste and the relative hazards of individual radionuclides. For the majority of waste shipments, the consequences of severe accidents would be less than those presented for the "worst-case" waste. The accident consequence assessment is intended to provide an estimate of the maximum potential impacts posed by a severe transportationrelated accident.

The severe accidents considered in the consequence assessment are characterized by extreme mechanical and thermal forces. In all cases, these accidents result in a release of radioactive material to the environment. The accidents correspond to those within the highest accident severity category, as described previously. These accidents represent low-probability high-consequence events. The probability of accidents of this magnitude occurring for each alternative depends on the total shipping distance for the alternative; however, accidents of this severity are expected to be extremely rare in general.

RISKIND was used for the accident consequence assessment for two reasons. First, the code has the ability to model the complex atmospheric (or site-specific) dispersion present in severe accidents. The atmospheric dispersion is modeled as an instantaneous release by using standard Gaussian puff methods. In addition, because severe accidents routinely involve fires, modeling of the potential radiological consequences takes into account physical phenomena resulting from the fire, such as buoyant plume rise. Second, RISKIND can be used to estimate the dose to MEIs in the vicinity of an accident. The location of the MEIs is determined by RISKIND on the basis of the atmospheric conditions assumed at the time of the accident and the thermal characteristics of the release. 


\section{A- 180}

\begin{tabular}{|c|c|c|c|}
\hline Shipping Site: RMI & $\begin{array}{l}\text { Total Volume } \\
(\mathrm{m} 3 / \mathrm{yr})\end{array}$ & $\begin{array}{l}\text { Total Mass } \\
(\mathrm{kg} / \mathrm{yr})\end{array}$ & $1.47 E+07$ \\
\hline & $\begin{array}{l}\text { Radionuclide } \\
\mathrm{T} 1-208 \\
\mathrm{~Pb}-212 \\
\mathrm{Bi}-212 \\
\mathrm{Po}-212 \\
\mathrm{Po}-216 \\
\mathrm{Ra}-224 \\
\mathrm{Ra}-228 \\
\mathrm{Ac}-228 \\
\mathrm{Th}-228 \\
\mathrm{Th}-231 \\
\mathrm{Th}-232 \\
\mathrm{Th}-234 \\
\mathrm{~Pa}-234 \\
\mathrm{~Pa}-234 \mathrm{~m} \\
\mathrm{U}-235 \\
\mathrm{U}-238\end{array}$ & \multicolumn{2}{|l|}{ 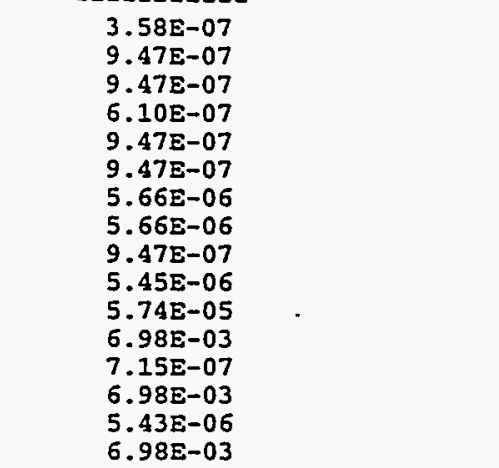 } \\
\hline Shipping Site: SLAC & $\begin{array}{l}\text { Total Volume } \\
\text { (n3/yr) }\end{array}$ & $\begin{array}{cc}\text { Total Mass } \\
(\mathrm{kg} / \mathrm{y} r)\end{array}$ & $7.45 E+05$ \\
\hline - & $\begin{array}{l}\text { Radionuclide } \\
\mathrm{Cr}-51 \\
\mathrm{Mn}-54 \\
\mathrm{Fe}-55 \\
\mathrm{Fe}-59 \\
\mathrm{Co}-58 \\
\mathrm{Co}-60 \\
\mathrm{Ni}-59 \\
\mathrm{Ni}-63 \\
\mathrm{Zr}-95\end{array}$ & $\begin{array}{c}\begin{array}{c}\text { Activity } \\
\text { (Ci/yI) }\end{array} \\
3.86 E-03 \\
7.36 \mathrm{E}-03 \\
2.74 \mathrm{E}-02 \\
2.48 \mathrm{E}-04 \\
9.88 \mathrm{E}-03 \\
7.74 \mathrm{E}-02 \\
3.54 \mathrm{E}-04 \\
5.04 \mathrm{E}-02 \\
3.01 \mathrm{E}-04\end{array}$ & \\
\hline
\end{tabular}


A-18I

\begin{tabular}{|c|c|c|c|}
\hline Shipping Sice: SNLA & $\begin{array}{l}\text { Total Volume } \\
(\mathrm{m} 3 / \mathrm{yr})\end{array}$ & $\begin{array}{l}\text { Total Mass } \\
(\mathrm{kg} / \mathrm{yr})\end{array}$ & $3.28 \mathrm{E}+05$ \\
\hline & $\begin{array}{l}\text { Radionuclide } \\
\mathrm{H}-3 \\
\mathrm{Co}-60 \\
\mathrm{Ni}-63 \\
\mathrm{Sr}-90 \\
\mathrm{Y}-90 \\
\mathrm{Tc}-99 \\
\mathrm{Ru}-106 \\
\mathrm{Rh}-106 \\
\mathrm{Sb}-125 \\
\mathrm{Te}-125 \mathrm{~m} \\
\mathrm{Cs}-134 \\
\mathrm{Cs}-137 \\
\mathrm{Ba}-137 \mathrm{~m} \\
\mathrm{Ce}-144 \\
\mathrm{Pr}-144 \\
\mathrm{Pr}-144 \mathrm{~m} \\
\mathrm{Pm}-147 \\
\mathrm{Sm}-151 \\
\mathrm{Eu}-152 \\
\mathrm{Eu}-154 \\
\mathrm{Eu}-155 \\
\mathrm{Tl}-208 \\
\mathrm{~Pb}-212 \\
\mathrm{Bi}-212 \\
\mathrm{Po}-212 \\
\mathrm{Po}-216 \\
\mathrm{Ra}-224 \\
\mathrm{Ra}-228 \\
\mathrm{Ac}-228 \\
\mathrm{Th}-228 \\
\mathrm{Th}-231 \\
\mathrm{Th}-232 \\
\mathrm{Th}-234 \\
\mathrm{~Pa}-234 \\
\mathrm{~Pa}-234 \mathrm{~m} \\
\mathrm{U}-235 \\
\mathrm{U}-238 \\
\mathrm{Pu}-238 \\
\mathrm{Pu}-239 \\
\mathrm{Pu}-240 \\
\mathrm{Pu}-241 \\
\mathrm{Am}-241 \\
\mathrm{Cm}-242 \\
\mathrm{Cm}-244 \\
\end{array}$ & \multicolumn{2}{|l|}{$\begin{array}{l}3.00 \mathrm{E}+00 \\
2.85 \mathrm{E}-05 \\
5.67 \mathrm{E}-05 \\
5.04 \mathrm{E}-03 \\
5.04 \mathrm{E}-03 \\
9.00 \mathrm{E}-07 \\
4.92 \mathrm{E}-04 \\
4.92 \mathrm{E}-04 \\
1.41 \mathrm{E}-04 \\
3.45 \mathrm{E}-05 \\
5.72 \mathrm{E}-04 \\
5.28 \mathrm{E}-03 \\
4.99 \mathrm{E}-03 \\
1.64 \mathrm{E}-03 \\
1.64 \mathrm{E}-03 \\
1.98 \mathrm{E}-05 \\
4.02 \mathrm{E}-03 \\
3.93 \mathrm{E}-05 \\
6.00 \mathrm{E}-07 \\
1.02 \mathrm{E}-04 \\
8.16 \mathrm{E}-05 \\
5.10 \mathrm{E}-06 \\
1.35 \mathrm{E}-05 \\
1.35 \mathrm{E}-05 \\
8.70 \mathrm{E}-06 \\
1.35 \mathrm{E}-05 \\
1.35 \mathrm{E}-05 \\
8.07 \mathrm{E}-05 \\
8.07 \mathrm{E}-05 \\
1.35 \mathrm{E}-05 \\
7.77 \mathrm{E}-05 \\
8.19 \mathrm{E}-04 \\
9.96 \mathrm{E}-02 \\
1.02 \mathrm{E}-05 \\
9.96 \mathrm{E}-02 \\
7.74 \mathrm{E}-05 \\
9.96 \mathrm{E}-02 \\
7.87 \mathrm{E}-02 \\
6.00 \mathrm{E}-03 \\
2.10 \mathrm{E}-02 \\
2.89 \mathrm{E}+00 \\
1.21 \mathrm{E}-04 \\
1.68 \mathrm{E}-03 \\
6.00 \mathrm{E}-04\end{array}$} \\
\hline \multirow[t]{2}{*}{ Shipping Site: SNLL } & $\begin{array}{l}\text { Total Volume } \\
(\mathrm{m} 3 / \mathrm{Yr})\end{array}$ & $\begin{array}{cc}\text { Total Mass } \\
(\mathrm{kg} / \mathrm{y})\end{array}$ & $9.73 E+04$ \\
\hline & $\begin{array}{l}\text { Radionuclide } \\
\mathrm{H}-3 \\
\mathrm{Fe}-55 \\
\mathrm{Co}-60 \\
\mathrm{TI}-208 \\
\mathrm{~Pb}-212 \\
\mathrm{Bi}-212 \\
\mathrm{Po}-212 \\
\mathrm{Po-216} \\
\mathrm{Ra}-224 \\
\mathrm{Ra}-228 \\
\mathrm{Ac}-228 \\
\mathrm{Th}-228 \\
\mathrm{Th}-231 \\
\mathrm{Th}-232 \\
\mathrm{Th}-234 \\
\mathrm{~Pa}-234 \\
\mathrm{~Pa}-234 \mathrm{~m} \\
\mathrm{U}-235 \\
\mathrm{U}-238\end{array}$ & \begin{tabular}{c}
$\begin{array}{c}\text { Activity } \\
\text { (Ci/yY) }\end{array}$ \\
\hdashline $2.78 E+04$ \\
$3.15 \mathrm{E}-01$ \\
$3.15 \mathrm{E}-01$ \\
$6.40 \mathrm{E}-05$ \\
$1.69 \mathrm{E}-04$ \\
$1.69 \mathrm{E}-04$ \\
$1.09 \mathrm{E}-04$ \\
$1.69 \mathrm{E}-04$ \\
$1.69 \mathrm{E}-04$ \\
$1.01 \mathrm{E}-03$ \\
$1.01 \mathrm{E}-03$ \\
$1.69 \mathrm{E}-04$ \\
$9.75 \mathrm{E}-04$ \\
$1.02 \mathrm{E}-02$ \\
$1.25 \mathrm{E}+00$ \\
$1.28 \mathrm{E}-04$ \\
$1.25 \mathrm{E}+00$ \\
$9.71 \mathrm{E}-04$ \\
$1.25 \mathrm{E}+00$
\end{tabular} & \\
\hline
\end{tabular}




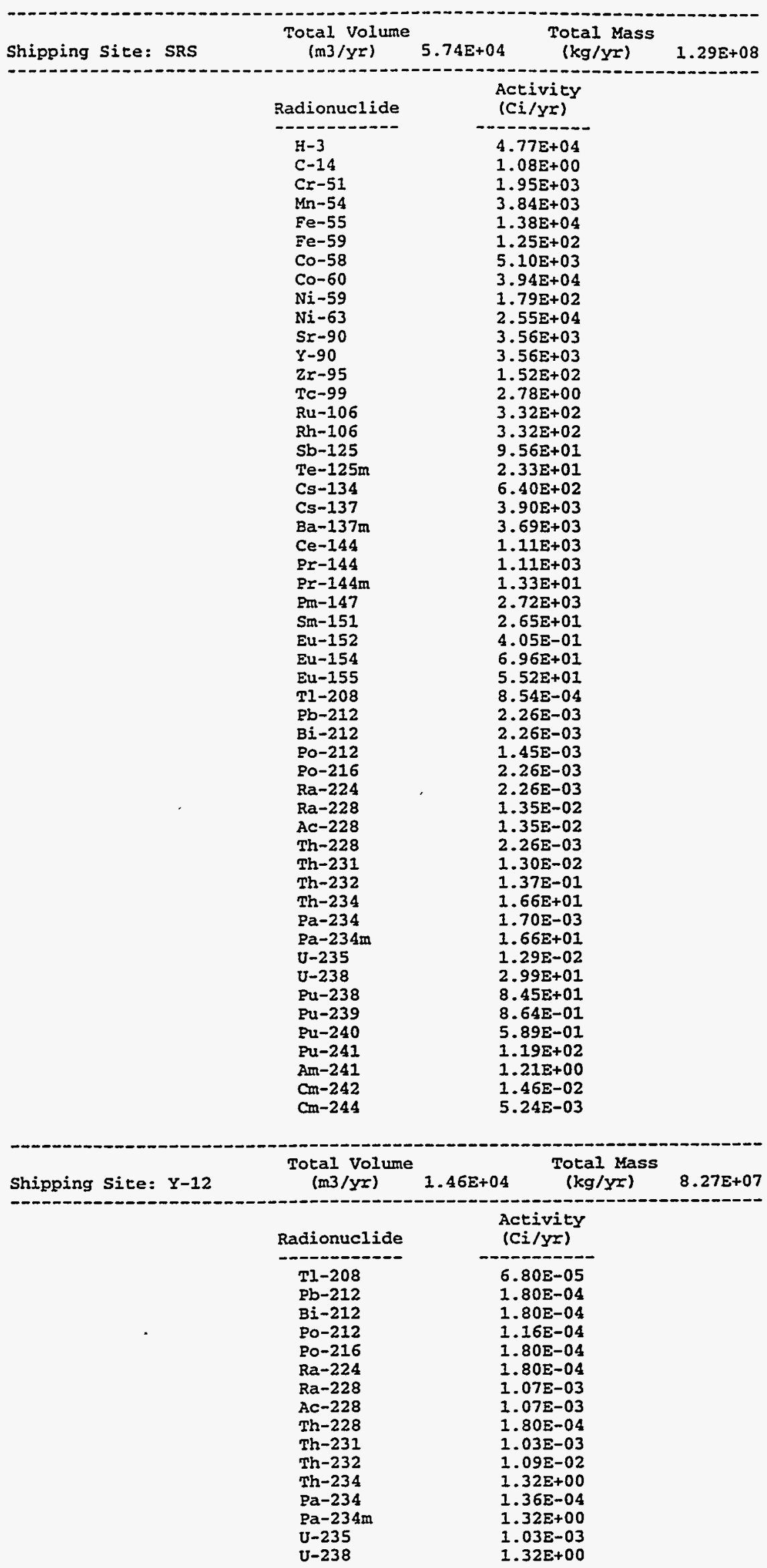




\section{A.22 WM LLW CENTRALIZED 2 ALTERNATIVE (CASE 8): ACTIVATED METALS}

Transported Material with Radionuclide Profile

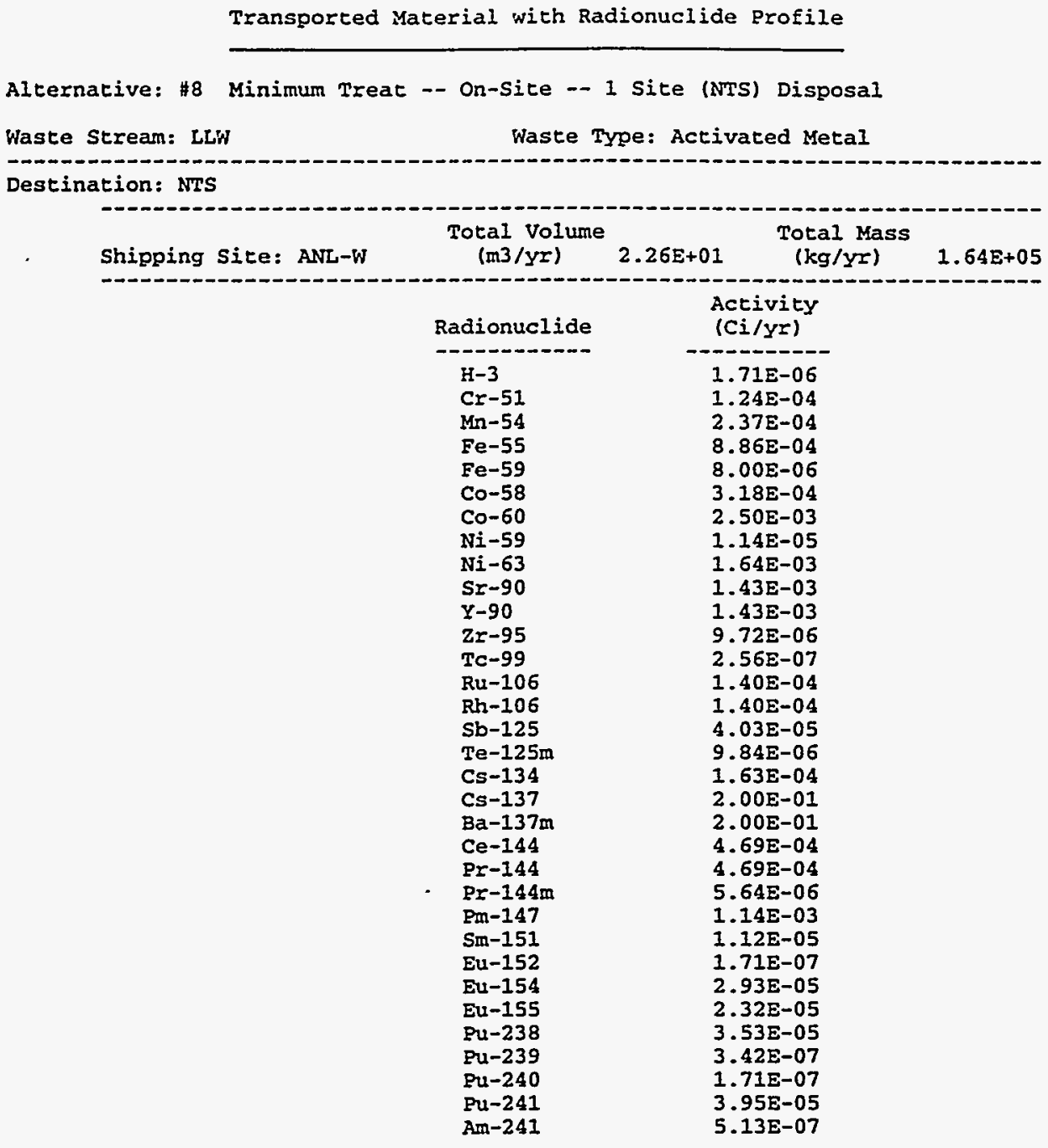


A- 184

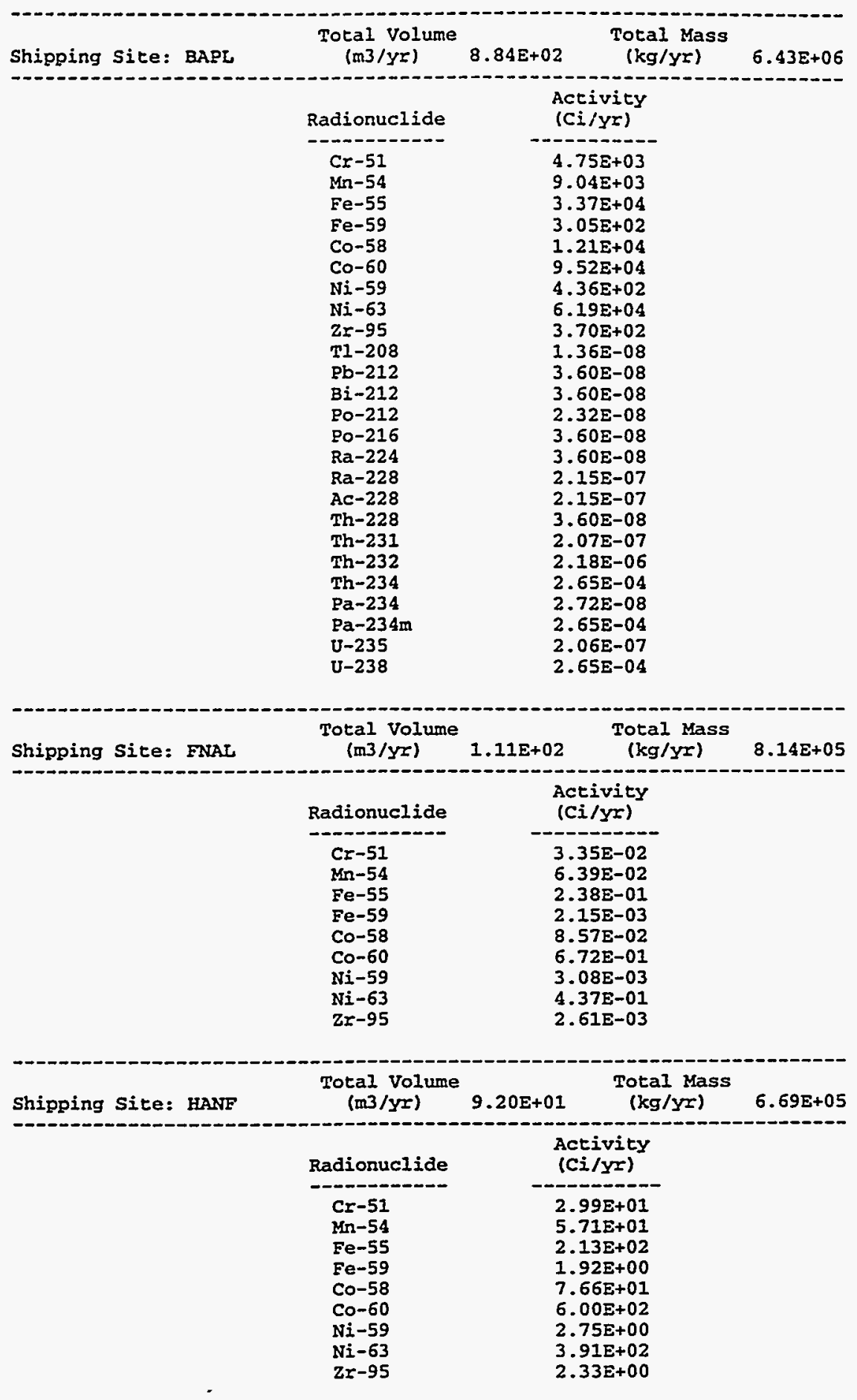


A- 185

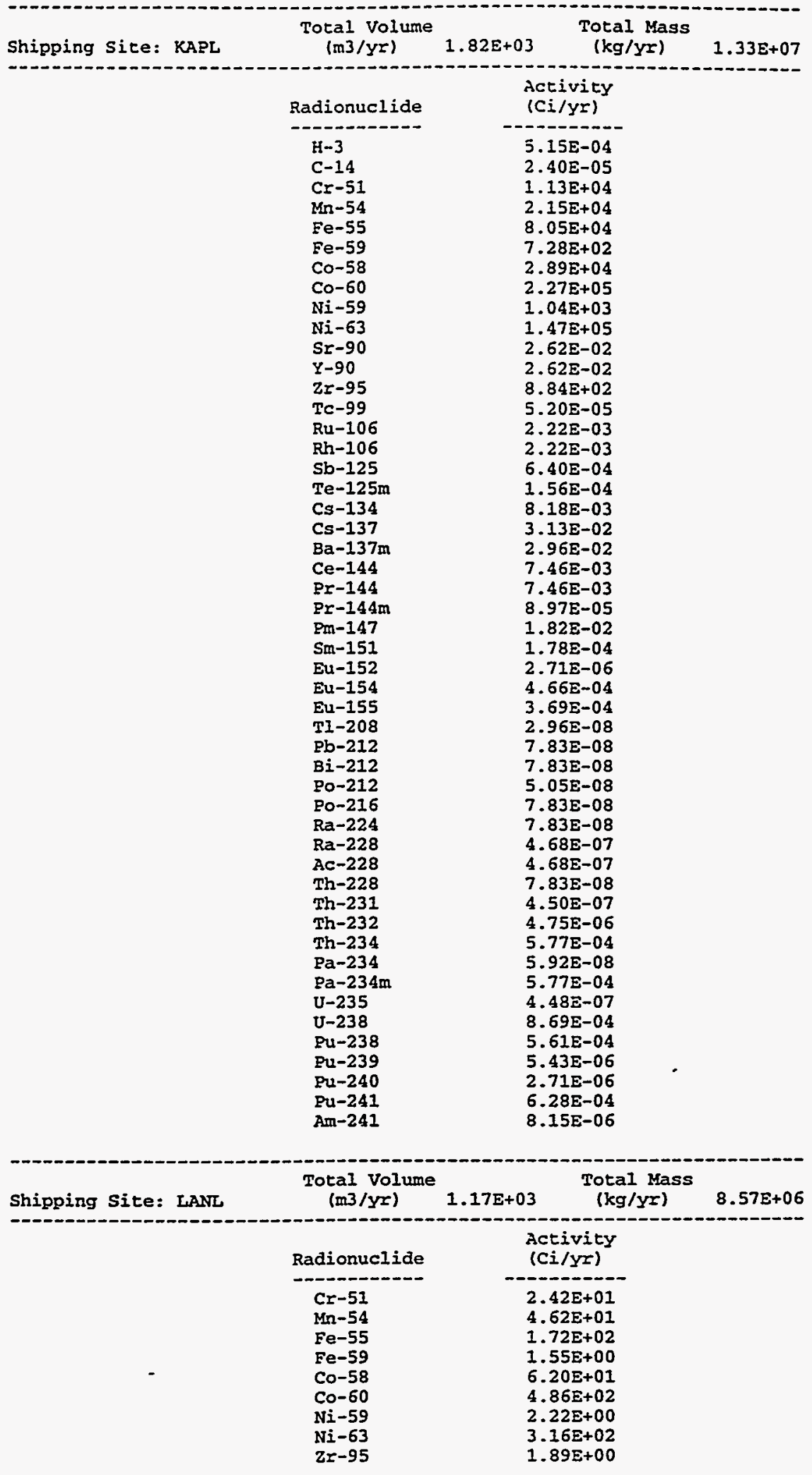




\begin{tabular}{|c|c|c|c|}
\hline Shipping Site: LBL & $\begin{array}{l}\text { Total volume } \\
(\mathrm{m} 3 / \mathrm{yr})\end{array}$ & $\begin{array}{c}\text { Total Mass } \\
\text { (kg/yr) }\end{array}$ & $2.87 \mathrm{E}+05$ \\
\hline & $\begin{array}{l}\text { Radionuclide } \\
\mathrm{Cr}-51 \\
\mathrm{Mn}-54 \\
\mathrm{Fe}-55 \\
\mathrm{Fe}-59 \\
\mathrm{Co}-58 \\
\mathrm{Co}-60 \\
\mathrm{Ni}-59 \\
\mathrm{Ni}-63 \\
\mathrm{Zr}-95\end{array}$ & \multicolumn{2}{|l|}{$\begin{array}{l}6.32 \mathrm{E}-02 \\
1.20 \mathrm{E}-01 \\
4.49 \mathrm{E}-01 \\
4.06 \mathrm{E}-03 \\
1.61 \mathrm{E}-01 \\
1.26 \mathrm{E}+00 \\
5.80 \mathrm{E}-03 \\
8.24 \mathrm{E}-01 \\
4.93 \mathrm{E}-03\end{array}$} \\
\hline \multirow[t]{2}{*}{ Shipping site: NRF } & $\begin{array}{l}\text { Total Volume } \\
\text { (m3/yr) }\end{array}$ & $\begin{array}{c}\text { Total Mass } \\
(\mathrm{kg} / \mathrm{yr})\end{array}$ & $9.40 \mathrm{E}+06$ \\
\hline & $\begin{array}{l}\text { Radionuclide } \\
\mathrm{Cr}-51 \\
\mathrm{Mn}-54 \\
\mathrm{Fe}-55 \\
\mathrm{Fe}-59 \\
\mathrm{Co}-58 \\
\mathrm{Co}-60 \\
\mathrm{Ni}-59 \\
\mathrm{Ni}-63 \\
\mathrm{Zr}-95\end{array}$ & \multicolumn{2}{|l|}{$\begin{array}{l}2.31 \mathrm{E}+03 \\
4.40 \mathrm{E}+03 \\
1.64 \mathrm{E}+04 \\
1.48 \mathrm{E}+02 \\
5.91 \mathrm{E}+03 \\
4.63 \mathrm{E}+04 \\
2.12 \mathrm{E}+02 \\
3.01 \mathrm{E}+04 \\
1.80 \mathrm{E}+02\end{array}$} \\
\hline \multirow[t]{2}{*}{ Shipping Site: SNLA } & $\begin{array}{l}\text { Total Volume } \\
(\mathrm{m} 3 / \mathrm{yr})\end{array}$ & $\begin{array}{c}\text { Total Mass } \\
(\mathrm{kg} / \mathrm{YI})\end{array}$ & $1.15 E+05$ \\
\hline & $\begin{array}{l}\text { Radionuclide } \\
\text { Cr-51 } \\
\mathrm{Mn}-54 \\
\mathrm{Fe}-55 \\
\mathrm{Fe}-59 \\
\mathrm{Co}-58 \\
\mathrm{Co}-60 \\
\mathrm{Ni}-59 \\
\mathrm{Ni}-63 \\
\mathrm{Zr}-95\end{array}$ & \multicolumn{2}{|l|}{$\begin{array}{l}6.54 \mathrm{E}-04 \\
1.24 \mathrm{E}-03 \\
4.64 \mathrm{E}-03 \\
4.20 \mathrm{E}-05 \\
1.67 \mathrm{E}-03 \\
1.31 \mathrm{E}-02 \\
6.00 \mathrm{E}-05 \\
8.53 \mathrm{E}-03 \\
5.10 \mathrm{E}-05\end{array}$} \\
\hline
\end{tabular}




\section{A.23 WM LLW CENTRALIZED 3 ALTERNATIVE (CASE 14): HETEROGENEOUS SOLIDS}

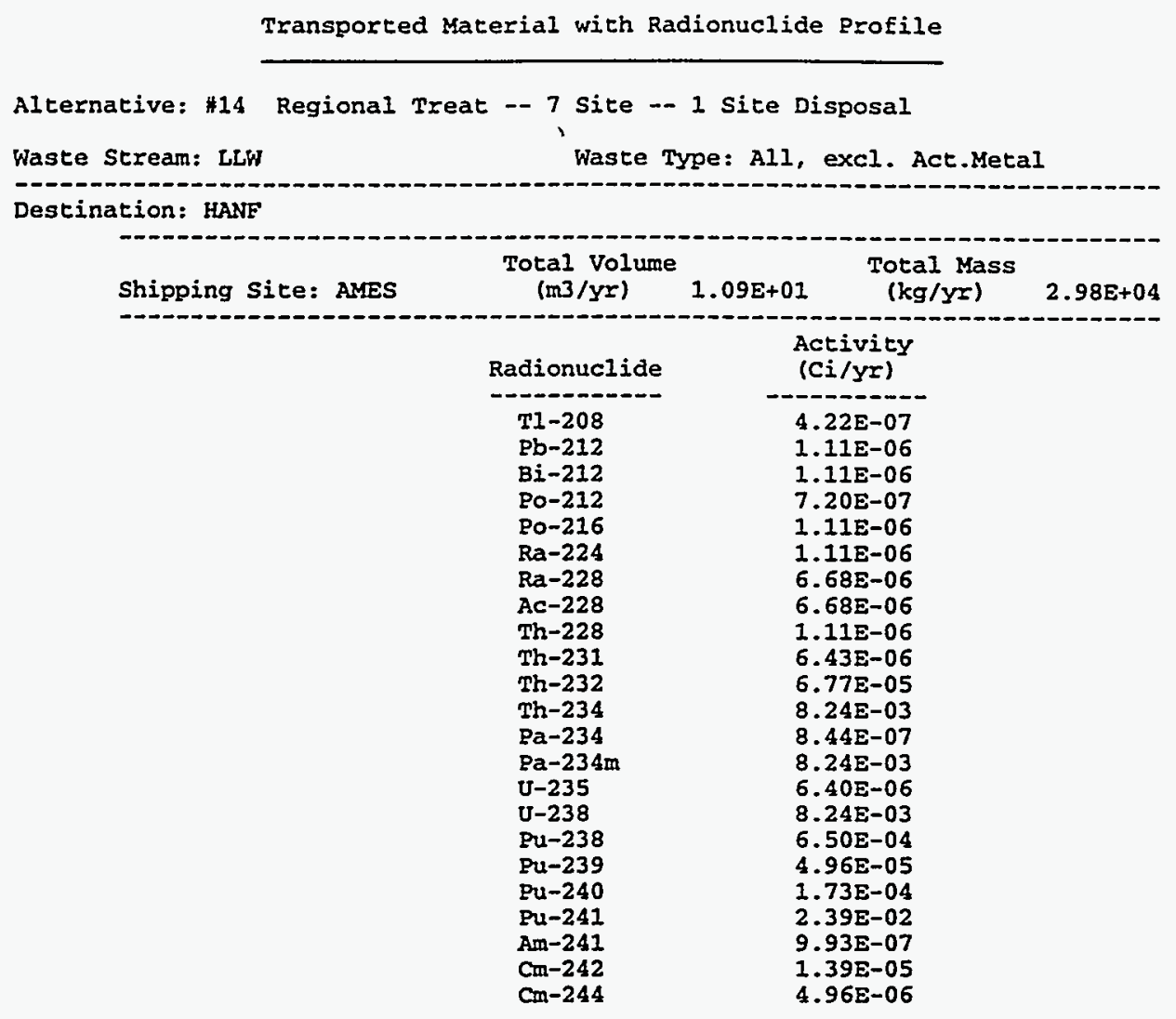




\begin{tabular}{|c|c|c|c|}
\hline Shipping Site: ANL-E & $\begin{array}{l}\text { Total Volume } \\
(\mathrm{m} 3 / \mathrm{yr})\end{array}$ & $\begin{array}{l}\text { Total Mass } \\
(\mathrm{kg} / \mathrm{y})\end{array}$ & $2.00 E+06$ \\
\hline & $\begin{array}{l}\text { Radionuclide } \\
\mathrm{H}-3 \\
\mathrm{C}-14 \\
\mathrm{Cr}-51 \\
\mathrm{Mn}-54 \\
\mathrm{Fe}-55 \\
\mathrm{Fe}-59 \\
\mathrm{Co}-58 \\
\mathrm{Co}-60 \\
\mathrm{Ni}-59 \\
\mathrm{Ni}-63 \\
\mathrm{Sr}-90 \\
\mathrm{Y}-90 \\
\mathrm{Zr}-95 \\
\mathrm{Tc}-99 \\
\mathrm{Ru}-106 \\
\mathrm{Rh}-106 \\
\mathrm{Sb}-125 \\
\mathrm{Te}-125 \mathrm{~m} \\
\mathrm{Cs}-134 \\
\mathrm{Cs}-137 \\
\mathrm{Ba}-137 \mathrm{~m} \\
\mathrm{Ce}-144 \\
\mathrm{Pr}-144 \\
\mathrm{Pr}-144 \mathrm{~m} \\
\mathrm{Pm}-147 \\
\mathrm{Sm}-151 \\
\text { Eu-152 } \\
\text { Eu-154 } \\
\text { Eu-155 } \\
\mathrm{U}-238 \\
\mathrm{Pu}-238 \\
\mathrm{Pu}-239 \\
\mathrm{Pu}-240 \\
\mathrm{Pu}-241 \\
\mathrm{Am}-241 \\
\end{array}$ & $\begin{array}{c}\text { Activicy } \\
(C i / y=) \\
1.33 \mathrm{E}+02 \\
7.91 \mathrm{E}-02 \\
2.87 \mathrm{E}+00 \\
1.43 \mathrm{E}+01 \\
2.04 \mathrm{E}+01 \\
1.84 \mathrm{E}-01 \\
1.55 \mathrm{E}+01 \\
8.15 \mathrm{E}+01 \\
2.63 \mathrm{E}-01 \\
3.77 \mathrm{E}+01 \\
3.33 \mathrm{E}+01 \\
3.33 \mathrm{E}+01 \\
2.24 \mathrm{E}-01 \\
1.62 \mathrm{E}-01 \\
2.16 \mathrm{E}+00 \\
2.16 \mathrm{E}+00 \\
6.21 \mathrm{E}-01 \\
1.51 \mathrm{E}-01 \\
2.09 \mathrm{E}+01 \\
4.75 \mathrm{E}+01 \\
4.50 \mathrm{E}+01 \\
7.24 \mathrm{E}+00 \\
7.24 \mathrm{E}+00 \\
8.70 \mathrm{E}-02 \\
1.76 \mathrm{E}+01 \\
1.72 \mathrm{E}-01 \\
2.63 \mathrm{E}-03 \\
4.52 \mathrm{E}-01 \\
3.58 \mathrm{E}-01 \\
9.63 \mathrm{E}-01 \\
5.44 \mathrm{E}-01 \\
5.27 \mathrm{E}-03 \\
2.63 \mathrm{E}-03 \\
6.09 \mathrm{E}-01 \\
7.91 \mathrm{E}-03\end{array}$ & \\
\hline Shipping Site: ANL-W & $\begin{array}{l}\text { Total Volume } \\
(\mathrm{m} 3 / \mathrm{yr})\end{array}$ & $\begin{array}{c}\text { Total Mass } \\
(\mathrm{kg} / \mathrm{yr})\end{array}$ & $1.83 \mathrm{E}+05$ \\
\hline & $\begin{array}{l}\text { Radionuclide } \\
\mathrm{H}-3 \\
\mathrm{Cr}-51 \\
\mathrm{Mn}-54 \\
\mathrm{Fe}-55 \\
\mathrm{Fe}-59 \\
\mathrm{Co}-58 \\
\mathrm{C}-60 \\
\mathrm{Ni}-59 \\
\mathrm{Ni}-63 \\
\mathrm{Zr}-95\end{array}$ & $\begin{array}{c}\begin{array}{c}\text { Activity } \\
(C i / y I)\end{array} \\
7.45 E-04 \\
3.58 E+03 \\
6.83 E+03 \\
2.54 E+04 \\
2.30 E+02 \\
9.16 E+03 \\
7.18 E+04 \\
3.29 E+02 \\
4.67 E+04 \\
2.79 E+02\end{array}$ & \\
\hline
\end{tabular}




\section{A- 189}

\begin{tabular}{|c|c|c|c|}
\hline Shipping Site: BAPL & $\begin{array}{l}\text { Total Volume } \\
\text { (m3/Yr) }\end{array}$ & $\begin{array}{c}\text { Total Mass } \\
(\mathrm{kg} / \mathrm{yr})\end{array}$ & $1.08 \mathrm{E}+06$ \\
\hline & $\begin{array}{l}\text { Radionuclide } \\
\mathrm{H}-3 \\
\mathrm{Co}-50 \\
\mathrm{Ni}-63 \\
\mathrm{Sr}-90 \\
\mathrm{Y}-90 \\
\mathrm{Tc}-99 \\
\mathrm{Ru}-106 \\
\mathrm{Rh}-106 \\
\mathrm{Sb}-125 \\
\mathrm{Te}-125 \mathrm{~m} \\
\mathrm{Cs}-134 \\
\mathrm{Cs}-137 \\
\mathrm{Ba}-137 \mathrm{~m} \\
\mathrm{Ce}-144 \\
\mathrm{Pr}-144 \\
\mathrm{Pr}-144 \mathrm{~m} \\
\mathrm{Pm}-147 \\
\mathrm{Sm}-151 \\
\mathrm{Eu}-152 \\
\mathrm{Eu}-154 \\
\mathrm{Eu}-155 \\
\mathrm{Tl}-208 \\
\mathrm{~Pb}-212 \\
\mathrm{Bi}-212 \\
\mathrm{Po}-212 \\
\mathrm{Po}-216 \\
\mathrm{Ra}-224 \\
\mathrm{Ra}-228 \\
\mathrm{Ac}-228 \\
\mathrm{Th}-228 \\
\mathrm{Th}-231 \\
\mathrm{Th}-232 \\
\mathrm{Th}-234 \\
\mathrm{~Pa}-234 \\
\mathrm{~Pa}-234 \mathrm{~m} \\
\mathrm{U}-235 \\
\mathrm{U}-238 \\
\mathrm{Pu}-238 \\
\mathrm{Pu}-239 \\
\mathrm{Pu}-240 \\
\mathrm{Pu}-241 \\
\mathrm{Am}-241 \\
\end{array}$ & $\begin{array}{c}\text { Activity } \\
\text { (Ci/YY) } \\
2.51 \mathrm{E}-03 \\
1.19 \mathrm{E}-02 \\
2.38 \mathrm{E}-02 \\
2.11 \mathrm{E}+00 \\
2.11 \mathrm{E}+00 \\
3.77 \mathrm{E}-04 \\
2.06 \mathrm{E}-01 \\
2.06 \mathrm{E}-01 \\
5.93 \mathrm{E}-02 \\
1.44 \mathrm{E}-02 \\
2.40 \mathrm{E}-01 \\
2.21 \mathrm{E}+00 \\
2.09 \mathrm{E}+00 \\
6.91 \mathrm{E}-01 \\
6.91 \mathrm{E}-01 \\
8.31 \mathrm{E}-03 \\
1.68 \mathrm{E}+00 \\
1.65 \mathrm{E}-02 \\
2.51 \mathrm{E}-04 \\
4.32 \mathrm{E}-02 \\
3.42 \mathrm{E}-02 \\
3.80 \mathrm{E}-06 \\
1.00 \mathrm{E}-05 \\
1.00 \mathrm{E}-05 \\
6.49 \mathrm{E}-06 \\
1.00 \mathrm{E}-05 \\
1.00 \mathrm{E}-05 \\
6.02 \mathrm{E}-05 \\
6.02 \mathrm{E}-05 \\
1.00 \mathrm{E}-05 \\
5.80 \mathrm{E}-05 \\
6.11 \mathrm{E}-04 \\
7.43 \mathrm{E}-02 \\
7.61 \mathrm{E}-06 \\
7.43 \mathrm{E}-02 \\
5.77 \mathrm{E}-05 \\
7.43 \mathrm{E}-02 \\
5.20 \mathrm{E}-02 \\
5.03 \mathrm{E}-04 \\
2.51 \mathrm{E}-04 \\
5.81 \mathrm{E}-02 \\
7.55 \mathrm{E}-04\end{array}$ & s \\
\hline Shipping Site: FNAL & $\begin{array}{l}\text { Total Volume } \\
\text { (m3/yr) }\end{array}$ & $\begin{array}{c}\text { Total Mass } \\
(\mathrm{kg} / \mathrm{y})\end{array}$ & $3.26 E+04$ \\
\hline - & $\begin{array}{l}\text { Radionuclide } \\
\mathrm{H}-3 \\
\mathrm{Cr}-51 \\
\mathrm{Mn}-54 \\
\mathrm{Fe}-55 \\
\mathrm{Fe}-59 \\
\mathrm{Co}-58 \\
\mathrm{Co}-60 \\
\mathrm{Ni}-59 \\
\mathrm{Ni}-63 \\
\mathrm{Zr}-95 \\
\mathrm{Tl}-208 \\
\mathrm{~Pb}-212 \\
\mathrm{Bi}-212 \\
\mathrm{PO}-212 \\
\mathrm{PO}-216 \\
\mathrm{Ra}-224 \\
\mathrm{Ra}-228 \\
\mathrm{Ac}-228 \\
\mathrm{Th}-228 \\
\mathrm{Th}-231 \\
\mathrm{Th}-232 \\
\mathrm{Th}-234 \\
\mathrm{~Pa}-234 \\
\mathrm{~Pa}-234 \mathrm{~m} \\
\mathrm{U}-235 \\
\mathrm{U}-238\end{array}$ & 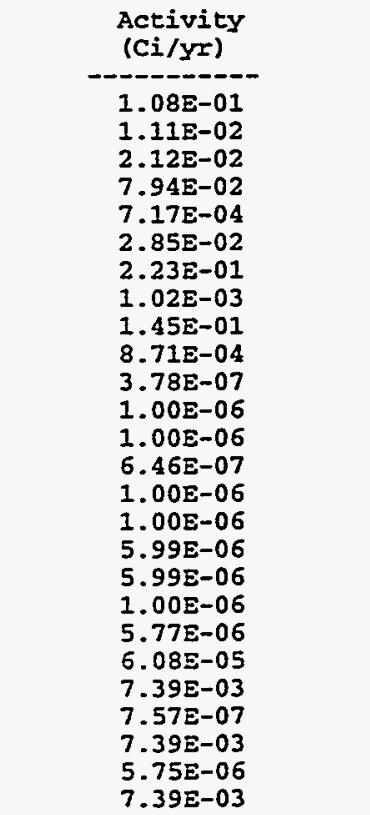 & \\
\hline
\end{tabular}


The accident consequences are calculated for both local populations and MEIs. The population dose includes the population within $80 \mathrm{~km}(50 \mathrm{mi})$ of the site of the accident. The exposure pathways considered are similar to those discussed previously for the accident risk assessment. Although remedial activities after the accident (e.g., evacuation or ground cleanup) would reduce the consequences of an accident, these activities were not given credit in the consequence assessment.

Because predicting the exact location of a severe transportation-related accident is impossible, separate accident consequences are calculated for accidents occurring in rural, suburban, and urban zones of population density. Moreover, to address the effects of the atmospheric conditions existing at the time of an accident, two different atmospheric conditions are considered. The first case assumes neutral atmospheric conditions, and the second assumes stable conditions. Atmospheric conditions are discussed further in Section 4.

\subsubsection{Vehicle-Related (Nonradiological) Accident Risk Assessment}

The vehicle-related accident risk refers to the potential for transportation-related accidents that directly result in fatalities that are not related to the cargo in the shipment. This risk represents fatalities from mechanical causes. State-specific rates for transportation-related fatalities are used in the assessment and are discussed in Section 4. Vehicle-related accident risks are calculated for each alternative by multiplying the total distance traveled in each state by the appropriate state rate for transportation-related fatalities. In all alternatives, the vehicle-related accident risks are calculated by using distances for round-trip shipment.

\subsection{REPRESENTATIVE ROUTE DETERMINATION METHOD}

Representative truck and rail routes were determined for all of the pairs of origin-and-destination sites specified by the alternative definitions. The routes were selected to be consistent with current routing practices and all applicable routing regulations and guidelines; however, because the routes were determined for the purposes of risk assessment, the routes do not necessarily represent the actual routes that would be used to transport LLW in the future.

The representative truck and rail routes were determined by using the routing models HIGHWAY 3.1 (Johnson et al. 1993a) and INTERLINE 5.0 (Johnson et al. 1993b), respectively. For both truck and rail transportation, the route characteristics that are most important to the radiological risk assessment include the total shipping distance between each origin-destination pair and the fractions of travel in rural, suburban, and urban zones of population density. The route selected determines the total potentially exposed population along a route and the expected 
A-190.

\begin{tabular}{|c|c|c|c|}
\hline \multirow{2}{*}{ Shipping Site: INEL } & $\begin{array}{c}\text { Total Volume } \\
\text { (m3/yr) }\end{array}$ & $\begin{array}{c}\text { Total Mass } \\
(\mathrm{kg} / \mathrm{yr})\end{array}$ & $1.64 \mathrm{E}+07$ \\
\hline & $\begin{array}{l}\text { Radionuclide } \\
\mathrm{H}-3 \\
\mathrm{Cr}-51 \\
\mathrm{Mn}-54 \\
\mathrm{Fe}-55 \\
\mathrm{Fe}-59 \\
\mathrm{Co}-58 \\
\mathrm{Co}-60 \\
\mathrm{Ni}-59 \\
\mathrm{Ni}-63 \\
\mathrm{Sr}-90 \\
\mathrm{Y}-90 \\
\mathrm{Zr}-95 \\
\mathrm{Tc}-99 \\
\mathrm{Ru}-106 \\
\mathrm{Rh}-106 \\
\mathrm{Sb}-125 \\
\mathrm{Te}-125 \mathrm{~m} \\
\mathrm{Cs}-134 \\
\mathrm{Cs}-137 \\
\mathrm{Ba}-137 \mathrm{~m} \\
\mathrm{Ce}-144 \\
\mathrm{Pr}-144 \\
\mathrm{Pr}-144 \mathrm{~m} \\
\mathrm{Pm}-147 \\
\mathrm{Sm}-151 \\
\mathrm{Eu}-152 \\
\mathrm{Eu}-154 \\
\mathrm{Eu}-155 \\
\mathrm{Pu}-238 \\
\mathrm{Pu}-239 \\
\mathrm{Pu}-240 \\
\mathrm{Pu}-241 \\
\mathrm{Am}-241 \\
\mathrm{Cm}-242 \\
\mathrm{Cm}-244\end{array}$ & $\begin{array}{c}\text { Activity } \\
\text { (Ci/yr) } \\
-1.56 \mathrm{y}+00 \\
1.02 \mathrm{E}+02 \\
1.94 \mathrm{E}+02 \\
7.24 \mathrm{E}+02 \\
6.55 \mathrm{E}+00 \\
2.60 \mathrm{E}+02 \\
2.04 \mathrm{E}+03 \\
9.35 \mathrm{E}+00 \\
1.33 \mathrm{E}+03 \\
5.01 \mathrm{E}+01 \\
5.01 \mathrm{E}+01 \\
7.95 \mathrm{E}+00 \\
8.96 \mathrm{E}-03 \\
4.90 \mathrm{E}+00 \\
4.90 \mathrm{E}+00 \\
1.40 \mathrm{E}+00 \\
3.43 \mathrm{E}-01 \\
5.69 \mathrm{E}+00 \\
5.25 \mathrm{E}+01 \\
4.97 \mathrm{E}+01 \\
1.64 \mathrm{E}+01 \\
1.64 \mathrm{E}+01 \\
1.97 \mathrm{E}-01 \\
4.00 \mathrm{E}+01 \\
3.91 \mathrm{E}-01 \\
5.97 \mathrm{E}-03 \\
1.02 \mathrm{E}+00 \\
8.12 \mathrm{E}-01 \\
1.23 \mathrm{E}+00 \\
1.19 \mathrm{E}-02 \\
5.97 \mathrm{E}-03 \\
1.38 \mathrm{E}+00 \\
1.79 \mathrm{E}-02 \\
4.88 \mathrm{E}-08 \\
1.74 \mathrm{E}-08\end{array}$ & . \\
\hline \multirow[t]{2}{*}{ Shipping Site: ITRI } & $\begin{array}{l}\text { Total Volume } \\
(\mathrm{m} 3 / \mathrm{Yr})\end{array}$ & $\begin{array}{l}\text { Total Mass } \\
(\mathrm{kg} / \mathrm{Yr})\end{array}$ & $1.53 \mathrm{E}+05$ \\
\hline & $\begin{array}{l}\text { Radionuclide } \\
\mathrm{H}-3 \\
\mathrm{C}-14 \\
\mathrm{Mn}-54 \\
\mathrm{Co}-58 \\
\mathrm{Co}-60 \\
\mathrm{Sr}-90 \\
\mathrm{Y}-90 \\
\mathrm{Tc}-99 \\
\mathrm{Cs}-134 \\
\mathrm{Cs}-137 \\
\mathrm{Ba}-137 \mathrm{~m} \\
\mathrm{U}-238\end{array}$ & $\begin{array}{c}\text { Activity } \\
(C i / y r)\end{array}$ & \\
\hline
\end{tabular}


A-19!

\begin{tabular}{|c|c|c|c|}
\hline Shipping Sice: $\mathrm{K}-25$ & $\begin{array}{l}\text { Total volume } \\
\text { (m3/yr) }\end{array}$ & $\begin{array}{cc}8.16 \mathrm{E}+01 & \begin{array}{c}\text { Total Mass } \\
(\mathrm{kg} / \mathrm{Yr})\end{array}\end{array}$ & $2.22 \mathrm{E}+05$ \\
\hline & $\begin{array}{l}\text { Radionuclide } \\
\mathrm{Tl}-208 \\
\mathrm{~Pb}-212 \\
\mathrm{Bi}-212 \\
\mathrm{Po-212} \\
\mathrm{Po-216} \\
\mathrm{Ra}-224 \\
\mathrm{Ra}-228 \\
\mathrm{Ac}-228 \\
\mathrm{Th}-228 \\
\mathrm{Th}-231 \\
\mathrm{Th}-232 \\
\mathrm{Th}-234 \\
\mathrm{~Pa}-234 \\
\mathrm{~Pa}-234 \mathrm{~m} \\
\mathrm{U}-235 \\
\mathrm{U}-238\end{array}$ & $\begin{array}{l}\begin{array}{c}\text { Activity } \\
(C i / Y z)\end{array} \\
-1.26 E-06 \\
3.34 \mathrm{E}-06 \\
3.34 \mathrm{E}-06 \\
2.15 \mathrm{E}-06 \\
3.34 \mathrm{E}-06 \\
3.34 \mathrm{E}-06 \\
1.99 \mathrm{E}-05 \\
1.99 \mathrm{E}-05 \\
3.34 \mathrm{E}-06 \\
1.92 \mathrm{E}-05 \\
2.02 \mathrm{E}-04 \\
2.46 \mathrm{E}-02 \\
2.52 \mathrm{E}-06 \\
2.46 \mathrm{E}-02 \\
1.91 \mathrm{E}-05 \\
2.46 \mathrm{E}-02\end{array}$ & \\
\hline Shipping Site: KAPL & $\begin{array}{l}\text { Total Volume } \\
(\mathrm{m} 3 / \mathrm{yr})\end{array}$ & $\begin{array}{c}\text { Total Mass } \\
(\mathrm{kg} / \mathrm{Yr})\end{array}$ & $5.36 E+05$ \\
\hline$\cdot$ & 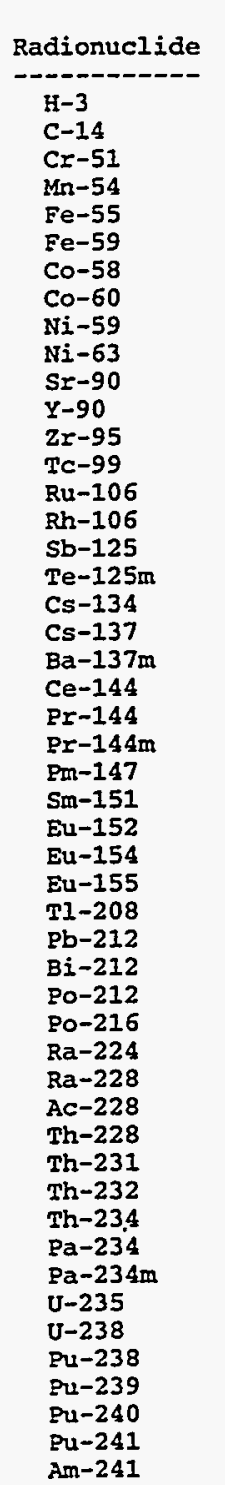 & \begin{tabular}{c} 
Activity \\
(Ci/Y工) \\
\hdashline$-14 \mathrm{-}-04$ \\
$5.14 \mathrm{E}-04$ \\
$2.40 \mathrm{E}-05$ \\
$5.11 \mathrm{E}-03$ \\
$1.24 \mathrm{E}-02$ \\
$3.63 \mathrm{E}-02$ \\
$3.28 \mathrm{E}-04$ \\
$1.55 \mathrm{E}-02$ \\
$1.09 \mathrm{E}-01$ \\
$4.69 \mathrm{E}-04$ \\
$6.70 \mathrm{E}-02$ \\
$2.58 \mathrm{E}-02$ \\
$2.58 \mathrm{E}-02$ \\
$3.99 \mathrm{E}-04$ \\
$5.20 \mathrm{E}-05$ \\
$2.19 \mathrm{E}-03$ \\
$2.19 \mathrm{E}-03$ \\
$6.31 \mathrm{E}-04$ \\
$1.54 \mathrm{E}-04$ \\
$8.14 \mathrm{E}-03$ \\
$3.09 \mathrm{E}-02$ \\
$2.92 \mathrm{E}-02$ \\
$7.35 \mathrm{E}-03$ \\
$7.35 \mathrm{E}-03$ \\
$8.84 \mathrm{E}-05$ \\
$1.79 \mathrm{E}-02$ \\
$1.75 \mathrm{E}-04$ \\
$2.67 \mathrm{E}-06$ \\
$4.59 \mathrm{E}-04$ \\
$3.64 \mathrm{E}-04$ \\
$4.50 \mathrm{E}-09$ \\
$1.19 \mathrm{E}-08$ \\
$1.19 \mathrm{E}-08$ \\
$7.60 \mathrm{E}-09$ \\
$1.19 \mathrm{E}-08$ \\
$1.19 \mathrm{E}-08$ \\
$7.11 \mathrm{E}-08$ \\
$7.11 \mathrm{E}-08$ \\
$1.19 \mathrm{E}-08$ \\
$6.84 \mathrm{E}-08$ \\
$7.21 \mathrm{E}-07$ \\
$8.77 \mathrm{E}-05$ \\
$8.90 \mathrm{E}-09$ \\
$8.77 \mathrm{E}-05$ \\
$6.82 \mathrm{E}-08$ \\
$3.79 \mathrm{E}-04$ \\
$5.53 \mathrm{E}-04$ \\
$5.35 \mathrm{E}-06$ \\
$2.57 \mathrm{E}-06$ \\
$5.18 \mathrm{E}-04$ \\
$3.03 \mathrm{E}-06$
\end{tabular} & \\
\hline
\end{tabular}




\begin{tabular}{|c|c|c|c|}
\hline Shipping Site: KCP & $\begin{array}{l}\text { Total Volume } \\
(\mathrm{m} 3 / \mathrm{yr})\end{array}$ & $\begin{array}{c}\text { Total Mass } \\
(\mathrm{kg} / \mathrm{yr})\end{array}$ & $7.23 E+03$ \\
\hline & $\begin{array}{l}\text { Radionuclide } \\
\mathrm{H}-3 \\
\mathrm{C}-14 \\
\mathrm{Mn}-54 \\
\mathrm{Co}-58 \\
\mathrm{Co}-60 \\
\mathrm{Sr}-90 \\
\mathrm{Y}-90 \\
\mathrm{Tc}-99 \\
\mathrm{Cs}-134 \\
\mathrm{Cs}-137 \\
\mathrm{Ba}-137 \mathrm{~m} \\
\mathrm{U}-238\end{array}$ & $\begin{array}{l}\text { Activity } \\
\text { (Ci/yr) } \\
-1.70 \mathrm{E}-02 \\
2.02 \\
1.44 \mathrm{E}-04 \\
1.62 \mathrm{E}-02 \\
1.50 \mathrm{E}-02 \\
4.34 \mathrm{E}-02 \\
2.04 \mathrm{E}-02 \\
2.04 \mathrm{E}-02 \\
2.89 \mathrm{E}-04 \\
3.37 \mathrm{E}-02 \\
4.44 \mathrm{E}-02 \\
4.20 \mathrm{E}-02 \\
1.76 \mathrm{E}-03\end{array}$ & \\
\hline Shipping Site: LANL & $\begin{array}{l}\text { Total Volume } \\
(\mathrm{m} 3 / \mathrm{y} r)\end{array}$ & $\begin{array}{cc}\text { Total Mass } \\
5.50 \mathrm{E}+03 & (\mathrm{~kg} / \mathrm{yr})\end{array}$ & $4.07 E+07$ \\
\hline - & 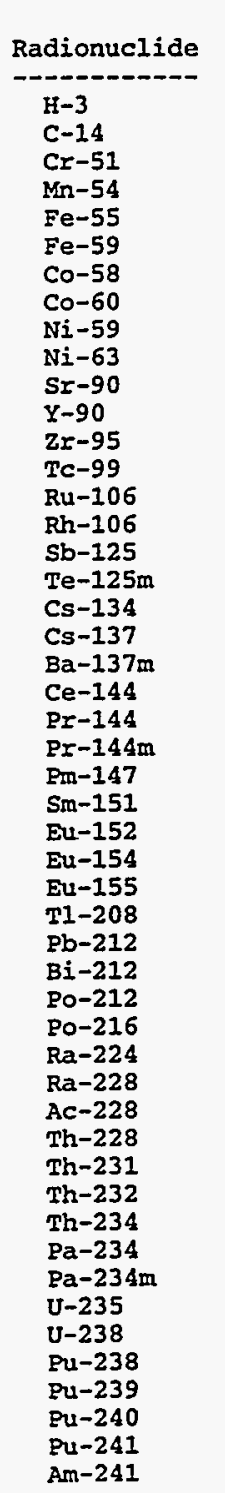 & \begin{tabular}{c} 
Activity \\
$(C i / y+)$ \\
- \hdashline$.--0-$ \\
$3.84 E+05$ \\
$1.83 E-01$ \\
$5.93 E+00$ \\
$3.19 E+01$ \\
$4.21 E+01$ \\
$3.81 E-01$ \\
$3.42 E+01$ \\
$5.40 E+02$ \\
$5.44 E-01$ \\
$8.05 E+02$ \\
$6.47 E+04$ \\
$6.47 E+04$ \\
$4.62 E-01$ \\
$1.19 E+01$ \\
$6.32 E+03$ \\
$6.32 E+03$ \\
$1.81 E+03$ \\
$4.43 E+02$ \\
$7.39 E+03$ \\
$6.79 E+04$ \\
$6.42 E+04$ \\
$2.11 E+04$ \\
$2.11 E+04$ \\
$2.54 E+02$ \\
$5.16 E+04$ \\
$5.04 E+02$ \\
$7.70 E+00$ \\
$1.32 E+03$ \\
$1.04 E+03$ \\
$1.69 E-02$ \\
$4.47 E-02$ \\
$4.47 E-02$ \\
$2.88 E-02$ \\
$4.47 E-02$ \\
$4.47 E-02$ \\
$2.67 E-01$ \\
$2.67 E-01$ \\
$4.47 E-02$ \\
$2.57 E-01$ \\
$2.71 E+00$ \\
$3.30 E+02$ \\
$3.38 E-02$ \\
$3.30 E+02$ \\
$2.56 E-01$ \\
$3.32 E+02$ \\
$1.59 E+03$ \\
$1.57 E+01$ \\
$8.94 E+00$ \\
$1.95 E+03$ \\
$2.31 E+01$
\end{tabular} & $\cdot$ \\
\hline
\end{tabular}


Destination: HANF

\begin{tabular}{|c|c|c|c|}
\hline Shipping Site: LANL & $\begin{array}{l}\text { Total Volume } \\
(\mathrm{m} 3 / \mathrm{yr})\end{array}$ & $\begin{array}{c}\text { Total Mass } \\
(\mathrm{kg} / \mathrm{y} \mathrm{r})\end{array}$ & $4.07 E+07$ \\
\hline & $\begin{array}{l}\text { Radionuclide } \\
\mathrm{Cm}-242 \\
\mathrm{Cm}-244\end{array}$ & $\begin{array}{c}\text { Activicy } \\
(\mathrm{Ci} / \mathrm{yr})\end{array}$ & \\
\hline Shipping Site: LBL & $\begin{array}{l}\text { Total Volume } \\
\text { (m3/yr) }\end{array}$ & $\begin{array}{l}\text { Total Mass } \\
(\mathrm{kg} / \mathrm{yr})\end{array}$ & $4.51 E+04$ \\
\hline & $\begin{array}{l}\text { Radionuclide } \\
\mathrm{H}-3 \\
\mathrm{C}-14 \\
\mathrm{Mn}-54 \\
\mathrm{Co}-58 \\
\mathrm{Co}-50 \\
\mathrm{~S}-90 \\
\mathrm{Y}-90 \\
\mathrm{Tc}-99 \\
\mathrm{Cs}-134 \\
\mathrm{Cs}-137 \\
\mathrm{Ba}-137 \mathrm{~m} \\
\mathrm{U}-238 \\
\mathrm{Cm}-244\end{array}$ & \begin{tabular}{c}
$\begin{array}{c}\text { Activity } \\
\text { (Ci/yr) }\end{array}$ \\
\hdashline $6.35 \mathrm{E}+02$ \\
$1.13 \mathrm{E}+00$ \\
$1.27 \mathrm{E}+02$ \\
$1.17 \mathrm{E}+02$ \\
$3.39 \mathrm{0}+02$ \\
$1.59 \mathrm{0}+02$ \\
$1.59 \mathrm{E}+02$ \\
$2.26 \mathrm{E}+00$ \\
$2.63 \mathrm{E}+02$ \\
$3.47 \mathrm{0}+02$ \\
$3.28 \mathrm{E}+02$ \\
$1.37 \mathrm{E}+01$ \\
$1.05 \mathrm{E}+01$
\end{tabular} & \\
\hline Shipping Site: LLNL & $\begin{array}{l}\text { Total volume } \\
(\mathrm{m} 3 / \mathrm{y} x)\end{array}$ & $\begin{array}{c}\text { Total Mass } \\
(\mathrm{kg} / \mathrm{yr})\end{array}$ & $1.06 E+06$ \\
\hline & $\begin{array}{l}\text { Radionuclide } \\
\mathrm{H}-3 \\
\mathrm{C}-14 \\
\mathrm{M} \mathrm{n}-54 \\
\mathrm{Co}-58 \\
\mathrm{Co}-60 \\
\mathrm{Ni}-63 \\
\mathrm{Sr}-90 \\
\mathrm{Y}-90 \\
\mathrm{Tc}-99 \\
\mathrm{Ru}-106 \\
\mathrm{Rh}-106 \\
\mathrm{Sb}-125 \\
\mathrm{Te}-125 \mathrm{~m} \\
\mathrm{Cs}-134 \\
\mathrm{Cs}-137 \\
\mathrm{Ba}-137 \mathrm{~m} \\
\mathrm{Ce}-144 \\
\mathrm{Pr}-144 \\
\mathrm{Pr}-144 \mathrm{~m} \\
\mathrm{Pm}-147 \\
\mathrm{Sm}-151 \\
\mathrm{Eu}-152 \\
\mathrm{Eu}-154 \\
\mathrm{Eu}-155 \\
\mathrm{U}-238 \\
\mathrm{Pu}-238 \\
\mathrm{Pu}-239 \\
\mathrm{Pu}-240 \\
\mathrm{Pu}-241 \\
\mathrm{Am}-241 \\
\mathrm{Cm}-242 \\
\mathrm{Cm}-244\end{array}$ & $\begin{array}{c}\text { Activity } \\
\text { (Ci/yI) } \\
3.33 \mathrm{E}+04 \\
1.55 \mathrm{E}-03 \\
1.74 \mathrm{E}-01 \\
1.61 \mathrm{E}-01 \\
4.69 \mathrm{E}-01 \\
4.89 \mathrm{E}-03 \\
6.54 \mathrm{E}-01 \\
6.54 \mathrm{E}-01 \\
3.18 \mathrm{E}-03 \\
4.24 \mathrm{E}-02 \\
4.24 \mathrm{E}-02 \\
1.21 \mathrm{E}-02 \\
2.97 \mathrm{E}-03 \\
4.11 \mathrm{E}-01 \\
9.33 \mathrm{E}-01 \\
8.82 \mathrm{E}-01 \\
1.42 \mathrm{E}-01 \\
1.42 \mathrm{E}-01 \\
1.70 \mathrm{E}-03 \\
3.46 \mathrm{E}-01 \\
3.39 \mathrm{E}-03 \\
5.17 \mathrm{E}-05 \\
8.87 \mathrm{E}-03 \\
7.03 \mathrm{E}-03 \\
1.88 \mathrm{x}-02 \\
7.85 \mathrm{E}-02 \\
5.28 \mathrm{E}-03 \\
1.81 \mathrm{E}-02 \\
2.50 \mathrm{E}+00 \\
2.58 \mathrm{E}-04 \\
1.44 \mathrm{E}-03 \\
5.17 \mathrm{E}-04\end{array}$ & \\
\hline
\end{tabular}




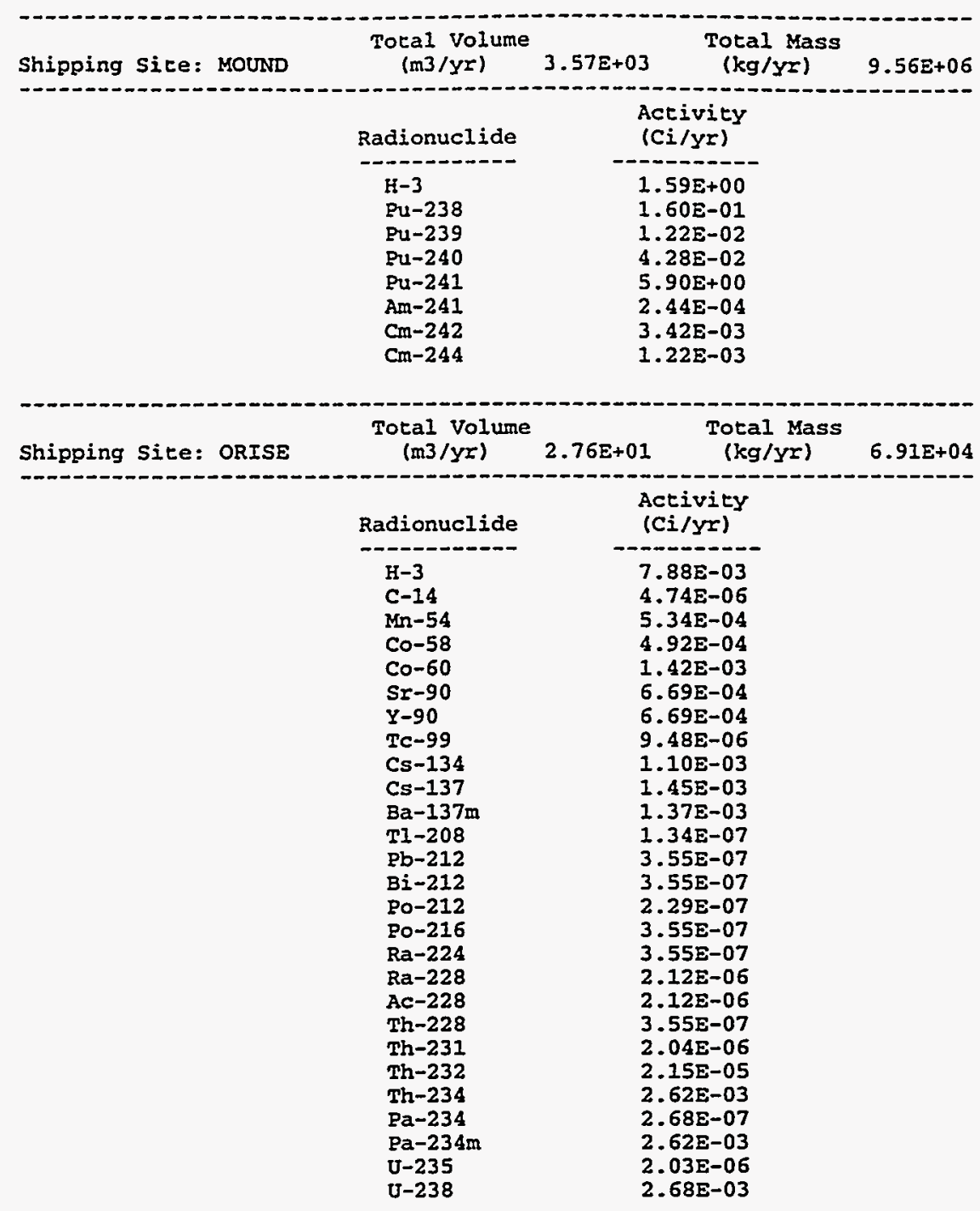




\begin{tabular}{|c|c|c|c|}
\hline Shipping Site: ORNL & $\begin{array}{l}\text { Total Volume } \\
(\mathrm{m} 3 / \mathrm{Y})\end{array}$ & $\begin{array}{l}\text { Total Mass } \\
(\mathrm{kg} / \mathrm{yr})\end{array}$ & $1.32 E+08$ \\
\hline & 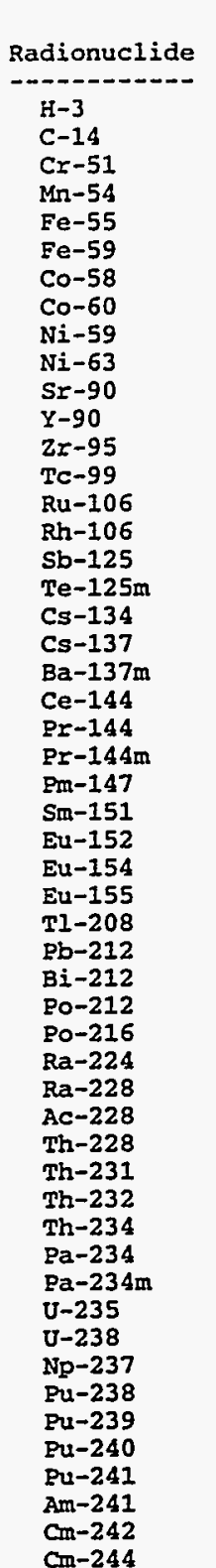 & $\begin{array}{l}\text { Activity } \\
(C i / y=) \\
-1.10 \mathrm{E}+00 \\
1.14 \mathrm{E}-05 \\
1.01 \mathrm{E}+01 \\
1.98 \mathrm{E}+01 \\
7.20 \mathrm{E}+01 \\
6.51 \mathrm{E}-01 \\
2.63 \mathrm{E}+01 \\
2.09 \mathrm{E}+02 \\
9.30 \mathrm{E}-01 \\
1.42 \mathrm{E}+02 \\
9.15 \mathrm{E}+02 \\
9.15 \mathrm{E}+02 \\
7.90 \mathrm{E}-01 \\
1.74 \mathrm{E}-01 \\
8.91 \mathrm{E}+01 \\
8.91 \mathrm{E}+01 \\
2.56 \mathrm{E}+01 \\
6.25 \mathrm{E}+00 \\
1.04 \mathrm{E}+02 \\
9.58 \mathrm{E}+02 \\
9.07 \mathrm{E}+02 \\
2.98 \mathrm{E}+02 \\
2.98 \mathrm{E}+02 \\
3.58 \mathrm{E}+00 \\
7.28 \mathrm{E}+02 \\
7.12 \mathrm{E}+00 \\
1.08 \mathrm{E}-01 \\
1.86 \mathrm{E}+01 \\
1.47 \mathrm{E}+01 \\
1.81 \mathrm{E}-04 \\
4.81 \mathrm{E}-04 \\
4.81 \mathrm{E}-04 \\
3.10 \mathrm{E}-04 \\
4.81 \mathrm{E}-04 \\
4.81 \mathrm{E}-04 \\
2.87 \mathrm{E}-03 \\
2.87 \mathrm{E}-03 \\
4.81 \mathrm{E}-04 \\
2.77 \mathrm{E}-03 \\
2.92 \mathrm{E}-02 \\
3.55 \mathrm{E}+00 \\
3.63 \mathrm{E}-04 \\
3.55 \mathrm{E}+00 \\
2.76 \mathrm{E}-03 \\
3.60 \mathrm{E}+00 \\
1.63 \mathrm{E}-03 \\
2.24 \mathrm{E}+01 \\
2.19 \mathrm{E}-01 \\
1.09 \mathrm{E}-01 \\
2.52 \mathrm{E}+01 \\
3.26 \mathrm{E}-01 \\
2.00 \mathrm{E}-05 \\
\end{array}$ & \\
\hline
\end{tabular}




\section{A-196}

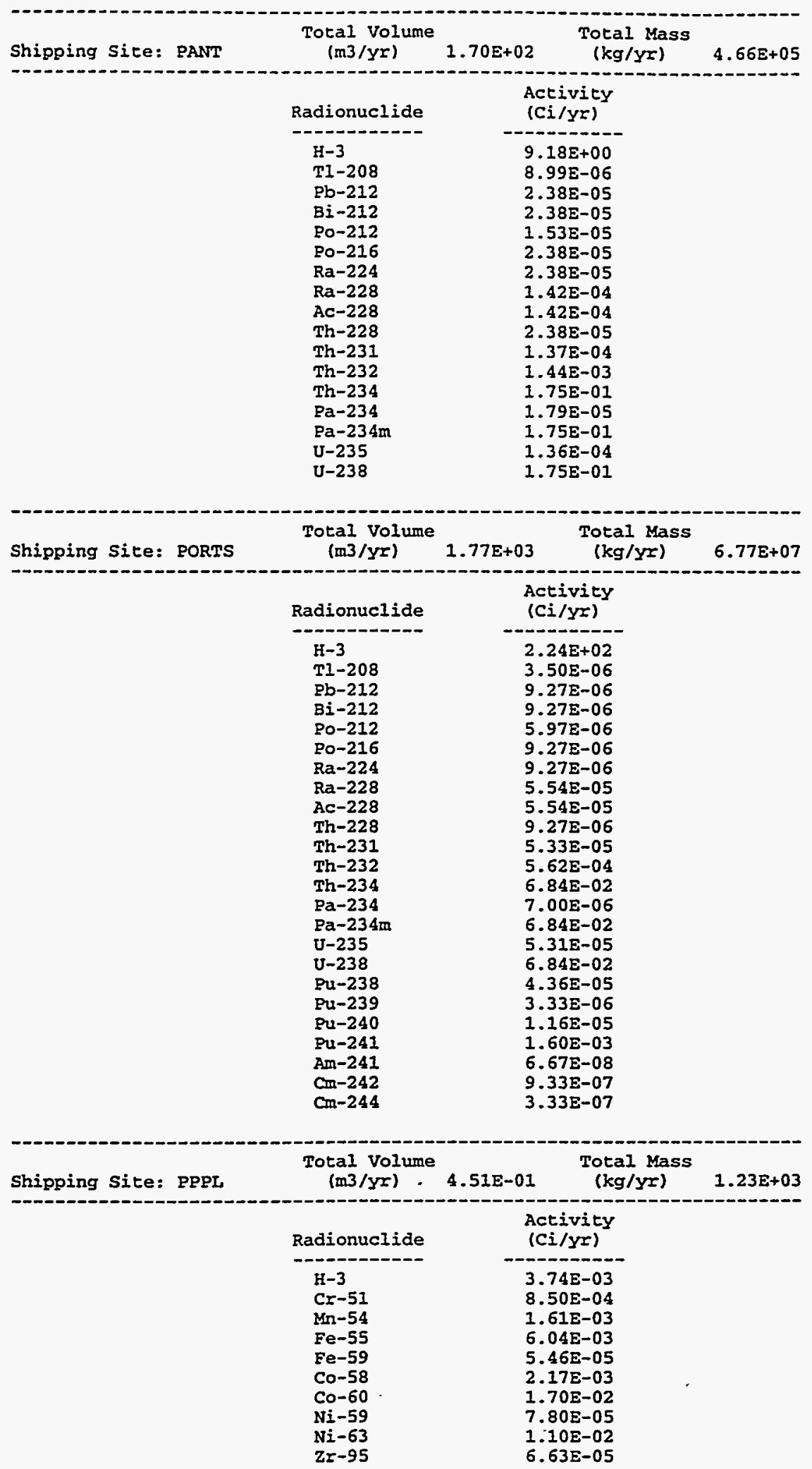




\begin{tabular}{|c|c|c|c|}
\hline Shipping Sice: Paducah & $\begin{array}{l}\text { Tocal volume } \\
(\mathrm{m} 3 / \mathrm{yr})\end{array}$ & $\begin{array}{l}\text { Total Mass } \\
(\mathrm{kg} / \mathrm{yr})\end{array}$ & $1.10 \mathrm{E}+07$ \\
\hline & $\begin{array}{l}\text { Radionuclide } \\
\mathrm{Tc}-99 \\
\mathrm{~T} 1-208 \\
\mathrm{~Pb}-212 \\
\mathrm{Bi}-212 \\
\mathrm{PO-212} \\
\mathrm{PO-216} \\
\mathrm{Ra}-224 \\
\mathrm{Ra}-228 \\
\mathrm{Ac}-228 \\
\mathrm{Th}-228 \\
\mathrm{Th}-231 \\
\mathrm{Th}-232 \\
\mathrm{Th}-234 \\
\mathrm{~Pa}-234 \\
\mathrm{~Pa}-234 \mathrm{~m} \\
\mathrm{U}-235 \\
\mathrm{U}-238 \\
\mathrm{~Np}-237 \\
\mathrm{Pu}-239\end{array}$ & $\begin{array}{c}\begin{array}{c}\text { Activity } \\
(C i / Y x)\end{array} \\
6.66 \mathrm{E}-03 \\
2.80 \mathrm{E}-05 \\
7.41 \mathrm{E}-05 \\
7.41 \mathrm{E}-05 \\
4.78 \mathrm{E}-05 \\
7.41 \mathrm{E}-05 \\
7.41 \mathrm{E}-05 \\
4.43 \mathrm{E}-04 \\
4.43 \mathrm{E}-04 \\
7.41 \mathrm{E}-05 \\
4.27 \mathrm{E}-04 \\
4.50 \mathrm{E}-03 \\
5.47 \mathrm{E}-01 \\
5.60 \mathrm{E}-05 \\
5.47 \mathrm{E}-01 \\
4.25 \mathrm{E}-04 \\
5.47 \mathrm{E}-01 \\
4.99 \mathrm{E}-03 \\
4.99 \mathrm{E}-03\end{array}$ & \\
\hline Shipping Site: RFP & $\begin{array}{l}\text { Total Volume } \\
(\mathrm{m} 3 / \mathrm{yr})\end{array}$ & $1.27 \mathrm{E}+03 \quad \begin{array}{c}\text { Total Mass } \\
(\mathrm{kg} / \mathrm{y} x)\end{array}$ & $6.52 \mathrm{E}+06$ \\
\hline & $\begin{array}{l}\text { Radionuclide } \\
\mathrm{Tl-208} \\
\mathrm{Pb}-212 \\
\mathrm{Bi}-212 \\
\mathrm{Po-212} \\
\mathrm{Po-216} \\
\mathrm{Ra}-224 \\
\mathrm{Ra}-228 \\
\mathrm{Ac-228} \\
\mathrm{Th}-228 \\
\mathrm{Th}-231 \\
\mathrm{Th}-232 \\
\mathrm{Th}-234 \\
\mathrm{~Pa}-234 \\
\mathrm{~Pa}-234 \mathrm{~m} \\
\mathrm{U}-235 \\
\mathrm{U}-238 \\
\mathrm{Pu}-238 \\
\mathrm{Pu}-239 \\
\mathrm{Pu}-240 \\
\mathrm{Pu}-241 \\
\mathrm{Am}-241 \\
\mathrm{Cm}-242 \\
\mathrm{Cm}-244\end{array}$ & 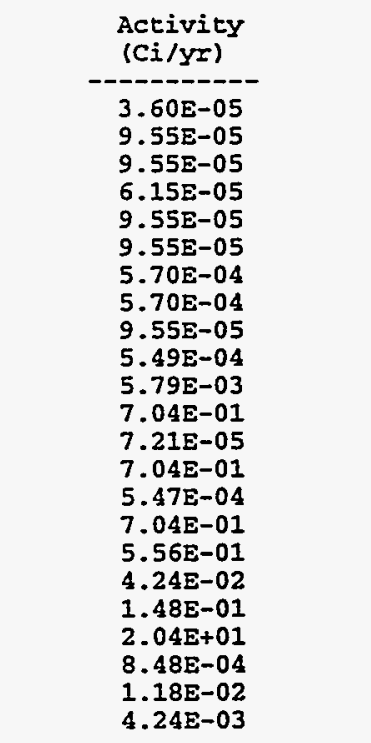 & \\
\hline Shipping Site: RMI & $\begin{array}{l}\text { Total Volume } \\
(\mathrm{m} 3 / \mathrm{y} x)\end{array}$ & $\begin{array}{c}\text { Total Mass } \\
(\mathrm{kg} / \mathrm{Yr})\end{array}$ & $1.45 E+07$ \\
\hline & $\begin{array}{l}\text { Radionuclide } \\
\mathrm{Pl}-208 \\
\mathrm{~Pb}-212 \\
\mathrm{Bi}-212 \\
\mathrm{Po-212} \\
\mathrm{Po-216} \\
\mathrm{Ra}-224 \\
\mathrm{Ra}-228 \\
\mathrm{Ac}-228 \\
\mathrm{Th}-228 \\
\mathrm{Th}-231 \\
\mathrm{Th}-232 \\
\mathrm{Th}-234 \\
\mathrm{~Pa}-234 \\
\mathrm{~Pa}-234 \mathrm{~m} \\
\mathrm{U}-235 \\
\mathrm{U}-238\end{array}$ & $\begin{array}{c}\begin{array}{c}\text { Activity } \\
(C i / y r)\end{array} \\
-3.38 \mathrm{E}-07 \\
8.96 \mathrm{E}-07 \\
8.96 \mathrm{E}-07 \\
5.77 \mathrm{E}-07 \\
8.96 \mathrm{E}-07 \\
8.96 \mathrm{E}-07 \\
5.35 \mathrm{E}-06 \\
5.35 \mathrm{E}-06 \\
8.96 \mathrm{E}-07 \\
5.15 \mathrm{E}-06 \\
5.43 \mathrm{E}-05 \\
6.61 \mathrm{E}-03 \\
6.77 \mathrm{E}-07 \\
6.61 \mathrm{E}-03 \\
5.13 \mathrm{E}-06 \\
6.61 \mathrm{E}-03\end{array}$ & \\
\hline
\end{tabular}




\begin{tabular}{|c|c|c|c|}
\hline Shipping Site: SLAC & $\begin{array}{l}\text { Total Volume } \\
(\mathrm{m} 3 / \mathrm{yr})\end{array}$ & $\begin{array}{l}\text { Total Mass } \\
(\mathrm{kg} / \mathrm{y} r)\end{array}$ & $7.45 \mathrm{E}+05$ \\
\hline & $\begin{array}{l}\text { Radionuclide } \\
\text { Cr-51 } \\
\mathrm{Mn}-54 \\
\mathrm{Fe}-55 \\
\mathrm{Fe}-59 \\
\mathrm{Co}-58 \\
\mathrm{Co}-60 \\
\mathrm{Ni}-59 \\
\mathrm{Ni}-63 \\
\mathrm{ZI}-95\end{array}$ & $\begin{array}{c}\begin{array}{c}\text { Activity } \\
(\mathrm{Ci} / \mathrm{yr})\end{array} \\
-3.86 \mathrm{E}-03 \\
7.36 \mathrm{E}-03 \\
2.74 \mathrm{E}-02 \\
2.48 \mathrm{E}-04 \\
9.88 \mathrm{E}-03 \\
7.74 \mathrm{E}-02 \\
3.54 \mathrm{E}-04 \\
5.04 \mathrm{E}-02 \\
3.01 \mathrm{E}-04\end{array}$ & \\
\hline Shipping Sice: SNLA & $\begin{array}{l}\text { Total Volume } \\
(\mathrm{m} 3 / \mathrm{y})\end{array}$ & $1.18 \mathrm{E}+02 \quad \begin{array}{c}\text { Total Mass } \\
(\mathrm{kg} / \mathrm{yr})\end{array}$ & $3.24 E+05$ \\
\hline-- & $\begin{array}{l}\text { Radionuclide } \\
\text { Co-60 } \\
\text { Ni-63 } \\
\text { Sr-90 } \\
\text { Y-90 } \\
\text { rc-99 } \\
\text { Ru-106 } \\
\text { Rh-106 } \\
\text { Sb-125 } \\
\text { Te-125m } \\
\text { Cs-134 } \\
\text { Cs-137 } \\
\text { Ba-137m } \\
\text { Ce-144 } \\
\text { Pr-144 } \\
\text { Pr-144m } \\
\text { Pm-147 } \\
\text { Sm-151 } \\
\text { Eu-152 } \\
\text { Eu-154 } \\
\text { Eu-155 } \\
\text { Pu-238 } \\
\text { Pu-239 } \\
\text { Pu-240 } \\
\text { Pu-241 } \\
\text { Am-241 } \\
\text { Cm-242 } \\
\text { Cm-244 }\end{array}$ & $\begin{array}{l}\text { Activity } \\
\text { (Ci/yr) } \\
3.00 \mathrm{E}+00 \\
2.85 \mathrm{E}-05 \\
5.67 \mathrm{E}-05 \\
5.04 \mathrm{E}-03 \\
5.04 \mathrm{E}-03 \\
9.00 \mathrm{E}-07 \\
4.92 \mathrm{E}-04 \\
4.92 \mathrm{E}-04 \\
1.41 \mathrm{E}-04 \\
3.45 \mathrm{E}-05 \\
5.72 \mathrm{E}-04 \\
5.28 \mathrm{E}-03 \\
4.99 \mathrm{E}-03 \\
1.64 \mathrm{E}-03 \\
1.64 \mathrm{E}-03 \\
1.98 \mathrm{E}-05 \\
4.02 \mathrm{E}-03 \\
3.93 \mathrm{E}-05 \\
6.00 \mathrm{E}-07 \\
1.02 \mathrm{E}-04 \\
8.16 \mathrm{E}-05 \\
7.87 \mathrm{E}-02 \\
6.00 \mathrm{E}-03 \\
2.10 \mathrm{E}-02 \\
2.89 \mathrm{E}+00 \\
1.21 \mathrm{E}-04 \\
1.68 \mathrm{E}-03 \\
6.00 \mathrm{E}-04\end{array}$ & -------- \\
\hline Shipping site: SNLL & $\begin{array}{l}\text { Total Volume } \\
\text { (m3/yx) }\end{array}$ & $\begin{array}{cc}\text { Total Mass } \\
(\mathrm{kg} / \mathrm{Yr})\end{array}$ & $9.36 \mathrm{E}+04$ \\
\hline - . & $\begin{array}{l}\text { Radionuclide } \\
\mathrm{H}-3 \\
\text { Fe-55 } \\
\mathrm{Co}-60 \\
\mathrm{~T} l-208 \\
\mathrm{~Pb}-212 \\
\mathrm{Bi}-212 \\
\mathrm{Po}-212 \\
\mathrm{Po}-216 \\
\mathrm{Ra}-224 \\
\mathrm{Ra}-228 \\
\mathrm{Ac}-228 \\
\mathrm{Th}-228 \\
\mathrm{Th}-231 \\
\mathrm{Th}-232 \\
\mathrm{Th}-234 \\
\mathrm{~Pa}-234 \\
\mathrm{~Pa}-234 \mathrm{~m} \\
\mathrm{U}-235 \\
\mathrm{U}-238\end{array}$ & $\begin{array}{c}\begin{array}{c}\text { Activity } \\
(C i / y x)\end{array} \\
-2.82 \mathrm{E}+04 \\
3.15 \mathrm{E}-01 \\
3.15 \mathrm{E}-01 \\
6.40 \mathrm{E}-05 \\
1.69 \mathrm{E}-04 \\
1.69 \mathrm{E}-04 \\
1.09 \mathrm{E}-04 \\
1.69 \mathrm{E}-04 \\
1.69 \mathrm{E}-04 \\
1.01 \mathrm{E}-03 \\
1.01 \mathrm{E}-03 \\
1.69 \mathrm{E}-04 \\
9.75 \mathrm{E}-04 \\
1.02 \mathrm{E}-02 \\
1.25 \mathrm{E}+00 \\
1.28 \mathrm{E}-04 \\
1.25 \mathrm{E}+00 \\
9.71 \mathrm{E}-04 \\
1.25 \mathrm{E}+00\end{array}$ & \\
\hline
\end{tabular}




\section{A-199}

\begin{tabular}{|c|c|c|c|}
\hline Shipping site: SRS & $\begin{array}{l}\text { Total Volume } \\
(\mathrm{m} 3 / \mathrm{yr})\end{array}$ & $\begin{array}{l}\text { Total Mass } \\
(\mathrm{kg} / \mathrm{yr})\end{array}$ & $1.305+08$ \\
\hline & 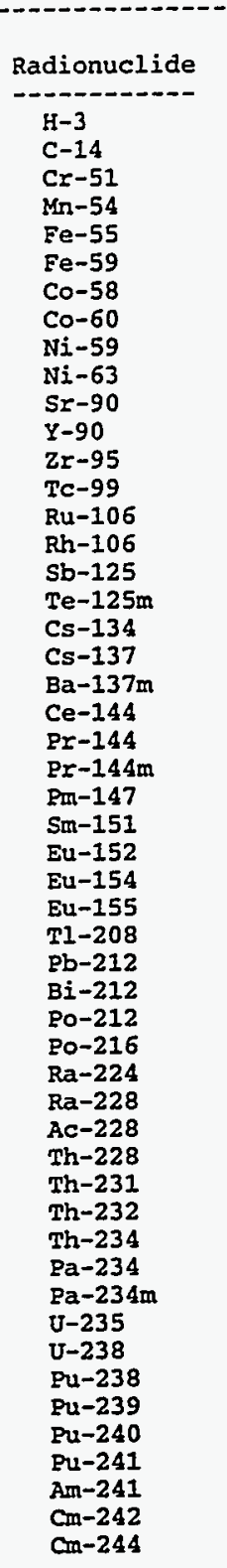 & 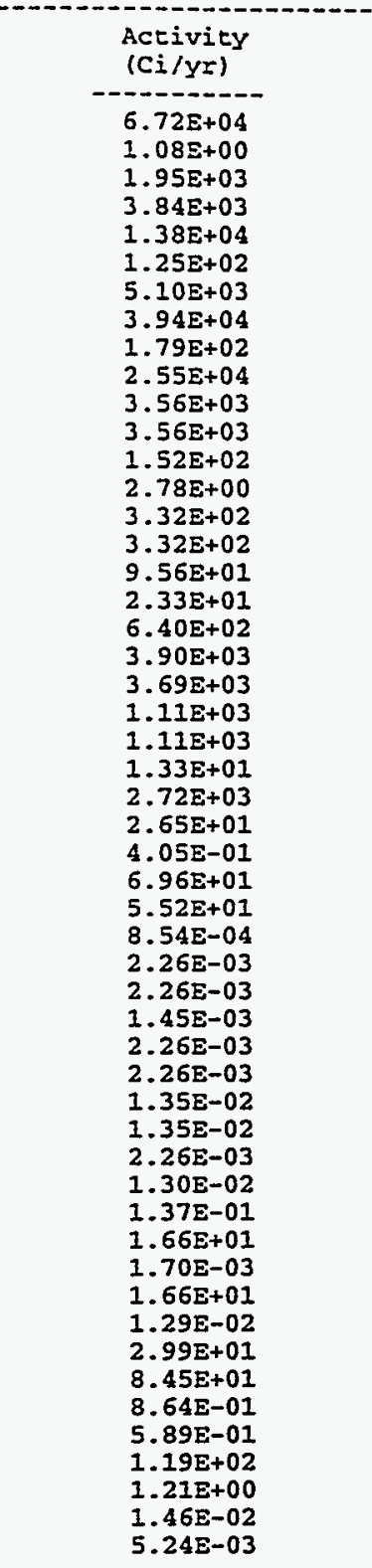 & \\
\hline Shipping Site: $Y-12$ & $\begin{array}{l}\text { Total Volume } \\
\text { (m3/yr) }\end{array}$ & $\begin{array}{l}\text { Total Mass } \\
\text { (kg/yI) }\end{array}$ & $1.51 E+06$ \\
\hline & $\begin{array}{l}\text { Radionuclide } \\
\mathrm{Tl}-208 \\
\mathrm{~Pb}-212 \\
\mathrm{Bi}-212 \\
\mathrm{Po}-212 \\
\mathrm{Po}-216 \\
\mathrm{Ra}-224 \\
\mathrm{Ra}-228 \\
\mathrm{Ac}-228 \\
\mathrm{Th}-228 \\
\mathrm{Th}-231 \\
\mathrm{Th}-232 \\
\mathrm{Th}-234 \\
\mathrm{~Pa}-234 \\
\mathrm{~Pa}-234 \mathrm{~m} \\
\mathrm{U}-235 \\
\mathrm{U}-238\end{array}$ & $\begin{array}{l}\begin{array}{l}\text { Activity } \\
\text { (Ci/y) }\end{array} \\
-2.58 E-06 \\
6.84 \mathrm{E}-06 \\
6.84 \mathrm{E}-06 \\
4.40 \mathrm{E}-06 \\
6.84 \mathrm{E}-06 \\
6.84 \mathrm{E}-06 \\
4.08 \mathrm{E}-05 \\
4.08 \mathrm{E}-05 \\
6.84 \mathrm{E}-06 \\
3.93 \mathrm{E}-05 \\
4.14 \mathrm{E}-04 \\
5.04 \mathrm{E}-02 \\
5.16 \mathrm{E}-06 \\
5.04 \mathrm{E}-02 \\
3.92 \mathrm{E}-05 \\
5.04 \mathrm{E}-02\end{array}$ & \\
\hline
\end{tabular}


frequency of transportation-related accidents. The route distances between major DOE sites are summarized in Tables 3.1 and 3.2 for truck and rail transportation, respectively.

\subsubsection{Truck Route Determination}

The HIGHWAY 3.1 computer program is used for predicting highway routes for transporting radioactive materials within the United States by truck. The HIGHWAY database is a computerized road atlas that currently describes approximately $386,243 \mathrm{~km}(240,000 \mathrm{mi})$ of roads. A complete description of the interstate highway system and of all U.S. highways is included in the database. In addition, most of the principal state highways and a number of local and community highways are also identified. The code is updated periodically to reflect current road conditions and has been benchmarked against reported mileages and observations of commercial trucking firms.

Routes are calculated within the model by minimizing the total impedance between the origin and the destination. The impedance is basically defined as a function of distance and driving time along a particular segment of highway. One of the special features of the HIGHWAY 3.1 model is its ability to calculate routes that maximize the use of interstate highways. This feature allows the user to predict routes for shipment of radioactive materials that conform to the transportation regulations of the U.S. Department of Transportation (DOT), specifically docket HM-164. The population densities along a route are derived from 1990 data from the U.S. Bureau of the Census. Rural, suburban, and urban areas are characterized according to the following breakdown: rural population densities range from $0-54$ persons $/ \mathrm{km}^{2}\left(0-139 / \mathrm{mi}^{2}\right)$, the suburban range is $55-1,284 / \mathrm{km}^{2}$ $\left(140-3,326 / \mathrm{mi}^{2}\right)$, and urban is taken to mean all population densities greater than $1,284 / \mathrm{km}^{2}$ $\left(3,326 / \mathrm{mi}^{2}\right)$.

\subsubsection{Rail Route Determination}

The INTERLINE 5.0 computer program is designed to simulate routing of the U.S. rail system. The INTERLINE database consists of 94 separate subnetworks and represents various competing rail companies in the United States. The database used by INTERLINE was originally based on data from the Federal Railroad Administration and reflected the U.S. railroad system in 1974. The database has since been expanded and modified over the past two decades. The code is updated periodically to reflect current track conditions and has been benchmarked against reported mileages and observations of commercial rail firms.

The INTERLINE 5.0 model uses a shortest-route algorithm that finds the path of minimum impedance within an individual subnetwork. A separate routine is used to find paths along the subnetworks. The routes chosen for this study used the standard assumptions in the INTERLINE model that simulate the process of selection that railroads would use to direct shipments of 
Destination: INEL

\begin{tabular}{|c|c|c|c|}
\hline Shipping Site: ANL-W & $\begin{array}{l}\text { Total Volume } \\
\text { (m3/yr) }\end{array}$ & $\begin{array}{cc}1.92 \mathrm{E}+02 & \begin{array}{c}\text { Total Mass } \\
(\mathrm{kg} / \mathrm{Yr})\end{array}\end{array}$ & $2.88 \mathrm{E}+04$ \\
\hline & \begin{tabular}{l} 
Radionuclide \\
\hdashline $\mathrm{H}-3$ \\
$\mathrm{Cr}-51$ \\
$\mathrm{Mn}-54$ \\
$\mathrm{Fe}-55$ \\
$\mathrm{Fe}-59$ \\
$\mathrm{Co}-58$ \\
$\mathrm{Co}-60$ \\
$\mathrm{Ni}-59$ \\
$\mathrm{Ni}-63$ \\
$\mathrm{Sr}-90$ \\
$\mathrm{y}-90$ \\
$\mathrm{Zr}-95$ \\
$\mathrm{Tc}-99$ \\
$\mathrm{Ru}-106$ \\
$\mathrm{Rh}-106$ \\
$\mathrm{Sb}-125$ \\
$\mathrm{Te}-125 \mathrm{~m}$ \\
$\mathrm{Cs}-134$ \\
$\mathrm{Cs}-137$ \\
$\mathrm{Ba}-137 \mathrm{~m}$ \\
$\mathrm{Ce}-144$ \\
$\mathrm{Pr}-144$ \\
$\mathrm{Pr}-144 \mathrm{~m}$ \\
$\mathrm{Pm}-147$ \\
$\mathrm{Sm}-151$ \\
$\mathrm{Eu}-152$ \\
$\mathrm{Eu}-154$ \\
$\mathrm{Eu}-155$ \\
$\mathrm{Pu}-238$ \\
$\mathrm{Pu}-239$ \\
$\mathrm{Pu}-240$ \\
$\mathrm{Pu}-241$ \\
$\mathrm{Am}-241$ \\
$\mathrm{Cm}-242$ \\
$\mathrm{Cm}-244$
\end{tabular} & $\begin{array}{c}\text { Activity } \\
(C i / y r) \\
-3.77 \mathrm{E}-05 \\
3.85 \mathrm{E}-03 \\
7.34 \mathrm{E}-03 \\
2.74 \mathrm{E}-02 \\
2.47 \mathrm{E}-04 \\
9.85 \mathrm{E}-03 \\
7.74 \mathrm{E}-02 \\
3.53 \mathrm{E}-04 \\
5.06 \mathrm{E}-02 \\
3.17 \mathrm{E}-02 \\
3.17 \mathrm{E}-02 \\
3.00 \mathrm{E}-04 \\
5.66 \mathrm{E}-06 \\
3.09 \mathrm{E}-03 \\
3.09 \mathrm{E}-03 \\
8.89 \mathrm{E}-04 \\
2.17 \mathrm{E}-04 \\
3.59 \mathrm{E}-03 \\
3.32 \mathrm{E}-02 \\
3.14 \mathrm{E}-02 \\
1.03 \mathrm{E}-02 \\
1.03 \mathrm{E}-02 \\
1.24 \mathrm{E}-04 \\
2.52 \mathrm{E}-02 \\
2.47 \mathrm{E}-04 \\
3.77 \mathrm{E}-06 \\
6.47 \mathrm{E}-04 \\
5.13 \mathrm{E}-04 \\
7.81 \mathrm{E}-04 \\
7.72 \mathrm{E}-06 \\
4.38 \mathrm{E}-06 \\
9.56 \mathrm{E}-04 \\
1.13 \mathrm{E}-05 \\
4.88 \mathrm{E}-08 \\
1.74 \mathrm{E}-08\end{array}$ & \\
\hline
\end{tabular}


Destination: LANL

\begin{tabular}{|c|c|c|c|}
\hline Shipping Sice: ITRI & $\begin{array}{l}\text { Total Volume } \\
(\mathrm{m} 3 / \mathrm{yr})\end{array}$ & $\begin{array}{l}\text { Total Mass } \\
(\mathrm{kg} / \mathrm{Yr})\end{array}$ & $1.17 E+04$ \\
\hline & $\begin{array}{l}\text { Radionuclide } \\
\mathrm{H}-3 \\
\mathrm{C}-14 \\
\mathrm{Mn}-54 \\
\mathrm{Co}-58 \\
\mathrm{Co}-60 \\
\mathrm{~S}-90 \\
\mathrm{Y}-90 \\
\mathrm{Tc}-99 \\
\mathrm{Cs}-134 \\
\mathrm{Cs}-137 \\
\text { Ba-137m } \\
\mathrm{U}-238\end{array}$ & $\begin{array}{c}\text { Activicy } \\
\text { (Ci/yr) } \\
3.73 E+00 \\
1.83 E-01 \\
2.06 E+01 \\
1.90 E+01 \\
5.51 E+01 \\
2.59 E+01 \\
2.59 E+01 \\
3.67 E-01 \\
4.27 E+01 \\
5.64 E+01 \\
5.33 E+01 \\
2.23 E+00\end{array}$ & \\
\hline Shipping Site: PANT & $\begin{array}{l}\text { Total Volume } \\
(\mathrm{m} 3 / \mathrm{yr})\end{array}$ & $\begin{array}{cc}\text { Total Mass } \\
(\mathrm{kg} / \mathrm{yr})\end{array}$ & $2.84 E+07$ \\
\hline & $\begin{array}{l}\text { Radionuclide } \\
\mathrm{H}-3 \\
\mathrm{~T} 1-208 \\
\mathrm{~Pb}-212 \\
\mathrm{Bi}-212 \\
\mathrm{PO}-212 \\
\mathrm{PO}-216 \\
\mathrm{Ra}-224 \\
\mathrm{Ra}-228 \\
\mathrm{Ac}-228 \\
\mathrm{Th}-228 \\
\mathrm{Th}-231 \\
\mathrm{Th}-232 \\
\mathrm{Th}-234 \\
\mathrm{~Pa}-234 \\
\mathrm{~Pa}-234 \mathrm{~m} \\
\mathrm{U}-235 \\
\mathrm{U}-238\end{array}$ & $\begin{array}{c}\begin{array}{c}\text { Activity } \\
(C i / y r)\end{array} \\
2.25 \mathrm{E}+02 \\
2.12 \mathrm{E}-04 \\
5.63 \mathrm{E}-04 \\
5.63 \mathrm{E}-04 \\
3.62 \mathrm{E}-04 \\
5.63 \mathrm{E}-04 \\
5.63 \mathrm{E}-04 \\
3.36 \mathrm{E}-03 \\
3.36 \mathrm{E}-03 \\
5.63 \mathrm{E}-04 \\
3.24 \mathrm{E}-03 \\
3.41 \mathrm{E}-02 \\
4.15 \mathrm{E}+00 \\
4.25 \mathrm{E}-04 \\
4.15 \mathrm{E}+00 \\
3.22 \mathrm{E}-03 \\
4.15 \mathrm{E}+00\end{array}$ & \\
\hline Shipping Site: SNLA & $\begin{array}{l}\text { Total Volume } \\
(\mathrm{m} 3 / \mathrm{Y} x)\end{array}$ & $\begin{array}{c}\text { Total Mass } \\
(\mathrm{kg} / \mathrm{y})\end{array}$ & $4.31 E+03$ \\
\hline & $\begin{array}{l}\text { Radionuclide } \\
\mathrm{Tl}-208 \\
\mathrm{~Pb}-212 \\
\mathrm{Bi}-212 \\
\mathrm{Po-212} \\
\mathrm{Po-216} \\
\mathrm{Ra}-224 \\
\mathrm{Ra}-228 \\
\mathrm{Ac-228} \\
\mathrm{Th}-228 \\
\mathrm{Th}-231 \\
\mathrm{Th}-232 \\
\mathrm{Th}-234 \\
\mathrm{~Pa}-234 \\
\mathrm{Pa-234m} \\
\mathrm{U}-235 \\
\mathrm{U}-238\end{array}$ & 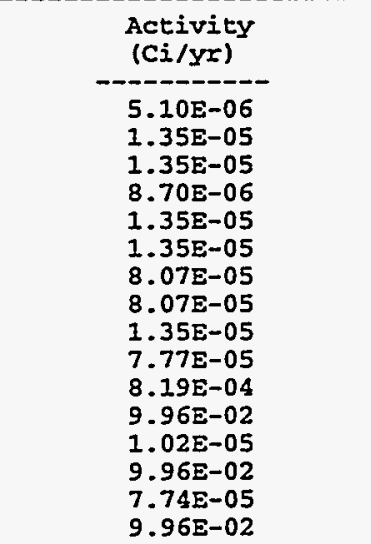 & \\
\hline
\end{tabular}


Destination: ORNL

\begin{tabular}{|c|c|c|c|}
\hline Shipping Site: $k-25$ & $\begin{array}{l}\text { Total volume } \\
(\mathrm{m} 3 / \mathrm{yr})\end{array}$ & $\begin{array}{l}\text { Total Mass } \\
(\mathrm{kg} / \mathrm{yr})\end{array}$ & $4.65 \mathrm{E}+07$ \\
\hline & $\begin{array}{c}\text { Radionuclide } \\
\mathrm{Tl-208} \\
\mathrm{Pb}-212 \\
\mathrm{Bi}-212 \\
\mathrm{Po}-212 \\
\mathrm{Po}-216 \\
\mathrm{Ra}-224 \\
\mathrm{Ra}-228 \\
\mathrm{Ac}-228 \\
\mathrm{Th}-228 \\
\mathrm{Th}-231 \\
\mathrm{Th}-232 \\
\mathrm{Th}-234 \\
\mathrm{~Pa}-234 \\
\mathrm{~Pa}-234 \mathrm{~m} \\
\mathrm{U}-235 \\
\mathrm{U}-238\end{array}$ & \multicolumn{2}{|l|}{$\begin{array}{l}1.05 \mathrm{E}-04 \\
2.79 \mathrm{E}-04 \\
2.79 \mathrm{E}-04 \\
1.79 \mathrm{E}-04 \\
2.79 \mathrm{E}-04 \\
2.79 \mathrm{E}-04 \\
1.66 \mathrm{E}-03 \\
1.66 \mathrm{E}-03 \\
2.79 \mathrm{E}-04 \\
1.60 \mathrm{E}-03 \\
1.69 \mathrm{E}-02 \\
2.05 \mathrm{E}+00 \\
2.10 \mathrm{E}-04 \\
2.05 \mathrm{E}+00 \\
1.60 \mathrm{E}-03 \\
2.05 \mathrm{E}+00\end{array}$} \\
\hline \multirow[t]{2}{*}{ Shipping Site: ORISE } & $\begin{array}{l}\text { Total Volume } \\
\text { (m3/yr) }\end{array}$ & $\begin{array}{cc}\text { Total Mass } \\
3.01 E+01 & (\mathrm{~kg} / \mathrm{yr})\end{array}$ & $4.52 E+03$ \\
\hline & $\begin{array}{l}\text { Radionuclide } \\
\mathrm{H}-3 \\
\mathrm{C}-14 \\
\mathrm{Mn}-54 \\
\mathrm{Co}-58 \\
\mathrm{Co}-60 \\
\mathrm{Sr}-90 \\
\mathrm{Y}-90 \\
\mathrm{Tc}-99 \\
\mathrm{Cs}-134 \\
\mathrm{Cs}-137 \\
\mathrm{Ba}-137 \mathrm{~m} \\
\mathrm{~T} \mathrm{I}-208 \\
\mathrm{~Pb}-212 \\
\mathrm{Bi}-212 \\
\mathrm{Po}-212 \\
\mathrm{Po}-216 \\
\mathrm{Ra}-224 \\
\mathrm{Ra}-228 \\
\mathrm{Ac}-228 \\
\text { Th-228 } \\
\text { Th-231 } \\
\text { Th-232 } \\
\text { Th-234 } \\
\text { Pa-234 } \\
\text { Pa-234m } \\
\mathrm{U}-235 \\
\mathrm{U}-238\end{array}$ & $\begin{array}{c}\begin{array}{c}\text { Activity } \\
\text { (Ci/yr) }\end{array} \\
1.22 \mathrm{E}-02 \\
7.23 \mathrm{E}-06 \\
8.15 \mathrm{E}-04 \\
7.52 \mathrm{E}-04 \\
2.17 \mathrm{E}-03 \\
1.02 \mathrm{E}-03 \\
1.02 \mathrm{E}-03 \\
1.44 \mathrm{E}-05 \\
1.68 \mathrm{E}-03 \\
2.22 \mathrm{E}-03 \\
2.10 \mathrm{E}-03 \\
2.05 \mathrm{E}-07 \\
5.42 \mathrm{E}-07 \\
5.42 \mathrm{E}-07 \\
3.49 \mathrm{E}-07 \\
5.42 \mathrm{E}-07 \\
5.42 \mathrm{E}-07 \\
3.24 \mathrm{E}-06 \\
3.24 \mathrm{E}-06 \\
5.42 \mathrm{E}-07 \\
3.12 \mathrm{E}-06 \\
3.29 \mathrm{E}-05 \\
4.00 \mathrm{E}-03 \\
4.10 \mathrm{E}-07 \\
4.00 \mathrm{E}-03 \\
3.11 \mathrm{E}-06 \\
4.09 \mathrm{E}-03\end{array}$ & \\
\hline
\end{tabular}




\begin{tabular}{|c|c|c|c|}
\hline Shipping Sire: Paducah & $\begin{array}{l}\text { Total Volume } \\
\text { (m3/yr) }\end{array}$ & $\begin{array}{c}\text { Total Mass } \\
(\mathrm{kg} / \mathrm{yr})\end{array}$ & $9.48 \mathrm{E}+05$ \\
\hline & $\begin{array}{l}\text { Radionuclide } \\
\text { Tc-99 } \\
\text { Tl-208 } \\
\mathrm{Pb}-212 \\
\mathrm{Bi}-212 \\
\mathrm{PO}-212 \\
\mathrm{PO}-216 \\
\mathrm{Ra}-224 \\
\mathrm{Ra}-228 \\
\mathrm{Ac}-228 \\
\mathrm{Th}-228 \\
\mathrm{Th}-231 \\
\mathrm{Th}-232 \\
\mathrm{Th}-234 \\
\mathrm{~Pa}-234 \\
\mathrm{~Pa}-234 \mathrm{~m} \\
\mathrm{U}-235 \\
\mathrm{U}-238 \\
\mathrm{~Np}-237 \\
\mathrm{Pu}-239\end{array}$ & $\begin{array}{c}\begin{array}{c}\text { Activity } \\
\text { (Ci/yr) }\end{array} \\
2.17 \mathrm{E}-03 \\
9.17 \mathrm{E}-06 \\
2.42 \mathrm{E}-05 \\
2.42 \mathrm{E}-05 \\
1.56 \mathrm{E}-05 \\
2.42 \mathrm{E}-05 \\
2.42 \mathrm{E}-05 \\
1.45 \mathrm{E}-04 \\
1.45 \mathrm{E}-04 \\
2.42 \mathrm{E}-05 \\
1.39 \mathrm{E}-04 \\
1.47 \mathrm{E}-03 \\
1.79 \mathrm{E}-01 \\
1.83 \mathrm{E}-05 \\
1.79 \mathrm{E}-01 \\
1.39 \mathrm{E}-04 \\
1.79 \mathrm{E}-01 \\
1.63 \mathrm{E}-03 \\
1.63 \mathrm{E}-03\end{array}$ & \\
\hline Shipping Site: $\mathrm{Y}-12$ & $\begin{array}{l}\text { Total volume } \\
(\mathrm{m} 3 / \mathrm{yr})\end{array}$ & $\begin{array}{l}\text { Total Mass } \\
(\mathrm{kg} / \mathrm{y} x)\end{array}$ & $8.12 \mathrm{E}+07$ \\
\hline & \begin{tabular}{l} 
Radionuclide \\
\hdashline $\mathrm{Tl-208}$ \\
$\mathrm{Pb}-212$ \\
$\mathrm{Bi}-212$ \\
$\mathrm{Po}-212$ \\
$\mathrm{Po}-216$ \\
$\mathrm{Ra}-224$ \\
$\mathrm{Ra}-228$ \\
$\mathrm{Ac}-228$ \\
$\mathrm{Th}-228$ \\
$\mathrm{Th}-231$ \\
$\mathrm{Th}-232$ \\
$\mathrm{Th}-234$ \\
$\mathrm{~Pa}-234$ \\
$\mathrm{~Pa}-234 \mathrm{~m}$ \\
$\mathrm{U}-235$ \\
$\mathrm{U}-238$
\end{tabular} & $\begin{array}{c}\begin{array}{c}\text { Activity } \\
\text { (Ci/yr) }\end{array} \\
6.54 \mathrm{E}-05 \\
1.73 \mathrm{E}-04 \\
1.73 \mathrm{E}-04 \\
1.11 \mathrm{E}-04 \\
1.73 \mathrm{E}-04 \\
1.73 \mathrm{E}-04 \\
1.03 \mathrm{E}-03 \\
1.03 \mathrm{E}-03 \\
1.73 \mathrm{E}-04 \\
9.96 \mathrm{E}-04 \\
1.05 \mathrm{E}-02 \\
1.27 \mathrm{E}+00 \\
1.30 \mathrm{E}-04 \\
1.27 \mathrm{E}+00 \\
9.92 \mathrm{E}-04 \\
1.27 \mathrm{E}+00\end{array}$ & \\
\hline
\end{tabular}


Destination: PORTS

\begin{tabular}{|c|c|c|c|}
\hline Shipping Sice: AMES & $\begin{array}{l}\text { Total Volume } \\
(\mathrm{m} 3 / \mathrm{yr})\end{array}$ & $\begin{array}{c}\text { Total Mass } \\
(\mathrm{kg} / \mathrm{yr})\end{array}$ & $1.00 \mathrm{E}+02$ \\
\hline & $\begin{array}{l}\text { Radionuclide } \\
\mathrm{Tl}-208 \\
\mathrm{~Pb}-212 \\
\mathrm{Bi}-212 \\
\mathrm{Po-212} \\
\mathrm{PO}-216 \\
\mathrm{Ra}-224 \\
\mathrm{Ra}-228 \\
\mathrm{Ac}-228 \\
\mathrm{Th}-228 \\
\mathrm{Th}-231 \\
\mathrm{Th}-232 \\
\mathrm{Th}-234 \\
\mathrm{~Pa}-234 \\
\mathrm{~Pa}-234 \mathrm{~m} \\
\mathrm{U}-235 \\
\mathrm{U}-238 \\
\mathrm{Pu}-238 \\
\mathrm{Pu}-239 \\
\mathrm{Pu}-240 \\
\mathrm{Pu}-241 \\
\mathrm{Am}-241 \\
\mathrm{Cm}-242 \\
\mathrm{Cm}-244\end{array}$ & $\begin{array}{c}\begin{array}{c}\text { Activity } \\
(C i / y r)\end{array} \\
-2.83 \mathrm{E}-08 \\
7.50 \mathrm{E}-08 \\
7.50 \mathrm{E}-08 \\
4.83 \mathrm{E}-08 \\
7.50 \mathrm{E}-08 \\
7.50 \mathrm{E}-08 \\
4.48 \mathrm{E}-07 \\
4.48 \mathrm{E}-07 \\
7.50 \mathrm{E}-08 \\
4.31 \mathrm{E}-07 \\
4.55 \mathrm{E}-06 \\
5.53 \mathrm{E}-04 \\
5.67 \mathrm{E}-08 \\
5.53 \mathrm{E}-04 \\
4.30 \mathrm{E}-07 \\
5.53 \mathrm{E}-04 \\
4.36 \mathrm{E}-05 \\
3.33 \mathrm{E}-06 \\
1.16 \mathrm{E}-05 \\
1.60 \mathrm{E}-03 \\
6.67 \mathrm{E}-08 \\
9.33 \mathrm{E}-07 \\
3.33 \mathrm{E}-07\end{array}$ & \\
\hline \multirow[t]{2}{*}{ Shipping Site: FNAL } & $\begin{array}{l}\text { Total volume } \\
\text { (m3/yr) }\end{array}$ & $\begin{array}{l}\text { Total Mass } \\
\text { (kg/yr) }\end{array}$ & $6.25 E+03$ \\
\hline & $\begin{array}{l}\text { Radionuclide } \\
\mathrm{H}-3\end{array}$ & $\begin{array}{c}\begin{array}{c}\text { Activity } \\
(C j / Y x)\end{array} \\
7.38 \mathrm{E}-02\end{array}$ & \\
\hline \multirow[t]{2}{*}{ Shipping Site: MOUND } & $\begin{array}{l}\text { Total volume } \\
\text { (m3/yr) }\end{array}$ & $\begin{array}{l}\text { Total Mass } \\
(\mathrm{kg} / \mathrm{Yr})\end{array}$ & $1.14 \mathrm{E}+05$ \\
\hline & $\begin{array}{l}\text { Radionuclide } \\
\mathrm{H}-3\end{array}$ & $\begin{array}{c}\begin{array}{c}\text { Activity } \\
\text { (Ci/yr) }\end{array} \\
1.34 \mathrm{E}+04\end{array}$ & \\
\hline
\end{tabular}




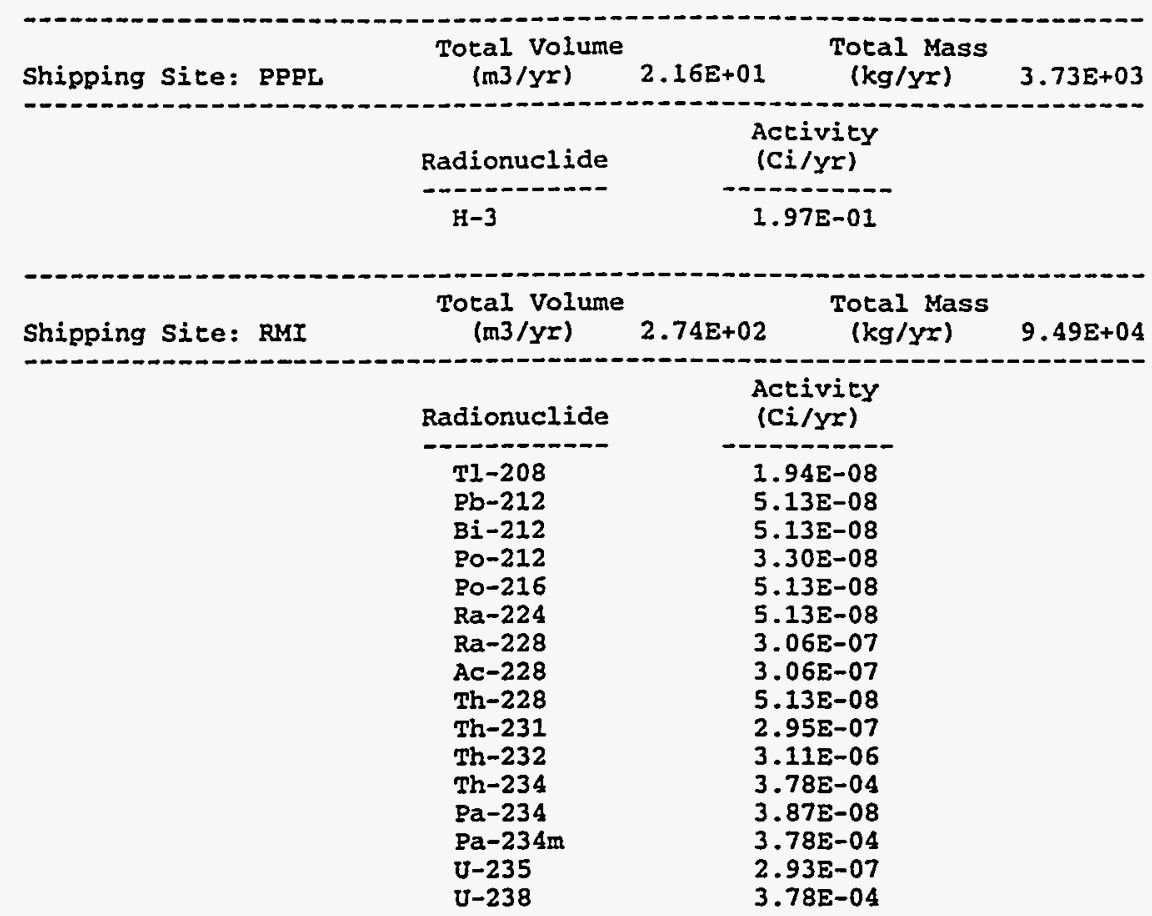

Destination: SRS

\begin{tabular}{|c|c|c|c|}
\hline Shipping Site: Pinellas & $\begin{array}{c}\text { Total Volune } \\
\left(\mathrm{m}^{3} / \mathrm{y}^{\prime}\right)\end{array}$ & $\begin{array}{c}\text { Total Mass } \\
(\mathrm{kg} / \mathrm{y} x)\end{array}$ & $1.91 \mathrm{E}+04$ \\
\hline & Radionuclide & $\begin{array}{c}\text { Activity } \\
\text { (Ci/yr) } \\
1.99 E+04\end{array}$ & \\
\hline
\end{tabular}




\section{A.24 WM LLW CENTRALIZED 3 ALTERNATIVE (CASE 14): ACTIVATED METALS}

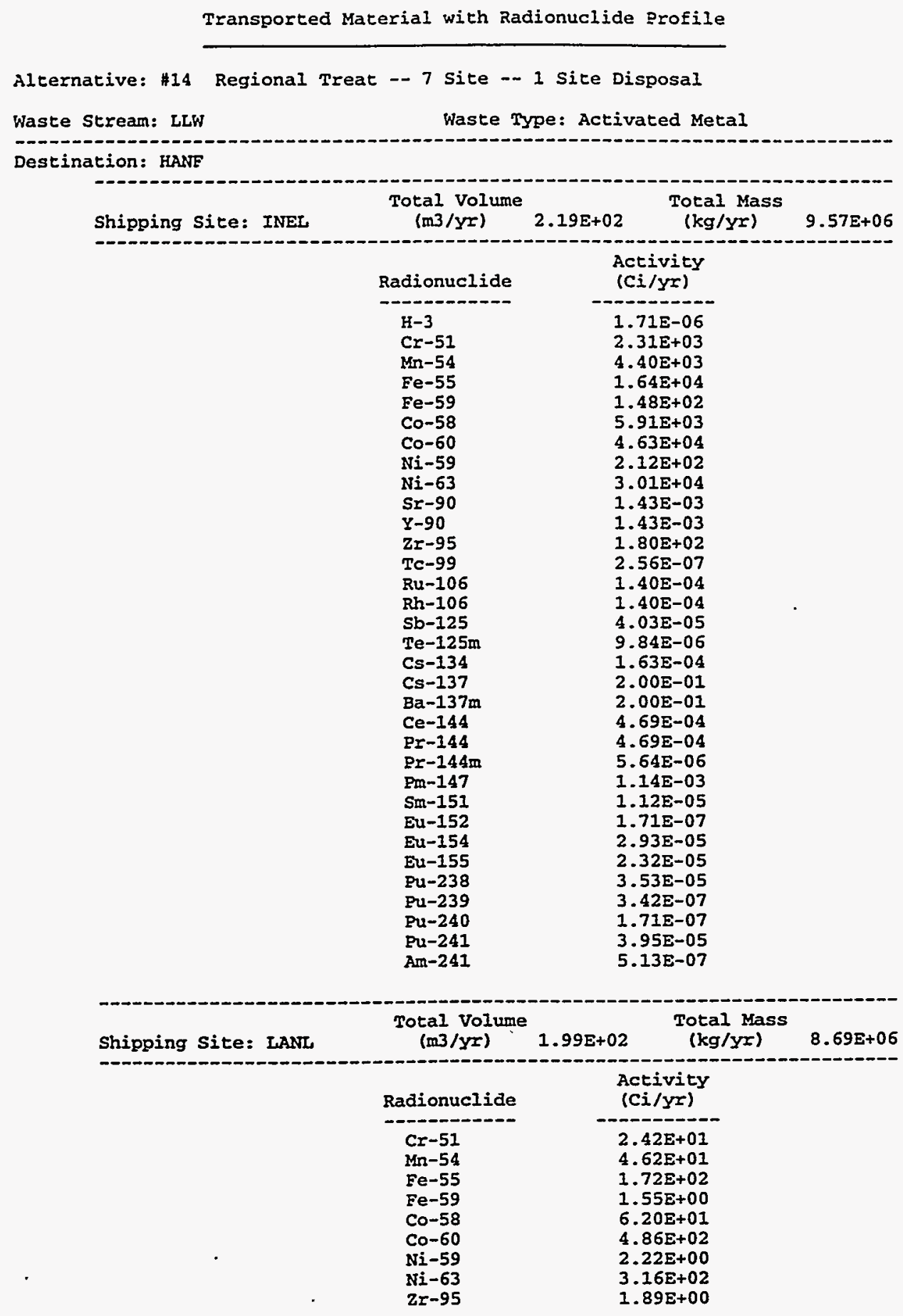




\begin{tabular}{|c|c|c|c|}
\hline Shipping Site: LBL & $\begin{array}{l}\text { Total Volume } \\
(\mathrm{m} 3 / \mathrm{yr})\end{array}$ & $\begin{array}{c}\text { Total Mass } \\
(\mathrm{kg} / \mathrm{yr})\end{array}$ & $2.87 E+05$ \\
\hline & $\begin{array}{l}\text { Radionuclide } \\
\mathrm{Cr}-51 \\
\mathrm{Mn}-54 \\
\mathrm{Fe}-55 \\
\mathrm{Fe}-59 \\
\mathrm{Co}-58 \\
\mathrm{Co}-60 \\
\mathrm{Ni}-59 \\
\mathrm{Ni}-63 \\
\mathrm{Zr}-95\end{array}$ & $\begin{array}{c}\begin{array}{c}\text { Activity } \\
\text { (Ci/yr) }\end{array} \\
6.32 \mathrm{E}-02 \\
1.20 \mathrm{E}-01 \\
4.49 \mathrm{E}-01 \\
4.06 \mathrm{E}-03 \\
1.61 \mathrm{E}-01 \\
1.26 \mathrm{E}+00 \\
5.80 \mathrm{E}-03 \\
8.24 \mathrm{E}-01 \\
4.93 \mathrm{E}-03\end{array}$ & \\
\hline Shipping Site: PORTS & $\begin{array}{l}\text { Total Volume } \\
(\mathrm{m} 3 / \mathrm{yr})\end{array}$ & 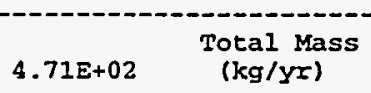 & $2.05 E+07$ \\
\hline . & 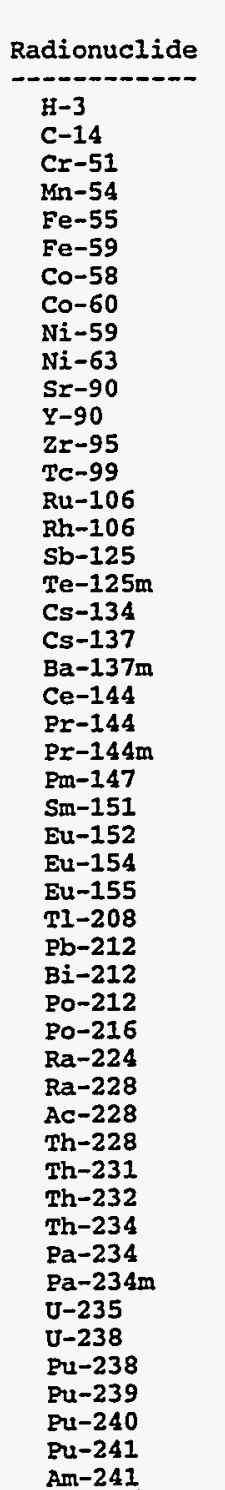 & 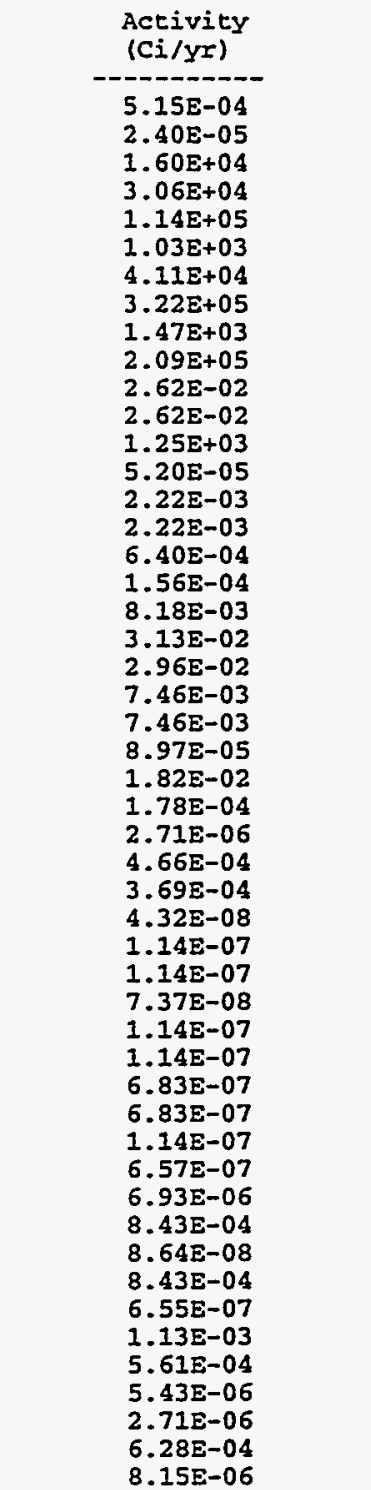 & . \\
\hline
\end{tabular}


Destination: INEL

\begin{tabular}{|c|c|c|c|}
\hline Shipping Site: ANL-W & $\begin{array}{l}\text { Total Volume } \\
(\mathrm{m} 3 / \mathrm{yr})\end{array}$ & $\begin{array}{l}\text { Total Mass } \\
(\mathrm{kg} / \mathrm{yr})\end{array}$ & $1.64 E+05$ \\
\hline & $\begin{array}{l}\text { Radionuclide } \\
\mathrm{H}-3 \\
\mathrm{Cr}-51 \\
\mathrm{Mn}-54 \\
\mathrm{Fe}-55 \\
\mathrm{Fe}-59 \\
\mathrm{Co}-58 \\
\mathrm{Co}-60 \\
\mathrm{~N}-59 \\
\mathrm{Ni}-63 \\
\mathrm{~S}-90 \\
\mathrm{Y}-90 \\
\mathrm{Zr}-95 \\
\mathrm{Tc}-99 \\
\mathrm{Ru}-106 \\
\mathrm{Rh}-106 \\
\mathrm{Sb}-125 \\
\mathrm{Te}-125 \mathrm{~m} \\
\mathrm{Cs}-134 \\
\mathrm{Cs}-137 \\
\mathrm{Ba}-137 \mathrm{~m} \\
\mathrm{Ce}-144 \\
\mathrm{Pr}-144 \\
\mathrm{Pr}-144 \mathrm{~m} \\
\mathrm{Pm}-147 \\
\mathrm{Sm}-151 \\
\text { Eu-152 } \\
\text { Eu-154 } \\
\text { Eu-155 } \\
\mathrm{Pu}-238 \\
\mathrm{Pu}-239 \\
\mathrm{Pu}-240 \\
\mathrm{Pu}-241 \\
\mathrm{Am-241}\end{array}$ & \multicolumn{2}{|l|}{$\begin{array}{l}1.71 \mathrm{E}-06 \\
1.24 \mathrm{E}-04 \\
2.37 \mathrm{E}-04 \\
8.86 \mathrm{E}-04 \\
8.00 \mathrm{E}-06 \\
3.18 \mathrm{E}-04 \\
2.50 \mathrm{E}-03 \\
1.14 \mathrm{E}-05 \\
1.64 \mathrm{E}-03 \\
1.43 \mathrm{E}-03 \\
1.43 \mathrm{E}-03 \\
9.72 \mathrm{E}-06 \\
2.56 \mathrm{E}-07 \\
1.40 \mathrm{E}-04 \\
1.40 \mathrm{E}-04 \\
4.03 \mathrm{E}-05 \\
9.84 \mathrm{E}-06 \\
1.63 \mathrm{E}-04 \\
2.00 \mathrm{E}-01 \\
2.00 \mathrm{E}-01 \\
4.69 \mathrm{E}-04 \\
4.69 \mathrm{E}-04 \\
5.64 \mathrm{E}-06 \\
1.14 \mathrm{E}-03 \\
1.12 \mathrm{E}-05 \\
1.71 \mathrm{E}-07 \\
2.93 \mathrm{E}-05 \\
2.32 \mathrm{E}-05 \\
3.53 \mathrm{E}-05 \\
3.42 \mathrm{E}-07 \\
1.71 \mathrm{E}-07 \\
3.95 \mathrm{E}-05 \\
5.13 \mathrm{E}-07\end{array}$} \\
\hline \multirow[t]{2}{*}{ Shipping Site: NRF } & $\begin{array}{l}\text { Total Volume } \\
\text { (m3/yr) }\end{array}$ & $\begin{array}{cc}\text { Total Mass } \\
(\mathrm{kg} / \mathrm{yr})\end{array}$ & $9.40 \mathrm{E}+06$ \\
\hline & $\begin{array}{l}\text { Radionuclide } \\
\mathrm{Cr}-51 \\
\mathrm{Mn}-54 \\
\mathrm{Fe}-55 \\
\mathrm{Fe}-59 \\
\mathrm{Co}-58 \\
\mathrm{Co}-60 \\
\mathrm{Ni}-59 \\
\mathrm{Ni}-63 \\
\mathrm{Zr}-95\end{array}$ & \begin{tabular}{c}
$\begin{array}{c}\text { Activity } \\
\text { (Ci/yr) }\end{array}$ \\
\hdashline $2.31 E+03$ \\
$4.40 E+03$ \\
$1.64 E+04$ \\
$1.48 E+02$ \\
$5.91 E+03$ \\
$4.63 E+04$ \\
$2.12 E+02$ \\
$3.01 E+04$ \\
$1.80 E+02$
\end{tabular} & \\
\hline
\end{tabular}


Destination: LANL

\begin{tabular}{|c|c|c|c|}
\hline Shipping Site: SNLA & $\begin{array}{l}\text { Total Volume } \\
(\mathrm{m} 3 / \mathrm{Yr})\end{array}$ & $\begin{array}{c}\text { Total Mass } \\
(\mathrm{kg} / \mathrm{Yr})\end{array}$ & $1.15 E+05$ \\
\hline & $\begin{array}{l}\text { Radionuclide } \\
\mathrm{Cr}-51 \\
\mathrm{Mn}-54 \\
\mathrm{Fe}-55 \\
\mathrm{Fe}-59 \\
\mathrm{Co}-58 \\
\mathrm{Co}-60 \\
\mathrm{Ni}-59 \\
\mathrm{Ni}-63 \\
\mathrm{Zr}-95\end{array}$ & $\begin{array}{c}\begin{array}{c}\text { Activity } \\
\text { (Ci/yr) }\end{array} \\
6.54 \mathrm{E}-04 \\
1.24 \mathrm{E}-03 \\
4.64 \mathrm{E}-03 \\
4.20 \mathrm{E}-05 \\
1.67 \mathrm{E}-03 \\
1.31 \mathrm{E}-02 \\
6.00 \mathrm{E}-05 \\
8.53 \mathrm{E}-03 \\
5.10 \mathrm{E}-05\end{array}$ & \\
\hline
\end{tabular}

Destination: PORTS

\begin{tabular}{|c|c|c|c|}
\hline Shipping Site: BAPL & $\begin{array}{l}\text { Total Volume } \\
(\mathrm{m} 3 / \mathrm{y} x)\end{array}$ & $\begin{array}{c}\text { Total Mass } \\
(\mathrm{kg} / \mathrm{yr})\end{array}$ & $6.43 \mathrm{E}+06$ \\
\hline & $\begin{array}{l}\text { Radionuclide } \\
\mathrm{Cr}-51 \\
\mathrm{Mn}-54 \\
\mathrm{Fe}-55 \\
\mathrm{Fe}-59 \\
\mathrm{Co}-58 \\
\mathrm{Co}-60 \\
\mathrm{Ni}-59 \\
\mathrm{Ni}-63 \\
\mathrm{Zr}-95 \\
\mathrm{Tl}-208 \\
\mathrm{~Pb}-212 \\
\mathrm{Bi}-212 \\
\mathrm{Po}-212 \\
\mathrm{Po-216} \\
\mathrm{Ra}-224 \\
\mathrm{Ra}-228 \\
\mathrm{Ac}-228 \\
\mathrm{Th}-228 \\
\mathrm{Th}-231 \\
\mathrm{Th}-232 \\
\mathrm{Th}-234 \\
\mathrm{~Pa}-234 \\
\mathrm{~Pa}-234 \mathrm{~m} \\
\mathrm{U}-235 \\
\mathrm{U}-238\end{array}$ & $\begin{array}{c}\begin{array}{c}\text { Activity } \\
(C i / y r)\end{array} \\
4.75 E+03 \\
9.04 E+03 \\
3.37 E+04 \\
3.05 E+02 \\
1.21 E+04 \\
9.52 E+04 \\
4.36 E+02 \\
6.19 E+04 \\
3.70 E+02 \\
1.36 E-08 \\
3.60 E-08 \\
3.60 E-08 \\
2.32 E-08 \\
3.60 E-08 \\
3.60 E-08 \\
2.15 E-07 \\
2.15 E-07 \\
3.60 E-08 \\
2.07 E-07 \\
2.18 E-06 \\
2.65 E-04 \\
2.72 E-08 \\
2.65 E-04 \\
2.06 E-07 \\
2.65 E-04\end{array}$ & \\
\hline Shipping site: FNAI & $\begin{array}{l}\text { Total volume } \\
\text { (m3/yx) }\end{array}$ & $\begin{array}{l}\text { Total Mass } \\
(\mathrm{kg} / \mathrm{y})\end{array}$ & $8.14 E+05$ \\
\hline & $\begin{array}{l}\text { Radionuclide } \\
\mathrm{Cr}-51 \\
\mathrm{Mn}-54 \\
\mathrm{Fe}-55 \\
\mathrm{Fe}-59 \\
\mathrm{Co}-58 \\
\mathrm{Co}-60 \\
\mathrm{Ni}-59 \\
\mathrm{Ni}-63 \\
\mathrm{Zr}-95\end{array}$ & $\begin{array}{c}\begin{array}{c}\text { Activity } \\
\text { (Ci/yr) }\end{array} \\
3.35 \mathrm{E}-02 \\
6.39 \mathrm{E}-02 \\
2.38 \mathrm{E}-01 \\
2.15 \mathrm{E}-03 \\
8.57 \mathrm{E}-02 \\
6.72 \mathrm{E}-01 \\
3.08 \mathrm{E}-03 \\
4.37 \mathrm{E}-01 \\
2.61 \mathrm{E}-03\end{array}$ & \\
\hline
\end{tabular}


TABLE 3.1 Truck Route Distances (mi) between Major DOE Sites ${ }^{\mathrm{a}}$

\begin{tabular}{|c|c|c|c|c|c|c|c|c|c|c|c|c|c|c|c|c|c|c|}
\hline & 䒺 & $\underset{\dot{z}}{\stackrel{\ddot{z}}{z}}$ & 产 & ป్ํ் & 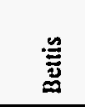 & $\overrightarrow{\mathbf{z}^{\prime}}$ & 节 & 咅 & $\begin{array}{l}\text { 忥 } \\
\text { 焉 }\end{array}$ & 总 & $\bar{\Xi}$ & $\tilde{\overline{\tilde{v}}}$ & $\stackrel{\text { 荄 }}{\dot{\Sigma}}$ & 苟 & $\stackrel{\dot{z}}{i}$ & $\underset{\underline{\Sigma}}{\dot{\Sigma}}$ & 咅 & है \\
\hline & 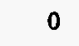 & 351 & 87 & 75 & 894 & 206 & 3 & 511 & 703 & 1287 & 1129 & 234 & 163 & $18+4$ & 853 & 136 & 54 & 1287 \\
\hline ANL & 351 & 0 & 582 & 348 & 567 & 874 & 36 & 94 & 1998 & 1582 & 1333 & 520 & 831 & 2139 & 2148 & 1431 & 317 & 582 \\
\hline ANL- & 1287 & 82 & 0 & 1906 & 2125 & 2437 & 1572 & 1842 & 599 & 0 & 1177 & 1325 & 2393 & 963 & 972 & 1144 & 875 & 0 \\
\hline BCL & 675 & 348 & 1906 & 0 & 223 & 653 & 380 & 113 & 2322 & 1906 & 1463 & 650 & 626 & 2463 & 2472 & 1552 & 72 & 1906 \\
\hline Betti & 894 & 567 & 2125 & 223 & 0 & 506 & 599 & 312 & 2541 & 2125 & 1682 & 869 & 543 & 2682 & 2691 & 1771 & 291 & 2125 \\
\hline BNL & 1206 & 874 & 2437 & 653 & 506 & 0 & 906 & 760 & 2853 & 2437 & 2113 & 1299 & 241 & 2994 & 3003 & 2201 & 721 & 2437 \\
\hline Ferm & 341 & 36 & 1572 & 380 & 599 & 906 & 0 & 326 & 1975 & 1572 & 1359 & 519 & 863 & 2129 & $2 ! 38$ & +21 & 349 & 1572 \\
\hline FEM & 11 & 294 & 1842 & 113 & 312 & 760 & 326 & 0 & 2258 & 1842 & 1399 & 586 & 733 & 2399 & 2408 & 488 & 49 & 1842 \\
\hline HS & 1703 & 1998 & 599 & 2322 & 2541 & 2853 & 1975 & 2258 & 0 & 599 & 1593 & 1741 & 2809 & 875 & 894 & 1560 & 291 & 599 \\
\hline INE & 1287 & 1582 & 0 & 1906 & 2125 & 2437 & 1572 & 1842 & 599 & 0 & 1177 & 1325 & 2393 & 963 & 972 & 1144 & 1875 & 0 \\
\hline ITRI & 1129 & 1333 & 1177 & 1463 & 1682 & 2113 & 1359 & 1399 & 1593 & 1177 & 0 & 895 & 2085 & 1194 & 1154 & 111 & 1432 & 1177 \\
\hline $\mathrm{KCP}$ & 234 & 520 & 1325 & 650 & 869 & 1299 & $519^{\circ}$ & $586^{\circ}$ & 1741 & 1325 & 895 & 0 & 1272 & 1881 & 1890 & 984 & 619 & 1325 \\
\hline $\mathrm{KAF}$ & 1163 & 831 & 2393 & 626 & 543 & 241 & 863 & 733 & 2809 & 2393 & 2085 & 1272 & 0 & 2950 & 2959 & 2174 & 694 & 2393 \\
\hline LBL & 1844 & 2139 & 963 & 2463 & 2682 & 2994 & $2129^{-4}$ & 2399 & 875 & 963 & 1194 & 1881. & 2950 & 0 & 45 & 1274 & 2432 & _.963 \\
\hline LLNL & 1853 & 2148 & 972 & 2472 & 2691 & 3003 & 2138 . & 2408 & 894 & 972 & 1154 & 1890 & 2959 & 45 & 0 & 1233 & 441 & $97 ?$ \\
\hline LANL & 1136 & 1431 & 1144 & 1552 & 1771 & 2201 & 1421 & 1488 & 1560 & 1144 & 111 & 984 & 2174 & 1274 & 1233 & 0 & 1521 & 114 \\
\hline Mound & 644 & 317 & 1875 & 72 & 291 & 721 & 349 & 49 & 2291 & 1875 & 1432 & 619 & 694 & 2432 & 2441 & 1521 & 0 & 1875 \\
\hline NRF & 1287 & 1582 & 0 & 1906 & 2125 & $2437^{\circ}$ & $1572^{\circ}$ & 1842 & 599 & 0 & 1177. & 1325 & 2393 & -963 & 972 & 1144 & $1875^{\circ}$ & 5 \\
\hline NTS & 1520 & 1815 & 712 & 2078 & 2297 & 2670 & 1805 & 2014 & 1128 & 712 & 918 & 1428 & 2626 & 719 & 678. & 997 & $204 ?$ & 712 \\
\hline ORISE & 887. & 571 & 2077 & 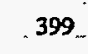 & $-586^{\circ}$ & 808 & 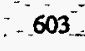 & $=299^{\circ}$ & 2493 & $20 \pi$ & 1420 & 752 & 872 & $2592^{-}$ & 2551 & 1509 & -335 & 2007 \\
\hline & 900 & 584 & $\begin{array}{r}2048 \\
-\end{array}$ & 412 & 563 & 821 & $=616$ & 312 & 2464 & 2048 & 1391 & 723 & 885 & 2563 & 2523 & 1480 & 348 & 2048 \\
\hline & $629^{\circ}$ & 385 & -1766 & & & & & $\begin{array}{r}409 \\
\end{array}$ & 2182 & 1766 & 1230 & $44 I_{-}$ & 1099 & 2322 & 2327 & 1319 & 441 & 1766 \\
\hline Pantex & 834 & 1038 & 1468 & 1168 & 1387 & 1817 & 1064 & 1104 & 1884 & 1468 & 313 & 600 & 1790 & 1485 & 1445 & 402 & 1137 & 1468 \\
\hline Pinellas & 1481 & 1204 & 2617 & 1065 & 1252 & 1329 & 1236 & 965 & 3033 & 2617 & 1959 & 1293 & $\therefore 1393^{-1}$ & 2945 & 2904. & 2048 & 1001 & 2617 \\
\hline PORTS & 755 & 428 & 1986 & 84 & 265. & 689 & 460 & 173. & 2402 & 1986 & 1543 & 730 & 688 & 2543 & 2552 & 1632 & 152 & 1986 \\
\hline${ }_{P P P L}$ & 1217 & 822. & 2448 & $546^{\circ}$ & $\because 398$ & -189 & 854 & 635 & $\therefore 2864$ & 2448 & 2006 & 1192 & 291. & 3005 & .3014 & 2094 & 614 & 2448 \\
\hline RMI & 751 & 419 & 1982. & 214 & 175 & 531 & 451 & 321 & 2398 & -1982 & 1673 & 860 & 416 & 2538 & 2547 & 1762 & 282 & 1982 \\
\hline $\mathbf{R F P}_{-}$ & +722 & 1017 & 716 & 1283 & 1500 & 1870 & 1005 & 1217 & 1132 & $\therefore 716$ & 483 & $63 i$ & 1827 & 1283 & 1292 & 452 & 1250 & 716 \\
\hline SNLNNM & 1120 & 1324 & 1168 & 1454 & 1673 & 2103 & 1350 & 1390 & 1584 & 1168 & 9 & 886 & 2076 & -1185 & 1145 & 102 & 1423 & 1168 \\
\hline SNLEC & & & $=972$ & 2472 & & 3003 & & 2408 & & & 1154 & 1890 & 2959 & 45 & 0 & 1233 & 2441 & $\infty$ \\
\hline SRS & 1175 & 892 & 2311 & 720 & 656 & 897 & 924 & 620 & 2727 & 2311 & 1653 & 987 & 961 & 2791 & 2750 & 1742 & 656 & 2311 \\
\hline$S H A C$ & 1885 & 2180 & 1004 & 2524 & 2723 & 3035 & $\begin{array}{r}2167 \\
-2\end{array}$ & 2440 & $\begin{array}{r}916 \\
-96\end{array}$ & 1004 & 1198 & 1939 & 2979 & 47 & 64 & 294 & 2473 & 1004 \\
\hline WVDP & 909 & 577 & 2140 & 372 & 257. & 492 & 609 & 479 . & 2556 & 2140 & 1832 & 1018 & 314 & 2697 & 2706 & 1921 & 440 & 2140 \\
\hline WIPP & 1301 & 1505 & 1759. & 1625 & 1813 & 2192 & $1531^{\circ}$ & 1526 & 2175 & $1759^{-}$ & 614 & 1067 & 2256 & 1509 & 468 & 693 & 1561 & 1759 \\
\hline $\mathrm{YM}$ & 1554 & 1849 & 746 & 2112 & 2331 & 2704 & 1839 & 2048 & 1162 & 746 & 952 & 1462 & 2660 & 753 & 712 & 1031 & 2081 & 746 \\
\hline
\end{tabular}


A-210

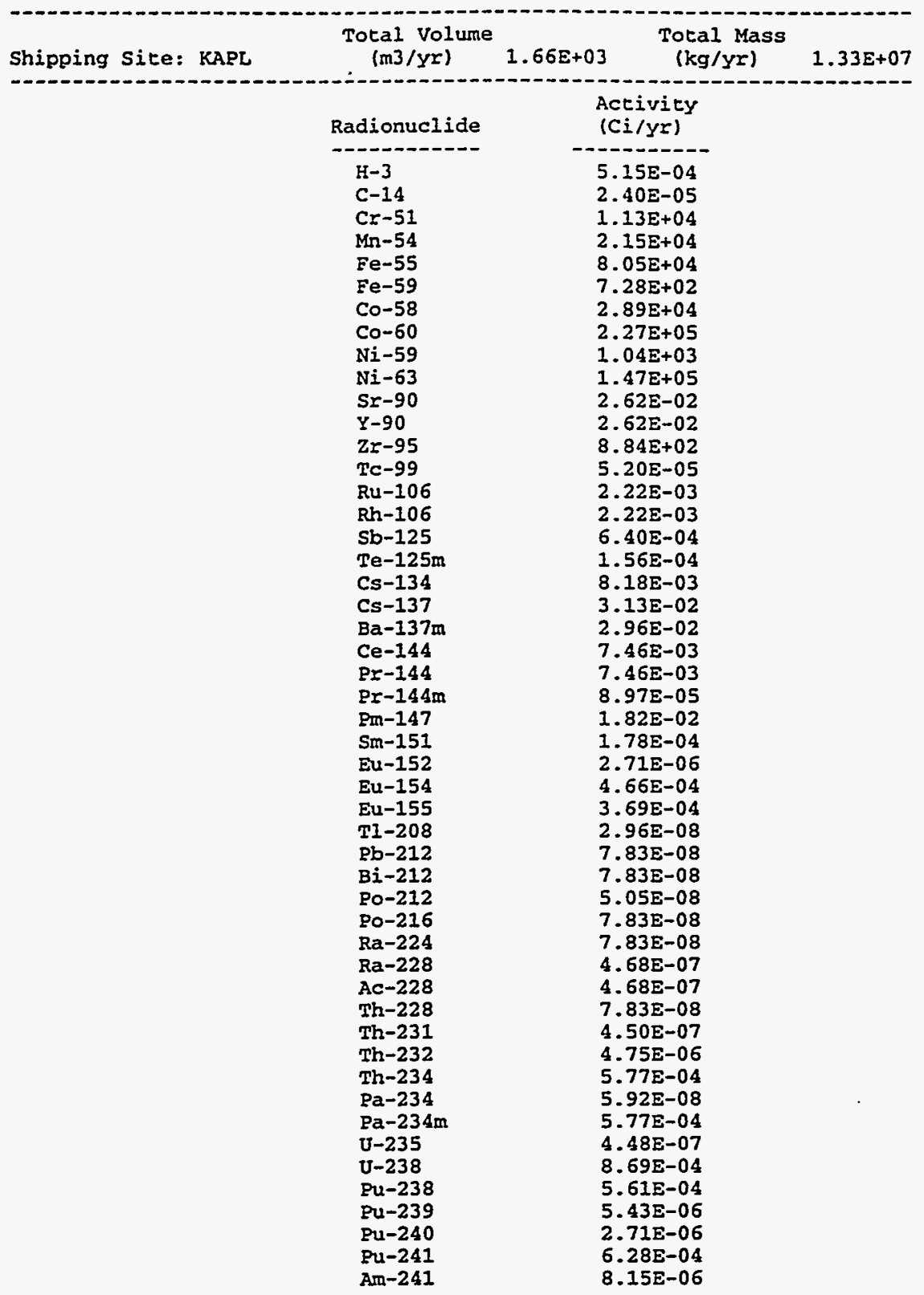




\section{A.25 WM LLW CENTRALIZED 4 ALTERNATIVE (CASE 14A): HETEROGENEOUS SOLIDS}

Transported Material with Radionuclide Profile

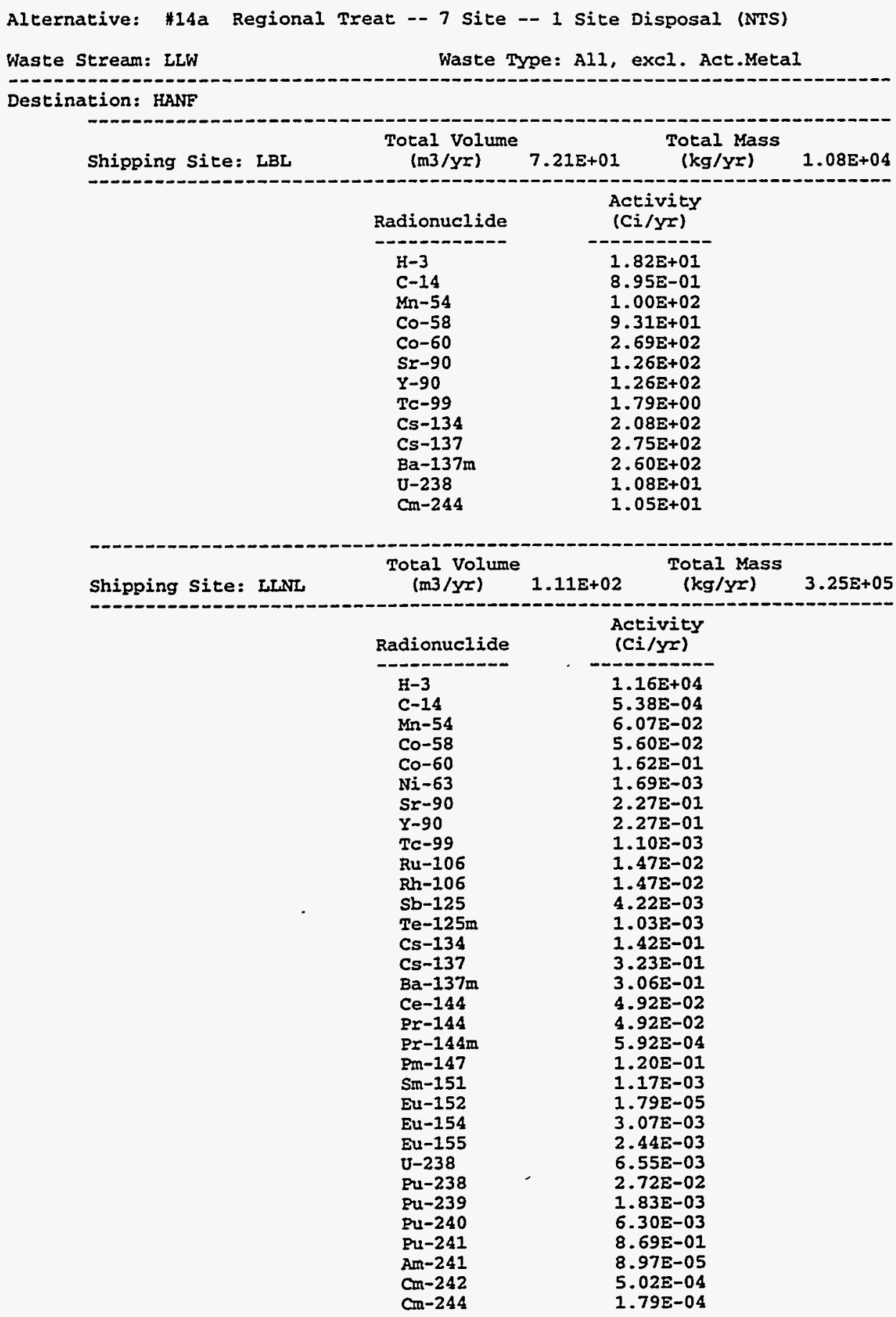


A-212

\begin{tabular}{|c|c|c|c|}
\hline Shipping Site: SLAC & $\begin{array}{l}\text { Total Volume } \\
\text { (m3/yr) }\end{array}$ & $\begin{array}{l}\text { Total Mass } \\
(\mathrm{kg} / \mathrm{yr})\end{array}$ & $1.24 E+01$ \\
\hline & $\begin{array}{l}\text { Radionuclide } \\
\mathrm{Cr}-51 \\
\mathrm{Mn}-54 \\
\mathrm{Fe}-55 \\
\mathrm{Fe}-59 \\
\mathrm{Co}-58 \\
\mathrm{Co}-60 \\
\mathrm{Ni}-59 \\
\mathrm{Ni}-63 \\
\mathrm{Zr}-95\end{array}$ & $\begin{array}{c}\begin{array}{c}\text { Activicy } \\
\text { (Ci/yr) }\end{array} \\
2.10 \mathrm{E}-07 \\
4.00 \mathrm{E}-07 \\
1.49 \mathrm{E}-06 \\
1.35 \mathrm{E}-08 \\
5.38 \mathrm{E}-07 \\
4.21 \mathrm{E}-06 \\
1.93 \mathrm{E}-08 \\
2.74 \mathrm{E}-06 \\
1.64 \mathrm{E}-08\end{array}$ & \\
\hline Shipping Site: SNLL & $\begin{array}{l}\text { Total Volume } \\
\text { (m3/yr) }\end{array}$ & $\begin{array}{cc} & \text { Total Mass } \\
2.28 \mathrm{E}+00 & (\mathrm{~kg} / \mathrm{Yr})\end{array}$ & $1.92 E+03$ \\
\hline & $\begin{array}{l}\text { Rađionuclide } \\
\mathrm{H}-3 \\
\mathrm{Fe}-55 \\
\mathrm{Co}-60 \\
\mathrm{Tl}-208 \\
\mathrm{~Pb}-212 \\
\mathrm{Bi}-212 \\
\mathrm{Po}-212 \\
\mathrm{Po}-216 \\
\mathrm{Ra}-224 \\
\mathrm{Ra}-228 \\
\mathrm{Ac}-228 \\
\mathrm{Th}-228 \\
\mathrm{Th}-231 \\
\mathrm{Th}-232 \\
\mathrm{Th}-234 \\
\mathrm{~Pa}-234 \\
\mathrm{~Pa}-234 \mathrm{~m} \\
\mathrm{U}-235 \\
\mathrm{U}-238\end{array}$ & \begin{tabular}{c}
$\begin{array}{c}\text { Activity } \\
(C i / y Y)\end{array}$ \\
\hdashline $2.82 E+04$ \\
$3.15 E-01$ \\
$3.15 E-01$ \\
$5.35 E-06$ \\
$1.41 E-05$ \\
$1.41 E-05$ \\
$9.13 E-06$ \\
$1.41 E-05$ \\
$1.41 E-05$ \\
$8.47 E-05$ \\
$8.47 E-05$ \\
$1.41 E-05$ \\
$8.15 E-05$ \\
$8.59 E-04$ \\
$1.04 E-01$ \\
$1.07 E-05$ \\
$1.04 E-01$ \\
$8.12 E-05$ \\
$1.04 E-01$
\end{tabular} & \\
\hline
\end{tabular}


Destination: INEL

\begin{tabular}{|c|c|c|c|}
\hline Shipping Site: ANL-W & $\begin{array}{l}\text { Total volume } \\
(\mathrm{m} 3 / \mathrm{Yr})\end{array}$ & $\begin{array}{l}\text { Toral Mass } \\
(\mathrm{kg} / \mathrm{y} z)\end{array}$ & $2.88 \mathrm{E}+04$ \\
\hline & \begin{tabular}{l} 
Radionuclide \\
\hdashline $\mathrm{H}-3$ \\
$\mathrm{Cr}-51$ \\
$\mathrm{Mn}-54$ \\
$\mathrm{Fe}-55$ \\
$\mathrm{Fe}-59$ \\
$\mathrm{Co}-58$ \\
$\mathrm{Co}-60$ \\
$\mathrm{Ni}-59$ \\
$\mathrm{Ni}-63$ \\
$\mathrm{Sr}-90$ \\
$\mathrm{Y}-90$ \\
$\mathrm{Zr}-95$ \\
$\mathrm{Tc}-99$ \\
$\mathrm{Ru}-106$ \\
$\mathrm{Rh}-106$ \\
$\mathrm{Sb}-125$ \\
$\mathrm{Te}-125 \mathrm{~m}$ \\
$\mathrm{Cs}-134$ \\
$\mathrm{Cs}-137$ \\
$\mathrm{Ba}-137 \mathrm{~m}$ \\
$\mathrm{Ce}-144$ \\
$\mathrm{Pr}-144$ \\
$\mathrm{Pr}-144 \mathrm{~m}$ \\
$\mathrm{Pm}-147$ \\
$\mathrm{Sm}-151$ \\
$\mathrm{Eu}-152$ \\
$\mathrm{Eu}-154$ \\
$\mathrm{Eu}-155$ \\
$\mathrm{Pu}-238$ \\
$\mathrm{Pu}-239$ \\
$\mathrm{Pu}-240$ \\
$\mathrm{Pu}-241$ \\
$\mathrm{Am}-241$ \\
$\mathrm{Cm}-242$ \\
$\mathrm{Cm}-244$
\end{tabular} & \begin{tabular}{c}
$\begin{array}{c}\text { Activity } \\
(C i / Y \mathrm{Y})\end{array}$ \\
\hdashline $3.77 \mathrm{E}-05$ \\
$3.85 \mathrm{E}-03$ \\
$7.34 \mathrm{E}-03$ \\
$2.74 \mathrm{E}-02$ \\
$2.47 \mathrm{E}-04$ \\
$9.85 \mathrm{E}-03$ \\
$7.74 \mathrm{E}-02$ \\
$3.53 \mathrm{E}-04$ \\
$5.06 \mathrm{E}-02$ \\
$3.17 \mathrm{E}-02$ \\
$3.17 \mathrm{E}-02$ \\
$3.00 \mathrm{E}-04$ \\
$5.66 \mathrm{E}-06$ \\
$3.09 \mathrm{E}-03$ \\
$3.09 \mathrm{E}-03$ \\
$8.89 \mathrm{E}-04$ \\
$2.17 \mathrm{E}-04$ \\
$3.59 \mathrm{E}-03$ \\
$3.32 \mathrm{E}-02$ \\
$3.14 \mathrm{E}-02$ \\
$1.03 \mathrm{E}-02$ \\
$1.03 \mathrm{E}-02$ \\
$1.24 \mathrm{E}-04$ \\
$2.52 \mathrm{E}-02$ \\
$2.47 \mathrm{E}-04$ \\
$3.77 \mathrm{E}-06$ \\
$6.47 \mathrm{E}-04$ \\
$5.13 \mathrm{E}-04$ \\
$7.81 \mathrm{E}-04$ \\
$7.72 \mathrm{E}-06$ \\
$4.38 \mathrm{E}-06$ \\
$9.56 \mathrm{E}-04$ \\
$1.13 \mathrm{E}-05$ \\
$4.88 \mathrm{E}-08$ \\
$1.74 \mathrm{E}-08$
\end{tabular} & \\
\hline
\end{tabular}


Destination: LANL

\begin{tabular}{|c|c|c|c|}
\hline Shipping Site: ITRI & $\begin{array}{l}\text { Total Volume } \\
(\mathrm{m} 3 / \mathrm{yx})\end{array}$ & $\begin{array}{c}\text { Total Mass } \\
(\mathrm{kg} / \mathrm{yc})\end{array}$ & $1.17 E+04$ \\
\hline & $\begin{array}{l}\text { Radionuclide } \\
\mathrm{H}-3 \\
\mathrm{C}-14 \\
\mathrm{Mn}-54 \\
\mathrm{Co}-58 \\
\mathrm{Co}-60 \\
\mathrm{Sr}-90 \\
\mathrm{Y}-90 \\
\mathrm{Tc}-99 \\
\mathrm{Cs}-134 \\
\mathrm{Cs}-137 \\
\mathrm{Ba}-137 \mathrm{~m} \\
\mathrm{U}-238\end{array}$ & \multicolumn{2}{|l|}{$\begin{array}{l}3.73 \mathrm{E}+00 \\
1.83 \mathrm{E}-01 \\
2.06 \mathrm{E}+01 \\
1.90 \mathrm{E}+01 \\
5.51 \mathrm{E}+01 \\
2.59 \mathrm{E}+01 \\
2.59 \mathrm{E}+01 \\
3.67 \mathrm{E}-01 \\
4.27 \mathrm{E}+01 \\
5.64 \mathrm{E}+01 \\
5.33 \mathrm{E}+01 \\
2.23 \mathrm{E}+00\end{array}$} \\
\hline \multirow[t]{2}{*}{ Shipping Site: PANT } & $\begin{array}{l}\text { Total Volume } \\
(\mathrm{m} 3 / \mathrm{yr})\end{array}$ & $\begin{array}{c}\text { Total Mass } \\
(\mathrm{kg} / \mathrm{yr})\end{array}$ & $2.84 E+07$ \\
\hline & $\begin{array}{l}\text { Radionuclide } \\
\mathrm{H}-3 \\
\mathrm{Tl}-208 \\
\mathrm{~Pb}-212 \\
\mathrm{Bi}-212 \\
\mathrm{PO-212} \\
\mathrm{PO}-216 \\
\mathrm{Ra}-224 \\
\mathrm{Ra}-228 \\
\mathrm{Ac}-228 \\
\mathrm{Th}-228 \\
\mathrm{Th}-231 \\
\mathrm{Th}-232 \\
\mathrm{Th}-234 \\
\mathrm{~Pa}-234 \\
\mathrm{~Pa}-234 \mathrm{~m} \\
\mathrm{U}-235 \\
\mathrm{U}-238\end{array}$ & \multicolumn{2}{|l|}{$\begin{array}{l}2.25 \mathrm{E}+02 \\
2.12 \mathrm{E}-04 \\
5.63 \mathrm{E}-04 \\
5.63 \mathrm{E}-04 \\
3.62 \mathrm{E}-04 \\
5.63 \mathrm{E}-04 \\
5.63 \mathrm{E}-04 \\
3.36 \mathrm{E}-03 \\
3.36 \mathrm{E}-03 \\
5.63 \mathrm{E}-04 \\
3.24 \mathrm{E}-03 \\
3.41 \mathrm{E}-02 \\
4.15 \mathrm{E}+00 \\
4.25 \mathrm{E}-04 \\
4.15 \mathrm{E}+00 \\
3.22 \mathrm{E}-03 \\
4.15 \mathrm{E}+00\end{array}$} \\
\hline \multirow[t]{2}{*}{ Shipping Site: SNLA } & $\begin{array}{l}\text { Total Volume } \\
\text { (m3/yr) }\end{array}$ & $\begin{array}{c}\text { Total Mass } \\
(\mathrm{kg} / \mathrm{Y} r)\end{array}$ & $4.31 E+03$ \\
\hline & $\begin{array}{l}\text { Radionuclide } \\
\mathrm{Tl}-208 \\
\mathrm{~Pb}-212 \\
\mathrm{Bi}-212 \\
\mathrm{PO}-212 \\
\mathrm{Po}-216 \\
\mathrm{Ra}-224 \\
\mathrm{Ra}-228 \\
\mathrm{Ac}-228 \\
\mathrm{Th}-228 \\
\mathrm{Th}-231 \\
\mathrm{Th}-232 \\
\mathrm{Th}-234 \\
\mathrm{~Pa}-234 \\
\mathrm{~Pa}-234 \mathrm{~m} \\
\mathrm{U}-235 \\
\mathrm{U}-238\end{array}$ & \multicolumn{2}{|l|}{$\begin{array}{l}5.10 \mathrm{E}-06 \\
1.35 \mathrm{E}-05 \\
1.35 \mathrm{E}-05 \\
8.70 \mathrm{E}-06 \\
1.35 \mathrm{E}-05 \\
1.35 \mathrm{E}-05 \\
8.07 \mathrm{E}-05 \\
8.07 \mathrm{E}-05 \\
1.35 \mathrm{E}-05 \\
7.77 \mathrm{E}-05 \\
8.19 \mathrm{E}-04 \\
9.96 \mathrm{E}-02 \\
1.02 \mathrm{E}-05 \\
9.96 \mathrm{E}-02 \\
7.74 \mathrm{E}-05 \\
9.96 \mathrm{E}-02\end{array}$} \\
\hline
\end{tabular}


Destination: NTS

\begin{tabular}{|c|c|c|c|}
\hline Shipping Site: AMES & $\begin{array}{l}\text { Total Volume } \\
(\mathrm{m} 3 / \mathrm{yr})\end{array}$ & $\begin{array}{c}\text { Total Mass } \\
(\mathrm{kg} / \mathrm{y})\end{array}$ & $2.98 \mathrm{E}+04$ \\
\hline & $\begin{array}{l}\text { Radionuclide } \\
\mathrm{Tl}-208 \\
\mathrm{~Pb}-212 \\
\mathrm{~B} \mathrm{i}-212 \\
\mathrm{Po}-212 \\
\mathrm{PO}-216 \\
\mathrm{Ra}-224 \\
\mathrm{Ra}-228 \\
\mathrm{Ac}-228 \\
\mathrm{Th}-228 \\
\mathrm{Th}-231 \\
\mathrm{Th}-232 \\
\mathrm{Th}-234 \\
\mathrm{~Pa}-234 \\
\mathrm{~Pa}-234 \mathrm{~m} \\
\mathrm{U}-235 \\
\mathrm{U}-238 \\
\mathrm{Pu}-238 \\
\mathrm{Pu}-239 \\
\mathrm{Pu}-240 \\
\mathrm{Pu}-241 \\
\mathrm{Am}-241 \\
\mathrm{Cm}-242 \\
\mathrm{Cm}-244\end{array}$ & \begin{tabular}{c}
$\begin{array}{c}\text { Activity } \\
(C i / y r)\end{array}$ \\
\hdashline $4.22 \mathrm{E}-07$ \\
$1.11 \mathrm{E}-06$ \\
$1.11 \mathrm{E}-06$ \\
$7.20 \mathrm{E}-07$ \\
$1.11 \mathrm{E}-06$ \\
$1.11 \mathrm{E}-06$ \\
$6.68 \mathrm{E}-06$ \\
$6.68 \mathrm{E}-06$ \\
$1.11 \mathrm{E}-06$ \\
$6.43 \mathrm{E}-06$ \\
$6.77 \mathrm{E}-05$ \\
$8.24 \mathrm{E}-03$ \\
$8.44 \mathrm{E}-07$ \\
$8.24 \mathrm{E}-03$ \\
$6.40 \mathrm{E}-06$ \\
$8.24 \mathrm{E}-03$ \\
$6.50 \mathrm{E}-04$ \\
$4.96 \mathrm{E}-05$ \\
$1.73 \mathrm{E}-04$ \\
$2.39 \mathrm{E}-02$ \\
$9.93 \mathrm{E}-07$ \\
$1.39 \mathrm{E}-05$ \\
$4.96 \mathrm{E}-06$
\end{tabular} & \\
\hline & $\begin{array}{l}\text { Total Volume } \\
\text { (m3/yr) }\end{array}$ & $\begin{array}{c}\text { Total Mass } \\
(\mathrm{kg} / \mathrm{yr})\end{array}$ & $2.00 E+06$ \\
\hline & $\begin{array}{l}\text { Radionuclide } \\
-1-3 \\
\mathrm{C}-14 \\
\mathrm{Cr}-51 \\
\mathrm{Mn}-54 \\
\mathrm{Fe}-55 \\
\mathrm{Fe}-59 \\
\mathrm{Co}-58 \\
\mathrm{Co}-60 \\
\mathrm{Ni}-59 \\
\mathrm{Ni}-63 \\
\mathrm{Sr}-90 \\
\mathrm{Y}-90 \\
\mathrm{Zr}-95 \\
\mathrm{Tc}-99 \\
\mathrm{Ru}-106 \\
\mathrm{Rh}-106 \\
\mathrm{Sb}-125 \\
\mathrm{Te}-125 \mathrm{~m} \\
\mathrm{Cs}-134 \\
\mathrm{Cs}-137 \\
\mathrm{Ba}-137 \mathrm{~m} \\
\mathrm{Ce}-144 \\
\mathrm{Pr}-144 \\
\mathrm{Pr}-144 \mathrm{~m} \\
\mathrm{Pm}-147 \\
\mathrm{Sm}-151 \\
\mathrm{Eu}-152 \\
\mathrm{Eu}-154 \\
\mathrm{Eu}-155 \\
\mathrm{U}-238 \\
\mathrm{Pu}-238 \\
\mathrm{Pu}-239 \\
\mathrm{Pu}-240 \\
\mathrm{Pu}-241 \\
\mathrm{Am}-241\end{array}$ & $\begin{array}{c}\text { Activity } \\
(C i / y r) \\
1.33 E+02 \\
7.91 E-02 \\
2.87 \mathrm{E}+00 \\
1.43 \mathrm{E}+01 \\
2.04 \mathrm{E}+01 \\
1.84 \mathrm{E}-01 \\
1.55 \mathrm{E}+01 \\
8.15 \mathrm{E}+01 \\
2.63 \mathrm{E}-01 \\
3.77 \mathrm{E}+01 \\
3.33 \mathrm{E}+01 \\
3.33 \mathrm{E}+01 \\
2.24 \mathrm{E}-01 \\
1.62 \mathrm{E}-01 \\
2.16 \mathrm{E}+00 \\
2.16 \mathrm{E}+00 \\
6.21 \mathrm{E}-01 \\
1.51 \mathrm{E}-01 \\
2.09 \mathrm{E}+01 \\
4.75 \mathrm{E}+01 \\
4.50 \mathrm{E}+01 \\
7.24 \mathrm{E}+00 \\
7.24 \mathrm{E}+00 \\
8.70 \mathrm{E}-02 \\
1.76 \mathrm{E}+01 \\
1.72 \mathrm{E}-01 \\
2.63 \mathrm{E}-03 \\
4.52 \mathrm{E}-01 \\
3.58 \mathrm{E}-01 \\
9.63 \mathrm{E}-01 \\
5.44 \mathrm{E}-01 \\
5.27 \mathrm{E}-03 \\
2.63 \mathrm{E}-03 \\
6.09 \mathrm{E}-01 \\
7.91 \mathrm{E}-03\end{array}$ & \\
\hline
\end{tabular}


A-216

\begin{tabular}{|c|c|c|c|}
\hline Shipping Site: ANL-W & $\begin{array}{l}\text { Tocal volume } \\
\text { (m3/yr) }\end{array}$ & $\begin{array}{l}\text { Total Mass } \\
(\mathrm{kg} / \mathrm{yr})\end{array}$ & $1.83 E+05$ \\
\hline & $\begin{array}{l}\text { Radionuclide } \\
\mathrm{K}-3 \\
\mathrm{Cr}-51 \\
\mathrm{Mn}-54 \\
\mathrm{Fe}-55 \\
\mathrm{Fe}-59 \\
\mathrm{Co}-58 \\
\mathrm{Co}-60 \\
\mathrm{Ni}-59 \\
\mathrm{Ni}-63 \\
\mathrm{Zr}-95\end{array}$ & $\begin{array}{l}\text { Activity } \\
\text { (Ci/yf) } \\
7.45 E-04 \\
3.58 E+03 \\
6.83 E+03 \\
2.54 E+04 \\
2.30 E+02 \\
9.16 E+03 \\
7.18 E+04 \\
3.29 E+02 \\
4.67 E+04 \\
2.79 E+02\end{array}$ & \\
\hline Shipping Site: BAPL & $\begin{array}{l}\text { Total volume } \\
\quad(\mathrm{m} 3 / y x)\end{array}$ & $\begin{array}{c}\text { Total Mass } \\
3.98 E+02 \quad(\mathrm{~kg} / \mathrm{yr})\end{array}$ & $1.08 \mathrm{E}+06$ \\
\hline & 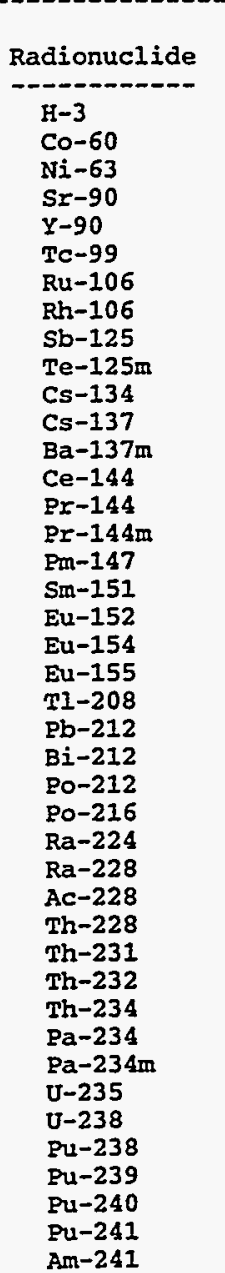 & $\begin{array}{l}\text { Activity } \\
\text { (Ci/Yr) } \\
2.51 \mathrm{E}-03 \\
1.19 \mathrm{E}-02 \\
2.38 \mathrm{E}-02 \\
2.11 \mathrm{E}+00 \\
2.11 \mathrm{E}+00 \\
3.77 \mathrm{E}-04 \\
2.06 \mathrm{E}-01 \\
2.06 \mathrm{E}-01 \\
5.93 \mathrm{E}-02 \\
1.44 \mathrm{E}-02 \\
2.40 \mathrm{E}-01 \\
2.21 \mathrm{E}+00 \\
2.09 \mathrm{E}+00 \\
6.91 \mathrm{E}-01 \\
6.91 \mathrm{E}-01 \\
8.31 \mathrm{E}-03 \\
1.68 \mathrm{E}+00 \\
1.65 \mathrm{E}-02 \\
2.51 \mathrm{E}-04 \\
4.32 \mathrm{E}-02 \\
3.42 \mathrm{E}-02 \\
3.80 \mathrm{E}-06 \\
1.00 \mathrm{E}-05 \\
1.00 \mathrm{E}-05 \\
6.49 \mathrm{E}-06 \\
1.00 \mathrm{E}-05 \\
1.00 \mathrm{E}-05 \\
6.02 \mathrm{E}-05 \\
6.02 \mathrm{E}-05 \\
1.00 \mathrm{E}-05 \\
5.80 \mathrm{E}-05 \\
6.11 \mathrm{E}-04 \\
7.43 \mathrm{E}-02 \\
7.61 \mathrm{E}-06 \\
7.43 \mathrm{E}-02 \\
5.77 \mathrm{E}-05 \\
7.43 \mathrm{E}-02 \\
5.20 \mathrm{E}-02 \\
5.03 \mathrm{E}-04 \\
2.51 \mathrm{E}-04 \\
5.81 \mathrm{E}-02 \\
7.55 \mathrm{E}-04\end{array}$ & \\
\hline
\end{tabular}




\begin{tabular}{|c|c|c|c|}
\hline Shipping Site: FNAL & $\begin{array}{l}\text { Total Volume } \\
\text { (m3/yr) }\end{array}$ & $\begin{array}{c}\text { Total Mass } \\
(\mathrm{kg} / \mathrm{yz})\end{array}$ & $3.26 E+04$ \\
\hline & \begin{tabular}{l} 
Radionuclide \\
\hdashline $\mathrm{H}-3$ \\
$\mathrm{Cr}-51$ \\
$\mathrm{Mn}-54$ \\
$\mathrm{Fe}-55$ \\
$\mathrm{Fe}-59$ \\
$\mathrm{Co}-58$ \\
$\mathrm{Co}-60$ \\
$\mathrm{Ni}-59$ \\
$\mathrm{Ni}-63$ \\
$\mathrm{Zr}-95$ \\
$\mathrm{~T}-208$ \\
$\mathrm{~Pb}-212$ \\
$\mathrm{Bi}-212$ \\
$\mathrm{Po}-212$ \\
$\mathrm{Po}-216$ \\
$\mathrm{Ra}-224$ \\
$\mathrm{Ra}-228$ \\
$\mathrm{Ac}-228$ \\
$\mathrm{Th}-228$ \\
$\mathrm{Th}-231$ \\
$\mathrm{Th}-232$ \\
$\mathrm{Th}-234$ \\
$\mathrm{~Pa}-234$ \\
$\mathrm{~Pa}-234 \mathrm{~m}$ \\
$\mathrm{U}-235$ \\
$\mathrm{U}-238$
\end{tabular} & \multicolumn{2}{|l|}{$\begin{array}{l}1.08 E-01 \\
1.11 E-02 \\
2.12 \mathrm{E}-02 \\
7.94 \mathrm{E}-02 \\
7.17 \mathrm{E}-04 \\
2.85 \mathrm{E}-02 \\
2.23 \mathrm{E}-01 \\
1.02 \mathrm{E}-03 \\
1.45 \mathrm{E}-01 \\
8.71 \mathrm{E}-04 \\
3.78 \mathrm{E}-07 \\
1.00 \mathrm{E}-06 \\
1.00 \mathrm{E}-06 \\
6.46 \mathrm{E}-07 \\
1.00 \mathrm{E}-06 \\
1.00 \mathrm{E}-06 \\
5.99 \mathrm{E}-06 \\
5.99 \mathrm{E}-06 \\
1.00 \mathrm{E}-06 \\
5.77 \mathrm{E}-06 \\
6.08 \mathrm{E}-05 \\
7.39 \mathrm{E}-03 \\
7.57 \mathrm{x}-07 \\
7.39 \mathrm{E}-03 \\
5.75 \mathrm{E}-06 \\
7.39 \mathrm{E}-03\end{array}$} \\
\hline \multirow[t]{2}{*}{ Shipping Site: HANE } & $\begin{array}{l}\text { Total Volume } \\
\text { (m3/yr) }\end{array}$ & $\begin{array}{c}\text { Total Mass } \\
(\mathrm{kg} / \mathrm{yr})\end{array}$ & $2.79 E+07$ \\
\hline & $\begin{array}{l}\text { Radionuclide } \\
\text { H-3 } \\
\mathrm{C}-14 \\
\mathrm{Cr}-51 \\
\mathrm{Mn}-54 \\
\mathrm{Fe}-55 \\
\mathrm{Fe}-59 \\
\mathrm{Co}-58 \\
\mathrm{Co}-60 \\
\mathrm{Ni}-59 \\
\mathrm{Ni}-63 \\
\mathrm{Sr}-90 \\
\mathrm{Y}-90 \\
\mathrm{Zr}-95 \\
\mathrm{TC}-99 \\
\mathrm{Ru}-106 \\
\mathrm{Rh}-106 \\
\mathrm{Sb}-125 \\
\mathrm{Te}-125 \mathrm{~m} \\
\mathrm{Cs}-134 \\
\mathrm{Cs}-137\end{array}$ & $\begin{array}{c}\begin{array}{c}\text { Activity } \\
(C i / y I)\end{array} \\
-1.17 E+04 \\
1.33 E-01 \\
6.33 E+01 \\
5.66 E+01 \\
2.08 E+02 \\
4.02 E+02 \\
3.63 E+00 \\
2.37 E+02 \\
1.41 E+03 \\
5.19 E+00 \\
7.60 E+02 \\
2.05 E+03 \\
2.05 E+03 \\
4.41 E+00 \\
2.13 E+00 \\
1.88 E+02 \\
1.88 E+02 \\
5.41 E+01 \\
1.32 E+01 \\
4.27 E+02 \\
2.29 E+03\end{array}$ & \\
\hline
\end{tabular}


A-218

Destination: NTS

\begin{tabular}{|c|c|c|c|}
\hline Shipping Site: HANF & $\begin{array}{l}\text { Toral Volume } \\
(\mathrm{m} 3 / \mathrm{Yr})\end{array}$ & $\begin{array}{l}\text { Total Mass } \\
(\mathrm{kg} / \mathrm{y} x)\end{array}$ & $2.79 E+07$ \\
\hline & $\begin{array}{l}\text { Radionuclide } \\
\mathrm{Ba}-137 \mathrm{~m} \\
\mathrm{Ce}-144 \\
\mathrm{Pr}-144 \\
\mathrm{Pr}-144 \mathrm{~m} \\
\mathrm{Pm}-147 \\
\mathrm{Sm}-151 \\
\mathrm{Eu}-152 \\
\mathrm{Eu}-154 \\
\mathrm{Eu}-155 \\
\mathrm{Tl}-208 \\
\mathrm{~Pb}-212 \\
\mathrm{Bi}-212 \\
\mathrm{Po}-212 \\
\mathrm{Po}-216 \\
\mathrm{Ra}-224 \\
\mathrm{Ra}-228 \\
\mathrm{Ac}-228 \\
\mathrm{Th}-228 \\
\mathrm{Th}-231 \\
\mathrm{Th}-232 \\
\mathrm{Th}-234 \\
\mathrm{~Pa}-234 \\
\mathrm{~Pa}-234 \mathrm{~m} \\
\mathrm{U}-235 \\
\mathrm{U}-238 \\
\mathrm{Pu}-238 \\
\mathrm{Pu}-239 \\
\mathrm{Pu}-240 \\
\mathrm{Pu}-241 \\
\mathrm{Am}-241 \\
\mathrm{Cm}-242 \\
\mathrm{Cm}-244\end{array}$ & 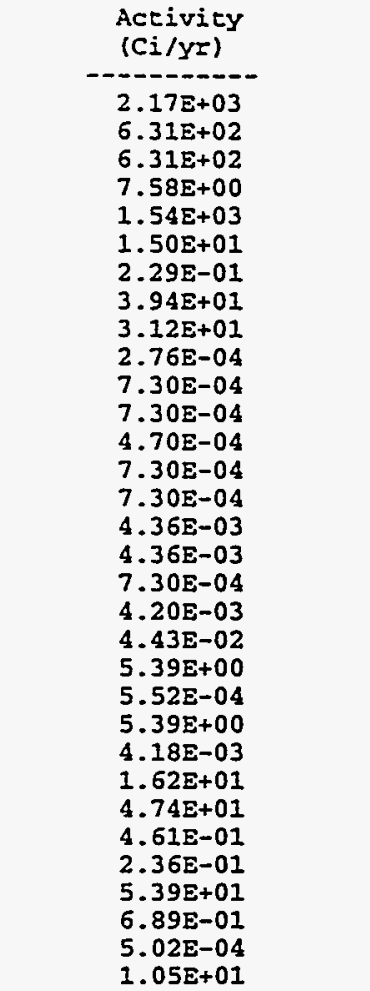 & \\
\hline Shipping Site: INEL & $\begin{array}{l}\text { Total Volume } \\
\text { (m3/yr) }\end{array}$ & $\begin{array}{cc}3.77 \mathrm{E}+03 & \begin{array}{c}\text { Total Mass } \\
(\mathrm{kg} / \mathrm{yr})\end{array}\end{array}$ & \\
\hline & $\begin{array}{l}\text { Radionuclide } \\
\mathrm{H}-3 \\
\mathrm{Cr}-51 \\
\mathrm{Mn}-54 \\
\mathrm{Fe}-55 \\
\mathrm{Fe}-59 \\
\mathrm{Co}-58 \\
\mathrm{Co}-60 \\
\mathrm{Ni}-59 \\
\mathrm{Ni}-63 \\
\mathrm{Sr}-90 \\
\mathrm{Y}-90 \\
\mathrm{Zr}-95 \\
\mathrm{Tc}-99 \\
\mathrm{Ru}-106 \\
\mathrm{Rh}-106 \\
\mathrm{Sb}-125 \\
\mathrm{Te}-125 \mathrm{~m} \\
\mathrm{Cs}-134 \\
\mathrm{Cs}-137 \\
\mathrm{Ba}-137 \mathrm{~m} \\
\mathrm{Ce}-144 \\
\mathrm{Pr}-144 \\
\mathrm{Pr}-144 \mathrm{~m} \\
\mathrm{Pm}-147 \\
\mathrm{Sm}-151 \\
\text { Eu-152 } \\
\text { Eu-154 } \\
\text { Eu-155 } \\
\mathrm{Pu}-238 \\
\mathrm{Pu}-239 \\
\mathrm{Pu}-240 \\
\mathrm{Pu}-241 \\
\mathrm{Am}-241 \\
\mathrm{Cm}-242 \\
\mathrm{Cm}-244\end{array}$ & 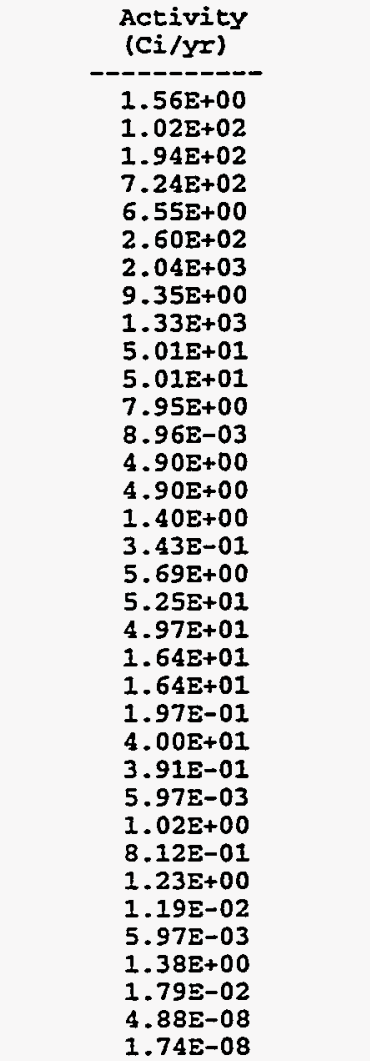 & \\
\hline
\end{tabular}




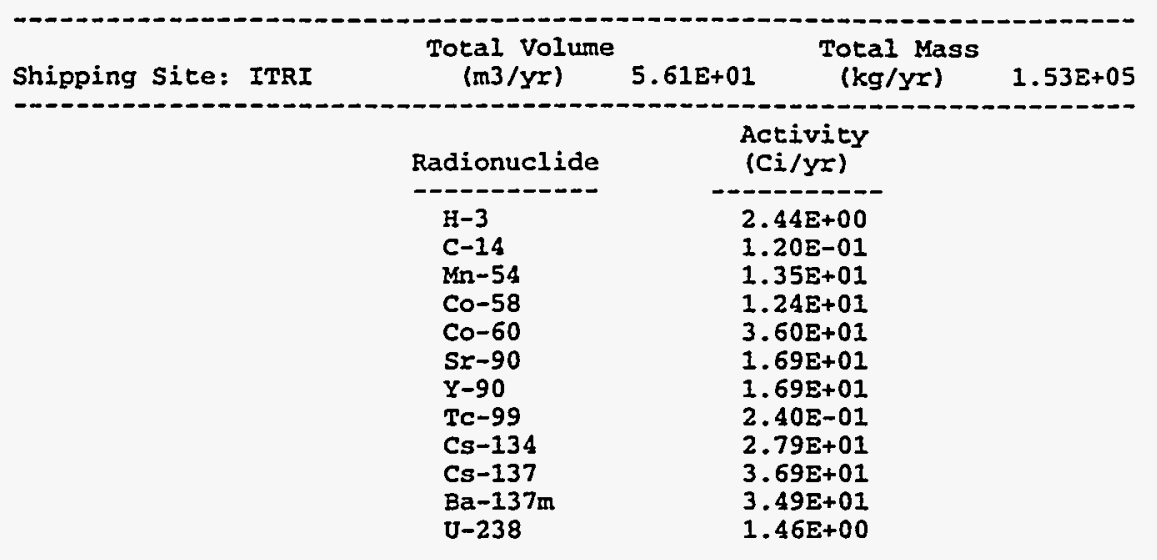




\section{CONTENTS}

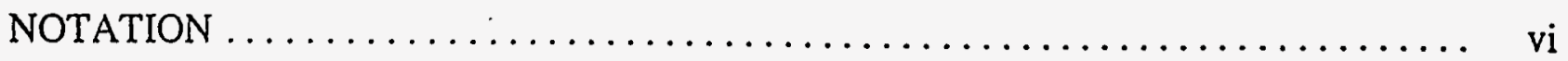

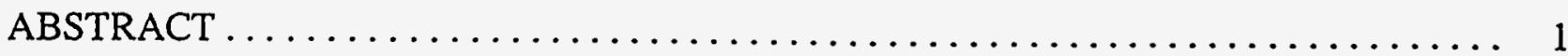

1 INTRODUCTION $\ldots \ldots \ldots \ldots \ldots \ldots \ldots \ldots \ldots \ldots \ldots \ldots \ldots \ldots \ldots \ldots \ldots \ldots \ldots \ldots$

2 LOW-LEVEL WASTE CHARACTERISTICS, ALTERNATIVES,

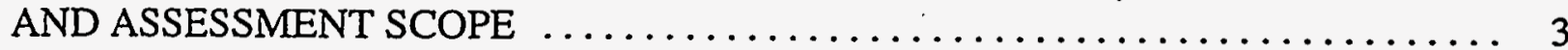

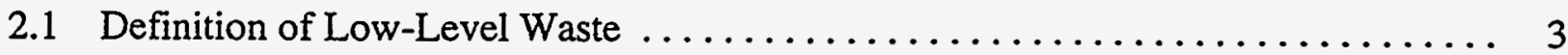

2.2 Low-Level Waste Alternatives for the WM PEIS $\ldots \ldots \ldots \ldots \ldots \ldots \ldots \ldots \ldots$

2.3 Characteristics of Low-Level Waste $\ldots \ldots \ldots \ldots \ldots \ldots \ldots \ldots \ldots \ldots . \ldots \ldots$

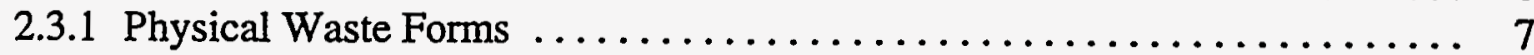

2.3.2 Radiological Profiles ........................... 7

2.4 Quantities and Characteristics of Transported Low-Level Waste by Case ....... 8

2.5 Scope of the Risk Assessment ............................. 9

2.5.1 Cargo-Related Impacts: Radiological ..................... 9

2.5.2 Vehicle-Related Impacts: Nonradiological . . . . . . . . . . . . . . 11

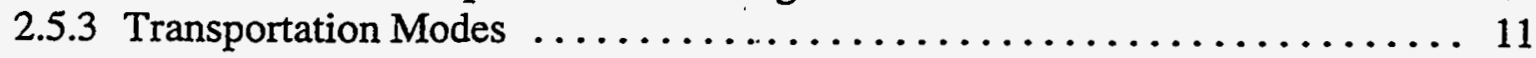

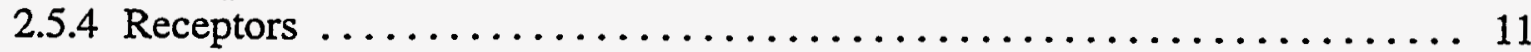

3 METHODS FOR CALCULATING TRANSPORTATION-RELATED RISKS . . . . . . 12

3.1 Routine Risk Assessment Method $\ldots \ldots \ldots \ldots \ldots \ldots \ldots \ldots \ldots \ldots \ldots \ldots \ldots \ldots \ldots$

3.1.1 Collective Population Risk ........................... 14

3.1.2 Maximally Exposed Individual Risk $\ldots \ldots \ldots \ldots \ldots \ldots \ldots \ldots \ldots \ldots$

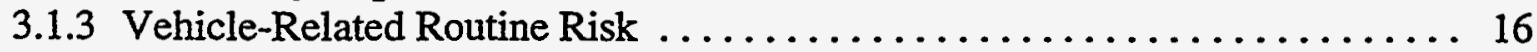

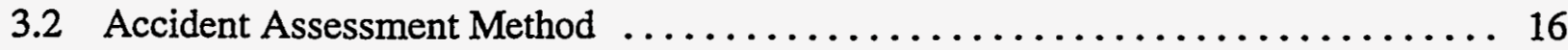

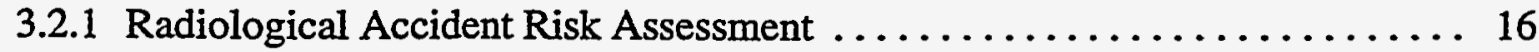

3.2.2 Radiological Accident Consequence Assessment ................. 18

3.2.3 Vehicle-Related Accident Risk Assessment . .................. 19

3.3 Representative Route Determination Method .................... 19

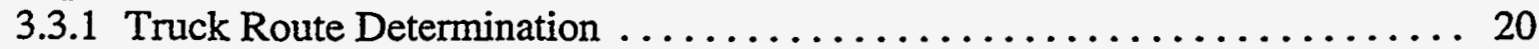

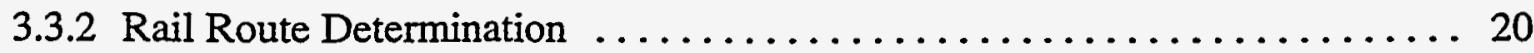

4 RISK ASSESSMENT ASSUMPTIONS AND PARAMETERS .............. 26

4.1 Shipping and Packaging Assumptions for Low-Level Waste $\ldots \ldots \ldots \ldots \ldots \ldots 26$

4.2 Accident Rates ...................................... 26 


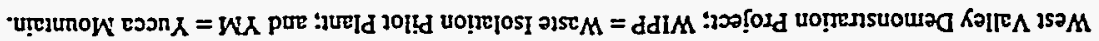

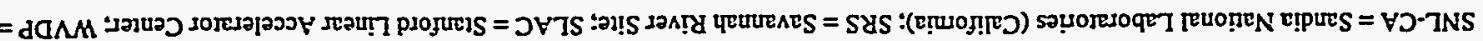

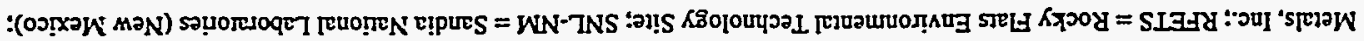

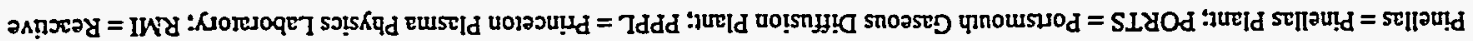

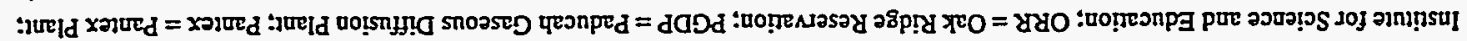

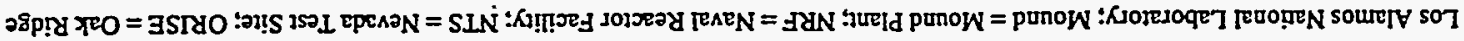

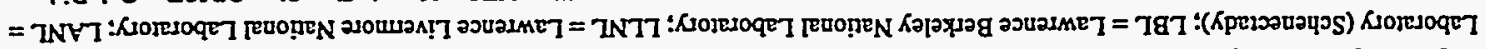

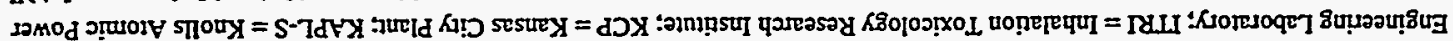

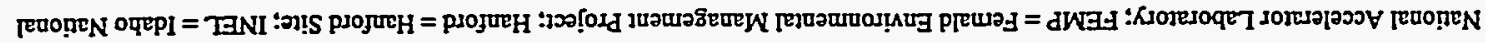
ก̣w

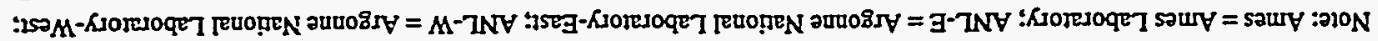

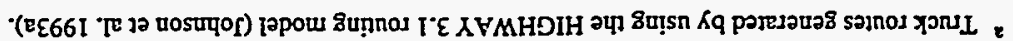

\begin{tabular}{|c|c|c|c|c|c|c|c|c|c|c|c|c|c|c|c|c|c|}
\hline 0 & 66हI & $\overline{L O t \bar{c}}$ & $\varepsilon L$ & $8+2$ & $\overline{c \mid l}$ & $\overline{\varepsilon \neq 6}$ & 898 & $8+\pi 2$ & 4592 & 612 & 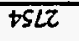 & 221 & 061 & 812 & 122 & $9 t$ & $\sqrt{2}$ \\
\hline 66દt & 0 & 0861 & 6ZSI & $t 2 S I$ & $89 b I-$ & $\operatorname{sos}$ & $\angle 90 \mathrm{I}$ & 2281 & $980 z$ & E9I. & ZgLI & c. & $8 S Z$ & I8EI & Olt] & "s9EI & ddIA \\
\hline$\angle 0 t \tau$ & $086 I$ & 0 & $8 \varepsilon \angle Z$ & દZ૦І & $90<\pi$ & $\varepsilon Z 81$ & ELSI & 291 & $b$ & $b$ & $6 I+I$ & LESI & $568^{\circ}$ & $99 L$ & $\dot{\varepsilon} \varsigma \dot{L}$ & ELẼ & disM \\
\hline$\varepsilon u$ & 6251 & $E l z$ & 0 & 0282 & & 8611 & 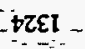 & $6 L S Z$ & $0 \varepsilon$ & 6852 & 6962 & 90SI & 6SEZ & $+8 s \mathcal{L}^{-}$ & $85 i$ & $6 \varepsilon L$ & DV7S. \\
\hline $8+t z$ & $t z S I$ & $\varepsilon 20 \mathrm{i}$ & $0 z 8 \overline{2}$ & 0 & osiz & $+9 I^{-}$ & 8191 & $9 \overline{z L}$ & $\angle 9 L$ & 45 & 029 & 8SEI & 895 & $\sigma \angle E$ & $69 \varepsilon$ & $t i t \tau$ & s\&s \\
\hline 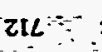 & $89+1$. & $90<z$ & $+0=$ & $\cos \angle \alpha^{--}$ & 0 & stits & $2 \sigma z r$ & $\angle t \leq z$ & $p t 0 \varepsilon$ & $z s s z^{2}$ & +062 & $\operatorname{sint}$ & $\angle z \varepsilon$ & £ֲst & ISST & $8 \angle 9$ & TNS \\
\hline$\varepsilon+6$ & 509 & $\varepsilon \overline{2} \mathrm{i}$ & 8611 & 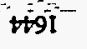 & stit & 0 & $+\angle \overrightarrow{L t}$ & 1991 & $\angle 66 \mathrm{I}$ & $\forall E S I$ & $056 \mathrm{I}$ & $\square 0 \varepsilon$ & $9 z z I$ & $z 8 \varepsilon \mathrm{i}$ & IIt I & 606 & WN-TNS \\
\hline $898^{-}$ & $\angle 9 O I$ & $E L S I$ & $\downarrow Z \varepsilon I^{-}$ & 8191 & $262 \mathrm{I}:$ & $\forall \angle t=-$ & $\mathbf{0}^{-}=$ & stor & $\varepsilon z 8 I$ & I9EI- & +261 & $t u$. & $z L O I$ & HSEL: & E8Et & $9 \varepsilon 8$ & SIA9 \\
\hline $8+2 \pi$ & ż8I & $29 \hat{i}$ & $6 L S Z$ & $9 \pi L$ & $L t s i$ & $+99 \mathrm{t}^{-}$ & sItI & 0 & 825 & $9 i z$ & I9ZI & 8LEI & $\angle 89$ & 809 & 565 & tIZZ & IWW \\
\hline$+592^{3}$ & 9802 & $68 t^{-}$ & $9+0 \varepsilon:$ & $\angle 9 L$ & DIOE & $266 \mathrm{I}$ & $\varepsilon Z 81$ & $825^{-}$ & 0 & 885 & ZSII & $0 \mathrm{LLI} \mathrm{I}^{-}$ & $600 \mathrm{I}$ & SIL & $20 L$ & 0292 & Iddd \\
\hline 2612 & 乙Е9I & $t \varepsilon t$ & +852 & $0 t s$ & zss & tESI & |वहा & $9 L z$ & 885 & 0 & $t z 01$ & $8+Z I$ & 0 & $\tau<\varepsilon$ & $8 \varsigma \varepsilon$ & $85 i 2$ & SLIOOd \\
\hline $\operatorname{tsc} z$ & zوLI & $61+i$ & 6962 & $029^{\circ}$ & $106 i$ & 0561 & t26I & I9ZI : & ZSII & $\$ 201$ & 0 & 499 I & $\$ \angle 8$ & $\$ 89$ & 269 & $0 z \varepsilon \pi$ & Eा|ग्!़d \\
\hline$\varepsilon \mapsto \tau \tau^{-}$ & $8 \overline{0 \varepsilon}$ & $\angle E S I$ & $905 i^{\circ}$ & 8SEI & SttI & toع & $i L i$ & $8 L E I$ & $0 \ddot{I} \angle I$ & $8+2 \mathrm{l}$ & $\$ 99 \mathrm{I}$ & 0 & $0 t 6$ & 9601 & SZII & $60 \mathrm{ZI}$ & ग)นred \\
\hline EOSI & 8521 & $5+8$ & $6 S E Z$ & 895 & $\angle Z E Z$ & 9221 & $z t_{0} t$ & $\angle 89^{\circ}$ & 6001 & $0^{-} \div$ & $D<8$. & 016. & 0 & $+0 \varepsilon$ & 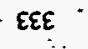 & 5981 & daDd \\
\hline s812 & โรEโ & 996 & $+8 s 2$ & $6 L \bar{\varepsilon}$ & $\varepsilon z \Sigma^{\circ}$ & $28 \varepsilon I$ & कSEI & 809 & SIL & $T \angle \varepsilon$ & $\$ 89^{\circ}$ & 9601 & $\overline{O E}$ & $0^{-1}$ & $\mathrm{OI}$ & IsIz & צ४⿻ \\
\hline$+1 z$ & olti & $\varepsilon S L$ & $+8 \Omega$ & $698:$ & I\$5 & $I I t I$ & E8EI & $56 S^{-}$ & $20 L$ & $8 S E$ & 269 & SZII & $\varepsilon \varepsilon \varepsilon$ & OI & 0 & 0812 & ISIYO \\
\hline 90 & S9EI & ELEC & $6 \varepsilon L$ & $t \operatorname{tb} i$ & $8 \angle 9$ & 606 & $9 \varepsilon 8$ & tizz & 0292 & $8 s i z$ & ozLZ & $60 Z I$ & t98I & ISIZ & 0812 & & SIN \\
\hline $9+L$ & $6 S L I$ & ob 12 & $+00 t$ & IโยZ & $\tau L 6$ & 8911 & 912 & $286 \mathrm{I}$ & $8+t z$ & $986 \mathrm{I}$ & $\angle 192$ & $89 t I$ & $99 L I$ & $8+0 \tau$ & $\angle \operatorname{coz}$ & LIL & JuN \\
\hline $180 \tau$ & I9SI & Ot & $\varepsilon<t \tau$ & 959 & $I t t z$ & $\Sigma Z+I$ & $0 s c 1$ & 28 & $\$ 19$ & $Z S I$ & 1001 & LEII & Itt & $t \varepsilon \varepsilon$ & ธ£Е & $\angle t O Z$ & unow \\
\hline IEOI & $\varepsilon 69$ & 1261 & $\$ 621$ & $\tau \neq L I$ & દ๕ZI & $20 \mathrm{I}$ & 2st & $29 L I$ & $\$ 60 \tau$ & zE9I & $8+0 z$ & 200 & 6IEI & $08+1$ & $605 \mathrm{sI}$ & $\angle 66$ & $N V T$ \\
\hline ZIL & $89+1$ & $90 L t$ & +9 & $O S \angle Z$ & 0 & SEII & 2621 & 52 & tIOE & zSS & 1062 & $\$ 1$ & $\angle Z \Omega Z$ & $\varepsilon \tau s \tau$ & ssc & $8 \angle 9$ & INTI \\
\hline$\varepsilon S L$ & $\cos I$ & $\angle 69 \tau$ & Lt & $16 L 2$ & st & S8II & E8ZІ & $8 \varepsilon S \tau$ & SOOE & $\varepsilon t s \tau$ & St6Z & 361 & $\tau \varepsilon \varepsilon$ & घ9ऽટ & $26 \Omega 2$ & 612 & 787 \\
\hline 0992 & $s$ & $\emptyset \mid \varepsilon$ & $6 L 62$ & 196 & 6562 & gLOZ & $L Z 81$ & 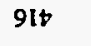 & 162 & 889 & $\varepsilon 6 \varepsilon I$ & $06 \angle 1$ & 6601 & $\$ 88$ & 288 & 9292 & $7 d \forall Y$ \\
\hline$z 9 t t$ & $\$ 01$ & 8101 & $6 \varepsilon 6 \mathrm{I}$ & $\angle 86$ & $068 \mathrm{I}$ & 988 & 9 & 098 & 2611 & OEL & $\varepsilon 6 Z 1$ & 009 & $I t$ & $\varepsilon Z L$ & 6 & 8271 & dכX \\
\hline 2S6 & t 19 & Zह81 & 8611 & $\varepsilon$ \&9I & $t S I t$ & 6 & 2 & $\mathrm{t}$ & $900 \tau$ & $\varepsilon \operatorname{st} I$ & 6561 & . & II & $6 \varepsilon 1$ & $2+1$ & 0 & I8.1] \\
\hline $9+L$ & SLI & 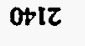 & +001 & Iโยน & $2 L 6$ & 8911 & $9 I L$ & Ir & $8+t \tau$ & $986 \mathrm{I}$ & $\angle 192$ & 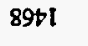 & $99 \mathrm{LI}$ & $800 z$ & $\angle 02$ & . & Igs \\
\hline 2911 & SLIT & $955 c$ & 916 & $L Z L Z$ & $\$ 68$ & $+8 S 1$ & zEI & $86 \varepsilon \bar{C}$ & 5982 & $200 \pi$ & ££О६ & 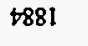 & 812 & $+9+2$ & $\varepsilon 6+\bar{c}$ & octi & IojurH \\
\hline $8+0 z$ & SI & $6 L t$ & $0 \nleftarrow z$ & 029 & $80+7$ & $06 \varepsilon 1$ & 177 & . & SE9 & $\tau$ & 370 & toII & $60 t$ & ZाE & 0 & $\$ 102$ & dWEA \\
\hline $6 £ 81$ & ESI & 609 & $\angle 912$ & +76 & 0017 & OSEI & $\mathrm{cml}$ & ISt & +58 & 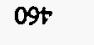 & 1 & 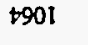 & $2 \pi$ & 919 & 600 & SO8I & ימטt \\
\hline toL2 & 12 & 260 & ડEOE & 160 & $\operatorname{cons}$ & Eolz & 0101 & 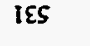 & 60 & 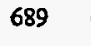 & EI & ti & SIII & I28 & 808 & $0\llcorner 9 \tau$ & TN8 \\
\hline เร£ & 81 & $\angle \Omega$ & $\varepsilon \widetilde{C} L \tau$ & 959 & $169 \ddot{c}$ & EL9I & osst & su & 0 & 6 & 21 & 2 & 969 & 258 & 985 & $\angle 6 \tau \tau$ & ספוג \\
\hline zIIZ & SZ9I & $z L \varepsilon$ & $\nabla Z \Omega$ & orL & 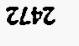 & $45+1$ & $\varepsilon 8 Z I$ & +12 & $9+5$ & 58 & \$901 & 8716 & 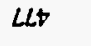 & 216 & 8 & $8 \angle 0$ & a \\
\hline $9+L$ & $6 S \angle 1$ & otic & $t 001$ & III & $\tau \angle 6$ & 8911 & 912 & 2861 & $8 t+z$ & 986I & $\angle 192$ & $89 \$ 1$ & $99 \angle I$ & $3+02$ & $\angle O E$ & $2 / L$ & N-TNY \\
\hline $6+81$ & SOSI & LLS & 0812 & 268 & 8012 & $t \tau \varepsilon I$ & $\angle \mathrm{HOI}$ & $61 t$ & $\tau z 8$ & $82 t$ & 5021 & $8 \varepsilon 01$ & 30 & (1) & $\angle S$ & S181 & $\mathrm{B}-\mathrm{TNV}$ \\
\hline tSSI & IOEI & 606 & 5881 & SLII & $\varepsilon \$ 81$ & očll & $\bar{c} \bar{L}$ & $S L$ & $\langle|\tau|$ & $S S L$ & $18+1$ & TE8 & 629 & 006 & $\angle 88$ & ozs1 & w \\
\hline $\mathfrak{z}$ & $\leqq$ & $\underset{\varliminf}{\underline{E}}$ & $\frac{c}{5}$ & $\pi$ & 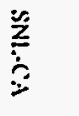 & 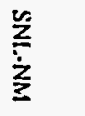 & 춫 & $\geqq$ & 巳 & ஓ & 言 & 竝 & 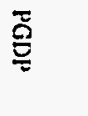 & $\approx$ & 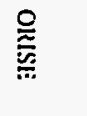 & $\bar{v}$ & \\
\hline
\end{tabular}


Destination: NTS

\begin{tabular}{|c|c|c|c|}
\hline Shipping Site: $\mathrm{k}-25$ & $\begin{array}{l}\text { Total volume } \\
(\mathrm{m} 3 / \mathrm{yr})\end{array}$ & $\begin{array}{c}\text { Total Mass } \\
(\mathrm{kg} / \mathrm{Yr})\end{array}$ & $2.22 E+05$ \\
\hline & $\begin{array}{l}\text { Radionuclide } \\
\mathrm{T} 1-208 \\
\mathrm{~Pb}-212 \\
\mathrm{Bi}-212 \\
\mathrm{Po-212} \\
\mathrm{Po-216} \\
\mathrm{Ra-224} \\
\mathrm{Ra-228} \\
\mathrm{Ac}-228 \\
\mathrm{Th}-228 \\
\mathrm{Th}-231 \\
\mathrm{Th}-232 \\
\mathrm{Th}-234 \\
\mathrm{~Pa}-234 \\
\mathrm{~Pa}-234 \mathrm{~m} \\
\mathrm{U}-235 \\
\mathrm{U}-238\end{array}$ & $\begin{array}{c}\begin{array}{c}\text { Activity } \\
(C i / y r)\end{array} \\
-2 .--0- \\
1.26 \mathrm{E}-06 \\
3.34 \mathrm{E}-06 \\
3.34 \mathrm{E}-06 \\
2.15 \mathrm{E}-06 \\
3.34 \mathrm{E}-06 \\
3.34 \mathrm{E}-06 \\
1.99 \mathrm{E}-05 \\
1.99 \mathrm{E}-05 \\
3.34 \mathrm{E}-06 \\
1.92 \mathrm{E}-05 \\
2.02 \mathrm{E}-04 \\
2.46 \mathrm{E}-02 \\
2.52 \mathrm{E}-06 \\
2.46 \mathrm{E}-02 \\
1.91 \mathrm{E}-05 \\
2.46 \mathrm{E}-02\end{array}$ & \\
\hline Shipping Site: KAPL & $\begin{array}{l}\text { Total volume } \\
(\mathrm{m} 3 / \mathrm{yr})\end{array}$ & $2.11 E+02 \quad \begin{array}{c}\text { Total Mass } \\
(\mathrm{kg} / \mathrm{yr})\end{array}$ & $5.36 \mathrm{E}+05$ \\
\hline & 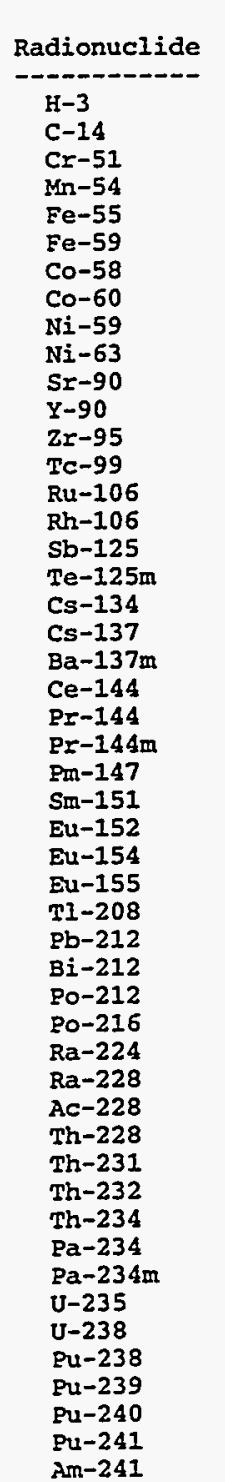 & 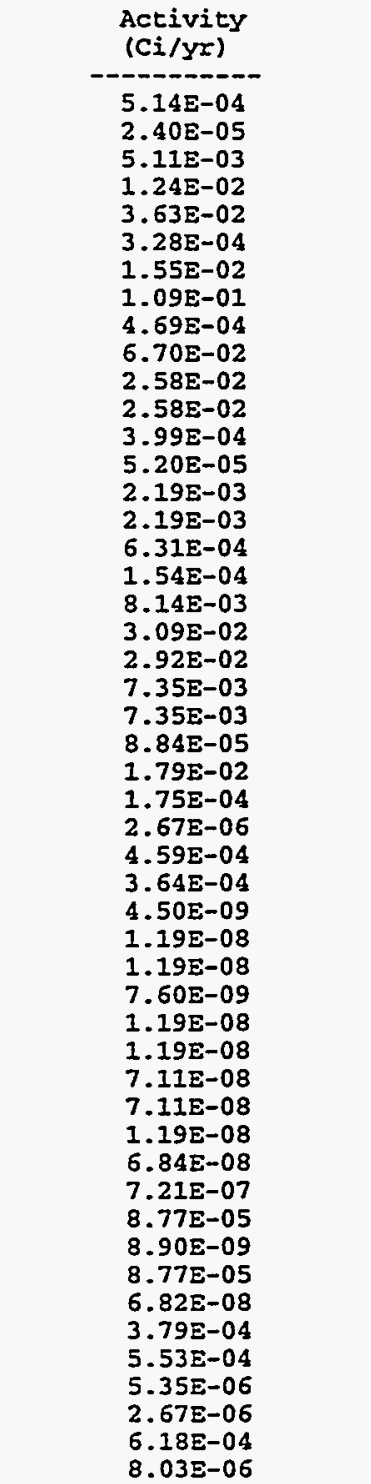 & \\
\hline
\end{tabular}




\begin{tabular}{|c|c|c|c|}
\hline Shipping Site: KCP & $\begin{array}{l}\text { Total Volume } \\
\text { (m3/yr) }\end{array}$ & $\begin{array}{l}\text { Total Mass } \\
(\mathrm{kg} / \mathrm{yr})\end{array}$ & $7.23 E+03$ \\
\hline & $\begin{array}{l}\text { Radionuclide } \\
\mathrm{H}-3 \\
\mathrm{C}-14 \\
\mathrm{Mn}-54 \\
\mathrm{Co}-58 \\
\mathrm{Co}-60 \\
\mathrm{~S} r-90 \\
Y-90 \\
\mathrm{Tc}-99 \\
\mathrm{Cs}-134 \\
\mathrm{Cs}-137 \\
\mathrm{Ba}-137 \mathrm{~m} \\
\mathrm{U}-238\end{array}$ & $\begin{array}{l}\text { Activity } \\
\text { (Ci/yr) } \\
2.70 \mathrm{E}-02 \\
1.44 \mathrm{E}-04 \\
1.62 \mathrm{E}-02 \\
1.50 \mathrm{E}-02 \\
4.34 \mathrm{E}-02 \\
2.04 \mathrm{E}-02 \\
2.04 \mathrm{E}-02 \\
2.89 \mathrm{E}-04 \\
3.37 \mathrm{E}-02 \\
4.44 \mathrm{E}-02 \\
4.20 \mathrm{E}-02 \\
1.76 \mathrm{E}-03\end{array}$ & \\
\hline Shipping Site: IANL & $\begin{array}{l}\text { Total Volume } \\
\text { (m3/yr) }\end{array}$ & $5.50 \mathrm{E}+03 \quad \begin{array}{c}\text { Total Mass } \\
\text { (kg/yr) }\end{array}$ & $4.07 E+07$ \\
\hline & 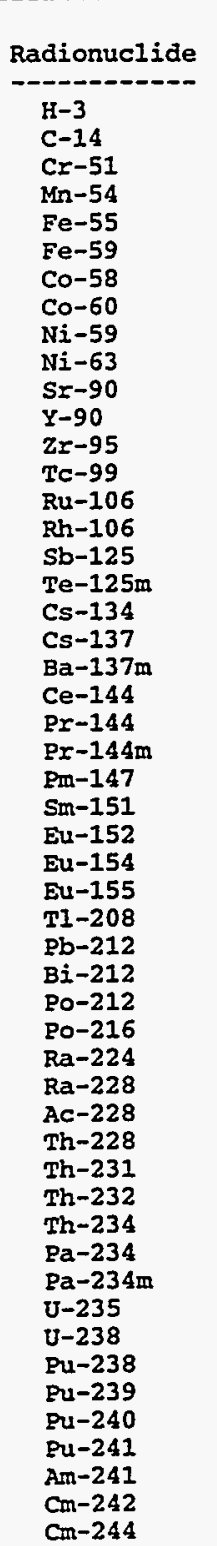 & 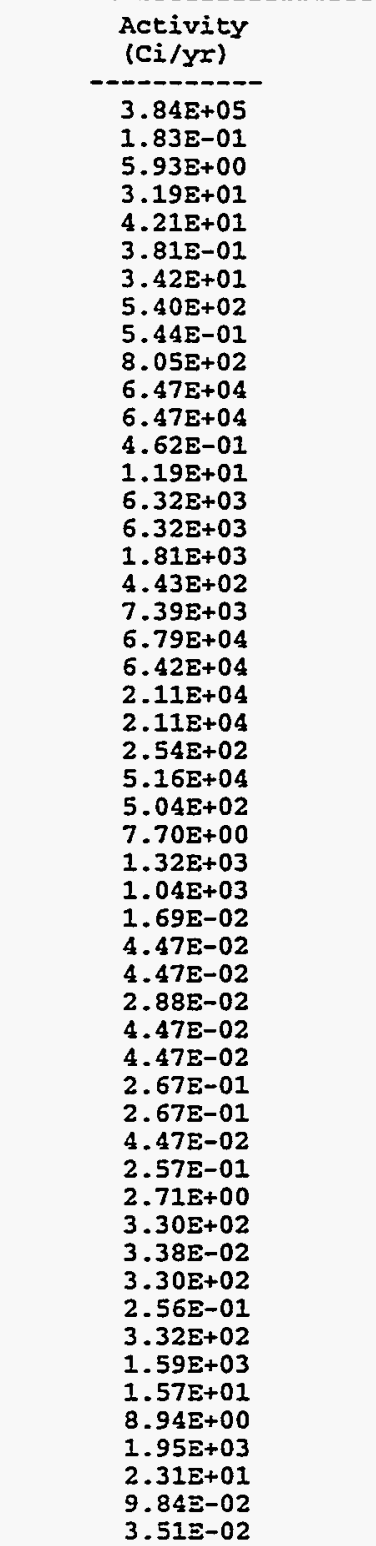 & • \\
\hline
\end{tabular}




\begin{tabular}{|c|c|c|c|}
\hline Shipping Site: LBL & $\begin{array}{l}\text { Total Volume } \\
(\mathrm{m} 3 / \mathrm{yr})\end{array}$ & $\begin{array}{cc}1.26 \mathrm{E}+01 & \begin{array}{c}\text { Total Mass } \\
(\mathrm{kg} / \mathrm{yr})\end{array}\end{array}$ & $3.43 E+04$ \\
\hline & $\begin{array}{l}\text { Radionuclide } \\
\mathrm{H}-3 \\
\mathrm{C}-14 \\
\mathrm{Mn}-54 \\
\mathrm{Co}-58 \\
\mathrm{Co}-60 \\
\mathrm{Sr}-90 \\
\mathrm{Y}-90 \\
\mathrm{Tc}-99 \\
\mathrm{Cs}-134 \\
\mathrm{Cs}-137 \\
\mathrm{Ba}-137 \mathrm{~m} \\
\mathrm{U}-238\end{array}$ & 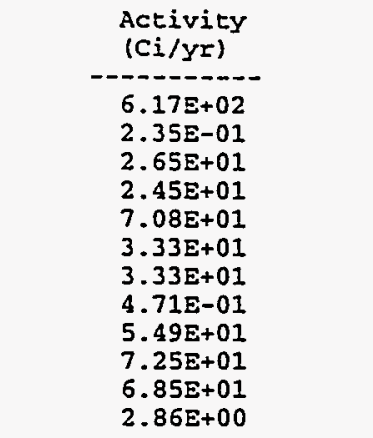 & \\
\hline \multirow[t]{2}{*}{ Shipping Site: LLNL } & $\begin{array}{l}\text { Total Volume } \\
\text { (m3/yr) }\end{array}$ & $2.98 \mathrm{E}+02 \quad \begin{array}{c}\text { Total Mass } \\
(\mathrm{kg} / \mathrm{yr})\end{array}$ & $7.42 \mathrm{E}+05$ \\
\hline & $\begin{array}{l}\text { Radionuclide } \\
\mathrm{H}-3 \\
\mathrm{C}-14 \\
\mathrm{Mn}-54 \\
\mathrm{Co}-58 \\
\mathrm{Co}-60 \\
\mathrm{Ni}-63 \\
\mathrm{Sr}-90 \\
\mathrm{y}-90 \\
\mathrm{Tc}-99 \\
\mathrm{Ru}-106 \\
\mathrm{Rh}-106 \\
\mathrm{Sb}-125 \\
\mathrm{Te}-125 \mathrm{~m} \\
\mathrm{Cs}-134 \\
\mathrm{Cs}-137 \\
\mathrm{Ba}-137 \mathrm{~m} \\
\mathrm{Ce}-144 \\
\mathrm{Pr}-144 \\
\mathrm{Pr}-144 \mathrm{~m} \\
\mathrm{Pm}-147 \\
\mathrm{Sm}-151 \\
\mathrm{Eu}-152 \\
\mathrm{Eu}-154 \\
\mathrm{Eu}-155 \\
\mathrm{U}-238 \\
\mathrm{Pu}-238 \\
\mathrm{Pu}-239 \\
\mathrm{Pu}-240 \\
\mathrm{Pu}-241 \\
\mathrm{Am}-241 \\
\mathrm{Cm}-242 \\
\mathrm{Cm}-244\end{array}$ & \begin{tabular}{c}
$\begin{array}{c}\text { Activity } \\
\text { (Ci/yI) }\end{array}$ \\
\hdashline $2.16 \mathrm{E}+04$ \\
$1.01 \mathrm{E}-03$ \\
$1.14 \mathrm{E}-01$ \\
$1.05 \mathrm{E}-01$ \\
$3.06 \mathrm{E}-01$ \\
$3.19 \mathrm{E}-03$ \\
$4.27 \mathrm{E}-01$ \\
$4.27 \mathrm{E}-01$ \\
$2.07 \mathrm{E}-03$ \\
$2.77 \mathrm{E}-02$ \\
$2.77 \mathrm{E}-02$ \\
$7.96 \mathrm{E}-03$ \\
$1.94 \mathrm{E}-03$ \\
$2.68 \mathrm{E}-01$ \\
$6.09 \mathrm{E}-01$ \\
$5.76 \mathrm{E}-01$ \\
$9.27 \mathrm{E}-02$ \\
$9.27 \mathrm{E}-02$ \\
$1.11 \mathrm{E}-03$ \\
$2.26 \mathrm{E}-01$ \\
$2.21 \mathrm{E}-03$ \\
$3.38 \mathrm{E}-05$ \\
$5.79 \mathrm{E}-03$ \\
$4.59 \mathrm{E}-03$ \\
$1.23 \mathrm{E}-02$ \\
$5.12 \mathrm{E}-02$ \\
$3.44 \mathrm{E}-03$ \\
$1.18 \mathrm{E}-02$ \\
$1.63 \mathrm{E}+00$ \\
$1.69 \mathrm{E}-04$ \\
$9.46 \mathrm{E}-04$ \\
$3.38 \mathrm{E}-04$
\end{tabular} & \\
\hline \multirow[t]{2}{*}{ Shipping Site: MOUND } & $\begin{array}{l}\text { Total Volume } \\
\left(\mathrm{m} 3 / \mathrm{yr}^{\prime}\right)\end{array}$ & $\begin{array}{l}\text { Total Mass } \\
(\mathrm{kg} / \mathrm{yr})\end{array}$ & $9.56 E+06$ \\
\hline & $\begin{array}{l}\text { Radionuclide } \\
\mathrm{H}-3 \\
\mathrm{Pu}-238 \\
\mathrm{Pu}-239 \\
\mathrm{Pu}-240 \\
\mathrm{Pu-241} \\
\mathrm{Am-241} \\
\mathrm{Cm}-242 \\
\mathrm{Cm}-244\end{array}$ & $\begin{array}{c}\begin{array}{c}\text { Activity } \\
\text { (Ci/yr) }\end{array} \\
1.59 \mathrm{E}+00 \\
1.60 \mathrm{E}-01 \\
1.22 \mathrm{E}-02 \\
4.28 \mathrm{E}-02 \\
5.90 \mathrm{E}+00 \\
2.44 \mathrm{E}-04 \\
3.42 \mathrm{E}-03 \\
1.22 \mathrm{E}-03\end{array}$ & \\
\hline
\end{tabular}


A-223

\begin{tabular}{|c|c|c|c|}
\hline Shipping Site: ORISE & $\begin{array}{l}\text { Total Volume } \\
(\mathrm{m} 3 / \mathrm{y} x)\end{array}$ & $\begin{array}{c}\text { Total Mass } \\
(\mathrm{kg} / \mathrm{Y} F)\end{array}$ & $6.91 E+04$ \\
\hline & $\begin{array}{l}\text { Radionuclide } \\
---3---- \\
\mathrm{C}-14 \\
\mathrm{Mn}-54 \\
\mathrm{Co}-58 \\
\mathrm{Co}-60 \\
\mathrm{~S}-90 \\
\mathrm{Y}-90 \\
\mathrm{Tc}-99 \\
\mathrm{Cs}-134 \\
\mathrm{Cs}-137 \\
\mathrm{Ba}-137 \mathrm{~m} \\
\mathrm{Tl}-208 \\
\mathrm{~Pb}-212 \\
\mathrm{Bi}-212 \\
\mathrm{Po}-212 \\
\mathrm{PO}-216 \\
\mathrm{Ra}-224 \\
\mathrm{Ra}-228 \\
\mathrm{Ac}-228 \\
\mathrm{Th}-228 \\
\mathrm{Th}-231 \\
\mathrm{Th}-232 \\
\mathrm{Th}-234 \\
\mathrm{~Pa}-234 \\
\mathrm{~Pa}-234 \mathrm{~m} \\
\mathrm{U}-235 \\
\mathrm{U}-238\end{array}$ & 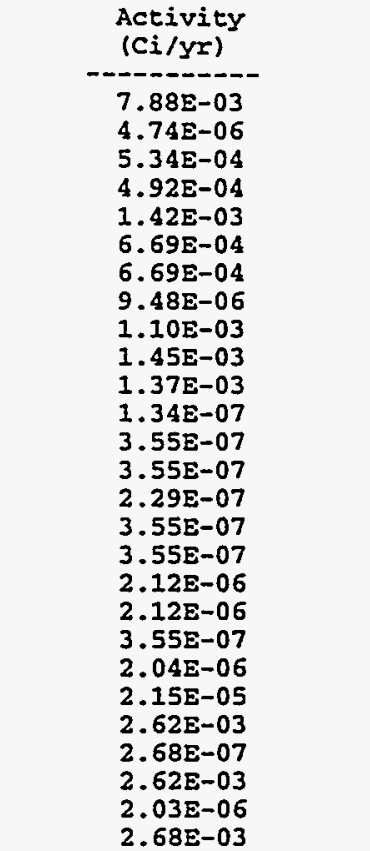 & \\
\hline
\end{tabular}


A-224

\begin{tabular}{|c|c|c|c|}
\hline Shipping Site: ORNL & $\begin{array}{l}\text { Total Volume } \\
\text { (m3/yr) }\end{array}$ & $\begin{array}{l}\text { Total Mass } \\
(\mathrm{kg} / \mathrm{yr})\end{array}$ & $1.32 E+08$ \\
\hline & $\begin{array}{l}\text { Radionuclide } \\
\mathrm{H}-3 \\
\mathrm{C}-14 \\
\mathrm{Cr}-51 \\
\mathrm{Mn}-54 \\
\mathrm{Fe}-55 \\
\mathrm{Fe}-59 \\
\mathrm{Co}-58 \\
\mathrm{Co}-60 \\
\mathrm{Ni}-59 \\
\mathrm{Ni}-63 \\
\mathrm{Sr}-90 \\
\mathrm{Y}-90 \\
\mathrm{Z}--95 \\
\mathrm{Tc}-99 \\
\mathrm{Ru}-106 \\
\mathrm{Rh}-106 \\
\mathrm{Sb}-125 \\
\mathrm{Te}-125 \mathrm{~m} \\
\mathrm{Cs}-134 \\
\mathrm{Cs}-137 \\
\mathrm{Ba}-137 \mathrm{~m} \\
\mathrm{Ce}-144 \\
\mathrm{Pr}-144 \\
\mathrm{Pr}-144 \mathrm{~m} \\
\mathrm{Pm}-147 \\
\mathrm{Sm}-151 \\
\mathrm{Eu}-152 \\
\mathrm{Eu}-154 \\
\mathrm{Eu}-155 \\
\mathrm{~T} 1-208 \\
\mathrm{~Pb}-212 \\
\mathrm{Bi}-212 \\
\mathrm{PO}-212 \\
\mathrm{Po-216} \\
\mathrm{Ra}-224 \\
\mathrm{Ra}-228 \\
\mathrm{Ac}-228 \\
\mathrm{Th}-228 \\
\mathrm{Th}-231 \\
\mathrm{Th}-232 \\
\mathrm{Th}-234 \\
\mathrm{~Pa}-234 \\
\mathrm{~Pa}-234 \mathrm{~m} \\
\mathrm{U}-235 \\
\mathrm{U}-238 \\
\mathrm{~Np}-237 \\
\mathrm{Pu}-238 \\
\mathrm{Pu}-239 \\
\mathrm{Pu}-240 \\
\mathrm{Pu}-241 \\
\mathrm{Am}-241 \\
\mathrm{Cm}-242 \\
\mathrm{Cm}-244 \\
\end{array}$ & 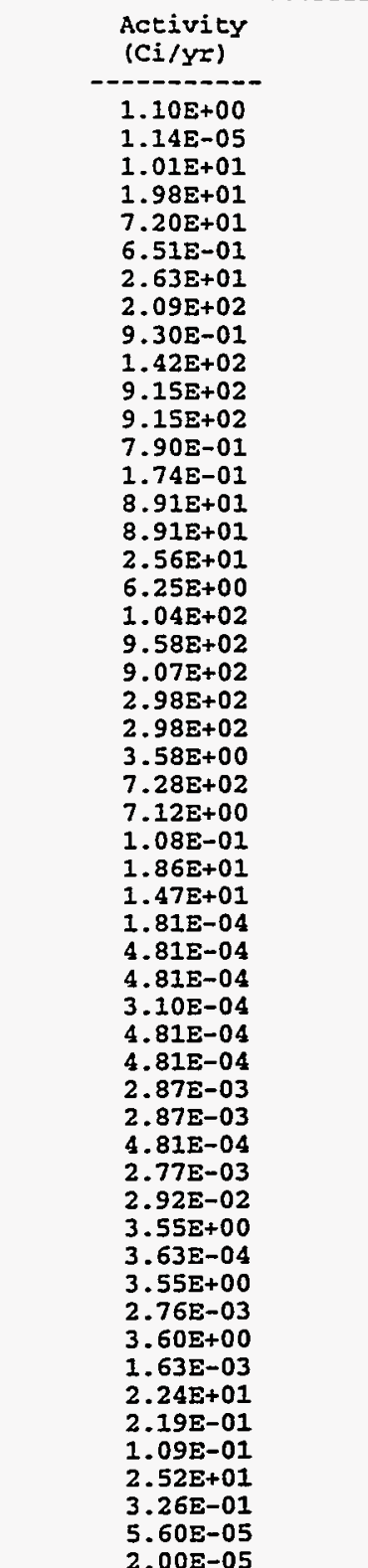 & \\
\hline
\end{tabular}




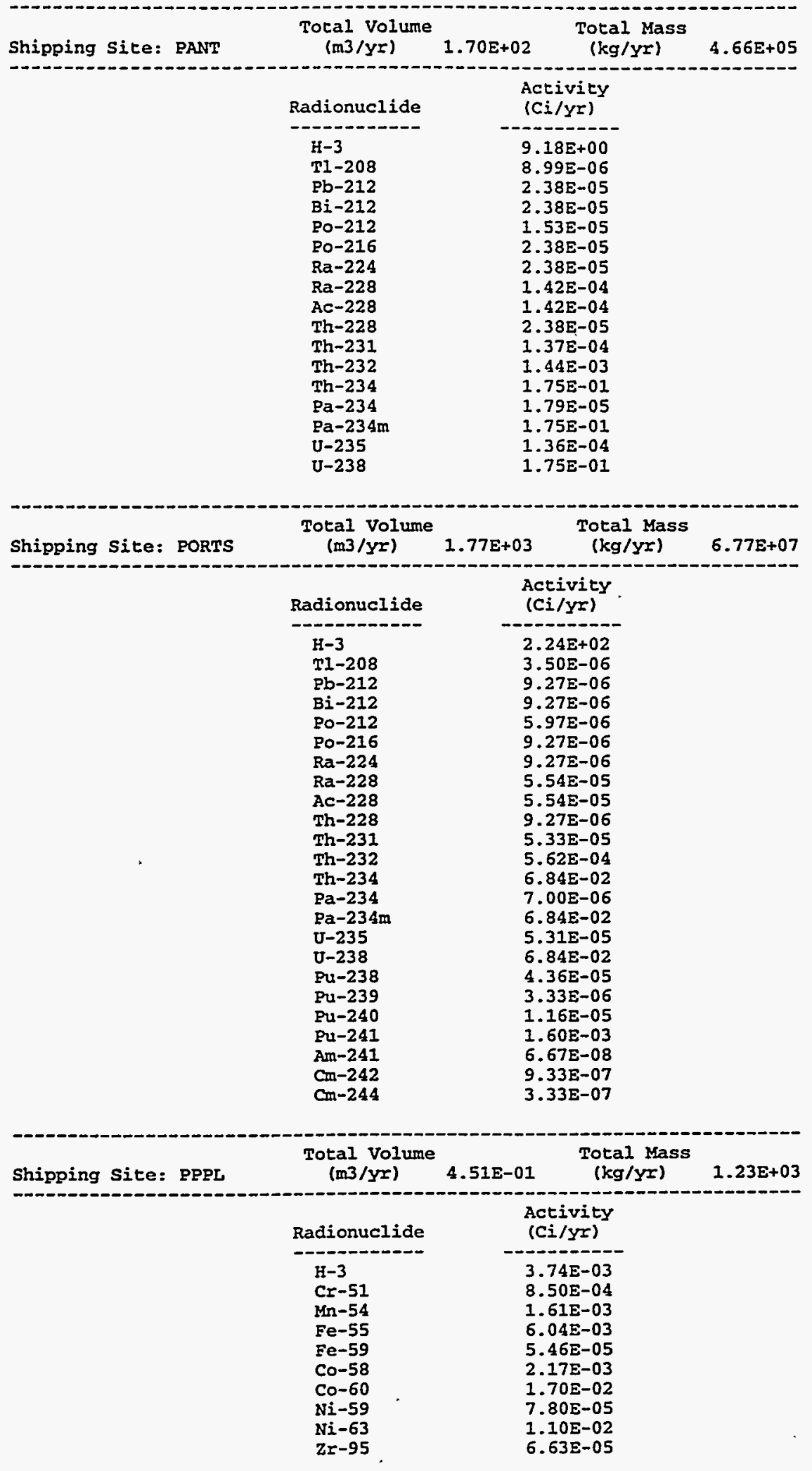




\begin{tabular}{|c|c|c|c|}
\hline Shipping Site: Paducah & $\begin{array}{l}\text { Total Volume } \\
(\mathrm{m} 3 / \mathrm{Yr})\end{array}$ & $\begin{array}{cc}4.04 \mathrm{E}+03 & \begin{array}{c}\text { Total Mass } \\
(\mathrm{kg} / \mathrm{yr})\end{array}\end{array}$ & $1.10 \mathrm{E}+07$ \\
\hline & $\begin{array}{l}\text { Radionuclide } \\
\text { Tc-99 } \\
\text { Tl-208 } \\
\text { Pb-212 } \\
\text { Bi-212 } \\
\text { Po-212 } \\
\text { Po-216 } \\
\text { Ra-224 } \\
\text { Ra-228 } \\
\text { Ac-228 } \\
\text { Th-228 } \\
\text { Th-231 } \\
\text { Th-232 } \\
\text { Th-234 } \\
\text { Pa-234 } \\
\text { Pa-234m } \\
\mathrm{U}-235 \\
\mathrm{U}-238 \\
\mathrm{~Np}-237 \\
\mathrm{Pu}-239\end{array}$ & $\begin{array}{c}\begin{array}{c}\text { Activity } \\
(C i / y r)\end{array} \\
-0.66 E-03 \\
6.80 E-05 \\
7.41 E-05 \\
7.41 E-05 \\
4.78 E-05 \\
7.41 E-05 \\
7.41 E-05 \\
4.43 E-04 \\
4.43 E-04 \\
7.41 E-05 \\
4.27 E-04 \\
4.50 E-03 \\
5.47 E-01 \\
5.60 E-05 \\
5.47 E-01 \\
4.25 E-04 \\
5.47 E-01 \\
4.99 E-03 \\
4.99 E-03\end{array}$ & \\
\hline Shipping Site: RFP & $\begin{array}{l}\text { Total Volume } \\
(\mathrm{m} 3 / y x)\end{array}$ & $\begin{array}{cc}1.27 E+03 & \begin{array}{c}\text { Total Mass } \\
(\mathrm{kg} / \mathrm{Yr})\end{array}\end{array}$ & $6.52 \mathrm{E}+06$ \\
\hline & $\begin{array}{l}\text { Radionuclide } \\
\mathrm{Tl}-208 \\
\mathrm{~Pb}-212 \\
\mathrm{Bi}-212 \\
\mathrm{Po-212} \\
\mathrm{Po-216} \\
\mathrm{Ra}-224 \\
\mathrm{Ra}-228 \\
\mathrm{Ac}-228 \\
\mathrm{Th}-228 \\
\mathrm{Th}-231 \\
\mathrm{Th}-232 \\
\mathrm{Th}-234 \\
\mathrm{~Pa}-234 \\
\mathrm{~Pa}-234 \mathrm{~m} \\
\mathrm{U}-235 \\
\mathrm{U}-238 \\
\mathrm{Pu}-238 \\
\mathrm{Pu}-239 \\
\mathrm{Pu}-240 \\
\mathrm{Pu}-241 \\
\mathrm{Am}-241 \\
\mathrm{Cm}-242 \\
\mathrm{Cm}-244\end{array}$ & $\begin{array}{c}\text { Activity } \\
\text { (Ci/yI) } \\
-3.60 \mathrm{E}-05 \\
9.55 \mathrm{E}-05 \\
9.55 \mathrm{E}-05 \\
6.15 \mathrm{E}-05 \\
9.55 \mathrm{E}-05 \\
9.55 \mathrm{E}-05 \\
5.70 \mathrm{E}-04 \\
5.70 \mathrm{E}-04 \\
9.55 \mathrm{E}-05 \\
5.49 \mathrm{E}-04 \\
5.79 \mathrm{E}-03 \\
7.04 \mathrm{E}-01 \\
7.21 \mathrm{E}-05 \\
7.04 \mathrm{E}-01 \\
5.47 \mathrm{E}-04 \\
7.04 \mathrm{E}-01 \\
5.56 \mathrm{E}-01 \\
4.24 \mathrm{E}-02 \\
1.48 \mathrm{E}-01 \\
2.04 \mathrm{E}+01 \\
8.48 \mathrm{E}-04 \\
1.18 \mathrm{E}-02 \\
4.24 \mathrm{E}-03\end{array}$ & \\
\hline Shipping Site: RMI & $\begin{array}{l}\text { Total Volume } \\
\text { (m3/yr) }\end{array}$ & $\begin{array}{cc}\text { Total Mass } \\
(\mathrm{kg} / \mathrm{yr})\end{array}$ & $1.45 E+07$ \\
\hline & $\begin{array}{l}\text { Radionuclide } \\
\mathrm{T} 1-208 \\
\mathrm{~Pb}-212 \\
\mathrm{Bi}-212 \\
\mathrm{PO}-212 \\
\mathrm{PO}-216 \\
\mathrm{Ra}-224 \\
\mathrm{Ra}-228 \\
\mathrm{Ac}-228 \\
\mathrm{Th}-228 \\
\mathrm{Th}-231 \\
\mathrm{Th}-232 \\
\mathrm{Th}-234 \\
\mathrm{~Pa}-234 \\
\mathrm{~Pa}-234 \mathrm{~m} \\
\mathrm{U}-235 \\
\mathrm{U}-238\end{array}$ & $\begin{array}{c}\begin{array}{c}\text { Activity } \\
(C i / y r)\end{array} \\
-3.38 \mathrm{E}-07 \\
8.96 \mathrm{E}-07 \\
8.96 \mathrm{E}-07 \\
5.77 \mathrm{E}-07 \\
8.96 \mathrm{E}-07 \\
8.96 \mathrm{E}-07 \\
5.35 \mathrm{E}-06 \\
5.35 \mathrm{E}-06 \\
8.96 \mathrm{E}-07 \\
5.15 \mathrm{E}-06 \\
5.43 \mathrm{E}-05 \\
6.61 \mathrm{E}-03 \\
6.77 \mathrm{E}-07 \\
6.61 \mathrm{E}-03 \\
5.13 \mathrm{E}-06 \\
6.61 \mathrm{E}-03\end{array}$ & \\
\hline
\end{tabular}


A. 227

\begin{tabular}{|c|c|c|c|}
\hline Shipping Sice: SLAC & $\begin{array}{l}\text { Total Volume } \\
(\mathrm{m} 3 / \mathrm{yr})\end{array}$ & $\begin{array}{l}\text { Total Mass } \\
(\mathrm{kg} / \mathrm{yr})\end{array}$ & $7.45 \mathrm{E}+05$ \\
\hline & $\begin{array}{l}\text { Radionuclide } \\
\mathrm{Cr}-51 \\
\mathrm{Mn}-54 \\
\mathrm{Fe}-55 \\
\mathrm{Fe}-59 \\
\mathrm{Co}-58 \\
\mathrm{Co}-60 \\
\mathrm{Ni}-59 \\
\mathrm{Ni}-63 \\
\mathrm{Z}-95\end{array}$ & $\begin{array}{c}\begin{array}{c}\text { Activity } \\
\text { (Ci/yY) }\end{array} \\
3.36 \mathrm{E}-03 \\
7.36 \mathrm{E}-03 \\
2.74 \mathrm{E}-02 \\
2.48 \mathrm{E}-04 \\
9.88 \mathrm{E}-03 \\
7.74 \mathrm{E}-02 \\
3.54 \mathrm{E}-04 \\
5.04 \mathrm{E}-02 \\
3.01 \mathrm{E}-04\end{array}$ & \\
\hline Shipping Sice: SNLA & $\begin{array}{l}\text { Total Volume } \\
(\mathrm{m} 3 / \mathrm{yr})\end{array}$ & $\begin{array}{c}\text { Total Mass } \\
(\mathrm{kg} / \mathrm{yr})\end{array}$ & $3.24 \mathrm{E}+05$ \\
\hline & $\begin{array}{l}\text { Radionuclide } \\
\mathrm{H}-3 \\
\mathrm{Co}-60 \\
\mathrm{Ni}-63 \\
\mathrm{Sr}-90 \\
\mathrm{Y}-90 \\
\mathrm{Tc}-99 \\
\mathrm{Ru}-106 \\
\mathrm{Rh}-106 \\
\mathrm{Sb}-125 \\
\mathrm{Te}-125 \mathrm{~m} \\
\mathrm{Cs}-134 \\
\mathrm{Cs}-137 \\
\mathrm{Ba}-137 \mathrm{~m} \\
\mathrm{Ce}-144 \\
\mathrm{Pr}-144 \\
\mathrm{Pr}-144 \mathrm{~m} \\
\mathrm{Pm}-147 \\
\mathrm{Sm}-151 \\
\mathrm{Eu}-152 \\
\mathrm{Eu}-154 \\
\mathrm{Eu}-155 \\
\mathrm{Pu}-238 \\
\mathrm{Pu}-239 \\
\mathrm{Pu}-240 \\
\mathrm{Pu}-241 \\
\mathrm{Am}-241 \\
\mathrm{Cm}-242 \\
\mathrm{Cm}-244\end{array}$ & $\begin{array}{c}\text { Activity } \\
\text { (Ci/yr) } \\
-1---- \\
3.00 E+00 \\
2.85 E-05 \\
5.67 E-05 \\
5.04 E-03 \\
5.04 E-03 \\
9.00 E-07 \\
4.92 E-04 \\
4.92 E-04 \\
1.41 E-04 \\
3.45 E-05 \\
5.72 E-04 \\
5.28 E-03 \\
4.99 E-03 \\
1.64 E-03 \\
1.64 E-03 \\
1.98 E-05 \\
4.02 E-03 \\
3.93 E-05 \\
6.00 E-07 \\
1.02 E-04 \\
8.16 E-05 \\
7.87 E-02 \\
6.00 E-03 \\
2.10 E-02 \\
2.89 E+00 \\
1.21 E-04 \\
1.68 E-03 \\
6.00 E-04\end{array}$ & . \\
\hline Shipping Site: SNLL & $\begin{array}{l}\text { Total Volume } \\
(\mathrm{m} 3 / \mathrm{y})\end{array}$ & $\begin{array}{cc}3.36 \mathrm{E}+01 & \begin{array}{c}\text { Total Mass } \\
(\mathrm{kg} / \mathrm{y} x)\end{array}\end{array}$ & $9.17 E+04$ \\
\hline & $\begin{array}{l}\text { Radionuclide } \\
\mathrm{H}-3 \\
\mathrm{Tl}-208 \\
\mathrm{~Pb}-212 \\
\mathrm{Bi}-212 \\
\mathrm{Po-212} \\
\mathrm{Po-216} \\
\mathrm{Ra}-224 \\
\mathrm{Ra}-228 \\
\mathrm{Ac}-228 \\
\mathrm{Th}-228 \\
\mathrm{Th}-231 \\
\mathrm{Th}-232 \\
\mathrm{Th}-234 \\
\mathrm{~Pa}-234 \\
\mathrm{~Pa}-234 \mathrm{~m} \\
\mathrm{U}-235 \\
\mathrm{U}-238\end{array}$ & 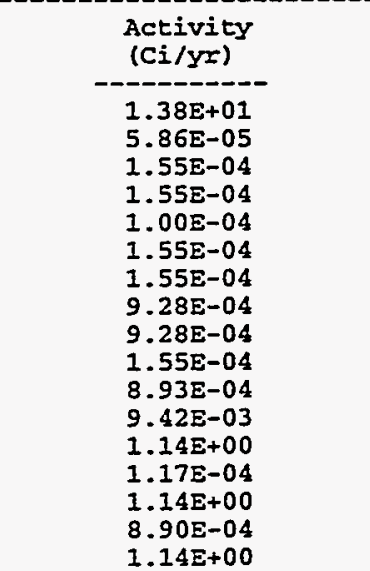 & \\
\hline
\end{tabular}




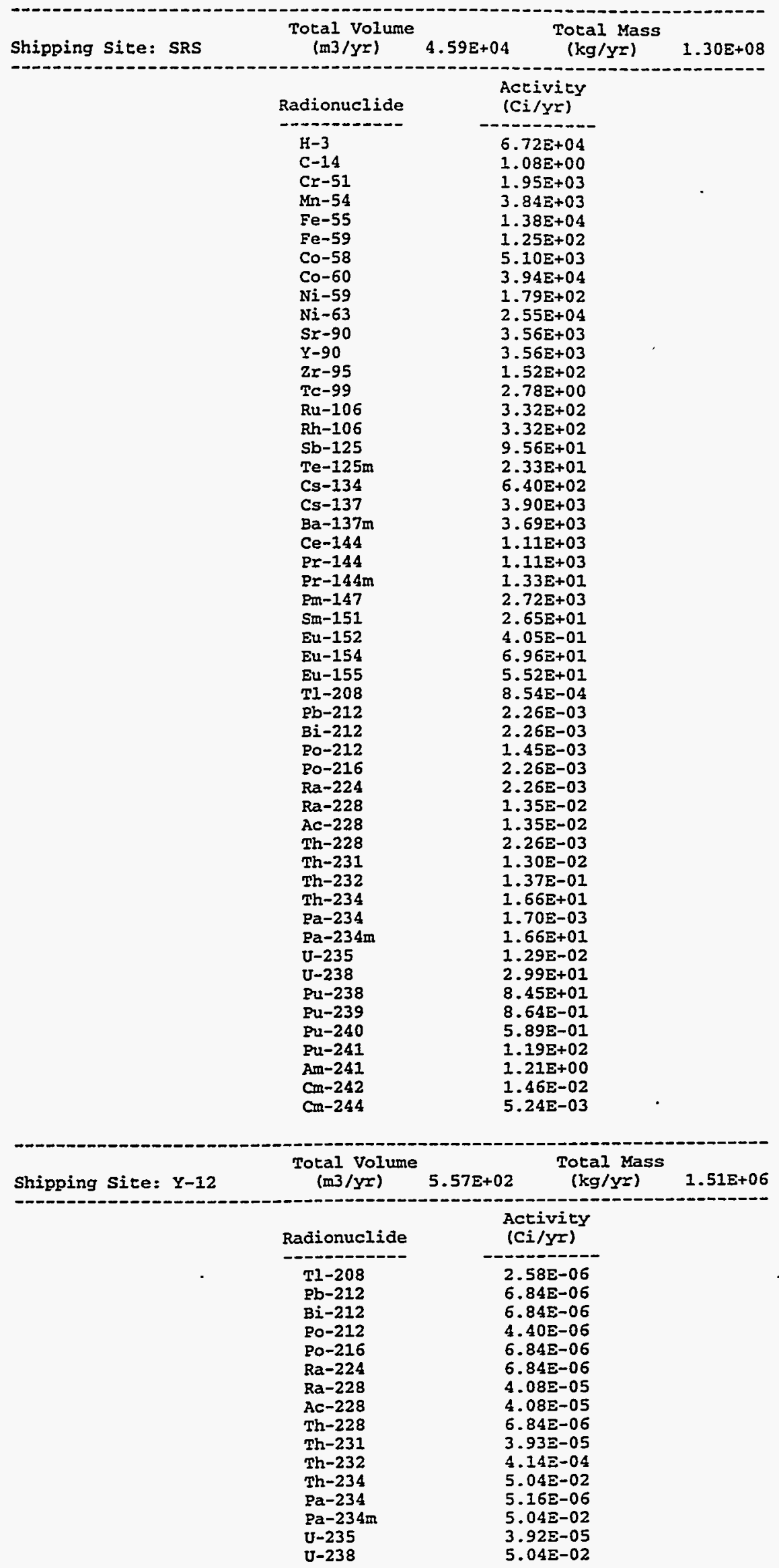


Destination: ORNL

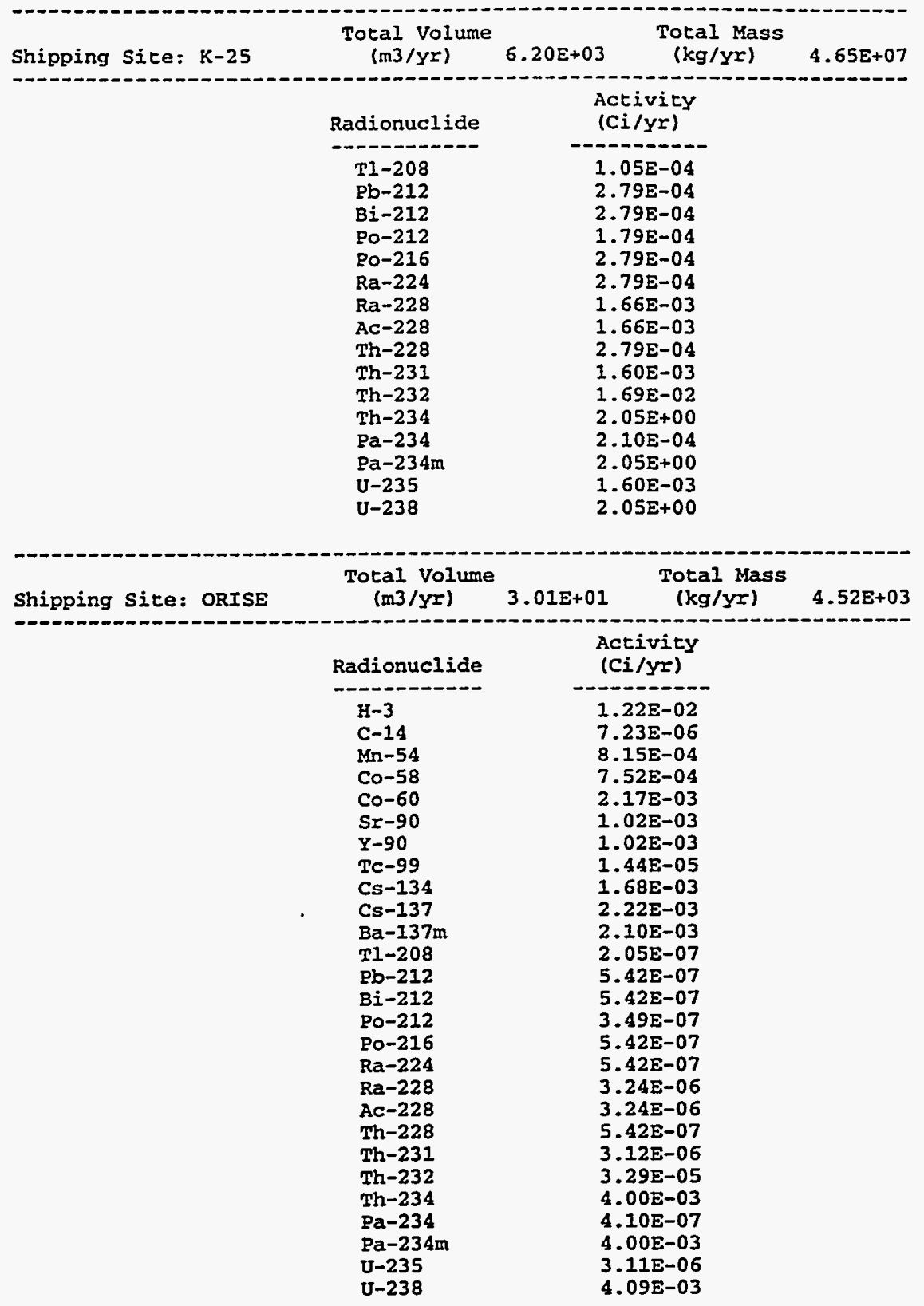


TABLE 3.2 Rail Route Distances (mi) between Major DOE Sites ${ }^{\mathrm{a}}$

\begin{tabular}{|c|c|c|c|c|c|c|c|c|c|c|c|c|c|c|c|c|c|c|}
\hline & $\frac{1}{3}$ & $\stackrel{\ddot{\dot{z}}}{\dot{\vec{z}}}$ & $\begin{array}{l}\frac{3}{3} \\
\frac{1}{z} \\
\end{array}$ & ป્త & 竞 & 岂 & 泀 & 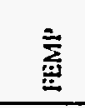 & $\begin{array}{l}\text { 롤 } \\
\text { 总 } \\
\end{array}$ & 岕 & $\underline{\underline{\underline{a}}}$ & $\hat{\bar{y}}$ & 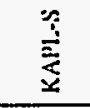 & $\vec{\exists}$ & $\stackrel{\bar{z}}{\Xi}$ & $\underset{\underline{z}}{\dot{z}}$ & $\overrightarrow{\underline{\Xi}}$ & 䓂 \\
\hline Ames & 0 & 329 & 1242 & 700 & 823 & 1365 & 291 & 717 & 1788 & 1242 & 1187 & 275 & 1126 & 1873 & 2018 & 1124 & 715 & 1242 \\
\hline ANL-E & 329 & 0 & 1655 & 401 & 518 & 1066 & 49 & 412 & 2201 & 1655 & 1351 & 439 & 827 & 2549 & 2506 & 1288 & 416 & 1655 \\
\hline ANL-W & 1242 & 1655 & 0 & 1942 & 2133 & 2607 & 1533 & 1907 & 658 & 0 & 1247 & 1238 & 2468 & 1102 & 1100 & 1179 & 1926 & 0 \\
\hline $\mathrm{BCL}$ & 700 & 401 & 1942 & 0 & 280 & 855 & 427 & 135 & 2488 & 1942 & 1759 & 753 & 615 & 2573 & 2718 & 1696 & 65 & 1942 \\
\hline Bertis & 823 & 518 & 2133 & 280 & 0 & 772 & 543 & 475 & 2611 & 2133 & 1857 & 943 & 533 & 2696 & 2840 & 1794 & 345 & 2133 \\
\hline BNL & 1365 & 1066 & 2607 & 855 & 772 & o & 1088 & 984 & 3153 & 2607 & 2414 & 1518 & 239 & 3238 & 3383 & 2351 & 920 & 2607 \\
\hline Fermi & 291 & 49 & 1533 & 427 & 543 & 1088 & 0 & 41 & 1971 & 1533 & 1356 & 453 & 853 & 2343 & 2341 & 1405 & 443 & 1533 \\
\hline FEMP & 717 & 412 & 1907 & 135 & 475 & 984 & 441 & 0 & 2505 & 1907 & 1751 & 717 & 745 & 2590 & 2735 & 1688 & 69 & 1907 \\
\hline Hanford & 1788 & 2201 & 658 & 2488 & 2611 & 3153 & 1971 & 2505 & 0 & 658 & 1793 & 1784 & 2914 & 986 & 973 & 1725 & 2472 & 658 \\
\hline INEL & 1242 & 1655 & 0 & 1942 & 2133 & 2607 & 1533 & 1907 & 658 & 0 & 1247 & 1238 & 2468 & 1102 & 1100 & 1179 & .1926 & 0 \\
\hline TRI & 1187 & 1351 & 1247 & 1759 & 1857 & 2414 & 1356 & 1751 & 1793 & 1247 & 0 & 932 & 2177 & 1266 & 1222 & 104 & 1767 & 1247 \\
\hline $\mathrm{KCP}$ & 275 & 439 & 1238 & 753 & 943 & 1518 & 453 & 717 & 1784 & $1238^{\circ}$ & 932 & 0 & $1250^{\circ}$ & 2016 & 2013 & 869 & 708 & 1238 \\
\hline KAPL & 1126 & 827 & 2468 & 615 & 533 & 239 & 853 & 745 & 2914 & 2468 & 217 & 1250 & 0 & 2999 & 3144 & 2122 & 680 & 2468 \\
\hline LBL & 1873 & 2549 & 1102 & 2573 & 2696 & 3238 & 2343 & 2590. & 986 & 1102 & 1266 & 2016 & 2999 & 0 & 46 & 1354 & 2717. & 1102 \\
\hline LLNL & 2018 & 2506 & 1100 & 2718 & 2840 & 3383 & 2341 & 2735 & 973 & 1100 & 1222 & 2013 & 3144 & 46 & 0 & 1326 & 2695 & 1100 \\
\hline LANL & 1124 & 1288 & 1179 & 1696 & 1794 & 2351 & 1405 & 1688 & 1725 & 1179 & $=104$ & $869-$ & 2122 & 1354 & 1326 & 0 & 1704 & 1179 \\
\hline Mound & 715 & 416 & 1926 & 65 & 345 & 920 & 443 & 69 & 2472 & 1926 & 1767 & 708 & 680 & 2717 & 2695 & 1704 & 0 & 1926 \\
\hline NRF & 1242 & 1655 & 0 & 1942 & 2133 & 2607 & .1533 & 1907 & 658 & 0 : & 1247 & $1238^{-}$ & 2468 & 1102 & 1100 & 1179 . & $=1926$ & $\therefore 0$ \\
\hline NTS & 1674 & 2348 & 756 & 2374 & 2496 & 3039 & 1997 & $239 !$ & 1302 & 756 & 1065 & 1670. & 2800 & 860. & 1370 & 1169 & 2386 & 756 \\
\hline ORISE & 956 & 651 & 2099 & 366 & 714 & 1221 & $679=$ & $\therefore 31$ & $2644^{\circ}$ & -2099 & $1989^{\circ}$ & 002 & $=.981$ & 2890 & 2868 & $1926 \ldots$ & . 301 & 2099: \\
\hline ORR & 954 & 649 & 2055 & 393 & 903 & 1152 & 682 & 358 & 2601 & 2055 & 1749 & 838 & 957 & 2686 & 2831 & 1686 & 328 & 2055 \\
\hline PGDP & 646 & 390 & 1699 & -581. & 010 & 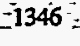 & 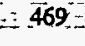 & $+\infty$ & - & 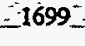 & $=1539$ & $\therefore 482$. & 1106 & 2490 & 2469 & 1476 & $\pi 564$ & $1699=$ \\
\hline Pantex & 809 & 972 & 1141 & 1381 & 1479 & 2035. & 977 & 1373 & 1686 & 1141 & 379 & 554 & 1807 & 1561 & 1534 & 483 & 1396 & 1141 \\
\hline Pineilas & 1623 & 1319 & 2721 & 1151 & 1293 & 1585 & 1344 & $=1116$ & -3267 & 2721 & 2079 & 1503 & 1390 & 3278 & 3491 & $2183=$ & $1085^{\circ}$ & 2721 \\
\hline PORTS & 727 & +22 & 1975 & 91 & 429. & -921 & 451 & 207 & 2515 & 1975 & 1761 & 758 & 681 & 2767 & 2745 & 1698 & 156 & 1975 \\
\hline PPPL" & 1197 & 898 & 2507 & 655 & 400 & 410 & $\therefore 24$ & 938 & 2985 & 2507 & 2248 & $1289^{-}$ & $214^{-}$ & 3298 & 3276 & 2186 & 719 & 2507. \\
\hline $\mathbf{R M I}$ & 717 & 418 & 2060 & 207 & 136 & 648 & 445 & 337 & 2505 & 2060 & 1769 & 842 & 408 & 2851 & 2829 & 1706. & 272. & 2060 . \\
\hline RFETS & 782 & 1194 & 738 & 1502: & 1692 & 2266 & 1016 & 1466 & 1284 & .738 & 572 & 778 & 2020 & -1320 & $\therefore 1394$ & $504=$ & $=1485$ & 738 \\
\hline SNL_NM & 1187 & 1351 & 1247 & 1759 & 1857 & 241 & 1356 & 175 & 1793 & 124 & 0 & 932 & 2177 & 1266 & $=1222$ & 104 & 1767 & 1247 \\
\hline SI & 018 & 2506 & 1100. & 2718 & Pes & 3383 & 23 & 138 & $=973$ & 1100 & 1222 & 2013 & 3144 & -46 & $=0$ & 1326 & 2695 & 1100 \\
\hline SRS & 1281 & 976 & 2407 & 740 & 947 & 1239 & 1001 & 774 & 2953 & 2407 & 2315 & 1161 & 1044 & 3192 & $=3183$ & 2252. & 744 & 2407 \\
\hline SLAC & 1924 & 2536 & 1160 & 2947 & 2746 & 3289 & 2393 & 2641 & 1036 & -1160 & 1253 & 2073 & $3050^{\circ}$ & $\because-56$ & $\therefore 60$ & 1357. & $=2930$ & 160 \\
\hline WVDP & 881 & 579 & 2123 & 370 . & 244 & 549 & 603 & $63 !$ & 2669 & 2123 & 1929 & 1033 & 309 & 2773 & 2898 & 1866 & 562 & 123 \\
\hline WIPP & 1115 & 1279 & 1447 & 1688 & 1785 & 2342 & 1284 & 1679 & 1993 & $144 \overline{7}$ & $=477$. & 861 & 2114 & 1660 & 1633 & 581 & 1703 & 447 \\
\hline $\mathrm{YM}$ & 1674 & 2348 & 756 & 2374 & 2496 & 3039 & 1997 & 2391 & 1302 & 756 & 1065 & 1670 & 2800 & 860 & 1370 & 1169 & 2386 & 756 \\
\hline
\end{tabular}


A-230

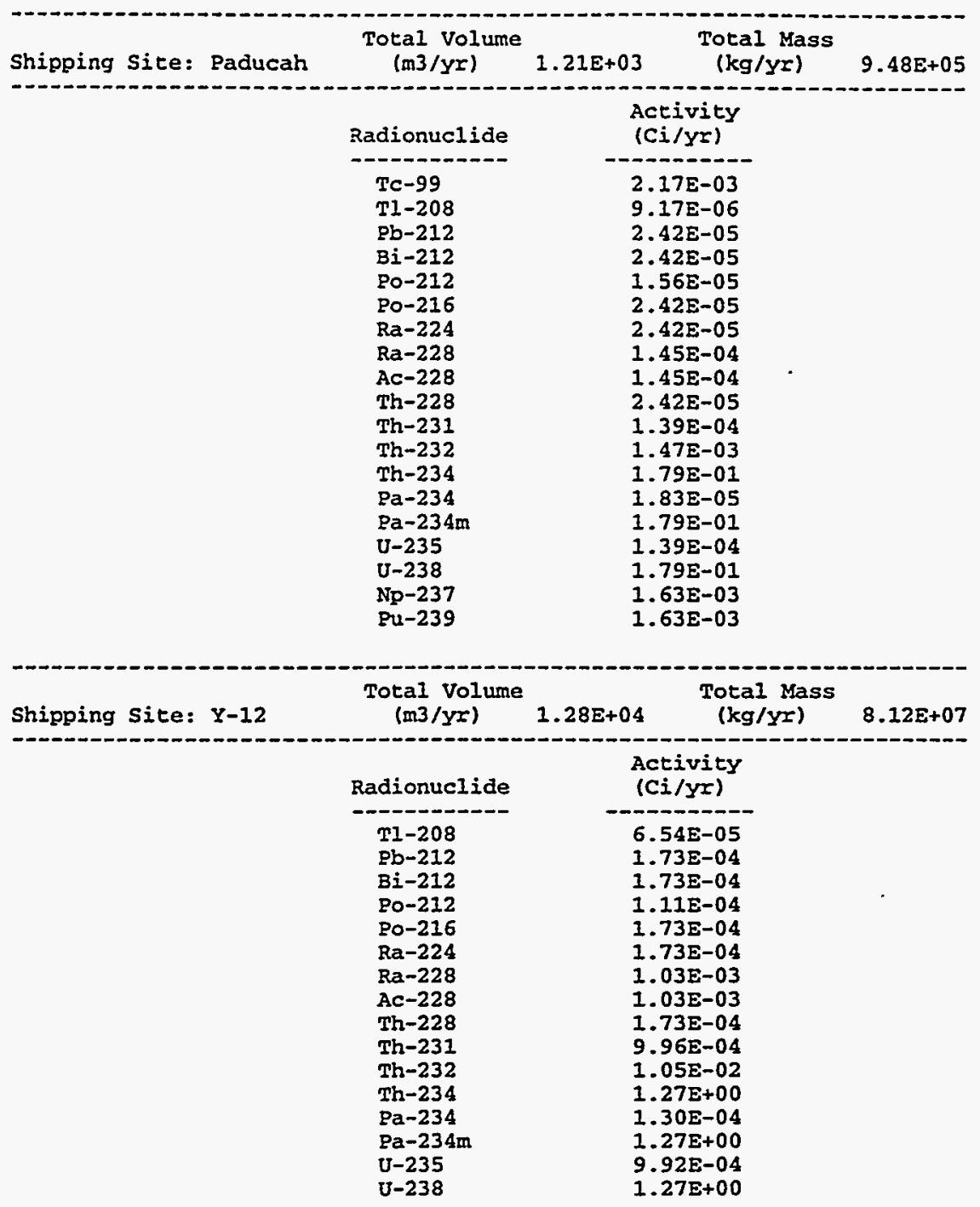


Destination: PORTS

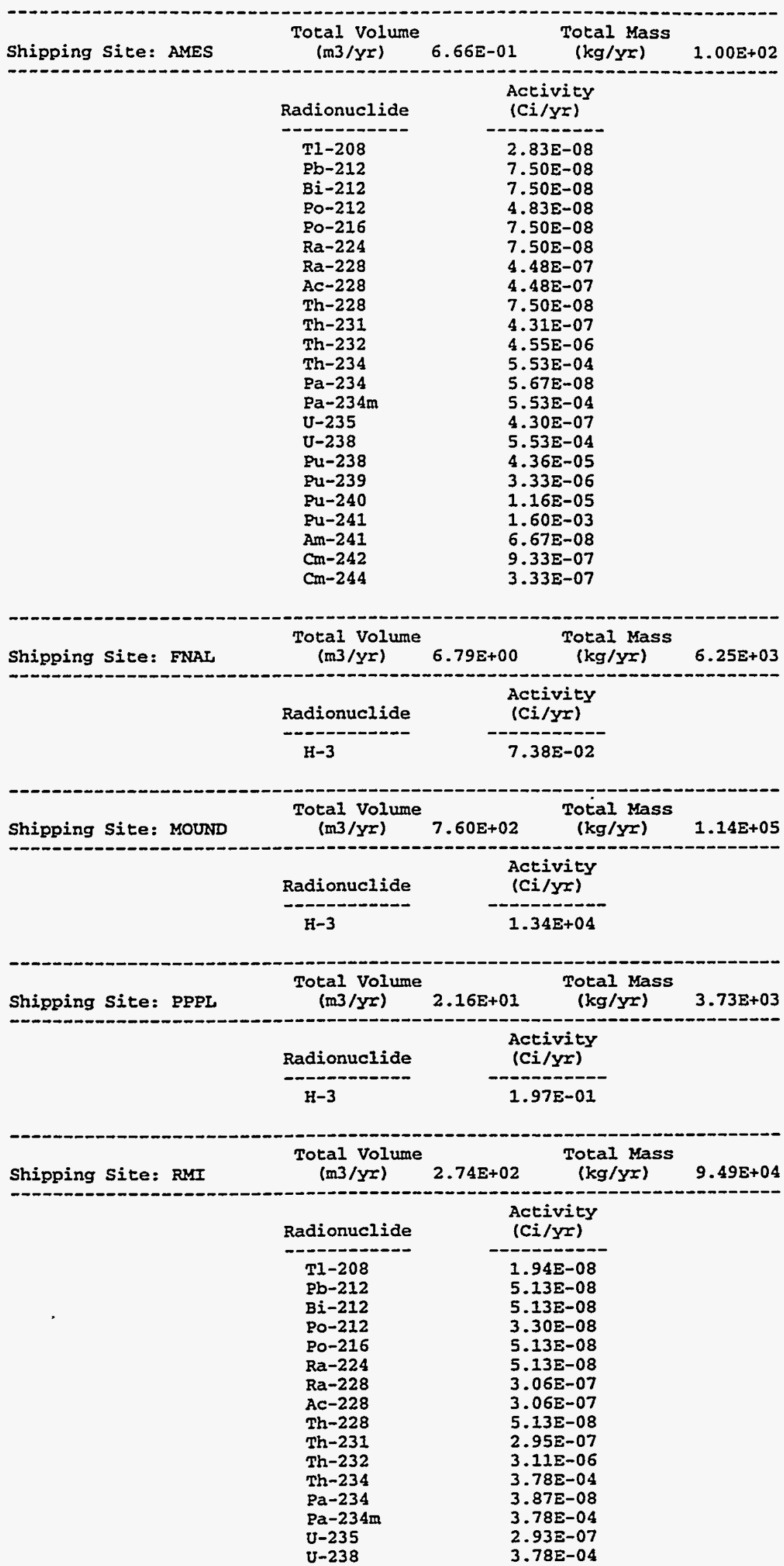


Destination: SRS

\begin{tabular}{|c|c|c|c|}
\hline Shipping Site: Pinellas & $\begin{array}{l}\text { Total Volume } \\
(\mathrm{m} 3 / \mathrm{yr})\end{array}$ & $\begin{array}{c}\text { Total Mass } \\
(\mathrm{kg} / \mathrm{yr})\end{array}$ & $1.91 \mathrm{E}+04$ \\
\hline & Radionuclide & $\begin{array}{l}\text { Activicy } \\
\text { (Ci/yr) } \\
1.99 \mathrm{E}+04\end{array}$ & ' \\
\hline
\end{tabular}




\section{A.26 WM LLW CENTRALIZED 4 ALTERNATIVE (CASE 14A): ACTIVATED METALS}

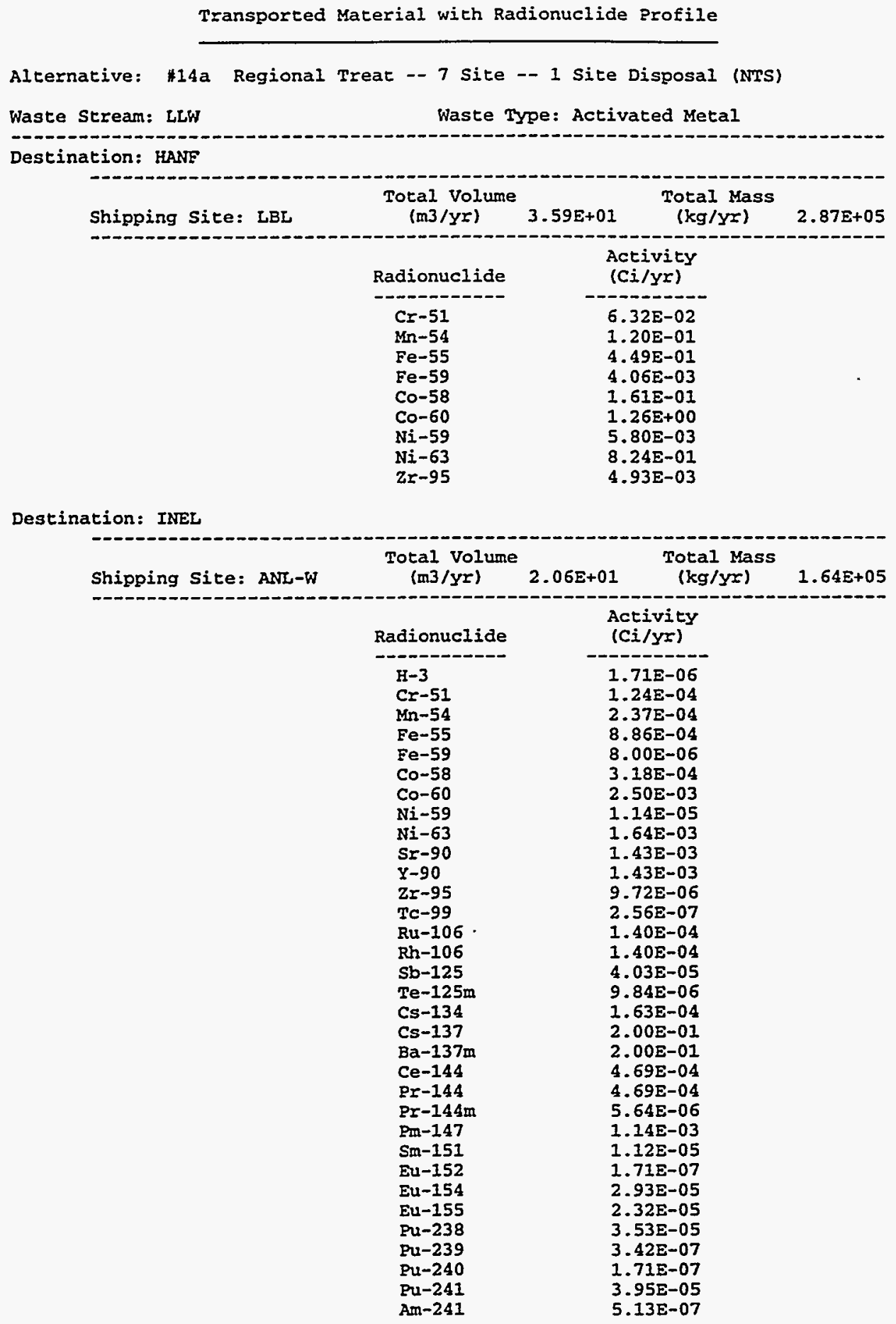


A-234

\begin{tabular}{|c|c|c|c|}
\hline Shipping Site: NRF & $\begin{array}{c}\text { Total Volume } \\
\left(\mathrm{m} 3 / \mathrm{yr}^{\mathrm{r}}\right)\end{array}$ & $\begin{array}{c}\text { Total Mass } \\
(\mathrm{kg} / \mathrm{YI})\end{array}$ & $9.40 \mathrm{E}+06$ \\
\hline & $\begin{array}{l}\text { Radionuclide } \\
\mathrm{Cr}-51 \\
\mathrm{Mn}-54 \\
\mathrm{Fe}-55 \\
\mathrm{Fe}-59 \\
\mathrm{Co}-58 \\
\mathrm{Co}-60 \\
\mathrm{Ni}-59 \\
\mathrm{Ni}-63 \\
\mathrm{Zr}-95\end{array}$ & \begin{tabular}{c}
$\begin{array}{c}\text { Activity } \\
\text { (Ci/yr) }\end{array}$ \\
\hdashline $2.31 \mathrm{E}+03$ \\
$4.40 \mathrm{E}+03$ \\
$1.64 \mathrm{E}+04$ \\
$1.48 \mathrm{E}+02$ \\
$5.91 \mathrm{E}+03$ \\
$4.63 \mathrm{E}+04$ \\
$2.12 \mathrm{E}+02$ \\
$3.01 \mathrm{E}+04$ \\
$1.80 \mathrm{E}+02$
\end{tabular} & \\
\hline
\end{tabular}

Destination: LANL

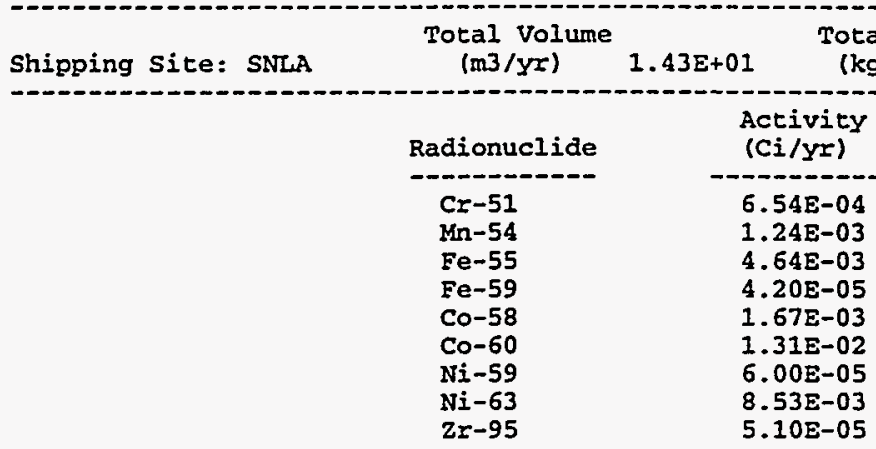

Destination: NTS

\begin{tabular}{|c|c|c|c|}
\hline Shipping Site: HANE & $\begin{array}{l}\text { Total Volume } \\
\text { (m3/yr) }\end{array}$ & $\begin{array}{c}\text { Total Mass } \\
(\mathrm{kg} / \mathrm{yr})\end{array}$ & $9.57 E+05$ \\
\hline & Radionuclide & $\begin{array}{l}\text { Activity } \\
\text { (Ci/yr). }\end{array}$ & \\
\hline & $\begin{array}{l}\mathrm{Cr}-51 \\
\mathrm{Mn}-54 \\
\mathrm{Fe}-55 \\
\mathrm{Fe}-59 \\
\mathrm{Co}-58 \\
\mathrm{Co}-60 \\
\mathrm{Ni}-59 \\
\mathrm{Ni}-63 \\
\mathrm{Zr}-95\end{array}$ & $\begin{array}{l}3.00 \mathrm{E}+01 \\
5.72 \mathrm{E}+01 \\
2.13 \mathrm{E}+02 \\
1.93 \mathrm{E}+00 \\
7.68 \mathrm{E}+01 \\
6.02 \mathrm{E}+02 \\
2.75 \mathrm{E}+00 \\
3.92 \mathrm{E}+02 \\
2.34 \mathrm{E}+00\end{array}$ & \\
\hline
\end{tabular}


A-235

\begin{tabular}{|c|c|c|c|}
\hline Shipping Sice: INEL & $\begin{array}{l}\text { Total Volume } \\
\text { (m3/yc) }\end{array}$ & $\begin{array}{l}\text { Total Mass } \\
\text { (kg/yr) }\end{array}$ & $9.57 \Sigma+06$ \\
\hline & $\begin{array}{l}\text { Radionuclide } \\
\mathrm{ir}-3 \\
\mathrm{Cr}-51 \\
\mathrm{Mn}-54 \\
\mathrm{Fe}-55 \\
\mathrm{Fe}-59 \\
\mathrm{Co}-58 \\
\mathrm{Co}-60 \\
\mathrm{Ni}-59 \\
\mathrm{Ni}-63 \\
\mathrm{Sr}-90 \\
\mathrm{Y}-90 \\
\mathrm{Zr}-95 \\
\mathrm{Tc}-99 \\
\mathrm{Ru}-106 \\
\mathrm{Rh}-106 \\
\mathrm{Sb}-125 \\
\mathrm{Te}-125 \mathrm{~m} \\
\mathrm{Cs}-134 \\
\mathrm{Cs}-137 \\
\mathrm{Ba}-137 \mathrm{~m} \\
\mathrm{Ce}-144 \\
\mathrm{Pr}-144 \\
\mathrm{Pr}-144 \mathrm{~m} \\
\mathrm{Pm}-147 \\
\mathrm{Sm}-151 \\
\mathrm{Eu}-152 \\
\mathrm{Eu}-154 \\
\mathrm{Eu}-155 \\
\mathrm{Pu}-238 \\
\mathrm{Pu}-239 \\
\mathrm{Pu}-240 \\
\mathrm{Pu}-241 \\
\mathrm{Am}-241 \\
\end{array}$ & $\begin{array}{l}\text { Activity } \\
\text { (Ci/yr) } \\
-1.71 \mathrm{E}-06 \\
2.31 \mathrm{E}+03 \\
4.40 \mathrm{E}+03 \\
1.64 \mathrm{E}+04 \\
1.48 \mathrm{E}+02 \\
5.91 \mathrm{E}+03 \\
4.63 \mathrm{E}+04 \\
2.12 \mathrm{E}+02 \\
3.01 \mathrm{E}+04 \\
1.43 \mathrm{E}-03 \\
1.43 \mathrm{E}-03 \\
1.80 \mathrm{E}+02 \\
2.56 \mathrm{E}-07 \\
1.40 \mathrm{E}-04 \\
1.40 \mathrm{E}-04 \\
4.03 \mathrm{E}-05 \\
9.84 \mathrm{E}-06 \\
1.63 \mathrm{E}-04 \\
2.00 \mathrm{E}-01 \\
2.00 \mathrm{E}-01 \\
4.69 \mathrm{E}-04 \\
4.69 \mathrm{E}-04 \\
5.64 \mathrm{E}-06 \\
1.14 \mathrm{E}-03 \\
1.12 \mathrm{E}-05 \\
1.71 \mathrm{E}-07 \\
2.93 \mathrm{E}-05 \\
2.32 \mathrm{E}-05 \\
3.53 \mathrm{E}-05 \\
3.42 \mathrm{E}-07 \\
1.71 \mathrm{E}-07 \\
3.95 \mathrm{E}-05 \\
5.13 \mathrm{E}-07\end{array}$ & \\
\hline Shipping Site: IANL & $\begin{array}{l}\text { Total Volume } \\
\text { (m3/yr) }\end{array}$ & $\begin{array}{cc}\text { Total Mass } \\
1.99 \mathrm{E}+02 & (\mathrm{~kg} / \mathrm{yr})\end{array}$ & $8.69 E+06$ \\
\hline & $\begin{array}{l}\text { Radionuclide } \\
\mathrm{Cr}-51 \\
\mathrm{Mr}-54 \\
\mathrm{Fe}-55 \\
\mathrm{Fe}-59 \\
\mathrm{Co}-58 \\
\mathrm{Co}-60 \\
\mathrm{Ni}-59 \\
\mathrm{Ni}-63 \\
\mathrm{Zr}-95\end{array}$ & $\begin{array}{c}\begin{array}{c}\text { Activity } \\
\text { (Ci/y工) }\end{array} \\
-2.42 \mathrm{E}+01 \\
4.62 \mathrm{E}+01 \\
1.72 \mathrm{E}+02 \\
1.55 \mathrm{E}+00 \\
6.20 \mathrm{E}+01 \\
4.86 \mathrm{E}+02 \\
2.22 \mathrm{E}+00 \\
3.16 \mathrm{E}+02 \\
1.89 \mathrm{E}+00\end{array}$ & \\
\hline
\end{tabular}


A-236

\begin{tabular}{|c|c|c|c|}
\hline Shipping Site: PORTS & $\begin{array}{l}\text { Total volume } \\
\text { (m3/yr) }\end{array}$ & $\begin{array}{c}\text { Total Mass } \\
(\mathrm{kg} / \mathrm{yr})\end{array}$ & $2.05 \mathrm{E}+07$ \\
\hline - & 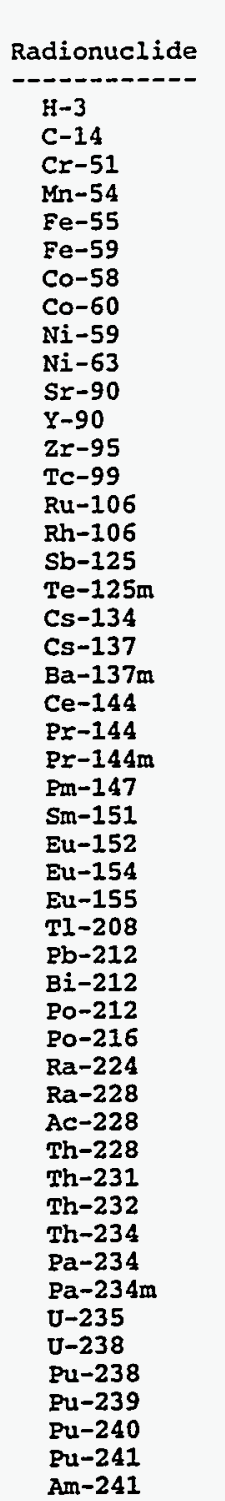 & 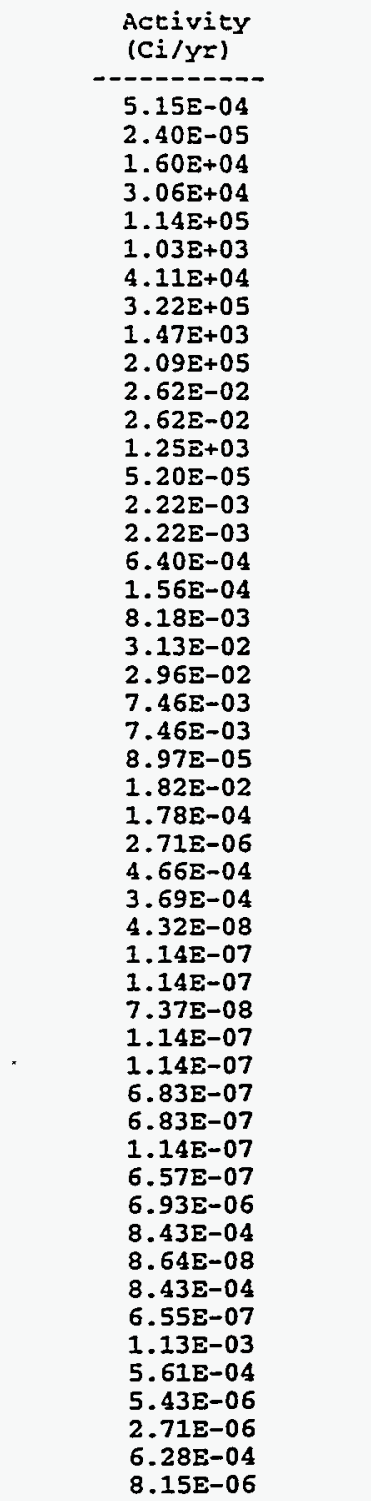 & \\
\hline
\end{tabular}




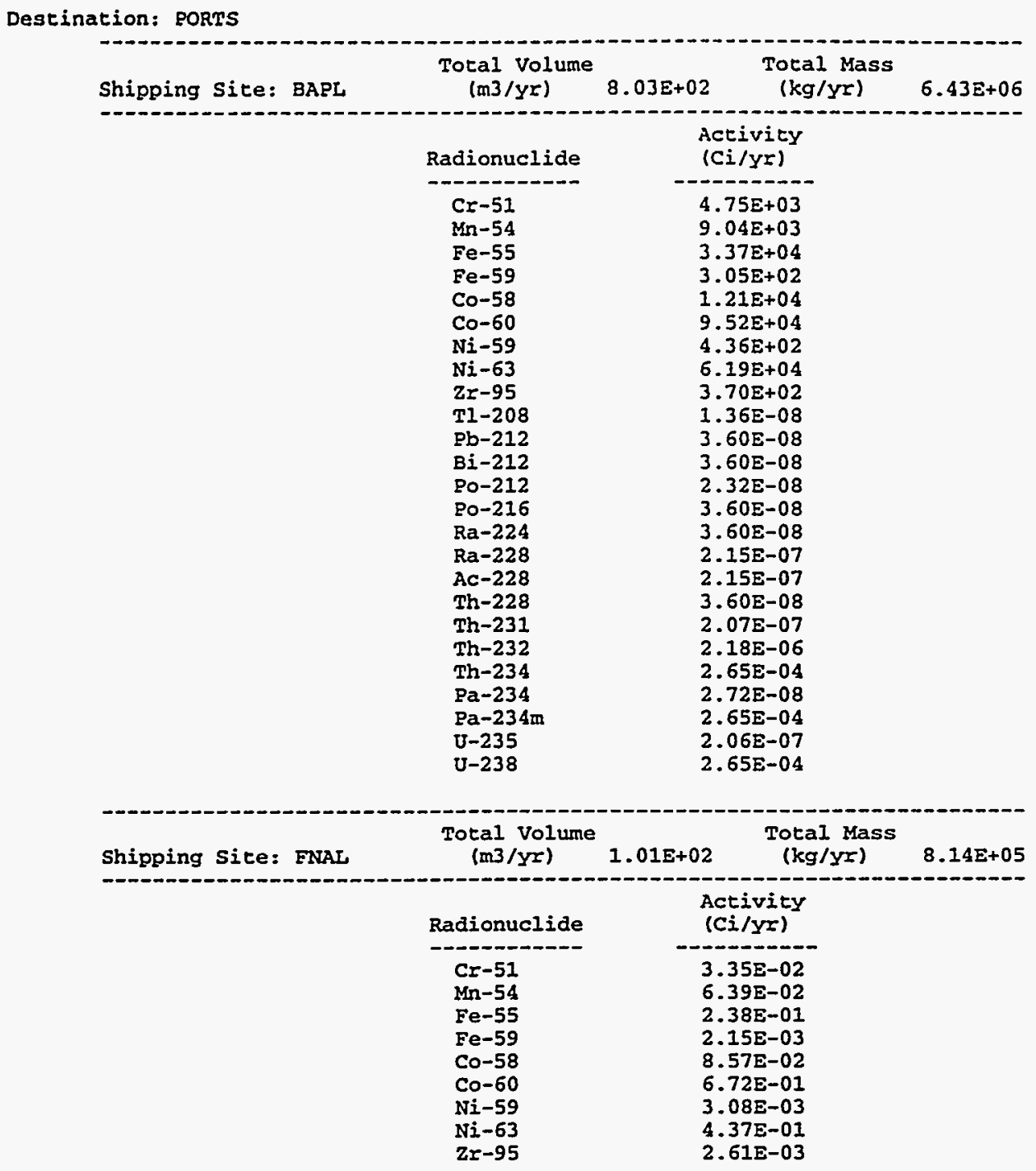


A-238

\begin{tabular}{|c|c|c|c|}
\hline Shipping Site: KARL & $\begin{array}{l}\text { Total Volume } \\
\text { (m3/yr) }\end{array}$ & $\begin{array}{c}\text { Total Mass } \\
(\mathrm{kg} / \mathrm{yr})\end{array}$ & $1.33 \mathrm{E}+07$ \\
\hline & 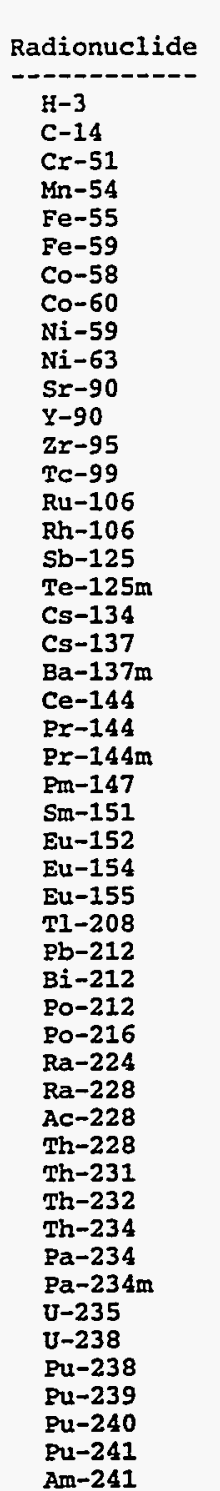 & 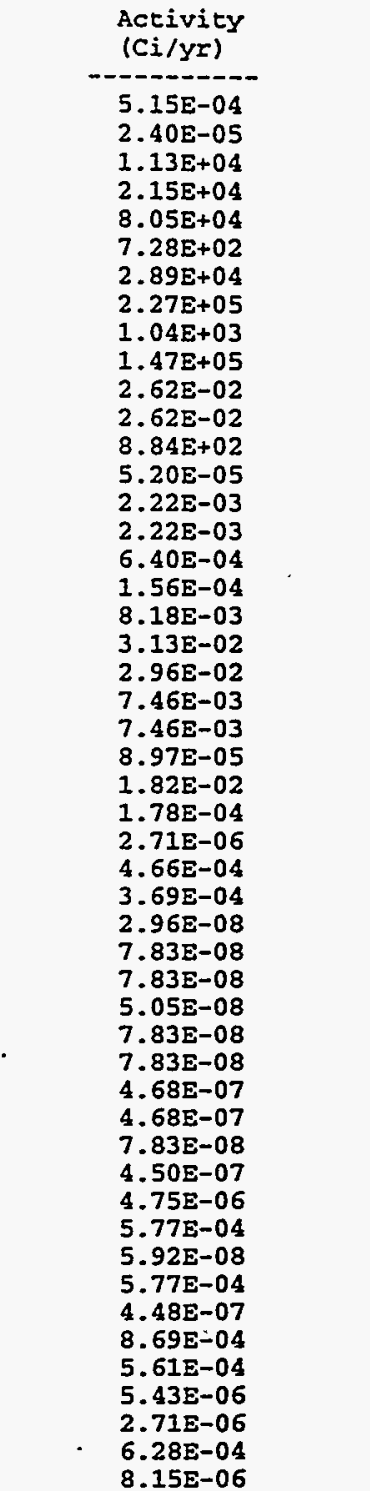 & . \\
\hline
\end{tabular}




\section{A.27 WM LLW CENTRALIZED 5 ALTERNATIVE (CASE 21): HETEROGENEOUS SOLIDS}

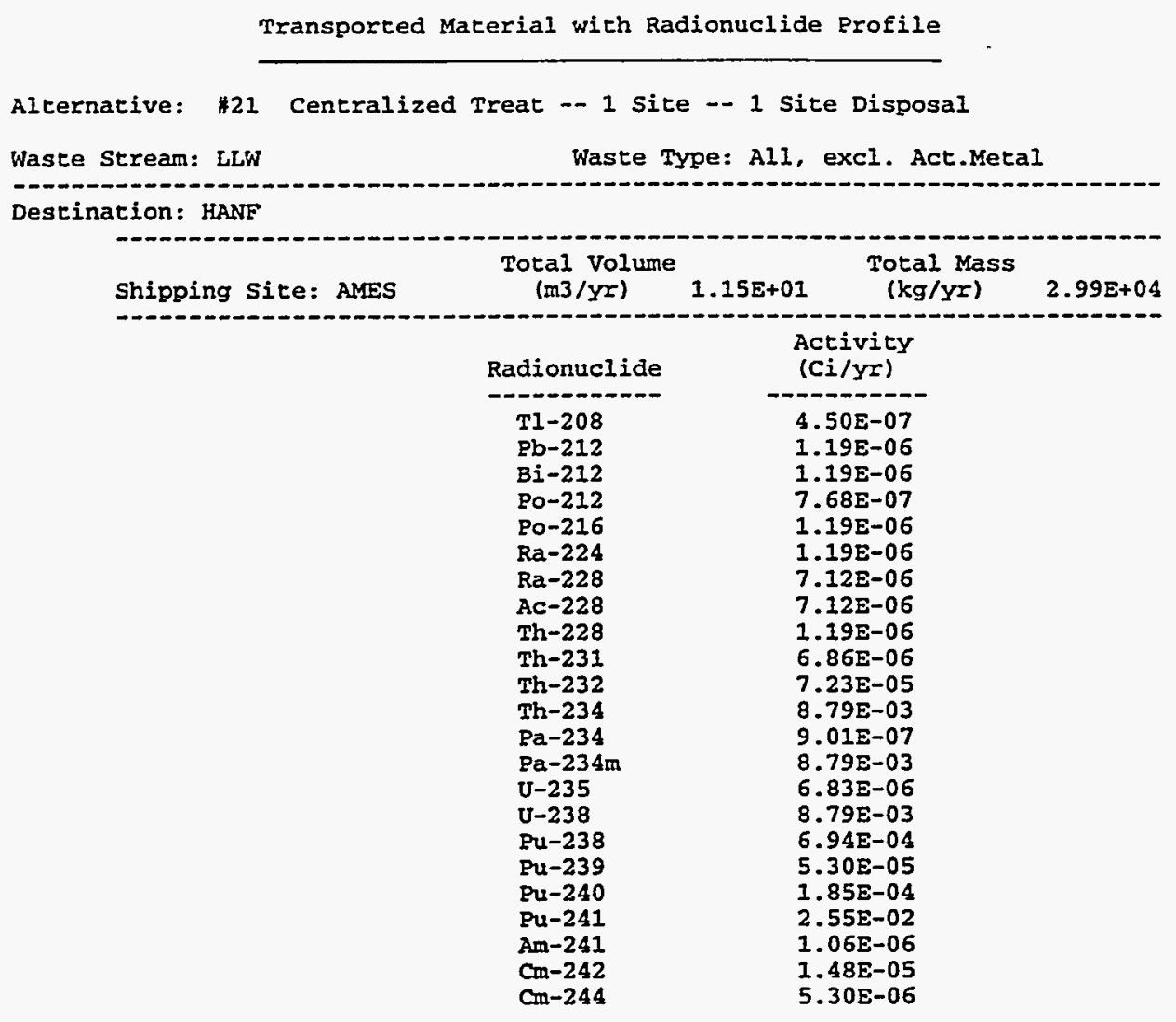


TABLE 3.2 (Cont.)

\begin{tabular}{|c|c|c|c|c|c|c|c|c|c|c|c|c|c|c|c|c|c|}
\hline & $\stackrel{\tilde{z}}{z}$ & $\frac{\text { 莞 }}{\frac{2}{5}}$ & 芩 & $\hat{\overline{0}}$ & 駦 & $\begin{array}{l}\hat{z} \\
\bar{z} \\
\underline{2}\end{array}$ & : & $\vec{a}$ & $=$ & 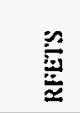 & 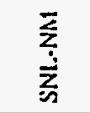 & 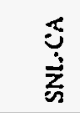 & & ְ̌ & $\sum_{3}^{\grave{i}}$ & $\frac{2}{\bar{z}}$ & 5 \\
\hline Ar & & 6 & 954 & $6+6$ & 309 & 23 & 727 & 197 & 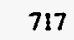 & 782 & 187 & 2018 & 281 & 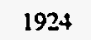 & 81 & (1. & 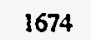 \\
\hline ANI & 108 & 651 & 649 & 390 & $7 ?$ & 1319 & 2 & 898 & 418 & 94 & 04 & 506 & 976 & 536 & 79 & 279 & 348 \\
\hline A & 1.00 & 199 & 55 & 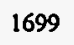 & 41 & 2721 & 975 & 2507 & . & 38 & 21 & 100 & 407 & 160 & 123 & 41 & 756 \\
\hline $\mathrm{BC}$ & 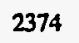 & 66 & 393 & 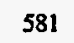 & 1381 & 1151 & 91 & 655 & 207 & 1502 & 1759 & 2718 & 740 & 2947 & 370 & 1688 & 2374 \\
\hline $\mathrm{Bc}$ & 0 & 14 & 903 & 16 & 479 & 1293 & 429 & 400 & 136 & 1692 & 1857 & 2840 & 947 & 274 & 244 & 1785 & 2496 \\
\hline BNL & 39 & 1221 & 1152 & 346 & 2035 & 1585 & 921 & 410 & 648 & 2266 & 2414 & 3383 & 239 & 3289 & 549 & 2342 & 3039 \\
\hline & 97 & 79 & 682 & 0 & 977 & 1344 & 451 & 924 & +3 & 1016 & 356 & 2341 & $\infty 1$ & 2393 & 603 & 28 & 1997 \\
\hline than & 91 & 31 & 358 & 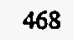 & 373 & 1116 & 207 & 930 & 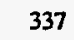 & 1466 & 1751 & 2735 & 774 & 2641 & 631 & 1679 & 2391 \\
\hline . & 02 & 44 & 2601 & $2+3$ & 1686 & 3267 & 515 & 2985 & 2505 & 1284 & 1793 & 973 & 2953 & 1036 & 2669 & 1993 & 1302 \\
\hline INEL & 756 & 999 & oss & 699 & 1141 & 2721 & 1975 & 2507 & 2060 & 738 & 247 & 1100 & 2407 & 1160 & 2123 & 1447 & 756 \\
\hline ITF & 65 & 989 & 1749 & 39 & 379 & 2079 & 1761 & 2248 & 1769 & 572 & 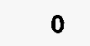 & 1222 & 315 & 1253 & 1929 & 477 & 065 \\
\hline $\mathrm{KCF}$ & 1670 & 881 & 838 & 482 & 554 & 1503 & 758 & 1289 & 842 & 778 & 932 & 2013 & 1161 & 2073 & 1033 & $861^{\circ}$ & 1670 \\
\hline & $\infty$ & 81 & 957 & 106 & 1807 & 1390 & 681 & 214 & 408 & 2027 & 2177 & 3144 & 1044 & 3050 & 309 & 211 & 2800 \\
\hline LBL & 0 & 390 & 2686 & 190 & 1561 & 3278 & 2767 & 3298 & 51 & 320 & 266 & 46 & 3192 & 56 & 2773 & 66 & 860 \\
\hline$L$ & 70 & 2868 & 2831 & 69 & 1534 & 3491 & 2745 & 3276 & 29 & 4 & 1222 & . & 183 & 0 & 98 & 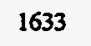 & 1370 \\
\hline $\mathrm{L}$ & 69 & 1926 & 1686 & 76 & 483 & 2183 & 1698 & 2186 & 06 & 4 & 104 & 326 & 2252 & 357 & 866 & 581 & 169 \\
\hline$M$ & 86 & 301 & 328 & 564 & 1396 & 1086 & 156 & 71 & 2 & 1485 & 1767 & 995 & 744 & 930 & 562 & 170 & 386 \\
\hline NRI & 86 & 2099 & 055 & 1699 & 1141 & 2721 & 1975 & 2507 & 206 & 738 & 247 & 1100 & 2407 & 1160 & 2123 & 1447 & 756 \\
\hline NTS & 0 & 2530 & 2487 & 2131 & 1376 & 3153 & 2401 & 2871 & 2391 & 987 & 1065 & 1370 & 2839 & 862 & 2554 & 1475 & 0 \\
\hline ORISE & 2530 & 0 & 40 & 632 & 1611 & $\overline{786}$. & 392 & 3176 & 575 & 1658 & 1989 & 2868 & 443 & 3103 & 889 & $1918^{\circ}$ & 2530 \\
\hline ORR & 2487 & 40 & 0 & 527 & 1371 & 797 & 442 & 760 & 600 & 1586 & 1749 & 2831 & 417 & 3031 & 889. & $1678=$ & 2487 \\
\hline PGDP & 2131 & -632 & 527 & $\div 0$ & 1103. & 1056 & 495 & 1145 & 698 & 1220 & 1539 & 2469 & 714 & 2597 & 861 & 1410 & 2131 \\
\hline Pantex & 1376 & 1611 & 1371 & 1103 & 0 & 1825 & 1382 & 1867 & 1387 & 465 & 379 & 1534 & 1937 & $1564 \mathrm{z}$ & 1551 & 307 & 1376 \\
\hline Pinella & 3153 & 786 & 797 & 1056 & 1825 & 0 & .1106 & 1207 & 1361 . & $2280^{\circ}$ & 2079 & 491 & 485 & $3280^{\circ}$ & 568 & 019 & 100 \\
\hline PORTS & 2401 & 392 & 442 & 495 & 1382 & 1106 & 0 & 838 & 279 & 1535 & 1761 & 745 & 655. & 2651 & 85 & 1689 & 2401 \\
\hline PPPL & 2871 & 1176 & 760 & 1145 & 1867 & 1207 & 838 & $\overline{0}$ & 511 & 66 & 48 & 3276 & 848 & 1 & 426 & 2185 & 2871 \\
\hline $\mathrm{RM}$ & 391 & 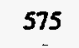 & ow & 698 & 1387 & 1361 & 279 & 511 & $\therefore 0$ & 619 & 1769. & 2829 & 920 & 2641 & 163 & 1705 & 2391 \\
\hline RF & 987 & 658 & 1586 & 1220 & -465 & 2280 & 1535 & 2066 & 1619 & $\because 0$ & $572=$ & 1394 & 1938 & 137 & 1782 & 769 & 887 \\
\hline & 1065 & 989 & 1749 & 1539 & 379 & 2079 & 1761 & 2248 & 1769 & 572 & 0 & 1222 & 231 & 1253 & 1929 & 477 & 1065 \\
\hline SNLCA & 1370 & 2868 & 2831 & 2469- & 1534 & 3491 & 2745 & 3276 & 2829 & -1394 & 1222 & $=0$ & 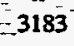 & 60 & 2898 & 1633 & 1370 \\
\hline SRS & 2839 & 443 & 417 & 714 & 1937 & 485 & 655 & 848 & 920 & 1938 & 2315 & 3183 & & 3194. & 1223 & 2243 & 2839 \\
\hline SLAC & 862 & 3103 & 3031 & 2597 & 1564 & 3280 & $265 i$ & 3121 & 2641 & 77 & 1253 & -60 & 3194 & $\because 0$ & 2804 & 1662 & 862 \\
\hline WVDP & 2554 & 889 & 889 & 801 & 1551 & 1568 & 585 & 426 & 163 & 1782 & 1929 & 2898 & 1223 & 2804 & 0 & 1858 & 2540 \\
\hline WIPP & 1475 & 1918 & 1678 & 410 & 307 & 2019 & -1689 & 2185 & 1705 & 769 & 477 & 1633 & 2243 & 1662 & 1858 & 0 & 1475 \\
\hline YM & 0 & 30 & 2487 & 31 & 376 & 3153 & 2401 & 2871 & 2391 & 987 & 1065 & 1370 & 2839 & 862 & $25+0$ & 1475 & 0 \\
\hline
\end{tabular}

${ }^{2}$ Rail routes generated by using the INTERLINE 5.0 routing model (Johnson et al. 1993b).

Note: Ames = Ames Laboratory; ANL-E = Argonne National Laboratory-East; ANL-W = Argonne National Laboratory-West: $\mathrm{BCL}=$ Battelle Columbus Laboratories; Bettis = Bettis Atomic Power Laboratory; BNL = Brookhaven National Laboratory; Fermi = Fermi National Accelerator Laboratory; FEMP = Fernald Environmental Management Project; Hanford = Hanford Site; INEI = Idaho National Engineering Laboratory; ITRI = Inhalation Toxicology Research Institute; KCP = Kansas City Plant; KAPL-S = Knolls Atomic Power Laboratory (Schenectady); LBL = Lawrence Berkeley National Laboratory; LLNL = Lawrence Livermore National Laboratory: LANL = Los Alamos National Laboratory; Mound = Mound Plant; NRF = Naval Reactor Facility: NTS = Nevada Test Site; ORISE = Oak Ridge Institute for Science and Education; ORR = Oak Ridge Reservation; PGDP = Paducah Gaseous Diffusion Plant; Pantex = Pantex Plant; Pinellas $=$ Pinellas Plant; PORTS $=$ Portsmouth Gaseous Diffusion Plant; PPPL = Princeton Plasma Physics Laboratory; RMI = Reactive Metals, Inc.; RFETS = Rocky Flats Environmental Technology Site; SNL-NM = Sandia National Laboratories (New Mexico); SNL.CA = Sandia National Laboratories (California); SRS = Savannah River Site; SLAC = Stanford Linear Accelerator Center, WVDP = West Valley Demonstration Project; WIPP = Waste Isolation Pilot Plant; and YM = Yucea Mountain. 
A-240

\begin{tabular}{|c|c|c|c|}
\hline Shipping Site: ANL-E & $\begin{array}{l}\text { Total volume } \\
(\mathrm{m} 3 / \mathrm{yr})\end{array}$ & $\begin{array}{c}\text { Total Mass } \\
(\mathrm{kg} / \mathrm{yr})\end{array}$ & $2.00 \mathrm{E}+06$ \\
\hline & $\begin{array}{l}\text { Radionuclide } \\
\mathrm{H}-3 \\
\mathrm{C}-14 \\
\mathrm{Cr}-51 \\
\mathrm{Mn}-54 \\
\mathrm{Fe}-55 \\
\mathrm{Fe}-59 \\
\mathrm{Co}-58 \\
\mathrm{Co}-60 \\
\mathrm{Ni}-59 \\
\mathrm{Ni}-63 \\
\mathrm{Sr}-90 \\
\mathrm{Y}-90 \\
\mathrm{Zr}-95 \\
\mathrm{Tc}-99 \\
\mathrm{Ru}-106 \\
\mathrm{Rh}-106 \\
\mathrm{Sb}-125 \\
\mathrm{Te}-125 \mathrm{~m} \\
\mathrm{Cs}-134 \\
\mathrm{Cs}-137 \\
\mathrm{Ba}-137 \mathrm{~m} \\
\mathrm{Ce}-144 \\
\mathrm{Pr}-144 \\
\mathrm{Pr}-144 \mathrm{~m} \\
\mathrm{Pm}-147 \\
\mathrm{Sm}-151 \\
\text { Eu-152 } \\
\mathrm{Eu}-154 \\
\mathrm{Eu}-155 \\
\mathrm{U}-238 \\
\mathrm{Pu}-238 \\
\mathrm{Pu}-239 \\
\mathrm{Pu}-240 \\
\mathrm{Pu}-241 \\
\mathrm{Am}-241\end{array}$ & $\begin{array}{c}\text { Activicy } \\
\text { (Ci/yr) } \\
-3.33 \mathrm{E}+02 \\
7.91 \mathrm{E}-02 \\
2.87 \mathrm{E}+00 \\
1.43 \mathrm{E}+01 \\
2.04 \mathrm{E}+01 \\
1.84 \mathrm{E}-01 \\
1.55 \mathrm{E}+01 \\
8.15 \mathrm{E}+01 \\
2.63 \mathrm{E}-01 \\
3.77 \mathrm{E}+01 \\
3.33 \mathrm{E}+01 \\
3.33 \mathrm{E}+01 \\
2.24 \mathrm{E}-01 \\
1.62 \mathrm{E}-01 \\
2.16 \mathrm{E}+00 \\
2.16 \mathrm{E}+00 \\
6.21 \mathrm{E}-01 \\
1.51 \mathrm{E}-01 \\
2.09 \mathrm{E}+01 \\
4.75 \mathrm{E}+01 \\
4.50 \mathrm{E}+01 \\
7.24 \mathrm{E}+00 \\
7.24 \mathrm{E}+00 \\
8.70 \mathrm{E}-02 \\
1.76 \mathrm{E}+01 \\
1.72 \mathrm{E}-01 \\
2.63 \mathrm{E}-03 \\
4.52 \mathrm{E}-01 \\
3.58 \mathrm{E}-01 \\
9.63 \mathrm{E}-01 \\
5.44 \mathrm{E}-01 \\
5.27 \mathrm{E}-03 \\
2.63 \mathrm{E}-03 \\
6.09 \mathrm{E}-01 \\
7.91 \mathrm{E}-03\end{array}$ & \\
\hline
\end{tabular}




\begin{tabular}{|c|c|c|c|}
\hline Shipping Site: ANL-W & $\begin{array}{l}\text { Total Volume } \\
(\mathrm{m} 3 / \mathrm{yr})\end{array}$ & $\begin{array}{c}\text { Total Mass } \\
(\mathrm{kg} / \mathrm{yr})\end{array}$ & $2.12 E+05$ \\
\hline & $\begin{array}{l}\text { Radionuclide } \\
\mathrm{H}-3 \\
\mathrm{Cr}-51 \\
\mathrm{Mn}-54 \\
\mathrm{Fe}-55 \\
\mathrm{Fe}-59 \\
\mathrm{Co}-58 \\
\mathrm{Co}-60 \\
\mathrm{Ni}-59 \\
\mathrm{Ni}-63 \\
\mathrm{Sr}-90 \\
\mathrm{Y}-90 \\
\mathrm{Zr}-95 \\
\mathrm{Tc}-99 \\
\mathrm{Ru}-106 \\
\mathrm{Rh}-106 \\
\mathrm{Sb}-125 \\
\mathrm{Te}-125 \mathrm{~m} \\
\mathrm{Cs}-134 \\
\mathrm{Cs}-137 \\
\mathrm{Ba}-137 \mathrm{~m} \\
\mathrm{Ce}-144 \\
\mathrm{Pr}-144 \\
\mathrm{Pr}-144 \mathrm{~m} \\
\mathrm{Pm}-147 \\
\mathrm{Sm}-151 \\
\mathrm{Eu}-152 \\
\mathrm{Eu}-154 \\
\mathrm{Eu}-155 \\
\mathrm{Pu}-238 \\
\mathrm{Pu}-239 \\
\mathrm{Pu}-240 \\
\mathrm{Pu}-241 \\
\mathrm{Am}-241 \\
\mathrm{Cm}-242 \\
\mathrm{Cm}-244\end{array}$ & 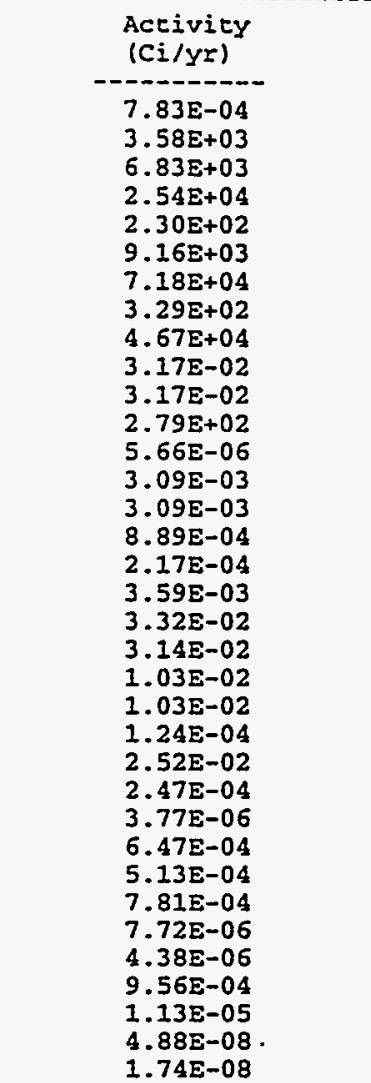 & \\
\hline
\end{tabular}




\begin{tabular}{|c|c|c|c|}
\hline Shipping Site: BAPL & $\begin{array}{l}\text { Total Volume } \\
\text { (m3/yr) }\end{array}$ & $\begin{array}{l}\text { Total Mass } \\
(\mathrm{kg} / \mathrm{yr})\end{array}$ & $1.08 \mathrm{E}+06$ \\
\hline & $\begin{array}{l}\text { Radionuclide } \\
\mathrm{H}-3 \\
\mathrm{Co}-60 \\
\mathrm{Ni}-63 \\
\mathrm{Sr}-90 \\
\mathrm{Y}-90 \\
\mathrm{Tc}-99 \\
\mathrm{Ru}-106 \\
\mathrm{Rh}-106 \\
\mathrm{Sb}-125 \\
\mathrm{Te}-125 \mathrm{~m} \\
\mathrm{Cs}-134 \\
\mathrm{Cs}-137 \\
\mathrm{Ba}-137 \mathrm{~m} \\
\mathrm{Ce}-144 \\
\mathrm{Pr}-144 \\
\mathrm{Pr}-144 \mathrm{~m} \\
\mathrm{Pm}-147 \\
\mathrm{Sm}-151 \\
\mathrm{Eu}-152 \\
\mathrm{Eu}-154 \\
\mathrm{Eu}-155 \\
\mathrm{~T} 1-208 \\
\mathrm{~Pb}-212 \\
\mathrm{Bi}-212 \\
\mathrm{Po}-212 \\
\mathrm{Po}-216 \\
\mathrm{Ra}-224 \\
\mathrm{Ra}-228 \\
\mathrm{Ac}-228 \\
\mathrm{Th}-228 \\
\mathrm{Th}-231 \\
\mathrm{Th}-232 \\
\mathrm{Th}-234 \\
\mathrm{~Pa}-234 \\
\mathrm{~Pa}-234 \mathrm{~m} \\
\mathrm{U}-235 \\
\mathrm{U}-238 \\
\mathrm{Pu}-238 \\
\mathrm{Pu}-239 \\
\mathrm{Pu}-240 \\
\mathrm{Pu}-241 \\
\mathrm{Am}-241 \\
\end{array}$ & 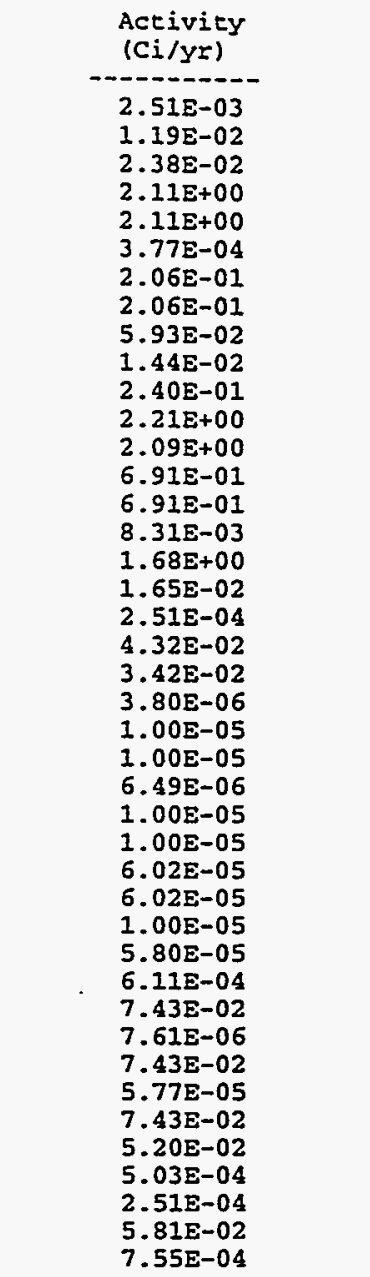 & \\
\hline & $\begin{array}{l}\text { Total Volume } \\
(\mathrm{m} 3 / \mathrm{yr})\end{array}$ & $\begin{array}{cc}\text { Total Mass } \\
(\mathrm{kg} / \mathrm{y} r)\end{array}$ & $3.89 \mathrm{E}+04$ \\
\hline & $\begin{array}{l}\text { Radionuclide } \\
\mathrm{H}-3 \\
\mathrm{Cr}-51 \\
\mathrm{Mn}-54 \\
\mathrm{Fe}-55 \\
\mathrm{Fe}-59 \\
\mathrm{Co}-58 \\
\mathrm{Co}-60 \\
\mathrm{Ni}-59 \\
\mathrm{Ni}-63 \\
\mathrm{Zr}-95 \\
\mathrm{TI}-208 \\
\mathrm{~Pb}-212 \\
\mathrm{Bi}-212 \\
\mathrm{Po}-212 \\
\mathrm{Po}-216 \\
\mathrm{Ra}-224 \\
\mathrm{Ra}-228 \\
\mathrm{Ac}-228 \\
\mathrm{Th}-228 \\
\mathrm{Th}-231 \\
\mathrm{Th}-232 \\
\mathrm{Th}-234 \\
\mathrm{~Pa}-234 \\
\mathrm{~Pa}-234 \mathrm{~m} \\
\mathrm{U}-235 \\
\mathrm{U}-238\end{array}$ & \begin{tabular}{c}
$\begin{array}{c}\text { Activity } \\
(C i / Y I)\end{array}$ \\
\hdashline $1.82 \mathrm{E}-01$ \\
$1.11 \mathrm{E}-02$ \\
$2.12 \mathrm{E}-02$ \\
$7.94 \mathrm{E}-02$ \\
$7.17 \mathrm{E}-04$ \\
$2.85 \mathrm{E}-02$ \\
$2.23 \mathrm{E}-01$ \\
$1.02 \mathrm{E}-03$ \\
$1.45 \mathrm{E}-01$ \\
$8.71 \mathrm{E}-04$ \\
$3.78 \mathrm{E}-07$ \\
$1.00 \mathrm{E}-06$ \\
$1.00 \mathrm{E}-06$ \\
$6.46 \mathrm{E}-07$ \\
$1.00 \mathrm{E}-06$ \\
$1.00 \mathrm{E}-06$ \\
$5.99 \mathrm{E}-06$ \\
$5.99 \mathrm{E}-06$ \\
$1.00 \mathrm{E}-06$ \\
$5.77 \mathrm{E}-06$ \\
$6.08 \mathrm{E}-05$ \\
$7.39 \mathrm{E}-03$ \\
$7.57 \mathrm{E}-07$ \\
$7.39 \mathrm{E}-03$ \\
$5.75 \mathrm{E}-06$ \\
$7.39 \mathrm{E}-03$
\end{tabular} & . \\
\hline
\end{tabular}


A-243

\begin{tabular}{|c|c|c|c|}
\hline Shipping Site: INEL & $\begin{array}{l}\text { Total Volume } \\
(\mathrm{m} 3 / \mathrm{yr})\end{array}$ & $\begin{array}{l}\text { Total Mass } \\
(\mathrm{kg} / \mathrm{y} x)\end{array}$ & $1.64 E+07$ \\
\hline & $\begin{array}{l}\text { Radionuclide } \\
\mathrm{H}-3 \\
\mathrm{Cr}-51 \\
\mathrm{Mn}-54 \\
\mathrm{Fe}-55 \\
\mathrm{Fe}-59 \\
\mathrm{Co}-58 \\
\mathrm{Co}-60 \\
\mathrm{Ni}-59 \\
\mathrm{Ni}-63 \\
\mathrm{Sr}-90 \\
\mathrm{Y}-90 \\
\mathrm{Zr}-95 \\
\mathrm{Tc}-99 \\
\mathrm{Ru}-106 \\
\mathrm{Rh}-106 \\
\mathrm{Sb}-125 \\
\mathrm{Te}-125 \mathrm{~m} \\
\mathrm{Cs}-134 \\
\mathrm{Cs}-137 \\
\mathrm{Ba}-137 \mathrm{~m} \\
\mathrm{Ce}-144 \\
\mathrm{Pr}-144 \\
\mathrm{Pr}-144 \mathrm{~m} \\
\mathrm{Pm}-147 \\
\mathrm{Sm}-151 \\
\mathrm{Eu}-152 \\
\mathrm{Eu}-154 \\
\mathrm{Eu}-155 \\
\mathrm{Pu}-238 \\
\mathrm{Pu}-239 \\
\mathrm{Pu}-240 \\
\mathrm{Pu}-241 \\
\mathrm{Am}-241\end{array}$ & \multicolumn{2}{|l|}{ 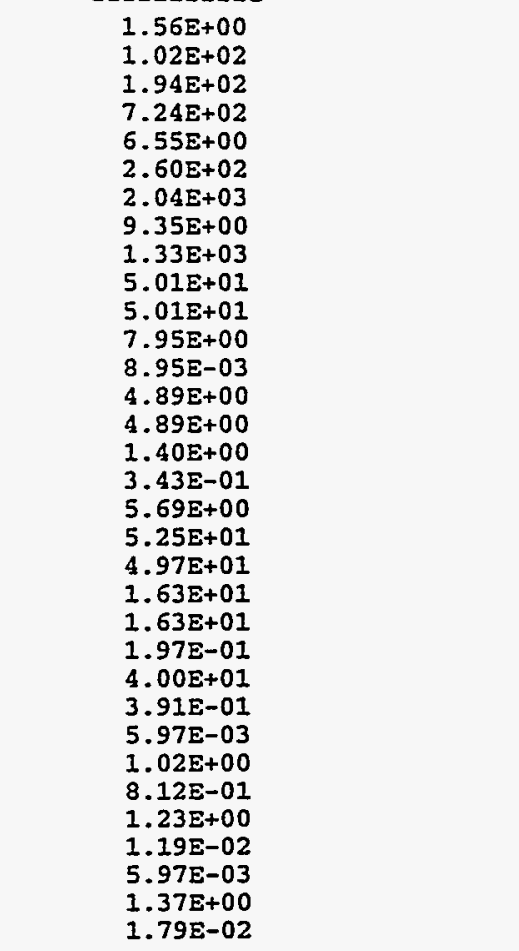 } \\
\hline \multirow[t]{2}{*}{ Shipping Site: IIRI } & $\begin{array}{l}\text { Toral Volume } \\
(\mathrm{m} 3 / \mathrm{yr})\end{array}$ & $\begin{array}{c}\text { Total Mass } \\
(\mathrm{kg} / \mathrm{yr})\end{array}$ & $1.64 \mathrm{E}+05$ \\
\hline & $\begin{array}{l}\text { Radionuclide } \\
\mathrm{H}-3 \\
\mathrm{C}-14 \\
\mathrm{Mn}-54 \\
\mathrm{Co}-58 \\
\mathrm{Co}-60 \\
\mathrm{~S}-90 \\
Y-90 \\
\mathrm{~T}-99 \\
\mathrm{Cs}-134 \\
\mathrm{Cs}-137 \\
\mathrm{Ba}-137 \mathrm{~m} \\
\mathrm{U}-238\end{array}$ & $\begin{array}{c}\begin{array}{c}\text { Activity } \\
\text { (Ci/yr) }\end{array} \\
-17 \mathrm{E}+00 \\
.17 \mathrm{0}-01 \\
3.03 \mathrm{E}-01 \\
3.41 \mathrm{E}+01 \\
3.15 \mathrm{E}+01 \\
9.12 \mathrm{E}+01 \\
4.28 \mathrm{E}+01 \\
4.28 \mathrm{E}+01 \\
6.07 \mathrm{E}-01 \\
7.07 \mathrm{E}+01 \\
9.33 \mathrm{E}+01 \\
8.82 \mathrm{E}+01 \\
3.69 \mathrm{E}+00\end{array}$ & \\
\hline
\end{tabular}




\begin{tabular}{|c|c|c|c|}
\hline Shipping Site: $k-25$ & $\begin{array}{l}\text { Total Volume } \\
(\mathrm{m} 3 / \mathrm{Yr})\end{array}$ & $\begin{array}{l}\text { Total Mass } \\
(\mathrm{kg} / \mathrm{yr})\end{array}$ & $4.67 E+07$ \\
\hline & $\begin{array}{l}\text { Radionuclide } \\
\mathrm{Tl}-208 \\
\mathrm{~Pb}-212 \\
\mathrm{Bi}-212 \\
\mathrm{Po}-212 \\
\mathrm{Po-216} \\
\mathrm{Ra}-224 \\
\mathrm{Ra}-228 \\
\mathrm{Ac}-228 \\
\mathrm{Th}-228 \\
\mathrm{Th}-231 \\
\mathrm{Th}-232 \\
\mathrm{Th}-234 \\
\mathrm{~Pa}-234 \\
\mathrm{~Pa}-234 \mathrm{~m} \\
\mathrm{U}-235 \\
\mathrm{U}-238\end{array}$ & $\begin{array}{c}\begin{array}{c}\text { Activity } \\
(C i / y r)\end{array} \\
-1.06 E-04 \\
2.82 \mathrm{E}-04 \\
2.82 \mathrm{E}-04 \\
1.82 \mathrm{E}-04 \\
2.82 \mathrm{E}-04 \\
2.82 \mathrm{E}-04 \\
1.68 \mathrm{E}-03 \\
1.68 \mathrm{E}-03 \\
2.82 \mathrm{E}-04 \\
1.62 \mathrm{E}-03 \\
1.71 \mathrm{E}-02 \\
2.08 \mathrm{E}+00 \\
2.13 \mathrm{E}-04 \\
2.08 \mathrm{E}+00 \\
1.62 \mathrm{E}-03 \\
2.08 \mathrm{E}+00\end{array}$ & \\
\hline Shipping Site: KAPL & $\begin{array}{l}\text { Total Volume } \\
(\mathrm{m} 3 / \mathrm{yx})\end{array}$ & $\begin{array}{cc}\text { Total Mass } \\
2.11 \mathrm{E}+02 \quad \mathrm{~kg} / \mathrm{Yr})\end{array}$ & $5.36 E+05$ \\
\hline . & 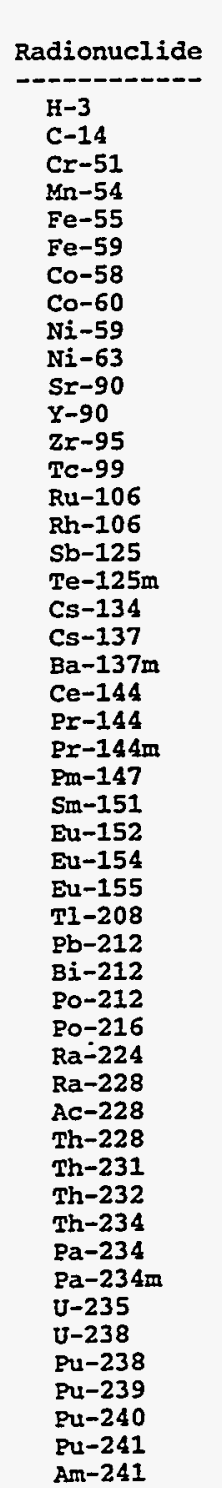 & 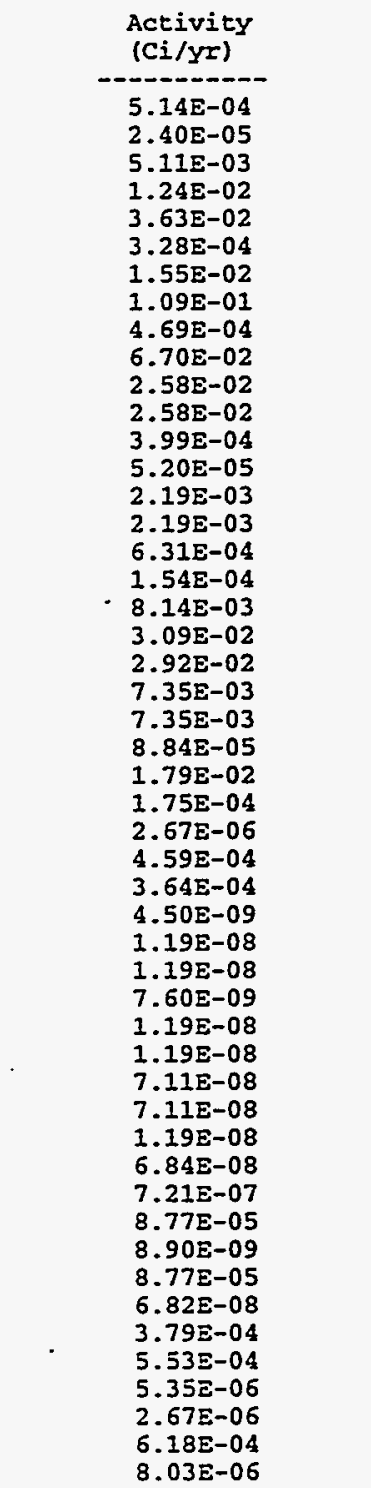 & \\
\hline
\end{tabular}


A-245

\begin{tabular}{|c|c|c|c|}
\hline Shipping Site: KCP & $\begin{array}{l}\text { Total Volume } \\
(\mathrm{m} 3 / \mathrm{y} r)\end{array}$ & $\begin{array}{l}\text { Total Mass } \\
(\mathrm{kg} / \mathrm{yr})\end{array}$ & $7.23 \mathrm{E}+03$ \\
\hline & $\begin{array}{l}\text { Radionuclide } \\
\mathrm{H}-3 \\
\mathrm{C}-14 \\
\mathrm{Mn}-54 \\
\mathrm{Co}-58 \\
\mathrm{Co}-60 \\
\mathrm{Sr}-90 \\
\mathrm{Y}-90 \\
\mathrm{TC}-99 \\
\mathrm{Cs}-134 \\
\mathrm{Cs}-137 \\
\mathrm{Ba}-137 \mathrm{~m} \\
\mathrm{U}-238\end{array}$ & $\begin{array}{c}\begin{array}{c}\text { Activity } \\
\text { (Ci/yr) }\end{array} \\
2.70 \mathrm{E}-02 \\
1.44 \mathrm{E}-04 \\
1.62 \mathrm{E}-02 \\
1.50 \mathrm{E}-02 \\
4.34 \mathrm{E}-02 \\
2.04 \mathrm{E}-02 \\
2.04 \mathrm{E}-02 \\
2.89 \mathrm{E}-04 \\
3.37 \mathrm{E}-02 \\
4.44 \mathrm{E}-02 \\
4.20 \mathrm{E}-02 \\
1.76 \mathrm{E}-03\end{array}$ & \\
\hline Shipping Site: LANL & $\begin{array}{l}\text { Total Volume } \\
(\mathrm{m} 3 / \mathrm{yr})\end{array}$ & $\begin{array}{cc}\text { 1.42E+04 } & \begin{array}{c}\text { Total Mass } \\
(\mathrm{kg} / \mathrm{Yr})\end{array}\end{array}$ & $1.22 \mathrm{E}+07$ \\
\hline & 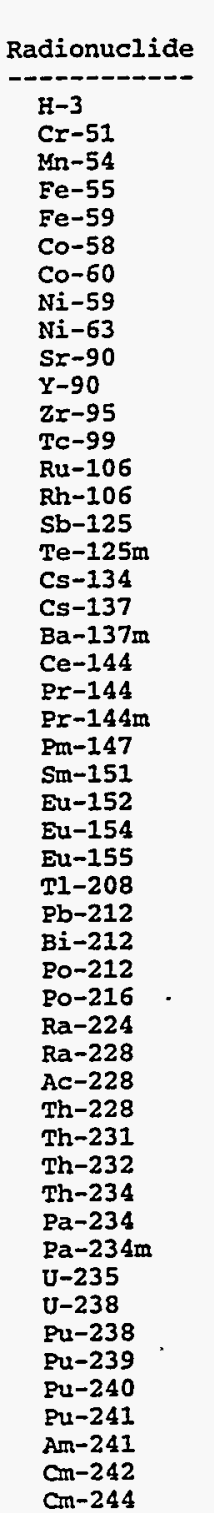 & 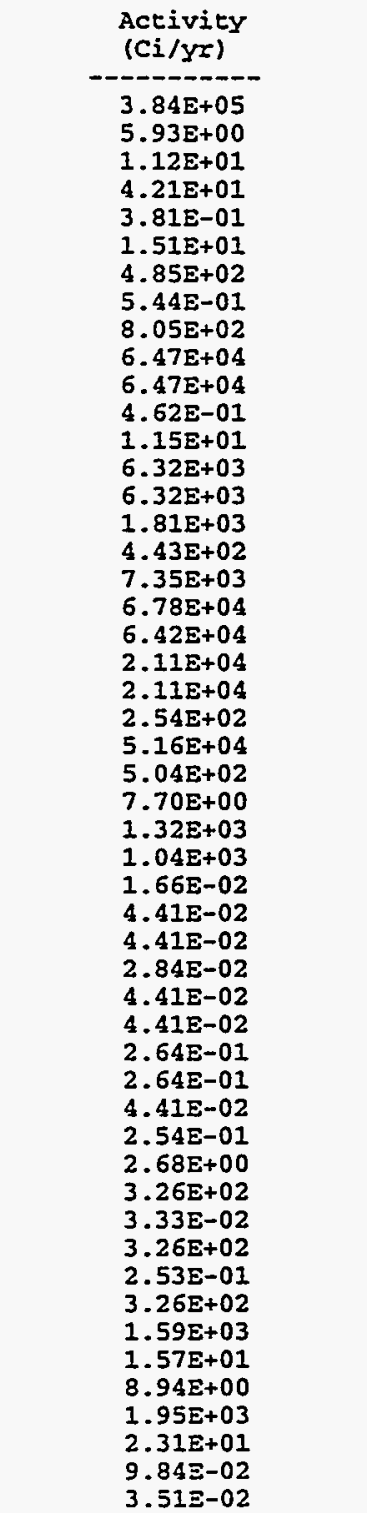 & \\
\hline
\end{tabular}




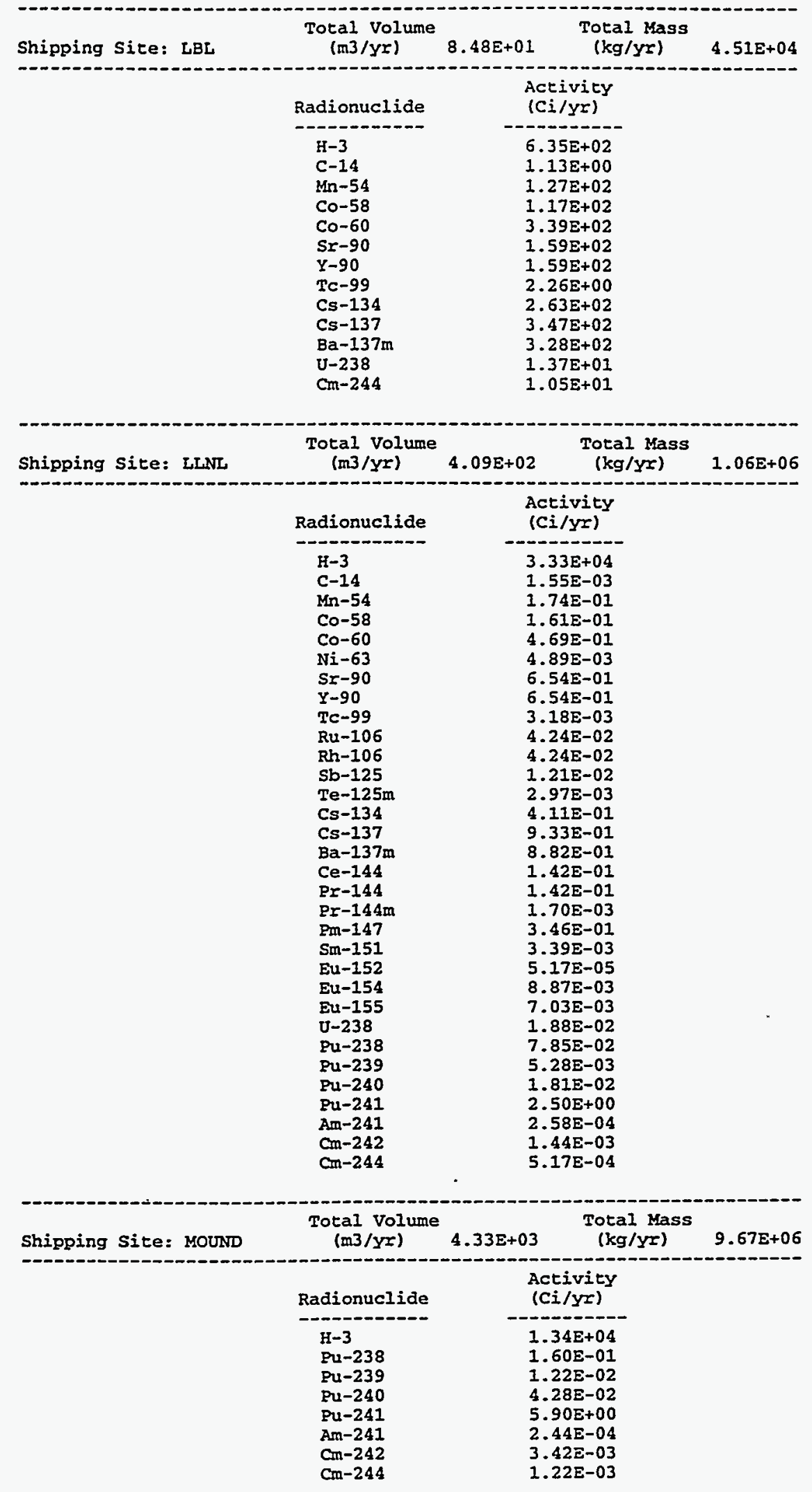




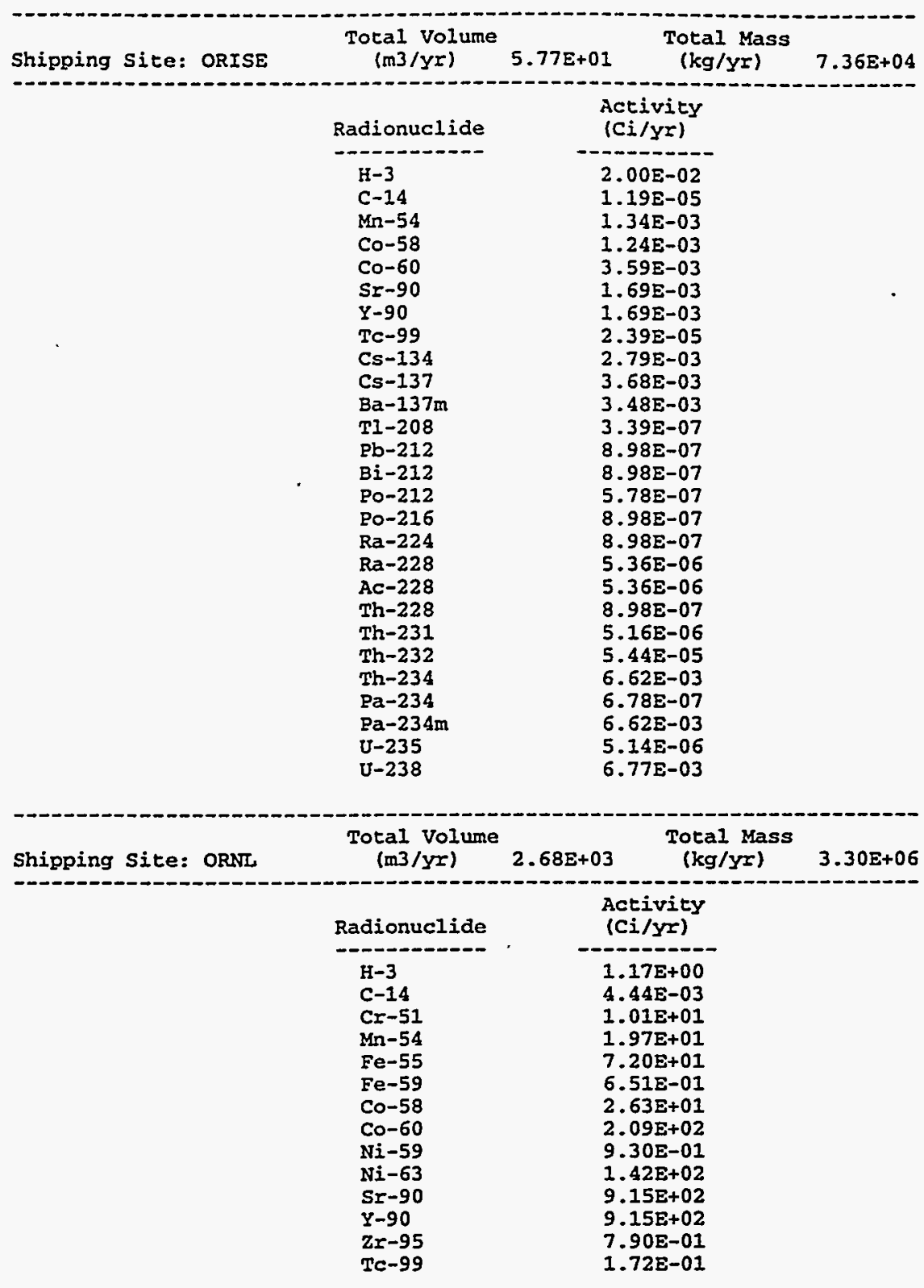




\begin{tabular}{|c|c|c|c|}
\hline Shipping Site: ORNL & $\begin{array}{l}\text { Total Volume } \\
\text { (m3/yr) }\end{array}$ & $\begin{array}{c}\text { Total Mass } \\
(\mathrm{kg} / \mathrm{yz})\end{array}$ & $3.305+06$ \\
\hline • & $\begin{array}{l}\text { Radionuclide } \\
\text { Ru-106 } \\
\text { Rh-106 } \\
\text { Sb-125 } \\
\text { Te-125m } \\
\text { Cs-134 } \\
\text { Cs-137 } \\
\text { Ba-137m } \\
\text { Ce-144 } \\
\text { Pr-144 } \\
\mathrm{Pr}-144 m \\
\mathrm{Pm}-147 \\
\mathrm{Sm}-151 \\
\mathrm{Eu}-152 \\
\mathrm{Eu}-154 \\
\mathrm{Eu}-155 \\
\mathrm{~T} 1-208 \\
\mathrm{~Pb}-212 \\
\mathrm{Bi}-212 \\
\mathrm{Po}-212 \\
\mathrm{Po}-216 \\
\mathrm{Ra}-224 \\
\mathrm{Ra}-228 \\
\mathrm{Ac}-228 \\
\mathrm{Th}-228 \\
\mathrm{Th}-231 \\
\mathrm{Th}-232 \\
\mathrm{Th}-234 \\
\mathrm{~Pa}-234 \\
\mathrm{~Pa}-234 \mathrm{~m} \\
\mathrm{U}-235 \\
\mathrm{U}-238 \\
\mathrm{Pu}-238 \\
\mathrm{Pu}-239 \\
\mathrm{Pu}-240 \\
\mathrm{Pu}-241 \\
\mathrm{Am}-241 \\
\mathrm{Cm}-242 \\
\mathrm{Cm}-244 \\
\end{array}$ & $\begin{array}{l}\text { Activity } \\
\text { (Ci/yr) } \\
8.91 \mathrm{E}+01 \\
8.91 \mathrm{E}+01 \\
2.56 \mathrm{E}+01 \\
6.25 \mathrm{E}+00 \\
1.04 \mathrm{E}+02 \\
9.58 \mathrm{E}+02 \\
9.07 \mathrm{E}+02 \\
2.98 \mathrm{E}+02 \\
2.98 \mathrm{E}+02 \\
3.58 \mathrm{E}+00 \\
7.28 \mathrm{E}+02 \\
7.12 \mathrm{E}+00 \\
1.08 \mathrm{E}-01 \\
1.86 \mathrm{E}+01 \\
1.47 \mathrm{E}+01 \\
1.70 \mathrm{E}-06 \\
4.50 \mathrm{E}-06 \\
4.50 \mathrm{E}-06 \\
2.90 \mathrm{E}-06 \\
4.50 \mathrm{E}-06 \\
4.50 \mathrm{E}-06 \\
2.69 \mathrm{E}-05 \\
2.69 \mathrm{E}-05 \\
4.50 \mathrm{E}-06 \\
2.59 \mathrm{E}-05 \\
2.73 \mathrm{E}-04 \\
3.31 \mathrm{E}-02 \\
3.40 \mathrm{E}-06 \\
3.31 \mathrm{E}-02 \\
2.58 \mathrm{E}-05 \\
8.72 \mathrm{E}-02 \\
2.24 \mathrm{E}+01 \\
2.17 \mathrm{E}-01 \\
1.09 \mathrm{E}-01 \\
2.52 \mathrm{E}+01 \\
3.26 \mathrm{E}-01 \\
5.60 \mathrm{E}-05 \\
2.00 \mathrm{E}-05\end{array}$ & \\
\hline Shipping Sice: RANT & $\begin{array}{l}\text { Radionuclide } \\
\mathrm{H}-3 \\
\mathrm{TI}-208 \\
\mathrm{~Pb}-212 \\
\mathrm{Bi}-212 \\
\mathrm{Po}-212 \\
\mathrm{Po}-216 \\
\mathrm{Ra}-224 \\
\mathrm{Ra}-228 \\
\mathrm{Ac}-228 \\
\mathrm{Th}-228 \\
\mathrm{Th}-231 \\
\mathrm{Th}-232 \\
\mathrm{Th}-234 \\
\mathrm{~Pa}-234 \\
\mathrm{~Pa}-234 \mathrm{~m} \\
\mathrm{U}-235 \\
\mathrm{U}-238\end{array}$ & $\begin{array}{c}\text { 3.97E+03 } \begin{array}{c}\text { Total Mass } \\
(\mathrm{kg} / \mathrm{yr})\end{array} \\
\begin{array}{c}\text { Activity } \\
(\mathrm{Ci} / \mathrm{Y} \text { ) }\end{array} \\
2.34 \mathrm{E}+02 \\
2.21 \mathrm{E}-04 \\
5.86 \mathrm{E}-04 \\
5.86 \mathrm{E}-04 \\
3.78 \mathrm{E}-04 \\
5.86 \mathrm{E}-04 \\
5.86 \mathrm{E}-04 \\
3.50 \mathrm{E}-03 \\
3.50 \mathrm{E}-03 \\
5.86 \mathrm{E}-04 \\
3.37 \mathrm{E}-03 \\
3.56 \mathrm{E}-02 \\
4.32 \mathrm{E}+00 \\
4.43 \mathrm{E}-04 \\
4.32 \mathrm{E}+00 \\
3.36 \mathrm{E}-03 \\
4.32 \mathrm{E}+00\end{array}$ & $2.88 \mathrm{E}+07$ \\
\hline
\end{tabular}




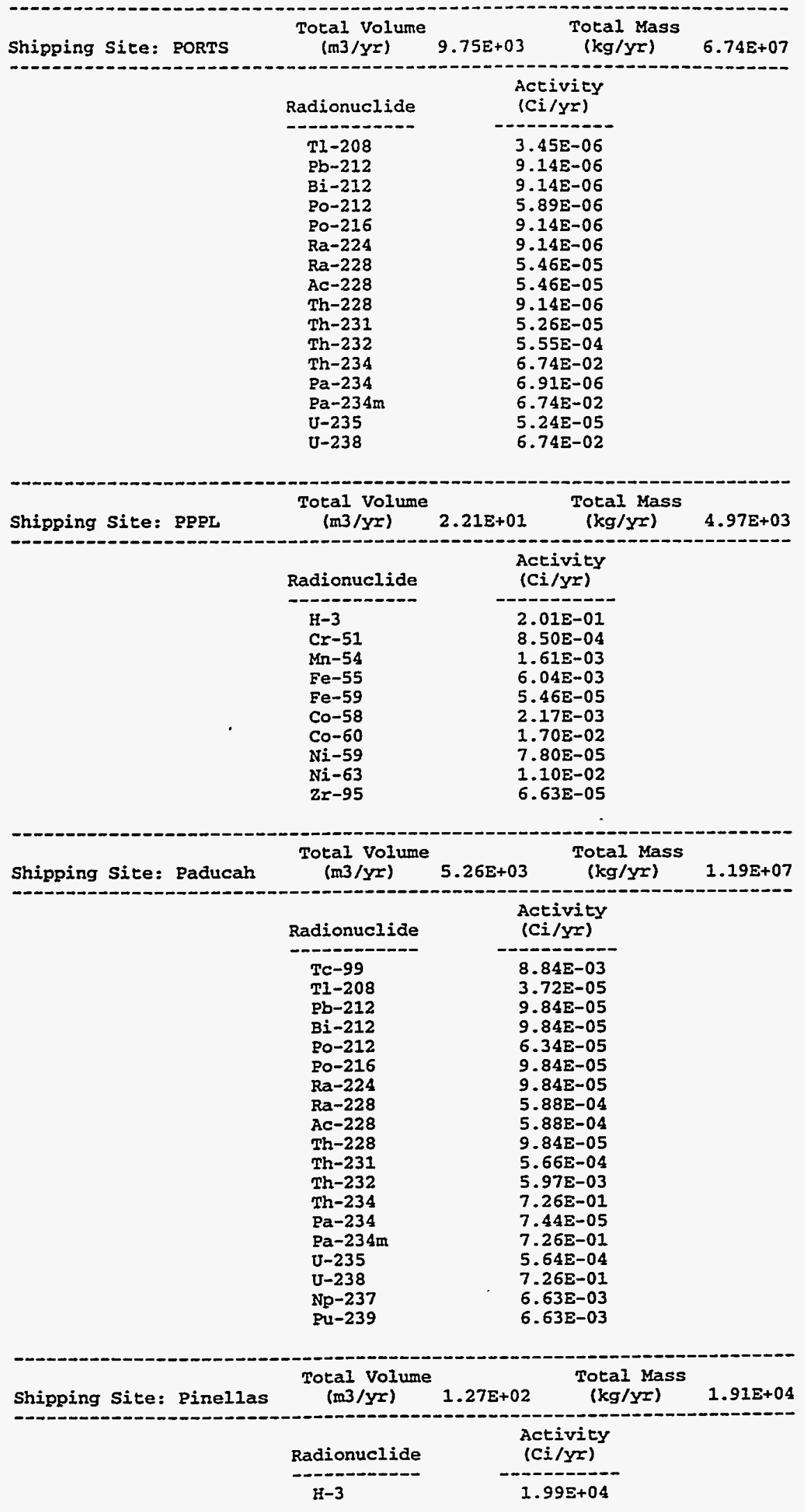


radioactive waste. For sites that do not have direct rail access, the rail siding nearest the site was used for routing purposes. The population densities along a route are derived from 1990 census data. Rural, suburban, and urban areas are characterized according to the following breakdown: rural population densities range from $0-54$ persons $/ \mathrm{km}^{2}\left(0-139 / \mathrm{mi}^{2}\right)$, the suburban range for population density is $55-1,284 / \mathrm{km}^{2}\left(140-3,326 / \mathrm{mi}^{2}\right)$, and urban is taken to mean all population densities greater than $1,284 / \mathrm{km}^{2}\left(3,326 / \mathrm{mi}^{2}\right)$. 
A-250

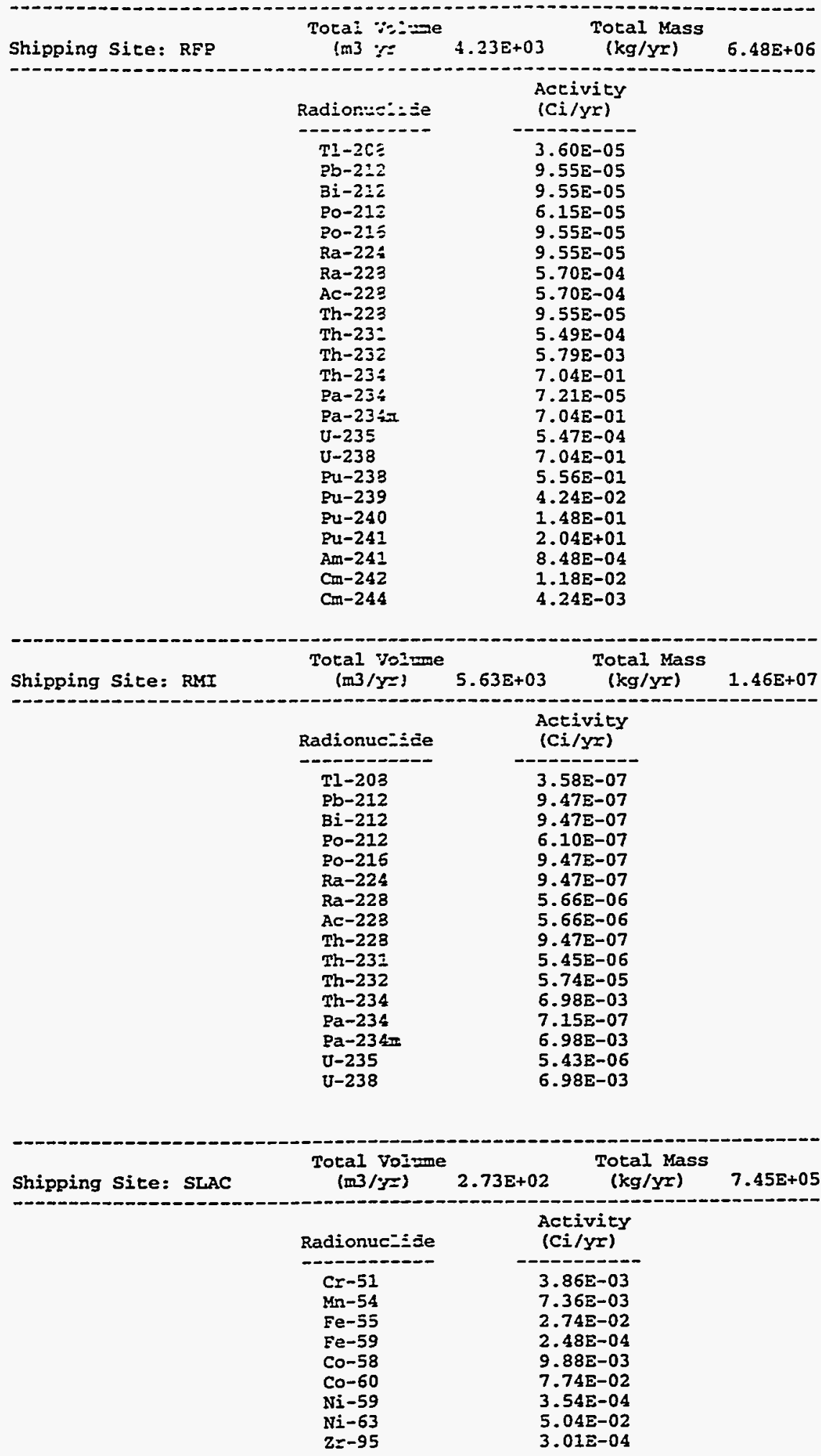




\begin{tabular}{|c|c|c|c|}
\hline Shipping Sice: SNLA & $\begin{array}{l}\text { Total Volume } \\
(\mathrm{m} 3 / \mathrm{y} \text { ) }\end{array}$ & $\begin{array}{l}\text { Total Mass } \\
(\mathrm{kg} / \mathrm{y} r)\end{array}$ & $3.28 \mathrm{E}+05$ \\
\hline & $\begin{array}{l}\text { Radionuclide } \\
\mathrm{H}-3 \\
\mathrm{Co}-60 \\
\mathrm{Ni}-63 \\
\mathrm{Sr}-90 \\
\mathrm{Y}-90 \\
\mathrm{Tc}-99 \\
\mathrm{Ru}-106 \\
\mathrm{Rh}-106 \\
\mathrm{Sb}-125 \\
\mathrm{Te}-125 \mathrm{~m} \\
\mathrm{Cs}-134 \\
\mathrm{Cs}-137 \\
\mathrm{Ba}-137 \mathrm{~m} \\
\mathrm{Ce}-144 \\
\mathrm{Pr}-144 \\
\mathrm{Pr}-144 \mathrm{~m} \\
\mathrm{Pm}-147 \\
\mathrm{Sm}-151 \\
\mathrm{Eu}-152 \\
\mathrm{Eu}-154 \\
\mathrm{Eu}-155 \\
\mathrm{~T} \mathrm{l}-208 \\
\mathrm{~Pb}-212 \\
\mathrm{Bi}-212 \\
\mathrm{Po}-212 \\
\mathrm{Po}-216 \\
\mathrm{Ra}-224 \\
\mathrm{Ra}-228 \\
\mathrm{Ac}-228 \\
\mathrm{Th}-228 \\
\mathrm{Th}-231 \\
\mathrm{Th}-232 \\
\mathrm{Th}-234 \\
\mathrm{~Pa}-234 \\
\mathrm{~Pa}-234 \mathrm{~m} \\
\mathrm{U}-235 \\
\mathrm{U}-238 \\
\mathrm{Pu}-238 \\
\mathrm{Pu}-239 \\
\mathrm{Pu}-240 \\
\mathrm{Pu}-241 \\
\mathrm{Am}-241 \\
\mathrm{Cm}-242 \\
\mathrm{Cm}-244 \\
\end{array}$ & 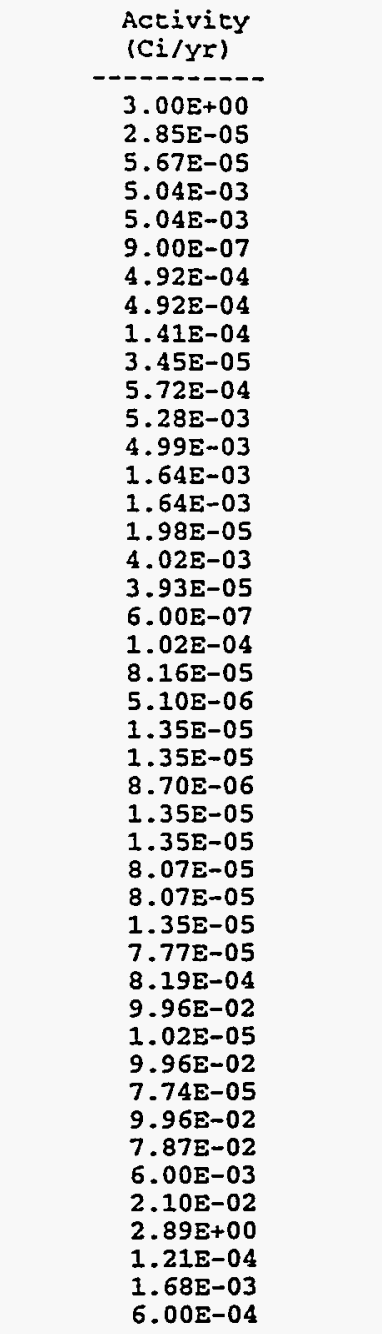 & \\
\hline Shipping Site: SNLL & $\begin{array}{l}\text { Total Volume } \\
\text { (m3/yr) }\end{array}$ & $\begin{array}{c}\text { Total Mass } \\
(\mathrm{kg} / \mathrm{yr})\end{array}$ & $9.36 E+04$ \\
\hline & $\begin{array}{l}\text { Radionuclide } \\
\mathrm{H}-3 \\
\mathrm{Fe}-55 \\
\mathrm{CO}-60 \\
\mathrm{~T} \mathrm{l}-208 \\
\mathrm{~Pb}-212 \\
\mathrm{~B}-212 \\
\mathrm{PO}-212 \\
\mathrm{PO}-216 \\
\mathrm{Ra}-224 \\
\mathrm{Ra}-228 \\
\mathrm{Ac}-228 \\
\mathrm{Th}-228 \\
\mathrm{Th}-231 \\
\mathrm{Th}-232 \\
\mathrm{Th}-234 \\
\mathrm{~Pa}-234 \\
\mathrm{~Pa}-234 \mathrm{~m} \\
\mathrm{U}-235 \\
\mathrm{U}-238\end{array}$ & \begin{tabular}{c}
$\begin{array}{c}\text { Activity } \\
\text { (Ci/yx) }\end{array}$ \\
\hdashline $2.82 \mathrm{E}+04$ \\
$3.15 \mathrm{E}-01$ \\
$3.15 \mathrm{E}-01$ \\
$6.40 \mathrm{E}-05$ \\
$1.69 \mathrm{E}-04$ \\
$1.69 \mathrm{E}-04$ \\
$1.09 \mathrm{E}-04$ \\
$1.69 \mathrm{E}-04$ \\
$1.69 \mathrm{E}-04$ \\
$1.01 \mathrm{E}-03$ \\
$1.01 \mathrm{E}-03$ \\
$1.69 \mathrm{E}-04$ \\
$9.75 \mathrm{E}-04$ \\
$1.02 \mathrm{E}-02$ \\
$1.25 \mathrm{E}+00$ \\
$1.28 \mathrm{E}-04$ \\
$1.25 \mathrm{E}+00$ \\
$9.71 \mathrm{E}-04$ \\
$1.25 \mathrm{E}+00$
\end{tabular} & \\
\hline
\end{tabular}




\begin{tabular}{|c|c|c|c|}
\hline Shipping Site: SRS & $\begin{array}{l}\text { Total Volume } \\
(\mathrm{m} 3 / \mathrm{yr})\end{array}$ & $\begin{array}{cc}5.62 \mathrm{E}+04 & \begin{array}{c}\text { Total Mass } \\
(\mathrm{kg} / \mathrm{y} 5)\end{array}\end{array}$ & $1.29 E+08$ \\
\hline & 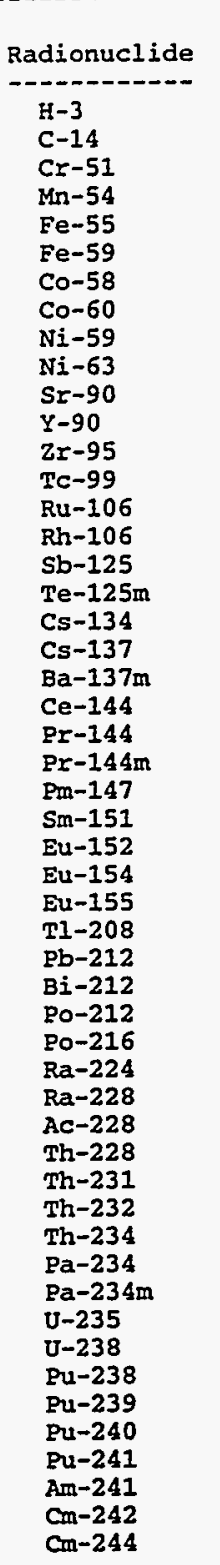 & 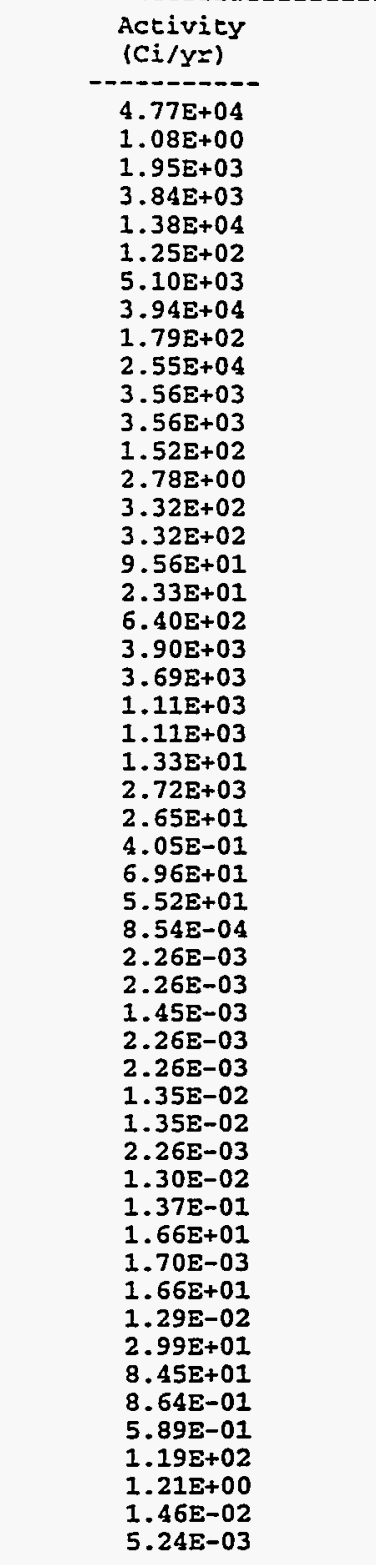 & \\
\hline Shipping Site: $Y-12$ & $\begin{array}{c}\text { Total Volume } \\
\text { (m3/yr) }\end{array}$ & $\begin{array}{cc}\text { Total Mass } \\
\text { 1.33E+04 }\end{array}$ & $8.27 E+07$ \\
\hline " & $\begin{array}{l}\text { Radionuclide } \\
\mathrm{Tl}-208 \\
\mathrm{~Pb}-212 \\
\mathrm{Bi}-212 \\
\mathrm{Po}-212 \\
\mathrm{Po}-216 \\
\mathrm{Ra}-224 \\
\mathrm{Ra}-228 \\
\mathrm{Ac}-228 \\
\mathrm{Th}-228 \\
\mathrm{Th}-231 \\
\mathrm{Th}-232 \\
\mathrm{Th}-234 \\
\mathrm{~Pa}-234 \\
\mathrm{~Pa}-234 \mathrm{~m} \\
\mathrm{U}-235 \\
\mathrm{U}-238\end{array}$ & $\begin{array}{l}\begin{array}{l}\text { Activity } \\
(\mathrm{Ci} / \mathrm{Yr})\end{array} \\
6.80 \mathrm{E}-05 \\
1.80 \mathrm{E}-04 \\
1.80 \mathrm{E}-04 \\
1.16 \mathrm{E}-04 \\
1.80 \mathrm{E}-04 \\
1.80 \mathrm{E}-04 \\
1.07 \mathrm{E}-03 \\
1.07 \mathrm{E}-03 \\
1.80 \mathrm{E}-04 \\
1.03 \mathrm{E}-03 \\
1.09 \mathrm{E}-02 \\
1.32 \mathrm{E}+00 \\
1.36 \mathrm{E}-04 \\
1.32 \mathrm{E}+00 \\
1.03 \mathrm{E}-03 \\
1.32 \mathrm{E}+00\end{array}$ & \\
\hline
\end{tabular}




\section{A.28 WM LLW CENTRALIZED 5 ALTERNATIVE (CASE 21): ACTIVATED METALS}

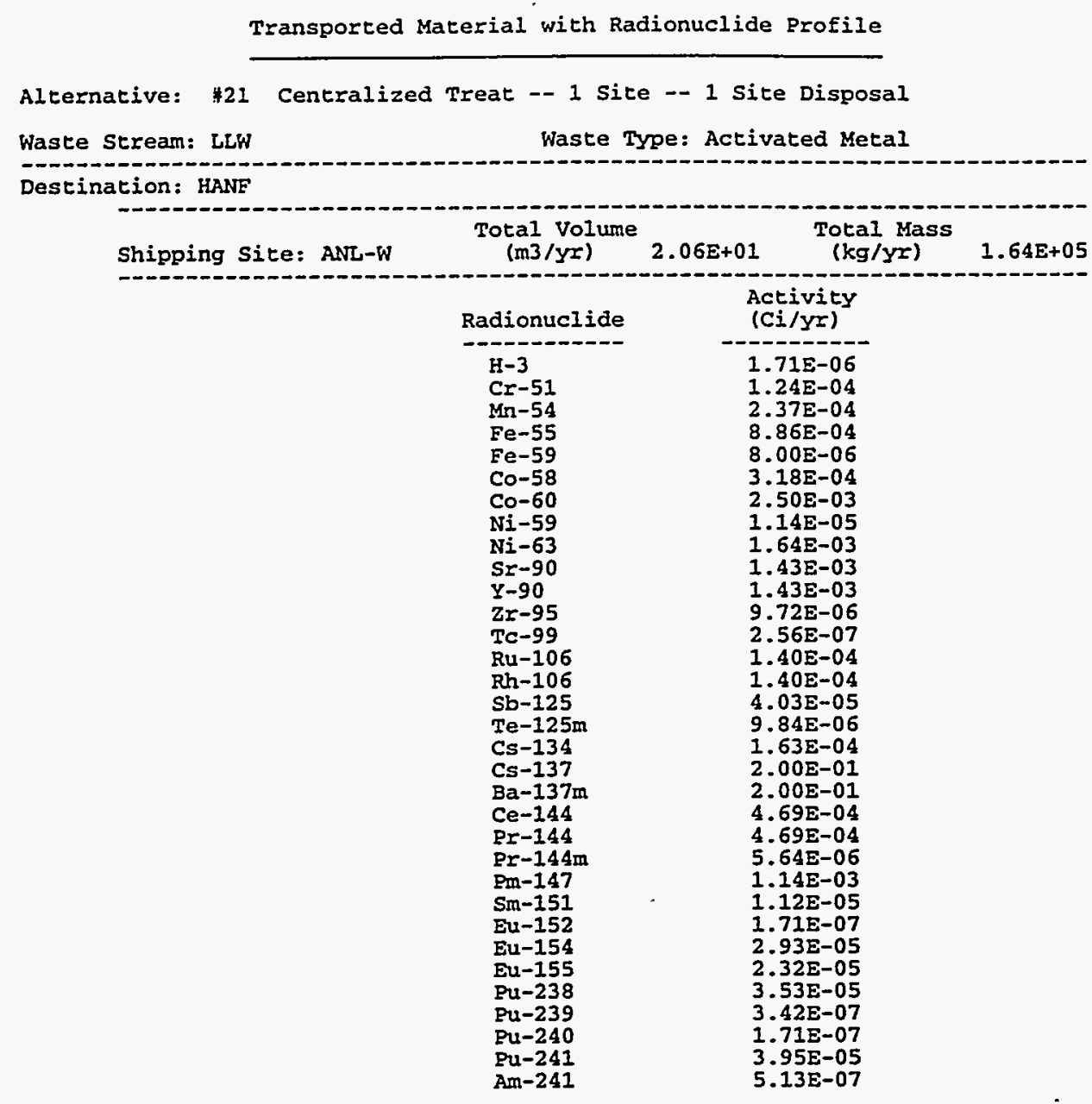




\begin{tabular}{|c|c|c|c|}
\hline Shipping sice: BAPL & $\begin{array}{l}\text { Total Volume } \\
(\mathrm{m} 3 / \mathrm{yr})\end{array}$ & $\begin{array}{l}\text { Total Mass } \\
\text { (kg/yr) }\end{array}$ & $6.43 \mathrm{E}+06$ \\
\hline & $\begin{array}{l}\text { Radionuclide } \\
\text { Cr-51 } \\
\text { Mn-54 } \\
\text { Fe-55 } \\
\text { Fe-59 } \\
\text { Co-58 } \\
\text { Co-60 } \\
\text { Ni-59 } \\
\text { Ni-63 } \\
\text { Zr-95 } \\
\text { T1-208 } \\
\text { Pb-212 } \\
\text { Bi-212 } \\
\text { Po-212 } \\
\text { Po-216 } \\
\text { Ra-224 } \\
\text { Ra-228 } \\
\text { Ac-228 } \\
\text { Th-228 } \\
\text { Th-231 } \\
\text { Th-232 } \\
\text { Th-234 } \\
\text { Pa-234 } \\
\text { Pa-234m } \\
\text { U-235 } \\
\text { U-238 }\end{array}$ & $\begin{array}{c}\text { Activity } \\
(C i / y x) \\
4.75 \mathrm{E}+03 \\
9.04 \mathrm{E}+03 \\
3.37 \mathrm{E}+04 \\
3.05 \mathrm{E}+02 \\
1.21 \mathrm{E}+04 \\
9.52 \mathrm{E}+04 \\
4.36 \mathrm{E}+02 \\
6.19 \mathrm{E}+04 \\
3.70 \mathrm{E}+02 \\
1.36 \mathrm{E}-08 \\
3.60 \mathrm{E}-08 \\
3.60 \mathrm{E}-08 \\
2.32 \mathrm{E}-08 \\
3.60 \mathrm{E}-08 \\
3.60 \mathrm{E}-08 \\
2.15 \mathrm{E}-07 \\
2.15 \mathrm{E}-07 \\
3.60 \mathrm{E}-08 \\
2.07 \mathrm{E}-07 \\
2.18 \mathrm{E}-06 \\
2.65 \mathrm{E}-04 \\
2.72 \mathrm{E}-08 \\
2.65 \mathrm{E}-04 \\
2.06 \mathrm{E}-07 \\
2.65 \mathrm{E}-04\end{array}$ & \\
\hline Shipping Site: ENAL & $\begin{array}{l}\text { Total Volume } \\
(\mathrm{m} 3 / \mathrm{y})\end{array}$ & $\begin{array}{l}\text { Toral Mass } \\
\text { (kg/y工) }\end{array}$ & $8.14 E+05$ \\
\hline & $\begin{array}{l}\text { Radionuclide } \\
\text { Cr-5I } \\
\text { Mn-54 } \\
\mathrm{Fe}-55 \\
\mathrm{Fe}-59 \\
\mathrm{Co}-58 \\
\mathrm{Co}-60 \\
\mathrm{Ni}-59 \\
\mathrm{Ni}-63 \\
\mathrm{Zr}-95\end{array}$ & $\begin{array}{c}\begin{array}{c}\text { Activity } \\
\text { (Ci/YI) }\end{array} \\
-3.35 \mathrm{E}-02 \\
6.39 \mathrm{E}-02 \\
2.38 \mathrm{E}-01 \\
2.15 \mathrm{E}-03 \\
8.57 \mathrm{E}-02 \\
6.72 \mathrm{E}-01 \\
3.08 \mathrm{E}-03 \\
4.37 \mathrm{E}-01 \\
2.61 \mathrm{E}-03\end{array}$ & \\
\hline
\end{tabular}


$A-255$

\begin{tabular}{|c|c|c|c|}
\hline Shipping Sice: KARL & $\begin{array}{l}\text { Total volume } \\
(\mathrm{m} 3 / \mathrm{y} r)\end{array}$ & $\begin{array}{cc}1.66 \mathrm{E}+03 & (\mathrm{~kg} / \mathrm{YY})\end{array}$ & $1.33 E+07$ \\
\hline & 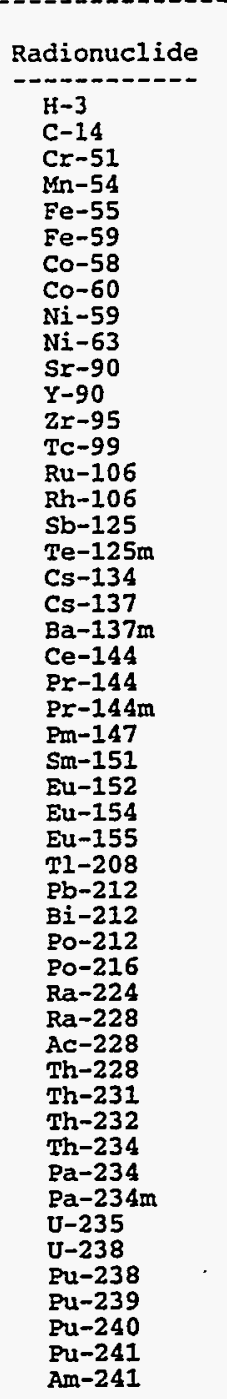 & $\begin{array}{l}\text { Activity } \\
\text { (Ci/YI) } \\
5.15 \mathrm{E}-04 \\
2.40 \mathrm{E}-05 \\
1.13 \mathrm{E}+04 \\
2.15 \mathrm{E}+04 \\
8.05 \mathrm{E}+04 \\
7.28 \mathrm{E}+02 \\
2.89 \mathrm{E}+04 \\
2.27 \mathrm{E}+05 \\
1.04 \mathrm{E}+03 \\
1.47 \mathrm{E}+05 \\
2.62 \mathrm{E}-02 \\
2.62 \mathrm{E}-02 \\
8.84 \mathrm{E}+02 \\
5.20 \mathrm{E}-05 \\
2.22 \mathrm{E}-03 \\
2.22 \mathrm{E}-03 \\
6.40 \mathrm{E}-04 \\
1.56 \mathrm{E}-04 \\
8.18 \mathrm{E}-03 \\
3.13 \mathrm{E}-02 \\
2.96 \mathrm{E}-02 \\
7.46 \mathrm{E}-03 \\
7.46 \mathrm{E}-03 \\
8.97 \mathrm{E}-05 \\
1.82 \mathrm{E}-02 \\
1.78 \mathrm{E}-04 \\
2.71 \mathrm{E}-06 \\
4.66 \mathrm{E}-04 \\
3.69 \mathrm{E}-04 \\
2.96 \mathrm{E}-08 \\
7.83 \mathrm{E}-08 \\
7.83 \mathrm{E}-08 \\
5.05 \mathrm{E}-08 \\
7.83 \mathrm{E}-08 \\
7.83 \mathrm{E}-08 \\
4.68 \mathrm{E}-07 \\
4.68 \mathrm{E}-07 \\
7.83 \mathrm{E}-08 \\
4.50 \mathrm{E}-07 \\
4.75 \mathrm{E}-06 \\
5.77 \mathrm{E}-04 \\
5.92 \mathrm{E}-08 \\
5.77 \mathrm{E}-04 \\
4.48 \mathrm{E}-07 \\
8.69 \mathrm{E}-04 \\
5.61 \mathrm{E}-04 \\
5.43 \mathrm{E}-06 \\
2.71 \mathrm{E}-06 \\
6.28 \mathrm{E}-04 \\
8.15 \mathrm{E}-06\end{array}$ & \\
\hline Shipping Site: IANL & $\begin{array}{l}\text { Total Volume } \\
\quad(\mathrm{m} 3 / \mathrm{yx})\end{array}$ & $\begin{array}{c}\text { Total Mass } \\
(\mathrm{kg} / \mathrm{Yr})\end{array}$ & $8.57 \mathrm{E}+06$ \\
\hline & $\begin{array}{l}\text { Radionuclide } \\
\text { Cr-51 } \\
\text { Mn-54 } \\
\text { Fe-55 } \\
\text { Fe-59 } \\
\text { Co-58 } \\
\text { Co-60 } \\
\text { Ni-59 } \\
\text { Ni-63 } \\
\text { Zr }-95\end{array}$ & $\begin{array}{c}\text { Activity } \\
\text { (Ci/YI) }\end{array}$ & \\
\hline
\end{tabular}




\begin{tabular}{|c|c|c|c|}
\hline Shipping Site: LBL & $\begin{array}{l}\text { Total Volume } \\
(\mathrm{m} 3 / \mathrm{y}) \mathrm{f}\end{array}$ & $\begin{array}{cc}\text { Total Mass } \\
(\mathrm{kg} / \mathrm{Y} \mathrm{I})\end{array}$ & $2.87 E+05$ \\
\hline & $\begin{array}{l}\text { Radionuclide } \\
\text { Cr-51 } \\
\text { Mn-54 } \\
\text { Fe-55 } \\
\text { Fe-59 } \\
\text { Co-58 } \\
\text { Co-60 } \\
N i-59 \\
N i-63 \\
\text { Zr-95 }\end{array}$ & $\begin{array}{l}\begin{array}{c}\text { Accivity } \\
\text { (Ci/yr) }\end{array} \\
-6.32 E-02 \\
6.20 \mathrm{E}-01 \\
1.49 \mathrm{E}-01 \\
4.06 \mathrm{I}-03 \\
4.061 \mathrm{E}-01 \\
1.61 \\
1.26 \mathrm{E}+00 \\
5.80 \mathrm{E}-03 \\
8.24 \mathrm{E}-01 \\
4.93 \mathrm{E}-03\end{array}$ & \\
\hline Shipping Site: NRF & $\begin{array}{l}\text { Total Volume } \\
\text { (m3/yr) }\end{array}$ & $\begin{array}{cc}\text { Total Mass } \\
(\mathrm{kg} / \mathrm{yr})\end{array}$ & $9.40 \mathrm{E}+06$ \\
\hline & $\begin{array}{l}\text { Radionuclide } \\
\mathrm{Cr}-51 \\
\mathrm{Mn}-54 \\
\mathrm{Fe}-55 \\
\mathrm{Fe}-59 \\
\mathrm{Co}-58 \\
\mathrm{Co}-60 \\
\mathrm{Ni}-59 \\
\mathrm{Ni}-63 \\
\mathrm{Zr}-95\end{array}$ & \begin{tabular}{l} 
Activity \\
(Ci/yr) \\
\hdashline $2.31 E+03$ \\
$4.40 E+03$ \\
$1.64 E+04$ \\
$1.48 E+02$ \\
$5.91 E+03$ \\
$4.63 E+04$ \\
$2.12 E+02$ \\
$3.01 E+04$ \\
$1.80 E+02$
\end{tabular} & \\
\hline Shipping Site: SMLA & $\begin{array}{l}\text { Total Volume } \\
(\mathrm{m} 3 / y x)\end{array}$ & $\begin{array}{c}\text { Total Mass } \\
(\mathrm{kg} / \mathrm{yr})\end{array}$ & $1.15 \mathrm{E}+05$ \\
\hline & $\begin{array}{l}\text { Radionuclide } \\
\mathrm{C} r-51 \\
\mathrm{Mn}-54 \\
\mathrm{Fe}-55 \\
\mathrm{Ee}-59 \\
\mathrm{Co}-58 \\
\mathrm{Co}-60 \\
\mathrm{Ni}-59 \\
\mathrm{Ni}-63 \\
\mathrm{Z}-95\end{array}$ & $\begin{array}{c}\begin{array}{c}\text { Activity } \\
\text { (Ci/yr) }\end{array} \\
-6.54 E-04 \\
6.54 \mathrm{E}-03 \\
1.24 \mathrm{E}-03 \\
4.64 \mathrm{E}-03 \\
4.20 \mathrm{E}-05 \\
1.67 \mathrm{E}-03 \\
1.31 \mathrm{E}-02 \\
6.00 \mathrm{E}-05 \\
8.53 \mathrm{E}-03 \\
5.10 \mathrm{E}-05\end{array}$ & \\
\hline
\end{tabular}


$B-1$

APPENDIX B:

TRANSPORTATION RISK ASSESSMENT RESULTS 
B-2 


\section{APPENDIX B:}

\section{TRANSPORTATION RISK ASSESSMENT RESULTS}

Detailed results of the collective population risk assessment for transportation of low-level waste (LLW) are presented in this appendix for the $14 \mathrm{WM}$ alternatives. For each alternative, results are presented separately for heterogeneous solids and activated metals. The total impacts of transportation for an alternative can be found by summing the quantities for heterogeneous solids and activated metals. In general, the quantity of activated metals transported is insignificant compared with the quantity of heterogeneous solids for each case.

The collective risk results are presented on an annual basis (cubic meters per year or kilograms per year). As stated previously, the Waste Management Programmatic Environmental Impact Statement (WM PEIS) considers a 20-year time frame for WM LLW. For most of the WM alternatives considered in the WM PEIS, the assumption was that shipments would occur uniformly over a 10-year period, which would allow an initial 10-year period to build treatment, storage, and disposal facilities. Sites would essentially store their own waste for the first 10 years and ship all waste over the subsequent 10 years; however, the assumption was that, for the No Action Alternative, shipments would be distributed uniformly over a 20 -year period because no new facilities would be required to be built. Therefore, although the total inventory considered is the same for all WM alternatives (current inventories plus 20 years of generation), the annual amounts of waste shipped, as reported in the WASTE_MGMT files in Appendix A, are a function of the assumption concerning the duration of the shipping campaigns for each alternative.

Because of the differences in the time frames noted and the uncertainties in the actual timing of future waste shipments, the transportation impacts for the various alternatives are best compared by examining the total impacts of transporting a specific inventory of waste, independent of the annual shipping rates. The total impacts of LLW transported for each alternative can be calculated by multiplying the annual shipment impacts by 20 years for the No Action Alternative and by 10 years for the other WM alternatives. The total impacts are provided in Section 5 of the main body of this report. 


\section{RISK ASSESSMENT ASSUMPTIONS AND PARAMETERS}

The majority of parameters and assumptions necessary to conduct the LLW transportation risk assessment are described in detail in Appendix E of the WM PEIS (DOE 1996). Supplemental information is provided subsequently for a number of the most important assessment parameters.

\subsection{SHIPPING AND PACKAGING ASSUMPTIONS FOR LOW-LEVEL WASTE}

Transportation of LLW is assumed to be in certified or certified-equivalent packagings, and exclusive-use vehicles are assumed to be used. Highway transportation is assumed to take place by legal-weight heavy-haul combination (tractor-trailer) trucks. Typically, Type A packages are transported on common flatbed or covered trailers. For transportation via truck, the maximum payload weight has been taken to be $19,958 \mathrm{~kg}(44,000 \mathrm{lb})$, on the basis of the DOT highway weight limitations and an average tractor-trailer weight of $16,329 \mathrm{~kg}(36,000 \mathrm{lb})$.

Rail transportation is assumed to take place by regular freight-train service. The use of special or dedicated train service was not considered in the analysis. For rail transportation, average payload weights for boxcars range from 45,359 to $68,039 \mathrm{~kg}(100,000-150,000 \mathrm{lb})$. A median payload weight of $54,431 \mathrm{~kg}(120,000 \mathrm{lb})$ has been assumed for purposes of assessment.

All LLW is assumed to be transported strong and tight or in Type A packaging, such as 208-L (55-gal) drums or standard waste boxes. Suitable Type A packagings are readily available from commercial sources. The number of shipments from a given site is calculated on the basis of projected site-specific information on waste inventory (weight) and on limitations on shipment capacity for each mode of transportation given previously. The effects of potential waste treatment, such as volume reduction or incineration, are reflected in changes in the density of the waste and are reflected in the WASTE_MGMT files in Appendix A. All shipments are assumed to be at the maximum weight limits for truck and rail shipments. On the basis of typical LLW densities, roughly eighty 208-L (55-gal) drums would be shipped per truck and 300 per railcar.

\subsection{ACCIDENT RATES}

For the calculation of accident risks, rates for vehicular accidents and for fatalities are taken from data provided by Saricks and Kvitek (1994). For each mode of transportation, accident rates are generically defined as the number of accident involvements (or fatalities) in a given year per unit of travel by that mode in that same year. Therefore, the rate is a fractional value, with accidentinvolvement count as the numerator of the fraction and vehicular activity (total traveled distance) as its denominator. Accident rates are generally determined for a multiyear period. For purposes of 


\section{B.1 WM LLW NO ACTION ALTERNATIVE (CASE 1): HETEROGENEOUS SOLIDS}

Table 1. Summary of Route Information

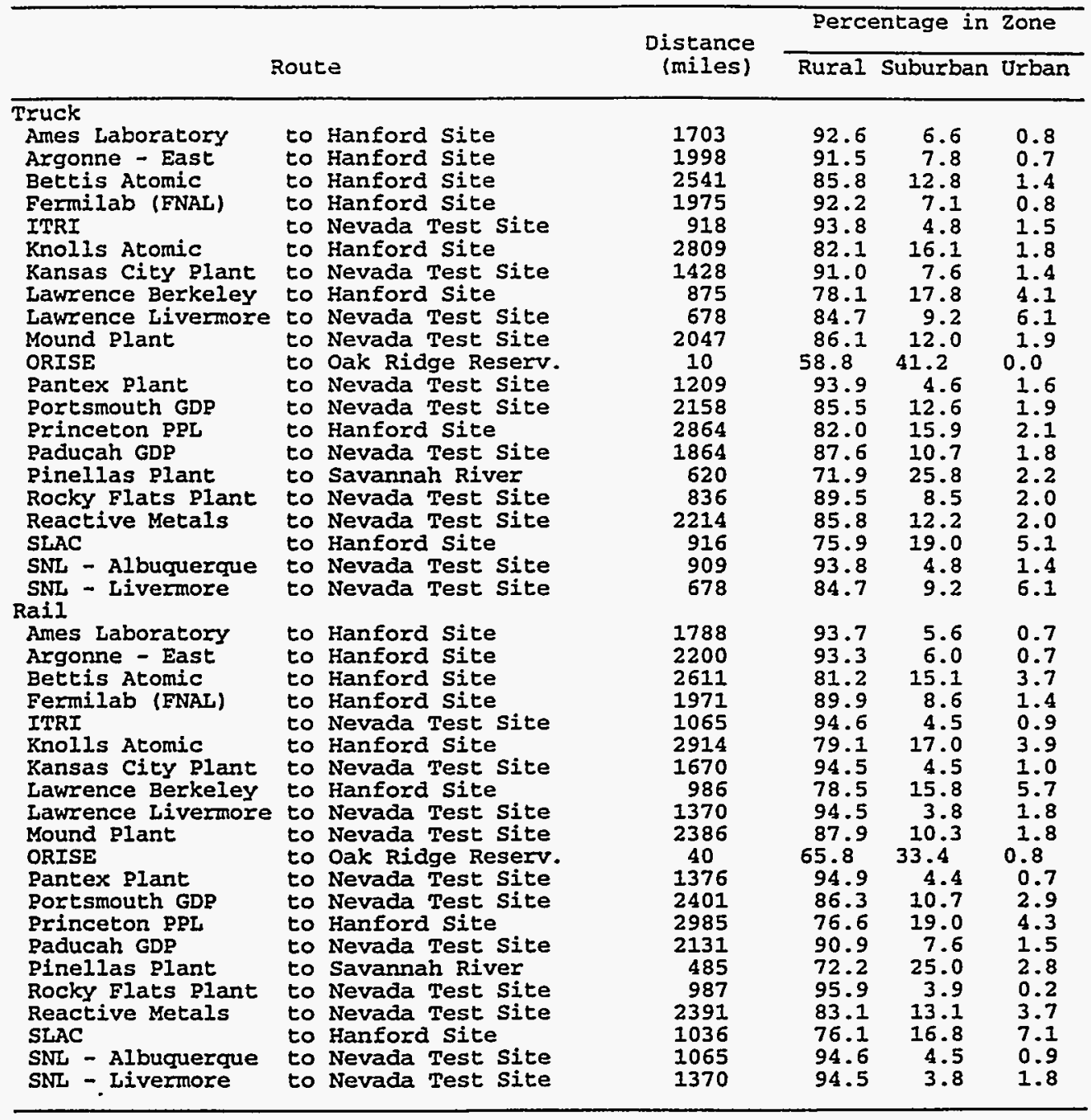




\section{B-5}

Table 2. Annual Number of Shipments and one-Way Shipment Distances

\begin{tabular}{|c|c|c|c|c|c|}
\hline \multirow[b]{2}{*}{ Route } & & \multicolumn{2}{|c|}{$\#$ of Shipments } & \multicolumn{2}{|c|}{ Total Mileage (mi) } \\
\hline & & Truck & Rail & Truck & Rail \\
\hline $\begin{array}{l}\text { Ames Laboratory } \\
\text { Argonne - East } \\
\text { Bettis Atomic } \\
\text { Fermilab (FNAL) } \\
\text { ITRI } \\
\text { Knolis Atomic } \\
\text { Kansas City Plant } \\
\text { Lawrence Berkeley } \\
\text { Lawrence Livernore } \\
\text { Mound Plant } \\
\text { ORISE } \\
\text { Pantex Plant } \\
\text { Portsmouth GDP } \\
\text { Princeton PPL } \\
\text { Paducah GDP } \\
\text { Pinellas Plant } \\
\text { Rocky Flats PIant } \\
\text { Reactive Metals } \\
\text { SLAC } \\
\text { SNL - Albuquerque } \\
\text { SNL - Livermore }\end{array}$ & $\begin{array}{l}\text { to Hanford Site } \\
\text { to Hanford Site } \\
\text { to Hanford Site } \\
\text { to Hanford Site } \\
\text { to Nevada Test Site } \\
\text { to Hanford Site } \\
\text { to Nevada Test Site } \\
\text { to Hanford Site } \\
\text { to Nevada Test Site } \\
\text { to Nevada Test Site } \\
\text { to Oak Ridge Reserv. } \\
\text { to Nevada Test Site } \\
\text { to Nevada Test Site } \\
\text { to Hanford Site } \\
\text { to Nevada Test Site } \\
\text { to Savannah River } \\
\text { to Nevada Test Site } \\
\text { to Nevada Test Site } \\
\text { to Hanford Site } \\
\text { to Nevada Test Site } \\
\text { to Nevada Test Site }\end{array}$ & $\begin{array}{r}1 \\
53 \\
29 \\
1 \\
5 \\
16 \\
1 \\
2 \\
16 \\
256 \\
1 \\
687 \\
1672 \\
1 \\
321 \\
2 \\
160 \\
365 \\
20 \\
9 \\
3\end{array}$ & $\begin{array}{r}1 \\
20 \\
11 \\
1 \\
2 \\
6 \\
1 \\
1 \\
6 \\
95 \\
1 \\
272 \\
637 \\
1 \\
120 \\
1 \\
60 \\
137 \\
8 \\
4 \\
1\end{array}$ & 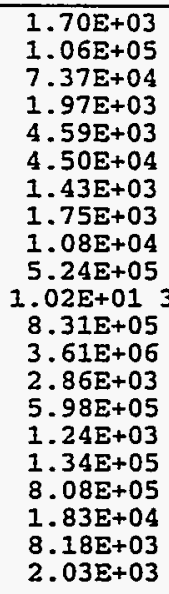 & $\begin{array}{l}1.79 E+03 \\
4.40 \mathrm{E}+04 \\
2.87 \mathrm{E}+04 \\
1.97 \mathrm{E}+03 \\
2.13 \mathrm{E}+03 \\
1.75 \mathrm{E}+04 \\
1.67 \mathrm{E}+03 \\
9.86 \mathrm{E}+02 \\
8.22 \mathrm{E}+03 \\
2.27 \mathrm{E}+05 \\
3.95 \mathrm{E}+01 \\
3.74 \mathrm{E}+05 \\
1.53 \mathrm{E}+06 \\
2.98 \mathrm{E}+03 \\
2.56 \mathrm{E}+05 \\
4.85 \mathrm{E}+02 \\
5.92 \mathrm{E}+04 \\
3.28 \mathrm{E}+05 \\
8.29 \mathrm{E}+03 \\
4.26 \mathrm{E}+03 \\
1.37 \mathrm{E}+03\end{array}$ \\
\hline Totals & & 3621 & 1386 & 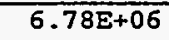 & $2.90 \mathrm{E}+06$ \\
\hline
\end{tabular}


Table 3. Incident-Free Dose per Shipment (person-rem/shipment)

\begin{tabular}{|c|c|c|c|c|c|}
\hline \multirow[b]{2}{*}{ Source/Route (s) } & \multirow[b]{2}{*}{ Crew } & \multicolumn{4}{|c|}{ General Public } \\
\hline & & off-Link & On-Link & Stops & Total \\
\hline $\begin{array}{l}\text { LLW(x) AL Eo HS } \\
\text { Ames Laboratory }\end{array}$ & $\begin{array}{lr}\text { to Hanford Sice } \\
\text { Truck } & 4.46 \mathrm{E}-02 \\
\text { Rail } & 1.03 \mathrm{E}-02\end{array}$ & $\begin{array}{l}1.62 \mathrm{E}-03 \\
4.69 \mathrm{E}-03\end{array}$ & $\begin{array}{l}5.39 \mathrm{E}-03 \\
1.46 \mathrm{E}-04\end{array}$ & $\begin{array}{l}4.77 \mathrm{E}-02 \\
8.98 \mathrm{E}-03\end{array}$ & $\begin{array}{l}5.47 \mathrm{E}-02 \\
1.38 \mathrm{E}-02\end{array}$ \\
\hline $\begin{array}{l}\text { LLW(x) AN to HS } \\
\text { Argonne - East }\end{array}$ & $\begin{array}{ll}\text { to Hanford Site } \\
\text { Truck } & 5.29 \mathrm{E}-02 \\
\text { Rail } & 1.21 \mathrm{E}-02\end{array}$ & $\begin{array}{l}1.97 \mathrm{E}-03 \\
5.75 \mathrm{E}-03\end{array}$ & $\begin{array}{l}6.28 \mathrm{E}-03 \\
1.82 \mathrm{E}-04\end{array}$ & $\begin{array}{l}5.59 \mathrm{E}-02 \\
1.03 \mathrm{E}-02\end{array}$ & $\begin{array}{l}6.42 \mathrm{E}-02 \\
1.62 \mathrm{E}-02\end{array}$ \\
\hline $\begin{array}{l}\text { LLW }(x) \text { BA to HS } \\
\text { Bettis Atomic }\end{array}$ & $\begin{array}{ll}\text { to Hanford Site } \\
\text { Truck } & 7.21 \mathrm{E}-02 \\
\text { Rail } & 1.38 \mathrm{E}-02\end{array}$ & $\begin{array}{l}4.36 E-03 \\
2.67 E-02\end{array}$ & $\begin{array}{l}9.77 \mathrm{E}-03 \\
4.53 \mathrm{E}-04\end{array}$ & $\begin{array}{l}7.11 \mathrm{E}-02 \\
1.15 \mathrm{E}-02\end{array}$ & $\begin{array}{l}8.53 E-02 \\
3.87 E-02\end{array}$ \\
\hline Fermilab (FNAL) & $\begin{array}{lr}\text { to Hanford Site } \\
\text { Truck } & 5.20 \mathrm{E}-02 \\
\text { Rail } & 1.11 \mathrm{E}-02\end{array}$ & $\begin{array}{l}1.92 \mathrm{E}-03 \\
9.07 \mathrm{E}-03\end{array}$ & $\begin{array}{l}6.25 \mathrm{E}-03 \\
2.12 \mathrm{E}-04\end{array}$ & $\begin{array}{l}5.53 \mathrm{E}-02 \\
9.55 \mathrm{E}-03\end{array}$ & $\begin{array}{l}6.35 E-02 \\
1.88 E-02\end{array}$ \\
\hline LLW $(x) \quad \mathrm{KA}$ to HS & $\begin{array}{ll}\text { to Nevada } & \text { Test Site } \\
\text { Truck } & 2.40 \mathrm{E}-02 \\
\text { Rail } & 7.32 \mathrm{E}-03\end{array}$ & $\begin{array}{l}1.05 E-03 \\
2.85 E-03\end{array}$ & $\begin{array}{l}3.25 E-03 \\
8.34 E-05\end{array}$ & $\begin{array}{l}2.57 E-02 \\
6.76 E-03\end{array}$ & $\begin{array}{l}3.00 \mathrm{E}-02 \\
9.69 \mathrm{E}-03\end{array}$ \\
\hline $\begin{array}{l}\text { LLW (x) KA to HS } \\
\text { Knolls Atomic }\end{array}$ & $\begin{array}{ll}\text { to Hanford Site } \\
\text { Truck } & 8.30 \mathrm{E}-02 \\
\text { Rail } & 1.50 \mathrm{E}-02\end{array}$ & $\begin{array}{l}6.03 \mathrm{E}-03 \\
3.18 \mathrm{E}-02\end{array}$ & $\begin{array}{l}1.19 \mathrm{E}-02 \\
5.40 \mathrm{E}-04\end{array}$ & $\begin{array}{l}7.87 \mathrm{E}-02 \\
1.25 \mathrm{E}-02\end{array}$ & $\begin{array}{l}9.66 \mathrm{E}-02 \\
4.48 \mathrm{E}-02\end{array}$ \\
\hline Kansas City Plant & $\begin{array}{ll}\text { to Nevada } & \text { Test Site } \\
\text { Truck } & 3.84 \mathrm{E}-02 \\
\text { Rail } & 9.85 \mathrm{E}-03\end{array}$ & $\begin{array}{l}1.90 \mathrm{E}-03 \\
4.98 \mathrm{E}-03\end{array}$ & $\begin{array}{l}5.17 E-03 \\
1.34 E-04\end{array}$ & $\begin{array}{l}4.00 \mathrm{E}-02 \\
8.62 \mathrm{E}-03\end{array}$ & $\begin{array}{l}4.70 \mathrm{E}-02 \\
1.37 \mathrm{E}-02\end{array}$ \\
\hline $\begin{array}{l}\text { LLW (x) LB to HS } \\
\text { Lawrence Berkeley }\end{array}$ & $\begin{array}{ll}\text { to Hanford Site } \\
\text { Truck } & 2.76 \mathrm{E}-02 \\
\text { Rail } & 6.99 \mathrm{E}-03\end{array}$ & $\begin{array}{l}3.02 \mathrm{E}-03 \\
1.38 \mathrm{E}-02\end{array}$ & $\begin{array}{l}5.17 E-03 \\
2.03 E-04\end{array}$ & $\begin{array}{l}2.45 E-02 \\
6.51 E-03\end{array}$ & $\begin{array}{l}3.27 \mathrm{E}-02 \\
2.05 \mathrm{E}-02\end{array}$ \\
\hline Iawrence Iivermore & $\begin{array}{ll}\text { to Nevada } & \text { Test Site } \\
\text { Truck } & 2.05 \mathrm{E}-02 \\
\text { Rail } & 8.60 \mathrm{E}-03\end{array}$ & $\begin{array}{l}2.57 E-03 \\
5.75 E-03\end{array}$ & $\begin{array}{l}4.65 E-03 \\
1.20 E-04\end{array}$ & $\begin{array}{l}1.90 E-02 \\
7.70 E-03\end{array}$ & $\begin{array}{l}2.62 \mathrm{E}-02 \\
1.36 \mathrm{E}-02\end{array}$ \\
\hline Mound Plant & $\begin{array}{ll}\text { to Nevada } & \text { Test Site } \\
\text { Truck } & 5.82 \mathrm{E}-02 \\
\text { Rail } & 1.28 \mathrm{E}-02\end{array}$ & $\begin{array}{r}\text { e } \\
3.93 \mathrm{E}-03 \\
1.36 \mathrm{E}-02\end{array}$ & $\begin{array}{l}8.53 \mathrm{E}-03 \\
2.90 \mathrm{E}-04\end{array}$ & $\begin{array}{l}5.73 \mathrm{E}-02 \\
1.08 \mathrm{E}-02\end{array}$ & $\begin{array}{l}6.98 \mathrm{E}-02 \\
2.47 \mathrm{E}-02\end{array}$ \\
\hline $\begin{array}{l}\text { LLW(x) OI to OR } \\
\text { ORISE } \\
\text { LLW(x) PP to NT }\end{array}$ & $\begin{array}{lr}\text { to Oak Ridge Reserv } \\
\text { Truck } & 3.63 \mathrm{E}-04 \\
\text { Rail } & 3.05 \mathrm{E}-03\end{array}$ & $\begin{array}{l}\mathrm{v} . \\
3.21 E-05 \\
3.36 \mathrm{E}-04\end{array}$ & $\begin{array}{l}4.23 \mathrm{E}-05 \\
8.77 \mathrm{E}-06\end{array}$ & $\begin{array}{l}2.86 \mathrm{E}-04 \\
3.60 \mathrm{E}-03\end{array}$ & $\begin{array}{l}3.60 \mathrm{E}-04 \\
3.95 \mathrm{E}-03\end{array}$ \\
\hline Pantex Plant & $\begin{array}{ll}\text { to Nevada Test Site } & \text { Truck } \\
\text { Truil } & 3.16 \mathrm{E}-02 \\
\text { Rail } & 8.62 \mathrm{E}-03\end{array}$ & $\begin{array}{l}\text { e } \\
1.43 E-03 \\
3.19 E-03\end{array}$ & $\begin{array}{l}4.34 \mathrm{E}-03 \\
1.03 \mathrm{E}-04\end{array}$ & $\begin{array}{l}3.38 \mathrm{E}-02 \\
7.72 \mathrm{E}-03\end{array}$ & $\begin{array}{l}3.96 \mathrm{E}-02 \\
1.10 \mathrm{E}-02\end{array}$ \\
\hline $\begin{array}{l}\text { LLW(x) Po to NT } \\
\text { Portsmouth GDP }\end{array}$ & $\begin{array}{ll}\text { to Nevada } & \text { Test Site } \\
\text { Truck } & 6.17 \mathrm{E}-02 \\
\text { Rail } & 1.29 \mathrm{E}-02\end{array}$ & $\begin{array}{r}4.18 \mathrm{E}-03 \\
1.88 \mathrm{E}-02\end{array}$ & $\begin{array}{l}8.96 \mathrm{E}-03 \\
3.36 \mathrm{E}-04\end{array}$ & $\begin{array}{l}6.04 E-02 \\
1.09 E-02\end{array}$ & $\begin{array}{l}7.36 \mathrm{E}-02 \\
3.01 \mathrm{E}-02\end{array}$ \\
\hline Princeton PPL & $\begin{array}{lr}\text { to Hanford Site } \\
\text { Truck } 8.51 \mathrm{E}-02 \\
\text { Rail } & 1.53 \mathrm{E}-02\end{array}$ & $\begin{array}{l}6.65 \mathrm{E}-03 \\
3.63 \mathrm{E}-02\end{array}$ & $\begin{array}{l}1.28 E-02 \\
6.03 E-04\end{array}$ & $\begin{array}{l}8.02 E-02 \\
1.27 E-02\end{array}$ & $\begin{array}{l}9.97 \mathrm{E}-02 \\
4.96 \mathrm{E}-02\end{array}$ \\
\hline Paducah GDP & $\begin{array}{ll}\text { to Nevada } & \text { Test Site } \\
\text { Truck } & 5.22 \mathrm{E}-02 \\
\text { Rail } & 1.18 \mathrm{E}-02\end{array}$ & $\begin{array}{r}3.26 E-03 \\
9.42 E-03\end{array}$ & $\begin{array}{l}7.47 \mathrm{E}-03 \\
2.18 \mathrm{E}-04\end{array}$ & $\begin{array}{l}5.22 \mathrm{E}-02 \\
1.00 \mathrm{E}-02\end{array}$ & $\begin{array}{l}6.29 \mathrm{E}-02 \\
1.97 \mathrm{E}-02\end{array}$ \\
\hline $\begin{array}{l}\text { LLW(x) PI to SR } \\
\text { Pinellas Plant }\end{array}$ & $\begin{array}{lr}\text { to Savannah River } \\
\text { Truck } & 2.02 \mathrm{E}-02 \\
\text { Rail } & 4.90 \mathrm{E}-03\end{array}$ & $\begin{array}{l}1.94 E-03 \\
5.13 E-03\end{array}$ & $\begin{array}{l}3.10 \mathrm{E}-03 \\
1.02 \mathrm{E}-04\end{array}$ & $\begin{array}{l}1.74 E-02 \\
4.97 E-03\end{array}$ & $\begin{array}{l}2.24 \mathrm{E}-02 \\
1.02 \mathrm{E}-02\end{array}$ \\
\hline $\begin{array}{l}\text { LLW (x) RF to NT } \\
\text { Rocky Flats plant }\end{array}$ & $\begin{array}{ll}\text { to Nevada } & \text { Test Site } \\
\text { Truck } & 2.30 \mathrm{E}-02 \\
\text { Rail } & 7.00 \mathrm{E}-03\end{array}$ & $\begin{array}{r}e \\
1.44 E-03 \\
1.29 E-03\end{array}$ & $\begin{array}{l}3.43 \mathrm{E}-03 \\
6.47 \mathrm{E}-05\end{array}$ & $\begin{array}{l}2.34 E-02 \\
6.52 E-03\end{array}$ & $\begin{array}{l}2.83 \mathrm{E}-02 \\
7.88 \mathrm{E}-03\end{array}$ \\
\hline $\begin{array}{l}\text { LLW(x) RM to NT } \\
\text { Reactive Metals }\end{array}$ & $\begin{array}{ll}\text { to Nevada } & \text { Test Site } \\
\text { Truck } & 6.33 \mathrm{E}-02 \\
\text { Rail } & 1.29 \mathrm{E}-02\end{array}$ & $\begin{array}{r}4.39 E-03 \\
2.35 \Xi-02\end{array}$ & $\begin{array}{l}9.38 \Xi-03 \\
3.91 \Xi-04\end{array}$ & $\begin{array}{l}6.20 \mathrm{E}-02 \\
1.08 \mathrm{E}-02\end{array}$ & $\begin{array}{l}7.58 \mathrm{E}-02 \\
3.47 \mathrm{E}-02\end{array}$ \\
\hline
\end{tabular}


LLW(X) ST to HS SLAC

LLW(x) SA tO NT SNL - Albuquerque

LLW(x) SL to $\mathrm{NT}$ SNL - Livermore to Hanford Site

$\begin{array}{llllll}\text { Truak } & 2.98 \mathrm{E}-02 & 3.70 \mathrm{E}-03 & 6.08 \mathrm{E}-03 & 2.56 \mathrm{E}-02 & 3.54 \mathrm{E}-02\end{array}$

Rail 7.20E-03 1.75E-02 2.40E-04 $6.67 \mathrm{E}-03 \quad 2.44 \mathrm{E}-02$

to Nevada Test Site

$\begin{array}{llllll}\text { Truck } & 2.37 \mathrm{E}-02 & 1.02 \mathrm{E}-03 & 3.18 \mathrm{E}-03 & 2.54 \mathrm{E}-02 & 2.97 \mathrm{E}-02\end{array}$

Rail 7.32E-03 2.85E-03 8.34E-05 6.76E-03 $9.69 \mathrm{E}-03$

to Nevada Test Site

$\begin{array}{lllllll}\text { Truck } & 2.05 \mathrm{E}-02 & 2.57 \mathrm{E}-03 & 4.65 \mathrm{E}-03 & 1.90 \mathrm{E}-02 & 2.62 \mathrm{E}-02\end{array}$

Rail $8.60 \mathrm{E}-03$ 5.75E-03 1.20E-04 $7.70 \mathrm{E}-03 \quad 1.36 \mathrm{E}-02$

Table 4. Accident Dose Risk per Shipment (person-rem/shipment)

\begin{tabular}{|c|c|c|c|}
\hline Source/Route & & Truck & Rail \\
\hline 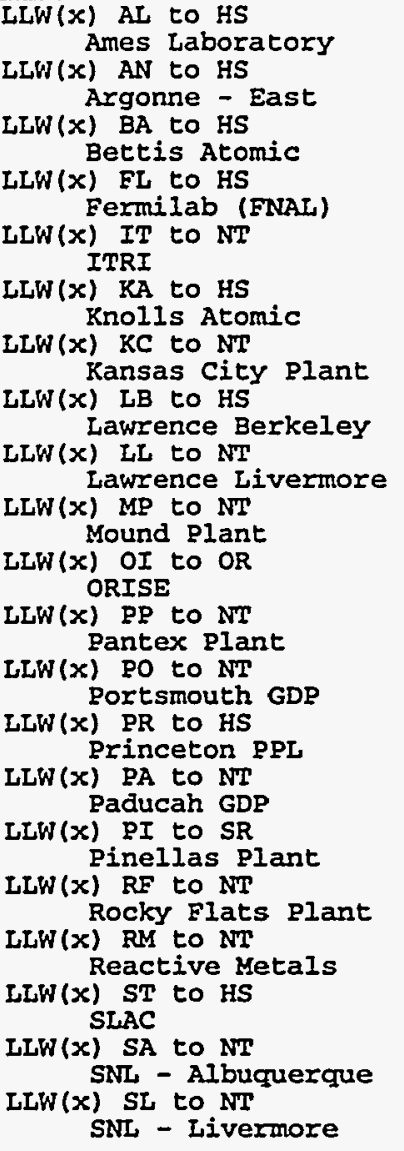 & $\begin{array}{l}\text { to Hanford Site } \\
\text { to Hanford Site } \\
\text { to Hanford Site } \\
\text { to Hanford Site } \\
\text { to Nevada Test Site } \\
\text { to Hanford Site } \\
\text { to Nevada Test Site } \\
\text { to Hanford Site } \\
\text { to Nevada Test Site } \\
\text { to Nevada Test Site } \\
\text { to Oak Ridge Reserv. } \\
\text { to Nevada Test Site } \\
\text { to Nevada Test Site } \\
\text { to Hanford Site } \\
\text { to Nevada Test Site } \\
\text { to Savannah River } \\
\text { to Nevada Test Site } \\
\text { to Nevada Test Site } \\
\text { to Hanford Site } \\
\text { to Nevada Test Site } \\
\text { to Nevada Test Site }\end{array}$ & $\begin{array}{l}5.20 E-06 \\
5.51 E-04 \\
4.37 E-05 \\
3.36 E-05 \\
2.18 E-03 \\
5.84 E-06 \\
1.27 E-05 \\
3.80 E-02 \\
7.11 E-06 \\
4.24 E-06 \\
1.12 E-07 \\
7.70 E-07 \\
2.36 E-08 \\
6.41 E-06 \\
1.21 E-06 \\
6.49 E-07 \\
4.77 E-06 \\
1.30 E-08 \\
4.22 E-07 \\
8.17 E-06 \\
3.63 E-05\end{array}$ & $\begin{array}{l}1.96 \mathrm{E}-06 \\
3.33 \mathrm{E}-04 \\
4.76 \mathrm{E}-05 \\
1.10 \mathrm{E}-05 \\
4.52 \mathrm{E}-04 \\
5.37 \mathrm{E}-06 \\
1.94 \mathrm{E}-06 \\
4.24 \mathrm{E}-02 \\
5.70 \mathrm{E}-06 \\
3.27 \mathrm{E}-06 \\
3.15 \mathrm{E}-08 \\
1.96 \mathrm{E}-07 \\
2.55 \mathrm{E}-08 \\
2.35 \mathrm{E}-06 \\
6.68 \mathrm{E}-07 \\
2.33 \mathrm{E}-07 \\
7.11 \mathrm{E}-07 \\
1.30 \mathrm{E}-08 \\
6.17 \mathrm{E}-07 \\
1.96 \mathrm{E}-06 \\
3.45 \mathrm{E}-05\end{array}$ \\
\hline
\end{tabular}


Table 5. Nonradiological Risk Factors per Shipment (Éatalities/shipment)

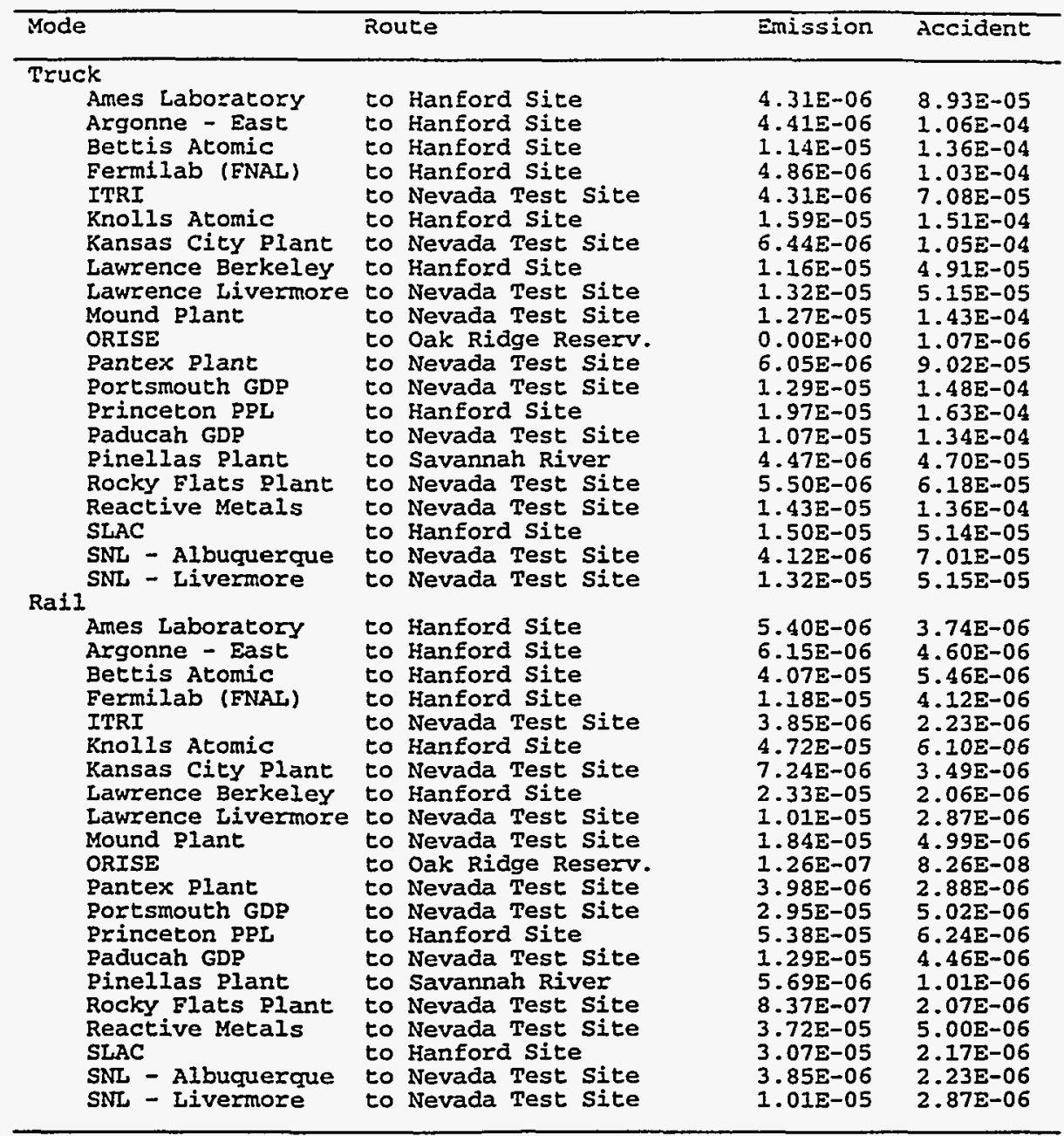

Table 6. Annual Incident-Free Dose for the Shipping Campaign (person-rem/yr)

\begin{tabular}{|c|c|c|c|c|c|c|}
\hline \multirow[b]{2}{*}{ Route } & & & \multicolumn{2}{|c|}{ Truck } & \multicolumn{2}{|c|}{ Rail } \\
\hline & & & Crew & Public & Crew & public \\
\hline $\begin{array}{l}\text { Ames Iaboratory } \\
\text { Argonne - East } \\
\text { Bettis Atomic } \\
\text { Fermilab (FNAL) } \\
\text { ITRI } \\
\text { Knolls Atomic } \\
\text { Kansas City Plant } \\
\text { Lawrence Berkeley } \\
\text { Lawrence Iivermore } \\
\text { Mound Plant } \\
\text { ORISE } \\
\text { Pantex Plant } \\
\text { Portsmouth GDP } \\
\text { Princeton PPL } \\
\text { Paducah GDP } \\
\text { Pinellas Plant } \\
\text { Rocky Flats Plant } \\
\text { Reactive Metals } \\
\text { SLAC } \\
\text { SNL - Albuquerque } \\
\text { SNL - Livermore }\end{array}$ & $\begin{array}{l}\text { to } \\
\text { to } \\
\text { to } \\
\text { to } \\
\text { to } \\
\text { to } \\
\text { to } \\
\text { to } \\
\text { to } \\
\text { to } \\
\text { to } \\
\text { to } \\
\text { to } \\
\text { to } \\
\text { to } \\
\text { to } \\
\text { to } \\
\text { to } \\
\text { to } \\
\text { to } \\
\text { to }\end{array}$ & $\begin{array}{l}\text { Hanford Site } \\
\text { Hanford Site } \\
\text { Hanford Site } \\
\text { Hanford Site } \\
\text { Nevada Test Site } \\
\text { Hanford Site } \\
\text { Nevada Test Site } \\
\text { Hanford Site } \\
\text { Nevada Test Site } \\
\text { Nevada Test Site } \\
\text { Oak Ridge Reserv. } \\
\text { Nevada Test Site } \\
\text { Nevada Test Site } \\
\text { Hanford Site } \\
\text { Nevada Test Site } \\
\text { Savannah River } \\
\text { Nevada Test Site } \\
\text { Nevada Test Site } \\
\text { Hanford Site } \\
\text { Nevada Test Site } \\
\text { Nevada Test Site }\end{array}$ & $\begin{array}{l}4.46 \mathrm{E}-02 \\
2.80 \mathrm{E}+00 \\
2.09 \mathrm{E}+00 \\
5.20 \mathrm{E}-02 \\
1.20 \mathrm{E}-01 \\
1.33 \mathrm{E}+00 \\
3.84 \mathrm{E}-02 \\
5.51 \mathrm{E}-02 \\
3.29 \mathrm{E}-01 \\
1.49 \mathrm{E}+01 \\
3.63 \mathrm{E}-04 \\
2.17 \mathrm{E}+01 \\
1.03 \mathrm{0}+02 \\
8.51 \mathrm{E}-02 \\
1.67 \mathrm{E}+01 \\
4.04 \mathrm{E}-02 \\
3.68 \mathrm{E}+00 \\
2.31 \mathrm{E}+01 \\
5.95 \mathrm{E}-01 \\
2.13 \mathrm{E}-01 \\
6.16 \mathrm{E}-02\end{array}$ & $\begin{array}{l}5.47 \mathrm{E}-02 \\
3.40 \mathrm{E}+00 \\
2.47 \mathrm{E}+00 \\
6.35 \mathrm{E}-02 \\
1.50 \mathrm{E}-01 \\
1.55 \mathrm{E}+00 \\
4.70 \mathrm{E}-02 \\
6.54 \mathrm{E}-02 \\
4.19 \mathrm{E}-01 \\
1.79 \mathrm{E}+01 \\
3.60 \mathrm{E}-04 \\
2.72 \mathrm{E}+01 \\
1.23 \mathrm{E}+02 \\
9.97 \mathrm{E}-02 \\
2.02 \mathrm{0}+01 \\
4.48 \mathrm{0}-02 \\
4.52 \mathrm{E}+00 \\
2.77 \mathrm{E}+01 \\
7.08 \mathrm{0}-01 \\
2.67 \mathrm{E}-01 \\
7.86 \mathrm{E}-02\end{array}$ & $\begin{array}{l}1.03 \mathrm{E}-02 \\
2.41 \mathrm{E}-01 \\
1.52 \mathrm{E}-01 \\
1.11 \mathrm{E}-02 \\
1.46 \mathrm{E}-02 \\
9.02 \mathrm{E}-02 \\
9.85 \mathrm{E}-03 \\
6.99 \mathrm{E}-03 \\
5.16 \mathrm{E}-02 \\
1.22 \mathrm{E}+00 \\
3.05 \mathrm{E}-03 \\
2.35 \mathrm{E}+00 \\
8.22 \mathrm{E}+00 \\
1.53 \mathrm{E}-02 \\
1.41 \mathrm{E}+00 \\
4.90 \mathrm{E}-03 \\
4.20 \mathrm{E}-01 \\
1.76 \mathrm{E}+00 \\
5.76 \mathrm{E}-02 \\
2.93 \mathrm{E}-02 \\
8.60 \mathrm{E}-03\end{array}$ & $\begin{array}{l}1.38 \mathrm{E}-02 \\
3.24 \mathrm{E}-01 \\
4.26 \mathrm{E}-01 \\
1.88 \mathrm{E}-02 \\
1.94 \mathrm{E}-02 \\
2.69 \mathrm{E}-01 \\
1.37 \mathrm{E}-02 \\
2.05 \mathrm{E}-02 \\
8.14 \mathrm{E}-02 \\
2.35 \mathrm{E}+00 \\
3.95 \mathrm{E}-03 \\
3.00 \mathrm{E}+00 \\
1.91 \mathrm{E}+01 \\
4.96 \mathrm{E}-02 \\
2.36 \mathrm{E}+00 \\
1.02 \mathrm{E}-02 \\
4.73 \mathrm{E}-01 \\
4.76 \mathrm{E}+00 \\
1.95 \mathrm{E}-01 \\
3.88 \mathrm{E}-02 \\
1.36 \mathrm{E}-02\end{array}$ \\
\hline
\end{tabular}


Table 7. Annual Accident Dose Risk for the Shipping Campaign (person-rem/yr)

\begin{tabular}{|c|c|c|c|}
\hline Route & & Truck & Rail \\
\hline $\begin{array}{l}\text { Ames Laboratory } \\
\text { Argonne - East } \\
\text { Bettis Atomic } \\
\text { Fermilab (INAL) } \\
\text { ITRI } \\
\text { Rnolls Atomic } \\
\text { Kansas City Plant } \\
\text { Lawrence Berkeley } \\
\text { Lawrence Livermore } \\
\text { Mound Plant } \\
\text { ORISE } \\
\text { Pantex Plant } \\
\text { Portsmouth GDP } \\
\text { Princeton PPL } \\
\text { Paducah GDP } \\
\text { Pinellas Plant } \\
\text { Rocky Flats Plant } \\
\text { Reactive Metals } \\
\text { SLAC } \\
\text { SNL - Albuquerque } \\
\text { SNL - Livermore }\end{array}$ & $\begin{array}{l}\text { to Hanford Site } \\
\text { to Hanford Site } \\
\text { to Hanford Site } \\
\text { to Hanford Site } \\
\text { to Nevada Test Site } \\
\text { to Hanford Site } \\
\text { to Nevada Test Site } \\
\text { to Hanford Site } \\
\text { to Nevada Test Site } \\
\text { to Nevada Test Site } \\
\text { to Oak Ridge Reserv. } \\
\text { to Nevada Test Site } \\
\text { to Nevada Test Site } \\
\text { to Hanford Site } \\
\text { to Nevada Test Site } \\
\text { to Savannah River } \\
\text { to Nevada Test Site } \\
\text { to Nevada Test Site } \\
\text { to Hanford Site } \\
\text { to Nevada Test Site } \\
\text { to Nevada Test Site }\end{array}$ & $\begin{array}{l}5.20 \mathrm{E}-06 \\
2.92 \mathrm{E}-02 \\
1.27 \mathrm{E}-03 \\
3.36 \mathrm{E}-05 \\
1.09 \mathrm{E}-02 \\
9.34 \mathrm{E}-05 \\
1.27 \mathrm{E}-05 \\
7.61 \mathrm{E}-02 \\
1.14 \mathrm{E}-04 \\
1.09 \mathrm{E}-03 \\
1.12 \mathrm{E}-07 \\
5.29 \mathrm{E}-04 \\
3.95 \mathrm{E}-05 \\
6.41 \mathrm{E}-06 \\
3.88 \mathrm{E}-04 \\
1.30 \mathrm{E}-06 \\
7.64 \mathrm{E}-04 \\
4.74 \mathrm{E}-06 \\
8.43 \mathrm{E}-06 \\
7.35 \mathrm{E}-05 \\
1.09 \mathrm{E}-04\end{array}$ & $\begin{array}{l}1.96 \mathrm{E}-06 \\
6.66 \mathrm{E}-03 \\
5.24 \mathrm{E}-04 \\
1.10 \mathrm{E}-05 \\
9.03 \mathrm{E}-04 \\
3.22 \mathrm{E}-05 \\
1.94 \mathrm{E}-06 \\
4.24 \mathrm{E}-02 \\
3.42 \mathrm{E}-05 \\
3.11 \mathrm{E}-04 \\
3.15 \mathrm{E}-08 \\
5.34 \mathrm{E}-05 \\
1.63 \mathrm{E}-05 \\
2.35 \mathrm{E}-06 \\
8.02 \mathrm{E}-05 \\
2.33 \mathrm{E}-07 \\
4.27 \mathrm{E}-05 \\
1.78 \mathrm{E}-06 \\
4.94 \mathrm{E}-06 \\
7.84 \mathrm{E}-06 \\
3.45 \mathrm{E}-05\end{array}$ \\
\hline Totals & & 1.21 & $\overline{5.11 E-02}$ \\
\hline
\end{tabular}

Table 8. Expected Annual Fatalities

for the Shipping Campaign

\begin{tabular}{lll}
\hline Exposure Group & Truck & Rail \\
\hline Radiological & & \\
Normal Crew & $7.6 \mathrm{E}-02$ & $6.4 \mathrm{E}-03$ \\
Normal Public & $1.1 \mathrm{E}-01$ & $1.7 \mathrm{E}-02$ \\
Accident Public & $6.0 \mathrm{E}-05$ & $2.6 \mathrm{E}-05$ \\
Nonradiological & & \\
Emission & $4.0 \mathrm{E}-02$ & $3.0 \mathrm{E}-02$ \\
Accident & $4.6 \mathrm{E}-01$ & $6.1 \mathrm{E}-03$ \\
\hline
\end{tabular}

Table 9. Expected Annual Cancer Incidence for the Shipping Campaign

\begin{tabular}{lll}
\hline Exposure Group & Truck & \multicolumn{1}{c}{ Rail } \\
\hline Radiological & & \\
$\quad$ Normal Crew & $2.7 E-01$ & $2.3 E-02$ \\
Normal Public & $3.9 E-01$ & $5.7 E-02$ \\
Accident Public & $2.1 \mathrm{E}-04$ & $8.7 \mathrm{E}-05$ \\
Nonradiological & $4.0 \mathrm{E}-02$ & $3.0 \mathrm{E}-02$ \\
Emission & $\mathrm{NA}$ & $\mathrm{NA}$ \\
Accident & &
\end{tabular}

Table 10. Expected Annual Genetic Effects for the Shipping Campaign

\begin{tabular}{lll}
\hline Exposure Group & Truck & Rail \\
\hline Radiological & & \\
Normal Crew & $1.1 E-02$ & $9.6 \mathrm{E}-04$ \\
Normal public & $2.3 \mathrm{E}-02$ & $3.4 \mathrm{E}-03$ \\
Accident Public & $3.6 \mathrm{E}-06$ & $1.3 \mathrm{E}-06$ \\
$\begin{array}{c}\text { Nonradiological } \\
\text { Emission }\end{array}$ & NA & NA \\
Accident & NA & NA \\
\hline
\end{tabular}




\section{B.2 WM LLW NO ACTION ALTERNATIVE (CASE 1): ACTIVATED METALS}

Table 1. Sumnary of Route Information

\begin{tabular}{|c|c|c|c|c|c|}
\hline & \multirow[b]{2}{*}{ Route } & \multirow{2}{*}{$\begin{array}{l}\text { Distance } \\
\text { (miles) }\end{array}$} & \multicolumn{3}{|c|}{ Percentage in zone } \\
\hline & & & -Rural & Suburban & Urban \\
\hline $\begin{array}{l}\text { Truck } \\
\text { Bettis Atomic } \\
\text { Fermilab (FNAL) } \\
\text { Knolls Atomic } \\
\text { Lawrence Berkeley } \\
\text { Naval Reactor Fac. } \\
\text { SNL - Albuquerque }\end{array}$ & $\begin{array}{l}\text { to Hanford Site } \\
\text { to Hanford Site } \\
\text { to Hanford Site } \\
\text { to Hanford Site } \\
\text { to Hanford Site } \\
\text { to Nevada Test Site }\end{array}$ & $\begin{array}{r}2541 \\
1975 \\
2809 \\
875 \\
599 \\
909\end{array}$ & $\begin{array}{l}85.8 \\
92.2 \\
82.1 \\
78.1 \\
91.3 \\
93.8\end{array}$ & $\begin{array}{r}12.8 \\
7.1 \\
16.1 \\
17.8 \\
7.6 \\
4.8\end{array}$ & $\begin{array}{l}1.4 \\
0.8 \\
1.8 \\
4.1 \\
1.1 \\
1.4\end{array}$ \\
\hline $\begin{array}{l}\text { Rail } \\
\text { Bettis Atomic } \\
\text { Fermilab (FNAL) } \\
\text { Knolls Atomic } \\
\text { Lawrence Berkeley } \\
\text { Naval Reactor Fac. } \\
\text { SNL - Albuquerque }\end{array}$ & $\begin{array}{l}\text { to Hanford Site } \\
\text { to Hanford Site } \\
\text { to Hanford Site } \\
\text { to Hanford Site } \\
\text { to Hanford Site } \\
\text { to Nevada Test Site }\end{array}$ & $\begin{array}{r}2611 \\
1971 \\
2914 \\
986 \\
658 \\
1065\end{array}$ & $\begin{array}{l}81.2 \\
89.9 \\
79.1 \\
78.5 \\
91.4 \\
94.6\end{array}$ & $\begin{array}{r}15.1 \\
8.6 \\
17.0 \\
15.8 \\
7.1 \\
4.5\end{array}$ & $\begin{array}{l}3.7 \\
1.4 \\
3.9 \\
5.7 \\
1.4 \\
0.9\end{array}$ \\
\hline
\end{tabular}

Table 2. Annual Number of Shipments and One-Way Shipment Distances

\begin{tabular}{|c|c|c|c|c|c|}
\hline \multirow[b]{2}{*}{ Route } & & \multicolumn{2}{|c|}{ \# of Shipments } & \multicolumn{2}{|c|}{ Total Mileage (mi) } \\
\hline & & Truck & Rail & Truck & Rail \\
\hline $\begin{array}{l}\text { Bettis Atomic } \\
\text { Fermilab (FNAL) } \\
\text { Knolls Atomic } \\
\text { Lawrence Berkeley } \\
\text { Naval Reactor Fac. } \\
\text { SNL - Albuquerque }\end{array}$ & $\begin{array}{l}\text { to Hanford Site } \\
\text { to Hanford Site } \\
\text { to Hanford Site } \\
\text { to Hanford Site } \\
\text { to Hanford Site } \\
\text { to Nevada Test Site }\end{array}$ & $\begin{array}{r}158 \\
20 \\
327 \\
8 \\
231 \\
3\end{array}$ & $\begin{array}{r}60 \\
8 \\
124 \\
3 \\
88 \\
2\end{array}$ & $\begin{array}{l}4.01 E+05 \\
3.95 E+04 \\
9.19 E+05 \\
7.00 E+03 \\
1.38 E+05 \\
2.73 E+03\end{array}$ & $\begin{array}{l}1.57 \mathrm{E}+05 \\
1.58 \mathrm{E}+04 \\
3.61 \mathrm{E}+05 \\
2.96 \mathrm{E}+03 \\
5.79 \mathrm{E}+04 \\
2.13 \mathrm{E}+03\end{array}$ \\
\hline Totals & & 747 & 285 & $1.51 E+06$ & $5.97 \mathrm{E}+05$ \\
\hline
\end{tabular}

Table 3. Incident-Free Dose per Shipment (person-rem/shipment)

\begin{tabular}{|c|c|c|c|c|c|}
\hline \multirow[b]{2}{*}{ Source/Route (s) } & \multirow[b]{2}{*}{ Crew } & \multicolumn{4}{|c|}{ General Public } \\
\hline & & Off-Link & On-Iink & Stops & TotaI \\
\hline $\begin{array}{l}\text { LLW(I) BA to HS } \\
\text { Bettis Atomic }\end{array}$ & $\begin{array}{ll}\text { to Hanford Site } \\
\text { Truck } & 7.21 \mathrm{E}-02 \\
\text { Rail } & 1.38 \mathrm{E}-02\end{array}$ & $\begin{array}{l}4.36 \mathrm{E}-03 \\
2.67 \mathrm{E}-02\end{array}$ & $\begin{array}{l}9.77 E-03 \\
4.53 E-04\end{array}$ & $\begin{array}{l}7.11 \mathrm{E}-02 \\
1.15 \mathrm{E}-02\end{array}$ & $\begin{array}{l}8.53 E-02 \\
3.87 E-02\end{array}$ \\
\hline $\begin{array}{l}\text { LIW(I) FL to HS } \\
\text { Fermilab (FNAL) }\end{array}$ & $\begin{array}{ll}\text { to Hanford Site } \\
\text { Truck } 5.20 \mathrm{E}-02 \\
\text { Rail } & 1.11 \mathrm{E}-02\end{array}$ & $\begin{array}{l}1.92 E-03 \\
9.07 E-03\end{array}$ & $\begin{array}{l}6.25 \mathrm{E}-03 \\
2.12 \mathrm{E}-04\end{array}$ & $\begin{array}{l}5.53 E-02 \\
9.55 E-03\end{array}$ & $\begin{array}{l}6.35 \mathrm{E}-02 \\
1.88 \mathrm{E}-02\end{array}$ \\
\hline $\begin{array}{l}\text { LLW(I) KA to HS } \\
\text { Knolls Atomic }\end{array}$ & $\begin{array}{lr}\text { to Hanford Site } \\
\text { Truck } & 8.30 \mathrm{E}-02 \\
\text { Rail } & 1.50 \mathrm{E}-02\end{array}$ & $\begin{array}{l}6.03 E-03 \\
3.18 E-02\end{array}$ & $\begin{array}{l}1.19 \mathrm{E}-02 \\
5.40 \mathrm{E}-04\end{array}$ & $\begin{array}{l}7.87 \mathrm{E}-02 \\
1.25 \mathrm{E}-02\end{array}$ & $\begin{array}{l}9.66 \mathrm{E}-02 \\
4.48 \mathrm{E}-02\end{array}$ \\
\hline $\begin{array}{l}\text { LLW(I) IB to HS } \\
\text { Lawrence Berkeley }\end{array}$ & $\begin{array}{ll} & \\
\text { to Hanford Site } \\
\text { Truck } & 2.76 \mathrm{E}-02 \\
\text { Rail } & 6.99 \mathrm{E}-03\end{array}$ & $\begin{array}{l}3.02 \mathrm{E}-03 \\
1.38 \mathrm{E}-02\end{array}$ & $\begin{array}{l}5.17 E-03 \\
2.03 E-04\end{array}$ & $\begin{array}{l}2.45 \mathrm{E}-02 \\
6.51 \mathrm{E}-03\end{array}$ & $\begin{array}{l}3.27 E-02 \\
2.05 E-02\end{array}$ \\
\hline $\begin{array}{l}\text { LLW(I) NR to HS } \\
\text { Naval Reactor Fac. }\end{array}$ & $\begin{array}{l}\text { to Hanford Site } \\
\text { Truck } \\
\text { Rail } \\
\text { R.60E-02 } \\
5.63 \mathrm{E}-03\end{array}$ & $\begin{array}{l}7.18 E-04 \\
2.83 E-03\end{array}$ & $\begin{array}{l}2.06 \mathrm{E}-03 \\
6.58 \mathrm{E}-05\end{array}$ & $\begin{array}{l}1.68 E-02 \\
5.51 \mathrm{E}-03\end{array}$ & $\begin{array}{l}1.95 \mathrm{E}-02 \\
8.40 \mathrm{E}-03\end{array}$ \\
\hline $\begin{array}{l}\text { LLW(I) SA to NT } \\
\text { SNL - Albuquerque }\end{array}$ & $\begin{array}{ll}\text { to Nevada } & \text { Test Sit } \\
\text { Truck } & 2.37 \mathrm{E}-02 \\
\text { Rail } & 7.32 \mathrm{E}-03\end{array}$ & $\begin{array}{l}\text { e } \\
1.02 E-03 \\
2.85 E-03\end{array}$ & $\begin{array}{l}3.18 \mathrm{E}-03 \\
8.34 \mathrm{E}-05\end{array}$ & $\begin{array}{l}2.54 \mathrm{E}-02 \\
6.76 \mathrm{E}-03\end{array}$ & $\begin{array}{l}2.97 \mathrm{E}-02 \\
9.69 \mathrm{E}-03\end{array}$ \\
\hline
\end{tabular}


Table 4. Accident Dose Risk per Shipment (person-rem/shipment)

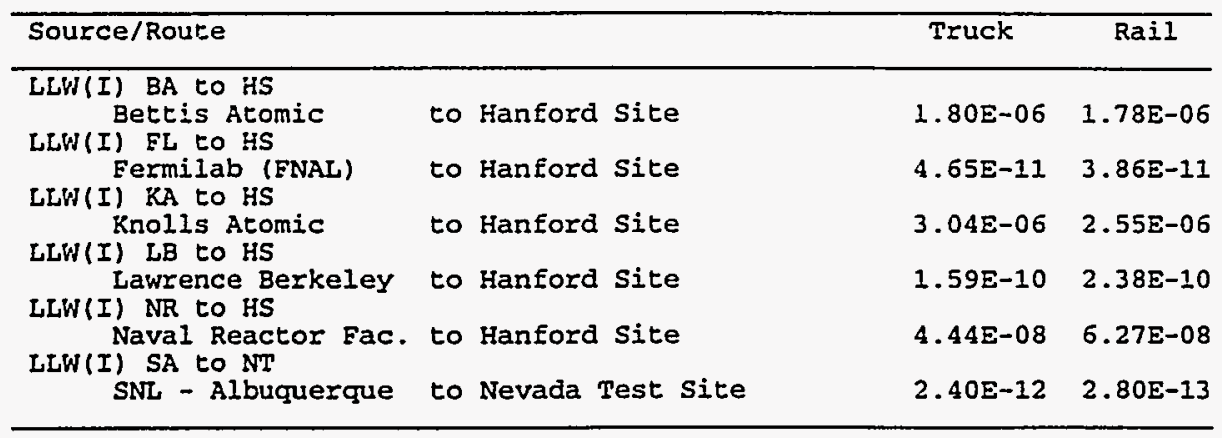

Table 5. Nonradiological Risk Factors per Shipment (Eatalities/shipment)

\begin{tabular}{llll}
\hline Mode & Route & Emission & Accident \\
\hline Truck & to Hanford Site & $1.14 \mathrm{E}-05$ & $1.36 \mathrm{E}-04$ \\
Bettis Atomic & to & $4.86 \mathrm{E}-06$ & $1.03 \mathrm{E}-04$ \\
Fermilab (FNAL) & to Hanford Site & $1.59 \mathrm{E}-05$ & $1.51 \mathrm{E}-04$ \\
Rnolls Atomic & to Hanford Site & $1.16 \mathrm{E}-05$ & $4.91 \mathrm{E}-05$ \\
Lawrence Berkeley & to Hanford Site & $2.19 \mathrm{E}-06$ & $3.07 \mathrm{E}-05$ \\
Naval Reactor Fac. to Hanford Site & $4.12 \mathrm{E}-06$ & $7.01 \mathrm{E}-05$ \\
SNL - Albuquerque & to Nevada Test Site & & \\
Rail & & $4.07 \mathrm{E}-05$ & $5.46 \mathrm{E}-06$ \\
Bettis Atomic & to Hanford Site & $1.18 \mathrm{E}-05$ & $4.12 \mathrm{E}-06$ \\
Fermilab (FNAL) & to Hanford Site & $4.72 \mathrm{E}-05$ & $6.10 \mathrm{E}-06$ \\
Rnolls Atomic & to Hanford Site & $2.33 \mathrm{E}-05$ & $2.06 \mathrm{E}-06$ \\
Lawrence Berkeley to Hanford Site & $3.98 \mathrm{E}-06$ & $1.38 \mathrm{E}-06$ \\
Naval Reactor Fac. to Hanford Site & $3.85 \mathrm{E}-06$ & $2.23 \mathrm{E}-06$ \\
SNL - Albuquerque & to Nevada Test Site & & \\
\hline
\end{tabular}

Table 6. Annual Incident-Free Dose for the Shipping Campaign (person-rem/yr)

\begin{tabular}{|c|c|c|c|c|c|}
\hline \multirow[b]{2}{*}{ Route } & & \multicolumn{2}{|c|}{ Truck } & \multicolumn{2}{|c|}{ Rail } \\
\hline & & Crew & Public & Crew & Public \\
\hline $\begin{array}{l}\text { Bettis Atomic } \\
\text { Fermilab (FNAL) } \\
\text { Knolis Atomic } \\
\text { Lawrence Berkeley } \\
\text { Naval Reactor Fac. } \\
\text { SNL - Albuquerque }\end{array}$ & $\begin{array}{l}\text { to Hanford Site } \\
\text { to Hanford Site } \\
\text { to Hanford Site } \\
\text { to Hanford Site } \\
\text { to Hanford Site } \\
\text { to Nevada Test Site }\end{array}$ & $\begin{array}{l}1.14 \mathrm{E}+01 \\
1.04 \mathrm{E}+00 \\
2.71 \mathrm{E}+01 \\
2.21 \mathrm{E}-01 \\
3.70 \mathrm{E}+00 \\
7.11 \mathrm{E}-02\end{array}$ & $\begin{array}{l}1.35 \mathrm{E}+01 \\
1.27 \mathrm{E}+00 \\
3.16 \mathrm{E}+01 \\
2.61 \mathrm{E}-01 \\
4.52 \mathrm{E}+00 \\
8.90 \mathrm{E}-02\end{array}$ & $\begin{array}{l}8.27 \mathrm{E}-01 \\
8.89 \mathrm{E}-02 \\
1.87 \mathrm{E}+00 \\
2.10 \mathrm{E}-02 \\
4.95 \mathrm{E}-01 \\
1.46 \mathrm{E}-02\end{array}$ & $\begin{array}{l}2.32 \mathrm{E}+00 \\
1.51 \mathrm{E}-01 \\
5.55 \mathrm{E}+00 \\
6.16 \mathrm{E}-02 \\
7.40 \mathrm{E}-01 \\
1.94 \mathrm{E}-02\end{array}$ \\
\hline Totals & & $4.35 E+01$ & $5.12 \mathrm{E}+01$ & $3.31 E+00$ & $8.85 E+00$ \\
\hline
\end{tabular}

Table 7. Annual Accident Dose Risk for the Shipping Campaign (person-rem/yr)

\begin{tabular}{llcc}
\hline Route & & Truck & Rail \\
\hline Bettis Atomic & to Hanford Site & $2.84 \mathrm{E}-04$ & $1.07 \mathrm{E}-04$ \\
Fermilab (FNAL) & to Hanford Site & $9.30 \mathrm{E}-10$ & $3.09 \mathrm{E}-10$ \\
Knolls Atomic & to Hanford Site & $9.95 \mathrm{E}-04$ & $3.16 \mathrm{E}-04$ \\
Lawrence Berkeley & to Hanford Site & $1.27 \mathrm{E}-09$ & $7.14 \mathrm{E}-10$ \\
Naval Reactor Fac. to Hanford Site & $1.02 \mathrm{E}-05$ & $5.52 \mathrm{E}-06$ \\
SNL - Albuquerque & to Nevada Test Site & $7.19 \mathrm{E}-12$ & $5.61 \mathrm{E}-13$ \\
\hline Totals & & $1.29 \mathrm{E}-03$ & $4.29 \mathrm{E}-04$ \\
\hline
\end{tabular}


Table 8. Expected Annual Fatalities for the Shipping Campaign

\begin{tabular}{lll}
\hline Exposure Group & Truck & Rail \\
\hline Radiological & $1.7 E-02$ & $1.3 E-03$ \\
Normal Crew & $2.6 \mathrm{E}-02$ & $4.4 \mathrm{E}-03$ \\
Normal Public & $6.4 \mathrm{E}-07$ & $2.1 \mathrm{E}-07$ \\
Accident Public & & \\
Nonradiological & $7.7 \mathrm{E}-03$ & $8.8 \mathrm{E}-03$ \\
Emission & $8.1 \mathrm{E}-02$ & $1.2 \mathrm{E}-03$ \\
Accident & & \\
\hline
\end{tabular}

Table 9. Expected Annual Cancer Incidence for the Shipping Campaign

\begin{tabular}{lll}
\hline Exposure Group & Truck & Rail \\
\hline Radiological & & \\
Normal Crew & $6.1 E-02$ & $4.6 E-03$ \\
Normal Public & $8.7 E-02$ & $1.5 E-02$ \\
Accident Public & $2.2 E-06$ & $7.3 E-07$ \\
Nonradiological & & \\
Emission & $7.7 E-03$ & $8.8 E-03$ \\
Accident & NA & NA \\
\hline
\end{tabular}

Table 10. Expected Annual Genetic Effects for the Shipping Campaign

\begin{tabular}{lll}
\hline Exposure Group & Truck & Rail \\
\hline Radiological & $2.6 E-03$ & $2.0 \mathrm{E}-04$ \\
Normal Crew & $5.1 \mathrm{E}-03$ & $8.8 \mathrm{E}-04$ \\
Normal Public & $1.3 \mathrm{E}-07$ & $4.3 \mathrm{E}-08$ \\
Accident Public & & \\
Nonradiological & NA & NA \\
Emission & NA & NA \\
Accident & \\
\hline
\end{tabular}




\section{B.3 WM LLW DECENTRALIZED ALTERNATIVE (CASE 2): HETEROGENEOUS SOLIDS}

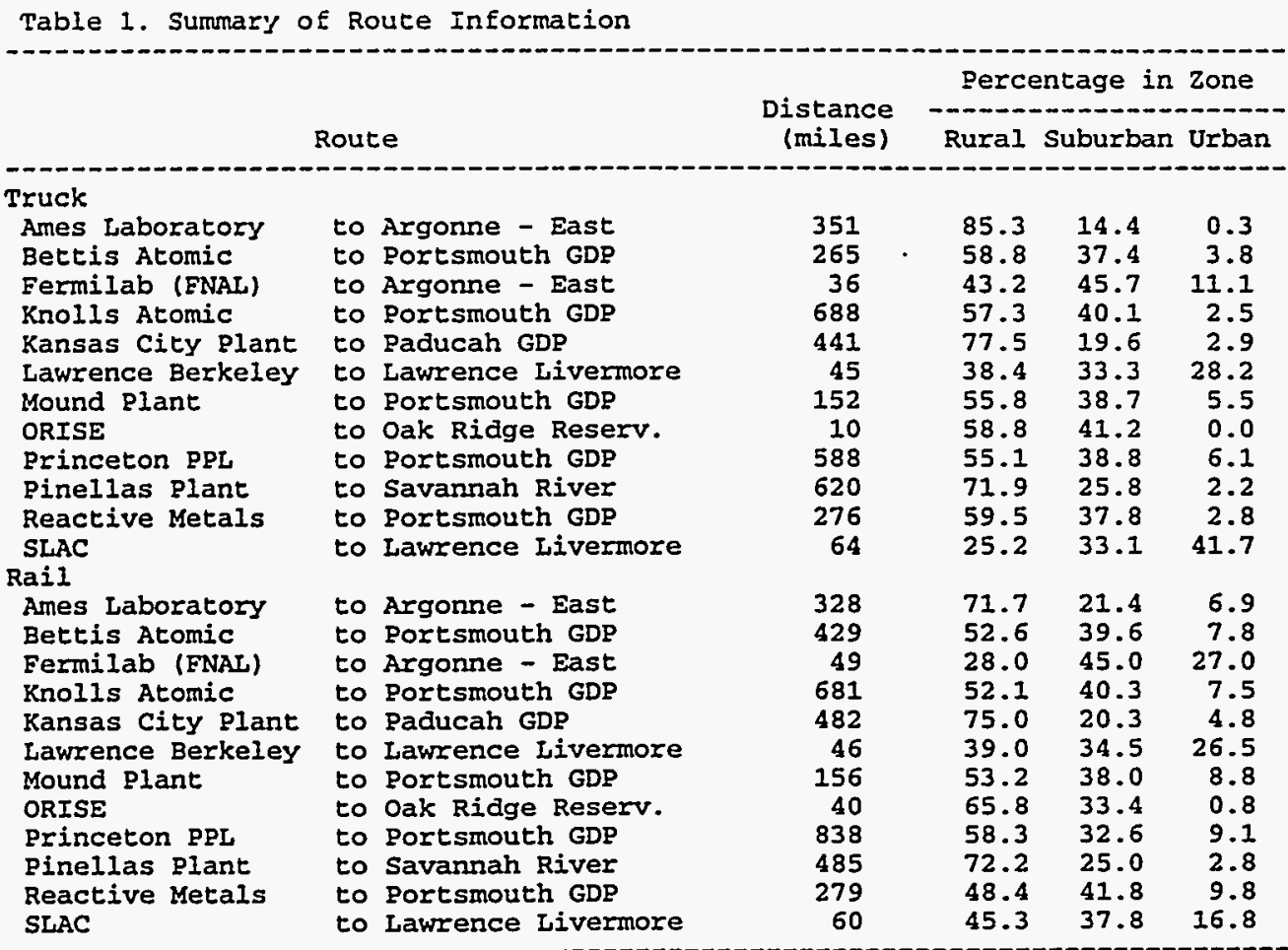

Table 2. Annual Number of Shipments and Total One-Way Shipment Distances

\begin{tabular}{|c|c|c|c|c|c|}
\hline \multirow[b]{2}{*}{ Route } & & \multicolumn{2}{|c|}{ \# of Shipments } & \multicolumn{2}{|c|}{ Total Mileage (mi) } \\
\hline & & Truck & Rail & Truck & Rail \\
\hline Ames Iaboratory & to Argonn & 2 & 1 & +02 & 02 \\
\hline Bettis Atomic & to Portsmouth GDP & 57 & $2 \overline{1}$ & $1.51 E+04$ & $9.01 E+03$ \\
\hline Fermilab (FNAL) & to Argonne - East & 3 & 2 & $1.08 \mathrm{E}+02$ & $9.78 \mathrm{E}+01$ \\
\hline Knolls Atomic & to Portsmouth GDP & 28 & 11 & $1.93 E+04$ & $7.50 \mathrm{E}+03$ \\
\hline Kansas City Plant & to Paducah GDP & 1 & 1 & $4.41 E+02$ & $4.82 \mathrm{E}+02$ \\
\hline Lawrence Berkeley & to Lawrence Livermore & 3 & 2 & $1.35 \mathrm{E}+02$ & $9.22 \mathrm{E}+01$ \\
\hline Mound Plant & to Portsmouth GDP & 512 & 190 & $7.79 E+04$ & $2.97 E+04$ \\
\hline ORISE & to Oak Ridge Reserv. & 5 & 2 & $5.10 E+01$ & $7.90 \mathrm{E}+01$ \\
\hline Princeton PPL & to Portsmouth GDP & 1 & 1 & $5.88 \mathrm{E}+02$ & $8.38 \mathrm{E}+02$ \\
\hline Pinellas plant & to Savannah River & 2 & 1 & $1.24 E+03$ & $4.85 E+02$ \\
\hline Reactive Metals & to Portsmouth GDP & 768 & 287 & $2.12 E+05$ & $8.01 E+04$ \\
\hline SLAC & to Lawrence Livermore & 39 & 15 & $2.50 \mathrm{E}+03$ & $9.00 \mathrm{E}+02$ \\
\hline Totals & & 1421 & 534 & $3.30 E+05$ & $1.30 \mathrm{E}+05$ \\
\hline
\end{tabular}


assessment, the total number of expected accidents or fatalities is calculated by multiplying the total shipping distance for a specific case by the appropriate accident or fatality rate.

For truck transportation, the rates presented in Saricks and Kvitek (1994) are specifically for heavy combination trucks involved in interstate commerce. Heavy combination trucks are rigs composed of a separable tractor unit containing the engine and one to three freight trailers connected to each other. Heavy combination trucks are typically used for shipments of radioactive waste. Saricks and Kvitek (1994) base their truck accident rates for each state on statistics compiled by the DOT Office of Motor Carriers for 1986-1988. Saricks and Kvitek (1994) present counts of accident involvement and of fatalities, estimated kilometers of travel by state, and the corresponding average accident involvement, fatality, and injury rates for the three years investigated. Fatalities are deaths (including crew members) that are attributable to the accident and that occurred at any time within 30 days thereafter. The truck accident rates are summarized in Table 4.1.

Rail accident rates are computed and presented similarly to truck accident rates in Saricks and Kvitek (1994); however, the unit of haulage is considered to be the railcar. Saricks and Kvitek (1994) base their state-specific rail accident involvement and fatality rates on statistics compiled by the Federal Railroad Administration for 1985-1988. Rail accident rates include both main-line accidents and those occurring in rail yards. The rail accident rates are summarized in Table 4.2.

\subsection{CHARACTERISTICS OF ACCIDENT RELEASES}

The transportation accident risk assessment depends on the fraction of material in a package that would be released or spilled to the environment during an accident, commonly referred to as the release fraction. The fraction of material released from a package is a function of the severity of the accident and the waste packaging; for instance, a low-impact accident, such as a "fender-bender," would not be expected to cause any release of material. Conversely, a very severe accident would be expected to release nearly all of the material in a shipment into the environment. The method used to characterize accident severities and the corresponding release fractions are described subsequently.

\subsubsection{Accident Severity Categories}

A method to characterize the potential severity of transportation-related accidents is described in an NRC report commonly referred to as NUREG-0170 (NRC 1977a). The NRC method divides the spectrum of transportation accident severities into eight categories. Other studies have divided the same accident spectrum into six categories (Wilmot 1981) and into 20 categories (Fischer et al. 1987); however, these studies focused primarily on accidents involving shipments of spent nuclear fuel and thus are not directly applicable to this assessment. 
Table 3. Incident-Free Dose per Shipment (person-rem/shipment)

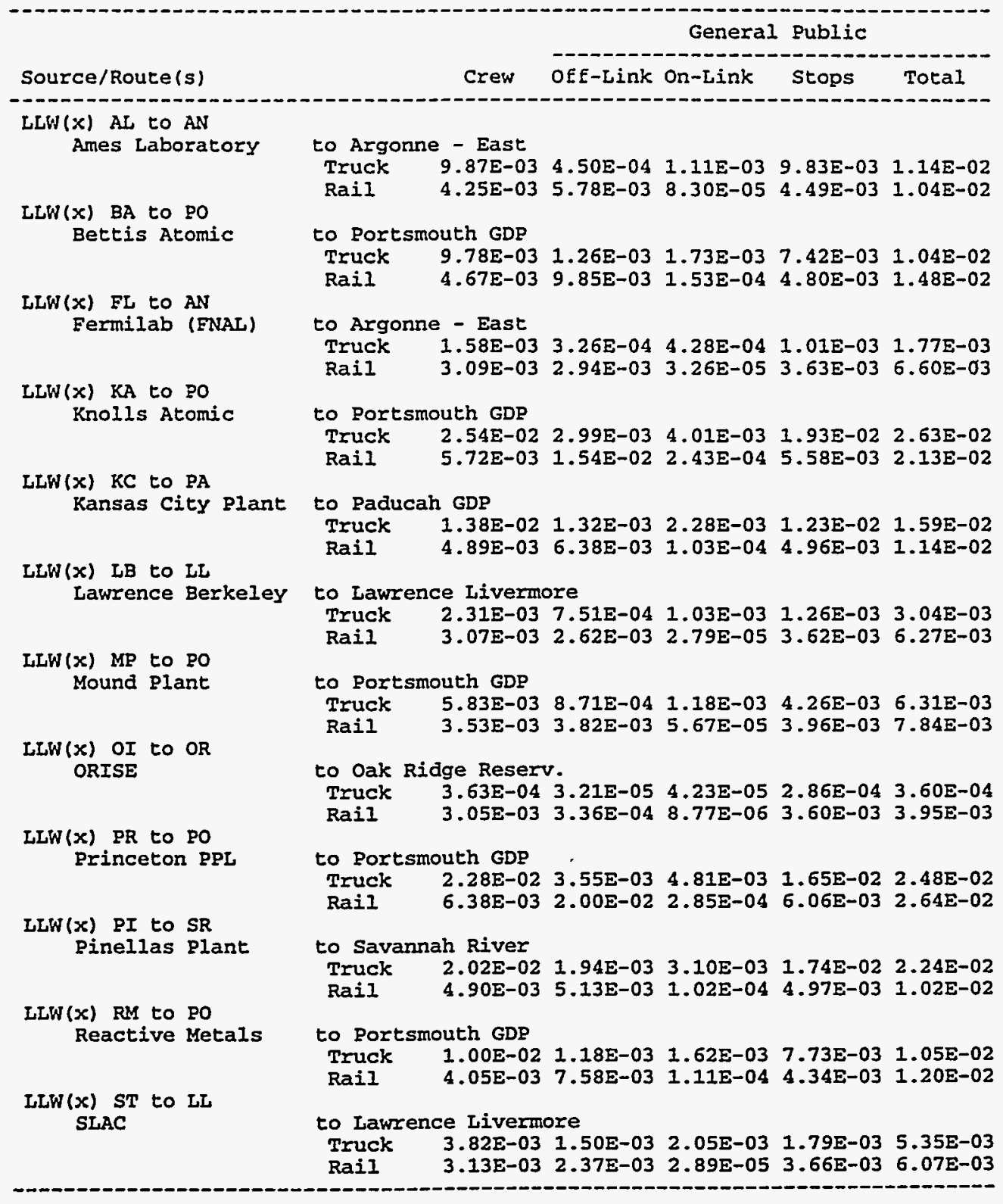




\section{$B-15$}

Table 4. Accident Dose Risk per Shipment (person-rem/shipment)

\begin{tabular}{|c|c|c|c|}
\hline \multicolumn{2}{|l|}{ Source/Route } & Truck & $\operatorname{Rail}$ \\
\hline LLW(x) AL to AN & & & \\
\hline $\begin{array}{l}\text { Ames Laboratory } \\
\text { LLW(x) BA to PO }\end{array}$ & to Argonne - East & $4.04 \mathrm{E}-06$ & $4.73 E-06$ \\
\hline $\begin{array}{l}\text { Bettis Atomic } \\
\text { LLW(x) FL to AN }\end{array}$ & to Portsmouth GDP & $6.25 E-06$ & $6.87 \mathrm{E}-06$ \\
\hline Fermilab (FNAL) & to Argonne - East & $4.66 \mathrm{E}-06$ & $3.23 E-06$ \\
\hline $\begin{array}{l}\text { Knolls Atomic } \\
L L W(x) \text { KC to PA }\end{array}$ & to Portsmouth GDP & $2.39 \mathrm{E}-06$ & $1.42 \mathrm{E}-06$ \\
\hline $\begin{array}{l}\text { Kansas City Plant } \\
\text { LLW(x) LB to LL }\end{array}$ & to Paducah GDP & $2.22 \mathrm{E}-05$ & $5.69 \mathrm{E}-06$ \\
\hline $\begin{array}{l}\text { Lawrence Berkeley } \\
\text { LLW(x) MP to Po }\end{array}$ & to Lawrence Livermore & $3.76 \mathrm{E}-03$ & $4.03 E-03$ \\
\hline $\begin{array}{l}\text { Mound plant } \\
\text { LLW(x) or to or }\end{array}$ & to Portsmouth GDP & $4.67 E-07$ & $3.08 \mathrm{E}-07$ \\
\hline $\begin{array}{l}\text { ORISE } \\
\text { LEW(x) PR to PO }\end{array}$ & to Oak Ridge Reserv. & $4.47 E-08$ & $3.16 \mathrm{E}-08$ \\
\hline $\begin{array}{l}\text { Princeton PPL } \\
\text { LLW(x) PI to } S R\end{array}$ & to Portsmouth GDP & $4.70 \mathrm{E}-06$ & $2.47 \mathrm{E}-06$ \\
\hline $\begin{array}{l}\text { Pinellas Plant } \\
\text { LLW(x) RM to PO }\end{array}$ & to Savannah River & $1.30 E-06$ & $4.65 E-07$ \\
\hline $\begin{array}{l}\text { Reactive Metals } \\
\text { LLW(x) ST to LI }\end{array}$ & to Portsmouth GDP & $2.26 \mathrm{E}-09$ & $1.74 \mathrm{E}-09$ \\
\hline SLAC & to Lawrence Livermore & $4.86 \mathrm{E}-08$ & $5.69 \mathrm{E}-08$ \\
\hline
\end{tabular}


Table 5. Nonradiological Risk Factors per Shipment (fatalities/shipment)

\begin{tabular}{|c|c|c|c|}
\hline Mode & Route & Emission & Accident \\
\hline \multicolumn{4}{|l|}{ Truck } \\
\hline Ames Laboratory & co Argonne - East & $3.22 \mathrm{E}-07$ & $1.87 E-05$ \\
\hline Bettis Atomic & to Portsmouth GDP & $3.22 \mathrm{E}-06$ & $1.33 \mathrm{E}-05$ \\
\hline Fermilab (FNAL) & to Argonne - East & $1.29 \mathrm{E}-06$ & $4.21 E-06$ \\
\hline Knolls Atomic & to Portsmouth GDP & $5.63 \mathrm{E}-06$ & $3.56 \mathrm{E}-05$ \\
\hline Kansas City Plant & to Paducah GDP & $4.12 E-06$ & $2.89 E-05$ \\
\hline Lawrence Berkeley & to Lawrence Livermore & $4.09 \mathrm{E}-06$ & $2.66 \mathrm{E}-06$ \\
\hline Mound Plant & to Portsmouth GDP & $2.70 \mathrm{E}-06$ & $6.66 \mathrm{E}-06$ \\
\hline ORISE & to Oak Ridge Reserv. & $0.00 E+00$ & $1.07 \mathrm{E}-06$ \\
\hline Princeton PPL & to Portsmouth GDP & $1.16 \mathrm{E}-05$ & $3.99 E-05$ \\
\hline Pinellas Plant & to Savannah River & $4.47 \mathrm{E}-06$ & $4.70 \mathrm{E}-05$ \\
\hline Reactive Metals & to Portsmouth GDP & $2.45 \mathrm{E}-06$ & $1.21 E-05$ \\
\hline SLAC & to Lawrence Livermore & $8.59 E-06$ & $3.47 E-06$ \\
\hline \multicolumn{4}{|l|}{ Rail } \\
\hline Ames Laboratory & to Argonne - East & $9.54 E-06$ & $6.87 E-07$ \\
\hline Bettis Atomic & to Portsmouth GDP & $1.40 \mathrm{E}-05$ & $8.98 \mathrm{E}-07$ \\
\hline Fermilab (FNAL) & to Axgonne - East & $5.52 \mathrm{E}-06$ & $1.02 \mathrm{E}-07$ \\
\hline Knolls Atomic & to Portsmouth GDP & $2.15 \mathrm{E}-05$ & $1.43 \mathrm{E}-06$ \\
\hline Kansas City Plant & to Paducah GDP & $9.58 \mathrm{E}-06$ & $1.01 \mathrm{E}-06$ \\
\hline Lawrence Berkeley & to Lawrence Livermore & $5.10 \mathrm{E}-06$ & $9.64 \mathrm{E}-08$ \\
\hline Mound Plant & to Portsmouth GDP & $5.73 \mathrm{E}-06$ & $3.27 \mathrm{E}-07$ \\
\hline ORISE & to Oak Ridge Reserv. & $1.26 \mathrm{E}-07$ & $8.26 \mathrm{E}-08$ \\
\hline Princeton PPL & to Portsmouth GDP & $3.18 \mathrm{E}-05$ & $1.75 \mathrm{E}-06$ \\
\hline Pinellas Plant & to Savannah River & $5.69 \mathrm{E}-06$ & $1.01 E-06$ \\
\hline Reactive Metals & to Portsmouth GDP & $1.14 \mathrm{E}-05$ & $5.84 E-07$ \\
\hline SLAC & to Lawxence Livermore & $4.23 \mathrm{E}-06$ & $1.26 \mathrm{E}-07$ \\
\hline
\end{tabular}

Table 6. Annual Incident-Free Dose for the Shipping Campaign (person-rem)

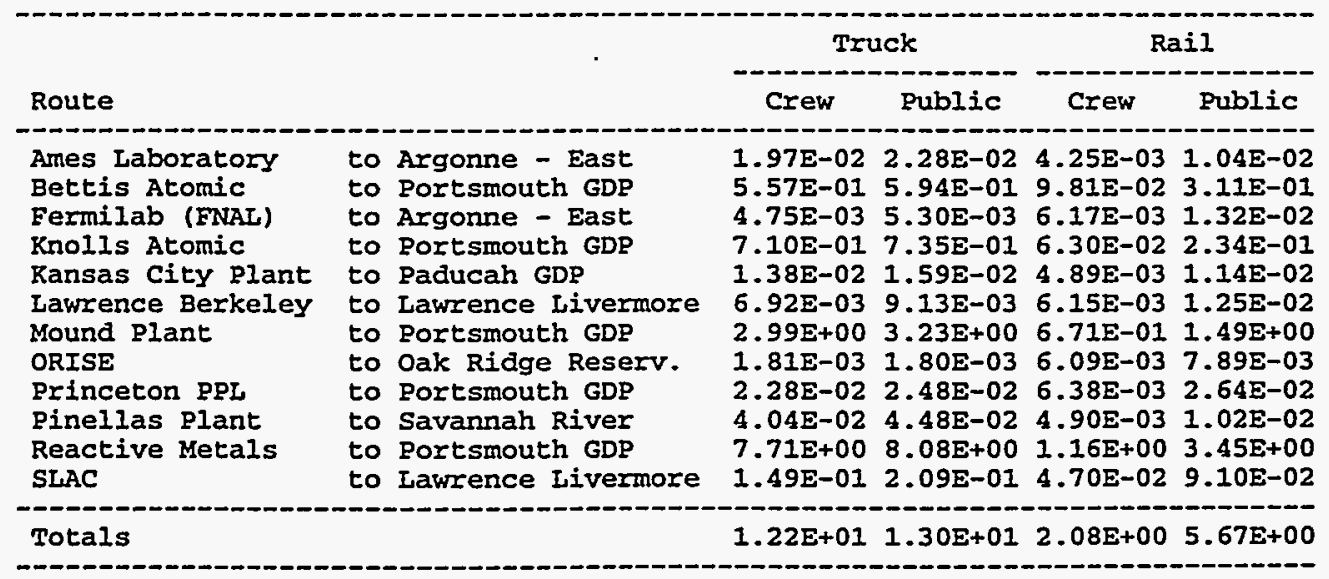




\section{$B-17$}

Table 7. Annual Accident Dose Risk for the Shipping Campaign (person-rem)

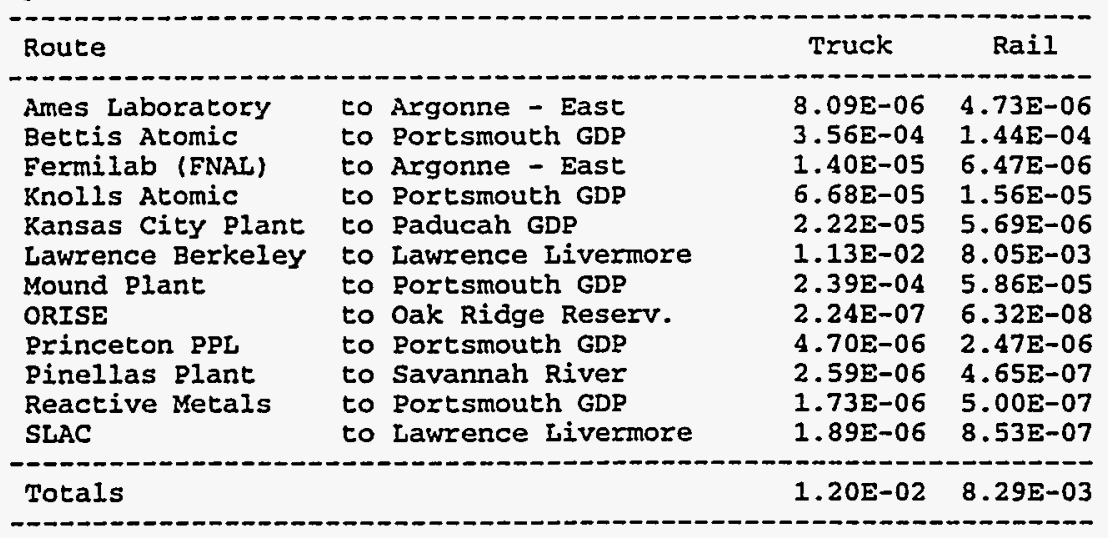

Table 8. Expected Annual Fatalities

for the Shipping Campaign

\begin{tabular}{lll} 
Exposure Group & Truck & Rail \\
\hline Radiological & & \\
$\quad$ Normal Crew & $4.9 E-03$ & $8.3 E-04$ \\
$\quad$ Normal Public & $6.5 E-03$ & $2.8 \mathrm{E}-03$ \\
$\quad$ Accident Public & $6.0 \mathrm{E}-06$ & $4.1 \mathrm{E}-06$ \\
Nonradiological & & \\
$\quad$ Enission & $4.0 \mathrm{E}-03$ & $5.0 \mathrm{E}-03$ \\
$\quad$ Accident & $1.5 \mathrm{E}-02$ & $2.7 \mathrm{E}-04$ \\
\hline
\end{tabular}

Table 9. Expected Annual Cancer Incidence for the Shipping Campaign

\begin{tabular}{lll}
\hline Exposure Group & Truck & Rail \\
\hline Radiological & & \\
$\quad$ Normal Crew & $1.7 E-02$ & $2.9 \mathrm{E}-03$ \\
$\quad$ Normal Public & $2.2 \mathrm{E}-02$ & $9.6 \mathrm{E}-03$ \\
$\quad$ Accident Public & $2.0 \mathrm{E}-05$ & $1.4 \mathrm{E}-05$ \\
Nonradiological & & \\
$\quad$ Emission & $4.0 \mathrm{E}-03$ & $5.0 \mathrm{E}-03$ \\
$\quad$ Accident & NA & NA \\
\hline
\end{tabular}

Table 10. Expected Annual Genetic Effects for the Shipping Campaign

\begin{tabular}{lll}
\hline Exposure Group & Truck & Rail \\
\hline Radiological & & \\
Normal Crew & $7.3 E-04$ & $1.2 E-04$ \\
Normal Public & $1.3 \mathrm{E}-03$ & $5.7 \mathrm{E}-04$ \\
Accident Public & $1.1 \mathrm{E}-06$ & $7.6 \mathrm{E}-07$ \\
Nonradiological & $\mathrm{N}$ & \\
Emission & NA & NA \\
Accident & NA & NA \\
\hline
\end{tabular}




\section{B.4 WM LLW DECENTRALIZED ALTERNATIVE (CASE 2): ACTIVATED METALS}

Table 1. Summary of Route Information

\begin{tabular}{|c|c|c|c|c|c|}
\hline \multicolumn{2}{|r|}{ Route } & $\begin{array}{l}\text { Distance } \\
\text { (miles) }\end{array}$ & $\begin{array}{r}\text { Perc } \\
\text { Rural }\end{array}$ & $\begin{array}{l}\text { Atage ir } \\
\text { Suburba }\end{array}$ & $\begin{array}{l}\text { Zone } \\
\text { Urban }\end{array}$ \\
\hline Truck & & & & & \\
\hline Bettis Atomic & to Portsmouth GDP & 265 & 58.8 & 37.4 & 3.8 \\
\hline Fermilab (FNAL) & to Argonne - East & 36 & 43.2 & 45.7 & 11.1 \\
\hline Knolls Atomic & to Portsmouth GDP & 688 & 57.3 & 40.1 & 2.5 \\
\hline Lawrence Berkeley & to Lawrence Livermore & 45 & 38.4 & 33.3 & 28.2 \\
\hline Rail & & & & & \\
\hline Bettis Atomic & to Portsmouth GDP & 429 & 52.6 & 39.6 & 7.8 \\
\hline Fermilab (FNAL) & to Argonne - East & 49 & 28.0 & 45.0 & 27.0 \\
\hline Knolls Atomic & to Portsmouth GDP & 681 & 52.1 & 40.3 & 7.5 \\
\hline Lawrence Berkeley & to Lawrence Iivermore & 46 & 39.0 & 34.5 & 26.5 \\
\hline
\end{tabular}

Table 2. Annual Number of Shipments and Total One-Way Shipment Distances

\begin{tabular}{|c|c|c|c|c|c|}
\hline \multirow[b]{2}{*}{ Route } & & \multicolumn{2}{|c|}{ \# of Shipments } & \multicolumn{2}{|c|}{ Total Mileage (mi) } \\
\hline & & Truck & Rail & Truck & Rail \\
\hline $\begin{array}{l}\text { Bettis Atomic } \\
\text { Fermilab (FNAL) } \\
\text { Rnolls Atomic } \\
\text { Lawrence Berkeley }\end{array}$ & $\begin{array}{l}\text { to Portsmouth GDP } \\
\text { to Argonne - East } \\
\text { to Portsmouth GDP } \\
\text { to Lawrence Livermore }\end{array}$ & $\begin{array}{r}316 \\
40 \\
650 \\
15\end{array}$ & $\begin{array}{r}120 \\
15 \\
246 \\
6\end{array}$ & $\begin{array}{l}8.37 E+04 \\
1.44 E+03 \\
4.47 E+05 \\
6.75 E+02\end{array}$ & $\begin{array}{l}5.15 \mathrm{E}+04 \\
7.34 \mathrm{E}+02 \\
1.68 \mathrm{E}+05 \\
2.77 \mathrm{E}+02\end{array}$ \\
\hline Totals & & 1021 & 387 & $5.33 \mathrm{E}+05$ & $2.20 E+05$ \\
\hline
\end{tabular}

Table 3. Incident-Free Dose per Shipment (person-rem/shipment)

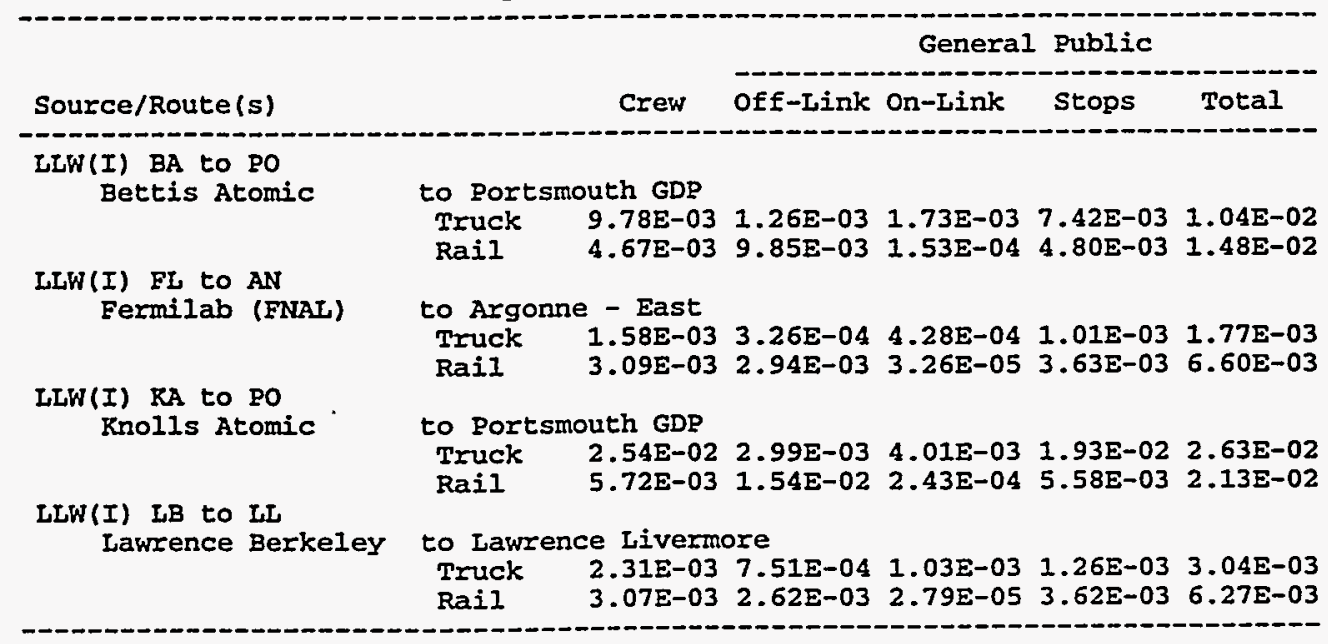


Table 4. Accident Dose Risk per Shipment (person-rem/shipment)

\begin{tabular}{|c|c|c|c|}
\hline Source/Route & & Truck & Rail \\
\hline $\begin{array}{l}\text { LLW(I) BA to PO } \\
\text { Bettis Acomic }\end{array}$ & to Portsmouth GDP & $3.62 E-07$ & $4.12 E-07$ \\
\hline $\begin{array}{l}\text { LLW(I) EL to AN } \\
\text { Fermilab (FNAL) } \\
\text { LLW(I) KA to go }\end{array}$ & to Argonne - East & $9.93 E-12$ & $1.22 \mathrm{E}-11$ \\
\hline $\begin{array}{l}\text { Knolls Atomic } \\
\text { LLW(I) LB to LL } \\
\text { Lawrence Berkeley }\end{array}$ & $\begin{array}{l}\text { to Portsmouth GDP } \\
\text { to Lawrence Livermore }\end{array}$ & $\begin{array}{l}1.63 \Xi-06 \\
1.27 \Xi-11\end{array}$ & $\begin{array}{l}1.01 \mathrm{E}-06 \\
2.29 \mathrm{E}-11\end{array}$ \\
\hline
\end{tabular}

Table 5. Nonradiological Risk Factors per Shipment (fatalities/shipment)

\begin{tabular}{|c|c|c|c|}
\hline Mode & Route & Emission & Accident \\
\hline \multicolumn{4}{|l|}{ Truck } \\
\hline Bettis Atomic & to Portsmouth GDP & $3.22 E-06$ & $1.33 E-05$ \\
\hline Fermilab (FNAL) & to Argonne - East & $1.29 \mathrm{E}-06$ & $4.21 E-06$ \\
\hline Rnolls Atomic & to Portsmouth GDP & $5.63 \mathrm{E}-06$ & $3.56 \mathrm{E}-05$ \\
\hline Lawrence Berkeley & to Lawrence Livermore & $4.09 E-06$ & $2.66 \mathrm{E}-06$ \\
\hline \multicolumn{4}{|l|}{ Rail } \\
\hline Bettis Atomic & to Portsmouth GDP & $1.40 \mathrm{E}-05$ & $8.98 \mathrm{E}-07$ \\
\hline Fermilab (FNAL) & to Argonne - East & $5.52 \mathrm{E}-06$ & $1.02 E-07$ \\
\hline Knolls Atomic & to Portsmouth GDP & $2.15 \mathrm{E}-05$ & $1.43 E-06$ \\
\hline Lawrence Berkeley & to Lawrence Livermore & $5.10 E-06$ & $9.64 \mathrm{E}-08$ \\
\hline
\end{tabular}

Table 6. Annual Incident-Free Dose for the Shipping Campaign (person-rem)

\begin{tabular}{|c|c|c|c|c|c|}
\hline \multirow[b]{2}{*}{ Route } & & \multicolumn{2}{|c|}{ Truck } & \multicolumn{2}{|c|}{ Rail } \\
\hline & & Crew & Public & Crew & Public \\
\hline $\begin{array}{l}\text { Bettis Atomic } \\
\text { Fermilab (FNAL) } \\
\text { Knolls Atomic } \\
\text { Lawrence Berkeley }\end{array}$ & $\begin{array}{l}\text { to Portsmouth GDP } \\
\text { to Argonne - East } \\
\text { to Portsmouth GDP } \\
\text { to Lawrence Iivermore }\end{array}$ & $\begin{array}{l}3.09 \mathrm{E}+00 \\
6.34 \mathrm{E}-02 \\
1.65 \mathrm{E}+01 \\
3.46 \mathrm{E}-02\end{array}$ & $\begin{array}{l}3.29 E+00 \\
7.06 E-02 \\
1.71 E+01 \\
4.57 E-02\end{array}$ & $\begin{array}{l}5.61 \mathrm{E}-01 \\
4.63 \mathrm{E}-02 \\
1.41 \mathrm{E}+00 \\
1.84 \mathrm{E}-02\end{array}$ & $\begin{array}{l}1.78 \mathrm{E}+00 \\
9.90 \mathrm{E}-02 \\
5.23 \mathrm{E}+00 \\
3.76 \mathrm{E}-02\end{array}$ \\
\hline Totals & & $1.97 E+01$ & $2.05 E+01$ & $2.03 \mathrm{E}+00$ & $7.14 \mathrm{E}+00$ \\
\hline
\end{tabular}

Table 7. Annual Accident Dose Risk for the Shipping Campaign (person-rem)

\begin{tabular}{llll} 
& & & \\
Route & & Truck & Rail \\
\hline Bettis Atomic & to Portsmouth GDP & $1.14 \mathrm{E}-04$ & $4.94 \mathrm{E}-05$ \\
Fermilab (FNAL) & to Argonne - East & $3.97 \mathrm{E}-10$ & $1.83 \mathrm{E}-10$ \\
Knolls Atomic & to Portsmouth GDP & $1.06 \mathrm{E}-03$ & $2.49 \mathrm{E}-04$ \\
Lawrence Berkeley to Lawrence Livermore & $1.90 \mathrm{E}-10$ & $1.37 \mathrm{E}-10$ \\
\hline Totals & & $1.17 \mathrm{E}-03$ & $2.98 \mathrm{E}-04$ \\
\hline
\end{tabular}


Table 8. Expected Annual Fatalities for the Shipping Campaign

\begin{tabular}{lll}
\hline Exposure Group & Truck & Rail \\
\hline Radiological & & \\
$\quad$ Normal Crew & $7.9 \mathrm{E}-03$ & $8.1 \mathrm{E}-04$ \\
$\quad$ Normal Public & $1.0 \mathrm{E}-02$ & $3.6 \mathrm{E}-03$ \\
$\quad$ Accident Public & $5.9 \mathrm{E}-07$ & $1.5 \mathrm{E}-07$ \\
Nonradiological & & \\
$\quad$ Emission & $4.8 \mathrm{E}-03$ & $7.1 \mathrm{E}-03$ \\
Accident & $2.8 \mathrm{E}-02$ & $4.6 \mathrm{E}-04$ \\
\hline
\end{tabular}

Table 9. Expected Annual Cancer Incidence for the Shipping Campaign

\begin{tabular}{lll}
\hline Exposure Group & Truck & \multicolumn{1}{c}{ Rail } \\
\hline Radiological & & \\
$\quad$ Normal Crew & $2.8 \mathrm{E}-02$ & $2.8 \mathrm{E}-03$ \\
$\quad \begin{array}{l}\text { Normal Public } \\
\text { Accident Public }\end{array}$ & $3.5 \mathrm{E}-02$ & $1.2 \mathrm{E}-02$ \\
$\begin{array}{c}\text { Nonradiological } \\
\text { Emission }\end{array}$ & $2.0 \mathrm{E}-06$ & $5.1 \mathrm{E}-07$ \\
$\quad$ Accident & $4.8 \mathrm{E}-03$ & $7.1 \mathrm{E}-03$ \\
\hline
\end{tabular}

Table 10. Expected Annual Genetic Effects for the Shipping Campaign

\begin{tabular}{lll}
\hline Exposure Group & Truck & Rail \\
\hline Radiological & & \\
Normal Crew & $1.2 \mathrm{E}-03$ & $1.2 \mathrm{E}-04$ \\
Normal Public & $2.0 \mathrm{E}-03$ & $7.1 \mathrm{E}-04$ \\
Accident Public & $1.2 \mathrm{E}-07$ & $3.0 \mathrm{E}-08$ \\
Nonradiological & & \\
Enission & $\mathrm{NA}$ & $\mathrm{NA}$ \\
Accident & $\mathrm{NA}$ & $\mathrm{NA}$ \\
\hline
\end{tabular}




\section{B.5 WM LLW REGIONALIZED 1 ALTERNATIVE (CASE 3): HETEROGENEOUS SOLIDS}

Table 1. Summary of Route Information

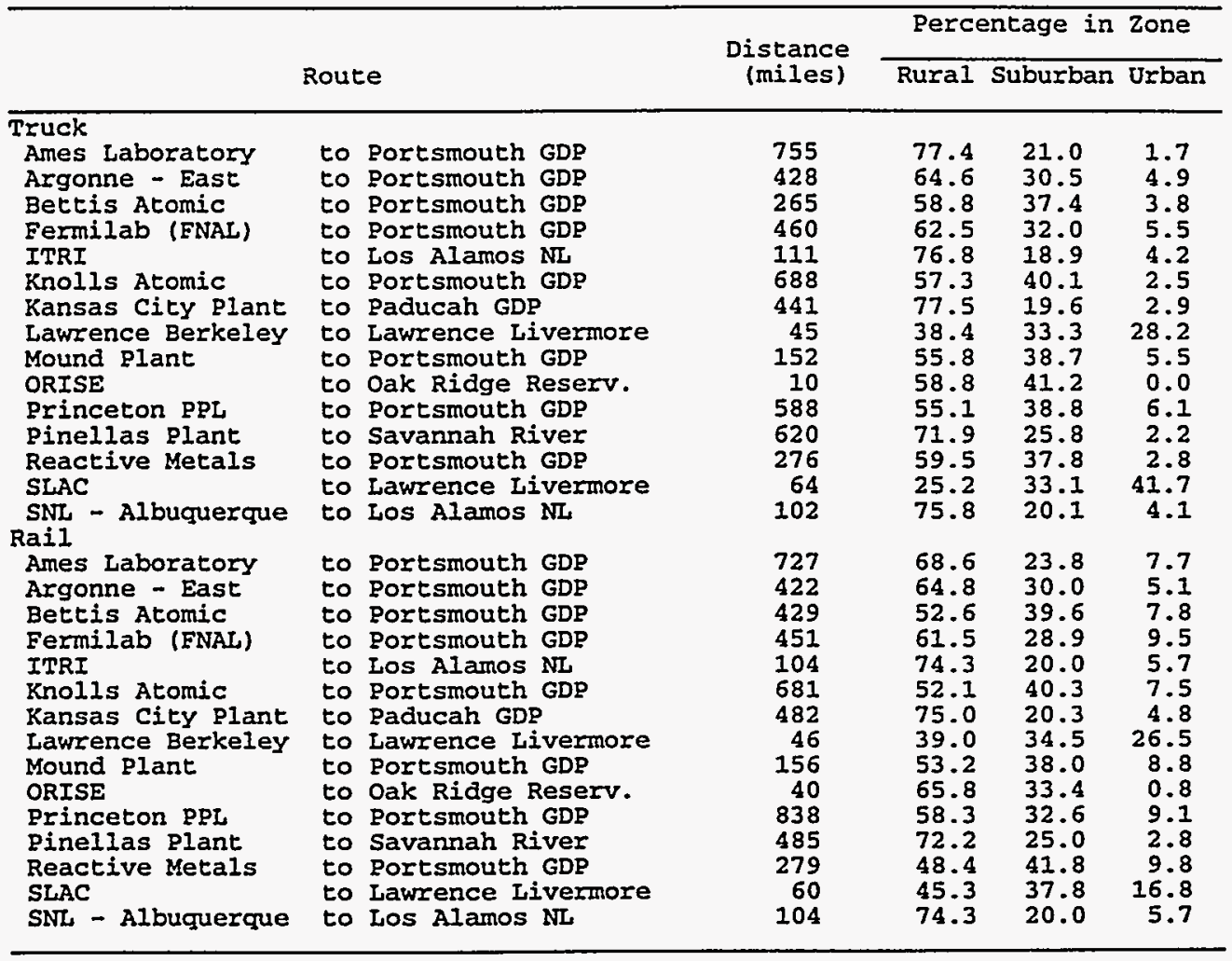

Table 2. Annual Number of Shipments and One-Way Shipment Distances

\begin{tabular}{|c|c|c|c|c|c|c|}
\hline \multirow[b]{2}{*}{ Route } & & & \multicolumn{2}{|c|}{ \# of Shipments } & \multicolumn{2}{|c|}{ Total Mileage (mi) } \\
\hline & & & Truck & Rail & Truck & Rail \\
\hline $\begin{array}{l}\text { Ames Laboratory } \\
\text { Argonne - East } \\
\text { Bettis Atomic } \\
\text { Fermilab (FNAL) } \\
\text { ITRI } \\
\text { Knolls Atomic } \\
\text { Kansas City Plant } \\
\text { Lawrence Berkeley } \\
\text { Mound Plant } \\
\text { ORISE } \\
\text { Princeton PPL } \\
\text { Pinellas Plant } \\
\text { Reactive Metals } \\
\text { SLAC } \\
\text { SNL - Albuquerque }\end{array}$ & $\begin{array}{l}\text { to } \\
\text { to } \\
\text { to } \\
\text { to } \\
\text { to } \\
\text { to } \\
\text { to } \\
\text { to } \\
\text { to } \\
\text { to } \\
\text { to } \\
\text { to } \\
\text { to } \\
\text { to } \\
\text { to }\end{array}$ & $\begin{array}{l}\text { Portsmouth GDP } \\
\text { Portsmouth GDP } \\
\text { Portsmouth GDP } \\
\text { Portsmouth GDP } \\
\text { Los Alamos NL } \\
\text { Portsmouth GDP } \\
\text { Paducah GDP } \\
\text { Lawrence Iivermore } \\
\text { Portsmouth GDP } \\
\text { Oak Ridge Reserv. } \\
\text { Portsmouth GDP } \\
\text { Savannah River } \\
\text { Portsmouth GDP } \\
\text { Lawrence Livermore } \\
\text { Los Alamos NL }\end{array}$ & $\begin{array}{r}2 \\
105 \\
57 \\
3 \\
10 \\
28 \\
1 \\
3 \\
512 \\
5 \\
1 \\
2 \\
768 \\
39 \\
17\end{array}$ & $\begin{array}{r}1 \\
39 \\
21 \\
2 \\
4 \\
11 \\
1 \\
2 \\
190 \\
2 \\
1 \\
1 \\
287 \\
15 \\
7\end{array}$ & $\begin{array}{l}1.51 \mathrm{E}+03 \\
4.50 \mathrm{E}+04 \\
1.51 \mathrm{E}+04 \\
1.38 \mathrm{E}+03 \\
1.11 \mathrm{E}+03 \\
1.93 \mathrm{E}+04 \\
4.41 \mathrm{E}+02 \\
1.35 \mathrm{E}+02 \\
7.79 \mathrm{E}+04 \\
5.10 \mathrm{E}+01 \\
5.88 \mathrm{E}+02 \\
1.24 \mathrm{E}+03 \\
2.12 \mathrm{E}+05 \\
2.50 \mathrm{E}+03 \\
1.73 \mathrm{E}+03\end{array}$ & $\begin{array}{l}7.27 E+02 \\
1.65 E+04 \\
9.01 E+03 \\
9.02 E+02 \\
4.16 E+02 \\
7.50 E+03 \\
4.82 E+02 \\
9.22 E+01 \\
2.97 E+04 \\
7.90 E+01 \\
8.38 E+02 \\
4.85 E+02 \\
8.01 E+04 \\
9.00 E+02 \\
7.28 E+02\end{array}$ \\
\hline Totals & & & 1553 & 584 & $3.80 E+05$ & $1.48 \mathrm{E}+05$ \\
\hline
\end{tabular}


Table 3. Incident-Free Dose per Shipment (person-rem/shipment)

\begin{tabular}{|c|c|c|c|c|c|}
\hline \multirow[b]{2}{*}{ Source/Route (s) } & \multirow[b]{2}{*}{ Crew } & \multicolumn{4}{|c|}{ General Public } \\
\hline & & off-Link & On-Link & Stops & Total \\
\hline $\begin{array}{l}\text { LLW(x) AL to PO } \\
\text { Ames Laboratory }\end{array}$ & $\begin{array}{lr}\text { to Portsmouth GDP } \\
\text { Truck } & 2.33 \mathrm{E}-02 \\
\text { Rail } & 5.91 \mathrm{E}-03\end{array}$ & $\begin{array}{l}1.86 \mathrm{E}-03 \\
1.42 \mathrm{E}-02\end{array}$ & $\begin{array}{l}2-03 \\
z-04\end{array}$ & $\begin{array}{l}2.11 \mathrm{E}-02 \\
5.72 \mathrm{E}-03\end{array}$ & $\begin{array}{l}2.63 E-02 \\
2.01 E-02\end{array}$ \\
\hline $\begin{array}{l}\text { LLW (x) AN to PO } \\
\text { Argonne - East }\end{array}$ & $\begin{array}{cc}\text { to Portsmouth GDP } \\
\text { Truck } & 1.53 \mathrm{E}-02 \\
\text { Rail } & 4.64 \mathrm{E}-03\end{array}$ & $\begin{array}{l}2.05 \mathrm{E}-03 \\
6.76 \mathrm{E}-03\end{array}$ & $\begin{array}{l}2.99 \mathrm{E}-03 \\
1.14 \mathrm{E}-04\end{array}$ & $\begin{array}{l}1.20 E-02 \\
4.78 E-03\end{array}$ & $\begin{array}{l}1.70 \mathrm{E}-02 \\
1.17 \mathrm{E}-02\end{array}$ \\
\hline $\begin{array}{l}\text { LLW }(x) \text { BA to Po } \\
\text { Bettis Atomic }\end{array}$ & $\begin{array}{ll} & \\
\text { to Portsmouth GDP } \\
\text { Truck } & 9.78 \mathrm{E}-03 \\
\text { Rail } & 4.67 \mathrm{E}-03\end{array}$ & $\begin{array}{l}1.26 \mathrm{E}-03 \\
9.85 \mathrm{E}-03\end{array}$ & $\begin{array}{l}1.73 E-03 \\
1.53 E-04\end{array}$ & $\begin{array}{l}7.42 \mathrm{E}-03 \\
4.80 \mathrm{E}-03\end{array}$ & $\begin{array}{l}1.04 \mathrm{E}-02 \\
1.48 \mathrm{E}-02\end{array}$ \\
\hline $\begin{array}{l}\text { LLW (x) FL to PO } \\
\text { Fermilab (FNAL) }\end{array}$ & $\begin{array}{lr}\text { to Portsmouth GDP } \\
\text { Truck } & 1.68 \mathrm{E}-02 \\
\text { Rail } & 4.76 \mathrm{E}-03\end{array}$ & $\begin{array}{l}2.40 \mathrm{E}-03 \\
1.08 \mathrm{E}-02\end{array}$ & $\begin{array}{l}3.43 \mathrm{E}-03 \\
1.48 \mathrm{E}-04\end{array}$ & $\begin{array}{l}1.29 E-02 \\
4.87 E-03\end{array}$ & $\begin{array}{l}1.87 \mathrm{E}-02 \\
1.58 \mathrm{E}-02\end{array}$ \\
\hline LLW (x) KA to po & $\begin{array}{cr}\text { to Los } & \text { Alamos NL } \\
\text { Truck } & 3.54 \mathrm{E}-03 \\
\text { Rail } & 3.31 \mathrm{E}-03\end{array}$ & $\begin{array}{l}4.00 \mathrm{E}-04 \\
1.55 \mathrm{E}-03\end{array}$ & $\begin{array}{l}6.71 \mathrm{E}-04 \\
2.36 \mathrm{E}-05\end{array}$ & $\begin{array}{l}3.11 E-03 \\
3.80 E-03\end{array}$ & $\begin{array}{l}4.18 E-03 \\
5.37 E-03\end{array}$ \\
\hline $\begin{array}{l}\text { LLW }(x) \text { KA to Po } \\
\text { Knolls Atomic }\end{array}$ & $\begin{array}{lr}\text { to Portsmouth GDP } \\
\text { Truck } & 2.54 \mathrm{E}-02 \\
\text { Rail } & 5.72 \mathrm{E}-03\end{array}$ & $\begin{array}{l}2.99 E-03 \\
1.54 E-02\end{array}$ & $\begin{array}{l}4.01 \mathrm{E}-03 \\
2.43 \mathrm{E}-04\end{array}$ & $\begin{array}{l}1.93 E-02 \\
5.58 E-03\end{array}$ & $\begin{array}{l}2.63 \mathrm{E}-02 \\
2.13 \mathrm{E}-02\end{array}$ \\
\hline Kansas City Plant & $\begin{array}{ll}\text { to Paducah GDP } \\
\text { Truck } & 1.38 \mathrm{E}-02 \\
\text { Raji } & 4.89 \mathrm{E}-03\end{array}$ & $\begin{array}{l}1 \cdot 32 \mathrm{E}-03 \\
6.38 \mathrm{E}-03\end{array}$ & $\begin{array}{l}2.28 \mathrm{E}-03 \\
1.03 \mathrm{E}-04\end{array}$ & $\begin{array}{l}1.23 E-02 \\
4.96 E-03\end{array}$ & $\begin{array}{l}1.59 \mathrm{E}-02 \\
1.14 \mathrm{E}-02\end{array}$ \\
\hline Lawrence Berkeley & $\begin{array}{ll}\text { to Lawrence Livermo } & \text { Liver } \\
\text { Truck } & 2.31 \mathrm{E}-03 \\
\text { Rail } & 3.07 \mathrm{E}-03\end{array}$ & $\begin{array}{l}\text { ore } \\
7.51 E-04 \\
2.62 \mathrm{E}-03\end{array}$ & $\begin{array}{l}\frac{1}{2} .03 E-03 \\
2.79 E-05\end{array}$ & $\begin{array}{l}1.26 \mathrm{E}-03 \\
3.62 \mathrm{E}-03\end{array}$ & $\begin{array}{l}3.04 E-03 \\
6.27 E-03\end{array}$ \\
\hline $\begin{array}{l}\text { LLW (x) MP to Po } \\
\text { Mound Plant }\end{array}$ & $\begin{array}{cr}\text { to Portsmouth GDP } \\
\text { Truck } & 5.83 \mathrm{E}-03 \\
\text { Rail } & 3.53 \mathrm{E}-03\end{array}$ & $\begin{array}{l}8.71 \mathrm{E}-04 \\
3.82 \mathrm{E}-03\end{array}$ & $\begin{array}{l}1.18 E-03 \\
5.67 E-05\end{array}$ & $\begin{array}{l}4.26 E-03 \\
3.96 E-03\end{array}$ & $\begin{array}{l}6.31 \mathrm{E}-03 \\
7.84 \mathrm{E}-03\end{array}$ \\
\hline LLW(x) PR to Po & $\begin{array}{cr}\text { to Oak Ridge Reserv } \\
\text { Truck } & 3.63 \mathrm{E}-04 \\
\text { Rail } & 3.05 \mathrm{E}-03\end{array}$ & $\begin{array}{l}3.21 E-05 \\
3.36 E-04\end{array}$ & $\begin{array}{l}4.23 E-05 \\
8.77 E-06\end{array}$ & $\begin{array}{l}2.86 \mathrm{E}-04 \\
3.60 \mathrm{E}-03\end{array}$ & $\begin{array}{l}3.60 \mathrm{E}-04 \\
3.95 \mathrm{E}-03\end{array}$ \\
\hline $\begin{array}{l}\text { LLW(x) PR to PO } \\
\text { Princeton PPL }\end{array}$ & $\begin{array}{lr}\text { to Portsmouth GDP } \\
\text { Truck } & 2.28 \mathrm{E}-02 \\
\text { Rail } & 6.38 \mathrm{E}-03\end{array}$ & $\begin{array}{l}3.55 E-03 \\
2.00 E-02\end{array}$ & $\begin{array}{l}4.81 E-03 \\
2.85 E-04\end{array}$ & $\begin{array}{l}1.65 \mathrm{E}-02 \\
6.06 \mathrm{E}-03\end{array}$ & $\begin{array}{l}2.48 E-02 \\
2.64 E-02\end{array}$ \\
\hline $\begin{array}{l}\text { LLW(x) PI to SR } \\
\text { Pinellas Plant }\end{array}$ & $\begin{array}{lr}\text { to Savannah River } \\
\text { Truck } & 2.02 \mathrm{E}-02 \\
\text { Rail } & \mathbf{4 . 9 0 \mathrm { E } - 0 3}\end{array}$ & $\begin{array}{l}1.94 \mathrm{E}-03 \\
5.13 \mathrm{E}-03\end{array}$ & $\begin{array}{l}3.10 \mathrm{E}-03 \\
1.02 \mathrm{E}-04\end{array}$ & $\begin{array}{l}1.74 \mathrm{E}-02 \\
4.97 \mathrm{E}-03\end{array}$ & $\begin{array}{l}2.24 \mathrm{E}-02 \\
1.02 \mathrm{E}-02\end{array}$ \\
\hline Reactive Metals & $\begin{array}{ll}\text { to Portsmouth GDP } \\
\text { Truck } & 1.00 \mathrm{E}-02 \\
\text { Rail } & 4.05 \mathrm{E}-03\end{array}$ & $\begin{array}{l}1.18 \mathrm{E}-03 \\
7.58 \mathrm{E}-03\end{array}$ & $\begin{array}{l}1.62 \mathrm{E}-03 \\
1.11 \mathrm{E}-04\end{array}$ & $\begin{array}{l}7.73 \mathrm{E}-03 \\
4.34 \mathrm{E}-03\end{array}$ & $\begin{array}{l}1.05 \mathrm{E}-02 \\
1.20 \mathrm{E}-02\end{array}$ \\
\hline $\begin{array}{c}\operatorname{LLW}(x) \text { ST to } L L \\
\text { SLAC }\end{array}$ & $\begin{array}{ll}\text { to Lawrence Livermo } \\
\text { Truck } & 3.82 \mathrm{E}-03 \\
\text { Rail } & 3.13 \mathrm{E}-03\end{array}$ & $\begin{array}{l}\text { ore } \\
1.50 E-03 \\
2.37 E-03\end{array}$ & $\begin{array}{l}2.05 \mathrm{E}-03 \\
2.89 \mathrm{E}-05\end{array}$ & $\begin{array}{l}1.79 \mathrm{E}-03 \\
3.66 \mathrm{E}-03\end{array}$ & $\begin{array}{l}5.35 E-03 \\
6.07 E-03\end{array}$ \\
\hline $\begin{array}{l}\text { LLW (x) SA to IAA } \\
\text { SNL - Albuquerque }\end{array}$ & $\begin{array}{cr}\text { to Ias Alamos NL } \\
\text { Truck } & 3.28 \mathrm{E}-03 \\
\text { Rail } & 3.31 \mathrm{E}-03\end{array}$ & $\begin{array}{l}3.70 \mathrm{E}-04 \\
1.55 \mathrm{E}-03\end{array}$ & $\begin{array}{l}6.13 \mathrm{E}-04 \\
2.36 \mathrm{E}-05\end{array}$ & $\begin{array}{l}2.86 \mathrm{E}-03 \\
3.80 \mathrm{E}-03\end{array}$ & $\begin{array}{l}3.84 E-03 \\
5.37 E-03\end{array}$ \\
\hline
\end{tabular}


Table 4. Accident Dose Risk per Shipment (person-rem/shipment)

\begin{tabular}{|c|c|c|c|}
\hline Source/Route & & Truck & Rail \\
\hline $\begin{array}{l}\text { LLW(x) AL to PO } \\
\text { Ames Laboraco } \\
\text { LLW(x) AN to PO } \\
\text { Argonne - East } \\
\text { LLW(x) BA to Po } \\
\text { BetEis Atomic } \\
\text { LLW(x) FL to PO } \\
\text { Fermilab (FNAL) } \\
\text { LLW(x) IT to LA } \\
\text { ITRI } \\
\text { LLW(x) KA to Po } \\
\text { KnoIls Atomic } \\
\text { LLW(x) RC to PA } \\
\text { Kansas City Plant } \\
\text { LLW(x) LB to LL } \\
\text { LaWrence Berkeley } \\
\text { LLW(x) MP to PO } \\
\text { Mound Plant } \\
\text { LLW(x) OI to OR } \\
\text { ORISE } \\
\text { LLW(x) PR to Po } \\
\text { Princeton PPL } \\
\text { LLW(x) PI to SR } \\
\text { Pinellas Plant } \\
\text { LLW(x) RM to PO } \\
\text { Reactive Metals } \\
\text { LLW(x) ST to LL } \\
\text { SLAC } \\
\text { LLW(x) SA to LA } \\
\text { SNL - Albuquerque }\end{array}$ & $\begin{array}{l}\text { to Portsmouth GDP } \\
\text { to Portsmouth GDP } \\
\text { to Portsmouth GDP } \\
\text { to Portsmouth GDP } \\
\text { to Los Alamos NL } \\
\text { to Portsmouth GDP } \\
\text { to Paducah GDP } \\
\text { to Lawrence Livermore } \\
\text { to Portsmouth GDP } \\
\text { to Oak Ridge Reserv. } \\
\text { to Portsmouth GDP } \\
\text { to Savannah River } \\
\text { to Portsmouth GDP } \\
\text { to Lawrence Livermore } \\
\text { to Los Alamos NL }\end{array}$ & $\begin{array}{l}7.75 E-06 \\
3.45 E-04 \\
6.25 E-06 \\
2.43 E-05 \\
1.53 E-03 \\
2.39 E-06 \\
2.31 E-05 \\
3.75 E-03 \\
4.67 E-07 \\
4.49 E-08 \\
4.70 E-06 \\
1.30 E-06 \\
2.26 E-09 \\
4.86 E-08 \\
5.39 E-06\end{array}$ & $\begin{array}{l}6.47 \mathrm{E}-06 \\
1.88 \mathrm{E}-04 \\
6.87 \mathrm{E}-06 \\
9.49 \mathrm{E}-06 \\
7.01 \mathrm{E}-05 \\
1.42 \mathrm{E}-06 \\
6.16 \mathrm{E}-06 \\
4.02 \mathrm{E}-03 \\
3.08 \mathrm{E}-07 \\
3.15 \mathrm{E}-08 \\
2.47 \mathrm{E}-06 \\
4.65 \mathrm{E}-07 \\
1.74 \mathrm{E}-09 \\
5.69 \mathrm{E}-08 \\
2.50 \mathrm{E}-07\end{array}$ \\
\hline
\end{tabular}

Table 5. Nonradiological Risk Factors per Shipment (fatalities/shipment)

\begin{tabular}{|c|c|c|c|}
\hline Mode & Route & Emission & Accident \\
\hline $\begin{array}{l}\text { Truck } \\
\text { Ames Laboratory } \\
\text { Argonne - East } \\
\text { Bettis Atomic } \\
\text { Eermilab (FNAL) } \\
\text { ITRI } \\
\text { Knolls Atomic } \\
\text { Kansas City Plant } \\
\text { Lawrence Berkeley } \\
\text { Mound Plant } \\
\text { orISE } \\
\text { Princeton PpL } \\
\text { Pinellas Plant } \\
\text { Reactive Metals } \\
\text { SLAC } \\
\text { SNL - Albuquerque } \\
\text { Rail } \\
\text { Ames Laboratory } \\
\text { Argonne - East } \\
\text { Bettis Atomic } \\
\text { Fermilab (FNAI) } \\
\text { ITRI } \\
\text { Knolls Atomic } \\
\text { Kansas City Plant } \\
\text { Lawrence Berkeley } \\
\text { Mound Plant } \\
\text { ORISE } \\
\text { Princeton ppL } \\
\text { Pinellas Plant } \\
\text { Reactive Metals } \\
\text { SLAC } \\
\text { SNL - Albuquerque }\end{array}$ & $\begin{array}{l}\text { to Portsmouth GDP } \\
\text { to Portsmouth GDP } \\
\text { to Portsmouth GDP } \\
\text { to Portsmouth GDP } \\
\text { to Los Alamos NL } \\
\text { to Portsmouth GDP } \\
\text { to Paducah GDP } \\
\text { to Lawrence Livermore } \\
\text { to Portsmouth GDP } \\
\text { to Oak Ridge Reserv. } \\
\text { to Portsmouth GDP } \\
\text { to Savannah River } \\
\text { to Portsmouth GDP } \\
\text { to Lawrence Livermore } \\
\text { to Los Alamos NL } \\
\text { to Portsmouth GDP } \\
\text { to Portsmouth GDP } \\
\text { to Portsmouth GDP } \\
\text { to Portsmouth GDP } \\
\text { to Los Alamos NL } \\
\text { to Portsmouth GDP } \\
\text { to Paducah GDP } \\
\text { to Lawrence Livermore } \\
\text { to Portsmouth GDP } \\
\text { to Oak Ridge Reserv. } \\
\text { to Portsmouth GDP } \\
\text { to Savannah River } \\
\text { to Portsmouth GDP } \\
\text { to Lawrence Livermore } \\
\text { to Los Alamos NL }\end{array}$ & $\begin{array}{l}4.02 E-06 \\
6.73 E-06 \\
3.22 E-06 \\
8.11 E-06 \\
1.51 E-06 \\
5.63 E-06 \\
4.12 E-06 \\
4.09 E-06 \\
2.70 E-06 \\
0.00 E+00 \\
1.16 E-05 \\
4.47 E-06 \\
2.45 E-06 \\
8.59 E-06 \\
1.35 E-06 \\
2.34 E-05 \\
9.08 E-06 \\
1.40 E-05 \\
1.80 E-05 \\
2.47 E-06 \\
2.15 E-05 \\
9.58 E-06 \\
5.10 E-06 \\
5.73 E-06 \\
1.26 E-07 \\
3.18 E-05 \\
5.69 E-06 \\
1.14 E-05 \\
4.23 E-06 \\
2.47 \Xi-06\end{array}$ & $\begin{array}{l}4.11 \mathrm{E}-05 \\
2.60 \mathrm{E}-05 \\
1.33 \mathrm{E}-05 \\
3.01 \mathrm{E}-05 \\
1.28 \mathrm{E}-05 \\
3.56 \mathrm{E}-05 \\
2.89 \mathrm{E}-05 \\
2.66 \mathrm{E}-06 \\
6.66 \mathrm{E}-06 \\
1.07 \mathrm{E}-06 \\
3.99 \mathrm{E}-05 \\
4.70 \mathrm{E}-05 \\
1.21 \mathrm{E}-05 \\
3.47 \mathrm{E}-06 \\
1.20 \mathrm{E}-05 \\
1.52 \mathrm{E}-06 \\
8.84 \mathrm{E}-07 \\
8.98 \mathrm{E}-07 \\
9.43 \mathrm{E}-07 \\
2.18 \mathrm{E}-07 \\
1.43 \mathrm{E}-06 \\
1.01 \mathrm{E}-06 \\
9.64 \mathrm{E}-08 \\
3.27 \mathrm{E}-07 \\
8.26 \mathrm{E}-08 \\
1.75 \mathrm{E}-06 \\
1.01 \mathrm{E}-06 \\
5.84 \mathrm{E}-07 \\
1.26 \mathrm{E}-07 \\
2.18 \mathrm{E}-07\end{array}$ \\
\hline
\end{tabular}


TABLE 4.1 Highway Combination-Truck Accident, Fatality, and Injury Rates by Road Type ${ }^{\mathrm{a}}$ Based on Reportable Interstate-Carrier Accidents, Reported Fatalities and Injuries, and Estimated Flows, 1986-1988 (10 ${ }^{-7}$ accidents and injuries per shipment-kilometer, $10^{-8}$ fatalities per shipment-kilometer)

\begin{tabular}{|c|c|c|c|c|c|c|c|c|c|c|c|c|c|c|c|}
\hline \multirow[b]{2}{*}{ State } & \multicolumn{5}{|c|}{ Accident-Involvument Rates } & \multicolumn{5}{|c|}{ Accident-Fatality Rates } & \multicolumn{5}{|c|}{ Accident-Injury Rules } \\
\hline & FAI-U & FAI-R & FAl & FAP & FAS & FAl-U & FAI-R & FAl & FAP & FAS & FAI-U & FAI-R & FAI & FAP & IFAS \\
\hline AL. & 4.68 & 1.26 & 1.85 & 5.16 & 3.96 & 3.29 & 1.84 & 2.09 & 6.34 & 7.84 & 4.66 & 1.24 & 1.83 & 5.62 & +.22 \\
\hline $\mathbf{A Z}$ & 2.71 & 1.60 & 1.76 & 2.12 & 1.45 & 3.56 & 2.13 & 2.33 & 4.97 & 1.20 & 2.89 & 1.65 & 1.82 & 2.20 & 2.77 \\
\hline$A R$ & 4.82 & 1.73 & 2.09 & 4.69 & 6.84 & 6.61 & 2.28 & 2.78 & 8.88 & 7.10 & 4.09 & 1.95 & 2.20 & 4.38 & 5.94 \\
\hline $\mathrm{CA}$ & 1.92 & 1.64 & 1.76 & 1.15 & 2.22 & 1.39 & 2.56 & 2.06 & 1.98 & 3.81 & 1.82 & 1.68 & 1.74 & 1.12 & 2.67 \\
\hline co & 6.28 & 2.76 & 3.60 & 4.11 & 4.42 & 3.38 & 2.45 & 2.67 & 6.58 & 5.83 & 5.47 & 2.82 & 3.45 & 3.95 & 3.67 \\
\hline CT & 2.67 & $4.60^{\mathrm{c}}$ & 3.23 & 2.56 & 9.09 & 1.01 & $5.11^{c}$ & 2.20 & 1.17 & 9.09 & 1.71 & $4.61^{\mathrm{c}}$ & 2.55 & 1.89 & 7.09 \\
\hline $\mathrm{DE}$ & 2.56 & .. & 2.56 & 7.35 & 4.81 & 1.66 & -- & 1.66 & $13.50^{\mathrm{c}}$ & 7.69 & 3.46 & .. & 3.46 & 7.18 & 3.08 \\
\hline FL. & 2.25 & 1.21 & 1.50 & 3.73 & 6.33 & 1.74 & 2.62 & 2.38 & 5.92 & 6.12 & 2.36 & 1.28 & 1.58 & +.12 & 7.92 \\
\hline$G A$ & 4.87 & 1.65 & 2.28 & 6.15 & 4.04 & 2.37 & 1.86 & 1.96 & 8.30 & 6.74 & 4.37 & 1.75 & 2.26 & 6.4 .3 & 3.71 \\
\hline ID & 1.73 & 2.30 & 2.22 & 4.93 & 2.29 & 0 & 2.06 & 1.78 & 7.46 & 2.54 & 1.18 & 2.12 & 1.99 & 3.54 & 1.69 \\
\hline IL & $8.75^{\mathrm{c}}$ & 1.76 & 3.53 & 6.40 & 1.78 & 5.33 & 1.39 & 2.38 & 7.84 & 2.30 & $8.23^{c}$ & 1.49 & 3.20 & 6.12 & 1.55 \\
\hline IN & 4.58 & 1.92 & 2.43 & 4.72 & 2.80 & 3.51 & 1.22 & 1.66 & 7.66 & 4.02 & 4.36 & 1.81 & 2.30 & 4.42 & 2.32 \\
\hline IA & 3.54 & 1.78 & 2.02 & 4.03 & 1.24 & 2.68 & 1.05 & 1.26 & 6.14 & 0.73 & 3.78 & 1.46 & 1.76 & 3.48 & 0.88 \\
\hline KS & 4.48 & 2.04 & 2.56 & 5.11 & 1.38 & 5.52 & 1.88 & 2.66 & 9.35 & 1.72 & 3.66 & 1.91 & 2.28 & 4.09 & 1.12 \\
\hline KY & 5.13 & 1.46 & 1.99 & 5.74 & 8.80 & 3.22 & 1.50 & 1.75 & 6.60 & 6.25 & 5.52 & 1.33 & 1.94 & 5.28 & 8.12 \\
\hline LA & 3.54 & 1.30 & 1.88 & 3.53 & 2.39 & 4.90 & 1.77 & 2.59 & 5.73 & 3.28 & 4.57 & 1.32 & 2.16 & 4.46 & 2.85 \\
\hline $\mathrm{ME}$ & $9.03^{\mathrm{c}}$ & 2.44 & 2.93 & 5.44 & 2.28 & 0 & 2.34 & 2.16 & 6.58 & 2.17 & 4.52 & 1.53 & 1.75 & 5.00 & 1.63 \\
\hline $\mathrm{MD}$ & 3.08 & 3.95 & 3.46 & 3.56 & 12,40 & 1.62 & 4.03 & 2.60 & 3.66 & 8.99 & 3.41 & 3.98 & $3.66^{\circ}$ & 4.32 & 13.40 \\
\hline MA & 1.42 & $6.47^{\circ}$ & 2.68 & 3.43 & $46.10^{c}$ & 1.30 & $6.23^{c}$ & 2.53 & 3.93 & $52.20^{c}$ & 1.13 & $4.99^{\mathrm{C}}$ & 2.09 & 3.02 & $43.90^{\circ}$ \\
\hline MI & 3.16 & 1.59 & 2.12 & 2.68 & 0.81 & 1.52 & 1.23 & 1.33 & 3.96 & 1.22 & 3.04 & 1.29 & 1.87 & 2.61 & 1.38 \\
\hline $\mathrm{MN}$ & 2.66 & 2.06 & 2.29 & 4.19 & 2.16 & 2.02 & 1.72 & 1.83 & 7.69 & 7.84 & 2.08 & 1.46 & 1.69 & 3.28 & 1.86 \\
\hline MS & 2.01 & 1.19 & 1.35 & 4.48 & 0.65 & 2.46 & 1.81 & 1.93 & 9.26 & 1.62 & 1.85 & 1.10 & 1.25 & 4.55 & 0.50 \\
\hline MO & 5.18 & 1.78 & 2.61 & 5.36 & 2.49 & 4.30 & 1.23 & 1.99 & 9.68 & 3.25 & 5.53 & 1.63 & 2.59 & 5.06 & 2.23 \\
\hline MT & $10.00^{e}$ & 2.52 & 2.89 & 5.38 & 1.02 & 3.12 & 1.44 & 1.52 & 7.97 & 2.04 & 4.69 & 1.79 & 1.93 & 3.95 & 0.20 \\
\hline NE & 6.27 & 1.77 & 2.09 & 3.62 & 0.99 & 6.58 & 1.10 & 1.43 & 5.75 & 0 & 6.58 & 1.17 & 1.50 & 3.54 & 0.54 \\
\hline NV & 6.33 & 1.57 & 1.97 & 4.35 & 3.17 & $8.89^{4}$ & 1.14 & 1.79 & i0.50 & 7.94 & 5.67 & 1.58 & 1.93 & 3.72 & 2.54 \\
\hline $\mathrm{NH}$ & 0.22 & 1.39 & 1.18 & 4.36 & 3.33 & 0 & 1.49 & 1.22 & 5.77 & 5.56 & 0 & 1.14 & 0.94 & 4.17 & 2.22 \\
\hline $\mathrm{NJ}$ & 2.77 & $7.65^{\mathrm{c}}$ & $4.24^{i}$ & 6.80 & 9.69 & 1.56 & $6.56^{\mathrm{c}}$ & 3.06 & 4.57 & 11.50 & 2.69 & $8.00^{i}$ & $4.28^{\mathrm{C}}$ & 6.86 & 11.30 \\
\hline NM & $9.64^{\mathrm{C}}$ & 1.92 & 2.35 & 4.77 & 12.20 & $9.01^{\mathrm{c}}$ & 1.93 & 2.32 & 6.99 & 5.56 & $8.92^{\mathrm{c}}$ & 1.86 & 2.25 & 4.62 & 10.60 \\
\hline NY & 5.69 & 2.93 & $3.98^{\mathrm{c}}$ & 3.16 & 9.48 & 2.04 & 1.38 & 1.63 & 4.61 & 10.30 & 4.49 & 2.56 & 3.28 & 2.71 & 10.00 \\
\hline
\end{tabular}


Table 6. Annual Incident-Free Dose for the Shipping Campaign (person-rem/yr)

\begin{tabular}{|c|c|c|c|c|c|c|}
\hline \multirow[b]{2}{*}{ Route } & & & \multicolumn{2}{|c|}{ Truck } & \multicolumn{2}{|c|}{ Rail } \\
\hline & & & Crew & Public & Crew & Public \\
\hline $\begin{array}{l}\text { Ames Laboratory } \\
\text { Argonne - East } \\
\text { Bettis Atomic } \\
\text { Fermilab (FNAL) } \\
\text { ITRI } \\
\text { Knolls Atomic } \\
\text { Kansas City Plant } \\
\text { Lawrence Berkeley } \\
\text { Mound Plant } \\
\text { ORISE } \\
\text { Princeton PPL } \\
\text { Pinellas Plant } \\
\text { Reactive Metals } \\
\text { SLAC } \\
\text { SNL - Albuquerque }\end{array}$ & $\begin{array}{l}\text { to } \\
\text { to } \\
\text { to } \\
\text { to } \\
\text { to } \\
\text { to } \\
\text { to } \\
\text { to } \\
\text { to } \\
\text { to } \\
\text { to } \\
\text { to } \\
\text { to } \\
\text { to } \\
\text { to }\end{array}$ & $\begin{array}{l}\text { Portsmouth GDP } \\
\text { Portsmouth GDP } \\
\text { Portsmouth GDP } \\
\text { Portsmouth GDP } \\
\text { Los Alamos NL } \\
\text { Portsmouth GDP } \\
\text { Paducah GDP } \\
\text { Lawrence Livermore } \\
\text { Portsmouth GDP } \\
\text { Oak Ridge Reserv. } \\
\text { Portsmouth GDP } \\
\text { Savannah River } \\
\text { Portsmouth GDP } \\
\text { Lawrence Livermore } \\
\text { Los Alamos NL }\end{array}$ & $\begin{array}{l}4.66 \mathrm{E}-02 \\
1.60 \mathrm{E}+00 \\
5.57 \mathrm{E}-01 \\
5.03 \mathrm{E}-02 \\
3.54 \mathrm{E}-02 \\
7.10 \mathrm{E}-01 \\
1.38 \mathrm{E}-02 \\
6.92 \mathrm{E}-03 \\
2.99 \mathrm{E}+00 \\
1.81 \mathrm{E}-03 \\
2.28 \mathrm{E}-02 \\
4.04 \mathrm{E}-02 \\
7.71 \mathrm{E}+00 \\
1.49 \mathrm{E}-01 \\
5.58 \mathrm{E}-02\end{array}$ & $\begin{array}{l}5.26 \mathrm{E}-02 \\
1.79 \mathrm{E}+00 \\
5.94 \mathrm{E}-01 \\
5.61 \mathrm{E}-02 \\
4.18 \mathrm{E}-02 \\
7.35 \mathrm{E}-01 \\
1.59 \mathrm{E}-02 \\
9.13 \mathrm{E}-03 \\
3.23 \mathrm{E}+00 \\
1.80 \mathrm{E}-03 \\
2.48 \mathrm{E}-02 \\
4.48 \mathrm{E}-02 \\
8.08 \mathrm{E}+00 \\
2.09 \mathrm{E}-01 \\
6.53 \mathrm{E}-02\end{array}$ & $\begin{array}{l}5.91 \mathrm{E}-03 \\
1.81 \mathrm{E}-01 \\
9.81 \mathrm{E}-02 \\
9.53 \mathrm{E}-03 \\
1.33 \mathrm{E}-02 \\
6.30 \mathrm{E}-02 \\
4.89 \mathrm{E}-03 \\
6.15 \mathrm{E}-03 \\
6.71 \mathrm{E}-01 \\
6.09 \mathrm{E}-03 \\
6.38 \mathrm{E}-03 \\
4.90 \mathrm{E}-03 \\
1.16 \mathrm{E}+00 \\
4.70 \mathrm{E}-02 \\
2.32 \mathrm{E}-02\end{array}$ & $\begin{array}{l}2.01 \mathrm{E}-02 \\
4.55 \mathrm{E}-01 \\
3.11 \mathrm{E}-01 \\
3.17 \mathrm{E}-02 \\
2.15 \mathrm{E}-02 \\
2.34 \mathrm{E}-01 \\
1.14 \mathrm{E}-02 \\
1.25 \mathrm{E}-02 \\
1.49 \mathrm{E}+00 \\
7.89 \mathrm{E}-03 \\
2.64 \mathrm{E}-02 \\
1.02 \mathrm{E}-02 \\
3.45 \mathrm{E}+00 \\
9.10 \mathrm{E}-02 \\
3.76 \mathrm{E}-02\end{array}$ \\
\hline $10 \mathrm{co}$ & & & & & & \\
\hline
\end{tabular}

Table 7. Annual Accident Dose Risk for the Shipping Campaign (person-rem/yr)

\begin{tabular}{llcc}
\hline Route & & Truck & Rail \\
\hline Ames Iaboratory & to Portsmouth GDP & $1.55 \mathrm{E}-05$ & $6.47 \mathrm{E}-06$ \\
Argonne - East & to Portsmouth GDP & $3.62 \mathrm{E}-02$ & $7.34 \mathrm{E}-03$ \\
Bettis Atomic & to Portsmouth GDP & $3.56 \mathrm{E}-04$ & $1.44 \mathrm{E}-04$ \\
Fermilab (FNAL) & to Portsmouth GDP & $7.30 \mathrm{E}-05$ & $1.90 \mathrm{E}-05$ \\
ITRI & to Los Alamos NL & $1.53 \mathrm{E}-02$ & $2.80 \mathrm{E}-04$ \\
Knolls Atomic & to Portsmouth GDP & $6.69 \mathrm{E}-05$ & $1.56 \mathrm{E}-05$ \\
Kansas City Plant & to Paducah GDP & $2.31 \mathrm{E}-05$ & $6.16 \mathrm{E}-06$ \\
Lawrence Berkeley & to Lawrence Livermore & $1.13 \mathrm{E}-02$ & $8.05 \mathrm{E}-03$ \\
Mound Plant & to Portsmouth GDP & $2.39 \mathrm{E}-04$ & $5.86 \mathrm{E}-05$ \\
ORISE & to Oak Ridge Reserv. & $2.24 \mathrm{E}-07$ & $6.30 \mathrm{E}-08$ \\
Princeton PPL & to Portsmouth GDP & $4.70 \mathrm{E}-06$ & $2.47 \mathrm{E}-06$ \\
Pinellas Plant & to Savannah River & $2.59 \mathrm{E}-06$ & $4.65 \mathrm{E}-07$ \\
Reactive Metais & to Portsmouth GDP & $1.73 \mathrm{E}-06$ & $5.00 \mathrm{E}-07$ \\
SLAC & to Lawrence Livermore & $1.89 \mathrm{E}-06$ & $8.53 \mathrm{E}-07$ \\
SNL - Albuquerque & to Los Alamos NL & $9.16 \mathrm{E}-05$ & $1.75 \mathrm{E}-06$ \\
\hline Totals & & $6.36 \mathrm{E}-02$ & $1.59 \mathrm{E}-02$ \\
\hline
\end{tabular}

Table 8. Expected Annual Fatalities

for the Shipping Campaign

\begin{tabular}{lll}
\hline Exposure Group & Truck & Rail \\
\hline Radiological & $5.6 E-03$ & $9.2 E-04$ \\
Normal Crew & $7.5 E-03$ & $3.1 E-03$ \\
Normal Public & $3.2 \mathrm{E}-05$ & $8.0 \mathrm{E}-06$ \\
Accident Public & & \\
Nonradiological & & \\
Emission & $1.8 \mathrm{E}-03$ & $5.5 \mathrm{E}-03$ \\
Accident & $1.8 \mathrm{E}-02$ & $3.1 \mathrm{E}-04$ \\
\hline
\end{tabular}

Table 9. Expected Annual Cancer Incidence for the Shipping Campaign

\begin{tabular}{|c|c|c|}
\hline Exposure Group & Truck & Rail \\
\hline $\begin{array}{l}\text { Radiological } \\
\text { Normal Crew } \\
\text { Normal Public } \\
\text { Accident Public } \\
\text { Nonradiological }\end{array}$ & $\begin{array}{l}2.0 \mathrm{E}-02 \\
2.5 \mathrm{E}-02 \\
1.1 \mathrm{E}-04\end{array}$ & $\begin{array}{l}3.2 E-03 \\
1.1 E-02 \\
2.7 E-05\end{array}$ \\
\hline $\begin{array}{l}\text { Nonradiological } \\
\text { Emission } \\
\text { Accident }\end{array}$ & $\begin{array}{l}4.8 \mathrm{E}-03 \\
\mathrm{NA}\end{array}$ & $\begin{array}{l}5.5 \mathrm{E}-03 \\
\mathrm{NA}\end{array}$ \\
\hline
\end{tabular}


Table 10. Expected Annual Genetic Effects for the Shipping Campaign

\begin{tabular}{lll}
\hline Exposure Group & Truck & Rail \\
\hline Radiological & & \\
Normal Crew & $8.4 \mathrm{E}-04$ & $1.4 \mathrm{E}-04$ \\
Normal Public & $1.5 \mathrm{E}-03$ & $6.2 \mathrm{E}-04$ \\
$\begin{array}{l}\text { Accident Public } \\
\text { Nonradiological } \\
\text { Emission }\end{array}$ & $3.1 \mathrm{E}-06$ & $6.8 \mathrm{E}-07$ \\
Accident & NA & NA \\
\hline
\end{tabular}




\section{B.6 WM LLW REGIONALIZED 1 ALTERNATIVE (CASE 3): ACTIVATED METALS}

Table 1. Summary of Route Information

\begin{tabular}{|c|c|c|c|c|c|}
\hline & \multirow[b]{2}{*}{ Route } & \multirow{2}{*}{$\begin{array}{c}\text { Distance } \\
\text { (miles) }\end{array}$} & \multicolumn{3}{|c|}{ Percentage in Zone } \\
\hline & & & Rural & Suburban & Urban \\
\hline $\begin{array}{l}\text { Truck } \\
\text { Bettis Atomic } \\
\text { Eermilab (FNAL) } \\
\text { Knolls Atomic } \\
\text { Lawrence Berkeley } \\
\text { SNL - Albuquerque } \\
\text { Rail }\end{array}$ & $\begin{array}{l}\text { to Portsmouth GDP } \\
\text { to Portsmouth GDP } \\
\text { to Portsmouth GDP } \\
\text { to Lawrence Livermore } \\
\text { to Los Alamos NL }\end{array}$ & $\begin{array}{r}265 \\
460 \\
688 \\
45 \\
102\end{array}$ & $\begin{array}{l}58.8 \\
62.5 \\
57.3 \\
38.4 \\
75.8\end{array}$ & $\begin{array}{l}37.4 \\
32.0 \\
40.1 \\
33.3 \\
20.1\end{array}$ & $\begin{array}{r}3.8 \\
5.5 \\
2.5 \\
28.2 \\
4.1\end{array}$ \\
\hline $\begin{array}{l}\text { Rail } \\
\text { Bettis Atomic } \\
\text { Fermilab (FNAL) } \\
\text { Knolls Atomic } \\
\text { Lawrence Berkeley } \\
\text { SNL - Albuquerque }\end{array}$ & $\begin{array}{l}\text { to Portsmouth GDP } \\
\text { to Portsmouth GDP } \\
\text { to Portsmouth GDP } \\
\text { to Lawrence Livermore } \\
\text { to Los Alamos NL }\end{array}$ & $\begin{array}{r}429 \\
451 \\
681 \\
46 \\
104\end{array}$ & $\begin{array}{l}52.6 \\
61.5 \\
52.1 \\
39.0 \\
74.3\end{array}$ & $\begin{array}{l}39.6 \\
28.9 \\
40.3 \\
34.5 \\
20.0\end{array}$ & $\begin{array}{r}7.8 \\
9.5 \\
7.5 \\
26.5 \\
5.7\end{array}$ \\
\hline
\end{tabular}

Table 2. Annual Number of Shipments and One-Way Shipment Distances

\begin{tabular}{|c|c|c|c|c|c|}
\hline \multirow[b]{2}{*}{ Route } & & \multicolumn{2}{|c|}{ \# of Shipments } & \multicolumn{2}{|c|}{ Total Mileage (mi) } \\
\hline & & Truck & Rail & Truck & Rail \\
\hline $\begin{array}{l}\text { Bettis Atomic } \\
\text { Fermilab (FNAL) } \\
\text { Knolis Atomic } \\
\text { Lawrence Berkeley } \\
\text { SNL - Albuquerque }\end{array}$ & $\begin{array}{l}\text { to Portsmouth GDP } \\
\text { to Portsmouth GDP } \\
\text { to Portsmouth GDP } \\
\text { to Lawrence Livermore } \\
\text { to Los Alamos NL }\end{array}$ & $\begin{array}{r}316 \\
40 \\
650 \\
15 \\
6\end{array}$ & $\begin{array}{r}120 \\
15 \\
246 \\
6 \\
3\end{array}$ & $\begin{array}{l}8.37 E+04 \\
1.84 E+04 \\
4.47 E+05 \\
6.75 E+02 \\
6.12 E+02\end{array}$ & $\begin{array}{l}5.15 \mathrm{E}+04 \\
6.76 \mathrm{E}+03 \\
1.68 \mathrm{E}+05 \\
2.77 \mathrm{E}+02 \\
3.12 \mathrm{E}+02\end{array}$ \\
\hline Totals & & 1027 & 390 & $5.51 \mathrm{E}+05$ & $2.26 \mathrm{E}+05$ \\
\hline
\end{tabular}

Table 3. Incident-Free Dose per Shipment (person-rem/shipment)

\begin{tabular}{|c|c|c|c|c|c|}
\hline \multirow[b]{2}{*}{ Source/Route (s) } & \multirow[b]{2}{*}{ Crew } & \multicolumn{4}{|c|}{ General Public } \\
\hline & & off-Iink & On-Link & Stops & Total \\
\hline $\begin{array}{l}\text { LLW(I) BA to PO } \\
\text { Bettis Atomic }\end{array}$ & $\begin{array}{lr}\text { to Portsmouth GDP } \\
\text { Truck } & 9.78 \mathrm{E}-03 \\
\text { Rail } & 4.67 \mathrm{E}-03\end{array}$ & $\begin{array}{l}1.26 \mathrm{E}-03 \\
9.85 \mathrm{E}-03\end{array}$ & $\begin{array}{l}1.73 \mathrm{E}-03 \\
1.53 \mathrm{E}-04\end{array}$ & $\begin{array}{l}7.42 \mathrm{E}-03 \\
4.80 \mathrm{E}-03\end{array}$ & $\begin{array}{l}1.04 E-02 \\
1.48 E-02\end{array}$ \\
\hline $\begin{array}{l}\text { LLW(I) FL to Po } \\
\text { Fermilab (FNAL) }\end{array}$ & $\begin{array}{ll} & \\
\text { to Portsmouth GDP } \\
\text { Truck } & 1.68 \mathrm{E}-02 \\
\text { Rail } & 4.76 \mathrm{E}-03\end{array}$ & $\begin{array}{l}2.40 \mathrm{E}-03 \\
1.08 \mathrm{E}-02\end{array}$ & $\begin{array}{l}3.43 E-03 \\
1.48 E-04\end{array}$ & $\begin{array}{l}1.29 \mathrm{E}-02 \\
4.87 \mathrm{E}-03\end{array}$ & $\begin{array}{l}1.87 \mathrm{E}-02 \\
1.58 \mathrm{E}-02\end{array}$ \\
\hline $\begin{array}{l}\text { LLW(I) RA to PO } \\
\text { Knolis Atomic }\end{array}$ & $\begin{array}{lr} & \\
\text { to Portsmouth GDP } \\
\text { Truck } & 2.54 \mathrm{E}-02 \\
\text { Rail } & 5.72 \mathrm{E}-03\end{array}$ & $\begin{array}{l}2.99 \mathrm{E}-03 \\
1.54 \mathrm{E}-02\end{array}$ & $\begin{array}{l}4.01 E-03 \\
2.43 E-04\end{array}$ & $\begin{array}{l}1.93 E-02 \\
5.58 E-03\end{array}$ & $\begin{array}{l}2.63 E-02 \\
2.13 E-02\end{array}$ \\
\hline $\begin{array}{l}\text { LLW(I) LB to LI } \\
\text { Lawrence Berkeley }\end{array}$ & $\begin{array}{ll} & \\
\text { to Lawrence Liverm } \\
\text { Truck } & 2.31 \mathrm{E}-03 \\
\text { Rail } & 3.07 \mathrm{E}-03\end{array}$ & $\begin{array}{l}\text { lore } \\
7.51 E-04 \\
2.62 E-03\end{array}$ & $\begin{array}{l}1.03 E-03 \\
2.79 E-05\end{array}$ & $\begin{array}{l}1.26 \mathrm{E}-03 \\
3.62 \mathrm{E}-03\end{array}$ & $\begin{array}{l}3.04 E-03 \\
6.27 E-03\end{array}$ \\
\hline $\begin{array}{l}\text { LLW(I) SA to LA } \\
\text { SNL - Albuquerque }\end{array}$ & $\begin{array}{cc}\text { to Los Alamos NL } \\
\text { Truck } & 3.28 \mathrm{E}-03 \\
\text { Rail } & 3.31 \mathrm{E}-03\end{array}$ & $\begin{array}{l}3.70 \mathrm{E}-04 \\
1.55 \mathrm{E}-03\end{array}$ & $\begin{array}{l}6.13 E-04 \\
2.36 \mathrm{E}-05\end{array}$ & $\begin{array}{l}2.86 E-03 \\
3.80 E-03\end{array}$ & $\begin{array}{l}3.84 E-03 \\
5.37 E-03\end{array}$ \\
\hline
\end{tabular}


Table 4. Accident Dose Risk per Shipment (person-rem/shipment)

\begin{tabular}{llll}
\hline Source/Route & & Truck & Rail \\
\hline LLW(I) BA to PO \\
$\begin{array}{c}\text { Bettis Atomic } \\
\text { LLW(I) FL to PO }\end{array}$ & to Portsmouth GDP & $3.62 \mathrm{E}-07$ & $4.12 \mathrm{E}-07$ \\
$\begin{array}{c}\text { Fermilab (FNAL) } \\
\text { LLW(I) KA to PO } \\
\text { Knolls Atomic }\end{array}$ & to Portsmouth GDP & $5.17 \mathrm{E}-11$ & $3.57 \mathrm{E}-11$ \\
$\begin{array}{c}\text { LLW(I) LB to LL } \\
\text { Lawrence Berkeley }\end{array}$ & to Portsmouth GDP & $1.63 \mathrm{E}-06$ & $1.01 \mathrm{E}-06$ \\
$\begin{array}{c}\text { LLW(I) SA to LA } \\
\text { SNL - Albuquerque }\end{array}$ & to Lowrence Livermore & $1.27 \mathrm{E}-11$ & $2.29 \mathrm{E}-11$ \\
\end{tabular}

Table 5. Nonradiological Risk Factors per Shipment

(fatalities/shipment)

\begin{tabular}{|c|c|c|c|}
\hline Mode & Route & Emission & Accident \\
\hline $\begin{array}{l}\text { Truck } \\
\text { Bettis Atomic } \\
\text { Fermilab (FNAL) } \\
\text { Knolls Atomic } \\
\text { Lawrence Berkeley } \\
\text { SNL - Albuquerque } \\
\text { Rail } \\
\text { Bettis Atomic } \\
\text { Fermilab (FNAL) } \\
\text { Knolls Atomic } \\
\text { Lawrence Berkeley } \\
\text { SNL - Albuquerque }\end{array}$ & $\begin{array}{l}\text { to Portsmouth GDP } \\
\text { to Portsmouth GDP } \\
\text { to Portsmouth GDP } \\
\text { to Lawrence Livermore } \\
\text { to Los Alamos NL } \\
\text { to Portsmouth GDP } \\
\text { to Portsmouth GDP } \\
\text { to Portsmouth GDP } \\
\text { to Lawrence Livermore } \\
\text { to Los Alamos NL }\end{array}$ & $\begin{array}{l}3.22 \mathrm{E}-06 \\
8.11 \mathrm{E}-06 \\
5.63 \mathrm{E}-06 \\
4.09 \mathrm{E}-06 \\
1.35 \mathrm{E}-06 \\
1.40 \mathrm{E}-05 \\
1.80 \mathrm{E}-05 \\
2.15 \mathrm{E}-05 \\
5.10 \mathrm{E}-06 \\
2.47 \mathrm{E}-06\end{array}$ & $\begin{array}{l}1.33 E-05 \\
3.01 E-05 \\
3.56 E-05 \\
2.66 E-06 \\
1.20 E-05 \\
8.98 E-07 \\
9.43 E-07 \\
1.43 E-06 \\
9.64 E-08 \\
2.18 E-07\end{array}$ \\
\hline
\end{tabular}

Table 6. Annual Incident-Free Dose for the Shipping Campaign (person-rem/yr)

\begin{tabular}{|c|c|c|c|c|c|}
\hline \multirow[b]{2}{*}{ Route } & & \multicolumn{2}{|c|}{ Truck } & \multicolumn{2}{|c|}{ Rail } \\
\hline & & Crew & Public & Crew & Public \\
\hline $\begin{array}{l}\text { Bettis Atomic } \\
\text { Fermilab (FNAL) } \\
\text { KnoIls Atomic } \\
\text { Lawrence Berkeley } \\
\text { SNL - Albuquerque }\end{array}$ & $\begin{array}{l}\text { to Portsmouth GDP } \\
\text { to Portsmouth GDP } \\
\text { to Portsmouth GDP } \\
\text { to Lawrence Iivermore } \\
\text { to Los Alamos NL }\end{array}$ & $\begin{array}{l}3.09 \mathrm{E}+00 \\
6.70 \mathrm{E}-01 \\
1.65 \mathrm{E}+01 \\
3.46 \mathrm{E}-02 \\
1.97 \mathrm{E}-02\end{array}$ & $\begin{array}{l}3.29 \mathrm{E}+00 \\
7.48 \mathrm{E}-01 \\
1.71 \mathrm{E}+01 \\
4.57 \mathrm{E}-02 \\
2.30 \mathrm{E}-02\end{array}$ & $\begin{array}{l}5.61 E-01 \\
7.14 E-02 \\
1.41 E+00 \\
1.84 E-02 \\
9.94 E-03\end{array}$ & $\begin{array}{l}1.78 \mathrm{E}+00 \\
2.37 \mathrm{E}-01 \\
5.23 \mathrm{E}+00 \\
3.76 \mathrm{E}-02 \\
1.61 \mathrm{E}-02\end{array}$ \\
\hline Totals & & $2.03 E+01$ & $2.12 \mathrm{E}+01$ & $2.07 E+00$ & $7.30 \mathrm{E}+00$ \\
\hline
\end{tabular}

Table 7. Annual Accident Dose Risk for the Shipping Campaign (person-rem/yr)

\begin{tabular}{llcc}
\hline Route & & Truck & Rail \\
\hline Bettis Atomic & to Portsmouth GDP & $1.14 \mathrm{E}-04$ & $4.94 \mathrm{E}-05$ \\
Fermilab (FNAL) & to Portsmouth GDP & $2.07 \mathrm{E}-09$ & $5.35 \mathrm{E}-10$ \\
Knolls Atomic & to Portsmouth GDP & $1.06 \mathrm{E}-03$ & $2.49 \mathrm{E}-04$ \\
Lawrence Berkeley & to Lawrence Livermore & $1.90 \mathrm{E}-10$ & $1.37 \mathrm{E}-10$ \\
SNL - Albuquerque & to Los Alamos NL & $1.03 \mathrm{E}-11$ & $1.95 \mathrm{E}-13$ \\
\hline Totals & & $1.17 \mathrm{E}-03$ & $2.98 \mathrm{E}-04$ \\
\hline
\end{tabular}

Table 8. Expected Annual Fatalities for the Shipping Campaign

\begin{tabular}{lll}
\hline Exposure Group & Truck & Rail \\
\hline Radiological & $8.1 \mathrm{E}-03$ & $8.3 \mathrm{E}-04$ \\
Normal Crew & $1.1 \mathrm{E}-02$ & $3.6 \mathrm{E}-03$ \\
Normal Public & $5.9 \mathrm{E}-07$ & $1.5 \mathrm{E}-07$ \\
Accident Public & $5.1 \mathrm{E}-03$ & $7.3 \mathrm{E}-03$ \\
Nonradiological & $2.9 \mathrm{E}-02$ & $4.7 \mathrm{E}-04$ \\
Emission & & \\
Accident & & \\
\hline
\end{tabular}


Table 9. Expected Annual Cancer Incidence for the Shipping Campaign

\begin{tabular}{lll}
\hline Exposure Group & Truck & Rail \\
\hline Radiological & & \\
Normal Crew & $2.8 E-02$ & $2.9 E-03$ \\
Normal Public & $3.6 E-02$ & $1.2 E-02$ \\
$\begin{array}{l}\text { Accident Public } \\
\text { Nonradiological } \\
\text { Emission }\end{array}$ & $2.0 E-06$ & $5.1 E-07$ \\
Accident & $5.1 E-03$ & $7.3 E-03$ \\
\hline
\end{tabular}

Table 10. Expected Annual Genetic Effects

for the Shipping Campaign

\begin{tabular}{|c|c|c|}
\hline Exposure Group & Truck & Rail \\
\hline $\begin{array}{l}\text { Radiological } \\
\text { Normal Crew } \\
\text { Normal Public } \\
\text { Accident Public } \\
\text { Nonradiological } \\
\text { Emission } \\
\text { Accident }\end{array}$ & $\begin{array}{l}1.2 \mathrm{E}-03 \\
2.1 \mathrm{E}-03 \\
1.2 \mathrm{E}-07 \\
\mathrm{NA} \\
\mathrm{NA}\end{array}$ & $\begin{array}{l}1.2 \mathrm{E}-04 \\
7.3 \mathrm{E}-04 \\
3.0 \mathrm{E}-08 \\
\mathrm{NA} \\
\mathrm{NA}\end{array}$ \\
\hline
\end{tabular}




\section{B.7 WM LLW REGIONALIZED 2 ALTERNATIVE (CASE 9): HETEROGENEOUS SOLIDS}

Table 1. Summary of Route Information

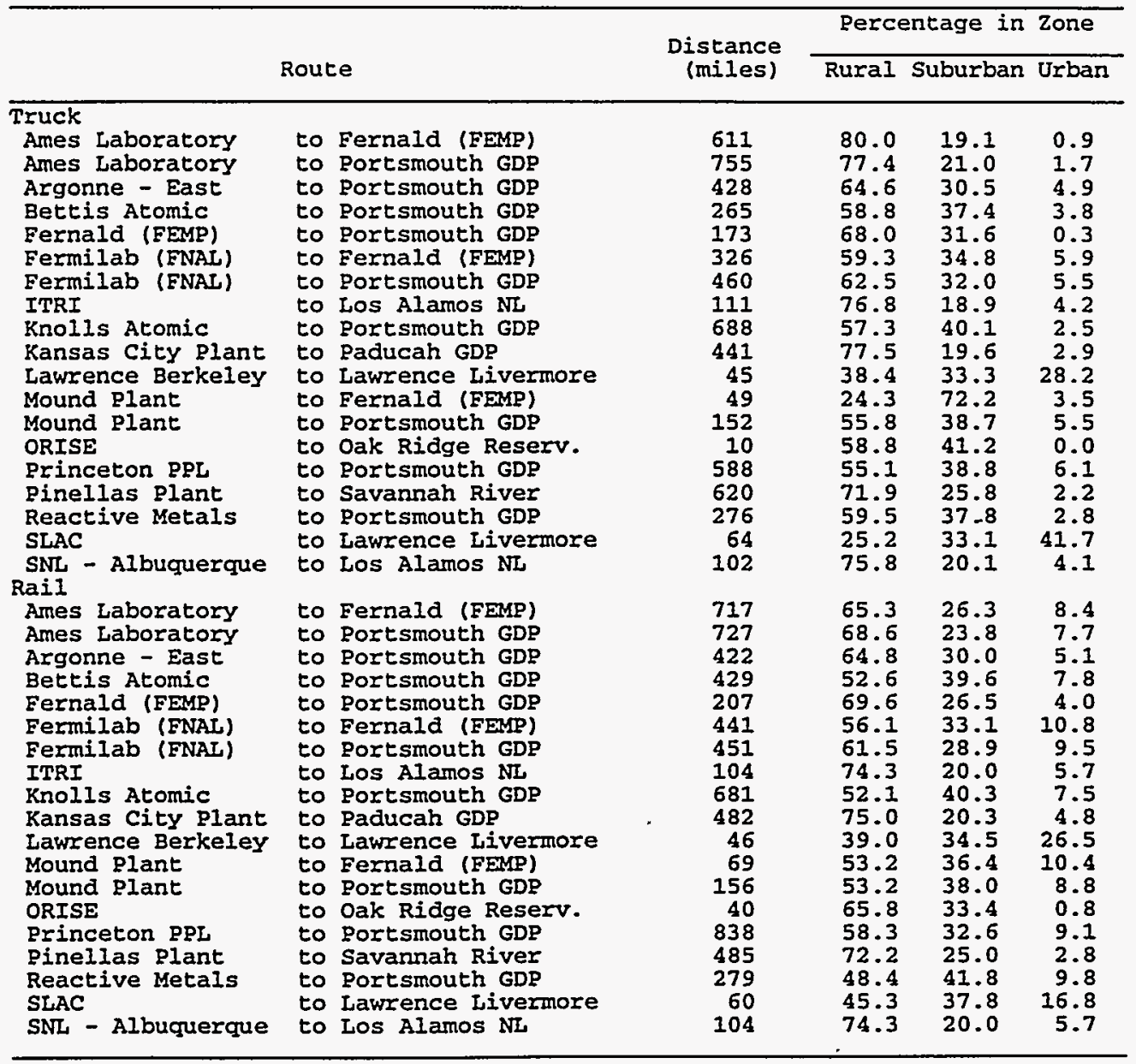

Table 2. Annual Number of Shipments and One-Way Shipment Distances

\begin{tabular}{|c|c|c|c|c|c|c|}
\hline \multirow[b]{2}{*}{ Route } & & & \multicolumn{2}{|c|}{ \# of Shipments } & \multicolumn{2}{|c|}{ Total Mileage (mi) } \\
\hline & & & Truck & Rail & Truck & Rail \\
\hline $\begin{array}{l}\text { Ames Laboratory } \\
\text { Ames Laboratory } \\
\text { Argonne - East } \\
\text { Bettis Atomic } \\
\text { Fernald (FEMP) } \\
\text { Fermilab (FNAL) } \\
\text { Fermilab (FNAL) } \\
\text { ITRI } \\
\text { Knolls Atomic } \\
\text { Kansas City Plant } \\
\text { Lawrence Berkeley } \\
\text { Mound Plant } \\
\text { Mound Plant } \\
\text { ORISE } \\
\text { Princeton PPL } \\
\text { Pinellas plant } \\
\text { Reactive Metals } \\
\text { SLAC } \\
\text { SNL - Albuquerque }\end{array}$ & $\begin{array}{l}\text { to } \\
\text { to } \\
\text { to } \\
\text { to } \\
\text { to } \\
\text { to } \\
\text { to } \\
\text { to } \\
\text { to } \\
\text { to } \\
\text { to } \\
\text { to } \\
\text { to } \\
\text { to } \\
\text { to } \\
\text { to } \\
\text { to } \\
\text { to } \\
\text { to }\end{array}$ & $\begin{array}{l}\text { Fernald (FEMP) } \\
\text { Portsmouth GDP } \\
\text { Portsmouth GDP } \\
\text { Portsmouth GDP } \\
\text { Portsmouth GDP } \\
\text { Fernald (FEMP) } \\
\text { Portsmouth GDP } \\
\text { Los Alamos NL } \\
\text { Portsmouth GDP } \\
\text { Paducah GDP } \\
\text { Lawrence Livermore } \\
\text { Fernald (FEMP) } \\
\text { Portsmouth GDP } \\
\text { Oak Ridge Reserv. } \\
\text { Portsmouth GDP } \\
\text { Savannah River } \\
\text { Portsmouth GDP } \\
\text { Lawrence Livermore } \\
\text { Los Alamos NL }\end{array}$ & $\begin{array}{r}1 \\
2 \\
105 \\
57 \\
7 \\
1 \\
2 \\
10 \\
28 \\
1 \\
3 \\
12 \\
496 \\
5 \\
1 \\
2 \\
761 \\
39 \\
17\end{array}$ & $\begin{array}{r}1 \\
1 \\
39 \\
21 \\
3 \\
1 \\
1 \\
4 \\
11 \\
1 \\
2 \\
5 \\
186 \\
2 \\
1 \\
1 \\
285 \\
15 \\
7\end{array}$ & 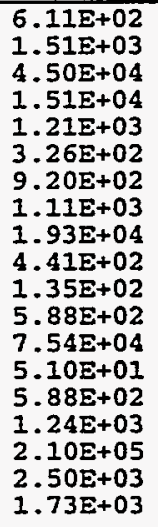 & $\begin{array}{l}7.17 E+02 \\
7.27 E+02 \\
1.65 E+04 \\
9.01 E+03 \\
6.22 E+02 \\
4.41 E+02 \\
4.51 E+02 \\
4.16 E+02 \\
7.50 E+03 \\
4.82 E+02 \\
9.22 E+01 \\
3.45 E+02 \\
2.90 E+04 \\
7.90 E+01 \\
8.38 E+02 \\
4.85 E+02 \\
7.95 E+04 \\
9.00 E+02 \\
7.28 E+02\end{array}$ \\
\hline Totals & & & 1550 & 587 & $3.78 \mathrm{E}+05$ & $1.49 \mathrm{E}+05$ \\
\hline
\end{tabular}


Table 3. Incident-Free Dose per Shipment (person-rem/shipment)

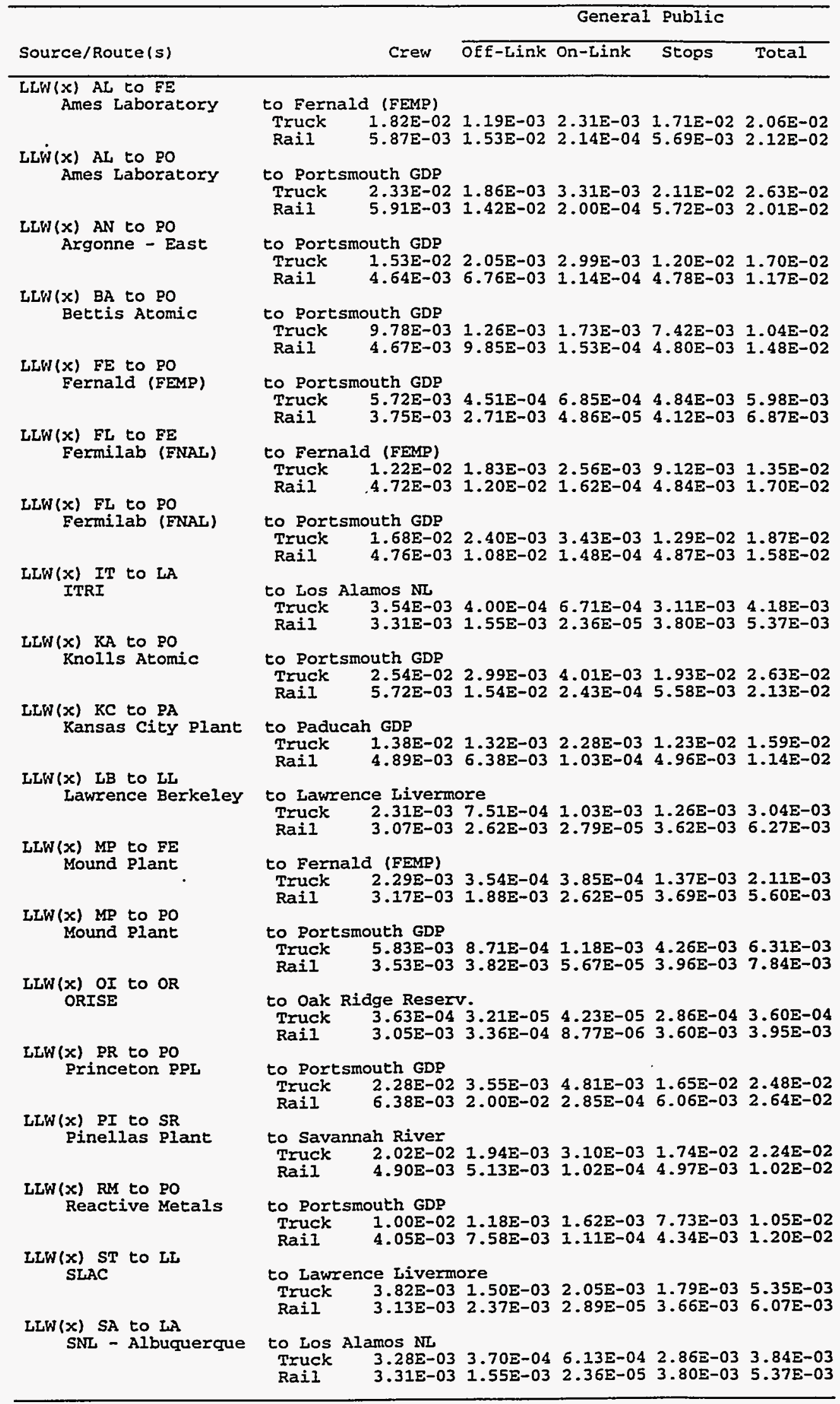


Table 4. Accident Dose Risk per Shipment (person-rem/shipment)

\begin{tabular}{|c|c|c|c|}
\hline Source/Route & & Truck & Rail \\
\hline 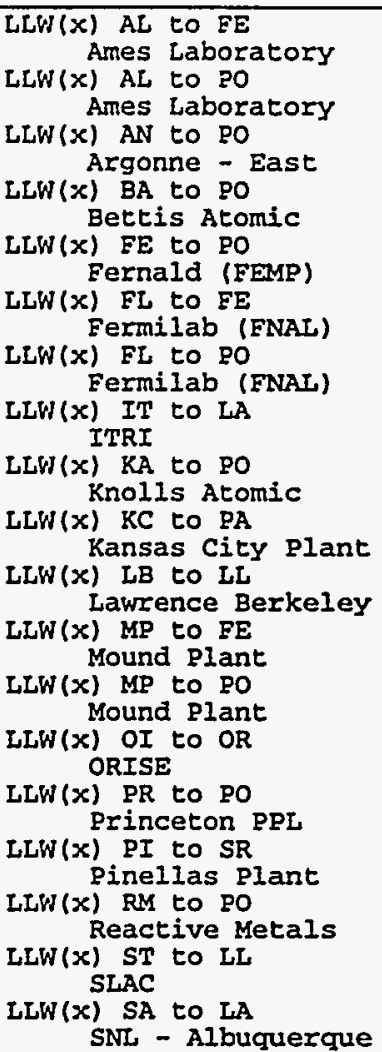 & $\begin{array}{l}\text { to Fernald (FEMP) } \\
\text { to Portsmouth GDP } \\
\text { to Portsmouth GDP } \\
\text { to Portsmouth GDP } \\
\text { to Portsmouth GDP } \\
\text { to Fernald (FEMP) } \\
\text { to Portsmouth GDP } \\
\text { to Los Alamos NL } \\
\text { to Portsmouth GDP } \\
\text { to Paducah GDP } \\
\text { to Lawrence Livermore } \\
\text { to Fernald (FEMP) } \\
\text { to Portsmouth GDP } \\
\text { to Oak Ridge Reserv. } \\
\text { to Portsmouth GDP } \\
\text { to Savannah River } \\
\text { to Portsmouth GDP } \\
\text { to Lawrence Livermore } \\
\text { to Los Alamos NL }\end{array}$ & $\begin{array}{l}8.59 \mathrm{E}-07 \\
7.28 \mathrm{E}-06 \\
3.45 \mathrm{E}-04 \\
6.25 \mathrm{E}-06 \\
2.24 \mathrm{E}-08 \\
1.60 \mathrm{E}-11 \\
3.67 \mathrm{E}-05 \\
1.53 \mathrm{E}-03 \\
2.39 \mathrm{E}-06 \\
2.31 \mathrm{E}-05 \\
3.75 \mathrm{E}-03 \\
3.56 \mathrm{E}-08 \\
4.81 \mathrm{E}-07 \\
4.49 \mathrm{E}-08 \\
4.70 \mathrm{E}-06 \\
1.30 \mathrm{E}-06 \\
2.28 \mathrm{E}-09 \\
4.86 \mathrm{E}-08 \\
5.39 \mathrm{E}-06\end{array}$ & $\begin{array}{l}4.13 E-07 \\
6.06 E-06 \\
1.88 E-04 \\
6.87 E-06 \\
1.15 E-08 \\
5.07 E-12 \\
1.89 E-05 \\
7.01 E-05 \\
1.42 E-06 \\
6.16 E-06 \\
4.02 E-03 \\
2.03 E-08 \\
3.13 E-07 \\
3.15 E-08 \\
2.47 E-06 \\
4.65 E-07 \\
1.75 E-09 \\
5.69 E-08 \\
2.50 E-07\end{array}$ \\
\hline
\end{tabular}


Table 5. Nonradiological Risk Factors per Shipment (fatalities/shipment)

\begin{tabular}{|c|c|c|c|}
\hline Mode & Route & Enissio: & $\therefore=c$ ident \\
\hline $\begin{array}{l}\text { Truck } \\
\text { Ames Laboratory } \\
\text { Ames Laboratory } \\
\text { Argonne - East } \\
\text { Bettis Atomic } \\
\text { Fernald (FgMP) } \\
\text { Fermilab (FNAL) } \\
\text { Fermilab (FNAL) } \\
\text { ITRI } \\
\text { Knolls Atomic } \\
\text { Kansas City Plant } \\
\text { Lawrence Berkeley } \\
\text { Mound Plant } \\
\text { Mound Plant } \\
\text { ORISE } \\
\text { Princeton PpL } \\
\text { Pinellas Plant } \\
\text { Reactive Metals } \\
\text { SLAC } \\
\text { SNL - Albuquerque } \\
\text { Rail } \\
\text { Ames Laboratory } \\
\text { Ames Laboratory } \\
\text { Argonne - East } \\
\text { Bettis Atomic } \\
\text { Fernald (FEMP) } \\
\text { Fermilab (FNAL) } \\
\text { Fermilab (FNAL) } \\
\text { ITRI } \\
\text { Knolls Atomic } \\
\text { Kansas City Plant } \\
\text { Lawrence Berkeley } \\
\text { Mound Plant } \\
\text { Mound Plant } \\
\text { ORISE } \\
\text { Princeton gPL } \\
\text { Pinellas plant } \\
\text { Reactive Metals } \\
\text { SLAC } \\
\text { SNL - Albuquerque }\end{array}$ & $\begin{array}{l}\text { to Fernald (FEMP) } \\
\text { to Portsmouth GDP } \\
\text { to Portsmouth GDP } \\
\text { to Portsmouth GDP } \\
\text { to Portsmouth GDP } \\
\text { to Fernald (FEMP) } \\
\text { to Portsmouth GDP } \\
\text { to Los Alamos NL } \\
\text { to Portsmouth GDP } \\
\text { to Paducah GDP } \\
\text { to Lawrence Livermore } \\
\text { to Fernald (FEMP) } \\
\text { to Portsmouth GDP } \\
\text { to Oak Ridge Reserv. } \\
\text { to Portsmouth GDP } \\
\text { to Savannah River } \\
\text { to Portsmouth GDP } \\
\text { to Lawrence Livermore } \\
\text { to Los Alamos NL } \\
\text { to Fernald (FEMP) } \\
\text { to Portsmouth GDP } \\
\text { to Portsmouth GDP } \\
\text { to Portsmouth GDP } \\
\text { to Portsmouth GDP } \\
\text { to Fernald (FEMP) } \\
\text { to Portsmouth GDP } \\
\text { to Los Alamos NL } \\
\text { to Portsmouth GDP } \\
\text { to Paducah GDP } \\
\text { to Lawrence Livermore } \\
\text { to Fernald (FEMP) } \\
\text { to Portsmouth GDP } \\
\text { to Oak Ridge Reserv. } \\
\text { to Portsmouth GDP } \\
\text { to Savannah River } \\
\text { to Portsmouth GDP } \\
\text { to Lawrence Livermore } \\
\text { to Los Alamos NL }\end{array}$ & 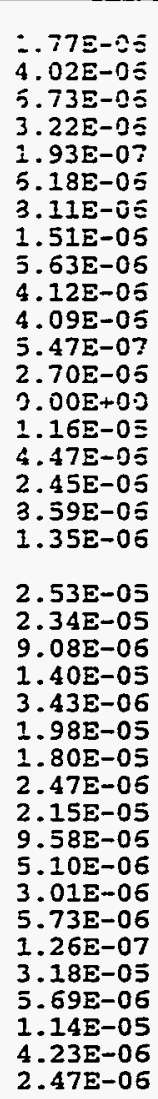 & 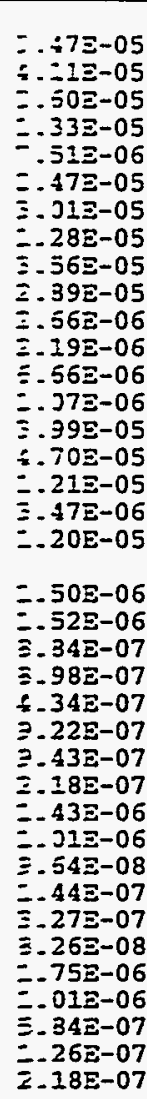 \\
\hline
\end{tabular}

Table 6. Annual Incident-Free Dose for the Shipping Campais= 'person-rem/yr)

\begin{tabular}{|c|c|c|c|c|c|c|}
\hline \multirow[b]{2}{*}{ Route } & & & \multicolumn{2}{|c|}{ Truck } & \multicolumn{2}{|c|}{ Rail } \\
\hline & & & Crew & Public & Crew & Public \\
\hline $\begin{array}{l}\text { Ames Laboratory } \\
\text { Ames Laboratory } \\
\text { Argonne - East } \\
\text { Bettis Atomic } \\
\text { Fernald (FEMP) } \\
\text { Fermilab (FNAL) } \\
\text { Fermilab (FNAL) } \\
\text { ITRI } \\
\text { Knolls Atomic } \\
\text { Kansas City Plant } \\
\text { Lawrence Berkeley } \\
\text { Mound Plant } \\
\text { Mound Plant } \\
\text { ORISE } \\
\text { Princeton PPL } \\
\text { Pinellas Plant } \\
\text { Reactive Metals } \\
\text { SLAC } \\
\text { SNL - Albuquerque }\end{array}$ & $\begin{array}{l}\text { to } \\
\text { to } \\
\text { to } \\
\text { to } \\
\text { to } \\
\text { to } \\
\text { to } \\
\text { to } \\
\text { to } \\
\text { to } \\
\text { to } \\
\text { to } \\
\text { to } \\
\text { to } \\
\text { to } \\
\text { to } \\
\text { to } \\
\text { to }\end{array}$ & $\begin{array}{l}\text { Fernald (FEMP) } \\
\text { Portsmouth GDP } \\
\text { Portsmouth GDP } \\
\text { Portsmouth GDP } \\
\text { Portsmouth GDP } \\
\text { Fernald (FEMP) } \\
\text { Portsmouth GDP } \\
\text { Los Alamos NL } \\
\text { Portsmouth GDP } \\
\text { Paducah GDP } \\
\text { Lawrence Livermore } \\
\text { Fernald (FEMP) } \\
\text { Portsmouth GDP } \\
\text { Oak Ridge Reserv. } \\
\text { Portsmouth GDP } \\
\text { Savannah River } \\
\text { Portsmouth GDP } \\
\text { Lawrence Livermore } \\
\text { Los Alamos NL }\end{array}$ & $\begin{array}{l}1.82 \mathrm{E}-02 \\
4.66 \mathrm{E}-02 \\
1.60 \mathrm{E}+00 \\
5.57 \mathrm{E}-01 \\
4.00 \mathrm{E}-02 \\
1.22 \mathrm{E}-02 \\
3.35 \mathrm{E}-02 \\
3.54 \mathrm{E}-02 \\
7.10 \mathrm{E}-01 \\
1.38 \mathrm{E}-02 \\
6.92 \mathrm{E}-03 \\
2.74 \mathrm{E}-02 \\
2.89 \mathrm{E}+00 \\
1.81 \mathrm{E}-03 \\
2.28 \mathrm{E}-02 \\
4.04 \mathrm{E}-02 \\
7.64 \mathrm{E}+00 \\
1.49 \mathrm{E}-01 \\
5.58 \mathrm{E}-02\end{array}$ & $\begin{array}{l}2.06 \mathrm{E}-02 \\
5.26 \mathrm{E}-02 \\
1.79 \mathrm{E}+00 \\
5.94 \mathrm{E}-02 \\
4.18 \mathrm{E}-02 \\
1.35 \mathrm{E}-02 \\
3.74 \mathrm{E}-02 \\
4.18 \mathrm{E}-02 \\
7.35 \mathrm{E}-0 \mathrm{0} \\
1.59 \mathrm{E}-02 \\
9.13 \mathrm{E}-0 \mathrm{5} \\
2.53 \mathrm{E}-02 \\
3.13 \mathrm{E}+00 \\
1.80 \mathrm{E}-05 \\
2.48 \mathrm{E}-02 \\
4.48 \mathrm{E}-02 \\
8.01 \mathrm{E}+09 \\
2.09 \mathrm{E}-0 \mathrm{0} \\
6.53 \mathrm{E}-02\end{array}$ & 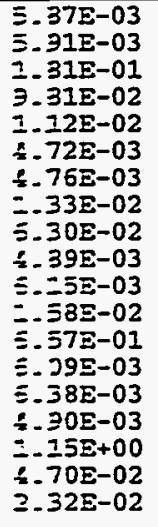 & $\begin{array}{l}2.12 \mathrm{E}-02 \\
2.01 \mathrm{E}-02 \\
4.55 \mathrm{E}-01 \\
3.11 \mathrm{E}-01 \\
2.06 \mathrm{E}-02 \\
1.70 \mathrm{E}-02 \\
1.58 \mathrm{E}-02 \\
2.15 \mathrm{E}-02 \\
2.34 \mathrm{E}-01 \\
1.14 \mathrm{E}-02 \\
1.25 \mathrm{E}-02 \\
2.80 \mathrm{E}-02 \\
1.46 \mathrm{E}+00 \\
7.89 \mathrm{E}-03 \\
2.64 \mathrm{E}-02 \\
1.02 \mathrm{E}-02 \\
3.43 \mathrm{E}+00 \\
9.10 \mathrm{E}-02 \\
3.76 \mathrm{E}-02\end{array}$ \\
\hline
\end{tabular}


Table 7. Annual Accident Dose Risk for the Shipping Campaign (person-rem/yr)

\begin{tabular}{|c|c|c|c|c|}
\hline Route & & & Truck & Rail \\
\hline $\begin{array}{l}\text { Ames Laboratory } \\
\text { Ames Laboratory } \\
\text { Argonne - East } \\
\text { Bettis Atomic } \\
\text { Fernald (FEMP) } \\
\text { Fermilab (FNAL) } \\
\text { Fermilab (FNAL) } \\
\text { ITRI } \\
\text { Knolls Atomic } \\
\text { Kansas City Plant } \\
\text { Lawrence Berkeley } \\
\text { Mound Plant } \\
\text { Mound Plant } \\
\text { ORISE } \\
\text { Princeton ppL } \\
\text { Pinellas plant } \\
\text { Reactive Metals } \\
\text { SLAC } \\
\text { SNL - Albuquerque }\end{array}$ & $\begin{array}{l}\text { to } \\
\text { to } \\
\text { to } \\
\text { to } \\
\text { to } \\
\text { to } \\
\text { to } \\
\text { to } \\
\text { to } \\
\text { to } \\
\text { to } \\
\text { to } \\
\text { to } \\
\text { to } \\
\text { to } \\
\text { to } \\
\text { to }\end{array}$ & $\begin{array}{l}\text { Fernald (FEMP) } \\
\text { Portsmouth GDP } \\
\text { Portsmouth GDP } \\
\text { Portsmouth GDP } \\
\text { Portsmouth GDP } \\
\text { Fernald (FEMP) } \\
\text { Portsmouth GDP } \\
\text { Los Alamos NL } \\
\text { Portsmouth GDP } \\
\text { Paducah GDP } \\
\text { Lawrence Iivermore } \\
\text { Fernald (FEMP) } \\
\text { Portsmouth GDP } \\
\text { Oak Ridge Reserv. } \\
\text { Portsmouth GDP } \\
\text { Savannah River } \\
\text { Portsmouth GDP } \\
\text { Lawrence Livermore } \\
\text { Los Alamos NL }\end{array}$ & $\begin{array}{l}8.59 \mathrm{E}-07 \\
1.46 \mathrm{E}-05 \\
3.62 \mathrm{E}-02 \\
3.56 \mathrm{E}-04 \\
1.57 \mathrm{E}-07 \\
1.60 \mathrm{E}-11 \\
7.34 \mathrm{E}-05 \\
1.53 \mathrm{E}-02 \\
6.69 \mathrm{E}-05 \\
2.31 \mathrm{E}-05 \\
1.13 \mathrm{E}-02 \\
4.27 \mathrm{E}-07 \\
2.39 \mathrm{E}-04 \\
2.24 \mathrm{E}-07 \\
4.70 \mathrm{E}-06 \\
2.59 \mathrm{E}-06 \\
1.73 \mathrm{E}-06 \\
1.89 \mathrm{E}-06 \\
9.16 \mathrm{E}-05\end{array}$ & $\begin{array}{l}4.13 E-07 \\
6.06 \mathrm{E}-06 \\
7.34 \mathrm{E}-03 \\
1.44 \mathrm{E}-04 \\
3.45 \mathrm{E}-08 \\
5.07 \mathrm{E}-12 \\
1.89 \mathrm{E}-05 \\
2.80 \mathrm{E}-04 \\
1.56 \mathrm{E}-05 \\
6.16 \mathrm{E}-06 \\
8.05 \mathrm{E}-03 \\
1.02 \mathrm{E}-07 \\
5.83 \mathrm{E}-05 \\
6.30 \mathrm{E}-08 \\
2.47 \mathrm{E}-06 \\
4.65 \mathrm{E}-07 \\
5.00 \mathrm{E}-07 \\
8.53 \mathrm{E}-07 \\
1.75 \mathrm{E}-06\end{array}$ \\
\hline theas & & & $6.36 \mathrm{E}-02$ & $1.59 \mathrm{E}-02$ \\
\hline
\end{tabular}

Table 8. Expected Annual Fatalities

for the Shipping Campaign

\begin{tabular}{lll}
\hline Exposure Group & Truck & Rail \\
\hline Radiological & $5.6 \mathrm{E}-03$ & $9.2 \mathrm{E}-04$ \\
Normal Crew & $7.4 \mathrm{E}-03$ & $3.1 \mathrm{E}-03$ \\
Normal Public & $3.2 \mathrm{E}-05$ & $8.0 \mathrm{E}-06$ \\
Accident Public & & \\
Nonradiological & & \\
Emission & & \\
Accident & $1.7 \mathrm{E}-03$ & $5.5 \mathrm{E}-03$ \\
& & $3.1 \mathrm{E}-04$ \\
\hline
\end{tabular}

Table 9. Expected Annual Cancer Incidence for the Shipping Campaign

\begin{tabular}{lll}
\hline Exposure Group & Truck & Rail \\
\hline Radiological & & \\
Normal Crew & $1.9 E-02$ & $3.2 \mathrm{E}-03$ \\
Normal Public & $2.5 \mathrm{E}-02$ & $1.1 \mathrm{E}-02$ \\
Accident Public & $1.1 \mathrm{E}-04$ & $2.7 \mathrm{E}-05$ \\
Nonradiological & $4.7 \mathrm{E}-03$ & $5.5 \mathrm{E}-03$ \\
Emission & $\mathrm{NA}$ & $\mathrm{NA}$ \\
\hline Accident & & \\
\hline
\end{tabular}

Table 10. Expected Annual Genetic Effects for the Shipping Campaign

\begin{tabular}{lll}
\hline Exposure Group & Truck & Rail \\
\hline Radiological & $8.3 E-04$ & $1.4 \mathrm{E}-04$ \\
Normal Crew & $1.5 \mathrm{E}-03$ & $6.2 \mathrm{E}-04$ \\
Normal Public & $3.1 \mathrm{E}-06$ & $6.8 \mathrm{E}-07$ \\
Accident Public & & \\
Nonradiological & NA & NA \\
Enission & NA & NA \\
Accident & & \\
\hline
\end{tabular}


TABLE 4.1 (Cont.)

\begin{tabular}{|c|c|c|c|c|c|c|c|c|c|c|c|c|c|c|c|}
\hline \multirow[b]{2}{*}{ State } & \multicolumn{5}{|c|}{ Accident-Involvement Rates } & \multicolumn{5}{|c|}{ Accident-Fatality Rates } & \multicolumn{5}{|c|}{ Accident-Injury Rates } \\
\hline & RAI-U & FAl-R & FAI & FAP & FAS & FAI-U & FAI-R & FAI & FAP & FAS & FAI-U & FAl-R & FAI & FAP & FAS \\
\hline NC & 5.92 & 2.28 & 2.97 & 5.17 & 6.37 & 5.08 & 2.92 & $3.33^{\mathrm{c}}$ & 6.71 & 11.00 & 6.37 & 2.19 & 2.99 & 5.53 & 6.22 \\
\hline $\mathrm{OH}$ & 3.16 & 2.27 & 2.52 & 4.42 & 11.00 & 1.41 & 1.32 & 1.35 & 6.07 & 9.88 & 2.85 & 2.02 & 2.25 & 4.40 & 1070 \\
\hline OK & 3.76 & 1.47 & 1.91 & 3.61 & 1.73 & 2.70 & 2.06 & 2.18 & 4.88 & 0.71 & 3.34 & 1.36 & 1.74 & 3.05 & 1.59 \\
\hline OR & 3.99 & 2.20 & 2.48 & 4.17 & 1.63 & 2.47 & 1.12 & 1.33 & 5.85 & 4.07 & 3.82 & 1.69 & 2.02 & 2.94 & 0.57 \\
\hline$P_{A}$ & 3.02 & 3.60 & 3.48 & 7.21 & 7.92 & 2.12 & 2.97 & 2.79 & 10.20 & 9.29 & 2.68 & 3.28 & 3.15 & 7.28 & 6.42 \\
\hline $\mathbf{R I}$ & 2.27 & 1.98 & 2.16 & 1.37 & 16.70 & 0.71 & 3.70 & 1.80 & 1.71 & $66.70^{c}$ & 3.12 & 2.35 & 2.84 & 0.86 & $23.30^{c}$ \\
\hline SC & 3.13 & 1.83 & 1.99 & 6.27 & 2.27 & 2.88 & 2.57 & 2.61 & 8.61 & 3.95 & 2.65 & 2.20 & 2.26 & 6.96 & 2.27 \\
\hline SD & $8.57^{c}$ & 2.09 & 2.18 & 3.94 & 1.49 & $14.30^{c}$ & 0.42 & 0.62 & 5.38 & 2.13 & 5.71 & 1.38 & 1.95 & 2.94 & 0 \\
\hline $\mathrm{TN}$ & 7.97 & 1.48 & 2.48 & 5.56 & 6.26 & 5.59 & 1.63 & 2.24 & 6.97 & 12.10 & $7.70^{\mathrm{C}}$ & 1.44 & 2.41 & 5.85 & 4.67 \\
\hline$T X$ & 2.74 & 1.56 & 2.00 & 2.78 & 1.09 & 1.93 & 1.97 & 1.95 & 4.77 & 1.84 & 2.53 & 1.42 & 1.83 & 2.53 & 0.92 \\
\hline $\mathrm{vr}$ & 0 & 1.38 & 1.33 & 6.30 & 6.80 & 0 & 0.43 & 0.42 & 3.36 & 24.00 & 0 & 1.08 & 1.04 & 6.13 & 4.40 \\
\hline VA & 2.63 & 2.54 & 2.56 & 4.67 & 5.03 & 1.91 & 1.76 & 1.80 & 7.28 & 7.73 & 2.49 & 2.46 & 2.47 & 5.39 & 4.81 \\
\hline WA & 1.61 & 2.50 & 2.10 & 2.62 & 0.73 & 0.80 & 1.47 & 1.17 & 2.54 & 0 & 1.49 & 2.14 & 1.85 & 2.11 & 0.51 \\
\hline wV & 2.95 & 3.10 & 3.07 & $11.70^{c}$ & 7.87 & 1.28 & 1.67 & 1.60 & $17.80^{\mathrm{C}}$ & 1.70 & 2.78 & 2.80 & 2.79 & $9.91^{\mathrm{C}}$ & 7.13 \\
\hline wI & 5.29 & 1.74 & 2.18 & 2.80 & 3.24 & 0.77 & 0.66 & 0.67 & 3.62 & 3.68 & 4.33 & 1.45 & 1.81 & 2.51 & 3.16 \\
\hline WY & 2.98 & 3.42 & 3.40 & 3.41 & 3.70 & 0 & 2.08 & 2.01 & 6.22 & 3.70 & 0 & 2.84 & 2.74 & 1.86 & 2.35 \\
\hline USA & 3.58 & 2.03 & 2.44 & 3.94 & 3.48 & 2.37 & 1.91 & 2.03 & 5.82 & 4.62 & 3.36 & 1.89 & 2.28 & 3.82 & 3.30 \\
\hline $0^{4}$ & 2.36 & 1.25 & 0.69 & 1.77 & 6.98 & 2.70 & 1.27 & 0.63 & 3.01 & 11.74 & 1.99 & 1.22 & 0.69 & 1.79 & 7.10 \\
\hline
\end{tabular}

- $\mathrm{PAI}=$ Federally aided interstalc

$$
\begin{aligned}
& U=\text { Urban } \\
& R=\text { Rural }
\end{aligned}
$$

IRAP a Federully aided primary

FAS a Federally aided secondary

- Includes only the 48 contiguous states, plus the totals for the United States.

- Rates are two or more standard deviations greater than the national average rate for that highway category,

d 0 s standard deviation.

Source: Saricks and Kvitek (1994). 


\section{B.8 WM LLW REGIONALIZED 2 ALTERNATIVE (CASE 9): ACTIVATED METALS}

Table 1. Summary of Rouce Information

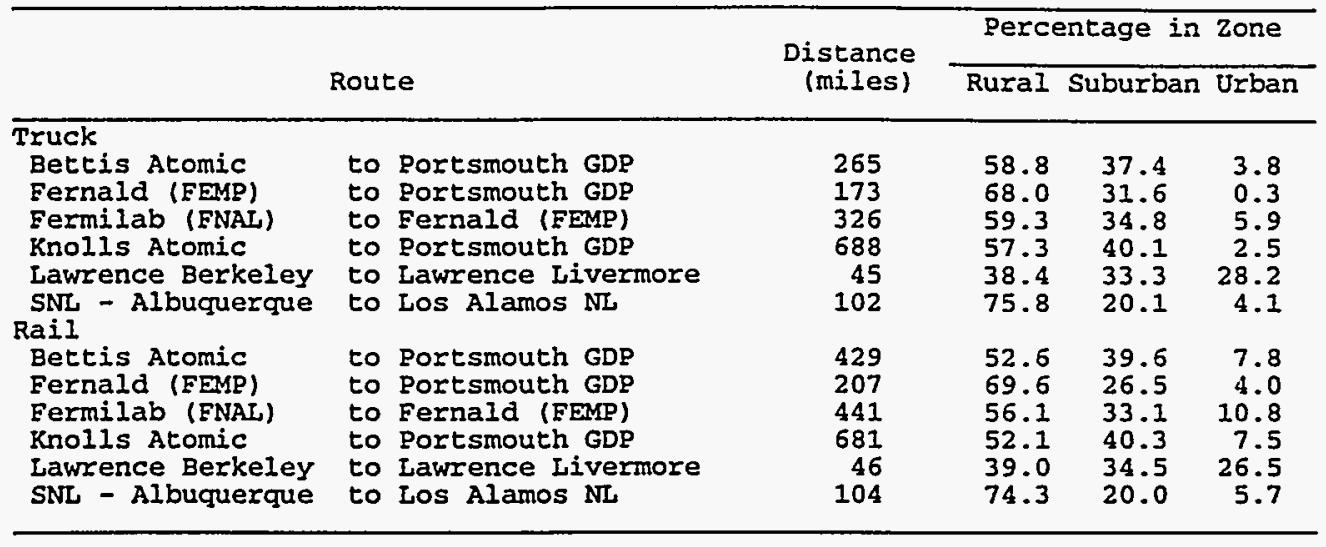

Table 2. Annual Number of Shipments and One-Way Shipment Distances

\begin{tabular}{|c|c|c|c|c|c|}
\hline \multirow[b]{2}{*}{ Route } & & \multicolumn{2}{|c|}{ \# of Shipments } & \multicolumn{2}{|c|}{ Total Mileage (mi) } \\
\hline & & Truck & Rail & Truck & Rail \\
\hline $\begin{array}{l}\text { Bettis Atomic } \\
\text { Fernald (FEMP) } \\
\text { Fermilab (FNAL) } \\
\text { Knolls Atomic } \\
\text { Lawrence Berkeley } \\
\text { SNL - Albuquerque }\end{array}$ & $\begin{array}{l}\text { to Portsmouth GDP } \\
\text { to Portsmouth GDP } \\
\text { to Fernald (FEMP) } \\
\text { to Portsmouth GDP } \\
\text { to Lawrence Iivermore } \\
\text { to Los Alamos NL }\end{array}$ & $\begin{array}{r}309 \\
31 \\
39 \\
639 \\
14 \\
6\end{array}$ & $\begin{array}{r}119 \\
14 \\
16 \\
245 \\
6 \\
3\end{array}$ & $\begin{array}{l}8.19 \mathrm{E}+04 \\
5.36 \mathrm{E}+03 \\
1.27 \mathrm{E}+04 \\
4.40 \mathrm{E}+05 \\
6.30 \mathrm{E}+02 \\
6.12 \mathrm{E}+02\end{array}$ & $\begin{array}{l}5.11 E+04 \\
2.90 E+03 \\
7.05 E+03 \\
1.67 E+05 \\
2.77 E+02 \\
3.12 E+02\end{array}$ \\
\hline Totals & & 1038 & 403 & $5.41 E+05$ & $2.29 E+05$ \\
\hline
\end{tabular}

Table 3. Incident-Free Dose per Shipment (person-rem/shipment)

\begin{tabular}{|c|c|c|c|c|c|}
\hline \multirow[b]{2}{*}{ Source/Route(s) } & \multirow[b]{2}{*}{ Crew } & \multicolumn{4}{|c|}{ General Public } \\
\hline & & off-Link & on-Link & stops & Total \\
\hline $\begin{array}{l}\text { LLW(I) BA to PO } \\
\text { Bettis Atomic }\end{array}$ & $\begin{array}{lr}\text { to Portsmouth GDP } \\
\text { Truck } & 9.78 \mathrm{E}-03 \\
\text { Rail } & 4.67 \mathrm{E}-03\end{array}$ & $\begin{array}{l}1.26 \mathrm{E}-03 \\
9.85 \mathrm{E}-03\end{array}$ & $\begin{array}{l}1.73 E-03 \\
1.53 E-04\end{array}$ & $\begin{array}{l}7.42 \mathrm{E}-03 \\
4.80 \mathrm{E}-03\end{array}$ & $\begin{array}{l}1.04 \mathrm{E}-02 \\
1.48 \mathrm{E}-02\end{array}$ \\
\hline $\begin{array}{l}\text { LLW(I) FE to PO } \\
\text { Fernald (FEMP) }\end{array}$ & $\begin{array}{lr} & \\
\text { to Portsmouth GDP } \\
\text { Truck } & 5.72 \mathrm{E}-03 \\
\text { Rail } & 3.75 \mathrm{E}-03\end{array}$ & $\begin{array}{l}4.51 \mathrm{E}-04 \\
2.71 \mathrm{E}-03\end{array}$ & $\begin{array}{l}6.85 \mathrm{E}-04 \\
4.86 \mathrm{E}-05\end{array}$ & $\begin{array}{l}4.84 \mathrm{E}-03 \\
4.12 \mathrm{E}-03\end{array}$ & $\begin{array}{l}5.98 \mathrm{E}-03 \\
6.87 \mathrm{E}-03\end{array}$ \\
\hline $\begin{array}{l}\text { LLW (I) FL to FE } \\
\text { Eermilab (ENAL) }\end{array}$ & $\begin{array}{ll}\text { to Fernald (FEMP) } \\
\text { Truck } & 1.22 \mathrm{E}-02 \\
\text { Rail } & 4.72 \mathrm{E}-03\end{array}$ & $\begin{array}{l}1.83 E-03 \\
1.20 E-02\end{array}$ & $\begin{array}{l}2.56 \mathrm{E}-03 \\
1.62 \mathrm{E}-04\end{array}$ & $\begin{array}{l}9.12 \mathrm{E}-03 \\
4.84 \mathrm{E}-03\end{array}$ & $\begin{array}{l}1.35 \mathrm{E}-02 \\
1.70 \mathrm{E}-02\end{array}$ \\
\hline $\begin{array}{l}\text { LLW(I) KA to Po } \\
\text { Knolls Atomic }\end{array}$ & $\begin{array}{lr} & \\
\text { to Portsmouth GDP } \\
\text { Truck } & 2.54 \mathrm{E}-02 \\
\text { Rail } & 5.72 \mathrm{E}-03\end{array}$ & $\begin{array}{l}2.99 \mathrm{E}-03 \\
1.54 \mathrm{E}-02\end{array}$ & $\begin{array}{l}4.01 \mathrm{E}-03 \\
2.43 \mathrm{E}-04\end{array}$ & $\begin{array}{l}1.93 \mathrm{E}-02 \\
5.58 \mathrm{E}-03\end{array}$ & $\begin{array}{l}2.63 E-02 \\
2.13 E-02\end{array}$ \\
\hline $\begin{array}{l}\text { LLW(I) IB to LL } \\
\text { Lawrence Berkeley }\end{array}$ & $\begin{array}{lr} & \\
\text { to Lawrence Livermo } & \text { Livek } \\
\text { Truck } & 2.31 \mathrm{E}-03 \\
\text { Rail } & 3.07 \mathrm{E}-03\end{array}$ & $\begin{array}{l}\text { ore } \\
7.51 \mathrm{E}-04 \\
2.62 \mathrm{E}-03\end{array}$ & $\begin{array}{l}1.03 E-03 \\
2.79 \mathrm{E}-05\end{array}$ & $\begin{array}{l}1.26 \mathrm{E}-03 \\
3.62 \mathrm{E}-03\end{array}$ & $\begin{array}{l}3.04 E-03 \\
6.27 E-03\end{array}$ \\
\hline $\begin{array}{l}\text { LLW(I) SA to LA } \\
\text { SNL - Albuquerque }\end{array}$ & $\begin{array}{ll} & \\
\text { to Ios Alamos } \mathrm{NL} \\
\text { Truck } & 3.28 \mathrm{E}-03 \\
\text { Rail } & 3.31 \mathrm{E}-03\end{array}$ & $\begin{array}{l}3.70 \mathrm{E}-04 \\
1.55 \mathrm{E}-03\end{array}$ & $\begin{array}{l}6.13 \mathrm{E}-04 \\
2.36 \mathrm{E}-05\end{array}$ & $\begin{array}{l}2.86 \mathrm{E}-03 \\
3.80 \mathrm{E}-03\end{array}$ & $\begin{array}{l}3.84 E-03 \\
5.37 E-03\end{array}$ \\
\hline
\end{tabular}


Table 4. Accident Dose Risk per Shipment (person-rem/shipment)

\begin{tabular}{|c|c|c|c|}
\hline Source/Roure & & Truck & Rail \\
\hline $\begin{array}{l}\text { LLW(I) BA Co PO } \\
\text { Bettis Atomic } \\
\text { LLW(I) EE to PO } \\
\text { Fernald (FEMP) } \\
\text { LLW(I) FL to FE } \\
\text { Fermilab (FNAL) } \\
\text { LLW(I) KA to PO } \\
\text { KnoIls Atomic } \\
\text { LLW(I) LB to LL } \\
\text { Lawrence Berkeley } \\
\text { LLW(I) SA to LA } \\
\text { SNL - Albuquerque }\end{array}$ & $\begin{array}{l}\text { to Portsmouth GDP } \\
\text { to Portsmouth GDP } \\
\text { to Fernald (FEMP) } \\
\text { to Portsmouth GDP } \\
\text { to Lawrence Livermore } \\
\text { to Los Alamos NL }\end{array}$ & $\begin{array}{l}3.70 \mathrm{E}-07 \\
1.43 \mathrm{E}-11 \\
4.58 \mathrm{E}-11 \\
1.66 \mathrm{E}-06 \\
1.36 \mathrm{E}-11 \\
1.72 \mathrm{E}-12\end{array}$ & $\begin{array}{l}4.16 \mathrm{E}-07 \\
7.55 \mathrm{E}-12 \\
3.57 \mathrm{E}-11 \\
1.02 \mathrm{E}-06 \\
2.29 \mathrm{E}-11 \\
6.51 \mathrm{E}-14\end{array}$ \\
\hline
\end{tabular}

Table 5. Nonradiological Risk Factors per Shipment (fatalities/shipment)

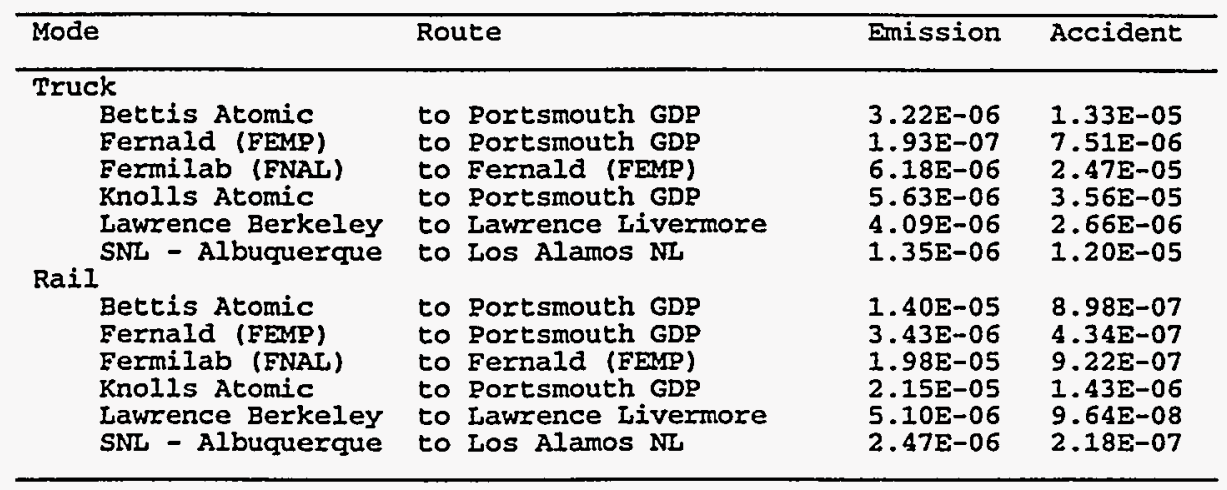

Table 6. Annual Incident-Free Dose for the Shipping Campaign (person-rem/yr)

\begin{tabular}{|c|c|c|c|c|c|}
\hline \multirow[b]{2}{*}{ Route } & & \multicolumn{2}{|c|}{ Truck } & \multicolumn{2}{|c|}{ Rail } \\
\hline & & Crew & Public & Crew & Public \\
\hline $\begin{array}{l}\text { Bettis Atomic } \\
\text { Fernald (FEMP) } \\
\text { Fermilab (FNAI) } \\
\text { Knolls Atomic } \\
\text { Lawrence Berkeley } \\
\text { SNL - Albuquerque }\end{array}$ & $\begin{array}{l}\text { to Portsmouth GDP } \\
\text { to Portsmouth GDP } \\
\text { to Fernald (FEMP) } \\
\text { to Portsmouth GDP } \\
\text { to Lawrence Iivermore } \\
\text { to Los Alamos NL }\end{array}$ & $\begin{array}{l}3.02 \mathrm{E}+00 \\
1.77 \mathrm{E}-01 \\
4.76 \mathrm{E}-01 \\
1.62 \mathrm{E}+01 \\
3.23 \mathrm{E}-02 \\
1.97 \mathrm{E}-02\end{array}$ & $\begin{array}{l}3.22 \mathrm{E}+00 \\
1.85 \mathrm{E}-01 \\
5.27 \mathrm{E}-01 \\
1.68 \mathrm{E}+01 \\
4.26 \mathrm{E}-02 \\
2.30 \mathrm{E}-02\end{array}$ & $\begin{array}{l}5.56 \mathrm{E}-01 \\
5.25 \mathrm{E}-02 \\
7.55 \mathrm{E}-02 \\
1.40 \mathrm{E}+00 \\
1.84 \mathrm{E}-02 \\
9.94 \mathrm{E}-03\end{array}$ & $\begin{array}{l}1.76 \mathrm{E}+00 \\
9.62 \mathrm{E}-02 \\
2.72 \mathrm{E}-01 \\
5.21 \mathrm{E}+00 \\
3.76 \mathrm{E}-02 \\
1.61 \mathrm{E}-02\end{array}$ \\
\hline Totals & & $1.99 \mathrm{E}+01$ & $2.08 \mathrm{E}+01$ & $2.11 \mathrm{E}+00$ & $7.39 \mathrm{E}+00$ \\
\hline
\end{tabular}

Table 7. Annual Accident Dose Risk for the Shipping Campaign (person-rem/yr)

\begin{tabular}{llcc}
\hline Route & & Truck & Rail \\
\hline Bettis Atomic & to Portsmouth GDP & $1.14 \mathrm{E}-04$ & $4.95 \mathrm{E}-05$ \\
Fernald (FEMP) & to Portsmouth GDP & $4.43 \mathrm{E}-10$ & $1.06 \mathrm{E}-10$ \\
Fermilab (FNAL) & to Fernald (FEMP) & $1.79 \mathrm{E}-09$ & $5.72 \mathrm{E}-10$ \\
Knolls Atomic & to Portsmouth GDP & $1.06 \mathrm{E}-03$ & $2.49 \mathrm{E}-04$ \\
Lawrence Berkeley & to Lawrence Livermore & $1.90 \mathrm{E}-10$ & $1.37 \mathrm{E}-10$ \\
SNL - Albuquerque & to Los Alamos NL & $1.03 \mathrm{E}-11$ & $1.95 \mathrm{E}-13$ \\
\hline Totals & & $1.17 \mathrm{E}-03$ & $2.99 \mathrm{E}-04$ \\
\hline
\end{tabular}


Table 8. Expected Annual Fatalities

for the Shipping Campaign

\begin{tabular}{llc}
\hline Exposure Group & Truck & Rail \\
\hline Radiological & & \\
$\quad$ Normal Crew & $8.0 \mathrm{E}-03$ & $8.5 \mathrm{E}-04$ \\
$\quad$ Normal Public & $1.0 \mathrm{E}-02$ & $3.7 \mathrm{E}-03$ \\
Accident Public & $5.9 \mathrm{E}-07$ & $1.5 \mathrm{E}-07$ \\
Nonradiological & & \\
Emission & $4.9 \mathrm{E}-03$ & $7.3 \mathrm{E}-03$ \\
Accident & $2.8 \mathrm{E}-02$ & $4.8 \mathrm{E}-04$ \\
\hline
\end{tabular}

Table 9. Expected Annual Cancer Incidence

for the Shipping Campaign

\begin{tabular}{lll}
\hline Exposure Group & Truck & Rail \\
\hline Radiological & & \\
Normal Crew & $2.8 \mathrm{E}-02$ & $3.0 \mathrm{E}-03$ \\
Normal Public & $3.5 \mathrm{E}-02$ & $1.3 \mathrm{E}-02$ \\
Accident Public & $2.0 \mathrm{E}-06$ & $5.1 \mathrm{E}-07$ \\
Nonradiological & & \\
Emission & $4.9 \mathrm{E}-03$ & $\mathbf{7 . 3 E}-03$ \\
Accident & $\mathrm{NA}$ & $\mathrm{NA}$ \\
\hline
\end{tabular}

Table 10. Expected Annual Genetic Effects for the Shipping Campaign

\begin{tabular}{lll}
\hline Exposure Group & Truck & Rail \\
\hline Radiological & & \\
Normal Crew & $1.2 \mathrm{E}-03$ & $1.3 \mathrm{E}-04$ \\
Normal Public & $2.1 \mathrm{E}-03$ & $7.4 \mathrm{E}-04$ \\
Accident Public & $1.2 \mathrm{E}-07$ & $3.0 \mathrm{E}-08$ \\
Nonradiological & & \\
Emission & NA & NA \\
Accident & NA & NA \\
\hline
\end{tabular}




\section{B.9 WM LLW REGIONALIZED 3 ALTERNATIVE (CASE 4): HETEROGENEOUS SOLIDS}

Table 1. Summary of Route Information

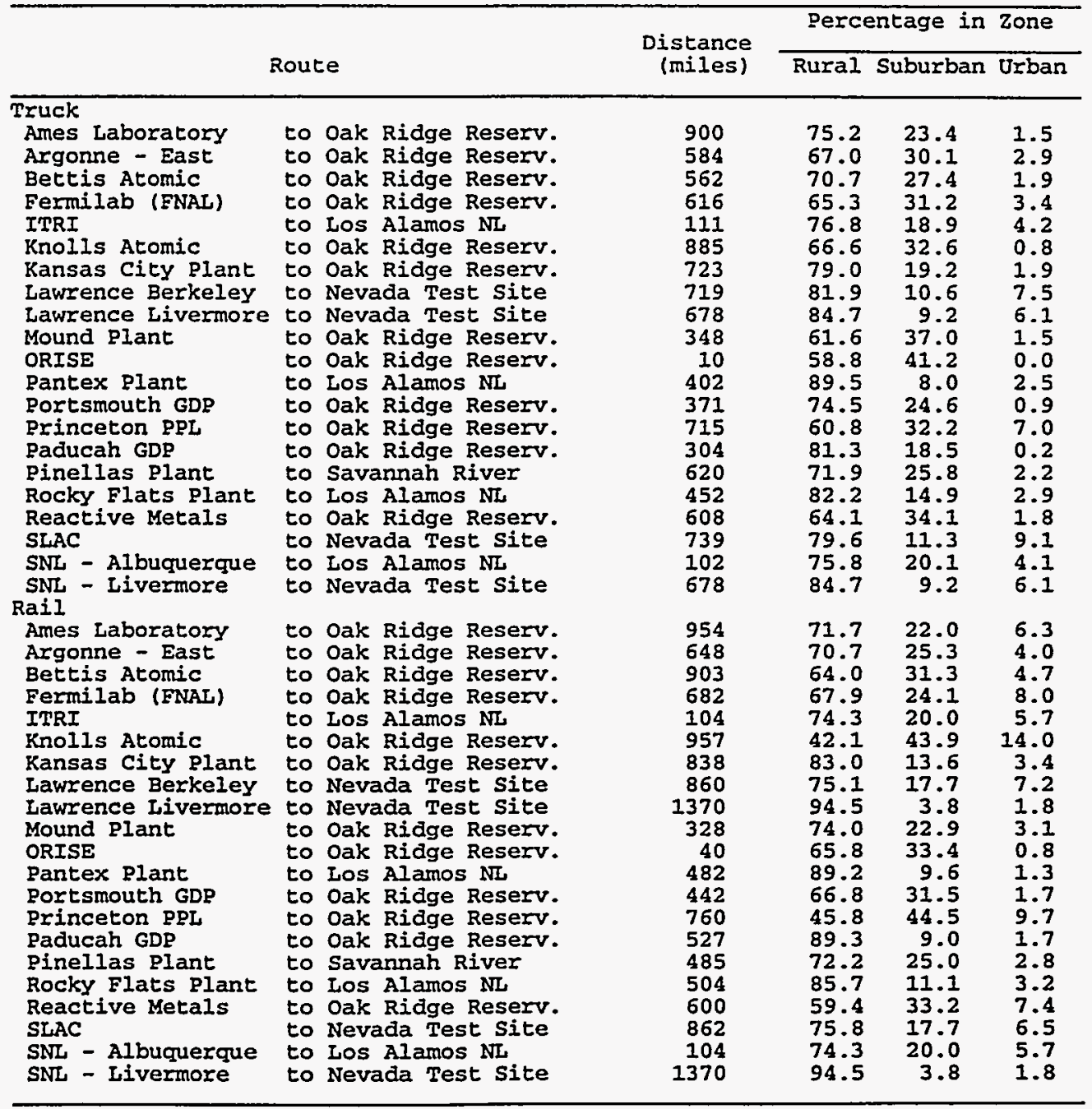




\section{$B-38$}

Table 2. Annual Number of Shipments and One-Way Shipment Distances

\begin{tabular}{|c|c|c|c|c|c|}
\hline \multirow[b]{2}{*}{ Route } & & \multicolumn{2}{|c|}{ \# of Shipments } & \multicolumn{2}{|c|}{ Total Mileage(mi) } \\
\hline & & Truck & Rail & Truck & Rail \\
\hline $\begin{array}{l}\text { Ames Laboratory } \\
\text { Argonne - East } \\
\text { Bettis Atomic } \\
\text { Fermilab (FNAL) } \\
\text { ITRI } \\
\text { Knolls Atomic } \\
\text { Ransas City Plant } \\
\text { Lawrence Berkeley } \\
\text { Lawrence Livermore } \\
\text { Mound Plant } \\
\text { ORISE } \\
\text { Pantex Plant } \\
\text { Portsmouth GDP } \\
\text { Princeton PDL } \\
\text { Paducah GDP } \\
\text { Pinellas Plant } \\
\text { Rocky Flats Plant } \\
\text { Reactive Metals } \\
\text { SLAC } \\
\text { SNL - Albuquerque } \\
\text { SNL - Livermore }\end{array}$ & $\begin{array}{l}\text { to Oak Ridge Reserv. } \\
\text { to Oak Ridge Reserv. } \\
\text { to Oak Ridge Reserv. } \\
\text { to Oak Ridge Reserv. } \\
\text { to Ios Alamos NL } \\
\text { to Oak Ridge Reserv. } \\
\text { to Oak Ridge Reserv. } \\
\text { to Nevada Test Site } \\
\text { to Nevada Test Site } \\
\text { to Oak Ridge Reserv. } \\
\text { to Oak Ridge Reserv. } \\
\text { to Los Alamos NL } \\
\text { to Oak Ridge Reserv. } \\
\text { to Oak Ridge Reserv. } \\
\text { to Oak Ridge Reserv. } \\
\text { to Savannah River } \\
\text { to Los Alamos NL } \\
\text { to Oak Ridge Reserv. } \\
\text { to Nevada Test Site } \\
\text { to Los Alamos NL } \\
\text { to Nevada Test Site }\end{array}$ & $\begin{array}{r}2 \\
105 \\
57 \\
3 \\
10 \\
28 \\
1 \\
3 \\
56 \\
512 \\
5 \\
1450 \\
3344 \\
1 \\
627 \\
2 \\
357 \\
768 \\
39 \\
17 \\
6\end{array}$ & $\begin{array}{r}3 \frac{1}{39} \\
21 \\
2 \\
4 \\
11 \\
1 \\
2 \\
21 \\
190 \\
2 \\
544 \\
1274 \\
1 \\
237 \\
1 \\
133 \\
287 \\
15 \\
7 \\
2\end{array}$ & $\begin{array}{l}1.80 \mathrm{E}+03 \\
6.13 \mathrm{E}+04 \\
3.21 \mathrm{E}+04 \\
1.85 \mathrm{E}+03 \\
1.11 \mathrm{E}+03 \\
2.48 \mathrm{E}+04 \\
7.23 \mathrm{E}+02 \\
2.16 \mathrm{E}+03 \\
3.80 \mathrm{E}+04 \\
1.78 \mathrm{E}+05 \\
5.10 \mathrm{E}+01 \\
5.83 \mathrm{E}+05 \\
1.24 \mathrm{E}+06 \\
7.15 \mathrm{E}+02 \\
1.91 \mathrm{E}+05 \\
1.24 \mathrm{E}+03 \\
1.61 \mathrm{E}+05 \\
4.67 \mathrm{E}+05 \\
2.88 \mathrm{E}+04 \\
1.73 \mathrm{E}+03 \\
4.07 \mathrm{E}+03\end{array}$ & $\begin{array}{l}9.54 \mathrm{E}+02 \\
2.53 \mathrm{E}+04 \\
1.90 \mathrm{E}+04 \\
1.36 \mathrm{E}+03 \\
4.16 \mathrm{E}+02 \\
1.05 \mathrm{E}+04 \\
8.38 \mathrm{E}+02 \\
1.72 \mathrm{E}+03 \\
2.88 \mathrm{E}+04 \\
6.23 \mathrm{E}+04 \\
7.90 \mathrm{E}+01 \\
2.62 \mathrm{E}+05 \\
5.63 \mathrm{E}+05 \\
7.60 \mathrm{E}+02 \\
1.25 \mathrm{E}+05 \\
4.85 \mathrm{E}+02 \\
6.70 \mathrm{E}+04 \\
1.72 \mathrm{E}+05 \\
1.29 \mathrm{E}+04 \\
7.28 \mathrm{E}+02 \\
2.74 \mathrm{E}+03\end{array}$ \\
\hline 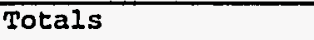 & & 7393 & 2795 & $3.02 \mathrm{E}+06$ & $1.36 \mathrm{E}+0 \overline{6}$ \\
\hline
\end{tabular}


Table 3. Incident-Free Dose per Shipment (person-rem/shipment)

\begin{tabular}{|c|c|c|c|c|c|}
\hline \multirow[b]{2}{*}{ Source/Route (s) } & \multirow[b]{2}{*}{ Crew } & \multicolumn{4}{|c|}{ General Public } \\
\hline & & ff-Link & On-Link & Stops & Total \\
\hline $\begin{array}{l}\mathrm{LW}(\mathrm{x}) \text { AL to } \mathrm{OR} \\
\text { Ames Laboratory }\end{array}$ & $\begin{array}{cc}\text { to Oak Ridge Reserv } \\
\text { Truck } & 2.83 \mathrm{E}-02 \\
\text { Rail } & 6.86 \mathrm{E}-03\end{array}$ & $\begin{array}{l}\text { v. } \\
2.29 \mathrm{E}-03 \\
1.58 \mathrm{E}-02\end{array}$ & $\begin{array}{l}3.92 E-03 \\
2.35 E-04\end{array}$ & $\begin{array}{l}2.52 \mathrm{E}-02 \\
6.42 \mathrm{E}-03\end{array}$ & $\begin{array}{c}3.14 E-02 \\
2.24 E-02\end{array}$ \\
\hline $\begin{array}{l}\text { LLW(x) AN to OR } \\
\text { Argonne - East }\end{array}$ & $\begin{array}{cc}\text { to Oak Ridge Reserv } \\
\text { Truck } & 2.00 \mathrm{E}-02 \\
\text { RaiI } & 5.59 \mathrm{E}-03\end{array}$ & $\begin{array}{r}v_{1} .19 \mathrm{E}-03 \\
8.34 \mathrm{E}-03\end{array}$ & $\begin{array}{l}3.27 \mathrm{E}-03 \\
1.48 \mathrm{E}-04\end{array}$ & $\begin{array}{l}1.63 \mathrm{E}-02 \\
5.48 \mathrm{E}-03\end{array}$ & $\begin{array}{l}2.18 \mathrm{E}-02 \\
1.40 \mathrm{E}-02\end{array}$ \\
\hline $\begin{array}{l}\text { LLW(x) BA to OR } \\
\text { Bettis Atomic }\end{array}$ & $\begin{array}{cc}\text { to Oak Ridge Reserv } \\
\text { Truck } & 1.85 \mathrm{E}-02 \\
\text { Rail } & 6.65 \mathrm{E}-03\end{array}$ & $\begin{array}{r}\text { v. } \\
1.72 \mathrm{E}-03 \\
1.40 \mathrm{E}-02\end{array}$ & $\begin{array}{l}2.71 E-03 \\
2.44 E-04\end{array}$ & $\begin{array}{l}1.57 \mathrm{E}-02 \\
6.26 \mathrm{E}-03\end{array}$ & $\begin{array}{l}2.02 \mathrm{E}-02 \\
2.05 \mathrm{E}-02\end{array}$ \\
\hline $\begin{array}{l}\operatorname{LLW}(x) \text { FL to } \text { OR } \\
\text { Fermilab (JNAL) }\end{array}$ & $\begin{array}{cc}\text { to Oak Ridge Reserv } \\
\text { Truck } & 2.15 \mathrm{E}-02 \\
\text { Rail } & 5.73 \mathrm{E}-03\end{array}$ & $\begin{array}{l}\text { v. } \\
\text { 2.53E-03 } \\
1.37 \mathrm{E}-02\end{array}$ & 3. & $\begin{array}{l}E-02 \\
E-03\end{array}$ & $\begin{array}{l}z-02 \\
E-02\end{array}$ \\
\hline $\begin{array}{l}\text { LLW(x) IT to LA } \\
\text { ITRI }\end{array}$ & $\begin{array}{cr}\text { to Los } & \text { Alamos } \mathrm{NL} \\
\text { Truck } & 3.54 \mathrm{E}-03 \\
\text { Rail } & 3.31 \mathrm{E}-03\end{array}$ & $\begin{array}{l}4.00 \mathrm{E}-04 \\
1.55 \mathrm{E}-03\end{array}$ & $\begin{array}{l}6.7 \\
2.3\end{array}$ & $\begin{array}{l}3.11 \mathrm{E}-03 \\
3.80 \mathrm{E}-03\end{array}$ & $\begin{array}{l}E-03 \\
E-03\end{array}$ \\
\hline $\begin{array}{l}\operatorname{LLW}(x) \text { KA to OR } \\
\text { Knolls Atomic }\end{array}$ & $\begin{array}{cc}\text { to Oak Ridge Reserv } \\
\text { Truck } & 2.98 \mathrm{E}-02 \\
\text { Rail } & 6.87 \mathrm{E}-03\end{array}$ & $\begin{array}{r}2.55 \mathrm{E}-03 \\
3.39 \mathrm{E}-02\end{array}$ & $\begin{array}{l}3.7 \\
4.4\end{array}$ & $\begin{array}{l}E-02 \\
E-03\end{array}$ & $\begin{array}{l}\varepsilon-02 \\
8-02\end{array}$ \\
\hline $\begin{array}{l}\text { LLW }(x) \text { KC to OR } \\
\text { Kansas City plant }\end{array}$ & $\begin{array}{ll}\text { to Oak Ridge Reserv } \\
\text { Truck } & 2.20 \mathrm{E}-02 \\
\text { Rail } & 6.38 \mathrm{E}-03\end{array}$ & $\begin{array}{r}\text { i. } \\
1.75 \mathrm{E}-03 \\
7.77 \mathrm{E}-03\end{array}$ & $\begin{array}{l}3.2 \\
1.3\end{array}$ & $\begin{array}{l}2.02 \mathrm{E}-02 \\
6.06 \mathrm{E}-03\end{array}$ & $\begin{array}{l}\varepsilon-02 \\
\varepsilon-02\end{array}$ \\
\hline $\begin{array}{l}\text { LLW(x) LB to NT } \\
\text { Lawrence Berkeley }\end{array}$ & $\begin{array}{ll} & \\
\text { to Nevada } & \text { Test Site } \\
\text { Truck } & 2.27 \mathrm{E}-02 \\
\text { RaiI } & 6.47 \mathrm{E}-03\end{array}$ & $\begin{array}{l}\text { e } \\
3.31 \mathrm{E}-03 \\
1.49 \mathrm{E}-02\end{array}$ & 2.6 & $\begin{array}{l}E-02 \\
E-03\end{array}$ & $\begin{array}{l}\varepsilon-02 \\
E-02\end{array}$ \\
\hline Lawrence Liv & $\begin{array}{ll}\text { to Nevada } & \text { Test Site } \\
\text { Truck } & 2.05 \mathrm{E}-02 \\
\text { Rai1 } & 8.60 \mathrm{E}-03\end{array}$ & $\begin{array}{l}2.57 \mathrm{E}-03 \\
5.75 \mathrm{E}-03\end{array}$ & 4 & $\begin{array}{l}E-02 \\
E-03\end{array}$ & $\begin{array}{l}E-02 \\
E-02\end{array}$ \\
\hline $\begin{array}{l}\text { ILW }(x) \text { MP to OR } \\
\text { Mound Plant }\end{array}$ & $\begin{array}{cc}\text { to Oak Ridge Reserv } \\
\text { Truck } & 1.23 \mathrm{E}-02 \\
\text { Rail } & 4.25 \mathrm{E}-03\end{array}$ & $\begin{array}{r}1.24 \mathrm{E}-03 \\
3.53 \mathrm{E}-03\end{array}$ & $\begin{array}{l}1.7 \\
6.6\end{array}$ & $\begin{array}{l}9.73 E-03 \\
4.49 E-03\end{array}$ & $\begin{array}{l}1.27 \mathrm{E}-02 \\
8.08 \mathrm{E}-03\end{array}$ \\
\hline ORISE & $\begin{array}{cc}\text { to Oak Ridge Reserv } \\
\text { Truck } & 3.63 E-04 \\
\text { Rail } & 3.05 E-03\end{array}$ & $\begin{array}{r}\text { v. } \\
3.21 \mathrm{E}-05 \\
3.36 \mathrm{E}-04\end{array}$ & $\begin{array}{l}4.2 \\
8.2\end{array}$ & & $:-04$ \\
\hline Pantex Plant & $\begin{array}{lr}\text { to Los } & \text { Alamos NL } \\
\text { Truck } & 1.11 \mathrm{E}-02 \\
\text { Rail } & 4.89 \mathrm{E}-03\end{array}$ & $\begin{array}{l}7.76 \mathrm{E}-04 \\
2.18 \mathrm{E}-03\end{array}$ & $\frac{1}{5.2}$ & $\begin{array}{l}1 \\
4\end{array}$ & $\begin{array}{l}-02 \\
-03\end{array}$ \\
\hline $\begin{array}{l}\text { LLW }(x) \text { PO to OR } \\
\text { Portsmouth GDP }\end{array}$ & $\begin{array}{ll}\text { to Oak Ridge Reserv } \\
\text { Truck } & 1.16 \mathrm{E}-02 \\
\text { Rail } & 4.73 \mathrm{E}-03\end{array}$ & $\begin{array}{l}\text { v.77E-04 } \\
4.37 \mathrm{E}-03\end{array}$ & 1. & 1. & $\begin{array}{l}E-02 \\
E-03\end{array}$ \\
\hline $\begin{array}{l}\text { LLW (x) PR to OR } \\
\text { Princeton PRL }\end{array}$ & $\begin{array}{cc}\text { to Oak Ridge Reserv } \\
\text { Truck } & 2.68 \mathrm{E}-02 \\
\text { Rail } & 6.05 \mathrm{E}-03\end{array}$ & $\begin{array}{r}\text { v. } \\
2.27 E-03 \\
2.10 E-02\end{array}$ & $\begin{array}{l}6.0 \\
3.1\end{array}$ & & $\begin{array}{l}8-02 \\
3-02\end{array}$ \\
\hline $\begin{array}{l}\text { LLW(x) PA to OR } \\
\text { Paducah GDP }\end{array}$ & $\begin{array}{cc}\text { to Oak Ridge Reserv } \\
\text { Truck } & 8.88 \mathrm{E}-03 \\
\text { Rail } & 5.08 \mathrm{E}-03\end{array}$ & $\begin{array}{r}\text { v. } \\
4.70 \mathrm{E}-04 \\
2.75 \mathrm{E}-03\end{array}$ & $\begin{array}{l}9.5 \\
5.5\end{array}$ & & $\begin{array}{l}8-03 \\
-03\end{array}$ \\
\hline $\begin{array}{l}\text { LLW(x) PI to SR } \\
\text { Pinellas Plant }\end{array}$ & $\begin{array}{lr}\text { to Savannah River } \\
\text { Truck } & 2.02 \mathrm{E}-02 \\
\text { Rail } & 4.90 \mathrm{E}-03\end{array}$ & $\begin{array}{l}1.94 \mathrm{E}-03 \\
5.13 \mathrm{E}-03\end{array}$ & 3. & 4. & $\begin{array}{l}E-02 \\
E-02\end{array}$ \\
\hline $\begin{array}{l}\text { LIW(x) RF to LA } \\
\text { Rocky Flats Plant }\end{array}$ & $\begin{array}{lr}\text { to Los } & \text { Alamos NL } \\
\text { Truck } & 1.35 \mathrm{E}-02 \\
\text { Rail } & 4.98 \mathrm{E}-03\end{array}$ & $\begin{array}{l}1.19 \mathrm{E}-03 \\
4.26 \mathrm{E}-03\end{array}$ & $\begin{array}{l}2.2 \\
7.3\end{array}$ & $\frac{1}{5}$ & $\begin{array}{l}1.61 \mathrm{E}-02 \\
9.36 \mathrm{E}-03\end{array}$ \\
\hline $\begin{array}{l}\text { LLW (x) RM to OR } \\
\text { Reactive Metals }\end{array}$ & $\begin{array}{cr}\text { to Oak Ridge Reserv } \\
\text { Truck } & 2.11 \mathrm{E}-02 \\
\text { Rail } & 5.38 \mathrm{E}-03\end{array}$ & $2.15 \mathrm{E}$ & 3 & $\frac{E-02}{E-03}$ & $\begin{array}{l}2.23 \mathrm{E}-02 \\
1.80 \mathrm{E}-02\end{array}$ \\
\hline
\end{tabular}




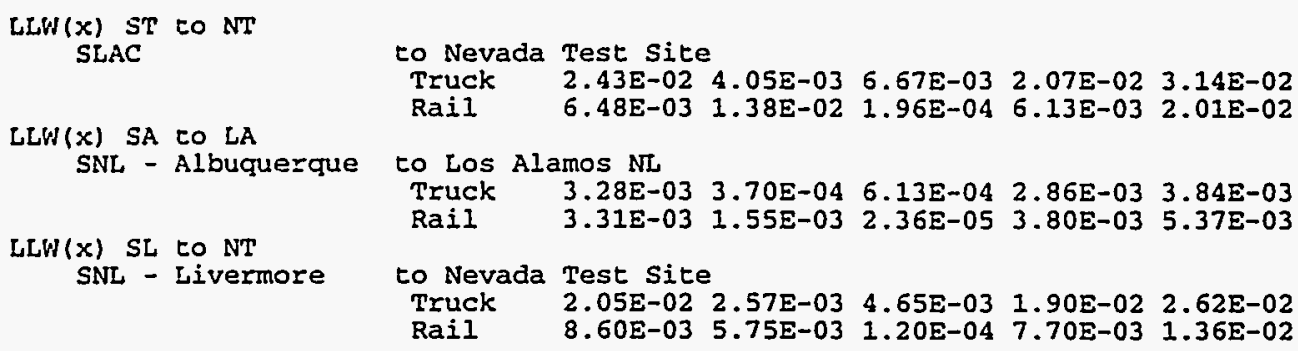

Table 4. Accident Dose Risk per Shipment (person-rem/shipment)

\begin{tabular}{|c|c|c|c|}
\hline Source/Route & & Truck & Rail \\
\hline 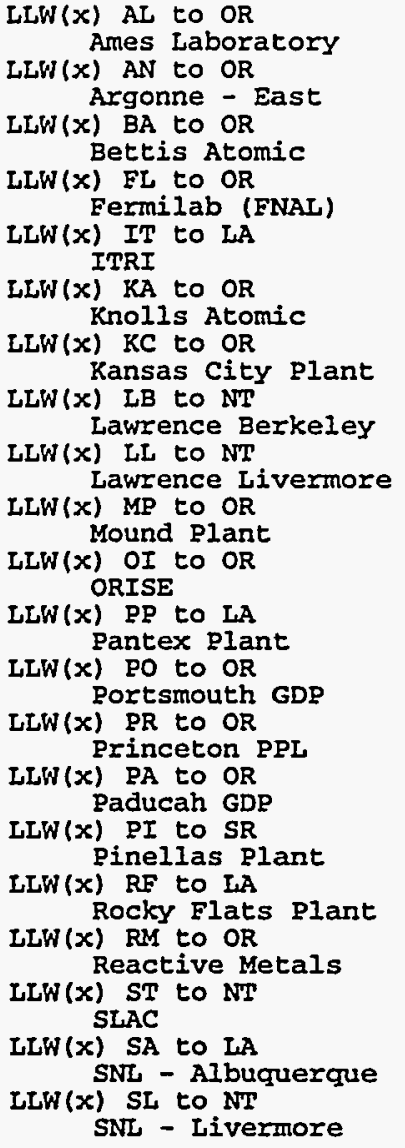 & $\begin{array}{l}\text { to Oak Ridge Reserv. } \\
\text { to Oak Ridge Reserv. } \\
\text { to Oak Ridge Reserv. } \\
\text { to Oak Ridge Reserv. } \\
\text { to Los Alamos NL } \\
\text { to Oak Ridge Reserv. } \\
\text { to Oak Ridge Reserv. } \\
\text { to Nevada Test Site } \\
\text { to Nevada Test Site } \\
\text { to Oak Ridge Reserv. } \\
\text { to Oak Ridge Reserv. } \\
\text { to Los Alamos NL } \\
\text { to Oak Ridge Reserv. } \\
\text { to Oak Ridge Reserv. } \\
\text { to Oak Ridge Reserv. } \\
\text { to Savannah River } \\
\text { to Los Alamos NL } \\
\text { to Oak Ridge Reserv. } \\
\text { to Nevada Test Site } \\
\text { to Los Alamos NL } \\
\text { to Nevada Test Site }\end{array}$ & $\begin{array}{l}9.48 \mathrm{E}-06 \\
5.16 \mathrm{E}-04 \\
9.94 \mathrm{E}-06 \\
3.56 \mathrm{E}-05 \\
1.53 \mathrm{E}-03 \\
2.13 \mathrm{E}-06 \\
3.55 \mathrm{E}-05 \\
2.56 \mathrm{E}-02 \\
4.06 \mathrm{E}-06 \\
1.07 \mathrm{E}-06 \\
4.49 \mathrm{E}-08 \\
5.53 \mathrm{E}-07 \\
7.22 \mathrm{E}-09 \\
5.74 \mathrm{E}-06 \\
3.12 \mathrm{E}-07 \\
1.30 \mathrm{E}-06 \\
4.65 \mathrm{E}-06 \\
5.68 \mathrm{E}-09 \\
2.14 \mathrm{E}-07 \\
5.39 \mathrm{E}-06 \\
3.63 \mathrm{E}-05\end{array}$ & $\begin{array}{l}6.83 \mathrm{E}-06 \\
2.26 \mathrm{E}-04 \\
1.12 \mathrm{E}-05 \\
1.16 \mathrm{E}-05 \\
7.01 \mathrm{E}-05 \\
3.16 \mathrm{E}-06 \\
7.40 \mathrm{E}-06 \\
2.61 \mathrm{E}-02 \\
3.26 \mathrm{E}-06 \\
3.06 \mathrm{E}-07 \\
3.15 \mathrm{E}-08 \\
9.15 \mathrm{E}-08 \\
3.57 \mathrm{E}-09 \\
2.32 \mathrm{E}-06 \\
1.08 \mathrm{E}-07 \\
4.65 \mathrm{E}-07 \\
5.34 \mathrm{E}-07 \\
2.86 \mathrm{E}-09 \\
3.39 \mathrm{E}-07 \\
2.50 \mathrm{E}-07 \\
3.45 \mathrm{E}-05\end{array}$ \\
\hline
\end{tabular}


Table 5. Nonradiological Risk Factors per Shipment (fatalicies/shipment)

\begin{tabular}{|c|c|c|c|}
\hline Mode & Route & Emission & Accident \\
\hline $\begin{array}{l}\text { Truck } \\
\text { Ames Laboratory } \\
\text { Argonne - East } \\
\text { Bettis Atomic } \\
\text { Fermilab (FNAL) } \\
\text { ITRI } \\
\text { Knolls Atomic } \\
\text { Ransas City Plant } \\
\text { Lawrence Berkeley } \\
\text { Lawrence Livermore } \\
\text { Mound Plant } \\
\text { ORISE } \\
\text { Pantex Plant } \\
\text { Portsmouth GDP } \\
\text { Princeton PPL } \\
\text { Paducah GDP } \\
\text { Pinellas Plant } \\
\text { Rocky Flats Plant } \\
\text { Reactive Metals } \\
\text { SLAC } \\
\text { SNL - Albuquerque } \\
\text { SNL - Livermore }\end{array}$ & $\begin{array}{l}\text { to Oak Ridge Reserv. } \\
\text { to Oak Ridge Reserv. } \\
\text { to Oak Ridge Reserv. } \\
\text { to Oak Ridge Reserv. } \\
\text { to Los Alamos NL } \\
\text { to Oak Ridge Reserv. } \\
\text { to Oak Ridge Reserv. } \\
\text { to Nevada Test Site } \\
\text { to Nevada Test Site } \\
\text { to Oak Ridge Reserv. } \\
\text { to Oak Ridge Reserv. } \\
\text { to Los Alamos NL } \\
\text { to Oak Ridge Reserv. } \\
\text { to Oak Ridge Reserv. } \\
\text { to Oak Ridge Reserv. } \\
\text { to Savannah River } \\
\text { to Los Alamos NL } \\
\text { to Oak Ridge Reserv. } \\
\text { to Nevada Test Site } \\
\text { to Los Alamos NL } \\
\text { to Nevada Test Site }\end{array}$ & $\begin{array}{l}4.25 \mathrm{E}-06 \\
5.38 \mathrm{E}-06 \\
3.41 \mathrm{E}-06 \\
6.76 \mathrm{E}-06 \\
1.51 \mathrm{E}-06 \\
2.16 \mathrm{E}-06 \\
4.31 \mathrm{E}-06 \\
1.73 \mathrm{E}-05 \\
1.32 \mathrm{E}-05 \\
1.64 \mathrm{E}-06 \\
0.00 \mathrm{E}+00 \\
3.28 \mathrm{E}-06 \\
1.09 \mathrm{E}-06 \\
1.60 \mathrm{E}-05 \\
1.93 \mathrm{E}-07 \\
4.47 \mathrm{E}-06 \\
4.25 \mathrm{E}-06 \\
3.57 \mathrm{E}-06 \\
2.17 \mathrm{E}-05 \\
1.35 \mathrm{E}-06 \\
1.32 \mathrm{E}-05\end{array}$ & $\begin{array}{l}5.79 E-05 \\
4.21 E-05 \\
3.71 E-05 \\
4.62 \mathrm{E}-05 \\
1.28 \mathrm{E}-05 \\
6.08 \mathrm{E}-05 \\
4.90 \mathrm{E}-05 \\
5.39 \mathrm{E}-05 \\
5.15 \mathrm{E}-05 \\
2.43 \mathrm{E}-05 \\
1.07 \mathrm{E}-06 \\
3.21 \mathrm{E}-05 \\
2.56 \mathrm{E}-05 \\
4.89 \mathrm{E}-05 \\
2.14 \mathrm{E}-05 \\
4.70 \mathrm{E}-05 \\
3.76 \mathrm{E}-05 \\
3.56 \mathrm{E}-05 \\
5.48 \mathrm{E}-05 \\
1.20 \mathrm{E}-05 \\
5.15 \mathrm{E}-05\end{array}$ \\
\hline $\begin{array}{l}\text { Rail } \\
\text { Ames Laboratory } \\
\text { Argonne - East } \\
\text { Bettis Atomic } \\
\text { Fermilab (FNAI) } \\
\text { ITRI } \\
\text { KnoIls Atomic } \\
\text { Kansas City Plant } \\
\text { Lawrence Berkeley } \\
\text { Lawrence Livermore } \\
\text { Mound Plant } \\
\text { ORISE } \\
\text { Pantex Plant } \\
\text { Portsmouth GDP } \\
\text { Princeton PDL } \\
\text { Paducah GDP } \\
\text { Pinellas PIant } \\
\text { Rocky Flats Plant } \\
\text { Reactive Metals } \\
\text { SLAC } \\
\text { SNL - Albuquerque } \\
\text { SNL - Livermore }\end{array}$ & $\begin{array}{l}\text { to Oak Ridge Reserv. } \\
\text { to Oak Ridge Reserv. } \\
\text { to Oak Ridge Reserv. } \\
\text { to Oak Ridge Reserv. } \\
\text { to Los Alamos NL } \\
\text { to Oak Ridge Reserv. } \\
\text { to Oak Ridge Reserv. } \\
\text { to Nevada Test Site } \\
\text { to Nevada Test Site } \\
\text { to Oak Ridge Reserv. } \\
\text { to Oak Ridge Reserv. } \\
\text { to Los Alamos NL } \\
\text { to Oak Ridge Reserv. } \\
\text { to Oak Ridge Reserv. } \\
\text { to Oak Ridge Reserv. } \\
\text { to Savannah River } \\
\text { to Los Alamos NL } \\
\text { to Oak Ridge Reserv. } \\
\text { to Nevada Test Site } \\
\text { to Los Alamos NL } \\
\text { to Nevada Test Site }\end{array}$ & $\begin{array}{l}2.52 E-05 \\
1.08 E-05 \\
1.78 E-05 \\
2.28 E-05 \\
2.47 E-06 \\
5.59 E-05 \\
1.18 E-05 \\
2.59 E-05 \\
1.01 E-05 \\
4.31 E-06 \\
1.26 E-07 \\
2.59 E-06 \\
3.18 E-06 \\
3.08 E-05 \\
3.81 E-06 \\
5.69 E-06 \\
6.78 E-06 \\
1.85 E-05 \\
2.35 E-05 \\
2.47 E-06 \\
1.01 E-05\end{array}$ & $\begin{array}{l}2.00 \mathrm{E}-06 \\
1.36 \mathrm{E}-06 \\
1.89 \mathrm{E}-06 \\
1.43 \mathrm{E}-06 \\
2.18 \mathrm{E}-07 \\
2.00 \mathrm{E}-06 \\
1.75 \mathrm{E}-06 \\
1.80 \mathrm{E}-06 \\
2.87 \mathrm{E}-06 \\
6.86 \mathrm{E}-07 \\
8.26 \mathrm{E}-08 \\
1.01 \mathrm{E}-06 \\
9.25 \mathrm{E}-07 \\
1.59 \mathrm{E}-06 \\
1.10 \mathrm{E}-06 \\
1.01 \mathrm{E}-06 \\
1.05 \mathrm{E}-06 \\
1.25 \mathrm{E}-06 \\
1.80 \mathrm{E}-06 \\
2.18 \mathrm{E}-07 \\
2.87 \mathrm{E}-06\end{array}$ \\
\hline
\end{tabular}

Table 6. Annual Incident-Free Dose for the Shipping Campaign (person-rem/yr)

\begin{tabular}{|c|c|c|c|c|c|c|}
\hline \multirow{2}{*}{ Route } & & & \multicolumn{2}{|c|}{ Truck } & \multicolumn{2}{|c|}{ Rail } \\
\hline & & & Crew & Public & Crew & Public \\
\hline $\begin{array}{l}\text { Anes Laboratory } \\
\text { Argonne - East } \\
\text { Bettis Atomic } \\
\text { Fermilab (FNAL) } \\
\text { ITRI } \\
\text { Rnolls Atomic } \\
\text { Kansas City Plant } \\
\text { Lawrence Berkeley } \\
\text { Lawrence Livermore } \\
\text { Mound Plant } \\
\text { ORISE } \\
\text { Pantex Plant } \\
\text { Portsmouth GDP } \\
\text { Princeton PPL } \\
\text { Paducah GDP } \\
\text { Pinellas Plant } \\
\text { Rocky Flats Plant } \\
\text { Reactive Metals } \\
\text { SLAC } \\
\text { SNL - Albuquerque } \\
\text { SNL - Livermore }\end{array}$ & $\begin{array}{l}\text { to } \\
\text { to } \\
\text { to } \\
\text { to } \\
\text { to } \\
\text { to } \\
\text { to } \\
\text { to } \\
\text { to } \\
\text { to } \\
\text { to } \\
\text { to } \\
\text { to } \\
\text { to } \\
\text { to } \\
\text { to } \\
\text { to } \\
\text { to } \\
\text { to } \\
\text { to } \\
\text { to }\end{array}$ & $\begin{array}{l}\text { Oak Ridge Reserv. } \\
\text { Oak Ridge Reserv. } \\
\text { Oak Ridge Reserv. } \\
\text { Oak Ridge Reserv. } \\
\text { Los Alamos NL } \\
\text { Oak Ridge Reserv. } \\
\text { Oak Ridge Reserv. } \\
\text { Nevada Test Site } \\
\text { Nevada Test Site } \\
\text { Oak Ridge Reserv. } \\
\text { Oak Ridge Reserv. } \\
\text { Los Alamos NL } \\
\text { Oak Ridge Reserv. } \\
\text { Oak Ridge Reserv. } \\
\text { Oak Ridge Reserv. } \\
\text { Savannah River } \\
\text { Los Alamos NL } \\
\text { Oak Ridge Reserv. } \\
\text { Nevada Test Site } \\
\text { Los Alamos NL } \\
\text { Nevada Test Site }\end{array}$ & $\begin{array}{l}5.66 \mathrm{E}-02 \\
2.10 \mathrm{E}+00 \\
1.05 \mathrm{E}+00 \\
6.45 \mathrm{E}-02 \\
3.54 \mathrm{E}-02 \\
8.33 \mathrm{E}-01 \\
2.20 \mathrm{E}-02 \\
6.81 \mathrm{E}-02 \\
1.15 \mathrm{E}+00 \\
6.28 \mathrm{E}+00 \\
1.81 \mathrm{E}-03 \\
1.62 \mathrm{E}+01 \\
3.90 \mathrm{E}+01 \\
2.68 \mathrm{E}-02 \\
5.57 \mathrm{E}+00 \\
4.04 \mathrm{E}-02 \\
4.83 \mathrm{E}+00 \\
1.62 \mathrm{E}+01 \\
9.46 \mathrm{E}-01 \\
5.58 \mathrm{E}-02 \\
1.23 \mathrm{E}-01\end{array}$ & $\begin{array}{l}6.28 E-02 \\
2.29 \mathrm{E}+00 \\
1.15 \mathrm{E}+00 \\
7.04 \mathrm{E}-02 \\
4.18 \mathrm{E}-02 \\
8.72 \mathrm{E}-01 \\
2.52 \mathrm{E}-02 \\
8.73 \mathrm{E}-02 \\
1.47 \mathrm{E}+00 \\
6.50 \mathrm{E}+00 \\
1.80 \mathrm{E}-03 \\
2.00 \mathrm{E}+01 \\
4.26 \mathrm{E}+01 \\
3.04 \mathrm{E}-02 \\
6.26 \mathrm{E}+00 \\
4.48 \mathrm{E}-02 \\
5.74 \mathrm{E}+00 \\
1.71 \mathrm{E}+01 \\
1.23 \mathrm{E}+00 \\
6.53 \mathrm{E}-02 \\
1.57 \mathrm{E}-01\end{array}$ & $\begin{array}{l}6.86 \mathrm{E}-03 \\
2.18 \mathrm{E}-01 \\
1.40 \mathrm{E}-01 \\
1.15 \mathrm{E}-02 \\
1.33 \mathrm{E}-02 \\
7.56 \mathrm{E}-02 \\
6.38 \mathrm{E}-03 \\
1.29 \mathrm{E}-02 \\
1.81 \mathrm{E}-01 \\
8.07 \mathrm{E}-01 \\
6.09 \mathrm{E}-03 \\
2.66 \mathrm{E}+00 \\
6.02 \mathrm{E}+00 \\
6.05 \mathrm{E}-03 \\
1.20 \mathrm{E}+00 \\
4.90 \mathrm{E}-03 \\
6.63 \mathrm{E}-01 \\
1.54 \mathrm{E}+00 \\
9.72 \mathrm{E}-02 \\
2.32 \mathrm{E}-02 \\
1.72 \mathrm{E}-02\end{array}$ & $\begin{array}{l}2.24 \mathrm{E}-02 \\
5.45 \mathrm{E}-01 \\
4.30 \mathrm{E}-01 \\
3.90 \mathrm{E}-02 \\
2.15 \mathrm{E}-02 \\
4.48 \mathrm{E}-01 \\
1.40 \mathrm{E}-02 \\
4.24 \mathrm{E}-02 \\
2.85 \mathrm{E}-01 \\
1.54 \mathrm{E}+00 \\
7.89 \mathrm{E}-03 \\
3.91 \mathrm{E}+00 \\
1.19 \mathrm{E}+01 \\
2.71 \mathrm{E}-02 \\
1.87 \mathrm{E}+00 \\
1.02 \mathrm{E}-02 \\
1.24 \mathrm{E}+00 \\
5.17 \mathrm{E}+00 \\
3.02 \mathrm{E}-01 \\
3.76 \mathrm{E}-02 \\
2.71 \mathrm{E}-02\end{array}$ \\
\hline Totals & & & $9.46 \mathrm{E} \div 01$ & $1.06 \mathrm{E}+02$ & $1.37 \mathrm{E}+01$ & $2.79 \mathrm{E}+01$ \\
\hline
\end{tabular}


Table 7. Annual Accident Dose Risk for the Shipping Campaign (person-rem/yr)

\begin{tabular}{|c|c|c|c|}
\hline Route & & Truck & Rail \\
\hline $\begin{array}{l}\text { Ames Laboratory } \\
\text { Argonne - East } \\
\text { Bettis Atomic } \\
\text { Eermilab (FNAL) } \\
\text { ITRI } \\
\text { Knolls Atomic } \\
\text { Ransas City Plant } \\
\text { Lawrence Berkeley } \\
\text { Lawrence Livermore } \\
\text { Mound Plant } \\
\text { ORISE } \\
\text { Pantex Plant } \\
\text { Portsmouth GDP } \\
\text { Princeton PPL } \\
\text { Paducah GDP } \\
\text { Pinellas Plant } \\
\text { Rocky Flats Plant } \\
\text { Reactive Metals } \\
\text { SLAC } \\
\text { SNL - Albuquerque } \\
\text { SNL - Livermore }\end{array}$ & $\begin{array}{l}\text { to Oak Ridge Reserv. } \\
\text { to Oak Ridge Reserv. } \\
\text { to Oak Ridge Reserv. } \\
\text { to Oak Ridge Reserv. } \\
\text { to Los Alamos NL } \\
\text { to Oak Ridge Reserv. } \\
\text { to Oak Ridge Reserv. } \\
\text { to Nevada Test Site } \\
\text { to Nevada Test Site } \\
\text { to Oak Ridge Reserv. } \\
\text { to Oak Ridge Reserv. } \\
\text { to Los Alamos NL } \\
\text { to Oak Ridge Reserv. } \\
\text { to Oak Ridge Reserv. } \\
\text { to Oak Ridge Reserv. } \\
\text { to Savannah River } \\
\text { to Los Alamos NL } \\
\text { to Oak Ridge Reserv. } \\
\text { to Nevada Test Site } \\
\text { to Ios Alamos NL } \\
\text { to Nevada Test Site }\end{array}$ & $\begin{array}{l}1.90 \mathrm{E}-05 \\
5.41 \mathrm{E}-02 \\
5.67 \mathrm{E}-04 \\
1.07 \mathrm{E}-04 \\
1.53 \mathrm{E}-02 \\
5.98 \mathrm{E}-05 \\
3.55 \mathrm{E}-05 \\
7.68 \mathrm{E}-02 \\
2.27 \mathrm{E}-04 \\
5.47 \mathrm{E}-04 \\
2.24 \mathrm{E}-07 \\
8.02 \mathrm{E}-04 \\
2.41 \mathrm{E}-05 \\
5.74 \mathrm{E}-06 \\
1.96 \mathrm{E}-04 \\
2.59 \mathrm{E}-06 \\
1.66 \mathrm{E}-03 \\
4.36 \mathrm{E}-06 \\
8.33 \mathrm{E}-06 \\
9.16 \mathrm{E}-05 \\
2.18 \mathrm{E}-04\end{array}$ & $\begin{array}{l}6.83 \mathrm{E}-06 \\
8.83 \mathrm{E}-03 \\
2.35 \mathrm{E}-04 \\
2.32 \mathrm{E}-05 \\
2.80 \mathrm{E}-04 \\
3.47 \mathrm{E}-05 \\
7.40 \mathrm{E}-06 \\
5.23 \mathrm{E}-02 \\
6.85 \mathrm{E}-05 \\
5.81 \mathrm{E}-05 \\
6.30 \mathrm{E}-08 \\
4.98 \mathrm{E}-05 \\
4.55 \mathrm{E}-06 \\
2.32 \mathrm{E}-06 \\
2.56 \mathrm{E}-05 \\
4.65 \mathrm{E}-07 \\
7.10 \mathrm{E}-05 \\
8.20 \mathrm{E}-07 \\
5.08 \mathrm{E}-06 \\
1.75 \mathrm{E}-06 \\
6.89 \mathrm{E}-05\end{array}$ \\
\hline Totals & & $1.51 \mathrm{E}-01$ & $6.20 \mathrm{E}-02$ \\
\hline
\end{tabular}

Table 8. Expected Annual Fatalities for the Shipping Campaign

\begin{tabular}{llc}
\hline Exposure Group & Truck & Rail \\
\hline Radiological & $3.8 \mathrm{E}-02$ & $5.5 \mathrm{E}-03$ \\
$\quad$ Normal Crew & $5.3 \mathrm{E}-02$ & $1.4 \mathrm{E}-02$ \\
$\quad$ Normal Public & $7.5 \mathrm{E}-05$ & $3.1 \mathrm{E}-05$ \\
$\begin{array}{c}\text { Accident Public } \\
\text { Nonradiological } \\
\text { Emission }\end{array}$ & $1.6 \mathrm{E}-02$ & $1.6 \mathrm{E}-02$ \\
Accident & $2.1 \mathrm{E}-01$ & $2.8 \mathrm{E}-03$ \\
\hline
\end{tabular}

Table 9. Expected Annual Cancer Incidence for the Shipping Campaign

\begin{tabular}{lll}
\hline Exposure Group & Truck & Rail \\
\hline Radiological & & \\
Normal Crew & $1.3 E-01$ & $1.9 \mathrm{E}-02$ \\
Normal Public & $1.8 \mathrm{E}-01$ & $4.7 \mathrm{E}-02$ \\
Accident Public & $2.6 \mathrm{E}-04$ & $1.1 \mathrm{E}-04$ \\
$\begin{array}{c}\text { Nonradiological } \\
\text { Emission }\end{array}$ & $1.6 \mathrm{E}-02$ & $1.6 \mathrm{E}-02$ \\
Accident & $\mathrm{NA}$ & $\mathrm{NA}$ \\
\hline
\end{tabular}

Table 10. Expected Annual Genetic Effects for the Shipping Campaign

\begin{tabular}{lll}
\hline Exposure Group & Truck & Rail \\
\hline Radiological & $5.7 E-03$ & $8.2 E-04$ \\
Normal Crew & $1.1 E-02$ & $2.8 E-03$ \\
Normal Public & $5.7 E-06$ & $1.6 \mathrm{E}-06$ \\
Accident Public & NA & NA \\
Nonradiological & NA & NA \\
Emission & & \\
Accident & &
\end{tabular}




\section{B.10 WM LLW REGIONALIZED 3 ALTERNATIVE (CASE 4): ACTIVATED METALS}

Table 1. Summary of Route Information

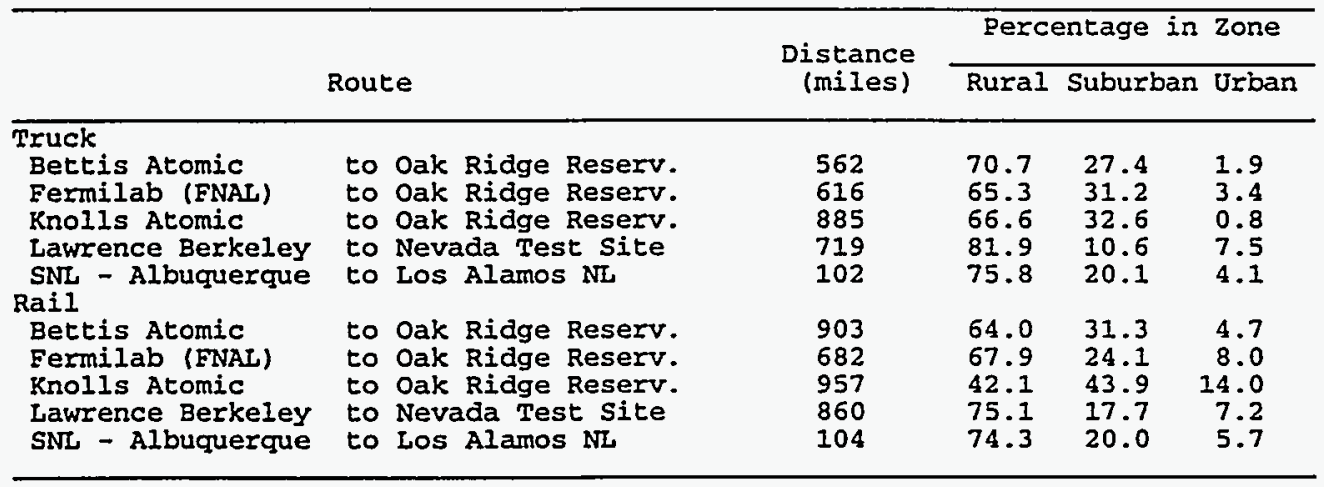

Table 2. Annual Number of Shipments and One-Way Shipment Distances

\begin{tabular}{|c|c|c|c|c|c|}
\hline \multirow[b]{2}{*}{ Route } & & \multicolumn{2}{|c|}{ \# of Shipments } & \multicolumn{2}{|c|}{ Total Mileage(mi) } \\
\hline & & Truck & Rail & Truck & Rail \\
\hline $\begin{array}{l}\text { Bettis Atomic } \\
\text { Eermilab (FNAL) } \\
\text { Knolls Atomic } \\
\text { Lawrence Berkeley } \\
\text { SNL - Albuquerque }\end{array}$ & $\begin{array}{l}\text { to Oak Ridge Reserv. } \\
\text { to Oak Ridge Reserv. } \\
\text { to Oak Ridge Reserv. } \\
\text { to Nevada Test Site } \\
\text { to Los Alamos NL }\end{array}$ & $\begin{array}{r}316 \\
40 \\
650 \\
15 \\
6\end{array}$ & $\begin{array}{r}120 \\
15 \\
246 \\
6 \\
3\end{array}$ & $\begin{array}{l}1.78 \mathrm{E}+05 \\
2.46 \mathrm{E}+04 \\
5.76 \mathrm{E}+05 \\
1.08 \mathrm{E}+04 \\
6.12 \mathrm{E}+02\end{array}$ & $\begin{array}{l}1.08 E+05 \\
1.02 E+04 \\
2.35 E+05 \\
5.16 E+03 \\
3.12 E+02\end{array}$ \\
\hline Totals & & 1027 & 390 & $7.89 E+05$ & $3.59 E+05$ \\
\hline
\end{tabular}

Table 3. Incident-Free Dose per Shipment (person-rem/shipment)

\begin{tabular}{|c|c|c|c|c|c|}
\hline \multirow[b]{2}{*}{ Source/Route(s) } & \multirow[b]{2}{*}{ Crew } & \multicolumn{4}{|c|}{ General Public } \\
\hline & & off-Link & On-Iink & Stops & Total \\
\hline $\begin{array}{l}\text { LLW(I) BA to OR } \\
\text { Bettis Atomic }\end{array}$ & $\begin{array}{cr}\text { to Oak Ridge Reser } \\
\text { Truck } & 1.85 \mathrm{E}-02 \\
\text { Rail } & 6.65 \mathrm{E}-03\end{array}$ & $\begin{array}{l}\text { I. } 72 \mathrm{E}-03 \\
1.40 \mathrm{E}-02\end{array}$ & $\begin{array}{l}2.71 E-03 \\
2.44 E-04\end{array}$ & $\begin{array}{l}1.57 \mathrm{E}-02 \\
6.26 \mathrm{E}-03\end{array}$ & $\begin{array}{l}2.02 \mathrm{E}-02 \\
2.05 \mathrm{E}-02\end{array}$ \\
\hline $\begin{array}{l}\text { ILW(I) FL to OR } \\
\text { Fermilab (ENAI) }\end{array}$ & $\begin{array}{cr}\text { to Oak Ridge Reser } \\
\text { Truck } & 2.15 \mathrm{E}-02 \\
\text { Rail } & 5.73 \mathrm{E}-03\end{array}$ & $\begin{array}{l}2.53 \mathrm{E}-03 \\
1.37 \mathrm{E}-02\end{array}$ & $\begin{array}{l}3.71 \mathrm{E}-03 \\
1.92 \mathrm{E}-04\end{array}$ & $\begin{array}{l}1.72 E-02 \\
5.58 E-03\end{array}$ & $\begin{array}{l}2.35 \mathrm{E}-02 \\
1.95 \mathrm{E}-02\end{array}$ \\
\hline $\begin{array}{l}\text { LLW(I) KA to OR } \\
\text { Knolis Atomic }\end{array}$ & $\begin{array}{cr}\text { to Oak Ridge Reser } \\
\text { Truck } & 2.98 \mathrm{E}-02 \\
\text { Rail } & 6.87 \mathrm{E}-03\end{array}$ & $\begin{array}{l}2.55 \mathrm{E}-03 \\
3.39 \mathrm{E}-02\end{array}$ & $\begin{array}{l}3.79 E-03 \\
4.49 E-04\end{array}$ & $\begin{array}{l}2.48 E-02 \\
6.42 E-03\end{array}$ & $\begin{array}{l}3.11 \mathrm{E}-02 \\
4.08 \mathrm{E}-02\end{array}$ \\
\hline $\begin{array}{l}\text { LLW(I) LB to NT } \\
\text { Lawrence Berkeley }\end{array}$ & $\begin{array}{ll}\text { to Nevada } & \text { Test Sit } \\
\text { Truck } & 2.27 \mathrm{E}-02 \\
\text { Rail } & 6.47 \mathrm{E}-03\end{array}$ & $\begin{array}{l}\text { e } \\
3.31 E-03 \\
1.49 E-02\end{array}$ & $\begin{array}{l}5.66 E-03 \\
2.04 E-04\end{array}$ & $\begin{array}{l}2.01 E-02 \\
6.13 E-03\end{array}$ & $\begin{array}{l}2.91 E-02 \\
2.12 E-02\end{array}$ \\
\hline $\begin{array}{l}\text { ILW (I) SA to IA } \\
\text { SNL - AIbuquerque }\end{array}$ & $\begin{array}{lr}\text { to Los Alamos } \mathrm{NL} \\
\text { Truck } & 3.28 \mathrm{E}-03 \\
\text { Rail } & 3.31 \mathrm{E}-03\end{array}$ & $\begin{array}{l}3.70 \mathrm{E}-04 \\
1.55 \mathrm{E}-03\end{array}$ & $\begin{array}{l}6.13 E-04 \\
2.36 E-05\end{array}$ & $\begin{array}{l}2.86 E-03 \\
3.80 E-03\end{array}$ & $\begin{array}{l}3.84 E-03 \\
5.37 E-03\end{array}$ \\
\hline
\end{tabular}


TABLE 4.2 Railroad-Freight-Car Accident Rates, by State, for 1986, 1987, 1988, and 1985-1988 Combined (10 ${ }^{-8}$ accidents per railcar-kilometer ${ }^{2}$ )

\begin{tabular}{|c|c|c|c|c|c|c|c|c|}
\hline \multirow[b]{2}{*}{ State $^{b}$} & \multicolumn{2}{|c|}{1986} & \multicolumn{2}{|c|}{1987} & \multicolumn{2}{|c|}{1988} & \multicolumn{2}{|c|}{$1985-88$} \\
\hline & Total & $\begin{array}{l}\text { Main } \\
\text { Only }\end{array}$ & Total & $\begin{array}{l}\text { Main } \\
\text { Only } \\
\end{array}$ & Total & $\begin{array}{l}\text { Main } \\
\text { Only }\end{array}$ & Total & $\begin{array}{l}\text { Main } \\
\text { Oniy }\end{array}$ \\
\hline$A L$ & 3.88 & 2.00 & 4.80 & 3.37 & 4.13 & 2.52 & 4.80 & 2.75 \\
\hline $\mathrm{AZ}$ & 1.40 & 1.05 & 1.30 & 1.08 & 1.44 & 1.18 & 1.75 & 1.30 \\
\hline AR & 5.05 & 2.59 & 4.27 & 2.32 & 8.34 & 3.94 & 6.78 & 3.54 \\
\hline $\mathrm{CA}$ & 5.10 & 2.64 & 3.30 & 1.98 & 4.32 & 2.30 & 5.10 & 2.51 \\
\hline $\mathrm{CO}$ & 1.44 & 1.01 & 0.86 & 0.40 & 2.55 & 1.51 & 1.73 & 1.02 \\
\hline$C T$ & 26.47 & 17.65 & 16.67 & 8.34 & 24.95 & 0 & $28.27^{c}$ & 10.10 \\
\hline $\mathrm{DE}$ & 27.44 & 9.15 & 25.23 & 16.82 & 18.04 & 18.04 & 17.71 & 11.07 \\
\hline DC & 0 & 0 & 316 & 316 & 0 & 0 & $117.09^{c}$ & $78.06^{\mathrm{c}}$ \\
\hline FL & 2.86 & 2.12 & 3.90 & 1.90 & 4.16 & 2.38 & 4.02 & 2.21 \\
\hline GA & 6.44 & 3.08 & 6.77 & 2.81 & 6.18 & 2.69 & 6.44 & 2.84 \\
\hline ID & 5.90 & 5.25 & 6.74 & 4.10 & 9.03 & 3.99 & 7.01 & 4.14 \\
\hline IL & 11.70 & 3.10 & 9.97 & 3.10 & 9.08 & 2.45 & 10.67 & 2.97 \\
\hline IN & 4.18 & 1.85 & 3.71 & 1.48 & 3.80 & 1.44 & 4.64 & 1.93 \\
\hline IA & 17.63 & 7.15 & 12.72 & 6.65 & 11.95 & 7.01 & 14.67 & 7.16 \\
\hline KS & 3.01 & 1.62 & 2.97 & 1.55 & 4.09 & 1.98 & 3.61 & 1.75 \\
\hline $\mathrm{KY}$ & 4.78 & 2.39 & 3.77 & 1.98 & 3.60 & 1.84 & 4.48 & 2.44 \\
\hline LA & 12.67 & 5.98 & 7.07 & 2.02 & 11.94 & 3.92 & 12.37 & 4.28 \\
\hline $\mathrm{ME}$ & 28.22 & 16.93 & 29.40 & 13.36 & 52.57 & 26.29 & $37.80^{c}$ & $18.53^{c}$ \\
\hline$M D$ & 6.07 & 3.54 & 5.55 & 2.44 & 4.55 & 1.24 & 5.62 & 2.58 \\
\hline MA & 12.33 & 5.87 & 10.06 & 3.71 & 7.31 & 2.81 & 11.65 & 4.97 \\
\hline MI & 18.34 & 8.18 & 15.10 & 4.42 & 15.52 & 7.45 & 16.47 & 7.19 \\
\hline $\mathrm{MN}$ & 8.63 & 2.64 & 6.24 & 3.30 & 7.86 & 3.63 & 8.48 & 3.16 \\
\hline MS & 15.16 & 11.20 & 12.93 & 9.84 & 8.47 & 7.14 & 11.52 & 8.51 \\
\hline MO & 4.59 & 2.12 & 3.60 & 1.87 & 5.05 & 2.87 & 5.28 & 2.56 \\
\hline $\mathrm{MT}$ & 1.35 & 1.01 & 1.24 & 0.81 & 2.11 & 1.17 & 1.73 & 1.10 \\
\hline $\mathrm{NE}$ & 4.04 & 2.11 & 4.81 & 2.49 & 4.46 & 2.23 & 4.63 & 2.56 \\
\hline NV & 3.27 & 1.87 & 1.29 & 0.86 & 3.97 & 2.65 & 3.23 & 2.19 \\
\hline $\mathrm{NH}$ & 6.60 & 6.60 & 17.52 & 11.68 & 24.27 & 16.18 & 21.45 & $17.16^{\mathrm{c}}$ \\
\hline $\mathrm{NJ}$ & 13.86 & 2.64 & 9.44 & 5.31 & 10.10 & 4.75 & 12.38 & 4.82 \\
\hline $\mathrm{NM}$ & 0.90 & 0.52 & 0.93 & 0.75 & 0.86 & 0.65 & 0.94 & 0.66 \\
\hline$N Y$ & 7.23 & 3.92 & 6.80 & 4.14 & 7.26 & 3.36 & 8.32 & 4.30 \\
\hline NC & 5.40 & 2.70 & 5.48 & 1.62 & 6.29 & 2.95 & 5.70 & 2.27 \\
\hline ND & 2.18 & 1.48 & 2.43 & 1.86 & 1.49 & 1.12 & 2.41 & 1.80 \\
\hline $\mathrm{OH}$ & 5.35 & 2.50 & 3.55 & 1.58 & 4.04 & 1.92 & 4.73 & 2.12 \\
\hline OK & 5.25 & 3.01 & 3.52 & 1.90 & 3.77 & 1.88 & 4.66 & 2.72 \\
\hline OR & 11.74 & 4.96 & 12.23 & 6.12 & 13.64 & 6.04 & 12.48 & 5.77 \\
\hline $\mathrm{PA}$ & 4.86 & 3.73 & 4.22 & 2.37 & 3.54 & 1.95 & 4.38 & 2.69 \\
\hline RI & 0 & 0 & 0 & 0 & 0 & 0 & $105.33^{c}$ & 0 \\
\hline
\end{tabular}


Table 4. Accident Dose Risk per Shipment (person-rem/shipment)

\begin{tabular}{|c|c|c|c|}
\hline Source/Route & & Truck & Rail \\
\hline $\begin{array}{l}\text { LLW(I) BA to OR } \\
\text { BetLis Atomic } \\
\text { LLW(I) FL to OR } \\
\text { Fermilab (ENAL) } \\
\text { LLW(I) KA to OR } \\
\text { Knolls Atomic } \\
\text { LLW(I) LB to NT } \\
\text { LaWrence Berkeley } \\
\text { LLW(I) SA to LA } \\
\text { SNL - Albuquerque }\end{array}$ & $\begin{array}{l}\text { to Oak Ridge Reserv. } \\
\text { to Oak Ridge Reserv. } \\
\text { to Oak Ridge Reserv. } \\
\text { to Nevada Test Site } \\
\text { to Los Alamos NL }\end{array}$ & $\begin{array}{l}7.27 \mathrm{E}-07 \\
7.58 \mathrm{E}-11 \\
1.47 \mathrm{E}-06 \\
8.23 \mathrm{E}-11 \\
1.72 \mathrm{E}-12\end{array}$ & $\begin{array}{l}6.36 \mathrm{E}-07 \\
4.36 \mathrm{E}-11 \\
2.29 \mathrm{E}-06 \\
1.45 \mathrm{E}-10 \\
6.51 \mathrm{E}-14\end{array}$ \\
\hline
\end{tabular}

Table 5. Nonradiological Risk Factors per Shipment

(fatalities/shipment)

\begin{tabular}{llll}
\hline Mode & Route & Emission & Accident \\
\hline Truck & & & \\
Bettis Atomic & to Oak Ridge Reserv. & $3.41 E-06$ & $3.71 \mathrm{E}-05$ \\
Fermilab (FNAL) & to Oak Ridge Reserv. & $6.76 \mathrm{E}-06$ & $4.62 \mathrm{E}-05$ \\
Knolis Atomic & to Oak Ridge Reserv. & $2.16 \mathrm{E}-06$ & $6.08 \mathrm{E}-05$ \\
Lawrence Berkeley & to Nevada Test Site & $1.73 \mathrm{E}-05$ & $5.39 \mathrm{E}-05$ \\
SNL - Albuquerque & to Los Alamos NL & $1.35 \mathrm{E}-06$ & $1.20 \mathrm{E}-05$ \\
Rail & & & \\
Bettis Atomic & to Oak Ridge Reserv. & $1.78 \mathrm{E}-05$ & $1.89 \mathrm{E}-06$ \\
Fermilab (FNAL) & to Oak Ridge Reserv. & $2.28 \mathrm{E}-05$ & $1.43 \mathrm{E}-06$ \\
Knolls Atomic & to Oak Ridge Reserv. & $5.59 \mathrm{E}-05$ & $2.00 \mathrm{E}-06$ \\
Lawrence Berkeley & to Nevada Test Site & $2.59 \mathrm{E}-05$ & $1.80 \mathrm{E}-06$ \\
SNL - Albuquerque & to Los Alamos NL & $2.47 \mathrm{E}-06$ & $2.18 \mathrm{E}-07$ \\
\hline
\end{tabular}

Table 6. Annual Incident-Free Dose for the Shipping Campaign (person-rem/yr)

\begin{tabular}{|c|c|c|c|c|c|}
\hline \multirow[b]{2}{*}{ Route } & & \multicolumn{2}{|c|}{ Truck } & \multicolumn{2}{|c|}{ Rail } \\
\hline & & Crew & Public & Crew & Public \\
\hline $\begin{array}{l}\text { Bettis Atomic } \\
\text { Fermilab (FNAL) } \\
\text { Knolis Atomic } \\
\text { Lawrence Berkeley } \\
\text { SNL - Albuquerque }\end{array}$ & $\begin{array}{l}\text { to Oak Ridge Reserv. } \\
\text { to Oak Ridge Reserv. } \\
\text { to Oak Ridge Reserv. } \\
\text { to Nevada Test Site } \\
\text { to Los Alamos NL }\end{array}$ & $\begin{array}{l}5.84 \mathrm{E}+00 \\
8.60 \mathrm{E}-01 \\
1.93 \mathrm{E}+01 \\
3.41 \mathrm{E}-01 \\
1.97 \mathrm{E}-02\end{array}$ & $\begin{array}{l}6.38 \mathrm{E}+00 \\
9.39 \mathrm{E}-01 \\
2.02 \mathrm{E}+01 \\
4.36 \mathrm{E}-01 \\
2.30 \mathrm{E}-02\end{array}$ & $\begin{array}{l}7.98 \mathrm{E}-01 \\
8.59 \mathrm{E}-02 \\
1.69 \mathrm{E}+00 \\
3.88 \mathrm{E}-02 \\
9.94 \mathrm{E}-03\end{array}$ & $\begin{array}{l}2.46 \mathrm{E}+00 \\
2.93 \mathrm{E}-01 \\
1.00 \mathrm{E}+01 \\
1.27 \mathrm{E}-01 \\
1.61 \mathrm{E}-02\end{array}$ \\
\hline Totals & & $2.64 \mathrm{E}+01$ & $2.80 \mathrm{E}+01$ & $2.62 \mathrm{E}+00$ & $1.29 \mathrm{E}+01$ \\
\hline
\end{tabular}

Table 7. Annual Accident Dose Risk for the Shipping Campaign (person-rem/yr)

\begin{tabular}{llcc}
\hline Route & & Truck & Rail \\
\hline Bettis Atomic & to Oak Ridge Reserv. & $2.30 \mathrm{E}-04$ & $7.63 \mathrm{E}-05$ \\
Fermilab (FNAI) & to Oak Ridge Reserv. & $3.03 \mathrm{E}-09$ & $6.54 \mathrm{E}-10$ \\
Knolls Atomic & to Oak Ridge Reserv. & $9.58 \mathrm{E}-04$ & $5.64 \mathrm{E}-04$ \\
Lawrence Berkeley & to Nevada Test Site & $1.23 \mathrm{E}-09$ & $8.71 \mathrm{E}-10$ \\
SNL - Albuquerque & to Los Alamos NL & $1.03 \mathrm{E}-11$ & $1.95 \mathrm{E}-13$ \\
\hline Totals & & $1.19 \mathrm{E}-03$ & $6.40 \mathrm{E}-04$ \\
\hline
\end{tabular}

Table 8. Expected Annual Fatalities

for the Shipping Campaign

\begin{tabular}{llc}
\hline Exposure Group & Truck & Rail \\
\hline Radiological & $1.1 \mathrm{E}-02$ & $1.0 \mathrm{E}-03$ \\
$\quad$ Normal Crew & $1.4 \mathrm{E}-02$ & $6.5 \mathrm{E}-03$ \\
Normal Public & $5.9 \mathrm{E}-07$ & $3.2 \mathrm{E}-07$ \\
Accident Public & $3.0 \mathrm{E}-03$ & $1.6 \mathrm{E}-02$ \\
$\begin{array}{c}\text { Nonradiological } \\
\text { Enission }\end{array}$ & $5.4 \mathrm{E}-02$ & $7.5 \mathrm{E}-04$ \\
Accident & \\
\hline
\end{tabular}


Table 9. Expected Annual Cancer Incidence for the Shipping Campaign

\begin{tabular}{lll}
\hline Exposure Group & Truck & Rail \\
\hline Radiological & & \\
$\quad$ Normal Crew & $3.7 \mathrm{E}-02$ & $3.7 \mathrm{E}-03$ \\
$\quad$ Normal Public & $4.8 \mathrm{E}-02$ & $2.2 \mathrm{E}-02$ \\
$\quad \begin{array}{cc}\text { Accident Public } \\
\text { Nonradiological } \\
\text { Emission }\end{array}$ & $2.0 \mathrm{E}-06$ & $1.1 \mathrm{E}-06$ \\
Accident & $3.0 \mathrm{E}-03$ & $\begin{array}{l}1.6 \mathrm{E}-02 \\
\mathrm{NA}\end{array}$ \\
\hline
\end{tabular}

Table 10. Expected Annual Genetic Effects for the Shipping Campaign

\begin{tabular}{lll}
\hline Exposure Group & Truck & Rail \\
\hline Radiological & & \\
Normal Crew & $1.6 \mathrm{E}-03$ & $1.6 \mathrm{E}-04$ \\
Normal Public & $2.8 \mathrm{E}-03$ & $1.3 \mathrm{E}-03$ \\
Accident Public & $1.2 \mathrm{E}-07$ & $6.4 \mathrm{E}-08$ \\
$\begin{array}{c}\text { Nonradiological } \\
\text { Emission }\end{array}$ & NA & NA \\
Accident & NA & NA \\
\hline
\end{tabular}




\section{B.11 WM LLW REGIONALIZED 4 ALTERNATIVE (CASE 12): HETEROGENEOUS SOLIDS}

Table 1. Summary of Route Information

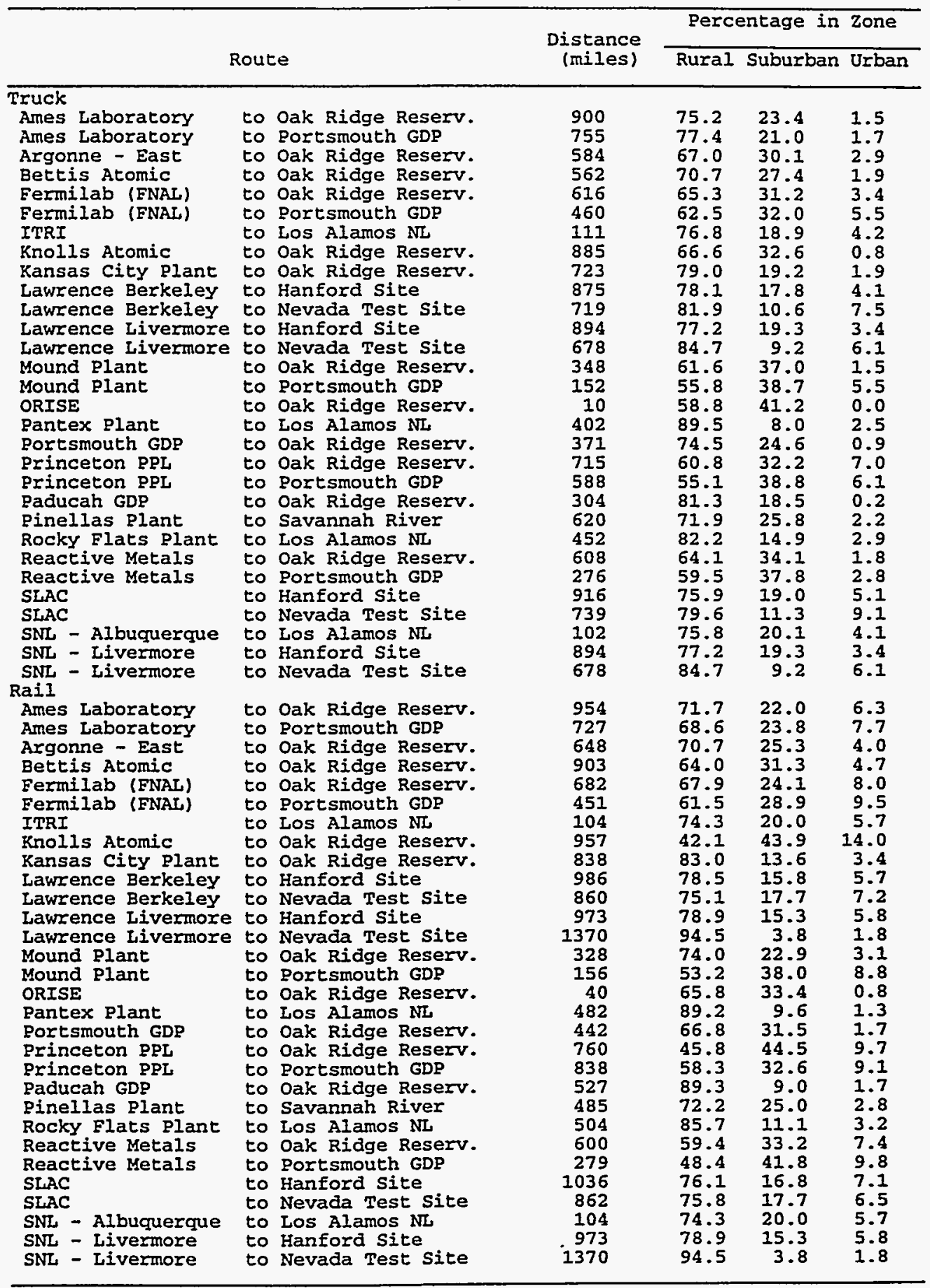


Table 2. Annual Number of Shipments and One-Way Shipment Distances

\begin{tabular}{|c|c|c|c|c|c|c|}
\hline \multirow{2}{*}{ Route } & & & \multicolumn{2}{|c|}{ \# of Shipments } & \multicolumn{2}{|c|}{ Total Mileage(mi) } \\
\hline & & & Truck & Rail & Truck & Rail \\
\hline $\begin{array}{l}\text { Ames Laboratory } \\
\text { Ames Laboratory } \\
\text { Argonne - East } \\
\text { Bettis Atomic } \\
\text { Fermilab (FNAL) } \\
\text { Fermilab (FNAL) } \\
\text { ITRI } \\
\text { Knolls Atomic } \\
\text { Kansas City Plant } \\
\text { Lawrence Berkeley } \\
\text { Lawrence Berkeley } \\
\text { Lawrence Livermore } \\
\text { Lawrence Livermore } \\
\text { Mound Plant } \\
\text { Mound Plant } \\
\text { ORISE } \\
\text { Pantex Plant } \\
\text { Portsmouth GDP } \\
\text { Princeton PPL } \\
\text { Princeton PPL } \\
\text { Paducah GDP } \\
\text { Pinellas Plant } \\
\text { Rocky Flats Plant } \\
\text { Reactive Metals } \\
\text { Reactive Metals } \\
\text { SLAC } \\
\text { SLAC } \\
\text { SNL - Albuquerque } \\
\text { SNL - Livermore } \\
\text { SNL - Livermore }\end{array}$ & $\begin{array}{l}\text { to } \\
\text { to } \\
\text { to } \\
\text { to } \\
\text { to } \\
\text { to } \\
\text { to } \\
\text { to } \\
\text { to } \\
\text { to } \\
\text { to } \\
\text { to } \\
\text { to } \\
\text { to } \\
\text { to } \\
\text { to } \\
\text { to } \\
\text { to } \\
\text { to } \\
\text { to } \\
\text { to } \\
\text { to } \\
\text { to } \\
\text { to } \\
\text { to } \\
\text { to } \\
\text { to } \\
\text { to } \\
\text { to } \\
\text { to }\end{array}$ & $\begin{array}{l}\text { Oak Ridge Reserv. } \\
\text { Port smouth GDP } \\
\text { Oak Ridge Reserv. } \\
\text { Oak Ridge Reserv. } \\
\text { Oak Ridge Reserv. } \\
\text { Portsmouth GDP } \\
\text { Los Alamos NL } \\
\text { Oak Ridge Reserv. } \\
\text { Oak Ridge Reserv. } \\
\text { Hanford Site } \\
\text { Nevada Test Site } \\
\text { Hanford Site } \\
\text { Nevada Test Site } \\
\text { Oak Ridge Reserv. } \\
\text { Portsmouth GDP } \\
\text { Oak Ridge Reserv. } \\
\text { Los Alamos NL } \\
\text { Oak Ridge Reserv. } \\
\text { Oak Ridge Reserv. } \\
\text { Portsmouth GDP } \\
\text { Oak Ridge Reserv. } \\
\text { Savannah River } \\
\text { Los Alamos NL } \\
\text { Oak Ridge Reserv. } \\
\text { Portsmouth GDP } \\
\text { Hanford Site } \\
\text { Nevada Test Site } \\
\text { Los Alamos NL } \\
\text { Hanford Site } \\
\text { Nevada Test Site }\end{array}$ & $\begin{array}{r}2 \\
1 \\
105 \\
57 \\
2 \\
1 \\
10 \\
28 \\
1 \\
2 \\
2 \\
17 \\
40 \\
496 \\
12 \\
5 \\
1418 \\
2967 \\
1 \\
1 \\
627 \\
2 \\
335 \\
745 \\
7 \\
1 \\
39 \\
17 \\
1 \\
5\end{array}$ & $\begin{array}{r}1 \\
1 \\
39 \\
21 \\
1 \\
1 \\
4 \\
11 \\
1 \\
1 \\
1 \\
7 \\
15 \\
186 \\
5 \\
2 \\
537 \\
1113 \\
1 \\
1 \\
235 \\
1 \\
123 \\
283 \\
3 \\
1 \\
15 \\
7 \\
1 \\
2\end{array}$ & 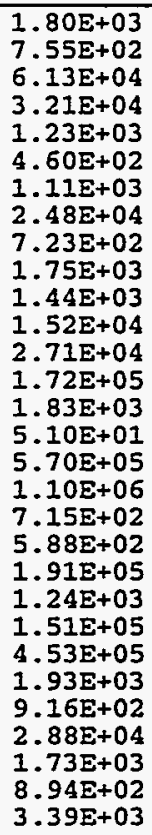 & 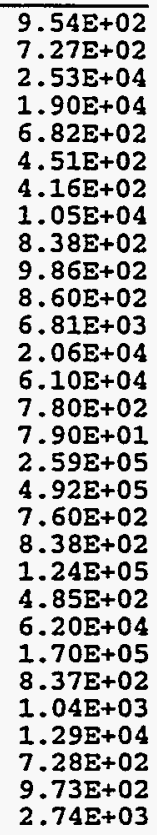 \\
\hline Totals & & & 6947 & 2620 & $2.85 E+06$ & $1.28 \mathrm{E}+06$ \\
\hline
\end{tabular}


Table 3. Incident-Free Dose per Shipment (person-rem/shipment)

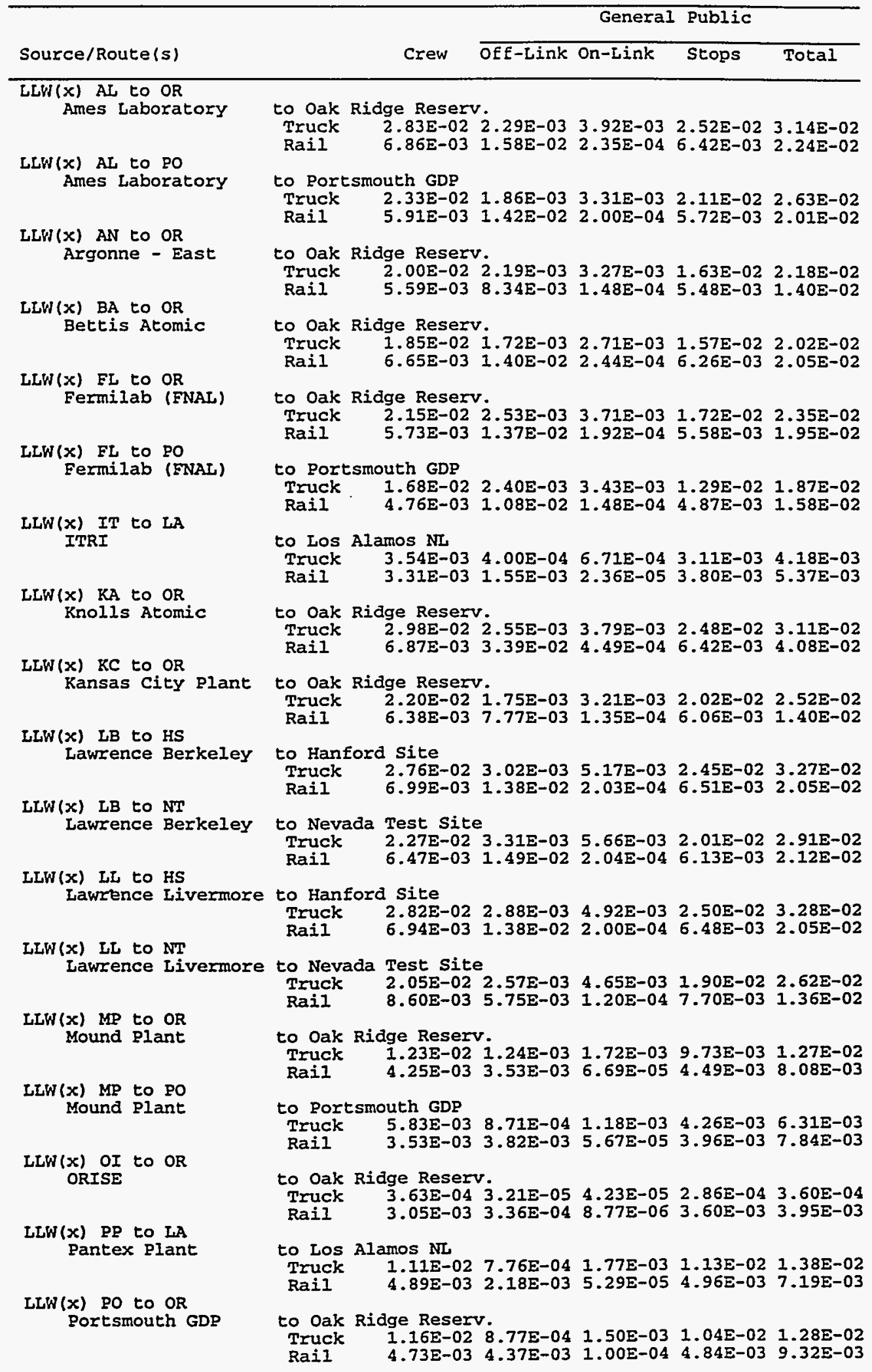




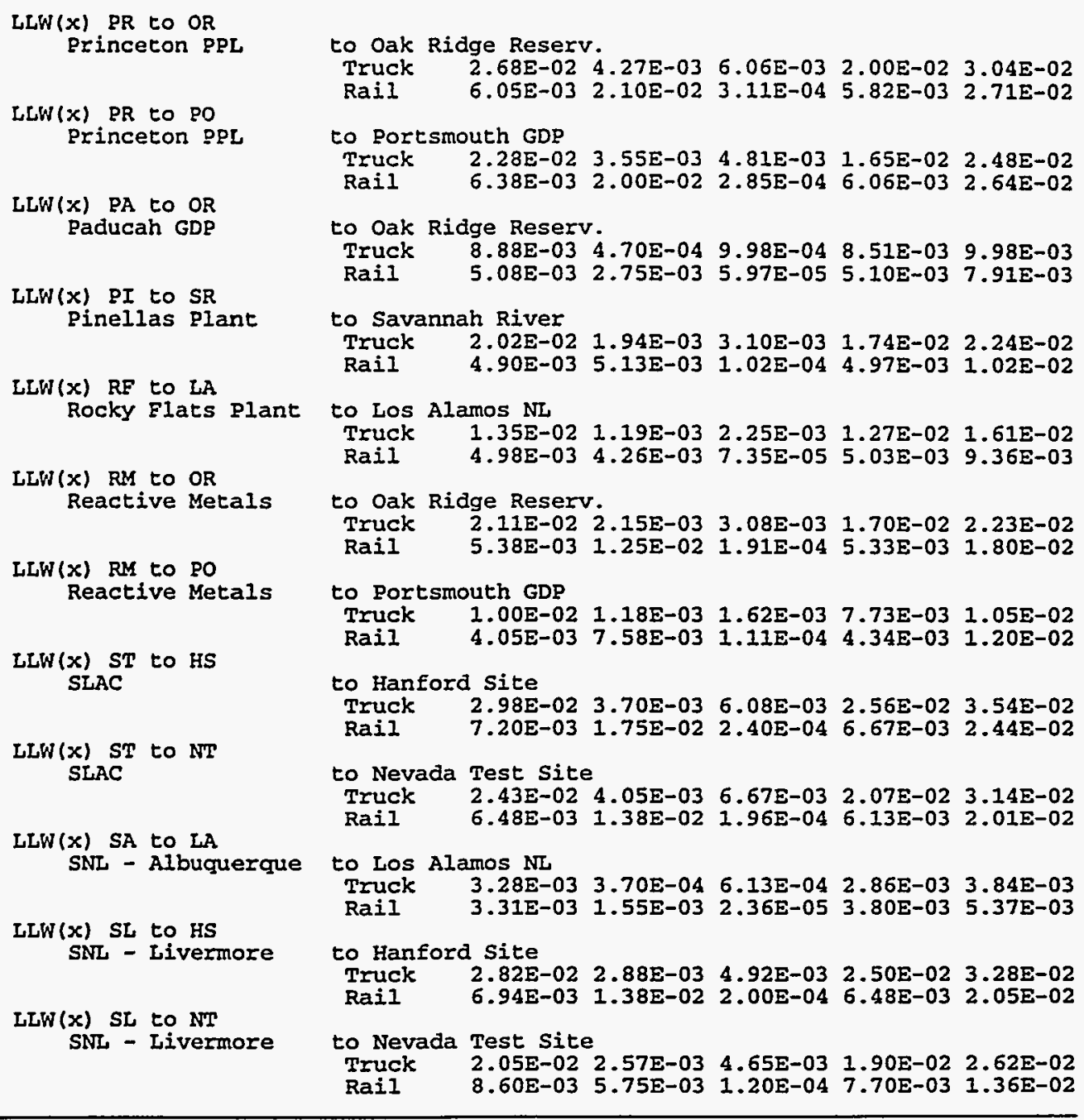


Table 4. Accident Dose Risk per Shipment (person-rem/shipment)

\begin{tabular}{|c|c|c|c|}
\hline Source/Route & & Truck & Rail \\
\hline 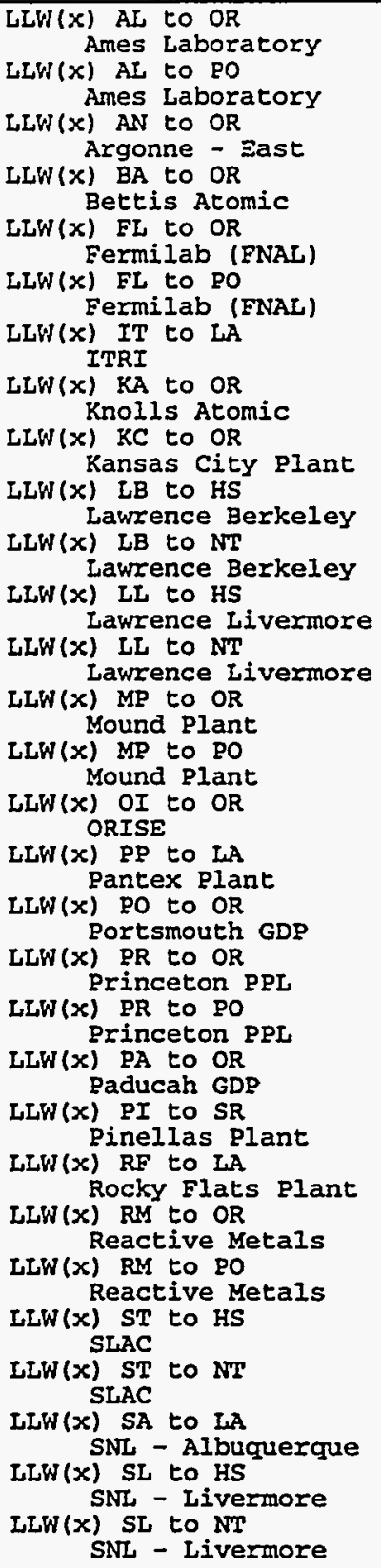 & $\begin{array}{l}\text { to Oak Ridge Reserv. } \\
\text { to Portsmouth GDP } \\
\text { to Oak Ridge Reserv. } \\
\text { to Oak Ridge Reserv. } \\
\text { to Oak Ridge Reserv. } \\
\text { to Portsmouth GDP } \\
\text { to Los Alamos NL } \\
\text { to Oak Ridge Reserv. } \\
\text { to Oak Ridge Reserv. } \\
\text { to Hanford Site } \\
\text { to Nevada Test Site } \\
\text { to Hanford Site } \\
\text { to Nevada Test Site } \\
\text { to Oak Ridge Reserv. } \\
\text { to Portsmouth GDP } \\
\text { to Oak Ridge Reserv. } \\
\text { to Los Alamos NL } \\
\text { to Oak Ridge Reserv. } \\
\text { to Oak Ridge Reserv. } \\
\text { to Portsmouth GDP } \\
\text { to Oak Ridge Reserv. } \\
\text { to Savannah River } \\
\text { to Los Alamos NL } \\
\text { to Oak Ridge Reserv. } \\
\text { to Portsmouth GDP } \\
\text { to Hanford Site } \\
\text { to Nevada Test Site } \\
\text { to Los Alamos NL } \\
\text { to Hanford Site } \\
\text { to Nevada Test Site }\end{array}$ & $\begin{array}{l}3.90 \mathrm{E}-06 \\
9.76 \mathrm{E}-07 \\
5.16 \mathrm{E}-04 \\
9.94 \mathrm{E}-06 \\
1.22 \mathrm{E}-05 \\
5.67 \mathrm{E}-05 \\
1.53 \mathrm{E}-03 \\
2.13 \mathrm{E}-06 \\
3.55 \mathrm{E}-05 \\
6.10 \mathrm{E}-02 \\
7.54 \mathrm{E}-03 \\
1.05 \mathrm{E}-05 \\
3.71 \mathrm{E}-06 \\
1.10 \mathrm{E}-06 \\
6.85 \mathrm{E}-08 \\
4.49 \mathrm{E}-08 \\
5.66 \mathrm{E}-07 \\
2.08 \mathrm{E}-08 \\
5.74 \mathrm{E}-06 \\
4.35 \mathrm{E}-11 \\
3.12 \mathrm{E}-07 \\
1.30 \mathrm{E}-06 \\
4.96 \mathrm{E}-06 \\
5.54 \mathrm{E}-09 \\
1.34 \mathrm{E}-08 \\
9.15 \mathrm{E}-10 \\
2.14 \mathrm{E}-07 \\
5.39 \mathrm{E}-06 \\
1.07 \mathrm{E}-04 \\
3.48 \mathrm{E}-05\end{array}$ & $\begin{array}{l}6.40 E-06 \\
4.06 E-07 \\
2.26 E-04 \\
1.12 E-05 \\
5.26 E-06 \\
1.47 E-05 \\
7.01 E-05 \\
3.16 E-06 \\
7.40 E-06 \\
6.80 E-02 \\
1.03 E-02 \\
1.34 E-05 \\
2.98 E-06 \\
3.11 E-07 \\
4.29 E-08 \\
3.15 E-08 \\
9.26 E-08 \\
1.05 E-08 \\
2.32 E-06 \\
2.17 E-11 \\
1.09 E-07 \\
4.65 E-07 \\
5.78 E-07 \\
2.75 E-09 \\
9.03 E-09 \\
5.37 E-10 \\
3.39 E-07 \\
2.50 E-07 \\
5.69 E-05 \\
2.74 E-05\end{array}$ \\
\hline
\end{tabular}


Table 5. Nonradiological Risk Eactors per Shipment (Étalicies/shipment)

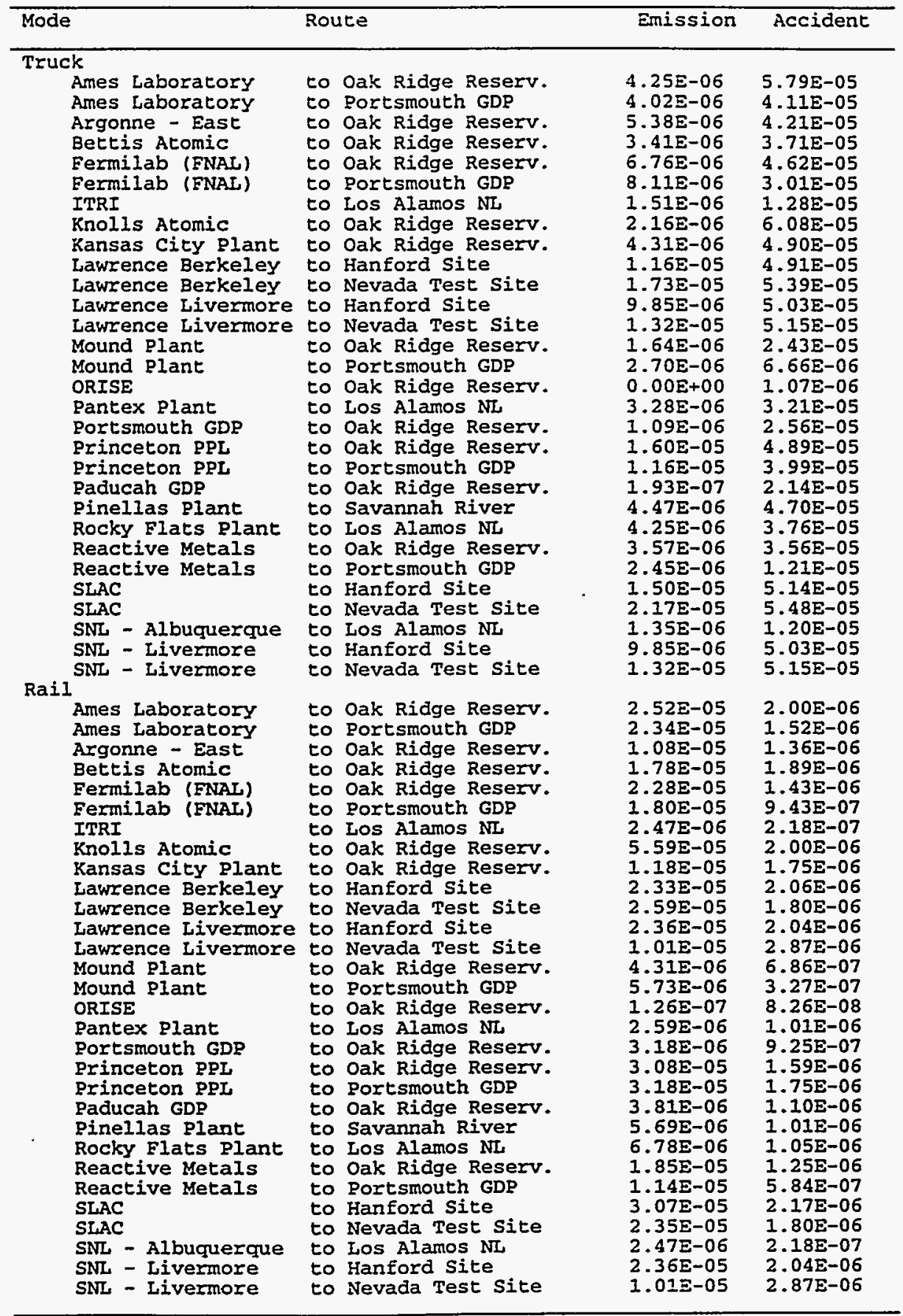


Table 6. Annual Incident-Free Dose for the Shipping Campaign (person-rem/yr)

\begin{tabular}{|c|c|c|c|c|c|c|}
\hline \multirow[b]{2}{*}{ Route } & & & \multicolumn{2}{|c|}{ Truck } & \multicolumn{2}{|l|}{ Rail } \\
\hline & & & Crew & Public & Crew & Public \\
\hline $\begin{array}{l}\text { Ames Laboratory } \\
\text { Ames Laboratory } \\
\text { Argonne - East } \\
\text { Bettis Atomic } \\
\text { Fermilab (FNAL) } \\
\text { Fermilab (FNAL) } \\
\text { ITRI } \\
\text { Knolls Atomic } \\
\text { Kansas City Plant } \\
\text { Lawrence Berkeley } \\
\text { Lawrence Berkeley } \\
\text { Lawrence Livermore } \\
\text { Lawrence Livermore } \\
\text { Mound Plant } \\
\text { Mound Plant } \\
\text { ORISE } \\
\text { Pantex Plant } \\
\text { Portsmouth GDP } \\
\text { Princeton PPL } \\
\text { Princeton pPL } \\
\text { Paducah GDP } \\
\text { PInellas Plant } \\
\text { Rocky Flats Plant } \\
\text { Reactive Metals } \\
\text { Reactive Metals } \\
\text { SLAC } \\
\text { SLAC - Albuquerque } \\
\text { SNL - Alivuere } \\
\text { SNL - Livermore } \\
\text { SNL - Livermore }\end{array}$ & $\begin{array}{l}\text { to } \\
\text { to } \\
\text { to } \\
\text { to } \\
\text { to } \\
\text { to } \\
\text { to } \\
\text { to } \\
\text { to } \\
\text { to } \\
\text { to } \\
\text { to } \\
\text { to } \\
\text { to } \\
\text { to } \\
\text { to } \\
\text { to } \\
\text { to } \\
\text { to } \\
\text { to } \\
\text { to } \\
\text { to } \\
\text { to } \\
\text { to } \\
\text { to } \\
\text { to } \\
\text { to } \\
\text { to } \\
\text { to } \\
\text { to }\end{array}$ & $\begin{array}{l}\text { Oak Ridge Reserv. } \\
\text { Portsmouth GDP } \\
\text { Oak Ridge Reserv. } \\
\text { Oak Ridge Reserv. } \\
\text { Oak Ridge Reserv. } \\
\text { Portsmouth GDP } \\
\text { Los Alamos NL } \\
\text { Oak Ridge Reserv. } \\
\text { Oak Ridge Reserv. } \\
\text { Hanford Site } \\
\text { Nevada Test Site } \\
\text { Hanford Site } \\
\text { Nevada Test Site } \\
\text { Oak Ridge Reserv. } \\
\text { Portsmouth GDP } \\
\text { Oak Ridge Reserv. } \\
\text { Los Alamos NL } \\
\text { Oak Ridge Reserv. } \\
\text { Oak Ridge Reserv. } \\
\text { Portsmouth GDP } \\
\text { Oak Ridge Reserv. } \\
\text { Savannah River } \\
\text { Los Alamos NL } \\
\text { Oak Ridge Reserv. } \\
\text { Portsmouth GDP } \\
\text { Hanford Site } \\
\text { Nevada Test Site } \\
\text { Los Alamos NL } \\
\text { Hanford Site } \\
\text { Nevada Test Site }\end{array}$ & $\begin{array}{l}5.66 \mathrm{E}-02 \\
2.33 \mathrm{E}-02 \\
2.10 \mathrm{E}+00 \\
1.05 \mathrm{E}+00 \\
4.30 \mathrm{E}-02 \\
1.68 \mathrm{E}-02 \\
3.54 \mathrm{E}-02 \\
8.33 \mathrm{E}-01 \\
2.20 \mathrm{E}-02 \\
5.51 \mathrm{E}-02 \\
4.54 \mathrm{E}-02 \\
4.79 \mathrm{E}-01 \\
8.22 \mathrm{E}-01 \\
6.09 \mathrm{E}+00 \\
7.00 \mathrm{E}-02 \\
1.81 \mathrm{E}-03 \\
1.58 \mathrm{E}+01 \\
3.46 \mathrm{E}+01 \\
2.58 \mathrm{E}-02 \\
2.28 \mathrm{E}-02 \\
5.57 \mathrm{E}+00 \\
4.04 \mathrm{E}-02 \\
4.53 \mathrm{E}+00 \\
1.57 \mathrm{E}+01 \\
7.02 \mathrm{E}-02 \\
2.98 \mathrm{E}-02 \\
9.46 \mathrm{E}-01 \\
5.58 \mathrm{E}-02 \\
2.82 \mathrm{E}-02 \\
1.03 \mathrm{E}-01\end{array}$ & $\begin{array}{l}6.28 \mathrm{E}-02 \\
2.63 \mathrm{E}-02 \\
2.29 \mathrm{E}+00 \\
1.15 \mathrm{E}+00 \\
4.70 \mathrm{E}-02 \\
1.87 \mathrm{E}-02 \\
4.18 \mathrm{E}-02 \\
8.72 \mathrm{E}-01 \\
2.52 \mathrm{E}-02 \\
6.54 \mathrm{E}-02 \\
5.82 \mathrm{E}-02 \\
5.58 \mathrm{E}-01 \\
1.05 \mathrm{E}+00 \\
6.30 \mathrm{E}+00 \\
7.58 \mathrm{E}-02 \\
1.80 \mathrm{E}-03 \\
1.96 \mathrm{E}+01 \\
3.78 \mathrm{E}+01 \\
3.04 \mathrm{E}-02 \\
2.48 \mathrm{E}-02 \\
6.26 \mathrm{E}+00 \\
4.48 \mathrm{E}-02 \\
5.39 \mathrm{E}+00 \\
1.66 \mathrm{E}+01 \\
7.37 \mathrm{E}-02 \\
3.54 \mathrm{E}-02 \\
1.23 \mathrm{E}+00 \\
6.53 \mathrm{E}-02 \\
3.28 \mathrm{E}-02 \\
1.31 \mathrm{E}-01\end{array}$ & $\begin{array}{l}6.86 \mathrm{E}-03 \\
5.91 \mathrm{E}-03 \\
2.18 \mathrm{E}-01 \\
1.40 \mathrm{E}-01 \\
5.73 \mathrm{E}-03 \\
4.76 \mathrm{E}-03 \\
1.33 \mathrm{E}-02 \\
7.56 \mathrm{E}-02 \\
6.38 \mathrm{E}-03 \\
6.99 \mathrm{E}-03 \\
6.47 \mathrm{E}-03 \\
4.86 \mathrm{E}-02 \\
1.29 \mathrm{E}-01 \\
7.90 \mathrm{E}-01 \\
1.77 \mathrm{E}-02 \\
6.09 \mathrm{E}-03 \\
2.63 \mathrm{E}+00 \\
5.26 \mathrm{E}+00 \\
6.05 \mathrm{E}-03 \\
6.38 \mathrm{E}-03 \\
1.19 \mathrm{E}+00 \\
4.90 \mathrm{E}-03 \\
6.13 \mathrm{E}-01 \\
1.52 \mathrm{E}+00 \\
1.21 \mathrm{E}-02 \\
7.20 \mathrm{E}-03 \\
9.72 \mathrm{E}-02 \\
2.32 \mathrm{E}-02 \\
6.94 \mathrm{E}-03 \\
1.72 \mathrm{E}-02\end{array}$ & $\begin{array}{l}2.24 \mathrm{E}-02 \\
2.01 \mathrm{E}-02 \\
5.45 \mathrm{E}-01 \\
4.30 \mathrm{E}-01 \\
1.95 \mathrm{E}-02 \\
1.58 \mathrm{E}-02 \\
2.15 \mathrm{E}-02 \\
4.48 \mathrm{E}-01 \\
1.40 \mathrm{E}-02 \\
2.05 \mathrm{E}-02 \\
2.12 \mathrm{E}-02 \\
1.43 \mathrm{E}-01 \\
2.03 \mathrm{E}-01 \\
1.50 \mathrm{E}+00 \\
3.92 \mathrm{E}-02 \\
7.89 \mathrm{E}-03 \\
3.86 \mathrm{E}+00 \\
1.04 \mathrm{E}+01 \\
2.71 \mathrm{E}-02 \\
2.64 \mathrm{E}-02 \\
1.86 \mathrm{E}+00 \\
1.02 \mathrm{E}-02 \\
1.15 \mathrm{E}+00 \\
5.10 \mathrm{E}+00 \\
3.61 \mathrm{E}-02 \\
2.44 \mathrm{E}-02 \\
3.02 \mathrm{E}-01 \\
3.76 \mathrm{E}-02 \\
2.05 \mathrm{E}-02 \\
2.71 \mathrm{E}-02\end{array}$ \\
\hline Totals & & & $8.92 \mathrm{E}+01$ & $9.99 E+01$ & $1.29 \mathrm{E}+01$ & $2.63 \mathrm{E}+01$ \\
\hline
\end{tabular}

Table 7. Annual Accident Dose Risk for the Shipping Campaign (person-rem/yr)

\begin{tabular}{|c|c|c|c|}
\hline Route & & Truck & Rail \\
\hline $\begin{array}{l}\text { Ames Laboratory } \\
\text { Ames Laboratory } \\
\text { Argonne - East } \\
\text { Bettis Atomic } \\
\text { Fermilab (FNAI) } \\
\text { Fermilab (FNAI) } \\
\text { ITRI } \\
\text { Knolls Atomic } \\
\text { Kansas City Plant } \\
\text { Lawrence Berkeley } \\
\text { Lawrence Berkeley } \\
\text { Lawrence Livermore } \\
\text { Lawrence Livermore } \\
\text { Mound Plant } \\
\text { Mound Plant } \\
\text { ORISE } \\
\text { Pantex Plant } \\
\text { Portsmouth GDP } \\
\text { Princeton PPL } \\
\text { Princeton PPL } \\
\text { Paducah GDP } \\
\text { Pinellas Plant } \\
\text { Rocky Flats Plant } \\
\text { Reactive Metals } \\
\text { Reactive Metals } \\
\text { SLAC } \\
\text { SLAC - Albuquerque } \\
\text { SNL - A L Livermore } \\
\text { SNL - L L Livermore } \\
\text { SNL - }\end{array}$ & $\begin{array}{l}\text { to Oak Ridge Reserv. } \\
\text { to Portsmouth GDP } \\
\text { to Oak Ridge Reserv. } \\
\text { to Oak Ridge Reserv. } \\
\text { to Oak Ridge Reserv. } \\
\text { to Portsmouth GDP } \\
\text { to Los Alamos NL } \\
\text { to Oak Ridge Reserv. } \\
\text { to Oak Ridge Reserv. } \\
\text { to Hanford Site } \\
\text { to Nevada Test Site } \\
\text { to Hanford Site } \\
\text { to Nevada Test Site } \\
\text { to Oak Ridge Reserv. } \\
\text { to Portsmouth GDP } \\
\text { to Oak Ridge Reserv. } \\
\text { to Los Alamos NL } \\
\text { to Oak Ridge Reserv. } \\
\text { to Oak Ridge Reserv. } \\
\text { to Portsmouth GDP } \\
\text { to Oak Ridge Reserv. } \\
\text { to Savannah River } \\
\text { to Los Alamos NL } \\
\text { to Oak Ridge Reserv. } \\
\text { to Portsmouth GDP } \\
\text { to Hanford Site } \\
\text { to Nevada Test Site } \\
\text { to Los Alamos NL } \\
\text { to Hanford Site } \\
\text { to Nevada Test Site }\end{array}$ & $\begin{array}{l}1.78 \mathrm{E}-05 \\
9.76 \mathrm{E}-07 \\
5.41 \mathrm{E}-02 \\
5.67 \mathrm{E}-04 \\
2.43 \mathrm{E}-05 \\
5.67 \mathrm{E}-05 \\
1.53 \mathrm{E}-02 \\
5.98 \mathrm{E}-05 \\
3.55 \mathrm{E}-05 \\
1.22 \mathrm{E}-01 \\
1.51 \mathrm{E}-02 \\
1.79 \mathrm{E}-04 \\
1.48 \mathrm{E}-04 \\
5.45 \mathrm{E}-04 \\
8.22 \mathrm{E}-07 \\
2.24 \mathrm{E}-07 \\
8.02 \mathrm{E}-04 \\
6.19 \mathrm{E}-05 \\
5.74 \mathrm{E}-06 \\
4.35 \mathrm{E}-11 \\
1.96 \mathrm{E}-04 \\
2.59 \mathrm{E}-06 \\
1.66 \mathrm{E}-03 \\
4.13 \mathrm{E}-06 \\
9.38 \mathrm{E}-08 \\
9.15 \mathrm{E}-10 \\
8.33 \mathrm{E}-06 \\
9.16 \mathrm{E}-05 \\
1.07 \mathrm{E}-04 \\
1.74 \mathrm{E}-04\end{array}$ & $\begin{array}{l}6.40 \mathrm{E}-06 \\
4.06 \mathrm{E}-07 \\
8.83 \mathrm{E}-03 \\
2.35 \mathrm{E}-04 \\
5.26 \mathrm{E}-06 \\
1.47 \mathrm{E}-05 \\
2.80 \mathrm{E}-04 \\
3.47 \mathrm{E}-05 \\
7.40 \mathrm{E}-06 \\
6.80 \mathrm{E}-02 \\
1.03 \mathrm{E}-02 \\
9.36 \mathrm{E}-05 \\
4.47 \mathrm{E}-05 \\
5.79 \mathrm{E}-05 \\
2.15 \mathrm{E}-07 \\
6.30 \mathrm{E}-08 \\
4.97 \mathrm{E}-05 \\
1.17 \mathrm{E}-05 \\
2.32 \mathrm{E}-06 \\
2.17 \mathrm{E}-11 \\
2.57 \mathrm{E}-05 \\
4.65 \mathrm{E}-07 \\
7.11 \mathrm{E}-05 \\
7.78 \mathrm{E}-07 \\
2.71 \mathrm{E}-08 \\
5.37 \mathrm{E}-10 \\
5.08 \mathrm{E}-06 \\
1.75 \mathrm{E}-06 \\
5.69 \mathrm{E}-05 \\
5.48 \mathrm{E}-05\end{array}$ \\
\hline Tota & & 2.11 & 8.8 \\
\hline
\end{tabular}


Table 8. Expected Annual Fatalities

for the Shipping Campaign

\begin{tabular}{llc}
\hline Exposure Group & Truck & Rail \\
\hline Radiological & $3.6 \mathrm{E}-02$ & $5.2 \mathrm{E}-03$ \\
$\quad$ Normal Crew & $5.0 \mathrm{E}-02$ & $1.3 \mathrm{E}-02$ \\
$\quad$ Normal Public & $1.1 \mathrm{E}-04$ & $4.4 \mathrm{E}-05$ \\
Accident Public & $1.6 \mathrm{E}-02$ & $1.5 \mathrm{E}-02$ \\
$\begin{array}{c}\text { Nonradiological } \\
\text { Emission }\end{array}$ & $2.0 \mathrm{E}-01$ & $2.7 \mathrm{E}-03$ \\
Accident & & \\
\hline
\end{tabular}

Table 9. Expected Annual Cancer Incidence for the Shipping Campaign

\begin{tabular}{|c|c|c|}
\hline Exposure Group & Truck & Rail \\
\hline $\begin{array}{l}\text { Radiological } \\
\text { Normal Crew } \\
\text { Normal Public } \\
\text { Accident Public } \\
\text { Nonradiological } \\
\text { Emission } \\
\text { Accident }\end{array}$ & $\begin{array}{l}1.2 \mathrm{E}-01 \\
1.7 \mathrm{E}-01 \\
3.6 \mathrm{E}-04 \\
1.6 \mathrm{E}-02 \\
\mathrm{NA}\end{array}$ & $\begin{array}{l}1.8 \mathrm{E}-02 \\
4.5 \mathrm{E}-02 \\
1.5 \mathrm{E}-04 \\
1.5 \mathrm{E}-02 \\
\mathrm{NA}\end{array}$ \\
\hline
\end{tabular}

Table 10. Expected Annual Genetic Effects for the Shipping Campaign

\begin{tabular}{lll}
\hline Exposure Group & Truck & Rail \\
\hline Radiological & $5.4 E-03$ & $7.7 \mathrm{E}-04$ \\
Normal Crew & $1.0 \mathrm{E}-02$ & $2.6 \mathrm{E}-03$ \\
Normal Public & $6.8 \mathrm{E}-06$ & $2.1 \mathrm{E}-06$ \\
Accident Public & & \\
Nonradiological & NA & NA \\
Emission & NA & NA \\
Accident & & \\
\hline
\end{tabular}


TABLE 4.2 (Cont.)

\begin{tabular}{|c|c|c|c|c|c|c|c|c|}
\hline \multirow[b]{2}{*}{ State $^{\mathrm{b}}$} & \multicolumn{2}{|c|}{1986} & \multicolumn{2}{|c|}{1987} & \multicolumn{2}{|c|}{1988} & \multicolumn{2}{|c|}{$1985-88$} \\
\hline & Total & $\begin{array}{l}\text { Main } \\
\text { Only }\end{array}$ & Total & $\begin{array}{l}\text { Main } \\
\text { Only }\end{array}$ & Total & $\begin{array}{l}\text { Main } \\
\text { Only }\end{array}$ & Total & $\begin{array}{l}\text { Main } \\
\text { Only }\end{array}$ \\
\hline SC & 4.64 & 2.16 & 4.74 & 3.62 & 5.03 & 3.71 & 5.11 & 3.31 \\
\hline SD & 8.16 & 6.27 & 14.98 & 14.49 & 7.27 & 6.36 & 10.19 & 9.09 \\
\hline TN & 4.88 & 1.69 & 4.67 & 1.89 & 7.56 & 2.02 & 5.59 & 1.88 \\
\hline$T X$ & 7.03 & 3.36 & 5.29 & 2.50 & 5.92 & 2.45 & 7.12 & 3.16 \\
\hline UT & 3.69 & 1.34 & 1.85 & 0.93 & 11.19 & 4.04 & 5.78 & 2.31 \\
\hline VT & 5.98 & 2.99 & 7.86 & 5.24 & 24.32 & 18.24 & 15.22 & 11.59 \\
\hline VA & 4.27 & 1.42 & 4.39 & 2.31 & 4.59 & 2.15 & 4.35 & 1.91 \\
\hline WA & 3.65 & 1.38 & 2.69 & 1.50 & 3.57 & 1.12 & 3.49 & 1.44 \\
\hline wV & 11.49 & 9.23 & 9.97 & 7.73 & 6.08 & 4.67 & 9.61 & 7.42 \\
\hline WI & 18.79 & 7.52 & 16.75 & 7.75 & 12.57 & 7.21 & 16.53 & 7.66 \\
\hline WY & 2.55 & 1.37 & 2.61 & 1.47 & 2.88 & 1.40 & 3.10 & 1.97 \\
\hline USA & 5.51 & 2.63 & 4.70 & 2.36 & 5.14 & 2.43 & 5.57 & 2.66 \\
\hline$\sigma^{d}$ & - & - & - & - & - & - & 21.48 & 11.12 \\
\hline
\end{tabular}

a Nationwide average fatality rate, using count of fatalities consistent with combination-truck fatality counts, is $6.50 \times 10^{-10}$ fatalities per railcar-kilometer. Nationwide average injury rate, using count of injuries consistent with combination-truck injury counts, is $7.83 \times 10^{-8}$ injuries per railcar-kilometer.

b Includes the 48 contiguous states, the District of Columbia, and the totals for the United States.

c Rates are one or more standard deviations greater than the national average rate for that category (i.e., all accidents on carrier-owned track or accidents on main lines only).

d $\sigma=$ standard deviation.

Source: Saricks and Kvitek (1994).

The NUREG-0170 scheme for accident classification is shown in Figures 4.1 and 4.2 for truck and rail transportation, respectively. Severity is described as a function of the magnitudes of the mechanical forces (impact) and thermal forces (fire) to which a package may be subjected during an accident. Because all accidents can be described in these terms, severity is independent of the specific accident sequence. In other words, any sequence of events that results in an accident in which a package is subjected to forces within a certain range of values is assigned to the accident severity category associated with that range. The scheme for accident severity is designed to take into account all credible transportation-related accidents, including accidents with low probability but high consequences and those with high probability but low consequences.

Each severity category represents a set of accident scenarios defined by a combination of mechanical and thermal forces. A conditional probability of occurrence - that is, the probability 


\section{B.12 WM LLW REGIONALIZED 4 ALTERNATIVE (CASE 12): ACTIVATED METALS}

Table 1. Sumnary of Rouce Information

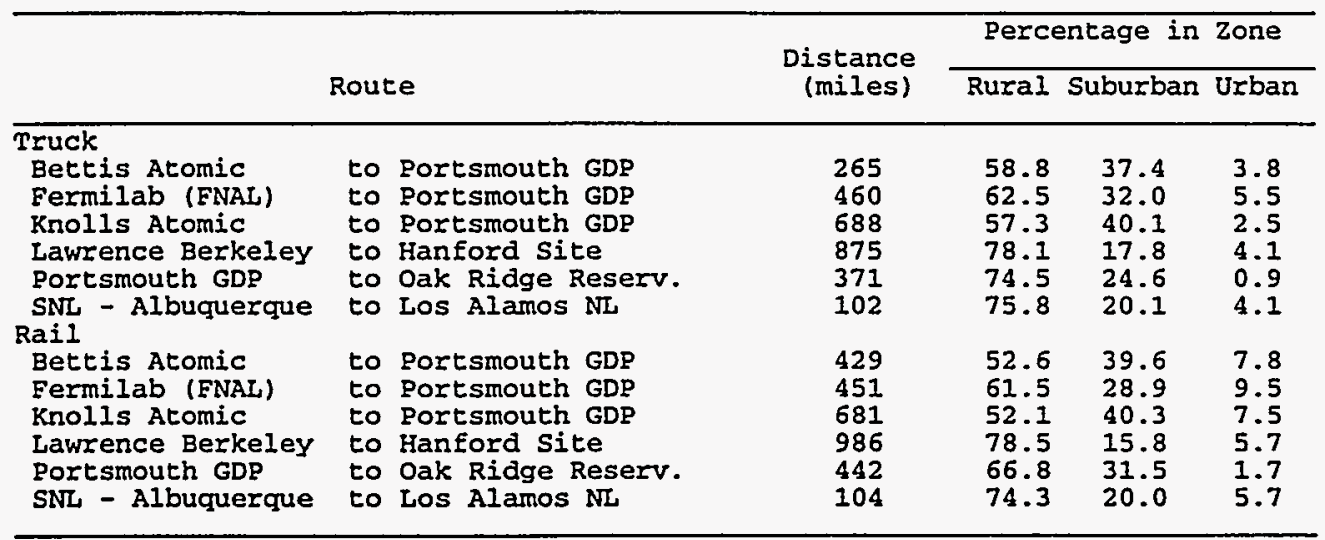

Table 2. Annual Number of Shipments and One-Way Shipment Distances

\begin{tabular}{|c|c|c|c|c|c|}
\hline \multirow[b]{2}{*}{ Route } & & \multicolumn{2}{|c|}{ \# of Shipments } & \multicolumn{2}{|c|}{ Total Mileage(mi) } \\
\hline & & Truck & $\operatorname{Rail}$ & Truck & Rail \\
\hline $\begin{array}{l}\text { Bettis Atomic } \\
\text { Fermilab (FNAL) } \\
\text { Knolls Atomic } \\
\text { Lawrence Berkeley } \\
\text { Portsmouth GDP } \\
\text { SNL - Albuquerque }\end{array}$ & $\begin{array}{l}\text { to Portsmouth GDP } \\
\text { to Portsmouth GDP } \\
\text { to Portsmouth GDP } \\
\text { to Hanford Site } \\
\text { to Oak Ridge Reserv. } \\
\text { to Los Alamos NL }\end{array}$ & $\begin{array}{r}309 \\
39 \\
639 \\
14 \\
785 \\
6\end{array}$ & $\begin{array}{r}119 \\
16 \\
245 \\
6 \\
337 \\
3\end{array}$ & $\begin{array}{l}8.19 \mathrm{E}+04 \\
1.79 \mathrm{E}+04 \\
4.40 \mathrm{E}+05 \\
1.22 \mathrm{E}+04 \\
2.91 \mathrm{E}+05 \\
6.12 \mathrm{E}+02\end{array}$ & $\begin{array}{l}5.11 E+04 \\
7.21 E+03 \\
1.67 E+05 \\
5.91 E+03 \\
1.49 E+05 \\
3.12 E+02\end{array}$ \\
\hline Totals & & 1792 & 726 & $8.43 E+05$ & $3.80 \mathrm{E}+05$ \\
\hline
\end{tabular}

Table 3. Incident-Free Dose per Shipment (person-rem/shipment)

\begin{tabular}{|c|c|c|c|c|c|}
\hline \multirow[b]{2}{*}{ Source/Route (s) } & \multirow[b]{2}{*}{ Crew } & \multicolumn{4}{|c|}{ General Public } \\
\hline & & off-Link & On-Link & Stops & Total \\
\hline $\begin{array}{l}\text { LLW(I) BA to Po } \\
\text { Bettis Atomic }\end{array}$ & $\begin{array}{lc}\text { to Portsmouth GDP } \\
\text { Truck } & 9.78 \mathrm{E}-03 \\
\text { Rail } & 4.67 \mathrm{E}-03\end{array}$ & $\begin{array}{l}1.26 \mathrm{E}-03 \\
9.85 \mathrm{E}-03\end{array}$ & $\begin{array}{l}1.73 E-03 \\
1.53 E-04\end{array}$ & $\begin{array}{l}7.42 \mathrm{E}-03 \\
4.80 \mathrm{E}-03\end{array}$ & $\begin{array}{l}1.04 \mathrm{E}-02 \\
1.48 \mathrm{E}-02\end{array}$ \\
\hline $\begin{array}{l}\text { LLW (I) FL to PO } \\
\text { Fermilab (FNAL) }\end{array}$ & $\begin{array}{cc}\text { to Portsmouth GDP } \\
\text { Truck } & 1.68 \mathrm{E}-02 \\
\text { Rail } & 4.76 \mathrm{E}-03\end{array}$ & $\begin{array}{l}2.40 \mathrm{E}-03 \\
1.08 \mathrm{E}-02\end{array}$ & $\begin{array}{l}3.43 \mathrm{E}-03 \\
1.48 \mathrm{E}-04\end{array}$ & $\begin{array}{l}1.29 \mathrm{E}-02 \\
4.87 \mathrm{E}-03\end{array}$ & $\begin{array}{l}1.87 \mathrm{E}-02 \\
1.58 \mathrm{E}-02\end{array}$ \\
\hline $\begin{array}{l}\text { LLW(I) KA to PO } \\
\text { Knolls Atomic }\end{array}$ & $\begin{array}{lr}\text { to Portsmouth GDP } \\
\text { Truck } & 2.54 \mathrm{E}-02 \\
\text { Rail } & 5.72 \mathrm{E}-03\end{array}$ & $\begin{array}{l}2.99 E-03 \\
1.54 E-02\end{array}$ & $\begin{array}{l}4.01 E-03 \\
2.43 E-04\end{array}$ & $\begin{array}{l}\frac{1}{5} .93 \mathrm{E}-02 \\
5.58 \mathrm{E}-03\end{array}$ & $\begin{array}{l}2.63 \mathrm{E}-02 \\
2.13 \mathrm{E}-02\end{array}$ \\
\hline $\begin{array}{l}\text { LLW(I) LB to HS } \\
\text { Lawrence Berkeley }\end{array}$ & $\begin{array}{ll}\text { to Hanford Site } \\
\text { Truck } & 2.76 \mathrm{E}-02 \\
\text { Rail } & 6.99 \mathrm{E}-03\end{array}$ & $\begin{array}{l}3.02 E-03 \\
1.38 E-02\end{array}$ & $\begin{array}{l}5.17 E-03 \\
2.03 E-04\end{array}$ & $\begin{array}{l}2.45 \mathrm{E}-02 \\
6.51 \mathrm{E}-03\end{array}$ & $\begin{array}{l}3.27 \mathrm{E}-02 \\
2.05 \mathrm{E}-02\end{array}$ \\
\hline $\begin{array}{l}\text { LLW (I) Po to OR } \\
\text { Portsmouth GDP }\end{array}$ & $\begin{array}{cc}\text { to Oak Ridge Reserv } \\
\text { Truck } & 1.16 \mathrm{E}-02 \\
\text { Rail } & 4.73 \mathrm{E}-03\end{array}$ & $\begin{array}{l}8.77 E-04 \\
4.37 E-03\end{array}$ & $\begin{array}{l}1.50 E-03 \\
1.00 E-04\end{array}$ & $\begin{array}{l}1.04 \mathrm{E}-02 \\
4.84 \mathrm{E}-03\end{array}$ & $\begin{array}{l}1.28 \mathrm{E}-02 \\
9.32 \mathrm{E}-03\end{array}$ \\
\hline $\begin{array}{l}\text { LLW(I) SA to LA } \\
\text { SNL - Albuquerque }\end{array}$ & $\begin{array}{cr}\text { to Los } & \text { Alamos } \mathrm{NL} \\
\text { Truck } & 3.28 \mathrm{E}-03 \\
\text { Rail } & 3.31 \mathrm{E}-03\end{array}$ & $\begin{array}{l}3.70 E-04 \\
1.55 E-03\end{array}$ & $\begin{array}{l}6.13 E-04 \\
2.36 \mathrm{E}-05\end{array}$ & $\begin{array}{l}2.86 \mathrm{E}-03 \\
3.80 \mathrm{E}-03\end{array}$ & $\begin{array}{l}3.84 E-03 \\
5.37 E-03\end{array}$ \\
\hline
\end{tabular}


Table 4. Accident Dose Risk per Shipment (person-rem/shipment)

\begin{tabular}{|c|c|c|c|}
\hline Source/Route & & Truck & Rail \\
\hline $\begin{array}{l}\text { LLW(I) BA to PO } \\
\text { Bettis ALomic } \\
\text { LLW(I) FL to PO } \\
\text { Fermilab (FNAL) } \\
\text { LLW(I) KA to PO } \\
\text { Knolls Atomic } \\
\text { LLW(I) LB to HS } \\
\text { LaWrence Berkeley } \\
\text { LLW(I) PO to OR } \\
\text { Portsmouth GDP } \\
\text { LLW(I) SA to LA } \\
\text { SNL - Albuquerque }\end{array}$ & $\begin{array}{l}\text { to Portsmouth GDP } \\
\text { to Portsmouth GDP } \\
\text { to Portsmouth GDP } \\
\text { to Hanford Site } \\
\text { to Oak Ridge Reserv. } \\
\text { to Los Alamos NL }\end{array}$ & $\begin{array}{l}3.70 \mathrm{E}-07 \\
5.29 \mathrm{E}-11 \\
1.66 \mathrm{E}-06 \\
1.81 \mathrm{E}-10 \\
8.34 \mathrm{E}-07 \\
1.72 \mathrm{E}-12\end{array}$ & $\begin{array}{l}4.16 \mathrm{E}-07 \\
3.35 \mathrm{E}-11 \\
1.02 \mathrm{E}-06 \\
2.37 \mathrm{E}-10 \\
3.69 \mathrm{E}-07 \\
6.51 \mathrm{E}-14\end{array}$ \\
\hline
\end{tabular}

Table 5. Nonradiological Risk Factors per Shipment (Eatalities/shipment)

\begin{tabular}{|c|c|c|c|}
\hline Mode & Route & Enission & Accident \\
\hline $\begin{array}{l}\text { Truck } \\
\text { Bettis Atomic } \\
\text { Fermilab (FNAL) } \\
\text { Knolis Atomic } \\
\text { Lawrence Berkeley } \\
\text { Portsmouth GDP } \\
\text { SNL - Albuquerque } \\
\text { Rail } \\
\text { Bettis Atomic } \\
\text { Fermilab (FNAI) } \\
\text { Knolis Atomic } \\
\text { Lawrence Berkeley } \\
\text { Portsmouth GDP } \\
\text { SNL - Albuquerque }\end{array}$ & $\begin{array}{l}\text { to Portsmouth GDP } \\
\text { to Portsmouth GDP } \\
\text { to Portsmouth GDP } \\
\text { to Hanford Site } \\
\text { to Oak Ridge Reserv. } \\
\text { to Los Alamos NL } \\
\text { to Portsmouth GDP } \\
\text { to Portsmouth GDP } \\
\text { to Portsmouth GDP } \\
\text { to Hanford Site } \\
\text { to Oak Ridge Reserv. } \\
\text { to Los Alamos NL }\end{array}$ & $\begin{array}{l}3.22 E-06 \\
8.11 E-06 \\
5.63 E-06 \\
1.16 E-05 \\
1.09 E-06 \\
1.35 E-06 \\
1.40 E-05 \\
1.80 E-05 \\
2.15 E-05 \\
2.33 E-05 \\
3.18 E-06 \\
2.47 E-06\end{array}$ & $\begin{array}{l}1.33 \mathrm{E}-05 \\
3.01 \mathrm{E}-05 \\
3.56 \mathrm{E}-05 \\
4.91 \mathrm{E}-05 \\
2.56 \mathrm{E}-05 \\
1.20 \mathrm{E}-05 \\
8.98 \mathrm{E}-07 \\
9.43 \mathrm{E}-07 \\
1.43 \mathrm{E}-06 \\
2.06 \mathrm{E}-06 \\
9.25 \mathrm{E}-07 \\
2.18 \mathrm{E}-07\end{array}$ \\
\hline
\end{tabular}

Table 6. Annual Incident-Free Dose for the Shipping Campaign (person-rem/yr)

\begin{tabular}{|c|c|c|c|c|c|}
\hline \multirow[b]{2}{*}{ Route } & & \multicolumn{2}{|c|}{ Truck } & \multicolumn{2}{|c|}{ Rail } \\
\hline & & Crew & Public & Crew & Public \\
\hline $\begin{array}{l}\text { Bettis Atomic } \\
\text { Fermilab (FNAL) } \\
\text { Knolls Atomic } \\
\text { Lawrence Berkeley } \\
\text { Portsmouth GDR } \\
\text { SNL - Albuquerque }\end{array}$ & $\begin{array}{l}\text { to Portsmouth GDP } \\
\text { to Portsmouth GDP } \\
\text { to Portsmouth GDP } \\
\text { to Hanford Site } \\
\text { to Oak Ridge Reserv. } \\
\text { to Los Alamos NL }\end{array}$ & $\begin{array}{l}3.02 \mathrm{E}+00 \\
6.54 \mathrm{E}-01 \\
1.62 \mathrm{E}+01 \\
3.86 \mathrm{E}-01 \\
9.14 \mathrm{E}+00 \\
1.97 \mathrm{E}-02\end{array}$ & $\begin{array}{l}3.22 \mathrm{E}+00 \\
7.30 \mathrm{E}-01 \\
1.68 \mathrm{E}+01 \\
4.58 \mathrm{E}-01 \\
1.00 \mathrm{E}+01 \\
2.30 \mathrm{E}-02\end{array}$ & $\begin{array}{l}5.56 \mathrm{E}-01 \\
7.62 \mathrm{E}-02 \\
1.40 \mathrm{E}+00 \\
4.20 \mathrm{E}-02 \\
1.59 \mathrm{E}+00 \\
9.94 \mathrm{E}-03\end{array}$ & $\begin{array}{l}1.76 \mathrm{E}+00 \\
2.53 \mathrm{E}-01 \\
5.21 \mathrm{E}+00 \\
1.23 \mathrm{E}-01 \\
3.14 \mathrm{E}+00 \\
1.61 \mathrm{E}-02\end{array}$ \\
\hline Totals & & $2.94 \mathrm{E}+01$ & $3.12 \mathrm{E}+01$ & $3.68 \mathrm{E}+00$ & $1.05 \mathrm{E}+01$ \\
\hline
\end{tabular}

Table 7. Annual Accident Dose Risk for the Shipping Campaign (person-rem/yr)

\begin{tabular}{llcc}
\hline Route & & Truck & Rail \\
\hline Bettis Atomic & to Portsmouth GDP & $1.14 \mathrm{E}-04$ & $4.95 \mathrm{E}-05$ \\
Fermilab (FNAL) & to Portsmouth GDP & $2.06 \mathrm{E}-09$ & $5.35 \mathrm{E}-10$ \\
Knolls Atomic & to Portsmouth GDP & $1.06 \mathrm{E}-03$ & $2.49 \mathrm{E}-04$ \\
Lawrence Berkeley & to Hanford Site & $2.53 \mathrm{E}-09$ & $1.42 \mathrm{E}-09$ \\
Portsmouth GDP & to Oak Ridge Reserv. & $6.55 \mathrm{E}-04$ & $1.24 \mathrm{E}-04$ \\
SNL - Albuquerque & to Los Alamos NL & $1.03 \mathrm{E}-11$ & $1.95 \mathrm{E}-13$ \\
\hline Totals & & $1.83 \mathrm{E}-03$ & $4.23 \mathrm{E}-04$ \\
\hline
\end{tabular}


Table 8. Expected Annual Fatalities for the Shipping Campaign

\begin{tabular}{llc}
\hline Exposure Group & Truck & Rail \\
\hline Radiological & & \\
Normal Crew & $1.2 \mathrm{E}-02$ & $1.5 \mathrm{E}-03$ \\
Normal Public & $1.6 \mathrm{E}-02$ & $5.3 \mathrm{E}-03$ \\
$\quad$ Accident Public & $9.1 \mathrm{E}-07$ & $2.1 \mathrm{E}-07$ \\
Nonradiological & & \\
Emission & $5.9 \mathrm{E}-03$ & $8.4 \mathrm{E}-03$ \\
Accident & $4.9 \mathrm{E}-02$ & $8.0 \mathrm{E}-04$ \\
\hline
\end{tabular}

Table 9. Expected Annual Cancer Incidence for the Shipping Campaign

\begin{tabular}{lll}
\hline Exposure Group & Truck & Rail \\
\hline Radiological & & \\
Normal Crew & $4.1 E-02$ & $5.2 E-03$ \\
Normal Public & $5.3 E-02$ & $1.8 E-02$ \\
Accident Public & $3.1 E-06$ & $7.2 E-07$ \\
$\begin{array}{c}\text { Nonradiological } \\
\text { Emission }\end{array}$ & $5.9 E-03$ & $8.4 E-03$ \\
Accident & NA & NA \\
\hline
\end{tabular}

Table 10. Expected Annual Genetic Effects for the Shipping Campaign

\begin{tabular}{lll}
\hline Exposure Group & Truck & Rail \\
\hline Radiological & & \\
Normal Crew & $1.8 \mathrm{E}-03$ & $2.2 \mathrm{E}-04$ \\
Normal Public & $3.1 \mathrm{E}-03$ & $1.1 \mathrm{E}-03$ \\
$\quad$ Accident Public & $1.8 \mathrm{E}-07$ & $4.2 \mathrm{E}-08$ \\
Nonradiological & & \\
Emission & NA & NA \\
Accident & NA & NA \\
\hline
\end{tabular}




\section{B.13 WM LLW REGIONALIZED 5 ALTERNATIVE (CASE 19): HETEROGENEOUS SOLIDS}

Table 1. Summary of Route Information

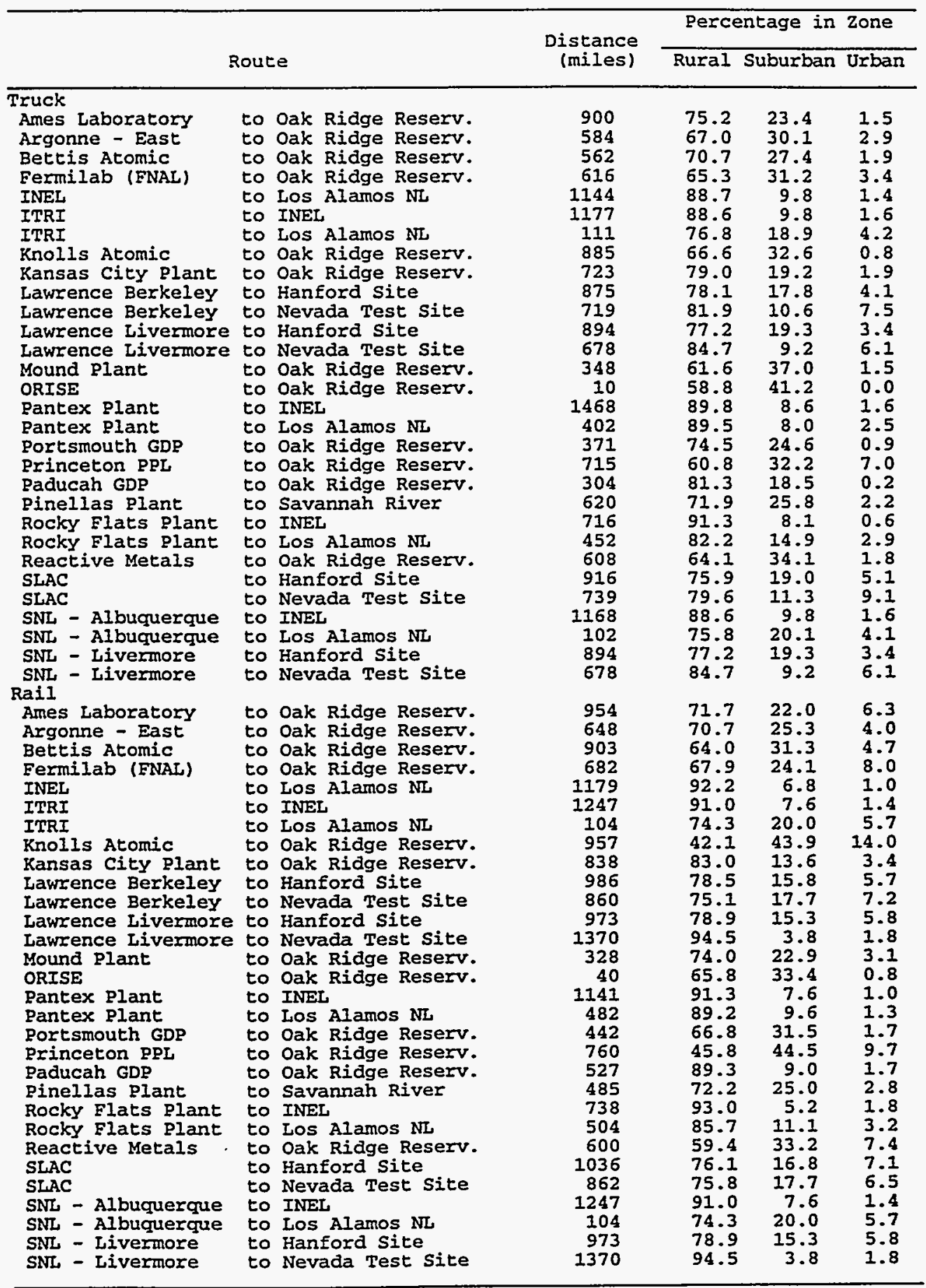




\section{$B-58$}

Table 2. Annual Number of Shipments and One-Way Shipment Distances

\begin{tabular}{|c|c|c|c|c|c|c|}
\hline \multirow[b]{2}{*}{ Route } & & & \multicolumn{2}{|c|}{ \# of Shipments } & \multicolumn{2}{|c|}{ Total Mileage (mi) } \\
\hline & & & Truck & Rail & Truck & Rail \\
\hline $\begin{array}{l}\text { Ames Laboratory } \\
\text { Argonne - East } \\
\text { Bettis Atomic } \\
\text { Fermilab (FNAL) } \\
\text { INEL } \\
\text { ITRI } \\
\text { ITRI } \\
\text { Knolls Atomic } \\
\text { Kansas City Plant } \\
\text { Lawrence Berkeley } \\
\text { Lawrence Berkeley } \\
\text { Lawrence Livermore } \\
\text { Lawrence Livermore } \\
\text { Mound Plant } \\
\text { ORISE } \\
\text { Pantex Plant } \\
\text { Pantex Plant } \\
\text { Portsmouth GDP } \\
\text { Princeton pPL } \\
\text { Paducah GDP } \\
\text { Pinellas Plant } \\
\text { Rocky Flats Plant } \\
\text { Rocky Flats Plant } \\
\text { Reactive Metals } \\
\text { SLAC } \\
\text { SLAC } \\
\text { SNL - Albuquerque } \\
\text { SNL - Albuquerque } \\
\text { SNL - Livermore } \\
\text { SNL - Livermore }\end{array}$ & $\begin{array}{l}\text { to } \\
\text { to } \\
\text { to } \\
\text { to } \\
\text { to } \\
\text { to } \\
\text { to } \\
\text { to } \\
\text { to } \\
\text { to } \\
\text { to } \\
\text { to } \\
\text { to } \\
\text { to } \\
\text { to } \\
\text { to } \\
\text { to } \\
\text { to } \\
\text { to } \\
\text { to } \\
\text { to } \\
\text { to } \\
\text { to } \\
\text { to } \\
\text { to } \\
\text { to } \\
\text { to } \\
\text { to } \\
\text { to }\end{array}$ & $\begin{array}{l}\text { Oak Ridge Reserv } \\
\text { Oak Ridge Reserv. } \\
\text { Oak Ridge Reserv. } \\
\text { Oak Ridge Reserv. } \\
\text { Ios Alamos NL } \\
\text { INEL } \\
\text { Los Alamos NL } \\
\text { Oak Ridge Reserv. } \\
\text { Oak Ridge Reserv. } \\
\text { Hanford Site } \\
\text { Nevada Test Site } \\
\text { Hanford Site } \\
\text { Nevada Test Site } \\
\text { Oak Ridge Reserv. } \\
\text { Oak Ridge Reserv. } \\
\text { INEL } \\
\text { Los Alamos NL } \\
\text { Oak Ridge Reserv. } \\
\text { Oak Ridge Reserv. } \\
\text { Oak Ridge Reserv. } \\
\text { Savannah River } \\
\text { INEL } \\
\text { Los Alamos NL } \\
\text { Oak Ridge Reserv. } \\
\text { Hanford Site } \\
\text { Nevada Test Site } \\
\text { INEL } \\
\text { Los Alamos NL } \\
\text { Hanford Site } \\
\text { Nevada Test Site }\end{array}$ & $\begin{array}{r}2 \\
105 \\
57 \\
3 \\
244 \\
2 \\
9 \\
28 \\
1 \\
2 \\
2 \\
17 \\
40 \\
516 \\
5 \\
1358 \\
25 \\
3250 \\
1 \\
627 \\
2 \\
212 \\
141 \\
761 \\
1 \\
39 \\
1 \\
17 \\
1 \\
5\end{array}$ & $\begin{array}{r}1 \\
39 \\
21 \\
1 \\
92 \\
1 \\
3 \\
11 \\
1 \\
1 \\
1 \\
7 \\
15 \\
190 \\
2 \\
528 \\
10 \\
1250 \\
1 \\
235 \\
1 \\
79 \\
54 \\
285 \\
1 \\
15 \\
1 \\
7 \\
1 \\
2\end{array}$ & $\begin{array}{l}1.80 E+03 \\
6.13 E+04 \\
3.21 E+04 \\
1.85 E+03 \\
2.79 E+05 \\
2.35 E+03 \\
9.99 E+02 \\
2.48 E+04 \\
7.23 E+02 \\
1.75 E+03 \\
1.44 E+03 \\
1.52 E+04 \\
2.71 E+04 \\
1.79 E+05 \\
5.10 E+01 \\
1.99 E+06 \\
1.01 E+04 \\
1.20 E+06 \\
7.15 E+02 \\
1.91 E+05 \\
1.24 E+03 \\
1.52 E+05 \\
6.37 E+04 \\
4.63 E+05 \\
9.16 E+02 \\
2.88 E+04 \\
1.17 E+03 \\
1.73 E+03 \\
8.94 E+02 \\
3.39 E+03\end{array}$ & 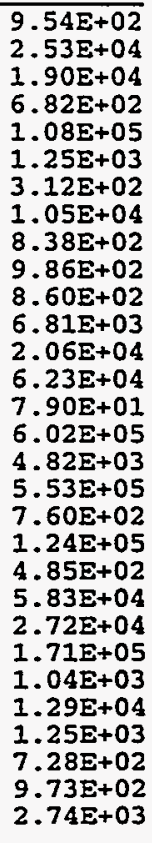 \\
\hline Totals & & & 7474 & 2856 & $4.75 E+06$ & $1.82 \mathrm{E}+06$ \\
\hline
\end{tabular}


Table 3. Incident-Free Dose per Shipment (person-rem/shipment)

\begin{tabular}{|c|c|c|c|c|c|}
\hline \multirow[b]{2}{*}{ Source/Route(s) } & \multirow[b]{2}{*}{ Crew } & \multicolumn{4}{|c|}{ General Public } \\
\hline & & Off-Link & On-Link & Stops & Total \\
\hline $\begin{array}{l}W(x) \text { AL to OR } \\
\text { Ames Laboracory }\end{array}$ & $\begin{array}{cr}\text { to Oak Ridge Reser } \\
\text { Truck } & 2.83 \mathrm{E}-02 \\
\text { Rail } & 6.86 \mathrm{E}-03\end{array}$ & $\begin{array}{l}\text { i. } \\
1.58 \mathrm{E}-03 \\
1.582\end{array}$ & $\begin{array}{l}3.92 \mathrm{E}-03 \\
2.35 \mathrm{E}-04\end{array}$ & $\begin{array}{l}2.52 E-02 \\
6.42 E-03\end{array}$ & $\begin{array}{l}3.14 E-02 \\
2.24 E-02\end{array}$ \\
\hline $\begin{array}{l}\text { LLW(x) AN to OR } \\
\text { Argonne - East }\end{array}$ & $\begin{array}{cr}\text { to Oak Ridge Reser } \\
\text { Truck } & 2.00 \mathrm{E}-02 \\
\text { Rail } & 5.59 \mathrm{E}-03\end{array}$ & $\begin{array}{l}2.19 \mathrm{E}-03 \\
8.34 \mathrm{E}-03\end{array}$ & $\begin{array}{l}3.27 E-03 \\
1.48 E-04\end{array}$ & $\begin{array}{l}1.63 \mathrm{E}-02 \\
5.48 \mathrm{E}-03\end{array}$ & $\begin{array}{l}2.18 \mathrm{E}-02 \\
1.40 \mathrm{E}-02\end{array}$ \\
\hline $\begin{array}{l}\operatorname{LLW}(x) \text { BA to OR } \\
\text { Bettis Atomic }\end{array}$ & $\begin{array}{cr}\text { to Oak Ridge Reser } \\
\text { Truck } \\
\text { Rail } & 1.85 \mathrm{E}-02 \\
6.65 \mathrm{E}-03\end{array}$ & $\begin{array}{l}\text { i. } \\
1.42 \mathrm{E}-03 \\
\text { 1.402-02 }\end{array}$ & $\begin{array}{l}2.71 E-03 \\
2.44 E-04\end{array}$ & $\begin{array}{l}1.57 \mathrm{E}-02 \\
6.26 \mathrm{E}-03\end{array}$ & $\begin{array}{l}2.02 \mathrm{E}-02 \\
2.05 \mathrm{E}-02\end{array}$ \\
\hline $\begin{array}{l}\operatorname{LLW}(x) \text { FL to OR } \\
\text { Fermilab (ENAL) }\end{array}$ & $\begin{array}{lr}\text { co Oak Ridge Reser } \\
\text { Truck } & 2.15 \mathrm{E}-02 \\
\text { Rail } & 5.73 \mathrm{E}-03\end{array}$ & $\begin{array}{l}2.53 \mathrm{E}-03 \\
1.37 \mathrm{E}-02\end{array}$ & $\begin{array}{l}3.71 E-03 \\
1.92 E-04\end{array}$ & $\begin{array}{l}1.72 \mathrm{E}-02 \\
5.58 \mathrm{E}-03\end{array}$ & $\begin{array}{l}E-02 \\
E-02\end{array}$ \\
\hline $\begin{array}{l}\text { LLW (x) IN to LA } \\
\text { INEL }\end{array}$ & $\begin{array}{cc}\text { to Los } & \text { Alamos NL } \\
\text { Truck } & 3.15 \mathrm{E}-02 \\
\text { RaiI } & 7.80 \mathrm{E}-03\end{array}$ & $\begin{array}{l}-03 \\
-03\end{array}$ & $\begin{array}{l}4.2 \\
1.0\end{array}$ & $\begin{array}{l}E-02 \\
z-03\end{array}$ & $\begin{array}{l}2-02 \\
8-02\end{array}$ \\
\hline LLW $\underset{\text { ITRI }}{(x)}$ IT to IN & $\begin{array}{l}\text { to INEL } \\
\text { Truck } \\
\text { Rail }\end{array}$ & $\begin{array}{l}-03 \\
-03\end{array}$ & $\begin{array}{l}4.5 \\
1.2\end{array}$ & $\begin{array}{l}E-02 \\
E-03\end{array}$ & $\begin{array}{l}E-02 \\
E-02\end{array}$ \\
\hline $\begin{array}{l}\text { LLW (x) IT to LA } \\
\text { ITRI }\end{array}$ & $\begin{array}{lr}\text { to Los } & \text { Alamos } \\
\text { Truck } & 3.5 \\
\text { Rail } & 3.3\end{array}$ & $\begin{array}{l}4.0 \\
1.5\end{array}$ & $\begin{array}{l}6.7 \\
2.3\end{array}$ & $\begin{array}{l}E-03 \\
E-03\end{array}$ & $\begin{array}{l}-03 \\
-03\end{array}$ \\
\hline $\begin{array}{l}\text { LLW(x) KA to OR } \\
\text { KnoIls Atomic }\end{array}$ & $\begin{array}{lr}\text { to Oak Ridge Reser } \\
\text { Truck } & 2.98 \mathrm{E}-02 \\
\text { Rail } & 6.87 \mathrm{E}-03\end{array}$ & $\begin{array}{l}\text { v. } \\
2.55 \mathrm{E}-03 \\
3.39 \mathrm{E}-02\end{array}$ & $\begin{array}{l}3.7 \\
4.4\end{array}$ & $\begin{array}{l}2.48 E-02 \\
6.42 E-03\end{array}$ & $\begin{array}{l}3.11 E-02 \\
4.08 E-02\end{array}$ \\
\hline $\begin{array}{l}\text { LLW(x) KC to OR } \\
\text { Kansas City Plant }\end{array}$ & $\begin{array}{cr}\text { to Oak Ridge Reser } \\
\text { Truck } & 2.20 \mathrm{E}-02 \\
\text { Rail } & 6.38 \mathrm{E}-03\end{array}$ & $\begin{array}{r}\text { v. } \\
i .7 \\
7.7\end{array}$ & $\begin{array}{l}3.2 \\
1.3\end{array}$ & $\begin{array}{l}2 E-02 \\
6 E-03\end{array}$ & $\begin{array}{l}2.52 \mathrm{E}-02 \\
1.40 \mathrm{E}-02\end{array}$ \\
\hline $\begin{array}{l}\text { LLW(x) LAA } \\
\text { INEL }\end{array}$ & $\begin{array}{cr}\text { to Los } & \text { Alamos NL } \\
\text { Truck } & 3.15 \mathrm{E}-02 \\
\text { Rail } & 7.80 \mathrm{E}-03\end{array}$ & $\begin{array}{l}1.7 \\
4.0\end{array}$ & $\begin{array}{l}4.2 \\
1.0\end{array}$ & $\begin{array}{l}E-02 \\
E-03\end{array}$ & $\begin{array}{l}-02 \\
-02\end{array}$ \\
\hline $\begin{array}{l}\text { LLW(x) LB to HS } \\
\text { Lawrence Berkeley }\end{array}$ & $\begin{array}{ll}\text { to Hanford Site } \\
\text { Truck } & 2.76 \mathrm{E}-02 \\
\text { Rail } & 6.99 \mathrm{E}-03\end{array}$ & $\begin{array}{l}3.02 \mathrm{E}-03 \\
1.38 \mathrm{E}-02\end{array}$ & $\begin{array}{l}5.17 \mathrm{E}-03 \\
2.03 \mathrm{E}-04\end{array}$ & $\begin{array}{l}2 \\
6\end{array}$ & -02 \\
\hline LIW(x) LI to gS & $\begin{array}{ll}\text { to Nevada } & \text { Test Sit } \\
\text { Truck } & 2.27 E-02 \\
\text { Rail } & 6.47 E-03\end{array}$ & $\begin{array}{l}\mathrm{e} \\
3.3 \\
1.4\end{array}$ & $\begin{array}{l}5.6 \\
2.0\end{array}$ & $\begin{array}{l}2 . \\
6\end{array}$ & $\begin{array}{l}8 \\
-02\end{array}$ \\
\hline $\begin{array}{l}\text { LLW (x) LI to HS } \\
\text { Lawrence Livermore }\end{array}$ & $\begin{array}{lr}\text { to Hanford Site } \\
\text { Truck } & 2.82 \mathrm{E}-02 \\
\text { Rail } & 6.94 \mathrm{E}-03\end{array}$ & $\begin{array}{l}2.8 \\
1.38\end{array}$ & $\begin{array}{l}4.5 \\
2.0\end{array}$ & $\begin{array}{l}2 . \\
6\end{array}$ & $:-02$ \\
\hline Lawrence Livermore & $\begin{array}{cc}\text { to Nevada } & \text { Test Sit } \\
\text { Truck } & 2.05 E-02 \\
\text { Rail } & 8.60 E-03\end{array}$ & $\begin{array}{l}\mathrm{e} \\
2.5 \\
5.7\end{array}$ & $\begin{array}{l}4 . \\
1 .\end{array}$ & 1 & -02 \\
\hline $\begin{array}{l}\text { LLW }(x) \text { MP to OR } \\
\text { Mound Plant }\end{array}$ & $\begin{array}{cr}\text { to Oak Ridge Reser } \\
\text { Truck } & 1.23 \mathrm{E}-02 \\
\text { Rail } & 4.25 \mathrm{E}-03\end{array}$ & $\begin{array}{r}\text { v. } \\
1.2 \\
3.5\end{array}$ & $\begin{array}{l}1.7 \\
6.6\end{array}$ & $\begin{array}{l}9.73 \mathrm{E}-03 \\
4.49 \mathrm{E}-03\end{array}$ & $\begin{array}{l}1.27 E-02 \\
8.08 E-03\end{array}$ \\
\hline $\begin{array}{c}\text { LIW( } x \text { ) OI to OR } \\
\text { ORISE }\end{array}$ & $\begin{array}{cr}\text { to Oak Ridge Reser } \\
\text { Truck } & 3.63 \mathrm{E}-04 \\
\text { Rail } & 3.05 \mathrm{E}-03\end{array}$ & $\begin{array}{l}v_{3} \\
3.21 E-05 \\
3.36 E-04\end{array}$ & $\begin{array}{l}4.2 \\
8.7\end{array}$ & 2 & $\begin{array}{l}8-04 \\
8-03\end{array}$ \\
\hline $\begin{array}{l}\text { LLW(x) PP to IN } \\
\text { Pantex Plant }\end{array}$ & $\begin{array}{l}\text { to INEL } \\
\text { Truck } \\
\text { Rail }\end{array}$ & $\begin{array}{l}2.24 E-03 \\
4.12 E-03\end{array}$ & $\begin{array}{l}5.63 \\
1.10\end{array}$ & $\begin{array}{l}4.11 \mathrm{E}-02 \\
6.99 \mathrm{E}-03\end{array}$ & $\begin{array}{l}4.90 \mathrm{E}-02 \\
1.12 \mathrm{E}-02\end{array}$ \\
\hline $\begin{array}{l}\text { LLW (x) PP to LA } \\
\text { Pantex Plant }\end{array}$ & $\begin{array}{cc}\text { to Los } & \text { Alamos NL } \\
\text { Truck } & 1.11 \mathrm{E}-02 \\
\text { Rail } & 4.89 \mathrm{E}-03\end{array}$ & & $\begin{array}{l}1.77 E-03 \\
5.29 E-05\end{array}$ & $\begin{array}{l}1.13 \mathrm{E}-02 \\
4.96 \mathrm{E}-03\end{array}$ & $\begin{array}{l}1.38 \mathrm{E}-02 \\
7.19 \mathrm{E}-03\end{array}$ \\
\hline
\end{tabular}


$\operatorname{LLW}(x)$ Po to OR Portsmouth GDP

LLW(x) PR to OR Princeton PQL

LLW(x) PA to OR Paducah GDP

LLW(x) PI to SR Pinelias Plant

LLW(x) RF to IN Rocky Flats Plant

LLW(x) RF to LA Rocky Flats Plant

LLW(x) RM to OR Reactive Metals

LLW(x) ST to HS SLAC

LLW(x) ST to NT SLAC

LLW(x) SA to IN SNL - Albuquerque

ILW(x) SA to IA SNL - Albuquerque

LLW(x) SL to 'HS SNL - Livermore

ILW(x) SL to NT SNL - Livermore to Oak Ridge Reserv.

Truck $1.16 \mathrm{E}-02$ 8.77E-04 1.50E-03 1.04E-02 $1.28 \mathrm{E}-02$

Rail 4.73E-03 4.37E-03 1.00E-04 4.84E-03 9.32E-03

to Oak Ridge Reserv.

$\begin{array}{llllll}\text { Truck } & 2.68 E-02 & 4.27 E-03 & 6.06 E-03 & 2.00 E-02 & 3.04 E-02\end{array}$

Rail $6.05 E-03 \quad 2.10 E-02 \quad 3.11 E-04 \quad 5.82 E-03 \quad 2.71 E-02$

to Oak Ridge Reserv.

Truck $\quad 8.88 \mathrm{E}-03 \quad 4.70 \mathrm{E}-04 \quad 9.98 \mathrm{E}-04 \quad 8.51 \mathrm{E}-03 \quad 9.98 \mathrm{E}-03$

Rail 5.08E-03 2.75E-03 5.97E-05 5.10E-03 7.91E-03

to Savannah River

$\begin{array}{lllllll}\text { Truck } & 2.02 \mathrm{E}-02 & 1.94 \mathrm{E}-03 & 3.10 \mathrm{E}-03 & 1.74 \mathrm{E}-02 & 2.24 \mathrm{E}-02\end{array}$

Rail $4.90 E-035.13 E-031.02 E-04 \quad 4.97 E-031.02 E-02$

to INEL

$\begin{array}{llllll}\text { Truck } & 1.90 \mathrm{E}-02 & 6.95 \mathrm{E}-04 & 2.22 \mathrm{E}-03 & 2.00 \mathrm{E}-02 & 2.30 \mathrm{E}-02\end{array}$

Rail $5.96 \mathrm{E}-03$ 3.32E-03 7.00E-05 5.75E-03 $9.14 \mathrm{E}-03$

to Los Alamos NL

$\begin{array}{llllll}\text { Truck } & 1.35 \mathrm{E}-02 & 1.19 \mathrm{E}-03 & 2.25 \mathrm{E}-03 & 1.27 \mathrm{E}-02 & 1.61 \mathrm{E}-02\end{array}$

Rail $4.98 \mathrm{E}-03 \quad 4.26 \mathrm{E}-03$ 7.35E-05 $5.03 \mathrm{E}-03$ 9.36E-03

to Oak Ridge Reserv.

$\begin{array}{llllll}\text { Truck } & 2.11 \mathrm{E}-02 & 2.15 \mathrm{E}-03 & 3.08 \mathrm{E}-03 & 1.70 \mathrm{E}-02 & 2.23 \mathrm{E}-02\end{array}$

Rail $5.38 \mathrm{E}-03$ 1.25E-02 $1.91 \mathrm{E}-04 \quad 5.33 \mathrm{E}-03 \quad 1.80 \mathrm{E}-02$

to Hanford Site

$\begin{array}{lllllll}\text { Truck } & 2.98 \mathrm{E}-02 & 3.70 \mathrm{E}-03 & 6.08 \mathrm{E}-03 & 2.56 \mathrm{E}-02 & 3.54 \mathrm{E}-02\end{array}$

Rail 7.20E-03 1.75E-02 2.40E-04 6.67E-03 2.44E-02

to Nevada Test Site

$\begin{array}{llllll}\text { Truck } & 2.43 E-02 & 4.05 E-03 & 6.67 E-03 & 2.07 E-02 & 3.14 E-02\end{array}$

Rail 6.48E-03 1.38E-02 1.96E-04 6.13E-03 2.01E-02

to INEL

$\begin{array}{lllllll}\text { Truck } & 3.22 \mathrm{E}-02 & 1.83 \mathrm{E}-03 & 4.46 \mathrm{E}-03 & 3.27 \mathrm{E}-02 & 3.90 \mathrm{E}-02\end{array}$

Rail $8.09 \mathrm{E}-03$ 5.30E-03 1.26E-04 7.32E-03 1.27E-02

to Los Alamos NL

$\begin{array}{lllllll}\text { Truck } & 3.28 \mathrm{E}-03 & 3.70 \mathrm{E}-04 & 6.13 \mathrm{E}-04 & 2.86 \mathrm{E}-03 & 3.84 \mathrm{E}-03\end{array}$

Rail $3.31 \mathrm{E}-03$ 1.55E-03 2.36E-05 3.80E-03 5.37E-03

to Hanford Site

$\begin{array}{llllll}\text { Truck 2.82E-02 } & 2.88 \mathrm{E}-03 & 4.92 \mathrm{E}-03 & 2.50 \mathrm{E}-02 & 3.28 \mathrm{E}-02\end{array}$ Rail 6.94E-03 1.38E-02 2.00E-04 6.48E-03 2.05E-02

to Nevada Test Site

$\begin{array}{llllll}\text { Truck } & 2.05 E-02 & 2.57 E-03 & 4.65 E-03 & 1.90 E-02 & 2.62 E-02\end{array}$ Rail $8.60 \mathrm{E}-03$ 5.75E-03 1.20E-04 $7.70 \mathrm{E}-03$ 1.36E-02 


\section{B-6I}

Table 4. Accident Dose Risk per Shipment (person-rem/shipment)

\begin{tabular}{|c|c|c|c|}
\hline Source/Route & & Truck & Rail \\
\hline 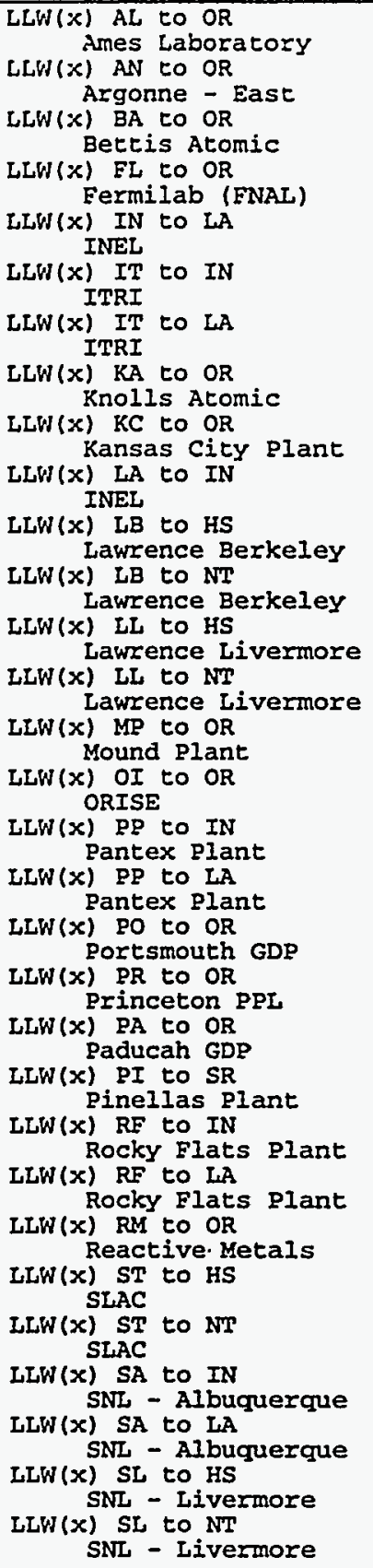 & $\begin{array}{l}\text { to Oak Ridge Reserv. } \\
\text { to Oak Ridge Reserv. } \\
\text { to Oak Ridge Reserv. } \\
\text { to Oak Ridge Reserv. } \\
\text { to Los Alamos NL } \\
\text { to INEL } \\
\text { to Los Alamos NL } \\
\text { to Oak Ridge Reserv. } \\
\text { to Oak Ridge Reserv. } \\
\text { to Los Alamos NL } \\
\text { to Hanford Site } \\
\text { to Nevada Test Sit } \\
\text { to Hanford Site } \\
\text { to Nevada Test Sit } \\
\text { to Oak Ridge Reserv. } \\
\text { to Oak Ridge Reserv. } \\
\text { to INEL } \\
\text { to Los Alamos NL } \\
\text { to Oak Ridge Reserv. } \\
\text { to Oak Ridge Reserv. } \\
\text { to Oak Ridge Reserv. } \\
\text { to Savannah River } \\
\text { to INEL } \\
\text { to Los Alamos NL } \\
\text { to Oak Ridge Reserv. } \\
\text { to Hanford Site } \\
\text { to Nevada Test Sit } \\
\text { to INEL } \\
\text { to Los Alamos NL } \\
\text { to Hanford Site } \\
\text { to }\end{array}$ & $\begin{array}{l}9.48 \mathrm{E}-06 \\
5.16 \mathrm{E}-04 \\
9.94 \mathrm{E}-06 \\
3.56 \mathrm{E}-05 \\
1.92 \mathrm{E}-01 \\
1.48 \mathrm{E}-02 \\
6.71 \mathrm{E}-04 \\
2.13 \mathrm{E}-06 \\
3.55 \mathrm{E}-05 \\
1.10 \mathrm{E}-01 \\
6.10 \mathrm{E}-02 \\
7.54 \mathrm{E}-03 \\
1.05 \mathrm{E}-05 \\
3.71 \mathrm{E}-06 \\
1.06 \mathrm{E}-06 \\
4.49 \mathrm{E}-08 \\
1.50 \mathrm{E}-06 \\
1.30 \mathrm{E}-06 \\
7.40 \mathrm{E}-09 \\
5.74 \mathrm{E}-06 \\
3.12 \mathrm{E}-07 \\
1.30 \mathrm{E}-06 \\
3.10 \mathrm{E}-06 \\
2.53 \mathrm{E}-06 \\
5.73 \mathrm{E}-09 \\
9.15 \mathrm{E}-10 \\
2.14 \mathrm{E}-07 \\
4.36 \mathrm{E}-05 \\
4.60 \mathrm{E}-06 \\
1.07 \mathrm{E}-04 \\
3.48 \mathrm{E}-05\end{array}$ & $\begin{array}{l}6.83 \mathrm{E}-06 \\
2.26 \mathrm{E}-04 \\
1.12 \mathrm{E}-05 \\
2.31 \mathrm{E}-05 \\
3.39 \mathrm{E}-02 \\
1.85 \mathrm{E}-03 \\
3.70 \mathrm{E}-05 \\
3.16 \mathrm{E}-06 \\
7.40 \mathrm{E}-06 \\
1.99 \mathrm{E}-02 \\
6.80 \mathrm{E}-02 \\
1.03 \mathrm{E}-02 \\
1.34 \mathrm{E}-05 \\
2.98 \mathrm{E}-06 \\
3.06 \mathrm{E}-07 \\
3.15 \mathrm{E}-08 \\
2.58 \mathrm{E}-07 \\
2.02 \mathrm{E}-07 \\
3.64 \mathrm{E}-09 \\
2.32 \mathrm{E}-06 \\
1.09 \mathrm{E}-07 \\
4.65 \mathrm{E}-07 \\
1.32 \mathrm{E}-06 \\
2.82 \mathrm{E}-07 \\
2.88 \mathrm{E}-09 \\
5.37 \mathrm{E}-10 \\
3.39 \mathrm{E}-07 \\
2.79 \mathrm{E}-06 \\
2.14 \mathrm{E}-07 \\
5.69 \mathrm{E}-05 \\
2.74 \mathrm{E}-05\end{array}$ \\
\hline
\end{tabular}


Table 5. Nonradiological Risk Factors per Shipment (Eatalities/shipment)

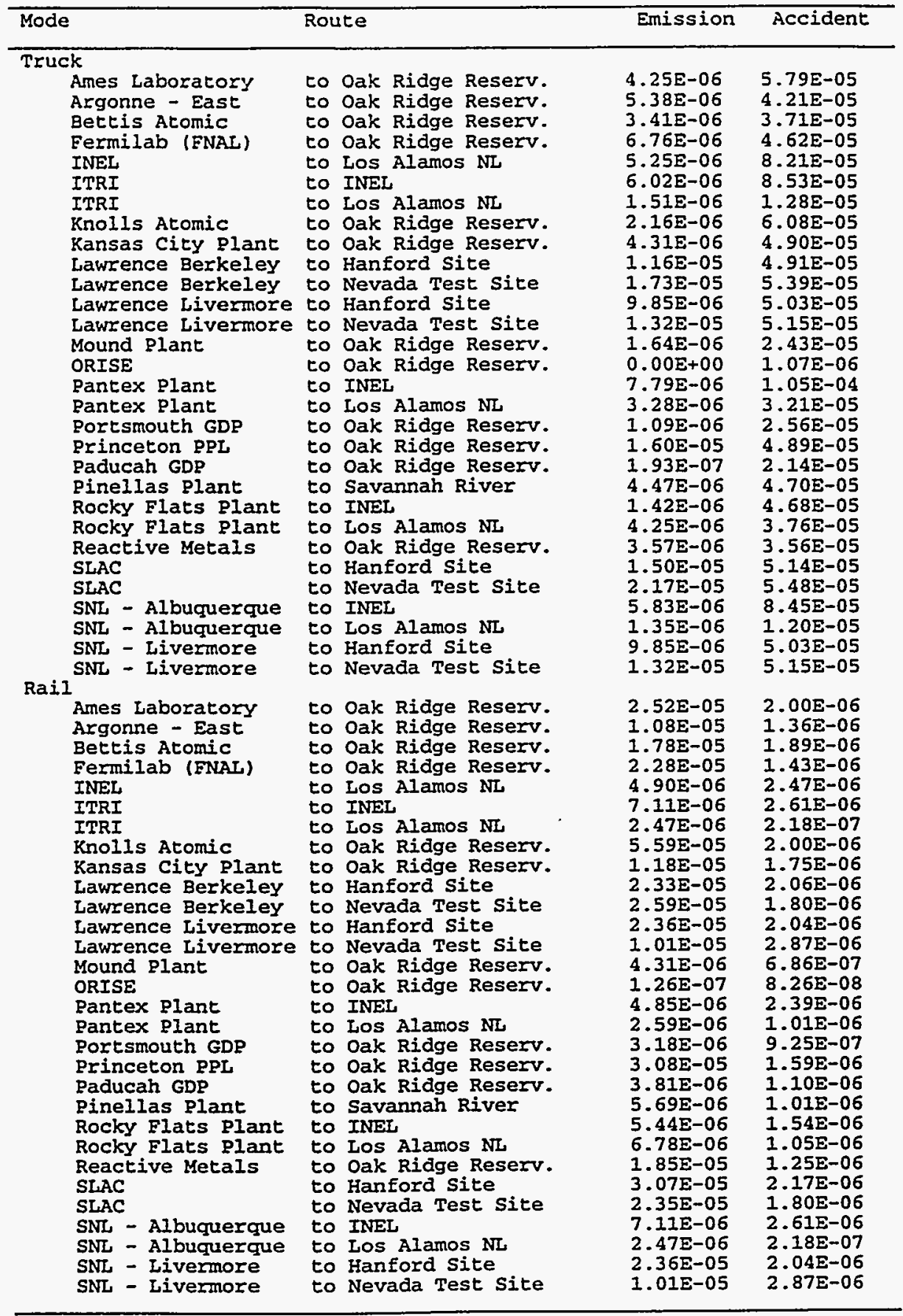


Table 6. Annual Incident-Free Dose for the Shipping Campaign (person-rem/yr)

\begin{tabular}{|c|c|c|c|c|c|c|}
\hline \multirow[b]{2}{*}{ Route } & & & \multicolumn{2}{|c|}{ Truck } & \multicolumn{2}{|l|}{ Rail } \\
\hline & & & Crew & Public & Crew & Public \\
\hline $\begin{array}{l}\text { Ames Laboratory } \\
\text { Argonne - East } \\
\text { Bettis Atomic } \\
\text { Fermilab (ENAL) } \\
\text { INEL } \\
\text { ITRI } \\
\text { ITRI } \\
\text { Knolls Atomic } \\
\text { Kansas City Plant } \\
\text { Lawrence Berkeley } \\
\text { Lawrence Berkeley } \\
\text { Lawrence Livermore } \\
\text { Lawrence Livermore } \\
\text { Mound Plant } \\
\text { ORISE } \\
\text { Pantex plant } \\
\text { Pantex Plant } \\
\text { Portsmouth GDP } \\
\text { Princeton ppI } \\
\text { Paducah GDP } \\
\text { Pinellas Plant } \\
\text { Rocky Flats Plant } \\
\text { Rocky Flats Plant } \\
\text { Reactive Metals } \\
\text { SLAC } \\
\text { SLAC - Albuquerque } \\
\text { SNL - Albuquer } \\
\text { SNL - Albuquerque } \\
\text { SNL - Livermore } \\
\text { SNL - Livermore }\end{array}$ & $\begin{array}{l}\text { to } \\
\text { to } \\
\text { to } \\
\text { to } \\
\text { to } \\
\text { to } \\
\text { to } \\
\text { to } \\
\text { to } \\
\text { to } \\
\text { to } \\
\text { to } \\
\text { to } \\
\text { to } \\
\text { to } \\
\text { to } \\
\text { to } \\
\text { to } \\
\text { to } \\
\text { to } \\
\text { to } \\
\text { to } \\
\text { to } \\
\text { to } \\
\text { to } \\
\text { to } \\
\text { to } \\
\text { to } \\
\text { to }\end{array}$ & $\begin{array}{l}\text { Oak Ridge Reserv. } \\
\text { Oak Ridge Reserv. } \\
\text { Oak Ridge Reserv. } \\
\text { Oak Ridge Reserv. } \\
\text { Los Alamos NL } \\
\text { INEL } \\
\text { Los Alamos NL } \\
\text { Oak Ridge Reserv. } \\
\text { Oak Ridge Reserv. } \\
\text { Hanford Site } \\
\text { Nevada Test Site } \\
\text { Hanford Site } \\
\text { Nevada Test Site } \\
\text { Oak Ridge Reserv. } \\
\text { Oak Ridge Reserv. } \\
\text { INEL } \\
\text { Los Alamos NL } \\
\text { Oak Ridge Reserv. } \\
\text { Oak Ridge Reserv. } \\
\text { Oak Ridge Reserv. } \\
\text { Savannah River } \\
\text { INEL } \\
\text { Los Alamos NL } \\
\text { Oak Ridge Reserv. } \\
\text { Hanford Site } \\
\text { Nevada Test Site } \\
\text { INEL } \\
\text { Los Alamos NL } \\
\text { Hanford Site } \\
\text { Nevada Test Site }\end{array}$ & $\begin{array}{l}5.66 \mathrm{E}-02 \\
2.10 \mathrm{E}+00 \\
1.05 \mathrm{E}+00 \\
6.45 \mathrm{E}-02 \\
7.68 \mathrm{E}+00 \\
6.50 \mathrm{E}-02 \\
3.19 \mathrm{E}-02 \\
8.33 \mathrm{E}-01 \\
2.20 \mathrm{E}-02 \\
5.51 \mathrm{E}-02 \\
4.54 \mathrm{E}-02 \\
4.79 \mathrm{E}-01 \\
8.22 \mathrm{E}-01 \\
6.33 \mathrm{E}+00 \\
1.81 \mathrm{E}-03 \\
5.45 \mathrm{E}+01 \\
2.79 \mathrm{E}-01 \\
3.79 \mathrm{E}+01 \\
2.68 \mathrm{E}-02 \\
5.57 \mathrm{E}+00 \\
4.04 \mathrm{E}-02 \\
4.02 \mathrm{E}+00 \\
1.91 \mathrm{E}+00 \\
1.61 \mathrm{E}+01 \\
2.98 \mathrm{E}-02 \\
9.46 \mathrm{E}-01 \\
3.22 \mathrm{E}-02 \\
5.58 \mathrm{E}-02 \\
2.82 \mathrm{E}-02 \\
1.03 \mathrm{E}-01\end{array}$ & $\begin{array}{l}6.28 \mathrm{E}-02 \\
2.29 \mathrm{E}+00 \\
1.15 \mathrm{E}+00 \\
7.04 \mathrm{E}-02 \\
9.28 \mathrm{E}+00 \\
7.87 \mathrm{E}-02 \\
3.76 \mathrm{E}-02 \\
8.72 \mathrm{E}-01 \\
2.52 \mathrm{E}-02 \\
6.54 \mathrm{E}-02 \\
5.82 \mathrm{E}-02 \\
5.58 \mathrm{E}-01 \\
1.05 \mathrm{E}+00 \\
6.55 \mathrm{E}+00 \\
1.80 \mathrm{E}-03 \\
6.65 \mathrm{E}+01 \\
3.45 \mathrm{E}-01 \\
4.14 \mathrm{E}+01 \\
3.04 \mathrm{E}-02 \\
6.26 \mathrm{E}+00 \\
4.48 \mathrm{E}-02 \\
4.87 \mathrm{E}+00 \\
2.27 \mathrm{E}+00 \\
1.69 \mathrm{E}+01 \\
3.54 \mathrm{E}-02 \\
1.23 \mathrm{E}+00 \\
3.90 \mathrm{E}-02 \\
6.53 \mathrm{E}-02 \\
3.28 \mathrm{E}-02 \\
1.31 \mathrm{E}-01\end{array}$ & $\begin{array}{l}6.86 \mathrm{E}-03 \\
2.18 \mathrm{E}-01 \\
1.40 \mathrm{E}-01 \\
5.73 \mathrm{E}-03 \\
7.18 \mathrm{E}-01 \\
8.09 \mathrm{E}-03 \\
9.94 \mathrm{E}-03 \\
7.56 \mathrm{E}-02 \\
6.38 \mathrm{E}-03 \\
6.99 \mathrm{E}-03 \\
6.47 \mathrm{E}-03 \\
4.86 \mathrm{E}-02 \\
1.29 \mathrm{E}-01 \\
8.07 \mathrm{E}-01 \\
6.09 \mathrm{E}-03 \\
4.03 \mathrm{E}+00 \\
4.89 \mathrm{E}-02 \\
5.91 \mathrm{E}+00 \\
6.05 \mathrm{E}-03 \\
1.19 \mathrm{E}+00 \\
4.90 \mathrm{E}-03 \\
4.71 \mathrm{E}-01 \\
2.69 \mathrm{E}-01 \\
1.53 \mathrm{E}+00 \\
7.20 \mathrm{E}-03 \\
9.72 \mathrm{E}-02 \\
8.09 \mathrm{E}-03 \\
2.32 \mathrm{E}-02 \\
6.94 \mathrm{E}-03 \\
1.72 \mathrm{E}-02\end{array}$ & $\begin{array}{l}2.24 \mathrm{E}-02 \\
5.45 \mathrm{E}-01 \\
4.30 \mathrm{E}-01 \\
1.95 \mathrm{E}-02 \\
1.03 \mathrm{E}+00 \\
1.27 \mathrm{E}-02 \\
1.61 \mathrm{E}-02 \\
4.48 \mathrm{E}-01 \\
1.40 \mathrm{E}-02 \\
2.05 \mathrm{E}-02 \\
2.12 \mathrm{E}-02 \\
1.43 \mathrm{E}-01 \\
2.03 \mathrm{E}-01 \\
1.54 \mathrm{E}+00 \\
7.89 \mathrm{E}-03 \\
5.92 \mathrm{E}+00 \\
7.19 \mathrm{E}-02 \\
1.16 \mathrm{E}+01 \\
2.71 \mathrm{E}-02 \\
1.86 \mathrm{E}+00 \\
1.02 \mathrm{E}-02 \\
7.22 \mathrm{E}-01 \\
5.05 \mathrm{E}-01 \\
5.13 \mathrm{E}+00 \\
2.44 \mathrm{E}-02 \\
3.02 \mathrm{E}-01 \\
1.27 \mathrm{E}-02 \\
3.76 \mathrm{E}-02 \\
2.05 \mathrm{E}-02 \\
2.71 \mathrm{E}-02\end{array}$ \\
\hline Totals & & & $1.41 \mathrm{E}+02$ & $1.62 \mathrm{E}+02$ & $1.58 \mathrm{E}+01$ & $3.08 \mathrm{E}+01$ \\
\hline
\end{tabular}

Table 7. Annual Accident Dose Risk for the Shipping Campaign (person-rem/yr)

\begin{tabular}{|c|c|c|c|c|}
\hline Route & & & Truck & Rail \\
\hline $\begin{array}{l}\text { Ames Laboratory } \\
\text { Argonne - East } \\
\text { Bettis Atomic } \\
\text { Fermilab (FNAI) } \\
\text { INEL } \\
\text { ITRI } \\
\text { ITRI } \\
\text { Knolls Atomic } \\
\text { Kansas City Plant } \\
\text { Lawrence Berkeley } \\
\text { Lawrence Berkeley } \\
\text { Lawrence Iivermore } \\
\text { Lawrence Livermore } \\
\text { Mound Plant } \\
\text { ORISE } \\
\text { Pantex Plant } \\
\text { Pantex Plant } \\
\text { Portsmouth GDP } \\
\text { Princeton PPL } \\
\text { Paducah GDP } \\
\text { Pinellas Plant } \\
\text { Rocky Flats Plant } \\
\text { Rocky Flats Plant } \\
\text { Reactive Metals } \\
\text { SLAC } \\
\text { SLAC } \\
\text { SNL - Albuquerque } \\
\text { SNL - Albuquerque } \\
\text { SNL - Livermore } \\
\text { SNL - Livermore }\end{array}$ & $\begin{array}{l}\text { to } \\
\text { to } \\
\text { to } \\
\text { to } \\
\text { to } \\
\text { to } \\
\text { to } \\
\text { to } \\
\text { to } \\
\text { to } \\
\text { to } \\
\text { to } \\
\text { to } \\
\text { to } \\
\text { to } \\
\text { to } \\
\text { to } \\
\text { to } \\
\text { to } \\
\text { to } \\
\text { to } \\
\text { to } \\
\text { to } \\
\text { to } \\
\text { to } \\
\text { to } \\
\text { to } \\
\text { to } \\
\text { to }\end{array}$ & $\begin{array}{l}\text { Oak Ridge Reserv. } \\
\text { Oak Ridge Reserv. } \\
\text { Oak Ridge Reserv. } \\
\text { Oak Ridge Reserv. } \\
\text { Los Alamos NL } \\
\text { INEI } \\
\text { Los Alamos NL } \\
\text { Oak Ridge Reserv. } \\
\text { Oak Ridge Reserv. } \\
\text { Hanford Site } \\
\text { Nevada Test Site } \\
\text { Hanford Site } \\
\text { Nevada Test Site } \\
\text { Oak Ridge Reserv. } \\
\text { Oak Ridge Reserv. } \\
\text { INEL } \\
\text { Los Alamos NL } \\
\text { Oak Ridge Reserv. } \\
\text { Oak Ridge Reserv. } \\
\text { Oak Ridge Reserv. } \\
\text { Savannah River } \\
\text { INEL } \\
\text { Los Alamos NL } \\
\text { Oak Ridge Reserv. } \\
\text { Hanford Site } \\
\text { Nevada Test Site } \\
\text { INEL } \\
\text { Los Alamos NL } \\
\text { Hanford Site } \\
\text { Nevada Test Site }\end{array}$ & $\begin{array}{l}1.90 \mathrm{E}-05 \\
5.41 \mathrm{E}-02 \\
5.67 \mathrm{E}-04 \\
1.07 \mathrm{E}-04 \\
3.42 \mathrm{E}+01 \\
2.95 \mathrm{E}-02 \\
6.04 \mathrm{E}-03 \\
5.98 \mathrm{E}-05 \\
3.55 \mathrm{E}-05 \\
1.22 \mathrm{E}-01 \\
1.51 \mathrm{E}-02 \\
1.79 \mathrm{E}-04 \\
1.48 \mathrm{E}-04 \\
5.46 \mathrm{E}-04 \\
2.24 \mathrm{E}-07 \\
2.03 \mathrm{E}-03 \\
3.25 \mathrm{E}-05 \\
2.40 \mathrm{E}-05 \\
5.74 \mathrm{E}-05 \\
1.96 \mathrm{E}-04 \\
2.59 \mathrm{E}-06 \\
6.56 \mathrm{E}-04 \\
3.56 \mathrm{E}-04 \\
4.36 \mathrm{E}-06 \\
9.15 \mathrm{E}-10 \\
8.33 \mathrm{E}-06 \\
4.36 \mathrm{E}-05 \\
7.83 \mathrm{E}-05 \\
1.07 \mathrm{E}-04 \\
1.74 \mathrm{E}-04\end{array}$ & $\begin{array}{l}6.83 \mathrm{E}-06 \\
8.83 \mathrm{E}-03 \\
2.35 \mathrm{E}-04 \\
2.31 \mathrm{E}-05 \\
2.30 \mathrm{E}+00 \\
1.85 \mathrm{E}-03 \\
1.11 \mathrm{E}-04 \\
3.47 \mathrm{E}-05 \\
7.40 \mathrm{E}-06 \\
6.80 \mathrm{E}-02 \\
1.03 \mathrm{E}-02 \\
9.36 \mathrm{E}-05 \\
4.47 \mathrm{E}-05 \\
5.81 \mathrm{E}-05 \\
6.30 \mathrm{E}-08 \\
1.36 \mathrm{E}-04 \\
2.02 \mathrm{E}-06 \\
4.55 \mathrm{E}-06 \\
2.32 \mathrm{E}-06 \\
2.57 \mathrm{E}-05 \\
4.65 \mathrm{E}-07 \\
1.04 \mathrm{E}-04 \\
1.53 \mathrm{E}-05 \\
8.20 \mathrm{E}-07 \\
5.37 \mathrm{E}-10 \\
5.08 \mathrm{E}-06 \\
2.79 \mathrm{E}-06 \\
1.50 \mathrm{E}-06 \\
5.69 \mathrm{E}-05 \\
5.48 \mathrm{E}-05\end{array}$ \\
\hline otals & & & $3.44 \Sigma+01$ & 2.35 \\
\hline
\end{tabular}




\section{CONTENTS (Cont.)}

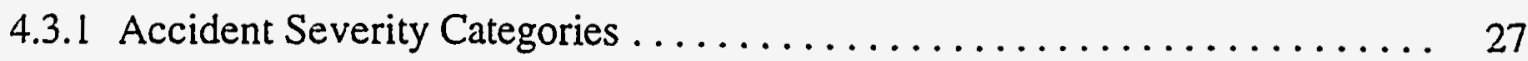

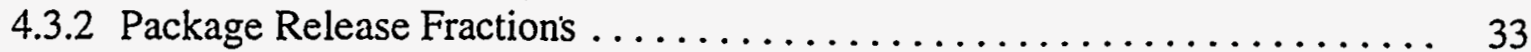

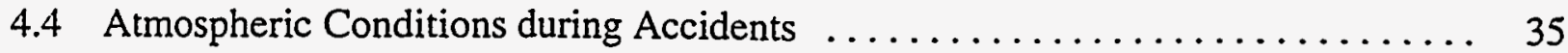

4.5 External Dose Rates from Shipments ...................... 36

4.6 Maximally Exposed Individual Exposure Scenarios ................. 37

4.7 General RADTRAN Input Parameters $\ldots \ldots \ldots \ldots \ldots \ldots \ldots \ldots \ldots \ldots \ldots \ldots \ldots \ldots \ldots \ldots$

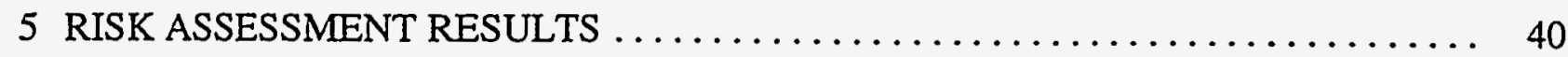

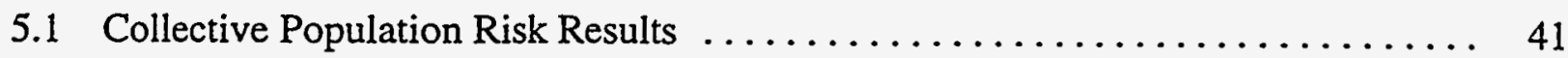

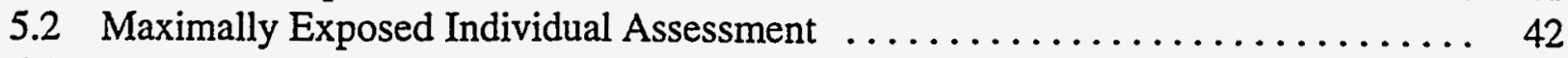

5.3 Accident Consequence Assessment $\ldots \ldots \ldots \ldots \ldots \ldots \ldots \ldots \ldots \ldots \ldots \ldots \ldots$

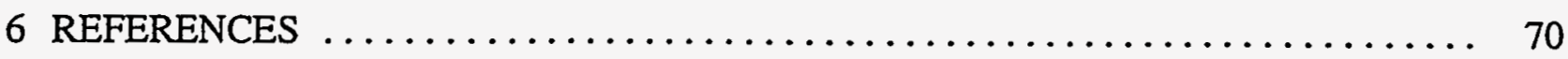

APPENDIX A: Transported Material Summary Files Generated by the WASTE_MGMT Model $\ldots \ldots \ldots \ldots \ldots \ldots \ldots \ldots \ldots \ldots \ldots \ldots$ A -1

APPENDIX B: Transportation Risk Assessment Results $\ldots \ldots \ldots \ldots \ldots \ldots \ldots \ldots$ B-1

\section{TABLES}

2.1 Summary of WM LLW Cases $\ldots \ldots \ldots \ldots \ldots \ldots \ldots \ldots \ldots \ldots \ldots \ldots \ldots \ldots \ldots \ldots \ldots \ldots$

3.1 Truck Route Distances between Major DOE Sites $\ldots \ldots \ldots \ldots \ldots \ldots \ldots \ldots .21$

3.2 Rail Route Distances between Major DOE Sites $\ldots \ldots \ldots \ldots \ldots \ldots \ldots \ldots \ldots$

4.1 Highway Combination-Truck Accident, Fatality, and Injury Rates by Road Type Based on Reportable Interstate-Carrier Accidents, Reported Fatalities and Injuries, and Estimated Flows, 1986-1988

4.2 Railroad-Freight-Car Accident Rates, by State, for 1986, 1987, 1988, and 1985-1988 Combined

4.3 Fractional Occurrences for Accidents by Severity Category and Population

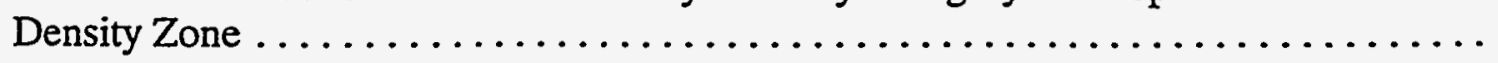




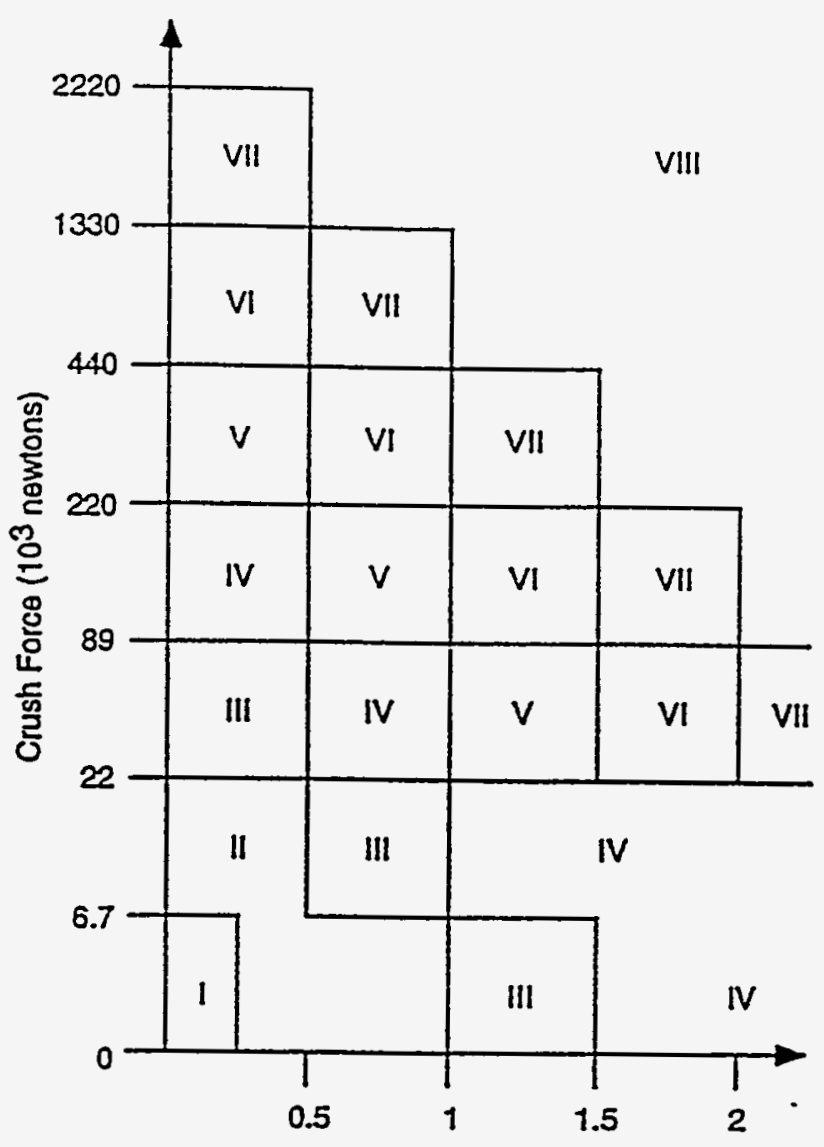

1300 Kelvin Fire Duration (hours)

FIGURE 4.1 Scheme for NUREG-0170 Classification by Accident Severity Category for Truck Accidents (Source: NRC 1977a)

that if an accident occurs, it is of a particular severity - is assigned to each category. The fractional occurrences for accidents by the accident severity category and the population density zone are shown in Table 4.3.

Category I accidents are the least severe but the most frequent, whereas Category VIII accidents are very severe but very infrequent. To determine the expected frequency of an accident of a given severity, the conditional probability in the category is multiplied by the baseline accident rate. Each population density zone has a distinct baseline accident rate and distribution of accident severities related to differences in average vehicular velocity, traffic density, and other factors, including location (rural, suburban, or urban).

For the accident consequence assessment, the doses were assessed for populations and individuals by assuming an accident of severity Category VIII. This accident severity category represents the most severe accident scenarios that can be postulated that would result in the largest 


\begin{tabular}{lll}
$\begin{array}{l}\text { Table 8. Expected Annual Fatalities } \\
\text { for the Shipping Campaign }\end{array}$ & \\
\hline Exposure Group & Truck & Rail \\
\hline Radiological & & \\
$\quad$ Normal Crew & $5.6 E-02$ & $6.3 E-03$ \\
Normal Public & $8.1 \mathrm{E}-02$ & $1.5 \mathrm{E}-02$ \\
Accident public & $1.7 \mathrm{E}-02$ & $1.2 \mathrm{E}-03$ \\
Nonradiological & $2.3 \mathrm{E}-02$ & $1.7 \mathrm{E}-02$ \\
$\quad$ Emission & $3.3 \mathrm{E}-01$ & $3.8 \mathrm{E}-03$ \\
\hline Accident & &
\end{tabular}

Table 9. Expected Annual Cancer Incidence for the Shipping Campaign

\begin{tabular}{lll}
\hline Exposure Group & Truck & Rail \\
\hline Radiological & $2.0 \mathrm{E}-01$ & $2.2 \mathrm{E}-02$ \\
Normal Crew & $2.8 \mathrm{E}-01$ & $5.2 \mathrm{E}-02$ \\
Normal Public & $5.9 \mathrm{E}-02$ & $4.1 \mathrm{E}-03$ \\
Accident Public & & \\
$\begin{array}{c}\text { Nonradiological } \\
\text { Emission }\end{array}$ & $2.3 \mathrm{E}-02$ & $1.7 \mathrm{E}-02$ \\
Accident & $\mathrm{NA}$ & $\mathrm{NA}$ \\
\hline
\end{tabular}

Table 10. Expected Annual Genetic Effects for the Shipping Campaign

\begin{tabular}{lll}
\hline Exposure Group & Truck & Rail \\
\hline Radiological & & \\
Normal Crew & $8.5 E-03$ & $9.5 E-04$ \\
Normal Public & $1.6 E-02$ & $3.1 E-03$ \\
Accident Public & $2.9 E-03$ & $1.8 E-04$ \\
Nonradiological & NA & NA \\
Emission & NA & NA \\
Accident & & \\
\hline
\end{tabular}




\section{B.14 WM LLW REGIONALIZED 5 ALTERNATIVE (CASE 19): ACTIVATED METALS}

Table 1. Summary of Route Information

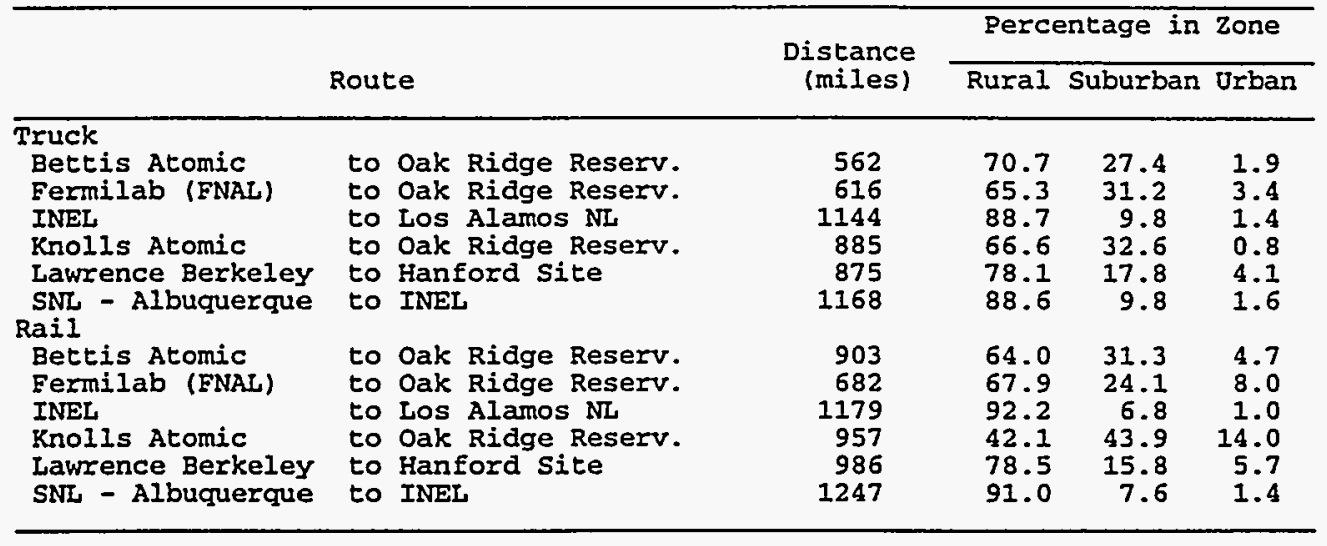

Table 2. Annual Number of Shipments and One-Way Shipment Distances

\begin{tabular}{|c|c|c|c|c|c|}
\hline \multirow[b]{2}{*}{ Route } & & \multicolumn{2}{|c|}{ \# of Shipments } & \multicolumn{2}{|c|}{ Total Mileage (mi) } \\
\hline & & Truck & Rail & Truck & Rail \\
\hline $\begin{array}{l}\text { Bettis Atomic } \\
\text { Fermilab (FNAL) } \\
\text { INEL } \\
\text { Rnolls Atomic } \\
\text { Lawrence Berkeley } \\
\text { SNL - Albuquerque }\end{array}$ & $\begin{array}{l}\text { to Oak Ridge Reserv. } \\
\text { to Oak Ridge Reserv. } \\
\text { to Los Alamos NL } \\
\text { to Oak Ridge Reserv. } \\
\text { to Hanford Site } \\
\text { to INEL }\end{array}$ & $\begin{array}{r}309 \\
39 \\
739 \\
639 \\
14 \\
6\end{array}$ & $\begin{array}{r}119 \\
16 \\
298 \\
245 \\
6 \\
3\end{array}$ & $\begin{array}{l}1.74 \mathrm{E}+05 \\
2.40 \mathrm{E}+04 \\
8.45 \mathrm{E}+05 \\
5.66 \mathrm{E}+05 \\
1.22 \mathrm{E}+04 \\
7.01 \mathrm{E}+03\end{array}$ & $\begin{array}{l}1.07 E+05 \\
1.09 E+04 \\
3.51 E+05 \\
2.34 E+05 \\
5.91 E+03 \\
3.74 E+03\end{array}$ \\
\hline Totals & & 1746 & 687 & $1.63 \mathrm{E}+06$ & $7.14 E+05$ \\
\hline
\end{tabular}

Table 3. Incident-Free Dose per Shipment (person-rem/shipment)

\begin{tabular}{|c|c|c|c|c|c|c|}
\hline \multirow[b]{2}{*}{ Source/Route (s) } & \multirow{2}{*}{\multicolumn{2}{|c|}{ Crew }} & \multicolumn{4}{|c|}{ General Public } \\
\hline & & & Off-Link & On-Link & Stops & Total \\
\hline $\begin{array}{l}\text { LLW(I) BA to OR } \\
\text { Bettis Atomic }\end{array}$ & $\begin{array}{l}\text { to Oak } \\
\text { Truck } \\
\text { Rail. }\end{array}$ & $\begin{array}{r}\text { Ridge Reserv } \\
1.85 \mathrm{E}-02 \\
6.65 \mathrm{E}-03\end{array}$ & $\begin{array}{l}1.72 \mathrm{E}-03 \\
1.40 \mathrm{E}-02\end{array}$ & $\begin{array}{l}2.71 E-03 \\
2.44 \mathrm{E}-04\end{array}$ & $\begin{array}{l}1.57 \mathrm{E}-02 \\
6.26 \mathrm{E}-03\end{array}$ & $\begin{array}{l}2.02 E-02 \\
2.05 E-02\end{array}$ \\
\hline $\begin{array}{l}\text { LLW(I) FL to OR } \\
\text { Fermilab (FNAL) }\end{array}$ & $\begin{array}{l}\text { to Oak } \\
\text { Truck } \\
\text { Rail }\end{array}$ & $\begin{array}{c}\text { Ridge Reserv } \\
2.15 \mathrm{E}-02 \\
5.73 \mathrm{E}-03\end{array}$ & $\begin{array}{l}2.53 \mathrm{E}-03 \\
1.37 \mathrm{E}-02\end{array}$ & $\begin{array}{l}3.71 \Xi-03 \\
1.92 \mathrm{E}-04\end{array}$ & $\begin{array}{l}1.72 \mathrm{E}-02 \\
5.58 \mathrm{E}-03\end{array}$ & $\begin{array}{l}2.35 E-02 \\
1.95 E-02\end{array}$ \\
\hline $\begin{array}{l}\text { LLW(I) IN to LA } \\
\text { INEL }\end{array}$ & $\begin{array}{l}\text { to Ios } \\
\text { Truck } \\
\text { Rail }\end{array}$ & $\begin{array}{r}\text { Alamos } \times \mathrm{NL} \\
3.15 \mathrm{E}-02 \\
7.80 \mathrm{E}-03\end{array}$ & $\begin{array}{l}1.73 \mathrm{E}-03 \\
4.00 \mathrm{E}-03\end{array}$ & $\begin{array}{l}4.27 E-03 \\
1.08 E-04\end{array}$ & $\begin{array}{l}3.20 \mathrm{E}-02 \\
7.11 \mathrm{E}-03\end{array}$ & $\begin{array}{l}3.80 \mathrm{E}-02 \\
1.12 \mathrm{E}-02\end{array}$ \\
\hline $\begin{array}{l}\text { LLW (I) KA to OR } \\
\text { Knolis Atomic }\end{array}$ & $\begin{array}{l}\text { to Oak } \\
\text { Truck } \\
\text { Rail }\end{array}$ & $\begin{array}{c}\text { Riage Reserv } \\
2.98 \mathrm{E}-02 \\
6.87 \mathrm{E}-03\end{array}$ & $\begin{array}{l}2.55 \mathrm{E}-03 \\
3.39 \mathrm{E}-02\end{array}$ & $\begin{array}{l}3.79 \mathrm{E}-03 \\
4.49 \mathrm{E}-04\end{array}$ & $\begin{array}{l}2.48 \mathrm{E}-02 \\
6.42 \mathrm{E}-03\end{array}$ & $\begin{array}{l}3.11 \mathrm{E}-02 \\
4.08 \mathrm{E}-02\end{array}$ \\
\hline $\begin{array}{l}\text { LLW(I) LAA to IN } \\
\text { INEL }\end{array}$ & $\begin{array}{l}\text { to Ios } \\
\text { Truck } \\
\text { Rail }\end{array}$ & $\begin{array}{l}\text { Alamos NL } \\
3.15 \mathrm{E}-02 \\
7.80 \mathrm{E}-03\end{array}$ & $\begin{array}{l}1.73 \mathrm{E}-03 \\
4.00 \mathrm{E}-03\end{array}$ & $\begin{array}{l}4.27 \mathrm{E}-03 \\
1.08 \mathrm{E}-04\end{array}$ & $\begin{array}{l}3.20 \mathrm{E}-02 \\
7.11 \mathrm{E}-03\end{array}$ & $\begin{array}{l}3.80 \mathrm{E}-02 \\
1.12 \mathrm{E}-02\end{array}$ \\
\hline $\begin{array}{l}\text { LLW(I) LB to HS } \\
\text { Lawrence Berkeley }\end{array}$ & $\begin{array}{l}\text { to Hanf } \\
\text { Truck } \\
\text { Rail }\end{array}$ & $\begin{array}{r}\text { ford Site } \\
2.76 \mathrm{E}-02 \\
6.99 \mathrm{E}-03\end{array}$ & $\begin{array}{l}3.02 \mathrm{E}-03 \\
1.38 \mathrm{E}-02\end{array}$ & $\begin{array}{l}5.17 \mathrm{E}-03 \\
2.03 \mathrm{E}-04\end{array}$ & $\begin{array}{l}2.45 \mathrm{E}-02 \\
6.51 \mathrm{E}-03\end{array}$ & $\begin{array}{l}3.27 E-02 \\
2.05 E-02\end{array}$ \\
\hline $\begin{array}{l}\text { LLW (I) SA to IN } \\
\text { SNL - Albuquerque }\end{array}$ & $\begin{array}{l}\text { to INEI } \\
\text { Truck } \\
\text { Rail }\end{array}$ & $\begin{array}{l}3.22 \mathrm{E}-02 \\
8.09 \mathrm{E}-03\end{array}$ & $\begin{array}{l}1.83 E-03 \\
5.30 E-03\end{array}$ & $\begin{array}{l}4.46 E-03 \\
1.26 E-04\end{array}$ & $\begin{array}{l}3.27 E-02 \\
7.32 E-03\end{array}$ & $\begin{array}{l}3.90 E-02 \\
1.27 E-02\end{array}$ \\
\hline
\end{tabular}


Table 4. Accident Dose Risk per Shipment (person-rem/shipment)

\begin{tabular}{|c|c|c|c|}
\hline Source/Roure & & Truck & Rail \\
\hline $\begin{array}{l}\text { LLW(I) BA to OR } \\
\text { Bettis Atomic } \\
\text { LLW(I) FL to OR } \\
\text { Fermi lab (FNAL) } \\
\text { LLW(I) IN to LA } \\
\text { INEL } \\
\text { LLW(I) KA to OR } \\
\text { KnOIls Atomic } \\
\text { LLW(I) LA to IN } \\
\text { INEL } \\
\text { LLW(I) LB to HS } \\
\text { Lawrence Berkeley } \\
\text { LLW(I) SA to IN } \\
\text { SNL - Albuquerque }\end{array}$ & $\begin{array}{l}\text { to Oak Ridge Reserv. } \\
\text { to Oak Ridge Reserv. } \\
\text { to Los Alamos NL } \\
\text { to Oak Ridge Reserv. } \\
\text { to Los Alamos NL } \\
\text { to Hanford Site } \\
\text { to INEL }\end{array}$ & $\begin{array}{l}7.44 \mathrm{E}-07 \\
7.76 \mathrm{E}-11 \\
3.54 \mathrm{E}-09 \\
1.50 \mathrm{E}-06 \\
2.80 \mathrm{E}-09 \\
1.81 \mathrm{E}-10 \\
5.42 \mathrm{E}-12\end{array}$ & $\begin{array}{l}6.41 \mathrm{E}-07 \\
4.09 \mathrm{E}-11 \\
4.78 \mathrm{E}-10 \\
2.30 \mathrm{E}-06 \\
4.24 \mathrm{E}-10 \\
2.37 \mathrm{E}-10 \\
6.53 \mathrm{E}-13\end{array}$ \\
\hline
\end{tabular}

Table 5. Nonradiological Risk Factors per Shipment (fatalities/shipment)

\begin{tabular}{llll}
\hline Mode & Route & Emission & Accident \\
\hline Truck & & & \\
Bettis Atomic & to Oak Ridge Reserv. & $3.41 E-06$ & $3.71 \mathrm{E}-05$ \\
Fermilab (FNAL) & to Oak Ridge Reserv. & $6.76 \mathrm{E}-06$ & $4.62 \mathrm{E}-05$ \\
INEL & to Los Alamos NL & $5.25 \mathrm{E}-06$ & $8.21 \mathrm{E}-05$ \\
Knolls Atomic & to Oak Ridge Reserv. & $2.16 \mathrm{E}-06$ & $6.08 \mathrm{E}-05$ \\
Lawrence Berkeley & to Hanford Site & $1.16 \mathrm{E}-05$ & $4.91 \mathrm{E}-05$ \\
SNL - Albuquerque & to INEL & $5.83 \mathrm{E}-06$ & $8.45 \mathrm{E}-05$ \\
Rail & & & \\
Bettis Atomic & to Oak Ridge Reserv. & $1.78 \mathrm{E}-05$ & $1.89 \mathrm{E}-06$ \\
Fermilab (FNAL) & to Oak Ridge Reserv. & $2.28 \mathrm{E}-05$ & $1.43 \mathrm{E}-06$ \\
INEL & to Los Alamos NL & $4.90 \mathrm{E}-06$ & $2.47 \mathrm{E}-06$ \\
Knolls Atomic & to Oak Ridge Reserv. & $5.59 \mathrm{E}-05$ & $2.00 \mathrm{E}-06$ \\
Lawrence Berkeley & to Hanford Site & $2.33 \mathrm{E}-05$ & $2.06 \mathrm{E}-06$ \\
SNL - Albuquerque & to INEL & $7.11 \mathrm{E}-06$ & $2.61 \mathrm{E}-06$ \\
\hline
\end{tabular}

Table 6. Annual Incident-Free Dose for the Shipping Campaign (person-rem/yr)

\begin{tabular}{|c|c|c|c|c|c|}
\hline \multirow[b]{2}{*}{ Route } & & \multicolumn{2}{|c|}{ Truck } & \multicolumn{2}{|c|}{ Rail } \\
\hline & & Crew & Public & Crew & Public \\
\hline $\begin{array}{l}\text { Bettis Atomic } \\
\text { Fermilab (FNAL) } \\
\text { INEL } \\
\text { Knolls Atomic } \\
\text { Lawrence Berkeley } \\
\text { SNL - Albuquerque }\end{array}$ & $\begin{array}{l}\text { to Oak Ridge Reserv. } \\
\text { to Oak Ridge Reserv. } \\
\text { to Los Alamos NL } \\
\text { to Oak Ridge Reserv. } \\
\text { to Hanford Site } \\
\text { to INEL }\end{array}$ & $\begin{array}{l}5 \cdot 71 E+00 \\
8.38 E-01 \\
2.33 E+01 \\
1.90 E+01 \\
3.86 E-01 \\
1.93 E-01\end{array}$ & $\begin{array}{l}6.24 \mathrm{E}+00 \\
9.16 \mathrm{E}-01 \\
2.81 \mathrm{E}+01 \\
1.99 \mathrm{E}+01 \\
4.58 \mathrm{E}-01 \\
2.34 \mathrm{E}-01\end{array}$ & $\begin{array}{l}7.91 \mathrm{E}-01 \\
9.16 \mathrm{E}-02 \\
2.32 \mathrm{E}+00 \\
1.68 \mathrm{E}+00 \\
4.20 \mathrm{E}-02 \\
2.43 \mathrm{E}-02\end{array}$ & $\begin{array}{l}2.44 \mathrm{E}+00 \\
3.12 \mathrm{E}-01 \\
3.34 \mathrm{E}+00 \\
9.99 \mathrm{E}+00 \\
1.23 \mathrm{E}-01 \\
3.82 \mathrm{E}-02\end{array}$ \\
\hline Totals & & $4.94 E+01$ & $5.58 E+01$ & $4.96 \mathrm{E}+00$ & $1.62 \mathrm{E}+01$ \\
\hline
\end{tabular}

Table 7. Annual Accident Dose Risk for the Shipping Campaign (person-rem/yr)

\begin{tabular}{|c|c|c|c|}
\hline Route & & Truck & Rail \\
\hline $\begin{array}{l}\text { Bettis Atomic } \\
\text { Eermilab (FNAL) } \\
\text { INEL } \\
\text { Rnolls Atomic } \\
\text { Lawrence Berkeley } \\
\text { SNL - Albuquerque }\end{array}$ & $\begin{array}{l}\text { to Oak Ridge Reserv. } \\
\text { to Oak Ridge Reserv. } \\
\text { to Los Alamos NL } \\
\text { to Oak Ridge Reserv. } \\
\text { to Hanford Site } \\
\text { to INEL }\end{array}$ & $\begin{array}{l}2.30 \mathrm{E}-04 \\
3.03 \mathrm{E}-09 \\
2.31 \mathrm{E}-06 \\
9.58 \mathrm{E}-04 \\
2.53 \mathrm{E}-09 \\
3.25 \mathrm{E}-11\end{array}$ & $\begin{array}{l}7.63 \mathrm{E}-05 \\
6.54 \mathrm{E}-10 \\
1.34 \mathrm{E}-07 \\
5.64 \mathrm{E}-04 \\
1.42 \mathrm{E}-09 \\
1.96 \mathrm{E}-12\end{array}$ \\
\hline Totals & & $1.19 \mathrm{E}-03$ & $6.41 \mathrm{E}-04$ \\
\hline
\end{tabular}


Table 8. Expected Annual Fatalities

for the Shipping Campaign

\begin{tabular}{llc}
\hline Exposure Group & Truck & Rail \\
\hline Radiological & & \\
Normal Crew & $2.0 \mathrm{E}-02$ & $2.0 \mathrm{E}-03$ \\
Normal Public & $2.8 \mathrm{E}-02$ & $8.1 \mathrm{E}-03$ \\
Accident Public & $6.0 \mathrm{E}-07$ & $3.2 \mathrm{E}-07$ \\
Nonradiological & & \\
Emission & $6.8 \mathrm{E}-03$ & $1.8 \mathrm{E}-02$ \\
Accident & $1.1 \mathrm{E}-01$ & $1.5 \mathrm{E}-03$ \\
\hline
\end{tabular}

Table 9. Expected Annual Cancer Incidence for the shipping Campaign

\begin{tabular}{lll}
\hline Exposure Group & Truck & Rail \\
\hline Radiological & & \\
Normal Crew & $6.9 \mathrm{E}-02$ & $6.9 \mathrm{E}-03$ \\
Normal Public & $9.5 \mathrm{E}-02$ & $2.8 \mathrm{E}-02$ \\
Accident Public & $2.0 \mathrm{E}-06$ & $1.1 \mathrm{E}-06$ \\
$\begin{array}{c}\text { Nonradiological } \\
\text { Emission }\end{array}$ & $6.8 \mathrm{E}-03$ & $1.8 \mathrm{E}-02$ \\
Accident & $\mathrm{NA}$ & $\mathrm{NA}$ \\
\hline
\end{tabular}

Table 10. Expected Annual Genetic Effects

for the Shipping Campaign

\begin{tabular}{lll}
\hline Exposure Group & Truck & Rail \\
\hline Radiological & $3.0 \mathrm{E}-03$ & $3.0 \mathrm{E}-04$ \\
Normal Crew & $5.6 \mathrm{E}-03$ & $1.6 \mathrm{E}-03$ \\
Normal Public & $1.2 \mathrm{E}-07$ & $6.4 \mathrm{E}-08$ \\
Accident Public & $\mathrm{NA}$ & $\mathrm{NA}$ \\
Nonradiological & $\mathrm{NA}$ & $\mathrm{NA}$ \\
Emission & $\mathrm{NAc}$ & \\
\hline
\end{tabular}


B.15 WM LLW REGIONALIZED 6 ALTERNATIVE (CASE 5): HETEROGENEOUS SOLIDS

Table 1. Sumnary of Route Information

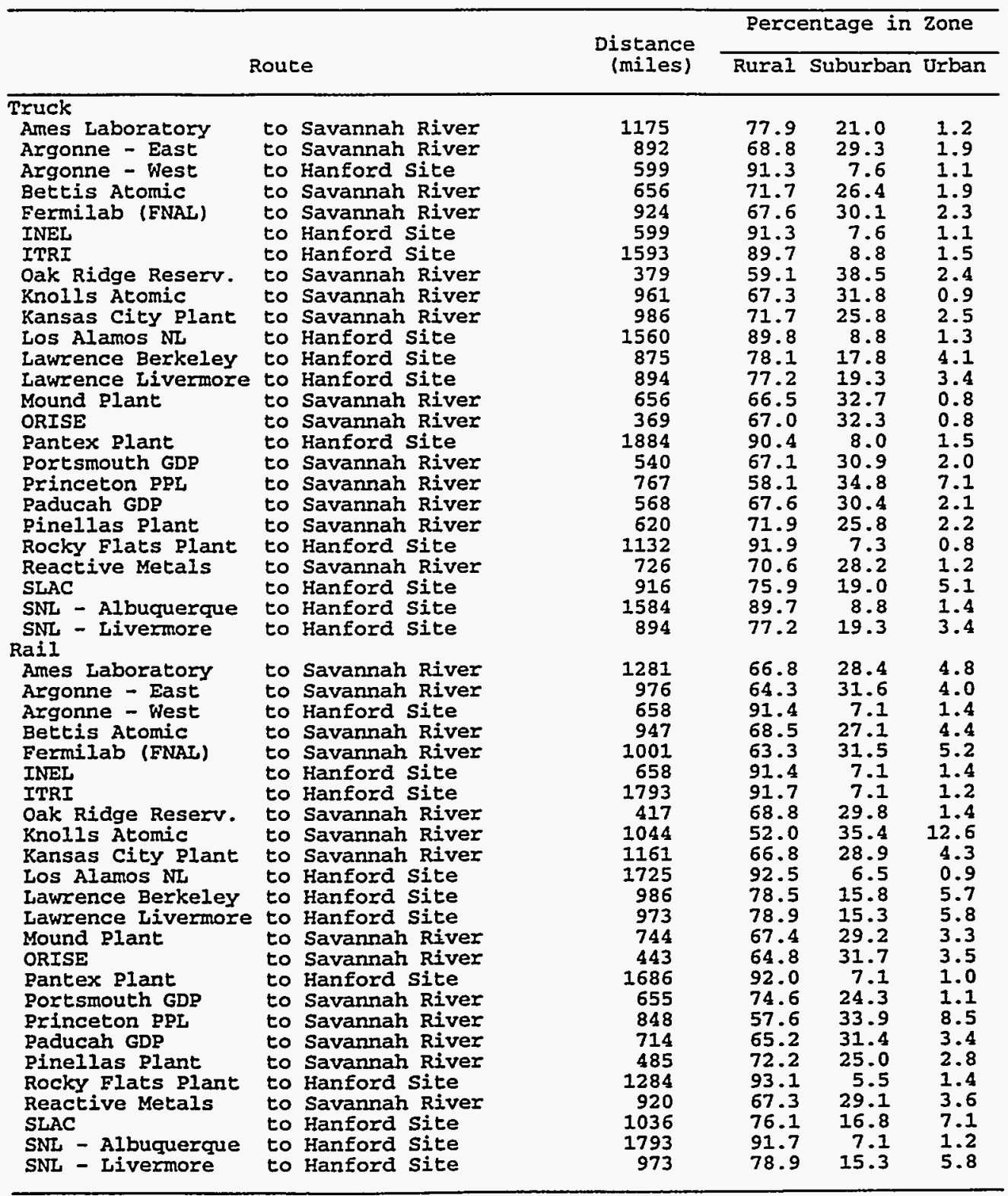


Table 2. Annual Number of Shipments and One-Way Shipment Distances

\begin{tabular}{|c|c|c|c|c|c|}
\hline \multirow[b]{2}{*}{ Route } & & \multicolumn{2}{|c|}{ \# of Shipments } & \multicolumn{2}{|c|}{ Total Mileage (mi) } \\
\hline & & Truck & Rail & Truck & Rail \\
\hline $\begin{array}{l}\text { Ames Laboratory } \\
\text { Argonne - East } \\
\text { Argonne - West } \\
\text { Bettis Atomic } \\
\text { Fermilab (FNAL) } \\
\text { INEL } \\
\text { ITRI } \\
\text { Oak Ridge Reserv. } \\
\text { Knolis Atomic } \\
\text { Kansas City Plant } \\
\text { Los Alamos NL } \\
\text { Lawrence Berkeley } \\
\text { Lawrence Livermore } \\
\text { Mound Plant } \\
\text { ORISE } \\
\text { Pantex Plant } \\
\text { Portsmouth GDP } \\
\text { Princeton PPL } \\
\text { Paducah GDP } \\
\text { Pinellas Plant } \\
\text { Rocky Flats Plant } \\
\text { Reactive Metals } \\
\text { SLAC } \\
\text { SNL - Albuquerque } \\
\text { SNL - Livermore }\end{array}$ & $\begin{array}{l}\text { to Savannah River } \\
\text { to Savannah River } \\
\text { to Hanford Site } \\
\text { to Savannah River } \\
\text { to Savannah River } \\
\text { to Hanford Site } \\
\text { to Hanford Site } \\
\text { to Savannah River } \\
\text { to Savannah River } \\
\text { to Savannah River } \\
\text { to Hanford Site } \\
\text { to Hanford Site } \\
\text { to Hanford Site } \\
\text { to Savannah River } \\
\text { to Savannah River } \\
\text { to Hanford Site } \\
\text { to Savannah River } \\
\text { to Savannah River } \\
\text { to Savannah River } \\
\text { to Savannah River } \\
\text { to Hanford Site } \\
\text { to Savannah River } \\
\text { to Hanford Site } \\
\text { to Hanford Sitte } \\
\text { to Hanford Site }\end{array}$ & $\begin{array}{r}2 \\
105 \\
13 \\
57 \\
3 \\
852 \\
10 \\
6542 \\
28 \\
1 \\
724 \\
3 \\
56 \\
512 \\
5 \\
1450 \\
3344 \\
1 \\
627 \\
2 \\
357 \\
768 \\
39 \\
17 \\
6\end{array}$ & $\begin{array}{r}1 \\
39 \\
5 \\
21 \\
2 \\
322 \\
4 \\
2486 \\
11 \\
1 \\
268 \\
2 \\
21 \\
190 \\
2 \\
544 \\
1274 \\
1 \\
237 \\
1 \\
133 \\
287 \\
15 \\
7 \\
2\end{array}$ & $\begin{array}{l}2.35 E+03 \\
9.36 E+04 \\
7.79 E+03 \\
3.74 E+04 \\
2.77 E+03 \\
5.10 E+05 \\
1.59 E+04 \\
2.48 E+06 \\
2.69 E+04 \\
9.86 E+02 \\
1.13 E+06 \\
2.62 E+03 \\
5.01 E+04 \\
3.36 E+05 \\
1.84 E+03 \\
2.73 E+06 \\
1.81 E+06 \\
7.67 E+02 \\
3.56 E+05 \\
1.24 E+03 \\
4.04 E+05 \\
5.57 E+05 \\
3.57 E+04 \\
2.69 E+04 \\
5.36 E+03\end{array}$ & 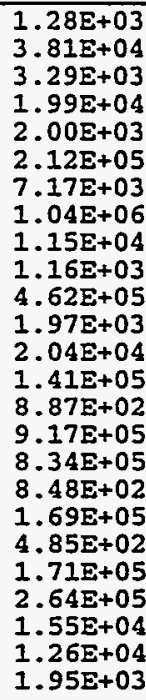 \\
\hline Totals & & 15524 & 5876 & $1.06 \mathrm{E}+07$ & $4.35 E+06$ \\
\hline
\end{tabular}


Table 3. Incident-Free Dose per Shipment (person-rem/shipment)

\begin{tabular}{|c|c|c|c|c|c|}
\hline \multirow[b]{2}{*}{ Source/Route (s) } & \multirow[b]{2}{*}{ Crew } & \multicolumn{4}{|c|}{ General Public } \\
\hline & & off-Link & On-Link & Stops & Total \\
\hline $\begin{array}{l}\text { LLW (x) AL Eo SR } \\
\text { Ames Laboratory }\end{array}$ & $\begin{array}{lr}\text { ro Savannah } & \text { River } \\
\text { Truck } & 3.59 \mathrm{E}-02 \\
\text { Rail } & 8.23 \mathrm{E}-03\end{array}$ & $\begin{array}{l}2.61 \mathrm{E}-03 \\
1.93 \mathrm{E}-02\end{array}$ & $\begin{array}{l}E-03 \\
E-04\end{array}$ & $\begin{array}{l}E-02 \\
E-03\end{array}$ & $\begin{array}{l}4.03 E-02 \\
2.70 E-02\end{array}$ \\
\hline $\begin{array}{l}\text { (x) AN } \\
\text { Argonne }\end{array}$ & $\begin{array}{lr}\text { to Savannah River } \\
\text { Truck } & 2.98 E-02 \\
\text { Rail } & 6.95 E-03\end{array}$ & $\varepsilon-03$ & $\begin{array}{l}4.37 \mathrm{E}-03 \\
2.55 \mathrm{E}-04\end{array}$ & $\begin{array}{l}E-02 \\
E-03\end{array}$ & $\begin{array}{l}3.22 \mathrm{E}-02 \\
2.07 \mathrm{E}-02\end{array}$ \\
\hline $\begin{array}{l}\text { LLW(x) AW to HS } \\
\text { Argonne - West }\end{array}$ & $\begin{array}{ll}\text { to Hanford Site } \\
\text { Truck } & 1.60 \mathrm{E}-02 \\
\text { Rail } & 5.63 \mathrm{E}-03\end{array}$ & $\begin{array}{l}7.18 E-04 \\
2.83 E-03\end{array}$ & $\begin{array}{l}2.06 E-03 \\
6.58 E-05\end{array}$ & $\begin{array}{l}\frac{1}{5} \cdot 68 \mathrm{E}-02 \\
5.51 \mathrm{E}-03\end{array}$ & $\begin{array}{l}1.95 E-02 \\
8.40 E-03\end{array}$ \\
\hline $\begin{array}{r}\text { LLW }(x) \text { BA } \\
\text { Bettis }\end{array}$ & $\begin{array}{lr}\text { to Savannah River } \\
\text { Truck } & 2.14 E-02 \\
\text { Rail } & 6.83 E-03\end{array}$ & $\begin{array}{l}1.9 \\
1.3\end{array}$ & $\begin{array}{l}3.1 \\
2.3\end{array}$ & $\begin{array}{l}1.84 \mathrm{E}-02 \\
6.39 \mathrm{E}-03\end{array}$ & $\begin{array}{l}2.35 E-02 \\
1.99 E-02\end{array}$ \\
\hline Fermilab (ENA & $\begin{array}{ll}\text { to Savannah River } \\
\text { Truck } & 3.13 \mathrm{E}-02 \\
\text { Rail } & 7.06 \mathrm{E}-03\end{array}$ & $\begin{array}{l}3.20 \mathrm{E}-03 \\
1.65 \mathrm{E}-02\end{array}$ & $\begin{array}{l}4.81 E-03 \\
2.79 E-04\end{array}$ & $\begin{array}{l}E-02 \\
E-03\end{array}$ & $\begin{array}{l}3.39 E-02 \\
2.33 E-02\end{array}$ \\
\hline 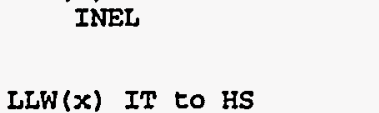 & $\begin{array}{ll}\text { to Hanford Site } \\
\text { Truck } & 1.60 \mathrm{E}-02 \\
\text { Rail } & 5.63 \mathrm{E}-03\end{array}$ & $\begin{array}{l}7.18 \\
2.8\end{array}$ & $\begin{array}{l}2.06 \mathrm{E}-03 \\
6.58 \mathrm{E}-05\end{array}$ & $\begin{array}{l}-02 \\
-03\end{array}$ & $\begin{array}{l}-02 \\
-03\end{array}$ \\
\hline & $\begin{array}{lr}\text { to Hanford Site } \\
\text { Truck } & 4.34 \mathrm{E}-02 \\
\text { Rail } & 1.04 \mathrm{E}-02\end{array}$ & $\begin{array}{l}2.30 \mathrm{E}-03 \\
6.90 \mathrm{E}-03\end{array}$ & $\begin{array}{l}5.9 \\
1.7\end{array}$ & $\begin{array}{l}4.46 E-02 \\
9.00 E-03\end{array}$ & $:-02$ \\
\hline $\begin{array}{l}\text { LLW (x) OR to SR } \\
\text { Oak Ridge Res }\end{array}$ & $\begin{array}{ll}\text { to Savannah River } \\
\text { Truck } & 1.38 \mathrm{E}-02 \\
\text { Rail } & 4.62 \mathrm{E}-03\end{array}$ & & $\begin{array}{l}2.1 \\
8.5\end{array}$ & $\frac{1}{4}$ & $\begin{array}{l}E-02 \\
E-03\end{array}$ \\
\hline Knolls Atomic & $\begin{array}{lr}\text { to Savannah River } \\
\text { Truck } & 3.22 \mathrm{E}-02 \\
\text { Rail } & 7.24 \mathrm{E}-03\end{array}$ & $\begin{array}{l}2.78 E-03 \\
3.25 E-02\end{array}$ & $\begin{array}{l}4.17 E-03 \\
4.25 E-04\end{array}$ & $\begin{array}{l}2.6 \\
6.6\end{array}$ & -02 \\
\hline Ransas City Plant & $\begin{array}{lr}\text { to Savannah River } \\
\text { Truck } & 3.23 \mathrm{E}-02 \\
\text { Rail } & 7.73 \mathrm{E}-03\end{array}$ & $\begin{array}{l}3.2 \\
1.6\end{array}$ & $\begin{array}{l}5.1 \\
2 .\end{array}$ & & $\begin{array}{l}-02 \\
-02\end{array}$ \\
\hline$V(x)$ IB to HS & $\begin{array}{lr}\text { to Hanford Site } \\
\text { Truck } \\
\text { Rail } & 4.24 \mathrm{E}-02 \\
& 1.01 \mathrm{E}-02\end{array}$ & $\begin{array}{l}2.16 \mathrm{E}-03 \\
5.60 \mathrm{E}-03\end{array}$ & $\begin{array}{l}5.6 \\
1.5\end{array}$ & $\begin{array}{l}-02 \\
-03\end{array}$ & $\begin{array}{l}-02 \\
-02\end{array}$ \\
\hline $\begin{array}{l}\text { LLW(x) LB to HS } \\
\text { Lawrence Berkeley }\end{array}$ & $\begin{array}{l}\text { Inford site } \\
2.76 \mathrm{E}-02 \\
6.99 \mathrm{E}-03\end{array}$ & $\begin{array}{l}2 E-03 \\
8 E-02\end{array}$ & $\begin{array}{l}5.17 E-03 \\
2.03 E-04\end{array}$ & 2. & -02 \\
\hline Lawrenc & $\begin{array}{lr}\text { to Hanford Site } \\
\text { Truck } & 2.82 \mathrm{E}-02 \\
\text { Rail } & 6.94 \mathrm{E}-03\end{array}$ & $\begin{array}{l}2.88 \mathrm{E}-03 \\
1.38 \mathrm{E}-02\end{array}$ & $\begin{array}{l}4.9 \\
2.0\end{array}$ & $\begin{array}{l}3-02 \\
3-03\end{array}$ & $\begin{array}{l}-02 \\
-02\end{array}$ \\
\hline $\operatorname{LLW}(x)$ & $\begin{array}{lr}\text { to Savannah River } \\
\text { Truck } & 2.21 \mathrm{E}-02 \\
\text { Rail } & 5.99 \mathrm{E}-03\end{array}$ & $\begin{array}{l}i .9 \\
9.2\end{array}$ & 2. & $\begin{array}{l}-02 \\
-03\end{array}$ & $\begin{array}{l}-02 \\
-02\end{array}$ \\
\hline $\operatorname{LLW}(\mathrm{x})$ OR & $\begin{array}{lr}\text { to Savannah River } \\
\text { Truck } & 1.24 \mathrm{E}-02 \\
\text { Rail } & 4.73 \mathrm{E}-03\end{array}$ & & 1 & $\begin{array}{l}-02 \\
-03\end{array}$ & $\begin{array}{l}:-02 \\
-02\end{array}$ \\
\hline $\begin{array}{l}\text { LLW (x) OR to SR } \\
\text { Oak Ridge Re }\end{array}$ & $\begin{array}{ll}\text { to Savannah River } \\
\text { Truck } & 1.38 \mathrm{E}-02 \\
\text { Rail } & 4.62 \mathrm{E}-03\end{array}$ & $\begin{array}{l}1.581 \\
3.761\end{array}$ & $\begin{array}{l}2.1 \\
8.9\end{array}$ & $\begin{array}{l}\varepsilon-02 \\
\varepsilon-03\end{array}$ & $\begin{array}{l}1.43 E-02 \\
8.61 E-03\end{array}$ \\
\hline Pantex Plant & $\begin{array}{lr}\text { to Hanford Site } \\
\text { Truck } & 5.10 \mathrm{E} \\
\text { Rail } & 9.92 \mathrm{E}\end{array}$ & 2 & $\begin{array}{l}7.01 \mathrm{E}-03 \\
1.56 \mathrm{E}-04\end{array}$ & $\begin{array}{l}5.27 E-02 \\
8.67 E-03\end{array}$ & $\begin{array}{l}6.24 \mathrm{E}-02 \\
1.45 \mathrm{E}-02\end{array}$ \\
\hline
\end{tabular}




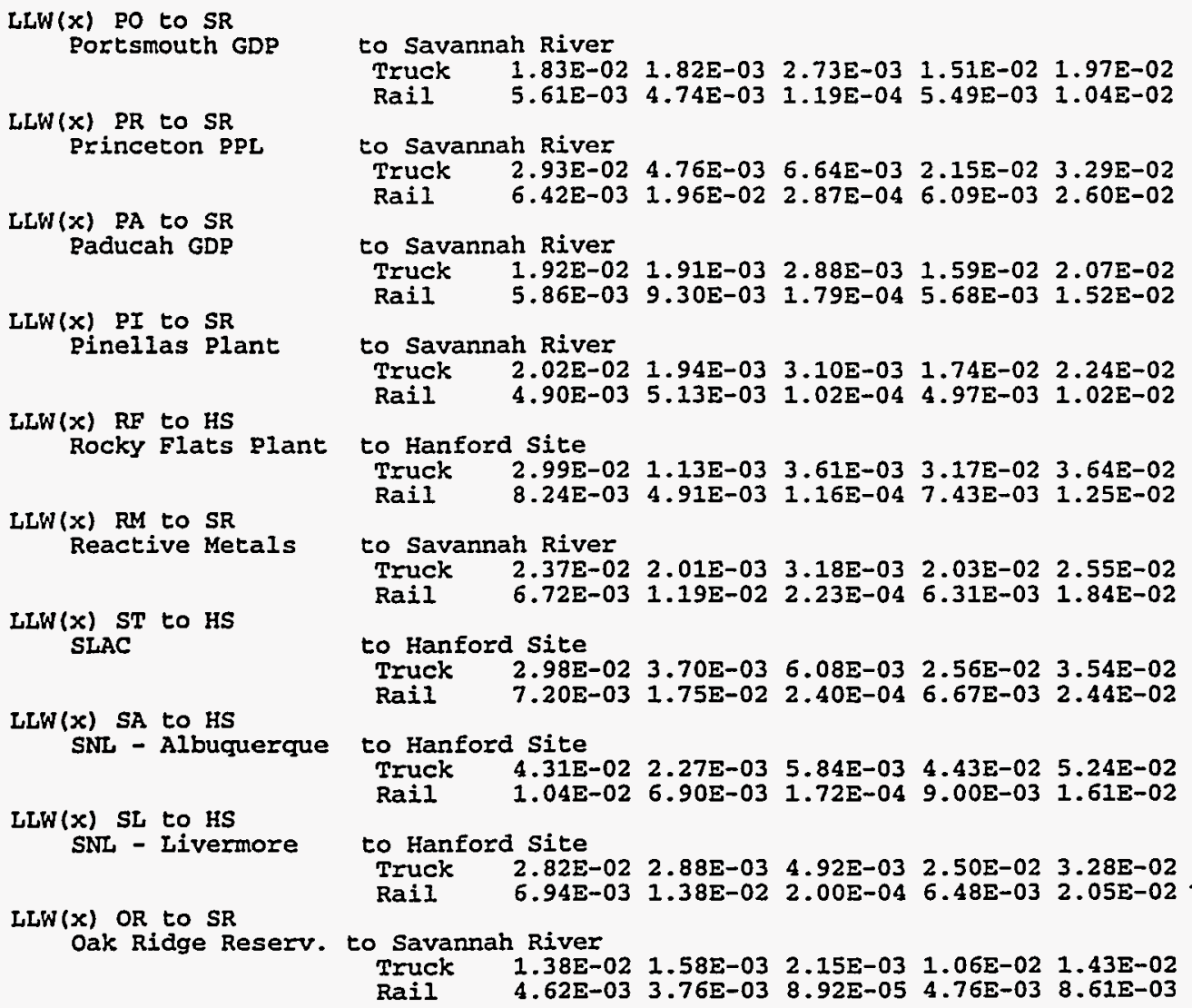


Table 4. Accident Dose Risk per Shipment (person-rem/shipment)

\begin{tabular}{|c|c|c|c|}
\hline Source/Route & & Truck & Rail \\
\hline 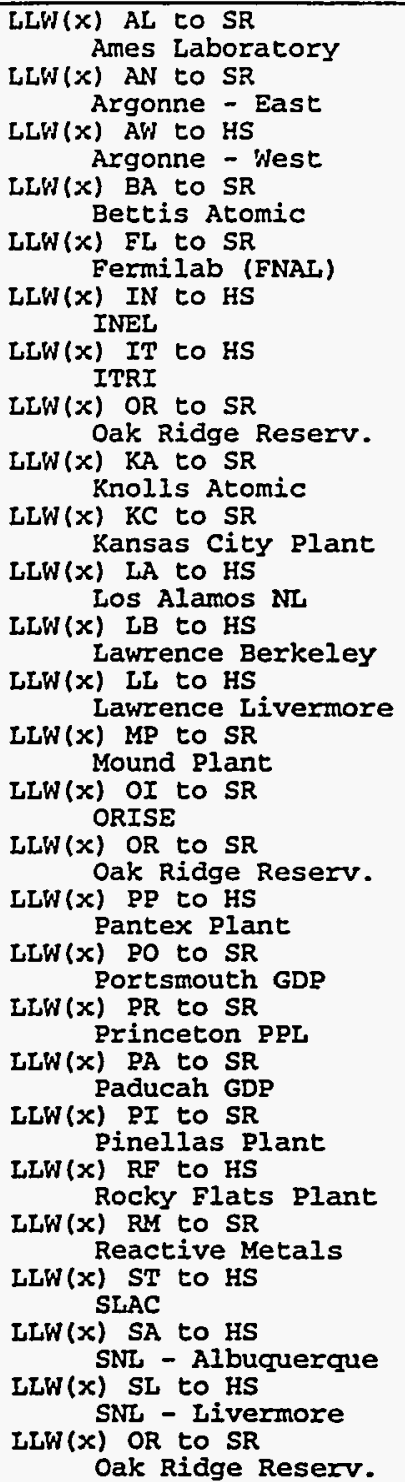 & $\begin{array}{l}\text { to Savannah River } \\
\text { to Savannah River } \\
\text { to Hanford Site } \\
\text { to Savannah River } \\
\text { to Savannah River } \\
\text { to Hanford Site } \\
\text { to Hanford Site } \\
\text { to Savannah River } \\
\text { to Savannah River } \\
\text { to Savannah River } \\
\text { to Hanford Site } \\
\text { to Hanford Site } \\
\text { to Hanford Site } \\
\text { to Savannah River } \\
\text { to Savannah River } \\
\text { to Savannah River } \\
\text { to Hanford Site } \\
\text { to Savannah River } \\
\text { to Savannah River } \\
\text { to Savannah River } \\
\text { to Savannah River } \\
\text { to Hanford Site } \\
\text { to Savannah River } \\
\text { to Hanford Site } \\
\text { to Hanford Site } \\
\text { to Hanford Site } \\
\text { to Savannah River }\end{array}$ & $\begin{array}{l}1.13 E-05 \\
6.76 E-04 \\
2.45 E-01 \\
9.72 E-06 \\
4.57 E-05 \\
1.10 E-04 \\
5.50 E-03 \\
1.66 E-07 \\
2.09 E-06 \\
5.50 E-05 \\
2.73 E-02 \\
5.07 E-02 \\
9.20 E-06 \\
1.62 E-06 \\
8.59 E-07 \\
1.66 E-07 \\
1.63 E-06 \\
8.36 E-09 \\
6.14 E-06 \\
7.70 E-07 \\
1.30 E-06 \\
3.44 E-06 \\
4.50 E-09 \\
4.30 E-07 \\
2.28 E-05 \\
8.79 E-05 \\
1.66 E-07\end{array}$ & $\begin{array}{l}8.56 \mathrm{E}-06 \\
4.55 \mathrm{E}-04 \\
3.44 \mathrm{E}-01 \\
9.30 \mathrm{E}-06 \\
1.69 \mathrm{E}-05 \\
1.56 \mathrm{E}-04 \\
1.57 \mathrm{E}-03 \\
4.85 \mathrm{E}-08 \\
3.04 \mathrm{E}-06 \\
1.34 \mathrm{E}-05 \\
9.38 \mathrm{E}-03 \\
4.24 \mathrm{E}-02 \\
1.28 \mathrm{E}-05 \\
8.64 \mathrm{E}-07 \\
4.56 \mathrm{E}-07 \\
4.85 \mathrm{E}-08 \\
4.96 \mathrm{E}-07 \\
3.71 \mathrm{E}-09 \\
2.15 \mathrm{E}-06 \\
4.23 \mathrm{E}-07 \\
4.65 \mathrm{E}-07 \\
2.49 \mathrm{E}-06 \\
3.34 \mathrm{E}-09 \\
6.58 \mathrm{E}-07 \\
7.55 \mathrm{E}-06 \\
1.40 \mathrm{E}-04 \\
4.85 \mathrm{E}-08\end{array}$ \\
\hline
\end{tabular}


Table 5. Nonradiological Risk Factors per Shipment (Eatalities/shipment)

\begin{tabular}{|c|c|c|c|}
\hline Mode & Route & Emission & Accident \\
\hline 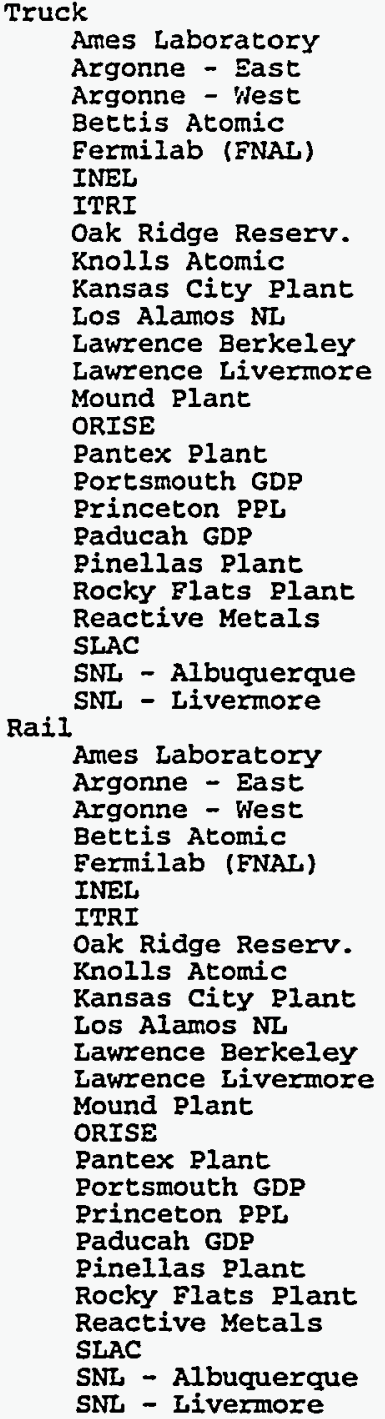 & $\begin{array}{l}\text { to Savannah River } \\
\text { to Savannah River } \\
\text { to Hanford Site } \\
\text { to Savannah River } \\
\text { to Savannah River } \\
\text { to Hanford Site } \\
\text { to Hanford Site } \\
\text { to Savannah River } \\
\text { to Savannah River } \\
\text { to Savannah River } \\
\text { to Hanford Site } \\
\text { to Hanford Site } \\
\text { to Hanford Site } \\
\text { to Savannah River } \\
\text { to Savannah River } \\
\text { to Hanford Site } \\
\text { to Savannah River } \\
\text { to Savannah River } \\
\text { to Savannah River } \\
\text { to Savannah River } \\
\text { to Hanford Site } \\
\text { to Savannah River } \\
\text { to Hanford Site } \\
\text { to Hanford Site } \\
\text { to Hanford Site } \\
\text { to Savannah River } \\
\text { to Savannah River } \\
\text { to Hanford Site } \\
\text { to Savannah River } \\
\text { to Savannah River } \\
\text { to Hanford Site } \\
\text { to Hanford Site } \\
\text { to Savannah River } \\
\text { to Savannah River } \\
\text { to Savannah River } \\
\text { to Hanford Site } \\
\text { to Hanford Site } \\
\text { to Hanford Site } \\
\text { to Savannah River } \\
\text { to Savannah River } \\
\text { to Hanford Site } \\
\text { to Savannah River } \\
\text { to Savannah River } \\
\text { to Savannah River } \\
\text { to Savannah River } \\
\text { to Hanford Site } \\
\text { to Savannah River } \\
\text { to Hanford Site } \\
\text { to Hanford Site } \\
\text { to Hanford Site }\end{array}$ & $\begin{array}{l}4.44 E-06 \\
5.41 E-06 \\
2.19 E-06 \\
3.99 E-06 \\
6.79 E-06 \\
2.19 E-06 \\
7.44 E-06 \\
2.96 E-06 \\
2.74 E-06 \\
7.89 E-06 \\
6.66 E-06 \\
1.16 E-05 \\
9.85 E-06 \\
1.67 E-06 \\
9.01 E-07 \\
9.21 E-06 \\
3.48 E-06 \\
1.74 E-05 \\
3.77 E-06 \\
4.47 E-06 \\
2.83 E-06 \\
2.80 E-06 \\
1.50 E-05 \\
7.24 E-06 \\
9.85 E-06 \\
2.57 E-05 \\
1.65 E-05 \\
3.98 E-06 \\
1.74 E-05 \\
2.18 E-05 \\
3.98 E-06 \\
9.04 E-06 \\
2.51 E-06 \\
5.51 E-05 \\
2.10 E-05 \\
6.82 E-06 \\
2.33 E-05 \\
2.36 E-05 \\
1.03 E-05 \\
6.53 E-06 \\
6.78 E-06 \\
3.01 E-06 \\
3.01 E-05 \\
1.01 E-05 \\
5.69 E-06 \\
7.36 E-06 \\
1.38 E-05 \\
3.07 E-05 \\
9.04 E-06 \\
2.36 E-05\end{array}$ & $\begin{array}{l}7.44 \mathrm{E}-05 \\
7.04 \mathrm{E}-05 \\
3.07 \mathrm{E}-05 \\
4.90 \mathrm{E}-05 \\
7.45 \mathrm{E}-05 \\
3.07 \mathrm{E}-05 \\
1.05 \mathrm{E}-04 \\
2.92 \mathrm{E}-05 \\
7.17 \mathrm{E}-05 \\
6.87 \mathrm{E}-05 \\
1.02 \mathrm{E}-04 \\
4.91 \mathrm{E}-05 \\
5.03 \mathrm{E}-05 \\
5.26 \mathrm{E}-05 \\
3.61 \mathrm{E}-05 \\
1.25 \mathrm{E}-04 \\
4.13 \mathrm{E}-05 \\
6.30 \mathrm{E}-05 \\
4.10 \mathrm{E}-05 \\
4.70 \mathrm{E}-05 \\
6.69 \mathrm{E}-05 \\
4.86 \mathrm{E}-05 \\
5.14 \mathrm{E}-05 \\
1.05 \mathrm{E}-04 \\
5.03 \mathrm{E}-05 \\
2.68 \mathrm{E}-06 \\
2.04 \mathrm{E}-06 \\
1.38 \mathrm{E}-06 \\
1.98 \mathrm{E}-06 \\
2.10 \mathrm{E}-06 \\
1.38 \mathrm{E}-06 \\
3.75 \mathrm{E}-06 \\
8.72 \mathrm{E}-07 \\
2.18 \mathrm{E}-06 \\
2.43 \mathrm{E}-06 \\
3.61 \mathrm{E}-06 \\
2.06 \mathrm{E}-06 \\
2.04 \mathrm{E}-06 \\
1.56 \mathrm{E}-06 \\
9.28 \mathrm{E}-07 \\
3.53 \mathrm{E}-06 \\
1.37 \mathrm{E}-06 \\
1.77 \mathrm{E}-06 \\
1.49 \mathrm{E}-06 \\
1.01 \mathrm{E}-06 \\
2.69 \mathrm{E}-06 \\
2.92 \mathrm{E}-06 \\
3.75 \mathrm{E}-06\end{array}$ \\
\hline
\end{tabular}




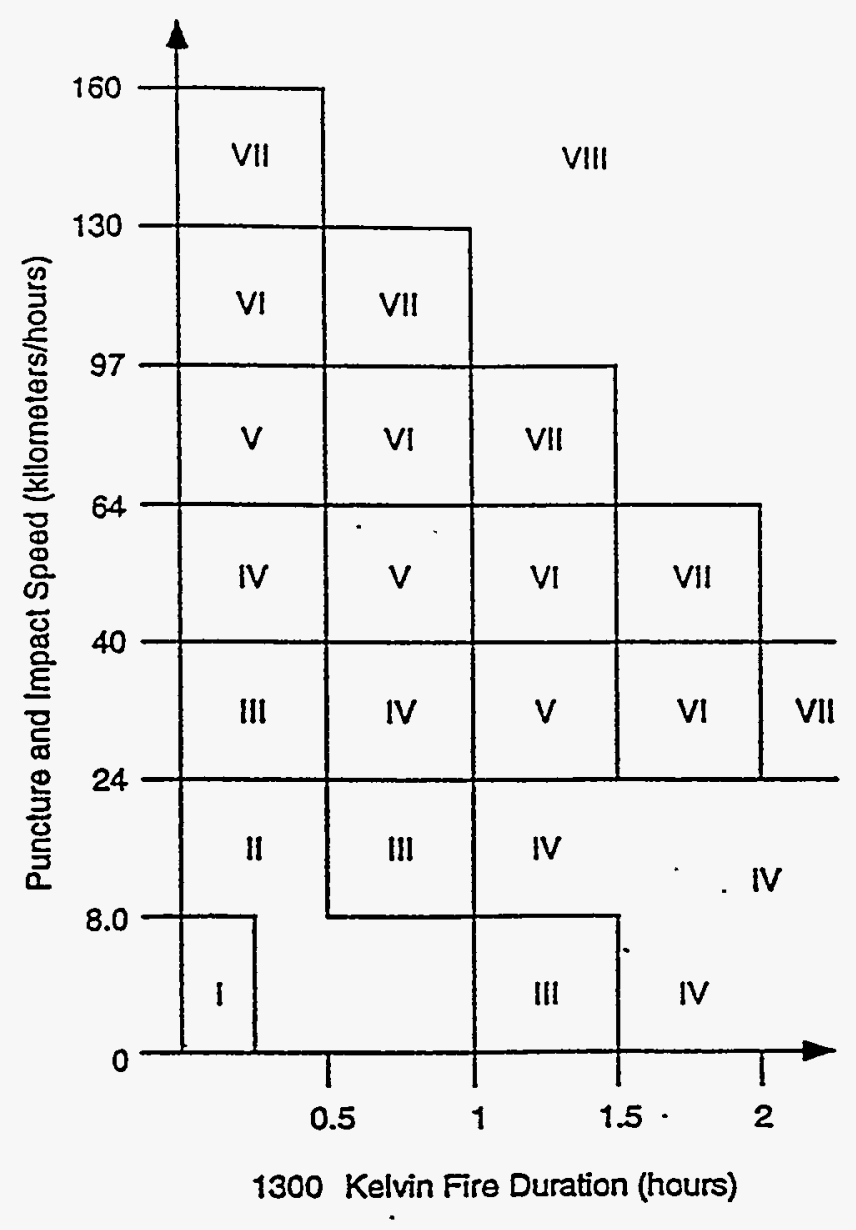

FIGURE 4.2 Scheme for NUREG-0170 Classification by Accident Severity Category for Rail Accidents (Source: NRC 1977a)

release of radioactive material. Accidents of this severity are extremely rare, occurring approximately once in every 70,000 truck or 100,000 rail accidents involving a shipment of radioactive waste. On the basis of national accident statistics (Saricks and Kvitek 1994), for every $1.6 \mathrm{~km}(1 \mathrm{mi})$ of loaded shipment, the probability of an accident of this severity is $6 \mathrm{E}-12$ for shipment by truck and 1E-12 for shipment by rail.

\subsubsection{Package Release Fractions}

Radiological consequences are calculated by assigning package release fractions to each accident severity category. The release fraction is defined as the fraction of the radioactive material in a package that could be released from the package as the result of an accident of a given severity. Release fractions take into account all mechanisms necessary to create release of radioactive material from a damaged package to the environment. Release fractions vary according to the type of package and the physical form of the waste. 
Table 6. Annual Incident-Free Dose for the Shipping Campaign (person-rem/yr)

\begin{tabular}{|c|c|c|c|c|c|c|}
\hline \multirow[b]{2}{*}{ Route } & & & \multicolumn{2}{|c|}{ Truck } & \multicolumn{2}{|c|}{ Rail } \\
\hline & & & Crew & Public & Crew & Public \\
\hline $\begin{array}{l}\text { Ames Laboratory } \\
\text { Argonne - East } \\
\text { Argonne - West } \\
\text { Bettis Atomic } \\
\text { Fermilab (FNAL) } \\
\text { INEL } \\
\text { ITRI } \\
\text { Oak Ridge Reserv. } \\
\text { Knolls Atomic } \\
\text { Kansas City Plant } \\
\text { Los Alamos NL } \\
\text { Lawrence Berkeley } \\
\text { Lawrence Livermore } \\
\text { Mound Plant } \\
\text { ORISE } \\
\text { Pantex Plant } \\
\text { Portsmouth GDP } \\
\text { Princeton PPL } \\
\text { Paducah GDP } \\
\text { Pinellas Plant } \\
\text { Rocky Flats Plant } \\
\text { Reactive Metals } \\
\text { SLAC } \\
\text { SNL - Albuquerque } \\
\text { SNL - Livermore }\end{array}$ & $\begin{array}{l}\text { to } \\
\text { to } \\
\text { to } \\
\text { to } \\
\text { to } \\
\text { to } \\
\text { to } \\
\text { to } \\
\text { to } \\
\text { to } \\
\text { to } \\
\text { to } \\
\text { to } \\
\text { to } \\
\text { to } \\
\text { to } \\
\text { to } \\
\text { to } \\
\text { to } \\
\text { to } \\
\text { to } \\
\text { to } \\
\text { to } \\
\text { to } \\
\text { to }\end{array}$ & $\begin{array}{l}\text { Savannah River } \\
\text { Savannah River } \\
\text { Hanford Site } \\
\text { Savannah River } \\
\text { Savannah River } \\
\text { Hanford Site } \\
\text { Hanford Site } \\
\text { Savannah River } \\
\text { Savannah River } \\
\text { Savannah River } \\
\text { Hanford Site } \\
\text { Hanford Site } \\
\text { Hanford Site } \\
\text { Savannah River } \\
\text { Savannah River } \\
\text { Hanford Site } \\
\text { Savannah River } \\
\text { Savannah River } \\
\text { Savannah River } \\
\text { Savannah River } \\
\text { Hanford Site } \\
\text { Savannah River } \\
\text { Hanford Site } \\
\text { Hanford Site } \\
\text { Hanford Site }\end{array}$ & $\begin{array}{l}7.18 \mathrm{E}-02 \\
3.13 \mathrm{E}+00 \\
2.08 \mathrm{E}-01 \\
1.22 \mathrm{E}+00 \\
9.38 \mathrm{E}-02 \\
1.36 \mathrm{E}+01 \\
4.34 \mathrm{E}-01 \\
9.01 \mathrm{E}+01 \\
9.00 \mathrm{E}-01 \\
3.23 \mathrm{E}-02 \\
3.07 \mathrm{E}+01 \\
8.27 \mathrm{E}-02 \\
1.58 \mathrm{E}+00 \\
1.13 \mathrm{E}+01 \\
6.18 \mathrm{E}-02 \\
7.40 \mathrm{E}+01 \\
6.12 \mathrm{E}+01 \\
2.93 \mathrm{E}-02 \\
1.20 \mathrm{E}+01 \\
4.04 \mathrm{E}-02 \\
1.07 \mathrm{E}+01 \\
1.82 \mathrm{E}+01 \\
1.16 \mathrm{E}+00 \\
7.33 \mathrm{E}-01 \\
1.69 \mathrm{E}-01\end{array}$ & $\begin{array}{l}8.05 \mathrm{E}-02 \\
3.38 \mathrm{E}+00 \\
2.54 \mathrm{E}-01 \\
1.34 \mathrm{E}+00 \\
1.02 \mathrm{E}-01 \\
1.67 \mathrm{E}+01 \\
5.28 \mathrm{E}-01 \\
9.38 \mathrm{E}+01 \\
9.48 \mathrm{E}-01 \\
3.59 \mathrm{E}-02 \\
3.73 \mathrm{E}+01 \\
9.80 \mathrm{E}-02 \\
1.84 \mathrm{E}+00 \\
1.18 \mathrm{E}+01 \\
6.48 \mathrm{E}-02 \\
9.05 \mathrm{E}+01 \\
6.58 \mathrm{E}+01 \\
3.29 \mathrm{E}-02 \\
1.30 \mathrm{E}+01 \\
4.48 \mathrm{E}-02 \\
1.30 \mathrm{E}+01 \\
1.96 \mathrm{E}+01 \\
1.38 \mathrm{E}+00 \\
8.92 \mathrm{E}-01 \\
1.97 \mathrm{E}-01\end{array}$ & $\begin{array}{l}8.23 \mathrm{E}-03 \\
2.71 \mathrm{E}-01 \\
2.81 \mathrm{E}-02 \\
1.43 \mathrm{E}-01 \\
1.41 \mathrm{E}-02 \\
1.81 \mathrm{E}+00 \\
4.15 \mathrm{E}-02 \\
1.15 \mathrm{E}+01 \\
7.96 \mathrm{E}-02 \\
7.73 \mathrm{E}-03 \\
2.70 \mathrm{E}+00 \\
1.40 \mathrm{E}-02 \\
1.46 \mathrm{E}-01 \\
1.14 \mathrm{E}+00 \\
9.46 \mathrm{E}-03 \\
5.40 \mathrm{E}+00 \\
7.15 \mathrm{E}+00 \\
6.42 \mathrm{E}-03 \\
1.39 \mathrm{E}+00 \\
4.90 \mathrm{E}-03 \\
1.10 \mathrm{E}+00 \\
1.93 \mathrm{E}+00 \\
1.08 \mathrm{E}-01 \\
7.25 \mathrm{E}-02 \\
1.39 \mathrm{E}-02\end{array}$ & $\begin{array}{l}2.70 \mathrm{E}-02 \\
8.06 \mathrm{E}-01 \\
4.20 \mathrm{E}-02 \\
4.17 \mathrm{E}-01 \\
4.66 \mathrm{E}-02 \\
2.71 \mathrm{E}+00 \\
6.43 \mathrm{E}-02 \\
2.14 \mathrm{E}+01 \\
4.36 \mathrm{E}-01 \\
2.39 \mathrm{E}-02 \\
3.90 \mathrm{E}+00 \\
4.10 \mathrm{E}-02 \\
4.30 \mathrm{E}-01 \\
2.89 \mathrm{E}+00 \\
2.17 \mathrm{E}-02 \\
7.91 \mathrm{E}+00 \\
1.32 \mathrm{E}+01 \\
2.60 \mathrm{E}-02 \\
3.59 \mathrm{E}+00 \\
1.02 \mathrm{E}-02 \\
1.66 \mathrm{E}+00 \\
5.29 \mathrm{E}+00 \\
3.66 \mathrm{E}-01 \\
1.12 \mathrm{E}-01 \\
4.10 \mathrm{E}-02\end{array}$ \\
\hline Totals & & & $32 \mathrm{E}+02$ & $3.73 \mathrm{E}+02$ & $3.51 E+01$ & $6.55 E+01$ \\
\hline
\end{tabular}

Table 7. Annual Accident Dose Risk for the Shipping Campaign (person-rem/yr)

\begin{tabular}{llcc}
\hline Route & & Truck & Rail \\
\hline Ames Laboratory & to Savannah River & $2.25 \mathrm{E}-05$ & $8.56 \mathrm{E}-06$ \\
Argonne - East & to Savannah River & $7.10 \mathrm{E}-02$ & $1.77 \mathrm{E}-02$ \\
Argonne - West & to Hanford Site & $3.18 \mathrm{E}+00$ & $1.72 \mathrm{E}+00$ \\
Bettis Atomic & to Savannah River & $5.54 \mathrm{E}-04$ & $1.95 \mathrm{E}-04$ \\
Fermilab (FNAL) & to Savannah River & $1.37 \mathrm{E}-04$ & $3.38 \mathrm{E}-05$ \\
INEL & to Hanford Site & $9.35 \mathrm{E}-02$ & $5.04 \mathrm{E}-02$ \\
ITRI & to Hanford Site & $5.50 \mathrm{E}-02$ & $6.27 \mathrm{E}-03$ \\
Oak Ridge Reserv. & to Savannah River & $1.09 \mathrm{E}-03$ & $1.21 \mathrm{E}-04$ \\
Rnolls Atomic & to Savannah River & $5.84 \mathrm{E}-05$ & $3.34 \mathrm{E}-05$ \\
Ransas City Plant & to Savannah River & $5.50 \mathrm{E}-05$ & $1.34 \mathrm{E}-05$ \\
Los Alamos NL & to Hanford Site & $1.98 \mathrm{E}+01$ & $2.51 \mathrm{E}+00$ \\
Lawrence Berkeley & to Hanford Site & $1.52 \mathrm{E}-01$ & $8.48 \mathrm{E}-02$ \\
Lawrence Livermore to Hanford Site & $5.15 \mathrm{E}-04$ & $2.70 \mathrm{E}-04$ \\
Mound Plant & to Savannah River & $8.30 \mathrm{E}-04$ & $1.64 \mathrm{E}-04$ \\
ORISE & to Savannah River & $4.30 \mathrm{E}-06$ & $9.11 \mathrm{E}-07$ \\
Pantex Plant & to Hanford Site & $2.36 \mathrm{E}-03$ & $2.70 \mathrm{E}-04$ \\
Portsmouth GDP & to Savannah River & $2.80 \mathrm{E}-05$ & $4.72 \mathrm{E}-06$ \\
Princeton PPL & to Savannah River & $6.14 \mathrm{E}-06$ & $2.15 \mathrm{E}-06$ \\
Paducah GDP & to Savannah River & $4.83 \mathrm{E}-04$ & $1.00 \mathrm{E}-04$ \\
Pinellas Plant & to Savannah River & $2.59 \mathrm{E}-06$ & $4.65 \mathrm{E}-07$ \\
Rocky Flats Plant & to Hanford Site & $1.23 \mathrm{E}-03$ & $3.31 \mathrm{E}-04$ \\
Reactive Metals & to Savannah River & $3.45 \mathrm{E}-06$ & $9.59 \mathrm{E}-07$ \\
SLAC & to Hanford Site & $1.68 \mathrm{E}-05$ & $9.87 \mathrm{E}-06$ \\
SNL - Albuquerque & to Hanford Site & $3.88 \mathrm{E}-04$ & $5.29 \mathrm{E}-05$ \\
SNL - Livermore & to Hanford Site & $5.27 \mathrm{E}-04$ & $2.79 \mathrm{E}-04$ \\
\hline Totals & & & \\
\hline
\end{tabular}




\begin{tabular}{llc}
$\begin{array}{l}\text { Table 8. Expected Annual Fatalities } \\
\text { for the Shipping Campaign }\end{array}$ & \\
\hline Exposure Group & Truck & Rail \\
\hline Radiological & & \\
$\quad$ Normal Crew & $1.3 \mathrm{E}-01$ & $1.4 \mathrm{E}-02$ \\
$\quad$ Normal Public & $1.9 \mathrm{E}-01$ & $3.3 \mathrm{E}-02$ \\
Accident Public & $1.2 \mathrm{E}-02$ & $2.2 \mathrm{E}-03$ \\
Nonradiological & & \\
Emission & $6.0 \mathrm{E}-02$ & $2.9 \mathrm{E}-02$ \\
Accident & $7.5 \mathrm{E}-01$ & $9.1 \mathrm{E}-03$ \\
\end{tabular}

Table 9. Expected Annual Cancer Incidence for the Shipping Campaign

\begin{tabular}{lll}
\hline Exposure Group & Truck & Rail \\
\hline Radiological & & \\
Normal Crew & $4.6 \mathrm{E}-01$ & $4.9 \mathrm{E}-02$ \\
Normal Public & $6.3 \mathrm{E}-01$ & $1.1 \mathrm{E}-01$ \\
Accident Public & $4.0 \mathrm{E}-02$ & $7.5 \mathrm{E}-03$ \\
Nonradiological & & \\
Emission & $6.0 \mathrm{E}-02$ & $2.9 \mathrm{E}-02$ \\
Accident & $\mathrm{NA}$ & $\mathrm{NA}$ \\
\hline
\end{tabular}

Table 10. Expected Annual Genetic Effects for the Shipping Campaign

\begin{tabular}{lll}
\hline Exposure Group & Truck & Rail \\
\hline Radiological & $2.0 \mathrm{E}-02$ & $2.1 \mathrm{E}-03$ \\
. Normal Crew & $3.7 \mathrm{E}-02$ & $6.5 \mathrm{E}-03$ \\
Normal Public & $2.0 \mathrm{E}-03$ & $3.7 \mathrm{E}-04$ \\
$\quad$ Accident Public & & $\mathrm{NA}$ \\
$\begin{array}{c}\text { Nonradiological } \\
\text { Emission }\end{array}$ & NA & NA \\
Accident & NA & \\
\hline
\end{tabular}




\section{B.16 WM LLW REGIONALIZED 6 ALTERNATIVE (CASE 5): ACTIVATED METALS}

Table 1. Summary of Route Information

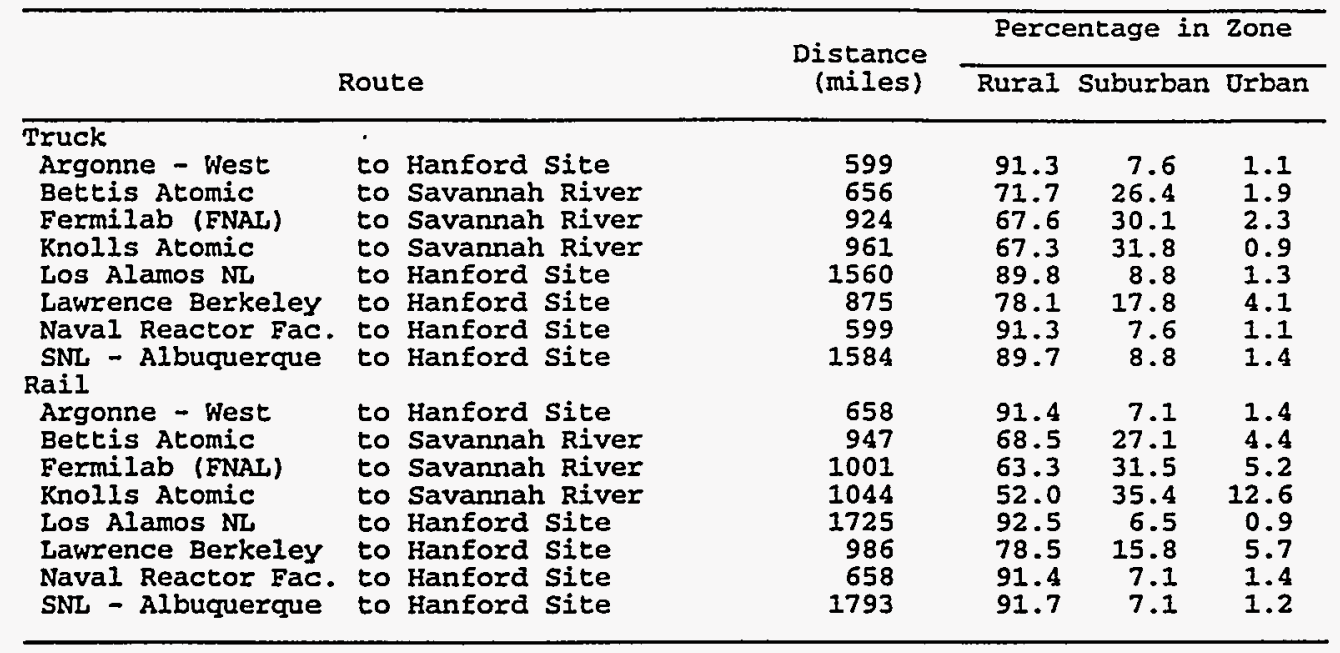

Table 2. Annual Number of Shipments and one-Way Shipment Distances

\begin{tabular}{|c|c|c|c|c|c|}
\hline \multirow[b]{2}{*}{ Route } & & \multicolumn{2}{|c|}{ \# of Shipments } & \multicolumn{2}{|c|}{ Total Mileage (mi) } \\
\hline & & Truck & Rail & Truck & Rail \\
\hline $\begin{array}{l}\text { Argonne - West } \\
\text { Bettis Atomic } \\
\text { Fermilab (FNAL) } \\
\text { Knolls Atomic } \\
\text { Los Alamos NL } \\
\text { Lawrence Berkeley } \\
\text { Naval Reactor Fac. } \\
\text { SNL - Albuquerque }\end{array}$ & $\begin{array}{l}\text { to Hanford Site } \\
\text { to Savannah River } \\
\text { to Savannah River } \\
\text { to Savannah River } \\
\text { to Hanford Site } \\
\text { to Hanford Site } \\
\text { to Hanford Site } \\
\text { to Hanford Site }\end{array}$ & $\begin{array}{r}99 \\
316 \\
40 \\
650 \\
418 \\
15 \\
461 \\
6\end{array}$ & $\begin{array}{r}4 \\
120 \\
15 \\
246 \\
159 \\
6 \\
175 \\
3\end{array}$ & $\begin{array}{l}5.39 E+03 \\
2.07 E+05 \\
3.70 E+04 \\
6.25 E+05 \\
6.52 E+05 \\
1.31 E+04 \\
2.76 E+05 \\
9.50 E+03\end{array}$ & $\begin{array}{l}2.63 \mathrm{E}+03 \\
1.14 \mathrm{E}+05 \\
1.50 \mathrm{E}+04 \\
2.57 \mathrm{E}+05 \\
2.74 \mathrm{E}+05 \\
5.91 \mathrm{E}+03 \\
1.15 \mathrm{E}+05 \\
5.38 \mathrm{E}+03\end{array}$ \\
\hline Totals & & 1915 & 728 & $1.82 \mathrm{E}+06$ & $7.89 E+05$ \\
\hline
\end{tabular}


Table 3. Incident-Free Dose per Shipment (person-rem/shipment)

\begin{tabular}{|c|c|c|c|c|c|}
\hline \multirow[b]{2}{*}{ Source/Route (s) } & \multirow[b]{2}{*}{ Crew } & \multicolumn{4}{|c|}{ General Public } \\
\hline & & Off-Link & On-Link & Stops & Total \\
\hline $\begin{array}{l}\text { LLW(I) AW to HS } \\
\text { Argonne - West }\end{array}$ & $\begin{array}{ll}\text { to Hanford Site } \\
\text { Truck } & 1.60 \mathrm{E}-02 \\
\text { Rail } & 5.63 \mathrm{E}-03\end{array}$ & $\begin{array}{l}7.18 \mathrm{E}-04 \\
2.83 \mathrm{E}-03\end{array}$ & $\begin{array}{l}2.06 \mathrm{E}-03 \\
6.58 \mathrm{E}-05\end{array}$ & $\begin{array}{l}1.68 \mathrm{E}-02 \\
5.51 \mathrm{E}-03\end{array}$ & $\begin{array}{l}1.95 \mathrm{E}-02 \\
8.40 \mathrm{E}-03\end{array}$ \\
\hline $\begin{array}{l}\text { LLW(I) BA to SR } \\
\text { Bettis Atomic }\end{array}$ & $\begin{array}{lr}\text { to Savannah River } \\
\text { Truck } & 2.14 \mathrm{E}-02 \\
\text { Rail } & 6.83 \mathrm{E}-03\end{array}$ & $\begin{array}{l}1.96 \mathrm{E}-03 \\
1.32 \mathrm{E}-02\end{array}$ & $\begin{array}{l}3.14 E-03 \\
2.31 E-04\end{array}$ & $\begin{array}{l}1.84 \mathrm{E}-02 \\
6.39 \mathrm{E}-03\end{array}$ & $\begin{array}{l}2.35 \mathrm{E}-02 \\
1.99 \mathrm{E}-02\end{array}$ \\
\hline $\begin{array}{l}\text { LLW(I) FL to SR } \\
\text { Fermilab (FNAL) }\end{array}$ & $\begin{array}{lr}\text { to Savannah River } \\
\text { Truck } & 3.13 \mathrm{E}-02 \\
\text { Rail } & 7.06 \mathrm{E}-03\end{array}$ & $\begin{array}{l}3.20 \mathrm{E}-03 \\
1.65 \mathrm{E}-02\end{array}$ & $\begin{array}{l}4.81 \mathrm{E}-03 \\
2.79 \mathrm{E}-04\end{array}$ & $\begin{array}{l}2.59 E-02 \\
6.56 E-03\end{array}$ & $\begin{array}{l}3.39 \mathrm{E}-02 \\
2.33 \mathrm{E}-02\end{array}$ \\
\hline $\begin{array}{l}\text { LLW(I) KA to SR } \\
\text { Knolis Atomic }\end{array}$ & $\begin{array}{lr} & \\
\text { to Savannah River } \\
\text { Truck } & 3.22 \mathrm{E}-02 \\
\text { Rail } & 7.24 \mathrm{E}-03\end{array}$ & $\begin{array}{l}2.78 \mathrm{E}-03 \\
3.25 \mathrm{E}-02\end{array}$ & $\begin{array}{l}4.17 \mathrm{E}-03 \\
4.25 \mathrm{E}-04\end{array}$ & $\begin{array}{l}2.69 \mathrm{E}-02 \\
6.69 \mathrm{E}-03\end{array}$ & $\begin{array}{l}3.38 E-02 \\
3.96 E-02\end{array}$ \\
\hline $\begin{array}{l}\text { LOS Alamos NL } \\
\text { Los }\end{array}$ & $\begin{array}{lr}\text { to Hanford Site } \\
\text { Truck } & 4.24 \mathrm{E}-02 \\
\text { Rail } & 1.01 \mathrm{E}-02\end{array}$ & $\begin{array}{l}2.16 \mathrm{E}-03 \\
5.60 \mathrm{E}-03\end{array}$ & $\begin{array}{l}5.65 E-03 \\
1.54 E-04\end{array}$ & $\begin{array}{l}4.37 \mathrm{E}-02 \\
8.79 \mathrm{E}-03\end{array}$ & $\begin{array}{l}5.15 \mathrm{E}-02 \\
1.45 \mathrm{E}-02\end{array}$ \\
\hline $\begin{array}{l}\text { LLW(I) LB to HS } \\
\text { Lawrence Berkeley }\end{array}$ & $\begin{array}{ll}\text { to Hanford } & \text { Site } \\
\text { Truck } & 2.76 \mathrm{E}-02 \\
\text { Rail } & 6.99 \mathrm{E}-03\end{array}$ & $\begin{array}{l}3.02 \mathrm{E}-03 \\
1.38 \mathrm{E}-02\end{array}$ & $\begin{array}{l}5.17 \mathrm{E}-03 \\
2.03 \mathrm{E}-04\end{array}$ & $\begin{array}{l}2.45 \mathrm{E}-02 \\
6.51 \mathrm{E}-03\end{array}$ & $\begin{array}{l}3.27 E-02 \\
2.05 E-02\end{array}$ \\
\hline $\begin{array}{l}\text { LLW(I) NR to HS } \\
\text { Naval Reactor Fac. }\end{array}$ & $\begin{array}{ll}\text { to Hanford Site } \\
\text { Truck } & 1.60 \mathrm{E}-02 \\
\text { Rail } & 5.63 \mathrm{E}-03\end{array}$ & $\begin{array}{l}7.18 \mathrm{E}-04 \\
2.83 \mathrm{E}-03\end{array}$ & $\begin{array}{l}2.06 \mathrm{E}-03 \\
6.58 \mathrm{E}-05\end{array}$ & $\begin{array}{l}1.68 \mathrm{E}-02 \\
5.51 \mathrm{E}-03\end{array}$ & $\begin{array}{l}1.95 E-02 \\
8.40 E-03\end{array}$ \\
\hline $\begin{array}{l}\text { LEW(I) SA to HS } \\
\text { SNL - Albuquerque }\end{array}$ & $\begin{array}{lr}\text { to Hanford Site } \\
\text { Truck } & 4.31 \mathrm{E}-02 \\
\text { Rail } & 1.04 \mathrm{E}-02\end{array}$ & $\begin{array}{l}2.27 E-03 \\
6.90 E-03\end{array}$ & $\begin{array}{l}5.84 \mathrm{E}-03 \\
1.72 \mathrm{E}-04\end{array}$ & $\begin{array}{l}4.43 \mathrm{E}-02 \\
9.00 \mathrm{E}-03\end{array}$ & $\begin{array}{l}5.24 \mathrm{E}-02 \\
1.61 \mathrm{E}-02\end{array}$ \\
\hline
\end{tabular}

Table 4. Accident Dose Risk per Shipment (person-rem/shipment)

\begin{tabular}{|c|c|c|c|}
\hline Source/Route & & Truck & Rail \\
\hline $\begin{array}{l}\text { LLW(I) AW to HS } \\
\text { Argonne - West } \\
\text { LLW(I) BA to SR } \\
\text { Bettis Atomic } \\
\text { LLW(I) FL to SR } \\
\text { Fermilab (FNAL) } \\
\text { LLW(I) KA to SR } \\
\text { Knolls Atomic } \\
\text { LLW(I) LA to HS } \\
\text { Los Alamos NL } \\
\text { LLW(I) IB to HS } \\
\text { Lawrence Berkeley } \\
\text { LLW(I) NR to HS } \\
\text { Naval Reactor Fac. } \\
\text { LLW(I) SA to HS } \\
\text { SNL - Albuquerque }\end{array}$ & $\begin{array}{l}\text { to Hanford Site } \\
\text { to Savannah River } \\
\text { to Savannah River } \\
\text { to Savannah River } \\
\text { to Hanford Site } \\
\text { to Hanford Site } \\
\text { to Hanford Site } \\
\text { to Hanford Site }\end{array}$ & $\begin{array}{l}8.15 \mathrm{E}-12 \\
7.03 \mathrm{E}-07 \\
9.75 \mathrm{E}-11 \\
1.44 \mathrm{E}-06 \\
3.10 \mathrm{E}-09 \\
1.69 \mathrm{E}-10 \\
4.44 \mathrm{E}-08 \\
6.06 \mathrm{E}-12\end{array}$ & $\begin{array}{l}9.58 \mathrm{E}-12 \\
6.43 \mathrm{E}-07 \\
6.35 \mathrm{E}-11 \\
2.20 \mathrm{E}-06 \\
8.88 \mathrm{E}-10 \\
2.37 \mathrm{E}-10 \\
6.34 \mathrm{E}-08 \\
1.32 \mathrm{E}-12\end{array}$ \\
\hline
\end{tabular}


Table 5. Nonradiological Risk Factors per Shipment (Eatalities/shipment)

\begin{tabular}{llll}
\hline Mode & Route & Emission & Accident \\
\hline Truck & & & \\
Argonne - West & to Hanford Site & $2.19 \mathrm{E}-06$ & $3.07 \mathrm{E}-05$ \\
Bettis Atomic & to Savannah River & $3.99 \mathrm{E}-06$ & $4.90 \mathrm{E}-05$ \\
Fermilab (FNAL) & to Savannah River & $6.79 \mathrm{E}-06$ & $7.45 \mathrm{E}-05$ \\
Knolls Atomic & to Savannah River & $2.74 \mathrm{E}-06$ & $7.17 \mathrm{E}-05$ \\
Los Alamos NL & to Hanford Site & $6.66 \mathrm{E}-06$ & $1.02 \mathrm{E}-04$ \\
Lawrence Berkeley & to Hanford Site & $1.16 \mathrm{E}-05$ & $4.91 \mathrm{E}-05$ \\
Naval Reactor Fac. & to Hanford Site & $2.19 \mathrm{E}-06$ & $3.07 \mathrm{E}-05$ \\
SNL - Albuquerque & to Hanford Site & $7.24 \mathrm{E}-06$ & $1.05 \mathrm{E}-04$ \\
Rail & & & \\
Argonne - West & to Hanford Site & $3.98 \mathrm{E}-06$ & $1.38 \mathrm{E}-06$ \\
Bettis Atomic & to Savannah River & $1.74 \mathrm{E}-05$ & $1.98 \mathrm{E}-06$ \\
Fermilab (FNAL) & to Savannah River & $2.18 \mathrm{E}-05$ & $2.10 \mathrm{E}-06$ \\
Knolls Atomic & to Savannah River & $5.51 \mathrm{E}-05$ & $2.18 \mathrm{E}-06$ \\
Los Alamos NL & to Hanford Site & $6.82 \mathrm{E}-06$ & $3.61 \mathrm{E}-06$ \\
Lawrence Berkeley & to Hanford Site & $2.33 \mathrm{E}-05$ & $2.06 \mathrm{E}-06$ \\
Naval Reactor Fac. to Hanford Site & $3.98 \mathrm{E}-06$ & $1.38 \mathrm{E}-06$ \\
SNL - Albuquerque & to Hanford Site & $9.04 \mathrm{E}-06$ & $3.75 \mathrm{E}-06$ \\
\hline
\end{tabular}

Table 6. Annual Incident-Free Dose for the Shipping Campaign (person-rem/yr)

\begin{tabular}{|c|c|c|c|c|c|}
\hline \multirow[b]{2}{*}{ Route } & & \multicolumn{2}{|c|}{ Truck } & \multicolumn{2}{|c|}{ Rail } \\
\hline & & Crew & Public & Crew & Public \\
\hline $\begin{array}{l}\text { Argonne - West } \\
\text { Bettis Atomic } \\
\text { Fermilab (FNAL) } \\
\text { Knolls Atomic } \\
\text { Los Alamos NL } \\
\text { Lawrence Berkeley } \\
\text { Naval Reactor Fac. } \\
\text { SNL - Albuquerque }\end{array}$ & $\begin{array}{l}\text { to Hanford Site } \\
\text { to Savannah River } \\
\text { to Savannah River } \\
\text { to Savannah River } \\
\text { to Hanford Site } \\
\text { to Hanford Site } \\
\text { to Hanford Site } \\
\text { to Hanford Site }\end{array}$ & $\begin{array}{l}1.44 \mathrm{E}-01 \\
6.75 \mathrm{E}+00 \\
1.25 \mathrm{E}+00 \\
2.09 \mathrm{E}+01 \\
1.77 \mathrm{E}+01 \\
4.14 \mathrm{E}-01 \\
7.37 \mathrm{E}+00 \\
2.59 \mathrm{E}-01\end{array}$ & $\begin{array}{l}1.76 \mathrm{E}-01 \\
7.42 \mathrm{E}+00 \\
1.36 \mathrm{E}+00 \\
2.20 \mathrm{E}+01 \\
2.15 \mathrm{E}+01 \\
4.90 \mathrm{E}-01 \\
9.01 \mathrm{E}+00 \\
3.15 \mathrm{E}-01\end{array}$ & $\begin{array}{l}2.25 \mathrm{E}-02 \\
8.20 \mathrm{E}-01 \\
1.06 \mathrm{E}-01 \\
1.78 \mathrm{E}+00 \\
1.60 \mathrm{E}+00 \\
4.20 \mathrm{E}-02 \\
9.85 \mathrm{E}-01 \\
3.11 \mathrm{E}-02\end{array}$ & $\begin{array}{l}3.36 \mathrm{E}-02 \\
2.38 \mathrm{E}+00 \\
3.50 \mathrm{E}-01 \\
9.75 \mathrm{E}+00 \\
2.31 \mathrm{E}+00 \\
1.23 \mathrm{E}-01 \\
1.47 \mathrm{E}+00 \\
4.82 \mathrm{E}-02\end{array}$ \\
\hline Totals & & $5.48 \mathrm{E}+01$ & $6.23 \mathrm{E}+01$ & כ. & $1.65 \mathrm{E}+01$ \\
\hline
\end{tabular}

Table 7. Annual Accident Dose Risk for the Shipping Campaign (person-rem/yr)

\begin{tabular}{llcc}
\hline Route & & Truck & Rail \\
\hline Argonne - West & to Hanford Site & $7.34 \mathrm{E}-11$ & $3.83 \mathrm{E}-11$ \\
Bettis Atomic & to Savannah River & $2.22 \mathrm{E}-04$ & $7.71 \mathrm{E}-05$ \\
Fermilab (FNAL) & to Savannah River & $3.90 \mathrm{E}-09$ & $9.53 \mathrm{E}-10$ \\
Rnolls Atomic & to Savannah River & $9.36 \mathrm{E}-04$ & $5.42 \mathrm{E}-04$ \\
Los Alamos NL & to Hanford Site & $1.29 \mathrm{E}-06$ & $1.41 \mathrm{E}-07$ \\
Lawrence Berkeley & to Hanford Site & $2.53 \mathrm{E}-09$ & $1.42 \mathrm{E}-09$ \\
Naval Reactor Fac. to Hanford Site & $2.05 \mathrm{E}-05$ & $1.11 \mathrm{E}-05$ \\
SNL - Albuquerque & to Hanford Site & $3.63 \mathrm{E}-11$ & $3.96 \mathrm{E}-12$ \\
\hline Totals & & & \\
\hline
\end{tabular}

Table 8. Expected Annual Fatalities

for the Shipping Campaign

\begin{tabular}{lll}
\hline Exposure Group & Truck & Rail \\
\hline Radiological & $2.2 \mathrm{E}-02$ & $2.2 \mathrm{E}-03$ \\
Normal Crew & $3.1 \mathrm{E}-02$ & $8.2 \mathrm{E}-03$ \\
Normal Public & $5.9 \mathrm{E}-07$ & $3.2 \mathrm{E}-07$ \\
Accident Public & $7.3 \mathrm{E}-03$ & $1.8 \mathrm{E}-02$ \\
Nonradiological & $1.2 \mathrm{E}-01$ & $1.7 \mathrm{E}-03$ \\
$\quad$ Emission & & \\
Accident & & \\
\hline
\end{tabular}


Table 9. Expected Annual Cancer Incidence for the Shipping Campaign

\begin{tabular}{|c|c|c|}
\hline Exposure Group & Truck & Rail \\
\hline $\begin{array}{l}\text { Radiological } \\
\text { Normal Crew } \\
\text { Normal Public } \\
\text { Accident Public } \\
\text { Nonradiological } \\
\text { Emission } \\
\text { Accident }\end{array}$ & $\begin{array}{l}7.7 E-02 \\
1.1 \mathrm{E}-01 \\
2.0 \mathrm{E}-06 \\
7.3 \mathrm{E}-03 \\
\mathrm{NA}\end{array}$ & $\begin{array}{l}7.5 E-03 \\
2.8 E-02 \\
1.1 E-06 \\
1.8 E-02 \\
N A\end{array}$ \\
\hline $\begin{array}{l}\text { Table } 10 \text { Expected Annual } \\
\text { for the Shipping Campaign }\end{array}$ & Genetic & Effects \\
\hline Exposure Group & Truck & Rail \\
\hline $\begin{array}{l}\text { Radiological } \\
\text { Normal Crew } \\
\text { Normal Public } \\
\text { Accident Public } \\
\text { Nonradiological } \\
\text { Emission } \\
\text { Accident }\end{array}$ & $\begin{array}{l}3.3 \mathrm{E}-03 \\
6.2 \mathrm{E}-03 \\
1.2 \mathrm{E}-07 \\
\mathrm{NA} \\
\mathrm{NA}\end{array}$ & $\begin{array}{l}3.2 \mathrm{E}-04 \\
1.6 \mathrm{E}-03 \\
6.3 \mathrm{E}-08 \\
\mathrm{NA} \\
\mathrm{NA}\end{array}$ \\
\hline
\end{tabular}




\section{B.17 WM LLW REGIONALIZED 7 ALTERNATIVE (CASE 6): HETEROGENEOUS SOLIDS}

Table 1. Summary of Route Information

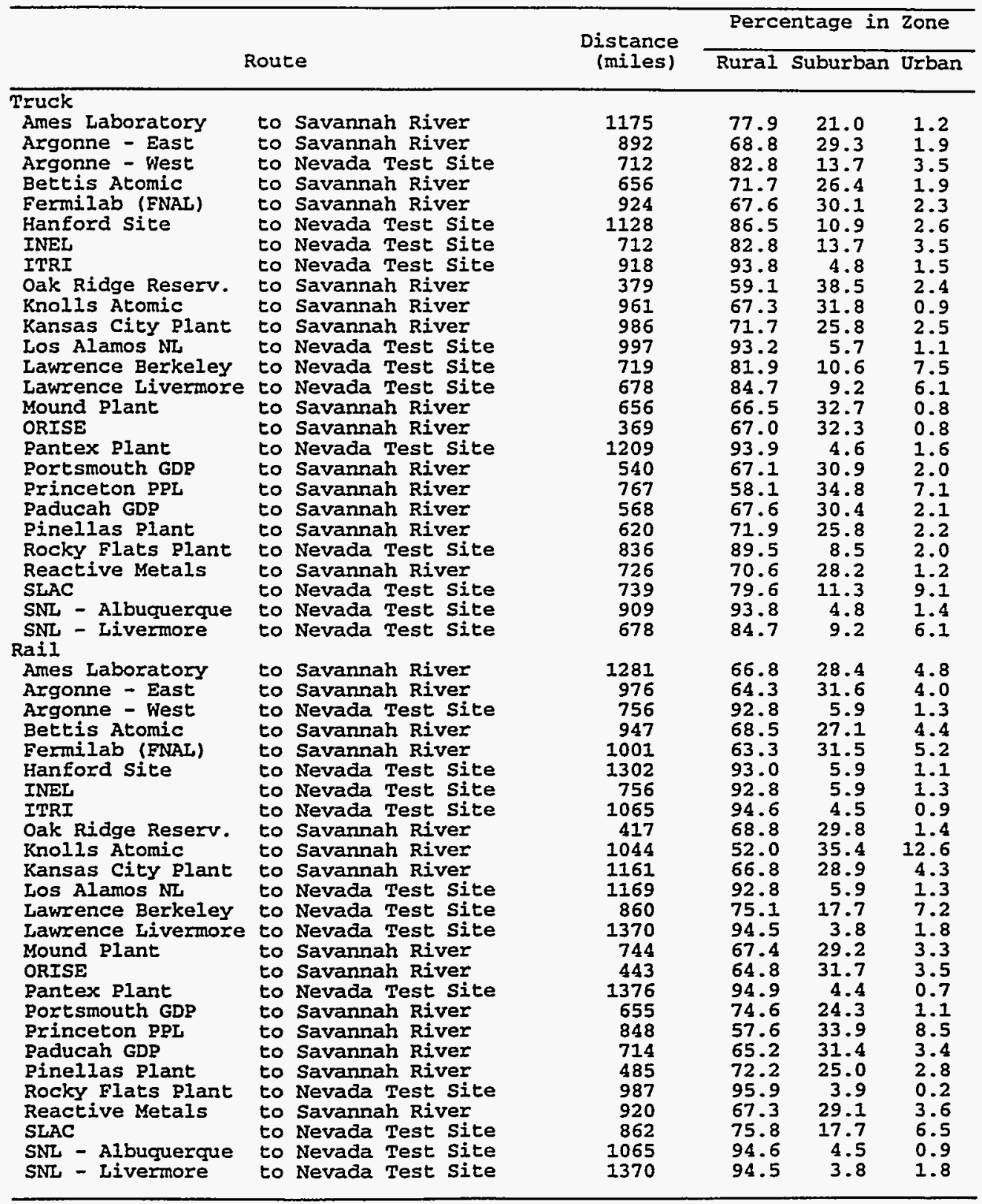


Table 2. Annual Number of Shipments and One-Way Shipment Distances

\begin{tabular}{|c|c|c|c|c|c|}
\hline \multirow[b]{2}{*}{ Route } & & \multicolumn{2}{|c|}{ \# of Shipments } & \multicolumn{2}{|c|}{ Total Mileage(mi) } \\
\hline & & Truck & Rail & Truck & Rail \\
\hline $\begin{array}{l}\text { Ames Laboratory } \\
\text { Argonne - East } \\
\text { Argonne - West } \\
\text { Bettis Atomic } \\
\text { Fermilab (FNAL) } \\
\text { Hanford Site } \\
\text { INEL } \\
\text { ITRI } \\
\text { Oak Ridge Reserv. } \\
\text { Knolls Atomic } \\
\text { Kansas City Plant } \\
\text { Los Alamos NL } \\
\text { Lawrence Berkeley } \\
\text { Lawrence Livermore } \\
\text { Mound Plant } \\
\text { ORISE } \\
\text { Pantex Plant } \\
\text { Portsmouth GDP } \\
\text { Princeton PPL } \\
\text { Paducah GDP } \\
\text { Pinellas Plant } \\
\text { Rocky Flats Plant } \\
\text { Reactive Metals } \\
\text { SLAC } \\
\text { SNL - Albuquerque } \\
\text { SNL - Livermore }\end{array}$ & $\begin{array}{l}\text { to Savannah River } \\
\text { to Savannah River } \\
\text { to Nevada Test Site } \\
\text { to Savannah River } \\
\text { to Savannah River } \\
\text { to Nevada Test Site } \\
\text { to Nevada Test Site } \\
\text { to Nevada Test Site } \\
\text { to Savannah River } \\
\text { to Savannah River } \\
\text { to Savannah River } \\
\text { to Nevada Test Site } \\
\text { to Nevada Test Site } \\
\text { to Nevada Test Site } \\
\text { to Savannah River } \\
\text { to Savannah River } \\
\text { to Nevada Test Site } \\
\text { to Savannah River } \\
\text { to Savannah River } \\
\text { to Savannah River } \\
\text { to Savannah River } \\
\text { to Nevada Test Site } \\
\text { to Savannah River } \\
\text { to Nevada Test Site } \\
\text { to Nevada Test Site } \\
\text { to Nevada Test Site }\end{array}$ & $\begin{array}{r}2 \\
105 \\
13 \\
57 \\
3 \\
1421 \\
852 \\
10 \\
6542 \\
28 \\
1 \\
724 \\
3 \\
56 \\
512 \\
5 \\
1450 \\
3344 \\
1 \\
627 \\
2 \\
357 \\
768 \\
39 \\
17 \\
6\end{array}$ & $\begin{array}{r}1 \\
39 \\
5 \\
21 \\
2 \\
531 \\
322 \\
4 \\
2486 \\
11 \\
1 \\
268 \\
2 \\
21 \\
190 \\
2 \\
544 \\
1274 \\
1 \\
237 \\
1 \\
133 \\
287 \\
15 \\
7 \\
2\end{array}$ & $\begin{array}{l}2.35 E+03 \\
9.36 E+04 \\
9.26 E+03 \\
3.74 E+04 \\
2.77 E+03 \\
1.60 E+06 \\
6.07 E+05 \\
9.18 E+03 \\
2.48 E+06 \\
2.69 E+04 \\
9.86 E+02 \\
7.22 E+05 \\
2.16 E+03 \\
3.80 E+04 \\
3.36 E+05 \\
1.84 E+03 \\
1.75 E+06 \\
1.81 E+06 \\
7.67 E+02 \\
3.56 E+05 \\
1.24 E+03 \\
2.98 E+05 \\
5.57 E+05 \\
2.88 E+04 \\
1.55 E+04 \\
4.07 E+03\end{array}$ & 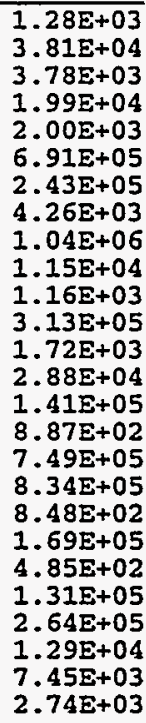 \\
\hline Totals & & 16945 & 6407 & $1.08 \Xi+07$ & $4.71 E+06$ \\
\hline
\end{tabular}


Table 3. Incident-Free Dose per Shipment (person-rem/shipment)

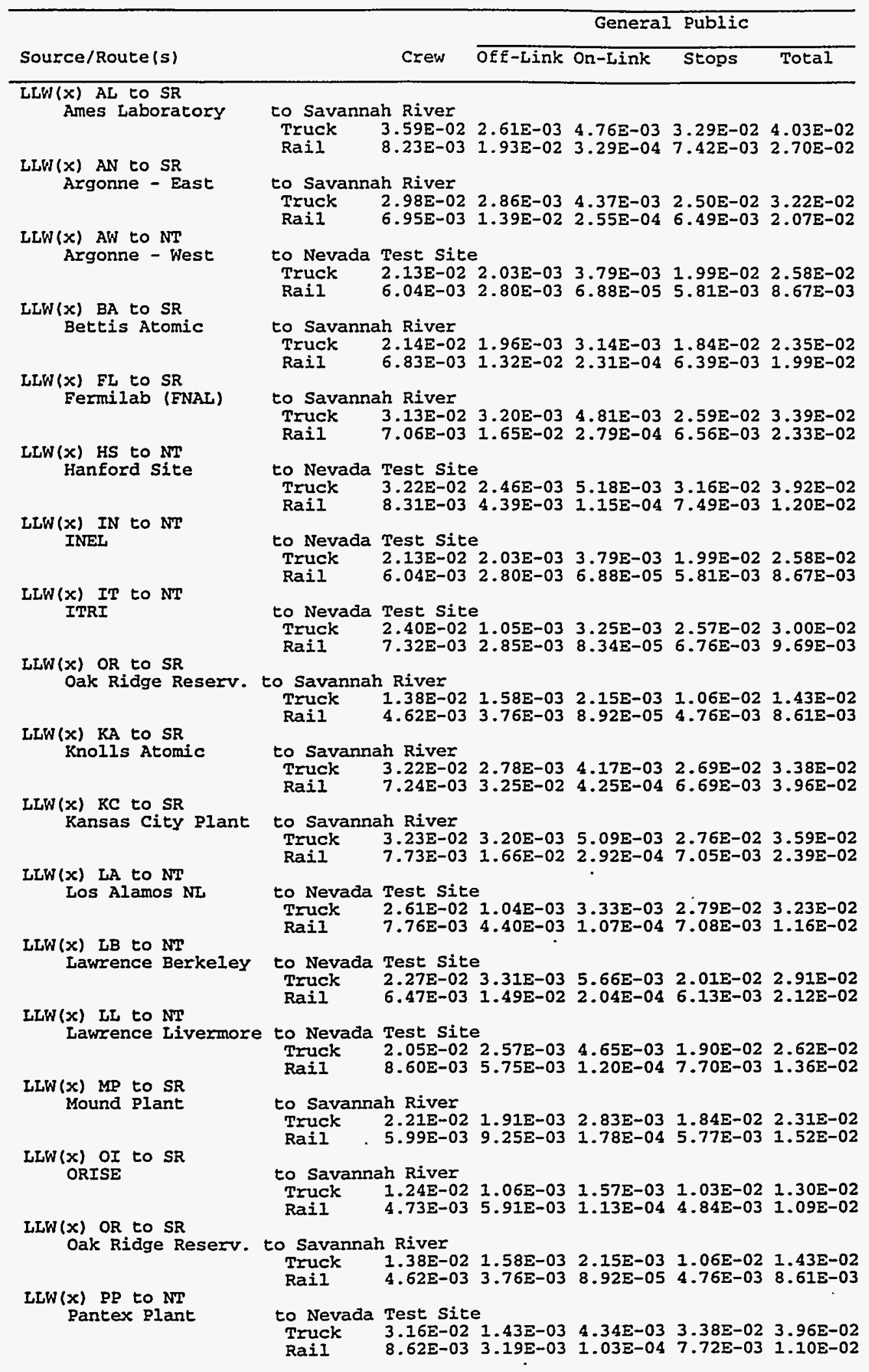




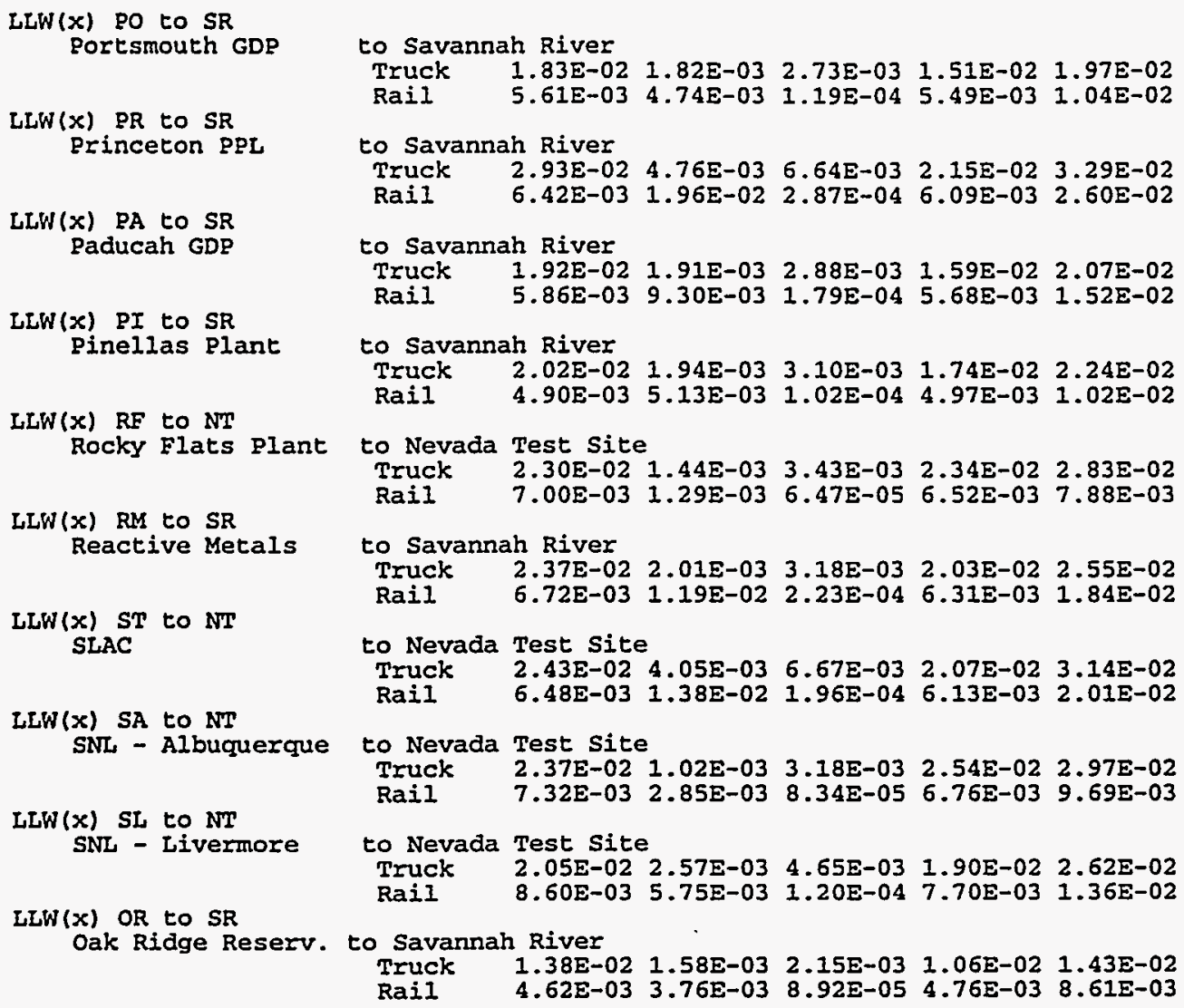


TABLE 4.3 Fractional Occurrences for Accidents by Severity Category and Population

Density Zone

\begin{tabular}{|c|c|c|c|c|}
\hline \multirow{2}{*}{$\begin{array}{l}\text { Severity } \\
\text { Category }\end{array}$} & \multirow{2}{*}{$\begin{array}{l}\text { Fractional } \\
\text { Occurrence }\end{array}$} & \multicolumn{3}{|c|}{$\begin{array}{c}\text { Fractional Occurrence } \\
\text { by Population Density Zone }\end{array}$} \\
\hline & & Rural & Suburban & Urban \\
\hline \multicolumn{5}{|l|}{ Truck } \\
\hline$I$ & $5.5 E-01$ & 0.1 & 0.1 & 0.8 \\
\hline II & $3.6 \mathrm{E}-01$ & 0.1 & 0.1 & 0.8 \\
\hline III & $7.0 E-02$ & 0.3 & 0.4 & 0.3 \\
\hline IV & $1.6 \mathrm{E}-02$ & 0.3 & 0.4 & 0.3 \\
\hline V & $2.8 \mathrm{E}-03$ & 0.5 & 0.3 & 0.2 \\
\hline VI & $1.1 E-03$ & 0.7 & 0.2 & 0.1 \\
\hline VII & $8.5 E-05$ & 0.8 & 0.1 & 0.1 \\
\hline VIII & $1.5 \mathrm{E}-05$ & 0.9 & 0.05 & 0.05 \\
\hline \multicolumn{5}{|l|}{ Rail } \\
\hline I & $5.0 \mathrm{E}-01$ & 0.1 & 0.1 & 0.8 \\
\hline$\Pi$ & $3.0 E-01$ & 0.1 & 0.1 & 0.8 \\
\hline III & $1.8 \mathrm{E}-01$ & 0.3 & 0.4 & 0.3 \\
\hline IV & $1.8 \mathrm{E}-02$ & 0.3 & 0.4 & 0.3 \\
\hline $\mathrm{V}$ & $1.8 \mathrm{E}-03$ & 0.5 & 0.3 & 0.2 \\
\hline VI & $1.3 E-04$ & 0.7 & 0.2 & 0.1 \\
\hline VII & $6.0 \mathrm{E}-05$ & 0.8 & 0.1 & 0.1 \\
\hline VIII & $1.0 \mathrm{E}-05$ & 0.9 & 0.05 & 0.05 \\
\hline
\end{tabular}

Source: NRC (1977a).

Representative waste package release fractions for accidents involving LLW shipments were taken from NUREG-0170 (NRC 1977a). The recommendations in NUREG-0170 are based on best engineering judgments and have been shown to provide conservative estimates of radioactive material releases following accidents. The release fractions used are those reported in NUREG-0170 for NRC Type A packages. Release fractions for accidents of each severity category are given in Table 4.4. As shown in Table 4.4 the amount of material released from the packaging ranges from zero for minor accidents to $100 \%$ for the most severe accidents.

Also important for the purposes of risk assessment are the fraction of the released material that can be entrained in an aerosol (part of an airborne radioactive plume) and the fraction of the aerosolized material that is also respirable (of a size that can be inhaled into the lungs). These 
Table 4. Accident Dose Risk per Shipment (person-rem/shipment)

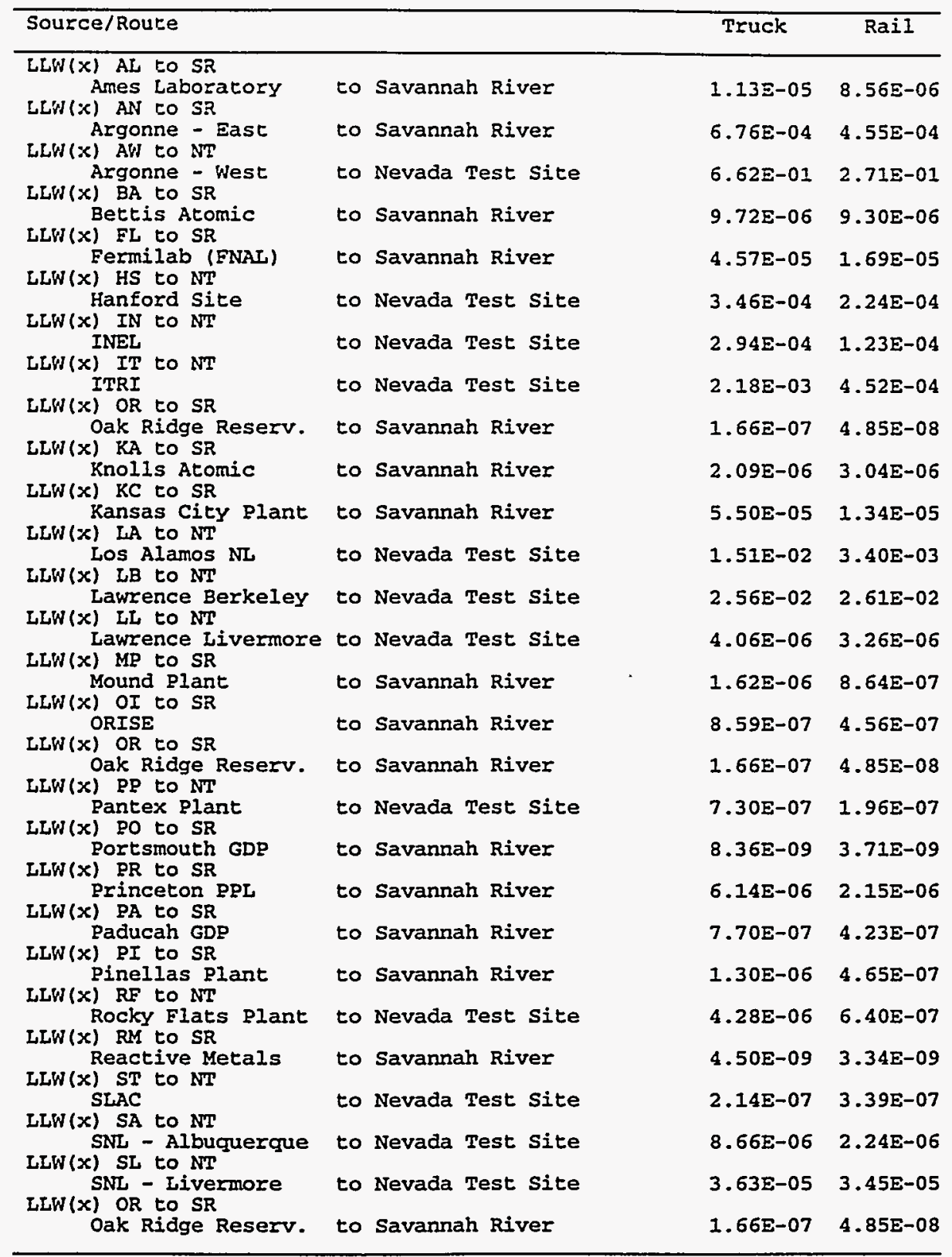


Table 5. Nonradiological Risk Factors per Shipment (fatalities/shipment)

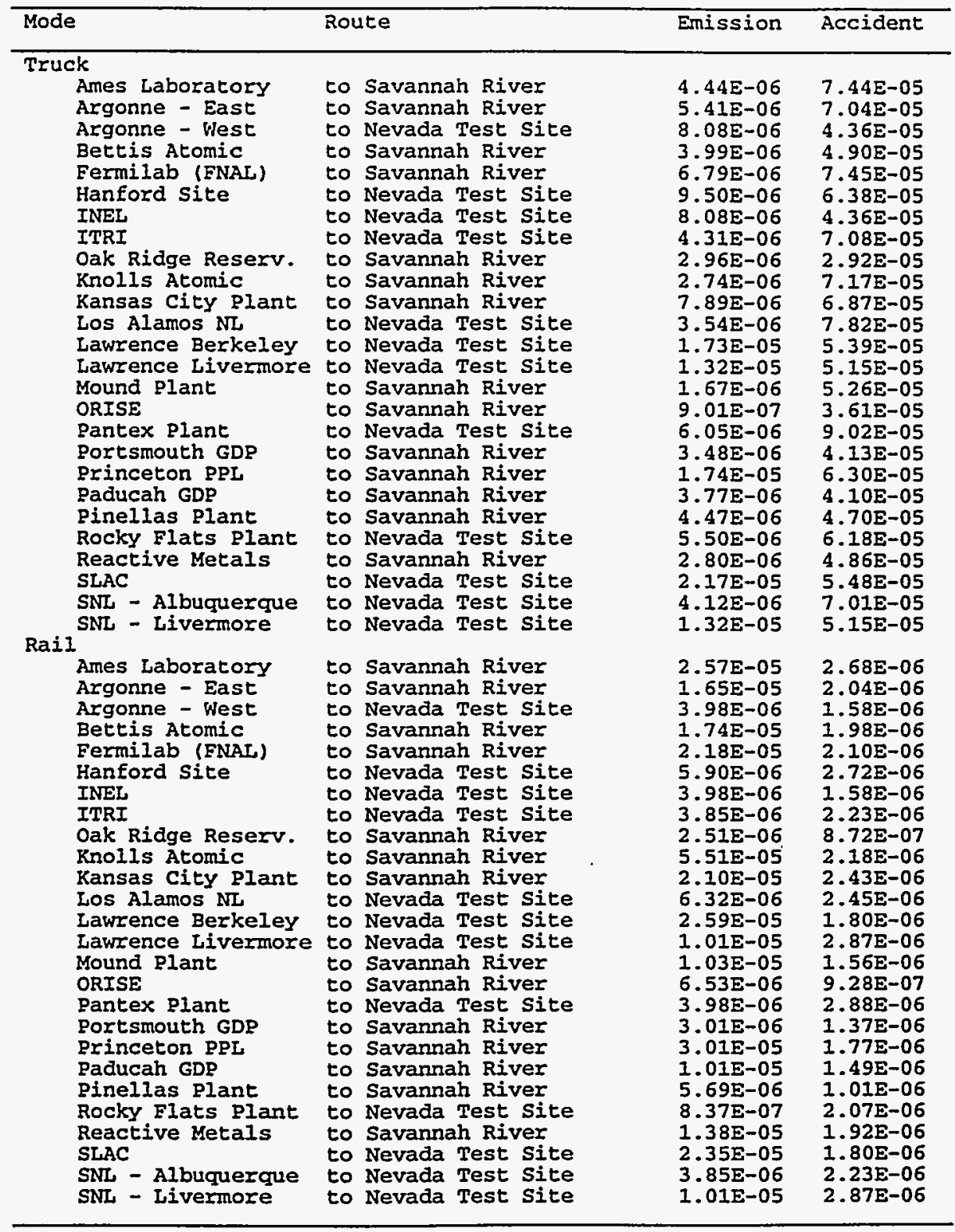


Table 6. Annual Incident-Free Dose for the Shipping Campaign (person-rem/yr)

\begin{tabular}{|c|c|c|c|c|c|c|}
\hline \multirow[b]{2}{*}{ Route } & & & \multicolumn{2}{|c|}{ Truck } & \multicolumn{2}{|c|}{ Rail } \\
\hline & & & Crew & Public & Crew & Public \\
\hline $\begin{array}{l}\text { Ames Laboratory } \\
\text { Argonne - East } \\
\text { Argonne - Hest } \\
\text { Bettis Atomic } \\
\text { Fermilab (FNA) } \\
\text { Hanford Site } \\
\text { INEL } \\
\text { ITRI } \\
\text { Oak Ridge Reserv. } \\
\text { Knolls Atomic } \\
\text { Kansas City Plant } \\
\text { Los Alamos NL } \\
\text { Lawrence Berkeley } \\
\text { Lawrence Livermore } \\
\text { Mound Plant } \\
\text { ORISE } \\
\text { Pantex Plant } \\
\text { Portsmouth GDP } \\
\text { Princeton pPL } \\
\text { Paducah GDP } \\
\text { Pinellas Plant } \\
\text { Rocky Flats Plant } \\
\text { Reactive Metals } \\
\text { SLAC } \\
\text { SNL - Albuquerque } \\
\text { SNL - Livermore }\end{array}$ & $\begin{array}{l}\text { co } \\
\text { to } \\
\text { to } \\
\text { to } \\
\text { to } \\
\text { to } \\
\text { to } \\
\text { to } \\
\text { to } \\
\text { to } \\
\text { to } \\
\text { to } \\
\text { to } \\
\text { to } \\
\text { to } \\
\text { to } \\
\text { to } \\
\text { to } \\
\text { to } \\
\text { to } \\
\text { to } \\
\text { to } \\
\text { to } \\
\text { to } \\
\text { to }\end{array}$ & $\begin{array}{l}\text { Savannah River } \\
\text { Savannah River } \\
\text { Nevada Test Site } \\
\text { Savannah River } \\
\text { Savannah River } \\
\text { Nevada Test Site } \\
\text { Nevada Test Site } \\
\text { Nevada Test Site } \\
\text { Savannah River } \\
\text { Savannah River } \\
\text { Savannah River } \\
\text { Nevada Test Site } \\
\text { Nevada Test Site } \\
\text { Nevada Test Site } \\
\text { Savannah River } \\
\text { Savannah River } \\
\text { Nevada Test Site } \\
\text { Savannah River } \\
\text { Savannah River } \\
\text { Savannah River } \\
\text { Savannah River } \\
\text { Nevada Test Site } \\
\text { Savannah River } \\
\text { Nevada Test Site } \\
\text { Nevada Test Site } \\
\text { Nevada Test Site }\end{array}$ & $\begin{array}{l}7.18 \mathrm{E}-02 \\
3.13 \mathrm{E}+00 \\
2.77 \mathrm{E}-01 \\
1.22 \mathrm{E}+00 \\
9.38 \mathrm{E}-02 \\
4.58 \mathrm{E}+01 \\
1.82 \mathrm{E}+01 \\
2.40 \mathrm{E}-01 \\
9.01 \mathrm{E}+01 \\
9.00 \mathrm{E}-01 \\
3.23 \mathrm{E}-02 \\
1.89 \mathrm{E}+01 \\
6.81 \mathrm{E}-02 \\
1.15 \mathrm{E}+00 \\
1.13 \mathrm{E}+01 \\
6.18 \mathrm{E}-02 \\
4.58 \mathrm{E}+01 \\
6.12 \mathrm{E}+01 \\
2.93 \mathrm{E}-02 \\
1.20 \mathrm{E}+01 \\
4.04 \mathrm{E}-02 \\
8.22 \mathrm{E}+00 \\
1.82 \mathrm{E}+01 \\
9.46 \mathrm{E}-01 \\
4.03 \mathrm{E}-01 \\
1.23 \mathrm{E}-01\end{array}$ & $\begin{array}{l}8.05 \mathrm{E}-02 \\
3.38 \mathrm{E}+00 \\
3.35 \mathrm{E}-01 \\
1.34 \mathrm{E}+00 \\
1.02 \mathrm{E}-01 \\
5.57 \mathrm{E}+01 \\
2.19 \mathrm{E}+01 \\
3.00 \mathrm{E}-01 \\
9.38 \mathrm{E}+01 \\
9.48 \mathrm{E}-01 \\
3.59 \mathrm{E}-02 \\
2.34 \mathrm{E}+01 \\
8.73 \mathrm{E}-02 \\
1.47 \mathrm{E}+00 \\
1.18 \mathrm{E}+01 \\
6.48 \mathrm{E}-02 \\
5.74 \mathrm{E}+01 \\
6.58 \mathrm{E}+01 \\
3.29 \mathrm{E}-02 \\
1.30 \mathrm{E}+01 \\
4.48 \mathrm{E}-02 \\
1.01 \mathrm{E}+01 \\
1.96 \mathrm{E}+01 \\
1.23 \mathrm{E}+00 \\
5.04 \mathrm{E}-01 \\
1.57 \mathrm{E}-01\end{array}$ & $\begin{array}{l}8.23 \mathrm{E}-03 \\
2.71 \mathrm{E}-01 \\
3.02 \mathrm{E}-02 \\
1.43 \mathrm{E}-01 \\
1.41 \mathrm{E}-02 \\
4.42 \mathrm{E}+00 \\
1.94 \mathrm{E}+00 \\
2.93 \mathrm{E}-02 \\
1.15 \mathrm{E}+01 \\
7.96 \mathrm{E}-02 \\
7.73 \mathrm{E}-03 \\
2.08 \mathrm{E}+00 \\
1.29 \mathrm{E}-02 \\
1.81 \mathrm{E}-01 \\
1.14 \mathrm{E}+00 \\
9.46 \mathrm{E}-03 \\
4.69 \mathrm{E}+00 \\
7.15 \mathrm{E}+00 \\
6.42 \mathrm{E}-03 \\
1.39 \mathrm{E}+00 \\
4.90 \mathrm{E}-03 \\
9.31 \mathrm{E}-01 \\
1.93 \mathrm{E}+00 \\
9.72 \mathrm{E}-02 \\
5.13 \mathrm{E}-02 \\
1.72 \mathrm{E}-02\end{array}$ & $\begin{array}{l}2.70 \mathrm{E}-02 \\
8.06 \mathrm{E}-01 \\
4.34 \mathrm{E}-02 \\
4.17 \mathrm{E}-01 \\
4.66 \mathrm{E}-02 \\
6.37 \mathrm{E}+00 \\
2.79 \mathrm{E}+00 \\
3.88 \mathrm{E}-02 \\
2.14 \mathrm{E}+01 \\
4.36 \mathrm{E}-01 \\
2.39 \mathrm{E}-02 \\
3.11 \mathrm{E}+00 \\
4.24 \mathrm{E}-02 \\
2.85 \mathrm{E}-01 \\
2.89 \mathrm{E}+00 \\
2.17 \mathrm{E}-02 \\
5.99 \mathrm{E}+00 \\
1.32 \mathrm{E}+01 \\
2.60 \mathrm{E}-02 \\
3.59 \mathrm{E}+00 \\
1.02 \mathrm{E}-02 \\
1.05 \mathrm{E}+00 \\
5.29 \mathrm{E}+00 \\
3.02 \mathrm{E}-01 \\
6.78 \mathrm{E}-02 \\
2.71 \mathrm{E}-02\end{array}$ \\
\hline cal & & & 02 & 02 & $8+01$ & 01 \\
\hline
\end{tabular}

Table 7. Annual Accident Dose Risk for the Shipping Campaign (person-rem/yr)

\begin{tabular}{|c|c|c|c|}
\hline Route & & Truck & Rail \\
\hline $\begin{array}{l}\text { Ames Laboratory } \\
\text { Argonne - East } \\
\text { Argonne - West } \\
\text { Bettis Atomic } \\
\text { Fermilab (FNAL) } \\
\text { Hanford Site } \\
\text { INEI } \\
\text { ITRI } \\
\text { Oak Ridge Reserv. } \\
\text { Knolls Atomic } \\
\text { Kansas City Plant } \\
\text { Ios Alamos NL } \\
\text { Lawrence Berkeley } \\
\text { Lawrence Livermore } \\
\text { Mound Plant } \\
\text { ORISE } \\
\text { Pantex Plant } \\
\text { Portsmouth GDP } \\
\text { Princeton PPL } \\
\text { Paducah GDP } \\
\text { Pinellas Plant } \\
\text { Rocky Flats Plant } \\
\text { Reactive Metals } \\
\text { SLAC } \\
\text { SNL - Albuquerque } \\
\text { SNL - Livermore }\end{array}$ & $\begin{array}{l}\text { to Savannah River } \\
\text { to Savannah River } \\
\text { to Nevada Test Site } \\
\text { to Savannah River } \\
\text { to Savannah River } \\
\text { to Nevada Test Site } \\
\text { to Nevada Test Site } \\
\text { to Nevada Test Site } \\
\text { to Savannah River } \\
\text { to Savannah River } \\
\text { to Savannah River } \\
\text { to Nevada Test Site } \\
\text { to Nevada Test Site } \\
\text { to Nevada Test Site } \\
\text { to Savannah River } \\
\text { to Savannah River } \\
\text { to Nevada Test Site } \\
\text { to Savannah River } \\
\text { to Savannah River } \\
\text { to Savannah River } \\
\text { to Savannah River } \\
\text { to Nevada Test Site } \\
\text { to Savannah River } \\
\text { to Nevada Test Site } \\
\text { to Nevada Test Site } \\
\text { to Nevada Test Site }\end{array}$ & $\begin{array}{l}2.25 \mathrm{E}-05 \\
7.10 \mathrm{E}-02 \\
8.60 \mathrm{E}+00 \\
5.54 \mathrm{E}-04 \\
1.37 \mathrm{E}-04 \\
4.92 \mathrm{E}-01 \\
2.50 \mathrm{E}-01 \\
2.18 \mathrm{E}-02 \\
1.09 \mathrm{E}-03 \\
5.84 \mathrm{E}-05 \\
5.50 \mathrm{E}-05 \\
1.10 \mathrm{E}+01 \\
7.68 \mathrm{E}-02 \\
2.27 \mathrm{E}-04 \\
8.30 \mathrm{E}-04 \\
4.30 \mathrm{E}-06 \\
1.06 \mathrm{E}-03 \\
2.80 \mathrm{E}-05 \\
6.14 \mathrm{E}-06 \\
4.83 \mathrm{E}-04 \\
2.59 \mathrm{E}-06 \\
1.53 \mathrm{E}-03 \\
3.45 \mathrm{E}-06 \\
8.33 \mathrm{E}-06 \\
1.47 \mathrm{E}-04 \\
2.18 \mathrm{E}-04\end{array}$ & 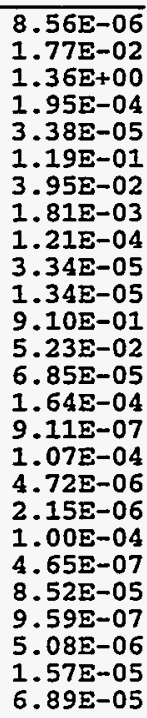 \\
\hline Totals & & $2.05 E+01$ & $2.50 E+00$ \\
\hline
\end{tabular}


Table 8. Expected Annual Fatalities for the Shipping Campaign

\begin{tabular}{llc}
\hline Exposure Group & Truck & Rail \\
\hline Radiological & $1.4 \mathrm{E}-01$ & $1.5 \mathrm{E}-02$ \\
$\quad$ Normal Crew & $1.9 \mathrm{E}-01$ & $3.4 \mathrm{E}-02$ \\
Normal Public & $1.0 \mathrm{E}-02$ & $1.2 \mathrm{E}-03$ \\
Accident Public & & \\
Nonradiological & $7.3 \mathrm{E}-02$ & $2.9 \mathrm{E}-02$ \\
$\quad$ Emission & $7.8 \mathrm{E}-01$ & $9.9 \mathrm{E}-03$ \\
Accident & & \\
\hline
\end{tabular}

Table 9. Expected Annual Cancer Incidence for the Shipping Campaign

\begin{tabular}{lll}
\hline Exposure Group & Truck & Rail \\
\hline Radiological & & \\
Normal Crew & $4.7 \mathrm{E}-01$ & $5.3 \mathrm{E}-02$ \\
Normal Public & $6.5 \mathrm{E}-01$ & $1.2 \mathrm{E}-01$ \\
Accident Public & $3.5 \mathrm{E}-02$ & $4.2 \mathrm{E}-03$ \\
Nonradiological & & \\
Emission & $7.3 \mathrm{E}-02$ & $2.9 \mathrm{E}-02$ \\
Accident & $\mathrm{NA}$ & $\mathrm{NA}$ \\
\hline
\end{tabular}

Table 10. Expected Annual Genetic Effects for the Shipping Campaign

\begin{tabular}{|c|c|c|}
\hline Exposure Group & Truck & Rail \\
\hline $\begin{array}{l}\text { Radiological } \\
\text { Normal Crew } \\
\text { Normal Public } \\
\text { Accident Public } \\
\text { Nonradiological } \\
\text { Emission } \\
\text { Accident }\end{array}$ & $\begin{array}{l}2.0 \mathrm{E}-02 \\
3.8 \mathrm{E}-02 \\
1.9 \mathrm{E}-03 \\
\mathrm{NA} \\
\mathrm{NA}\end{array}$ & $\begin{array}{l}2.3 \mathrm{E}-03 \\
6.8 \mathrm{E}-03 \\
2.2 \mathrm{E}-04 \\
\mathrm{NA} \\
\mathrm{NA}\end{array}$ \\
\hline
\end{tabular}




\section{B.18 WM LLW REGIONALIZED 7 ALTERNATIVE (CASE 6): ACTIVATED METALS}

Table 1. Summary of Route Information

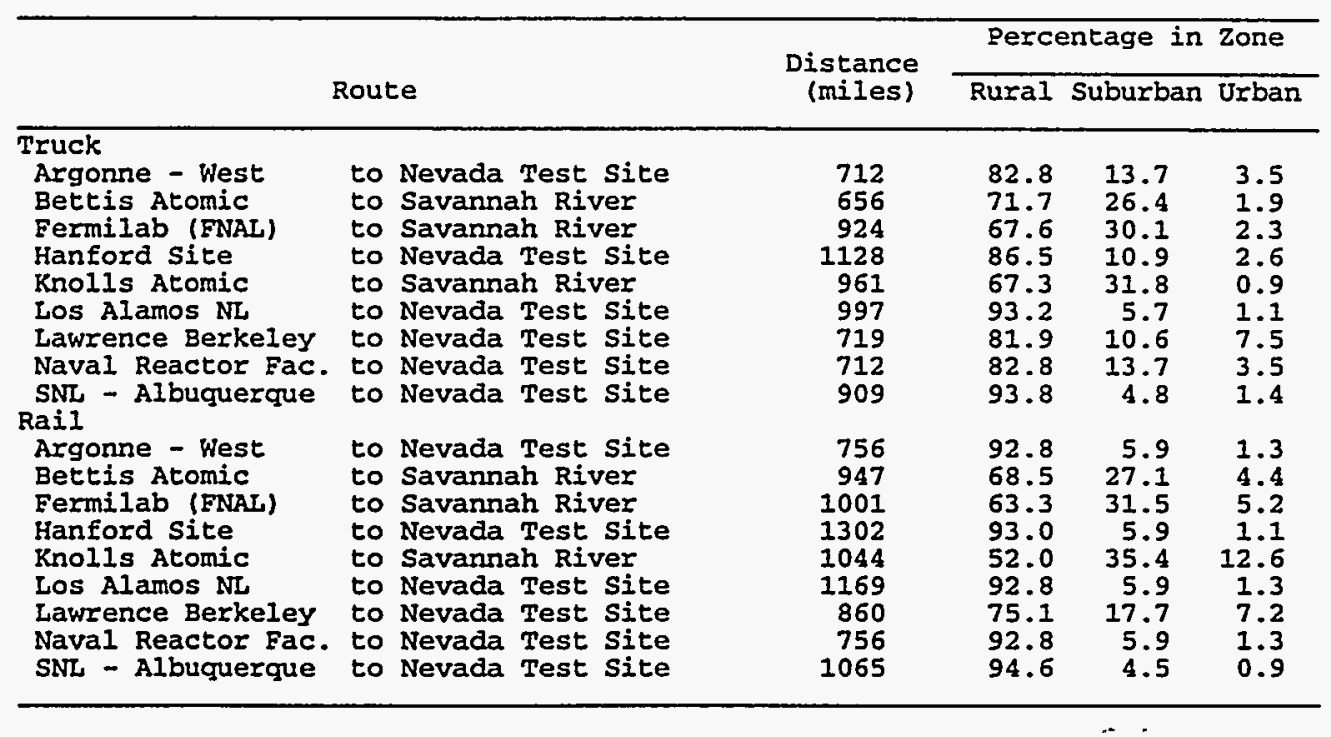

Table 2. Annual Number of Shipments and One-Way Shipment Distances.

\begin{tabular}{|c|c|c|c|c|c|}
\hline \multirow[b]{2}{*}{ Route } & & \multicolumn{2}{|c|}{ \# of Shipments } & \multicolumn{2}{|c|}{ Total Mileage(mi) } \\
\hline & & Truck & Rail & Truck & Rail \\
\hline $\begin{array}{l}\text { Argonne - West } \\
\text { Bettis Atomic } \\
\text { Fermilab (FNAL) } \\
\text { Hanford Site } \\
\text { Knolis Atomic } \\
\text { Los Alamos NL } \\
\text { Lawrence Berkeley } \\
\text { Naval Reactor Fac. } \\
\text { SNL - Albuquerque }\end{array}$ & $\begin{array}{l}\text { to Nevada Test Site } \\
\text { to Savannah River } \\
\text { to Savannah River } \\
\text { to Nevada Test Site } \\
\text { to Savannah River } \\
\text { to Nevada Test Site } \\
\text { to Nevada Test Site } \\
\text { to Nevada Test Site } \\
\text { to Nevada Test Site }\end{array}$ & $\begin{array}{r}99 \\
316 \\
40 \\
33 \\
650 \\
418 \\
15 \\
461 \\
6\end{array}$ & $\begin{array}{r}4 \\
120 \\
15 \\
13 \\
246 \\
159 \\
6 \\
175 \\
3\end{array}$ & $\begin{array}{l}6.41 E+03 \\
2.07 E+05 \\
3.70 E+04 \\
3.72 E+04 \\
6.25 E+05 \\
4.17 E+05 \\
1.08 E+04 \\
3.28 E+05 \\
5.45 E+03\end{array}$ & $\begin{array}{l}3.02 E+03 \\
1.14 E+05 \\
1.50 E+04 \\
1.69 E+04 \\
2.57 E+05 \\
1.86 E+05 \\
5.16 E+03 \\
1.32 E+05 \\
3.19 E+03\end{array}$ \\
\hline Totals & & 1948 & 741 & $1.67 \mathrm{E}+06$ & $7.32 \mathrm{E}+05$ \\
\hline
\end{tabular}


Table 3. Incident-Free Dose per Shipment (person-rem/shipment)

\begin{tabular}{|c|c|c|c|c|c|c|}
\hline \multirow[b]{2}{*}{ Source/Roure (s) } & & \multirow[b]{2}{*}{ Crew } & \multicolumn{4}{|c|}{ General Public } \\
\hline & & & OEf-Link & On-Link & Stops & Total \\
\hline $\begin{array}{l}\text { LLW(I) AW to NT } \\
\text { Argonne - West }\end{array}$ & $\begin{array}{l}\text { to Nevada } \\
\text { Truck } \\
\text { Rail }\end{array}$ & $\begin{array}{l}\text { Test Site } \\
2.13 \mathrm{E}-02 \\
6.04 \mathrm{E}-03\end{array}$ & $\begin{array}{l}2.03 \mathrm{E}-03 \\
2.80 \mathrm{E}-03\end{array}$ & $\begin{array}{l}3.7 \\
6.8\end{array}$ & $\begin{array}{l}1.99 \mathrm{E}-02 \\
5.81 \mathrm{E}-03\end{array}$ & $\begin{array}{l}2.58 \mathrm{E}-02 \\
8.67 \mathrm{E}-03\end{array}$ \\
\hline $\begin{array}{l}\text { LLW(I) BA to SR } \\
\text { Bettis Atomic }\end{array}$ & $\begin{array}{l}\text { to Savanna } \\
\text { Truck } \\
\text { Rail }\end{array}$ & $\begin{array}{c}\text { ah River } \\
2.14 \mathrm{E}-02 \\
6.83 \mathrm{E}-03\end{array}$ & $\begin{array}{l}1.96 \mathrm{E}-03 \\
1.32 \mathrm{E}-02\end{array}$ & $\begin{array}{l}3.14 \mathrm{E}-03 \\
2.31 \mathrm{E}-04\end{array}$ & $\begin{array}{l}1.84 E-02 \\
6.39 E-03\end{array}$ & $\begin{array}{l}2.35 \mathrm{E}-02 \\
1.99 \mathrm{E}-02\end{array}$ \\
\hline $\begin{array}{l}\text { LLW(I) FL to SR } \\
\text { Fermilab (FNAL) }\end{array}$ & $\begin{array}{l}\text { to Savanna } \\
\text { Truck } \\
\text { Rail }\end{array}$ & $\begin{array}{l}\text { ah River } \\
3.13 \mathrm{E}-02 \\
7.06 \mathrm{E}-03\end{array}$ & $\begin{array}{l}3.20 \mathrm{E}-03 \\
1.65 \mathrm{E}-02\end{array}$ & $\begin{array}{l}4.81 \mathrm{E}-03 \\
2.79 \mathrm{E}-04\end{array}$ & $\begin{array}{l}2.59 \mathrm{E}-02 \\
6.56 \mathrm{E}-03\end{array}$ & $\begin{array}{l}3.39 \mathrm{E}-02 \\
2.33 \mathrm{E}-02\end{array}$ \\
\hline $\begin{array}{l}\text { LLW(I) HS to NT } \\
\text { Hanford Site }\end{array}$ & $\begin{array}{l}\text { to Nevada } \\
\text { Truck } \\
\text { Rail }\end{array}$ & $\begin{array}{l}\text { Test Site } \\
3.22 \mathrm{E}-02 \\
8.31 \mathrm{E}-03\end{array}$ & $\begin{array}{l}2.46 \mathrm{E}-03 \\
4.39 \mathrm{E}-03\end{array}$ & $\begin{array}{l}5.18 \mathrm{E}-03 \\
1.15 \mathrm{E}-04\end{array}$ & $\begin{array}{l}3.16 \mathrm{E}-02 \\
7.49 \mathrm{E}-03\end{array}$ & $\begin{array}{l}3.92 \mathrm{E}-02 \\
1.20 \mathrm{E}-02\end{array}$ \\
\hline $\begin{array}{l}\text { LLW (I) KA to SR } \\
\text { Knolls Atomic }\end{array}$ & $\begin{array}{l}\text { to Savanna } \\
\text { Truck } \\
\text { Rail }\end{array}$ & $\begin{array}{l}\text { ah River } \\
3.22 \mathrm{E}-02 \\
7.24 \mathrm{E}-03\end{array}$ & $\begin{array}{l}2.78 \mathrm{E}-03 \\
3.25 \mathrm{E}-02\end{array}$ & $\begin{array}{l}4.17 \mathrm{E}-03 \\
4.25 \mathrm{E}-04\end{array}$ & $\begin{array}{l}2.69 \mathrm{E}-02 \\
6.69 \mathrm{E}-03\end{array}$ & $\begin{array}{l}3.38 \mathrm{E}-02 \\
3.96 \mathrm{E}-02\end{array}$ \\
\hline $\begin{array}{l}\text { LLW(I) LA to NT } \\
\text { LOS Alamos NL }\end{array}$ & $\begin{array}{l}\text { to Nevada } \\
\text { Truck } \\
\text { Rail }\end{array}$ & $\begin{array}{l}\text { Test site } \\
2.61 \mathrm{E}-02 \\
7.76 \mathrm{E}-03\end{array}$ & $\begin{array}{l}1.04 \mathrm{E}-03 \\
4.40 \mathrm{E}-03\end{array}$ & $\begin{array}{l}3.33 \mathrm{E}-03 \\
1.07 \mathrm{E}-04\end{array}$ & $\begin{array}{l}2.79 \mathrm{E}-02 \\
7.08 \mathrm{E}-03\end{array}$ & $\begin{array}{l}3.23 E-02 \\
1.16 E-02\end{array}$ \\
\hline $\begin{array}{l}\text { LLW(I) LB to NT } \\
\text { Lawrence Berkeley }\end{array}$ & $\begin{array}{l}\text { to Nevada } \\
\text { Truck } \\
\text { Rail }\end{array}$ & $\begin{array}{l}\text { Test Site } \\
2.27 E-02 \\
6.47 E-03\end{array}$ & $\begin{array}{l}3.31 \mathrm{E}-03 \\
1.49 \mathrm{E}-02\end{array}$ & $\begin{array}{l}5.66 \mathrm{E}-03 \\
2.04 \mathrm{E}-04\end{array}$ & $\begin{array}{l}2.01 \mathrm{E}-02 \\
6.13 \mathrm{E}-03\end{array}$ & $\begin{array}{l}2.91 \mathrm{E}-02 \\
2.12 \mathrm{E}-02\end{array}$ \\
\hline $\begin{array}{l}\text { LLW(I) NR to NT } \\
\text { Naval Reactor Fac. }\end{array}$ & $\begin{array}{l}\text { to Nevada } \\
\text { Truck } \\
\text { Rail }\end{array}$ & $\begin{array}{l}\text { Test Site } \\
2.13 E-02 \\
6.04 E-03\end{array}$ & $\begin{array}{l}2.03 E-03 \\
2.80 E-03\end{array}$ & $\begin{array}{l}3.79 \mathrm{E}-03 \\
6.88 \mathrm{E}-05\end{array}$ & $\begin{array}{l}1.99 \mathrm{E}-02 \\
5.81 \mathrm{E}-03\end{array}$ & $\begin{array}{l}2.58 \mathrm{E}-02 \\
8.67 \mathrm{E}-03\end{array}$ \\
\hline $\begin{array}{l}\text { LLW(I) SA to NT } \\
\text { SNL - Albuquerque }\end{array}$ & $\begin{array}{l}\text { to Nevada } \\
\text { Truck } \\
\text { Rail }\end{array}$ & $\begin{array}{l}\text { Test Site } \\
2.37 \pm-02 \\
7.32 E-03\end{array}$ & $\begin{array}{l}1.02 E-03 \\
2.85 E-03\end{array}$ & $\begin{array}{l}3.1 \\
8.3\end{array}$ & $\begin{array}{l}4 \mathrm{E}-02 \\
6 \mathrm{E}-03\end{array}$ & $\begin{array}{l}2.97 E-02 \\
9.69 \mathrm{E}-03\end{array}$ \\
\hline
\end{tabular}

Table 4. Accident Dose Risk per Shipment (person-rem/shipment)

\begin{tabular}{|c|c|c|c|}
\hline Source/Route & & Truck & $\operatorname{Rail}$ \\
\hline $\begin{array}{l}\text { LLW(I) AW to NT } \\
\text { Argonne - West } \\
\text { LLW(I) BA to SR } \\
\text { Bettis Atomic } \\
\text { LLW(I) FL to SR } \\
\text { Fermilab (FNAL) } \\
\text { LLW(I) HS to NT } \\
\text { Hanford Site } \\
\text { LLW(I) RA to SR } \\
\text { Rnolls Atomic } \\
\text { LLW(I) LA to NT } \\
\text { Ios Alamos NL } \\
\text { LLW(I) LB to NT } \\
\text { Lawrence Berkeley } \\
\text { LLW(I) NR to NT } \\
\text { Naval Reactor Fac. } \\
\text { LLW(I) SA to NT } \\
\text { SNL - Albuquerque }\end{array}$ & $\begin{array}{l}\text { to Nevada Test Site } \\
\text { to Savannah River } \\
\text { to Savannah River } \\
\text { to Nevada Test Site } \\
\text { to Savannah River } \\
\text { to Nevada Test Site } \\
\text { to Nevada Test Site } \\
\text { to Nevada Test Site } \\
\text { to Nevada Test Site }\end{array}$ & $\begin{array}{l}2.03 \mathrm{E}-11 \\
7.03 \mathrm{E}-07 \\
9.75 \mathrm{E}-11 \\
2.71 \mathrm{E}-08 \\
1.44 \mathrm{E}-06 \\
1.78 \mathrm{E}-09 \\
8.23 \mathrm{E}-11 \\
1.20 \mathrm{E}-07 \\
2.40 \mathrm{E}-12\end{array}$ & $\begin{array}{l}7.25 \mathrm{E}-12 \\
6.43 \mathrm{E}-07 \\
6.35 \mathrm{E}-11 \\
1.57 \mathrm{E}-08 \\
2.20 \mathrm{E}-06 \\
3.07 \mathrm{E}-10 \\
1.45 \mathrm{E}-10 \\
4.99 \mathrm{E}-08 \\
3.74 \mathrm{E}-13\end{array}$ \\
\hline
\end{tabular}


Table 5. Nonradiological Risk Factors per Shipment (Eatalities/shipment)

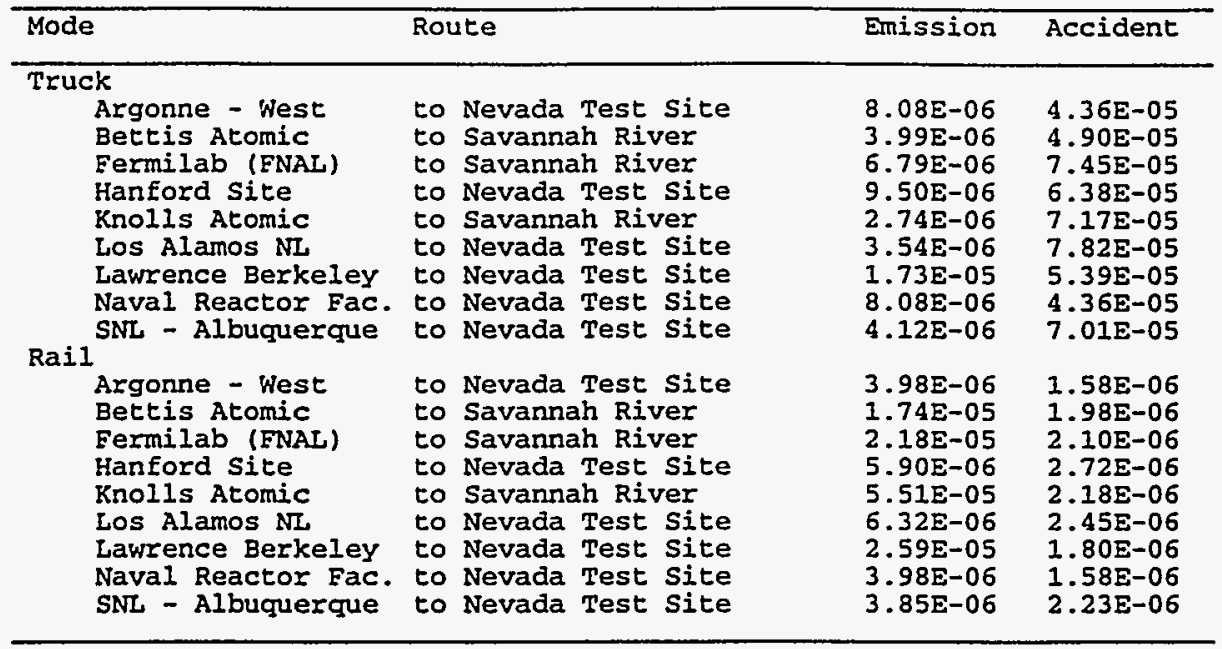

Table 6. Annual Incident-Free Dose for the Shipping Campaign (person-rem/yr)

\begin{tabular}{|c|c|c|c|c|c|}
\hline \multirow[b]{2}{*}{ Route } & & \multicolumn{2}{|c|}{ Truck } & \multicolumn{2}{|c|}{ Rail } \\
\hline & & Crew & $\overline{\text { Public }}$ & Crew & Public \\
\hline $\begin{array}{l}\text { Argonne - West } \\
\text { Bettis Atomic } \\
\text { Fermilab (FNAL) } \\
\text { Hanford Site } \\
\text { Knolls Atomic } \\
\text { Los Alamos NL } \\
\text { Lawrence Berkeley } \\
\text { Naval Reactor Fac. } \\
\text { SNL - Albuquerque }\end{array}$ & $\begin{array}{l}\text { to Nevada Test Site } \\
\text { to Savannah River } \\
\text { to Savannah River } \\
\text { to Nevada Test Site } \\
\text { to Savannah River } \\
\text { to Nevada Test Site } \\
\text { to Nevada Test Site } \\
\text { to Nevada Test Site } \\
\text { to Nevada Test Site }\end{array}$ & $\begin{array}{l}1.92 \mathrm{E}-01 \\
6.75 \mathrm{E}+00 \\
1.25 \mathrm{E}+00 \\
1.06 \mathrm{E}+00 \\
2.09 \mathrm{E}+01 \\
1.09 \mathrm{E}+01 \\
3.41 \mathrm{E}-01 \\
9.83 \mathrm{E}+00 \\
1.42 \mathrm{E}-01\end{array}$ & $\begin{array}{l}2.32 \mathrm{E}-01 \\
7.42 \mathrm{E}+00 \\
1.36 \mathrm{E}+00 \\
1.29 \mathrm{E}+00 \\
2.20 \mathrm{E}+01 \\
1.35 \mathrm{E}+01 \\
4.36 \mathrm{E}-01 \\
1.19 \mathrm{E}+01 \\
1.78 \mathrm{E}-01\end{array}$ & $\begin{array}{l}2.41 \mathrm{E}-02 \\
8.20 \mathrm{E}-01 \\
1.06 \mathrm{E}-01 \\
1.08 \mathrm{E}-01 \\
1.78 \mathrm{E}+00 \\
1.23 \mathrm{E}+00 \\
3.88 \mathrm{E}-02 \\
1.06 \mathrm{E}+00 \\
2.20 \mathrm{E}-02\end{array}$ & $\begin{array}{l}3.47 \mathrm{E}-02 \\
2.38 \mathrm{E}+00 \\
3.50 \mathrm{E}-01 \\
1.56 \mathrm{E}-01 \\
9.75 \mathrm{E}+00 \\
1.84 \mathrm{E}+00 \\
1.27 \mathrm{E}-01 \\
1.52 \mathrm{E}+00 \\
2.91 \mathrm{E}-02\end{array}$ \\
\hline Totals & & $5.14 \mathrm{E}+01$ & $5.83 E+01$ & $5.19 E+00$ & $1.62 \mathrm{E}+01$ \\
\hline
\end{tabular}

Table 7. Annual Accident Dose Risk for the Shipping Campaign (person-rem/yr)

\begin{tabular}{llcc}
\hline Route & & Truck & RaiI \\
\hline Argonne - West & to Nevada Test Site & $1.82 \mathrm{E}-10$ & $2.90 \mathrm{E}-11$ \\
Bettis Atomic & to Savannah River & $2.22 \mathrm{E}-04$ & $7.71 \mathrm{E}-05$ \\
Fermilab (FNAL) & to Savannah River & $3.90 \mathrm{E}-09$ & $9.53 \mathrm{E}-10$ \\
Hanford Site & to Nevada Test Site & $8.95 \mathrm{E}-07$ & $2.05 \mathrm{E}-07$ \\
Knolls Atomic & to Savannah River & $9.36 \mathrm{E}-04$ & $5.42 \mathrm{E}-04$ \\
Los Alamos N. & to Nevada Test Site & $7.46 \mathrm{E}-07$ & $4.89 \mathrm{E}-08$ \\
Lawrence Berkeley to Nevada Test Site & $1.23 \mathrm{E}-09$ & $8.71 \mathrm{E}-10$ \\
Naval Reactor Fac. to Nevada Test Site & $5.53 \mathrm{E}-05$ & $8.73 \mathrm{E}-06$ \\
SNL - Albuquerque & to Nevada Test Site & $1.44 \mathrm{E}-11$ & $1.12 \mathrm{E}-12$ \\
\hline Totals & & & \\
\hline
\end{tabular}

Table 8. Expected Annual Fatalities for the Shipping Campaign

\begin{tabular}{|c|c|c|}
\hline Exposure Group & Truck & Rail \\
\hline $\begin{array}{l}\text { Radiological } \\
\text { Normal Crew } \\
\text { Normal Public } \\
\text { Accident Public } \\
\text { Nonradiological }\end{array}$ & $\begin{array}{l}2.1 E-02 \\
2.9 E-02 \\
6.1 E-07\end{array}$ & $\begin{array}{l}2.1 \mathrm{E}-03 \\
8.1 \mathrm{E}-03 \\
3.1 \mathrm{E}-07\end{array}$ \\
\hline $\begin{array}{l}\text { Emission } \\
\text { Accident }\end{array}$ & $\begin{array}{l}9.2 \mathrm{E}-03 \\
1.2 \mathrm{E}-01\end{array}$ & $\begin{array}{l}1.8 \mathrm{E}-02 \\
1.5 \mathrm{E}-03\end{array}$ \\
\hline
\end{tabular}


Table 9. Expected Annual Cancer Incidence for the Shipping Campaign

\begin{tabular}{lll}
\hline Exposure Group & Truck & Rail \\
\hline Radiological & & \\
Normal Crew & $7.2 \mathrm{E}-02$ & $7.3 \mathrm{E}-03$ \\
Normal Public & $9.9 \mathrm{E}-02$ & $2.8 \mathrm{E}-02$ \\
Accident Public & $2.1 \mathrm{E}-06$ & $1.1 \mathrm{E}-06$ \\
$\begin{array}{c}\text { Nonradiological } \\
\text { Emission }\end{array}$ & $9.2 \mathrm{E}-03$ & $1.8 \mathrm{E}-02$ \\
Accident & $\mathrm{NA}$ & $\mathrm{NA}$ \\
\hline
\end{tabular}

Table 10. Expected Annual Genetic Effects for the Shipping Campaign

\begin{tabular}{lll}
\hline Exposure Group & Truck & Rail \\
\hline Radiological & & \\
Normal Crew & $3.1 E-03$ & $3.1 E-04$ \\
Normal Public & $5.8 E-03$ & $1.6 E-03$ \\
Accident Public & $1.2 E-07$ & $6.3 E-08$ \\
$\begin{array}{c}\text { Nonradiological } \\
\text { Emission }\end{array}$ & NA & NA \\
Accident & NA & NA \\
\hline
\end{tabular}




\section{B.19 WM LLW CENTRALIZED 1 ALTERNATIVE (CASE 7): HETEROGENEOUS SOLIDS}

Table 1. Summary of Route Information

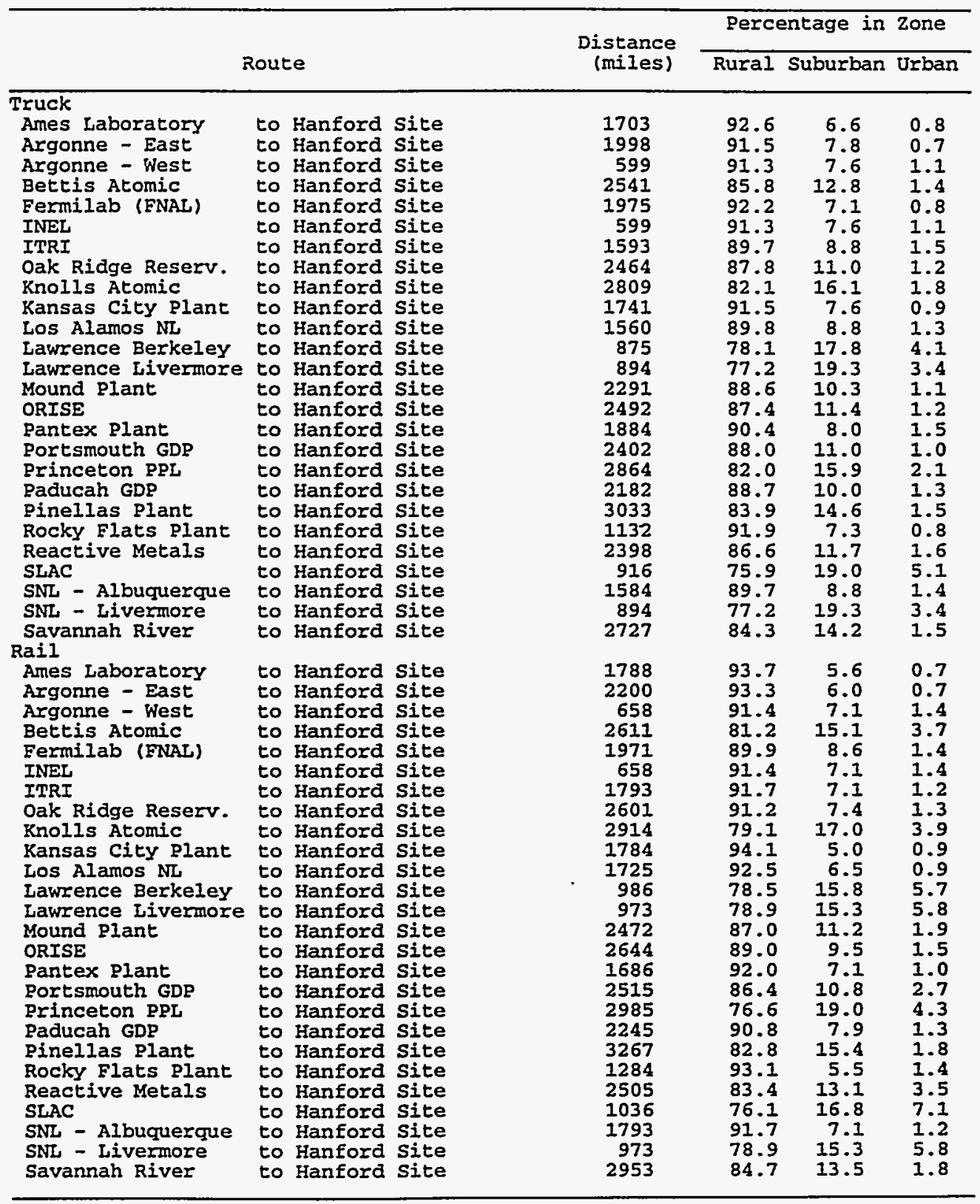


Table 2. Annual Number of Shipments and One-Way Shipment Distances

\begin{tabular}{|c|c|c|c|c|c|}
\hline \multirow[b]{2}{*}{ Route } & & \multicolumn{2}{|c|}{ \# of Shipments } & \multicolumn{2}{|c|}{ Total Mileage (mi) } \\
\hline & & Truck & Rail & Truck & Rail \\
\hline $\begin{array}{l}\text { Ames Laboratory } \\
\text { Argonne - East } \\
\text { Argonne - West } \\
\text { Bettis Atomic } \\
\text { Fermilab (FNAL) } \\
\text { INEL } \\
\text { ITRI } \\
\text { Oak Ridge Reserv. } \\
\text { Knolls Atomic } \\
\text { Kansas City Plant } \\
\text { Los Alamos NL } \\
\text { Lawrence Berkeley } \\
\text { Lawrence Livermore } \\
\text { Mound Plant } \\
\text { ORISE } \\
\text { Pantex Plant } \\
\text { Portsmouth GDP } \\
\text { Princeton pPL } \\
\text { Paducah GDP } \\
\text { Pinellas Plant } \\
\text { Rocky Flats Plant } \\
\text { Reactive Metals } \\
\text { SLAC - Albuquerque } \\
\text { SNL - Albuguer } \\
\text { SNL - Livermore } \\
\text { Savannah River }\end{array}$ & 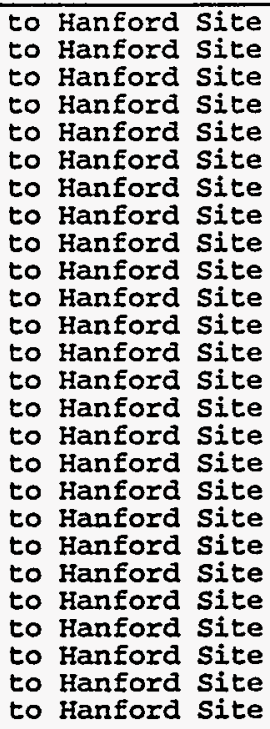 & $\begin{array}{r}2 \\
105 \\
13 \\
57 \\
3 \\
852 \\
10 \\
6542 \\
28 \\
1 \\
724 \\
3 \\
56 \\
512 \\
5 \\
1450 \\
3344 \\
1 \\
627 \\
2 \\
357 \\
768 \\
39 \\
17 \\
6 \\
6834\end{array}$ & $\begin{array}{r}31 \\
39 \\
5 \\
21 \\
2 \\
322 \\
4 \\
2486 \\
11 \\
1 \\
268 \\
2 \\
21 \\
190 \\
2 \\
544 \\
1274 \\
1 \\
237 \\
1 \\
133 \\
287 \\
15 \\
7 \\
2 \\
2540\end{array}$ & 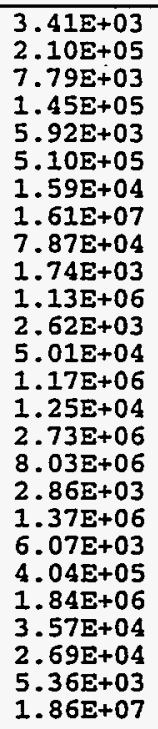 & $\begin{array}{l}1.79 \mathrm{E}+03 \\
8.58 \mathrm{E}+04 \\
3.29 \mathrm{E}+03 \\
5.48 \mathrm{E}+04 \\
3.94 \mathrm{E}+03 \\
2.12 \mathrm{E}+05 \\
7.17 \mathrm{E}+03 \\
6.47 \mathrm{E}+06 \\
3.21 \mathrm{E}+04 \\
1.78 \mathrm{E}+03 \\
4.62 \mathrm{E}+05 \\
1.97 \mathrm{E}+03 \\
2.04 \mathrm{E}+04 \\
4.70 \mathrm{E}+05 \\
5.29 \mathrm{E}+03 \\
9.17 \mathrm{E}+05 \\
3.20 \mathrm{E}+06 \\
2.98 \mathrm{E}+03 \\
5.32 \mathrm{E}+05 \\
3.27 \mathrm{E}+03 \\
1.71 \mathrm{E}+05 \\
7.39 \mathrm{E}+05 \\
1.55 \mathrm{E}+04 \\
1.26 \mathrm{E}+04 \\
1.95 \mathrm{E}+03 \\
7.50 \mathrm{E}+06\end{array}$ \\
\hline Totals & & 22358 & 8416 & $5.26 E+07$ & $2.09 E+07$ \\
\hline
\end{tabular}


fractions depend on the physical form of the waste material. As stated previously, the LLW assessment considered two physical waste forms: heterogeneous solids and activated metals. The aerosolized and respirable fractions for these two forms are given in Table 4.5.

\subsection{ATMOSPHERIC CONDITIONS DURING ACCIDENTS}

Radioactive material released to the atmosphere is transported by the wind. Dispersion or dilution of the radioactive material in the air depends on meteorologic conditions at the time of the accident. Because predicting the specific location of an off-site transportation-related accident is impossible, generic atmospheric conditions were selected for the accident risk and consequence assessments.

For the accident risk assessment, neutral weather conditions (represented by Pasquill Stability Class D with a wind speed of $4 \mathrm{~m} / \mathrm{s}$ [9 mph]) were assumed. Because neutral meteorologic conditions constitute the most frequently occurring atmospheric stability conditions in the United States, these conditions are most likely to be present in the event of an accident involving a LLW shipment. On the basis of observations from National Weather Service surface meteorologic stations at over 300 locations in the United States, on an annual average, neutral conditions (represented by Pasquill Classes $C$ and D) occur about one-half of the time, while stable (Pasquill Classes E and F) and unstable (Pasquill Classes $A$ and B) conditions occur about one-third and one-sixth of the time, respectively (Doty et al. 1976). The neutral category predominates in all seasons but most frequently in the winter (nearly $60 \%$ of the observations). For the accident consequence assessment, doses were assessed under both neutral and stable (Pasquill Stability Class $F$ with a wind speed of $1 \mathrm{~m} / \mathrm{s}$ [2.2 mph]) atmospheric conditions. The results calculated for neutral conditions represent the most likely consequences, and the results for stable conditions represent a "worst-case" weather situation (i.e., conditions that result in the least amount of dilution and the highest air concentrations of radioactive material).
TABLE 4.4 Estimated Release Fractions for LLW Packages under Various Accident Severity Categories

\begin{tabular}{ll}
\hline $\begin{array}{l}\text { Severity } \\
\text { Category }\end{array}$ & $\begin{array}{c}\text { Release } \\
\text { Fraction }^{\mathrm{a}}\end{array}$ \\
\hline $\begin{array}{c}\text { Truck } \\
\text { I }\end{array}$ & 0 \\
II & 0.01 \\
III & 0.1 \\
IV & 1.0 \\
V & 1.0 \\
VI & 1.0 \\
VII & 1.0 \\
VIII & 1.0 \\
& \\
Rail & \\
I & 0 \\
II & 0.01 \\
III & 0.1 \\
IV & 1.0 \\
V & 1.0 \\
VI & 1.0 \\
VII & 1.0 \\
VIII & 1.0 \\
\hline a Values are for total material \\
release fraction (the fraction \\
of material in a package \\
released to the environment \\
during an accident). \\
Sutce
\end{tabular}

Source: NRC (1977a). 
Table 3. Incident-Free Dose per Shipment (person-rem/shipment)

\begin{tabular}{|c|c|c|c|c|c|}
\hline \multirow[b]{2}{*}{ Source/Route (s) } & \multirow[b]{2}{*}{ Crew } & \multicolumn{4}{|c|}{ General Public } \\
\hline & & $\overline{\text { Off-Link }}$ & On-Link & Stops & Total \\
\hline $\begin{array}{l}\text { LLW }(x) \text { AL to HS } \\
\text { Ames Laboratory }\end{array}$ & $\begin{array}{ll}\text { Eo Hanford Site } \\
\text { Truck } & 4.46 \mathrm{E}-02 \\
\text { Rail } & 1.03 \mathrm{E}-02\end{array}$ & $\begin{array}{l}1.62 \mathrm{E}-03 \\
4.69 \mathrm{E}-03\end{array}$ & $\begin{array}{l}5.3 \\
1.4\end{array}$ & $\begin{array}{l}4.77 E-02 \\
8.98 E-03\end{array}$ & $\begin{array}{l}5.47 E-02 \\
1.38 E-02\end{array}$ \\
\hline $\begin{array}{l}\text { LLW(x) AN to HS } \\
\text { Argonne - East }\end{array}$ & $\begin{array}{lr}\text { to Hanford Site } \\
\text { Truck } & 5.29 E-02 \\
\text { Rail } & 1.21 E-02\end{array}$ & $\begin{array}{l}1.97 \mathrm{E}-03 \\
5.75 \mathrm{E}-03\end{array}$ & $\begin{array}{l}6.28 \mathrm{E}-03 \\
1.82 \mathrm{E}-04\end{array}$ & $\begin{array}{l}5.59 \mathrm{E}-02 \\
1.03 \mathrm{E}-02\end{array}$ & $\begin{array}{l}6.42 \mathrm{E}-02 \\
1.62 \mathrm{E}-02\end{array}$ \\
\hline $\begin{array}{l}\text { LLW (x) AW to HS } \\
\text { Argonne - West }\end{array}$ & $\begin{array}{ll}\text { to Hanford Site } \\
\text { Truck } & 1.60 \mathrm{E}-02 \\
\text { Rail } & 5.63 \mathrm{E}-03\end{array}$ & $\begin{array}{l}7.18 \mathrm{E}-04 \\
2.83 \mathrm{E}-03\end{array}$ & $\begin{array}{l}2.0 \\
6.5\end{array}$ & $\begin{array}{l}E-02 \\
E-03\end{array}$ & $\begin{array}{l}1.95 E-02 \\
8.40 E-03\end{array}$ \\
\hline $\begin{array}{l}\text { LLW(x) BA to HS } \\
\text { Bettis Atomic }\end{array}$ & $\begin{array}{ll}\text { co Hanford Site } \\
\text { Truck } & 7.21 \mathrm{E}-02 \\
\text { Rail } & 1.38 \mathrm{E}-02\end{array}$ & $\begin{array}{l}4.36 \mathrm{E}-03 \\
2.67 \mathrm{E}-02\end{array}$ & $\begin{array}{l}9.7 \\
4.5\end{array}$ & $\begin{array}{l}E-02 \\
E-02\end{array}$ & $\begin{array}{l}8.53 E-02 \\
3.87 E-02\end{array}$ \\
\hline $\begin{array}{l}\text { LLW(x) FL to HS } \\
\text { Fermilab (FNAL) }\end{array}$ & 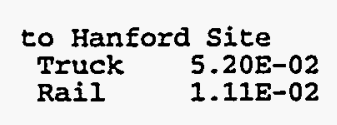 & $\begin{array}{l}1.92 \mathrm{E}-03 \\
9.07 \mathrm{E}-03\end{array}$ & $\begin{array}{l}6.2 \\
2.1\end{array}$ & $\begin{array}{l}E-02 \\
E-03\end{array}$ & $\begin{array}{l}6.35 \mathrm{E}-02 \\
1.88 \mathrm{E}-02\end{array}$ \\
\hline INEL & $\begin{array}{lr}\text { to Hanford Site } \\
\text { Truck } & 1.60 \mathrm{E}-02 \\
\text { Rail } & 5.63 \mathrm{E}-03\end{array}$ & $\begin{array}{l}7.1 \\
2.8\end{array}$ & $\begin{array}{l}2.5 \\
6.5\end{array}$ & -02 & $\begin{array}{l}-02 \\
-03\end{array}$ \\
\hline HUW(×) OR to HS & $\begin{array}{ll}\text { to Hanford Site } \\
\text { Truck } & 4.34 \mathrm{E}-02 \\
\text { Rail } & 1.04 \mathrm{E}-02\end{array}$ & $\begin{array}{l}2.30 \mathrm{E}-03 \\
6.90 \mathrm{E}-03\end{array}$ & $\begin{array}{l}5.9 \\
1.7\end{array}$ & $\begin{array}{l}-02 \\
-03\end{array}$ & $\begin{array}{l}-02 \\
-02\end{array}$ \\
\hline $\begin{array}{l}\text { LLW(x) OR to HS } \\
\text { Oak Ridge Rese }\end{array}$ & $\begin{array}{cl}\text { to Hanford } & \text { Site } \\
\text { Truck } & 6.83 \mathrm{E}-02 \\
\text { Rail } & 1.37 \mathrm{E}-02\end{array}$ & $\begin{array}{l}3.65 E-03 \\
1.08 E-02\end{array}$ & $\begin{array}{l}8.9 \\
2.5\end{array}$ & -02 & $\begin{array}{l}-02 \\
-02\end{array}$ \\
\hline LLW (x) KC to HS & $\begin{array}{ll}\text { to Hanford Site } \\
\text { Truck } & 8.30 \mathrm{~B}-02 \\
\text { Rail } & 1.50 \mathrm{E}-02\end{array}$ & $\begin{array}{l}6.03 \\
3.18\end{array}$ & $\frac{1}{5}$. & $\begin{array}{l}-02 \\
-02\end{array}$ & $\begin{array}{l}-02 \\
-02\end{array}$ \\
\hline $\begin{array}{l}\text { LLW (x) RC to HS } \\
\text { Ransas City plant }\end{array}$ & $\begin{array}{r}\text { ford Site } \\
4.62 \mathrm{E}-02 \\
1.03 \mathrm{E}-02\end{array}$ & $\begin{array}{l}1.90 \mathrm{E}-03 \\
5.00 \mathrm{E}-03\end{array}$ & $\begin{array}{l}5.7 \\
1.4\end{array}$ & $\begin{array}{l}-02 \\
-03\end{array}$ & $\begin{array}{r}-02 \\
-02\end{array}$ \\
\hline 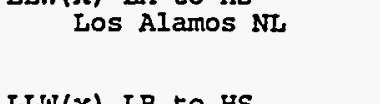 & $\begin{array}{lr}\text { to Hanford Site } \\
\text { Truck } & 4.24 \mathrm{E}-02 \\
\text { Rail } & 1.01 \mathrm{E}-02\end{array}$ & $\begin{array}{l}2.16 E-03 \\
5.60 E-03\end{array}$ & $\begin{array}{l}5.6 \\
1.5\end{array}$ & & $\begin{array}{l}-02 \\
-02\end{array}$ \\
\hline $\begin{array}{l}\text { LLW (x) LB to HS } \\
\text { Lawrence Berkeley }\end{array}$ & $\begin{array}{r}\text { ford site } \\
2.76 \mathrm{E}-02 \\
6.99 \mathrm{E}-03\end{array}$ & $\begin{array}{l}3.02 \mathrm{E}-03 \\
1.38 \mathrm{E}-02\end{array}$ & 5.1 & $\begin{array}{l}-02 \\
-03\end{array}$ & $\begin{array}{l}-02 \\
-02\end{array}$ \\
\hline Lawre: & $\begin{array}{lr}\text { to Hanford } \mathrm{si} \\
\text { Truck } & 2.8 \\
\text { Rail } & 6.9\end{array}$ & $\begin{array}{l}2.88 E-03 \\
1.38 E-02\end{array}$ & $\begin{array}{l}4.5 \\
2.0\end{array}$ & $\begin{array}{l}-02 \\
-03\end{array}$ & $\begin{array}{l}-02 \\
-02\end{array}$ \\
\hline $\begin{array}{l}\text { LLW (x) MP to HS } \\
\text { Mound Plant }\end{array}$ & $\begin{array}{r}\text { ford Site } \\
6.29 \mathrm{E}-02 \\
1.32 \mathrm{E}-02\end{array}$ & $\begin{array}{l}3.13 E-03 \\
1.47 E-02\end{array}$ & 8. & $\begin{array}{l}-02 \\
-02\end{array}$ & $\begin{array}{l}-02 \\
-02\end{array}$ \\
\hline $\begin{array}{l}\text { LLW }(x) \text { OI to HS } \\
\text { ORISE }\end{array}$ & $\begin{array}{r}\text { ford Site } \\
6.94 \mathrm{E}-02 \\
1.39 \mathrm{E}-02\end{array}$ & $\begin{array}{l}3.76 \\
1.30\end{array}$ & 9. & $\begin{array}{l}-02 \\
-02\end{array}$ & $:-02$ \\
\hline $\begin{array}{l}\text { LLW }(x) \text { OR to HS } \\
\text { Oak Ridge Re }\end{array}$ & $\begin{array}{cc}\text { to Hanford Site } \\
\text { Truck } & 6.83 \mathrm{E} \\
\text { Rail } & 1.37 \mathrm{E}\end{array}$ & $\begin{array}{l}3.6 \\
1.0\end{array}$ & 8 & $\begin{array}{r}-02 \\
-02\end{array}$ & -02 \\
\hline $\begin{array}{l}\text { LLW }(x) \text { PP to HS } \\
\text { Pantex Plant }\end{array}$ & $\begin{array}{lr}\text { to Hanford Site } \\
\text { Truck } & 5.10 \mathrm{E}-02 \\
\text { Rail } & 9.92 \mathrm{E}-03\end{array}$ & $\begin{array}{l}2.68 \\
5.71\end{array}$ & 7. & $\begin{array}{l}8-02 \\
8-03\end{array}$ & $=02$ \\
\hline $\begin{array}{l}\text { LLW(x) PO to HS } \\
\text { Portsmouth GDP }\end{array}$ & $\begin{array}{r}\text { ford } \mathrm{Si} \\
6.6 \\
1.3\end{array}$ & $\begin{array}{l}3.3 \\
1.8\end{array}$ & 3. & $\begin{array}{l}6.72 E-02 \\
1.12 E-02\end{array}$ & $\begin{array}{l}7.91 \Xi-02 \\
3.04 E-02\end{array}$ \\
\hline
\end{tabular}




\begin{tabular}{|c|c|c|c|c|c|}
\hline $\begin{array}{l}\text { LLW(x) PR to HS } \\
\text { Princeton PPL }\end{array}$ & $\begin{array}{lr}\text { to Hanford Site } \\
\text { Truck } & 8.51 \mathrm{E}-02 \\
\text { Rail } & 1.53 \mathrm{E}-02\end{array}$ & $\begin{array}{l}6.65 \mathrm{E}-03 \\
3.63 \mathrm{E}-02\end{array}$ & $\begin{array}{l}1.28 E-02 \\
6.03 E-04\end{array}$ & $\begin{array}{l}8.02 \mathrm{E}-02 \\
1.27 \mathrm{E}-02\end{array}$ & $\begin{array}{l}9.97 \mathrm{E}-02 \\
4.96 \mathrm{E}-02\end{array}$ \\
\hline $\begin{array}{l}\text { LLW (x) PA CO HS } \\
\text { Paducah GDP }\end{array}$ & $\begin{array}{lr}\text { to Hanford Site } \\
\text { Truck } & 6.00 \mathrm{E}-02 \\
\text { Rail } & 1.22 \mathrm{E}-02\end{array}$ & $\begin{array}{l}3.22 \mathrm{E}-03 \\
9.43 \mathrm{E}-03\end{array}$ & $\begin{array}{l}8.02 E-03 \\
2.28 E-04\end{array}$ & $\begin{array}{l}6.11 E-02 \\
1.04 E-02\end{array}$ & $\begin{array}{l}7.23 E-02 \\
2.01 E-02\end{array}$ \\
\hline $\begin{array}{l}\text { LLW(x) PI to HS } \\
\text { Pinelias Plant }\end{array}$ & $\begin{array}{lr}\text { to Hanford Site } \\
\text { Truck } & 8.77 \mathrm{E}-02 \\
\text { Rail } & 1.65 \mathrm{E}-02\end{array}$ & $\begin{array}{l}5.74 \mathrm{E}-03 \\
2.19 \mathrm{E}-02\end{array}$ & $\begin{array}{l}1.21 E-02 \\
4.79 E-04\end{array}$ & $\begin{array}{l}8.49 \mathrm{E}-02 \\
1.35 \mathrm{E}-02\end{array}$ & $\begin{array}{l}1.03 \mathrm{E}-01 \\
3.59 \mathrm{E}-02\end{array}$ \\
\hline $\begin{array}{l}\text { LLW }(x) \text { RF to HS } \\
\text { Rocky Flats Plant }\end{array}$ & $\begin{array}{lr}\text { to Hanford Site } \\
\text { Truck } & 2.99 \mathrm{E}-02 \\
\text { Rail } & 8.24 \mathrm{E}-03\end{array}$ & $\begin{array}{l}1.13 \mathrm{E}-03 \\
4.91 \mathrm{E}-03\end{array}$ & $\begin{array}{l}3.61 \mathrm{E}-03 \\
1.16 \mathrm{E}-04\end{array}$ & $\begin{array}{l}3.17 \mathrm{E}-02 \\
7.43 \mathrm{E}-03\end{array}$ & $\begin{array}{l}3.64 \mathrm{E}-02 \\
1.25 \mathrm{E}-02\end{array}$ \\
\hline $\begin{array}{l}\text { LLW(x) RM to HS } \\
\text { Reactive Metals }\end{array}$ & $\begin{array}{ll}\text { to Hanford Site } \\
\text { Truck } & 6.76 \mathrm{E}-02 \\
\text { Rail } & 1.33 \mathrm{E}-02\end{array}$ & $\begin{array}{l}4.22 E-03 \\
2.35 E-02\end{array}$ & $\begin{array}{l}9.51 \mathrm{E}-03 \\
4.01 \mathrm{E}-04\end{array}$ & $\begin{array}{l}6.71 \mathrm{E}-02 \\
1.12 \mathrm{E}-02\end{array}$ & $\begin{array}{l}8.09 \mathrm{E}-02 \\
3.51 \mathrm{E}-02\end{array}$ \\
\hline $\begin{array}{l}\text { LLW(x) ST to HS } \\
\text { SLAC }\end{array}$ & $\begin{array}{lr}\text { to Hanford Site } \\
\text { Truck } & 2.98 \mathrm{E}-02 \\
\text { Rail } & 7.20 \mathrm{E}-03\end{array}$ & $\begin{array}{l}3.70 \mathrm{E}-03 \\
1.75 \mathrm{E}-02\end{array}$ & $\begin{array}{l}6.08 \mathrm{E}-03 \\
2.40 \mathrm{E}-04\end{array}$ & $\begin{array}{l}2.56 \mathrm{E}-02 \\
6.67 \mathrm{E}-03\end{array}$ & $\begin{array}{l}3.54 \mathrm{E}-02 \\
2.44 \mathrm{E}-02\end{array}$ \\
\hline $\begin{array}{l}\text { LLW (x) SA to HS } \\
\text { SNL - Albuquerque }\end{array}$ & $\begin{array}{lr}\text { to Hanford Site } \\
\text { Truck } & 4.31 \mathrm{E}-02 \\
\text { Rail } & 1.04 \mathrm{E}-02\end{array}$ & $\begin{array}{l}2.27 E-03 \\
6.90 E-03\end{array}$ & $\begin{array}{l}5.84 \mathrm{E}-03 \\
1.72 \mathrm{E}-04\end{array}$ & $\begin{array}{l}4.43 \mathrm{E}-02 \\
9.00 \mathrm{E}-03\end{array}$ & $\begin{array}{l}5.24 E-02 \\
1.61 E-02\end{array}$ \\
\hline $\begin{array}{l}\text { LLW(x) SL to HS } \\
\text { SNL - Livermore }\end{array}$ & $\begin{array}{lr}\text { to Hanford Site } \\
\text { Truck } & 2.82 \mathrm{E}-02 \\
\text { Rail } & 6.94 \mathrm{E}-03\end{array}$ & $\begin{array}{l}2.88 \mathrm{E}-03 \\
1.38 \mathrm{E}-02\end{array}$ & $\begin{array}{l}4.92 \mathrm{E}-03 \\
2.00 \mathrm{E}-04\end{array}$ & $\begin{array}{l}2.50 \mathrm{E}-02 \\
6.48 \mathrm{E}-03\end{array}$ & $\begin{array}{l}3.28 \mathrm{E}-02 \\
2.05 \mathrm{E}-02\end{array}$ \\
\hline $\begin{array}{l}\text { LLW(x) SR to HS } \\
\text { Savannah River }\end{array}$ & $\begin{array}{lr}\text { to Hanford Site } \\
\text { Truck } & 7.86 \mathrm{E}-02 \\
\text { Rail } & 1.52 \mathrm{E}-02\end{array}$ & $\begin{array}{l}5.10 \mathrm{E}-03 \\
1.85 \mathrm{E}-02\end{array}$ & $\begin{array}{l}1.08 \mathrm{E}-02 \\
4.05 \mathrm{E}-04\end{array}$ & $\begin{array}{l}7.64 \mathrm{E}-02 \\
1.26 \mathrm{E}-02\end{array}$ & $\begin{array}{l}9.23 \mathrm{E}-02 \\
3.15 \mathrm{E}-02\end{array}$ \\
\hline $\begin{array}{l}\text { LLW (x) OR to HS } \\
\text { Oak Ridge Reserv. }\end{array}$ & $\begin{array}{cl}\text { to Hanford } & \text { Site } \\
\text { Truck } & 6.83 \mathrm{E}-02 \\
\text { Rail } & 1.37 \mathrm{E}-02\end{array}$ & $\begin{array}{l}3.65 \mathrm{E}-03 \\
1.08 \mathrm{E}-02\end{array}$ & $\begin{array}{l}8.95 E-03 \\
2.59 E-04\end{array}$ & $\begin{array}{l}6.90 \mathrm{E}-02 \\
1.15 \mathrm{E}-02\end{array}$ & $\begin{array}{l}8.16 \Xi-02 \\
2.26 E-02\end{array}$ \\
\hline
\end{tabular}


Table 4. Accident Dose Risk per Shipment (person-rem/shipment)

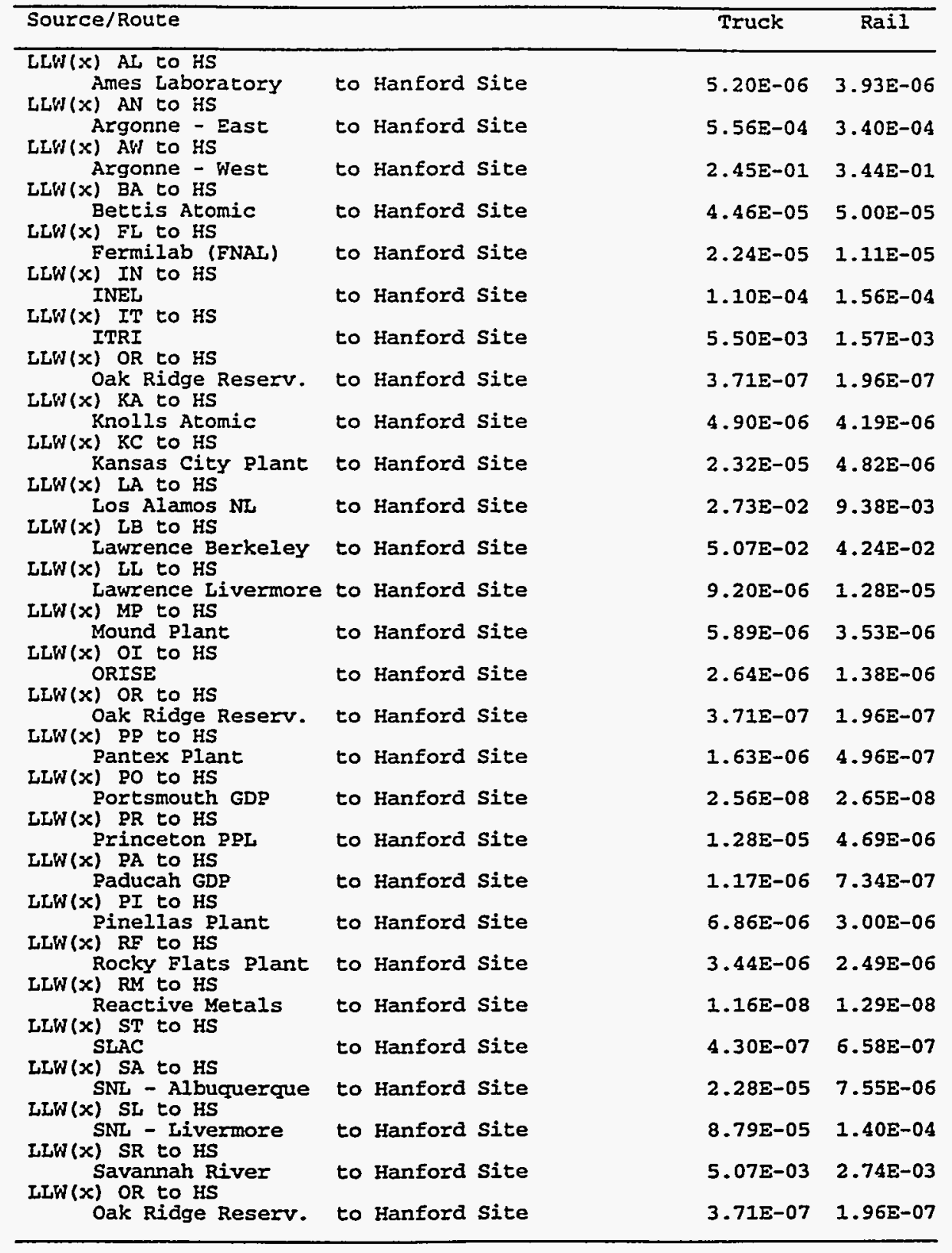


Table 5. Nonradiological Risk Factors per Shipment (Eatalities/shipment)

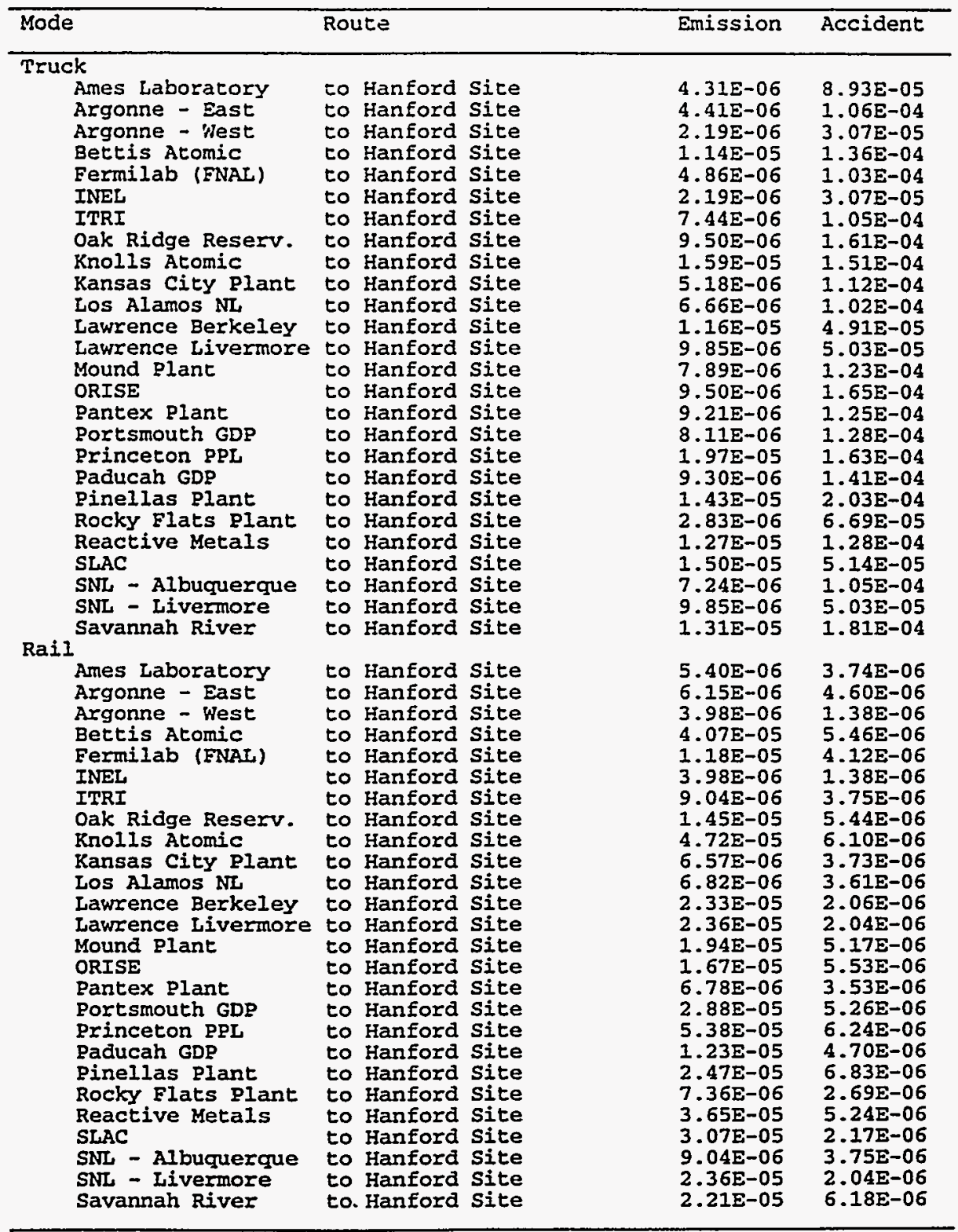


Table 6. Annual Incident-Free Dose for the Shipping Campaign (person-rem/yr)

\begin{tabular}{|c|c|c|c|c|c|c|}
\hline \multirow[b]{2}{*}{ Route } & & & \multicolumn{2}{|c|}{ Truck } & \multicolumn{2}{|c|}{ Rail } \\
\hline & & & Crew & Public & Crew & Public \\
\hline $\begin{array}{l}\text { Ames Laboratory } \\
\text { Argonne - East } \\
\text { Argonne - Nest } \\
\text { Bettis Atomic } \\
\text { Fermilab (FNAL) } \\
\text { INEL } \\
\text { ITRI } \\
\text { Oak Ridge Reserv. } \\
\text { Knolls Atomic } \\
\text { Kansas City Plant } \\
\text { Los Alamos NR } \\
\text { Lawrence Berkeley } \\
\text { Lawrence Livermore } \\
\text { Mound Plant } \\
\text { ORISE } \\
\text { Pantex Plant } \\
\text { Portsmouth GDP } \\
\text { Princeton PPL } \\
\text { Paducah GDP } \\
\text { Pinellas Plant } \\
\text { Rocky Flats Plant } \\
\text { Reactive Metals } \\
\text { SLAC } \\
\text { SNL - Albuquerque } \\
\text { SNL - Livermore } \\
\text { Savannah River }\end{array}$ & 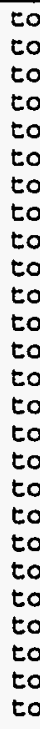 & $\begin{array}{l}\text { Hanford Site } \\
\text { Hanford Site } \\
\text { Hanford Site } \\
\text { Hanford Site } \\
\text { Hanford Site } \\
\text { Hanford Site } \\
\text { Hanford Site } \\
\text { Hanford Site } \\
\text { Fanford Site } \\
\text { Hanford Site } \\
\text { Hanford Site } \\
\text { Hanford Site } \\
\text { Hanford Site } \\
\text { Hanford Site } \\
\text { Hanford Site } \\
\text { Hanford Site } \\
\text { Hanford Site } \\
\text { Hanford Site } \\
\text { Hanford Site } \\
\text { Hanford Site } \\
\text { Hanford Site } \\
\text { Hanford Site } \\
\text { Hanford Site } \\
\text { Hanford Site } \\
\text { Hanford Site } \\
\text { Hanford Site }\end{array}$ & $\begin{array}{l}8.93 \mathrm{E}-02 \\
5.55 \mathrm{E}+00 \\
2.08 \mathrm{E}-01 \\
4.11 \mathrm{E}+00 \\
1.56 \mathrm{E}-01 \\
1.36 \mathrm{E}+01 \\
4.34 \mathrm{E}-01 \\
4.47 \mathrm{E}+02 \\
2.32 \mathrm{E}+00 \\
4.62 \mathrm{E}-02 \\
3.07 \mathrm{E}+01 \\
8.27 \mathrm{E}-02 \\
1.58 \mathrm{E}+00 \\
3.22 \mathrm{E}+01 \\
3.47 \mathrm{E}-01 \\
7.40 \mathrm{E}+01 \\
2.22 \mathrm{E}+02 \\
8.51 \mathrm{E}-02 \\
3.76 \mathrm{E}+01 \\
1.75 \mathrm{E}-01 \\
1.07 \mathrm{E}+01 \\
5.19 \mathrm{E}+01 \\
1.16 \mathrm{E}+00 \\
7.33 \mathrm{E}-01 \\
1.69 \mathrm{E}-01 \\
5.37 \mathrm{E}+02\end{array}$ & $\begin{array}{l}1.09 \mathrm{E}-01 \\
6.74 \mathrm{E}+00 \\
2.54 \mathrm{E}-01 \\
4.86 \mathrm{E}+00 \\
1.90 \mathrm{E}-01 \\
1.67 \mathrm{E}+01 \\
5.28 \mathrm{E}-01 \\
5.34 \mathrm{E}+02 \\
2.70 \mathrm{E}+00 \\
5.64 \mathrm{E}-02 \\
3.73 \mathrm{E}+01 \\
9.80 \mathrm{E}-02 \\
1.84 \mathrm{E}+00 \\
3.86 \mathrm{E}+01 \\
4.13 \mathrm{E}-01 \\
9.05 \mathrm{E}+01 \\
2.65 \mathrm{E}+02 \\
9.97 \mathrm{E}-02 \\
4.53 \mathrm{E}+01 \\
2.05 \mathrm{E}-01 \\
1.30 \mathrm{E}+01 \\
6.21 \mathrm{E}+01 \\
1.38 \mathrm{E}+00 \\
8.92 \mathrm{E}-01 \\
1.97 \mathrm{E}-01 \\
6.31 \mathrm{E}+02\end{array}$ & $\begin{array}{l}1.03 E-02 \\
4.70 E-01 \\
2.81 E-02 \\
2.89 \mathrm{E}-01 \\
2.22 \mathrm{E}-02 \\
1.81 \mathrm{E}+00 \\
4.15 \mathrm{E}-02 \\
3.41 \mathrm{E}+01 \\
1.65 \mathrm{E}-01 \\
1.03 \mathrm{E}-02 \\
2.70 \mathrm{E}+00 \\
1.40 \mathrm{E}-02 \\
1.46 \mathrm{E}-01 \\
2.51 \mathrm{E}+00 \\
2.78 \mathrm{E}-02 \\
5.40 \mathrm{E}+00 \\
1.70 \mathrm{E}+01 \\
1.53 \mathrm{E}-02 \\
2.90 \mathrm{E}+00 \\
1.65 \mathrm{E}-02 \\
1.10 \mathrm{E}+00 \\
3.83 \mathrm{E}+00 \\
1.08 \mathrm{E}-01 \\
7.25 \mathrm{E}-02 \\
1.39 \mathrm{E}-02 \\
3.86 \mathrm{E}+01\end{array}$ & $\begin{array}{l}1.38 \mathrm{E}-02 \\
6.31 \mathrm{E}-01 \\
4.20 \mathrm{E}-02 \\
8.13 \mathrm{E}-01 \\
3.77 \mathrm{E}-02 \\
2.71 \mathrm{E}+00 \\
6.43 \mathrm{E}-02 \\
5.61 \mathrm{E}+01 \\
4.93 \mathrm{E}-01 \\
1.41 \mathrm{E}-02 \\
3.90 \mathrm{E}+00 \\
4.10 \mathrm{E}-02 \\
4.30 \mathrm{E}-01 \\
4.96 \mathrm{E}+00 \\
4.98 \mathrm{E}-02 \\
7.91 \mathrm{E}+00 \\
3.88 \mathrm{E}+01 \\
4.96 \mathrm{E}-02 \\
4.75 \mathrm{E}+00 \\
3.59 \mathrm{E}-02 \\
1.66 \mathrm{E}+00 \\
1.01 \mathrm{E}+01 \\
3.66 \mathrm{E}-01 \\
1.12 \mathrm{E}-01 \\
4.10 \mathrm{E}-02 \\
8.00 \mathrm{E}+01\end{array}$ \\
\hline Totals & & & $1.47 \mathrm{E}+03$ & $1.75 E+03$ & $1.11 E+02$ & $2.14 E+02$ \\
\hline
\end{tabular}

Table 7. Annual Accident Dose Risk for the Shipping Campaign (person-rem/yr)

\begin{tabular}{llcc}
\hline Route & & Truck & Rail \\
\hline Ames Laboratory & to Hanford Site & $1.04 \mathrm{E}-05$ & $3.93 \mathrm{E}-06$ \\
Argonne - East & to Hanford Site & $5.84 \mathrm{E}-02$ & $1.33 \mathrm{E}-02$ \\
Argonne - West & to Hanford Site & $3.18 \mathrm{E}+00$ & $1.72 \mathrm{E}+00$ \\
Bettis Atomic & to Hanford Site & $2.54 \mathrm{E}-03$ & $1.05 \mathrm{E}-03$ \\
Fermilab (FNAL) & to Hanford Site & $6.73 \mathrm{E}-05$ & $2.22 \mathrm{E}-05$ \\
INEL & to Hanford Site & $9.35 \mathrm{E}-02$ & $5.04 \mathrm{E}-02$ \\
ITRI & to Hanford Site & $5.50 \mathrm{E}-02$ & $6.27 \mathrm{E}-03$ \\
Oak Ridge Reserv. & to Hanford Site & $2.43 \mathrm{E}-03$ & $4.87 \mathrm{E}-04$ \\
Knolls Atomic & to Hanford Site & $1.37 \mathrm{E}-04$ & $4.61 \mathrm{E}-05$ \\
Ransas City Plant & to Hanford Site & $2.32 \mathrm{E}-05$ & $4.82 \mathrm{E}-06$ \\
Los Alamos NL & to Hanford Site & $1.98 \mathrm{E}+01$ & $2.51 \mathrm{E}+00$ \\
Lawrence Berkeley & to Hanford Site & $1.52 \mathrm{E}-01$ & $8.48 \mathrm{E}-02$ \\
Lawrence Livermore to Hanford Site & $5.15 \mathrm{E}-04$ & $2.70 \mathrm{E}-04$ \\
Mound Plant & to Hanford Site & $3.01 \mathrm{E}-03$ & $6.71 \mathrm{E}-04$ \\
ORISE & to Hanford Site & $1.32 \mathrm{E}-05$ & $2.77 \mathrm{E}-06$ \\
Pantex Plant & to Hanford Site & $2.36 \mathrm{E}-03$ & $2.70 \mathrm{E}-04$ \\
Portsmouth GDP & to Hanford Site & $8.56 \mathrm{E}-05$ & $3.38 \mathrm{E}-05$ \\
Princeton PPL & to Hanford Site & $1.28 \mathrm{E}-05$ & $4.69 \mathrm{E}-06$ \\
Paducah GDP & to Hanford Site & $7.35 \mathrm{E}-04$ & $1.74 \mathrm{E}-04$ \\
Pinellas Plant & to Hanford Site & $1.37 \mathrm{E}-05$ & $3.00 \mathrm{E}-06$ \\
Rocky Flats Plant & to Hanford Site & $1.23 \mathrm{E}-03$ & $3.31 \mathrm{E}-04$ \\
Reactive Metals & to Hanford Site & $8.90 \mathrm{E}-06$ & $3.69 \mathrm{E}-06$ \\
SLAC & to Hanford Site & $1.68 \mathrm{E}-05$ & $9.87 \mathrm{E}-06$ \\
SNL - Albuquerque & to Hanford Site & $3.88 \mathrm{E}-04$ & $5.29 \mathrm{E}-05$ \\
SNL - Livermore & to Hanford Site & $5.27 \mathrm{E}-04$ & $2.79 \mathrm{E}-04$ \\
Savannah River & to Hanford Site & $3.46 \mathrm{E}+01$ & $6.96 \mathrm{E}+00$ \\
\hline Totals & & & \\
\hline
\end{tabular}


Table 8. Expected Annual Fatalities for the Shipping Campaign

\begin{tabular}{lll}
\hline Exposure Group & Truck & Rail \\
\hline Radiological & & \\
Normal Crew & $5.9 E-01$ & $4.5 \mathrm{E}-02$ \\
Normal Public & $8.8 \mathrm{E}-01$ & $1.1 \mathrm{E}-01$ \\
Accident Public & $2.9 \mathrm{E}-02$ & $5.7 \mathrm{E}-03$ \\
Nonradiological & & \\
Emission & $2.2 \mathrm{E}-01$ & $1.6 \mathrm{E}-01$ \\
Accident & $3.3 \mathrm{E}+00$ & $4.4 \mathrm{E}-02$ \\
\hline
\end{tabular}

Table 9. Expected Annual Cancer Incidence for the Shipping Campaign

\begin{tabular}{lll}
\hline Exposure Group & Truck & Rail \\
\hline Radiological & & \\
Normal Crew & $2.1 \mathrm{E}+00$ & $1.6 \mathrm{E}-01$ \\
Normal Public & $3.0 \mathrm{E}+00$ & $3.6 \mathrm{E}-01$ \\
$\quad$ Accident Public & $9.9 \mathrm{E}-02$ & $1.9 \mathrm{E}-02$ \\
Nonradiological & & \\
$\quad$ Emission & $2.2 \mathrm{E}-01$ & $1.6 \mathrm{E}-01$ \\
Accident & $\mathrm{NA}$ & $\mathrm{NA}$ \\
\hline
\end{tabular}

Table 10. Expected Annual Genetic Effects for the Shipping Campaign

\begin{tabular}{lll}
\hline Exposure Group & Truck & Rail \\
\hline Radiological & & \\
Normal Crew & $8.8 E-02$ & $6.7 E-03$ \\
Normal Public & $1.8 E-01$ & $2.1 E-02$ \\
Accident Public & $5.3 E-03$ & $1.0 \mathrm{E}-03$ \\
Nonradiological & & \\
Emission & NA & NA \\
Accident & NA & NA \\
\hline
\end{tabular}




\section{B.20 WM LLW CENTRALIZED 1 ALTERNATIVE (CASE 7): ACTIVATED METALS}

Table 1. Summary of Route Information

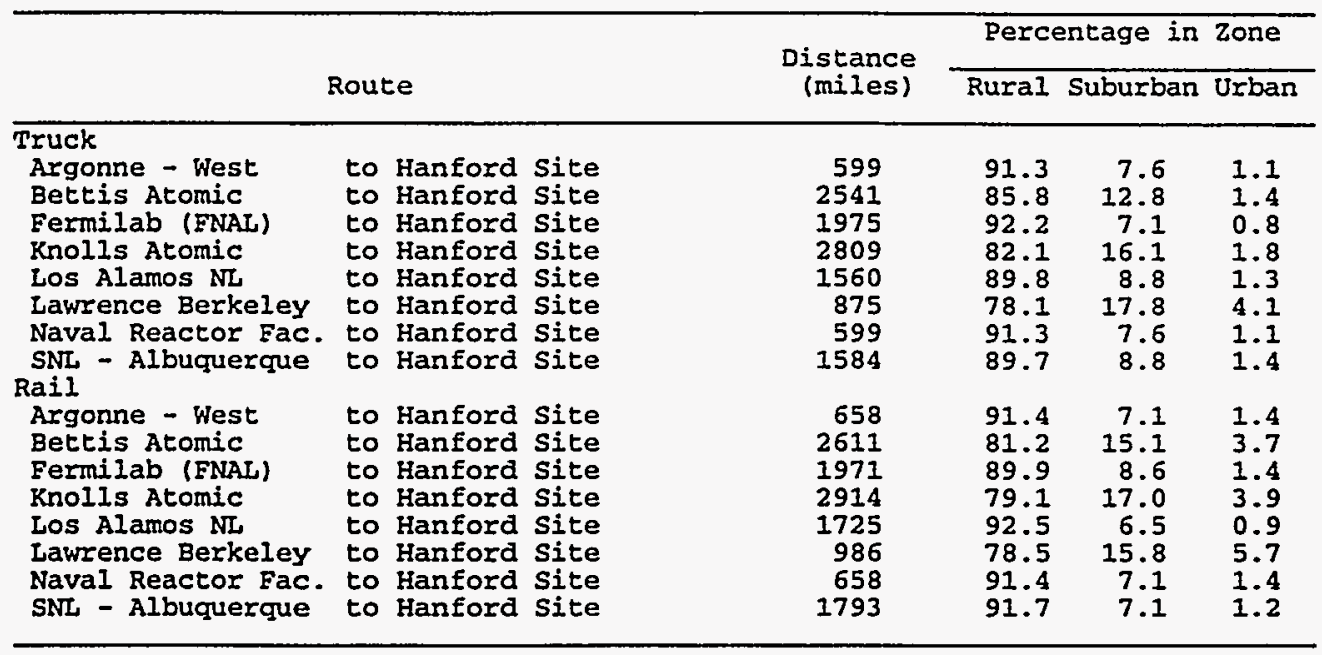

Table 2. Annual Number of Shipments and One-Way Shipment Distances

\begin{tabular}{|c|c|c|c|c|c|}
\hline \multirow[b]{2}{*}{ Route } & & \multicolumn{2}{|c|}{ \# of Shipments } & \multicolumn{2}{|c|}{ Total Mileage(mi) } \\
\hline & & Truck & Rail & Truck & Rail \\
\hline $\begin{array}{l}\text { Argonne - West } \\
\text { Bettis Atomic } \\
\text { Fermilab (FNAL) } \\
\text { Knolls Atomic } \\
\text { Los Alamos NL } \\
\text { Lawrence Berkeley } \\
\text { Naval Reactor Fac. } \\
\text { SNL - Albuquerque }\end{array}$ & $\begin{array}{l}\text { to Hanford Site } \\
\text { to Hanford Site } \\
\text { to Hanford Site } \\
\text { to Hanford Site } \\
\text { to Hanford Site } \\
\text { to Hanford Site } \\
\text { to Hanford Site } \\
\text { to Hanford Site }\end{array}$ & $\begin{array}{r}319 \\
416 \\
650 \\
418 \\
15 \\
461 \\
6\end{array}$ & $\begin{array}{r}4 \\
120 \\
15 \\
246 \\
159 \\
6 \\
175 \\
3\end{array}$ & $\begin{array}{l}5.39 \mathrm{E}+03 \\
8.03 \mathrm{E}+05 \\
7.90 \mathrm{E}+04 \\
1.83 \mathrm{E}+06 \\
6.52 \mathrm{E}+05 \\
1.31 \mathrm{E}+04 \\
2.76 \mathrm{E}+05 \\
9.50 \mathrm{E}+03\end{array}$ & $\begin{array}{l}2.63 \mathrm{E}+03 \\
3.13 \mathrm{E}+05 \\
2.96 \mathrm{E}+04 \\
7.17 \mathrm{E}+05 \\
2.74 \mathrm{E}+05 \\
5.91 \mathrm{E}+03 \\
1.15 \mathrm{E}+05 \\
5.38 \mathrm{E}+03\end{array}$ \\
\hline Totals & & 1915 & 728 & $3.66 \mathrm{E}+06$ & $1.46 \mathrm{E}+06$ \\
\hline
\end{tabular}


Table 3. Incident-Free Dose per Shipment (person-rem/shipment)

\begin{tabular}{|c|c|c|c|c|c|}
\hline \multirow[b]{2}{*}{ Source/Route (s) } & \multirow[b]{2}{*}{ Crew } & \multicolumn{4}{|c|}{ General Public } \\
\hline & & OEf-Link & On-Link & Stops & Total \\
\hline $\begin{array}{l}\text { LW(I) AW to HS } \\
\text { Argonne - Hest }\end{array}$ & 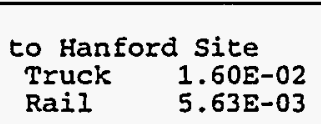 & $\begin{array}{l}E-04 \\
E-03\end{array}$ & $\begin{array}{l}2.06 E-03 \\
6.58 E-05\end{array}$ & $\begin{array}{l}1.68 E-02 \\
5.51 E-03\end{array}$ & $\begin{array}{l}1.95 \mathrm{E}-02 \\
8.40 \mathrm{E}-03\end{array}$ \\
\hline $\begin{array}{l}\text { LLW(I) BA to HS } \\
\text { Bettis Atomic }\end{array}$ & $\begin{array}{lr}\text { to Hanford Site } \\
\text { Truck } & 7.21 \mathrm{E}-02 \\
\text { Rail } & 1.38 \mathrm{E}-02\end{array}$ & $\begin{array}{l}4.36 E-03 \\
2.67 E-02\end{array}$ & $\begin{array}{l}9.77 \mathrm{E}-03 \\
4.53 \mathrm{E}-04\end{array}$ & $\begin{array}{l}7.11 \mathrm{E}-02 \\
1.15 \mathrm{E}-02\end{array}$ & $\begin{array}{l}8.53 \mathrm{E}-02 \\
3.87 \mathrm{E}-02\end{array}$ \\
\hline $\begin{array}{l}\text { LLW(I) FL to HS } \\
\text { Fermilab (FNAL) }\end{array}$ & $\begin{array}{lr}\text { to Hanford Site } \\
\text { Truck } & 5.20 \mathrm{E}-02 \\
\text { Rail } & 1.11 \mathrm{E}-02\end{array}$ & $\begin{array}{l}1.92 E-03 \\
9.07 E-03\end{array}$ & $\begin{array}{l}6.25 E-03 \\
2.12 E-04\end{array}$ & $\begin{array}{l}5.53 \mathrm{E}-02 \\
9.55 \mathrm{E}-03\end{array}$ & $\begin{array}{l}6.35 \mathrm{E}-02 \\
1.88 \mathrm{E}-02\end{array}$ \\
\hline $\begin{array}{l}\text { ILW(I) KA to HS } \\
\text { Knolls Atomic }\end{array}$ & $\begin{array}{lr}\text { to Hanford Site } \\
\text { Truck } & 8.30 \mathrm{E}-02 \\
\text { Rail } & 1.50 \mathrm{E}-02\end{array}$ & $\begin{array}{l}6.03 E-03 \\
3.18 E-02\end{array}$ & $\begin{array}{l}1.19 \mathrm{E}-02 \\
5.40 \mathrm{E}-04\end{array}$ & $\begin{array}{l}7.87 \mathrm{E}-02 \\
1.25 \mathrm{E}-02\end{array}$ & $\begin{array}{l}9.66 \\
4.48\end{array}$ \\
\hline $\begin{array}{l}\text { LLW (I) LA to HS } \\
\text { LOS Alamos NL }\end{array}$ & $\begin{array}{l}\text { to Hanford Site } \\
\text { Truck } \\
\text { Rail } \\
4.24 \mathrm{E}-02 \\
1.01 \mathrm{E}-02\end{array}$ & $\begin{array}{l}2.16 E-03 \\
5.60 E-03\end{array}$ & $\begin{array}{l}5.6 \\
1.5\end{array}$ & $\begin{array}{l}E-02 \\
E-03\end{array}$ & $\begin{array}{l}5.15 \mathrm{E}-02 \\
1.45 \mathrm{E}-02\end{array}$ \\
\hline $\begin{array}{l}\text { LLW(I) LB to HS } \\
\text { Lawrence Berkeley }\end{array}$ & $\begin{array}{lr}\text { to Hanford Site } \\
\text { Truck } & 2.76 \mathrm{E}-02 \\
\text { Rail } & 6.99 \mathrm{E}-03\end{array}$ & $\begin{array}{l}-03 \\
-02\end{array}$ & & $\begin{array}{l}8-02 \\
8-03\end{array}$ & $\begin{array}{l}3.27 \mathrm{E}-\mathrm{C} \\
2.05 \mathrm{E}-\mathrm{C}\end{array}$ \\
\hline $\begin{array}{l}\text { LLW(I) NR to HS } \\
\text { Naval Reactor Fac. }\end{array}$ & $\begin{array}{ll}\text { to Hanford Site } \\
\text { Truck } & 1.60 \mathrm{E}-02 \\
\text { Rail } & 5.63 \mathrm{E}-03\end{array}$ & $\begin{array}{l}7.18 E-04 \\
2.83 E-03\end{array}$ & $\begin{array}{l}2.06 E-03 \\
6.58 E-05\end{array}$ & $\begin{array}{l}1.68 \mathrm{E}-02 \\
5.51 \mathrm{E}-03\end{array}$ & $\begin{array}{l}1.95 \mathrm{E}-02 \\
8.40 \mathrm{E}-03\end{array}$ \\
\hline $\begin{array}{l}\text { ILW(I) SA to HS } \\
\text { SNL - Albuquerque }\end{array}$ & $\begin{array}{ll}\text { to Hanford Site } \\
\text { Truck } & 4.31 \mathrm{E}-02 \\
\text { Rail } & 1.04 \mathrm{E}-02\end{array}$ & $\begin{array}{l}2.27 E-03 \\
6.90 \mathrm{E}-03\end{array}$ & $\begin{array}{l}5.84 \mathrm{E}-03 \\
1.72 \mathrm{E}-04\end{array}$ & $\begin{array}{l}4.43 \mathrm{E}-02 \\
9.00 \mathrm{E}-03\end{array}$ & $\begin{array}{l}5.24 \mathrm{E}-02 \\
1.61 \mathrm{E}-02\end{array}$ \\
\hline
\end{tabular}

Table 4. Accident Dose Risk per Shipment (person-rem/shipment)

\begin{tabular}{|c|c|c|c|}
\hline Source/Route & & Truck & Rail \\
\hline $\begin{array}{l}\text { LLW(I) AW to HS } \\
\text { Argonne - West } \\
\text { LLW(I) BA to HS } \\
\text { Bettis Atomic } \\
\text { LLW(I) FL to HS } \\
\text { Fermilab (FNAL) } \\
\text { LLW(I) KA to HS } \\
\text { Knolls Atomic } \\
\text { LLW(I) LA to HS } \\
\text { Los Alamos NL } \\
\text { LLW(I) LB to HS } \\
\text { Lawrence Berkeley } \\
\text { LLW(I) NR to HS } \\
\text { Naval Reactor Fac. } \\
\text { ILW(I) SA to HS } \\
\text { SNL - Albuquerque }\end{array}$ & $\begin{array}{l}\text { to Hanford Site } \\
\text { to Hanford Site } \\
\text { to Hanford Site } \\
\text { to Hanford Site } \\
\text { to Hanford Site } \\
\text { to Hanford Site } \\
\text { to Hanford Site } \\
\text { to Hanford Site }\end{array}$ & $\begin{array}{l}8.15 \mathrm{E}-12 \\
1.80 \mathrm{E}-06 \\
4.65 \mathrm{E}-11 \\
3.07 \mathrm{E}-06 \\
3.10 \mathrm{E}-09 \\
1.69 \mathrm{E}-10 \\
4.44 \mathrm{E}-08 \\
6.06 \mathrm{E}-12\end{array}$ & $\begin{array}{l}9.58 \mathrm{E}-12 \\
1.78 \mathrm{E}-06 \\
4.12 \mathrm{E}-11 \\
2.58 \mathrm{E}-06 \\
8.88 \mathrm{E}-10 \\
2.37 \mathrm{E}-10 \\
6.34 \mathrm{E}-08 \\
1.32 \mathrm{E}-12\end{array}$ \\
\hline
\end{tabular}


Table 5. Nonradiological Risk Factors per Shipment (fatalities/shipment)

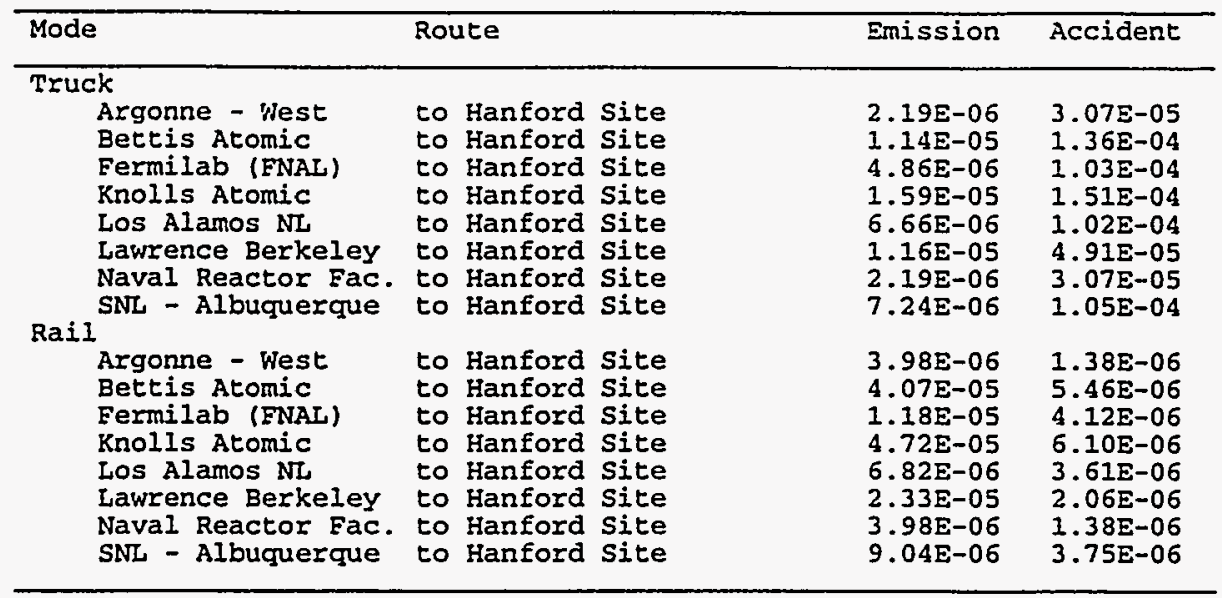

Table 6. Annual Incident-Free Dose for the Shipping Campaign (person-rem/yr)

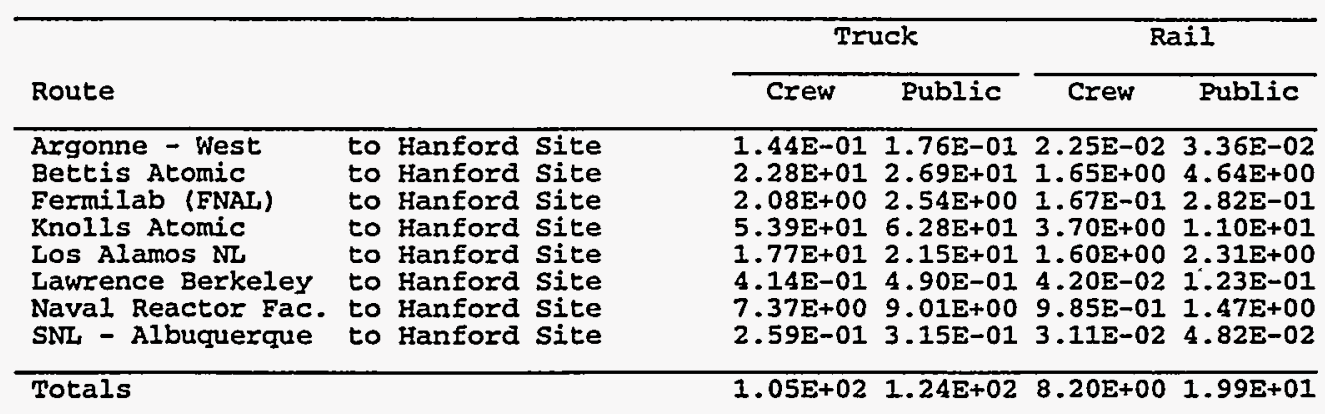

Table 7. Annual Accident Dose Risk for the Shipping Campaign (person-rem/yr)

\begin{tabular}{llcc}
\hline Route & & Truck & Rail \\
\hline Argonne - West & to Hanford Site & $7.34 \mathrm{E}-11$ & $3.83 \mathrm{E}-11$ \\
Bettis Atomic & to Hanford Site & $5.68 \mathrm{E}-04$ & $2.13 \mathrm{E}-04$ \\
Fermilab (FNAL) & to Hanford Site & $1.86 \mathrm{E}-09$ & $6.17 \mathrm{E}-10$ \\
Rnolls Atomic & to Hanford Site & $1.99 \mathrm{E}-03$ & $6.36 \mathrm{E}-04$ \\
Los Alamos NL & to Hanford Site & $1.29 \mathrm{E}-06$ & $1.41 \mathrm{E}-07$ \\
Lawrence Berkeley & to Hanford Site & $2.53 \mathrm{E}-09$ & $1.42 \mathrm{E}-09$ \\
Naval Reactor Fac. to Hanford Site & $2.05 \mathrm{E}-05$ & $1.11 \mathrm{E}-05$ \\
SNL - Albuquerque & to Hanford Site & $3.63 \mathrm{E}-11$ & $3.96 \mathrm{E}-12$ \\
\hline Totals & & & \\
\hline
\end{tabular}

Table 8. Expected Annual Fatalities for the Shipping Campaign

\begin{tabular}{|c|c|c|}
\hline Exposure Group & Truck & Rail \\
\hline $\begin{array}{l}\text { Radiological } \\
\text { Normal Crew } \\
\text { Normal Public } \\
\text { Accident Public } \\
\text { Nonradiological }\end{array}$ & $\begin{array}{l}4.2 E-02 \\
6.2 E-02 \\
1.3 E-06\end{array}$ & $\begin{array}{l}3.3 E-03 \\
1.0 E-02 \\
4.3 E-07\end{array}$ \\
\hline $\begin{array}{l}\text { Emission } \\
\text { Accident }\end{array}$ & $\begin{array}{l}1.8 E-02 \\
2.0 E-01\end{array}$ & $\begin{array}{l}1.9 \mathrm{E}-02 \\
3.1 \mathrm{E}-03\end{array}$ \\
\hline
\end{tabular}


Table 9. Expected Annual Cancer Incidence Eor the Shipping Campaign

\begin{tabular}{|c|c|c|}
\hline Exposure Group & Truck & Rail \\
\hline $\begin{array}{l}\text { Radiological } \\
\text { Normal Crew } \\
\text { Normal public } \\
\text { Accident Public }\end{array}$ & $\begin{array}{l}1.5 E-01 \\
2.1 E-01 \\
4.4 E-06\end{array}$ & $\begin{array}{l}1.1 \mathrm{E}-02 \\
3.4 \mathrm{E}-02 \\
1.5 \mathrm{E}-06\end{array}$ \\
\hline $\begin{array}{l}\text { Nonradiological } \\
\text { Emission } \\
\text { Accident }\end{array}$ & $\frac{1.8 E-02}{N A}$ & $\begin{array}{l}1.9 \mathrm{E}-02 \\
\mathrm{NA}\end{array}$ \\
\hline
\end{tabular}

Table 10. Expected Annual Genetic Effects for the Shipping Campaign

\begin{tabular}{lll}
\hline Exposure Group & Truck & Rail \\
\hline Radiological & & \\
Normal Crew & $6.3 \mathrm{E}-03$ & $4.9 \mathrm{E}-04$ \\
Normal Public & $1.2 \mathrm{E}-02$ & $2.0 \mathrm{E}-03$ \\
Accident Public & $2.6 \mathrm{E}-07$ & $8.6 \mathrm{E}-08$ \\
Nonradiological & & \\
Emission & $\mathrm{NA}$ & $\mathrm{NA}$ \\
Accident & $\mathrm{NA}$ & $\mathrm{NA}$ \\
\hline
\end{tabular}


TABLE 4.5 Aerosolized and Respirable Material Release Fractions for $\mathbf{L L W}^{\mathbf{a}}$

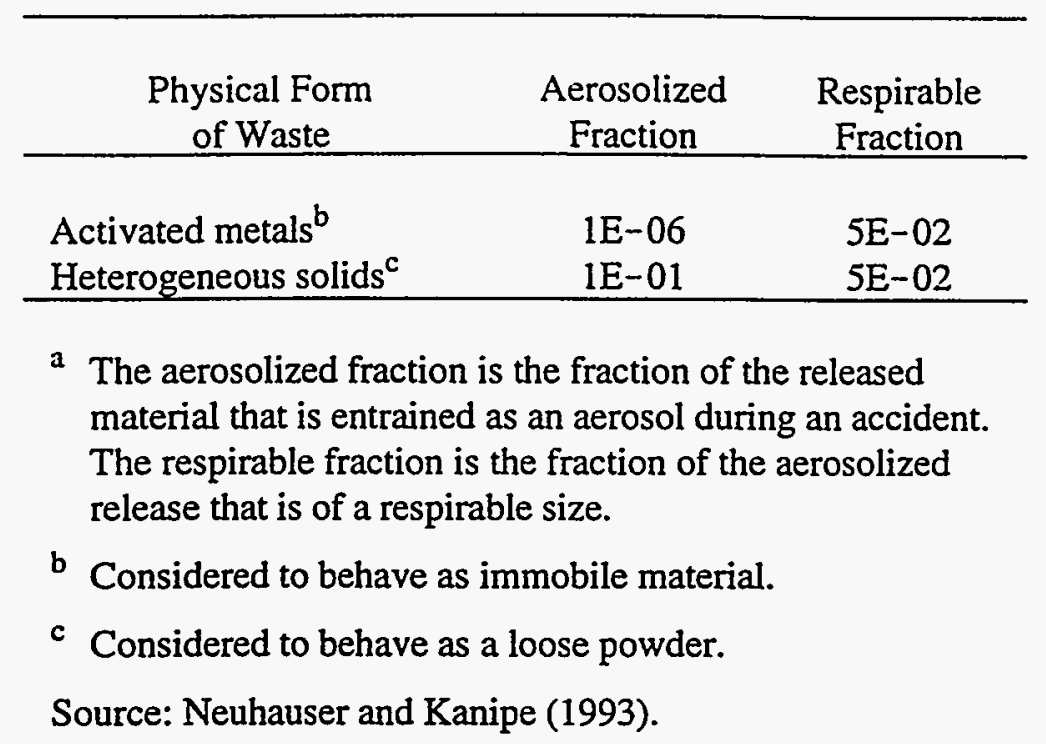

\subsection{EXTERNAL DOSE RATES FROM SHIPMENTS}

The dose (and, correspondingly, the risk) to populations during routine transportation is directly proportional to the assumed external dose rate from the shipment. The actual dose rate from the shipment is a complex function of the composition and configuration of shielding and containment materials used in the waste packaging, the geometry of the loaded shipment, and the characteristics of the waste material itself. Representative shipment dose rates have been developed and used in the WM PEIS LLW transportation analysis. In practice, external dose rates will vary not only from site to site and from waste type to waste type, but also from shipment to shipment at a given site.

For WM LLW shipments, the external dose rates from historical waste shipments were investigated by using the Shipment Mobility/Accountability Collection (SMAC) system (Morris 1993). The SMAC database contains information on unclassified commercial freight shipments made by the DOE and its contractors. The information available in the SMAC database is collected from site shipping and receiving documents. Available information for shipments of radioactive materials includes the types of material shipped, the number of packages in each shipment, shipment weights, external dose rates, and package isotopic inventories. Approximately two-thirds of all DOE unclassified shipments are estimated to be reported to the SMAC system.

Shipment information from the SMAC database was examined for fiscal years 1983-1993 (Morris 1993). Information was provided for three general categories of radioactive material: irradiated fuel, "other" highway route controlled quantities, and LLW. (The material categories chosen were dictated by the format in which data are submitted and entered into the SMAC database 


\section{B.21 WM LLW CENTRALIZED 2 ALTERNATIVE (CASE 8): HETEROGENEOUS SOLIDS}

Table 1. Summary of Route Information

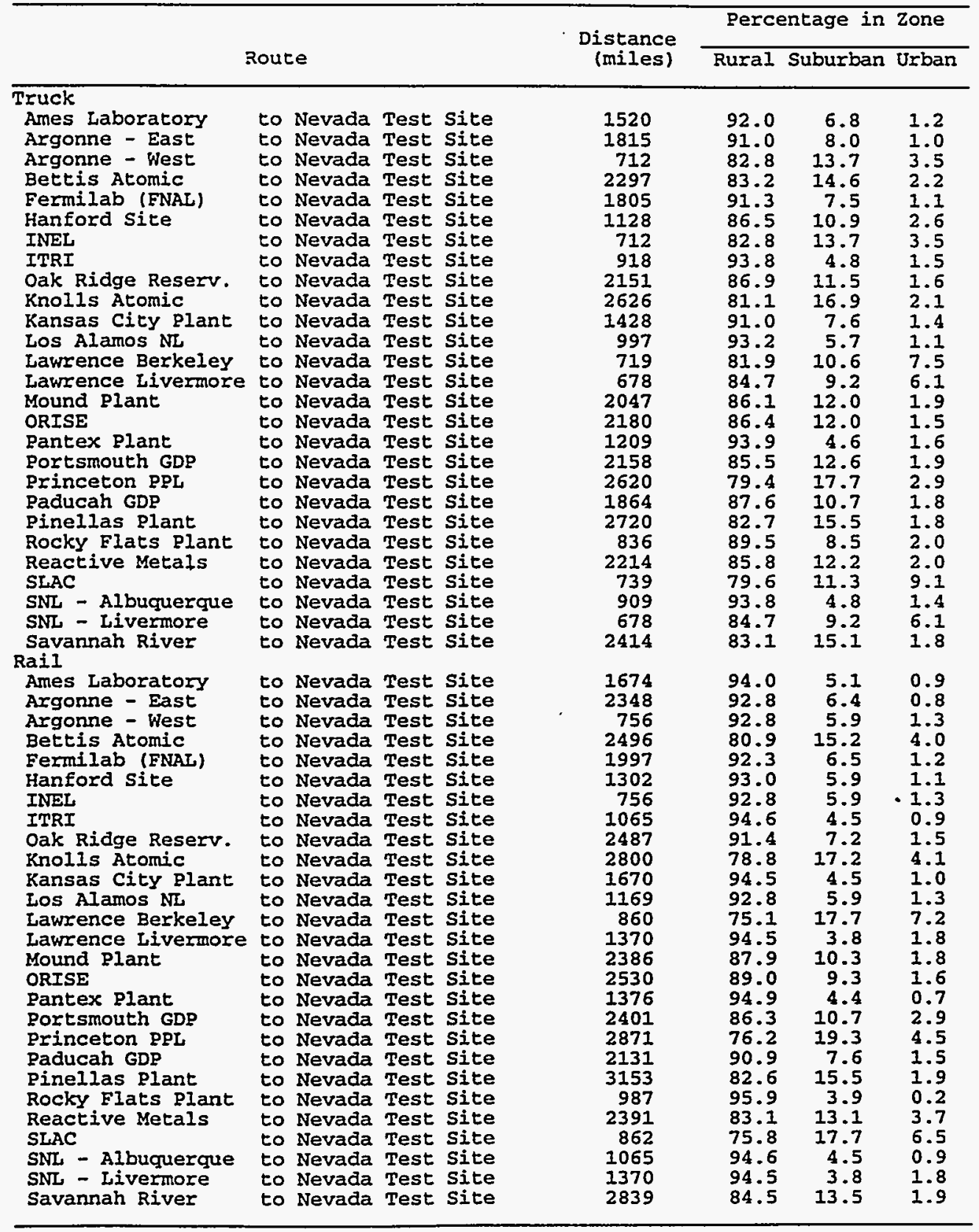


Table 2. Annual Number of Shipments and One-Way Shipment Distances

\begin{tabular}{|c|c|c|c|c|c|c|}
\hline \multirow[b]{2}{*}{ Route } & & & \multicolumn{2}{|c|}{ \# of Shipments } & \multicolumn{2}{|c|}{ Total Mileage(mi) } \\
\hline & & & Truck & Rail & Truck & Rail \\
\hline $\begin{array}{l}\text { Ames Laboratory } \\
\text { Argonne - East } \\
\text { Argonne - West } \\
\text { Bettis Atomic } \\
\text { Fermilab (FNAL) } \\
\text { Hanford Site } \\
\text { INEL } \\
\text { ITRI } \\
\text { Oak Ridge Reserv. } \\
\text { Knolls Atomic } \\
\text { Ransas City Plant } \\
\text { Los Alamos NL } \\
\text { Lawrence Berkeley } \\
\text { Lawrence Livermore } \\
\text { Mound Plant } \\
\text { ORISE } \\
\text { Pantex Plant } \\
\text { Portsmouth GDP } \\
\text { Princeton PPL } \\
\text { Paducah GDP } \\
\text { Pinellas Plant } \\
\text { Rocky Flats Plant } \\
\text { Reactive Metals } \\
\text { SLAC } \\
\text { SNL - Albuquerque } \\
\text { SNL - Livermore } \\
\text { Savannah River }\end{array}$ & $\begin{array}{l}\text { to Nevada } \\
\text { to Nevada } \\
\text { to Nevada } \\
\text { to Nevada } \\
\text { to Nevada } \\
\text { to Nevada } \\
\text { to Nevada } \\
\text { to Nevada } \\
\text { to Nevada } \\
\text { to Nevada } \\
\text { to Nevada } \\
\text { to Nevada } \\
\text { to Nevada } \\
\text { to Nevada } \\
\text { to Nevada } \\
\text { to Nevada } \\
\text { to Nevada } \\
\text { to Nevada } \\
\text { to Nevada } \\
\text { to Nevada } \\
\text { to Nevada } \\
\text { to Nevada } \\
\text { to Nevada } \\
\text { to Nevada } \\
\text { to Nevada } \\
\text { to Nevada } \\
\text { to Nevada }\end{array}$ & $\begin{array}{l}\text { Test Site } \\
\text { Test Site } \\
\text { Test Site } \\
\text { Test Site } \\
\text { Test Site } \\
\text { Test Site } \\
\text { Test Site } \\
\text { Test Site } \\
\text { Test Site } \\
\text { Test Site } \\
\text { Test Site } \\
\text { Test Site } \\
\text { Test Site } \\
\text { Test Site } \\
\text { Test Site } \\
\text { Test Site } \\
\text { Test Site } \\
\text { Test Site } \\
\text { Test Site } \\
\text { Test Site } \\
\text { Test Site } \\
\text { Test Site } \\
\text { Test Site } \\
\text { Test Site } \\
\text { Test Site } \\
\text { Test Site } \\
\text { Test Site }\end{array}$ & $\begin{array}{r}2 \\
105 \\
13 \\
57 \\
3 \\
1421 \\
852 \\
10 \\
6542 \\
28 \\
1 \\
724 \\
3 \\
56 \\
512 \\
5 \\
1450 \\
3344 \\
1 \\
627 \\
2 \\
357 \\
768 \\
39 \\
17 \\
6 \\
6834\end{array}$ & $\begin{array}{r}17 \\
39 \\
5 \\
21 \\
2 \\
531 \\
322 \\
4 \\
2486 \\
11 \\
1 \\
268 \\
2 \\
21 \\
190 \\
2 \\
544 \\
1274 \\
1 \\
237 \\
1 \\
133 \\
287 \\
15 \\
7 \\
2 \\
2540\end{array}$ & $\begin{array}{l}3.04 E+03 \\
1.91 E+05 \\
9.26 E+03 \\
1.31 E+05 \\
5.41 E+03 \\
1.60 E+06 \\
6.07 E+05 \\
9.18 E+03 \\
1.41 E+07 \\
7.35 E+04 \\
1.43 E+03 \\
7.22 E+05 \\
2.16 E+03 \\
3.80 E+04 \\
1.05 E+06 \\
1.09 E+04 \\
1.75 E+06 \\
7.22 E+06 \\
2.62 E+03 \\
1.17 E+06 \\
5.44 E+03 \\
2.98 E+05 \\
1.70 E+06 \\
2.88 E+04 \\
1.55 E+04 \\
4.07 E+03 \\
1.65 E+07\end{array}$ & $\begin{array}{l}1.67 \mathrm{E}+03 \\
9.16 \mathrm{E}+04 \\
3.78 \mathrm{E}+03 \\
5.24 \mathrm{E}+04 \\
3.99 \mathrm{E}+03 \\
6.91 \mathrm{E}+05 \\
2.43 \mathrm{E}+05 \\
4.26 \mathrm{E}+03 \\
6.18 \mathrm{E}+06 \\
3.08 \mathrm{E}+04 \\
1.67 \mathrm{E}+03 \\
3.13 \mathrm{E}+05 \\
1.72 \mathrm{E}+03 \\
2.88 \mathrm{E}+04 \\
4.53 \mathrm{E}+05 \\
5.06 \mathrm{E}+03 \\
7.49 \mathrm{E}+05 \\
3.06 \mathrm{E}+06 \\
2.87 \mathrm{E}+03 \\
5.05 \mathrm{E}+05 \\
3.15 \mathrm{E}+03 \\
1.31 \mathrm{E}+05 \\
6.86 \mathrm{E}+05 \\
1.29 \mathrm{E}+04 \\
7.45 \mathrm{E}+03 \\
2.74 \mathrm{E}+03 \\
7.21 \mathrm{E}+06\end{array}$ \\
\hline Totals & & & 23779 & 8947 & $4.72 E+07$ & $2.05 E+07$ \\
\hline
\end{tabular}


Table 3. Incident-Free Dose per Shipment (person-rem/shipment)

\begin{tabular}{|c|c|c|c|c|c|c|}
\hline \multirow[b]{2}{*}{ Source/Route (s) } & & \multirow[b]{2}{*}{ Crew } & \multicolumn{4}{|c|}{ General Public } \\
\hline & & & OEE-Link & On-Link & Stops & Total \\
\hline $\begin{array}{l}\text { LLW }(x) \text { AL to NT } \\
\text { Ames Laboratory }\end{array}$ & $\begin{array}{l}\text { to Nevada } \\
\text { Truck } \\
\text { Rail }\end{array}$ & $\begin{array}{l}\text { Test Site } \\
4.03 \mathrm{E}-02 \\
9.87 \mathrm{E}-03\end{array}$ & $\begin{array}{l}\mathrm{e} \\
1.78 \mathrm{E}-03 \\
4.68 \mathrm{E}-03\end{array}$ & $\begin{array}{l}5.2 \\
1.3\end{array}$ & $\begin{array}{l}4.25 E-02 \\
8.63 E-03\end{array}$ & $\begin{array}{l}E-02 \\
E-02\end{array}$ \\
\hline $\begin{array}{l}\text { LLW (x) AN to NT } \\
\text { Argonne - East }\end{array}$ & $\begin{array}{l}\text { to Nevada } \\
\text { Truck } \\
\text { Rail }\end{array}$ & $\begin{array}{l}\text { Test Site } \\
4.86 \mathrm{E}-02 \\
1.27 \mathrm{E}-02\end{array}$ & $\begin{array}{r}e \\
2.13 E-03 \\
6.96 E-03\end{array}$ & $\begin{array}{l}6.15 \mathrm{E}-03 \\
2.04 \mathrm{E}-04\end{array}$ & $\begin{array}{l}5.08 \mathrm{E}-02 \\
1.07 \mathrm{E}-02\end{array}$ & $\begin{array}{l}5.91 \mathrm{E}-02 \\
1.79 \mathrm{E}-02\end{array}$ \\
\hline $\begin{array}{l}\text { LLW }(x) \text { AW to NT } \\
\text { Argonne - West }\end{array}$ & $\begin{array}{l}\text { to Nevada } \\
\text { Truck } \\
\text { Rail }\end{array}$ & $\begin{array}{l}\text { Test Site } \\
2.13 \mathrm{E}-02 \\
6.04 \mathrm{E}-03\end{array}$ & $\begin{array}{l}\text { e } \\
2.0 \\
2.8\end{array}$ & $\begin{array}{l}3.7 \\
6.8\end{array}$ & & $\begin{array}{l}-02 \\
-03\end{array}$ \\
\hline $\begin{array}{l}\text { LLW (x) BA to NT } \\
\text { Bettis Atomic }\end{array}$ & $\begin{array}{l}\text { to } ~ \\
\text { Try } \\
\text { Rai }\end{array}$ & $\begin{array}{l}\text { Test Site } \\
6.75 E-02 \\
1.33 E-02\end{array}$ & $\begin{array}{r}5.17 E-03 \\
2.67 E-02\end{array}$ & $\begin{array}{l}1.02 \mathrm{E}-02 \\
4.43 \mathrm{E}-04\end{array}$ & $\begin{array}{l}8-02 \\
-02\end{array}$ & $\begin{array}{l}7.97 \mathrm{E}-02 \\
3.83 \mathrm{E}-02\end{array}$ \\
\hline $\begin{array}{l}\text { LLW (x) FL to NT } \\
\text { Fermilab (FNAL) }\end{array}$ & $\begin{array}{l}\text { to Nevada } \\
\text { Truck } \\
\text { Rail }\end{array}$ & $\begin{array}{l}\text { Test } \\
4.82 \\
1.12\end{array}$ & $\begin{array}{r}2.15 E-03 \\
7.26 E-03\end{array}$ & $\begin{array}{l}6.20 \mathrm{E}-03 \\
1.85 \mathrm{E}-04\end{array}$ & $\begin{array}{l}5.05 \mathrm{E}-02 \\
9.63 \mathrm{E}-03\end{array}$ & $\begin{array}{l}-02 \\
8-02\end{array}$ \\
\hline $\begin{array}{l}\text { LLW }(x) \text { HS to NT } \\
\text { Hanford Site }\end{array}$ & $\begin{array}{l}\text { to Nevada } \\
\text { Truck } \\
\text { Rail }\end{array}$ & $\begin{array}{l}\text { Tes } \\
3.2 \\
8.3\end{array}$ & $\begin{array}{l}2.46 E-03 \\
4.39 E-03\end{array}$ & & & \\
\hline $\begin{array}{l}\text { LLW(x) IN to NT } \\
\text { INEL }\end{array}$ & $\begin{array}{l}\text { to } N \\
\text { Tru } \\
\text { Rai }\end{array}$ & $\begin{array}{l}\text { Tes } \\
2.1 \\
6.0\end{array}$ & $\begin{array}{r}\text { e } \\
2.0 \\
2.8\end{array}$ & & & \\
\hline$\underset{\text { ITRI }}{\operatorname{LLW}(x)}$ IT to $\mathrm{NT}$ & $\begin{array}{l}\text { to } \\
\text { Tr? } \\
\text { Ra: }\end{array}$ & $\begin{array}{l}\text { Test site } \\
2.40 \mathrm{E}-02 \\
7.32 \mathrm{E}-03\end{array}$ & $\begin{array}{l}1.05 E-03 \\
2.85 E-03\end{array}$ & & & \\
\hline $\begin{array}{l}\text { LLW(x) OR to NT } \\
\text { Oak Ridge Reserv. }\end{array}$ & $\begin{array}{l}\text { to Ne } \\
\text { Tru } \\
\text { Rai. }\end{array}$ & $\begin{array}{r}\text { Test Site } \\
6.04 \mathrm{E}-02 \\
1.33 \mathrm{E}-02\end{array}$ & $\begin{array}{l}3.66 \mathrm{E}-03 \\
1.08 \mathrm{E}-02\end{array}$ & $\begin{array}{l}-03 \\
-04\end{array}$ & & $\begin{array}{l}8-02 \\
3-02\end{array}$ \\
\hline $\begin{array}{l}\text { LLW (x) KA to NT } \\
\text { Knolls Atomic }\end{array}$ & $\begin{array}{l}\text { to Nevada } \\
\text { Truck } \\
\text { Rail }\end{array}$ & $\begin{array}{l}\text { Tes } \\
7.8 \\
1.8\end{array}$ & $\begin{array}{l}e \\
6.2 \\
3.1\end{array}$ & $\frac{1}{5}$ & & \\
\hline $\begin{array}{l}\text { LLW (x) KC to NT } \\
\text { Kansas City Plant }\end{array}$ & $\begin{array}{l}\text { to Nevada } \\
\text { Truck } \\
\text { Rail }\end{array}$ & $\begin{array}{l}\text { Tes } \\
3.8 \\
9.8\end{array}$ & $\begin{array}{r}1.90 \mathrm{E}-03 \\
4.98 \mathrm{E}-03\end{array}$ & $\begin{array}{l}5.1 \\
1.3\end{array}$ & & $\begin{array}{l}4.7 \\
1.3\end{array}$ \\
\hline TB to NT & $\begin{array}{l}\text { to } N \\
\text { Tra } \\
\text { Rai }\end{array}$ & $\begin{array}{l}\text { Test } \\
2.61 \\
7.76\end{array}$ & $\begin{array}{l}1.0 \\
4.4\end{array}$ & & & \\
\hline $\begin{array}{l}l(x) \text { LB to } \mathrm{NT} \\
\text { Lawrence Ber] }\end{array}$ & $\begin{array}{l}\text { to } 1 \\
\text { Tri } \\
\text { Ras }\end{array}$ & $\begin{array}{l}\text { Test Site } \\
2.27 \mathrm{E}-02 \\
6.47 \mathrm{E}-03\end{array}$ & $\begin{array}{l}3.3 \\
1.4\end{array}$ & & $\begin{array}{l}\varepsilon-02 \\
E-03\end{array}$ & $\begin{array}{l}E-02 \\
E-02\end{array}$ \\
\hline Lawrence Live & $\begin{array}{l}\text { to } N \\
\text { Tru } \\
\text { Rai }\end{array}$ & $\begin{array}{l}\text { Tes } \\
2.0 \\
8.6\end{array}$ & $\begin{array}{l}\text { e } \\
2.57 E-03 \\
5.75 E-03\end{array}$ & & & \\
\hline $\begin{array}{r}\text { LLW }(x) \text { MP to } \mathrm{NT} \\
\text { Mound Plant }\end{array}$ & $\begin{array}{l}\text { to } 1 \\
\text { Tru } \\
\text { Raj }\end{array}$ & $\begin{array}{l}\text { Test Sit } \\
5.82 \mathrm{E}-02 \\
1.28 \mathrm{E}-02\end{array}$ & $\begin{array}{l}\text { e } \\
3.9 \\
1.3\end{array}$ & & & $\begin{array}{l}-02 \\
-02\end{array}$ \\
\hline $\begin{array}{l}\text { LLW }(x) \text { OI } \\
\text { ORISE }\end{array}$ & $\begin{array}{l}\text { to Nevada } \\
\text { Truck } \\
\text { Rail }\end{array}$ & $\begin{array}{l}\text { Test Sitt } \\
6.15 \mathrm{E}-02 \\
1.34 \mathrm{E}-02\end{array}$ & $\begin{array}{l}3.77 E-03 \\
1.30 E-02\end{array}$ & & & $\begin{array}{l}-02 \\
-02\end{array}$ \\
\hline $\begin{array}{l}\text { LLW (x) OR to NT } \\
\text { Oak Ridge Res }\end{array}$ & $\begin{array}{l}\text { Nevada } 1 \\
\text { Truck } \\
\text { Rail }\end{array}$ & $\begin{array}{r}\text { Test Site } \\
6.04 \mathrm{E}-02 \\
1.33 \mathrm{E}-02\end{array}$ & $\begin{array}{l}3.66 \mathrm{E}-03 \\
1.08 \mathrm{E}-02\end{array}$ & 8 & & $\begin{array}{l}7.22 \mathrm{E}-02 \\
2.22 \mathrm{E}-02\end{array}$ \\
\hline $\begin{array}{l}\text { LLW (x) PP to NT } \\
\text { Pantex plant }\end{array}$ & $\begin{array}{l}\text { to Nev } \\
\text { Truck } \\
\text { Rail }\end{array}$ & $\begin{array}{l}\text { Test Site } \\
3.16 \mathrm{E}-02 \\
8.62 \mathrm{E}-03\end{array}$ & SE-0s & & $\begin{array}{l}3.38 \mathrm{E}-02 \\
7.72 \mathrm{E}-03\end{array}$ & $\begin{array}{l}3.96 \mathrm{E}-02 \\
1.10 \mathrm{E}-02\end{array}$ \\
\hline
\end{tabular}




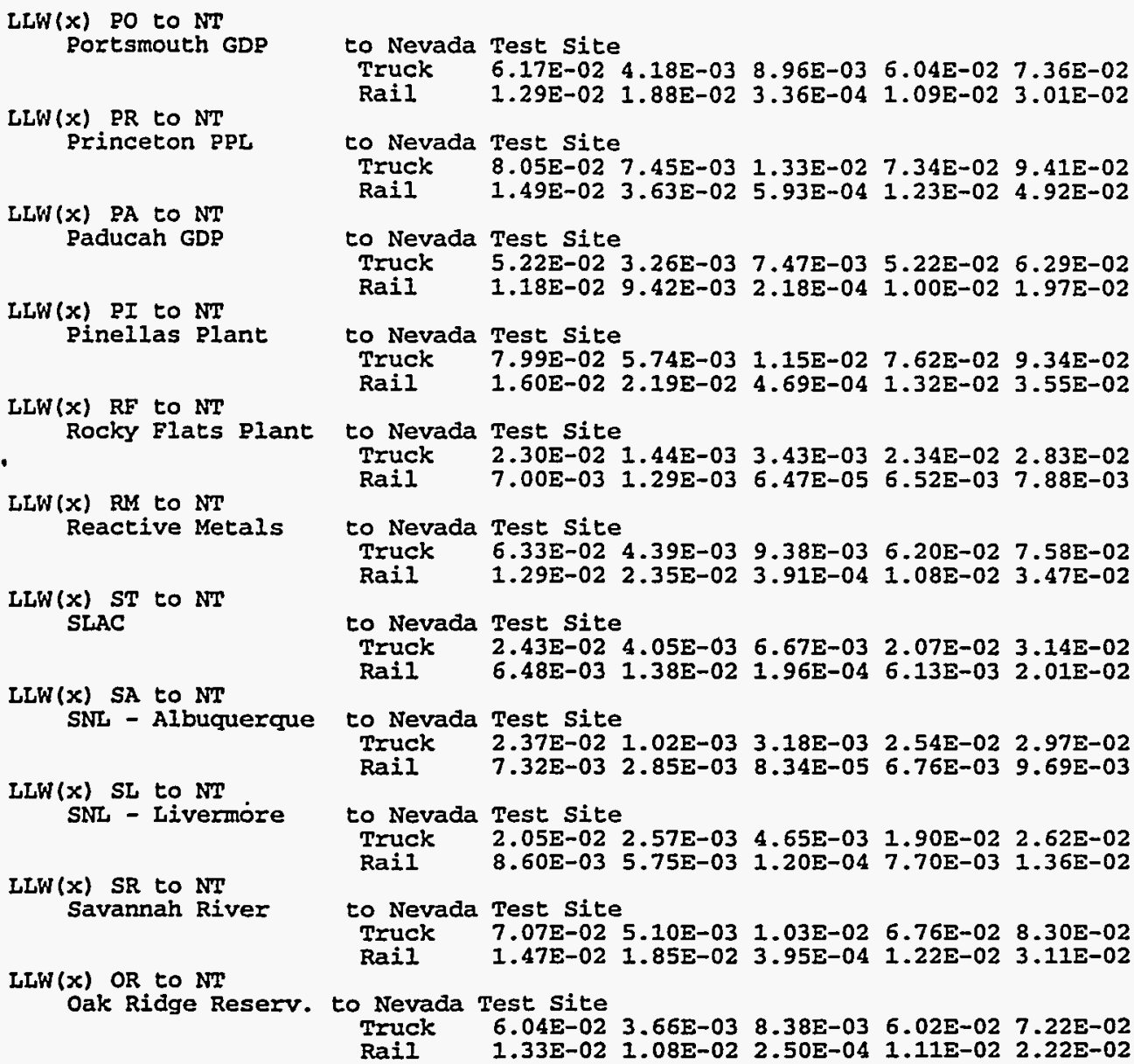


Table 4. Accident Dose Risk per Shipment (person-rem/shipment)

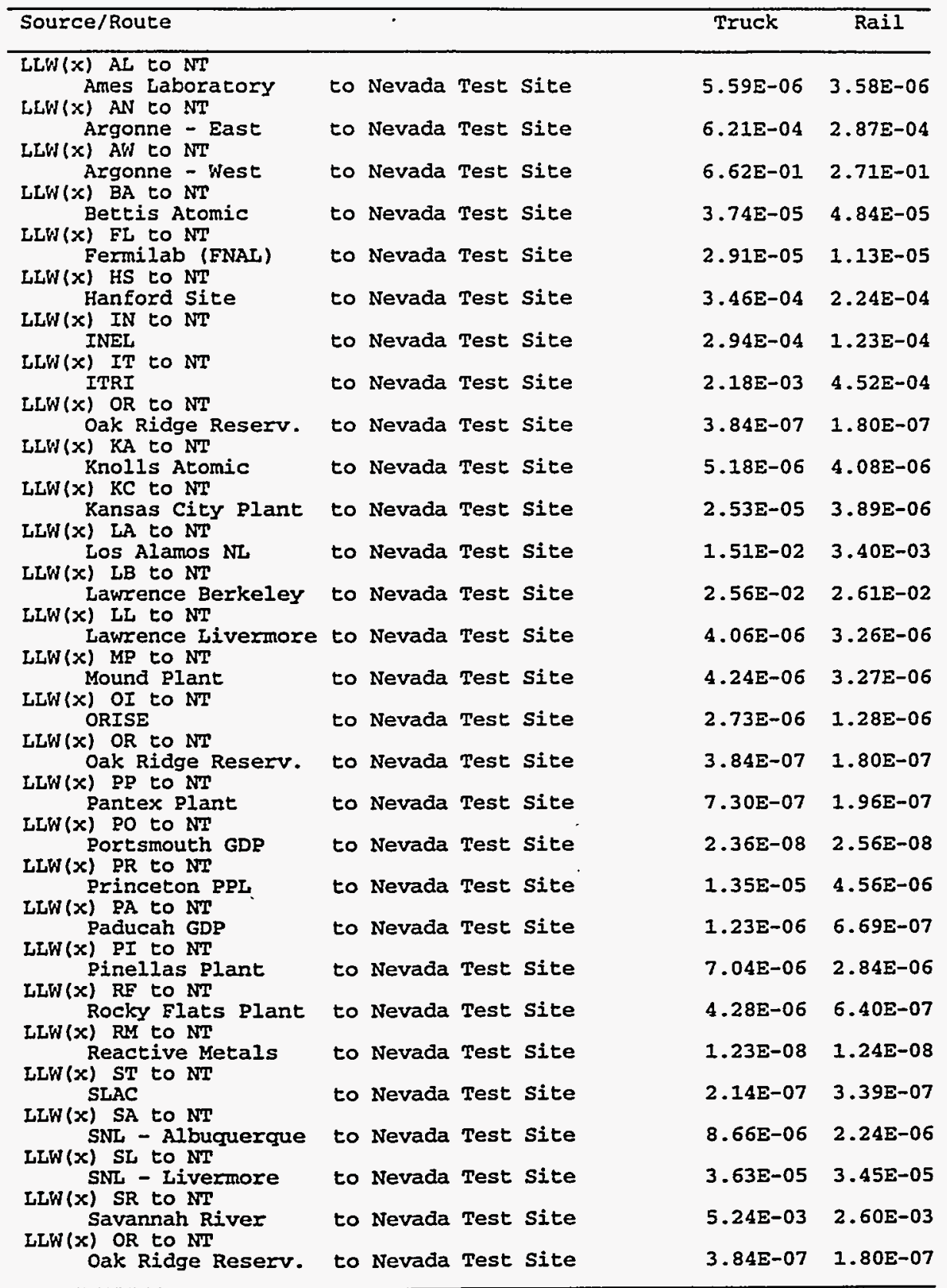


Table 5. Nonradiological Risk Factors per Shipment (Eatalicies/shipment)

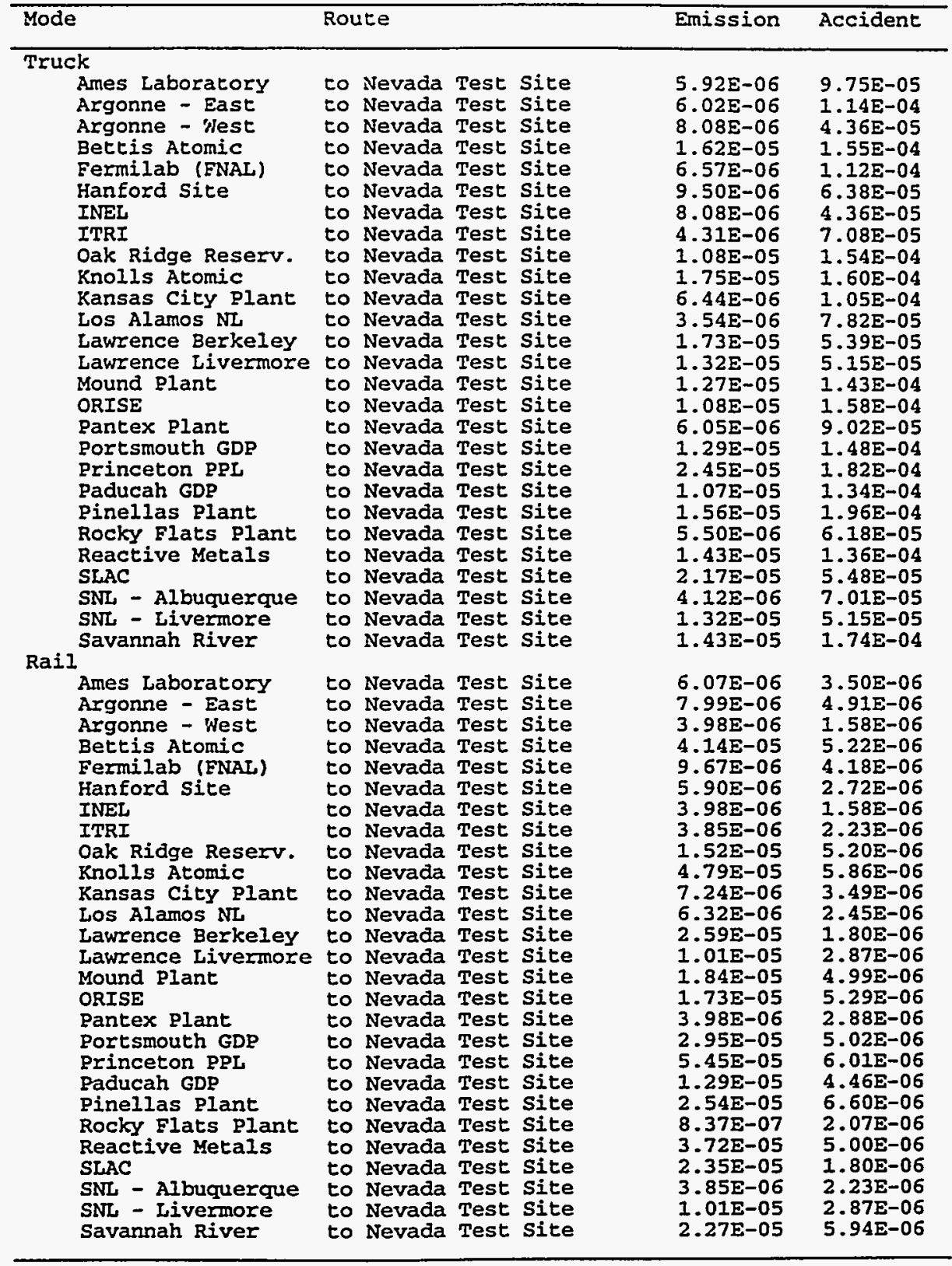


Table 6. Annual Incident-Free Dose for the Shipping Campaign (person-rem/yr)

\begin{tabular}{|c|c|c|c|c|c|c|c|}
\hline \multirow[b]{2}{*}{ Route } & & & & \multicolumn{2}{|c|}{ Truck } & \multicolumn{2}{|c|}{ Rail } \\
\hline & & & & Crew & $\overline{\text { Public }}$ & Crew & Public \\
\hline $\begin{array}{l}\text { Ames Laboratory } \\
\text { Argonne - East } \\
\text { Argonne - West } \\
\text { Bettis Atomic } \\
\text { Fermilab (FNAL) } \\
\text { Hanford Site } \\
\text { INEL } \\
\text { ITRI } \\
\text { Oak Ridge Reserv. } \\
\text { Knolls Atomic } \\
\text { Kansas City Plant } \\
\text { Los Alamos NL } \\
\text { Lawrence Berkeley } \\
\text { Lawrence Livermore } \\
\text { Mound Plant } \\
\text { ORISE } \\
\text { Pantex Plant } \\
\text { Portsmouth GDP } \\
\text { Princeton PPL } \\
\text { Paducah GDP } \\
\text { Pinellas Plant } \\
\text { Rocky Flats Plant } \\
\text { Reactive Metals } \\
\text { SLAC } \\
\text { SNL - Albuguerque } \\
\text { SNL - Livermore } \\
\text { Savannah River }\end{array}$ & $\begin{array}{l}\text { to } \\
\text { to } \\
\text { to } \\
\text { to } \\
\text { to } \\
\text { to } \\
\text { to } \\
\text { to } \\
\text { to } \\
\text { to } \\
\text { to } \\
\text { to } \\
\text { to } \\
\text { to } \\
\text { to } \\
\text { to } \\
\text { to } \\
\text { to } \\
\text { to } \\
\text { to } \\
\text { to } \\
\text { to } \\
\text { to } \\
\text { to } \\
\text { to } \\
\text { to }\end{array}$ & $\begin{array}{l}\text { Nevada } \\
\text { Nevada } \\
\text { Nevada } \\
\text { Nevada } \\
\text { Nevada } \\
\text { Nevada } \\
\text { Nevada } \\
\text { Nevada } \\
\text { Nevada } \\
\text { Nevada } \\
\text { Nevada } \\
\text { Nevada } \\
\text { Nevada } \\
\text { Nevada } \\
\text { Nevada } \\
\text { Nevada } \\
\text { Nevada } \\
\text { Nevada } \\
\text { Nevada } \\
\text { Nevada } \\
\text { Nevada } \\
\text { Nevada } \\
\text { Nevada } \\
\text { Nevada } \\
\text { Nevada } \\
\text { Nevada } \\
\text { Nevada }\end{array}$ & $\begin{array}{l}\text { Test Site } \\
\text { Test Site } \\
\text { Test Site } \\
\text { Test Site } \\
\text { Test Site } \\
\text { Test Site } \\
\text { Test Site } \\
\text { Test Site } \\
\text { Test Site } \\
\text { Test Site } \\
\text { Test Site } \\
\text { Test Site } \\
\text { Test Site } \\
\text { Test Site } \\
\text { Test Site } \\
\text { Test Site } \\
\text { Test Site } \\
\text { Test Site } \\
\text { Test Site } \\
\text { Test Site } \\
\text { Test Site } \\
\text { Test Site } \\
\text { Test Site } \\
\text { Test Site } \\
\text { Test Site } \\
\text { Test Site } \\
\text { Test Site }\end{array}$ & $\begin{array}{l}8.06 \mathrm{E}-02 \\
5.10 \mathrm{E}+00 \\
2.77 \mathrm{E}-01 \\
3.85 \mathrm{E}+00 \\
1.44 \mathrm{E}-01 \\
4.58 \mathrm{E}+01 \\
1.82 \mathrm{E}+01 \\
2.40 \mathrm{E}-01 \\
3.95 \mathrm{E}+02 \\
2.20 \mathrm{E}+00 \\
3.84 \mathrm{E}-02 \\
1.89 \mathrm{E}+01 \\
6.81 \mathrm{E}-02 \\
1.15 \mathrm{E}+00 \\
2.98 \mathrm{E}+01 \\
3.08 \mathrm{E}-01 \\
4.58 \mathrm{E}+01 \\
2.06 \mathrm{E}+02 \\
8.05 \mathrm{E}-02 \\
3.27 \mathrm{E}+01 \\
1.60 \mathrm{E}-01 \\
8.22 \mathrm{E}+00 \\
4.86 \mathrm{E}+01 \\
9.46 \mathrm{E}-01 \\
4.03 \mathrm{E}-01 \\
1.23 \mathrm{E}-01 \\
4.83 \mathrm{E}+02\end{array}$ & $\begin{array}{l}9.92 \mathrm{E}-02 \\
6.20 \mathrm{E}+00 \\
3.35 \mathrm{E}-01 \\
4.54 \mathrm{E}+00 \\
1.77 \mathrm{E}-01 \\
5.57 \mathrm{E}+01 \\
2.19 \mathrm{E}+01 \\
3.00 \mathrm{E}-01 \\
4.73 \mathrm{E}+02 \\
2.56 \mathrm{E}+00 \\
4.70 \mathrm{E}-02 \\
2.34 \mathrm{E}+01 \\
8.73 \mathrm{E}-02 \\
1.47 \mathrm{E}+00 \\
3.57 \mathrm{E}+01 \\
3.67 \mathrm{E}-01 \\
5.74 \mathrm{E}+01 \\
2.46 \mathrm{E}+02 \\
9.41 \mathrm{E}-02 \\
3.94 \mathrm{E}+01 \\
1.87 \mathrm{E}-01 \\
1.01 \mathrm{E}+01 \\
5.82 \mathrm{E}+01 \\
1.23 \mathrm{E}+00 \\
5.04 \mathrm{E}-01 \\
1.57 \mathrm{E}-01 \\
5.67 \mathrm{E}+02\end{array}$ & $\begin{array}{l}9.87 \mathrm{E}-03 \\
4.95 \mathrm{E}-01 \\
3.02 \mathrm{E}-02 \\
2.79 \mathrm{E}-01 \\
2.24 \mathrm{E}-02 \\
4.42 \mathrm{E}+00 \\
1.94 \mathrm{E}+00 \\
2.93 \mathrm{E}-02 \\
3.30 \mathrm{E}+01 \\
1.60 \mathrm{E}-01 \\
9.85 \mathrm{E}-03 \\
2.08 \mathrm{E}+00 \\
1.29 \mathrm{E}-02 \\
1.81 \mathrm{E}-01 \\
2.44 \mathrm{E}+00 \\
2.69 \mathrm{E}-02 \\
4.69 \mathrm{E}+00 \\
1.64 \mathrm{E}+01 \\
1.49 \mathrm{E}-02 \\
2.79 \mathrm{E}+00 \\
1.60 \mathrm{E}-02 \\
9.31 \mathrm{E}-01 \\
3.69 \mathrm{E}+00 \\
9.72 \mathrm{E}-02 \\
5.13 \mathrm{E}-02 \\
1.72 \mathrm{E}-02 \\
3.74 \mathrm{E}+01\end{array}$ & $\begin{array}{l}1.34 \mathrm{E}-02 \\
6.97 \mathrm{E}-01 \\
4.34 \mathrm{E}-02 \\
8.05 \mathrm{E}-01 \\
3.41 \mathrm{E}-02 \\
6.37 \mathrm{E}+00 \\
2.79 \mathrm{E}+00 \\
3.88 \mathrm{E}-02 \\
5.52 \mathrm{E}+01 \\
4.89 \mathrm{E}-01 \\
1.37 \mathrm{E}-02 \\
3.11 \mathrm{E}+00 \\
4.24 \mathrm{E}-02 \\
2.85 \mathrm{E}-01 \\
4.70 \mathrm{E}+00 \\
4.90 \mathrm{E}-02 \\
5.99 \mathrm{E}+00 \\
3.83 \mathrm{E}+01 \\
4.92 \mathrm{E}-02 \\
4.66 \mathrm{0}+00 \\
3.55 \mathrm{E}-02 \\
1.05 \mathrm{0}+00 \\
9.96 \mathrm{E}+00 \\
3.02 \mathrm{E}-01 \\
6.78 \mathrm{E}-02 \\
2.71 \mathrm{E}-02 \\
7.91 \mathrm{E}+01\end{array}$ \\
\hline Totals & & & & 3 & 3 & -02 & $\overline{102}$ \\
\hline
\end{tabular}

Table 7. Annual Accident Dose Risk for the Shipping Campaign (person-rem/Yr)

\begin{tabular}{|c|c|c|c|c|}
\hline Route & & & Truck & Rail \\
\hline $\begin{array}{l}\text { Ames Laboratory } \\
\text { Argonne - East } \\
\text { Argonne - West } \\
\text { Bettis Atomic } \\
\text { Fermilab (ENAL) } \\
\text { Hanford Site } \\
\text { INEI } \\
\text { ITRI } \\
\text { Oak Ridge Reserv. } \\
\text { Knolls Atomic } \\
\text { Kansas City plant } \\
\text { Ios Alamos NL } \\
\text { Lawrence Berkeley } \\
\text { Lawrence Livermore } \\
\text { Mound PIant } \\
\text { ORISE } \\
\text { Pantex Plant } \\
\text { Portsmouth GDP } \\
\text { Princeton PPL } \\
\text { Paducah GDP } \\
\text { Pinellas Plant } \\
\text { Rocky Flats Plant } \\
\text { Reactive Metals } \\
\text { SLAC } \\
\text { SNL - Albuquerque } \\
\text { SNL - Livermore } \\
\text { Savannah River }\end{array}$ & $\begin{array}{l}\text { to Nevada } \\
\text { to Nevada } \\
\text { to Nevada } \\
\text { to Nevada } \\
\text { to Nevada } \\
\text { to Nevada } \\
\text { to Nevada } \\
\text { to Nevada } \\
\text { to Nevada } \\
\text { to Nevada } \\
\text { to Nevada } \\
\text { to Nevada } \\
\text { to Nevada } \\
\text { to Nevada } \\
\text { to Nevada } \\
\text { to Nevada } \\
\text { to Nevada } \\
\text { to Nevada } \\
\text { to Nevada } \\
\text { to Nevada } \\
\text { to Nevada } \\
\text { to Nevada } \\
\text { to Nevada } \\
\text { to Nevada } \\
\text { to Nevada } \\
\text { to Nevada } \\
\text { to Nevada }\end{array}$ & $\begin{array}{l}\text { Test Site } \\
\text { Test Site } \\
\text { Test Site } \\
\text { Test site } \\
\text { Test site } \\
\text { Test Site } \\
\text { Test Site } \\
\text { Test Site } \\
\text { Test Site } \\
\text { Test Site } \\
\text { Test Site } \\
\text { Test Site } \\
\text { Test Site } \\
\text { Test Site } \\
\text { Test Site } \\
\text { Test Site } \\
\text { Test Site } \\
\text { Test Site } \\
\text { Test Site } \\
\text { Test Site } \\
\text { Test Site } \\
\text { Test Site } \\
\text { Test Site } \\
\text { Test Site } \\
\text { Test Site } \\
\text { Test Site } \\
\text { Test Site }\end{array}$ & $\begin{array}{l}1.12 E-05 \\
6.52 E-02 \\
8.60 E+00 \\
2.13 E-03 \\
8.72 E-05 \\
4.92 E-01 \\
2.50 E-01 \\
2.18 E-02 \\
2.51 E-03 \\
1.45 E-04 \\
2.53 E-05 \\
1.10 E+01 \\
7.68 E-02 \\
2.27 E-04 \\
2.17 E-03 \\
1.36 E-05 \\
1.06 E-03 \\
7.90 E-05 \\
1.35 E-05 \\
7.71 E-04 \\
1.41 E-05 \\
1.53 E-03 \\
9.48 E-06 \\
8.33 E-06 \\
1.47 E-04 \\
2.18 E-04 \\
3.58 E+01\end{array}$ & $\begin{array}{l}3.58 E-06 \\
1.12 E-02 \\
1.36 E+00 \\
1.02 E-03 \\
2.26 E-05 \\
1.19 E-01 \\
3.95 E-02 \\
1.81 E-03 \\
4.48 E-04 \\
4.48 E-05 \\
3.89 E-06 \\
9.10 E-01 \\
5.23 E-02 \\
6.85 E-05 \\
6.21 E-04 \\
2.57 E-06 \\
1.07 E-04 \\
3.26 E-05 \\
4.56 E-06 \\
1.59 E-04 \\
2.84 E-06 \\
8.52 E-05 \\
3.56 E-06 \\
5.08 E-06 \\
1.57 E-05 \\
6.89 E-05 \\
6.60 E+00\end{array}$ \\
\hline Totals & & & $5.63 E+01$ & $9.09 E+00$ \\
\hline
\end{tabular}




\section{$B-111$}

\begin{tabular}{lll}
$\begin{array}{l}\text { Table 8. Expected Annual Fatalities } \\
\text { for the Shipping Campaign }\end{array}$ & \\
\hline Exposure Group & Truck & Rail \\
\hline $\begin{array}{c}\text { Radiological } \\
\text { Normal Crew }\end{array}$ & $5.4 E-01$ & $4.4 E-02$ \\
Normal Public & $8.0 \mathrm{E}-01$ & $1.1 \mathrm{E}-01$ \\
$\begin{array}{c}\text { Accident Public } \\
\text { Nonradiological }\end{array}$ & $2.8 \mathrm{E}-02$ & $4.5 \mathrm{E}-03$ \\
Emission & $2.7 \mathrm{E}-01$ & $1.6 \mathrm{E}-01$ \\
Accident & $3.3 \mathrm{E}+00$ & $4.3 \mathrm{E}-02$ \\
\end{tabular}

Table 9. Expected Annual Cancer Incidence for the Shipping Campaign

\begin{tabular}{lll}
\hline Exposure Group & Truck & Rail \\
\hline Radiological & & \\
Normal Crew & $1.9 \mathrm{E}+00$ & $1.6 \mathrm{E}-01$ \\
Normal Public & $2.7 \mathrm{E}+00$ & $3.6 \mathrm{E}-01$ \\
Accident Public & $9.6 \mathrm{E}-02$ & $1.5 \mathrm{E}-02$ \\
Nonradiological & $2.7 \mathrm{E}-01$ & $1.6 \mathrm{E}-01$ \\
$\quad$ Emission & $\mathrm{NA}$ & $\mathrm{NA}$ \\
\hline Accident & & \\
\hline
\end{tabular}

Table 10. Expected Annual Genetic Effects for the Shipping Campaign

\begin{tabular}{lll}
\hline Exposure Group & Truck & Rail \\
\hline Radiological & & \\
Normal Crew & $8.1 E-02$ & $6.7 \mathrm{E}-03$ \\
Normal Public & $1.6 \mathrm{E}-01$ & $2.1 \mathrm{E}-02$ \\
Accident Public & $5.3 \mathrm{E}-03$ & $\mathbf{8 . 5 \mathrm { E } - 0 4}$ \\
$\begin{array}{l}\text { Nonradiological } \\
\text { Emission }\end{array}$ & NA & NA \\
Accident & NA & NA \\
\hline
\end{tabular}




\section{B.22 WM LLW CENTRALIZED 2 ALTERNATIVE (CASE 8): ACTIVATED METALS}

Table 1. Summary of Route Information

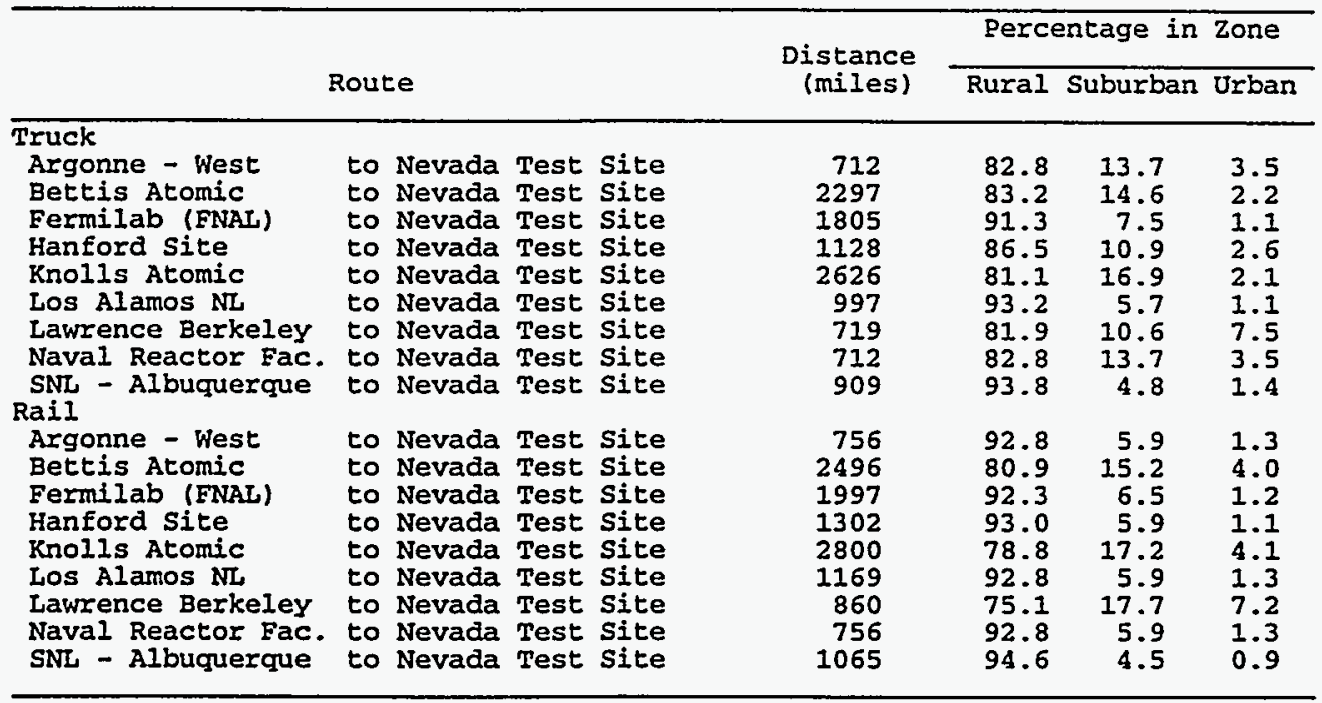

Table 2. Annual Number of Shipments and One-Way Shipment Distances

\begin{tabular}{|c|c|c|c|c|c|}
\hline \multirow[b]{2}{*}{ Route } & & \multicolumn{2}{|c|}{ \# of Shipments } & \multicolumn{2}{|c|}{ Total Mileage (mi) } \\
\hline & & Truck & Rail & Truck & Rail \\
\hline $\begin{array}{l}\text { Argonne - West } \\
\text { Bettis Atomic } \\
\text { Fermilab (FNAL) } \\
\text { Hanford Site } \\
\text { Rnolis Atomic } \\
\text { Los Alamos NL } \\
\text { Lawrence Berkeley } \\
\text { Naval Reactor Fac. } \\
\text { SNL - Albuquerque }\end{array}$ & $\begin{array}{l}\text { to Nevada Test Site } \\
\text { to Nevada Test Site } \\
\text { to Nevada Test Site } \\
\text { to Nevada Test Site } \\
\text { to Nevada Test Site } \\
\text { to Nevada Test Site } \\
\text { to Nevada Test Site } \\
\text { to Nevada Test Site } \\
\text { to Nevada Test Site }\end{array}$ & $\begin{array}{r}316 \\
40 \\
33 \\
650 \\
418 \\
15 \\
461 \\
6\end{array}$ & $\begin{array}{r}4 \\
120 \\
15 \\
13 \\
246 \\
159 \\
6 \\
175 \\
3\end{array}$ & $\begin{array}{l}6.41 \mathrm{E}+03 \\
7.26 \mathrm{E}+05 \\
7.22 \mathrm{E}+04 \\
3.72 \mathrm{E}+04 \\
1.71 \mathrm{E}+06 \\
4.17 \mathrm{E}+05 \\
1.08 \mathrm{E}+04 \\
3.28 \mathrm{E}+05 \\
5.45 \mathrm{E}+03\end{array}$ & $\begin{array}{l}3.02 \mathrm{E}+03 \\
3.00 \mathrm{E}+05 \\
3.00 \mathrm{E}+04 \\
1.69 \mathrm{E}+04 \\
6.89 \mathrm{E}+05 \\
1.86 \mathrm{E}+05 \\
5.16 \mathrm{E}+03 \\
1.32 \mathrm{E}+05 \\
3.19 \mathrm{E}+03\end{array}$ \\
\hline Totals & & 1948 & 741 & $3.31 E+06$ & $1.36 \mathrm{E}+06$ \\
\hline
\end{tabular}


Table 3. Incident-Free Dose per Shipment (person-rem/shipment)

\begin{tabular}{|c|c|c|c|c|c|c|}
\hline \multirow[b]{2}{*}{ Source/Route (s) } & & \multirow[b]{2}{*}{ Crew } & \multicolumn{4}{|c|}{ General Public } \\
\hline & & & off-Link & On-Link & Stops & Total \\
\hline $\begin{array}{l}\text { LLW(I) AW to NT } \\
\text { Argonne - West }\end{array}$ & $\begin{array}{l}\text { to Nevada } \\
\text { Truck } \\
\text { Rail }\end{array}$ & $\begin{array}{l}\text { Test Site } \\
2.13 \mathrm{E}-02 \\
6.04 \mathrm{E}-03\end{array}$ & $\begin{array}{l}2.03 E-03 \\
2.80 E-03\end{array}$ & $\begin{array}{l}3.79 \mathrm{E}-03 \\
6.88 \mathrm{E}-05\end{array}$ & $\begin{array}{l}1.99 \mathrm{E}-02 \\
5.81 \mathrm{E}-03\end{array}$ & $\begin{array}{l}2.58 E-02 \\
8.67 E-03\end{array}$ \\
\hline $\begin{array}{l}\text { LLW(I) BA to NT } \\
\text { Bettis Atomic }\end{array}$ & $\begin{array}{l}\text { to Nevada } \\
\text { Truck } \\
\text { Rail }\end{array}$ & $\begin{array}{l}\text { Test Site } \\
6.75 E-02 \\
1.33 E-02\end{array}$ & $\begin{array}{l}\text { e } \\
2.17 E-03 \\
2.67 \mathrm{E}-02\end{array}$ & $\begin{array}{l}1.02 \mathrm{E}-02 \\
4.43 \mathrm{E}-04\end{array}$ & $\begin{array}{l}-02 \\
-02\end{array}$ & $\begin{array}{l}7.97 \mathrm{E}-02 \\
3.83 \mathrm{E}-02\end{array}$ \\
\hline $\begin{array}{l}\text { LLW(I) FL to NT } \\
\text { Eermilab (FNAL) }\end{array}$ & $\begin{array}{l}\text { to Nevada } \\
\text { Truck } \\
\text { Rail }\end{array}$ & $\begin{array}{l}\text { Test Site } \\
4.82 \mathrm{E}-02 \\
1.12 \mathrm{E}-02\end{array}$ & $\begin{array}{l}2.15 \mathrm{E}-03 \\
7.26 \mathrm{E}-03\end{array}$ & $\begin{array}{l}6.20 \mathrm{E}-03 \\
1.85 \mathrm{E}-04\end{array}$ & $\begin{array}{l}5.05 \mathrm{E}-02 \\
9.63 \mathrm{E}-03\end{array}$ & $\begin{array}{l}5.89 E-02 \\
1.71 E-02\end{array}$ \\
\hline $\begin{array}{l}\text { LLW(I) HS to NT } \\
\text { Hanford Site }\end{array}$ & $\begin{array}{l}\text { to Nevada } \\
\text { Truck } \\
\text { Rail }\end{array}$ & $\begin{array}{l}\text { Test Site } \\
3.22 \mathrm{E}-02 \\
8.31 \mathrm{E}-03\end{array}$ & $\begin{array}{l}2.46 \mathrm{E}-03 \\
4.39 \mathrm{E}-03\end{array}$ & $\begin{array}{l}5.18 \mathrm{E}-03 \\
1.15 \mathrm{E}-04\end{array}$ & $\begin{array}{l}3.16 \mathrm{E}-02 \\
7.49 \mathrm{E}-03\end{array}$ & $\begin{array}{l}3.92 \mathrm{E}-02 \\
1.20 \mathrm{E}-02\end{array}$ \\
\hline $\begin{array}{l}\text { LLW(I) KA to NT } \\
\text { Knolls Atomic }\end{array}$ & $\begin{array}{l}\text { to Nevada } \\
\text { Truck } \\
\text { Rail }\end{array}$ & $\begin{array}{l}\text { Test Site } \\
7.86 \mathrm{E}-02 \\
1.46 \mathrm{E}-02\end{array}$ & $\begin{array}{l}6.20 \mathrm{E}-03 \\
3.18 \mathrm{E}-02\end{array}$ & $\begin{array}{l}1.18 \mathrm{E}-02 \\
5.30 \mathrm{E}-04\end{array}$ & $\begin{array}{l}7.35 \mathrm{E}-02 \\
1.21 \mathrm{E}-02\end{array}$ & $\begin{array}{l}9.15 \mathrm{E}-02 \\
4.44 \mathrm{E}-02\end{array}$ \\
\hline $\begin{array}{l}\text { LLW(I) LA to NT } \\
\text { LOS Alamos NL }\end{array}$ & $\begin{array}{l}\text { to Nevada } \\
\text { Truck } \\
\text { Rail }\end{array}$ & $\begin{array}{l}\text { Test Site } \\
2.61 \mathrm{E}-02 \\
7.76 \mathrm{E}-03\end{array}$ & $\begin{array}{l}1.04 \mathrm{E}-03 \\
4.40 \mathrm{E}-03\end{array}$ & $\begin{array}{l}3.33 \mathrm{E}-03 \\
1.07 \mathrm{E}-04\end{array}$ & $\begin{array}{l}2.79 \mathrm{E}-02 \\
7.08 \mathrm{E}-03\end{array}$ & $\begin{array}{l}3.23 \mathrm{E}-02 \\
1.16 \mathrm{E}-02\end{array}$ \\
\hline $\begin{array}{l}\text { LLW(I) LB to NT } \\
\text { Lawrence Berkeley }\end{array}$ & $\begin{array}{l}\text { to Nevada } \\
\text { Truck } \\
\text { Rail }\end{array}$ & $\begin{array}{l}\text { Test Site } \\
2.27 \mathrm{E}-02 \\
6.47 \mathrm{E}-03\end{array}$ & $\begin{array}{l}\text { e } \\
3.31 E-03 \\
1.49 E-02\end{array}$ & $\begin{array}{l}5.66 \mathrm{E}-03 \\
2.04 \mathrm{E}-04\end{array}$ & $\begin{array}{l}2.01 E-02 \\
6.13 E-03\end{array}$ & $\begin{array}{l}2.91 E-02 \\
2.12 E-02\end{array}$ \\
\hline $\begin{array}{l}\text { LLW(I) NR to NT } \\
\text { Naval Reactor Fac. }\end{array}$ & $\begin{array}{l}\text { to Nevada } \\
\text { Truck } \\
\text { Rail }\end{array}$ & $\begin{array}{l}\text { Test Site } \\
2.13 \mathrm{E}-02 \\
6.04 \mathrm{E}-03\end{array}$ & $\begin{array}{r}2.03 E-03 \\
2.80 E-03\end{array}$ & $\begin{array}{l}3.79 E-03 \\
6.88 E-05\end{array}$ & $\begin{array}{l}1.99 \mathrm{E}-02 \\
5.81 \mathrm{E}-03\end{array}$ & $\begin{array}{l}2.58 \mathrm{E}-02 \\
8.67 \mathrm{E}-03\end{array}$ \\
\hline $\begin{array}{l}\text { LLW(I) SA to NT } \\
\text { SNL - Albuquerque }\end{array}$ & $\begin{array}{l}\text { to Nevada } \\
\text { Truck } \\
\text { Rail }\end{array}$ & $\begin{array}{l}\text { Test Site } \\
2.37 \mathrm{E}-02 \\
7.32 \mathrm{E}-03\end{array}$ & $\begin{array}{l}\text { e } \\
\frac{1.02 E-03}{2.85 E-03}\end{array}$ & $\begin{array}{l}3.18 E-03 \\
8.34 E-05\end{array}$ & $\begin{array}{l}2.54 \mathrm{E}-02 \\
6.76 \mathrm{E}-03\end{array}$ & $\begin{array}{l}2.97 \mathrm{E}-02 \\
9.69 \mathrm{E}-03\end{array}$ \\
\hline
\end{tabular}

Table 4. Accident Dose Risk per Shipment (person-rem/shipment)

\begin{tabular}{|c|c|c|c|}
\hline Source/Route & & Truck & Rail \\
\hline $\begin{array}{l}\text { LLW(I) AW to NT } \\
\text { Argonne - West } \\
\text { LLW(I) BA to NT } \\
\text { Bettis Atomic } \\
\text { LLW(I) FL to NT } \\
\text { Fermilab (FNAI) } \\
\text { LLW(I) HS to NT } \\
\text { Hanford Site } \\
\text { LLW(I) KA to NT } \\
\text { Rnolls Atomic } \\
\text { LLW(I) LA to NT } \\
\text { Los Alamos NL } \\
\text { LLW(I) LB to NT } \\
\text { LaWrence Berkeley } \\
\text { LLW(I) NR to NT } \\
\text { Naval Reactor Fac. } \\
\text { LLW(I) SA to NT } \\
\text { SNL - Albuquerque }\end{array}$ & $\begin{array}{l}\text { to Nevada Test Site } \\
\text { to Nevada Test Site } \\
\text { to Nevada Test Site } \\
\text { to Nevada Test Site } \\
\text { to Nevada Test Site } \\
\text { to Nevada Test Site } \\
\text { to Nevada Test Site } \\
\text { to Nevada Test Site } \\
\text { to Nevada Test Site }\end{array}$ & $\begin{array}{l}2.03 E-11 \\
1.92 E-06 \\
6.08 E-11 \\
2.71 E-08 \\
3.29 E-06 \\
1.78 E-09 \\
8.23 E-11 \\
1.20 E-07 \\
2.40 E-12\end{array}$ & $\begin{array}{l}7.25 \mathrm{E}-12 \\
1.72 \mathrm{E}-06 \\
4.06 \mathrm{E}-11 \\
1.57 \mathrm{E}-08 \\
2.51 \mathrm{E}-06 \\
3.07 \mathrm{E}-10 \\
1.45 \mathrm{E}-10 \\
4.99 \mathrm{E}-08 \\
3.74 \mathrm{E}-13\end{array}$ \\
\hline
\end{tabular}


and are not consistent with the definitions of waste types used in the WM PEIS.) Of the $15,000 \mathrm{LLW}$ shipments recorded in the SMAC database, approximately 2,500 reported external dose rates. The average dose rate reported was approximately $1 \mathrm{mrem} / \mathrm{h}$ measured at $1 \mathrm{~m}(3.3 \mathrm{ft})$ from the surface of a shipment. This value was used for future WM LLW shipments for the WM PEIS analysis.

\subsection{MAXIMALLY EXPOSED INDIVIDUAL EXPOSURE SCENARIOS}

The risk to MEIs has been estimated for a number of hypothetical exposure scenarios for LLW shipments. The receptors include crew members, departure inspectors, and members of the public exposed during traffic obstructions (traffic jam), while working at a service station, or by living near a treatment, storage, or disposal site. The dose and risk to MEIs were calculated for given distances and durations of exposure. The distances and durations of exposure for each receptor are similar to those given in previous transportation assessments (DOE 1987b, 1990a). The scenarios for exposure are not meant to be exhaustive but were selected to provide a range of potential situations for exposure. The assumptions for exposure scenarios are as follows:

- Crew Members. Truck and rail crew members are assumed to be occupational radiation workers and would be monitored by a dosimetry program. Therefore, the maximum allowable dose would be $5 \mathrm{rem} / \mathrm{yr}$. As an administrative procedure, the DOE limits doses to DOE workers to $2 \mathrm{rem} / \mathrm{yr}$ (DOE 1992).

- Inspectors (Truck and Rail). Inspectors are assumed to be either federal or state vehicle inspectors. Inspectors are not assumed to be monitored by a dosimetry program. An average exposure distance of $3 \mathrm{~m}(10 \mathrm{ft})$ and an exposure duration of 30 minutes are assumed.

- Rail-Yard Crew Member. A rail-yard crew member is not assumed to be monitored by a dosimetry program. An average exposure distance of $10 \mathrm{~m}$ $(33 \mathrm{ft})$ and an exposure duration of two hours are assumed.

- Resident (Truck and Rail). A resident is assumed to live $30 \mathrm{~m}$ (98 ft) from a site entrance route (truck or rail). Shipments pass at an average speed of $24 \mathrm{~km} / \mathrm{h}(15 \mathrm{mph})$, and the resident is exposed unshielded. Cumulative doses are assessed for each site on the basis of the number of shipments entering or exiting the site, with the assumption that the resident is present for $100 \%$ of the shipments. 


\section{$B-11+$}

Table 5. Nonradiological Risk Factors per Shipment

(facalities/shipment)

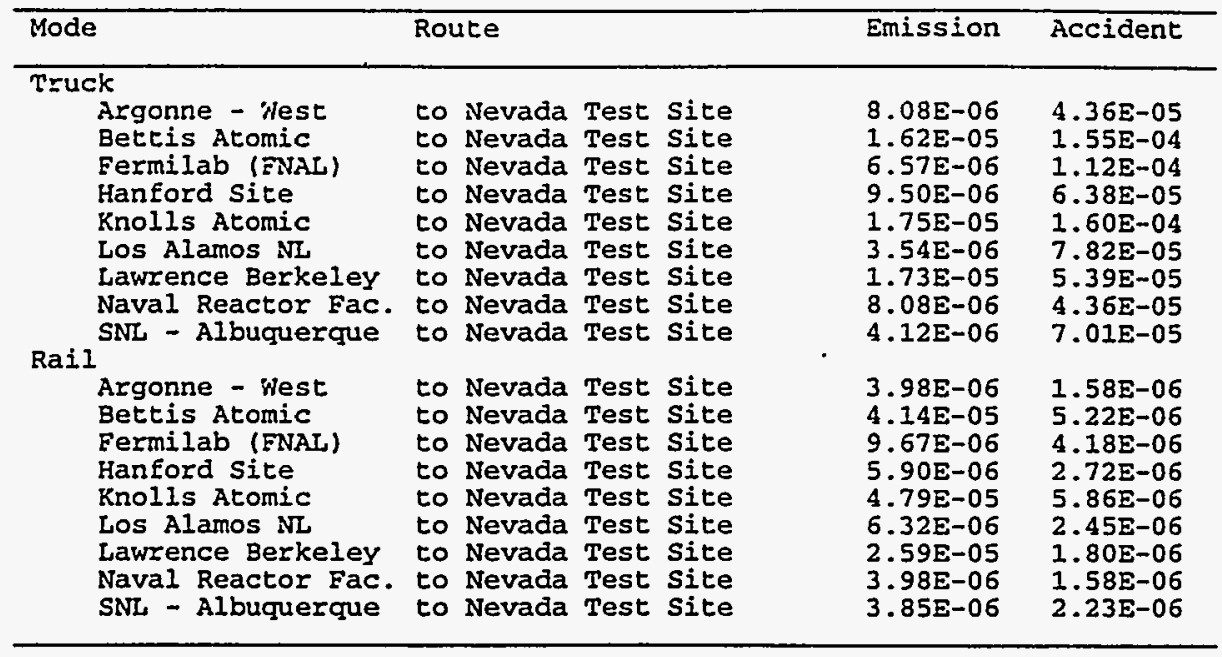

Table 6. Annual Incident-Free Dose for the Shipping Campaign (person-rem/yr)

\begin{tabular}{|c|c|c|c|c|c|}
\hline \multirow[b]{2}{*}{ Route } & & \multicolumn{2}{|c|}{ Truck } & \multicolumn{2}{|c|}{ Rail } \\
\hline & & Crew & Public & Crew & Public \\
\hline $\begin{array}{l}\text { Argonne - West } \\
\text { Bettis Atomic } \\
\text { Fermilab (FNAL) } \\
\text { Hanford Site } \\
\text { Rnolls Atomic } \\
\text { Los Alamos NL } \\
\text { Lawrence Berkeley } \\
\text { Naval Reactor Fac. } \\
\text { SNL - Albuquerque }\end{array}$ & $\begin{array}{ll}\text { to Nevada } & \text { Test Site } \\
\text { to Nevada } & \text { Test Site } \\
\text { to Nevada } & \text { Test Site } \\
\text { to Nevada } & \text { Test Site } \\
\text { to Nevada } & \text { Test Site } \\
\text { to Nevada Test Site } \\
\text { to Nevada Test Site } \\
\text { to Nevada Test Site } \\
\text { to Nevada Test Site }\end{array}$ & $\begin{array}{l}1.92 \mathrm{E}-01 \\
2.13 \mathrm{E}+01 \\
1.93 \mathrm{E}+00 \\
1.06 \mathrm{E}+00 \\
5.11 \mathrm{E}+01 \\
1.09 \mathrm{E}+01 \\
3.41 \mathrm{E}-01 \\
9.83 \mathrm{E}+00 \\
1.42 \mathrm{E}-01\end{array}$ & $\begin{array}{l}2.32 \mathrm{E}-01 \\
2.52 \mathrm{E}+01 \\
2.35 \mathrm{E}+00 \\
1.29 \mathrm{E}+00 \\
5.95 \mathrm{E}+01 \\
1.35 \mathrm{E}+01 \\
4.36 \mathrm{E}-01 \\
1.19 \mathrm{E}+01 \\
1.78 \mathrm{E}-01\end{array}$ & $\begin{array}{l}2.41 \mathrm{E}-02 \\
1.60 \mathrm{E}+00 \\
1.68 \mathrm{E}-01 \\
1.08 \mathrm{E}-01 \\
3.58 \mathrm{E}+00 \\
1.23 \mathrm{E}+00 \\
3.88 \mathrm{E}-02 \\
1.06 \mathrm{E}+00 \\
2.20 \mathrm{E}-02\end{array}$ & $\begin{array}{l}3.47 \mathrm{E}-02 \\
4.60 \mathrm{E}+00 \\
2.56 \mathrm{E}-01 \\
1.56 \mathrm{E}-01 \\
1.09 \mathrm{E}+01 \\
1.84 \mathrm{E}+00 \\
1.27 \mathrm{E}-01 \\
1.52 \mathrm{E}+00 \\
2.91 \mathrm{E}-02\end{array}$ \\
\hline Totals & & $9.68 \mathrm{E}+01$ & $1.15 \mathrm{E}+02$ & $7.83 E+00$ & $1.95 \mathrm{E}+01$ \\
\hline
\end{tabular}

Table 7. Annual Accident Dose Risk for the Shipping Campaign (person-rem/yr)

\begin{tabular}{llccc}
\hline Route & & & Truck & Rail \\
\hline Argonne - West & to Nevada Test Site & $1.82 \mathrm{E}-10$ & $2.90 \mathrm{E}-11$ \\
Bettis Atomic & to Nevada Test Site & $6.08 \mathrm{E}-04$ & $2.06 \mathrm{E}-04$ \\
Fermilab (FNAI) & to Nevada Test Site & $2.43 \mathrm{E}-09$ & $6.09 \mathrm{E}-10$ \\
Hanford Site & to Nevada Test Site & $8.95 \mathrm{E}-07$ & $2.05 \mathrm{E}-07$ \\
Knolls Atomic & to Nevada Test Site & $2.14 \mathrm{E}-03$ & $6.18 \mathrm{E}-04$ \\
Los Alamos NL & to Nevada Test Site & $7.46 \mathrm{E}-07$ & $4.89 \mathrm{E}-08$ \\
Lawrence Berkeley & to Nevada Test Site & $1.23 \mathrm{E}-09$ & $8.71 \mathrm{E}-10$ \\
Naval Reactor Fac. to Nevada Test Site & $5.53 \mathrm{E}-05$ & $8.73 \mathrm{E}-06$ \\
SNL - Albuquerque & to Nevada Test Site & $1.44 \mathrm{E}-11$ & $1.12 \mathrm{E}-12$ \\
& & & & \\
\hline Totals & & & & \\
\hline
\end{tabular}

Table 8. Expected Annual Fatalities

for the Shipping Campaign

\begin{tabular}{llc}
\hline Exposure Group & Truck & Rail \\
\hline RadiologicaI & $3.9 \mathrm{E}-02$ & $3.1 \mathrm{E}-03$ \\
Normal Crew & $5.7 \mathrm{E}-02$ & $9.7 \mathrm{E}-03$ \\
Normal Public & $1.4 \mathrm{E}-06$ & $4.2 \mathrm{E}-07$ \\
Accident Public & $2.3 \mathrm{E}-02$ & $1.9 \mathrm{E}-02$ \\
Nonradiological & $2.1 \mathrm{E}-01$ & $2.9 \mathrm{E}-03$ \\
$\quad$ Emission & & \\
Accident & &
\end{tabular}




\section{$B-115$}

Table 9. Expected Annual Cancer Incidence for the Shipping Campaign

\begin{tabular}{lll}
\hline Exposure Group & Truck & Rail \\
\hline Radiological & & \\
$\quad$ Normal Crew & $1.4 E-01$ & $1.1 E-02$ \\
$\quad$ Normal Public & $1.9 E-01$ & $3.3 E-02$ \\
$\begin{array}{c}\text { Accident Public } \\
\text { Nonradiological }\end{array}$ & $4.8 \mathrm{E}-06$ & $1.4 \mathrm{E}-06$ \\
$\begin{array}{l}\text { Emission } \\
\text { Accident }\end{array}$ & $2.3 \mathrm{E}-02$ & $1.9 \mathrm{E}-02$ \\
& $\mathrm{NA}$ & $\mathrm{NA}$ \\
\hline
\end{tabular}

Table 10. Expected Annual Genetic Effects for the Shipping Campaign

\begin{tabular}{lll}
\hline Exposure Group & Truck & Rail \\
\hline Radiological & & \\
Normal Crew & $5.8 E-03$ & $4.7 E-04$ \\
Normal Public & $1.1 E-02$ & $1.9 E-03$ \\
Accident Public & $2.8 E-07$ & $8.4 E-08$ \\
Nonradiological & & \\
Emission & NA & NA \\
Accident & NA & NA \\
\hline
\end{tabular}




\section{B.23 WM LLW CENTRALIZED 3 ALTERNATIVE (CASE 14): HETEROGENEOUS SOLIDS}

Table 1. Summary of Route Information

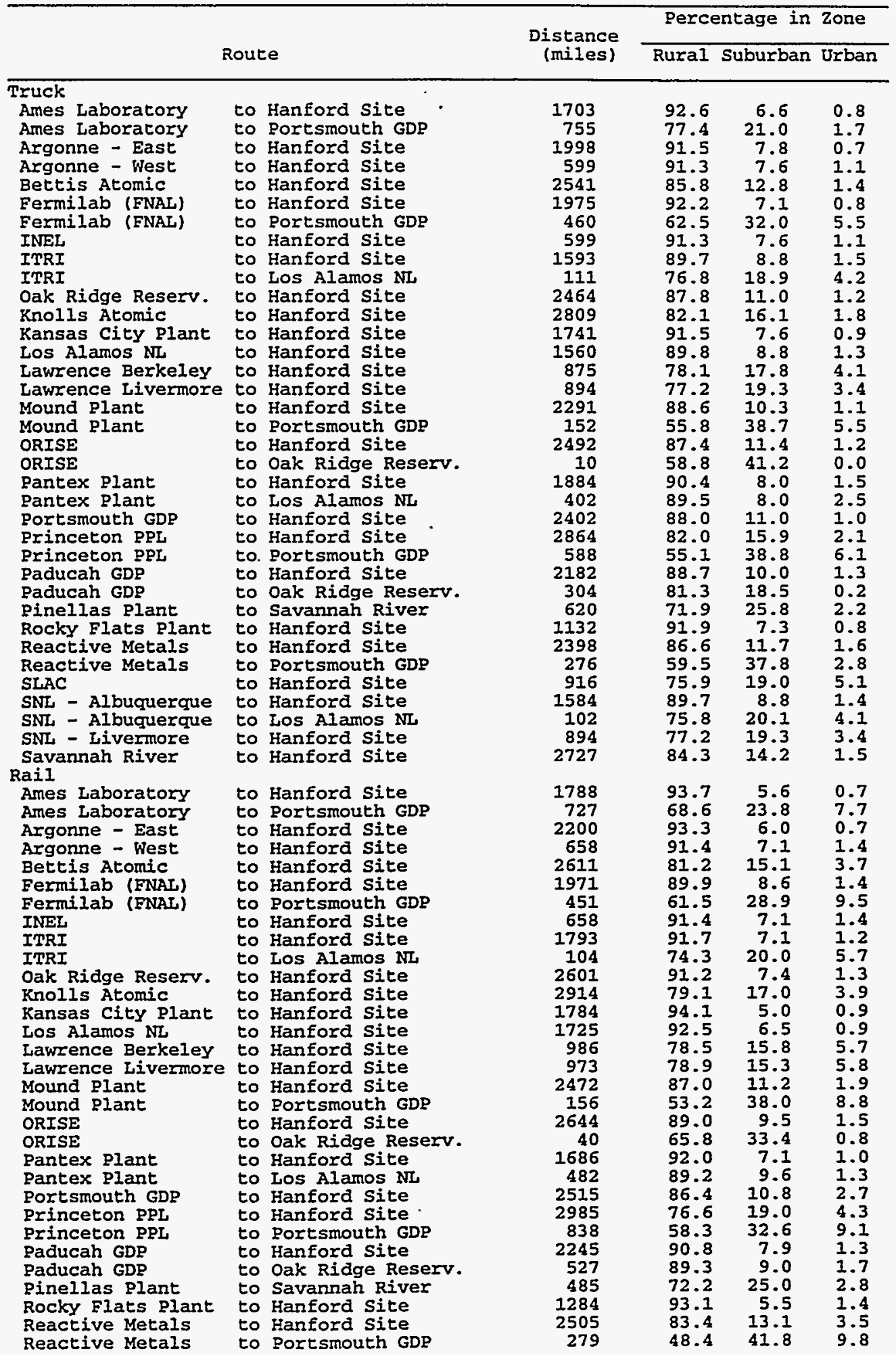


SLAC

SNL - Albuquerque

SNL - Albuquerque

SNL - Livermore

to Hanford Site

1036

76.1

to Hanford Site

to Los Alamos NI

to Hanford Site

1793

91.7

74.3

16.8

7.1

Savannah River

to Hanford Site

973
2953

$84.7 \quad 15.3$

5.7

5.8

$\begin{array}{llll}2953 & 84.7 & 13.5 & 1.8\end{array}$

Table 2. Annual Number of Shipments and One-Way Shipment Distances

\begin{tabular}{|c|c|c|c|c|c|c|}
\hline \multirow[b]{2}{*}{ Route } & & & \multicolumn{2}{|c|}{ \# of Shipments } & \multicolumn{2}{|c|}{ Total Mileage (mi) } \\
\hline & & & Truck & Rail & Truck & Rail \\
\hline $\begin{array}{l}\text { Ames Laboratory } \\
\text { Ames Laboratory } \\
\text { Argonne - East } \\
\text { Argonne - West } \\
\text { Bettis Atomic } \\
\text { Fermilab (FNAL) } \\
\text { Fermilab (FNAL) } \\
\text { INEL } \\
\text { ITRI } \\
\text { ITRI } \\
\text { Oak Ridge Reserv. } \\
\text { Knolls Atomic } \\
\text { Kansas City Plant } \\
\text { Los Alamos NL } \\
\text { Lawrence Berkeley } \\
\text { Lawrence Livermore } \\
\text { Mound Plant } \\
\text { Mound Plant } \\
\text { ORISE } \\
\text { ORISE } \\
\text { Pantex Plant } \\
\text { Pantex Plant } \\
\text { Portsmouth GDP } \\
\text { Princeton PPL } \\
\text { Princeton PPL } \\
\text { Paducah GDP } \\
\text { Paducah GDP } \\
\text { Pinellas Plant } \\
\text { RockY Elats Plant } \\
\text { Reactive Metals } \\
\text { Reactive Metals } \\
\text { SLAC } \\
\text { SNL - Albuquerque } \\
\text { SNL - Albuquerque } \\
\text { SNL - Livermore } \\
\text { Savannah River }\end{array}$ & $\begin{array}{l}\text { to } \\
\text { to } \\
\text { to } \\
\text { to } \\
\text { to } \\
\text { to } \\
\text { to } \\
\text { to } \\
\text { to } \\
\text { to } \\
\text { to } \\
\text { to } \\
\text { to } \\
\text { to } \\
\text { to } \\
\text { to } \\
\text { to } \\
\text { to } \\
\text { to } \\
\text { to } \\
\text { to } \\
\text { to } \\
\text { to } \\
\text { to } \\
\text { to } \\
\text { to } \\
\text { to } \\
\text { to } \\
\text { to } \\
\text { to } \\
\text { to } \\
\text { to } \\
\text { to } \\
\text { to } \\
\text { to } \\
\text { to }\end{array}$ & $\begin{array}{l}\text { Hanford Site } \\
\text { Portsmouth GDP } \\
\text { Hanford Site } \\
\text { Hanford Site } \\
\text { Hanford Site } \\
\text { Hanford Site } \\
\text { Portsmouth GDP } \\
\text { Hanford Site } \\
\text { Hanford Site } \\
\text { Los Alamos NL } \\
\text { Hanford Site } \\
\text { Hanford Site } \\
\text { Hanford Site } \\
\text { Hanford Site } \\
\text { Hanford Site } \\
\text { Hanford Site } \\
\text { Hanford Site } \\
\text { Portsmouth GDP } \\
\text { Hanford Site } \\
\text { Oak Ridge Reserv. } \\
\text { Hanford Site } \\
\text { Los Alamos NL } \\
\text { Hanford Site } \\
\text { Hanford Site } \\
\text { Portsmouth GDP } \\
\text { Hanford Site } \\
\text { Oak Ridge Reserv. } \\
\text { Savannah River } \\
\text { Hanford Site } \\
\text { Hanford Site } \\
\text { Portsmouth GDP } \\
\text { Hanford Site } \\
\text { Hanford Site } \\
\text { Los Alamos NL } \\
\text { Hanford Site } \\
\text { Hanford Site }\end{array}$ & $\begin{array}{r}2 \\
1 \\
105 \\
10 \\
57 \\
2 \\
1 \\
820 \\
9 \\
2 \\
6067 \\
28 \\
1 \\
1965 \\
3 \\
56 \\
496 \\
12 \\
4 \\
1 \\
25 \\
1358 \\
2950 \\
1 \\
7 \\
578 \\
57 \\
2 \\
335 \\
745 \\
7 \\
39 \\
17 \\
1 \\
5 \\
6750\end{array}$ & $\begin{array}{r}1 \\
1 \\
39 \\
4 \\
21 \\
1 \\
1 \\
315 \\
3 \\
1 \\
2425 \\
11 \\
1 \\
764 \\
2 \\
21 \\
186 \\
5 \\
2 \\
1 \\
10 \\
528 \\
1107 \\
1 \\
1 \\
215 \\
21 \\
1 \\
123 \\
283 \\
3 \\
15 \\
7 \\
1 \\
2 \\
2522\end{array}$ & 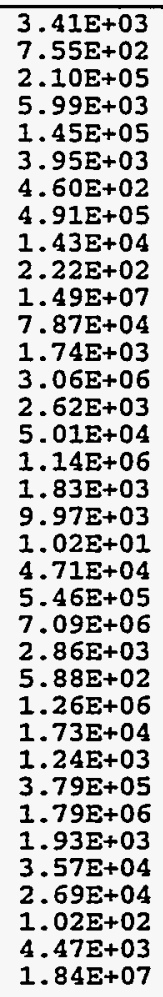 & $\begin{array}{l}1.79 E+03 \\
7.27 E+02 \\
8.58 E+04 \\
2.63 E+03 \\
5.48 E+04 \\
1.97 E+03 \\
4.51 E+02 \\
2.07 E+05 \\
5.38 E+03 \\
1.04 E+02 \\
6.31 E+06 \\
3.21 E+04 \\
1.78 E+03 \\
1.32 E+06 \\
1.97 E+03 \\
2.04 E+04 \\
4.60 E+05 \\
7.80 E+02 \\
5.29 E+03 \\
3.95 E+01 \\
1.69 E+04 \\
2.55 E+05 \\
2.78 E+06 \\
2.98 E+03 \\
8.38 E+02 \\
4.83 E+05 \\
1.11 E+04 \\
4.85 E+02 \\
1.58 E+05 \\
7.09 E+05 \\
8.37 E+02 \\
1.55 E+04 \\
1.26 E+04 \\
1.04 E+02 \\
1.95 E+03 \\
7.45 E+06\end{array}$ \\
\hline Totals & & & 2513 & 645 & $4.98 \mathrm{E}+07$ & $.04 \mathrm{E}+07$ \\
\hline
\end{tabular}


Table 3. Incident-Free Dose per Shipment (person-rem/shipment)

\begin{tabular}{|c|c|c|c|c|c|}
\hline \multirow[b]{2}{*}{ Source/Route (s) } & \multirow[b]{2}{*}{ Crew } & \multicolumn{4}{|c|}{ General Public } \\
\hline & & $\overline{\text { off-Link }}$ & On-Link & Stops & Total \\
\hline $\begin{array}{l}W(x) \text { AL to HS } \\
\text { Ames Laboratory }\end{array}$ & $\begin{array}{ll}\text { to Hanford Site } \\
\text { Truck } & 4.46 \mathrm{E}-02 \\
\text { Rail } & 1.03 \mathrm{E}-02\end{array}$ & $\begin{array}{l}1.62 \mathrm{E}-03 \\
4.69 \mathrm{E}-03\end{array}$ & $\begin{array}{l}5.3 \\
1.4\end{array}$ & $\begin{array}{l}4.77 E-02 \\
8.98 E-03\end{array}$ & $\begin{array}{l}5.47 \mathrm{E}-02 \\
1.38 \mathrm{E}-02\end{array}$ \\
\hline $\begin{array}{l}\text { LLW(x) AL to PO } \\
\text { Ames Laboratory }\end{array}$ & $\begin{array}{lr}\text { to Portsmouth GDP } \\
\text { Truck } & 2.33 \mathrm{E}-02 \\
\text { Rail } & 5.91 \mathrm{E}-03\end{array}$ & $\begin{array}{l}1.86 \mathrm{E}-03 \\
1.42 \mathrm{E}-02\end{array}$ & $\begin{array}{l}3-03 \\
3-04\end{array}$ & $\begin{array}{l}2.11 E-02 \\
5.72 E-03\end{array}$ & $\begin{array}{l}2.63 \mathrm{E}-02 \\
2.01 \mathrm{E}-02\end{array}$ \\
\hline $\begin{array}{l}\text { LLW(x) AN to HS } \\
\text { Argonne - East }\end{array}$ & $\begin{array}{lr}\text { to Hanford Site } \\
\text { Truck } & 5.29 \mathrm{E}-02 \\
\text { Rail } & 1.21 \mathrm{E}-02\end{array}$ & $\begin{array}{l}1.97 E-03 \\
5.75 E-03\end{array}$ & $\begin{array}{l}6.2 \\
1.8\end{array}$ & $\begin{array}{l}5.59 E-02 \\
1.03 E-02\end{array}$ & $\begin{array}{l}6.42 \mathrm{E}-02 \\
1.62 \mathrm{E}-02\end{array}$ \\
\hline lest & $\begin{array}{ll}\text { to Hanford Site } \\
\text { Truck } & 1.60 \mathrm{E}-02 \\
\text { Rail } & 5.63 \mathrm{E}-03\end{array}$ & $\begin{array}{l}7.18 \mathrm{E}-04 \\
2.83 \mathrm{E}-03\end{array}$ & $\begin{array}{l}2.08 \\
6.58\end{array}$ & $\begin{array}{l}1.68 E-02 \\
5.51 E-03\end{array}$ & $\begin{array}{l}1.95 \mathrm{E}-02 \\
8.40 \mathrm{E}-03\end{array}$ \\
\hline LLW(x) FL to HS & $\begin{array}{ll}\text { to Hanford Site } \\
\text { Truck } & 7.21 \mathrm{E}-02 \\
\text { Rail } & 1.38 \mathrm{E}-02\end{array}$ & $\begin{array}{l}4.36 E-03 \\
2.67 E-02\end{array}$ & $\begin{array}{l}9.77 E-03 \\
4.53 E-04\end{array}$ & $\begin{array}{l}7.11 \mathrm{E}-02 \\
1.15 \mathrm{E}-02\end{array}$ & $\begin{array}{l}8.53 E-02 \\
3.87 E-02\end{array}$ \\
\hline $\begin{array}{l}\text { LLW(x) FL to HS } \\
\text { Eermilab (ENAL) }\end{array}$ & $\begin{array}{ll}\text { to Hanford Site } \\
\text { Truck } & 5.20 \mathrm{E}-02 \\
\text { Rail } & 1.11 \mathrm{E}-02\end{array}$ & $\begin{array}{l}1.92 \\
9.07\end{array}$ & $\begin{array}{l}6.2 \\
2.1\end{array}$ & $\begin{array}{l}5.53 \mathrm{E}-02 \\
9.55 \mathrm{E}-03\end{array}$ & $\begin{array}{l}5 E-02 \\
8 E-02\end{array}$ \\
\hline Fermilab (FN & $\begin{array}{lr}\text { to Portsmouth GDP } \\
\text { Truck } & 1.68 \mathrm{E}-02 \\
\text { Rail } & 4.76 \mathrm{E}-03\end{array}$ & $\begin{array}{l}2.4 \\
1.0\end{array}$ & $\begin{array}{l}3.4 \\
1.4\end{array}$ & $\begin{array}{l}E-02 \\
E-03\end{array}$ & $\begin{array}{l}1.87 E-02 \\
1.58 E-02\end{array}$ \\
\hline $\begin{array}{l}\text { LLW(x) IN to HS } \\
\text { INEL }\end{array}$ & $\begin{array}{l}\text { to Hanford Site } \\
\text { Truck } \\
\text { Rail } \\
\text { R.60E-02 } \\
\end{array}$ & $\begin{array}{l}7.1 \\
2.8\end{array}$ & $\begin{array}{l}2.0 \\
6.5\end{array}$ & $\begin{array}{l}E-02 \\
E-03\end{array}$ & $\begin{array}{l}E-02 \\
E-03\end{array}$ \\
\hline 20 & $\begin{array}{lr}\text { to Hanford Site } \\
\text { Truck } & 4.34 \mathrm{E}-02 \\
\text { Rail } & 1.04 \mathrm{E}-02\end{array}$ & $\begin{array}{l}2.30 \\
6.90\end{array}$ & $\begin{array}{l}5.9 \\
1.7\end{array}$ & & $\begin{array}{l}5.28 \mathrm{E}-02 \\
1.61 \mathrm{E}-02\end{array}$ \\
\hline TrW (x) OR to HS & $\begin{array}{cr}\text { to Los } & \text { Alamos NL } \\
\text { Truck } & 3.54 \mathrm{E}-03 \\
\text { Rail } & 3.31 \mathrm{E}-03\end{array}$ & $\begin{array}{l}4.0 \\
1.5\end{array}$ & 6. & $\begin{array}{l}E-03 \\
E-03\end{array}$ & $\begin{array}{l}8 E-03 \\
7 E-03\end{array}$ \\
\hline $\begin{array}{l}\text { LLW (x) OR to HS } \\
\text { Oak Ridge Res }\end{array}$ & $\begin{array}{ll}\text { to Hanford } & \text { Site } \\
\text { Truck } & 6.83 \mathrm{E}-02 \\
\text { Rail } & 1.37 \mathrm{E}-02\end{array}$ & $\begin{array}{l}3.65 \\
1.08\end{array}$ & $\begin{array}{l}8.9 \\
2.5\end{array}$ & $\begin{array}{l}\varepsilon-02 \\
E-02\end{array}$ & $\begin{array}{l}-02 \\
-02\end{array}$ \\
\hline M & $\begin{array}{l}\text { ford Site } \\
8.30 \mathrm{E}-02 \\
1.50 \mathrm{E}-02\end{array}$ & & $\begin{array}{l}1.3 \\
5.4\end{array}$ & $\begin{array}{l}-02 \\
-02\end{array}$ & $\begin{array}{l}5 E-02 \\
E-02\end{array}$ \\
\hline TTW $(x)$ TA to หS & $\begin{array}{lr}\text { to Hanford Site } \\
\text { Truck } & 4.62 \mathrm{E}-02 \\
\text { Rail } & 1.03 \mathrm{E}-02\end{array}$ & $\begin{array}{l}1.9 \\
5.0\end{array}$ & $\begin{array}{l}5.7 \\
1.4\end{array}$ & $\begin{array}{l}-02 \\
-03\end{array}$ & $\begin{array}{l}5.64 \mathrm{E}-02 \\
1.41 \mathrm{E}-02\end{array}$ \\
\hline $\begin{array}{l}\text { LLW }(x) \text { LA to HS } \\
\text { Los Alamos } \mathrm{NL}\end{array}$ & $\begin{array}{lr}\text { to Hanford Site } \\
\text { Truck } & 4.24 \mathrm{E}-02 \\
\text { Rail } & 1.01 \mathrm{E}-02\end{array}$ & $\begin{array}{l}2.16 \mathrm{E}-03 \\
5.60 \mathrm{E}-03\end{array}$ & $\begin{array}{l}5.65 \\
1.54\end{array}$ & $\begin{array}{l}E-02 \\
E-03\end{array}$ & $\begin{array}{l}5.15 \mathrm{E}-02 \\
1.45 \mathrm{E}-02\end{array}$ \\
\hline $\begin{array}{l}\text { LLW }(x) \text { LB to HS } \\
\text { Lawrence Berkeley }\end{array}$ & $\begin{array}{lr}\text { to Hanford Site } \\
\text { Truck } & 2.76 \mathrm{E}-02 \\
\text { Rail } & 6.99 \mathrm{E}-03\end{array}$ & $\begin{array}{l}3.02 \\
1.3\end{array}$ & $\begin{array}{l}5.1 \\
2.0\end{array}$ & $\begin{array}{l}-02 \\
-03\end{array}$ & $\begin{array}{l}3.27 E-02 \\
2.05 E-02\end{array}$ \\
\hline (x) & $\begin{array}{lr}\text { to Hanford. Site } \\
\text { Truck } & 2.82 \mathrm{E}-02 \\
\text { Rail } & 6.94 \mathrm{E}-03\end{array}$ & $\begin{array}{l}2.8 \\
1.3\end{array}$ & $\begin{array}{l}4.9 \\
2.0\end{array}$ & $\begin{array}{l}0 E-02 \\
8 E-03\end{array}$ & $\begin{array}{l}3.28 \mathrm{E}-02 \\
2.05 \mathrm{E}-02\end{array}$ \\
\hline Mound Plant & $\begin{array}{ll}\text { to Hanford Site } \\
\text { Truck } & 6.29 \mathrm{E}-02 \\
\text { Rail } & 1.32 \mathrm{E}-02\end{array}$ & $\begin{array}{l}3.13 \mathrm{E}-03 \\
1.47 \mathrm{E}-02\end{array}$ & $\begin{array}{l}8.05 \\
3.13\end{array}$ & $\begin{array}{l}6.41 E-02 \\
1.11 E-02\end{array}$ & $\begin{array}{l}7.53 E-02 \\
2.61 E-02\end{array}$ \\
\hline $\begin{array}{l}\text { LLW (x) MP to Po } \\
\text { Mound Plant }\end{array}$ & $\begin{array}{cr}\text { to Portsmouth GDP } \\
\text { Truck } & 5.83 \mathrm{E}-03 \\
\text { Rail } & 3.53 \mathrm{E}-03\end{array}$ & $\begin{array}{l}8.71 E-04 \\
3.82 E-03\end{array}$ & $\begin{array}{l}1.18 \mathrm{E}-03 \\
5.67 \mathrm{E}-05\end{array}$ & $\begin{array}{l}4.26 \mathrm{E}-03 \\
3.96 \mathrm{E}-03\end{array}$ & $\begin{array}{l}6.31 E-03 \\
7.84 E-03\end{array}$ \\
\hline
\end{tabular}


LLW(x) OI TO HS ORISE

LLW(X) OI tO OR ORISE

LLW(x) OR to HS

Oak Ridge Reserv. to Hanford Site

LLG(x) PP to HS Pantex Plant

LLW(x) PP to LA Pantex Plant

LLW(x) PO to HS Portsmouth GDP

LLW (x) PR to HS Princeton PPL

LLW(x) PR to PO Princeton PPL

LLW(x) PA to HS Paducah GDP

LLW(x) PA to OR Paducah GDP

LLW(x) PI to SR Pinellas plane

LLW(x) RF to HS Rocky Flats Plant

LLW(x) RM to HS Reactive Metals

$L L W(x)$ RM to Po Reactive Metals

LLW(x) ST to HS SLAC

LLW(x) SA to HS SNL - Albuquerque

LLW(x) SA to LA SNL - Albuquerque

LLW(x) SL to HS SNL - Livermore

LLW(x) SR to HS Savannah River

LLW(x) OR to HS Oak Ridge Reserv. to Hanford Site

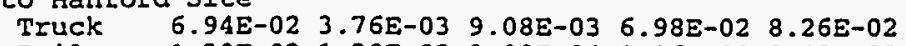
Rail $1.39 \mathrm{E}-02 \quad 1.30 \mathrm{E}-02 \quad 2.98 \mathrm{E}-04$ 1.16E-02 2.49E-02

to Oak Ridge Reserv

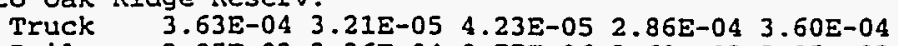
Rail 3.05E-03 3.36E-04 8.77E-06 3.60E-03 3.95E-03

Truck $\quad 6.83 \mathrm{E}-02 \quad 3.65 \mathrm{E}-03 \quad 8.95 \mathrm{E}-03 \quad 6.90 \mathrm{E}-02 \quad 8.16 \mathrm{E}-02$

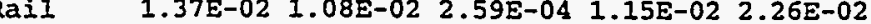

to Hanford site

$\begin{array}{llllll}\text { Truck } 5.10 E-02 & 2.68 E-03 & 7.01 E-03 & 5.27 E-02 & 6.24 E-02\end{array}$

Rail $9.92 \mathrm{E}-03 \quad 5.71 \mathrm{E}-03 \quad 1.56 \mathrm{E}-04 \quad 8.67 \mathrm{E}-03 \quad 1.45 \mathrm{E}-02$

to Los Alamos NL

$\begin{array}{llllll}\text { Truck } & 1.11 \mathrm{E}-02 & 7.76 \mathrm{E}-04 & 1.77 \mathrm{E}-03 & 1.13 \mathrm{E}-02 & 1.38 \mathrm{E}-02\end{array}$

Rail $4.89 \mathrm{E}-03 \quad 2.18 \mathrm{E}-03 \quad 5.29 \mathrm{E}-05 \quad 4.96 \mathrm{E}-03 \quad 7.19 \mathrm{E}-03$

to Hanford Site

$\begin{array}{llllll}\text { Truck } & 6.63 \mathrm{E}-02 & 3.38 \mathrm{E}-03 & 8.48 \mathrm{E}-03 & 6.72 \mathrm{E}-02 & 7.91 \mathrm{E}-02\end{array}$

Rail 1.34E-02 1.89E-02 $3.46 \mathrm{E}-04 \quad 1.12 \mathrm{E}-02 \quad 3.04 \mathrm{E}-02$

to Hanford Site

Truck $\quad 8.51 \mathrm{E}-02 \quad 6.65 \mathrm{E}-03 \quad 1.28 \mathrm{E}-02 \quad 8.02 \mathrm{E}-02 \quad 9.97 \mathrm{E}-02$

Rail 1.53E-02 3.63E-02 6.03E-04 1.27E-02 4.96E-02

to Portsmouth GDP

$\begin{array}{llllll}\text { Truck } & 2.28 \mathrm{E}-02 & 3.55 \mathrm{E}-03 & 4.81 \mathrm{E}-03 & 1.65 \mathrm{E}-02 & 2.48 \mathrm{E}-02\end{array}$ Rail $6.38 \mathrm{E}-03$ 2.00E-02 $2.85 \mathrm{E}-04$ 6.06E-03 $2.64 \mathrm{E}-02$

to Hanford Site

$\begin{array}{llllll}\text { Truck } & 6.00 \mathrm{E}-02 & 3.22 \mathrm{E}-03 & 8.02 \mathrm{E}-03 & 6.11 \mathrm{E}-02 & 7.23 \mathrm{E}-02\end{array}$ Rail 1.22E-02 9.43E-03 2.28E-04 1.04E-02 2.01E-02

to Oak Ridge Reserv.

$\begin{array}{llllll}\text { Truck } & 8.88 \mathrm{E}-03 & 4.70 \mathrm{E}-04 & 9.98 \mathrm{E}-04 & 8.51 \mathrm{E}-03 & 9.98 \mathrm{E}-03\end{array}$ Rail $5.08 \mathrm{E}-03 \quad 2.75 \mathrm{E}-03$ 5.97E-05 5.10E-03 $7.91 \mathrm{E}-03$

to Savannah River

$\begin{array}{llllll}\text { Truck } 2.02 E-02 & 1.94 E-03 & 3.10 E-03 & 1.74 E-02 & 2.24 E-02\end{array}$ Rail 4.90E-03 5.13E-03 1.02E-04.4.97E-03 1.02E-02

to Hanford Site

Truck 2.99E-02 1.13E-03 3.61E-03 $3.17 \mathrm{E}-02 \quad 3.64 \mathrm{E}-02$

Rail $8.24 \mathrm{E}-03 \quad 4.91 \mathrm{E}-03 \quad 1.16 \mathrm{E}-04 \quad 7.43 \mathrm{E}-03 \quad 1.25 \mathrm{E}-02$

to Hanford Site

$\begin{array}{llllll}\text { Truck } & 6.76 \mathrm{E}-02 & 4.22 \mathrm{E}-03 & 9.51 \mathrm{E}-03 & 6.71 \mathrm{E}-02 & 8.09 \mathrm{E}-02\end{array}$

Rail $\quad 1.33 \mathrm{E}-02 \quad 2.35 \mathrm{E}-02 \quad 4.01 \mathrm{E}-04 \quad 1.12 \mathrm{E}-02 \quad 3.51 \mathrm{E}-02$

to Portsmouth GDP

Truck $1.00 \mathrm{E}-02 \quad 1.18 \mathrm{E}-03 \quad 1.62 \mathrm{E}-03 \quad 7.73 \mathrm{E}-03 \quad 1.05 \mathrm{E}-02$

Rail $4.05 E-03 \quad 7.58 E-03 \quad 1.11 E-04 \quad 4.34 E-03 \quad 1.20 E-02$

to Hanford Site

$\begin{array}{lllllll}\text { Truck } & 2.98 \mathrm{E}-02 & 3.70 \mathrm{E}-03 & 6.08 \mathrm{E}-03 & 2.56 \mathrm{E}-02 & 3.54 \mathrm{E}-02\end{array}$

$\begin{array}{llllll}\text { Rail } 7.20 \mathrm{E}-03 & 1.75 \mathrm{E}-02 & 2.40 \mathrm{E}-04 & 6.67 \mathrm{E}-03 & 2.44 \mathrm{E}-02\end{array}$

to Hanford Site

$\begin{array}{llllll}\text { Truck } & 4.31 \mathrm{E}-02 & 2.27 \mathrm{E}-03 & 5.84 \mathrm{E}-03 & 4.43 \mathrm{E}-02 & 5.24 \mathrm{E}-02\end{array}$

Rail $\quad 1.04 \mathrm{E}-02 \quad 6.90 \mathrm{E}-03 \quad 1.72 \mathrm{E}-04 \quad 9.00 \mathrm{E}-03 \quad 1.61 \mathrm{E}-02$

to Los Alamos NL

$\begin{array}{lllllll}\text { Truck } & 3.28 \mathrm{E}-03 & 3.70 \mathrm{E}-04 & 6.13 \mathrm{E}-04 & 2.86 \mathrm{E}-03 & 3.84 \mathrm{E}-03\end{array}$

Rail $\quad 3.31 \mathrm{E}-03 \quad 1.55 \mathrm{E}-03 \quad 2.36 \mathrm{E}-05 \quad 3.80 \mathrm{E}-03 \quad 5.37 \mathrm{E}-03$

to Fanford Site

$\begin{array}{llllllllll}\text { Truck } & 2.82 \mathrm{E}-02 & 2.88 \mathrm{E}-03 & 4.92 \mathrm{E}-03 & 2.50 \mathrm{E}-02 & 3.28 \mathrm{E}-02\end{array}$

Rail $6.94 \mathrm{E}-03 \quad 1.38 \mathrm{E}-02 \quad 2.00 \mathrm{E}-04 \quad 6.48 \mathrm{E}-03 \quad 2.05 \mathrm{E}-02$

to Hanford site

$\begin{array}{llllll}\text { Truck } & 7.86 \mathrm{E}-02 & 5.10 \mathrm{E}-03 & 1.08 \mathrm{E}-02 & 7.64 \mathrm{E}-02 & 9.23 \mathrm{E}-02\end{array}$

Rail 1.52E-02 1.85E-02 4.05E-04 1.26E-02 3.15E-02

to Hanford Site

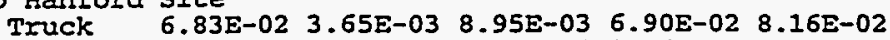

Rail 1.37E-02 $1.08 E-02 \quad 2.59 E-04 \quad 1.15 E-02 \quad 2.26 E-02$ 
Table 4. Accident Dose Risk per Shipment (person-rem/shipment)

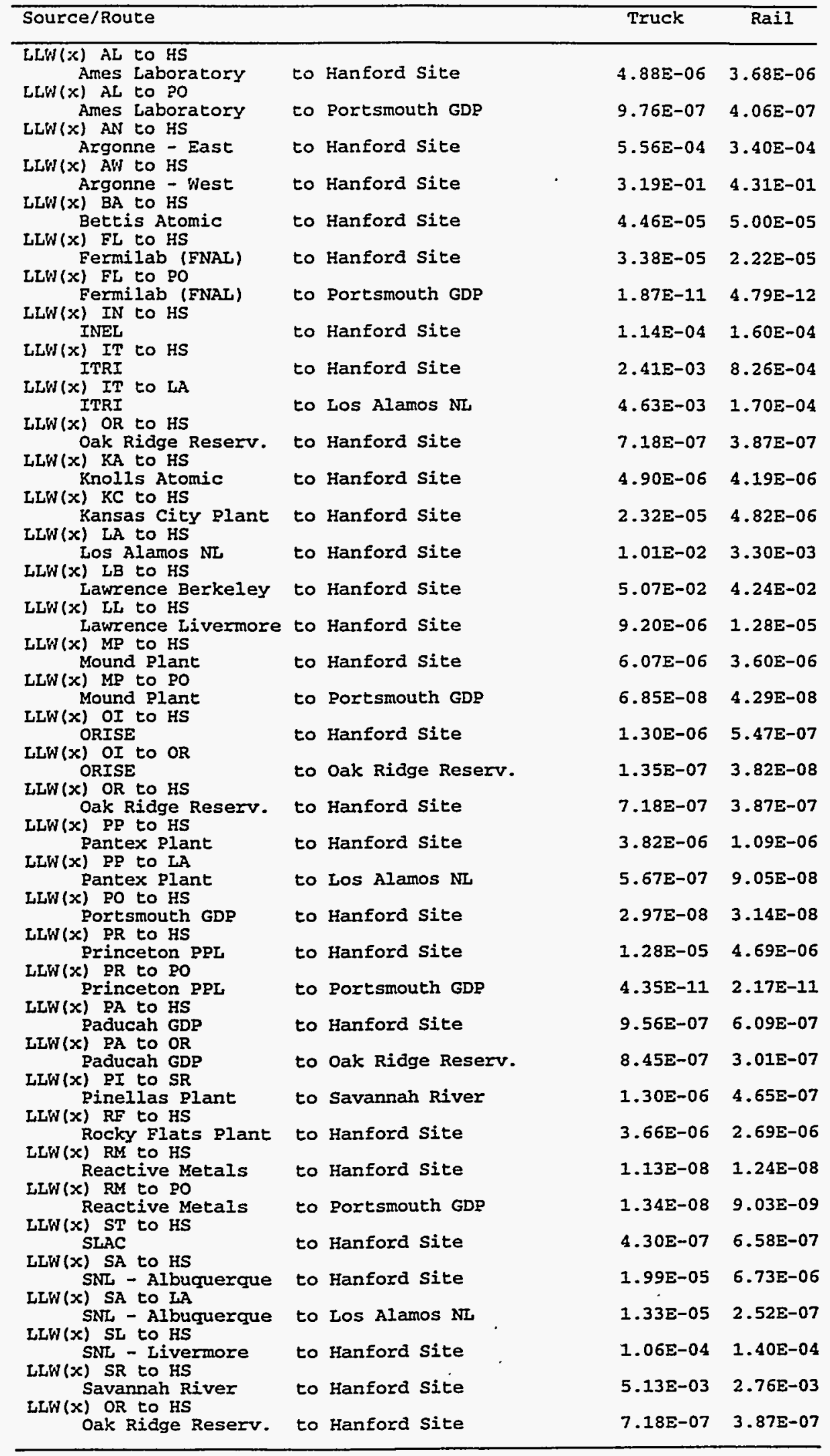


Table 5. Nonradiological Risk Factors per Shipment (fatalities/shipment)

\begin{tabular}{|c|c|c|c|}
\hline Mode & Route & Emission & Accident \\
\hline $\begin{array}{l}\text { Truck } \\
\text { Ames Laboratory } \\
\text { Ames Laboracory } \\
\text { Argonne - East } \\
\text { Argonne - West } \\
\text { Bettis Atomic } \\
\text { Fermilab (FNAL) } \\
\text { Fermilab (FNAL) } \\
\text { INEL } \\
\text { ITRI } \\
\text { ITRI } \\
\text { Oak Ridge Reserv. } \\
\text { Knolls Atomic } \\
\text { Kansas City Plant } \\
\text { Los Alamos NL } \\
\text { Lawrence Berkeley } \\
\text { Lawrence Livermore } \\
\text { Mound Plant } \\
\text { Mound Plant } \\
\text { ORISE } \\
\text { ORISE } \\
\text { Pantex Plant } \\
\text { Pantex Plant } \\
\text { Portsmouth GDP } \\
\text { Princeton PPL } \\
\text { Princeton PPL } \\
\text { Paducah GDP } \\
\text { Paducah GDP } \\
\text { Pinellas Plant } \\
\text { Rocky Flats Plant } \\
\text { Reactive Metals } \\
\text { Reactive Metals } \\
\text { SLAC } \\
\text { SNL - Albuquerque } \\
\text { SNL - Albuquerque } \\
\text { SNL - Livermore } \\
\text { Savannah River }\end{array}$ & $\begin{array}{l}\text { to Hanford Site } \\
\text { to Portsmouth GDP } \\
\text { to Hanford Site } \\
\text { to Hanford Site } \\
\text { to Hanford Site } \\
\text { to Hanford Site } \\
\text { to Portsmouth GDP } \\
\text { to Hanford Site } \\
\text { to Hanford Site } \\
\text { to Los Alamos NL } \\
\text { to Hanford Site } \\
\text { to Hanford Site } \\
\text { to Hanford Site } \\
\text { to Hanford Site } \\
\text { to Hanford Site } \\
\text { to Hanford Site } \\
\text { to Hanford Site } \\
\text { to Portsmouth GDP } \\
\text { to Hanford Site } \\
\text { to Oak Ridge Reserv. } \\
\text { to Hanford Site } \\
\text { to Los Alamos ND } \\
\text { to Hanford Site } \\
\text { to Hanford Site } \\
\text { to Portsmouth GDP } \\
\text { to Hanford Site } \\
\text { to Oak Ridge Reserv. } \\
\text { to Savannah River } \\
\text { to Hanford Site } \\
\text { to Hanford Site } \\
\text { to Portsmouth GDP } \\
\text { to Hanford Site } \\
\text { to Hanford Site } \\
\text { to Los Alamos NL } \\
\text { to Hanford Site } \\
\text { to Hanford Site }\end{array}$ & $\begin{array}{l}4.31 \mathrm{E}-06 \\
4.02 \mathrm{E}-06 \\
4.41 \mathrm{E}-06 \\
2.19 \mathrm{E}-06 \\
1.14 \mathrm{E}-05 \\
4.86 \mathrm{E}-06 \\
8.11 \mathrm{E}-06 \\
2.19 \mathrm{E}-06 \\
7.44 \mathrm{E}-06 \\
1.51 \mathrm{E}-06 \\
9.50 \mathrm{E}-06 \\
1.59 \mathrm{E}-05 \\
5.18 \mathrm{E}-06 \\
6.66 \mathrm{E}-06 \\
1.16 \mathrm{E}-05 \\
9.85 \mathrm{E}-06 \\
7.89 \mathrm{E}-06 \\
2.70 \mathrm{E}-06 \\
9.50 \mathrm{E}-06 \\
0.00 \mathrm{E}+00 \\
9.21 \mathrm{E}-06 \\
3.28 \mathrm{E}-06 \\
8.11 \mathrm{E}-06 \\
1.97 \mathrm{E}-05 \\
1.16 \mathrm{E}-05 \\
9.30 \mathrm{E}-06 \\
1.93 \mathrm{E}-07 \\
4.47 \mathrm{E}-06 \\
2.83 \mathrm{E}-06 \\
1.27 \mathrm{E}-05 \\
2.45 \mathrm{E}-06 \\
1.50 \mathrm{E}-05 \\
7.24 \mathrm{E}-06 \\
1.35 \mathrm{E}-06 \\
9.85 \mathrm{E}-06 \\
1.31 \mathrm{E}-05\end{array}$ & $\begin{array}{l}8.93 \mathrm{E}-05 \\
4.11 \mathrm{E}-05 \\
1.06 \mathrm{E}-04 \\
3.07 \mathrm{E}-05 \\
1.36 \mathrm{E}-04 \\
1.03 \mathrm{E}-04 \\
3.01 \mathrm{E}-05 \\
3.07 \mathrm{E}-05 \\
1.05 \mathrm{E}-04 \\
1.28 \mathrm{E}-05 \\
1.61 \mathrm{E}-04 \\
1.51 \mathrm{E}-04 \\
1.12 \mathrm{E}-04 \\
1.02 \mathrm{E}-04 \\
4.91 \mathrm{E}-05 \\
5.03 \mathrm{E}-05 \\
1.23 \mathrm{E}-04 \\
6.66 \mathrm{E}-06 \\
1.65 \mathrm{E}-04 \\
1.07 \mathrm{E}-06 \\
1.25 \mathrm{E}-04 \\
3.21 \mathrm{E}-05 \\
1.28 \mathrm{E}-04 \\
1.63 \mathrm{E}-04 \\
3.99 \mathrm{E}-05 \\
1.41 \mathrm{E}-04 \\
2.14 \mathrm{E}-05 \\
4.70 \mathrm{E}-05 \\
6.69 \mathrm{E}-05 \\
1.28 \mathrm{E}-04 \\
1.21 \mathrm{E}-05 \\
5.14 \mathrm{E}-05 \\
1.05 \mathrm{E}-04 \\
1.20 \mathrm{E}-05 \\
5.03 \mathrm{E}-05 \\
1.81 \mathrm{E}-04\end{array}$ \\
\hline $\begin{array}{l}\text { Ames Laboratory } \\
\text { Ames Laboratory } \\
\text { Argonne - East } \\
\text { Argonne - West } \\
\text { Bettis Atomic } \\
\text { Fermilab (FNAL) } \\
\text { Fermilab (FNAI) } \\
\text { INEL } \\
\text { ITRI } \\
\text { ITRI } \\
\text { Oak Ridge Reserv. } \\
\text { Rnolls Atomic } \\
\text { Ransas City Plant } \\
\text { Ios Alamos NL } \\
\text { Lawrence Berkeley } \\
\text { Iawrence Livermore } \\
\text { Mound Plant } \\
\text { Mound Plant } \\
\text { ORISE } \\
\text { ORISE } \\
\text { Pantex Plant } \\
\text { Pantex Plant } \\
\text { Portsmouth GDP } \\
\text { Princeton PPI } \\
\text { Princeton PPL } \\
\text { Paducah GDP } \\
\text { Paducah GDP } \\
\text { Pinellas Plant } \\
\text { Rocky Flats Plant } \\
\text { Reactive Metals } \\
\text { Reactive Metals } \\
\text { SLAC } \\
\text { SNL - Albuquerque } \\
\text { SNL - Albuguerque } \\
\text { SNL - Livermore } \\
\text { Savannah River }\end{array}$ & $\begin{array}{l}\text { to Hanford Site } \\
\text { to Portsmouth GDP } \\
\text { to Hanford Site } \\
\text { to Hanford Site } \\
\text { to Hanford Site } \\
\text { to Hanford Site } \\
\text { to Portsmouth GDP } \\
\text { to Hanford Site } \\
\text { to Hanford Site } \\
\text { to Los Alamos NI } \\
\text { to Hanford Site } \\
\text { to Hanford Site } \\
\text { to Hanford Site } \\
\text { to Hanford Site } \\
\text { to Hanford Site } \\
\text { to Hanford Site } \\
\text { to Hanford Site } \\
\text { to Portsmouth GDP } \\
\text { to Hanford Site } \\
\text { to Oak Ridge Reserv. } \\
\text { to Hanford Site } \\
\text { to Los Alamos NL } \\
\text { to Hanford Site } \\
\text { to Hanford Site } \\
\text { to Portsmouth GDP } \\
\text { to Hanford Site } \\
\text { to Oak Ridge Reserv. } \\
\text { to Savannah River } \\
\text { to Hanford Site } \\
\text { to Hanford Site } \\
\text { to Portsmouth GDP } \\
\text { to Hanford Site } \\
\text { to Hanford Site } \\
\text { to Los Alamos NL } \\
\text { to Hanford Site } \\
\text { to Hanford Site }\end{array}$ & $\begin{array}{l}5.40 \mathrm{E}-06 \\
2.34 \mathrm{E}-05 \\
6.15 \mathrm{E}-06 \\
3.98 \mathrm{E}-06 \\
4.07 \mathrm{E}-05 \\
1.18 \mathrm{E}-05 \\
1.80 \mathrm{E}-05 \\
3.98 \mathrm{E}-06 \\
9.04 \mathrm{E}-06 \\
2.47 \mathrm{E}-06 \\
1.45 \mathrm{E}-05 \\
4.72 \mathrm{E}-05 \\
6.57 \mathrm{E}-06 \\
6.82 \mathrm{E}-06 \\
2.33 \mathrm{E}-05 \\
2.36 \mathrm{E}-05 \\
1.94 \mathrm{E}-05 \\
5.73 \mathrm{E}-06 \\
1.67 \mathrm{E}-05 \\
1.26 \mathrm{E}-07 \\
6.78 \mathrm{E}-06 \\
2.59 \mathrm{E}-06 \\
2.88 \mathrm{E}-05 \\
5.38 \mathrm{E}-05 \\
3.18 \mathrm{E}-05 \\
1.23 \mathrm{E}-05 \\
3.81 \mathrm{E}-06 \\
5.69 \mathrm{E}-06 \\
7.36 \mathrm{E}-06 \\
3.65 \mathrm{E}-05 \\
1.14 \mathrm{E}-05 \\
3.07 \mathrm{E}-05 \\
9.04 \mathrm{E}-06 \\
2.47 \mathrm{E}-06 \\
2.36 \mathrm{E}-05 \\
2.21 \mathrm{E}-05\end{array}$ & $\begin{array}{l}3.74 E-06 \\
1.52 \mathrm{E}-06 \\
4.60 \mathrm{E}-06 \\
1.38 \mathrm{E}-06 \\
5.46 \mathrm{E}-06 \\
4.12 \mathrm{E}-06 \\
9.43 \mathrm{E}-07 \\
1.38 \mathrm{E}-06 \\
3.75 \mathrm{E}-06 \\
2.18 \mathrm{E}-07 \\
5.44 \mathrm{E}-06 \\
5.10 \mathrm{E}-06 \\
3.73 \mathrm{E}-06 \\
3.61 \mathrm{E}-06 \\
2.06 \mathrm{E}-06 \\
2.04 \mathrm{E}-06 \\
5.17 \mathrm{E}-06 \\
3.27 \mathrm{E}-07 \\
5.53 \mathrm{E}-06 \\
8.26 \mathrm{E}-08 \\
3.53 \mathrm{E}-06 \\
1.01 \mathrm{E}-06 \\
5.26 \mathrm{E}-06 \\
6.24 \mathrm{E}-06 \\
1.75 \mathrm{E}-06 \\
4.70 \mathrm{E}-06 \\
1.10 \mathrm{E}-06 \\
1.01 \mathrm{E}-06 \\
2.69 \mathrm{E}-06 \\
5.24 \mathrm{E}-06 \\
5.84 \mathrm{E}-07 \\
2.17 \mathrm{E}-06 \\
3.75 \mathrm{E}-06 \\
2.18 \mathrm{E}-07 \\
2.04 \mathrm{E}-06 \\
6.18 \mathrm{E}-06\end{array}$ \\
\hline
\end{tabular}


Table 6. Annual Incident-Free Dose for the Shipping Campaign (person-rem/yr)

\begin{tabular}{|c|c|c|c|c|c|c|}
\hline \multirow[b]{2}{*}{ Route } & & & \multicolumn{2}{|c|}{ Truck } & \multicolumn{2}{|c|}{ Rail } \\
\hline & & & Crew & Public & Crew & Public \\
\hline $\begin{array}{l}\text { Ames Laboratory } \\
\text { Ames Laboratory } \\
\text { Argonne - East } \\
\text { Argonne - West } \\
\text { Bettis Atomic } \\
\text { Fermilab (FNAL) } \\
\text { Fermilab (FNAL) } \\
\text { INEL } \\
\text { ITRI } \\
\text { ITRI } \\
\text { Oak Riage Reserv. } \\
\text { Rnolis Atomic } \\
\text { Kansas City Plant } \\
\text { Los Alamos NL } \\
\text { Lawrence Berkeley } \\
\text { Lawrence Livermore } \\
\text { Mound Plant } \\
\text { Mound Plant } \\
\text { ORISE } \\
\text { ORISE } \\
\text { Pantex Plant } \\
\text { Pantex Plant } \\
\text { Portsmouth GDP } \\
\text { Princeton PPL } \\
\text { Princeton PPL } \\
\text { Paducah GDP } \\
\text { Paducah GDP } \\
\text { Pinellas Plant } \\
\text { Rocky Flats Plant } \\
\text { Reactive Metals } \\
\text { Reactive Metals } \\
\text { SLAC } \\
\text { SNL - Albuquerque } \\
\text { SNL - Albuquerque } \\
\text { SNL - Livermore } \\
\text { Savannah River }\end{array}$ & $\begin{array}{l}\text { to } \\
\text { to } \\
\text { to } \\
\text { to } \\
\text { to } \\
\text { to } \\
\text { to } \\
\text { to } \\
\text { to } \\
\text { to } \\
\text { to } \\
\text { to } \\
\text { to } \\
\text { to } \\
\text { to } \\
\text { to } \\
\text { to } \\
\text { to } \\
\text { to } \\
\text { to } \\
\text { to } \\
\text { to } \\
\text { to } \\
\text { to } \\
\text { to } \\
\text { to } \\
\text { to } \\
\text { to } \\
\text { to } \\
\text { to } \\
\text { to } \\
\text { to } \\
\text { to } \\
\text { to }\end{array}$ & $\begin{array}{l}\text { Hanford Site } \\
\text { Portsmouth GDP } \\
\text { Hanford Site } \\
\text { Hanford Site } \\
\text { Hanford Site } \\
\text { Hanford Site } \\
\text { Portsmouth GDP } \\
\text { Hanford Site } \\
\text { Hanford Site } \\
\text { Los Alamos NL } \\
\text { Hanford Site } \\
\text { Hanford Site } \\
\text { Hanford Site } \\
\text { Hanford Site } \\
\text { Hanford Site } \\
\text { Hanford Site } \\
\text { Hanford Site } \\
\text { Portsmouth GDP } \\
\text { Hanford Site } \\
\text { Oak Ridge Reserv. } \\
\text { Hanford Site } \\
\text { Los Alamos NL } \\
\text { Hanford Site } \\
\text { Hanford Site } \\
\text { Portsmouth GDP } \\
\text { Hanford Site } \\
\text { Oak Ridge Reserv. } \\
\text { Savannah River } \\
\text { Hanford Site } \\
\text { Hanford Site } \\
\text { Portsmouth GDP } \\
\text { Hanford Site } \\
\text { Hanford Site } \\
\text { Los Alamos NL } \\
\text { Hanford Site } \\
\text { Hanford Site }\end{array}$ & $\begin{array}{l}8.93 \mathrm{E}-02 \\
2.33 \mathrm{E}-02 \\
5.55 \mathrm{E}+00 \\
1.60 \mathrm{E}-01 \\
4.11 \mathrm{E}+00 \\
1.04 \mathrm{E}-01 \\
1.68 \mathrm{E}-02 \\
1.31 \mathrm{E}+01 \\
3.91 \mathrm{E}-01 \\
7.08 \mathrm{E}-03 \\
4.14 \mathrm{E}+02 \\
2.32 \mathrm{E}+00 \\
4.62 \mathrm{E}-02 \\
8.33 \mathrm{E}+01 \\
8.27 \mathrm{E}-02 \\
1.58 \mathrm{E}+00 \\
3.12 \mathrm{E}+01 \\
7.00 \mathrm{E}-02 \\
2.78 \mathrm{E}-01 \\
3.63 \mathrm{E}-04 \\
1.28 \mathrm{E}+00 \\
1.51 \mathrm{E}+01 \\
1.96 \mathrm{E}+02 \\
8.51 \mathrm{E}-02 \\
2.28 \mathrm{E}-02 \\
3.47 \mathrm{E}+01 \\
5.06 \mathrm{E}-01 \\
4.04 \mathrm{E}-02 \\
1.00 \mathrm{E}+01 \\
5.04 \mathrm{E}+01 \\
7.02 \mathrm{E}-02 \\
1.16 \mathrm{E}+00 \\
7.33 \mathrm{E}-01 \\
3.28 \mathrm{E}-03 \\
1.41 \mathrm{E}-01 \\
5.30 \mathrm{E}+02\end{array}$ & $\begin{array}{l}1.09 \mathrm{E}-01 \\
2.63 \mathrm{E}-02 \\
6.74 \mathrm{E}+00 \\
1.95 \mathrm{E}-01 \\
4.86 \mathrm{E}+00 \\
1.27 \mathrm{E}-01 \\
1.87 \mathrm{E}-02 \\
1.60 \mathrm{E}+01 \\
4.75 \mathrm{E}-01 \\
8.36 \mathrm{E}-03 \\
4.95 \mathrm{E}+02 \\
2.70 \mathrm{E}+00 \\
5.64 \mathrm{E}-02 \\
1.01 \mathrm{E}+02 \\
9.80 \mathrm{E}-02 \\
1.84 \mathrm{E}+00 \\
3.74 \mathrm{E}+01 \\
7.58 \mathrm{E}-02 \\
3.31 \mathrm{E}-01 \\
3.60 \mathrm{E}-04 \\
1.56 \mathrm{E}+00 \\
1.87 \mathrm{E}+01 \\
2.33 \mathrm{E}+02 \\
9.97 \mathrm{E}-02 \\
2.48 \mathrm{E}-02 \\
4.18 \mathrm{E}+01 \\
5.69 \mathrm{E}-01 \\
4.48 \mathrm{E}-02 \\
1.22 \mathrm{E}+01 \\
6.02 \mathrm{E}+01 \\
7.37 \mathrm{E}-02 \\
1.38 \mathrm{E}+00 \\
8.92 \mathrm{E}-01 \\
3.84 \mathrm{E}-03 \\
1.64 \mathrm{E}-01 \\
6.23 \mathrm{E}+02\end{array}$ & $\begin{array}{l}1.03 \mathrm{E}-02 \\
5.91 \mathrm{E}-03 \\
4.70 \mathrm{E}-01 \\
2.25 \mathrm{E}-02 \\
2.89 \mathrm{E}-01 \\
1.11 \mathrm{E}-02 \\
4.76 \mathrm{E}-03 \\
1.77 \mathrm{E}+00 \\
3.11 \mathrm{E}-02 \\
3.31 \mathrm{E}-03 \\
3.33 \mathrm{E}+01 \\
1.65 \mathrm{E}-01 \\
1.03 \mathrm{E}-02 \\
7.70 \mathrm{E}+00 \\
1.40 \mathrm{E}-02 \\
1.46 \mathrm{E}-01 \\
2.45 \mathrm{E}+00 \\
1.77 \mathrm{E}-02 \\
2.78 \mathrm{E}-02 \\
3.05 \mathrm{E}-03 \\
9.92 \mathrm{E}-02 \\
2.58 \mathrm{E}+00 \\
1.48 \mathrm{E}+01 \\
1.53 \mathrm{E}-02 \\
6.38 \mathrm{E}-03 \\
2.63 \mathrm{E}+00 \\
1.07 \mathrm{E}-01 \\
4.90 \mathrm{E}-03 \\
1.01 \mathrm{E}+00 \\
3.77 \mathrm{E}+00 \\
1.21 \mathrm{E}-02 \\
1.08 \mathrm{E}-01 \\
7.25 \mathrm{E}-02 \\
3.31 \mathrm{E}-03 \\
1.39 \mathrm{E}-02 \\
3.83 \mathrm{E}+01\end{array}$ & $\begin{array}{l}1.38 \mathrm{E}-02 \\
2.01 \mathrm{E}-02 \\
6.31 \mathrm{E}-01 \\
3.36 \mathrm{E}-02 \\
8.13 \mathrm{E}-01 \\
1.88 \mathrm{E}-02 \\
1.58 \mathrm{E}-02 \\
2.65 \mathrm{E}+00 \\
4.82 \mathrm{E}-02 \\
5.37 \mathrm{E}-03 \\
5.47 \mathrm{E}+01 \\
4.93 \mathrm{E}-01 \\
1.41 \mathrm{E}-02 \\
1.11 \mathrm{E}+01 \\
4.10 \mathrm{E}-02 \\
4.30 \mathrm{E}-01 \\
4.86 \mathrm{E}+00 \\
3.92 \mathrm{E}-02 \\
4.98 \mathrm{E}-02 \\
3.95 \mathrm{E}-03 \\
1.45 \mathrm{E}-01 \\
3.80 \mathrm{E}+00 \\
3.37 \mathrm{E}+01 \\
4.96 \mathrm{E}-02 \\
2.64 \mathrm{E}-02 \\
4.31 \mathrm{E}+00 \\
1.66 \mathrm{E}-01 \\
1.02 \mathrm{E}-02 \\
1.53 \mathrm{E}+00 \\
9.93 \mathrm{E}+00 \\
3.61 \mathrm{E}-02 \\
3.66 \mathrm{E}-01 \\
1.12 \mathrm{E}-01 \\
5.37 \mathrm{E}-03 \\
4.10 \mathrm{E}-02 \\
7.94 \mathrm{E}+01\end{array}$ \\
\hline
\end{tabular}


Table 7. Annual Accident Dose Risk for the Shipping Campaign (person-rem/yr)

\begin{tabular}{|c|c|c|c|}
\hline Route & & Truck & Rail \\
\hline $\begin{array}{l}\text { Ames Laboratory } \\
\text { Ames Laboratory } \\
\text { Argonne - East } \\
\text { Argonne - West } \\
\text { Bettis Atomic } \\
\text { Fermilab (FNAL) } \\
\text { Fermilab (FNAL) } \\
\text { INEL } \\
\text { ITRI } \\
\text { ITRI } \\
\text { Oak Ridge Reserv. } \\
\text { Knolls Atomic } \\
\text { Kansas City Plant } \\
\text { Los Alamos NL } \\
\text { Lawrence Berkeley } \\
\text { Lawrence Livermore } \\
\text { Mound Plant } \\
\text { Mound Plant } \\
\text { ORISE } \\
\text { ORISE } \\
\text { Pantex PIant } \\
\text { Pantex Plant } \\
\text { Portsmouth GDP } \\
\text { Princeton PPL } \\
\text { Princeton PPL } \\
\text { Paducah GDP } \\
\text { Paducah GDP } \\
\text { Pinellas Plant } \\
\text { Rocky Flats Plant } \\
\text { Reactive Metals } \\
\text { Reactive Metals } \\
\text { SLAC } \\
\text { SNL - Albuquerque } \\
\text { SNL - Albuquerque } \\
\text { SNL - Iivermore } \\
\text { Savannah River } \\
\text { And }\end{array}$ & $\begin{array}{l}\text { to Hanford Site } \\
\text { to Portsmouth GDP } \\
\text { to Hanford Site } \\
\text { to Hanford Site } \\
\text { to Hanford Site } \\
\text { to Hanford Site } \\
\text { to Portsmouth GDP } \\
\text { to Hanford Site } \\
\text { to Hanford Site } \\
\text { to Los Alamos NL } \\
\text { to Hanford Site } \\
\text { to Hanford Site } \\
\text { to Hanford Site } \\
\text { to Hanford Site } \\
\text { to Hanford Site } \\
\text { to Hanford Site } \\
\text { to Hanford Site } \\
\text { to Portsmouth GDP } \\
\text { to Hanford Site } \\
\text { to Oak Ridge Reserv. } \\
\text { to Hanford Site } \\
\text { to Los Alamos NL } \\
\text { to Hanford Site } \\
\text { to Hanford Site } \\
\text { to Portsmouth GDP } \\
\text { to Hanford Site } \\
\text { to Oak Ridge Reserv. } \\
\text { to Savannah River } \\
\text { to Hanford Site } \\
\text { to Hanford Site } \\
\text { to Portsmouth GDP } \\
\text { to Hanford Site } \\
\text { to Hanford Site } \\
\text { to Los Alamos NL } \\
\text { to Hanford Site } \\
\text { to Hanford Site }\end{array}$ & $\begin{array}{l}9.76 \mathrm{E}-06 \\
9.76 \mathrm{E}-07 \\
5.84 \mathrm{E}-02 \\
3.19 \mathrm{E}+00 \\
2.54 \mathrm{E}-03 \\
6.76 \mathrm{E}-05 \\
1.87 \mathrm{E}-11 \\
9.38 \mathrm{E}-02 \\
2.17 \mathrm{E}-02 \\
9.26 \mathrm{E}-03 \\
4.36 \mathrm{E}-03 \\
1.37 \mathrm{E}-04 \\
2.32 \mathrm{E}-05 \\
1.98 \mathrm{E}+01 \\
1.52 \mathrm{E}-01 \\
5.15 \mathrm{E}-04 \\
3.01 \mathrm{E}-03 \\
8.22 \mathrm{E}-07 \\
5.20 \mathrm{E}-06 \\
1.35 \mathrm{E}-07 \\
9.56 \mathrm{E}-05 \\
7.70 \mathrm{E}-04 \\
8.77 \mathrm{E}-05 \\
1.28 \mathrm{E}-05 \\
4.35 \mathrm{E}-11 \\
5.53 \mathrm{E}-04 \\
4.82 \mathrm{E}-05 \\
2.59 \mathrm{E}-06 \\
1.23 \mathrm{E}-03 \\
8.42 \mathrm{E}-06 \\
9.38 \mathrm{E}-08 \\
1.68 \mathrm{E}-05 \\
3.39 \mathrm{E}-04 \\
1.33 \mathrm{E}-05 \\
5.28 \mathrm{E}-04 \\
3.46 \mathrm{E}+01\end{array}$ & $\begin{array}{l}3.68 E-06 \\
4.06 E-07 \\
1.33 E-02 \\
1.72 E+00 \\
1.05 E-03 \\
2.22 E-05 \\
4.79 E-12 \\
5.04 E-02 \\
2.48 E-03 \\
1.70 E-04 \\
9.40 E-04 \\
4.61 E-05 \\
4.82 E-06 \\
2.52 E+00 \\
8.48 E-02 \\
2.70 E-04 \\
6.69 E-04 \\
2.15 E-07 \\
1.09 E-06 \\
3.82 E-08 \\
1.09 E-05 \\
4.78 E-05 \\
3.47 E-05 \\
4.69 E-06 \\
2.17 E-11 \\
1.31 E-04 \\
6.32 E-06 \\
4.65 E-07 \\
3.31 E-04 \\
3.50 E-06 \\
2.71 E-08 \\
9.87 E-06 \\
4.71 E-05 \\
2.52 E-07 \\
2.79 E-04 \\
6.96 E+00\end{array}$ \\
\hline & & 5. & 1. \\
\hline
\end{tabular}

Table 8. Expected Annual Fatalities for the Shipping Campaign

\begin{tabular}{lll}
\hline Exposure Group & Truck & Rail \\
\hline Radiological & & \\
Normal Crew & $5.6 \mathrm{E}-01$ & $4.4 \mathrm{E}-02$ \\
Normal Public & $8.3 \mathrm{E}-01$ & $1.0 \mathrm{E}-01$ \\
Accident Public & $2.9 \mathrm{E}-02$ & $5.7 \mathrm{E}-03$ \\
Nonradiological & & \\
Emission & $2.1 \mathrm{E}-01$ & $1.5 \mathrm{E}-01$ \\
Accident & $3.1 \mathrm{E}+00$ & $4.3 \mathrm{E}-02$ \\
\hline
\end{tabular}

Table 9. Expected Annual Cancer Incidence for the Shipping Campaign

\begin{tabular}{lll}
\hline Exposure Group & Truck & Rail \\
\hline Radiological & & \\
Normal Crew & $2.0 \mathrm{E}+00$ & $1.5 \mathrm{E}-01$ \\
Normal Public & $2.8 \mathrm{E}+00$ & $3.6 \mathrm{E}-01$ \\
Accident Public & $9.9 \mathrm{E}-02$ & $1.9 \mathrm{E}-02$ \\
Nonradiological & $2.1 \mathrm{E}-01$ & $1.5 \mathrm{E}-01$ \\
Emission & $\mathrm{NA}$ & $\mathrm{NA}$ \\
Accident & &. \\
\hline
\end{tabular}


- Person in Traffic Obstruction (Truck and Rail). A person is assumed to be stopped next to a waste shipment (e.g., because of traffic slowdown). The person is assumed to be exposed unshielded at a distance of $1 \mathrm{~m}(3.3 \mathrm{ft})$ for a duration of 30 minutes.

- Person at Truck Service Station. A person is assumed to be exposed at an average distance of $20 \mathrm{~m}$ (66 ft) for a duration of two hours. This receptor could be a worker at a truck stop.

- Resident Near a Rail Stop. A resident is assumed to live near a rail classification yard. The resident is assumed to be exposed unshielded at a distance of $200 \mathrm{~m}$ ( $656 \mathrm{ft})$ for a duration of 20 hours.

The largest uncertainty in predicting the dose to MEIs during transportation involves determining the frequency of occurrence of exposures. This difficulty results from the uncertainties in future shipment schedules and route selection and from the inherent uncertainty in predicting the frequency of random or chance events. For instance, that an individual may be stopped in traffic next to a shipment of radioactive waste is conceivable; however, predicting how often the same individual would experience this event is difficult. Therefore, for the majority of receptors considered, doses are assessed on a per-event basis. To account for possible multiple exposures, ranges of realistic total doses are discussed qualitatively. One exception is the calculation of the dose to a hypothetical resident living near an entrance route to a storage or disposal site. For these residents, total doses are calculated on the basis of the number of shipments entering or exiting each site for each alternative.

\subsection{GENERAL RADTRAN INPUT PARAMETERS}

In addition to the specific parameters discussed previously, values for a number of general parameters must be specified within the RADTRAN code. These general parameters define basic characteristics of the shipment and traffic and are specific to the mode of transportation. The user's manual for the RADTRAN code (Neuhauser and Kanipe 1993) contains derivations and descriptions of these parameters. The general RADTRAN input parameters used in the transportation risk assessment are summarized in Table 4.6. 


\section{$B-124$}

Table 10. Expected Annual Genetic Effects for the Shipping Campaign

\begin{tabular}{lll}
\hline Exposure Group & Truck & Rail \\
\hline Radiological & & \\
Normal Crew & $8.4 \mathrm{E}-02$ & $6.6 \mathrm{E}-03$ \\
Normal Public & $1.7 \mathrm{E}-01$ & $2.1 \mathrm{E}-02$ \\
Accident Public & $5.3 \mathrm{E}-03$ & $1.0 \mathrm{E}-03$ \\
Nonradiological & & \\
Emission & NA & NA \\
Accident & NA & NA \\
\hline
\end{tabular}




\section{B.24 WM LLW CENTRALIZED 3 ALTERNATIVE (CASE 14): ACTIVATED METALS}

Table 1. Summary of Route Information

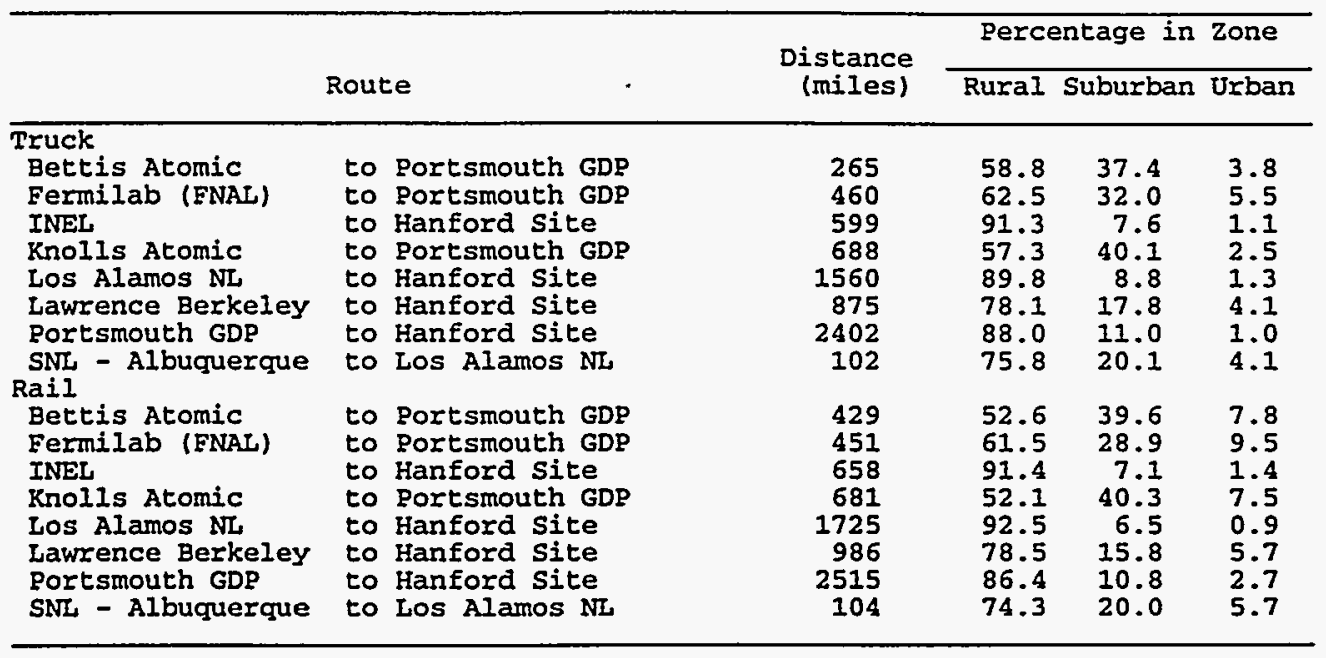

Table 2. Annual Number of Shipments and One-Way Shipment Distances

\begin{tabular}{|c|c|c|c|c|c|}
\hline \multirow[b]{2}{*}{ Route } & & \multicolumn{2}{|c|}{ \# of Shipments } & \multicolumn{2}{|c|}{ Total Mileage(mi) } \\
\hline & & Truck & RaiI & Truck & Rail \\
\hline $\begin{array}{l}\text { Bettis Atomic } \\
\text { Fermilab (FNAL) } \\
\text { INEL } \\
\text { Knolls Atomic } \\
\text { Los Alamos NL } \\
\text { Lawrence Berkeley } \\
\text { Portsmouth GDP } \\
\text { SNL - Albuquerque }\end{array}$ & $\begin{array}{l}\text { to Portsmouth GDP } \\
\text { to Portsmouth GDP } \\
\text { to Hanford Site } \\
\text { to Portsmouth GDP } \\
\text { to Hanford Site } \\
\text { to Hanford Site } \\
\text { to Hanford Site } \\
\text { to Los Alamos NL }\end{array}$ & $\begin{array}{r}309 \\
39 \\
365 \\
639 \\
332 \\
14 \\
785 \\
6\end{array}$ & $\begin{array}{r}119 \\
16 \\
157 \\
245 \\
143 \\
6 \\
337 \\
3\end{array}$ & $\begin{array}{l}8.19 \mathrm{E}+04 \\
1.79 \mathrm{E}+04 \\
2.19 \mathrm{E}+05 \\
4.40 \mathrm{E}+05 \\
5.18 \mathrm{E}+05 \\
1.22 \mathrm{E}+04 \\
1.89 \mathrm{E}+06 \\
6.12 \mathrm{E}+02\end{array}$ & $\begin{array}{l}5.11 E+04 \\
7.21 E+03 \\
1.03 E+05 \\
1.67 E+05 \\
2.47 E+05 \\
5.91 E+03 \\
8.48 E+05 \\
3.12 E+02\end{array}$ \\
\hline Totals & & 2489 & 1026 & $3.17 E+06$ & $1.43 E+06$ \\
\hline
\end{tabular}


Table 3. Incident-Free Dose per Shipment (person-rem/shipment)

\begin{tabular}{|c|c|c|c|c|c|}
\hline \multirow[b]{2}{*}{ Source/Route (s) } & \multirow[b]{2}{*}{ Crew } & \multicolumn{4}{|c|}{ General Public } \\
\hline & & off-Link & On-Iink & Stops & Total \\
\hline \multirow[t]{2}{*}{$\begin{array}{l}\text { LLW(I) BA to PO } \\
\text { Bettis Atomic }\end{array}$} & to Portsmouth GDP & & & & \\
\hline & $\begin{array}{ll}\text { Truck } & 9.78 \mathrm{E}-03 \\
\text { Rail } & 4.67 \mathrm{E}-03\end{array}$ & $\begin{array}{l}1.26 \mathrm{E}-03 \\
9.85 \mathrm{E}-03\end{array}$ & $\begin{array}{l}1.73 \mathrm{E}-03 \\
1.53 \mathrm{E}-04\end{array}$ & $\begin{array}{l}7.42 \mathrm{E}-03 \\
4.80 \mathrm{E}-03\end{array}$ & $\begin{array}{l}1.04 \mathrm{E}-02 \\
1.48 \mathrm{E}-02\end{array}$ \\
\hline \multirow[t]{2}{*}{$\begin{array}{l}\text { LLW(I) FL to PO } \\
\text { Fermilab (FNAL) }\end{array}$} & to Portsmouth GDP & & & & \\
\hline & $\begin{array}{ll}\text { Truck } & 1.68 \mathrm{E}-02 \\
\text { Rail } & 4.76 \mathrm{E}-03\end{array}$ & $\begin{array}{l}2.40 \mathrm{E}-03 \\
1.08 \mathrm{E}-02\end{array}$ & $\begin{array}{l}3.43 \mathrm{E}-03 \\
1.48 \mathrm{E}-04\end{array}$ & $\begin{array}{l}1.29 E-02 \\
4.87 E-03\end{array}$ & $\begin{array}{l}1.87 \mathrm{E}-02 \\
1.58 \mathrm{E}-02\end{array}$ \\
\hline \multirow[t]{2}{*}{$\begin{array}{l}\text { LLW(I) IN to HS } \\
\text { INEL }\end{array}$} & NLE & & & & \\
\hline & $\begin{array}{ll}\text { Truck } & 1.60 \mathrm{E}-02 \\
\text { Rail } & 5.63 \mathrm{E}-03\end{array}$ & $\begin{array}{l}7.18 \mathrm{E}-04 \\
2.83 \mathrm{E}-03\end{array}$ & $\begin{array}{l}2.06 \mathrm{E}-03 \\
6.58 \mathrm{E}-05\end{array}$ & $\begin{array}{l}1.68 \mathrm{E}-02 \\
5.51 \mathrm{E}-03\end{array}$ & $\begin{array}{l}1.95 \mathrm{E}-02 \\
8.40 \mathrm{E}-03\end{array}$ \\
\hline \multirow[t]{2}{*}{$\begin{array}{l}\text { LIW(I) KA to Po } \\
\text { Knolls Atomic }\end{array}$} & tsmouth GDP & & & & \\
\hline & $\begin{array}{ll}\text { Truck } & 2.54 \mathrm{E}-02 \\
\text { Rail } & 5.72 \mathrm{E}-03\end{array}$ & $\begin{array}{l}2.99 E-03 \\
1.54 E-02\end{array}$ & $\begin{array}{l}4.01 E-03 \\
2.43 E-04\end{array}$ & $\begin{array}{l}1.93 \mathrm{E}-02 \\
5.58 \mathrm{E}-03\end{array}$ & $\begin{array}{l}2.63 \mathrm{E}-02 \\
2.13 \mathrm{E}-02\end{array}$ \\
\hline \multirow[t]{2}{*}{$\begin{array}{l}\text { LLW (I) LA to HS } \\
\text { IOS Alamos NL }\end{array}$} & to Hanford Site & & & & \\
\hline & $\begin{array}{ll}\text { Truck } & 4.24 \mathrm{E}-02 \\
\text { RaiI } & 1.01 \mathrm{E}-02\end{array}$ & $\begin{array}{l}2.16 \mathrm{E}-03 \\
5.60 \mathrm{E}-03\end{array}$ & $\begin{array}{l}5.65 \mathrm{E}-03 \\
1.54 \mathrm{E}-04\end{array}$ & $\begin{array}{l}4.37 E-02 \\
8.79 E-03\end{array}$ & $\begin{array}{l}5.15 \mathrm{E}-02 \\
1.45 \mathrm{E}-02\end{array}$ \\
\hline \multirow{2}{*}{$\begin{array}{l}\text { LLW(I) IB to HS } \\
\text { Lawrence Berkeley }\end{array}$} & ord $s$ & & & & \\
\hline & $\begin{array}{ll}\text { Truck } & 2.76 \mathrm{E}-02 \\
\text { Rail } & 6.99 \mathrm{E}-03\end{array}$ & $\begin{array}{l}3.02 \mathrm{E}-03 \\
1.38 \mathrm{E}-02\end{array}$ & $\begin{array}{l}5.17 E-03 \\
2.03 E-04\end{array}$ & $\begin{array}{l}2.45 \mathrm{E}-02 \\
6.51 \mathrm{E}-03\end{array}$ & $\begin{array}{l}3.27 E-02 \\
2.05 E-02\end{array}$ \\
\hline \multirow[t]{2}{*}{$\begin{array}{l}\text { LLW(I) PO to HS } \\
\text { Portsmouth GDP }\end{array}$} & d si & & & & \\
\hline & $\begin{array}{ll}\text { Truck } & 6.63 \mathrm{E}-02 \\
\text { Rail } & 1.34 \mathrm{E}-02\end{array}$ & $\begin{array}{l}3.38 \mathrm{E}-03 \\
1.89 \mathrm{E}-02\end{array}$ & $\begin{array}{l}8.48 \mathrm{E}-03 \\
3.46 \mathrm{E}-04\end{array}$ & $\begin{array}{l}6.72 \mathrm{E}-02 \\
1.12 \mathrm{E}-02\end{array}$ & $\begin{array}{l}7.91 \mathrm{E}-02 \\
3.04 \mathrm{E}-02\end{array}$ \\
\hline \multirow[t]{2}{*}{$\begin{array}{l}\text { LLW(I) SA to LA } \\
\text { SNL - Albuquerque }\end{array}$} & S Al & & & & \\
\hline & $\begin{array}{ll}\text { Truck } & 3.28 \mathrm{E}-03 \\
\text { Rail } & 3.31 \mathrm{E}-03\end{array}$ & $\begin{array}{l}3.70 \mathrm{E}-04 \\
1.55 \mathrm{E}-03\end{array}$ & $\begin{array}{l}6.13 \mathrm{E}-04 \\
2.36 \mathrm{E}-05\end{array}$ & $\begin{array}{l}2.86 \mathrm{E}-03 \\
3.80 \mathrm{E}-03\end{array}$ & $\begin{array}{l}3.84 E-03 \\
5.37 E-03\end{array}$ \\
\hline
\end{tabular}

Table 4. Accident Dose Risk per Shipment (person-rem/shipment)

\begin{tabular}{|c|c|c|c|}
\hline Source/Route & & Truck & Rail \\
\hline $\begin{array}{l}\text { LLW(I) BA to PO } \\
\text { Bettis Atomic } \\
\text { LLW(I) FL to PO } \\
\text { Fermilab (FNAL) } \\
\text { LLW(I) IN to HS } \\
\text { INEL } \\
\text { LLW(I) RA to Po } \\
\text { Knolls Atomic } \\
\text { LLW(I) LA to HS } \\
\text { LOS Alamos NL } \\
\text { LLW(I) LB to HS } \\
\text { Lawrence Berkeley } \\
\text { LLW(I) PO to HS } \\
\text { Portsmouth GDP } \\
\text { LLW(I) SA to LA } \\
\text { SNL - Albuquerque }\end{array}$ & $\begin{array}{l}\text { to Portsmouth GDP } \\
\text { to Portsmouth GDP } \\
\text { to Hanford Site } \\
\text { to. Portsmouth GDP } \\
\text { to Hanford Site } \\
\text { to Hanford Site } \\
\text { to Hanford Site } \\
\text { to Los Alamos NL }\end{array}$ & $\begin{array}{l}3.70 \mathrm{E}-07 \\
5.29 \mathrm{E}-11 \\
5.63 \mathrm{E}-08 \\
1.66 \mathrm{E}-06 \\
3.90 \mathrm{E}-09 \\
1.81 \mathrm{E}-10 \\
2.14 \mathrm{E}-06 \\
1.72 \mathrm{E}-12\end{array}$ & $\begin{array}{l}4.16 \mathrm{E}-07 \\
3.35 \mathrm{E}-11 \\
7.06 \mathrm{E}-08 \\
1.02 \mathrm{E}-06 \\
9.87 \mathrm{E}-10 \\
2.37 \mathrm{E}-10 \\
1.80 \mathrm{E}-06 \\
6.51 \mathrm{E}-14\end{array}$ \\
\hline
\end{tabular}


Table 5. Nonradiological Risk Factors per Shipment (fatalities/shipment)

\begin{tabular}{llll}
\hline Mode & Route & Emission & Accident \\
\hline Truck & & $3.22 E-06$ & $1.33 E-05$ \\
Bettis Atomic & to Portsmouth GDP & $8.11 E-06$ & $3.01 \mathrm{E}-05$ \\
Fermilab (FNAL) & to Portsmouth GDP & $2.19 \mathrm{E}-06$ & $3.07 \mathrm{E}-05$ \\
INEL & to Hanford Site & $5.63 \mathrm{E}-06$ & $3.56 \mathrm{E}-05$ \\
Knolls Atomic & to Portsmouth GDP & $6.66 \mathrm{E}-06$ & $1.02 \mathrm{E}-04$ \\
Los Alamos NL & to Hanford Site & $1.16 \mathrm{E}-05$ & $\mathbf{4 . 9 1 E - 0 5}$ \\
Lawrence Berkeley & to Hanford Site & $8.11 \mathrm{E}-06$ & $1.28 \mathrm{E}-04$ \\
Portsmouth GDP & to Hanford Site & $1.35 \mathrm{E}-06$ & $1.20 \mathrm{E}-05$ \\
SNL - Albuquerque. & to Los Alamos NL & & \\
Rail & & $1.40 \mathrm{E}-05$ & $8.98 \mathrm{E}-07$ \\
Bettis Atomic & to Portsmouth GDP & $1.80 \mathrm{E}-05$ & $9.43 \mathrm{E}-07$ \\
Fermilab (FNAL) & to Portsmouth GDP & $3.98 \mathrm{E}-06$ & $1.38 \mathrm{E}-06$ \\
INEL & to Hanford Site & $2.15 \mathrm{E}-05$ & $1.43 \mathrm{E}-06$ \\
Knolis Atomic & to Portsmouth GDP & $6.82 \mathrm{E}-06$ & $3.61 \mathrm{E}-06$ \\
Los Alamos NL & to Hanford Site & $2.33 \mathrm{E}-05$ & $2.06 \mathrm{E}-06$ \\
Lawrence Berkeley & to Hanford Site & $2.88 \mathrm{E}-05$ & $5.26 \mathrm{E}-06$ \\
Portsmouth GDP & to Hanford Site & $2.47 \mathrm{E}-06$ & $2.18 \mathrm{E}-07$ \\
SNL - Albuquerque & to Los Alamos NL & & \\
\hline
\end{tabular}

Table 6. Annual Incident-Free Dose for the Shipping Campaign (person-rem/yr)

\begin{tabular}{|c|c|c|c|c|c|}
\hline \multirow[b]{2}{*}{ Route } & & \multicolumn{2}{|c|}{ Truck } & \multicolumn{2}{|c|}{ Rail } \\
\hline & & Crew & Public & .Crew & Public \\
\hline $\begin{array}{l}\text { Bettis Atomic } \\
\text { Fermilab (FNAL) } \\
\text { INEL } \\
\text { Rnolis Atomic } \\
\text { Los Alamos NL } \\
\text { Lawrence Berkeley } \\
\text { Portsmouth GDP } \\
\text { SNL - Albuquerque }\end{array}$ & $\begin{array}{l}\text { to Portsmouth GDP } \\
\text { to Portsmouth GDP } \\
\text { to Hanford Site } \\
\text { to Portsmouth GDP } \\
\text { to Hanford Site } \\
\text { to Hanford Site } \\
\text { to Hanford Site } \\
\text { to Los Alamos NL }\end{array}$ & $\begin{array}{l}3.02 E+00 \\
6.54 \mathrm{E}-01 \\
5.84 \mathrm{E}+00 \\
1.62 \mathrm{E}+01 \\
1.41 \mathrm{E}+01 \\
3.86 \mathrm{E}-01 \\
5.21 \mathrm{E}+01 \\
1.97 \mathrm{E}-02\end{array}$ & $\begin{array}{l}3.22 \mathrm{E}+00 \\
7.30 \mathrm{E}-01 \\
7.13 \mathrm{E}+00 \\
1.68 \mathrm{E}+01 \\
1.71 \mathrm{E}+01 \\
4.58 \mathrm{E}-01 \\
6.21 \mathrm{E}+01 \\
2.30 \mathrm{E}-02\end{array}$ & $\begin{array}{l}5.56 \mathrm{E}-01 \\
7.62 \mathrm{E}-02 \\
8.83 \mathrm{E}-01 \\
1.40 \mathrm{E}+00 \\
1.44 \mathrm{E}+00 \\
4.20 \mathrm{E}-02 \\
4.51 \mathrm{E}+00 \\
9.94 \mathrm{E}-03\end{array}$ & $\begin{array}{l}1.76 \mathrm{E}+00 \\
2.53 \mathrm{E}-01 \\
1.32 \mathrm{E}+00 \\
5.21 \mathrm{E}+00 \\
2.08 \mathrm{E}+00 \\
1.23 \mathrm{E}-01 \\
1.03 \mathrm{E}+01 \\
1.61 \mathrm{E}-02\end{array}$ \\
\hline Totals & & $9.23 E+0$ & $1.08 \mathrm{E}+02$ & $8.92 E+00$ & $2.10 \mathrm{E}+01$ \\
\hline
\end{tabular}

Table 7. Annual Accident Dose Risk for the Shipping Campaign (person-rem/yr)

\begin{tabular}{llcc}
\hline Route & & Truck & Rail \\
\hline Bettis Atomic & to Portsmouth GDP & $1.14 \mathrm{E}-04$ & $4.95 \mathrm{E}-05$ \\
Fermilab (FNAL) & to Portsmouth GDP & $2.06 \mathrm{E}-09$ & $5.35 \mathrm{E}-10$ \\
INEL & to Hanford Site & $2.06 \mathrm{E}-05$ & $1.11 \mathrm{E}-05$ \\
Knolls Atomic & to Portsmouth GDP & $1.06 \mathrm{E}-03$ & $2.49 \mathrm{E}-04$ \\
Los Alamos NL & to Hanford Site & $1.29 \mathrm{E}-06$ & $1.41 \mathrm{E}-07$ \\
Lawrence Berkeley & to Hanford Site & $2.53 \mathrm{E}-09$ & $1.42 \mathrm{E}-09$ \\
Portsmouth GDP & to Hanford Site & $1.68 \mathrm{E}-03$ & $6.06 \mathrm{E}-04$ \\
SNL - Albuquerque & to Los Alamos NL & $1.03 \mathrm{E}-11$ & $1.95 \mathrm{E}-13$ \\
& & $2.87 \mathrm{E}-03$ & $9.16 \mathrm{E}-04$ \\
\hline
\end{tabular}

Table 8. Expected Annual Fatalities for the Shipping Campaign

\begin{tabular}{lll}
\hline Exposure Group & Truck & Rail \\
\hline Radiological & $\cdot 3 \mathrm{EE}-02$ & $3.6 \mathrm{E}-03$ \\
Normal Crew & $5.4 \mathrm{E}-02$ & $1.1 \mathrm{E}-02$ \\
Normal Public & $1.4 \mathrm{E}-06$ & $4.6 \mathrm{E}-07$ \\
Accident Public & & \\
Nonradiological & $1.4 \mathrm{E}-02$ & $1.9 \mathrm{E}-02$ \\
Emission & $1.7 \mathrm{E}-01$ & $3.0 \mathrm{E}-03$ \\
Accident & & \\
\hline
\end{tabular}


Table 9. Expected Annual Cancer Incidence for the Shipping Campaign

\begin{tabular}{lll}
\hline Exposure Group & Truck & Rail \\
\hline Radiological & & \\
$\quad$ Normal Crew & $1.3 \mathrm{E}-01$ & $1.2 \mathrm{E}-02$ \\
$\quad$ Normal Public & $1.8 \mathrm{E}-01$ & $3.6 \mathrm{E}-02$ \\
$\quad \begin{array}{cc}\text { Accident Public } \\
\text { Nonradiological } \\
\text { Emission }\end{array}$ & $4.9 \mathrm{E}-06$ & $1.6 \mathrm{E}-06$ \\
Accident & $1.4 \mathrm{E}-02$ & $1.9 \mathrm{E}-02$ \\
\hline
\end{tabular}

Table 10. Expected Annual Genetic Effects for the Shipping Campaign

\begin{tabular}{lll}
\hline Exposure Group & Truck & Rail \\
\hline Radiological & & \\
Normal Crew & $5.5 E-03$ & $5.4 \mathrm{E}-04$ \\
Normal Public & $1.1 \mathrm{E}-02$ & $2.1 \mathrm{E}-03$ \\
Accident Public & $2.9 \mathrm{E}-07$ & $9.2 \mathrm{E}-08$ \\
$\begin{array}{l}\text { Nonradiological } \\
\text { Emission }\end{array}$ & NA & NA \\
Accident & NA & NA \\
\hline
\end{tabular}




\section{B.25 WM LLW CENTRALIZED 4 ALTERNATIVE (CASE 14A): HETEROGENEOUS SOLIDS}

Table 1. Summary of Route Information

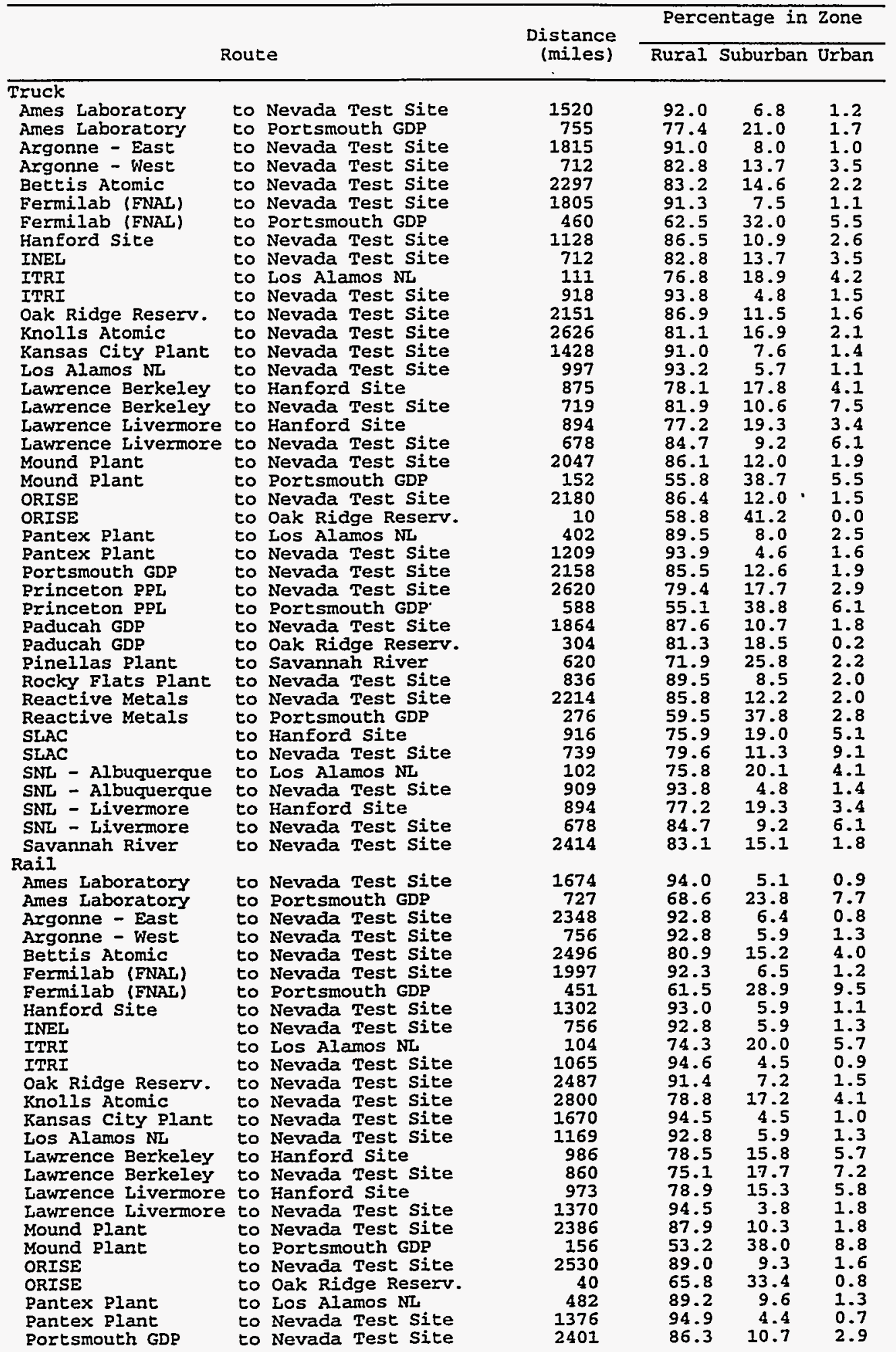




\begin{tabular}{|c|c|c|c|c|c|c|}
\hline $\begin{array}{l}\text { Princeton PPL } \\
\text { Princeton PPL } \\
\text { Paducah GDP } \\
\text { Paducah GDP } \\
\text { Pinellas Plant } \\
\text { Rocky Flats Plant } \\
\text { Reactive Metals } \\
\text { Reactive Metals } \\
\text { SLAC } \\
\text { SLAC } \\
\text { SNL - Albuquerque } \\
\text { SNL - Albuquerque } \\
\text { SNL - Livermore } \\
\text { SNL - Livermore } \\
\text { Savannah River }\end{array}$ & $\begin{array}{l}\text { to } \\
\text { to } \\
\text { to } \\
\text { to } \\
\text { to } \\
\text { to } \\
\text { to } \\
\text { to } \\
\text { to } \\
\text { to } \\
\text { to } \\
\text { to } \\
\text { to } \\
\text { to } \\
\text { to }\end{array}$ & $\begin{array}{l}\text { Nevada Test Site } \\
\text { Portsmouth GDP } \\
\text { Nevada Test Site } \\
\text { Oak Ridge Reserv. } \\
\text { Savannah River } \\
\text { Nevada Test Site } \\
\text { Nevada Test Site } \\
\text { Portsmouth GDP } \\
\text { Hanford Site } \\
\text { Nevada Test Site } \\
\text { Los Alamos NL } \\
\text { Nevada Test Site } \\
\text { Hanford Site } \\
\text { Nevada Test Site } \\
\text { Nevada Test Site }\end{array}$ & $\begin{array}{r}2871 \\
838 \\
2131 \\
527 \\
485 \\
987 \\
2391 \\
279 \\
1036 \\
862 \\
104 \\
1065 \\
973 \\
1370 \\
2839\end{array}$ & $\begin{array}{l}76.2 \\
58.3 \\
90.9 \\
89.3 \\
72.2 \\
95.9 \\
83.1 \\
48.4 \\
76.1 \\
75.8 \\
74.3 \\
94.6 \\
78.9 \\
94.5 \\
84.5\end{array}$ & $\begin{array}{r}19.3 \\
32.6 \\
7.6 \\
9.0 \\
25.0 \\
3.9 \\
13.1 \\
41.8 \\
16.8 \\
17.7 \\
20.0 \\
4.5 \\
15.3 \\
3.8 \\
13.5\end{array}$ & $\begin{array}{l}4.5 \\
9.1 \\
1.5 \\
1.7 \\
2.8 \\
0.2 \\
3.7 \\
9.8 \\
7.1 \\
6.5 \\
5.7 \\
0.9 \\
5.8 \\
1.8 \\
1.9\end{array}$ \\
\hline
\end{tabular}

Table 2. Annual Number of Shipments and One-Way Shipment Distances

\begin{tabular}{|c|c|c|c|c|c|c|}
\hline \multirow{2}{*}{ Route } & & & \multicolumn{2}{|c|}{ \# of Shipments } & \multicolumn{2}{|c|}{ Total Mileage(mi) } \\
\hline & & & Truck & Rail & Truck & Rail \\
\hline 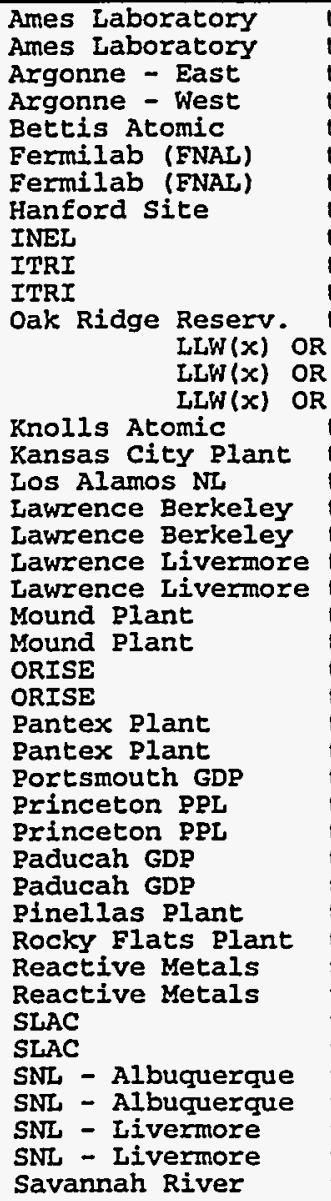 & $\begin{array}{l}\text { to } \\
\text { to } \\
\text { to } \\
\text { to } \\
\text { to } \\
\text { to } \\
\text { to } \\
\text { to } \\
\text { to } \\
\text { to } \\
\text { to } \\
\text { to } \\
\text { to } \\
\text { to } \\
\text { to } \\
\text { to } \\
\text { to } \\
\text { to } \\
\text { to } \\
\text { to } \\
\text { to } \\
\text { to } \\
\text { to } \\
\text { to } \\
\text { to } \\
\text { to } \\
\text { to } \\
\text { to } \\
\text { to } \\
\text { to } \\
\text { to } \\
\text { to } \\
\text { to } \\
\text { to } \\
\text { to } \\
\text { to } \\
\text { to } \\
\text { to } \\
\text { to } \\
\text { to } \\
\text { to } \\
\text { to } \\
\text { to }\end{array}$ & $\begin{array}{l}\text { Nevada Test Site } \\
\text { Portsmouth GDP } \\
\text { Nevada Test Site } \\
\text { Nevada Test Site } \\
\text { Nevada Test Site } \\
\text { Nevada Test Site } \\
\text { Portsmouth GDP } \\
\text { Nevada Test Site } \\
\text { Nevada Test Site } \\
\text { Los Alamos NL } \\
\text { Nevada Test Site } \\
\text { Nevada Test Site } \\
\text { NT } \\
\text { NT } \\
\text { NT } \\
\text { Nevada Test Site } \\
\text { Nevada Test Site } \\
\text { Nevada Test Site } \\
\text { Hanford Site } \\
\text { Nevada Test Site } \\
\text { Hanford Site } \\
\text { Nevada Test Site } \\
\text { Nevada Test Site } \\
\text { Portsmouth GDP } \\
\text { Nevada Test Site } \\
\text { Oak Ridge Reserv. } \\
\text { Los Alamos NL } \\
\text { Nevada Test Site } \\
\text { Nevada Test Site } \\
\text { Nevada Test Site } \\
\text { Portsmouth GDP } \\
\text { Nevada Test Site } \\
\text { Oak Ridge Reserv. } \\
\text { Savannah River } \\
\text { Nevada Test Site } \\
\text { Nevada Test Site } \\
\text { Portsmouth GDP } \\
\text { Hanford Site } \\
\text { Nevada Test Site } \\
\text { Los Alamos NL } \\
\text { Nevada Test Site } \\
\text { Hanford Site } \\
\text { Nevada Test Site } \\
\text { Nevada Test site }\end{array}$ & $\begin{array}{r}2 \\
1 \\
105 \\
10 \\
57 \\
2 \\
1 \\
1 \\
1363 \\
820 \\
2 \\
9 \\
6067 \\
12 \\
5975 \\
80 \\
28 \\
1 \\
1965 \\
2 \\
2 \\
17 \\
40 \\
496 \\
12 \\
4 \\
1 \\
1358 \\
25 \\
2950 \\
1 \\
1 \\
578 \\
57 \\
2 \\
335 \\
745 \\
7 \\
1 \\
39 \\
1 \\
17 \\
1 \\
5 \\
6750\end{array}$ & $\begin{array}{r}1 \\
1 \\
39 \\
4 \\
21 \\
1 \\
1 \\
520 \\
315 \\
1 \\
3 \\
2425 \\
5 \\
2390 \\
30 \\
11 \\
1 \\
764 \\
1 \\
1 \\
7 \\
15 \\
186 \\
5 \\
2 \\
1 \\
528 \\
10 \\
1107 \\
1 \\
1 \\
215 \\
21 \\
1 \\
123 \\
283 \\
3 \\
1 \\
15 \\
1 \\
7 \\
1 \\
2 \\
2522\end{array}$ & 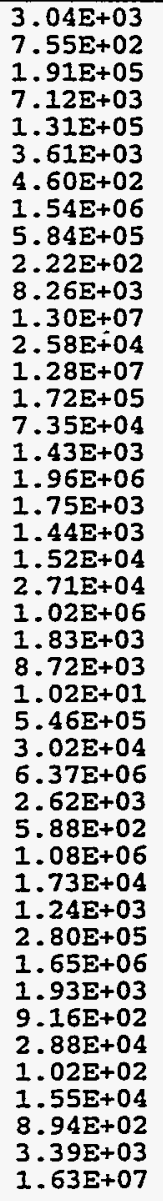 & 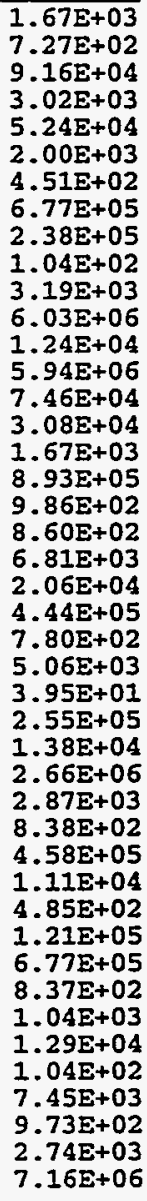 \\
\hline Totals & & & 23880 & 9168 & $4.49 E+07$ & $1.99 \mathrm{E}+07$ \\
\hline
\end{tabular}


Table 3. Incident-Free Dose per Shipment (person-rem/shipment)

\begin{tabular}{|c|c|c|c|c|c|c|}
\hline \multirow[b]{2}{*}{ Source/Route (s) } & & \multirow[b]{2}{*}{ Crew } & \multicolumn{4}{|c|}{ General Public } \\
\hline & & & off-Iink & On-Link & Stops & Total \\
\hline $\begin{array}{l}\text { LLW }(x) \text { AL to NT } \\
\text { Ames Laboratory }\end{array}$ & $\begin{array}{l}\text { to Nevada } \\
\text { Truck } \\
\text { Rail }\end{array}$ & $\begin{array}{l}\text { Test Site } \\
4.03 \mathrm{E}-02 \\
9.87 \mathrm{E}-03\end{array}$ & $\begin{array}{l}\text { e } \\
4.7\end{array}$ & $\begin{array}{l}-03 \\
-04\end{array}$ & $\begin{array}{l}E-02 \\
\varepsilon-03\end{array}$ & $\begin{array}{l}E-02 \\
z-02\end{array}$ \\
\hline $\begin{array}{l}\text { LLW(x) AL to Po } \\
\text { Ames Laboratory }\end{array}$ & $\begin{array}{l}\text { to Portsmo } \\
\text { Truck } \\
\text { Rail }\end{array}$ & $\begin{array}{l}\text { outh GDP } \\
2.33 \mathrm{E}-02 \\
5.91 \mathrm{E}-03\end{array}$ & 1.8 & & $\begin{array}{l}8 \\
-02 \\
-03\end{array}$ & $\begin{array}{l}2.63 \mathrm{E}-02 \\
2.01 \mathrm{E}-02\end{array}$ \\
\hline LTW(x) AW to NT & $\begin{array}{l}\text { to Nev } \\
\text { Truck } \\
\text { Rail }\end{array}$ & $\begin{array}{l}\text { Test Site } \\
4.86 \mathrm{E}-02 \\
1.27 \mathrm{E}-02\end{array}$ & $\begin{array}{l}2.13 \\
6.96\end{array}$ & -03 & $\begin{array}{l}5.08 \mathrm{E}-02 \\
1.07 \mathrm{E}-02\end{array}$ & $\begin{array}{l}5.91 \mathrm{E}-02 \\
1.79 \mathrm{E}-02\end{array}$ \\
\hline $\begin{array}{l}\text { LLW (x) AW to NT } \\
\text { Argonne - West }\end{array}$ & $\begin{array}{l}\text { Eo Nevada } \\
\text { Truck } \\
\text { Rail }\end{array}$ & $\begin{array}{l}\text { Test Site } \\
2.13 \mathrm{E}-02 \\
6.04 \mathrm{E}-03\end{array}$ & $\begin{array}{l}2.03 E-03 \\
2.80 E-03\end{array}$ & $\begin{array}{l}3.79 \mathrm{E}-03 \\
6.88 \mathrm{E}-05\end{array}$ & $\begin{array}{l}1.99 E-02 \\
5.81 E-03\end{array}$ & $\begin{array}{l}2.58 \mathrm{E}-02 \\
8.67 \mathrm{E}-03\end{array}$ \\
\hline LLW(x) FL to NT & $\begin{array}{l}\text { to Nevada } \\
\text { Truck } \\
\text { Rail }\end{array}$ & $\begin{array}{l}\text { Test Site } \\
6.75 \mathrm{E}-02 \\
1.33 \mathrm{E}-02\end{array}$ & $\begin{array}{l}5.17 \mathrm{E}-03 \\
2.67 \mathrm{E}-02\end{array}$ & $\begin{array}{l}1.02 \mathrm{E}-02 \\
4.43 \mathrm{E}-04\end{array}$ & $\begin{array}{l}6.43 E-02 \\
1.12 E-02\end{array}$ & $\begin{array}{l}7.97 \mathrm{E}-02 \\
3.83 \mathrm{E}-02\end{array}$ \\
\hline $\begin{array}{l}\operatorname{LLW}(x) \text { FL to } \mathrm{NT} \\
\text { Fermilab (FNAL) }\end{array}$ & $\begin{array}{l}\text { to Nevada } \\
\text { Truck } \\
\text { Rail }\end{array}$ & $\begin{array}{l}\text { Test Site } \\
4.82 \mathrm{E}-02 \\
1.12 \mathrm{E}-02\end{array}$ & 2.1 & $:-03$ & $\begin{array}{l}E-02 \\
E-03\end{array}$ & $\begin{array}{l}E-02 \\
E-02\end{array}$ \\
\hline 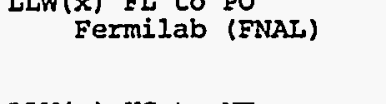 & $\begin{array}{l}\text { to Portsmo } \\
\text { Truck } \\
\text { Rail }\end{array}$ & $\begin{array}{c}\text { outh GDP } \\
1.68 \mathrm{E}-02 \\
4.76 \mathrm{E}-03\end{array}$ & $\begin{array}{l}2.40 \mathrm{E}-03 \\
1.08 \mathrm{E}-02\end{array}$ & $\begin{array}{l}3.43 \mathrm{E}-03 \\
1.48 \mathrm{E}-04\end{array}$ & $\begin{array}{l}1.29 \mathrm{E}-02 \\
4.87 \mathrm{E}-03\end{array}$ & $\begin{array}{l}1.87 E-02 \\
1.58 E-02\end{array}$ \\
\hline $\begin{array}{l}\text { LLW }(x) \text { HS to NT } \\
\text { Hanford site }\end{array}$ & $\begin{array}{l}\text { to N } \\
\text { Tru } \\
\text { Rai. }\end{array}$ & $\begin{array}{l}\text { Test Site } \\
3.22 \mathrm{E}-02 \\
8.31 \mathrm{E}-03\end{array}$ & $\begin{array}{l}2.46 \mathrm{E}-03 \\
4.39 \mathrm{E}-03\end{array}$ & $\begin{array}{l}:-03 \\
:-04\end{array}$ & $\begin{array}{l}E-02 \\
E-03\end{array}$ & $\begin{array}{l}-02 \\
-02\end{array}$ \\
\hline$T T W(x)$ TT to To & $\begin{array}{l}\text { to Nevada } \\
\text { Truck } \\
\text { Rail }\end{array}$ & $\begin{array}{l}\text { Test Site } \\
2.13 E-02 \\
6.04 E-03\end{array}$ & $\begin{array}{l}2.03 E-03 \\
2.80 E-03\end{array}$ & & & $\begin{array}{l}-02 \\
-03\end{array}$ \\
\hline $\begin{array}{l}\text { LLW(x) IT to LA } \\
\text { ITRI }\end{array}$ & $\begin{array}{l}\text { to Ios Ala } \\
\text { Truck } \\
\text { Rail }\end{array}$ & $\begin{array}{l}\text { amos NL } \\
3.54 \mathrm{E}-03 \\
3.31 \mathrm{E}-03\end{array}$ & $\begin{array}{l}4.00 \mathrm{E}-04 \\
1.55 \mathrm{E}-03\end{array}$ & & $\begin{array}{l}\varepsilon-03 \\
E-03\end{array}$ & 4.1 \\
\hline ITRI & $\begin{array}{l}\text { to Nevada } \\
\text { Truck } \\
\text { Rail }\end{array}$ & $\begin{array}{l}\text { Test Site } \\
2.40 \mathrm{E}-02 \\
7.32 \mathrm{E}-03\end{array}$ & $\begin{array}{l}1.05 E-03 \\
2.85 E-03\end{array}$ & $\begin{array}{l}-03 \\
-05\end{array}$ & & \\
\hline LLW $(2$ & $\begin{array}{l}\text { to Nevada T } \\
\text { Truck } \\
\text { Rail }\end{array}$ & $\begin{array}{r}\text { Test Site } \\
6.04 \mathrm{E}-02 \\
1.33 \mathrm{E}-02\end{array}$ & 3.6 & & $\begin{array}{l}6.02 \mathrm{E}-02 \\
1.11 \mathrm{E}-02\end{array}$ & $\begin{array}{l}7.22 \\
2.22\end{array}$ \\
\hline$T H W(x) R C$ to NT & $\begin{array}{l}\text { to Ne } \\
\text { True } \\
\text { Rail }\end{array}$ & $\begin{array}{l}\text { Test Site } \\
7.86 \mathrm{E}-02 \\
1.46 \mathrm{E}-02\end{array}$ & 6. & $\begin{array}{l}02 \\
04\end{array}$ & $\begin{array}{l}7.35 \mathrm{E}-02 \\
1.21 \mathrm{E}-02\end{array}$ & $\begin{array}{l}-02 \\
-02\end{array}$ \\
\hline $\begin{array}{l}\text { LLW(x) RC to NT } \\
\text { Kansas City plant }\end{array}$ & $\begin{array}{l}\text { to Nevada } \\
\text { Truck } \\
\text { Rail }\end{array}$ & $\begin{array}{l}\text { Test Sitt } \\
3.84 \mathrm{E}-02 \\
9.85 \mathrm{E}-03\end{array}$ & $\begin{array}{l}1.90 E-03 \\
4.98 E-03\end{array}$ & $\begin{array}{l}5.1 \\
1.3\end{array}$ & $\begin{array}{l}E-02 \\
8-03\end{array}$ & $\begin{array}{l}-02 \\
8-02\end{array}$ \\
\hline Los Alamos NL & $\begin{array}{l}\text { to Nevada } \\
\text { Truck } \\
\text { Rail }\end{array}$ & $\begin{array}{l}\text { Test Site } \\
2.61 \mathrm{E}-02 \\
7.76 \mathrm{E}-03\end{array}$ & $\begin{array}{l}1.04 \mathrm{E}-03 \\
4.40 \mathrm{E}-03\end{array}$ & 3.3 & $\begin{array}{l}-02 \\
-03\end{array}$ & $:-02$ \\
\hline $\begin{array}{l}\text { LLW }(x) \text { LB to HS } \\
\text { Lawrence Berkeley }\end{array}$ & $\begin{array}{l}\text { to Hanfo } \\
\text { Truck } \\
\text { Rail }\end{array}$ & $\begin{array}{l}\text { d Site } \\
2.76 E-02 \\
6.99 E-03\end{array}$ & $\begin{array}{l}3.02 \mathrm{E}-03 \\
1.38 \mathrm{E}-02\end{array}$ & $\begin{array}{l}5.17 E-03 \\
2.03 E-04\end{array}$ & $\begin{array}{l}-02 \\
-03\end{array}$ & -02 \\
\hline $\begin{array}{l}\text { LLW (x) LB to NT } \\
\text { Lawrence Berkeley }\end{array}$ & $\begin{array}{l}\text { to Nevada } \\
\text { Truck } \\
\text { Rail }\end{array}$ & $\begin{array}{l}\text { Test } \\
2.27 \\
6.47\end{array}$ & & $\begin{array}{l}5.6 \\
2.0\end{array}$ & $\begin{array}{l}2.01 \mathrm{E}-02 \\
6.13 \mathrm{E}-03\end{array}$ & $\begin{array}{l}2.91 \mathrm{E}-02 \\
2.12 \mathrm{E}-02\end{array}$ \\
\hline $\begin{array}{l}\text { LLW(x) II to HS } \\
\text { Lawrence Livermore }\end{array}$ & $\begin{array}{l}\text { to } \mathrm{H} \\
\text { Truc } \\
\text { Rai. }\end{array}$ & $\begin{array}{l}\text { Site } \\
2.82 \mathrm{E}-02 \\
6.94 \mathrm{E}-03\end{array}$ & $\begin{array}{l}E-03 \\
E-02\end{array}$ & & $\begin{array}{l}0 E-02 \\
8 E-03\end{array}$ & $\begin{array}{l}3.28 E-02 \\
2.05 E-02\end{array}$ \\
\hline
\end{tabular}




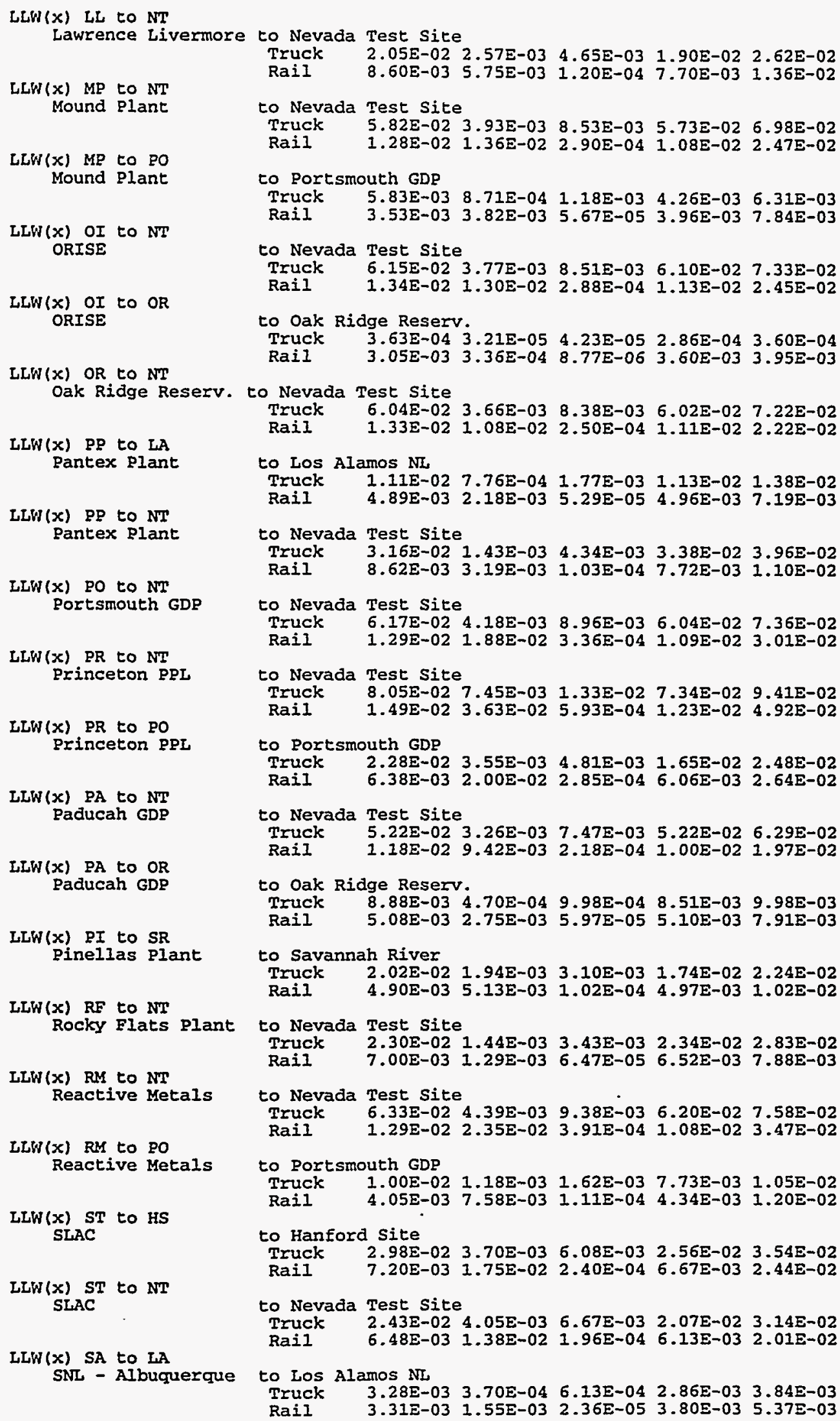




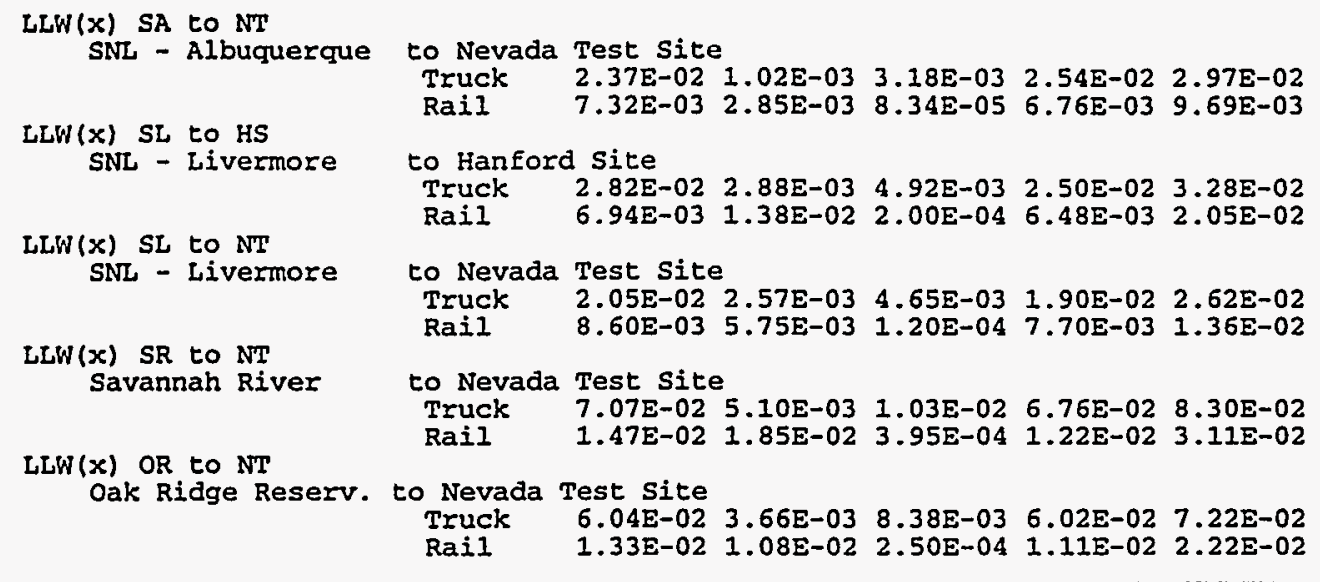


TABLE 4.6 General RADTRAN Input Parameters for the LLW Assessment

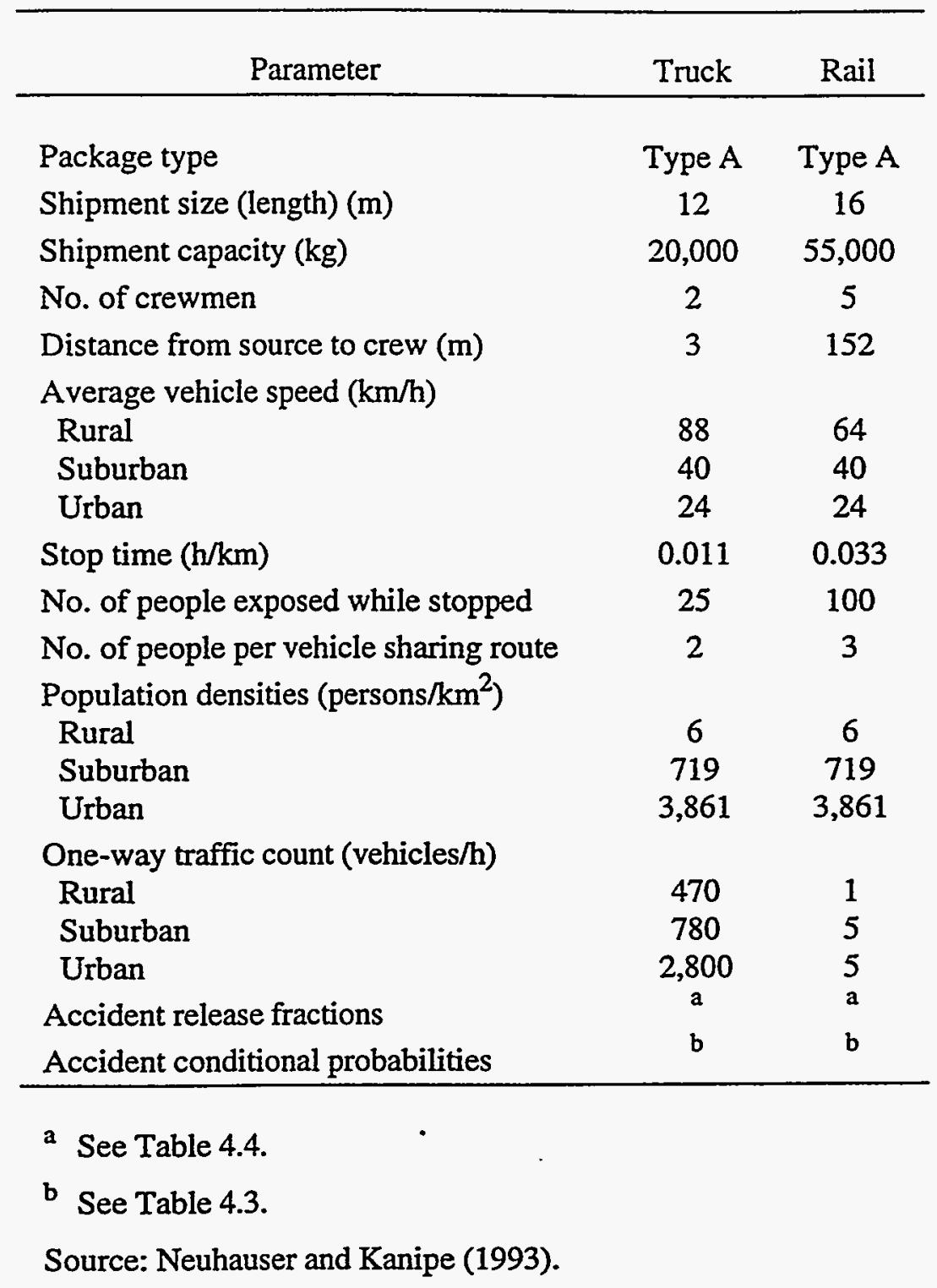


Table 4. Accident Dose Risk per Shipment (person-rem/shipment)

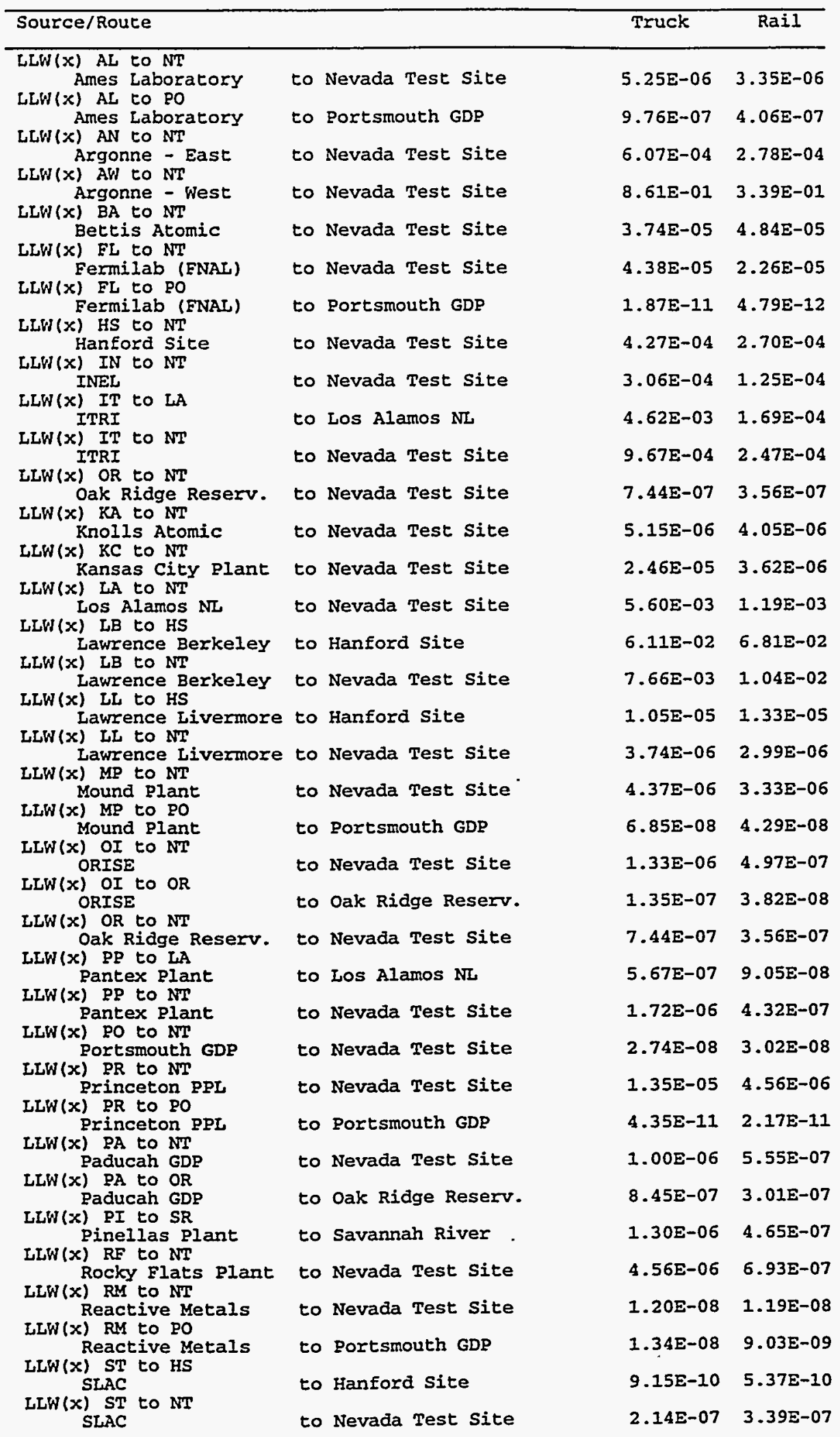




\section{$B-135$}

LLW(x) SA tO LA

SNL - Albuquerque to Los Alamos NL

LLW (x) SA to NT

SNL - Albuquerque to Nevada Test Site

LLW(x) SL to HS

SNL - Iivermore to Hanford Site

LLW (x) SL to NT

SNL - Livermore to Nevada Test Site

to Nevada Test Site

Savannah River

LLW $(x)$ OR to NT

Oak Ridge Reserv.

to Nevada Test Site

$1.33 E-05 \quad 2.52 E-07$

7.53E-06 2.01E-06

1.07E-04 5.69E-05

3.48E-05 2.74E-05

$5.34 E-03 \quad 2.62 E-03$

$7.44 \mathrm{E}-07 \quad 3.56 \mathrm{E}-07$ 
Table 5. Nonradiological Risk Eactors per Shipment (Eatalicies/shipment)

\begin{tabular}{|c|c|c|c|}
\hline Mode & Roule & Emission & Accident \\
\hline $\begin{array}{l}\text { Truck } \\
\text { Ames Laboratory } \\
\text { Ames Laboratory } \\
\text { Argonne - East } \\
\text { Argonne - ilest } \\
\text { Bettis Atomic } \\
\text { Fermilab (INAL) } \\
\text { Fermilab (FNAL) } \\
\text { Hanford Site } \\
\text { INEL } \\
\text { ITRI } \\
\text { ITRI } \\
\text { Oak Ridge Reserv. } \\
\text { Rnolis Atomic } \\
\text { Kansas City Plant } \\
\text { Los Alamos NL } \\
\text { Lawrence Berkeley } \\
\text { Lawrence Berkeley } \\
\text { Lawrence Livermore } \\
\text { Lawrence Livermore } \\
\text { Mound Plant } \\
\text { Mound Plant } \\
\text { ORISE } \\
\text { ORISE } \\
\text { Pantex Plant } \\
\text { Pantex Plant } \\
\text { Portsmouth GDP } \\
\text { Princeton PPL } \\
\text { Princeton PPL } \\
\text { Paducah GDP } \\
\text { Paducah GDP } \\
\text { Pinellas Plant } \\
\text { Rocky Flats PIant } \\
\text { Reactive Metals } \\
\text { Reactive Metals } \\
\text { SLAC } \\
\text { SLAC } \\
\text { SNL - Albuquerque } \\
\text { SNL - Albuquerque } \\
\text { SNL - Livermore } \\
\text { SNL - Livermore } \\
\text { Savannah River }\end{array}$ & $\begin{array}{l}\text { to Nevada Test Site } \\
\text { to Portsmouth GDP } \\
\text { to Nevada Test Site } \\
\text { to Nevada Test Site } \\
\text { to Nevada Test Site } \\
\text { to Nevada Test Site } \\
\text { to Portsmouth GDP } \\
\text { to Nevada Test Site } \\
\text { to Nevada Test Site } \\
\text { to Los Alamos NL } \\
\text { to Nevada Test Site } \\
\text { to Nevada Test Site } \\
\text { to Nevada Test Site } \\
\text { to Nevada Test Site } \\
\text { to Nevada Test Site } \\
\text { to Hanford Site } \\
\text { to Nevada Test Site } \\
\text { to Hanford Site } \\
\text { to Nevada Test Site } \\
\text { to Nevada Test Site } \\
\text { to Portsmouth GDP } \\
\text { to Nevada Test Site } \\
\text { to Oak Ridge Reserv. } \\
\text { to Los Alamos NL } \\
\text { to Nevada Test Site } \\
\text { to Nevada Test Site } \\
\text { to Nevada Test Site } \\
\text { to Portsmouth GDP } \\
\text { to Nevada Test Site } \\
\text { to Oak Ridge Reserv. } \\
\text { to Savannah River } \\
\text { to Nevada Test Site } \\
\text { to Nevada Test Site } \\
\text { to Portsmouth GDP } \\
\text { to Hanford Sitte } \\
\text { to Nevada Test Site } \\
\text { to Los Alamos NL } \\
\text { to Nevada Test Site } \\
\text { to Hanford Site } \\
\text { to Nevada Test Site } \\
\text { to Nevada Test Site }\end{array}$ & $\begin{array}{l}5.92 \mathrm{E}-06 \\
4.02 \mathrm{E}-06 \\
6.02 \mathrm{E}-06 \\
8.08 \mathrm{E}-06 \\
1.62 \mathrm{E}-05 \\
6.57 \mathrm{E}-06 \\
8.11 \mathrm{E}-06 \\
9.50 \mathrm{E}-06 \\
8.08 \mathrm{E}-06 \\
1.51 \mathrm{E}-06 \\
4.31 \mathrm{E}-06 \\
1.08 \mathrm{E}-05 \\
1.75 \mathrm{E}-05 \\
6.44 \mathrm{E}-06 \\
3.54 \mathrm{E}-06 \\
1.16 \mathrm{E}-05 \\
1.73 \mathrm{E}-05 \\
9.85 \mathrm{E}-06 \\
1.32 \mathrm{E}-05 \\
1.27 \mathrm{E}-05 \\
2.70 \mathrm{E}-06 \\
1.08 \mathrm{E}-05 \\
0.00 \mathrm{E}+00 \\
3.28 \mathrm{E}-06 \\
6.05 \mathrm{E}-06 \\
1.29 \mathrm{E}-05 \\
2.45 \mathrm{E}-05 \\
1.16 \mathrm{E}-05 \\
1.07 \mathrm{E}-05 \\
1.93 \mathrm{E}-07 \\
4.47 \mathrm{E}-06 \\
5.50 \mathrm{E}-06 \\
1.43 \mathrm{E}-05 \\
2.45 \mathrm{E}-06 \\
1.50 \mathrm{E}-05 \\
2.17 \mathrm{E}-05 \\
1.35 \mathrm{E}-06 \\
4.12 \mathrm{E}-06 \\
9.85 \mathrm{E}-06 \\
1.32 \mathrm{E}-05 \\
1.43 \mathrm{E}-05\end{array}$ & $\begin{array}{l}9.75 \mathrm{E}-05 \\
4.11 \mathrm{E}-05 \\
1.14 \mathrm{E}-04 \\
4.36 \mathrm{E}-05 \\
1.55 \mathrm{E}-04 \\
1.12 \mathrm{E}-04 \\
3.01 \mathrm{E}-05 \\
6.38 \mathrm{E}-05 \\
4.36 \mathrm{E}-05 \\
1.28 \mathrm{E}-05 \\
7.08 \mathrm{E}-05 \\
1.54 \mathrm{E}-04 \\
1.60 \mathrm{E}-04 \\
1.05 \mathrm{E}-04 \\
7.82 \mathrm{E}-05 \\
4.91 \mathrm{E}-05 \\
5.39 \mathrm{E}-05 \\
5.03 \mathrm{E}-05 \\
5.15 \mathrm{E}-05 \\
1.43 \mathrm{E}-04 \\
6.66 \mathrm{E}-06 \\
1.58 \mathrm{E}-04 \\
1.07 \mathrm{E}-06 \\
3.21 \mathrm{E}-05 \\
9.02 \mathrm{E}-05 \\
1.48 \mathrm{E}-04 \\
1.82 \mathrm{E}-04 \\
3.99 \mathrm{E}-05 \\
1.34 \mathrm{E}-04 \\
2.14 \mathrm{E}-05 \\
4.70 \mathrm{E}-05 \\
6.18 \mathrm{E}-05 \\
1.36 \mathrm{E}-04 \\
1.21 \mathrm{E}-05 \\
5.14 \mathrm{E}-05 \\
5.48 \mathrm{E}-05 \\
1.20 \mathrm{E}-05 \\
7.01 \mathrm{E}-05 \\
5.03 \mathrm{E}-05 \\
5.15 \mathrm{E}-05 \\
1.74 \mathrm{E}-04\end{array}$ \\
\hline $\begin{array}{l}\text { RaiI } \\
\text { Ames Laboratory } \\
\text { Ames Laboratory } \\
\text { Argonne - East } \\
\text { Argonne - West } \\
\text { Bettis Atomic } \\
\text { Fermilab (FNAI) } \\
\text { Fermilab (FNAI) } \\
\text { Hanford Site } \\
\text { INEL } \\
\text { ITRI } \\
\text { ITRI } \\
\text { Oak Ridge Reserv. } \\
\text { Knolls Atomic } \\
\text { Kansas City Plant } \\
\text { Los Alamos NL } \\
\text { Lawrence Berkeley } \\
\text { Lawrence Berkeley } \\
\text { Lawrence Livermore } \\
\text { Lawrence Livermore } \\
\text { Mound Plant } \\
\text { Mound Plant } \\
\text { ORISE } \\
\text { ORISE } \\
\text { Pantex Plant } \\
\text { Pantex Plant } \\
\text { Portsmouth GDP } \\
\text { Princeton PPL } \\
\text { Princeton PPL } \\
\text { Paducah GDP } \\
\text { Paducah GD } \\
\text { Pinellas Plant } \\
\text { Rocky Elats Plant }\end{array}$ & $\begin{array}{l}\text { to Nevada Test Site } \\
\text { to Portsmouth GDP } \\
\text { to Nevada Test Site } \\
\text { to Nevada Test Sitte } \\
\text { to Nevada Test Sitte } \\
\text { to Nevada Test Sitte } \\
\text { to Portsmouth GDP } \\
\text { to Nevada Test Site } \\
\text { to Nevada Test Site } \\
\text { to Ios Alamos NL } \\
\text { to Nevada Test Site } \\
\text { to Nevada Test Site } \\
\text { to Nevada Test Site } \\
\text { to Nevada Test Site } \\
\text { to Nevada Test Site } \\
\text { to Hanford Site } \\
\text { to Nevada Test Site } \\
\text { to Hanford Site } \\
\text { to Nevada Test Site } \\
\text { to Nevada Test Site } \\
\text { to Portsmouth GDP } \\
\text { to Nevada Test Site } \\
\text { to Oak Ridge Reserv. } \\
\text { to Los Alamos NL } \\
\text { to Nevada Test Site } \\
\text { to Nevada Test Site } \\
\text { to Nevada Test Site } \\
\text { to Portsmouth GDP } \\
\text { to Nevada Test Site } \\
\text { to Oak Ridge Reserv. } \\
\text { to Savannah River } \\
\text { to Nevada Test Site }\end{array}$ & $\begin{array}{l}6.07 \mathrm{E}-06 \\
2.34 \mathrm{E}-05 \\
7.99 \mathrm{E}-06 \\
3.98 \mathrm{E}-06 \\
4.14 \mathrm{E}-05 \\
9.67 \mathrm{E}-06 \\
1.80 \mathrm{E}-05 \\
5.90 \mathrm{E}-06 \\
3.98 \mathrm{E}-06 \\
2.47 \mathrm{E}-06 \\
3.85 \mathrm{E}-06 \\
1.52 \mathrm{E}-05 \\
4.79 \mathrm{E}-05 \\
7.24 \mathrm{E}-06 \\
6.32 \mathrm{E}-06 \\
2.33 \mathrm{E}-05 \\
2.59 \mathrm{E}-05 \\
2.36 \mathrm{E}-05 \\
1.01 \mathrm{E}-05 \\
1.84 \mathrm{E}-05 \\
5.73 \mathrm{E}-06 \\
1.73 \mathrm{E}-05 \\
1.26 \mathrm{E}-07 \\
2.59 \mathrm{E}-06 \\
3.98 \mathrm{E}-06 \\
2.95 \mathrm{E}-05 \\
5.45 \mathrm{E}-05 \\
3.18 \mathrm{E}-05 \\
1.29 \mathrm{E}-05 \\
3.81 \mathrm{E}-06 \\
5.69 \mathrm{E}-06 \\
8.37 \mathrm{E}-07\end{array}$ & $\begin{array}{l}3.50 \mathrm{E}-06 \\
1.52 \mathrm{E}-06 \\
4.91 \mathrm{E}-06 \\
1.58 \mathrm{E}-06 \\
5.22 \mathrm{E}-06 \\
4.18 \mathrm{E}-06 \\
9.43 \mathrm{E}-07 \\
2.72 \mathrm{E}-06 \\
1.58 \mathrm{E}-06 \\
2.18 \mathrm{E}-07 \\
2.23 \mathrm{E}-06 \\
5.20 \mathrm{E}-06 \\
5.86 \mathrm{E}-06 \\
3.49 \mathrm{E}-06 \\
2.45 \mathrm{E}-06 \\
2.06 \mathrm{E}-06 \\
1.80 \mathrm{E}-06 \\
2.04 \mathrm{E}-06 \\
2.87 \mathrm{E}-06 \\
4.99 \mathrm{E}-06 \\
3.27 \mathrm{E}-07 \\
5.29 \mathrm{E}-06 \\
8.26 \mathrm{E}-08 \\
1.01 \mathrm{E}-06 \\
2.88 \mathrm{E}-06 \\
5.02 \mathrm{E}-06 \\
6.01 \mathrm{E}-06 \\
1.75 \mathrm{E}-06 \\
4.46 \mathrm{E}-06 \\
1.10 \mathrm{E}-06 \\
1.01 \mathrm{E}-06 \\
2.07 \mathrm{E}-06\end{array}$ \\
\hline
\end{tabular}




Reactive Metals
Reactive Metals
SLAC
SLAC
SNL - Albuquerque
SNL - Albuquerque
SNL - Livermore
SNL - Livermore
Savannah River

Reactive Metals

SLAC

SNL - Albuquerque

Savannah River

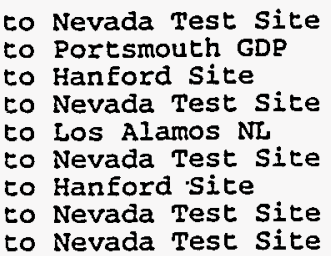

co Nevada Test Site to Portsmouth GDP to Hanford Site to Nevada Test Site to Los Alamos NL to Nevada Test Site to Hanford Site to Nevada Test Site to Nevada Test Site
3. $72 E-05$
5. $00 \mathrm{E}-06$
$1.14 \mathrm{E}-05$
5. $84 \mathrm{E}-07$
. 07E-05
2.35E-05
2. $47 \mathrm{E}-06$
$2.17 \mathrm{E}-06$
1. $80 \mathrm{E}-06$
2.18E-07
3.85E-06 2.23E-05
2.36E-05 2.04E-06
1.01E-05 2.87E-06
2.27E-05 5.94E-06

Table 6. Annual Incident-Free Dose for the Shipping Campaign (person-rem/yr)

\begin{tabular}{|c|c|c|c|c|c|c|}
\hline \multirow[b]{2}{*}{ Route } & & & \multicolumn{2}{|c|}{ Truck } & \multicolumn{2}{|l|}{ Rail } \\
\hline & & & Crew & Public & Crew & Public \\
\hline 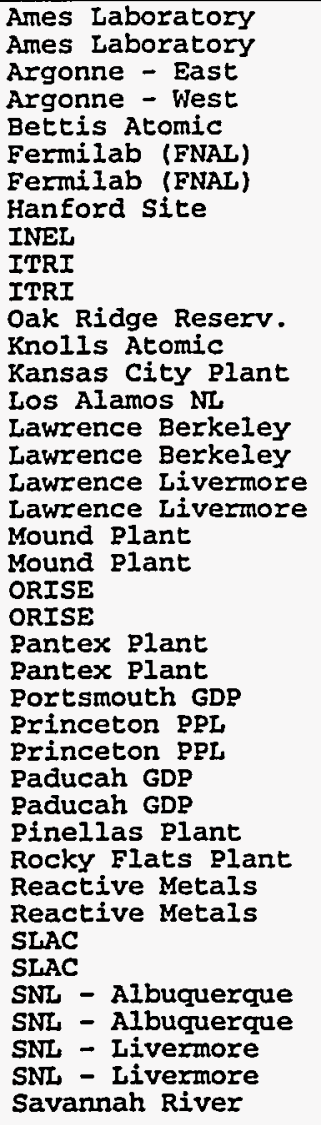 & $\begin{array}{l}\text { to } \\
\text { to } \\
\text { to } \\
\text { to } \\
\text { to } \\
\text { to } \\
\text { to } \\
\text { to } \\
\text { to } \\
\text { to } \\
\text { to } \\
\text { to } \\
\text { to } \\
\text { to } \\
\text { to } \\
\text { to } \\
\text { to } \\
\text { to } \\
\text { to } \\
\text { to } \\
\text { to } \\
\text { to } \\
\text { to } \\
\text { to } \\
\text { to } \\
\text { to } \\
\text { to } \\
\text { to } \\
\text { to } \\
\text { to } \\
\text { to } \\
\text { to } \\
\text { to } \\
\text { to } \\
\text { to } \\
\text { to } \\
\text { to } \\
\text { to } \\
\text { to } \\
\text { to } \\
\text { to }\end{array}$ & $\begin{array}{l}\text { Nevada Test Site } \\
\text { Portsmouth GDP } \\
\text { Nevada Test Site } \\
\text { Nevada Test Site } \\
\text { Nevada Test Site } \\
\text { Nevada Test Site } \\
\text { Portsmouth GDP } \\
\text { Nevada Test Site } \\
\text { Nevada Test Site } \\
\text { Los Alamos NL } \\
\text { Nevada Test Site } \\
\text { Nevada Test Site } \\
\text { Nevada Test Site } \\
\text { Nevada Test Site } \\
\text { Nevada Test Site } \\
\text { Hanford Site } \\
\text { Nevada Test Site } \\
\text { Hanford Site } \\
\text { Nevada Test Site } \\
\text { Nevada Test Site } \\
\text { Portsmouth GDP } \\
\text { Nevada Test Site } \\
\text { Oak Ridge Reserv. } \\
\text { Los Alamos NL } \\
\text { Nevada Test Site } \\
\text { Nevada Test Site } \\
\text { Nevada Test Site } \\
\text { Portsmouth GDP } \\
\text { Nevada Test Site } \\
\text { Oak Ridge Reserv. } \\
\text { Savannah River } \\
\text { Nevada Test Site } \\
\text { Nevada Test Site } \\
\text { Portsmouth GDP } \\
\text { Hanford Site } \\
\text { Nevada Test Site } \\
\text { Los Alamos NL } \\
\text { Nevada Test Site } \\
\text { Hanford Site } \\
\text { Nevada Test Site } \\
\text { Nevada Test Site }\end{array}$ & $\begin{array}{l}8.06 \mathrm{E}-02 \\
2.33 \mathrm{E}-02 \\
5.10 \mathrm{E}+00 \\
2.13 \mathrm{E}-01 \\
3.85 \mathrm{E}+00 \\
9.63 \mathrm{E}-02 \\
1.68 \mathrm{E}-02 \\
4.39 \mathrm{E}+01 \\
1.75 \mathrm{E}+01 \\
7.08 \mathrm{E}-03 \\
2.16 \mathrm{E}-01 \\
3.67 \mathrm{E}+02 \\
2.20 \mathrm{E}+00 \\
3.84 \mathrm{E}-02 \\
5.12 \mathrm{E}+01 \\
5.51 \mathrm{E}-02 \\
4.54 \mathrm{E}-02 \\
4.79 \mathrm{E}-01 \\
8.22 \mathrm{E}-01 \\
2.89 \mathrm{E}+01 \\
7.00 \mathrm{E}-02 \\
2.46 \mathrm{E}-01 \\
3.63 \mathrm{E}-04 \\
1.51 \mathrm{E}+01 \\
7.89 \mathrm{E}-01 \\
1.82 \mathrm{E}+02 \\
8.05 \mathrm{E}-02 \\
2.28 \mathrm{E}-02 \\
3.01 \mathrm{E}+01 \\
5.06 \mathrm{E}-01 \\
4.04 \mathrm{E}-02 \\
7.71 \mathrm{E}+00 \\
4.72 \mathrm{E}+01 \\
7.02 \mathrm{E}-02 \\
2.98 \mathrm{E}-02 \\
9.46 \mathrm{E}-01 \\
3.28 \mathrm{E}-03 \\
4.03 \mathrm{E}-01 \\
2.82 \mathrm{E}-02 \\
1.03 \mathrm{E}-01 \\
4.77 \mathrm{E}+02\end{array}$ & $\begin{array}{l}9.92 \mathrm{E}-02 \\
2.63 \mathrm{E}-02 \\
6.20 \mathrm{E}+00 \\
2.58 \mathrm{E}-01 \\
4.54 \mathrm{E}+00 \\
1.18 \mathrm{E}-01 \\
1.87 \mathrm{E}-02 \\
5.35 \mathrm{E}+01 \\
2.11 \mathrm{E}+01 \\
8.36 \mathrm{E}-03 \\
2.70 \mathrm{E}-01 \\
4.38 \mathrm{E}+02 \\
2.56 \mathrm{E}+00 \\
4.70 \mathrm{E}-02 \\
6.34 \mathrm{E}+01 \\
6.54 \mathrm{E}-02 \\
5.82 \mathrm{E}-02 \\
5.58 \mathrm{E}-01 \\
1.05 \mathrm{E}+00 \\
3.46 \mathrm{E}+01 \\
7.58 \mathrm{E}-02 \\
2.93 \mathrm{E}-01 \\
3.60 \mathrm{E}-04 \\
1.87 \mathrm{E}+01 \\
9.90 \mathrm{E}-01 \\
2.17 \mathrm{E}+02 \\
9.41 \mathrm{E}-02 \\
2.48 \mathrm{E}-02 \\
3.64 \mathrm{E}+01 \\
5.69 \mathrm{E}-01 \\
4.48 \mathrm{E}-02 \\
9.47 \mathrm{E}+00 \\
5.64 \mathrm{E}+01 \\
7.37 \mathrm{E}-02 \\
3.54 \mathrm{E}-02 \\
1.23 \mathrm{E}+00 \\
3.84 \mathrm{E}-03 \\
5.04 \mathrm{E}-01 \\
3.28 \mathrm{E}-02 \\
1.31 \mathrm{E}-01 \\
5.60 \mathrm{E}+02\end{array}$ & $\begin{array}{l}9.87 \mathrm{E}-03 \\
5.91 \mathrm{E}-03 \\
4.95 \mathrm{E}-01 \\
2.41 \mathrm{E}-02 \\
2.79 \mathrm{E}-01 \\
1.12 \mathrm{E}-02 \\
4.76 \mathrm{E}-03 \\
4.32 \mathrm{E}+00 \\
1.90 \mathrm{E}+00 \\
3.31 \mathrm{E}-03 \\
2.20 \mathrm{E}-02 \\
3.21 \mathrm{E}+01 \\
1.60 \mathrm{E}-01 \\
9.85 \mathrm{E}-03 \\
5.93 \mathrm{E}+00 \\
6.99 \mathrm{E}-03 \\
6.47 \mathrm{E}-03 \\
4.86 \mathrm{E}-02 \\
1.29 \mathrm{E}-01 \\
2.39 \mathrm{E}+00 \\
1.77 \mathrm{E}-02 \\
2.69 \mathrm{E}-02 \\
3.05 \mathrm{E}-03 \\
2.58 \mathrm{E}+00 \\
8.62 \mathrm{E}-02 \\
1.43 \mathrm{E}+01 \\
1.49 \mathrm{E}-02 \\
6.38 \mathrm{E}-03 \\
2.53 \mathrm{E}+00 \\
1.07 \mathrm{E}-01 \\
4.90 \mathrm{E}-03 \\
8.61 \mathrm{E}-01 \\
3.64 \mathrm{E}+00 \\
1.21 \mathrm{E}-02 \\
7.20 \mathrm{E}-03 \\
9.72 \mathrm{E}-02 \\
3.31 \mathrm{E}-03 \\
5.13 \mathrm{E}-02 \\
6.94 \mathrm{E}-03 \\
1.72 \mathrm{E}-02 \\
3.71 \mathrm{E}+01\end{array}$ & $\begin{array}{l}1.34 \mathrm{E}-02 \\
2.01 \mathrm{E}-02 \\
6.97 \mathrm{E}-01 \\
3.47 \mathrm{E}-02 \\
8.05 \mathrm{E}-01 \\
1.71 \mathrm{E}-02 \\
1.58 \mathrm{E}-02 \\
6.24 \mathrm{E}+00 \\
2.73 \mathrm{E}+00 \\
5.37 \mathrm{E}-03 \\
2.91 \mathrm{E}-02 \\
5.38 \mathrm{E}+01 \\
4.89 \mathrm{E}-01 \\
1.37 \mathrm{E}-02 \\
8.86 \mathrm{E}+00 \\
2.05 \mathrm{E}-02 \\
2.12 \mathrm{E}-02 \\
1.43 \mathrm{E}-01 \\
2.03 \mathrm{E}-01 \\
4.60 \mathrm{E}+00 \\
3.92 \mathrm{E}-02 \\
4.90 \mathrm{E}-02 \\
3.95 \mathrm{E}-03 \\
3.80 \mathrm{E}+00 \\
1.10 \mathrm{E}-01 \\
3.33 \mathrm{E}+01 \\
4.92 \mathrm{E}-02 \\
2.64 \mathrm{E}-02 \\
4.23 \mathrm{E}+00 \\
1.66 \mathrm{E}-01 \\
1.02 \mathrm{E}-02 \\
9.69 \mathrm{E}-01 \\
9.83 \mathrm{E}+00 \\
3.61 \mathrm{E}-02 \\
2.44 \mathrm{E}-02 \\
3.02 \mathrm{E}-01 \\
5.37 \mathrm{E}-03 \\
6.78 \mathrm{E}-02 \\
2.05 \mathrm{E}-02 \\
2.71 \mathrm{E}-02 \\
7.85 \mathrm{E}+01\end{array}$ \\
\hline Totals & & & $1.28 \mathrm{E}+03$ & $1.53 E+03$ & $1.09 \mathrm{E}+02$ & $2.10 \mathrm{E}+02$ \\
\hline
\end{tabular}


Table 7. Annual Accident Dose Risk for the Shipping Campaign (person-rem/yr)

\begin{tabular}{|c|c|c|c|}
\hline Route & & Truck & Rail \\
\hline 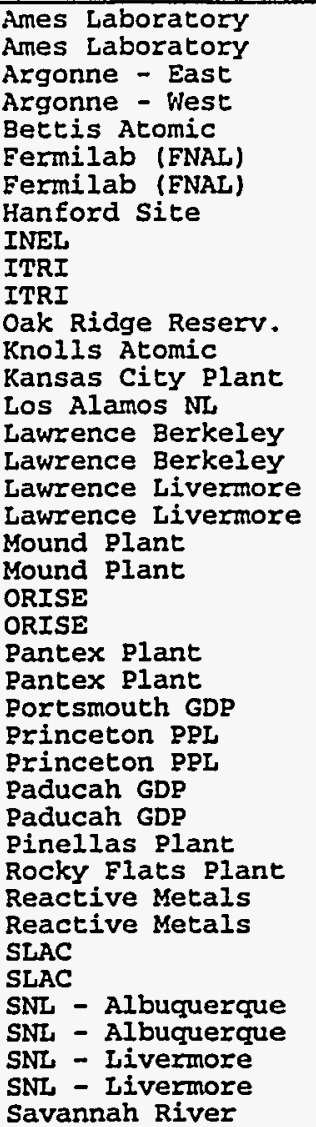 & $\begin{array}{l}\text { to Nevada Test Site } \\
\text { to Portsmouth GDP } \\
\text { to Nevada Test Site } \\
\text { to Nevada Test Site } \\
\text { to Nevada Test Site } \\
\text { to Nevada Test Site } \\
\text { to Portsmouth GDP } \\
\text { to Nevada Test Site } \\
\text { to Nevada Test Site } \\
\text { to Los Alamos NL } \\
\text { to Nevada Test Site } \\
\text { to Nevada Test Site } \\
\text { to Nevada Test Site } \\
\text { to Nevada Test Site } \\
\text { to Nevada Test Site } \\
\text { to Hanford Site } \\
\text { to Nevada Test Site } \\
\text { to Hanford Site } \\
\text { to Nevada Test Site } \\
\text { to Nevada Test Site } \\
\text { to Portsmouth GDP } \\
\text { to Nevada Test Site } \\
\text { to Oak Ridge Reserv. } \\
\text { to Los Alamos NL } \\
\text { to Nevada Test Site } \\
\text { to Nevada Test Site } \\
\text { to Nevada Test Site } \\
\text { to Portsmouth GDP } \\
\text { to Nevada Test Site } \\
\text { to Oak Ridge Reserv. } \\
\text { to Savannah River } \\
\text { to Nevada Test Site } \\
\text { to Nevada Test Site } \\
\text { to Portsmouth GDP } \\
\text { to Hanford Site } \\
\text { to Nevada Test Site } \\
\text { to Los Alamos NL } \\
\text { to Nevada Test Site } \\
\text { to Hanford Site } \\
\text { to Nevada Test Site } \\
\text { to Nevada Test Site }\end{array}$ & $\begin{array}{l}1.05 \mathrm{E}-05 \\
9.76 \mathrm{E}-07 \\
6.37 \mathrm{E}-02 \\
8.61 \mathrm{E}+00 \\
2.13 \mathrm{E}-03 \\
8.76 \mathrm{E}-05 \\
1.87 \mathrm{E}-11 \\
5.82 \mathrm{E}-01 \\
2.51 \mathrm{E}-01 \\
9.23 \mathrm{E}-03 \\
8.70 \mathrm{E}-03 \\
4.51 \mathrm{E}-03 \\
1.44 \mathrm{E}-04 \\
2.46 \mathrm{E}-05 \\
1.10 \mathrm{E}+01 \\
1.22 \mathrm{E}-01 \\
1.53 \mathrm{E}-02 \\
1.79 \mathrm{E}-04 \\
1.49 \mathrm{E}-04 \\
2.17 \mathrm{E}-03 \\
8.22 \mathrm{E}-07 \\
5.33 \mathrm{E}-06 \\
1.35 \mathrm{E}-07 \\
7.70 \mathrm{E}-04 \\
4.29 \mathrm{E}-05 \\
8.08 \mathrm{E}-05 \\
1.35 \mathrm{E}-05 \\
4.35 \mathrm{E}-11 \\
5.80 \mathrm{E}-04 \\
4.82 \mathrm{E}-05 \\
2.59 \mathrm{E}-06 \\
1.53 \mathrm{E}-03 \\
8.98 \mathrm{E}-06 \\
9.38 \mathrm{E}-08 \\
9.15 \mathrm{E}-10 \\
8.33 \mathrm{E}-06 \\
1.33 \mathrm{E}-05 \\
1.28 \mathrm{E}-04 \\
1.07 \mathrm{E}-04 \\
1.74 \mathrm{E}-04 \\
3.61 \mathrm{E}+01\end{array}$ & $\begin{array}{l}3.35 \mathrm{E}-06 \\
4.06 \mathrm{E}-07 \\
1.08 \mathrm{E}-02 \\
1.36 \mathrm{E}+00 \\
1.02 \mathrm{E}-03 \\
2.26 \mathrm{E}-05 \\
4.79 \mathrm{E}-12 \\
1.41 \mathrm{E}-01 \\
3.95 \mathrm{E}-02 \\
1.69 \mathrm{E}-04 \\
7.41 \mathrm{E}-04 \\
8.64 \mathrm{E}-04 \\
4.46 \mathrm{E}-05 \\
3.62 \mathrm{E}-06 \\
9.12 \mathrm{E}-01 \\
6.81 \mathrm{E}-02 \\
1.04 \mathrm{E}-02 \\
9.34 \mathrm{E}-05 \\
4.49 \mathrm{E}-05 \\
6.19 \mathrm{E}-04 \\
2.15 \mathrm{E}-07 \\
9.93 \mathrm{E}-07 \\
3.82 \mathrm{E}-08 \\
4.78 \mathrm{E}-05 \\
4.32 \mathrm{E}-06 \\
3.34 \mathrm{E}-05 \\
4.56 \mathrm{E}-06 \\
2.17 \mathrm{E}-11 \\
1.19 \mathrm{E}-04 \\
6.32 \mathrm{E}-06 \\
4.65 \mathrm{E}-07 \\
8.53 \mathrm{E}-05 \\
3.38 \mathrm{E}-06 \\
2.71 \mathrm{E}-08 \\
5.37 \mathrm{E}-10 \\
5.08 \mathrm{E}-06 \\
2.52 \mathrm{E}-07 \\
1.40 \mathrm{E}-05 \\
5.69 \mathrm{E}-05 \\
5.48 \mathrm{E}-05 \\
6.62 \mathrm{E}+00\end{array}$ \\
\hline & & & 9.1 \\
\hline
\end{tabular}

Table 8. Expected Annual Fatalities

for the Shipping Campaign

\begin{tabular}{llc}
\hline Exposure Group & Truck & Rail \\
\hline Radiological & & \\
Normal Crew & $5.1 E-01$ & $4.4 E-02$ \\
Normal Public & $7.6 \mathrm{E}-01$ & $1.1 \mathrm{E}-01$ \\
Accident Public & $2.8 \mathrm{E}-02$ & $4.6 \mathrm{E}-03$ \\
Nonradiological & & \\
Emission & $2.6 \mathrm{E}-01$ & $1.6 \mathrm{E}-01$ \\
Accident & $3.2 \mathrm{E}+00$ & $4.2 \mathrm{E}-02$ \\
\hline
\end{tabular}

Table 9. Expected Annual Cancer Incidence for the Shipping Campaign

\begin{tabular}{lll}
\hline Exposure Group & Truck & Rail \\
\hline Radiological & & \\
Normal Crew & $1.8 \mathrm{E}+00$ & $1.5 \mathrm{E}-01$ \\
Normal Public & $2.6 \mathrm{E}+00$ & $3.6 \mathrm{E}-01$ \\
Accident Public & $9.6 \mathrm{E}-02$ & $1.6 \mathrm{E}-02$ \\
Nonradiological & $2.6 \mathrm{E}-01$ & $1.6 \mathrm{E}-01$ \\
Emission & $\mathrm{NA}$ & $\mathrm{NA}$ \\
\hline Accident & & \\
\hline
\end{tabular}


Table 10. Expected Annual Genetic Effects for the Shipping Campaign

\begin{tabular}{lll}
\hline Exposure Group & Truck & Rail \\
\hline Radiological & & \\
Normal Crew & $7.7 \mathrm{E}-02$ & $6.6 \mathrm{E}-03$ \\
Normal Public & $1.5 \mathrm{E}-01$ & $2.1 \mathrm{E}-02$ \\
Accident Public & $5.4 \mathrm{E}-03$ & $8.6 \mathrm{E}-04$ \\
Nonradiological & NA & NA \\
Enission & NA & NA \\
Accident & & \\
\hline
\end{tabular}




\section{B.26 WM LLW CENTRALIZED 4 ALTERNATIVE (CASE 14A): ACTIVATED METALS}

Table 1. Summary of Route Information

\begin{tabular}{|c|c|c|c|c|c|}
\hline & \multirow[b]{2}{*}{ Route } & \multirow{2}{*}{$\begin{array}{l}\text { Distance } \\
\text { (miles) }\end{array}$} & \multicolumn{3}{|c|}{ Percentage in Zone } \\
\hline & & & Rural & Suburban & Urban \\
\hline $\begin{array}{l}\text { Truck } \\
\text { Bettis Atomic } \\
\text { Fermilab (FNAL) } \\
\text { Hanford Site } \\
\text { INEL } \\
\text { Knolis Atomic } \\
\text { Los Alamos NL } \\
\text { Lawrence Berkeley } \\
\text { Portsmouth GDP } \\
\text { SNL - Albuquerque }\end{array}$ & $\begin{array}{l}\text { to Portsmouth GDP } \\
\text { to Portsmouth GDP } \\
\text { to Nevada Test Site } \\
\text { to Nevada Test Site } \\
\text { to Portsmouth GDP } \\
\text { to Nevada Test Site } \\
\text { to Hanford Site } \\
\text { to Nevada Test Site } \\
\text { to Los Alamos NL }\end{array}$ & $\begin{array}{r}265 \\
460 \\
1128 \\
712 \\
688 \\
997 \\
875 \\
2158 \\
102\end{array}$ & $\begin{array}{l}58.8 \\
62.5 \\
86.5 \\
82.8 \\
57.3 \\
93.2 \\
78.1 \\
85.5 \\
75.8\end{array}$ & $\begin{array}{r}37.4 \\
32.0 \\
10.9 \\
13.7 \\
40.1 \\
5.7 \\
17.8 \\
12.6 \\
20.1\end{array}$ & $\begin{array}{l}3.8 \\
5.5 \\
2.6 \\
3.5 \\
2.5 \\
1.1 \\
4.1 \\
1.9 \\
4.1\end{array}$ \\
\hline $\begin{array}{l}\text { Bettis Atomic } \\
\text { Fermilab (FNAL) } \\
\text { Hanford Site } \\
\text { INEL } \\
\text { Knolls Atomic } \\
\text { Los Alamos NL } \\
\text { Lawrence Berkeley } \\
\text { Portsmouth GDP } \\
\text { SNL - Albuquerque }\end{array}$ & $\begin{array}{l}\text { to Portsmouth GDP } \\
\text { to Portsmouth GDP } \\
\text { to Nevada Test Site } \\
\text { to Nevada Test Site } \\
\text { to Portsmouth GDP } \\
\text { to Nevada Test Site } \\
\text { to Hanford Site } \\
\text { to Nevada Test Site } \\
\text { to Los Alamos NL }\end{array}$ & $\begin{array}{r}429 \\
451 \\
1302 \\
756 \\
681 \\
1169 \\
986 \\
2401 \\
104\end{array}$ & $\begin{array}{l}52.6 \\
61.5 \\
93.0 \\
92.8 \\
52.1 \\
92.8 \\
78.5 \\
86.3 \\
74.3\end{array}$ & $\begin{array}{r}39.6 \\
28.9 \\
5.9 \\
5.9 \\
40.3 \\
5.9 \\
15.8 \\
10.7 \\
20.0\end{array}$ & $\begin{array}{l}7.8 \\
9.5 \\
1.1 \\
1.3 \\
7.5 \\
1.3 \\
5.7 \\
2.9 \\
5.7\end{array}$ \\
\hline
\end{tabular}

Table 2. Annual Number of Shipments and One-Way Shipment Distances

\begin{tabular}{|c|c|c|c|c|c|}
\hline \multirow[b]{2}{*}{ Route } & & \multicolumn{2}{|c|}{ \# of Shipments } & \multicolumn{2}{|c|}{ Total Mileage (mi) } \\
\hline & & Truck & Rail & Truck & Rail \\
\hline $\begin{array}{l}\text { Bettis Atomic } \\
\text { Fermilab (FNAL) } \\
\text { Hanford Site } \\
\text { INEL } \\
\text { Knolls Atomic } \\
\text { Los Alamos NL } \\
\text { Lawrence Berkeley } \\
\text { Portsmouth GDP } \\
\text { SNL - Albuquerque }\end{array}$ & $\begin{array}{l}\text { to Portsmouth GDP } \\
\text { to Portsmouth GDP } \\
\text { to Nevada Test Site } \\
\text { to Nevada Test Site } \\
\text { to Portsmouth GDP } \\
\text { to Nevada Pest Site } \\
\text { to Hanford Site } \\
\text { to Nevada Test Site } \\
\text { to Los Alamos NL }\end{array}$ & $\begin{array}{r}309 \\
39 \\
37 \\
365 \\
639 \\
332 \\
14 \\
785 \\
6\end{array}$ & $\begin{array}{r}119 \\
16 \\
16 \\
157 \\
245 \\
143 \\
6 \\
337 \\
3\end{array}$ & $\begin{array}{l}8.19 E+04 \\
1.79 E+04 \\
4.17 E+04 \\
2.60 E+05 \\
4.40 E+05 \\
3.31 E+05 \\
1.22 E+04 \\
1.69 E+06 \\
6.12 E+02\end{array}$ & $\begin{array}{l}5.11 \mathrm{E}+04 \\
7.21 \mathrm{E}+03 \\
2.08 \mathrm{E}+04 \\
1.19 \mathrm{E}+05 \\
1.67 \mathrm{E}+05 \\
1.67 \mathrm{E}+05 \\
5.91 \mathrm{E}+03 \\
8.09 \mathrm{E}+05 \\
3.12 \mathrm{E}+02\end{array}$ \\
\hline Totals & & 2526 & 1042 & $2.88 \mathrm{E}+06$ & $1.35 \mathrm{E}+06$ \\
\hline
\end{tabular}


Table 3. Incident-Free Dose per Shipment (person-rem/shipment)

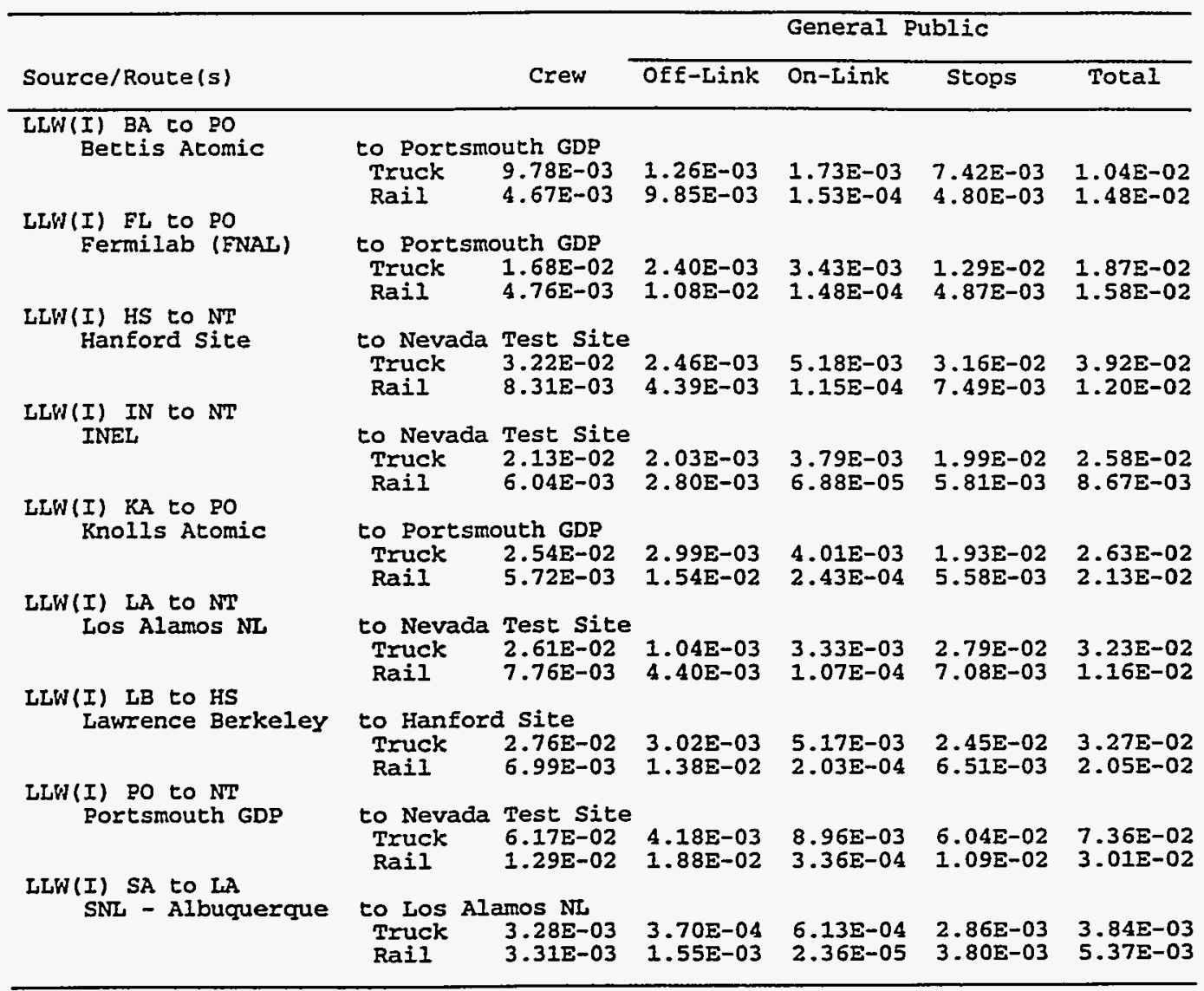

Table 4. Accident Dose Risk per Shipment (person-rem/shipment)

\begin{tabular}{|c|c|c|c|}
\hline Source/Route & & Truck & Rail \\
\hline $\begin{array}{l}\text { LLW(I) BA to PO } \\
\text { Bettis Atomic } \\
\text { LLW(I) FL to PO } \\
\text { Fermilab (FNAL) } \\
\text { LLW(I) HS to NT } \\
\text { Hanford Site } \\
\text { LLW(I) IN to NI } \\
\text { INEL to } \\
\text { LLW(I) RA to PO } \\
\text { Rnolls Atomic } \\
\text { LLW(I) LA to NT } \\
\text { LOS Alamos NL } \\
\text { LLW(I) LB to HS } \\
\text { Lawrence Berkeley } \\
\text { LLW(I) PO to NT } \\
\text { Portsmouth GDP } \\
\text { LLW(I) SA to LA } \\
\text { SNL - Albuquerque }\end{array}$ & $\begin{array}{l}\text { to Portsmouth GDP } \\
\text { to Portsmouth GDP } \\
\text { to Nevada Test Site } \\
\text { to Nevada Test Site } \\
\text { to Portsmouth GDP } \\
\text { to Nevada Test Site } \\
\text { to Hanford Site } \\
\text { to Nevada Test Site } \\
\text { to Los Alamos NL }\end{array}$ & $\begin{array}{l}3.70 \mathrm{E}-07 \\
5.29 \mathrm{E}-11 \\
2.43 \mathrm{E}-08 \\
1.52 \mathrm{E}-07 \\
1.66 \mathrm{E}-06 \\
2.25 \mathrm{E}-09 \\
1.81 \mathrm{E}-10 \\
2.31 \mathrm{E}-06 \\
1.72 \mathrm{E}-12\end{array}$ & $\begin{array}{l}4.16 \mathrm{E}-07 \\
3.35 \mathrm{E}-11 \\
1.28 \mathrm{E}-08 \\
5.55 \mathrm{E}-08 \\
1.02 \mathrm{E}-06 \\
3.42 \mathrm{E}-10 \\
2.37 \mathrm{E}-10 \\
1.73 \mathrm{E}-06 \\
6.51 \mathrm{E}-14\end{array}$ \\
\hline
\end{tabular}


Table 5. Nonradiological Risk Factors per Shipment (fatalities/shipment)

\begin{tabular}{|c|c|c|c|}
\hline Mode & Route & Emission & Accident \\
\hline $\begin{array}{l}\text { Truck } \\
\text { Bettis Atomic } \\
\text { Fermilab (FNAL) } \\
\text { Hanford Site } \\
\text { INEL } \\
\text { Knolis Atomic } \\
\text { Los Alamos NL } \\
\text { Lawrence Berkeley } \\
\text { Portsmouth GDP } \\
\text { SNL - Albuquerque } \\
\text { Rail } \\
\text { Bettis Atomic } \\
\text { Fermilab (FNAL) } \\
\text { Hanford Site } \\
\text { INEL } \\
\text { Knolls Atomic } \\
\text { Los Alamos NL } \\
\text { Lawrence Berkeley } \\
\text { Portsmouth GDP } \\
\text { SNL - Albuquerque }\end{array}$ & $\begin{array}{l}\text { to Portsmouth GDP } \\
\text { to Portsmouth GDP } \\
\text { to Nevada Test Site } \\
\text { to Nevada Test Site } \\
\text { to Portsmouth GDP } \\
\text { to Nevada Test Site } \\
\text { to Hanford Site } \\
\text { to Nevada Test Site } \\
\text { to Los Alamos NL } \\
\text { to Portsmouth GDP } \\
\text { to Portsmouth GDP } \\
\text { to Nevada Test Site } \\
\text { to Nevada Test Site } \\
\text { to Portsmouth GDP } \\
\text { to Nevada Test Site } \\
\text { to Hanford Site } \\
\text { to Nevada Test Site } \\
\text { to Los Alamos NL }\end{array}$ & $\begin{array}{l}3.22 \mathrm{E}-06 \\
8.11 \mathrm{E}-06 \\
9.50 \mathrm{E}-06 \\
8.08 \mathrm{E}-06 \\
5.63 \mathrm{E}-06 \\
3.54 \mathrm{E}-06 \\
1.16 \mathrm{E}-05 \\
1.29 \mathrm{E}-05 \\
1.35 \mathrm{E}-06 \\
1.40 \mathrm{E}-05 \\
1.80 \mathrm{E}-05 \\
5.90 \mathrm{E}-06 \\
3.98 \mathrm{E}-06 \\
2.15 \mathrm{E}-05 \\
6.32 \mathrm{E}-06 \\
2.33 \mathrm{E}-05 \\
2.95 \mathrm{E}-05 \\
2.47 \mathrm{E}-06\end{array}$ & $\begin{array}{l}1.33 E-05 \\
3.01 E-05 \\
6.38 E-05 \\
4.36 E-05 \\
3.56 E-05 \\
7.82 E-05 \\
4.91 E-05 \\
1.48 E-04 \\
1.20 E-05 \\
8.98 E-07 \\
9.43 E-07 \\
2.72 E-06 \\
1.58 E-06 \\
1.43 E-06 \\
2.45 E-06 \\
2.06 E-06 \\
5.02 E-06 \\
2.18 E-07\end{array}$ \\
\hline
\end{tabular}

Table 6. Annual Incident-Free Dose for the Shipping Campaign (person-rem/yr)

\begin{tabular}{|c|c|c|c|c|c|}
\hline \multirow[b]{2}{*}{ Route } & & \multicolumn{2}{|c|}{ Truck } & \multicolumn{2}{|c|}{ Rail } \\
\hline & & Crew & Public & Crew & Public \\
\hline $\begin{array}{l}\text { Bettis Atomic } \\
\text { Fermilab (FNAL) } \\
\text { Hanford Site } \\
\text { INEL } \\
\text { Knolis Atomic } \\
\text { Los Alamos NL } \\
\text { Lawrence Berkeley } \\
\text { Portsmouth GDP } \\
\text { SNL - Albuquerque }\end{array}$ & $\begin{array}{l}\text { to Portsmouth GDP } \\
\text { to Portsmouth GDP } \\
\text { to Nevada Test Site } \\
\text { to Nevada Test Site } \\
\text { to Portsmouth GDP } \\
\text { to Nevada Test Site } \\
\text { to Hanford Site } \\
\text { to Nevada Test Site } \\
\text { to Los Alamos NL }\end{array}$ & $\begin{array}{l}3.02 \mathrm{E}+00 \\
6.54 \mathrm{E}-01 \\
1.19 \mathrm{E}+00 \\
7.78 \mathrm{E}+00 \\
1.62 \mathrm{E}+01 \\
8.66 \mathrm{E}+00 \\
3.86 \mathrm{E}-01 \\
4.84 \mathrm{E}+01 \\
1.97 \mathrm{E}-02\end{array}$ & $\begin{array}{l}3.22 \mathrm{E}+00 \\
7.30 \mathrm{E}-01 \\
1.45 \mathrm{E}+00 \\
9.40 \mathrm{E}+00 \\
1.68 \mathrm{E}+01 \\
1.07 \mathrm{E}+01 \\
4.58 \mathrm{E}-01 \\
5.77 \mathrm{E}+01 \\
2.30 \mathrm{E}-02\end{array}$ & $\begin{array}{l}5.56 \mathrm{E}-01 \\
7.62 \mathrm{E}-02 \\
1.33 \mathrm{E}-01 \\
9.48 \mathrm{E}-01 \\
1.40 \mathrm{E}+00 \\
1.11 \mathrm{E}+00 \\
4.20 \mathrm{E}-02 \\
4.35 \mathrm{E}+00 \\
9.94 \mathrm{E}-03\end{array}$ & $\begin{array}{l}1.76 \mathrm{E}+00 \\
2.53 \mathrm{E}-01 \\
1.92 \mathrm{E}-01 \\
1.36 \mathrm{E}+00 \\
5.21 \mathrm{E}+00 \\
1.66 \mathrm{E}+00 \\
1.23 \mathrm{E}-01 \\
1.01 \mathrm{E}+01 \\
1.61 \mathrm{E}-02\end{array}$ \\
\hline Totals & & $8.64 E+01$ & $1.01 E+02$ & $8.62 \mathrm{E}+00$ & $2.07 E+01$ \\
\hline
\end{tabular}

Table 7. Annual Accident Dose Risk for the Shipping Campaign (person-rem/yr)

\begin{tabular}{llcc}
\hline Route & & Truck & Rail \\
\hline Bettis Atomic & to Portsmouth GDP & $1.14 \mathrm{E}-04$ & $4.95 \mathrm{E}-05$ \\
Fermilab (FNAL) & to Portsmouth GDP & $2.06 \mathrm{E}-09$ & $5.35 \mathrm{E}-10$ \\
Hanford Site & to Nevada Test Site & $8.98 \mathrm{E}-07$ & $2.05 \mathrm{E}-07$ \\
INEL & to Nevada Test Site & $5.56 \mathrm{E}-05$ & $8.72 \mathrm{E}-06$ \\
Rnolls Atomic & to Portsmouth GDP & $1.06 \mathrm{E}-03$ & $2.49 \mathrm{E}-04$ \\
Los Alamos NL & to Nevada Test Site & $7.46 \mathrm{E}-07$ & $4.88 \mathrm{E}-08$ \\
Lawrence Berkeley & to Hanford Site & $2.53 \mathrm{E}-09$ & $1.42 \mathrm{E}-09$ \\
Portsmouth GDP & to Nevada Test Site & $1.81 \mathrm{E}-03$ & $5.81 \mathrm{E}-04$ \\
SNL - Albuquerque & to Los Alamos NL & $1.03 \mathrm{E}-11$ & $1.95 \mathrm{E}-13$ \\
& & & \\
\hline Totals & & & \\
\end{tabular}

Table 8. Expected Annual Fatalities

for the Shipping Campaign

\begin{tabular}{lll}
\hline Exposure Group & Truck & Rail \\
\hline Radiological & $3.5 E-02$ & $3.4 E-03$ \\
Normal Crew & $5.0 E-02$ & $1.0 E-02$ \\
Normal Public & $1.5 E-06$ & $4.4 E-07$ \\
Accident Public & $2.0 E-02$ & $1.9 E-02$ \\
Nonradiological & $1.9 E-01$ & $2.8 E-03$ \\
Enission & & \\
Accident & &
\end{tabular}


Table 9. Expected Annual Cancer Incidence for the Shipping Campaign

\begin{tabular}{lll}
\hline Exposure Group & Truck & Rail \\
\hline Radiological & & \\
$\quad$ Normal Crew & $1.2 \mathrm{E}-01$ & $1.2 \mathrm{E}-02$ \\
$\quad$ Normal Public & $1.7 \mathrm{E}-01$ & $3.5 \mathrm{E}-02$ \\
$\quad$ Accident Public & $5.2 \mathrm{E}-06$ & $1.5 \mathrm{E}-06$ \\
$\begin{array}{c}\text { Nonradiological } \\
\text { Emission }\end{array}$ & $2.0 \mathrm{E}-02$ & $1.9 \mathrm{E}-02$ \\
Accident & $\mathrm{NA}$ & $\mathrm{NA}$ \\
\hline
\end{tabular}

Table 10. Expected Annual Genetic Effects for the Shipping Campaign

\begin{tabular}{lll}
\hline Exposure Group & Truck & Rail \\
\hline Rađiological & & \\
Normal Crew & $5.2 \mathrm{E}-03$ & $5.2 \mathrm{E}-04$ \\
Normal Public & $1.0 \mathrm{E}-02$ & $2.1 \mathrm{E}-03$ \\
Accident Public & $3.0 \mathrm{E}-07$ & $8.9 \mathrm{E}-08$ \\
Nonradiological & & \\
Emission & NA & NA \\
Accident & NA & NA \\
\hline
\end{tabular}




\section{RISK ASSESSMENT RESULTS}

The results of the LLW transportation risk assessment are summarized in this section for the 14 WM alternatives defined in Section 2.2. Although the method for risk assessment and the important assumptions used for the assessment have been presented in detail in previous sections of this report, a brief overview of the risk assessment process is provided to aid the reader in interpreting the results of risk assessment.

The impacts of transportation are calculated in three areas: (1) collective population risks during routine conditions and accidents for each case, (2) risks to MEIs during routine conditions for each case, and (3) consequences to individuals and populations after the most severe accidents involving a release of radioactive material. These three areas are detailed in the following paragraphs:

- Collective Population Risk. The collective population risk is a measure of the total risk posed to society as a whole by the alternative being considered. For the collective population risk assessment, the persons exposed are considered as a group, without specifying individual receptors. The collective population risk is used as the primary means to compare the various alternatives.

Collective population risks are calculated from both vehicle- and cargo-related causes for routine transportation and accidents. Vehicle-related risks are independent of the cargo in the shipment and include risks from vehicular exhaust emissions and traffic accidents (fatalities caused by physical trauma). Vehicle-related risks are presented in terms of estimated fatalities for each case.

For radioactive material, cargo-related risk refers to the risk posed by the radioactive nature of the material. The collective population risks are calculated for each alternative by using RADTRAN 4 . The RADTRAN 4 calculations for population risk take into account both the consequences and the probabilities of potential exposure-causing events (such as accidents). The accident risk values are referred to as "dose risk" because they incorporate the probabilities of a spectrum of accidents. The collective population risks are presented in terms of the total dose (person-rem) to workers and to members of the public for each alternative. The collective population risks are also presented in terms of the estimated number of fatal latent-cancer cases, the number of latent-cancer cases (incidence), and the number of severe genetic effects by using the health risk conversion factors from ICRP Publication 60 (ICRP 1991). 


\section{B.27 WM LLW CENTRALIZED 5 ALTERNATIVE (CASE 21): HETEROGENEOUS SOLIDS}

Table 1. Summary of Route Information

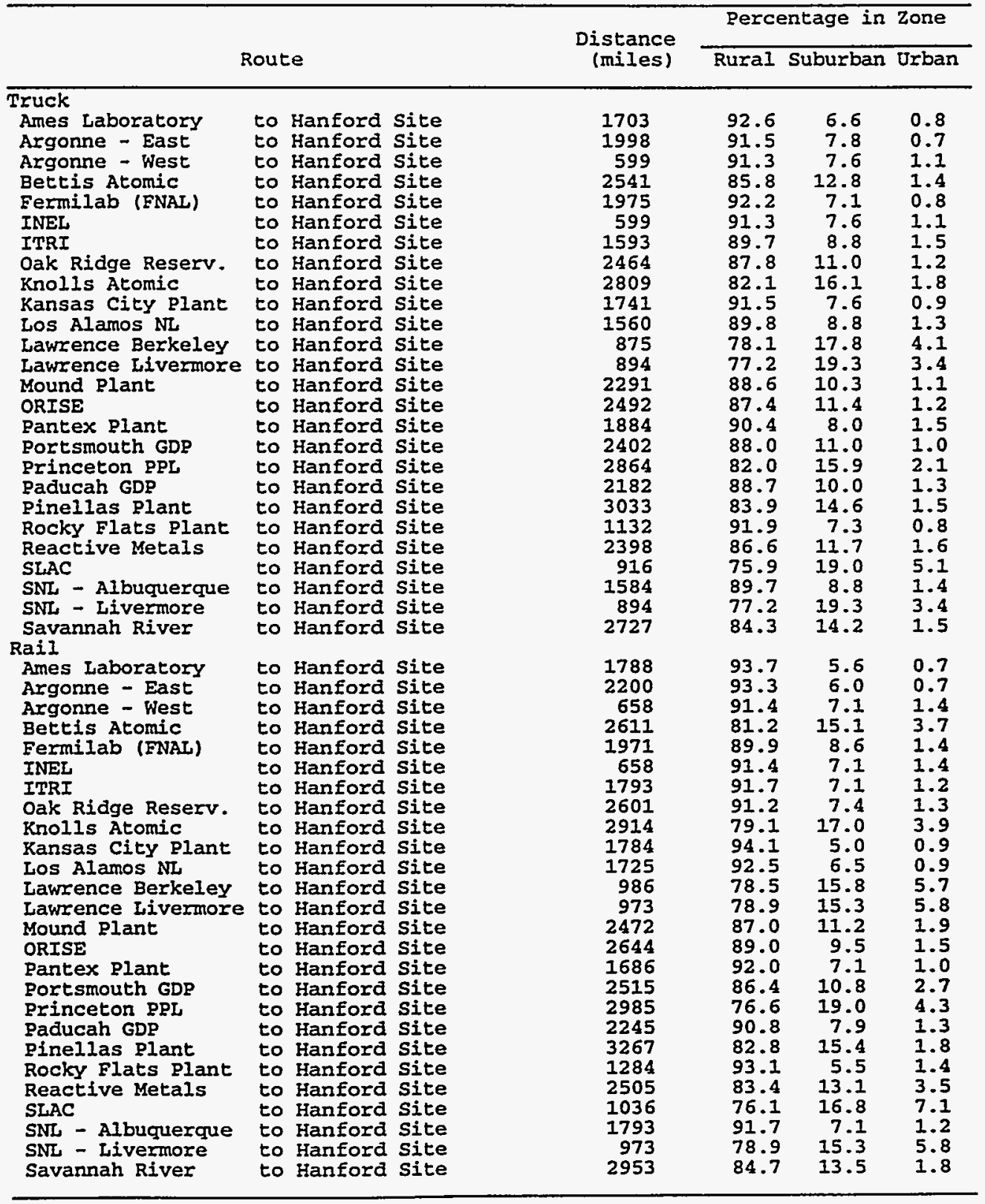




\section{$B-145$}

Table 2. Annual Number of Shipments and One-Way Shipment Distances

\begin{tabular}{|c|c|c|c|c|c|c|}
\hline \multirow[b]{2}{*}{ Roure } & & & \multicolumn{2}{|c|}{ \# of Shipments } & \multicolumn{2}{|c|}{ Total Mileage (mi) } \\
\hline & & & Truck & Rail & Truck & Rail \\
\hline $\begin{array}{l}\text { Ames Laboratory } \\
\text { Argonne - East } \\
\text { Argonne - West } \\
\text { Bettis Atomic } \\
\text { Fermilab (FNAL) } \\
\text { INEL } \\
\text { ITRI } \\
\text { Oak Ridge Reserv. } \\
\text { Knolls Atomic } \\
\text { Kansas City Plant } \\
\text { Los Alamos NL } \\
\text { Lawrence Berkeley } \\
\text { Lawrence Livermore } \\
\text { Mound Plant } \\
\text { ORISE } \\
\text { Pantex Plant } \\
\text { Portsmouth GDP } \\
\text { Princeton PRL } \\
\text { Paducah GDP } \\
\text { Pinellas Plant } \\
\text { Rocky Flats Plant } \\
\text { Reactive Metals } \\
\text { SIAC } \\
\text { SNL - Albuquerque } \\
\text { SNL - Livermore } \\
\text { Savannah River }\end{array}$ & $\begin{array}{l}\text { to } \\
\text { to } \\
\text { to } \\
\text { to } \\
\text { to } \\
\text { to } \\
\text { to } \\
\text { to } \\
\text { to } \\
\text { to } \\
\text { to } \\
\text { to } \\
\text { to } \\
\text { to } \\
\text { to } \\
\text { to } \\
\text { to } \\
\text { to } \\
\text { to } \\
\text { to } \\
\text { to } \\
\text { to } \\
\text { to } \\
\text { to } \\
\text { to } \\
\text { to }\end{array}$ & $\begin{array}{l}\text { Hanford Site } \\
\text { Hanford Site } \\
\text { Hanford Site } \\
\text { Hanford Site } \\
\text { Hanford Site } \\
\text { Hanford Site } \\
\text { Hanford Site } \\
\text { Hanford Site } \\
\text { Hanford Site } \\
\text { Hanford Site } \\
\text { Hanford Site } \\
\text { Hanford Site } \\
\text { Hanford Site } \\
\text { Hanford Site } \\
\text { Hanford Site } \\
\text { Hanford Site } \\
\text { Hanford Site } \\
\text { Hanford Site } \\
\text { Hanford Site } \\
\text { Hanford Site } \\
\text { Hanford Site } \\
\text { Hanford Site } \\
\text { Hanford Site } \\
\text { Hanford Site } \\
\text { Hanford Site } \\
\text { Hanford Site }\end{array}$ & $\begin{array}{r}2 \\
105 \\
13 \\
57 \\
3 \\
857 \\
10 \\
6584 \\
28 \\
1 \\
710 \\
3 \\
56 \\
516 \\
5 \\
1418 \\
3250 \\
1 \\
627 \\
2 \\
353 \\
761 \\
39 \\
17 \\
5 \\
6854\end{array}$ & $\begin{array}{r}19 \\
39 \\
5 \\
21 \\
1 \\
320 \\
4 \\
2489 \\
11 \\
1 \\
265 \\
2 \\
21 \\
190 \\
2 \\
537 \\
1250 \\
1 \\
235 \\
1 \\
132 \\
285 \\
15 \\
7 \\
2 \\
2532\end{array}$ & $\begin{array}{l}3.41 \mathrm{E}+03 \\
2.10 \mathrm{E}+05 \\
7.79 \mathrm{E}+03 \\
1.45 \mathrm{E}+05 \\
5.92 \mathrm{E}+03 \\
5.13 \mathrm{E}+05 \\
1.59 \mathrm{E}+04 \\
1.62 \mathrm{E}+07 \\
7.87 \mathrm{E}+04 \\
1.74 \mathrm{E}+03 \\
1.11 \mathrm{E}+06 \\
2.62 \mathrm{E}+03 \\
5.01 \mathrm{E}+04 \\
1.18 \mathrm{E}+06 \\
1.25 \mathrm{E}+04 \\
2.67 \mathrm{E}+06 \\
7.81 \mathrm{E}+06 \\
2.86 \mathrm{E}+03 \\
1.37 \mathrm{E}+06 \\
6.07 \mathrm{E}+03 \\
3.99 \mathrm{E}+05 \\
1.82 \mathrm{E}+06 \\
3.57 \mathrm{E}+04 \\
2.69 \mathrm{E}+04 \\
4.47 \mathrm{E}+03 \\
1.87 \mathrm{E}+07\end{array}$ & $\begin{array}{l}1.79 \mathrm{E}+03 \\
8.58 \mathrm{E}+04 \\
3.29 \mathrm{E}+03 \\
5.48 \mathrm{E}+04 \\
1.97 \mathrm{E}+03 \\
2.11 \mathrm{E}+05 \\
7.17 \mathrm{E}+03 \\
6.47 \mathrm{E}+06 \\
3.21 \mathrm{E}+04 \\
1.78 \mathrm{E}+03 \\
4.57 \mathrm{E}+05 \\
1.97 \mathrm{E}+03 \\
2.04 \mathrm{E}+04 \\
4.70 \mathrm{E}+05 \\
5.29 \mathrm{E}+03 \\
9.06 \mathrm{E}+05 \\
3.14 \mathrm{E}+06 \\
2.98 \mathrm{E}+03 \\
5.28 \mathrm{E}+05 \\
3.27 \mathrm{E}+03 \\
1.70 \mathrm{E}+05 \\
7.14 \mathrm{E}+05 \\
1.55 \mathrm{E}+04 \\
1.26 \mathrm{E}+04 \\
1.95 \mathrm{E}+03 \\
7.48 \mathrm{E}+06\end{array}$ \\
\hline Totals & & & 22277 & 8369 & $5.24 E+07$ & $2.08 E+07$ \\
\hline
\end{tabular}


Table 3. Incident-Free Dose per Shipment (person-rem/shipment)

\begin{tabular}{|c|c|c|c|c|c|}
\hline \multirow[b]{2}{*}{ Source/Route (s) } & \multirow[b]{2}{*}{ Crew } & \multicolumn{4}{|c|}{ General Public } \\
\hline & & Off-Link & On-Link & Stops & Total \\
\hline $\begin{array}{l}\text { LLW(x) AL to HS } \\
\text { Ames Laboratory }\end{array}$ & $\begin{array}{lr}\text { to Hanford Site } \\
\text { Truck } & 4.46 \mathrm{E}-02 \\
\text { Rail } & 1.03 \mathrm{E}-02\end{array}$ & $\begin{array}{l}1.62 \mathrm{E}-03 \\
4.69 \mathrm{E}-03\end{array}$ & $\begin{array}{l}5.39 \mathrm{E}-03 \\
1.46 \mathrm{E}-04\end{array}$ & $\begin{array}{l}4.77 E-02 \\
8.98 E-03\end{array}$ & $\begin{array}{l}5.47 \mathrm{E}-02 \\
1.38 \mathrm{E}-02\end{array}$ \\
\hline $\begin{array}{l}\text { LLW(x) AN to HS } \\
\text { Argonne - East }\end{array}$ & $\begin{array}{lr}\text { to Hanford Site } \\
\text { Truck } 5.29 \mathrm{E}-02 \\
\text { Rail } & 1.21 \mathrm{E}-02\end{array}$ & $\begin{array}{l}1.97 \mathrm{E}-03 \\
5.75 \mathrm{E}-03\end{array}$ & $\begin{array}{l}E-03 \\
E-04\end{array}$ & $\begin{array}{l}5.59 \mathrm{E}-02 \\
1.03 \mathrm{E}-02\end{array}$ & $\begin{array}{l}6.42 \mathrm{E}-02 \\
1.62 \mathrm{E}-02\end{array}$ \\
\hline $\begin{array}{l}\text { LLW(x) AW to HS } \\
\text { Argonne - West }\end{array}$ & 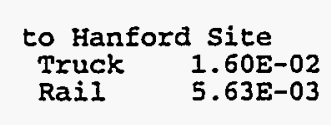 & $\begin{array}{l}7.18 \mathrm{E}-04 \\
2.83 \mathrm{E}-03\end{array}$ & $\begin{array}{l}2.06 \mathrm{E}-03 \\
6.58 \mathrm{E}-05\end{array}$ & $\begin{array}{l}E-02 \\
E-03\end{array}$ & $\begin{array}{l}1.95 \mathrm{E}-02 \\
8.40 \mathrm{E}-03\end{array}$ \\
\hline $\begin{array}{l}\text { LLW (x) BA to HS } \\
\text { Bettis Atomic }\end{array}$ & $\begin{array}{lr}\text { to Hanford Site } \\
\text { Truck } & 7.21 \mathrm{E}-02 \\
\text { Rail } & 1.38 \mathrm{E}-02\end{array}$ & $\begin{array}{l}4.36 \mathrm{E}-03 \\
2.67 \mathrm{E}-02\end{array}$ & $\begin{array}{l}9.77 E-03 \\
4.53 E-04\end{array}$ & $\begin{array}{l}7.11 \mathrm{E}-02 \\
1.15 \mathrm{E}-02\end{array}$ & $\begin{array}{l}8.53 E-02 \\
3.87 E-02\end{array}$ \\
\hline LLW(x) IN to HS & $\begin{array}{lr}\text { to Hanford Site } \\
\text { Truck } & 5.20 \mathrm{E}-02 \\
\text { Rail } & 1.11 \mathrm{E}-02\end{array}$ & $\begin{array}{l}1.92 \mathrm{E}-03 \\
9.07 \mathrm{E}-03\end{array}$ & $\begin{array}{l}6.2 \\
2.1\end{array}$ & $\begin{array}{l}-02 \\
-03\end{array}$ & $\begin{array}{l}6.35 \mathrm{E}-02 \\
1.88 \mathrm{E}-02\end{array}$ \\
\hline $\begin{array}{l}\text { LLW (x) IN to HS } \\
\text { INEL }\end{array}$ & $\begin{array}{ll}\text { to Hanford Site } \\
\text { Truck } & 1.60 \mathrm{E}-02 \\
\text { Rail } & 5.63 \mathrm{E}-03\end{array}$ & $\begin{array}{l}7.18 E-04 \\
2.83 E-03\end{array}$ & $\begin{array}{l}2.0 \\
6.5\end{array}$ & & \\
\hline$\underset{\text { ITRI }}{\operatorname{LLW}(x) \operatorname{IT}}$ & $\begin{array}{ll}\text { to Hanford Site } \\
\text { Truck } & 4.34 \mathrm{E}-02 \\
\text { Rail } & 1.04 \mathrm{E}-02\end{array}$ & $\begin{array}{l}2.30 \mathrm{E}-03 \\
6.90 \mathrm{E}-03\end{array}$ & $\begin{array}{l}5.9 \\
1.7\end{array}$ & & $\begin{array}{l}E-02 \\
E-02\end{array}$ \\
\hline$T H(Y)$ KA to HS & $\begin{array}{cl}\text { to Hanford } & \text { Site } \\
\text { Truck } & 6.83 \mathrm{E}-02 \\
\text { Rail } & 1.37 \mathrm{E}-02\end{array}$ & $\begin{array}{l}3.6 \\
1.0\end{array}$ & $\begin{array}{l}8.5 \\
2.5\end{array}$ & & $\begin{array}{l}8.16 \mathrm{E}-02 \\
2.26 \mathrm{E}-02\end{array}$ \\
\hline $\begin{array}{l}\text { LLW }(x) \text { KA to HS } \\
\text { Knolls Atomic }\end{array}$ & $\begin{array}{lr}\text { to Hanford Site } \\
\text { Truck } & 8.30 \mathrm{E}-02 \\
\text { Rail } & 1.50 \mathrm{E}-02\end{array}$ & $\begin{array}{l}6.03 E-03 \\
3.18 E-02\end{array}$ & $\begin{array}{l}1.19 \mathrm{E}-02 \\
5.40 \mathrm{E}-04\end{array}$ & & $\begin{array}{l}9.66 \mathrm{E}-02 \\
4.48 \mathrm{E}-02\end{array}$ \\
\hline $\begin{array}{l}\text { LLW }(x) \text { KC to HS } \\
\text { Ransas City PI }\end{array}$ & $\begin{array}{l}\text { Site } \\
4.62 \mathrm{E}-02 \\
1.03 \mathrm{E}-02\end{array}$ & $\begin{array}{l}1.9 \\
5.0\end{array}$ & $\begin{array}{l}5.74 E-03 \\
1.44 E-04\end{array}$ & & $\begin{array}{l}5.64 E-02 \\
1.41 E-02\end{array}$ \\
\hline $\operatorname{LLW}(x)$ IB to HS & $\begin{array}{l}\text { to Hanford Site } \\
\text { Truck } \\
\text { Rail } \\
\text { Ra.24E-02 } \\
\end{array}$ & $\begin{array}{l}2.1 \\
5.6\end{array}$ & $\begin{array}{l}5.8 \\
1.5\end{array}$ & $\begin{array}{l}4.3 \\
8.7\end{array}$ & $\begin{array}{l}-02 \\
-02\end{array}$ \\
\hline $\begin{array}{l}\text { LLW (x) LB to HS } \\
\text { Lawrence Berkeley }\end{array}$ & $\begin{array}{lr}\text { to Hanford Site } \\
\text { Truck } & 2.76 \mathrm{E}-02 \\
\text { Rail } & 6.99 \mathrm{E}-03\end{array}$ & $\begin{array}{l}3.02 \mathrm{E}-03 \\
1.38 \mathrm{E}-02\end{array}$ & $\begin{array}{l}5.17 E-03 \\
2.03 E-04\end{array}$ & $\begin{array}{l}2.5 \\
6.5\end{array}$ & $\begin{array}{l}3.27 \mathrm{E}-02 \\
2.05 \mathrm{E}-02\end{array}$ \\
\hline $\begin{array}{l}\text { LLW(x) LL to HS } \\
\text { Lawrence Liv }\end{array}$ & $\begin{array}{r}\text { ford Site } \\
2.82 \mathrm{E}-02 \\
6.94 \mathrm{E}-03\end{array}$ & $\begin{array}{l}2.88 \mathrm{E}-03 \\
1.38 \mathrm{E}-02\end{array}$ & $\begin{array}{l}4.92 \mathrm{E}-03 \\
2.00 \mathrm{E}-04\end{array}$ & & $\begin{array}{l}3.28 \mathrm{E}-02 \\
2.05 \mathrm{E}-02\end{array}$ \\
\hline $\begin{array}{l}\text { LLW (x) MP to HS } \\
\text { Mound Plant }\end{array}$ & \begin{tabular}{lr}
\multicolumn{1}{l}{ to Hanford Site } \\
Truck & $6.29 \mathrm{E}-02$ \\
Rail & $1.32 \mathrm{E}-02$
\end{tabular} & $\begin{array}{l}3.13 \mathrm{E}-03 \\
1.47 \mathrm{E}-02\end{array}$ & $\begin{array}{l}8.05 E-03 \\
3.13 E-04\end{array}$ & $\begin{array}{l}6 \\
1\end{array}$ & $\begin{array}{l}E-02 \\
E-02\end{array}$ \\
\hline $\begin{array}{l}\text { LLW(x) OI to HS } \\
\text { ORISE }\end{array}$ & $\begin{array}{ll}\text { to Hanford Site } \\
\text { Truck } & 6.94 \mathrm{E}-02 \\
\text { Rail } & 1.39 \mathrm{E}-02\end{array}$ & $\begin{array}{l}3.76 \mathrm{E}-03 \\
1.30 \mathrm{E}-02\end{array}$ & $\begin{array}{l}9.08 \mathrm{E}-03 \\
2.98 \mathrm{E}-04\end{array}$ & $\begin{array}{l}6.98 \mathrm{E}-02 \\
1.16 \mathrm{E}-02\end{array}$ & $\begin{array}{l}8.26 \mathrm{E}-02 \\
2.49 \mathrm{E}-02\end{array}$ \\
\hline $\begin{array}{l}\text { LLW (x) OR to HS } \\
\text { Oak Ridge Re }\end{array}$ & $\begin{array}{cl}\text { to Hanford } & \text { Site } \\
\text { Truck } & 6.83 \mathrm{E}-02 \\
\text { Rail } & 1.37 \mathrm{E}-02\end{array}$ & $\begin{array}{l}3.65 \mathrm{E}-03 \\
1.08 \mathrm{E}-02\end{array}$ & $\begin{array}{l}8.95 \mathrm{E}-03 \\
2.59 \mathrm{E}-04\end{array}$ & $\begin{array}{l}6.9 \\
1.1\end{array}$ & $\begin{array}{l}8.16 \mathrm{E}-02 \\
2.26 \mathrm{E}-02\end{array}$ \\
\hline $\begin{array}{l}\text { LLW(x) PP to HS } \\
\text { Pantex Plant }\end{array}$ & $\begin{array}{lr}\text { to Hanford Site } \\
\text { Truck } & 5.10 \mathrm{E}-02 \\
\text { Rail } & 9.92 \mathrm{E}-03\end{array}$ & $\begin{array}{l}2.68 \mathrm{E}-03 \\
5.71 \mathrm{E}-03\end{array}$ & $\begin{array}{l}7.01 \mathrm{E}-03 \\
1.56 \mathrm{E}-04\end{array}$ & $\begin{array}{l}5.27 \mathrm{E}-02 \\
8.67 \mathrm{E}-03\end{array}$ & $\begin{array}{l}6.24 \mathrm{E}-02 \\
1.45 \mathrm{E}-02\end{array}$ \\
\hline $\begin{array}{l}\text { LLW (x) BO to HS } \\
\text { Portsmouth GDP }\end{array}$ & $\begin{array}{lr}\text { to Hanford Site } \\
\text { Truck } & 6.63 \mathrm{E} \\
\text { Rail } & 1.34 \mathrm{E}\end{array}$ & $\begin{array}{l}3.38 \mathrm{E}-03 \\
1.89 \mathrm{E}-02\end{array}$ & $\begin{array}{l}8.48 \mathrm{E}-03 \\
3.46 \mathrm{E}-04\end{array}$ & $\begin{array}{l}6.72 \mathrm{E}-02 \\
1.12 \mathrm{E}-02\end{array}$ & $\begin{array}{l}7.91 \mathrm{E}-02 \\
3.04 \mathrm{E}-02\end{array}$ \\
\hline
\end{tabular}




\begin{tabular}{|c|c|c|c|c|c|}
\hline Crul(2) DA to & $\begin{array}{l}\text { to Hanford site } \\
\text { Truck } \\
\text { Rail } \\
\text { Rais1E-02 } \\
\end{array}$ & $\begin{array}{l}6.65 \mathrm{E}-03 \\
3.63 \mathrm{E}-02\end{array}$ & $\begin{array}{l}1.28 \mathrm{E}-02 \\
6.03 \mathrm{E}-04\end{array}$ & $\begin{array}{l}8.02 \mathrm{E}-02 \\
1.27 \mathrm{E}-02\end{array}$ & $\begin{array}{l}9.97 \mathrm{E}-02 \\
4.96 \mathrm{E}-02\end{array}$ \\
\hline $\begin{array}{l}\text { LLW (x) PA to HS } \\
\text { Paducah GDP }\end{array}$ & $\begin{array}{ll}\text { to Hanford Site } \\
\text { Truck } & 6.00 \mathrm{E}-02 \\
\text { Rail } & 1.22 \mathrm{E}-02\end{array}$ & $\begin{array}{l}3.22 \mathrm{E}-03 \\
9.43 \mathrm{E}-03\end{array}$ & $\begin{array}{l}8.02 \mathrm{E}-03 \\
2.28 \mathrm{E}-04\end{array}$ & $\begin{array}{l}6.11 \mathrm{E}-02 \\
1.04 \mathrm{E}-02\end{array}$ & $\begin{array}{l}7.23 \mathrm{E}-02 \\
2.01 \mathrm{E}-02\end{array}$ \\
\hline $\begin{array}{l}\text { LLW(x) PI to HS } \\
\text { Pinelias Plant }\end{array}$ & $\begin{array}{ll}\text { to Hanford Site } \\
\text { Truck } & 8.77 \mathrm{E}-02 \\
\text { Rail } & 1.65 \mathrm{E}-02\end{array}$ & $\begin{array}{l}5.74 \mathrm{E}-03 \\
2.19 \mathrm{E}-02\end{array}$ & $\begin{array}{l}1.21 E-02 \\
4.79 E-04\end{array}$ & $\begin{array}{l}8.49 \mathrm{E}-02 \\
1.35 \mathrm{E}-02\end{array}$ & $\begin{array}{l}1.03 \mathrm{E}-01 \\
3.59 \mathrm{E}-02\end{array}$ \\
\hline $\begin{array}{l}\text { LLW(x) RF to HS } \\
\text { Rocky Flats plant }\end{array}$ & $\begin{array}{ll}\text { to Hanford Site } \\
\text { Truck } & 2.99 \mathrm{E}-02 \\
\text { Rail } & 8.24 \mathrm{E}-03\end{array}$ & $\begin{array}{l}1.13 \mathrm{E}-03 \\
4.91 \mathrm{E}-03\end{array}$ & $\begin{array}{l}3.61 \mathrm{E}-03 \\
1.16 \mathrm{E}-04\end{array}$ & $\begin{array}{l}3.17 \mathrm{E}-02 \\
7.43 \mathrm{E}-03\end{array}$ & $\begin{array}{l}3.64 \mathrm{E}-02 \\
1.25 \mathrm{E}-02\end{array}$ \\
\hline $\begin{array}{l}\text { LLW (x) RM to HS } \\
\text { Reactive Metals }\end{array}$ & $\begin{array}{ll}\text { to Hanford Site } \\
\text { Truck } & 6.76 \mathrm{E}-02 \\
\text { Rail } & 1.33 \mathrm{E}-02\end{array}$ & $\begin{array}{l}4.22 E-03 \\
2.35 E-02\end{array}$ & $\begin{array}{l}9.51 \mathrm{E}-03 \\
4.01 \mathrm{E}-04\end{array}$ & $\begin{array}{l}6.71 \mathrm{E}-02 \\
1.12 \mathrm{E}-02\end{array}$ & $\begin{array}{l}8.09 \mathrm{E}-02 \\
3.51 \mathrm{E}-02\end{array}$ \\
\hline $\begin{array}{l}\text { LLW(x) } \\
\text { SLAC }\end{array}$ & $\begin{array}{lr}\text { to Hanford Site } \\
\text { Truck } & 2.98 \mathrm{E}-02 \\
\text { Rail } & 7.20 \mathrm{E}-03\end{array}$ & $\begin{array}{l}3.70 \mathrm{E}-03 \\
1.75 \mathrm{E}-02\end{array}$ & $\begin{array}{l}6.08 \mathrm{E}-03 \\
2.40 \mathrm{E}-04\end{array}$ & $\begin{array}{l}2.56 \mathrm{E}-02 \\
6.67 \mathrm{E}-03\end{array}$ & $\begin{array}{l}3.54 \mathrm{E}-02 \\
2.44 \mathrm{E}-02\end{array}$ \\
\hline $\begin{array}{l}\text { LLW(x) SA to HS } \\
\text { SNL - Albuquerque }\end{array}$ & $\begin{array}{ll}\text { to Hanford Site } \\
\text { Truck } & 4.31 \mathrm{E}-02 \\
\text { Rail } & 1.04 \mathrm{E}-02\end{array}$ & $\begin{array}{l}2.27 \mathrm{E}-03 \\
6.90 \mathrm{E}-03\end{array}$ & $\begin{array}{l}5.84 \mathrm{E}-03 \\
1.72 \mathrm{E}-04\end{array}$ & $\begin{array}{l}4.43 \mathrm{E}-02 \\
9.00 \mathrm{E}-03\end{array}$ & $\begin{array}{l}5.24 E-02 \\
1.61 E-02\end{array}$ \\
\hline $\begin{array}{l}\text { LLW(x) SL to HS } \\
\text { SNL - Livermore }\end{array}$ & $\begin{array}{ll}\text { to Hanford Site } \\
\text { Truck } & 2.82 \mathrm{E}-02 \\
\text { Rail } & 6.94 \mathrm{E}-03\end{array}$ & $\begin{array}{l}2.88 E-03 \\
1.38 E-02\end{array}$ & $\begin{array}{l}4.92 E-03 \\
2.00 E-04\end{array}$ & $\begin{array}{l}2.50 \mathrm{E}-02 \\
6.48 \mathrm{E}-03\end{array}$ & $\begin{array}{l}3.28 E-02 \\
2.05 E-02\end{array}$ \\
\hline $\begin{array}{l}\text { LLW (x) SR to HS } \\
\text { Savannah River }\end{array}$ & $\begin{array}{ll}\text { to Hanford Site } \\
\text { Truck } & 7.86 \mathrm{E}-02 \\
\text { Rail } & 1.52 \mathrm{E}-02\end{array}$ & $\begin{array}{l}5.10 \mathrm{E}-03 \\
1.85 \mathrm{E}-02\end{array}$ & $\begin{array}{l}1.08 E-02 \\
4.05 E-04\end{array}$ & $\begin{array}{l}7.64 \mathrm{E}-02 \\
1.26 \mathrm{E}-02\end{array}$ & $\begin{array}{l}9.23 E-02 \\
3.15 E-02\end{array}$ \\
\hline $\begin{array}{l}\text { LLW (x) OR to HS } \\
\text { Oak Ridge Reserv. }\end{array}$ & $\begin{array}{cl}\text { to Hanford } & \text { Site } \\
\text { Truck } & 6.83 \mathrm{E}-02 \\
\text { Rail } & 1.37 \mathrm{E}-02\end{array}$ & $\begin{array}{l}3.65 \mathrm{E}-03 \\
1.08 \mathrm{E}-02\end{array}$ & $\begin{array}{l}8.95 \mathrm{E}-03 \\
2.59 \mathrm{E}-04\end{array}$ & $\begin{array}{l}6.90 \mathrm{E}-02 \\
1.15 \mathrm{E}-02\end{array}$ & $\begin{array}{l}8.16 \mathrm{E}-02 \\
2.26 \mathrm{E}-02\end{array}$ \\
\hline
\end{tabular}


Table 4. Accident Dose Risk per Shipment (person-rem/shipment)

\begin{tabular}{|c|c|c|c|c|}
\hline Source/Roure & & & Truck & Rail \\
\hline $\begin{array}{l}\text { LLW(x) AL to HS } \\
\text { Ames Laboratory } \\
\text { LLW(x) AN to HS } \\
\text { Argonne - East } \\
\text { LLW(x) AW to HS } \\
\text { Argonne - West } \\
\text { LLW(x) BA to HS } \\
\text { Bettis Atomic } \\
\text { LLW(x) FL to HS } \\
\text { Fermilab (FNAL) } \\
\text { LLW(x) IN to HS } \\
\text { INEL } \\
\text { LLW(x) IT to HS } \\
\text { ITRI to HS } \\
\text { LLW(x) OR to HS } \\
\text { Oak Ridge Reserv. } \\
\text { LLW(x) KA to HS } \\
\text { Knolls Atomic } \\
\text { LLW(x) KC to HS } \\
\text { Kansas Citty Plant } \\
\text { LLW(x) IA to HS } \\
\text { LOS Alamos NL } \\
\text { LLW(x) IB to HS } \\
\text { Lawrence Berkeley } \\
\text { LLW(x) IL to HS } \\
\text { Lawrence Livermore } \\
\text { LLW(x) MP to HS } \\
\text { Mound Plant } \\
\text { LLW(x) OI to HS } \\
\text { ORISE } \\
\text { LLW(x) OR to HS } \\
\text { Oak Ridge Reserv. } \\
\text { LLW(x) PP to HS } \\
\text { Pantex Plant } \\
\text { LLW(x) PO to HS } \\
\text { Portsmouth GDP } \\
\text { LLW(x) PR to HS } \\
\text { Princeton PPL } \\
\text { LLW(x) PA to HS } \\
\text { Paducah GDP } \\
\text { LLW(x) PI to HS } \\
\text { Pinellas Plant } \\
\text { LLW(x) RF to HS } \\
\text { Rocky Flats Plant } \\
\text { LLW(x) RM to HS } \\
\text { Reactive Metals } \\
\text { LLW(x) ST to HS } \\
\text { SLAC to HS } \\
\text { LLW(x) SA to HS } \\
\text { SNL - Albuquerque } \\
\text { LLW(x) SL to HS } \\
\text { SNL - Iivermore } \\
\text { LLW(x) SR to HS } \\
\text { Savannah River } \\
\text { LLW(x) OR to HS } \\
\text { Oak Ridge Reserv. }\end{array}$ & $\begin{array}{l}\text { to Hanford Site } \\
\text { to Hanford Site } \\
\text { to Hanford Site } \\
\text { to Hanford Site } \\
\text { to Hanford Site } \\
\text { to Hanford Site } \\
\text { to Hanford Site } \\
\text { to Hanford Site } \\
\text { to Hanford Site } \\
\text { to Hanford Site } \\
\text { to Hanford Site } \\
\text { to Hanford Site } \\
\text { to Hanford Site } \\
\text { to Hanford Site } \\
\text { to Hanford Site } \\
\text { to Hanford Site } \\
\text { to Hanford Site } \\
\text { to Hanford Site } \\
\text { to Hanford Site } \\
\text { to Hanford Site } \\
\text { to Hanford Site } \\
\text { to Hanford Site } \\
\text { to Hanford Site } \\
\text { to Hanford Site } \\
\text { to Hanford Site } \\
\text { to Hanford Site } \\
\text { to Hanford Site } \\
\text { to Hanford Site }\end{array}$ & . & $\begin{array}{l}5.20 \mathrm{E}-06 \\
5.56 \mathrm{E}-04 \\
2.45 \mathrm{E}-01 \\
4.46 \mathrm{E}-05 \\
2.24 \mathrm{E}-05 \\
1.09 \mathrm{E}-04 \\
5.50 \mathrm{E}-03 \\
3.63 \mathrm{E}-07 \\
4.90 \mathrm{E}-06 \\
2.32 \mathrm{E}-05 \\
2.79 \mathrm{E}-02 \\
5.07 \mathrm{E}-02 \\
9.20 \mathrm{E}-06 \\
5.83 \mathrm{E}-06 \\
2.64 \mathrm{E}-06 \\
3.63 \mathrm{E}-07 \\
1.67 \mathrm{E}-06 \\
2.62 \mathrm{E}-08 \\
1.28 \mathrm{E}-05 \\
1.17 \mathrm{E}-06 \\
6.86 \mathrm{E}-06 \\
3.48 \mathrm{E}-06 \\
1.17 \mathrm{E}-08 \\
4.30 \mathrm{E}-07 \\
2.28 \mathrm{E}-05 \\
1.06 \mathrm{E}-04 \\
5.05 \mathrm{E}-03 \\
3.63 \mathrm{E}-07\end{array}$ & $\begin{array}{l}3.93 \mathrm{E}-06 \\
3.40 \mathrm{E}-04 \\
3.44 \mathrm{E}-01 \\
5.00 \mathrm{E}-05 \\
2.22 \mathrm{E}-05 \\
1.57 \mathrm{E}-04 \\
1.57 \mathrm{E}-03 \\
1.97 \mathrm{E}-07 \\
4.19 \mathrm{E}-06 \\
4.82 \mathrm{E}-06 \\
9.49 \mathrm{E}-03 \\
4.24 \mathrm{E}-02 \\
1.28 \mathrm{E}-05 \\
3.53 \mathrm{E}-06 \\
1.38 \mathrm{E}-06 \\
1.97 \mathrm{E}-07 \\
5.03 \mathrm{E}-07 \\
2.70 \mathrm{E}-08 \\
4.69 \mathrm{E}-06 \\
7.40 \mathrm{E}-07 \\
3.00 \mathrm{E}-06 \\
2.51 \mathrm{E}-06 \\
1.30 \mathrm{E}-08 \\
6.58 \mathrm{E}-07 \\
7.55 \mathrm{E}-06 \\
1.40 \mathrm{E}-04 \\
2.74 \mathrm{E}-03 \\
1.97 \mathrm{E}-07\end{array}$ \\
\hline
\end{tabular}


Table 5. Nonradiological Risk Factors per Shipment (fatalities/shipment)

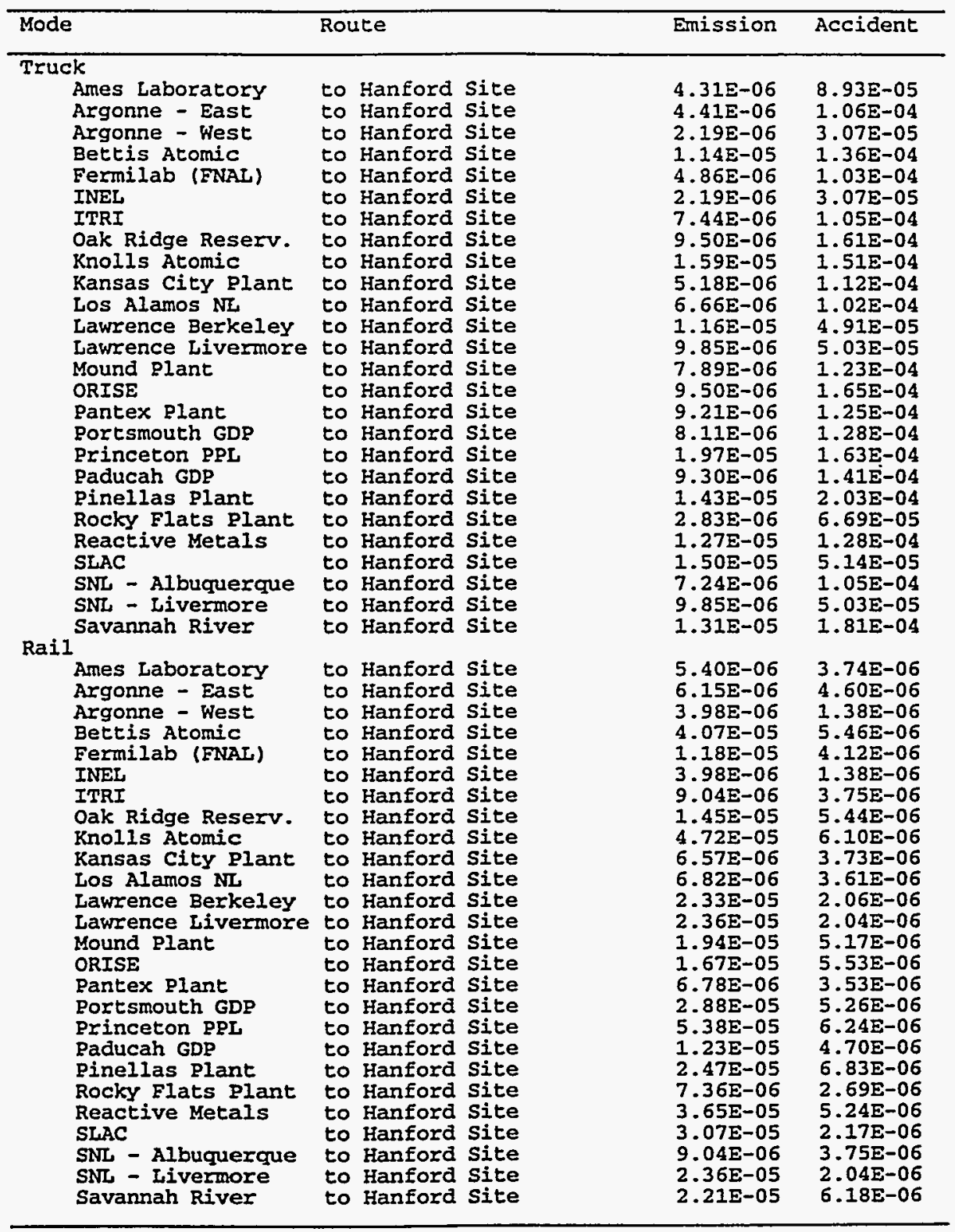


Table 6. Annual Incident-Free Dose for the Shipping Campaign (person-rem/yr)

\begin{tabular}{|c|c|c|c|c|c|}
\hline \multirow[b]{2}{*}{ Route } & & \multicolumn{2}{|c|}{ Truck } & \multicolumn{2}{|c|}{ Rail } \\
\hline & & Crew & $\overline{\text { Public }}$ & Crew & Public \\
\hline $\begin{array}{l}\text { Ames Laboratory } \\
\text { Argonne - East } \\
\text { Argonne - West } \\
\text { Bettis Atomic } \\
\text { Fermilab (FNAL) } \\
\text { INEL } \\
\text { ITRI } \\
\text { Oak Ridge Reserv. } \\
\text { Knolls Atomic } \\
\text { Kansas City Plant } \\
\text { Los Alamos NL } \\
\text { Lawrence Berkeley } \\
\text { Lawrence Livermore } \\
\text { Mound Plant } \\
\text { ORISE } \\
\text { Pantex Plant } \\
\text { Portsmouth GDP } \\
\text { Princeton PPL } \\
\text { Paducah GDP } \\
\text { PineIlas Plant } \\
\text { RockY Flats Plant } \\
\text { Reactive Metals } \\
\text { SLAC } \\
\text { SNL - Albuquerque } \\
\text { SNL - Livermore } \\
\text { Savannah River }\end{array}$ & $\begin{array}{l}\text { to Hanford Site } \\
\text { to Hanford Site } \\
\text { to Hanford Site } \\
\text { to Hanford Site } \\
\text { to Hanford Site } \\
\text { to Hanford Site } \\
\text { to Hanford Site } \\
\text { to Hanford Site } \\
\text { to Hanford Site } \\
\text { to Hanford Site } \\
\text { to Hanford Site } \\
\text { to Hanford Site } \\
\text { to Hanford Site } \\
\text { to Hanford Site } \\
\text { to Hanford Site } \\
\text { to Hanford Site } \\
\text { to Hanford Site } \\
\text { to Hanford Site } \\
\text { to Hanford Site } \\
\text { to Hanford Site } \\
\text { to Hanford Site } \\
\text { to Hanford Site } \\
\text { to Hanford Site } \\
\text { to Hanford Site } \\
\text { to Hanford Site } \\
\text { to Hanford Site }\end{array}$ & $\begin{array}{l}8.93 E-02 \\
5.55 E+00 \\
2.08 E-01 \\
4.11 E+00 \\
1.56 E-01 \\
1.37 E+01 \\
4.34 E-01 \\
4.50 E+02 \\
2.32 E+00 \\
4.62 E-02 \\
3.01 E+01 \\
8.27 E-02 \\
1.58 E+00 \\
3.24 E+01 \\
3.47 E-01 \\
7.23 E+01 \\
2.16 E+02 \\
8.51 E-02 \\
3.76 E+01 \\
1.75 E-01 \\
1.05 E+01 \\
5.15 E+01 \\
1.16 E+00 \\
7.33 E-01 \\
1.41 E-01 \\
5.39 E+02\end{array}$ & $\begin{array}{l}1.09 \mathrm{E}-01 \\
6.74 \mathrm{E}+00 \\
2.54 \mathrm{E}-01 \\
4.86 \mathrm{E}+00 \\
1.90 \mathrm{E}-01 \\
1.68 \mathrm{E}+01 \\
5.28 \mathrm{E}-01 \\
5.37 \mathrm{E}+02 \\
2.70 \mathrm{E}+00 \\
5.64 \mathrm{E}-02 \\
3.66 \mathrm{E}+01 \\
9.80 \mathrm{E}-02 \\
1.84 \mathrm{E}+00 \\
3.89 \mathrm{E}+01 \\
4.13 \mathrm{E}-01 \\
8.85 \mathrm{E}+01 \\
2.57 \mathrm{E}+02 \\
9.97 \mathrm{E}-02 \\
4.53 \mathrm{E}+01 \\
2.05 \mathrm{E}-01 \\
1.29 \mathrm{E}+01 \\
6.15 \mathrm{E}+01 \\
1.38 \mathrm{E}+00 \\
8.92 \mathrm{E}-01 \\
1.64 \mathrm{E}-01 \\
6.32 \mathrm{E}+02\end{array}$ & $\begin{array}{l}1.03 \mathrm{E}-02 \\
4.70 \mathrm{E}-01 \\
2.81 \mathrm{E}-02 \\
2.89 \mathrm{E}-01 \\
1.11 \mathrm{E}-02 \\
1.80 \mathrm{E}+00 \\
4.15 \mathrm{E}-02 \\
3.42 \mathrm{E}+01 \\
1.65 \mathrm{E}-01 \\
1.03 \mathrm{E}-02 \\
2.67 \mathrm{E}+00 \\
1.40 \mathrm{E}-02 \\
1.46 \mathrm{E}-01 \\
2.51 \mathrm{E}+00 \\
2.78 \mathrm{E}-02 \\
5.33 \mathrm{E}+00 \\
1.67 \mathrm{E}+01 \\
1.53 \mathrm{E}-02 \\
2.88 \mathrm{E}+00 \\
1.65 \mathrm{E}-02 \\
1.09 \mathrm{E}+00 \\
3.80 \mathrm{E}+00 \\
1.08 \mathrm{E}-01 \\
7.25 \mathrm{E}-02 \\
1.39 \mathrm{E}-02 \\
3.85 \mathrm{E}+01\end{array}$ & $\begin{array}{l}1.38 \mathrm{E}-02 \\
6.31 \mathrm{E}-01 \\
4.20 \mathrm{E}-02 \\
8.13 \mathrm{E}-01 \\
1.88 \mathrm{E}-02 \\
2.69 \mathrm{E}+00 \\
6.43 \mathrm{E}-02 \\
5.62 \mathrm{E}+01 \\
4.93 \mathrm{E}-01 \\
1.41 \mathrm{E}-02 \\
3.85 \mathrm{E}+00 \\
4.10 \mathrm{E}-02 \\
4.30 \mathrm{E}-01 \\
4.96 \mathrm{E}+00 \\
4.98 \mathrm{E}-02 \\
7.81 \mathrm{E}+00 \\
3.80 \mathrm{E}+01 \\
4.96 \mathrm{E}-02 \\
4.71 \mathrm{E}+00 \\
3.59 \mathrm{E}-02 \\
1.65 \mathrm{E}+00 \\
1.00 \mathrm{E}+01 \\
3.66 \mathrm{E}-01 \\
1.12 \mathrm{E}-01 \\
4.10 \mathrm{E}-02 \\
7.98 \mathrm{E}+01\end{array}$ \\
\hline Totals & & $1.47 \mathrm{E}+03$ & $1.75 \mathrm{E}+03$ & $1.11 E+02$ & $2.13 E+02$ \\
\hline
\end{tabular}

Table 7. Annual Accident Dose Risk for the Shipping Campaign (person-rem/Yr)

\begin{tabular}{llcc}
\hline Route & & Truck & Rail \\
\hline Ames Laboratory & to Hanford Site & $1.04 \mathrm{E}-05$ & $3.93 \mathrm{E}-06$ \\
Argonne - East & to Hanford Site & $5.84 \mathrm{E}-02$ & $1.33 \mathrm{E}-02$ \\
Argonne - West & to Hanford Site & $3.18 \mathrm{E}+00$ & $1.72 \mathrm{E}+00$ \\
Bettis Atomic & to Hanford Site & $2.54 \mathrm{E}-03$ & $1.05 \mathrm{E}-03$ \\
Fermilab (FNAI) & to Hanford Site & $6.73 \mathrm{E}-05$ & $2.22 \mathrm{E}-05$ \\
INEL & to Hanford Site & $9.37 \mathrm{E}-02$ & $5.04 \mathrm{E}-02$ \\
ITRI & to Hanford Site & $5.50 \mathrm{E}-02$ & $6.27 \mathrm{E}-03$ \\
Oak Ridge Reserv. & to Hanford Site & $2.39 \mathrm{E}-03$ & $4.90 \mathrm{E}-04$ \\
Knol1s Atomic & to Hanford Site & $1.37 \mathrm{E}-04$ & $4.61 \mathrm{E}-05$ \\
Kansas City Plant & to Hanford Site & $2.32 \mathrm{E}-05$ & $4.82 \mathrm{E}-06$ \\
Los Alamos NL & to Hanford Site & $1.98 \mathrm{E}+01$ & $2.52 \mathrm{E}+00$ \\
Lawrence Berkeley & to Hanford Site & $1.52 \mathrm{E}-01$ & $8.48 \mathrm{E}-02$ \\
Lawrence Livermore to Hanford Site & $5.15 \mathrm{E}-04$ & $2.70 \mathrm{E}-04$ \\
Mound Plant & to Hanford Site & $3.01 \mathrm{E}-03$ & $6.71 \mathrm{E}-04$ \\
ORISE & to Hanford Site & $1.32 \mathrm{E}-05$ & $2.77 \mathrm{E}-06$ \\
Pantex Plant & to Hanford Site & $2.36 \mathrm{E}-03$ & $2.70 \mathrm{E}-04$ \\
Portsmouth GDP & to Hanford Site & $8.53 \mathrm{E}-05$ & $3.38 \mathrm{E}-05$ \\
Princeton PPL & to Hanford Site & $1.28 \mathrm{E}-05$ & $4.69 \mathrm{E}-06$ \\
Paducah GDP & to Hanford Site & $7.35 \mathrm{E}-04$ & $1.74 \mathrm{E}-04$ \\
Pinellas Plant & to Hanford Site & $1.37 \mathrm{E}-05$ & $3.00 \mathrm{E}-06$ \\
Rocky Flats Plant & to Hanford Site & $1.23 \mathrm{E}-03$ & $3.31 \mathrm{E}-04$ \\
Reactive Metals & to Hanford Site & $8.89 \mathrm{E}-06$ & $3.69 \mathrm{E}-06$ \\
SLAC & to Hanford Site & $1.68 \mathrm{E}-05$ & $9.87 \mathrm{E}-06$ \\
SNL - Albuquerque & to Hanford Site & $3.88 \mathrm{E}-04$ & $5.29 \mathrm{E}-05$ \\
SNL - Livermore & to Hanford Site & $5.28 \mathrm{E}-04$ & $2.79 \mathrm{E}-04$ \\
Savannah River & to Hanford Site & $3.46 \mathrm{E}+01$ & $6.94 \mathrm{E}+00$ \\
\hline Totals & & $5.80 \mathrm{E}+01$ & $1.13 \mathrm{E}+01$ \\
\hline
\end{tabular}


Table 8. Expected Annual Fatalities

for the Shipping Campaign

\begin{tabular}{lcc}
\hline Exposure Group & Truck & Rail \\
\hline Radiological & $5.9 E-01$ & $4.4 E-02$ \\
Normal Crew & $8.7 E-01$ & $1.1 \mathrm{E}-01$ \\
Normal Public & $2.9 \mathrm{E}-02$ & $5.7 \mathrm{E}-03$ \\
Accident Public & & \\
Nonradiological & & \\
Emission & $2.2 \mathrm{E}-01$ & $1.6 \mathrm{E}-01$ \\
Accident & $3.3 \mathrm{E}+00$ & $4.4 \mathrm{E}-02$ \\
\hline
\end{tabular}

Table 9. Expected Annual Cancer Incidence for the Shipping Campaign

\begin{tabular}{lll}
\hline Exposure Group & Truck & Rail \\
\hline Radiological & & \\
Normal Crew & $2.1 \mathrm{E}+00$ & $1.6 \mathrm{E}-01$ \\
Normal Public & $3.0 \mathrm{E}+00$ & $3.6 \mathrm{E}-01$ \\
Accident Public & $9.9 \mathrm{E}-02$ & $1.9 \mathrm{E}-02$ \\
$\begin{array}{c}\text { Nonradiological } \\
\text { Emission }\end{array}$ & $2.2 \mathrm{E}-01$ & $1.6 \mathrm{E}-01$ \\
Accident & $\mathrm{NA}$ & $\mathrm{NA}$ \\
\hline
\end{tabular}

Table 10. Expected Annual Genetic Effects for the Shipping Campaign

\begin{tabular}{lll}
\hline Exposure Group & Truck & Rail \\
\hline Radiological & & \\
Normal Crew & $8.8 E-02$ & $6.7 E-03$ \\
Normal public & $1.7 E-01$ & $2.1 E-02$ \\
Accident Public & $5.3 E-03$ & $1.0 \mathrm{E}-03$ \\
Nonradiological & $\mathrm{NA}$ & $\mathrm{NA}$ \\
Emission & $\mathrm{NA}$ & $\mathrm{NA}$ \\
Accident & & \\
\hline
\end{tabular}




\section{B.28 WM LLW CENTRALIZED 5 ALTERNATIVE (CASE 21): ACTIVATED METALS}

Table 1. Summary of Route Information

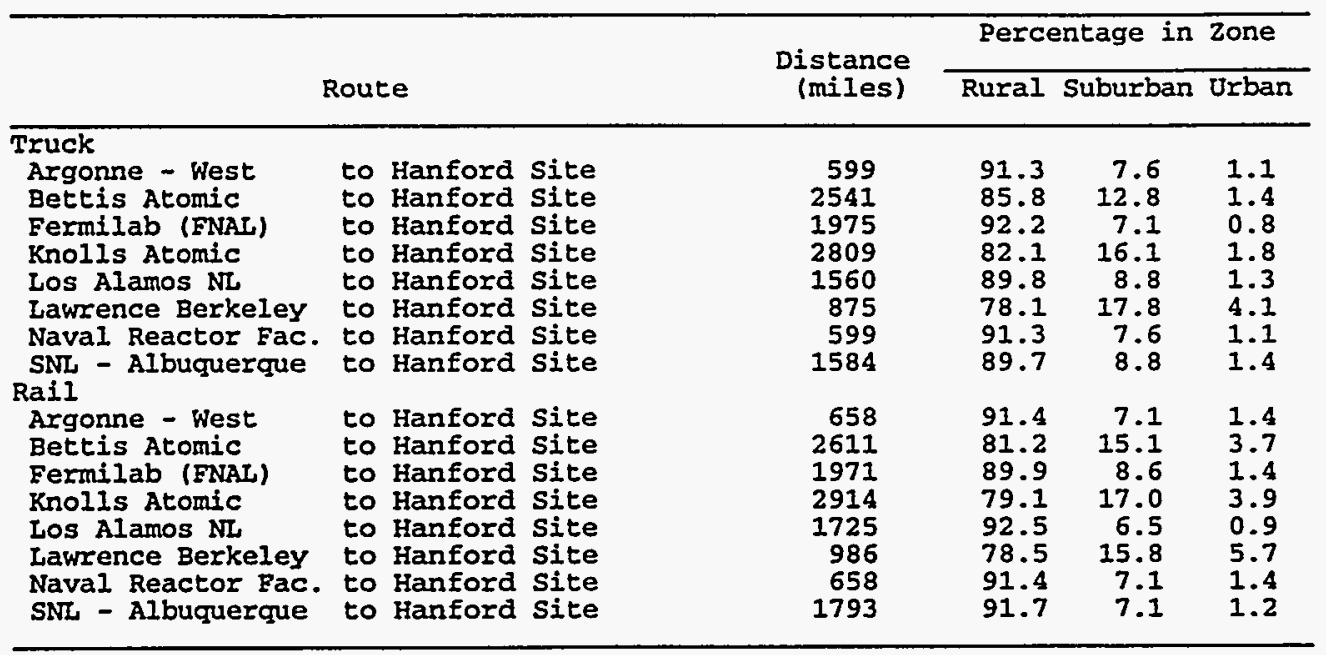

Table 2. Annual Number of Shipments and One-Way Shipment Distances

\begin{tabular}{|c|c|c|c|c|c|}
\hline \multirow[b]{2}{*}{ Route } & & \multicolumn{2}{|c|}{ \# of Shipments } & \multicolumn{2}{|c|}{ Total Mileage(mi) } \\
\hline & & Truck & Rail & Truck & Rail \\
\hline $\begin{array}{l}\text { Argonne - West } \\
\text { Bettis Atomic } \\
\text { Fermilab (FNAL) } \\
\text { Rnolis Atomic } \\
\text { Los Alamos NL } \\
\text { Lawrence Berkeley } \\
\text { Naval Reactor Fac. } \\
\text { SNL - Albuquerque }\end{array}$ & $\begin{array}{l}\text { to Hanford Site } \\
\text { to Hanford Site } \\
\text { to Hanford Site } \\
\text { to Hanford Site } \\
\text { to Hanford Site } \\
\text { to Hanford Site } \\
\text { to Hanford Site } \\
\text { to Hanford Site }\end{array}$ & $\begin{array}{r}8 \\
309 \\
39 \\
639 \\
412 \\
14 \\
450 \\
6\end{array}$ & $\begin{array}{r}4 \\
119 \\
16 \\
245 \\
158 \\
6 \\
178 \\
3\end{array}$ & $\begin{array}{l}4.79 \mathrm{E}+03 \\
7.85 \mathrm{E}+05 \\
7.70 \mathrm{E}+04 \\
1.80 \mathrm{E}+06 \\
6.43 \mathrm{E}+05 \\
1.22 \mathrm{E}+04 \\
2.70 \mathrm{E}+05 \\
9.50 \mathrm{E}+03\end{array}$ & $\begin{array}{l}2.63 E+03 \\
3.11 E+05 \\
3.15 E+04 \\
7.14 E+05 \\
2.73 E+05 \\
5.91 E+03 \\
1.17 E+05 \\
5.38 E+03\end{array}$ \\
\hline Totals & & 1877 & 729 & $3.60 E+06$ & $1.46 \mathrm{E}+06$ \\
\hline
\end{tabular}


Table 3. Incident-Free Dose per Shipment (person-rem/shipment)

\begin{tabular}{|c|c|c|c|c|c|}
\hline \multirow[b]{2}{*}{ Source/Route (s) } & \multirow[b]{2}{*}{ Crew } & \multicolumn{4}{|c|}{ General Public } \\
\hline & & off-Link & On-Link & Stops & Total \\
\hline $\begin{array}{l}\text { LLW(I) AW to HS } \\
\text { Argonne - Hest }\end{array}$ & $\begin{array}{lr}\text { to Hanford Site } \\
\text { Truck } & 1.60 \mathrm{E}-02 \\
\text { Raji } & 5.63 \mathrm{E}-03\end{array}$ & $\begin{array}{l}7.18 \mathrm{E}-04 \\
2.83 \mathrm{E}-03\end{array}$ & $\begin{array}{l}2.06 \mathrm{E}-03 \\
6.58 \mathrm{E}-05\end{array}$ & $\begin{array}{l}1.68 \mathrm{E}-02 \\
5.51 \mathrm{E}-03\end{array}$ & $\begin{array}{l}1.95 E-02 \\
8.40 E-03\end{array}$ \\
\hline $\begin{array}{l}\text { LLW(I) BA to HS } \\
\text { Bettis Atomic }\end{array}$ & $\begin{array}{ll}\text { to Hanford Site } \\
\text { Truck } & 7.21 \mathrm{E}-02 \\
\text { Rail } & 1.38 \mathrm{E}-02\end{array}$ & $\begin{array}{l}4.36 \mathrm{E}-03 \\
2.67 \mathrm{E}-02\end{array}$ & $\begin{array}{l}9.77 \mathrm{E}-03 \\
4.53 \mathrm{E}-04\end{array}$ & $\begin{array}{l}7.11 \mathrm{E}-02 \\
1.15 \mathrm{E}-02\end{array}$ & $\begin{array}{l}8.53 \mathrm{E}-02 \\
3.87 \mathrm{E}-02\end{array}$ \\
\hline $\begin{array}{l}\text { LLW(I) FL to HS } \\
\text { Fermilab (FNAL) }\end{array}$ & $\begin{array}{ll}\text { to Hanford Site } \\
\text { Truck } & 5.20 \mathrm{E}-02 \\
\text { Rail } & 1.11 \mathrm{E}-02\end{array}$ & $\begin{array}{l}1.92 \mathrm{E}-03 \\
9.07 \mathrm{E}-03\end{array}$ & $\begin{array}{l}6.25 E-03 \\
2.12 E-04\end{array}$ & $\begin{array}{l}5.53 \mathrm{E}-02 \\
9.55 \mathrm{E}-03\end{array}$ & $\begin{array}{l}6.35 \mathrm{E}-02 \\
1.88 \mathrm{E}-02\end{array}$ \\
\hline $\begin{array}{l}\text { LLW(I) KA to HS } \\
\text { Knolls Atomic }\end{array}$ & $\begin{array}{lr}\text { to Hanford Site } \\
\text { Truck } & 8.30 \mathrm{E}-02 \\
\text { Rail } & 1.50 \mathrm{E}-02\end{array}$ & $\begin{array}{l}6.03 \mathrm{E}-03 \\
3.18 \mathrm{E}-02\end{array}$ & $\begin{array}{l}1.19 E-02 \\
5.40 E-04\end{array}$ & $\begin{array}{l}7.87 \mathrm{E}-02 \\
1.25 \mathrm{E}-02\end{array}$ & $\begin{array}{l}9.66 \mathrm{E}-02 \\
4.48 \mathrm{E}-02\end{array}$ \\
\hline $\begin{array}{l}\text { LLW(I) LA to HS } \\
\text { LOS Alamos NL }\end{array}$ & $\begin{array}{lr}\text { to Hanford Site } \\
\text { Truck } & 4.24 \mathrm{E}-02 \\
\text { Rail } & 1.01 \mathrm{E}-02\end{array}$ & $\begin{array}{l}2.16 \mathrm{E}-03 \\
5.60 \mathrm{E}-03\end{array}$ & $\begin{array}{l}5.65 E-03 \\
1.54 E-04\end{array}$ & $\begin{array}{l}4.37 E-02 \\
8.79 E-03\end{array}$ & $\begin{array}{l}5.15 \mathrm{E}-02 \\
1.45 \mathrm{E}-02\end{array}$ \\
\hline $\begin{array}{l}\text { LLW(I) LB to HS } \\
\text { Lawrence Berkeley }\end{array}$ & $\begin{array}{lr}\text { to Hanford Site } \\
\text { Truck } & 2.76 \mathrm{E}-02 \\
\text { Rail } & 6.99 \mathrm{E}-03\end{array}$ & $\begin{array}{l}3.02 \mathrm{E}-03 \\
1.38 \mathrm{E}-02\end{array}$ & $\begin{array}{l}5.17 E-03 \\
2.03 E-04\end{array}$ & $\begin{array}{l}2.45 E-02 \\
6.51 E-03\end{array}$ & $\begin{array}{l}3.27 \mathrm{E}-02 \\
2.05 \mathrm{E}-02\end{array}$ \\
\hline $\begin{array}{l}\text { LLW(I) NR to HS } \\
\text { Naval Reactor Fac. }\end{array}$ & $\begin{array}{ll}\text { to Hanford Site } \\
\text { Truck } & 1.60 \mathrm{E}-02 \\
\text { Rail } & 5.63 \mathrm{E}-03\end{array}$ & $\begin{array}{l}7.18 \mathrm{E}-04 \\
2.83 \mathrm{E}-03\end{array}$ & $\begin{array}{l}2.06 \mathrm{E}-03 \\
6.58 \mathrm{E}-05\end{array}$ & $\begin{array}{l}1.68 \mathrm{E}-02 \\
5.51 \mathrm{E}-03\end{array}$ & $\begin{array}{l}1.95 \mathrm{E}-02 \\
8.40 \mathrm{E}-03\end{array}$ \\
\hline $\begin{array}{l}\text { LLW(I) SA to HS } \\
\text { SNL - Albuquerque }\end{array}$ & $\begin{array}{ll} & \\
\text { to Hanford Site } \\
\text { Truck } & 4.31 \mathrm{E}-02 \\
\text { Rail } & 1.04 \mathrm{E}-02\end{array}$ & $\begin{array}{l}2.27 \Sigma-03 \\
6.90 \mathrm{E}-03\end{array}$ & $\begin{array}{l}5.84 \mathrm{E}-03 \\
1.72 \mathrm{E}-04\end{array}$ & $\begin{array}{l}4.43 \mathrm{E}-02 \\
9.00 \mathrm{E}-03\end{array}$ & $\begin{array}{l}5.24 \mathrm{E}-02 \\
1.61 \mathrm{E}-02\end{array}$ \\
\hline
\end{tabular}

Table 4. Accident Dose Risk per Shipment (person-rem/shipment)

\begin{tabular}{|c|c|c|c|}
\hline Source/Route & & Truck & Rail \\
\hline $\begin{array}{l}\text { LLW(I) AW to HS } \\
\text { Argonne - West } \\
\text { LLW(I) BA to HS } \\
\text { Bettis Atomic } \\
\text { LLW(I) FL to HS } \\
\text { Fermilab (FNAL) } \\
\text { LLW(I) KA to HS } \\
\text { Knolls Atomic } \\
\text { LLW(I) LA to HS } \\
\text { Los Alamos NL } \\
\text { LLW(I) LB to HS } \\
\text { Lawrence Berkeley } \\
\text { LLW(I) NR to HS } \\
\text { Naval Reactor Fac. } \\
\text { LLW(I) SA to HS } \\
\text { SNL - Albuquerque }\end{array}$ & $\begin{array}{l}\text { to Hanford Site } \\
\text { to Hanford Site } \\
\text { to Hanford Site } \\
\text { to Hanford Site } \\
\text { to Hanford Site } \\
\text { to Hanford Site } \\
\text { to Hanford Site } \\
\text { to Hanford Site }\end{array}$ & $\begin{array}{l}9.18 \mathrm{E}-12 \\
1.84 \mathrm{E}-06 \\
4.76 \mathrm{E}-11 \\
3.12 \mathrm{E}-06 \\
3.15 \mathrm{E}-09 \\
1.81 \mathrm{E}-10 \\
4.57 \mathrm{E}-08 \\
6.06 \mathrm{E}-12\end{array}$ & $\begin{array}{l}9.58 \mathrm{E}-12 \\
1.79 \mathrm{E}-06 \\
3.86 \mathrm{E}-11 \\
2.60 \mathrm{E}-06 \\
8.94 \mathrm{E}-10 \\
2.37 \mathrm{E}-10 \\
6.22 \mathrm{E}-08 \\
1.32 \mathrm{E}-12\end{array}$ \\
\hline
\end{tabular}


- Maximally Exposed Individuals during Routine Conditions. During the routine transportation of radioactive waste, specific individuals may be exposed to radiation in the vicinity of a shipment. The risk to these individuals has been estimated for a number of hypothetical exposure-causing events by using RISKIND. The receptors include transportation crew members, inspectors, and members of the public exposed during traffic delays, while working at a service station, or while living near a DOE site. The assumptions about exposure are given in Section 4.6. The scenarios for exposure are not meant to be exhaustive but were selected to provide a range of potential exposures.

For the majority of the individual receptors considered, doses are assessed and presented on a per-event basis - no attempt is made to estimate the frequency of exposure-causing events, although the range of possible exposures is qualitatively discussed. However, one exception is the calculation of the dose to a hypothetical resident living near the entrance route to a treatment, storage, or disposal site. For these residents, cumulative doses are calculated on the basis of the total number of shipments entering or exiting each site for each alternative.

- Accident Consequence Assessment. The RISKIND code is used to provide a detailed assessment of the consequences of the most severe transportationrelated accidents involving LLW shipments. Whereas the RADTRAN 4 collective accident risk assessment considers the entire range of accident severities and their related probabilities, the RISKIND accident consequence assessment assumes that an accident of the highest severity category (Category VIII) has occurred. The consequences, in terms of committed dose (rem) and latent-cancer fatalities, are calculated for both exposed populations and individuals in the vicinity of an accident.

\subsection{COLLECTIVE POPULATION RISK RESULTS}

The results of the collective population risk assessment are summarized in this section. The summarized results present the total transportation impacts that would be incurred across the DOE complex for each alternative from all LLW shipments associated with that alternative. The summarized results do not indicate the quantity of waste that would be shipped between specific sites for a given alternative; however, detailed results of assessment are presented in Appendix B. The detailed results include route information, the estimated number of annual shipments, and "pershipment" risk factors (an estimate of the risk for a single shipment between two specific sites) for shipments between all origin-destination pairs considered by each alternative. 
Table 5. Nonradiological Risk Factors per Shipment (fatalities/shipment)

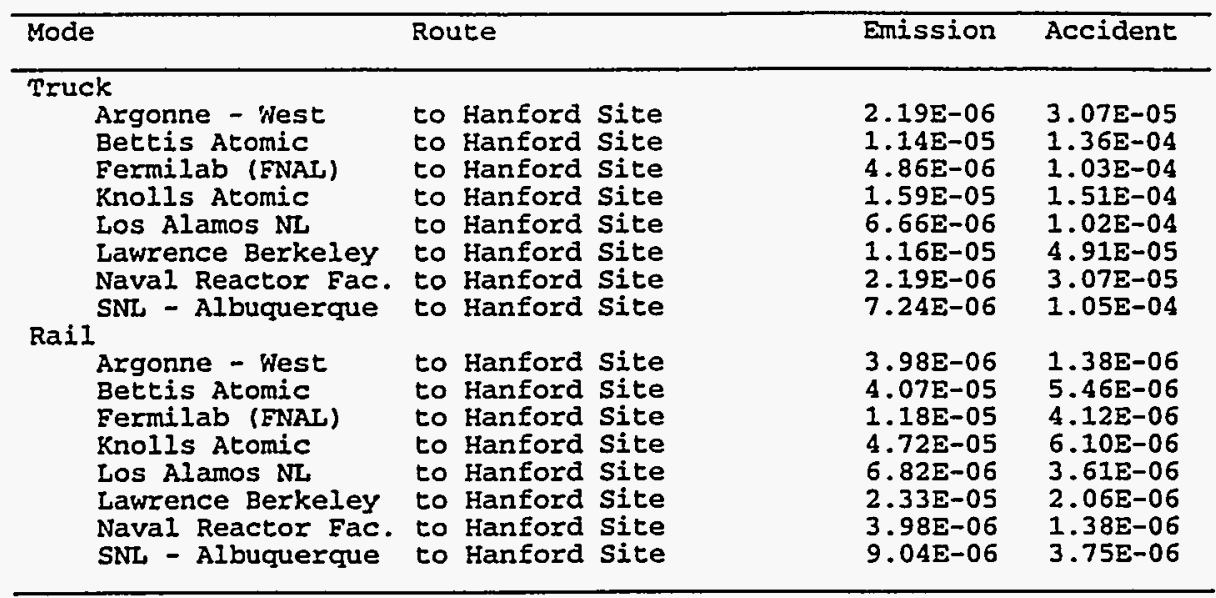

Table 6. Annual Incident-Free Dose for the Shipping Campaign (person-rem/yr)

\begin{tabular}{|c|c|c|c|c|c|}
\hline \multirow[b]{2}{*}{ Route } & & \multicolumn{2}{|c|}{ Truck } & \multicolumn{2}{|c|}{ Rail } \\
\hline & & Crew & Public & Crew & Public \\
\hline $\begin{array}{l}\text { Argonne - West } \\
\text { Bettis Atomic } \\
\text { Fermilab (FNAL) } \\
\text { Knolls Atomic } \\
\text { Los Alamos NL } \\
\text { Lawrence Berkeley } \\
\text { Naval Reactor Fac. } \\
\text { SNL - Albuquerque }\end{array}$ & $\begin{array}{l}\text { to Hanford Site } \\
\text { to Hanford Site } \\
\text { to Hanford Site } \\
\text { to Hanford Site } \\
\text { to Hanford Site } \\
\text { to Hanford Site } \\
\text { to Hanford Site } \\
\text { to Hanford Site }\end{array}$ & $\begin{array}{l}7.28 \mathrm{E}-01 \\
2.23 \mathrm{E}+01 \\
2.03 \mathrm{E}+00 \\
5.30 \mathrm{E}+01 \\
1.75 \mathrm{E}+01 \\
3.86 \mathrm{E}-01 \\
7.20 \mathrm{E}+00 \\
2.59 \mathrm{E}-01\end{array}$ & $\begin{array}{l}1.56 \mathrm{E}-01 \\
2.63 \mathrm{E}+01 \\
2.47 \mathrm{E}+00 \\
6.17 \mathrm{E}+01 \\
2.12 \mathrm{E}+01 \\
4.58 \mathrm{E}-01 \\
8.80 \mathrm{E}+00 \\
3.15 \mathrm{E}-01\end{array}$ & $\begin{array}{l}2.25 \mathrm{E}-02 \\
1.64 \mathrm{E}+00 \\
1.78 \mathrm{E}-01 \\
3.68 \mathrm{E}+00 \\
1.59 \mathrm{E}+00 \\
4.20 \mathrm{E}-02 \\
1.00 \mathrm{E}+00 \\
3.11 \mathrm{E}-02\end{array}$ & $\begin{array}{l}3.36 \mathrm{E}-02 \\
4.61 \mathrm{E}+00 \\
3.01 \mathrm{E}-01 \\
1.10 \mathrm{E}+01 \\
2.30 \mathrm{E}+00 \\
1.23 \mathrm{E}-01 \\
1.50 \mathrm{E}+00 \\
4.82 \mathrm{E}-02\end{array}$ \\
\hline Totals & & $1.03 \mathrm{E}+02$ & $1.21 E+02$ & $8.19 E+00$ & $1.99 \mathrm{E}+01$ \\
\hline
\end{tabular}

Table 7. Annual Accident Dose Risk for the Shipping Campaign (person-rem/yr)

\begin{tabular}{llcc}
\hline Route & & Truck & Rail \\
\hline Argonne - West & to Hanford Site & $7.34 \mathrm{E}-11$ & $3.83 \mathrm{E}-11$ \\
Bettis Atomic & to Hanford Site & $5.68 \mathrm{E}-04$ & $2.13 \mathrm{E}-04$ \\
Fermilab (FNAL) & to Hanford Site & $1.86 \mathrm{E}-09$ & $6.17 \mathrm{E}-10$ \\
Rnolis Atomic & to Hanford Site & $1.99 \mathrm{E}-03$ & $6.36 \mathrm{E}-04$ \\
Los Alamos NL & to Hanford Site & $1.30 \mathrm{E}-06$ & $1.41 \mathrm{E}-07$ \\
Lawrence Berkeley to Hanford Site & $2.53 \mathrm{E}-09$ & $1.42 \mathrm{E}-09$ \\
Naval Reactor Fac. to Hanford Site & $2.06 \mathrm{E}-05$ & $1.11 \mathrm{E}-05$ \\
SNL - Albuquerque & to Hanford Site & $3.63 \mathrm{E}-11$ & $3.96 \mathrm{E}-12$ \\
\hline Totals & & $2.58 \mathrm{E}-03$ & $8.61 \mathrm{E}-04$ \\
\hline
\end{tabular}

Table 8. Expected Annual Fatalities for the Shipping Campaign

\begin{tabular}{lll}
\hline Exposure Group & Truck & Rail \\
\hline Radiological & & \\
Normal Crew & $4.1 E-02$ & $3.3 E-03$ \\
Normal Public & $6.1 E-02$ & $9.9 E-03$ \\
Accident Public & $1.3 E-06$ & $4.3 \mathrm{E}-07$ \\
Nonradiological & $1.8 \mathrm{E}-02$ & $1.9 \mathrm{E}-02$ \\
Emission & $2.0 \mathrm{E}-01$ & $3.1 \mathrm{E}-03$ \\
Accident & & \\
\hline
\end{tabular}


Table 9. Expected Annual Cancer Incidence for the Shipping Campaign

\begin{tabular}{lll}
\hline Exposure Group & Truck & Rail \\
\hline Radiological & & \\
Normal Crew & $1.4 \mathrm{E}-01$ & $1.1 \mathrm{E}-02$ \\
$\quad$ Oormal Public & $2.1 \mathrm{E}-01$ & $3.4 \mathrm{E}-02$ \\
$\quad$ Accident Public & $4.4 \mathrm{E}-06$ & $1.5 \mathrm{E}-06$ \\
Nonradiological & $1.8 \mathrm{E}-02$ & $1.9 \mathrm{E}-02$ \\
$\quad$ Emission & $\mathrm{NA}$ & $\mathrm{NA}$ \\
Accident & & \\
\hline
\end{tabular}

Table 10. Expected Annual Genetic Effects for the Shipping Campaign

\begin{tabular}{lll}
\hline Exposure Group & Truck & Rail \\
\hline Radiological & $6.2 \mathrm{E}-03$ & $4.9 \mathrm{E}-04$ \\
Normal Crew & $1.2 \mathrm{E}-02$ & $2.0 \mathrm{E}-03$ \\
Normal public & $2.6 \mathrm{E}-07$ & $8.7 \mathrm{E}-08$ \\
Accident Public & $\mathrm{NA}$ & $\mathrm{NA}$ \\
Nonradiological & NA & NA \\
$\quad$ Emission & & \\
Accident & &
\end{tabular}




\section{$B-156$}




\section{TABLES (Cont.)}

4.4 Estimated Release Fractions for LLW Packages under Various Accident Severity Categories .................................... 35

4.5 Aerosolized and Respirable Material Release Fractions for LLW ............ 36

4.6 General RADTRAN Input Parameters for the LLW Assessment ............... 39

5.1 Total Number of Shipments and One-Way Shipping Distances

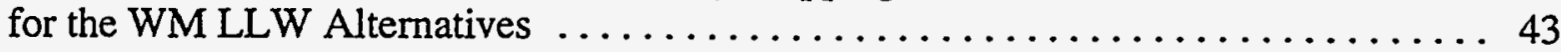

5.2 Total Routine Dose for the WM LLW Alternatives $\ldots \ldots \ldots \ldots \ldots \ldots \ldots$

5.3 Total Accident Dose Risk for the WM LLW Alternatives $\ldots \ldots \ldots \ldots \ldots \ldots \ldots 4$

5.4 Total Estimated Fatalities for the WM LLW Alternatives $\ldots \ldots \ldots \ldots \ldots \ldots$

5.5 Total Estimated Cancer Incidence for the WM LLW Alternatives ............ 50

5.6 Total Estimated Genetic Effects for the WM LLW Alternatives ............. 54

5.7 Estimated Routine Doses and Lifetime Risk of Fatal Cancer to MEIs from Shipments of WM LLW ...................... 58

5.8 Cumulative Dose and Lifetime Risk to MEIs Living along a Site Entrance Route for WM LLW Shipments ........................ 59

5.9 Estimated Consequences for the Most Severe Accidents Involving

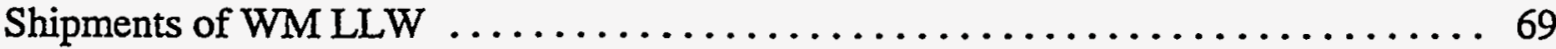

\section{FIGURES}

3.1 Technical Approach for the LLW Transportation Radiological

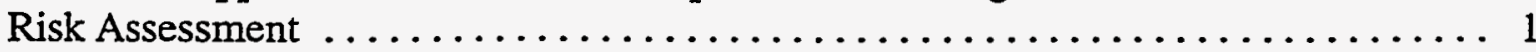

4.1 Scheme for NUREG-0170 Classification by Accident Severity Category for Truck Accidents

4.2 Scheme for NUREG-0170 Classification by Accident Severity Category

for Rail Accidents 
The collective risk results for the 14 WM LLW alternatives are summarized in a series of tables. The collective risk results were calculated with the RADTRAN 4 computer code by using the WASTE_MGMT files provided in Appendix A as the primary input. The summarized results present the total impacts from shipping current inventories plus 20 years of WM LLW generation. As stated previously, the average annual risk (or number of shipments) can be estimated by dividing the summarized results by the duration of the shipping campaigns. For the No Action Alternative, shipments would be distributed uniformly over a 20-year period; however, for all other alternatives, shipments would occur uniformly over a 10-year period, with the assumption of a 10 -year period to build TSD facilities.

The total number of shipments and shipment mileages are presented for each of the WM LLW alternatives in Table 5.1. The numbers of shipments were calculated by using the inventory data presented in Appendix $A$ and the assumptions concerning representative shipment sizes presented in Section 4.1. The total incident-free (routine) dose for each alternative is presented in Table 5.2 in terms of person-rem. The routine doses are presented separately for crew members and for members of the public along the transportation routes; these doses result from exposures to external radiation in the vicinity of a shipment. The total accident dose risk for each alternative is presented in Table 5.3. The estimates of accident dose risk depend on the specific radionuclides present in each shipment and take into account the probability of accident occurrences and the distribution of accident severities. Finally, impacts are presented in terms of human health risks in Tables 5.4 through 5.6. The health risks are presented for cargo-related (radiological) and vehiclerelated (nonradiological) causes. All impacts are presented in two columns, with the first column assuming all truck shipments and the second assuming all rail shipments.

\subsection{MAXIMALLY EXPOSED INDIVIDUAL ASSESSMENT}

Doses to individuals during routine transportation have been estimated for each of the receptor scenarios described in Section 4.6 for WM LLW shipments. The estimated doses to individuals exposed to WM LLW shipments are presented in Table 5.7. The doses are calculated by assuming an external dose rate of $1 \mathrm{mrem} / \mathrm{h}$ at $1 \mathrm{~m}$ (3.3 ft) from a shipment and are reported on a perevent basis (rem per event). The total dose for repeated exposures can be estimated by multiplying the per-event dose by the number of exposures.

The potential exists for significant individual exposures if multiple exposure-causing events occur during WM LLW shipments; for instance, the dose to a person stuck in traffic next to a shipment for 30 minutes is estimated to be $0.5 \mathrm{mrem}$. If the duration of exposure is longer, the dose would rise proportionally. It is conceivable that a person could receive a dose on the order of 2-10 mrem while stopped in traffic next to a WM LLW shipment. In addition, a person working at a truck service station could receive an increased dose if trucks use the same stops repeatedly. If a truck-stop worker is present for 100 shipment stops (at the distance and duration given previously), 
TABLE 5.1 Total Number of Shipments and One-Way Shipping Distances for the WMI LLW Alternatives ${ }^{a}$

\begin{tabular}{lrrrrrr}
\hline & \multicolumn{2}{c}{ No. of Shipments } & & \multicolumn{2}{c}{ Total Mileage (mi) } \\
\cline { 2 - 3 } \cline { 5 - 6 } \multicolumn{1}{c}{ WM LLW Alternative } & \multicolumn{1}{c}{ Truck } & Rail & & Truck & Rail \\
\hline & & & & & & \\
No Action (Case 1) & 87,360 & 33,420 & & $1.66 \mathrm{E}+08$ & $6.99 \mathrm{E}+07$ \\
Decentralized (Case 2) & 24,420 & 9,210 & & $8.63 \mathrm{E}+06$ & $3.50 \mathrm{E}+06$ \\
Regionalized 1 (Case 3) & 25,800 & 9,740 & & $9.31 \mathrm{E}+06$ & $3.74 \mathrm{E}+06$ \\
Regionalized 2 (Case 9) & 25,880 & 9,900 & & $9.19 \mathrm{E}+06$ & $3.78 \mathrm{E}+06$ \\
Regionalized 3 (Case 4) & 84,200 & 31,850 & & $3.81 \mathrm{E}+07$ & $1.72 \mathrm{E}+07$ \\
Regionalized 4 (Case 12) & 87,390 & 33,460 & & $3.69 \mathrm{E}+07$ & $1.66 \mathrm{E}+07$ \\
Regionalized 5 (Case 19) & 92,200 & 35,430 & & $6.38 \mathrm{E}+07$ & $2.53 \mathrm{E}+07$ \\
Regionalized 6 (Case 5) & 174,390 & 66,040 & & $1.24 \mathrm{E}+08$ & $5.14 \mathrm{E}+07$ \\
Regionalized 7 (Case 6) & 188,930 & 71,480 & & $1.25 \mathrm{E}+08$ & $5.44 \mathrm{E}+07$ \\
Centralized 1 (Case 7) & 242,730 & 91,440 & & $5.63 \mathrm{E}+08$ & $2.24 \mathrm{E}+08$ \\
Centralized 2 (Case 8) & 257,270 & 96,880 & & $5.05 \mathrm{E}+08$ & $2.19 \mathrm{E}+08$ \\
Centralized 3 (Case 14) & 250,020 & 96,710 & & $5.30 \mathrm{E}+08$ & $2.18 \mathrm{E}+08$ \\
Centralized 4 (Case 14a) & 264,060 & 102,100 & & $4.78 \mathrm{E}+08$ & $2.12 \mathrm{E}+08$ \\
Centralized 5 (Case 21) & 241,540 & 90,980 & & $5.60 \mathrm{E}+08$ & $2.23 \mathrm{E}+08$ \\
\hline
\end{tabular}

a For each case, impacts are calculated and presented separately assuming exclusive truck or exclusive rail shipments. Therefore, the impacts for truck and rail modes should not be added together.

b The number of shipments is calculated by assuming that truck and rail shipments consist of $19,958 \mathrm{~kg}(44,000 \mathrm{lb})$ and $54,431 \mathrm{~kg}(120,000 \mathrm{lb})$ of LLW, respectively.

the estimated dose is on the order of 2 mrem. Administrative controls could be instituted to control the location and duration of truck stops if multiple exposures were to happen routinely. The probability of multiple exposures increases as the amount of waste transportation increases.

The potential cumulative dose to a resident living along a site entrance route has been estimated and is summarized in Table 5.8 for each WM LLW alternative. The cumulative dose assumes that a resident is present for every shipment either entering or exiting a site and is unshielded at a distance of $30 \mathrm{~m}$ ( $98 \mathrm{ft}$ ) from the roadway. The assessment considers 20 years of shipments for WM LLW. The lifetime risk of fatality due to cancer has been calculated by multiplying the doses by the ICRP health risk conversion factor of $5 \mathrm{E}-04$ fatal cancers per rem for members of the public. The estimated annual dose to a resident would be well below the annual limit of 100 mrem specified for members of the public (DOE 1990b). 
TABLE 5.2 Total Routine (Incident-Free) Dose for the WM LLW Alternatives ${ }^{\text {a }}$

\begin{tabular}{|c|c|c|c|c|}
\hline \multirow[b]{3}{*}{ WM LLW Alternative } & \multicolumn{4}{|c|}{ Dose (person-rem) } \\
\hline & \multicolumn{2}{|c|}{ Truck } & \multicolumn{2}{|c|}{ Rail } \\
\hline & Crew $^{\mathrm{b}}$ & Public $^{c}$ & Crew $^{b}$ & Public ${ }^{c}$ \\
\hline No Action (Case 1) & $4.69 E+03$ & $5.62 E+03$ & $3.88 E+02$ & $8.49 E+02$ \\
\hline Decentralized (Case 2) & $3.19 E+02$ & $3.35 E+02$ & $4.11 E+01$ & $1.28 \mathrm{E}+02$ \\
\hline Regionalized 1 (Case 3) & $3.43 E+02$ & $3.62 E+02$ & $4.37 \mathrm{E}+01$ & $1.35 \mathrm{E}+02$ \\
\hline Regionalized 2 (Case 9) & $3.38 \mathrm{E}+02$ & $3.57 \mathrm{E}+02$ & $4.42 \mathrm{E}+01$ & $1.36 \mathrm{E}+02$ \\
\hline Regionalized 3 (Case 4) & $1.21 E+03$ & $1.34 \mathrm{E}+03$ & $1.63 E+02$ & $4.08 \mathrm{E}+02$ \\
\hline Regionalized 4 (Case 12) & $1.19 E+03$ & $1.31 E+03$ & $1.66 \mathrm{E}+02$ & $3.68 \mathrm{E}+02$ \\
\hline Regionalized 5 (Case 19) & $1.90 \mathrm{E}+03$ & $2.18 \mathrm{E}+03$ & $2.08 \mathrm{E}+02$ & $4.70 \mathrm{E}+02$ \\
\hline Regionalized 6 (Case 5) & $3.87 E+03$ & $4.35 E+03$ & $4.05 \mathrm{E}+02$ & $8.20 \mathrm{E}+02$ \\
\hline Regionalized 7 (Case 6) & $3.89 E+03$ & $4.41 E+03$ & $4.33 E+02$ & $8.45 E+02$ \\
\hline Centralized 1 (Case 7) & $1.58 \mathrm{E}+04$ & $1.87 \mathrm{E}+04$ & $1.19 \mathrm{E}+03$ & $2.34 \mathrm{E}+03$ \\
\hline Centralized 2 (Case 8) & $1.45 \mathrm{E}+04$ & $1.72 E+04$ & $1.19 E+03$ & $2.34 \mathrm{E}+03$ \\
\hline Centralized 3 (Case 14) & $1.49 E+04$ & $1.77 E+04$ & $1.19 E+03$ & $2.31 E+03$ \\
\hline Centralized 4 (Case 14a) & 1.37E+04 & $1.63 E+04$ & $1.18 \mathrm{E}+03$ & $2.31 E+03$ \\
\hline Centralized 5 (Case 21) & $1.57 \mathrm{E}+04$ & $1.87 \mathrm{E}+04$ & $1.19 \mathrm{E}+03$ & $2.33 \mathrm{E}+03$ \\
\hline
\end{tabular}

a Routine doses result from exposure to external radiation in the vicinity of a loaded shipment. The external dose rate is assumed to be $1 \mathrm{mrem} / \mathrm{h}$ at $1 \mathrm{~m}(3.3 \mathrm{ft})$ from a WM LLW shipment. For each case, impacts are calculated and presented separately assuming exclusive truck or exclusive rail shipments. Therefore, the impacts for truck and rail modes should not be added together.

b The crew includes truck drivers, rail crew members, and rail workers in marshaling or classification yards.

c Members of the public include persons in vehicles sharing transportation routes, persons living along routes, and persons exposed at shipment stops.

\subsection{ACCIDENT CONSEQUENCE ASSESSMENT}

The RISKIND (Yuan et al. 1993) code is used to provide a detailed assessment of the consequences of the most severe transportation-related accidents for WM LLW shipments. Whereas the RADTRAN 4 accident risk assessment considers the entire range of accident severities and their related probabilities, the RISKIND accident consequence assessment assumes that an accident of the highest credible severity has occurred.

Accident consequences are presented for a shipment of waste that represents the highest potential radiological risk if an accident occurs. This "worst-case" waste is identified by screening 
TABLE 5.3 Total Accident Dose Risk for the WMI LLW Alternatives ${ }^{a, b}$

\begin{tabular}{lll}
\hline & \multicolumn{2}{c}{$\begin{array}{c}\text { Dose Risk } \\
\text { (person-rem) }\end{array}$} \\
\cline { 2 - 3 } \multicolumn{1}{c}{ WM LLW Alternative } & Truck & Rail \\
\hline No Action (Case 1) & $2.45 \mathrm{E}+00$ & $1.03 \mathrm{E}+00$ \\
Decentralized (Case 2) & $1.32 \mathrm{E}-01$ & $8.59 \mathrm{E}-02$ \\
Regionalized 1 (Case 3) & $6.48 \mathrm{E}-01$ & $1.62 \mathrm{E}-01$ \\
Regionalized 2 (Case 9) & $6.48 \mathrm{E}-01$ & $1.62 \mathrm{E}-01$ \\
Regionalized 3 (Case 4) & $1.52 \mathrm{E}+00$ & $6.26 \mathrm{E}-01$ \\
Regionalized 4 (Case 12) & $2.13 \mathrm{E}+00$ & $8.86 \mathrm{E}-01$ \\
Regionalized 5 (Case 19) & $3.44 \mathrm{E}+02$ & $2.39 \mathrm{E}+01$ \\
Regionalized 6 (Case 5) & $2.33 \mathrm{E}+02$ & $4.40 \mathrm{E}+01$ \\
Regionalized 7 (Case 6) & $2.05 \mathrm{E}+02$ & $2.50 \mathrm{E}+01$ \\
Centralized 1 (Case 7) & $5.80 \mathrm{E}+02$ & $1.14 \mathrm{E}+02$ \\
Centralized 2 (Case 8) & $5.63 \mathrm{E}+02$ & $9.09 \mathrm{E}+01$ \\
Centralized 3 (Case 14) & $5.80 \mathrm{E}+02$ & $1.14 \mathrm{E}+02$ \\
Centralized 4 (Case 14a) & $5.67 \mathrm{E}+02$ & $9.16 \mathrm{E}+01$ \\
Centralized 5 (Case 21) & $5.80 \mathrm{E}+02$ & $1.13 \mathrm{E}+02$ \\
\hline
\end{tabular}

a The calculation of accident dose risk takes into account the dose to the public following accidents involving the release of radioactive material to the environment, the probability of accident occurrences, and the distribution of accident severities.

b For each case, impacts are calculated and presented separately assuming exclusive truck or exclusive rail shipments. Therefore, the impacts for truck and rail modes should not be added together.

the site-specific radiological waste characteristics (i.e., activity concentrations) developed for the WM PEIS, taking into account physical waste forms and the relative hazards of individual radionuclides. For the majority of waste shipments, the consequences of severe accidents would be less than those presented for the "worst-case" waste. The accident consequence assessment is intended to provide an estimate of the maximum potential impacts posed by a severe transportation accident involving a given waste type. The assessment is not meant to be used as a basis for comparison among the different alternatives.

As stated previously, the accident consequences were calculated for transportationrelated accidents that result in the maximum release of radioactive material (accident severity 
TABLE 5.4 Total Estimated Fatalities for the WM LLW Alternatives ${ }^{a, b}$

\begin{tabular}{|c|c|c|c|}
\hline \multirow[b]{2}{*}{ WM LLW Alternative } & \multirow[b]{2}{*}{ Exposure Group } & \multicolumn{2}{|c|}{ Fatalities } \\
\hline & & Truck & Rail \\
\hline \multirow[t]{7}{*}{ No Action (Case 1) } & $\begin{array}{l}\text { Cargo-related } \\
\text { (radiological) }\end{array}$ & & \\
\hline & Routine crew & $1.9 \mathrm{E}+00$ & $1.5 \mathrm{E}-01$ \\
\hline & Routine public & $2.7 \mathrm{E}+00$ & $4.3 E-01$ \\
\hline & Accident public & $1.2 \mathrm{E}-03$ & $5.2 \mathrm{E}-04$ \\
\hline & $\begin{array}{l}\text { Vehicle-related } \\
\text { (nonradiological) }\end{array}$ & & \\
\hline & Emission & $9.5 E-01$ & $7.8 \mathrm{E}-01$ \\
\hline & Accident & $1.1 E+01$ & $1.5 \mathrm{E}-01$ \\
\hline \multirow[t]{7}{*}{ Decentralized (Case 2) } & $\begin{array}{l}\text { Cargo-related } \\
\text { (radiological) }\end{array}$ & & \\
\hline & Routine crew & $1.3 \mathrm{E}-01$ & $1.6 \mathrm{E}-02$ \\
\hline & Routine public & $1.7 E-01$ & $6.4 \mathrm{E}-02$ \\
\hline & Accident public & $6.6 \mathrm{E}-05$ & $4.3 \mathrm{E}-05$ \\
\hline & $\begin{array}{l}\text { Vehicle-related } \\
\text { (nonradiological) }\end{array}$ & & \\
\hline & Emission & $8.8 \mathrm{E}-02$ & $1.2 \mathrm{E}-01$ \\
\hline & Accident & 4.3E-01 & $7.3 \mathrm{E}-03$ \\
\hline \multirow[t]{7}{*}{ Regionalized 1 (Case 3) } & $\begin{array}{l}\text { Cargo-related } \\
\text { (radiological) }\end{array}$ & & \\
\hline & Routine crew & $1.4 \mathrm{E}-01$ & $1.8 \mathrm{E}-02$ \\
\hline & Routine public & $1.9 \mathrm{E}-01$ & $6.7 \mathrm{E}-02$ \\
\hline & Accident public & $3.3 \mathrm{E}-04$ & $8.2 E-05$ \\
\hline & $\begin{array}{l}\text { Vehicle-related } \\
\text { (nonradiological) }\end{array}$ & & \\
\hline & Emission & $9.9 \mathrm{E}-02$ & $1.3 \mathrm{E}-01$ \\
\hline & Accident & $4.7 E-01$ & $7.8 \mathrm{E}-03$ \\
\hline \multirow[t]{7}{*}{ Regionalized 2 (Case 9) } & $\begin{array}{l}\text { Cargo-related } \\
\text { (radiological) }\end{array}$ & & \\
\hline & Routine crew & $1.4 \mathrm{E}-01$ & $1.8 \mathrm{E}-02$ \\
\hline & Routine public & $1.7 \mathrm{E}-01$ & $6.8 \mathrm{E}-02$ \\
\hline & Accident public & $3.3 \mathrm{E}-04$ & 8.2E-05 \\
\hline & $\begin{array}{l}\text { Vehicle-related } \\
\text { (nonradiological) }\end{array}$ & & \\
\hline & Emission & $9.6 \mathrm{E}-02$ & $1.3 \mathrm{E}-01$ \\
\hline & Accident & $4.6 \mathrm{E}-01$ & $7.9 E-03$ \\
\hline
\end{tabular}


TABLE 5.4 (Cont.)

\begin{tabular}{|c|c|c|c|}
\hline \multirow[b]{2}{*}{ WM LLW Alternative } & \multirow[b]{2}{*}{ Exposure Group } & \multicolumn{2}{|c|}{ Fatalities } \\
\hline & & Truck & Rail \\
\hline \multirow[t]{7}{*}{ Regionalized 3 (Case 4) } & $\begin{array}{l}\text { Cargo-related } \\
\text { (radiological) }\end{array}$ & & \\
\hline & Routine crew & $4.9 \mathrm{E}-01$ & $6.5 E-02$ \\
\hline & Routine public & $6.7 \mathrm{E}-01$ & $2.1 E-01$ \\
\hline & Accident public & $7.6 \mathrm{E}-04$ & $3.1 E-04$ \\
\hline & $\begin{array}{l}\text { Vehicle-related } \\
\text { (nonradiological) }\end{array}$ & & \\
\hline & Emission & $1.9 \mathrm{E}-01$ & $3.2 \mathrm{E}-01$ \\
\hline & Accident & $2.6 \mathrm{E}+00$ & $3.6 \mathrm{E}-02$ \\
\hline \multirow[t]{7}{*}{ Regionalized 4 (Case 12) } & $\begin{array}{l}\text { Cargo-related } \\
\text { (radiological) }\end{array}$ & & \\
\hline & Routine crew & $4.8 \mathrm{E}-01$ & $6.7 E-02$ \\
\hline & Routine public & $6.6 \mathrm{E}-01$ & $1.8 \mathrm{E}-01$ \\
\hline & Accident public & $1.1 \mathrm{E}-03$ & $4.4 \mathrm{E}-04$ \\
\hline & $\begin{array}{l}\text { Vehicle-related } \\
\text { (nonradiological) }\end{array}$ & & \\
\hline & Emission & $2.2 \mathrm{E}-01$ & $2.3 \mathrm{E}-01$ \\
\hline & Accident & $2.5 \mathrm{E}+00$ & $3.5 \mathrm{E}-02$ \\
\hline \multirow[t]{7}{*}{ Regionalized 5 (Case 19) } & $\begin{array}{l}\text { Cargo-related } \\
\text { (radiological) }\end{array}$ & & \\
\hline & Routine crew & $7.6 \mathrm{E}-01$ & $8.3 E-02$ \\
\hline & Routine public & $1.1 \mathrm{E}+\infty$ & $2.3 E-01$ \\
\hline & Accident public & $1.7 \mathrm{E}-01$ & $1.2 E-02$ \\
\hline & $\begin{array}{l}\text { Vehicle-related } \\
\text { (nonradiological) }\end{array}$ & & \\
\hline & Emission & $3.0 \mathrm{E}-01$ & $3.5 \mathrm{E}-01$ \\
\hline & Accident & $4.4 \mathrm{E}+00$ & $5.3 \mathrm{E}-02$ \\
\hline \multirow[t]{7}{*}{ Regionalized 6 (Case 5) } & $\begin{array}{l}\text { Cargo-related } \\
\text { (radiological) }\end{array}$ & & \\
\hline & Routine crew & $1.5 \mathrm{E}+00$ & $1.6 \mathrm{E}-01$ \\
\hline & Routine public & $2.2 E+00$ & 4.1E-01 \\
\hline & Accident public & $1.2 \mathrm{E}-01$ & 2.2E-02 \\
\hline & $\begin{array}{l}\text { Vehicle-related } \\
\text { (nonradiological) }\end{array}$ & & \\
\hline & Emission & $6.7 \mathrm{E}-01$ & $4.7 \mathrm{E}-01$ \\
\hline & Accident & $8.7 E+00$ & $1.1 \mathrm{E}-01$ \\
\hline
\end{tabular}


TABLE 5.4 (Cont.)

\begin{tabular}{|c|c|c|c|}
\hline \multirow[b]{2}{*}{ WM LLW Alternative } & \multirow[b]{2}{*}{ Exposure Group } & \multicolumn{2}{|c|}{ Fatalities } \\
\hline & & Truck & Rail \\
\hline \multirow[t]{7}{*}{ Regionalized 7 (Case 6) } & $\begin{array}{l}\text { Cargo-related } \\
\text { (radiological) }\end{array}$ & & \\
\hline & Routine crew & $1.6 E+00$ & $1.7 \mathrm{E}-01$ \\
\hline & Routine public & $2.2 \mathrm{E}+00$ & 4.2E-01 \\
\hline & Accident public & $1.0 \mathrm{E}-01$ & $1.2 \mathrm{E}-02$ \\
\hline & $\begin{array}{l}\text { Vehicle-related } \\
\text { (nonradiological) }\end{array}$ & & \\
\hline & Emission & $8.2 \mathrm{E}-01$ & $4.7 \mathrm{E}-01$ \\
\hline & Accident & $9.0 \mathrm{E}+00$ & $1.1 \mathrm{E}-01$ \\
\hline \multirow[t]{7}{*}{ Centralized 1 (Case 7) } & $\begin{array}{l}\text { Cargo-related } \\
\text { (radiological) }\end{array}$ & & \\
\hline & Routine crew & $6.3 E+00$ & $4.8 \mathrm{E}-01$ \\
\hline & Routine public & $9.4 \mathrm{E}+00$ & $1.2 \mathrm{E}+00$ \\
\hline & Accident public & $2.9 \mathrm{E}-01$ & $5.7 \mathrm{E}-02$ \\
\hline & $\begin{array}{l}\text { Vehicle-related } \\
\text { (nonradiological) }\end{array}$ & & \\
\hline & Emission & $2.4 \mathrm{E}+00$ & $1.8 \mathrm{E}+00$ \\
\hline & Accident & $3.5 \mathrm{E}+01$ & 4.7E-01 \\
\hline \multirow[t]{7}{*}{ Centralized 2 (Case 8) } & $\begin{array}{l}\text { Cargo-related } \\
\text { (radiological) }\end{array}$ & & \\
\hline & Routine crew & $5.8 \mathrm{E}+00$ & 4.7E-01 \\
\hline & Routine public & $8.6 \mathrm{E}+00$ & $1.2 \mathrm{E}+00$ \\
\hline & Accident public & $2.8 \mathrm{E}-01$ & $4.5 \mathrm{E}-02$ \\
\hline & $\begin{array}{l}\text { Vehicle-related } \\
\text { (nonradiological) }\end{array}$ & & \\
\hline & Emission & $2.9 \mathrm{E}+00$ & $1.8 \mathrm{E}+\infty$ \\
\hline & Accident & $3.5 \mathrm{E}+01$ & $4.6 \mathrm{E}-01$ \\
\hline \multirow[t]{7}{*}{ Centralized 3 (Case 14) } & $\begin{array}{l}\text { Cargo-related } \\
\text { (radiological) }\end{array}$ & & \\
\hline & Routine crew & $5.6 \mathrm{E}+00$ & $4.8 \mathrm{E}-01$ \\
\hline & Routine public & $8.8 \mathrm{E}+00$ & $1.1 \mathrm{E}+00$ \\
\hline & Accident public & $2.9 \mathrm{E}-01$ & 5.7E-02 \\
\hline & $\begin{array}{l}\text { Vehicle-related } \\
\text { (nonradiological) }\end{array}$ & & \\
\hline & Emission & $2.2 \mathrm{E}+00$ & $1.7 E+\infty$ \\
\hline & Accident & $3.3 \mathrm{E}+01$ & $4.6 \mathrm{E}-0 \mathrm{I}$ \\
\hline
\end{tabular}


TABLE 5.4 (Cont.)

\begin{tabular}{|c|c|c|c|}
\hline \multirow[b]{2}{*}{ WM LLW Alternative } & \multirow[b]{2}{*}{ Exposure Group } & \multicolumn{2}{|c|}{ Fatalities } \\
\hline & & Truck & Rail \\
\hline \multirow[t]{7}{*}{ Centralized 4 (Case 14a) } & $\begin{array}{l}\text { Cargo-related } \\
\text { (radiological) }\end{array}$ & & \\
\hline & Routine crew & $5.5 \mathrm{E}+00$ & $4.7 \mathrm{E}-01$ \\
\hline & Routine public & $8.1 E+00$ & $1.2 \mathrm{E}+00$ \\
\hline & Accident public & $2.8 \mathrm{E}-01$ & $4.6 \mathrm{E}-02$ \\
\hline & $\begin{array}{l}\text { Vehicle-related } \\
\text { (nonradiological) }\end{array}$ & & \\
\hline & Emission & $2.8 \mathrm{E}+00$ & $1.8 \mathrm{E}+00$ \\
\hline & Accident & $3.4 \mathrm{E}+01$ & $4.5 \mathrm{E}-01$ \\
\hline \multirow[t]{7}{*}{ Centralized 5 (Case 21) } & $\begin{array}{l}\text { Cargo-related } \\
\text { (radiological) }\end{array}$ & & \\
\hline & Routine crew & $6.3 E+00$ & $4.7 \mathrm{E}-01$ \\
\hline & Routine public & $9.3 E+00$ & $1.2 \mathrm{E}+00$ \\
\hline & Accident public & $2.9 \mathrm{E}-01$ & $5.7 \mathrm{E}-02$ \\
\hline & $\begin{array}{l}\text { Vehicle-related } \\
\text { (nonradiological) }\end{array}$ & & \\
\hline & Emission & $2.4 \mathrm{E}+00$ & $1.8 \mathrm{E}+00$ \\
\hline & Accident & $3.5 \mathrm{E}+01$ & 4.7E-01 \\
\hline
\end{tabular}

a Latent-cancer fatalities are calculated by multiplying dose by the ICRP Publication 60 (ICRP 1991) health risk conversion factors of $4 E-04$ and 5E-04 fatal cancers per person-rem for workers and for the public, respectively.

b For each alternative, impacts are calculated and presented separately assuming exclusive truck or exclusive rail shipments. Therefore, the impacts for truck and rail modes should not be added together.

Category VIII). For these accidents, the assumptions were that all of the material in the shipment would be released from its packaging, that $10 \%$ would be entrained as an aerosol, and that $5 \%$ of the aerosol would be respirable.

During screening, the WM LLW from the Argonne National Laboratory-West (ANL-W) site was found to result in the highest transportation accident doses. The accident consequence results from RISKIND for WM LLW shipments are presented in Table 5.9. The population doses are for a uniform population density within an $80-\mathrm{km}(50-\mathrm{mi})$ radius of accidents occurring in rural, suburban, and urban population density zones. The location of the MEI after an accident is determined on the basis of atmospheric conditions and the buoyant characteristics of the released 
TABLE 5.5 Total Estimated Cancer Incidence for the WM LLW Alternatives ${ }^{\mathbf{a}, \mathbf{b}}$

\begin{tabular}{|c|c|c|c|}
\hline \multirow[b]{2}{*}{ WM LLW Alternative } & \multirow[b]{2}{*}{ Exposure Group } & \multicolumn{2}{|c|}{ Cancer Cases } \\
\hline & & Truck & Rail \\
\hline \multirow[t]{7}{*}{ No Action (Case 1) } & $\begin{array}{l}\text { Cargo-related } \\
\text { (radiological) }\end{array}$ & & \\
\hline & Routine crew & $6.6 \mathrm{E}+00$ & $5.5 \mathrm{E}-01$ \\
\hline & Routine public & $9.5 E+00$ & $1.4 E+00$ \\
\hline & Accident public & $4.2 \mathrm{E}-03$ & $1.8 \mathrm{E}-03$ \\
\hline & $\begin{array}{l}\text { Vehicle-related } \\
\text { (nonradiological) }\end{array}$ & & \\
\hline & Emission & $9.5 \mathrm{E}-01$ & $7.8 \mathrm{E}-01$ \\
\hline & Accident & $\mathrm{NA}^{\mathrm{c}}$ & NA \\
\hline \multirow[t]{7}{*}{ Decentralized (Case 2) } & $\begin{array}{l}\text { Cargo-related } \\
\text { (radiological) }\end{array}$ & & \\
\hline & Routine crew & $4.5 \mathrm{E}-01$ & $5.7 \mathrm{E}-02$ \\
\hline & Routine public & $5.1 \mathrm{E}-01$ & $2.2 \mathrm{E}-01$ \\
\hline & Accident public & $2.2 \mathrm{E}-04$ & $1.5 \mathrm{E}-0.4$ \\
\hline & $\begin{array}{l}\text { Vehicle-related } \\
\text { (nonradiological) }\end{array}$ & & \\
\hline & Emission & $8.8 \mathrm{E}-02$ & $1.2 E-01$ \\
\hline & Accident & NA & NA \\
\hline \multirow[t]{7}{*}{ Regionalized 1 (Case 3) } & $\begin{array}{l}\text { Cargo-related } \\
\text { (radiological) }\end{array}$ & & \\
\hline & Routine crew & $4.8 \mathrm{E}-01$ & $6.1 E-02$ \\
\hline & Routine public & $6.1 \mathrm{E}-01$ & $2.3 E-01$ \\
\hline & Accident public & $1.1 \mathrm{E}-03$ & $2.8 \mathrm{E}-04$ \\
\hline & $\begin{array}{l}\text { Vehicle-related } \\
\text { (nonradiological) }\end{array}$ & & \\
\hline & Emission & $9.9 \mathrm{E}-02$ & $1.3 E-01$ \\
\hline & Accident & NA & NA \\
\hline \multirow[t]{7}{*}{ Regionalized 2 (Case 9) } & $\begin{array}{l}\text { Cargo-related } \\
\text { (radiological) }\end{array}$ & & \\
\hline & Routine crew & $4.7 \mathrm{E}-0 \mathrm{I}$ & $6.2 \mathrm{E}-02$ \\
\hline & Routine public & $6.0 \mathrm{E}-01$ & $2.4 \mathrm{E}-01$ \\
\hline & Accident public & $1.1 \mathrm{E}-03$ & $2.8 \mathrm{E}-04$ \\
\hline & $\begin{array}{l}\text { Vehicle-related } \\
\text { (nonradiological) }\end{array}$ & & \\
\hline & Emission & $9.6 \mathrm{E}-02$ & $1.3 \mathrm{E}-01$ \\
\hline & Accident & NA & NA \\
\hline
\end{tabular}


TABLE 5.5 (Cont.)

\begin{tabular}{|c|c|c|c|}
\hline \multirow[b]{2}{*}{ WM LLW Alternative } & \multirow[b]{2}{*}{ Exposure Group } & \multicolumn{2}{|c|}{ Cancer Cases } \\
\hline & & Truck & Rail \\
\hline \multirow[t]{7}{*}{ Regionalized 3 (Case 4) } & $\begin{array}{l}\text { Cargo-related } \\
\text { (radiological) }\end{array}$ & & \\
\hline & Routine crew & $1.7 \mathrm{E}+00$ & $2.3 \mathrm{E}-01$ \\
\hline & Routine public & $2.3 \mathrm{E}+00$ & $6.9 \mathrm{E}-01$ \\
\hline & Accident public & $2.6 \mathrm{E}-03$ & 1.1E-03 \\
\hline & $\begin{array}{l}\text { Vehicle-related } \\
\text { (nonradiological) }\end{array}$ & & \\
\hline & Emission & $1.9 \mathrm{E}-01$ & $3.2 E-01$ \\
\hline & Accident & $\mathrm{NA}$ & $\mathrm{NA}$ \\
\hline \multirow[t]{7}{*}{ Regionalized 4 (Case 12) } & $\begin{array}{l}\text { Cargo-related } \\
\text { (radiological) }\end{array}$ & & \\
\hline & Routine crew & $1.6 \mathrm{E}+00$ & $2.3 E-01$ \\
\hline & Routine public & $2.2 E+00$ & $6.3 \mathrm{E}-01$ \\
\hline & Accident public & $3.6 \mathrm{E}-03$ & $1.5 E-03$ \\
\hline & $\begin{array}{l}\text { Vehicle-related } \\
\text { (nonradiological) }\end{array}$ & & \\
\hline & Emission & $2.2 \mathrm{E}-01$ & $2.3 \mathrm{E}-01$ \\
\hline & Accident & NA & NA \\
\hline \multirow[t]{7}{*}{ Regionalized 5 (Case 19) } & $\begin{array}{l}\text { Cargo-related } \\
\text { (radiological) }\end{array}$ & & \\
\hline & Routine crew & $2.7 \mathrm{E}+00$ & $2.9 \mathrm{E}-01$ \\
\hline & Routine public & $3.8 \mathrm{E}+00$ & $8.0 E-01$ \\
\hline & Accident public & $5.9 \mathrm{E}-01$ & $4.1 E-02$ \\
\hline & $\begin{array}{l}\text { Vehicle-related } \\
\text { (nonradiological) }\end{array}$ & & \\
\hline & Emission & $3.0 \mathrm{E}-01$ & $3.5 E-01$ \\
\hline & Accident & NA & NA \\
\hline \multirow[t]{7}{*}{ Regionalized 6 (Case 5) } & $\begin{array}{l}\text { Cargo-related } \\
\text { (radiological) }\end{array}$ & & \\
\hline & Routine crew & $5.4 \mathrm{E}+00$ & $5.6 \mathrm{E}-01$ \\
\hline & Routine public & $7.4 \mathrm{E}+00$ & $1.4 \mathrm{E}+00$ \\
\hline & Accident public & $4.0 \mathrm{E}-01$ & $7.5 \mathrm{E}-02$ \\
\hline & $\begin{array}{l}\text { Vehicle-related } \\
\text { (nonradiological) }\end{array}$ & & \\
\hline & Emission & $6.7 \mathrm{E}-01$ & 4.7E-01 \\
\hline & Accident & $\mathrm{NA}$ & $\mathrm{NA}$ \\
\hline
\end{tabular}




\section{NOTATION}

The following is a list of the acronyms, initialisms, and abbreviations (including units of measure) used in this document. Some acronyms used only in tables are defined in those tables.

\section{ACRONYMS, INITIALISMS, AND ABBREVIATIONS}

$\begin{array}{ll}\text { ANL-E } & \text { Argonne National Laboratory-East } \\ \text { ANL-W } & \text { Argonne National Laboratory-West } \\ \text { BNL } & \text { Brookhaven National Laboratory } \\ \text { DOE } & \text { U.S. Department of Energy } \\ \text { DOT } & \text { U.S. Department of Transportation } \\ \text { FEMP } & \text { Fernald Environmental Management Project } \\ \text { Hanford } & \text { Hanford Site } \\ \text { ICRP } & \text { International Commission on Radiological Protection } \\ \text { INEL } & \text { Idaho National Engineering Laboratory } \\ \text { LANL } & \text { Los Alamos National Laboratory } \\ \text { LLNL } & \text { Lawrence Livermore National Laboratory } \\ \text { LLW } & \text { low-level waste } \\ \text { MEI } & \text { maximally exposed individual } \\ \text { NRC } & \text { U.S. Nuclear Regulatory Commission } \\ \text { NTS } & \text { Nevada Test Site } \\ \text { ORR } & \text { Oak Ridge Reservation } \\ \text { Pantex } & \text { Pantex Plant } \\ \text { PEIS } & \text { Programmatic Environmental Impact Statement } \\ \text { PGDP } & \text { Paducah Gaseous Diffusion Plant } \\ \text { PORTS } & \text { Portsmouth Gaseous Diffusion Plant } \\ \text { RFETS } & \text { Rocky Flats Environmental Technology Site } \\ \text { SMAC } & \text { Shipment Mobility/Accountability Collection } \\ \text { SNL-NM } & \text { Sandia National Laboratories (New Mexico) } \\ \text { SRS } & \text { Savannah River Site } \\ \text { TSD } & \text { treatment, storage, and disposal } \\ \text { WM } & \text { Waste Management } \\ \text { WVDP } & \text { West Valley Demonstration Project } \\ & \end{array}$

\section{UNITS OF MEASURE}

$\begin{array}{ll}\mathrm{ft} & \text { foot (feet) } \\ \mathrm{g} & \text { gram(s) } \\ \text { gal } & \text { gallon(s) } \\ \mathrm{h} & \text { hour(s) } \\ \mathrm{kg} & \text { kilogram(s) }\end{array}$


TABLE 5.5 (Cont.)

\begin{tabular}{|c|c|c|c|}
\hline \multirow[b]{2}{*}{ WM LLW Alternative } & \multirow[b]{2}{*}{ Exposure Group } & \multicolumn{2}{|c|}{ Cancer Cases } \\
\hline & & Truck & Rail \\
\hline \multirow[t]{7}{*}{ Regionalized 7 (Case 6) } & $\begin{array}{l}\text { Cargo-related } \\
\text { (radiological) }\end{array}$ & & \\
\hline & Routine crew & $5.4 E+00$ & $6.0 \mathrm{E}-01$ \\
\hline & Routine public & 7.5E+00 & $1.5 \mathrm{E}+00$ \\
\hline & Accident public & $3.5 \mathrm{E}-01$ & $4.2 \mathrm{E}-02$ \\
\hline & $\begin{array}{l}\text { Vehicle-related } \\
\text { (nonradiological) }\end{array}$ & & \\
\hline & Emission & $8.2 \mathrm{E}-01$ & $4.7 \mathrm{E}-01$ \\
\hline & Accident & NA & NA \\
\hline \multirow[t]{7}{*}{ Centralized 1 (Case 7) } & $\begin{array}{l}\text { Cargo-related } \\
\text { (radiological) }\end{array}$ & & \\
\hline & Routine crew & $2.2 \mathrm{E}+01$ & $1.7 \mathrm{E}+00$ \\
\hline & Routine public & $3.2 E+01$ & $3.9 \mathrm{E}+00$ \\
\hline & Accident public & $9.9 \mathrm{E}-01$ & $1.9 \mathrm{E}-01$ \\
\hline & $\begin{array}{l}\text { Vehicle-related } \\
\text { (nonradiological) }\end{array}$ & & \\
\hline & Emission & $2.4 \mathrm{E}+00$ & $1.8 \mathrm{E}+00$ \\
\hline & Accident & NA & NA \\
\hline \multirow[t]{7}{*}{ Centralized 2 (Case 8) } & $\begin{array}{l}\text { Cargo-related } \\
\text { (radiological) }\end{array}$ & & \\
\hline & Routine crew & $2.0 \mathrm{E}+01$ & $1.7 \mathrm{E}+00$ \\
\hline & Routine public & $2.9 \mathrm{E}+01$ & $3.9 E+00$ \\
\hline & Accident public & $9.6 \mathrm{E}-01$ & $1.5 \mathrm{E}-01$ \\
\hline & $\begin{array}{l}\text { Vehicle-related } \\
\text { (nonradiological) }\end{array}$ & & \\
\hline & Emission & $2.9 \mathrm{E}+00$ & $1.8 \mathrm{E}+00$ \\
\hline & Accident & NA & NA \\
\hline \multirow[t]{7}{*}{ Centralized 3 (Case 14) } & $\begin{array}{l}\text { Cargo-related } \\
\text { (radiological) }\end{array}$ & & \\
\hline & Routine crew & $2.1 E+01$ & $1.6 \mathrm{E}+00$ \\
\hline & Routine public & $3.0 \mathrm{E}+01$ & $4.0 \mathrm{E}+00$ \\
\hline & Accident public & $9.9 E-01$ & $1.9 \mathrm{E}-01$ \\
\hline & $\begin{array}{l}\text { Vehicle-related } \\
\text { (nonradiological) }\end{array}$ & & \\
\hline & Emission & $2.2 E+00$ & $1.7 \div 00$ \\
\hline & Accident & $\mathrm{NA}$ & NA \\
\hline
\end{tabular}


TABLE 5.5 (Cont.)

\begin{tabular}{|c|c|c|c|}
\hline \multirow[b]{2}{*}{ WM LLW Alternative } & \multirow[b]{2}{*}{ Exposure Group } & \multicolumn{2}{|c|}{ Cancer Cases } \\
\hline & & Truck & Rail \\
\hline \multirow[t]{7}{*}{ Centralized 4 (Case 14a) } & $\begin{array}{l}\text { Cargo-related } \\
\text { (radiological) }\end{array}$ & & \\
\hline & Routine crew & $1.9 \mathrm{E}+01$ & $1.6 \mathrm{E}+00$ \\
\hline & Routine public & $2.8 \mathrm{E}+01$ & $4.0 \mathrm{E}+00$ \\
\hline & Accident public & $9.6 \mathrm{E}-01$ & $1.6 \mathrm{E}-01$ \\
\hline & $\begin{array}{l}\text { Vehicle-related } \\
\text { (nonradiological) }\end{array}$ & & \\
\hline & Emission & $2.8 \mathrm{E}+00$ & $1.8 \mathrm{E}+00$ \\
\hline & Accident & NA & NA \\
\hline \multirow[t]{7}{*}{ Centralized 5 (Case 21) } & $\begin{array}{l}\text { Cargo-related } \\
\text { (radiological) }\end{array}$ & & \\
\hline & Routine crew & $2.2 \mathrm{E}+01$ & $1.7 \mathrm{E}+00$ \\
\hline & Routine public & $3.2 \mathrm{E}+01$ & $3.9 E+00$ \\
\hline & Accident public & $9.9 \mathrm{E}-01$ & $1.9 E-01$ \\
\hline & $\begin{array}{l}\text { Vehicle-related } \\
\text { (nonradiological) }\end{array}$ & & \\
\hline & Emission & $2.4 \mathrm{E}+00$ & $1.8 \mathrm{E}+00$ \\
\hline & Accident & NA & NA \\
\hline
\end{tabular}

a Latent-cancer incidences are calculated by multiplying dose by the ICRP Publication 60 (ICRP 1991) health risk conversion factors of 1.4E-03 and 1.7E-03 cancer cases per person-rem for workers and for the public, respectively.

b For each alternative, impacts are calculated and presented separately assuming exclusive truck or exclusive rail shipments. Therefore, the impacts for truck and rail modes should not be added together.

c NA $=$ not applicable.

plume. The locations of maximum exposure are approximately $160 \mathrm{~m}(525 \mathrm{ft})$ and $400 \mathrm{~m}(1,312 \mathrm{ft})$ from the accident site for neutral and stable weather conditions, respectively. The dose to the MEI is independent of the location of the accident. The maximum dose to an individual (approximately 7 rem for a rail accident under unfavorable weather conditions) has a potential lifetime fatal-cancer risk of $4 \mathrm{E}-03$.

The accident consequence results for LLW from ANL-W should be considered extremely conservative for the majority of WM LLW shipments for a number of reasons. First, the LLW from ANL-W represents less than $1 \%$ by volume of the total WM LLW generated annually within the 
TABLE 5.6 Total Estimated Genetic Effects for the WM LLW Alternatives $^{\mathbf{a}, \mathbf{b}}$

\begin{tabular}{|c|c|c|c|}
\hline \multirow[b]{2}{*}{ WM LLW Alternative } & \multirow[b]{2}{*}{ Exposure Group } & \multicolumn{2}{|c|}{ Genetic Effects } \\
\hline & & Truck & Rail \\
\hline \multirow[t]{7}{*}{ No Action (Case I) } & $\begin{array}{l}\text { Cargo-related } \\
\text { (radiological) }\end{array}$ & & \\
\hline & Routine crew & $2.7 \mathrm{E}-01$ & $2.3 \mathrm{E}-02$ \\
\hline & Routine public & $5.6 \mathrm{E}-01$ & $8.6 \mathrm{E}-02$ \\
\hline & Accident public & $7.5 \mathrm{E}-05$ & 2.7E-05 \\
\hline & $\begin{array}{l}\text { Vehicle-related } \\
\text { (nonradiological) }\end{array}$ & & \\
\hline & Emission & $N A^{c}$ & NA \\
\hline & Accident & $\mathrm{NA}$ & $\mathrm{NA}$ \\
\hline \multirow[t]{7}{*}{ Decentralized (Case 2) } & $\begin{array}{l}\text { Cargo-related } \\
\text { (radiological) }\end{array}$ & & \\
\hline & Routine crew & $1.9 \mathrm{E}-02$ & $2.4 \mathrm{E}-03$ \\
\hline & Routine public & $3.3 \mathrm{E}-02$ & $1.3 \mathrm{E}-02$ \\
\hline & Accident public & $1.2 \mathrm{E}-05$ & $7.9 \mathrm{E}-06$ \\
\hline & $\begin{array}{l}\text { Vehicle-related } \\
\text { (nonradiological) }\end{array}$ & & \\
\hline & Emission & NA & NA \\
\hline & Accident & NA & NA \\
\hline \multirow[t]{7}{*}{ Regionalized 1 (Case 3) } & $\begin{array}{l}\text { Cargo-related } \\
\text { (radiological) }\end{array}$ & & \\
\hline & Routine crew & $2.0 \mathrm{E}-02$ & $2.6 \mathrm{E}-03$ \\
\hline & Routine public & $3.6 \mathrm{E}-02$ & $1.4 \mathrm{E}-02$ \\
\hline & Accident public & $3.2 \mathrm{E}-05$ & 7.1E-06 \\
\hline & $\begin{array}{l}\text { Vehicle-related } \\
\text { (nonradiological) }\end{array}$ & & \\
\hline & Emission & NA & NA \\
\hline & Accident & NA & NA \\
\hline \multirow[t]{7}{*}{ Regionalized 2 (Case 9) } & $\begin{array}{l}\text { Cargo-related } \\
\text { (radiological) }\end{array}$ & & \\
\hline & Routine crew & $2.0 \mathrm{E}-02$ & $2.7 \mathrm{E}-03$ \\
\hline & Routine public & $3.6 \mathrm{E}-02$ & $1.4 \mathrm{E}-02$ \\
\hline & Accident public & $3.2 E-05$ & $7.1 \mathrm{E}-06$ \\
\hline & $\begin{array}{l}\text { Vehicle-related } \\
\text { (nonradiological) }\end{array}$ & & \\
\hline & Emission & NA & NA \\
\hline & Accident & NA & NA \\
\hline
\end{tabular}


TABLE 5.6 (Cont.)

\begin{tabular}{|c|c|c|c|}
\hline \multirow[b]{2}{*}{ WM LLW Alternative } & \multirow[b]{2}{*}{ Exposure Group } & \multicolumn{2}{|c|}{ Genetic Effects } \\
\hline & & Truck & Rail \\
\hline \multirow[t]{7}{*}{ Regionalized 3 (Case 4) } & $\begin{array}{l}\text { Cargo-related } \\
\text { (radiological) }\end{array}$ & & \\
\hline & Routine crew & $7.3 \mathrm{E}-02$ & $9.8 \mathrm{E}-03$ \\
\hline & Routine public & $1.4 \mathrm{E}-01$ & $4.1 E-02$ \\
\hline & Accident public & $5.8 \mathrm{E}-05$ & $1.7 E-05$ \\
\hline & $\begin{array}{l}\text { Vehicle-related } \\
\text { (nonradiological) }\end{array}$ & & \\
\hline & Emission & NA & $\mathrm{NA}$ \\
\hline & Accident & NA & NA \\
\hline \multirow[t]{7}{*}{ Regionalized 4 (Case 12) } & $\begin{array}{l}\text { Cargo-related } \\
\text { (radiological) }\end{array}$ & & \\
\hline & Routine crew & $7.2 \mathrm{E}-02$ & $9.9 \mathrm{E}-03$ \\
\hline & Routine public & $1.3 \mathrm{E}-01$ & $3.7 \mathrm{E}-02$ \\
\hline & Accident public & $7.0 \mathrm{E}-05$ & $2.1 \mathrm{E}-05$ \\
\hline & $\begin{array}{l}\text { Vehicle-related } \\
\text { (nonradiological) }\end{array}$ & & \\
\hline & Emission & $\mathrm{NA}$ & NA \\
\hline & Accident & NA & NA \\
\hline \multirow[t]{7}{*}{ Regionalized 5 (Case 19) } & $\begin{array}{l}\text { Cargo-related } \\
\text { (radiological) }\end{array}$ & & \\
\hline & Routine crew & $1.2 \mathrm{E}-01$ & $1.2 \mathrm{E}-02$ \\
\hline & Routine public & $2.2 \mathrm{E}-01$ & 4.7E-02 \\
\hline & Accident public & $2.9 \mathrm{E}-02$ & $1.8 \mathrm{E}-03$ \\
\hline & $\begin{array}{l}\text { Vehicle-related } \\
\text { (nonradiological) }\end{array}$ & & \\
\hline & Emission & $\mathrm{NA}$ & NA \\
\hline & Accident & NA & NA \\
\hline \multirow[t]{7}{*}{ Regionalized 6 (Case 5) } & $\begin{array}{l}\text { Cargo-related } \\
\text { (radiological) }\end{array}$ & & \\
\hline & Routine crew & $2.3 E-01$ & $2.4 \mathrm{E}-02$ \\
\hline & Routine public & $4.3 E-01$ & $8.1 E-02$ \\
\hline & Accident public & $2.0 \mathrm{E}-02$ & $3.7 \mathrm{E}-03$ \\
\hline & $\begin{array}{l}\text { Vehicle-related } \\
\text { (nonradiological) }\end{array}$ & & \\
\hline & Emission & $\mathrm{NA}$ & $\mathrm{NA}$ \\
\hline & Accident & $\mathrm{NA}$ & NA \\
\hline
\end{tabular}


TABLE 5.6 (Cont.)

\begin{tabular}{|c|c|c|c|}
\hline \multirow[b]{2}{*}{ WM LLW Alternative } & \multirow[b]{2}{*}{ Exposure Group } & \multicolumn{2}{|c|}{ Genetic Effects } \\
\hline & & Truck & Rail \\
\hline \multirow[t]{7}{*}{ Regionalized 7 (Case 6) } & $\begin{array}{l}\text { Cargo-related } \\
\text { (radiological) }\end{array}$ & & \\
\hline & Routine crew & $2.3 E-01$ & $2.6 \mathrm{E}-02$ \\
\hline & Routine public & $4.4 \mathrm{E}-01$ & $8.4 E-02$ \\
\hline & Accident public & $1.9 \mathrm{E}-02$ & $2.2 \mathrm{E}-03$ \\
\hline & $\begin{array}{l}\text { Vehicle-related } \\
\text { (nonradiological) }\end{array}$ & & \\
\hline & Emission & NA & NA \\
\hline & Accident & NA & NA \\
\hline \multirow[t]{7}{*}{ Centralized 1 (Case 7) } & $\begin{array}{l}\text { Cargo-related } \\
\text { (radiological) }\end{array}$ & & \\
\hline & Routine crew & $9.4 \mathrm{E}-01$ & $7.2 \mathrm{E}-02$ \\
\hline & Routine public & $1.9 \mathrm{E}+00$ & $2.3 \mathrm{E}-01$ \\
\hline & Accident public & $5.3 \mathrm{E}-02$ & $1.0 \mathrm{E}-02$ \\
\hline & $\begin{array}{l}\text { Vehicle-related } \\
\text { (nonradiological) }\end{array}$ & & \\
\hline & Emission & NA & NA \\
\hline & Accident & NA & NA \\
\hline \multirow[t]{7}{*}{ Centralized 2 (Case 8) } & $\begin{array}{l}\text { Cargo-related } \\
\text { (radiological) }\end{array}$ & & \\
\hline & Routine crew & $8.7 \mathrm{E}-01$ & $7.2 \mathrm{E}-02$ \\
\hline & Routine public & $1.7 \mathrm{E}+00$ & $2.3 \mathrm{E}-01$ \\
\hline & Accident public & $5.3 \mathrm{E}-02$ & $8.5 \mathrm{E}-03$ \\
\hline & $\begin{array}{l}\text { Vehicle-related } \\
\text { (nonradiological) }\end{array}$ & & \\
\hline & Emission & NA & NA \\
\hline & Accident & NA & NA \\
\hline \multirow[t]{7}{*}{ Centralized 3 (Case 14) } & $\begin{array}{l}\text { Cargo-related } \\
\text { (radiological) }\end{array}$ & & \\
\hline & Routine crew & $9.0 \mathrm{E}-01$ & $7.1 \mathrm{E}-02$ \\
\hline & Routine public & $1.8 E+\infty 0$ & $2.3 \mathrm{E}-01$ \\
\hline & Accident public & $5.3 E-02$ & $1.0 \mathrm{E}-02$ \\
\hline & $\begin{array}{l}\text { Vehicle-related } \\
\text { (nonradiological) }\end{array}$ & & \\
\hline & Emission & NA & NA \\
\hline & Accident & NA & NA \\
\hline
\end{tabular}


TABLE 5.6 (Cont.)

\begin{tabular}{|c|c|c|c|}
\hline \multirow[b]{2}{*}{ WM LLW Alternative } & \multirow[b]{2}{*}{ Exposure Group } & \multicolumn{2}{|c|}{ Genetic Effects } \\
\hline & & Truck & Rail \\
\hline \multirow[t]{7}{*}{ Centralized 4 (Case 14a) } & $\begin{array}{l}\text { Cargo-related } \\
\text { (radiological) }\end{array}$ & & \\
\hline & Routine crew & $8.2 \mathrm{E}-01$ & $7.1 \mathrm{E}-02$ \\
\hline & Routine public & $1.6 \mathrm{E}+00$ & $2.3 \mathrm{E}-01$ \\
\hline & Accident public & $5.4 \mathrm{E}-02$ & $8.6 E-03$ \\
\hline & $\begin{array}{l}\text { Vehicle-related } \\
\text { (nonradiological) }\end{array}$ & & \\
\hline & Emission & NA & NA \\
\hline & Accident & NA & NA \\
\hline \multirow[t]{7}{*}{ Centralized 5 (Case 21) } & $\begin{array}{l}\text { Cargo-related } \\
\text { (radiological) }\end{array}$ & & \\
\hline & Routine crew & $9.4 \mathrm{E}-01$ & $7.2 \mathrm{E}-02$ \\
\hline & Routine public & $1.8 \mathrm{E}+00$ & $2.3 \mathrm{E}-01$ \\
\hline & Accident public & $5.3 E-02$ & $1.0 \mathrm{E}-02$ \\
\hline & $\begin{array}{l}\text { Vehicle-related } \\
\text { (nonradiological) }\end{array}$ & & \\
\hline & Emission & NA & NA \\
\hline & Accident & NA & NA \\
\hline
\end{tabular}

a Genetic effects are calculated by multiplying dose by the ICRP Publication 60 (ICRP 1991) health risk conversion factors of $6 E-05$ and $1 E-04$ adverse genetic effects per person-rem for workers and for the public, respectively.

b For each alternative, impacts are calculated and presented separately assuming exclusive truck or exclusive rail shipments. Therefore, the impacts for truck and rail modes should not be added together.

c NA = not applicable.

DOE. Only about two truck shipments would be required each year to transport ANL-W waste to an off-site facility for treatment, storage, or disposal. Therefore, a severe LLW accident involving LLW from ANL-W is unlikely. Second, the accident dose results for LLW from ANL-W are at least a factor of 10 greater than those for LLW from other sites, primarily because of the large cobalt-60 content of the ANL-W waste. The "average" accident consequences would be much less than those presented here. 
TABLE 5.7 Estimated Routine Doses and Lifetime Risk of Fatal Cancer to MEIs from Shipments of WMI LLW (per Exposure Event) ${ }^{2}$

\begin{tabular}{|c|c|c|c|c|}
\hline \multirow[b]{2}{*}{ Receptor $^{b}$} & \multicolumn{2}{|c|}{ Dose (rem) } & \multicolumn{2}{|c|}{ Lifetime Risk $^{c}$} \\
\hline & Truck & Rail & Truck & Rail \\
\hline \multicolumn{5}{|l|}{ Workers } \\
\hline Crew member & d & d & d & d \\
\hline Inspector & $1.5 \mathrm{E}-04$ & $1.5 \mathrm{E}-04$ & $6 \mathrm{E}-08$ & $6 \mathrm{E}-08$ \\
\hline Rail-yard crew member & $N A^{e}$ & $7.9 \mathrm{E}-05$ & NA & $3 E-08$ \\
\hline \multicolumn{5}{|l|}{ Public } \\
\hline Resident & $1.6 \mathrm{E}-08$ & $1.6 E-08$ & $8 \mathrm{E}-12$ & $8 \mathrm{E}-12$ \\
\hline Person in traffic jam & $5.0 \mathrm{E}-04$ & $5.0 \mathrm{E}-04$ & $3 E-07$ & $3 \mathrm{E}-07$ \\
\hline $\begin{array}{l}\text { Person at service } \\
\text { station }\end{array}$ & $2.1 \mathrm{E}-05$ & NA & 1E-08 & NA \\
\hline Resident near rail stop & NA & $1.1 E-06$ & NA & $6 E-10$ \\
\hline
\end{tabular}

a Dose rate is assumed to be $1 \mathrm{mrem} / \mathrm{h}$ at $1 \mathrm{~m}(3.3 \mathrm{ft})$ from a WM LLW shipment.

b Receptor assumptions are described in Section 4.6.

c Lifetime risk of fatal cancer is based on ICRP Publication 60 (ICRP 1991) health risk conversion factors of 4E-04 and 5E-04 fatal cancers per person-rem for workers and for members of the public, respectively.

d The DOE administrative control level limits doses to DOE workers to $2 \mathrm{rem} / \mathrm{yr}$. For a representative shipment of $1,609 \mathrm{~km}(1,000 \mathrm{mi})$ and an average speed of $80 \mathrm{~km} / \mathrm{h}(50 \mathrm{mph})$, the dose to a driver would be approximately $40 \mathrm{mrem}$ if the dose rate in the crew compartment were at the regulatory limit of $2 \mathrm{mrem} / \mathrm{h}$. Dose rates are typically much less than $2 \mathrm{mrem} / \mathrm{h}$ in the crew compartment.

e $\mathrm{NA}=$ not applicable. 
TABLE 5.8 Cumulative Dose and Lifetime Risk to MEIs Living along a Site Entrance Route for WM LLW Shipments (Current Inventories plus 20 Years of Generation) $^{\mathrm{a}}$

\begin{tabular}{|c|c|c|c|c|c|c|}
\hline \multirow[b]{2}{*}{$\begin{array}{c}\text { Alternative } \\
\text { and Site }\end{array}$} & \multicolumn{3}{|c|}{ All Truck } & \multicolumn{3}{|c|}{ All Rail } \\
\hline & $\begin{array}{c}\text { Total } \\
\text { Shipments }\end{array}$ & $\begin{array}{l}\text { Dose } \\
\text { (rem) }\end{array}$ & $\begin{array}{c}\text { Risk } \\
\text { (Fatal Cancer) }\end{array}$ & $\begin{array}{c}\text { Total } \\
\text { Shipments }\end{array}$ & $\begin{array}{l}\text { Dose } \\
\text { (rem) }\end{array}$ & $\begin{array}{c}\text { Risk } \\
\text { (Fatal Cancer) }^{\mathrm{e}}\end{array}$ \\
\hline \multicolumn{7}{|l|}{$\begin{array}{l}\text { No Action } \\
\text { (Case I) }\end{array}$} \\
\hline NTS & 69.960 & $1.1 \mathrm{E}-03$ & $5.6 \mathrm{E}-07$ & 26,740 & $4.3 \mathrm{E}-04$ & $2.2 \mathrm{E}-07$ \\
\hline PORTS & 33.440 & $5.4 \mathrm{E}-04$ & $2.7 \mathrm{E}-07$ & 12,740 & 2.1E-0.4 & $1.0 \mathrm{E}-07$ \\
\hline Hanford & 17,340 & $2.8 \mathrm{E}-04$ & I. $4 \mathrm{E}-07$ & 6.640 & 1.IE-04 & $5.3 \mathrm{E}-08$ \\
\hline Pantex & 13,740 & $2.2 \mathrm{E}-04$ & 1.1E-07 & 5.440 & $8.8 \mathrm{E}-05$ & $4.4 \mathrm{E}-08$ \\
\hline RMI & 7.300 & $1.2 \mathrm{E}-04$ & $5.9 E-08$ & 2,740 & $4.4 \mathrm{E}-05$ & $2.2 \mathrm{E}-08$ \\
\hline KAPL-S & 6,860 & 1.1E-04 & $5.5 \mathrm{E}-08$ & 2,600 & $4.2 \mathrm{E}-05$ & $2.1 \mathrm{E}-08$ \\
\hline PGDP & 6,420 & $1.0 \mathrm{E}-04$ & $5.2 \mathrm{E}-08$ & 2,400 & $3.9 \mathrm{E}-05$ & $1.9 \mathrm{E}-08$ \\
\hline Mound & 5,120 & $8.2 \mathrm{E}-05$ & 4.1E-08 & 1,900 & $3.1 \mathrm{E}-05$ & $1.5 E-08$ \\
\hline NRF & 4,620 & $7.4 \mathrm{E}-05$ & 3.7E-08 & 1,760 & $2.8 \mathrm{E}-05$ & $1.4 E-08$ \\
\hline Betris & 3,740 & $6.0 \mathrm{E}-05$ & $3.0 \mathrm{E}-08$ & 1,420 & $2.3 \mathrm{E}-05$ & 1.1E-08 \\
\hline RFETS & 3,200 & 5.2E-05 & $2.6 \mathrm{E}-08$ & 1,200 & $1.9 E-05$ & $9.7 \mathrm{E}-09$ \\
\hline ANL-E & 1,060 & $1.7 \mathrm{E}-05$ & $8.5 E-09$ & 400 & $6.4 \mathrm{E}-06$ & 3.2E-09 \\
\hline Fermi & 420 & $6.8 \mathrm{E}-06$ & $3.4 E-09$ & 180 & $2.9 \mathrm{E}-06$ & $1.4 \mathrm{E}-09$ \\
\hline SLAC & 400 & $6.4 \mathrm{E}-06$ & 3.2E-09 & 160 & $2.6 \mathrm{E}-06$ & $1.3 \mathrm{E}-09$ \\
\hline LLNL & 320 & $5.2 \mathrm{E}-06$ & $2.6 \mathrm{E}-09$ & 120 & $1.9 \mathrm{E}-06$ & $9.7 \mathrm{E}-10$ \\
\hline SNL-NM & 240 & $3.9 \mathrm{E}-06$ & $1.9 \mathrm{E}-09$ & 120 & $1.9 \mathrm{E}-06$ & $9.7 E-10$ \\
\hline LBL & 200 & $3.2 \mathrm{E}-06$ & $1.6 E-09$ & 80 & $1.3 E-06$ & $6.4 \mathrm{E}-10$ \\
\hline ITRI & 100 & $1.6 \mathrm{E}-06$ & $8.1 E-10$ & 40 & $6.4 \mathrm{E}-07$ & $3.2 \mathrm{E}-10$ \\
\hline SNL-CA & 60 & $9.7 \mathrm{E}-07$ & $4.8 \mathrm{E}-10$ & 20 & $3.2 \mathrm{E}-07$ & $1.6 \mathrm{E}-10$ \\
\hline SRS & 40 & $6.4 \mathrm{E}-07$ & $3.2 E-10$ & 20 & $3.2 \mathrm{E}-07$ & $1.6 \mathrm{E}-10$ \\
\hline Pinellas & 40 & $6.4 \mathrm{E}-07$ & 3.2E-10 & 20 & 3.2E-07 & $1.6 \mathrm{E}-10$ \\
\hline PPPL & 20 & $3.2 \mathrm{E}-07$ & $1.6 \mathrm{E}-10$ & 20 & $3.2 \mathrm{E}-07$ & $1.6 \mathrm{E}-10$ \\
\hline ORR & 20 & $3.2 \mathrm{E}-07$ & $1.6 \mathrm{E}-10$ & 20 & $3.2 \mathrm{E}-07$ & $1.6 \mathrm{E}-10$ \\
\hline ORISE & 20 & $3.2 \mathrm{E}-07$ & $1.6 \mathrm{E}-10$ & 20 & $3.2 \mathrm{E}-07$ & $1.6 \mathrm{E}-10$ \\
\hline $\mathrm{KCP}$ & 20 & $3.2 \mathrm{E}-07$ & $1.6 \mathrm{E}-10$ & 20 & $3.2 \mathrm{E}-07$ & $1.6 \mathrm{E}-10$ \\
\hline Ames & 20 & $3.2 \mathrm{E}-07$ & $1.6 \mathrm{E}-10$ & 20 & $3.2 \mathrm{E}-07$ & $1.6 E-10$ \\
\hline \multicolumn{7}{|l|}{$\begin{array}{l}\text { Decentralized } \\
\text { (Case 2) }\end{array}$} \\
\hline PORTS & 23,320 & $3.7 \mathrm{E}-04$ & $1.9 E-07$ & 8.760 & $1.4 \mathrm{E}-0.4$ & $7.0 E-08$ \\
\hline RMI & 7,680 & $1.2 \mathrm{E}-04$ & $6.2 \mathrm{E}-08$ & 2.870 & $4.6 \mathrm{E}-05$ & 2.3E-08 \\
\hline KAPL-S & 6,780 & $1.1 \mathrm{E}-04$ & $5.5 \mathrm{E}-08$ & 2.570 & $4.1 E-05$ & 2.1E-08 \\
\hline Mound & 5,120 & 8.2E-05 & $4.1 \mathrm{E}-08$ & 1.900 & $3.1 E-05$ & $1.5 \mathrm{E}-08$ \\
\hline Betris & 3,730 & $6.0 \mathrm{E}-05$ & $3.0 \mathrm{E}-08$ & 1,410 & $2.3 \mathrm{E}-05$ & 1.1E-08 \\
\hline LLNL & 570 & $9.2 \mathrm{E}-06$ & $4.6 E-09$ & 230 & $3.7 \mathrm{E}-06$ & 1.9E-09 \\
\hline ANL-E & 450 & $7.2 \mathrm{E}-06$ & $3.6 \mathrm{E}-09$ & 180 & $2.9 \mathrm{E}-06$ & $1.4 \mathrm{E}-09$ \\
\hline Fermi & 430 & $6.9 \mathrm{E}-06$ & $3.5 \mathrm{E}-09$ & 170 & 2.7E-06 & $1 .+E-09$ \\
\hline SLAC & 390 & $6.3 \mathrm{E}-06$ & $3.1 E-09$ & 150 & 2.4E-06 & 1.2E-09 \\
\hline LBL & 180 & $2.9 E-06$ & $1.4 \mathrm{E}-09$ & 80 & $1.3 E-06$ & $6.4 E-10$ \\
\hline
\end{tabular}


TABLE 5.8 (Cont.)

\begin{tabular}{|c|c|c|c|c|c|c|}
\hline \multirow[b]{2}{*}{$\begin{array}{l}\text { Altemative and } \\
\text { Site }^{b}\end{array}$} & \multicolumn{3}{|c|}{ All Truck } & \multicolumn{3}{|c|}{ All Rail } \\
\hline & $\begin{array}{c}\text { Total } \\
\text { Shipments }\end{array}$ & $\begin{array}{l}\text { Dose } \\
\text { (rem) }\end{array}$ & $\begin{array}{c}\text { Risk } \\
\text { (Fatal Cancer) }^{c}\end{array}$ & $\begin{array}{c}\text { Total } \\
\text { Shipments }\end{array}$ & $\begin{array}{l}\text { Dose } \\
\text { (rem) }\end{array}$ & $\begin{array}{c}\text { Risk } \\
\text { (Fatal Cancer) }^{c}\end{array}$ \\
\hline ORR & 50 & 8.1E-07 & $4.0 \mathrm{E}-10$ & 20 & 3.2E-07 & $1.6 \mathrm{E}-10$ \\
\hline ORISE & 50 & 8. $1 E-07$ & $4.0 E-10$ & 20 & $3.2 \mathrm{E}-07$ & $1.6 \mathrm{E}-10$ \\
\hline SRS & 20 & $3.2 \mathrm{E}-07$ & $1.6 \mathrm{E}-10$ & 10 & $1.6 \mathrm{E}-07$ & 8.IE-II \\
\hline Pinellas & 20 & $3.2 \mathrm{E}-07$ & $1.6 \mathrm{E}-10$ & 10 & $1.6 \mathrm{E}-07$ & 8.|E-1) \\
\hline Ames & 20 & $3.2 \mathrm{E}-07$ & $1.6 \mathrm{E}-10$ & 10 & $1.6 E-07$ & 8. IE-1I \\
\hline PPPL & 10 & $1.6 \mathrm{E}-07$ & 8.1E-11 & 10 & $1.6 \mathrm{E}-07$ & 8.IE-II \\
\hline PGDP & 10 & $1.6 \mathrm{E}-07$ & $8.1 E-11$ & 10 & $1.6 E-07$ & $8.1 E-11$ \\
\hline $\mathrm{KCP}$ & 10 & $1.6 \mathrm{E}-07$ & 8.1E-11 & 10 & $1.6 \mathrm{E}-07$ & $8.1 E-11$ \\
\hline \multicolumn{7}{|l|}{$\begin{array}{l}\text { Regionalized I } \\
\text { (Case 3) }\end{array}$} \\
\hline PORTS & 24.820 & $4.0 \mathrm{E}-04$ & $2.0 \mathrm{E}-07$ & 9.330 & $1.5 \mathrm{E}-04$ & $7.5 \mathrm{E}-08$ \\
\hline RMI & 7.680 & $1.2 \mathrm{E}-04$ & $6.2 \mathrm{E}-08$ & 2.870 & $4.6 \mathrm{E}-05$ & $2.3 \mathrm{E}-08$ \\
\hline KAPL-S & 6.780 & 1.1E-04 & $5.5 \mathrm{E}-08$ & 2.570 & $4.1 \mathrm{E}-05$ & 2.1E-08 \\
\hline Mound & 5.120 & $8.2 \mathrm{E}-05$ & $4.1 \mathrm{E}-08$ & 1.900 & $3.1 E-05$ & $1.5 \mathrm{E}-08$ \\
\hline Bettis & 3.730 & $6.0 \mathrm{E}-05$ & $3.0 \mathrm{E}-08$ & 1,410 & $2.3 \mathrm{E}-05$ & $1.1 \mathrm{E}-08$ \\
\hline ANL-E & 1,050 & $1.7 \mathrm{E}-05$ & 8.5E-09 & 390 & $6.3 \mathrm{E}-06$ & 3.1E-09 \\
\hline LLNL & 570 & $9.2 \mathrm{E}-06$ & $4.6 \mathrm{E}-09$ & 230 & $3.7 \mathrm{E}-06$ & $1.9 \mathrm{E}-09$ \\
\hline Fermi & 430 & $6.9 \mathrm{E}-06$ & $3.5 \mathrm{E}-09$ & 170 & 2.7E-06 & $1.4 E-09$ \\
\hline SLAC & 390 & $6.3 \mathrm{E}-06$ & $3.1 E-09$ & 150 & $2.4 \mathrm{E}-06$ & $1.2 \mathrm{E}-09$ \\
\hline LANL & 330 & $5.3 \mathrm{E}-06$ & 2.7E-09 & 140 & 2.3E-06 & $1.1 \mathrm{E}-09$ \\
\hline SNL-NM & 230 & $3.7 \mathrm{E}-06$ & $1.9 \mathrm{E}-09$ & 100 & $1.6 \mathrm{E}-06$ & $8.1 E-10$ \\
\hline LBL & 180 & $2.9 \mathrm{E}-06$ & $1.4 \mathrm{E}-09$ & 80 & $1.3 E-06$ & $6.4 \mathrm{E}-10$ \\
\hline ITRI & 100 & $1.6 \mathrm{E}-06$ & 8.1E-10 & 40 & $6.4 E-07$ & $3.2 E-10$ \\
\hline ORR & 50 & $8.1 E-07$ & $4.0 \mathrm{E}-10$ & 20 & $3.2 \mathrm{E}-07$ & $1.6 \mathrm{E}-10$ \\
\hline ORISE & 50 & 8.1E-07 & $4.0 \mathrm{E}-10$ & 20 & $3.2 \mathrm{E}-07$ & $1.6 \mathrm{E}-10$ \\
\hline SRS & 20 & 3.2E-07 & $1.6 \mathrm{E}-10$ & 10 & $1.6 \mathrm{E}-07$ & $8.1 E-11$ \\
\hline Pinellas & 20 & $3.2 \mathrm{E}-07$ & $1.6 \mathrm{E}-10$ & 10 & $1.6 \mathrm{E}-07$ & $8.1 E-11$ \\
\hline Ames & 20 & $3.2 \mathrm{E}-07$ & $1.6 \mathrm{E}-10$ & 10 & $1.6 \mathrm{E}-07$ & $8.1 \mathrm{E}-11$ \\
\hline PPPL & 10 & $1.6 E-07$ & 8.1E-11 & 10 & $1.6 \mathrm{E}-07$ & $8.1 E-11$ \\
\hline PGDP & 10 & $1.6 \mathrm{E}-07$ & 8.1E-11 & 10 & $1.6 \mathrm{E}-07$ & $8.1 E-11$ \\
\hline $\mathrm{KCP}$ & 10 & $1.6 \mathrm{E}-07$ & 8.1E-11 & 10 & $1.6 \mathrm{E}-07$ & $8.1 E-11$ \\
\hline \multicolumn{7}{|l|}{$\begin{array}{l}\text { Regionalized } 2 \\
\text { (Case 9) }\end{array}$} \\
\hline PORTS & 24,380 & $3.9 \mathrm{E}-04$ & $2.0 \mathrm{E}-07$ & 9,260 & $1.5 \mathrm{E}-0.4$ & 7.5E-08 \\
\hline RMI & 7,610 & 1.2E-04 & $6.1 E-08$ & 2.850 & $4.6 \mathrm{E}-05$ & $2.3 \mathrm{E}-08$ \\
\hline KAPL-S & 6,670 & $1.1 E-04$ & $5.4 \mathrm{E}-08$ & 2,560 & $4.1 \mathrm{E}-05$ & $2.1 \mathrm{E}-08$ \\
\hline Mound & 5,080 & 8.2E-05 & 4. $1 E-08$ & 1.910 & $3.1 E-05$ & $1.5 \mathrm{E}-08$ \\
\hline Betris & 3,660 & $5.9 E-05$ & $2.9 \mathrm{E}-08$ & 1,400 & $2.3 \mathrm{E}-05$ & $1.1 \mathrm{E}-08$ \\
\hline ANL-E & 1,050 & $1.7 E-05$ & 8.5E-09 & 390 & $6.3 \mathrm{E}-06$ & $3.1 E-09$ \\
\hline FEMP & 910 & $1.5 \mathrm{E}-05$ & 7.3E-09 & 400 & $6.4 \mathrm{E}-06$ & 3.2E-09 \\
\hline LLNL & 560 & $9.0 \mathrm{E}-06$ & $4.5 \mathrm{E}-09$ & 230 & $3.7 \mathrm{E}-06$ & $1.9 \mathrm{E}-09$ \\
\hline Fermi & 420 & $6.8 E-06$ & $3.4 \mathrm{E}-09$ & 180 & $2.9 \mathrm{E}-06$ & $1.4 \mathrm{E}-09$ \\
\hline
\end{tabular}


TABLE 5.8 (Cont.)

\begin{tabular}{|c|c|c|c|c|c|c|}
\hline \multirow[b]{2}{*}{$\begin{array}{l}\text { Altemative and } \\
\text { Site }^{\mathrm{b}}\end{array}$} & \multicolumn{3}{|c|}{ All Truck } & \multicolumn{3}{|c|}{ All Rail } \\
\hline & $\begin{array}{c}\text { Total } \\
\text { Shipments }\end{array}$ & $\begin{array}{l}\text { Dose } \\
\text { (rem) }\end{array}$ & $\begin{array}{c}\text { Risk } \\
\text { (Fatal Cancer) }\end{array}$ & $\begin{array}{c}\text { Total } \\
\text { Shipments }\end{array}$ & $\begin{array}{l}\text { Dose } \\
\text { (rem) }\end{array}$ & $\begin{array}{c}\text { Risk } \\
\text { (Fatal Cancer) }^{*}\end{array}$ \\
\hline SLAC & 390 & $6.3 \mathrm{E}-06$ & 3.1E-09 & 150 & $2.4 E-06$ & $1.2 E-09$ \\
\hline LANL & 330 & $5.3 \mathrm{E}-06$ & $2.7 \mathrm{E}-09$ & 140 & 2.3E-06 & $1.1 E-09$ \\
\hline SNL-NM & 230 & $3.7 \mathrm{E}-06$ & $1.9 \mathrm{E}-09$ & 100 & $1.6 \mathrm{E}-06$ & $8.1 E-10$ \\
\hline LBL & 170 & 2.7E-06 & $1.4 \mathrm{E}-09$ & 80 & $1.3 \mathrm{E}-06$ & $6.4 \mathrm{E}-10$ \\
\hline ITRI & 100 & $1.6 \mathrm{E}-06$ & $8.1 E-10$ & 40 & $6.4 E-07$ & $3.2 \mathrm{E}-10$ \\
\hline ORR & 50 & 8.1E-07 & $4.0 \mathrm{E}-10$ & 20 & 3.2E-07 & $1.6 \mathrm{E}-10$ \\
\hline ORISE & 50 & $8.1 E-07$ & $4.0 \mathrm{E}-10$ & 20 & 3.2E-07 & $1.6 E-10$ \\
\hline Ames & 30 & $4.8 \mathrm{E}-07$ & $2.4 \mathrm{E}-10$ & 20 & $3.2 \mathrm{E}-07$ & $1.6 E-10$ \\
\hline SRS & 20 & $3.2 \mathrm{E}-07$ & $1.6 \mathrm{E}-10$ & 10 & $1.6 \mathrm{E}-07$ & $8.1 E-11$ \\
\hline Pinellas & 20 & $3.2 \mathrm{E}-07$ & $1.6 \mathrm{E}-10$ & 10 & $1.6 \mathrm{E}-07$ & $8.1 E-11$ \\
\hline PPPL & 10 & $1.6 \mathrm{E}-07$ & $8.1 E-11$ & 10 & $1.6 \mathrm{E}-07$ & $8.1 E-11$ \\
\hline PGDP & 10 & $1.6 \mathrm{E}-07$ & $8.1 E-11$ & 10 & $1.6 E-07$ & $8.1 E-11$ \\
\hline $\mathrm{KCP}$ & 10 & $1.6 \mathrm{E}-07$ & $8.1 E-11$ & 10 & $1.6 \mathrm{E}-07$ & $8.1 E-11$ \\
\hline \multicolumn{7}{|l|}{$\begin{array}{l}\text { Regionalized } 3 \\
\text { (Case 4) }\end{array}$} \\
\hline ORR & 64,590 & $1.0 \mathrm{E}-03$ & $5.2 \mathrm{E}-07$ & 24,470 & $3.9 \mathrm{E}-04$ & $2.0 \mathrm{E}-07$ \\
\hline PORTS & 33,440 & $5.4 \mathrm{E}-04$ & 2.7E-07 & 12,740 & $2.1 \mathrm{E}-04$ & $1.0 E-07$ \\
\hline LANL & 18,400 & $3.0 \mathrm{E}-04$ & $1.5 \mathrm{E}-07$ & 6,910 & $1.1 \mathrm{E}-04$ & $5.6 \mathrm{E}-08$ \\
\hline Pantex & 14,500 & 2.3E-04 & $1.2 \mathrm{E}-07$ & 5,440 & $8.8 \mathrm{E}-05$ & $4.4 E-08$ \\
\hline RMI & 7,680 & $1.2 \mathrm{E}-04$ & $6.2 \mathrm{E}-08$ & 2,870 & $4.6 \mathrm{E}-05$ & $2.3 E-08$ \\
\hline KAPL-S & 6,780 & $1.1 \mathrm{E}-04$ & $5.5 \mathrm{E}-08$ & 2,570 & $4.1 \mathrm{E}-05$ & 2.1E-08 \\
\hline PGDP & 6,270 & $1.0 \mathrm{E}-04$ & $5.0 \mathrm{E}-08$ & 2,370 & $3.8 \mathrm{E}-05$ & $1.9 E-08$ \\
\hline Mound & 5,120 & $8.2 \mathrm{E}-05$ & $4.1 \mathrm{E}-08$ & 1,900 & $3.1 E-05$ & $1.5 E-08$ \\
\hline Bettis & 3,730 & $6.0 \mathrm{E}-05$ & $3.0 \mathrm{E}-08$ & 1,410 & $2.3 E-05$ & $1.1 \mathrm{E}-08$ \\
\hline RFETS & 3,570 & 5.7E-05 & $2.9 \mathrm{E}-08$ & 1,330 & 2.1E-05 & $1.1 \mathrm{E}-08$ \\
\hline NTS & 1,190 & $1.9 \mathrm{E}-05$ & $9.6 \mathrm{E}-09$ & 460 & $7.4 \mathrm{E}-06$ & $3.7 \mathrm{E}-09$ \\
\hline ANL-E & 1,050 & $1.7 \mathrm{E}-05$ & 8.5E-09 & 390 & $6.3 \mathrm{E}-06$ & $3.1 \mathrm{E}-09$ \\
\hline LLNL & 560 & $9.0 \mathrm{E}-06$ & $4.5 \mathrm{E}-09$ & 210 & $3.4 E-06$ & $1.7 E-09$ \\
\hline Fermi & 430 & $6.9 \mathrm{E}-06$ & $3.5 \mathrm{E}-09$ & 170 & $2.7 \mathrm{E}-06$ & $1 .+E-09$ \\
\hline SLAC & 390 & $6.3 \mathrm{E}-06$ & $3.1 E-09$ & 150 & $2.4 E-06$ & $1.2 \mathrm{E}-09$ \\
\hline SNL-NMI & 230 & $3.7 \mathrm{E}-06$ & $1.9 E-09$ & 100 & $1.6 E-06$ & $8.1 E-10$ \\
\hline LBL & 180 & $2.9 \mathrm{E}-06$ & $1.4 \mathrm{E}-09$ & 80 & $1.3 E-06$ & $6.4 E-10$ \\
\hline ITRI & 100 & $1.6 \mathrm{E}-06$ & $8.1 E-10$ & 40 & $6.4 \mathrm{E}-07$ & 3.2E-10 \\
\hline SNL-CA & 60 & $9.7 \mathrm{E}-07$ & $4.8 \mathrm{E}-10$ & 20 & $3.2 \mathrm{E}-07$ & $1.6 \mathrm{E}-10$ \\
\hline ORISE & 50 & $8.1 E-07$ & $4.0 \mathrm{E}-10$ & 20 & $3.2 \mathrm{E}-07$ & $1.6 \mathrm{E}-10$ \\
\hline SRS & 20 & 3.2E-07 & $1.6 \mathrm{E}-10$ & 10 & $1.6 \mathrm{E}-07$ & $8.1 E-11$ \\
\hline Pinellas & 20 & $3.2 \mathrm{E}-07$ & $1.6 \mathrm{E}-10$ & 10 & $1.6 \mathrm{E}-07$ & 8.IE-1I \\
\hline Ames & 20 & 3.2E-07 & $1.6 \mathrm{E}-10$ & 10 & $1.6 E-07$ & $8.1 E-11$ \\
\hline PPPL & 10 & $1.6 \mathrm{E}-07$ & $8.1 E-11$ & 10 & $1.6 \mathrm{E}-07$ & $8.1 E-11$ \\
\hline $\mathrm{KCP}$ & 10 & $1.6 \mathrm{E}-07$ & $8.1 E-11$ & 10 & $1.6 \mathrm{E}-07$ & $8.1 E-11$ \\
\hline
\end{tabular}




$\begin{array}{ll}\mathrm{km} & \text { kilometers(s) } \\ \mathrm{km}^{2} & \text { square kilometer(s) } \\ \mathrm{L} & \text { liter(s) } \\ \mathrm{lb} & \text { pounds(s) } \\ \mathrm{m} & \text { meter(s) } \\ \mathrm{mi} & \text { mile(s) } \\ \mathrm{mi}^{2} & \text { square mile(s) } \\ \mathrm{mph} & \text { mile(s) per hour } \\ \mathrm{mrem} & \text { millirem } \\ \mathrm{nCi} & \text { nanocurie(s) } \\ \mathrm{rem} & \text { roentgen-equivalent man } \\ \mathrm{s} & \text { second(s) } \\ \mathrm{yr} & \text { year(s) }\end{array}$


TABLE 5.8 (Cont.)

\begin{tabular}{|c|c|c|c|c|c|c|}
\hline \multirow[b]{2}{*}{$\begin{array}{c}\text { Allemative and } \\
\text { Site }^{\mathrm{b}} \\
\end{array}$} & \multicolumn{3}{|c|}{ All Truck } & \multicolumn{3}{|c|}{ All Rail } \\
\hline & $\begin{array}{c}\text { Total } \\
\text { Shipments }\end{array}$ & $\begin{array}{l}\text { Dose } \\
\text { (rem) }\end{array}$ & $\begin{array}{c}\text { Risk } \\
\text { (Fatal Cancer) }\end{array}$ & $\begin{array}{c}\text { Total } \\
\text { Shipments }\end{array}$ & $\begin{array}{l}\text { Dose } \\
\text { (rem) }\end{array}$ & $\begin{array}{c}\text { Risk } \\
\text { (Fatal Cancer) }^{\circ}\end{array}$ \\
\hline \multicolumn{7}{|l|}{$\begin{array}{l}\text { Regionalized }+ \\
\text { (Case 12) }\end{array}$} \\
\hline ORR & 58,210 & $9.4 \mathrm{E}-04$ & $4.7 \mathrm{E}-07$ & 22.310 & $3.6 \mathrm{E}-04$ & $1.8 \mathrm{E}-07$ \\
\hline PORTS & +7.610 & 7.7E-04 & $3.8 \mathrm{E}-07$ & 18.410 & $3.0 \mathrm{E}-04$ & $1.5 E-07$ \\
\hline LANL & 17.860 & $2.9 \mathrm{E}-04$ & 1.4E-07 & 6,740 & $1.1 E-04$ & $5.4 E-08$ \\
\hline Pantex & 14,180 & $2.3 \mathrm{E}-04$ & 1. $1 E-07$ & 5.370 & $8.6 \mathrm{E}-0.5$ & $4.3 E-08$ \\
\hline RMI & 7,520 & $1.2 \mathrm{E}-04$ & $6.1 E-08$ & 2,860 & $4.6 E-05$ & $2.3 \mathrm{E}-08$ \\
\hline KAPL-S & 6.670 & 1.IE-04 & $5.4 \mathrm{E}-08$ & 2,560 & $4.1 E-05$ & $2.1 E-08$ \\
\hline PGDP & 6,270 & $1.0 \mathrm{E}-04$ & $5.0 \mathrm{E}-08$ & 2,350 & $3.8 E-05$ & $1.9 \mathrm{E}-08$ \\
\hline Mound & 5,080 & $8.2 \mathrm{E}-05$ & 4.1E-08 & 1,910 & $3.1 E-05$ & $1.5 \mathrm{E}-08$ \\
\hline Bettis & 3.660 & $5.9 \mathrm{E}-05$ & $2.9 \mathrm{E}-08$ & 1,400 & $2.3 \mathrm{E}-0.5$ & 1.1E-08 \\
\hline RFETS & 3,350 & $5.4 \mathrm{E}-05$ & 2.7E-08 & 1,230 & $2.0 \mathrm{E}-05$ & $9.9 \mathrm{E}-09$ \\
\hline ANL-E & 1.050 & $1.7 \mathrm{E}-05$ & 8.5E-09 & 390 & $6.3 \mathrm{E}-06$ & $3.1 \mathrm{E}-09$ \\
\hline NTS & 860 & $1.4 E-05$ & $6.9 \mathrm{E}-09$ & 330 & $5.3 \mathrm{E}-06$ & 2.7E-09 \\
\hline LLNL & 570 & $9.2 \mathrm{E}-06$ & $4.6 \mathrm{E}-09$ & 220 & $3.5 \mathrm{E}-06$ & $1.8 \mathrm{E}-09$ \\
\hline Fermi & 420 & $6.8 \mathrm{E}-06$ & $3.4 \mathrm{E}-09$ & 180 & $2.9 \mathrm{E}-06$ & $1.4 E-09$ \\
\hline SLAC & 400 & $6.4 \mathrm{E}-06$ & $3.2 \mathrm{E}-09$ & 160 & $2.6 \mathrm{E}-06$ & $1.3 \mathrm{E}-09$ \\
\hline Hanford & 350 & $5.6 \mathrm{E}-06$ & 2.8E-09 & 160 & $2.6 \mathrm{E}-06$ & $1.3 E-09$ \\
\hline SNL-NM & 230 & $3.7 \mathrm{E}-06$ & $1.9 \mathrm{E}-09$ & 100 & $1.6 \mathrm{E}-06$ & $8.1 E-10$ \\
\hline LBL & 180 & $2.9 \mathrm{E}-06$ & $1.4 \mathrm{E}-09$ & 80 & $1.3 \mathrm{E}-06$ & $6.4 \mathrm{E}-10$ \\
\hline ITRI & 100 & $1.6 \mathrm{E}-06$ & 8.1E-10 & 40 & $6.4 \mathrm{E}-07$ & $3.2 \mathrm{E}-10$ \\
\hline SNL-CA & 60 & $9.7 \mathrm{E}-07$ & $4.8 \mathrm{E}-10$ & 30 & $4.8 \mathrm{E}-07$ & $2.4 E-10$ \\
\hline ORISE & 50 & $8.1 E-07$ & $4.0 \mathrm{E}-10$ & 20 & $3.2 E-07$ & $1.6 \mathrm{E}-10$ \\
\hline Ames & 30 & $4.8 \mathrm{E}-07$ & $2.4 \mathrm{E}-10$ & 20 & $3.2 \mathrm{E}-07$ & $1.6 E-10$ \\
\hline PPPL & 20 & $3.2 \mathrm{E}-07$ & $1.6 \mathrm{E}-10$ & 20 & $3.2 \mathrm{E}-07$ & $1.6 \mathrm{E}-10$ \\
\hline SRS & 20 & $3.2 \mathrm{E}-07$ & $1.6 \mathrm{E}-10$ & 10 & $1.6 \mathrm{E}-07$ & 8.1E-11 \\
\hline Pinellas & 20 & $3.2 \mathrm{E}-07$ & $1.6 \mathrm{E}-10$ & 10 & $1.6 \mathrm{E}-07$ & $8.1 E-11$ \\
\hline $\mathrm{KCP}$ & 10 & $1.6 \mathrm{E}-07$ & $8.1 E-11$ & 10 & $1.6 \mathrm{E}-07$ & $8.1 E-11$ \\
\hline \multicolumn{7}{|l|}{$\begin{array}{l}\text { Regionalized } 5 \\
\text { (Case 19) }\end{array}$} \\
\hline ORR & 63.430 & $1.0 \mathrm{E}-03$ & $5.1 \mathrm{E}-07$ & 24,170 & $3.9 E-04$ & $1.9 \mathrm{E}-07$ \\
\hline PORTS & 32,500 & 5.2E-04 & $2.6 \mathrm{E}-07$ & 12,500 & $2.0 \mathrm{E}-04$ & $1.0 \mathrm{E}-07$ \\
\hline INEL & 25,620 & $4.1 \mathrm{E}-04$ & 2.1E-07 & 10.020 & $1.6 \mathrm{E}-04$ & 8.1E-08 \\
\hline Pantex & 13,830 & $2.2 \mathrm{E}-04$ & $1.1 E-07$ & 5.380 & 8.7E-05 & 4.3E-08 \\
\hline LANL & 11,750 & $1.9 \mathrm{E}-04$ & $9.5 \mathrm{E}-08$ & 4,640 & $7.5 \mathrm{E}-05$ & $3.7 \mathrm{E}-08$ \\
\hline RMI & 7,610 & $1.2 \mathrm{E}-04$ & $6.1 \mathrm{E}-08$ & 2.850 & $4.6 \mathrm{E}-05$ & $2.3 \mathrm{E}-08$ \\
\hline KAPL-S & 6,670 & 1.1E-04 & 5.4E-08 & 2,560 & $4.1 \mathrm{E}-05$ & 2.1E-08 \\
\hline PGDP & 6,270 & $1.0 \mathrm{E}-04$ & $5.0 \mathrm{E}-08$ & 2,350 & $3.8 \mathrm{E}-05$ & $1.9 \mathrm{E}-08$ \\
\hline Mound & 5,160 & 8.3E-05 & $4.2 \mathrm{E}-08$ & 1.900 & $3.1 \mathrm{E}-05$ & 1.5E-08 \\
\hline Bettis & 3.660 & $5.9 \mathrm{E}-05$ & $2.9 \mathrm{E}-08$ & 1.400 & $2.3 \mathrm{E}-05$ & $1.1 E-08$ \\
\hline RFETS & 3.530 & $5.7 \mathrm{E}-05$ & $2.8 \mathrm{E}-08$ & 1,330 & $2.1 E-05$ & $1.1 E-08$ \\
\hline ANL-E & 1,050 & $1.7 \mathrm{E}-05$ & 8.5E-09 & 390 & $6.3 \mathrm{E}-06$ & $3.1 \mathrm{E}-09$ \\
\hline
\end{tabular}


TABLE 5.8 (Cont.)

\begin{tabular}{|c|c|c|c|c|c|c|}
\hline \multirow[b]{2}{*}{$\begin{array}{c}\text { Altemative and } \\
\text { Site }^{b}\end{array}$} & \multicolumn{3}{|c|}{ All Truck } & \multicolumn{3}{|c|}{ All Rail } \\
\hline & $\begin{array}{c}\text { Total } \\
\text { Shipments }\end{array}$ & $\begin{array}{l}\text { Dose } \\
\text { (rem) }\end{array}$ & $\begin{array}{c}\text { Risk } \\
\text { (Fatal Cancer) }\end{array}$ & $\begin{array}{c}\text { Total } \\
\text { Shipments }\end{array}$ & $\begin{array}{l}\text { Dose } \\
\text { (rem) }\end{array}$ & $\begin{array}{c}\text { Risk } \\
\text { (Fatal Cancer) }^{\mathrm{c}}\end{array}$ \\
\hline NTS & 860 & $1.4 E-05$ & $6.9 \mathrm{E}-09$ & 330 & $5.3 E-06$ & $2.7 \mathrm{E}-09$ \\
\hline LLNL & 570 & $9.2 \mathrm{E}-06$ & $4.6 \mathrm{E}-09$ & 220 & $3.5 \mathrm{E}-06$ & $1.8 \mathrm{E}-09$ \\
\hline Fermi & +20 & $6.8 \mathrm{E}-06$ & $3.4 \mathrm{E}-09$ & 170 & $2.7 \mathrm{E}-06$ & $1.4 \mathrm{E}-09$ \\
\hline SLAC & 400 & $6.4 \mathrm{E}-06$ & $3.2 \mathrm{E}-09$ & 160 & 2.6E-06 & $1.3 \mathrm{E}-09$ \\
\hline Hanford & 350 & $5.6 \mathrm{E}-06$ & $2.8 \mathrm{E}-09$ & 160 & $2.6 \mathrm{E}-06$ & $1.3 \mathrm{E}-09$ \\
\hline SiNL-NM & 240 & $3.9 \mathrm{E}-06$ & $1.9 \mathrm{E}-09$ & 110 & $1.8 \mathrm{E}-06$ & $8.9 E-10$ \\
\hline LBL & 180 & $2.9 \mathrm{E}-06$ & $1.4 \mathrm{E}-09$ & 80 & $1.3 \mathrm{E}-06$ & $6.4 \mathrm{E}-10$ \\
\hline ITRI & 110 & $1.8 \mathrm{E}-06$ & $8.9 \mathrm{E}-10$ & 40 & $6.4 \mathrm{E}-07$ & 3.2E-10 \\
\hline SNL-CA & 60 & $9.7 \mathrm{E}-07$ & $4.8 \mathrm{E}-10$ & 30 & $4.8 \mathrm{E}-07$ & $2.4 E-10$ \\
\hline ORISE & 50 & $8.1 E-07$ & $4.0 \mathrm{E}-10$ & 20 & $3.2 \mathrm{E}-07$ & $1.6 \mathrm{E}-10$ \\
\hline SRS & 20 & $3.2 \mathrm{E}-07$ & $1.6 \mathrm{E}-10$ & 10 & $1.6 \mathrm{E}-07$ & $8.1 E-11$ \\
\hline Pinellas & 20 & $3.2 \mathrm{E}-07$ & $1.6 \mathrm{E}-10$ & 10 & $1.6 \mathrm{E}-07$ & 8.1E-11 \\
\hline Ames & 20 & $3.2 \mathrm{E}-07$ & $1.6 \mathrm{E}-10$ & 10 & $1.6 \mathrm{E}-07$ & $8.1 E-11$ \\
\hline PPPL & 10 & $1.6 \mathrm{E}-07$ & $8.1 \mathrm{E}-11$ & 10 & $1.6 \mathrm{E}-07$ & $8.1 E-11$ \\
\hline $\mathrm{KCP}$ & 10 & $1.6 \mathrm{E}-07$ & $8.1 E-11$ & 10 & $1.6 \mathrm{E}-07$ & $8.1 E-11$ \\
\hline \multicolumn{7}{|l|}{$\begin{array}{l}\text { Regionalized } 6 \\
\text { (Case 5) }\end{array}$} \\
\hline SRS & 130,030 & $2.1 \mathrm{E}-03$ & $1.0 \mathrm{E}-06$ & 49,340 & $7.9 \mathrm{E}-04$ & $4.0 \mathrm{E}-07$ \\
\hline ORR & 65,420 & $1.1 \mathrm{E}-03$ & $5.3 \mathrm{E}-07$ & 24,860 & $4.0 \mathrm{E}-04$ & $2.0 \mathrm{E}-07$ \\
\hline Hanford & 44,360 & 7.1E-04 & $3.6 \mathrm{E}-07$ & 16,700 & 2.7E-04 & $1.3 \mathrm{E}-07$ \\
\hline PORTS & 33,440 & $5.4 \mathrm{E}-04$ & 2.7E-07 & 12,740 & $2.1 \mathrm{E}-04$ & $1.0 \mathrm{E}-07$ \\
\hline Pantex & 14,500 & $2.3 \mathrm{E}-04$ & $1.2 E-07$ & 5,440 & $8.8 \mathrm{E}-05$ & $4.4 \mathrm{E}-08$ \\
\hline LANL & 11,420 & $1.8 \mathrm{E}-04$ & $9.2 \mathrm{E}-08$ & 4,270 & $6.9 \mathrm{E}-05$ & $3.4 \mathrm{E}-08$ \\
\hline INEL & 8,520 & $1.4 \mathrm{E}-04$ & $6.9 \mathrm{E}-08$ & 3.220 & $5.2 E-05$ & $2.6 \mathrm{E}-08$ \\
\hline RMI & 7,680 & $1.2 \mathrm{E}-04$ & $6.2 \mathrm{E}-08$ & 2,870 & $4.6 \mathrm{E}-05$ & $2.3 E-08$ \\
\hline KAPL-S & 6,780 & 1.1E-04 & 5.5E-08 & 2,570 & $4.1 \mathrm{E}-05$ & 2.1E-08 \\
\hline PGDP & 6,270 & $1.0 \mathrm{E}-04$ & $5.0 \mathrm{E}-08$ & 2,370 & $3.8 \mathrm{E}-05$ & $1.9 \mathrm{E}-08$ \\
\hline Mound & 5,120 & $8.2 \mathrm{E}-05$ & $4.1 E-08$ & 1.900 & $3.1 E-05$ & $1.5 \mathrm{E}-08$ \\
\hline NRF & 4,610 & $7.4 \mathrm{E}-05$ & 3.7E-08 & 1.750 & $2.8 \mathrm{E}-05$ & $1.4 \mathrm{E}-08$ \\
\hline Bettis & 3,730 & $6.0 \mathrm{E}-05$ & $3.0 \mathrm{E}-08$ & 1,410 & $2.3 \mathrm{E}-05$ & $1.1 E-08$ \\
\hline RFETS & 3,570 & $5.7 \mathrm{E}-05$ & 2.9E-08 & 1,330 & 2.1E-05 & $1.1 \mathrm{E}-08$ \\
\hline ANVL-E & 1,050 & $1.7 \mathrm{E}-05$ & 8.5E-09 & 390 & $6.3 \mathrm{E}-06$ & 3.1E-09 \\
\hline LLNL & 560 & $9.0 \mathrm{E}-06$ & $4.5 \mathrm{E}-09$ & 210 & $3.4 \mathrm{E}-06$ & $1.7 \mathrm{E}-09$ \\
\hline Fermi & 430 & $6.9 E-06$ & 3.5E-09 & 170 & 2.7E-06 & $1.4 \mathrm{E}-09$ \\
\hline SLAC & 390 & $6.3 \mathrm{E}-06$ & $3.1 E-09$ & 150 & 2.+E-06 & $1.2 \mathrm{E}-09$ \\
\hline SNL-NM & 230 & 3.7E-06 & $1.9 \mathrm{E}-09$ & 100 & $1.6 \mathrm{E}-06$ & 8.1E-10 \\
\hline ANL-W & 220 & $3.5 \mathrm{E}-06$ & $1.8 \mathrm{E}-09$ & 90 & $1.4 E-06$ & 7.2E-10 \\
\hline LBL & 180 & $2.9 E-06$ & $1.4 \mathrm{E}-09$ & 80 & $1.3 \mathrm{E}-06$ & $6.4 E-10$ \\
\hline ITRI & 100 & $1.6 \mathrm{E}-06$ & 8.1E-10 & .40 & $6.4 \mathrm{E}-07$ & 3.2E-10 \\
\hline SNL-CA & 60 & $9.7 \mathrm{E}-07$ & $4.8 \mathrm{E}-10$ & 20 & 3.2E-07 & $1.6 \mathrm{E}-10$ \\
\hline ORISE & 50 & \$.1E-07 & $4.0 \mathrm{E}-10$ & 20 & 3.2E-07 & $1.6 E-10$ \\
\hline Pinellas & 20 & 3.2E-07 & $1.6 \mathrm{E}-10$ & 10 & $1.6 \mathrm{E}-07$ & $8.1 E-11$ \\
\hline
\end{tabular}


TABLE 5.8 (Cont.)

\begin{tabular}{|c|c|c|c|c|c|c|}
\hline \multirow[b]{2}{*}{$\begin{array}{l}\text { Altemative and } \\
\text { Site }^{b}\end{array}$} & \multicolumn{3}{|c|}{ All Truck } & \multicolumn{3}{|c|}{ All Rail } \\
\hline & $\begin{array}{c}\text { Total } \\
\text { Shipments }\end{array}$ & $\begin{array}{l}\text { Dose } \\
\text { (rem) }\end{array}$ & $\begin{array}{c}\text { Risk } \\
\text { (Fatal Cancer) }\end{array}$ & $\begin{array}{c}\text { Total } \\
\text { Shipments }\end{array}$ & $\begin{array}{l}\text { Dose } \\
\text { (rem) }\end{array}$ & $\begin{array}{c}\text { Risk } \\
\text { (Fatal Cancer) }^{*}\end{array}$ \\
\hline Ames & 20 & $3.2 \mathrm{E}-07$ & $1.6 \mathrm{E}-10$ & 10 & $1.6 \mathrm{E}-07$ & $8.1 E-11$ \\
\hline PPPL & 10 & $1.6 \mathrm{E}-07$ & $8.1 E-11$ & 10 & $1.6 \mathrm{E}-07$ & $8.1 E-11$ \\
\hline $\mathrm{KCP}$ & 10 & $1.6 \mathrm{E}-07$ & $8.1 E-11$ & 10 & $1.6 \mathrm{E}-07$ & $8.1 E-11$ \\
\hline \multicolumn{7}{|l|}{$\begin{array}{l}\text { Regionalized } 7 \\
\text { (Case 6) }\end{array}$} \\
\hline SRS & 130,030 & $2.1 E-03$ & $1.0 \mathrm{E}-06$ & 49.340 & $7.9 \mathrm{E}-0.4$ & $4.0 \mathrm{E}-07$ \\
\hline ORR & 65,420 & $1.1 E-03$ & $5.3 \mathrm{E}-07$ & 24,860 & $4.0 \mathrm{E}-04$ & $2.0 \mathrm{E}-07$ \\
\hline NTS & 58,900 & $9.5 \mathrm{E}-04$ & $4.7 \mathrm{E}-07$ & 22.140 & $3.6 \mathrm{E}-04$ & $1.8 \mathrm{E}-07$ \\
\hline PORTS & 33,440 & $5.4 \mathrm{E}-04$ & 2.7E-07 & 12.740 & 2.IE-04 & $1.0 \mathrm{E}-07$ \\
\hline Hanford & 14,540 & $2.3 E-04$ & $1.2 \mathrm{E}-07$ & 5.440 & $8.8 \mathrm{E}-05$ & 4.4E-08 \\
\hline Pantex & 14,500 & $2.3 E-04$ & $1.2 \mathrm{E}-07$ & 5.440 & $8.8 \mathrm{E}-05$ & $4.4 E-08$ \\
\hline LANL & 11,420 & $1.8 \mathrm{E}-04$ & $9.2 \mathrm{E}-08$ & 4,270 & $6.9 \mathrm{E}-05$ & $3.4 E-08$ \\
\hline INEL & 8.520 & $1.4 \mathrm{E}-04$ & $6.9 E-08$ & 3.220 & $5.2 \mathrm{E}-05$ & $2.6 \mathrm{E}-08$ \\
\hline RMI & 7,680 & $1.2 \mathrm{E}-04$ & $6.2 \mathrm{E}-08$ & 2,870 & $4.6 \mathrm{E}-05$ & $2.3 E-08$ \\
\hline KAPL-S & 6,780 & $1.1 \mathrm{E}-04$ & $5.5 \mathrm{E}-08$ & 2,570 & 4.IE-05 & $2.1 \mathrm{E}-08$ \\
\hline PGDP & 6,270 & $1.0 \mathrm{E}-04$ & $5.0 \mathrm{E}-08$ & 2,370 & $3.8 \mathrm{E}-05$ & $1.9 E-08$ \\
\hline Mound & 5,120 & $8.2 E-05$ & $4.1 \mathrm{E}-08$ & 1,900 & $3.1 E-05$ & $1.5 E-08$ \\
\hline NRF & 4,610 & $7.4 \mathrm{E}-05$ & $3.7 \mathrm{E}-08$ & 1,750 & $2.8 E-05$ & $1.4 E-08$ \\
\hline Bettis & 3,730 & $6.0 \mathrm{E}-05$ & $3.0 \mathrm{E}-08$ & 1,410 & $2.3 \mathrm{E}-05$ & $1.1 E-08$ \\
\hline RFETS & 3,570 & $5.7 \mathrm{E}-05$ & $2.9 \mathrm{E}-08$ & 1,330 & 2.1E-05 & $1.1 \mathrm{E}-08$ \\
\hline ANL-E & 1.050 & $1.7 \mathrm{E}-05$ & $8.5 \mathrm{E}-09$ & 390 & $6.3 \mathrm{E}-06$ & $3.1 E-09$ \\
\hline LLNL & 560 & $9.0 \mathrm{E}-06$ & 4.5E-09 & 210 & $3.4 \mathrm{E}-06$ & $1.7 \mathrm{E}-09$ \\
\hline Fermi & 430 & $6.9 \mathrm{E}-06$ & $3.5 \mathrm{E}-09$ & 170 & $2.7 \mathrm{E}-06$ & 1.4E-09 \\
\hline SLAC & 390 & $6.3 \mathrm{E}-06$ & $3.1 \mathrm{E}-09$ & 150 & $2.4 \mathrm{E}-06$ & $1.2 \mathrm{E}-09$ \\
\hline SNL-NM & 230 & 3.7E-06 & $1.9 \mathrm{E}-09$ & 100 & $1.6 \mathrm{E}-06$ & $8.1 E-10$ \\
\hline ANL-W & 220 & $3.5 \mathrm{E}-06$ & $1.8 \mathrm{E}-09$ & 90 & $1.4 E-06$ & 7.2E-10 \\
\hline LBL & 180 & 2.9E-06 & $1.4 \mathrm{E}-09$ & 80 & $1.3 E-06$ & $6.4 \mathrm{E}-10$ \\
\hline ITRI & 100 & $1.6 \mathrm{E}-06$ & 8.1E-10 & 40 & $6.4 \mathrm{E}-07$ & $3.2 \mathrm{E}-10$ \\
\hline SNL-CA & 60 & 9.7E-07 & $4.8 \mathrm{E}-10$ & 20 & $3.2 E-07$ & $1.6 \mathrm{E}-10$ \\
\hline ORISE & 50 & $8.1 E-07$ & $4.0 \mathrm{E}-10$ & 20 & $3.2 \mathrm{E}-07$ & $1.6 \mathrm{E}-10$ \\
\hline Pinellas & 20 & 3.2E-07 & $1.6 \mathrm{E}-10$ & 10 & $1.6 \mathrm{E}-07$ & $8.1 E-11$ \\
\hline Ames & 20 & $3.2 \mathrm{E}-07$ & $1.6 \mathrm{E}-10$ & 10 & $1.6 \mathrm{E}-07$ & $8.1 E-11$ \\
\hline PPPL & 10 & $1.6 \mathrm{E}-07$ & $8.1 E-11$ & 10 & $1.6 \mathrm{E}-07$ & $8.1 \mathrm{E}-11$ \\
\hline $\mathrm{KCP}$ & 10 & $1.6 \mathrm{E}-07$ & 8.1E-11 & 10 & $1.6 \mathrm{E}-07$ & $8.1 E-11$ \\
\hline \multicolumn{7}{|l|}{$\begin{array}{l}\text { Centralized } 1 \\
\text { (Case 7) }\end{array}$} \\
\hline Hanford & 242,730 & $3.9 \mathrm{E}-03$ & 2.0E-06 & $91,+40$ & $1.5 \mathrm{E}-03$ & $7.4 \mathrm{E}-07$ \\
\hline SRS & 68,340 & $1.1 \mathrm{E}-03$ & $5.5 \mathrm{E}-07$ & 25.400 & $4.1 E-04$ & $2.0 E-07$ \\
\hline ORR & 65,420 & $1.1 \mathrm{E}-03$ & $5.3 \mathrm{E}-07$ & 24,860 & $4.0 \mathrm{E}-0.4$ & $2.0 E-07$ \\
\hline PORTS & 33.440 & $5.4 E-04$ & 2.7E-07 & 12,740 & 2.IE-04 & $1.0 E-07$ \\
\hline Pantex & 14,500 & $2.3 E-04$ & $1.2 \mathrm{E}-07$ & 5.440 & $8.8 E-05$ & $+.4 E-08$ \\
\hline LANL & 11,420 & $1.8 E-04$ & $9.2 \mathrm{E}-08$ & 4.270 & $6.9 E-05$ & $3.4 E-08$ \\
\hline
\end{tabular}


TABLE 5.8 (Cont.)

\begin{tabular}{|c|c|c|c|c|c|c|}
\hline \multirow[b]{2}{*}{$\begin{array}{c}\text { Alternative and } \\
\text { Site }^{b}\end{array}$} & \multicolumn{3}{|c|}{ All Truck } & \multicolumn{3}{|c|}{ All Rail } \\
\hline & $\begin{array}{c}\text { Total } \\
\text { Shipments }\end{array}$ & $\begin{array}{l}\text { Dose } \\
\text { (rem) }\end{array}$ & $\begin{array}{c}\text { Risk } \\
\text { (Fatal Cancer) }^{\mathbf{2}}\end{array}$ & $\begin{array}{c}\text { Total } \\
\text { Shipments }\end{array}$ & $\begin{array}{l}\text { Dose } \\
\text { (rem) }\end{array}$ & $\begin{array}{c}\text { Risk } \\
\text { (Fatal Cancer) }\end{array}$ \\
\hline INEL & 8.520 & $1.4 \mathrm{E}-04$ & $6.9 \mathrm{E}-08$ & 3.220 & $5.2 E-05$ & $2.6 \mathrm{E}-08$ \\
\hline RMI & 7,680 & $1.2 \mathrm{E}-04$ & $6.2 \mathrm{E}-08$ & 2.870 & $4.6 \mathrm{E}-05$ & 2.3E-08 \\
\hline KAPL-S & 6.780 & 1.1E-04 & $5.5 \mathrm{E}-08$ & 2.570 & $4.1 E-05$ & 2.IE-08 \\
\hline PGDP & 6.270 & $1.0 \mathrm{E}-04$ & $5.0 \mathrm{E}-08$ & 2.370 & $3.8 \mathrm{E}-05$ & $1.9 \mathrm{E}-08$ \\
\hline Mound & 5.120 & $8.2 \mathrm{E}-05$ & 4.1E-08 & 1,900 & $3.1 E-05$ & $1.5 \mathrm{E}-08$ \\
\hline NRF & $\$ .610$ & $7.4 \mathrm{E}-05$ & $3.7 \mathrm{E}-08$ & 1.750 & $2.8 \mathrm{E}-05$ & $1.4 E-08$ \\
\hline Bettis & 3.730 & $6.0 \mathrm{E}-05$ & 3.0E-08 & 1,410 & $2.3 \mathrm{E}-05$ & 1. $1 E-08$ \\
\hline RFETS & 3,570 & $5.7 \mathrm{E}-05$ & $2.9 \mathrm{E}-08$ & 1.330 & $2.1 E-05$ & 1. IE-08 \\
\hline ANL-E & 1,050 & $1.7 \mathrm{E}-05$ & 8.5E-09 & 390 & $6.3 E-06$ & $3.1 E-09$ \\
\hline LLNL & 560 & $9.0 \mathrm{E}-06$ & $4.5 \mathrm{E}-09$ & 210 & $3.4 \mathrm{E}-06$ & $1.7 \mathrm{E}-09$ \\
\hline Fermi & 430 & $6.9 E-06$ & $3.5 \mathrm{E}-09$ & 170 & $2.7 \mathrm{E}-06$ & $1.4 E-09$ \\
\hline SLAC & 390 & $6.3 \mathrm{E}-06$ & $3.1 E-09$ & 150 & $2.4 \mathrm{E}-06$ & 1.2E-09 \\
\hline SNL-NM & 230 & $3.7 \mathrm{E}-06$ & $1.9 \mathrm{E}-09$ & 100 & $1.6 \mathrm{E}-06$ & 8.1E-10 \\
\hline ANL-W & 220 & $3.5 \mathrm{E}-06$ & $1.8 \mathrm{E}-09$ & 90 & $1.4 \mathrm{E}-06$ & 7.2E-10 \\
\hline LBL & 180 & $2.9 E-06$ & $1.4 \mathrm{E}-09$ & 80 & $1.3 E-06$ & $6 .+E-10$ \\
\hline ITRI & 100 & $1.6 \mathrm{E}-06$ & 8.1E-10 & 40 & $6.4 \mathrm{E}-07$ & 3.2E-10 \\
\hline SNL-CA & 60 & 9.7E-07 & $4.8 \mathrm{E}-10$ & 20 & $3.2 \mathrm{E}-07$ & $1.6 \mathrm{E}-10$ \\
\hline ORISE & 50 & $8.1 \mathrm{E}-07$ & $4.0 \mathrm{E}-10$ & 20 & 3.2E-07 & $1.6 \mathrm{E}-10$ \\
\hline Pinellas & 20 & $3.2 \mathrm{E}-07$ & $1.6 \mathrm{E}-10$ & 10 & $1.6 \mathrm{E}-07$ & 8.1E-11 \\
\hline Ames & 20 & $3.2 \mathrm{E}-07$ & $1.6 \mathrm{E}-10$ & 10 & $1.6 \mathrm{E}-07$ & $8.1 E-11$ \\
\hline PPPL & 10 & $1.6 E-07$ & $8.1 E-11$ & 10 & $1.6 \mathrm{E}-07$ & $8.1 E-11$ \\
\hline $\mathrm{KCP}$ & 10 & $1.6 \mathrm{E}-07$ & $8.1 \mathrm{E}-11$ & 10 & $1.6 \mathrm{E}-07$ & $8.1 E-11$ \\
\hline \multicolumn{7}{|l|}{$\begin{array}{l}\text { Centralized } 2 \\
\text { (Case 8) }\end{array}$} \\
\hline NTS & 257,270 & $4.1 E-03$ & 2.1E-06 & 96,880 & $1.6 \mathrm{E}-03$ & 7.8E-07 \\
\hline SRS & 68,340 & $1.1 \mathrm{E}-03$ & $5.5 \mathrm{E}-07$ & 25,400 & $4.1 \mathrm{E}-0.4$ & $2.0 E-07$ \\
\hline ORR & 65,420 & $1.1 \mathrm{E}-03$ & $5.3 \mathrm{E}-07$ & 24,860 & $4.0 \mathrm{E}-0.4$ & $2.0 \mathrm{E}-07$ \\
\hline PORTS & 33,440 & $5.4 \mathrm{E}-04$ & $2.7 \mathrm{E}-07$ & 12,740 & $2.1 \mathrm{E}-0.4$ & $1.0 \mathrm{E}-07$ \\
\hline Hanford & 14,540 & $2.3 \mathrm{E}-04$ & $1.2 \mathrm{E}-07$ & 5.440 & $8.8 \mathrm{E}-05$ & $4.4 E-08$ \\
\hline Pantex & 14,500 & $2.3 \mathrm{E}-04$ & $1.2 \mathrm{E}-07$ & 5,440 & $8.8 \mathrm{E}-05$ & $4.4 E-08$ \\
\hline LANL & 11,420 & $1.8 \mathrm{E}-04$ & $9.2 E-08$ & 4,270 & $6.9 \mathrm{E}-05$ & $3.4 E-08$ \\
\hline INEL & 8,520 & $1 .+E-04$ & $6.9 \mathrm{E}-08$ & 3.220 & $5.2 \mathrm{E}-05$ & 2.6E-08 \\
\hline RMII & 7,680 & 1.2E-04 & $6.2 \mathrm{E}-08$ & 2.870 & $4.6 \mathrm{E}-05$ & $2.3 \mathrm{E}-08$ \\
\hline KAPL-S & 6,780 & $1.1 E-04$ & $5.5 \mathrm{E}-08$ & 2,570 & $4.1 E-05$ & 2.1E-08 \\
\hline PGDP & 6,270 & $1.0 \mathrm{E}-04$ & $5.0 \mathrm{E}-08$ & 2.370 & $3.8 \mathrm{E}-05$ & $1.9 \mathrm{E}-08$ \\
\hline Mound & 5,120 & $8.2 \mathrm{E}-05$ & 4. $1 E-08$ & 1.900 & $3.1 \mathrm{E}-05$ & $1.5 \mathrm{E}-08$ \\
\hline NRF & 4,610 & 7.4E-05 & $3.7 \mathrm{E}-08$ & 1.750 & $2.8 E-05$ & $1.4 \mathrm{E}-08$ \\
\hline Bettis & 3.730 & $6.0 \mathrm{E}-05$ & $3.0 \mathrm{E}-08$ & $1 .+10$ & $2.3 E-05$ & $1.1 \mathrm{E}-08$ \\
\hline RFETS & 3,570 & $5.7 \mathrm{E}-05$ & $2.9 E-08$ & 1.330 & 2.IE-05 & $1.1 E-08$ \\
\hline ANL-E & 1,050 & $1.7 E-05$ & 8.5E-09 & 390 & $6.3 \mathrm{E}-06$ & $3.1 E-09$ \\
\hline LL.YL & 560 & $9.0 \mathrm{E}-06$ & $+.5 \mathrm{E}-09$ & 210 & $3 .+\mathrm{E}-06$ & $1.7 \mathrm{E}-09$ \\
\hline Fermi & 430 & $6.9 \mathrm{E}-06$ & $3.5 \mathrm{E}-09$ & 170 & $2.7 \mathrm{E}-06$ & $1.4 E-09$ \\
\hline
\end{tabular}


TABLE 5.8 (Cont.)

\begin{tabular}{|c|c|c|c|c|c|c|}
\hline \multirow[b]{2}{*}{$\begin{array}{l}\text { Alternative and } \\
\text { Site }\end{array}$} & \multicolumn{3}{|c|}{ All Truck } & \multicolumn{3}{|c|}{ All Rail } \\
\hline & $\begin{array}{c}\text { Total } \\
\text { Shipments }\end{array}$ & $\begin{array}{l}\text { Dose } \\
\text { (rem) }\end{array}$ & $\begin{array}{c}\text { Risk } \\
\text { (Fatal Cancer) }^{c}\end{array}$ & $\begin{array}{c}\text { Total } \\
\text { Shipments }\end{array}$ & $\begin{array}{l}\text { Dose } \\
\text { (rem) }\end{array}$ & $\begin{array}{c}\text { Risk } \\
\text { (Fatal Cancer) }\end{array}$ \\
\hline SLAC & 390 & $6.3 \mathrm{E}-06$ & $3.1 E-09$ & 150 & 2.4E-06 & $1.2 E-09$ \\
\hline SNL-NM & 230 & $3.7 \mathrm{E}-06$ & $1.9 \mathrm{E}-09$ & 100 & $1.6 \mathrm{E}-06$ & $8.1 E-10$ \\
\hline ANL-W & 220 & $3.5 \mathrm{E}-06$ & $1.8 \mathrm{E}-09$ & 90 & $1.4 \mathrm{E}-06$ & 7.2E-10 \\
\hline LBL & 180 & $2.9 \mathrm{E}-06$ & $1.4 \mathrm{E}-09$ & 80 & $1.3 \mathrm{E}-06$ & $6.4 \mathrm{E}-10$ \\
\hline ITRI & 100 & $1.6 \mathrm{E}-06$ & 8.1E-10 & 40 & $6.4 E-07$ & $3.2 E-10$ \\
\hline SiNL-CA & 60 & $9.7 \mathrm{E}-07$ & $4.8 \mathrm{E}-10$ & 20 & $3.2 \mathrm{E}-07$ & $1.6 \mathrm{E}-10$ \\
\hline ORISE & 50 & $8.1 \mathrm{E}-07$ & $4.0 \mathrm{E}-10$ & 20 & 3.2E-07 & $1.6 \mathrm{E}-10$ \\
\hline Pinellas & 20 & $3.2 \mathrm{E}-07$ & $1.6 \mathrm{E}-10$ & 10 & $1.6 \mathrm{E}-07$ & 8.1E-11 \\
\hline Ames & 20 & $3.2 \mathrm{E}-07$ & $1.6 \mathrm{E}-10$ & 10 & $1.6 \mathrm{E}-07$ & $8.1 E-11$ \\
\hline PPPL & 10 & $1.6 \mathrm{E}-07$ & $8.1 E-11$ & 10 & $1.6 \mathrm{E}-07$ & 8.1E-11 \\
\hline $\mathrm{KCP}$ & 10 & $1.6 \mathrm{E}-07$ & $8.1 E-11$ & 10 & $1.6 \mathrm{E}-07$ & $8.1 E-11$ \\
\hline \multicolumn{7}{|l|}{$\begin{array}{l}\text { Centralized } 3 \\
\text { (Case 14) }\end{array}$} \\
\hline Hanford & 225,660 & $3.6 \mathrm{E}-03$ & $1.8 \mathrm{E}-06$ & 87,240 & 1.4E-03 & $7.0 E-07$ \\
\hline SRS & 67,520 & $1.1 \mathrm{E}-03$ & $5.4 \mathrm{E}-07$ & 25,230 & $4.1 E-04$ & $2.0 \mathrm{E}-07$ \\
\hline ORR & 61,250 & $9.9 \mathrm{E}-04$ & $4.9 \mathrm{E}-07$ & 24,470 & $3.9 \mathrm{E}-04$ & 2.0E-07 \\
\hline PORTS & 47,440 & $7.6 \mathrm{E}-04$ & $3.8 \mathrm{E}-07$ & 18,350 & $3.0 \mathrm{E}-04$ & $1.5 \mathrm{E}-07$ \\
\hline LANL & 36,640 & $5.9 \mathrm{E}-04$ & $2.9 \mathrm{E}-07$ & 14,400 & $2.3 \mathrm{E}-04$ & 1.2E-07 \\
\hline Pantex & 13,830 & $2.2 \mathrm{E}-04$ & $1.1 E-07$ & 5,380 & $8.7 E-05$ & 4.3E-08 \\
\hline INEL & 11,850 & $1.9 \mathrm{E}-04$ & $9.5 \mathrm{E}-08$ & 4,720 & $7.6 \mathrm{E}-05$ & $3.8 \mathrm{E}-08$ \\
\hline RMI & 7,520 & 1.2E-04 & 6.1E-08 & 2,860 & $4.6 \mathrm{E}-05$ & 2.3E-08 \\
\hline KAPL-S & 6,670 & 1.1E-04 & $5.4 \mathrm{E}-08$ & 2,560 & $4.1 E-05$ & 2.1E-08 \\
\hline PGDP & 6,350 & $1.0 \mathrm{E}-04$ & $5.1 E-08$ & 2,360 & $3.8 \mathrm{E}-05$ & $1.9 \mathrm{E}-08$ \\
\hline Mound & 5,080 & $8.2 \mathrm{E}-05$ & 4.1E-08 & 1,910 & $3.1 E-05$ & $1.5 E-08$ \\
\hline Bettis & 3,660 & $5.9 E-05$ & $2.9 \mathrm{E}-08$ & 1,400 & $2.3 E-05$ & $1.1 \mathrm{E}-08$ \\
\hline RFETS & 3,350 & $5.4 \mathrm{E}-05$ & $2.7 \mathrm{E}-08$ & 1,230 & $2.0 \mathrm{E}-05$ & $9.9 E-09$ \\
\hline ANL-E & 1,050 & $1.7 \mathrm{E}-05$ & $8.5 E-09$ & 390 & $6.3 \mathrm{E}-06$ & $3.1 E-09$ \\
\hline LLNL & 560 & $9.0 \mathrm{E}-06$ & $4.5 \mathrm{E}-09$ & 210 & $3.4 \mathrm{E}-06$ & $1.7 \mathrm{E}-09$ \\
\hline Fermi & 420 & $6.8 \mathrm{E}-06$ & $3.4 \mathrm{E}-09$ & 180 & $2.9 \mathrm{E}-06$ & $1.4 \mathrm{E}-09$ \\
\hline SLAC & 390 & $6.3 \mathrm{E}-06$ & $3.1 E-09$ & 150 & $2.4 \mathrm{E}-06$ & $1.2 \mathrm{E}-09$ \\
\hline SNL-NM & 240 & $3.9 \mathrm{E}-06$ & $1.9 \mathrm{E}-09$ & 110 & $1.8 \mathrm{E}-06$ & $8.9 E-10$ \\
\hline LBL & 170 & $2.7 \mathrm{E}-06$ & $1.4 \mathrm{E}-09$ & 80 & $1.3 \mathrm{E}-06$ & $6.4 E-10$ \\
\hline ITRI & 110 & $1.8 \mathrm{E}-06$ & $8.9 E-10$ & 40 & $6 .+E-07$ & $3.2 \mathrm{E}-10$ \\
\hline ANL-W & 100 & $1.6 \mathrm{E}-06$ & 8.1E-10 & 40 & $6.4 \mathrm{E}-07$ & 3.2E-10 \\
\hline ORISE & 50 & $8.1 \mathrm{E}-07$ & $4.0 \mathrm{E}-10$ & 30 & $4.8 \mathrm{E}-07$ & $2.4 \mathrm{E}-10$ \\
\hline SNL-CA & 50 & $8.1 E-07$ & $4.0 \mathrm{E}-10$ & 20 & $3.2 \mathrm{E}-07$ & $1.6 \mathrm{E}-10$ \\
\hline Ames & 30 & $4.8 \mathrm{E}-07$ & $2.4 \mathrm{E}-10$ & 20 & $3.2 \mathrm{E}-07$ & $1.6 \mathrm{E}-10$ \\
\hline PPPL & 20 & 3.2E-07 & $1.6 \mathrm{E}-10$ & 20 & 3.2E-07 & $1.6 E-10$ \\
\hline Pinellas & 20 & $3.2 \mathrm{E}-07$ & $1.6 \mathrm{E}-10$ & 10 & $1.6 \mathrm{E}-07$ & 8.1E-11 \\
\hline $\mathrm{KCP}$ & 10 & $1.6 \mathrm{E}-07$ & $8.1 E-11$ & 10 & $1.6 \mathrm{E}-07$ & 8.1E-11 \\
\hline
\end{tabular}


TABLE 5.8 (Cont.)

\begin{tabular}{|c|c|c|c|c|c|c|}
\hline \multirow[b]{2}{*}{$\begin{array}{l}\text { Alternative and } \\
\text { Site }^{\mathrm{b}}\end{array}$} & \multicolumn{3}{|c|}{ All Truck } & \multicolumn{3}{|c|}{ All Rail } \\
\hline & $\begin{array}{c}\text { Total } \\
\text { Shipments }\end{array}$ & $\begin{array}{l}\text { Dose } \\
\text { (rem) }\end{array}$ & $\begin{array}{c}\text { Risk } \\
\text { (Fatal Cancer) }^{\text {E }}\end{array}$ & $\begin{array}{c}\text { Total } \\
\text { Shipments }\end{array}$ & $\begin{array}{l}\text { Dose } \\
\text { (rem) }\end{array}$ & $\begin{array}{c}\text { Risk } \\
\text { (Fatal Cancer) }\end{array}$ \\
\hline \multicolumn{7}{|l|}{$\begin{array}{l}\text { Centralized } 4 \\
\text { (Case 14a) }\end{array}$} \\
\hline NTS & 239,350 & $3.9 \mathrm{E}-03$ & $1.9 \mathrm{E}-06$ & 92.470 & $1.5 \mathrm{E}-03$ & $7.4 E-07$ \\
\hline SRS & 67.520 & $1.1 E-03$ & $5.4 E-07$ & 25.230 & 4.1E-04 & 2.0E-07 \\
\hline ORR & 61,250 & $9.9 E-04$ & $4.9 \mathrm{E}-07$ & 24,470 & $3.9 \mathrm{E}-04$ & $2.0 \mathrm{E}-07$ \\
\hline PORTS & $47 .+40$ & $7.6 \mathrm{E}-04$ & $3.8 \mathrm{E}-07$ & 18.350 & $3.0 \mathrm{E}-04$ & $1.5 \mathrm{E}-07$ \\
\hline LANL & 36.640 & $5.9 \mathrm{E}-04$ & $2.9 \mathrm{E}-07$ & 14,400 & $2.3 \mathrm{E}-04$ & $1.2 \mathrm{E}-07$ \\
\hline Hanford & 14,350 & $2.3 \mathrm{E}-04$ & $1.2 \mathrm{E}-07$ & 5,520 & $8.9 \mathrm{E}-05$ & $4.4 \mathrm{E}-08$ \\
\hline Pantex & 13.830 & $2.2 \mathrm{E}-04$ & $1.1 \mathrm{E}-07$ & 5,380 & 8.7E-05 & $4.3 \mathrm{E}-08$ \\
\hline INEL & 11.850 & $1.9 \mathrm{E}-04$ & $9.5 \mathrm{E}-08$ & 4,720 & $7.6 \mathrm{E}-05$ & $3.8 \mathrm{E}-08$ \\
\hline RMI & 7.520 & $1.2 \mathrm{E}-04$ & $6.1 \mathrm{E}-08$ & 2,860 & $4.6 \mathrm{E}-05$ & 2.3E-08 \\
\hline KAPL-S & 6.670 & 1.1E-04 & $5.4 \mathrm{E}-08$ & 2.560 & $4.1 E-05$ & $2.1 \mathrm{E}-08$ \\
\hline PGDP & 6,350 & $1.0 \mathrm{E}-04$ & $5.1 \mathrm{E}-08$ & 2,360 & $3.8 \mathrm{E}-05$ & $1.9 E-08$ \\
\hline Mound & 5,080 & 8.2E-05 & 4.1E-08 & 1,910 & $3.1 \mathrm{E}-05$ & $1.5 \mathrm{E}-08$ \\
\hline Bettis & 3,660 & $5.9 \mathrm{E}-05$ & $2.9 \mathrm{E}-08$ & 1,400 & $2.3 \mathrm{E}-05$ & $1.1 E-08$ \\
\hline RFETS & 3,350 & $5.4 \mathrm{E}-05$ & $2.7 \mathrm{E}-08$ & 1,230 & $2.0 \mathrm{E}-05$ & $9.9 E-09$ \\
\hline ANL-E & 1.050 & $1.7 \mathrm{E}-05$ & $8.5 \mathrm{E}-09$ & 390 & $6.3 \mathrm{E}-0.6$ & $3.1 E-09$ \\
\hline LLNL & 570 & $9.2 E-06$ & $4.6 \mathrm{E}-09$ & 220 & $3.5 \mathrm{E}-06$ & $1.8 \mathrm{E}-09$ \\
\hline Fermi & 420 & $6.8 E-06$ & $3.4 \mathrm{E}-09$ & 180 & $2.9 \mathrm{E}-06$ & $1.4 \mathrm{E}-09$ \\
\hline SLAC & 400 & $6.4 \mathrm{E}-06$ & 3.2E-09 & 160 & $2.6 \mathrm{E}-06$ & $1.3 E-09$ \\
\hline SNL-NM & 240 & $3.9 \mathrm{E}-06$ & $1.9 \mathrm{E}-09$ & 110 & $1.8 \mathrm{E}-06$ & $8.9 E-10$ \\
\hline LBL & 180 & $2.9 \mathrm{E}-06$ & $1.4 \mathrm{E}-09$ & 80 & $1.3 \mathrm{E}-06$ & $6.4 E-10$ \\
\hline ITRI & 110 & $1.8 \mathrm{E}-06$ & $8.9 \mathrm{E}-10$ & 40 & $6.4 \mathrm{E}-07$ & $3.2 \mathrm{E}-10$ \\
\hline ANL-W & 100 & $1.6 \mathrm{E}-06$ & $8.1 \mathrm{E}-10$ & 40 & $6.4 \mathrm{E}-07$ & 3.2E-10 \\
\hline SNL-CA & 60 & 9.7E-07 & $4.8 \mathrm{E}-10$ & 30 & $4.8 \mathrm{E}-07$ & $2.4 \mathrm{E}-10$ \\
\hline ORISE & 50 & $8.1 \mathrm{E}-07$ & $4.0 \mathrm{E}-10$ & 30 & $4.8 \mathrm{E}-07$ & $2.4 \mathrm{E}-10$ \\
\hline Ames & 30 & $4.8 \mathrm{E}-07$ & $2.4 \mathrm{E}-10$ & 20 & $3.2 \mathrm{E}-07$ & $1.6 \mathrm{E}-10$ \\
\hline PPPL & 20 & $3.2 \mathrm{E}-07$ & $1.6 \mathrm{E}-10$ & 20 & 3.2E-07 & $1.6 \mathrm{E}-10$ \\
\hline Pinellas & 20 & $3.2 \mathrm{E}-07$ & $1.6 E-10$ & 10 & $1.6 \mathrm{E}-07$ & $8.1 \mathrm{E}-11$ \\
\hline $\mathrm{KCP}$ & 10 & $1.6 \mathrm{E}-07$ & $8.1 E-11$ & 10 & $1.6 E-07$ & $8.1 E-11$ \\
\hline \multicolumn{7}{|l|}{$\begin{array}{l}\text { Centralized } 5 \\
\text { (Case 21) }\end{array}$} \\
\hline Hanford & 241,540 & $3.9 \mathrm{E}-03$ & $1.9 \mathrm{E}-06$ & 90.980 & $1.5 \mathrm{E}-03$ & $7.3 E-07$ \\
\hline SRS & 68,540 & $1.1 \mathrm{E}-03$ & $5.5 \mathrm{E}-07$ & 25,320 & $4.1 \mathrm{E}-04$ & $2.0 \mathrm{E}-07$ \\
\hline ORR & 65,840 & $1.1 \mathrm{E}-03$ & $5.3 E-07$ & 24,890 & $4.0 \mathrm{E}-04$ & $2.0 \mathrm{E}-07$ \\
\hline PORTS & 32,500 & $5.2 \mathrm{E}-04$ & $2.6 \mathrm{E}-07$ & 12,500 & $2.0 \mathrm{E}-04$ & $1.0 \mathrm{E}-07$ \\
\hline Pantex & 14,180 & $2.3 \mathrm{E}-04$ & $1.1 \mathrm{E}-07$ & 5.370 & $8.6 \mathrm{E}-05$ & $4.3 \mathrm{E}-08$ \\
\hline LANL & 11,220 & $1.8 \mathrm{E}-04$ & $9.0 \mathrm{E}-08$ & 4.230 & $6.8 \mathrm{E}-05$ & $3.4 E-08$ \\
\hline INEL & 8.570 & $1.4 \mathrm{E}-04$ & $6.9 \mathrm{E}-08$ & 3.200 & $5.2 E-05$ & $2.6 \mathrm{E}-08$ \\
\hline R.MI & 7,610 & 1.2E-04 & $6.1 \mathrm{E}-08$ & 2,850 & $4.6 \mathrm{E}-0.5$ & $2.3 E-08$ \\
\hline KAPL-S & 6.670 & $1.1 E-04$ & $5.4 E-08$ & 2.560 & $4.1 \mathrm{E}-05$ & 2.1E-08 \\
\hline PGDP & 6.270 & $1.0 \mathrm{E}-04$ & $5.0 \mathrm{E}-08$ & 2.350 & $3.8 \mathrm{E}-05$ & $1.9 E-08$ \\
\hline
\end{tabular}


TABLE 5.8 (Cont.)

\begin{tabular}{|c|c|c|c|c|c|c|}
\hline \multirow[b]{2}{*}{$\begin{array}{l}\text { Altemative and } \\
\text { Site }\end{array}$} & \multicolumn{3}{|c|}{ All Truck } & \multicolumn{3}{|c|}{ All Rail } \\
\hline & $\begin{array}{c}\text { Total } \\
\text { Shipments }\end{array}$ & $\begin{array}{l}\text { Dose } \\
\text { (rem) }\end{array}$ & $\begin{array}{c}\text { Risk } \\
\text { (Fatal Cancer) } \\
\end{array}$ & $\begin{array}{c}\text { Total } \\
\text { Shipments }\end{array}$ & $\begin{array}{l}\text { Dose } \\
\text { (rem) }\end{array}$ & $\begin{array}{c}\text { Risk } \\
\text { (Fatal Cancer) }^{\circ}\end{array}$ \\
\hline Mound & 5,160 & 8.3E-05 & $4.2 \mathrm{E}-08$ & 1.900 & $3.1 E-05$ & $1.5 \mathrm{E}-08$ \\
\hline NRF & 4,500 & $7.2 \mathrm{E}-05$ & $3.6 \mathrm{E}-08$ & 1.780 & $2.9 E-05$ & $1.4 E-08$ \\
\hline Bettis & 3.660 & $5.9 \mathrm{E}-05$ & $2.9 \mathrm{E}-08$ & 1.400 & 2.3E-05 & $1.1 E-08$ \\
\hline RFETS & 3,530 & $5.7 \mathrm{E}-05$ & $2.8 \mathrm{E}-08$ & 1.320 & 2.1E-05 & $1.1 E-08$ \\
\hline ANL-E & 1.050 & $1.7 \mathrm{E}-05$ & $8.5 \mathrm{E}-09$ & 390 & $6.3 \mathrm{E}-06$ & $3.1 \mathrm{E}-09$ \\
\hline LLNL & 560 & $9.0 \mathrm{E}-06$ & 4.5E-09 & 210 & $3.4 E-06$ & $1.7 \mathrm{E}-09$ \\
\hline Fermi & 420 & $6.8 \mathrm{E}-06$ & $3.4 E-09$ & 170 & 2.7E-06 & $1.4 E-09$ \\
\hline SLAC & 390 & $6.3 \mathrm{E}-06$ & $3.1 \mathrm{E}-09$ & 150 & $2.4 E-06$ & $1.2 \mathrm{E}-09$ \\
\hline SNL-NM & 230 & $3.7 \mathrm{E}-06$ & $1.9 E-09$ & 100 & $1.6 \mathrm{E}-06$ & 8.1E-10 \\
\hline ANL -W & 210 & $3.4 \mathrm{E}-06$ & $1.7 \mathrm{E}-09$ & 90 & $1.4 \mathrm{E}-06$ & $7.2 \mathrm{E}-10$ \\
\hline LBL & 170 & $2.7 \mathrm{E}-06$ & $1.4 \mathrm{E}-09$ & 80 & $1.3 \mathrm{E}-06$ & $6.4 \mathrm{E}-10$ \\
\hline ITRI & 100 & $1.6 \mathrm{E}-06$ & 8. IE-10 & 40 & $6.4 E-07$ & $3.2 \mathrm{E}-10$ \\
\hline SNL-CA & 50 & 8.1E-07 & $4.0 \mathrm{E}-10$ & 20 & 3.2E-07 & $1.6 E-10$ \\
\hline ORISE & 50 & $8.1 E-07$ & $4.0 \mathrm{E}-10$ & 20 & 3.2E-07 & $1.6 \mathrm{E}-10$ \\
\hline Pinellas & 20 & $3.2 \mathrm{E}-07$ & $1.6 \mathrm{E}-10$ & 10 & $1.6 E-07$ & 8.1E-11 \\
\hline Ames & 20 & $3.2 \mathrm{E}-07$ & $1.6 \mathrm{E}-10$ & 10 & $1.6 \mathrm{E}-07$ & $8.1 E-11$ \\
\hline PPPL & 10 & $1.6 \mathrm{E}-07$ & $8.1 E-11$ & 10 & $1.6 \mathrm{E}-07$ & $8.1 E-11$ \\
\hline $\mathrm{KCP}$ & 10 & $1.6 \mathrm{E}-07$ & 8.1E-11 & 10 & $1.6 \mathrm{E}-07$ & 8.1E-11 \\
\hline
\end{tabular}

a The external dose rate is assumed to be $1 \mathrm{mrem} / \mathrm{h}$ at $1 \mathrm{~m}(3.3 \mathrm{ft})$ for all shipments. The resident is assumed to be present for all shipments that enter or exit the site. Shipments are assumed to pass at a distance of $30 \mathrm{~m}(98 \mathrm{ft})$ and an average speed of $24 \mathrm{~km} / \mathrm{h}(15 \mathrm{mph})$.

b Note: Ames = Ames National Laboratory; ANL-E = Argonne National Laboratory-East; ANL-W = Argonne National Laboratory-West; Bettis = Bettis Atomic Power Laboratory; BNL = Brookhaven National Laboratory; Fermi = Fermi National Accelerator Laboratory; Hanford $=$ Hanford Sire; INEL = Idaho National Engineering Laboratory: ITRI = Inhalation Toxicology Research Institute; $\mathrm{KCP}=$ Kansas City Plant; KAPL-S = Knolls Atomic Power Laboratory (Schenectady); LBL = Lawrence Berkeley National Laboralory; LLNL = Lawrence Livermore National Laboratory; LANL $=$ Los Alamos National Laboratory; Mound $=$ Mound Plant; NRF $=$ Naval Reactor Facility; NTS = Nevada Test Site; ORISE = Oak Ridge Institute for Science and Education; ORR = Oak Ridge Reservation: PGDP = Paducah Gaseous Diffusion Plant; Pantex = Pantex Plant; Pinellas = Pinellas Plant; PORTS = Portsmouth Gaseous Diffusion Plant; PPPL = Princeton Plasma Physics Laboratory; RMI = Reactive Metals, Inc., RFETS = Rocky Flats Environmental Technology Site; SNL-NM = Sandia National Laboratories (New Mexico); SNL-CA = Sandia National Laboratories (Califomia); SRS = Savannah River Site; SLAC = Stanford Linear Accelerator Center; and WVDP = West Valley Demonstration Project.

c The risk of fatal cancer is calculated by using the ICRP Publication 60 (ICRP 1991) health risk conversion factor of $5 E-04$ fatal cancers per person-rem for members of the public. 


\section{TABLE 5.9 Estimated Consequences for the Most Severe Accidents Involving Shipments of WM LLW}

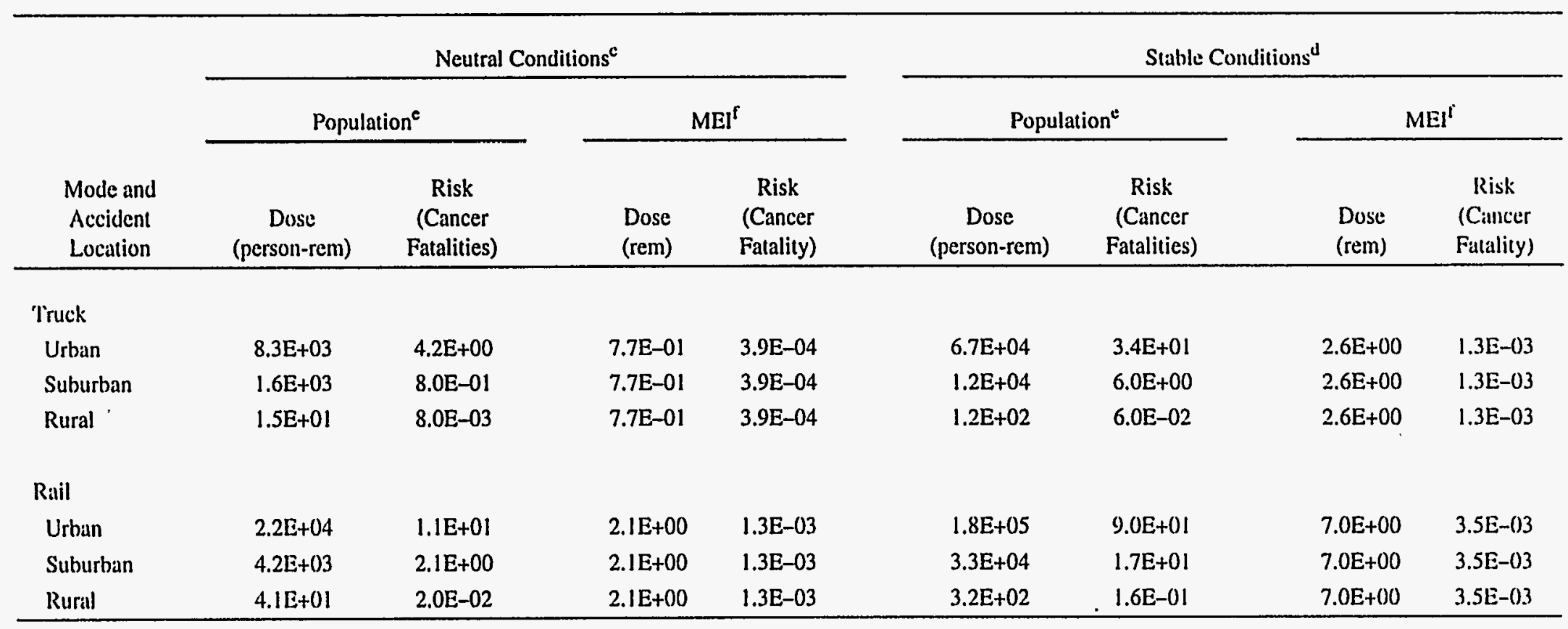

:The most severe accidents correspond to the highest NUREG-0170 accident severity category (Category VIII) (NRC 1977a). Results are reported lor WM L.LW from ANL-W, which was found to result in the highest potential accident doses. The assumptions were that $100 \%$ of the radioactive material would be released Irom its packaging in an accident, that $10 \%$ of the release would be entrained in an aerosol, and that $5 \%$ of the aerosolized release would be respirable.

1) Buoyant plume rise resulting from fire for a severe accident was included in the exposure model.

c Neutral weather conditions result in moderate dispersion and dilution of the released plume. Neutral conditions were taken to be Pascuill Stability Class D with at wind speed of $4 \mathrm{~m} / \mathrm{s}(9 \mathrm{mph})$. Neutral conditions occur approximalely $50 \%$ of the time in the United States.

¿ Stable weather conditions result in minimal dispersion and dilution of the released plume and are thus unfavorable. Stable conditions were taken to be Pasyuill Stability Class F with a wind speed of $1 \mathrm{~m} / \mathrm{s}(2.2 \mathrm{mph})$. Stable conditions occur approximately one-third of the time in the United States.

- Populations extend at a uniform population density to a radius of $80 \mathrm{~km}(50 \mathrm{mi})$ from the accident site. Population exposure patlıways include actice inhalation; acute cloudshine; groundshine; resuspended inhalation; resuspended cloudshine; and ingestion of food, including initially contaminated food (rural only). No decontamination or mitigative actions are taken.

$f$ The MEI is assumed to be at the location of maximum exposure. The locations of maximum exposure would be $160 \mathrm{~m}(525 \mathrm{ft})$ and $400 \mathrm{~m}(1,312 \mathrm{ft})$ from the accident site under neutral and stable atmospheric conditions, respectively. Individual exposure pathways include acute inhalation, acute cloudshine, and groundshine during passage of the plume. No ingested dose is considered. 


\section{REFERENCES}

DOE: See U.S. Department of Energy.

Doty, S.R., et al., 1976, A Climatological Analysis of Pasquill Stability Categories Based on 'STAR' Summaries, National Oceanic and Atmospheric Administration, National Climatic Center, Asheville, N.C., April.

Fischer, L.E., et al., 1987, Shipping Container Response to Severe Highway and Railway Accident Conditions, NUREG/CR-4829, UCID-20733, prepared by Lawrence Livermore National Laboratory, Livermore, Calif., for U.S. Nuclear Regulatory Commission, Washington, D.C.

Goyette, M.L., and Dolak, D. A., 1996, Low-Level Waste Inventory, Characteristics, Generation, and Facility Assessment for Treatment, Storage, and Disposal Alternatives Considered in the U.S. Department of Energy Waste Management Programmatic Environmental Impact Statement, ANL/EAD/TM-20, Argonne National Laboratory, Argonne, Ill., Dec.

ICRP: See International Commission on Radiological Protection.

International Commission on Radiological Protection, 1977, "Recommendations of the International Commission on Radiological Protection," ICRP publication 26, Annals of the ICRP 1(3), Pergamon Press, New York, N.Y.

International Commission on Radiological Protection, 1991, "1990 Recommendations of the International Commission on Radiological Protection," ICRP publication 60, Annals of the ICRP 21 (1-3), Pergamon Press, New York, N.Y.

Johnson, P.E., et al., 1993a, HIGHWAY 3.1, An Enhanced Transportation Routing Model: Program Description, Methodology, and Revised User's Manual, ORNL/TM-12124, Oak Ridge National Laboratory, Oak Ridge, Tenn., March.

Johnson, P.E., et al., 1993b, INTERLINE 5.0, An Expanded Railroad Routing Model: Program Description, Methodology, and Revised User's Manual, ORNL/TM-12090, Oak Ridge National Laboratory, Oak Ridge, Tenn., March.

Kotek, T.J., et al., 1996, WASTE_MGMT: A Computer Model for Calculation of Waste Loads, Profiles, and Emissions, ANL/EAD/TM-30, Argonne National Laboratory, Argonne, Ill., Dec. 
Monette, F.A., et al., 1996a, Supplemental Information Related to Risk Assessment for the Off-Site Transportation of Transuranic Waste for the U.S. Department of Energy Waste Management Programmatic Environmental Impact Statement, ANL/EAD/TM-27, Argonne National Laboratory, Argonne, Ill., Dec.

Monette, F.A., et al., 1996b, Risk Assessment for the Off-Site Transportation of High-Level Waste for the U.S. Department of Energy Waste Management Programmatic Environmental Impact Statement, ANL/EAD/TM-21, Argonne National Laboratory, Argonne, Ill., Dec.

Monette, F.A., et al., 1996c, Supplemental Information Related to Risk Assessment for the Off-Site Transportation of Low-Level Mixed Waste for the U.S. Department of Energy Waste Management Programmatic Environmental Impact Statement, ANL/EAD/TM-35, Argonne National Laboratory, Argonne, Ill., Dec.

Morris, 1993, personal communication from Morris (Science Applications International Corp.) to F.A. Monette (Argonne National Laboratory), Dec. 9.

Neuhauser, K.S., and F.L. Kanipe, 1993, RADTRAN 4: Volume II. Technical Manual, SAND89-2370, Sandia National Laboratories, Albuquerque, N.M., Aug.

NRC: See U.S. Nuclear Regulatory Commission.

Rao, R.K., et al., 1982, Non-Radiological Impacts of Transporting Radioactive Material, SAND811703, TTC-0236, Sandia National Laboratories, Albuquerque, N.M.

Saricks, C., and T. Kvitek, 1994, Longitudinal Review of State-Level Accident Statistics for Carriers of Interstate Freight, ANL/ESD/TM-68, Argonne National Laboratory, Argonne, Ill., Mar.

U.S. Department of Energy, 1986, Environmental Assessment: Yucca Mountain, Nevada, Nuclear Waste Policy Act, DOE/RW-0073, Office of Civilian Radioactive Waste Management, Washington, D.C., May.

U.S. Department of Energy, 1987a, Final Environmental Impact Statement: Disposal of Hanford Defense High-Level, Transuranic and Tank Wastes, DOE/EIS-0113, Washington, D.C., Dec.

U.S. Department of Energy, 1987b, Analysis of Radiation Doses from Operation of Postulated Commercial Spent Fuel Transportation Systems, DOE-CH/TPO-001, Pacific Northwest Laboratory, Richland, Wash., Nov.

U.S. Department of Energy, 1988a, External Dose Rate Conversion Factors for Calculation of Dose to the Public, DOE/EH-0070, Office of Environment, Safety, and Health, Washington, D.C. 

U.S. Department of Energy, 1988b, Internal Dose Conversion Factors for Calculation of Dose to the Public, DOE/EH-0071, Office of Environment, Safety, and Health, Washington, D.C.

U.S. Department of Energy, 1990a, Supplemental Environmental Impact Statement: Waste Isolation Pilot Plant, DOE/EIS-0026-FS, Washington, D.C., Jan.

U.S. Department of Energy, 1990b, "Radiation Protection of the Public and the Environment," DOE Order 5400.5.

U.S. Department of Energy, 1992, "Public Affairs Policy and Planning Requirements for Emergencies," DOE Order 5500.4A.

U.S. Department of Energy, 1996, Office of Waste Management Programmatic Environmental Impact Statement for Managing Treatment, Storage, and Disposal of Radioactive and Hazardous Waste, DOE/EIS-0200-F, Office of Environmental Management, Washington, D.C.

U.S. Nuclear Regulatory Commission, 1977a, Final Environmental Statement on the Transportation of Radioactive Material by Air and Other Modes, NUREG-0170, Washington, D.C.

U.S. Nuclear Regulatory Commission, 1977b, Calculation of Annual Dose to Man from Routine Releases of Reactor Effluents for the Purpose of Evaluating Compliance with 10 CFR Part 50, Appendix I, Rev. I, Regulatory Guide 1.109, Washington, D.C.

Wilmot, E.L., 1981, Transportation Accident Scenarios for Commercial Spent Fuel, SAND80-2124, Sandia National Laboratories, Albuquerque, N.M.

Yuan, Y.C., et al., 1993, RISKIND: A Computer Program for Calculating Radiological Consequences and Health Risks from Transportation of Spent Nuclear Fuel, ANL/EAIS-6-Rev. 0, Argonne National Laboratory, Argonne, Ill., Feb. 


\section{$A-1$}

APPENDIX A:

TRANSPORTED MATERIAL SUMMARY FILES GENERATED BY THE WASTE_MGMT MODEL 
A-2 


\section{APPENDIX A:}

\section{TRANSPORTED MATERIAL SUMMARY FILES GENERATED BY THE WASTE_MGMT MODEL}

The WASTE_MGMT model has been developed at Argonne National Laboratory to support analyses of risks and costs for all alternatives for each waste type considered in the Waste Management Programmatic Environmental Impact Statement (WM PEIS) (Kotek et al. 1996). One output of the WASTE_MGMT model consists of the annual quantity, physical form, and radiological characteristics of the waste shipped between sites for each case. The WASTE_MGMT output files are used directly as input to the transportation risk assessment.

The WASTE_MGMT transportation summary files generated for the low-level waste (LLW) transportation assessment are presented in this appendix, Appendix A. For each case, shipment summaries are presented separately for heterogeneous solids and activated metals. The total quantity of shipments for a case can be found by summing the quantities for heterogeneous solids and activated metals. In general, the quantity of activated metals transported is insignificant compared with the quantity of heterogeneous solids for each case.

The WASTE_MGMT files report the quantity of waste shipped on an annual basis (cubic meters per year or kilograms per year). As stated previously, the WM PEIS considers a 20-year time frame for WM LLW. For most of the WM alternatives considered in the WM PEIS, the assumption was that shipments would occur uniformly over a 10-year period, which would allow an initial 10-year period to build treatment, storage, and disposal facilities. Sites would essentially store their own waste for the first 10 years and ship all waste over the subsequent 10 years; however, the assumption was that for the No Action Alternative shipments would be distributed uniformly over a 20-year period because no new facilities would be required to be built. Therefore, although the total inventory considered is the same for all WM alternatives (current inventories plus 20 years of generation), the annual amounts of waste shipped, as reported in the WASTE_MGMT files in Appendix $\mathrm{A}$, are a function of the assumption concerning the duration of the shipping campaigns for each alternative.

Because of the differences in the time frames noted and the uncertainties in the actual timing of future waste shipments, the transportation impacts for the various alternatives are best compared by examining the total impacts of transporting a specific inventory of waste, independent of the annual shipping rates. The total amount of LLW transported for each alternative can be calculated by multiplying the annual shipment quantities (reported in the WASTE_MGMT files in Appendix A) by 20 years for the No Action Alternative and by 10 years for the other WM alternatives. 


\section{A.1 WM LLW NO ACTION ALTERNATIVE (CASE 1): HETEROGENEOUS SOLIDS}

Transported Material with Radionuclide Profile

\begin{tabular}{|c|c|c|c|}
\hline \multicolumn{4}{|l|}{ Alternative: "l No Action } \\
\hline Destination: HANF & & & \\
\hline Shipping Site: AMES & $\begin{array}{l}\text { Total Volume } \\
(\mathrm{m} 3 / \mathrm{y} x)\end{array}$ & $\begin{array}{cc}5.83 \mathrm{E}+00 & \begin{array}{c}\text { Total Mass } \\
(\mathrm{kg} / \mathrm{Yr})\end{array}\end{array}$ & $1.49 E+04$ \\
\hline & \begin{tabular}{l} 
Radionuclide \\
\hdashline $\mathrm{T} 1-208$ \\
$\mathrm{~Pb}-212$ \\
$\mathrm{Bi}-212$ \\
$\mathrm{Po}-212$ \\
$\mathrm{Po}-216$ \\
$\mathrm{Ra}-224$ \\
$\mathrm{Ra}-228$ \\
$\mathrm{Ac}-228$ \\
$\mathrm{Th}-228$ \\
$\mathrm{Th}-231$ \\
$\mathrm{Th}-232$ \\
$\mathrm{Th}-234$ \\
$\mathrm{~Pa}-234$ \\
$\mathrm{~Pa}-234 \mathrm{~m}$ \\
$\mathrm{U}-235$ \\
$\mathrm{U}-238$ \\
$\mathrm{Pu}-238$ \\
$\mathrm{Pu}-239$ \\
$\mathrm{Pu}-240$ \\
$\mathrm{Pu}-241$ \\
$\mathrm{Am}-241$ \\
$\mathrm{Cm}-242$ \\
$\mathrm{Cm}-244$
\end{tabular} & $\begin{array}{c}\begin{array}{c}\text { Activity } \\
(C i / y r)\end{array} \\
-2.25 \mathrm{E}-07 \\
5.96 \mathrm{E}-07 \\
5.96 \mathrm{E}-07 \\
3.84 \mathrm{E}-07 \\
5.96 \mathrm{E}-07 \\
5.96 \mathrm{E}-07 \\
3.56 \mathrm{E}-06 \\
3.56 \mathrm{E}-06 \\
5.96 \mathrm{E}-07 \\
3.43 \mathrm{E}-06 \\
3.61 \mathrm{E}-05 \\
4.39 \mathrm{E}-03 \\
4.50 \mathrm{E}-07 \\
4.39 \mathrm{E}-03 \\
3.41 \mathrm{E}-06 \\
4.39 \mathrm{E}-03 \\
3.47 \mathrm{E}-04 \\
2.65 \mathrm{E}-05 \\
9.27 \mathrm{E}-05 \\
1.27 \mathrm{E}-02 \\
5.30 \mathrm{E}-07 \\
7.42 \mathrm{E}-06 \\
2.65 \mathrm{E}-06\end{array}$ & \\
\hline
\end{tabular}


A-5

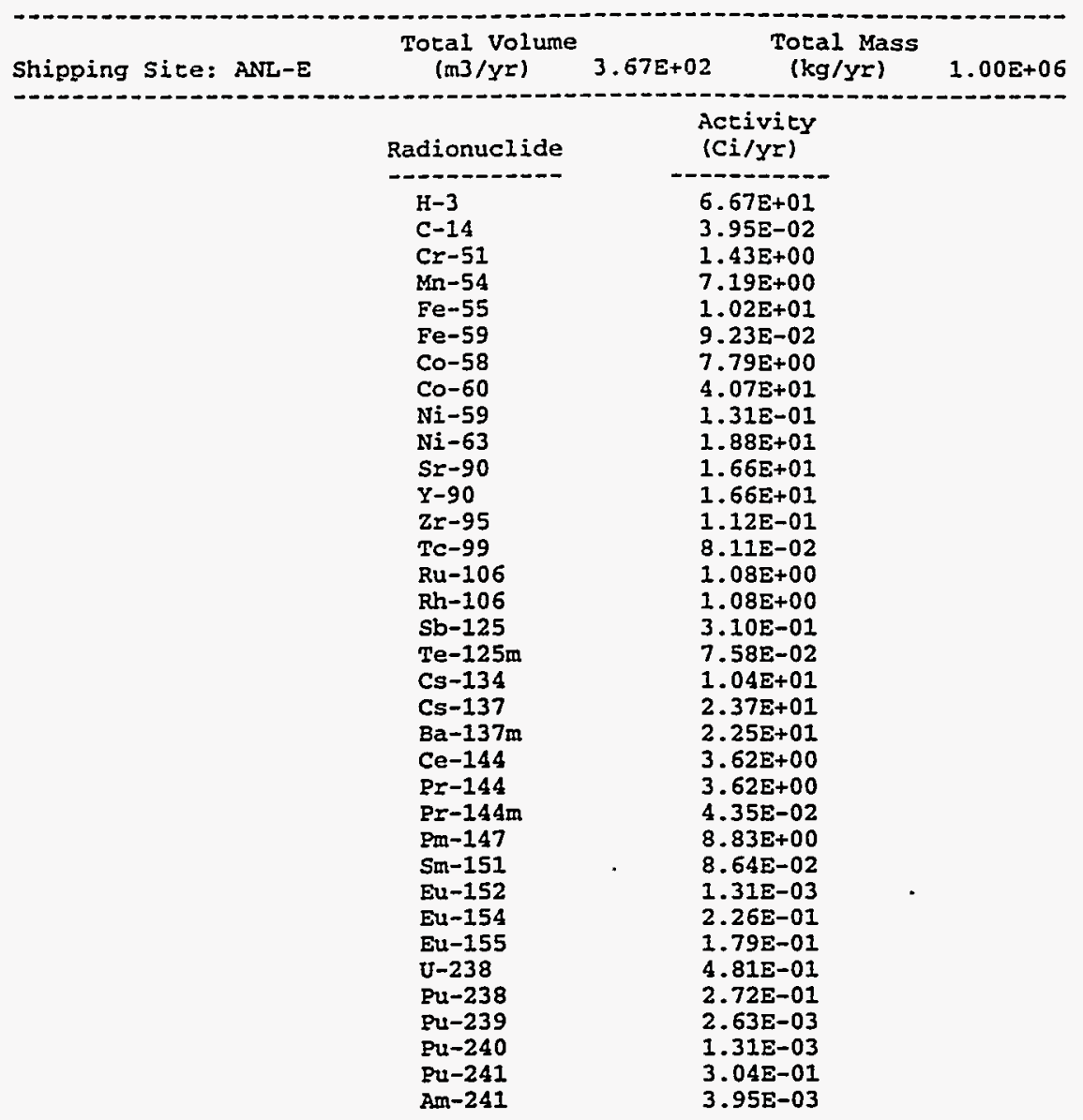


A-6

\begin{tabular}{|c|c|c|c|}
\hline \multirow[t]{2}{*}{ Shipping Site: BAPL } & $\begin{array}{l}\text { Total volume } \\
(\mathrm{m} 3 / \mathrm{yr})\end{array}$ & $\begin{array}{l}\text { Total Mass } \\
(\mathrm{kg} / \mathrm{Yr})\end{array}$ & $5.43 \mathrm{E}+05$ \\
\hline & $\begin{array}{l}\text { Radionuclide } \\
\mathrm{H}-3 \\
\mathrm{Co}-60 \\
\mathrm{Ni}-63 \\
\mathrm{Sr}-90 \\
\mathrm{Y}-90 \\
\mathrm{Tc}-99 \\
\mathrm{Ru}-106 \\
\mathrm{Rh}-106 \\
\mathrm{Sb}-125 \\
\mathrm{Te}-125 \mathrm{~m} \\
\mathrm{Cs}-134 \\
\mathrm{Cs}-137 \\
\mathrm{Ba}-137 \mathrm{~m} \\
\mathrm{Ce}-144 \\
\mathrm{Pr}-144 \\
\mathrm{Pr}-144 \mathrm{~m} \\
\mathrm{Pm}-147 \\
\mathrm{Sm}-151 \\
\mathrm{Eu}-152 \\
\mathrm{Eu}-154 \\
\mathrm{Eu}-155 \\
\mathrm{TI}-208 \\
\mathrm{~Pb}-212 \\
\mathrm{Bi}-212 \\
\mathrm{Po}-212 \\
\mathrm{PO}-216 \\
\mathrm{Ra}-224 \\
\mathrm{Ra}-228 \\
\mathrm{Ac}-228 \\
\mathrm{Th}-228 \\
\mathrm{Th}-231 \\
\mathrm{Th}-232 \\
\mathrm{Th}-234 \\
\mathrm{~Pa}-234 \\
\mathrm{~Pa}-234 \mathrm{~m} \\
\mathrm{U}-235 \\
\mathrm{U}-238 \\
\mathrm{Pu}-238 \\
\mathrm{Pu}-239 \\
\mathrm{Pu}-240 \\
\mathrm{Pu}-241 \\
\mathrm{Am}-241 \\
\end{array}$ & $\begin{array}{l}\text { Activity } \\
\text { (Ci/yE) } \\
1.25 \mathrm{E}-03 \\
1.98 \mathrm{-}-03 \\
5.98 \mathrm{E}-03 \\
1.19 \mathrm{E}-02 \\
1.05 \mathrm{E}+00 \\
1.05 \mathrm{E}+00 \\
1.88 \mathrm{E}-04 \\
1.03 \mathrm{E}-01 \\
1.03 \mathrm{E}-01 \\
2.96 \mathrm{E}-02 \\
7.24 \mathrm{E}-03 \\
1.20 \mathrm{E}-01 \\
1.10 \mathrm{E}+00 \\
1.04 \mathrm{E}+00 \\
3.45 \mathrm{E}-01 \\
3.45 \mathrm{E}-01 \\
4.15 \mathrm{E}-03 \\
8.44 \mathrm{E}-01 \\
8.25 \mathrm{E}-03 \\
1.25 \mathrm{E}-04 \\
2.16 \mathrm{E}-02 \\
1.71 \mathrm{E}-02 \\
1.90 \mathrm{E}-06 \\
5.04 \mathrm{E}-06 \\
5.04 \mathrm{E}-06 \\
3.24 \mathrm{E}-06 \\
5.04 \mathrm{E}-06 \\
5.04 \mathrm{E}-06 \\
3.01 \mathrm{E}-05 \\
3.01 \mathrm{E}-05 \\
5.04 \mathrm{E}-06 \\
2.90 \mathrm{E}-05 \\
3.05 \mathrm{E}-04 \\
3.71 \mathrm{E}-02 \\
3.80 \mathrm{E}-06 \\
3.71 \mathrm{E}-02 \\
2.88 \mathrm{E}-05 \\
3.71 \mathrm{E}-02 \\
2.60 \mathrm{E}-02 \\
2.51 \mathrm{E}-04 \\
1.25 \mathrm{E}-04 \\
2.90 \mathrm{E}-02 \\
3.77 \mathrm{E}-04\end{array}$ & \\
\hline \multirow[t]{2}{*}{ Shipping Site: FNAL } & $\begin{array}{l}\text { Total Volume } \\
\text { (m3/yr) }\end{array}$ & $\begin{array}{cc} & \text { Total Mass } \\
1.15 \mathrm{E}+01 & (\mathrm{~kg} / \mathrm{yr})\end{array}$ & $1.26 \mathrm{E}+04$ \\
\hline & $\begin{array}{l}\text { Radionuclide } \\
-\mathrm{H}-3 \\
\mathrm{Cr}-51 \\
\mathrm{Mn}-54 \\
\mathrm{Fe}-55 \\
\mathrm{Fe}-59 \\
\mathrm{Co}-58 \\
\mathrm{Co}-60 \\
\mathrm{~N} \mathrm{i}-59 \\
\mathrm{Ni}-63 \\
\mathrm{Zr}-95 \\
\mathrm{Tl}-208 \\
\mathrm{~Pb}-212 \\
\mathrm{Bi}-212 \\
\mathrm{Po-212} \\
\mathrm{PO}-216 \\
\mathrm{Ra}-224 \\
\mathrm{Ra}-228 \\
\mathrm{Ac}-228 \\
\mathrm{Th}-228 \\
\mathrm{Th}-231 \\
\mathrm{Th}-232 \\
\mathrm{Th}-234 \\
\mathrm{~Pa}-234 \\
\mathrm{~Pa}-234 \mathrm{~m} \\
\mathrm{U}-235 \\
\mathrm{U}-238\end{array}$ & \begin{tabular}{c}
$\begin{array}{c}\text { Activity } \\
\text { (Ci/yI) }\end{array}$ \\
\hdashline $9.15 E-02$ \\
$5.58 E-03$ \\
$1.06 E-02$ \\
$3.97 E-02$ \\
$3.58 E-04$ \\
$1.42 E-02$ \\
$1.11 E-01$ \\
$5.12 E-04$ \\
$7.28 E-02$ \\
$4.35 E-04$ \\
$1.89 E-07$ \\
$5.01 E-07$ \\
$5.01 E-07$ \\
$3.23 E-07$ \\
$5.01 E-07$ \\
$5.01 E-07$ \\
$2.99 E-06$ \\
$2.99 E-06$ \\
$5.01 E-07$ \\
$2.88 E-06$ \\
$3.04 E-05$ \\
$3.69 E-03$ \\
$3.79 E-07$ \\
$3.69 E-03$ \\
$2.87 E-06$ \\
$3.69 E-03$
\end{tabular} & \\
\hline
\end{tabular}




\section{A-7}

\begin{tabular}{|c|c|c|c|}
\hline Shipping Sice: KAPL & $\begin{array}{l}\text { Total volume } \\
(\mathrm{m} 3 / \mathrm{yr})\end{array}$ & $\begin{array}{c}\text { Total Mass } \\
(\mathrm{kg} / \mathrm{yr})\end{array}$ & $2.98 E+05$ \\
\hline - & 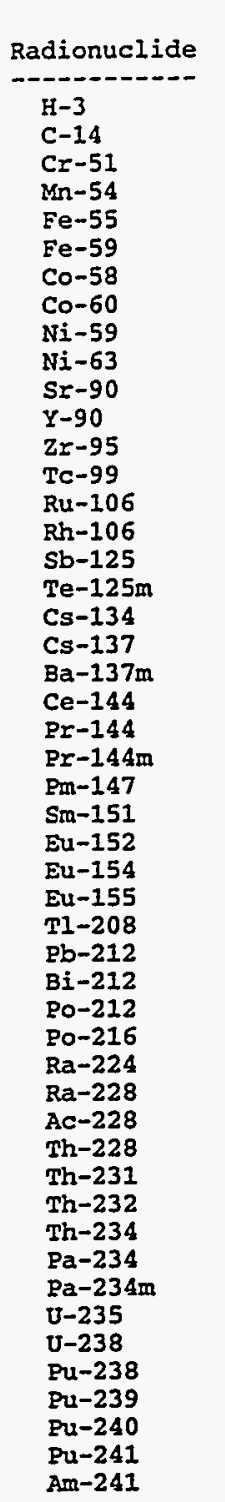 & 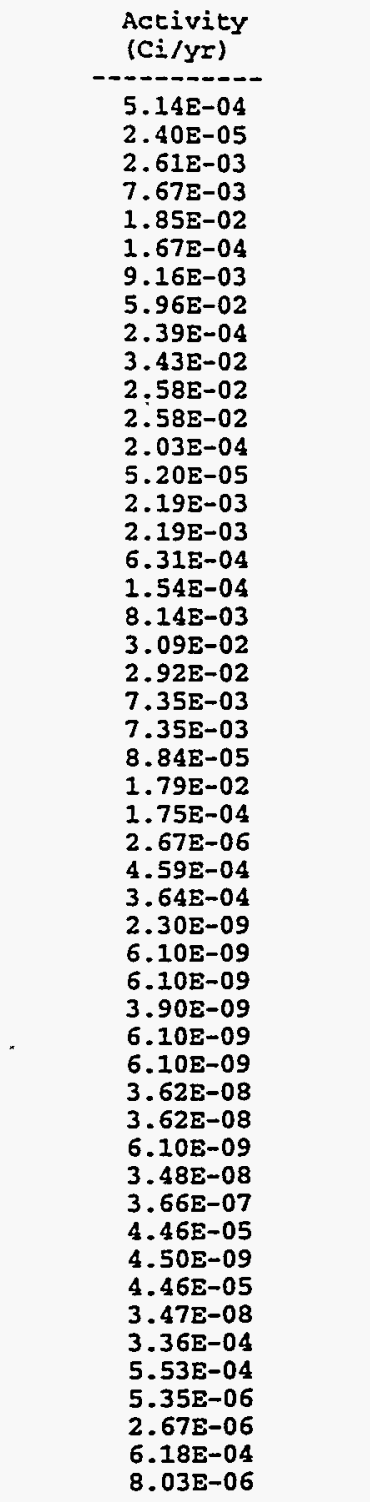 & \\
\hline \multirow[t]{2}{*}{ Shipping Site: LBL } & $\begin{array}{l}\text { Total Volume } \\
\text { (m3/yr) }\end{array}$ & $\begin{array}{l}\text { Total Mass } \\
(\mathrm{kg} / \mathrm{yr})\end{array}$ & $3.69 E+04$ \\
\hline & $\begin{array}{l}\text { Radionuclide } \\
\mathrm{E}-3 \\
\mathrm{C}-14 \\
\mathrm{Mn}-54 \\
\mathrm{Co-58} \\
\mathrm{Co}-60 \\
\mathrm{~S}-90 \\
\mathrm{Y}-90 \\
\mathrm{Tc}-99 \\
\mathrm{Cs}-134 \\
\mathrm{Cs}-137 \\
\mathrm{Ba}-137 \mathrm{~m} \\
\mathrm{U}-238 \\
\mathrm{Cm}-244\end{array}$ & \begin{tabular}{c}
$\begin{array}{c}\text { Activity } \\
\text { (Ci/yr) }\end{array}$ \\
\hdashline $7.44 E+02$ \\
$5.65 E-01$ \\
$6.37 E+01$ \\
$5.88 E+01$ \\
$1.69 E+02$ \\
$7.99 E+01$ \\
$7.99 E+01$ \\
$1.13 E+00$ \\
$1.31 E+02$ \\
$1.73 E+02$ \\
$1.64 E+02$ \\
$6.88 E+00$ \\
$5.27 E+00$
\end{tabular} & \\
\hline
\end{tabular}


A-8

\begin{tabular}{|c|c|c|c|}
\hline Shipping Sice: PPPL & $\begin{array}{l}\text { Total Volume } \\
\left(\mathrm{m}^{3} / \mathrm{yr}\right)\end{array}$ & $\begin{array}{c}\text { Total Mass } \\
(\mathrm{kg} / \mathrm{yr})\end{array}$ & $2.48 \mathrm{E}+03$ \\
\hline & $\begin{array}{l}\text { Radionuclide } \\
\mathrm{H}-3 \\
\mathrm{Cr}-51 \\
\mathrm{Mn}-54 \\
\mathrm{Fe}-55 \\
\mathrm{Fe}-59 \\
\mathrm{Co}-58 \\
\mathrm{Co}-60 \\
\mathrm{Ni}-59 \\
\mathrm{Ni}-63 \\
\mathrm{Zr}-95\end{array}$ & $\begin{array}{c}\begin{array}{c}\text { Activity } \\
\text { (Ci/yr) }\end{array} \\
1.00 \mathrm{E}-01 \\
4.25 \mathrm{E}-04 \\
8.09 \mathrm{E}-04 \\
3.02 \mathrm{E}-03 \\
2.73 \mathrm{E}-05 \\
1.08 \mathrm{E}-03 \\
8.51 \mathrm{E}-03 \\
3.90 \mathrm{E}-05 \\
5.54 \mathrm{E}-03 \\
3.31 \mathrm{E}-05\end{array}$ & \\
\hline Shipping Site: SLAC & $\begin{array}{l}\text { Total Volume } \\
(\mathrm{m} 3 / \mathrm{yr})\end{array}$ & $\begin{array}{cc}1.36 \mathrm{E}+02 & \text { Total Mass } \\
(\mathrm{kg} / \mathrm{yr})\end{array}$ & $3.72 E+05$ \\
\hline & $\begin{array}{l}\text { Radionuclide } \\
\text { Cr-51 } \\
\mathrm{Mn}-54 \\
\mathrm{Fe}-55 \\
\mathrm{Fe}-59 \\
\mathrm{Co}-58 \\
\mathrm{Co}-60 \\
\mathrm{Ni}-59 \\
\mathrm{Ni}-63 \\
\mathrm{Zr}-95\end{array}$ & $\begin{array}{c}\text { Activity } \\
\text { (Ci/yx) }\end{array}$ & \\
\hline
\end{tabular}


Destination: INEL

\begin{tabular}{|c|c|c|c|}
\hline Shipping Site: ANL-W & $\begin{array}{l}\text { Total Volume } \\
(\mathrm{m} 3 / \mathrm{yr})\end{array}$ & $\begin{array}{l}\text { Total Mass } \\
(\mathrm{kg} / \mathrm{yr})\end{array}$ & $1.07 \mathrm{E}+05$ \\
\hline - & 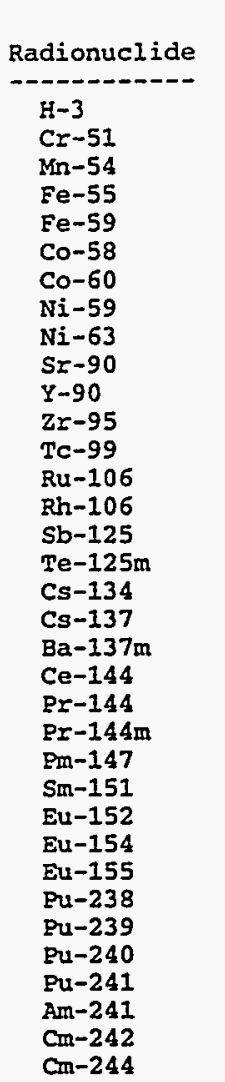 & \begin{tabular}{c}
$\begin{array}{c}\text { Activity } \\
(C i / y r)\end{array}$ \\
\hdashline $7.64 \mathrm{E}-04$ \\
$1.79 \mathrm{E}+03$ \\
$3.41 \mathrm{E}+03$ \\
$1.27 \mathrm{E}+04$ \\
$1.15 \mathrm{E}+02$ \\
$4.58 \mathrm{E}+03$ \\
$3.59 \mathrm{E}+04$ \\
$1.64 \mathrm{E}+02$ \\
$2.33 \mathrm{E}+04$ \\
$1.58 \mathrm{E}-02$ \\
$1.58 \mathrm{E}-02$ \\
$1.39 \mathrm{E}+02$ \\
$2.83 \mathrm{E}-06$ \\
$1.54 \mathrm{E}-03$ \\
$1.54 \mathrm{E}-03$ \\
$4.44 \mathrm{E}-04$ \\
$1.08 \mathrm{E}-04$ \\
$1.79 \mathrm{E}-03$ \\
$1.66 \mathrm{E}-02$ \\
$1.57 \mathrm{E}-02$ \\
$5.18 \mathrm{E}-03$ \\
$5.18 \mathrm{E}-03$ \\
$6.22 \mathrm{E}-05$ \\
$1.26 \mathrm{E}-02$ \\
$1.23 \mathrm{E}-04$ \\
$1.88 \mathrm{E}-06$ \\
$3.23 \mathrm{E}-04$ \\
$2.56 \mathrm{E}-04$ \\
$3.90 \mathrm{E}-04$ \\
$3.86 \mathrm{E}-06$ \\
$2.19 \mathrm{E}-06$ \\
$4.78 \mathrm{E}-04$ \\
$5.66 \mathrm{E}-06$ \\
$2.44 \mathrm{E}-08$ \\
$8.70 \mathrm{E}-09$
\end{tabular} & \\
\hline Shipping Site: ITRI & $\begin{array}{l}\text { Total volume } \\
\text { (m3/yr) }\end{array}$ & $\begin{array}{cc}\text { Total Mass } \\
(\mathrm{kg} / \mathrm{Yr})\end{array}$ & $8.23 E+04$ \\
\hline & \begin{tabular}{l} 
Radionuclide \\
\hdashline $\mathrm{H}-3$ \\
$\mathrm{C}-14$ \\
$\mathrm{Mn}-54$ \\
$\mathrm{Co}-58$ \\
$\mathrm{Co}-60$ \\
$\mathrm{Sr}-90$ \\
$\mathrm{Y}-90$ \\
$\mathrm{TC}-99$ \\
$\mathrm{Cs}-134$ \\
$\mathrm{Cs}-137$ \\
$\mathrm{Ba}-137 \mathrm{~m}$ \\
$\mathrm{U}-238$
\end{tabular} & $\begin{array}{c}\begin{array}{c}\text { Activity } \\
\text { (Ci/yr) }\end{array} \\
3.08 \mathrm{E}+00 \\
1.51 \mathrm{E}-01 \\
1.70 \mathrm{E}+01 \\
1.57 \mathrm{E}+01 \\
4.56 \mathrm{E}+01 \\
2.14 \mathrm{E}+01 \\
2.14 \mathrm{E}+01 \\
3.03 \mathrm{E}-01 \\
3.53 \mathrm{E}+01 \\
4.66 \mathrm{E}+01 \\
4.41 \mathrm{E}+01 \\
1.84 \mathrm{E}+00\end{array}$ & \\
\hline Shipping Site: $\mathrm{KCP}$ & $\begin{array}{l}\text { Total volume } \\
(\mathrm{m} 3 / \mathrm{yr})\end{array}$ & $\begin{array}{l}\text { Total Mass } \\
(\mathrm{kg} / \mathrm{yr})\end{array}$ & $3.61 E+03$ \\
\hline & $\begin{array}{l}\text { Radionuclide } \\
\mathrm{H}-3 \\
\mathrm{C}-14 \\
\mathrm{Mn}-54 \\
\mathrm{Co}-58 \\
\mathrm{Co}-60 \\
\mathrm{Sr}-90 \\
\mathrm{Y}-90 \\
\mathrm{Tc}-99 \\
\mathrm{Cs}-134 \\
\mathrm{Cs}-137 \\
\mathrm{Ba}-137 \mathrm{~m} \\
\mathrm{U}-238\end{array}$ & \begin{tabular}{c} 
Activity \\
(Ci/Yr) \\
\hdashline $1.35 E-02$ \\
$7.23 E-05$ \\
$8.14 E-03$ \\
$7.52 E-03$ \\
$2.17 E-02$ \\
$1.02 E-02$ \\
$1.02 E-02$ \\
$1.44 \Xi-04$ \\
$1.68 E-02$ \\
$2.22 \Xi-02$ \\
$2.10 E-02$ \\
$8.80 E-04$
\end{tabular} & \\
\hline
\end{tabular}




\title{
SUPPLEMENTAL INFORMATION RELATED TO RISK ASSESSMENT FOR THE OFF-SITE TRANSPORTATION OF LOW-LEVEL WASTE FOR THE U.S. DEPARTMENT OF ENERGY WASTE MANAGEMENT PROGRAMMATIC ENVIRONMENTAL IMPACT STATEMENT
}

by

F.A. Monette, B.M. Biwer, D.J. LePoire, and S.Y. Chen

\begin{abstract}
This report presents supplemental information to support the human health risk assessment conducted for the transportation of low-level waste (LLW) in support of the U.S. Department of Energy Waste Management Programmatic Environmental Impact Statement (WM PEIS). Detailed descriptions of the transportation health risk assessment method and results of the assessment are presented in Appendix E of the WM PEIS and are not repeated in this report. This report presents additional information that is not presented in Appendix $E$ but that was needed to conduct the transportation risk assessment for Waste Management (WM) LLW. Included are definitions of the LLW alternatives considered in the WM PEIS, data related to the inventory and to the physical and radiological characteristics of WM LLW, an overview of the risk assessment method, and detailed results of the assessment for each WM LLW alternative considered.
\end{abstract}

\section{INTRODUCTION}

This report presents supplemental information to support the human health risk assessment conducted for the transportation of low-level waste (LLW) for the U.S. Department of Energy (DOE) Waste Management Programmatic Environmental Impact Statement (WM PEIS) (DOE 1996). Detailed descriptions of the transportation health risk assessment method, major assumptions, and packaging and routing determinations, as well as a summary of the results of the transportation assessment, are presented in Appendix E of the WM PEIS (DOE 1996) and are not repeated in this report. This report presents additional information that is not presented in Appendix E but that was needed to conduct the transportation risk assessment. This report should be read in conjunction with Appendix $\mathrm{E}$ to provide a complete description of the transportation risk assessment conducted for LLW. Similar supporting reports have been prepared for the transportation assessments conducted for the WM PEIS alternatives for high-level waste, low-level mixed waste, and transuranic waste (Monette et al. 1996a-c). 


\begin{tabular}{|c|c|c|c|}
\hline Shipping Sice: LLNL & $\begin{array}{l}\text { Total volume } \\
(\mathrm{m} 3 / \mathrm{yr})\end{array}$ & 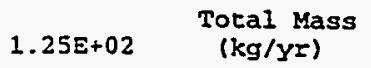 & $2.91 z+05$ \\
\hline & $\begin{array}{l}\text { Radionuclide } \\
\mathrm{H}-3 \\
\mathrm{C}-14 \\
\mathrm{Mn}-54 \\
\mathrm{Co}-58 \\
\mathrm{Co}-60 \\
\mathrm{Ni}-63 \\
\mathrm{Sr}-90 \\
\mathrm{Y}-90 \\
\mathrm{Tc}-99 \\
\mathrm{Ru}-106 \\
\mathrm{Rh}-106 \\
\mathrm{Sb}-125 \\
\mathrm{Te}-125 \mathrm{~m} \\
\mathrm{Cs}-134 \\
\mathrm{Cs}-137 \\
\mathrm{Ba}-137 \mathrm{~m} \\
\mathrm{Ce}-144 \\
\mathrm{Pr}-144 \\
\mathrm{Pr}-144 \mathrm{~m} \\
\mathrm{Pm}-147 \\
\mathrm{Sm}-151 \\
\mathrm{Eu}-152 \\
\mathrm{Eu}-154 \\
\mathrm{Eu}-155 \\
\mathrm{U}-238 \\
\mathrm{Pu}-238 \\
\mathrm{Pu}-239 \\
\mathrm{Pu}-240 \\
\mathrm{Pu}-241 \\
\mathrm{Am}-241 \\
\mathrm{Cm}-242 \\
\mathrm{Cm}-244\end{array}$ & $\begin{array}{c}\text { Activity } \\
\text { (Ci/yz) } \\
-1.68 \mathrm{E}+04 \\
7.76 \mathrm{E}-04 \\
8.74 \mathrm{E}-02 \\
8.07 \mathrm{E}-02 \\
2.34 \mathrm{E}-01 \\
2.44 \mathrm{E}-03 \\
3.27 \mathrm{E}-01 \\
3.27 \mathrm{E}-01 \\
1.59 \mathrm{E}-03 \\
2.12 \mathrm{E}-02 \\
2.12 \mathrm{E}-02 \\
6.09 \mathrm{E}-03 \\
1.48 \mathrm{E}-03 \\
2.05 \mathrm{E}-01 \\
4.66 \mathrm{E}-01 \\
4.41 \mathrm{E}-01 \\
7.10 \mathrm{E}-02 \\
7.10 \mathrm{E}-02 \\
8.54 \mathrm{E}-04 \\
1.73 \mathrm{E}-01 \\
1.69 \mathrm{E}-03 \\
2.58 \mathrm{E}-05 \\
4.43 \mathrm{E}-03 \\
3.51 \mathrm{E}-03 \\
9.44 \mathrm{E}-03 \\
3.92 \mathrm{E}-02 \\
2.64 \mathrm{E}-03 \\
9.08 \mathrm{E}-03 \\
1.25 \mathrm{E}+00 \\
1.29 \mathrm{E}-04 \\
7.24 \mathrm{E}-04 \\
2.58 \mathrm{E}-04\end{array}$ & \\
\hline Shipping Site: MOUND & $\begin{array}{l}\text { Total volume } \\
\text { (m3/yr) }\end{array}$ & $\begin{array}{c}\text { Total Mass } \\
(\mathrm{kg} / \mathrm{Yr})\end{array}$ & $4.83 E+06$ \\
\hline & $\begin{array}{l}\text { Radionuclide } \\
\mathrm{H}-3 \\
\mathrm{Pu}-238 \\
\mathrm{Pu}-239 \\
\mathrm{Pu}-240 \\
\mathrm{Pu}-241 \\
\mathrm{Am}-241 \\
\mathrm{Cm}-242 \\
\mathrm{Cm}-244\end{array}$ & $\begin{array}{l}6.70 E+03 \\
8.02 E-02 \\
6.12 E-03 \\
2.14 E-02 \\
2.95 E+00 \\
1.22 E-04 \\
1.71 E-03 \\
6.12 E-04\end{array}$ & \\
\hline \multirow[t]{2}{*}{ Shipping site: PANT } & $\begin{array}{l}\text { Total volume } \\
\text { (m3/yr) }\end{array}$ & $\begin{array}{c}\text { Total Mass } \\
(\mathrm{kg} / \mathrm{yr})\end{array}$ & $1.44 \mathrm{E}+07$ \\
\hline & $\begin{array}{l}\text { Radionuclide } \\
\mathrm{H}-3 \\
\mathrm{~T} \mathrm{-}-208 \\
\mathrm{~Pb}-212 \\
\mathrm{Bi}-212 \\
\mathrm{PO}-212 \\
\mathrm{Po}-216 \\
\mathrm{Ra}-224 \\
\mathrm{Ra}-228 \\
\mathrm{Ac}-228 \\
\mathrm{Th}-228 \\
\mathrm{Th}-231 \\
\mathrm{Th}-232 \\
\mathrm{Th}-234 \\
\mathrm{~Pa}-234 \\
\mathrm{~Pa}-234 \mathrm{~m} \\
\mathrm{U}-235 \\
\mathrm{U}-238\end{array}$ & $\begin{array}{l}1.17 \mathrm{E}+02 \\
1.11 \mathrm{E}-04 \\
2.93 \mathrm{E}-04 \\
2.93 \mathrm{E}-04 \\
1.89 \mathrm{E}-04 \\
2.93 \mathrm{E}-04 \\
2.93 \mathrm{E}-04 \\
1.75 \mathrm{E}-03 \\
1.75 \mathrm{E}-03 \\
2.93 \mathrm{E}-04 \\
1.69 \mathrm{E}-03 \\
1.78 \mathrm{E}-02 \\
2.16 \mathrm{E}+00 \\
2.22 \mathrm{E}-04 \\
2.16 \mathrm{E}+00 \\
1.68 \mathrm{E}-03 \\
2.16 \mathrm{E}+00\end{array}$ & \\
\hline
\end{tabular}




\section{A-II}

\begin{tabular}{|c|c|c|c|}
\hline Shipping Sice: PORTS & $\begin{array}{l}\text { Toral Volume } \\
(\mathrm{m} 3 / \mathrm{yr})\end{array}$ & $\begin{array}{c}\text { Toral Mass } \\
(\mathrm{kg} / \mathrm{yr})\end{array}$ & $3.36 E+07$ \\
\hline & $\begin{array}{l}\text { Radionuclide } \\
\mathrm{Tl}-208 \\
\mathrm{~Pb}-212 \\
\mathrm{Bi}-212 \\
\mathrm{PO}-212 \\
\mathrm{PO}-216 \\
\mathrm{Ra}-224 \\
\mathrm{Ra}-228 \\
\mathrm{Ac}-228 \\
\mathrm{Th}-228 \\
\mathrm{Th}-231 \\
\mathrm{Th}-232 \\
\mathrm{Th}-234 \\
\mathrm{~Pa}-234 \\
\mathrm{~Pa}-234 \mathrm{~m} \\
\mathrm{U}-235 \\
\mathrm{U}-238\end{array}$ & $\begin{array}{c}\begin{array}{l}\text { Activity } \\
\text { (Ci/yr) }\end{array} \\
1.72 \mathrm{E}-06 \\
4.57 \mathrm{E}-06 \\
4.57 \mathrm{E}-06 \\
2.94 \mathrm{E}-06 \\
4.57 \mathrm{E}-06 \\
4.57 \mathrm{E}-06 \\
2.73 \mathrm{E}-05 \\
2.73 \mathrm{E}-05 \\
4.57 \mathrm{E}-06 \\
2.63 \mathrm{E}-05 \\
2.77 \mathrm{E}-04 \\
3.37 \mathrm{E}-02 \\
3.45 \mathrm{E}-06 \\
3.37 \mathrm{E}-02 \\
2.62 \mathrm{E}-05 \\
3.37 \mathrm{E}-02\end{array}$ & \\
\hline \multirow[t]{2}{*}{ Shipping Site: Paducah } & $\begin{array}{l}\text { TotaI Volume } \\
\text { (m3/yr) }\end{array}$ & $\begin{array}{l}\text { Total Mass } \\
(\mathrm{kg} / \mathrm{yx})\end{array}$ & $6.11 E+06$ \\
\hline & $\begin{array}{l}\text { Radionuclide } \\
\text { Tc-99 } \\
\mathrm{TI}-208 \\
\mathrm{~Pb}-212 \\
\mathrm{Bi}-212 \\
\mathrm{Po}-212 \\
\mathrm{PO}-216 \\
\mathrm{Ra}-224 \\
\mathrm{Ra}-228 \\
\mathrm{Ac}-228 \\
\mathrm{Th}-228 \\
\mathrm{Th}-231 \\
\mathrm{Th}-232 \\
\mathrm{Th}-234 \\
\mathrm{~Pa}-234 \\
\mathrm{~Pa}-234 \mathrm{~m} \\
\mathrm{U}-235 \\
\mathrm{U}-238 \\
\mathrm{~Np}-237 \\
\mathrm{Pu}-239\end{array}$ & $\begin{array}{c}\begin{array}{c}\text { Activity } \\
\text { (Ci/yr) }\end{array} \\
4.46 \mathrm{E}-03 \\
1.88 \mathrm{E}-05 \\
4.97 \mathrm{E}-05 \\
4.97 \mathrm{E}-05 \\
3.20 \mathrm{E}-05 \\
4.97 \mathrm{E}-05 \\
4.97 \mathrm{E}-05 \\
2.97 \mathrm{E}-04 \\
2.97 \mathrm{E}-04 \\
4.97 \mathrm{E}-05 \\
2.86 \mathrm{E}-04 \\
3.01 \mathrm{E}-03 \\
3.67 \mathrm{E}-01 \\
3.76 \mathrm{E}-05 \\
3.67 \mathrm{E}-01 \\
2.85 \mathrm{E}-04 \\
3.67 \mathrm{E}-01 \\
3.35 \mathrm{E}-03 \\
3.35 \mathrm{E}-03\end{array}$ & \\
\hline Shipping Site: RFP & $\begin{array}{l}\text { Total volume } \\
(\mathrm{m} 3 / \mathrm{yr})\end{array}$ & $\begin{array}{c}\text { Total Mass } \\
\text { (kg/yz) }\end{array}$ & $3.08 \mathrm{E}+06$ \\
\hline & $\begin{array}{l}\text { Radionuclide } \\
\mathrm{Tl}-208 \\
\mathrm{~Pb}-212 \\
\mathrm{Bi}-212 \\
\mathrm{Po}-212 \\
\mathrm{Po}-216 \\
\mathrm{Ra}-224 \\
\mathrm{Ra}-228 \\
\mathrm{Ac}-228 \\
\mathrm{Th}-228 \\
\mathrm{Th}-231 \\
\mathrm{Th}-232 \\
\mathrm{Th}-234 \\
\mathrm{~Pa}-234 \\
\mathrm{~Pa}-234 \mathrm{~m} \\
\mathrm{U}-235 \\
\mathrm{U}-238 \\
\mathrm{Pu}-238 \\
\mathrm{Pu}-239 \\
\mathrm{Pu}-240 \\
\mathrm{Pu}-241 \\
\mathrm{Am}-241 \\
\mathrm{Cm}-242 \\
\mathrm{Cm}-244\end{array}$ & \begin{tabular}{c} 
Activity \\
(Ci/YX) \\
- \hdashline.---- \\
$1.80 E-05$ \\
$4.77 E-05$ \\
$4.77 E-05$ \\
$3.07 E-05$ \\
$4.77 E-05$ \\
$4.77 E-05$ \\
$2.85 E-04$ \\
$2.85 E-04$ \\
$4.77 E-05$ \\
$2.74 E-04$ \\
$2.89 E-03$ \\
$3.52 E-01$ \\
$3.60 E-05$ \\
$3.52 E-01$ \\
$2.73 E-04$ \\
$3.52 E-01$ \\
$2.78 E-01$ \\
$2.12 E-02$ \\
$7.42 E-02$ \\
$1.02 E+01$ \\
$4.24 E-04$ \\
$5.94 E-03$ \\
$2.12 E-03$
\end{tabular} & \\
\hline
\end{tabular}




\begin{tabular}{|c|c|c|c|}
\hline Shipping Site: RMI & $\begin{array}{l}\text { Total Volume } \\
(\mathrm{m} 3 / \mathrm{yr})\end{array}$ & $\begin{array}{c}\text { Total Mass } \\
\left(\mathrm{kg} / \mathrm{yr}^{\mathrm{r}}\right)\end{array}$ & $7.03 E+06$ \\
\hline & \begin{tabular}{l} 
Radionuclide \\
\hdashline $\mathrm{Tl}-208$ \\
$\mathrm{~Pb}-212$ \\
$\mathrm{Bi}-212$ \\
$\mathrm{PO}-212$ \\
$\mathrm{Po}-216$ \\
$\mathrm{Ra}-224$ \\
$\mathrm{Ra}-228$ \\
$\mathrm{Ac}-228$ \\
$\mathrm{Th}-228$ \\
$\mathrm{Th}-231$ \\
$\mathrm{Th}-232$ \\
$\mathrm{Th}-234$ \\
$\mathrm{~Pa}-234$ \\
$\mathrm{~Pa}-234 \mathrm{~m}$ \\
$\mathrm{U}-235$ \\
$\mathrm{U}-238$
\end{tabular} & \begin{tabular}{c}
$\begin{array}{c}\text { Activicy } \\
\text { (Ci/yr) }\end{array}$ \\
\hdashline $1.79 \mathrm{E}-07$ \\
$4.73 \mathrm{E}-07$ \\
$4.73 \mathrm{E}-07$ \\
$3.05 \mathrm{E}-07$ \\
$4.73 \mathrm{E}-07$ \\
$4.73 \mathrm{E}-07$ \\
$2.83 \mathrm{E}-06$ \\
$2.83 \mathrm{E}-06$ \\
$4.73 \mathrm{E}-07$ \\
$2.72 \mathrm{E}-06$ \\
$2.87 \mathrm{E}-05$ \\
$3.49 \mathrm{E}-03$ \\
$3.58 \mathrm{E}-07$ \\
$3.49 \mathrm{E}-03$ \\
$2.71 \mathrm{E}-06$ \\
$3.49 \mathrm{E}-03$
\end{tabular} & \\
\hline Shipping Site: SNLA & $\begin{array}{l}\text { Total Volume } \\
(\mathrm{m} 3 / \mathrm{y} x)\end{array}$ & $\begin{array}{c}\text { Total Mass } \\
(\mathrm{kg} / \mathrm{yr})\end{array}$ & $1.64 \mathrm{E}+05$ \\
\hline & 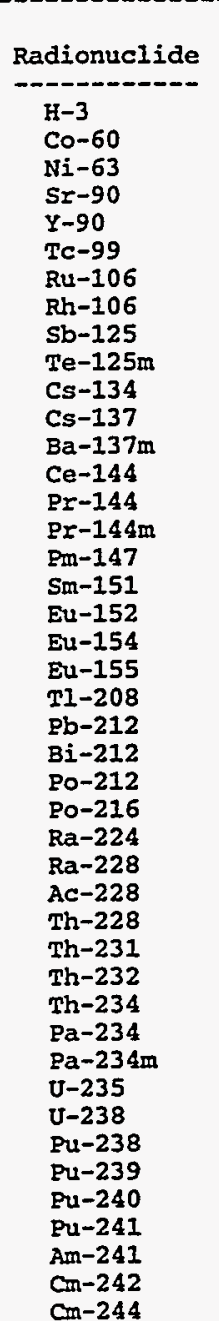 & $\begin{array}{c}\text { Activity } \\
\text { (Ci/yr) } \\
1.50 \mathrm{y}+00 \\
1.42 \mathrm{E}-05 \\
2.83 \mathrm{E}-05 \\
2.52 \mathrm{E}-03 \\
2.52 \mathrm{E}-03 \\
4.50 \mathrm{E}-07 \\
2.46 \mathrm{E}-04 \\
2.46 \mathrm{E}-04 \\
7.06 \mathrm{E}-05 \\
1.72 \mathrm{E}-05 \\
2.86 \mathrm{E}-04 \\
2.64 \mathrm{E}-03 \\
2.49 \mathrm{E}-03 \\
8.23 \mathrm{E}-04 \\
8.23 \mathrm{E}-04 \\
9.90 \mathrm{E}-05 \\
2.01 \mathrm{E}-03 \\
1.96 \mathrm{E}-05 \\
3.00 \mathrm{E}-07 \\
5.14 \mathrm{E}-05 \\
4.08 \mathrm{E}-05 \\
2.55 \mathrm{E}-06 \\
6.75 \mathrm{E}-06 \\
6.75 \mathrm{E}-06 \\
4.35 \mathrm{E}-06 \\
6.75 \mathrm{E}-06 \\
6.75 \mathrm{E}-06 \\
4.03 \mathrm{E}-05 \\
4.03 \mathrm{E}-05 \\
6.75 \mathrm{E}-06 \\
3.88 \mathrm{E}-05 \\
4.09 \mathrm{E}-04 \\
4.98 \mathrm{E}-02 \\
5.10 \mathrm{E}-06 \\
4.98 \mathrm{E}-02 \\
3.87 \mathrm{E}-05 \\
4.98 \mathrm{E}-02 \\
3.93 \mathrm{E}-02 \\
3.00 \mathrm{E}-03 \\
1.05 \mathrm{E}-02 \\
1.44 \mathrm{E}+00 \\
6.09 \mathrm{E}-05 \\
8.40 \mathrm{E}-04 \\
3.00 \mathrm{E}-04\end{array}$ & \\
\hline
\end{tabular}




\begin{tabular}{|c|c|c|c|}
\hline Shipping Sice: SNLL & $\begin{array}{l}\text { Tocal Volume } \\
\left(\mathrm{m} 3 / y^{\mathrm{r}}\right)\end{array}$ & $\begin{array}{c}\text { Total Mass } \\
(\mathrm{kg} / \mathrm{yr})\end{array}$ & $4.685+04$ \\
\hline & $\begin{array}{l}\text { Radionuclide } \\
\mathrm{H}-3 \\
\mathrm{Fe}-55 \\
\mathrm{Co}-60 \\
\mathrm{Tl}-208 \\
\mathrm{~Pb}-212 \\
\mathrm{Bi}-212 \\
\mathrm{Po}-212 \\
\mathrm{Po-216} \\
\mathrm{Ra}-224 \\
\mathrm{Ra}-228 \\
\mathrm{Ac}-228 \\
\mathrm{Th}-228 \\
\mathrm{Th}-231 \\
\mathrm{Th}-232 \\
\mathrm{Th}-234 \\
\mathrm{~Pa}-234 \\
\mathrm{~Pa}-234 \mathrm{~m} \\
\mathrm{U}-235 \\
\mathrm{U}-238\end{array}$ & \begin{tabular}{c}
$\begin{array}{c}\text { Activity } \\
\text { (Ci/yr) }\end{array}$ \\
\hdashline $1.41 \mathrm{E}+04$ \\
$1.57 \mathrm{E}-01$ \\
$1.57 \mathrm{E}-01$ \\
$3.20 \mathrm{E}-05$ \\
$8.47 \mathrm{E}-05$ \\
$8.47 \mathrm{E}-05$ \\
$5.46 \mathrm{E}-05$ \\
$8.47 \mathrm{E}-05$ \\
$8.47 \mathrm{E}-05$ \\
$5.06 \mathrm{E}-04$ \\
$5.05 \mathrm{E}-04$ \\
$8.47 \mathrm{E}-05$ \\
$4.87 \mathrm{E}-04$ \\
$5.14 \mathrm{E}-03$ \\
$6.25 \mathrm{E}-01$ \\
$6.40 \mathrm{E}-05$ \\
$6.25 \mathrm{E}-01$ \\
$4.85 \mathrm{E}-04$ \\
$6.25 \mathrm{E}-01$
\end{tabular} & \\
\hline
\end{tabular}


Ation: ORNL

\begin{tabular}{|c|c|c|c|}
\hline Shipping Site: $\mathrm{K}-25$ & $\begin{array}{l}\text { Total Volume } \\
(\mathrm{m} 3 / \mathrm{yr})\end{array}$ & $\begin{array}{cc}3.43 \mathrm{E}+03 & \text { Total Mass } \\
(\mathrm{kg} / \mathrm{y} x)\end{array}$ & $2.33 E+07$ \\
\hline & $\begin{array}{l}\text { Radionuclide } \\
\mathrm{T} 1-208 \\
\mathrm{~Pb}-212 \\
\mathrm{Bi}-212 \\
\mathrm{PO}-212 \\
\mathrm{Po-216} \\
\mathrm{Ra}-224 \\
\mathrm{Ra}-228 \\
\mathrm{Ac}-228 \\
\mathrm{Th}-228 \\
\mathrm{Th}-231 \\
\mathrm{Th}-232 \\
\mathrm{Th}-234 \\
\mathrm{~Pa}-234 \\
\mathrm{~Pa}-234 \mathrm{~m} \\
\mathrm{U}-235 \\
\mathrm{U}-238\end{array}$ & \begin{tabular}{c}
$\begin{array}{c}\text { Activity } \\
\text { (Ci/yr) }\end{array}$ \\
\hdashline $5.33 E-05$ \\
$1.41 E-04$ \\
$1.41 E-04$ \\
$9.10 E-05$ \\
$1.41 E-04$ \\
$1.41 E-04$ \\
$8.44 E-04$ \\
$8.44 E-04$ \\
$1.41 E-04$ \\
$8.13 E-04$ \\
$8.57 E-03$ \\
$1.04 E+00$ \\
$1.06 E-04$ \\
$1.04 E+00$ \\
$8.10 E-04$ \\
$1.04 E+00$
\end{tabular} & \\
\hline Shipping Site: ORISE & $\begin{array}{l}\text { Total volume } \\
\text { (m3/yr) }\end{array}$ & $\begin{array}{cc} & \text { Total Mass } \\
2.59 E+01 & (\mathrm{~kg} / \mathrm{Yr})\end{array}$ & $1.58 \mathrm{E}+04$ \\
\hline & $\begin{array}{l}\text { Radionuclide } \\
\mathrm{H}-3 \\
\mathrm{C}-14 \\
\mathrm{Mn}-54 \\
\mathrm{Co-58} \\
\mathrm{Co}-60 \\
\mathrm{Sr}-90 \\
\mathrm{Y}-90 \\
\mathrm{Tc}-99 \\
\mathrm{Cs}-134 \\
\mathrm{Cs}-137 \\
\mathrm{Ba}-137 \mathrm{~m} \\
\mathrm{~T}-208 \\
\mathrm{~Pb}-212 \\
\mathrm{Bi}-212 \\
\mathrm{Po-212} \\
\mathrm{Po-216} \\
\mathrm{Ra}-224 \\
\mathrm{Ra}-228 \\
\mathrm{Ac}-228 \\
\mathrm{Th}-228 \\
\mathrm{Th}-231 \\
\mathrm{Th}-232 \\
\mathrm{Th}-234 \\
\mathrm{~Pa}-234 \\
\mathrm{Pa-234m} \\
\mathrm{U}-235 \\
\mathrm{U}-238\end{array}$ & $\begin{array}{c}\begin{array}{c}\text { Activity } \\
(C i / y r)\end{array} \\
1.01 \mathrm{E}-02 \\
5.98 \mathrm{E}-06 \\
6.74 \mathrm{E}-04 \\
6.22 \mathrm{E}-04 \\
1.79 \mathrm{E}-03 \\
8.46 \mathrm{E}-04 \\
8.46 \mathrm{E}-04 \\
1.19 \mathrm{E}-05 \\
1.39 \mathrm{E}-03 \\
1.84 \mathrm{E}-03 \\
1.74 \mathrm{E}-03 \\
1.69 \mathrm{E}-07 \\
4.49 \mathrm{E}-07 \\
4.49 \mathrm{E}-07 \\
2.89 \mathrm{E}-07 \\
4.49 \mathrm{E}-07 \\
4.49 \mathrm{E}-07 \\
2.68 \mathrm{E}-06 \\
2.68 \mathrm{E}-06 \\
4.49 \mathrm{E}-07 \\
2.58 \mathrm{E}-06 \\
2.72 \mathrm{E}-05 \\
3.31 \mathrm{E}-03 \\
3.39 \mathrm{E}-07 \\
3.31 \mathrm{E}-03 \\
2.57 \mathrm{E}-06 \\
3.38 \mathrm{E}-03\end{array}$ & - \\
\hline Shipping Site: $Y-12$ & $\begin{array}{l}\text { Total Volume } \\
\text { (m3/yr) }\end{array}$ & $\begin{array}{cc}7.19 \mathrm{E}+03 & \begin{array}{c}\text { Potal Mass } \\
(\mathrm{kg} / \mathrm{yr})\end{array}\end{array}$ & $4.13 E+07$ \\
\hline & $\begin{array}{l}\text { Radionuclide } \\
-\mathrm{T} 1-208 \\
\mathrm{~Pb}-212 \\
\mathrm{Bi}-212 \\
\mathrm{Po}-212 \\
\mathrm{Po}-216 \\
\mathrm{Ra}-224 \\
\mathrm{Ra}-228 \\
\mathrm{Ac}-228 \\
\mathrm{Th}-228 \\
\mathrm{Th}-231 \\
\mathrm{Th}-232 \\
\mathrm{Th}-234 \\
\mathrm{~Pa}-234 \\
\mathrm{~Pa}-234 \mathrm{~m} \\
\mathrm{U}-235 \\
\mathrm{U}-238\end{array}$ & \begin{tabular}{c}
$\begin{array}{c}\text { Activity } \\
\text { (Ci/yz) }\end{array}$ \\
\hdashline $3.40 \mathrm{E}-05$ \\
$9.00 \mathrm{E}-05$ \\
$9.00 \mathrm{E}-05$ \\
$5.80 \mathrm{E}-05$ \\
$9.00 \mathrm{E}-05$ \\
$9.00 \mathrm{E}-05$ \\
$5.38 \mathrm{E}-04$ \\
$5.38 \mathrm{E}-04$ \\
$9.00 \mathrm{E}-05$ \\
$5.18 \mathrm{E}-04$ \\
$5.46 \mathrm{E}-03$ \\
$6.63 \mathrm{E}-01$ \\
$6.80 \mathrm{E}-05$ \\
$6.63 \mathrm{E}-01$ \\
$5.16 \mathrm{E}-04$ \\
$6.63 \mathrm{E}-01$
\end{tabular} & \\
\hline
\end{tabular}


Destination: SRS

\begin{tabular}{|c|c|c|c|}
\hline Shipping Site: Pinellas & $\begin{array}{l}\text { Total Volume } \\
(\mathrm{m} 3 / \mathrm{yr})\end{array}$ & $\begin{array}{c}\text { Total Mass } \\
(\mathrm{kg} / \mathrm{yr})\end{array}$ & $9.57 E+03$ \\
\hline & Radionuclide & $\begin{array}{l}\text { Activity } \\
\text { (Ci/yr) }\end{array}$ & \\
\hline & $\mathrm{H}-3$ & $9.96 \mathrm{E}+03$ & \\
\hline
\end{tabular}




\section{A.2 WM LLW NO ACTION ALTERNATIVE (CASE 1): ACTIVATED METALS}

Transported Material with Radionuclide Profile

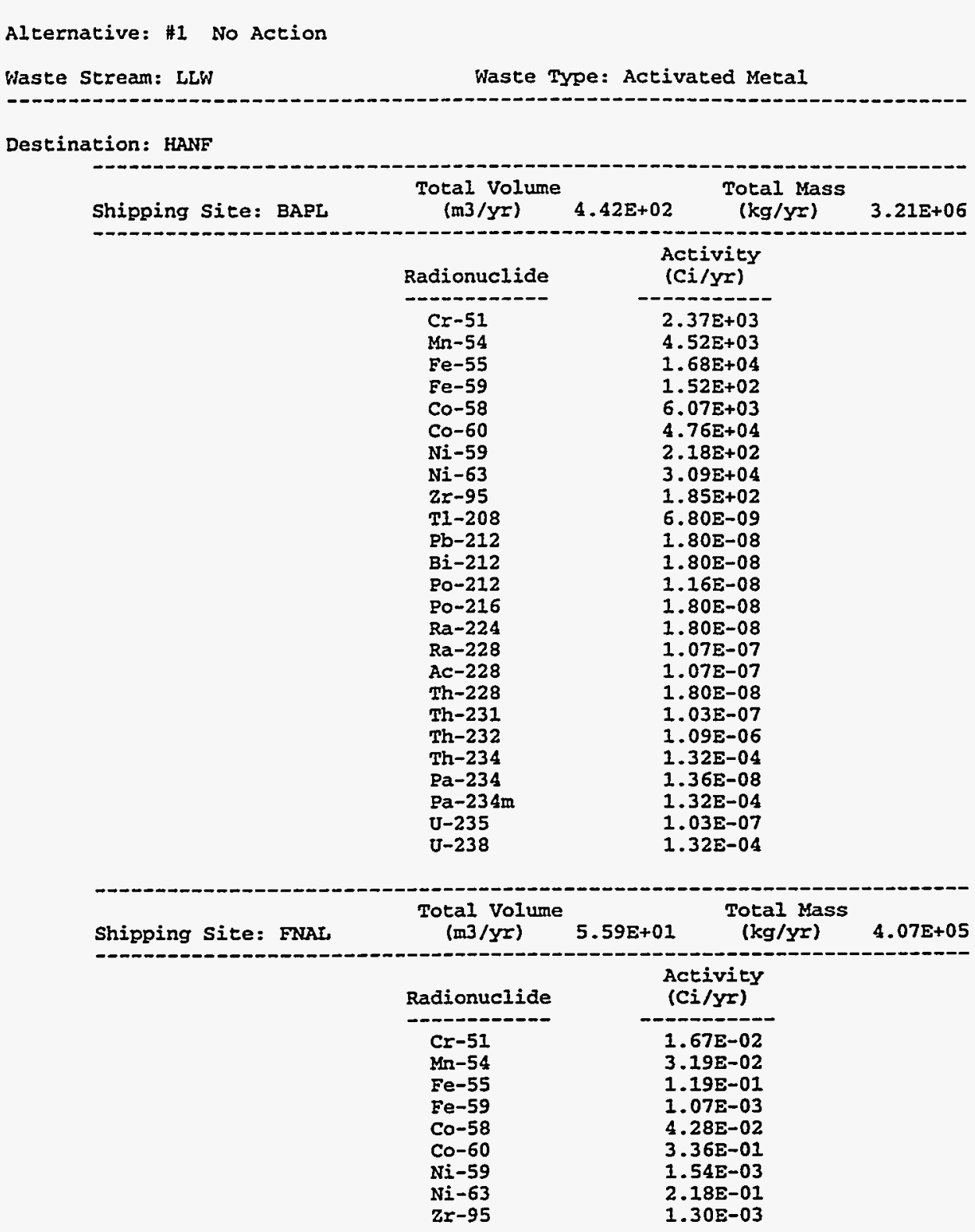




\begin{tabular}{|c|c|c|c|}
\hline Shipping Sice: KAPL & $\begin{array}{l}\text { Total Volume } \\
(\mathrm{m} 3 / \mathrm{Yr})\end{array}$ & $\begin{array}{c}\text { Total Mass } \\
\text { ( } \mathrm{kg} / \mathrm{Yr})\end{array}$ & $6.65 \Sigma+06$ \\
\hline & $\begin{array}{l}\text { Radionuclide } \\
\mathrm{H}-3 \\
\mathrm{C}-14 \\
\mathrm{Cr}-51 \\
\mathrm{Mn}-54 \\
\mathrm{Fe}-55 \\
\mathrm{Fe}-59 \\
\mathrm{Co}-58 \\
\mathrm{Co}-60 \\
\mathrm{Ni}-59 \\
\mathrm{Ni}-63 \\
\mathrm{Sr}-90 \\
\mathrm{Y}-90 \\
\mathrm{Zr}-95 \\
\mathrm{TC}-99 \\
\mathrm{Ru}-106 \\
\mathrm{Rh}-106 \\
\mathrm{Sb}-125 \\
\mathrm{Te}-125 \mathrm{~m} \\
\mathrm{Cs}-134 \\
\mathrm{Cs}-137 \\
\mathrm{Ba}-137 \mathrm{~m} \\
\mathrm{Ce}-144 \\
\mathrm{Pr}-144 \\
\mathrm{Pr}-144 \mathrm{~m} \\
\mathrm{Pm}-147 \\
\mathrm{Sm}-151 \\
\mathrm{Eu}-152 \\
\mathrm{Eu}-154 \\
\mathrm{Eu}-155 \\
\mathrm{~T} \mathrm{H}-208 \\
\mathrm{~Pb}-212 \\
\mathrm{Bi}-212 \\
\mathrm{Po-212} \\
\mathrm{Po-216} \\
\mathrm{Ra}-224 \\
\mathrm{Ra}-228 \\
\mathrm{Ac}-228 \\
\mathrm{Th}-228 \\
\mathrm{Th}-231 \\
\mathrm{Th}-232 \\
\mathrm{Th}-234 \\
\mathrm{~Pa}-234 \\
\mathrm{~Pa}-234 \mathrm{~m} \\
\mathrm{U}-235 \\
\mathrm{U}-238 \\
\mathrm{Pu}-238 \\
\mathrm{Pu}-239 \\
\mathrm{Pu}-240 \\
\mathrm{Pu}-241 \\
\mathrm{Am}-241 \\
\end{array}$ & 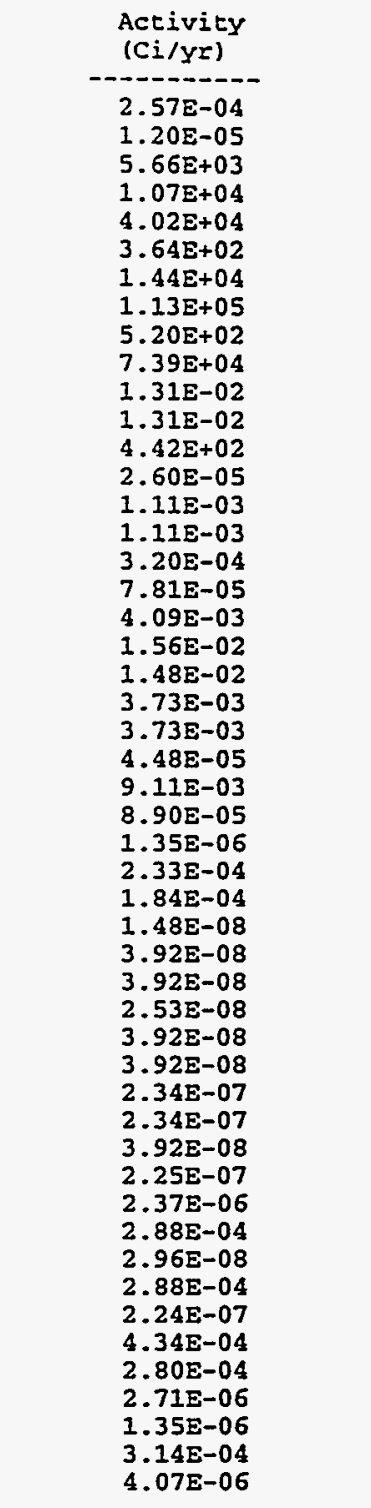 & \\
\hline Shipping Site: IBL & $\begin{array}{l}\text { Total Volume } \\
(\mathrm{m} 3 / \mathrm{y})\end{array}$ & $\begin{array}{cc}\text { Total Mass } \\
(\mathrm{kg} / \mathrm{y})\end{array}$ & $1.43 E+05$ \\
\hline & $\begin{array}{l}\text { Radionuclide } \\
\text { Cr-51 } \\
\text { Mn-54 } \\
\text { Fe-55 } \\
\text { Fe-59 } \\
\text { Co-58 } \\
\text { Co-60 } \\
\mathrm{Ni}-59 \\
\mathrm{Ni}-63 \\
\mathrm{Z}-95\end{array}$ & $\begin{array}{l}\text { Activity } \\
\text { (Ci/yz) } \\
3.16 \mathrm{E}-02 \\
6.02 \mathrm{E}-02 \\
2.24 \mathrm{E}-01 \\
2.03 \mathrm{E}-03 \\
8.08 \mathrm{E}-02 \\
6.33 \mathrm{E}-01 \\
2.90 \mathrm{E}-03 \\
4.12 \mathrm{E}-01 \\
2.46 \mathrm{E}-03\end{array}$ & 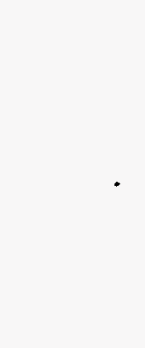 \\
\hline
\end{tabular}


A- 18

\begin{tabular}{|c|c|c|c|}
\hline Shipping Site: NRF & $\begin{array}{c}\text { Total Volume } \\
(\mathrm{m} 3 / \mathrm{yr})\end{array}$ & $\begin{array}{c}\text { Total Mass } \\
(\mathrm{kg} / \mathrm{yr})\end{array}$ & $4.70 E+06$ \\
\hline & $\begin{array}{l}\text { Radionuclide } \\
\mathrm{Cr}-51 \\
\mathrm{Mn}-54 \\
\mathrm{Fe}-55 \\
\mathrm{Fe}-59 \\
\mathrm{Co}-58 \\
\mathrm{Co}-60 \\
\mathrm{Ni}-59 \\
\mathrm{Ni}-63 \\
\mathrm{Z}-95\end{array}$ & $\begin{array}{c}\begin{array}{c}\text { Activity } \\
\text { (Ci/yr) }\end{array} \\
1.15 E+03 \\
2.20 \mathrm{E}+03 \\
8.22 \mathrm{E}+03 \\
7.43 \mathrm{E}+01 \\
2.95 \mathrm{E}+03 \\
2.31 \mathrm{E}+04 \\
1.06 \mathrm{E}+02 \\
1.50 \mathrm{E}+04 \\
9.02 \mathrm{E}+01\end{array}$ & \\
\hline
\end{tabular}


Destination: INEL

\begin{tabular}{|c|c|c|c|}
\hline Shipping Sice: ANL-W & $\begin{array}{l}\text { Total Volume } \\
\text { (m3/yr) }\end{array}$ & $\begin{array}{c}\text { Total Mass } \\
(\mathrm{kg} / \mathrm{yr})\end{array}$ & $8.24 \mathrm{E}+04$ \\
\hline & $\begin{array}{l}\text { Radionuclide } \\
\mathrm{H}-3 \\
\mathrm{Cr}-51 \\
\mathrm{Mn}-54 \\
\mathrm{Fe}-55 \\
\mathrm{Fe}-59 \\
\mathrm{Co}-58 \\
\mathrm{Co}-60 \\
\mathrm{Ni}-59 \\
\mathrm{Ni}-63 \\
\mathrm{Sr}-90 \\
\mathrm{Y}-90 \\
\mathrm{Zr}-95 \\
\mathrm{Tc}-99 \\
\mathrm{Ru}-106 \\
\mathrm{Rh}-106 \\
\mathrm{Sb}-125 \\
\mathrm{Te}-125 \mathrm{~m} \\
\mathrm{Cs}-134 \\
\mathrm{Cs}-137 \\
\mathrm{Ba}-137 \mathrm{~m} \\
\mathrm{Ce}-144 \\
\mathrm{Pr}-144 \\
\mathrm{Pr}-144 \mathrm{~m} \\
\mathrm{Pm}-147 \\
\mathrm{Sm}-151 \\
\mathrm{Eu}-152 \\
\mathrm{Eu}-154 \\
\mathrm{Eu}-155 \\
\mathrm{Pu}-238 \\
\mathrm{Pu}-239 \\
\mathrm{Pu}-240 \\
\mathrm{Pu}-241 \\
\mathrm{Am}-241\end{array}$ & $\begin{array}{c}\text { Activity } \\
\text { (Ci/yr) } \\
8.56 \mathrm{E}-07 \\
6.23 \mathrm{E}-05 \\
1.18 \mathrm{E}-04 \\
4.43 \mathrm{E}-04 \\
4.00 \mathrm{E}-06 \\
1.59 \mathrm{E}-04 \\
1.25 \mathrm{E}-03 \\
5.71 \mathrm{E}-06 \\
8.21 \mathrm{E}-04 \\
7.19 \mathrm{E}-04 \\
7.19 \mathrm{E}-04 \\
4.86 \mathrm{E}-06 \\
1.28 \mathrm{E}-07 \\
7.01 \mathrm{E}-05 \\
7.01 \mathrm{E}-05 \\
2.01 \mathrm{E}-05 \\
4.92 \mathrm{E}-06 \\
8.16 \mathrm{E}-05 \\
1.00 \mathrm{E}-01 \\
1.00 \mathrm{E}-01 \\
2.34 \mathrm{E}-04 \\
2.34 \mathrm{E}-04 \\
2.82 \mathrm{E}-06 \\
5.73 \mathrm{E}-04 \\
5.60 \mathrm{E}-06 \\
8.56 \mathrm{E}-08 \\
1.46 \mathrm{E}-05 \\
1.16 \mathrm{E}-05 \\
1.76 \mathrm{E}-05 \\
1.71 \mathrm{E}-07 \\
8.56 \mathrm{E}-08 \\
1.97 \mathrm{E}-05 \\
2.56 \mathrm{E}-07\end{array}$ & \\
\hline
\end{tabular}

Destination: NTS

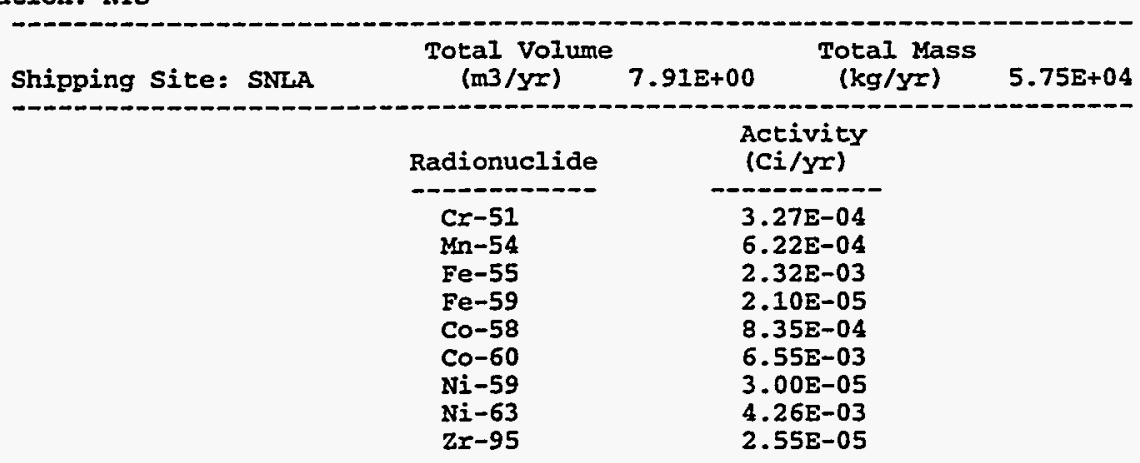

